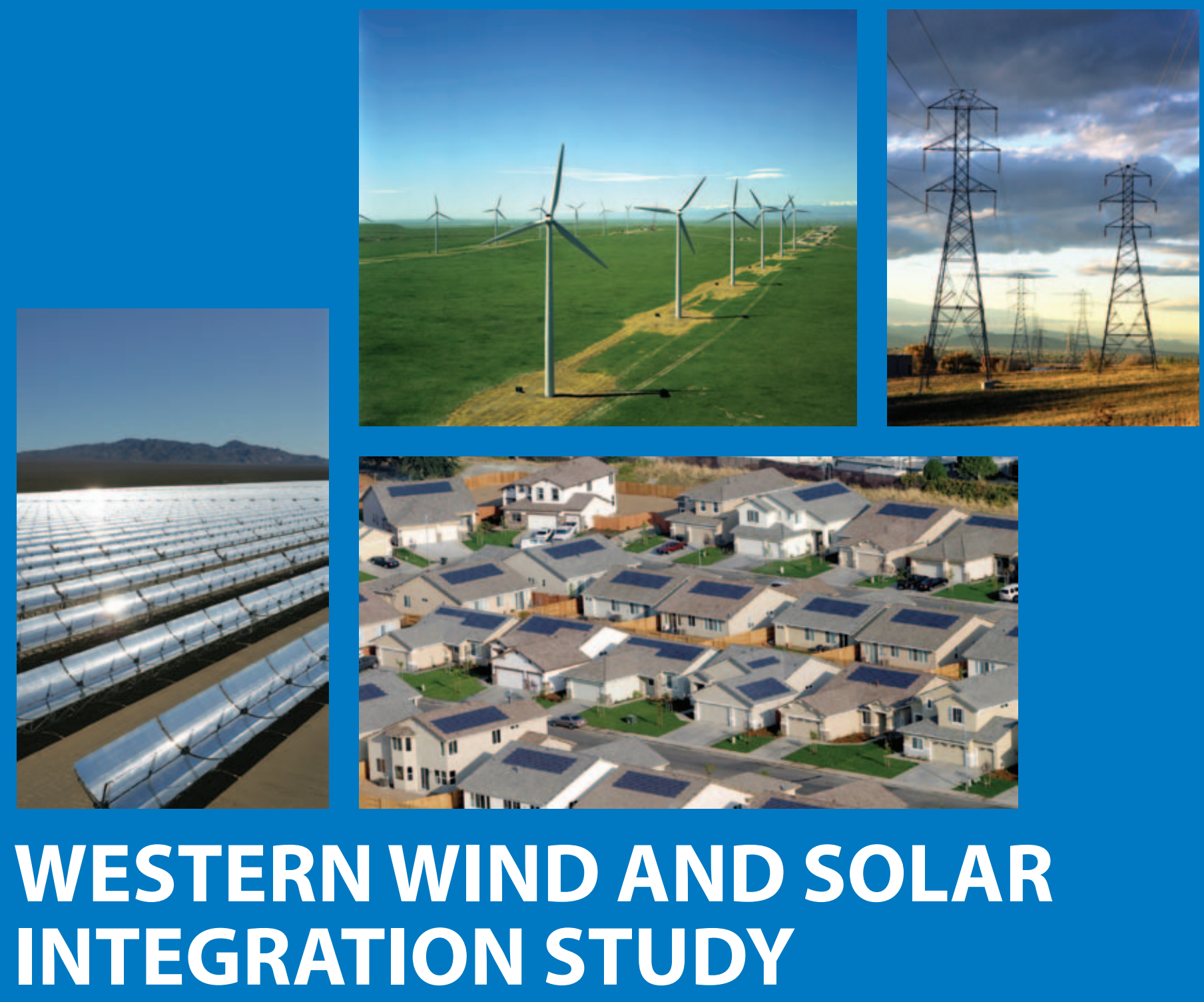

PREPARED FOR:

The National Renewable Energy Laboratory

A national laboratory of the U.S. Department of Energy

PREPARED BY:

GE Energy

MAY 2010 



\title{
WESTERN WIND AND SOLAR INTEGRATION STUDY
}

\author{
May 2010 \\ Prepared for NREL by GE Energy \\ 1 River Road \\ Schenectady, New York 12345 \\ Technical Monitor: Debra Lew \\ Prepared under Subcontract No. AAM-8-77557-01
}

Subcontract Report

NREL/SR-550-47434

National Renewable Energy Laboratory

1617 Cole Boulevard, Golden, Colorado 80401

303-275-3000 • www.nrel.gov

NREL is a national laboratory of the U.S. Department of Energy

Office of Energy Efficiency and Renewable Energy

Operated by the Alliance for Sustainable Energy, LLC

Contract No. DE-AC36-08GO28308

Cover photos (from left to right): PIX \# 15996, 08604, 10927 and 14898 


\section{NOTICE}

This report was prepared as an account of work sponsored by an agency of the United States government. Neither the United States government nor any agency thereof, nor any of their employees, makes any warranty, express or implied, or assumes any legal liability or responsibility for the accuracy, completeness, or usefulness of any information, apparatus, product, or process disclosed, or represents that its use would not infringe privately owned rights. Reference herein to any specific commercial product, process, or service by trade name, trademark, manufacturer, or otherwise does not necessarily constitute or imply its endorsement, recommendation, or favoring by the United States government or any agency thereof. The views and opinions of authors expressed herein do not necessarily state or reflect those of the United States government or any agency thereof.

Available electronically at http: / / www.osti.gov/ bridge

Available for a processing fee to U.S. Department of Energy and its contractors, in paper, from:

U.S. Department of Energy

Office of Scientific and Technical Information

P.O. Box 62

Oak Ridge, TN 37831-0062

phone: 865.576 .8401

fax: 865.576 .5728

email: mailto:reports@adonis.osti.gov

Available for sale to the public, in paper, from:

U.S. Department of Commerce

National Technical Information Service

5285 Port Royal Road

Springfield, VA 22161

phone: 800.553 .6847

fax: 703.605 .6900

email: orders@ntis.fedworld.gov

online ordering: http:/ / www.ntis.gov/ ordering.htm

Printed on paper containing at least 50\% wastepaper, including $20 \%$ postconsumer waste 


\section{Table of Contents}

List of Figures......................................................................................................................................... vii

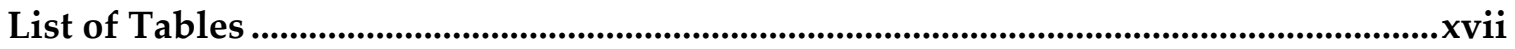

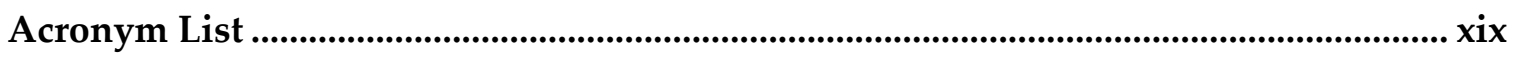

Acknowledgments...............................................................................................................................

Executive Summary ................................................................................................................. ES-1

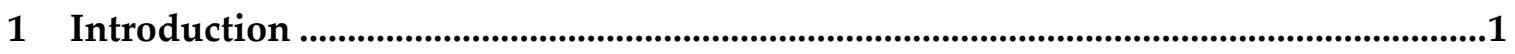

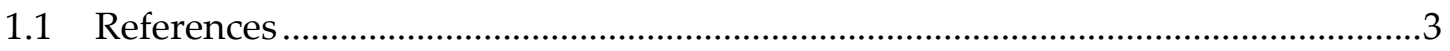

2 Objectives and Technical Approach .....................................................................................5

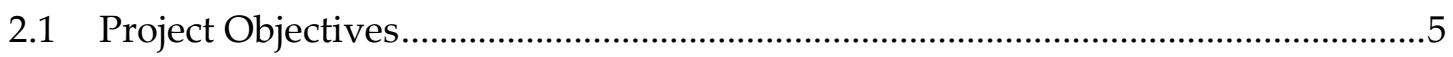

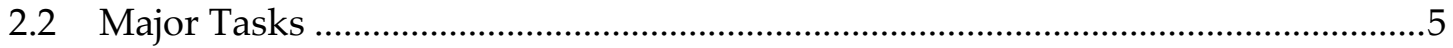

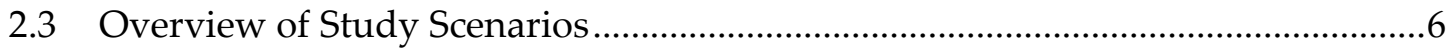

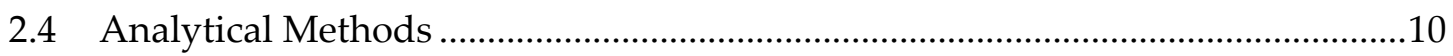

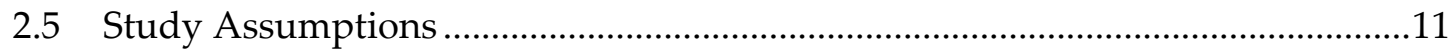

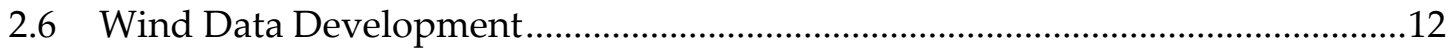

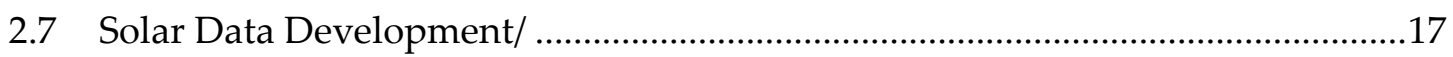

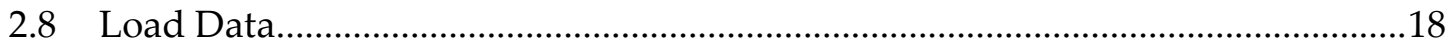

2.9 What This Study Does and Does Not Cover ........................................................19

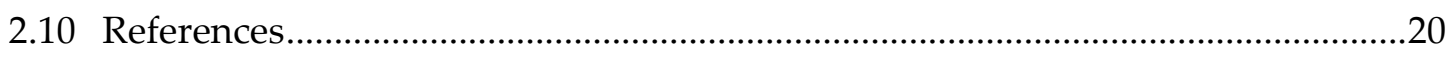

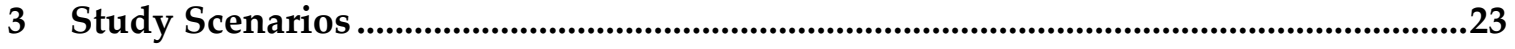

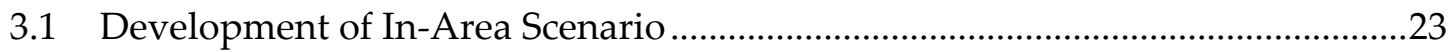

3.1.1 Objectives of the Site Selection Process ........................................................23

3.1.2 Available Wind and Solar Sites ..................................................................24

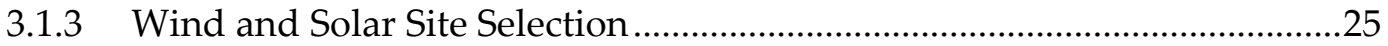

3.1.4 Transmission Zones and Areas .................................................................28

3.2 Development of Mega-Project Scenario …................................................................

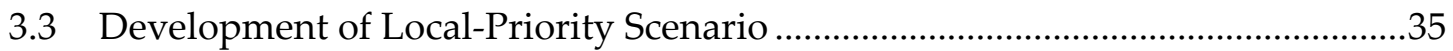

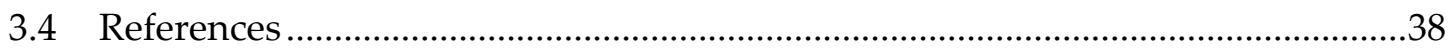

4 Statistical Analysis: Seasonal, Monthly, and Daily Trends.........................................39

4.1 Monthly Energy from Wind and Solar ..............................................................40 
4.1.1 Year-to-Year Comparison .............................................................................

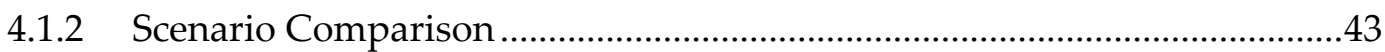

4.1.3 State-by-State Comparison ......................................................................... 44

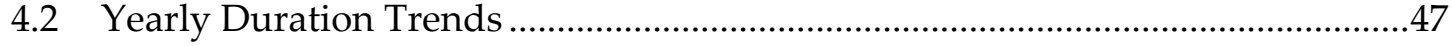

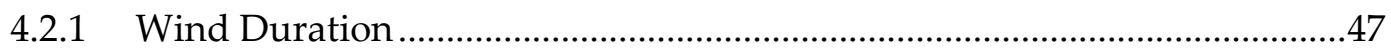

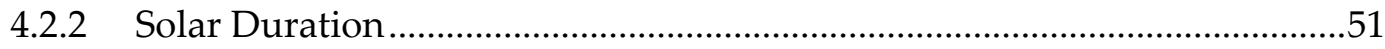

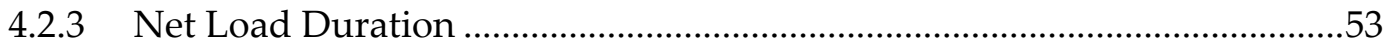

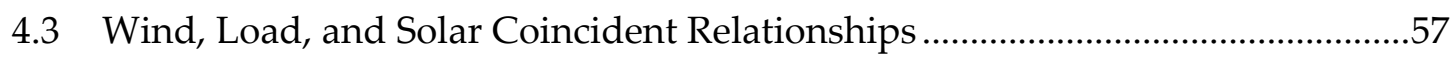

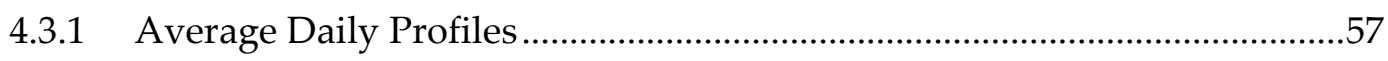

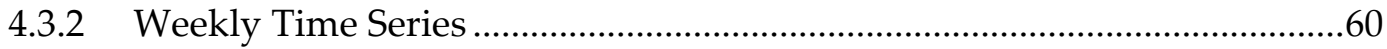

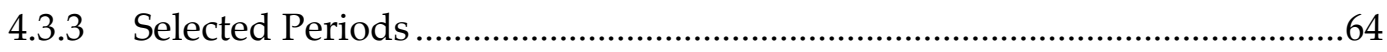

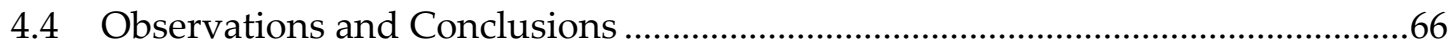

5 Statistical Analysis: Hourly Variability and Day-Ahead Predictability ...................69

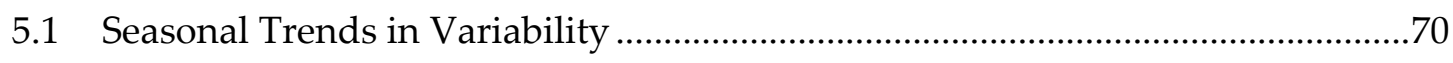

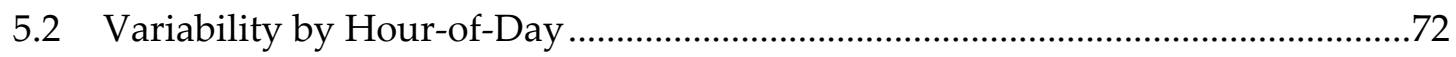

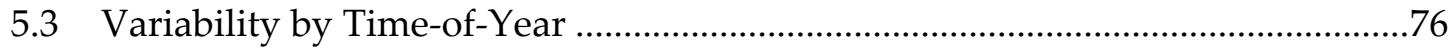

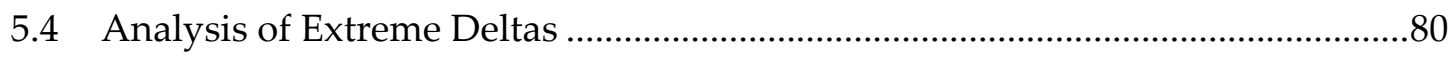

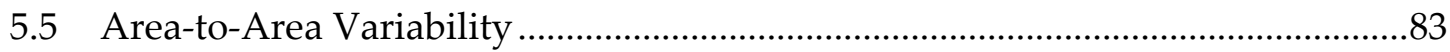

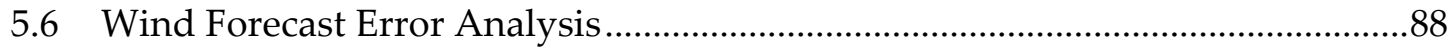

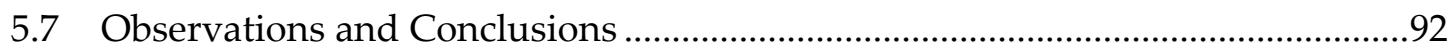

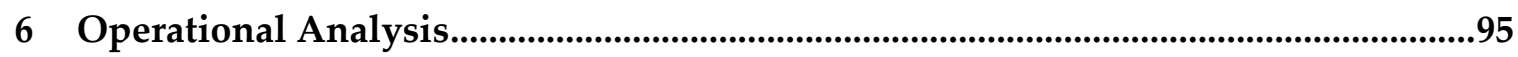

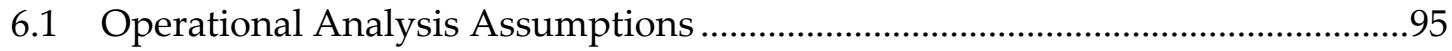

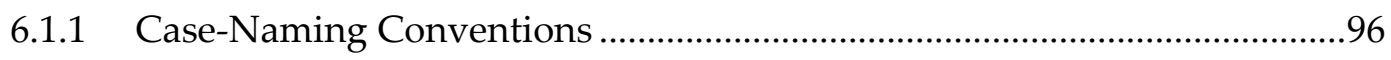

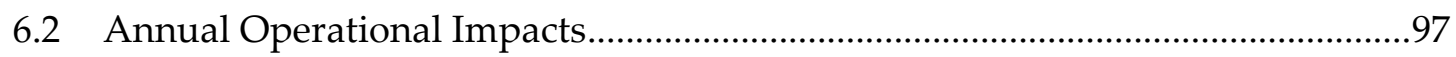

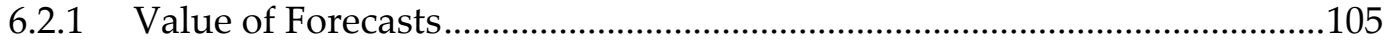

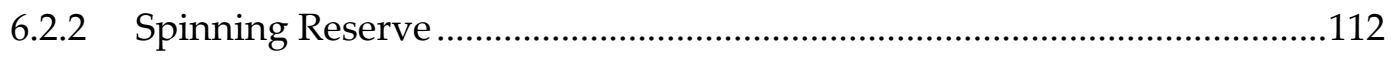

6.2.3 Annual Profile Sensitivities..................................................................118

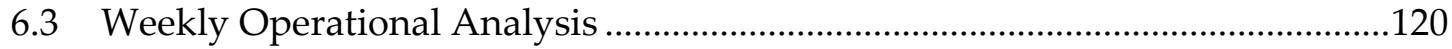

6.4 Additional Operational Sensitivities................................................................ 130

6.4.1 Variations in Renewable Unit Siting..........................................................130

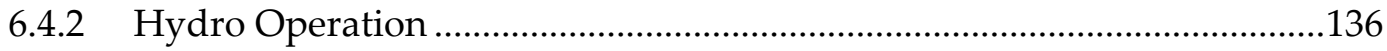




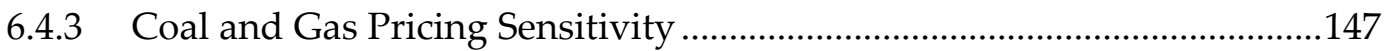

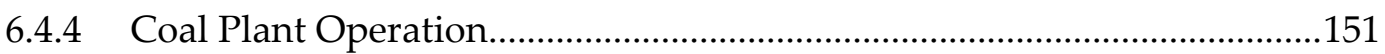

6.4.5 Balancing Area Consolidation .................................................................158

6.4.6 Transmission Expansion Sensitivity …........................................................159

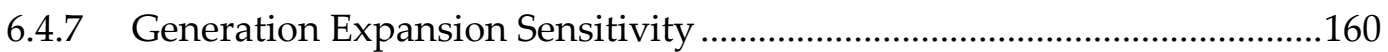

6.5 Operational Analysis Observations and Conclusions.........................................162

7 Sub-hourly Operational Analysis.......................................................................................165

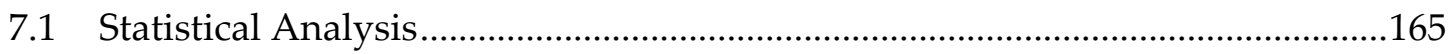

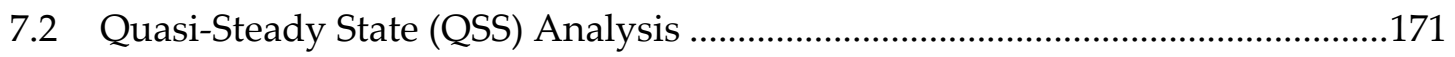

7.2.1 Overview of Algorithm ........................................................................... 171

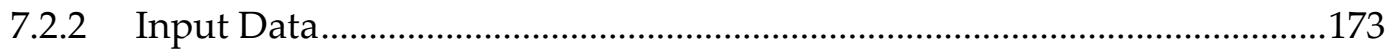

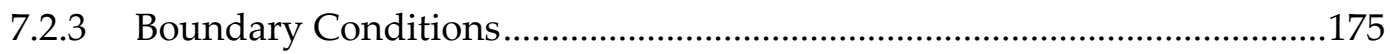

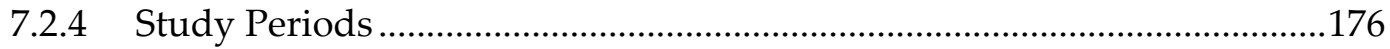

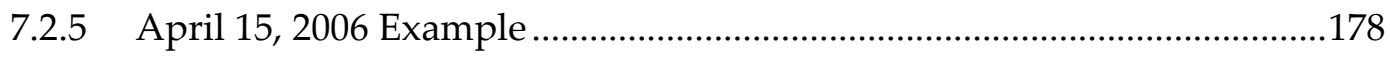

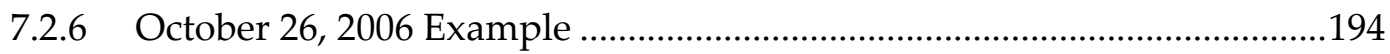

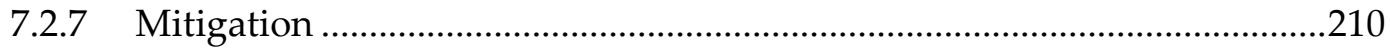

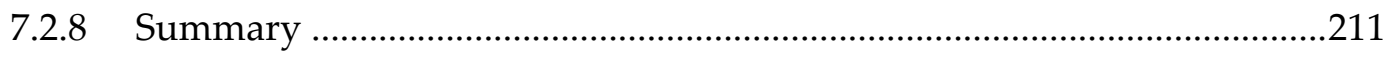

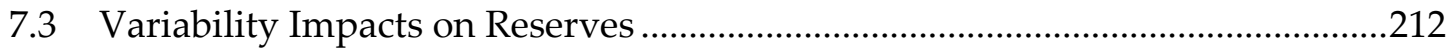

7.3.1 Existing Reserve Rules and Practices ........................................................212

7.3.2 Load and Net Load Variability ...................................................................214

7.3.3 Relationship to Existing Reserve Rules ....................................................219

7.3.4 Net Load Variability Relationship to Load and Wind Levels...................221

7.3.5 Reserve Time Frames and Other Reserve Drivers ......................................234

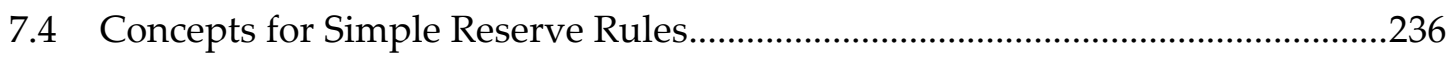

7.4.1 Load and Wind Level Based Rules .............................................................237

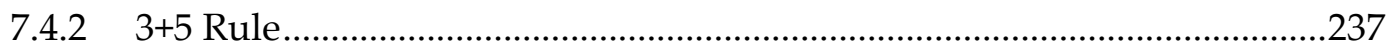

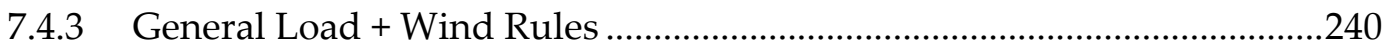

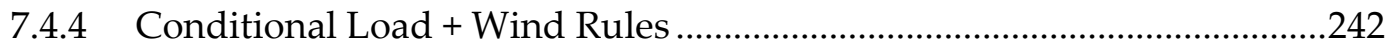

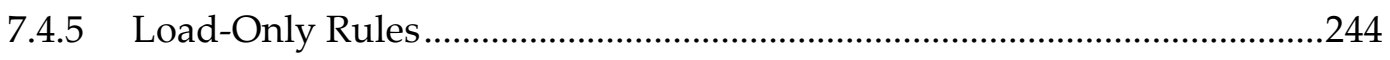

7.4.6 Summary and Trends for Simplified Reserve Rules ................................247

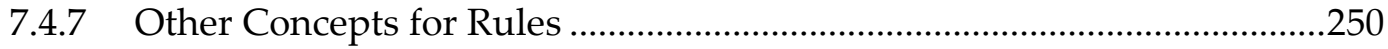


7.5 Reserve Performance. .253

7.5.1 Hourly Reserves at Dispatch ....................................................................253

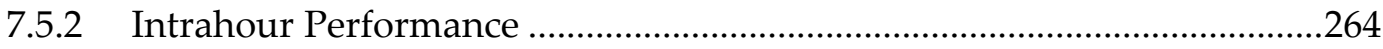

7.5.3 Non-Spinning Reserve Performance ………............................................220

7.5.4 Demand Participation in Reserves ............................................................22

7.5.5 Energy Storage Contribution to Reserves ................................................2.

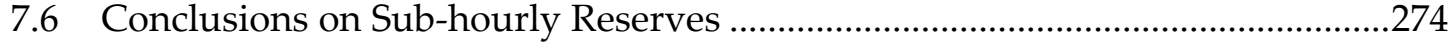

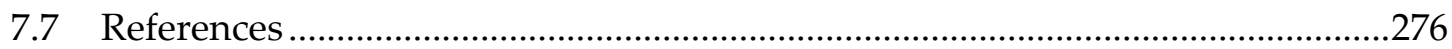

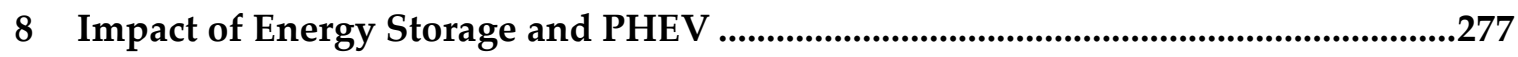

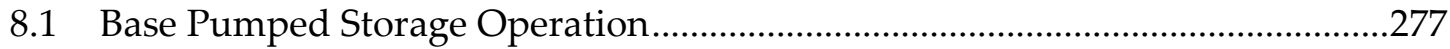

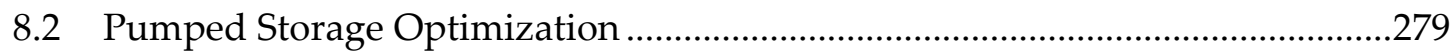

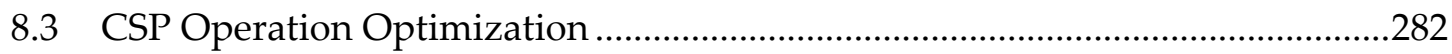

8.4 Plug-in Hybrid Electric Vehicles, PHEV ..........................................................28

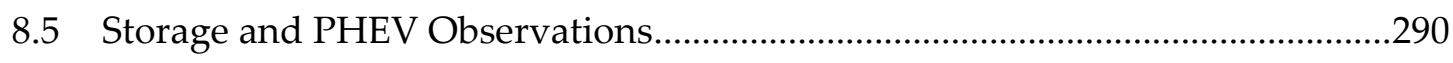

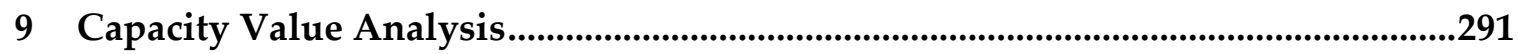

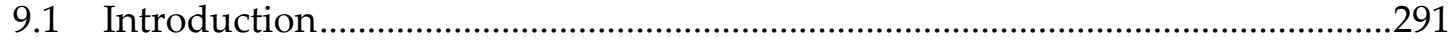

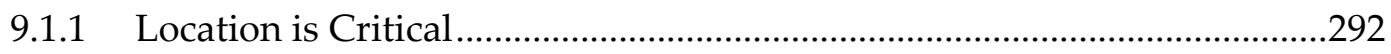

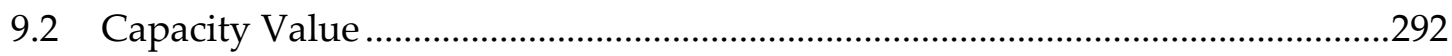

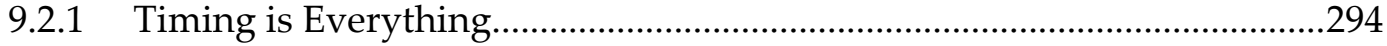

9.2.2 Hourly and Unserved Energy Measures....................................................296

9.3 Capacity Value Variation by Scenario ..................................................................298

9.4 Capacity Value Variation by Shape Year ...............................................................300

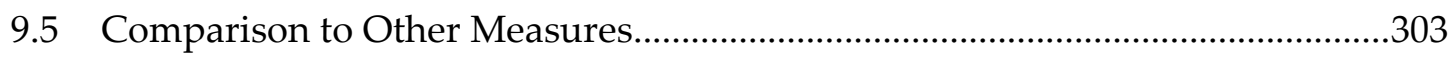

9.5.1 ELCC in a Multi-Area Environment ..........................................................304

9.6 Yet Another Calculation Method ………..................................................................304

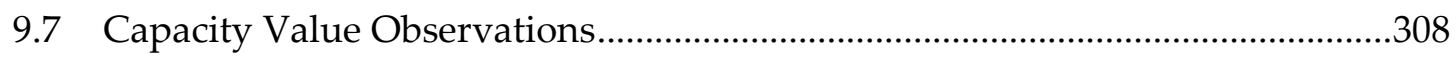

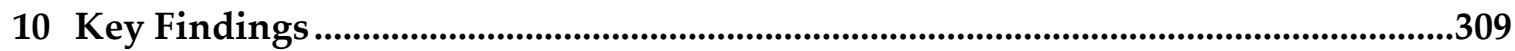

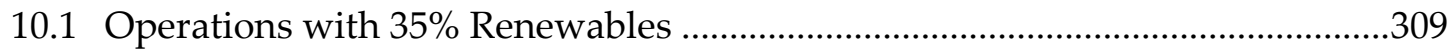

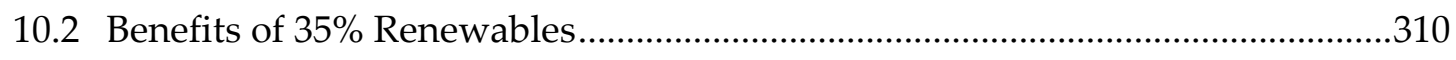

10.3 What Changes are Needed to Integrate 35\% Renewables? .................................310

10.3.1 Balancing Area Cooperation is Essential ....................................................310 
10.3.2 Sub-Hourly Scheduling is Critical ..... 311

10.4 What is the Impact of Uncertainty and Variability?...............................................

10.4.1 Using Forecasts Results in the Biggest Impact on the System ...................312

10.4.2 Extreme Forecast Errors and Contingency Reserve Shortfalls...................312

10.4.3 How Often is Wind Curtailed? .....................................................................313

10.4.4 Are Additional Reserves Necessary to Cover Variability?.........................313

10.5 What are the Major Differences Between the Scenarios?.....................................313

10.5.1 Is New Long Distance Transmission Needed? .........................................314

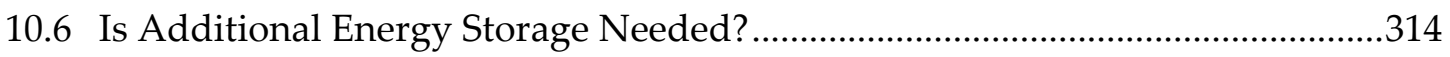

10.7 What is the Benefit of Flexibility in the Generation Fleet? ..................................315

10.7.1 Load Following by Hydro Plants is Helpful ............................................315

10.7.2 Constraints on Coal Plants Result in Higher Operating Costs ..................315

10.8 How do Renewables Contribute to Resource Adequacy?...................................316

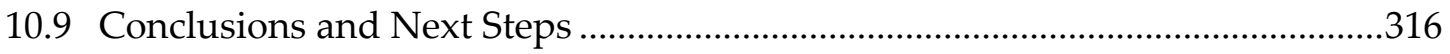

Appendix A. Tables of Wind+Solar MW by State; Siting Algorithm Overview.. A-1

Appendix B. One-hour Statistical Trend Analysis Plots ......................... B-1

Appendix C. One-hour Statistical Delta Analysis Plots.......................... C-1

Appendix D. MAPS Flow-Duration............................................ D

Appendix E. Ten-minute Statistical Analysis Plots............................. E-1

Appendix F. Quasi Steady State Plots.......................................... F-1

Appendix G. Reserves......................................................... G-1 



\section{List of Figures}

Figure 1.1 Geographic Footprint of WestConnect Utilities ............................................................2

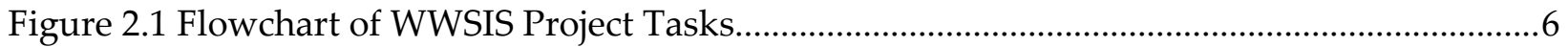

Figure 2.2 Statistical Analysis Showing Data Seam at Hour 16 of Every Third Day .....................13

Figure 2.3 Annual Wind Energy Forecast Error by Area ...............................................................14

Figure 2.4 Monthly Wind Forecast Error in WECC ....................................................................14

Figure 2.5 Examples from Wind Dataset Validation Analysis - Meso-Scale Model Results Versus

Actual Texas Wind Plant Output (top) And Actual WestConnect Wind Plant Output

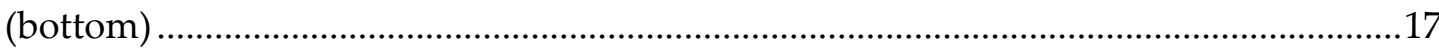

Figure 3.1 All Available Wind Sites in WECC (Each Grey Dot Represents $30 \mathrm{MW}$ )...................24

Figure 3.2 All Available Solar Sites in WECC (Each Grey Dot Represents $100 \mathrm{MW}$ )..................25

Figure 3.3 Overview of In-Area Scenario with 30\% Wind Energy and 5\% Solar Energy ..............27

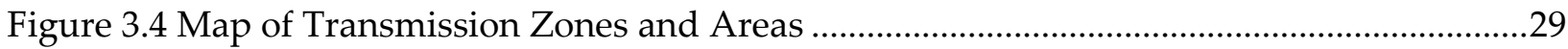

Figure 3.5 WECC Transmission Paths and Ratings (in MW) .........................................................31

Figure 3.6 Map of Mega-Project Scenario with Transmission Additions ......................................34

Figure 3.7 Map of Local-Priority Scenario with Transmission Additions ......................................36

Figure 4.1 Graphical Representation of Statistical Study Permutations and Most Often Used

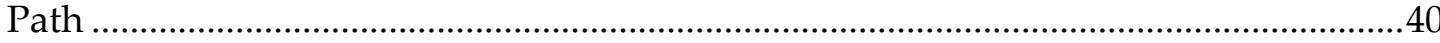

Figure 4.2 Total In-Footprint Monthly Energy from Wind and Solar for 2004 - 2006 (30\% In-Area

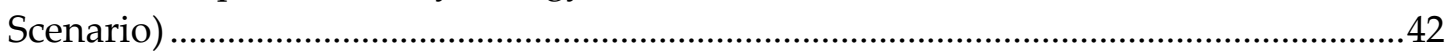

Figure 4.3 Total In-Footprint Monthly Energy from Wind and Solar for 2004 - 2006 (30\% In-Area

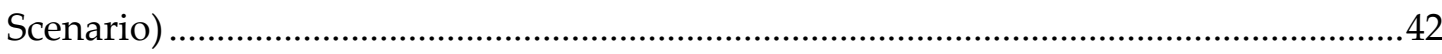

Figure 4.4 2006 Total In-Footprint Monthly Energy from Wind and Solar for All Scenarios (30\%

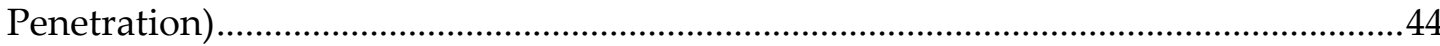

Figure 4.5 2006 Percent In-Footprint Monthly Energy from Wind and Solar for All Scenarios

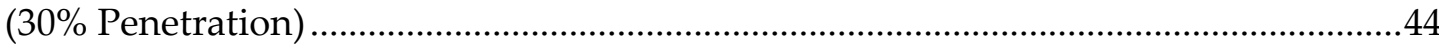

Figure 4.6 2006 Arizona Total and Percent Monthly Energy from Wind and Solar (30\% Local-

Priority Scenario)

Figure 4.7 2006 Wyoming Total and Percent Monthly Energy from Wind and Solar (30\% Local-

Priority Scenario) .45

Figure 4.8 2006 Study Footprint Wind Duration Plots for All Three Scenarios (30\% Penetration)47 Figure 4.9 2006 Study Footprint Wind Duration Plots Normalized as \% of Nameplate Wind.....48 Figure 4.10 2006 Study Footprint Wind Duration Plots for All Three Penetration Levels (Local-

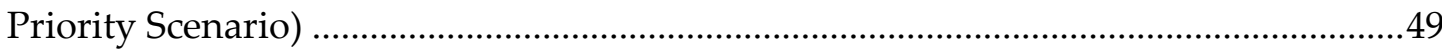

Figure 4.11 2006 Study Footprint Wind Duration Plots Normalized as \% of Nameplate Wind...49 Figure 4.122006 In-Footprint Area Wind Duration Plots for the 30\% Local-Priority Scenario ....50 Figure 4.132006 In-Footprint Area Wind Duration Plots Normalized as \% of Nameplate Wind50 Figure 4.14 2006 Study Footprint CSP Duration and Penetration for the 30\% Local-Priority

Scenario .51

Figure 4.15 2006 Study Footprint PV Duration and Penetration for the 30\% Local-Priority

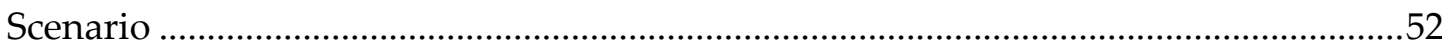

Figure 4.16 Study Footprint 2006 Net Load Duration for the In-Area Scenario.............................53 
Figure 4.17 Study Footprint 2006 Net Load Duration for All Scenarios (30\% Penetration) ..........54

Figure 4.18 Arizona 2006 Net Load Duration for the In-Area Scenario .........................................55

Figure 4.19 New Mexico 2006 Net Load Duration for the Local-Priority Scenario.........................56

Figure 4.20 Wyoming 2006 Net Load Duration for the Mega-Project Scenario.............................56

Figure 4.21 2006 Study Footprint Load, Wind, and Solar Average Daily Profiles by Seasonal

Month for the 30\% Local-Priority Scenario .....................................................................58

Figure 4.22 Arizona Load, Wind, and Solar Average Daily Profiles for January and July, 30\% LP

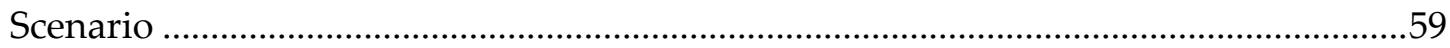

Figure 4.23 Nevada Load, Wind, and Solar Average Daily Profiles for January and July, 30\% LP

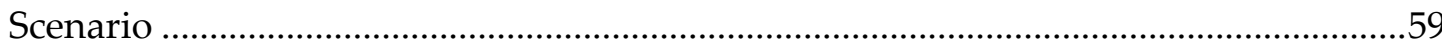

Figure 4.24 Wyoming Load, Wind, and Solar Average Daily Profiles for January and July, 30\%

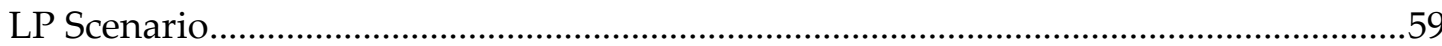

Figure 4.25 Study Footprint Total Load, Wind, and Solar for a Week in July 2006 30\% Local-

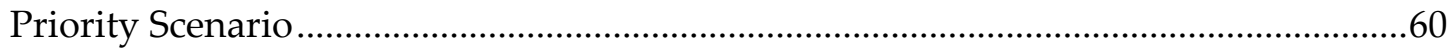

Figure 4.26 Study Footprint Total Load, Net Load for a Week in July 2006 30\% Local-Priority

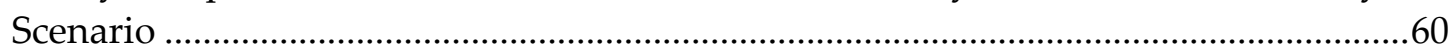

Figure 4.27 Study Footprint Net Load for a Week in July 2006 for 10\%, 20\% and 30\% Local-

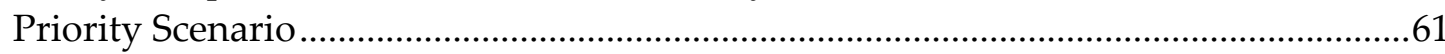

Figure 4.28 Study Footprint Total Load, Wind, and Solar for a Week in April 2006 30\% Local-

Priority Scenario.

Figure 4.29 Study Footprint Total Load, Net Load for a Week in April 2006 30\% Local-Priority

Scenario

Figure 4.30 Study Footprint Net Load for a Week in April 2006 for 10\%, 20\%, and 30\% Local-

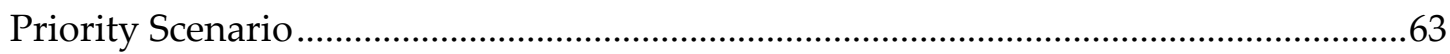

Figure 4.31 Study Footprint Net Load for November $14^{\text {th }}$, 2006, 30\% Local-Priority Scenario .....64

Figure 4.32 Arizona Net Load for November $14^{\text {th }}, 2006,30 \%$ Local-Priority Scenario ...................64

Figure 4.33 Study Footprint Net Load for June $6^{\text {th }}, 2006,30 \%$ Local-Priority Scenario ..................65

Figure 4.34 Colorado-East Footprint Net Load for June $6^{\text {th }}, 2006$, 30\% Local-Priority Scenario ...65

Figure 4.35 Study Footprint Total Load, Net Load for April 2006, 30\% Local-Priority Scenario..66

Figure 5.1 Relationship Between Standard Deviation and Area Under the Standard Normal

Curve.....

Figure 5.2 2006 Study Footprint Wind Deltas vs. Load Deltas by Season for 30\% Local-Priority

Scenario

Figure 5.3 2006 Study Footprint Wind Deltas vs. Load Deltas by Scenario for 30\% Penetration .71

Figure 5.4 Study Footprint Average Daily Profile of Deltas Over Year 2006, 10\% Local-Priority

Scenario

Figure 5.5 Study Footprint Average Daily Profile of Deltas Over Year 2006, 20\% Local-Priority

Scenario

Figure 5.6 Study Footprint Average Daily Profile of Deltas Over Year 2006, 30\% Local-Priority

Scenario

Figure 5.7 Study Footprint Average Daily Profile of Deltas Over April 2006, 30\% Local-Priority

Scenario 
Figure 5.8 Average Daily Profile of Load and Net Load Deltas Over 2006 for Selected Areas, $30 \%$ Local-Priority Scenario.

Figure 5.9 Study Footprint and Wyoming Net Load for January $8^{\text {th }}$, 2006, 30\% Local-Priority Scenario .76

Figure 5.10 Timing of Extreme Load-Alone Positive Deltas in Study Footprint for 2006.............77

Figure 5.11 Timing of Extreme Net Load Positive Deltas in Study Footprint for 2006, 10\% LP

Scenario .78

Figure 5.12 Timing of Extreme Net Load Positive Deltas in Study Footprint for 2006, 20\% LP

Scenario .78

Figure 5.13 Timing of Extreme Net Load Positive Deltas in Study Footprint for 2006, 30\% LP

Scenario .78

Figure 5.14 Timing of Extreme Net Load Negative Deltas in Study Footprint for 2006 Local-

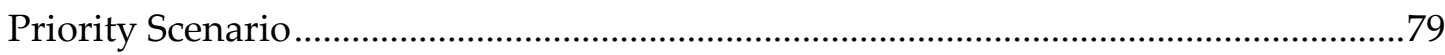

Figure 5.15 2006 Study Area (SA) or Footprint Distribution of Net Load Deltas for Local-Priority

Scenario).

Figure 5.16 Study Area (SA) or Footprint Distribution of Extreme Hourly Net Load Deltas 2006

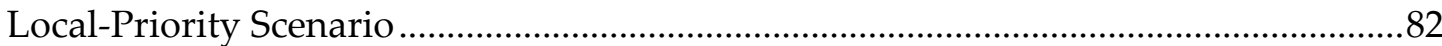

Figure 5.17 Percent Increase in Net Load Delta Sigma in All Areas for In-Area Scenario. .83

Figure 5.18 Percent Increase in Net Load Negative and Positive Deltas in All Areas for In-Area

Scenario

Figure 5.19 2006 Duration of Extreme Hourly Net Load Deltas in All Areas for 30\% Wind Local-

Priority Scenario

Figure 5.20 Distribution of Footprint Day-Ahead Wind Forecast Errors for 2006 10\%, 20\%, 30\%

LP Scenario. .88

Figure 5.21 Scatter Plot of Wind Forecast Errors vs. Load for Study Footprint 30\% LP Scenario 89 Figure 5.22 Scatter Plot of Wind Forecast Errors vs. Wind and Net Load for Study Footprint 30\%

LP Scenario .90

Figure 5.23 Duration of Day-Ahead Wind Forecast Errors for Areas and Total Footprint, 2006 $30 \%$ LP Scenario .91

Figure 5.24 Study Footprint Next Day Wind Forecast Errors vs. Forecast, 30\% Local-Priority Scenario .92

Figure 6.1 Annual WECC Spot Price Duration Curve, Perfect Forecast, In-Area Scenarios .........98

Figure 6.2 Annual WECC Spot Price Duration Curve, S-o-A Forecast, In-Area Scenarios ...........99

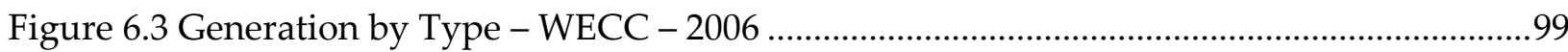

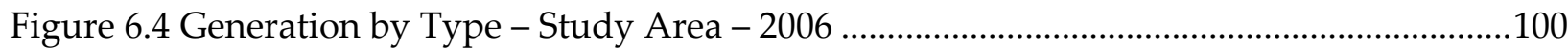

Figure 6.5 Total WECC Operating Costs (\$B) - 2006...................................................................100

Figure 6.6 Operating Cost Savings (\$B) - WECC - 2006 .............................................................101

Figure 6.7 Operating Cost Savings Per MWh of Renewable Energy, WECC - 2006 ...................101

Figure 6.8 Incremental Savings per Incremental Renewable Energy (\$/MWh). WECC - 2006...102

Figure 6.9 Average Hourly Exports From Study Footprint.........................................................102

Figure 6.10 Impact of Renewables in Neighboring Areas, all of WECC ......................................103

Figure 6.11 Coal Plant Statistics, Local-Priority Scenario ...........................................................104

Figure 6.12 Combined-Cycle Plant Statistics, Local-Priority Scenario..........................................104 


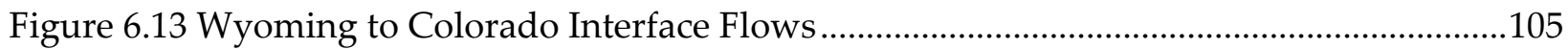

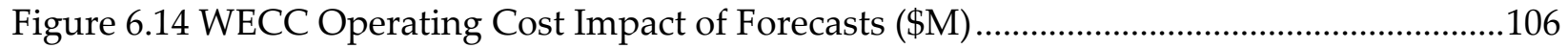

Figure 6.15 Operating Cost Impact of Forecast (\$/MWh) ..........................................................106

Figure 6.16 Impact of Forecast on Curtailed Energy and Contingency Reserve Shortfalls .........107

Figure 6.17 WECC Operating Cost Impact of No Forecasts versus a S-o-A Forecast $(\$ \mathrm{M})$.........108

Figure 6.18 WECC Operating Cost Impact of No Forecasts versus a S-o-A Forecast (\$/MWh)..108

Figure 6.19 WECC Impact of No Forecast on Spilled and Unserved Energy ..............................109

Figure 6.20 Total Generation, Study Area - 2006 .......................................................................109

Figure 6.21 Generator Revenue, Study Area - 2006 ........................................................110

Figure 6.22 Incremental Value of Renewable Energy Based on Spot Price Revenue, Study Area111 Figure 6.23 Incremental Value of Renewable Energy Based on Spot Price Revenue, WECC .....111

Figure 6.24 Total Emission Reductions, WECC - 2006..........................................................112

Figure 6.25 Total Emission Reductions Per MWh of Renewable Generation, WECC - 2006 ......112

Figure 6.26 Adjusted Wind Forecast Error, I30R .........................................................................113

Figure 6.27 Curtailed Energy and Contingency Reserve Shortfall When Discounting Forecast 114 Figure 6.28 Distribution of Contingency Reserve Shortfall vs. Discounting of Wind Forecasts.114 Figure 6.29 Hours of Interruption vs. MW of Demand Response Participation ..........................115

Figure 6.30 Annual Spot Price Duration Curve - I30R - Impact of Discounting Forecast ..........115

Figure 6.31 Total WECC Operating Costs as a Function of Discounting the Wind Forecast (\$M)116

Figure 6.32 Reduced Unserved Energy with Increased Reserves Committed for Wind

Generation. 116

Figure 6.33 Cost of Reducing Contingency Reserve Shortfall by Increasing Reserve Commitment

for Renewables. .117

Figure 6.34 Cost of Increasing Committed Spinning Reserve Per MWh of Wind Generation (\$/MWh) .118

Figure 6.35 Generation by Type, WECC, by Case and Shape Year ..............................................118

Figure 6.36 Variation in Renewable Generation by Weather Year for 30\% Case .........................119

Figure 6.37 Incremental Savings Per Incremental Renewable Energy, WECC.............................119

Figure 6.38 Total Generation, Study Area, for Different Annual Shapes .....................................120

Figure 6.39 Emission Reductions, Study Area, for Different Annual Shapes ..............................120

Figure 6.40 Week of April 10th - Hourly Load, Renewables and Exports for 10\% Case.............121

Figure 6.41 Study Area Dispatch - Week of April 10th - No Renewables...................................121

Figure 6.42 Study Area Dispatch - Week of April 10th - 10\% R Case ............................................122

Figure 6.43 Study Area - Week of April 10th - Load and Renewables by Penetration ...............123

Figure 6.44 Study Area - Week of April 10th - Net Load by Penetration.....................................123

Figure 6.45 Study Area - Week of April 10th - Net Loads Plus Exports by Penetration.............124

Figure 6.46 Study Area Dispatch - Week of April 10th - 20\% R Case ...........................................124

Figure 6.47 Study Area Dispatch - Week of April 10th - 30\% R Case ...........................................125

Figure 6.48 Change in Operation - Week of April 10 th .................................................................125

Figure 6.49 Study Area - Week of July 10th - Renewables by Penetration .................................126

Figure 6.50 Study Area - Week of April 10th - Net Load by Penetration......................................126

Figure 6.51 Study Area - Week of April 10th - Net Load Plus Exports by Penetration ..............127

Figure 6.52 Study Area Dispatch - Week of July 10th - No Wind ..............................................127 
Figure 6.53 Study Area Dispatch - Week of July 10th - 10\% R Case ...........................................128

Figure 6.54 Study Area Dispatch - Week of July 10th - 20\% R Case ............................................128

Figure 6.55 Study Area Dispatch - Week of July 10th - 30\% R Case ............................................129

Figure 6.56 Change in Operation - Week of July 10th ............................................................129

Figure 6.57 Generation by Type, Study Area, Multiple Scenarios ................................................130

Figure 6.58 Annual Spot Price Duration Curve - S-o-A Forecast, In-Area Scenario ....................131

Figure 6.59 Annual Spot Price Duration Curve - S-o-A Forecast, Local-Priority Scenario ..........131

Figure 6.60 Annual Spot Price Duration Curve - S-o-A Forecast, Mega-Project Scenario...........132

Figure 6.61 Annual Spot Price Duration Curve, Perfect Forecast, 30\% Scenarios........................132

Figure 6.62 Annual Spot Price Duration Curve, S-o-A Forecast, 30\% Scenarios..........................133

Figure 6.63 Total Generation, Study Area, Multiple Scenarios....................................................133

Figure 6.64 Operating Cost Savings Per MWh of Renewable Energy, WECC .............................134

Figure 6.65 Incremental Savings Per Incremental Renewable Energy, WECC.............................134

Figure 6.66 Incremental Savings Per Incremental Renewable Energy, WECC, In-Area Scenario135

Figure 6.67 Incremental Savings Per Incremental Renewable Energy, WECC, Local-Priority

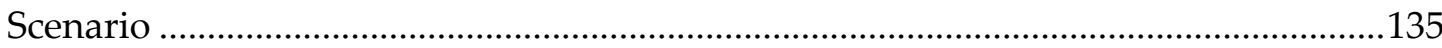

Figure 6.68 Incremental Savings Per Incremental Renewable Energy, WECC, Mega-Project

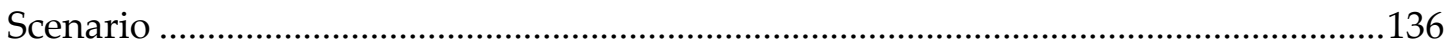

Figure 6.69 Hydro Operation - Week of April 10 th .....................................................................137

Figure 6.70 Hydro Operation - Week of July 10th..........................................................................137

Figure 6.71 Annual Hydro Generation Duration Curve, WECC....................................................138

Figure 6.72 Annual Hydro Hourly Delta Duration Curve, WECC …............................................138

Figure 6.73 Spot Price Impact of Hydro Operation ........................................................................139

Figure 6.74 Operating Cost Increase for Dispatching Hydro on Load Alone vs. Net Load, WECC140

Figure 6.75 Glen Canyon Capacity vs. Elevation ......................................................................141

Figure 6.76 Historical Elevation of Lake Powell .......................................................................... 141

Figure 6.77 Net Monthly Generation at Glen Canyon ..............................................................142

Figure 6.78 MAPS Daily Simulation of Glen Canyon with No Wind ...........................................142

Figure 6.79 MAPS Monthly Simulation of Glen Canyon with No Wind .....................................143

Figure 6.80 Historical Maximum Operation of Glen Canyon ........................................................143

Figure 6.81 Historical Maximum and Average Operation of Glen Canyon ................................144

Figure 6.82 Impact of Flat Hydro Operation on WECC Operating Costs .....................................145

Figure 6.83 Incremental Operating Cost Impact of Flat Hydro Operation at Various Wind

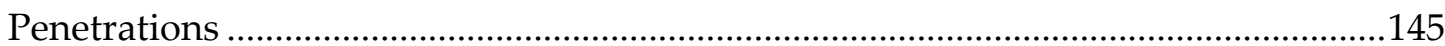

Figure 6.84 Spot Price Impact of Flat Hydro Operation................................................................146

Figure 6.85 Spot Price Impact of Flat Hydro Operation by Penetration........................................146

Figure 6.86 Historical Henry Hub Natural Gas Prices ................................................................147

Figure 6.87 Coal vs. Combined-Cycle Cost Comparison with \$30/ton CO2 Tax .........................148

Figure 6.88 Generation by Type with Gas Price Reductions, WECC ...........................................149

Figure 6.89 Emissions with Gas Price Reductions, WECC ........................................................149

Figure 6.90 Emission Reductions with Gas Price Reductions ................................................150

Figure 6.91 Impact of Gas Price Reductions on Operations Savings, I30 Case............................150

Figure 6.92 Typical Minimum Operating Ratings of Coal Units as a Percent of Capacity ..........151 
Figure 6.93 Number of Cycles Below Operating Levels - 100-MW Coal Unit ............................152

Figure 6.94 Number of Cycles Below Operating Levels - 350-MW Coal Unit .............................152

Figure 6.95 Number of Cycles Below Operating Levels - 500-MW Coal Unit ............................153

Figure 6.96 Cycles Per Month Below 80\% or 90\% of Rating for 500-MW Coal Unit at 30\%

Penetration

Figure 6.97 Operation of 500-MW Coal Unit Week of April 10th at 30\% Penetration, In-Area

Scenario

Figure 6.98 Spilled Energy as a Function of Coal Minimum Operating Points...........................154

Figure 6.99 Unserved Energy as a Function of Coal Minimum Operating Points.......................155

Figure 6.100 Average System Spot Price vs. Coal Minimums .....................................................155

Figure 6.101 WECC Operating Costs vs. Coal Minimums ........................................................156

Figure 6.102 Increased WECC Operating Costs Over Coal at 40\% Minimum Rating..................156

Figure 6.103 WECC Coal Plant Revenue versus Coal Unit Minimum Operating Points ............157

Figure 6.104 Impact of Coal Minimums on WECC Emissions...................................................157

Figure 6.105 Spot Price Impact of Balancing Area Consolidation, 10\% Penetration. ...................158

Figure 6.106 Curtailed Energy and Contingency Reserve Shortfall w/o Transmission Expansion160

Figure 6.107 Savings Reductions with No Transmission Expansion ...........................................160

Figure 6.108 Emission Impact of Reduced Capacity Additions...................................................161

Figure 6.109 Operating Cost Impact of Reduced Capacity Additions........................................161

Figure 7.1 10-minute Wind Deltas vs. Load Deltas by Season for Study Area (2006, 30\%, Local-

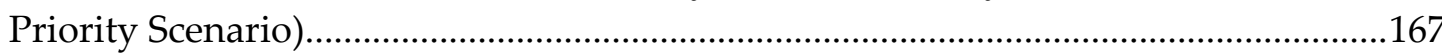

Figure 7.2 Timing of Maximum Daily 10-Min Positive Deltas for Study Area (2006, Local-

Priority Scenario).

Figure 7.3 Timing of Maximum Daily 10-Min Negative Deltas for Study Area (2006, Local-

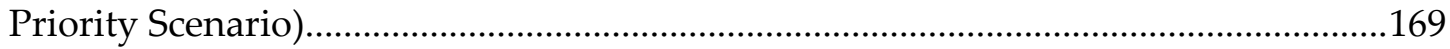

Figure 7.4 Distribution of Net Load Deltas for Study Area (2006, Local-Priority Scenario)........170

Figure 7.5 Distribution of Extreme Hourly Wind Deltas for 2006 (2006, Local-Priority Scenario)171

Figure 7.6 Study Footprint Characteristics for April QSS Period, Local-Priority, 30\% Wind

Penetration

Figure 7.7 Study Footprint Characteristics for October QSS Period, Local-Priority, 30\% Wind

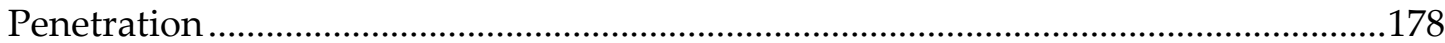

Figure 7.8. Arizona Input Profiles, Local-Priority, April QSS Study Period................................179

Figure 7.9 Arizona Conventional Generation Hourly Schedule, Local-Priority, April QSS Study

Period.

Figure 7.10 Arizona Export Schedule, Local-Priority, April QSS Study Period...........................181

Figure 7.11 Arizona Dispatch, 10\% Wind, Local-Priority, April QSS Study Period.....................183

Figure 7.12. Arizona Regulation, 10\% Wind, Local-Priority, April QSS Study Period. ...............183

Figure 7.13 Arizona Totals, 10\% Wind, Local-Priority, April QSS Study Period.........................185

Figure 7.14 Arizona Regulation and ACE, 10\% Wind, Local-Priority, April QSS Study Period.185

Figure 7.15 Arizona Dispatch, 20\% Wind, Local-Priority, April QSS Study Period....................186

Figure 7.16 Arizona Dispatch, 20/20\% Wind, Local-Priority, April QSS Study Period................187

Figure 7.17 Arizona Dispatch, 30\% Wind, Local-Priority, April QSS Study Period .....................187

Figure 7.18 Arizona Regulation, 20\% Wind, Local-Priority, April QSS Study Period. ................189 
Figure 7.19 Arizona Regulation, 20/20\% Wind, Local-Priority, April QSS Study Period............189 Figure 7.20 Arizona Regulation, 30\% Wind, Local-Priority, April QSS Study Period. ................190 Figure 7.21 Arizona ACE, All Penetration Levels, Local-Priority, April QSS Study Period. ......191 Figure 7.22 Arizona Wind Curtailment, April QSS Study Period................................................192 Figure 7.23 Arizona Regulation and ACE, 30\% Wind with Curtailment, Local-Priority, April

QSS Study Period..........................................................................................................192

Figure 7.24 Transmission Area ACEs, 30\% Wind, Local-Priority, April QSS Study Period........193 Figure 7.25 Footprint ACEs with Cooperation and Consolidation, 30\% Wind, Local-Priority,

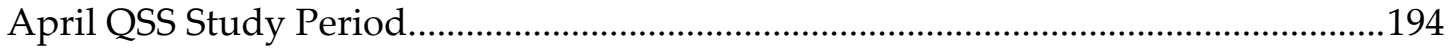

Figure 7.26 Colorado-East Input Profiles, Local-Priority, October QSS Study Period.................195 Figure 7.27 Colorado-East Conventional Generation Hourly Schedule, Local-Priority, October QSS Study Period 196

Figure 7.28 Colorado-East Export Schedule, Local-Priority, October QSS Study Period. ...........196 Figure 7.29 Colorado-East Dispatch, 10\% Wind, Local-Priority, October QSS Study Period. ....198 Figure 7.30 Colorado-East Dispatch, 20\% Wind, Local-Priority, October QSS Study Period. ....198 Figure 7.31. Colorado-East Dispatch, 20/20\% Wind, Local-Priority, October QSS Study Period.199 Figure 7.32 Colorado-East Dispatch, 30\% Wind, Local-Priority, October QSS Study Period. ....199 Figure 7.33 Colorado-East Regulation, 10\% Wind, Local-Priority, October QSS Study Period..200 Figure 7.34 Colorado-East Regulation, 20\% Wind, Local-Priority, October QSS Study Period..201 Figure 7.35. Colorado-East Regulation, 20/20\% Wind, Local-Priority, October QSS Study Period.201 Figure 7.36 Colorado-East Regulation, 30\% Wind, Local-Priority, October QSS Study Period..202 Figure 7.37 Colorado-East ACEs, All Penetration Levels, Local-Priority, October QSS Study

Period 203

Figure 7.38 Colorado-East Dispatch, Continuous Schedule, 30\% Wind, Local-Priority, October QSS Study Period. 204

Figure 7.39 Colorado-East Regulation, Continuous Schedule, 30\% Wind, Local-Priority, October QSS Study Period. 205

Figure 7.40. Colorado-East Dispatch, Perfect Forecast, 30\% Wind, Local-Priority, October QSS

Study Period. 205

Figure 7.41. Colorado-East Regulation, Perfect Forecast, 30\% Wind, Local-Priority, October QSS Study Period. 206

Figure 7.42. Colorado-East Combined-Cycle Usage, 30\% Wind, Local-Priority, October QSS Study Period. 207

Figure 7.43 Colorado-East Combined-Cycle Usage, All Penetration Levels, Local-Priority,

October QSS Study Period.............................................................................................208

Figure 7.44. Colorado-East Combined-Cycle Usage, Forecast and Schedule Variations, 30\%

Wind, Local-Priority, October QSS Study Period. 208

Figure 7.45. Colorado-East Combined-Cycle RMS Usage Index, Forecast and Schedule Variations, 30\% Wind, Local-Priority, October QSS Study Period..............................209

Figure 7.46 Local Priority Case - 10-Minute Wind Variability for 2006 ......................................2215

Figure 7.47 Local Priority 10-Minute Net Load Variability (10-minute $\Delta \sigma$ in MW) for 2006 ....216

Figure 7.48 10-Minute Variability: Wind and Net Load - 3 Scenarios........................................2217

Figure 7.49 10-Minute Wind Variability: Normalized to Installed Rating .................................218 
Figure 7.50 Arizona and Wyoming: Average (MW) vs. Sigma of 10-Minute Delta (10-minute $\Delta \sigma$

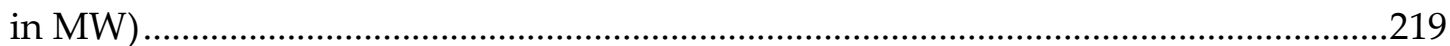

Figure 7.51 10-Minute Variability (10-minute $\Delta \sigma$ in percent of peak load) .................................220

Figure 7.52 Load-Wind-Net Load Relationship for L30 System .................................................222

Figure 7.53 Relationship Between Load Duration and Load Range for Local-Priority Scenario223

Figure 7.54 Operations L30: Hours of Load and Wind Combinations .......................................224

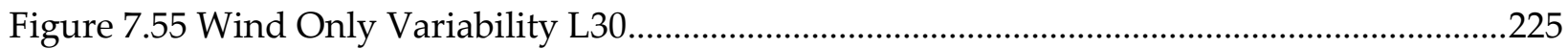

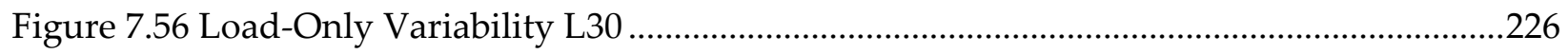

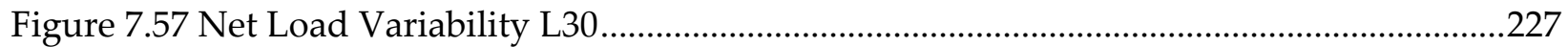

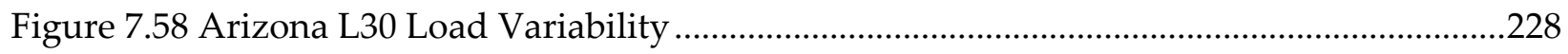

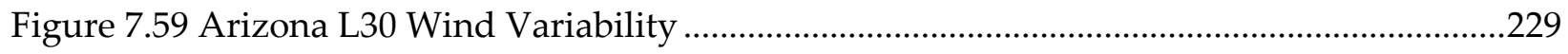

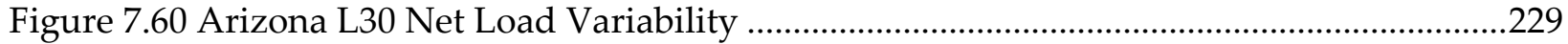

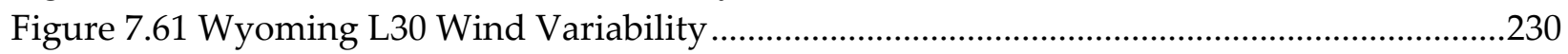

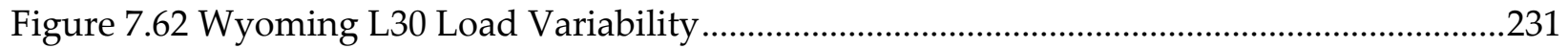

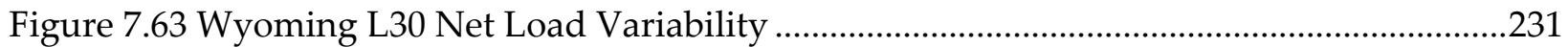

Figure 7.64 Wyoming L30 Time Space .....................................................................................232

Figure 7.65 Wyoming L30 Net Load Variability: $3 \Delta \sigma$ vs. $\mu+3 \Delta \sigma$-Rise........................................233

Figure 7.66 Average Reserve Requirements for Local Priority 30\% Scenario...............................234

Figure 7.67 Net Load Variability in Different Time Frames - Various GE Studies.......................235

Figure 7.68 Regulation Reserve Requirements (by Area) .........................................................236

Figure 7.69 Study Footprint L30 3\% Load Plus 5\% Wind Rule...................................................238

Figure 7.70 Arizona L30 3\% Load Plus 5\% Wind Rule ...................................................................239

Figure 7.71 Study Footprint L30 Best RMS Fit X\% Load Plus Y\% Wind Rule..............................241

Figure 7.72 Arizona L30 Best RMS Fit X\% Load Plus Y\% Wind Rule...........................................242

Figure 7.73 Study Foot Print L30 X\% Load Plus Y\% Conditional Wind Rule ..............................243

Figure 7.74 Arizona L30 X\% Load Plus Y\% Conditional Wind Rule ............................................244

Figure 7.75 Arizona L30 Load-Only Variability...........................................................................245

Figure 7.76 Arizona L30 Scenario Load-Only Variability ..........................................................246

Figure 7.77 Regulation Reserve Requirements for Load-Only (by Area) ...................................247

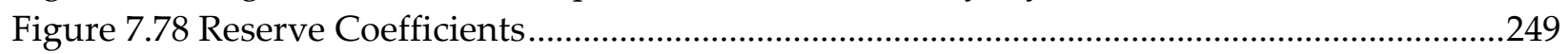

Figure 7.79 X\% Load and Y\% Wind Reserve Rule - Relationships to Penetration ......................249

Figure 7.80 Comparison of Peak Reserve Requirement Rules (by Area) ....................................250

Figure 7.81 Study Footprint - Polynomial Fits to Net Load Variability (L30 Scenario)...............251

Figure 7.82 Study Footprint - Polynomial Fits to Wind Variability ..............................................252

Figure 7.83 L30R Reserve Duration (Best Fit X+Y Conditional rules) ........................................2254

Figure 7.84 Reserve Margins: Comparison to No Wind and Solar ............................................255

Figure 7.85 Impact of Load and Wind Levels on Variability Reserve Margins ...........................256

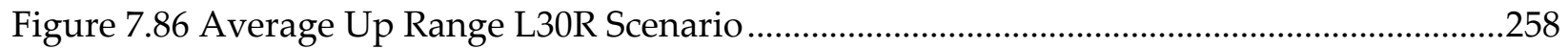

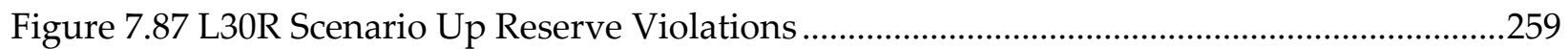

Figure 7.88 Up Reserves vs. Forecast Error (Full Year) ............................................................260

Figure 7.89 Up Reserves vs. Wind Forecast Error (Selected Load - Wind Pairs) .........................260

Figure 7.90 L30R Scenario Down Reserve Violations.................................................................261

Figure 7.91 L30R Arizona Reserve Duration - Best fit rules …....................................................262 


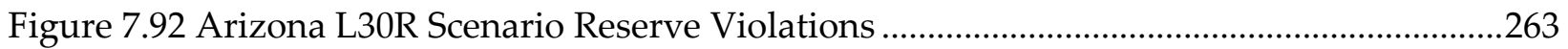

Figure 7.93 Wyoming L30R Scenario Up Reserve Violations .....................................................264

Figure 7.94 Arizona L30R Scenario April 15, 2009 Trajectory .....................................................266

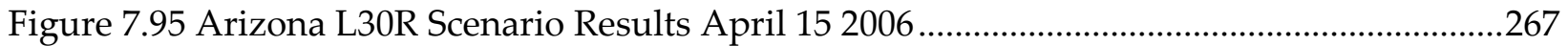

Figure 7.96 Arizona L30R Scenario October 262006 Trajectory .................................................267

Figure 7.97 Arizona L30R Scenario Results October 262006 ....................................................268

Figure 7.98 Wyoming L30R Scenario April 15, 2006 Trajectory …............................................268

Figure 7.99 Wyoming L30R Scenario - Regulation Allocation Detail - April 15 Results ............269

Figure 7.100 Wyoming L30R Net Load Variability October 262006 Trajectory..........................269

Figure 7.101 Wyoming L30R Scenario October 26, 2006 Results .................................................270

Figure 7.102 Comparison of Quick Start Reserves..................................................................272

Figure 7.103 Comparison of Combined Reserve Margins ...........................................................22

Figure 7.104 Relationship of Wind Drops and Quick-start Reserves to Wind Power Level ......273

Figure 8.1 PSH Annual Duration Curve .................................................................................278

Figure 8.2 PSH Operation with Discounted Pumping Costs .....................................................278

Figure 8.3 Operational Impact of Forcing PSH Operation ..........................................................279

Figure 8.4 Arizona Spot Prices, Week of April 10th, Perfect Forecasting......................................280

Figure 8.5 Arizona Spot Prices, Week of April 10th, S-o-A Forecasting.......................................280

Figure 8.6 Incremental Value of a 100-MW PSH Plant ...............................................................282

Figure 8.7 Sample Energy Shift for CSP "Optimal" Dispatch.........................................................283

Figure 8.8 Monthly CSP Energy by Zone..................................................................................284

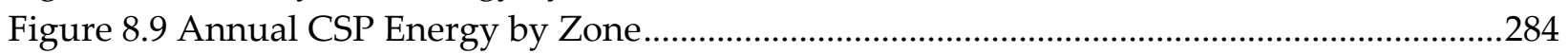

Figure 8.10 Annual CSP Revenue Increase vs. Hours of Storage ................................................285

Figure 8.11 Annual CSP Revenue Increase by Zone Due to Optimal Dispatch. ..........................286

Figure 8.12 Monthly CSP \% Revenue Increase by Zone Due to Optimal Dispatch .....................287

Figure 8.13 Total Monthly CSP Revenue Increase .......................................................................287

Figure 8.14 Distribution of PHEV Charging Energy …...............................................................289

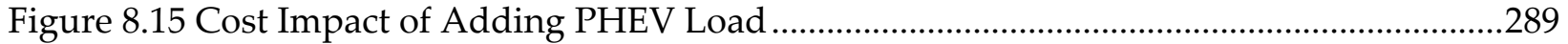

Figure 8.16 Operational Impact of Renewable Energy with PHEV .............................................290

Figure 9.1 Study Area Risk vs. Capacity Additions .....................................................................293

Figure 9.2 Study Area Risk with Renewable Additions ..........................................................293

Figure 9.3 Hourly Average Wind and Load Shape .................................................................294

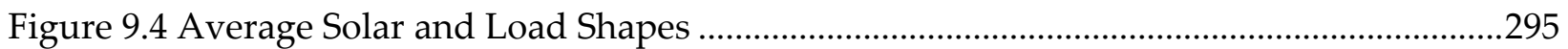

Figure 9.5 Study Area Total Monthly Wind and Solar Energy ....................................................296

Figure 9.6 Study Area Risk in Hours/Year...................................................................................297

Figure 9.7 Study Area Risk in Unserved Energy ........................................................................297

Figure 9.8 Wind Capacity Values by Scenario, 2006 Shapes, Perfect Capacity ...........................299

Figure 9.9 CSP with Storage Capacity Values by Scenario, 2006 Shapes, Perfect Capacity .........300

Figure 9.10 PV Capacity Values by Scenario, 2006 Shapes, Perfect Capacity ...............................300

Figure 9.11 Annual and Monthly Variation in Renewable Energy ...............................................301

Figure 9.12 Capacity Value for Wind, Perfect Capacity, Daily LOLE, All Years .........................301

Figure 9.13 Capacity Value for CSP with 6 Hours of Storage, Perfect Capacity, Daily LOLE, All

Years. 
Figure 9.14 Capacity Value for PV, Perfect Capacity, Daily LOLE, All Years .............................302 Figure 9.15 Combined Capacity Value for Wind, CSP and PV, Perfect Capacity, Daily LOLE, All

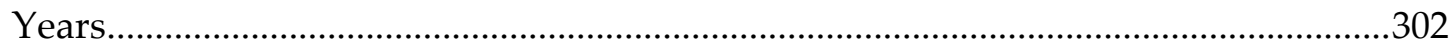

Figure 9.16 Capacity Value for Wind Only, 2006 Load Shapes, Random Wind Shapes.............305

Figure 9.17 Capacity Value for CSP with Storage, 2006 Load Shapes, Random Solar Shapes ....306

Figure 9.18 Capacity Value for PV Only, 2006 Load Shapes, Random Solar Shapes ...................306

Figure 9.19 Capacity Value for Wind, CSP and PV, 2006 Load Shapes, Random Wind and Solar

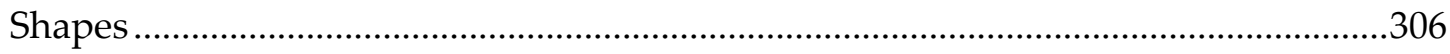

Figure 9.20 Comparison of Wind Capacity Values, Random and Aligned Shapes .....................307

Figure 9.21 Comparison of CSP Capacity Values, Random and Aligned Shapes........................307

Figure 9.22 Comparison of PV Capacity Values, Random and Aligned Shapes..........................308 


\section{List of Tables}

Table 2.1 Wind and Solar Energy Penetrations for WWSIS Scenarios.............................................7

Table 2.2 Summary of Aggregated Wind and Solar MW Ratings by State for WWSIS

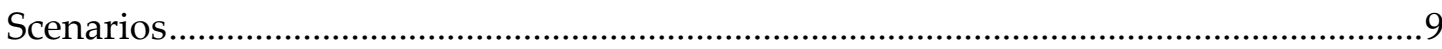

Table 3.1 Annual Wind Energy by State for Three Years, 30\% In-Area Scenario ........................22

Table 3.2 Energy Summary for 30\% In-Area Scenario ................................................................28

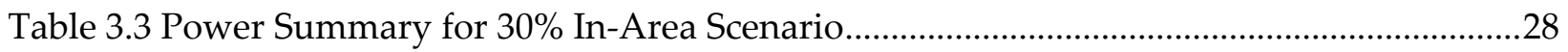

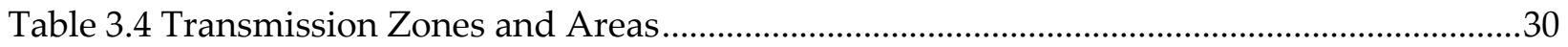

Table 3.5 Selected WECC Transmission Path Ratings....................................................................

Table 3.6 Energy Summary for 30\% Mega-Project Scenario..............................................................35

Table 3.7 Power Summary for 30\% Mega-Project Scenario ...............................................................35

Table 3.8 Summary of Capital Costs included in Scenario Development ........................................37

Table 3.9 Energy Summary for 30\% Local-Priority Scenario .........................................................38

Table 3.10 Power Summary for 30\% Local-Priority Scenario.......................................................38

Table 4.1 Monthly Wind and Solar Energy Summary for 2004, 2005, and 2006, 30\% In-

Area Scenario

Table 4.2 Summary of 2006 Percent Monthly Energy from Wind and Solar (30\% Wind

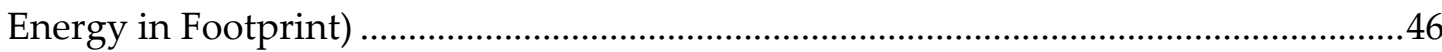

Table 5.1 Summary of 2006 Footprint Net Load Variability for the Local-Priority Scenario ........80

Table 5.2 Summary Statistics for In-Footprint States, Total Footprint and WECC for All

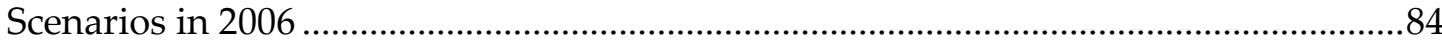

Table 5.3 Summary of Forecast Errors by Area, 30\% Local-Priority Scenario ...............................91

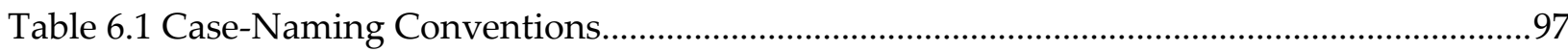

Table 6.2 Wyoming to Colorado Interface Flow Statistics........................................................105

Table 7.1 10-minute Net Load Variability by Area, Footprint and WECC....................................166

Table 7.2 Summary Statistics for 10-Minute Delta for Wyoming .................................................167

Table 7.3. Generic Ramp Rate Data by Unit Type....................................................................174

Table 7.4. Regulation Requirement by Transmission Area .......................................................174

Table 7.5 Approximate CPS2 L10 Limits by Transmission Area ..............................................175

Table 7.6 Economic Dispatch Priority for Arizona during April QSS Study Period. ...................181

Table 7.7 Regulation Priority and Capability for Arizona During April QSS Study

Period

Table 7.8 Economic Dispatch Priority for Colorado-East during October QSS Study

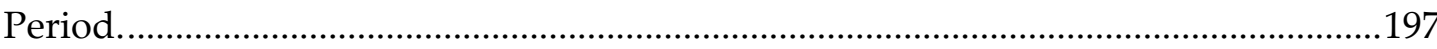

Table 7.9 Regulation Priority and Capability for Colorado-East during October QSS Study Period.

Table 7.10. Colorado-East Regulation at Capability Limits........................................................202

Table 7.11. Combined-Cycle RMS Usage Index....................................................................209

Table 7.12. Maximum Arizona Wind Curtailment in Single Worst Event for Various Penetration Levels, Local-Priority, April QSS Study Period.

Table 7.13 Maximum Colorado-East Load Participation for Single Worst Forecast Error for Various Penetration Levels, Local-Priority, October QSS Study Period. 


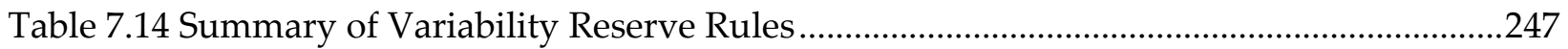

Table 8.1 Potential Load Increase from PHEV by State ............................................................28

Table 9.1 Renewable Capacities By Type .......................................................................................294

Table 9.2 Renewable Capacity Values By Type, 2006 Shapes, Perfect Capacity, Daily

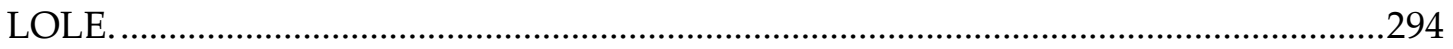

Table 9.3 Renewable Capacity Values By Type, 2006 Shapes, Perfect Capacity, Hourly

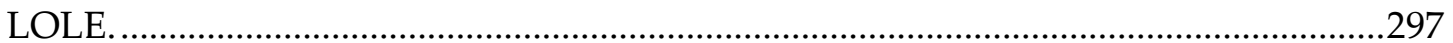

Table 9.4 Renewable Capacity Values By Type, 2006 Shapes, Perfect Capacity ..........................298

Table 9.5 Renewable Capacity Values By Type, 2006 Shapes, Perfect Capacity, Average

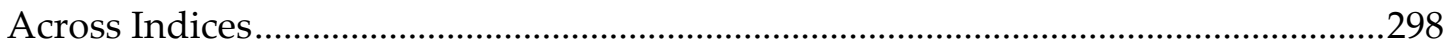

Table 9.6 Renewable Capacity Values By Type, Perfect Capacity, Daily LOLE, By Scenario. 


\section{Acronym List}

\begin{tabular}{|c|c|}
\hline ACE & Area Control Error \\
\hline AGC & Automatic Generation Control \\
\hline ADI & ACE Diversity Interchange \\
\hline BOP & Balance of Plant \\
\hline CAES & Compressed Air Energy Storage \\
\hline CPS2 & Control Performance Standard 2 \\
\hline $\mathrm{CO} 2$ & Carbon Dioxide \\
\hline CSP & Concentrating Solar Plants \\
\hline $\mathrm{DOE}$ & U.S. Department of Energy \\
\hline DSM & Demand Side Management \\
\hline EWITS & Eastern Wind Integration and Transmission Study \\
\hline LOLE & Loss of Load Expectation \\
\hline LOLP & Loss of Load Probability \\
\hline MAE & Mean Absolute Error \\
\hline MAPS & Multi Area Production Simulation \\
\hline MARS & Multi Area Reliability Simulation \\
\hline NAU & Northern Arizona University \\
\hline NCAR/NCEP & National Center for Atmospheric Research / National Center for Environmental Prediction \\
\hline NERC & North American Electric Reliability Corporation \\
\hline NOX & Nitrogen Oxides \\
\hline NREL & National Renewable Energy Laboratory \\
\hline NWP & Numerical Weather Prediction \\
\hline PHEV & Plug-in Hybrid Electric Vehicle \\
\hline PSH & Pumped Storage Hydro \\
\hline PUC & Public Utility Commission \\
\hline PV & Photovoltaic \\
\hline QSS & Quasi-steady state \\
\hline RMSE & Root Mean Square Error \\
\hline SCORE & Statistical Correction to Output from Record Extension \\
\hline S-o-A & State of the Art \\
\hline SOX & Sulfur Oxides \\
\hline SUNY & State University of New York \\
\hline TRC & Technical Review Committee \\
\hline WECC & Western Electric Coordinating Council \\
\hline WRF & Weather Research and Forecasting \\
\hline WWSIS & Western Wind and Solar Integration Study \\
\hline
\end{tabular}




\section{ACKNOWLEDGMENTS}

The National Renewable Energy Laboratory (NREL) thanks the U.S. Department of Energy (DOE) for sponsoring the Western Wind and Solar Integration Study. Contributors include:

PROJECT MANAGERS

Debra Lew

NREL

Richard Piwko

GE Energy

STUDY TEAM

GE Nicholas Miller, Kara Clark, Gary Jordan, Lavelle Freeman, Zhi Gao, Shakeer Meeran, Ekrem Gursoy, Miaolei Shao, Ryan Konopinski, Amanvir Chahal, Glenn Haringa, David Burnham, Kristen Bleyman

NREL Michael Milligan, Brian Parsons, Yih-Huei Wan, Erik Ela, Donna Heimiller, George Scott, Kirsten Orwig, Ray George, Nate Blair, Mark Mehos, Craig Turchi, Paul Denholm, Marc Schwartz, Dennis Elliott, Steve Haymes, Dave Corbus, Tony Markel

3TIER Group

Exeter Associates

Northern Arizona University

State University of New York at Albany and Clean Power Research

WESTCONNECT

TECHNICAL REVIEW COMMITTEE

Arizona Public Service

El Paso Electric

NV Energy

Public Service of New Mexico

Salt River Project

Tri-State Generation and Transmission

Tucson Electric Power

Western Area Power Administration

Xcel Energy

American Wind Energy Association
Consultant
Enernex Corporation
Lawrence Berkeley National Laboratory
Renewable Energy Consulting
Sandia National Laboratory
University College Dublin
U.S. Department of Energy
Utility Wind Integration Group
Western Electricity Coordinating Council
Western Interstate Energy Board
WindLogics

Cameron Potter, Bart Nijssen

Kevin Porter, Sari Fink

Tom Acker, Karin Wadsack, Carson Pete, Jason Kemper and Mark Bielecki

Richard Perez, Tom Hoff

Charlie Reinhold

Finally, we thank Bruce Green (NREL) and Mark Schroder (Purple Sage Design) for designing this Executive Summary and the full report. 


\section{INTRODUCTION}

The focus of the Western Wind and Solar Integration Study (WWSIS) is to investigate the operational impact of up to 35\% energy penetration of wind, photovoltaics (PVs), and concentrating solar power (CSP) on the power system operated by the WestConnect group of utilities in Arizona, Colorado, Nevada, New Mexico, and Wyoming ${ }^{1}$. WWSIS was conducted over two and a half years by a team of researchers in wind power, solar power, and utility operations, with oversight from technical experts in these fields. This report discusses the development of data inputs, the design of scenarios to address key issues, and the analysis and sensitivity studies that were conducted to answer questions about the integration of wind and solar power on the grid.

\section{WESTCONNECT}

WestConnect is a group of transmission providers that are working collaboratively on initiatives to improve wholesale electricity markets in the West. Participants include Arizona Public Service, El Paso Electric Co., NV Energy, Public Service of New Mexico, Salt River Project, Tri-State Generation and Transmission Cooperative, Tucson Electric Power, Western Area Power Administration, and Xcel Energy.

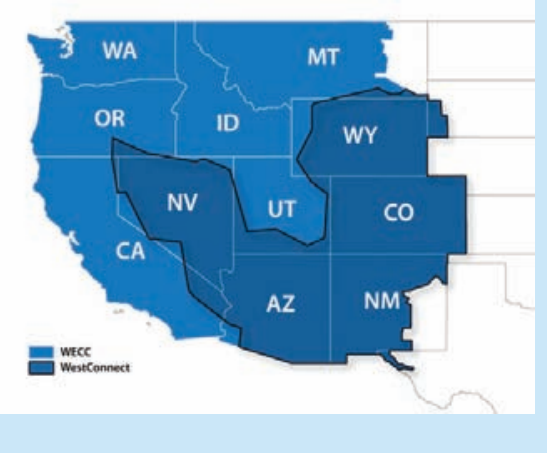

The technical analysis performed in this study shows that it is operationally feasible for WestConnect to accommodate $30 \%$ wind and $5 \%$ solar energy penetration, assuming the following changes to current practice could be made over time:

- Substantially increase balancing area cooperation or consolidation, real or virtual;

- Increase the use of sub-hourly scheduling for generation and interchanges;

- Increase utilization of transmission;

- Enable coordinated commitment and economic dispatch of generation over wider regions;

- Incorporate state-of-the-art wind and solar forecasts in unit commitment and grid operations;

- Increase the flexibility of dispatchable generation where appropriate (e.g., reduce minimum generation levels, increase ramp rates, reduce start/stop costs or minimum down time);

- Commit additional operating reserves as appropriate;

- Build transmission as appropriate to accommodate renewable energy expansion;

- Target new or existing demand response programs (load participation) to accommodate increased variability and uncertainty;

- Require wind plants to provide down reserves.

In addition, suggestions for follow-on work to further explore these and additional mitigation options are listed in the Conclusions and Next Steps section.

${ }^{1}$ WestConnect also includes utilities in California, but these were not included in WWSIS because California had already com pleted a renewable energy integration study for the state. 


\section{BACKGROUND}

WWSIS and its sister study, the Eastern Wind Integration and Transmission Study (EWITS), follow the U.S. Department of Energy's (DOE) 20\% Wind Energy by 2030 Study that considered the benefits, costs, and challenges associated with sourcing $20 \%$ of the nation's energy from wind power by 2030 $[1,2]$. The study found that while proactive measures were required,

\section{BALANCING AREAS}

Balancing areas are responsible for balancing load and generation within a defined area and maintaining scheduled interchanges with other balancing areas.

no insurmountable barriers to reaching 20\% wind were identified. Thus, DOE and the National Renewable Energy Laboratory (NREL) embarked upon WWSIS and EWITS to examine, in much greater depth, whether there were technical or physical barriers in operating the grid with $20 \%$ wind. Solar power was included in WWSIS due to the significant solar resources and solar development in the West.

Four of the five states in WestConnect have Renewables Portfolio Standards (RPS) that require $15-30 \%$ of annual electricity sales to come from renewable sources by 20202025. Additionally, WWSIS models the entire western interconnection, examining the operating impact of up to $23 \%$ penetration of wind and solar in the rest of the Western Electricity Coordinating Council (WECC). Most of the states in WECC have similar RPS requirements and renewable energy growth in the region has been significant.

The study was designed to answer questions that utilities, Public Utility Commissions, developers, and regional planning organizations had about renewable energy use in the West:

- What is the operating impact of up to $35 \%$ renewable energy penetration and how can this be accommodated?

- How does geographic diversity help to mitigate variability?

- How do local resources compare to remote, higher quality resources delivered by long distance transmission?

- Can balancing area cooperation mitigate variability?

- How should reserve requirements be modified to account for the variability in wind and solar?

- What is the benefit of integrating wind and solar forecasting into grid operations?

- How can hydro generation help with integration of renewables?

WWSIS and its sister study EWITS build upon a large body of work on wind integration [3-9]. Previous studies examined specific utilities or states, looking at the impact of wind on operations in the regulation (seconds to minutes), load following (minutes to hours), and unit commitment (hours to days) time frames. In these studies, hypothetical wind and transmission build-outs were typically added to the existing system, which was simulated or statistically analyzed over these time 
frames. These studies generally consider the impact of the variability of wind (due to varying weather) and the uncertainty of wind (due to our inability to perfectly forecast the weather). Even if the weather and the wind could be perfectly forecast,

\section{STUDY ASSUMPTIONS SCENARIO DEVELOPMENT:}

- Specific energy targets for each of three technologies: wind, PV, and CSP were fixed. For example, wind sites could not be traded out for CSP sites.

- A number of capital cost assumptions in 2008 dollars were used in determining the different geographic scenarios: wind at $\$ 2000 / \mathrm{kW}, \mathrm{PV}$ at $\$ 4000 / \mathrm{kW}$, CSP with thermal storage at $\$ 4000 / \mathrm{kW}$, transmission at $\$ 1600 / \mathrm{MW}$-mile, and transmission losses at $1 \%$ per 100 miles. No tax credits are assumed or included.

- The geographic scenarios considered different interstate transmission build-outs and included these costs in the scenarios. Incremental intra-state transmission build-outs were not specified in this analysis. Existing transmission capacity is assumed to be unavailable for new renewable energy generation only for the scenario development process.

- New transmission was undersized: 0.7 MW of new transmission was added for each 1.0 MW of remote generation.

\section{PRODUCTION SIMULATION ANALYSIS:}

- All study results are in 2017 nominal dollars with $2 \%$ escalation per year.

- $\quad$ 2/MBTU coal; \$9.50/MBTU natural gas.

- Carbon dioxide costs were assumed to be $\$ 30 /$ metric ton of $\mathrm{CO}_{2}$.

- Except in cases where specified, extensive balancing area cooperation is assumed (see box on page 19).

- The production simulation analysis assumes that all units are economically committed and dispatched while respecting existing and new transmission limits and generator cycling capabilities and minimum turndowns.

- Existing available transmission capacity is accessible to renewable generation.

- Generation equivalent to $6 \%$ of load is held as contingency reserves - half is spinning and half is non-spinning.

- The balance of generation was not optimized for renewables. Rather, a business-as-usual capacity expansion met projected load growth in 2017. Renewable energy capacity was added to this mix, so the system analyzed is overbuilt by the amount of capacity value of the renewable plants.

- Increased O\&M of conventional generators due to increased ramping and cycling was not included due to lack of data.

- Renewable energy plant O\&M costs are not included. Wind and solar are considered pricetakers.

- The hydro modeling did not reflect the specific climatic patterns of 2004, 2005, and 2006, but rather a 10-year long term average flow per month.

- The sub-hourly modeling assumes a 5-minute economic dispatch. 
grid operators would still have to accommodate wind's variability. It is important to note that operators already manage variability and uncertainty in the load; wind and solar add to that variability and uncertainty.

WWSIS was funded by DOE and was managed by NREL. The main partner in this study was WestConnect. The project team included 3TIER Group (wind power dataset, and wind and solar forecasts), State University of New York at Albany/Clean Power Research (solar radiation dataset), Exeter Associates (data collection), Northern Arizona University (wind validation and hydro), NREL (wind validation, and PV and CSP power datasets), and GE (scenarios, and main technical/economic analysis). A Technical Review Committee (TRC), composed of members of WestConnect utilities, western utility organizations, and industry and technical experts, met eight times to review technical results and progress. A broader stakeholder group, open to the public, met five times to ensure study direction and results were relevant to western grid issues. Interim and final results of this study have been vetted in approximately 30 public forums.

The study examined grid operation for the year 2017. That is, system loads and generation expansion were projected to represent year 2017. While 35\% renewable energy penetration was not expected by 2017, this year was selected in order to start with a realistic model of the transmission grid. The study examined inter-annual operability by modeling operations for year 2017 three times, using historical load and weather patterns from years 2004, 2005, and 2006.

\section{WHAT THIS STUDY DOES AND DOES NOT COVER}

While this study undertakes detailed analysis and modeling of the power system, it was meant to be a complement to other in-depth studies:

- WWSIS is an operations study, not a transmission planning study, although different scenarios model different interstate transmission expansion options.

- WWSIS is not a cost-benefit analysis, even though wind and solar capital costs were incorporated in scenario development. Rather WWSIS focuses on the variable operational costs and savings due to fuel and emissions.

- WWSIS is not a reliability study, although analysis of the capacity value of wind and solar was conducted to assess their contributions to resource adequacy. A full complement of planning and operational electrical studies would be required to more accurately understand and identify system impacts.

- WWSIS does not address dynamic stability issues.

- WWSIS does not attempt to optimize the balance between wind and solar resources. Wind and solar levels were fixed independently.

In 2017, it is anticipated that WestConnect and WECC will operate differently from current practice. WWSIS assumed the following changes from current operational practice: 
- Production simulations of WECC grid operations assume least-cost economic dispatch in which all generation resources are shared equally and not committed to specific loads. Except for California and Alberta, WECC currently utilizes a bilateral contract market with long and short-term contracts in which resources are contracted out to meet specific loads.

- Other than California and Alberta, WECC currently operates as 37 separate balancing areas that utilize these bilateral contracts to balance their areas. Except where specified, this study assumes five regional balancing areas in WECC (Arizona-New Mexico, Rocky Mountain, Pacific Northwest, Canada and California). WWSIS does not consider any power purchase agreements, including those for renewables².

- Except for California and Alberta, transmission in WECC is primarily contractually obligated and utilized. Existing available transmission capacity may be contractually obligated and not accessible to other generation. This study assumes that existing available transmission capacity is accessible to other generation on a short-term, non-firm basis.

- Pricing developed by production cost modeling can vary widely from bilateral contract prices, and was not aligned or calibrated with current bilateral contract prices. The incremental operations and maintenance (O\&M) costs in the report do not necessarily replicate escalated current costs in the Western Interconnection.

In addition to these caveats, there are reasons that the study results tend toward the conservative:

- WWSIS did not model a more flexible non-renewable balance of generation than what exists and is planned in WECC today. If 20-35\% variable generation were to be planned in WECC, more flexible generation would be likely planned as well, reducing the challenge that wind and solar place on operation in this study.

- This study modeled the grid for the year 2017. If WWSIS were conducted for a later year when $35 \%$ renewables would be more plausible, the power system would likely have a larger load, more flexible balance of generation, and more transmission, all of which would help to accommodate the renewables.

- The wind dataset used was conservative in terms of overestimating the actual variability found in measured wind plant output.

- The base assumption of $\$ 9.50$ / MBTU for gas means that gas is displaced, which leaves coal (which in the West, is less flexible than gas) to accommodate the variability of the renewables.

2 Thus, throughout this work, costs specifically and solely refer only to variable costs, i.e., fuel plus O\&M plus carbon tax, that are incurred during operation. Prices paid to individual generators are not reported. 


\section{SCENARIOS}

\section{WIND, SOLAR, AND LOAD DATA}

About $75 \mathrm{GW}$ of wind generation sites were required for the study scenarios. Because there are not adequate measurements of wind speed or wind power to model this amount of wind generation, 3TIER Group employed a mesoscale Numerical Weather Prediction (NWP) Model to essentially recreate the weather in a 3-dimensional physical representation of the atmosphere in the western U.S. for the years 2004-2006. They then sampled this model at a 2-km, 10-minute resolution and modeled wind plants throughout this region, based on a Vestas V90 3-MW turbine. 3TIER Group also developed day-ahead wind forecasts for each hour. Over $960 \mathrm{GW}$ of wind sites were modeled. The wind dataset is publicly available [10, 11].

Similarly, a lack of solar irradiance or power measurements led to the use of a satellite cloud cover model to simulate the United States at a 10-km, hourly resolution [12]. Day-ahead hourly solar forecasts were also developed [10]. PV was modeled in 100MW blocks as distributed generation on rooftops because modeling information for large, central station PV plants was not available at the time of the study. Over 15 GW of PV plants were included in the dataset. Ten-minute variability was subsequently added to the aggregate hourly outputs to create the 10-minute PV data.

CSP was modeled as 100-MW blocks of parabolic trough plants with six hours of thermal storage. Over $200 \mathrm{GW}$ of CSP plants were modeled in the dataset. Because the CSP with thermal storage produces a very stable output, the 10-minute dataset was created simply by interpolating the hourly dataset.

Hourly load-profile data for all operating areas in WECC were obtained from a Ventyx database, and 10-minute load data were derived by interpolating the hourly data. 


\section{SCENARIO DESCRIPTION}

The WWSIS used a multidimensional scenario-based study approach to evaluate:

- Different levels of energy penetration for wind and solar generation, ranging from $11 \%$ to $35 \%$;

- Different geographic locations for the wind and solar resources;

- A wide array of sensitivities to assess issues such as fuel costs, operating reserve levels, unit commitment strategies, storage alternatives, balancing area size, etc.

Table 1 shows the four levels of wind and solar energy penetration assumed for the study scenarios. The Preselected case includes that wind and solar capacity which was installed by the end of 2008 . The $10 \%$ case includes $10 \%$ wind energy (relative to total annual load energy) and 1\% solar energy (solar consisted of 70\% CSP and 30\% PV) in the study footprint, as well as the rest of WECC. The $20 \%$ case includes $20 \%$ wind energy and 3\% solar energy in the study footprint, with 10\% wind energy and $1 \%$ solar energy in the rest of WECC. The $20 / 20 \%$ case includes $20 \%$ wind energy and $3 \%$ solar energy in the study footprint, as well as the rest of WECC. The $30 \%$ case included 30\% wind energy and 5\% solar energy in the study footprint, with $20 \%$ wind energy and 3\% solar energy in the rest of WECC.

\begin{tabular}{|l|c|c|c|c|c|}
\hline \multicolumn{1}{|c|}{ TABLE 1 - WIND AND SOLAR ENERGY PENETRATIONS FOR WWSIS CASES WITH NAMING CONVENTION IN BLUE. } \\
\hline CASE NAME & \multicolumn{2}{|c|}{ IN FOOTPRINT } & \multicolumn{3}{c|}{ REST OF WECC } \\
\hline NAME & $\begin{array}{c}\text { WIND } \\
\text { SOLAR }\end{array}$ & WIND & SOLAR & \multicolumn{2}{c|}{ WIND SOLAR } \\
\hline PRE-SELECTED CASE & $3 \% *$ & $3 \%$ & $*$ & $2 \%$ & $*$ \\
\hline $10 \%$ CASE & $11 \%$ & $10 \%$ & $1 \%$ & $10 \%$ & $1 \%$ \\
\hline $20 \%$ CASE & $23 \%$ & $20 \%$ & $3 \%$ & $10 \%$ & $1 \%$ \\
\hline $20 / 20 \%$ CASE & $23 \%$ & $20 \%$ & $3 \%$ & $20 \%$ & $3 \%$ \\
\hline $30 \%$ CASE & $35 \%$ & $30 \%$ & $5 \%$ & $20 \%$ & $3 \%$ \\
\hline
\end{tabular}

\section{* Existing solar embedded in load}

Three geographic scenarios were developed to examine the tradeoff between: 1) local resources that are closer to load, but have lower capacity factors and 2) remote resources that have higher capacity factors, but require long distance transmission to access loads. An algorithm was developed to select sites based on energy value, capacity value, and geographic diversity according to criteria developed for each scenario. Figure 1 shows maps of the study scenarios for the $30 \%$ case. Total nameplate ratings of wind generation for each state are shown in blue; solar MW ratings are shown in red. New transmission lines to increase interstate transfer capability are shown in black. Significant intra-state transmission also needs to be built to bring the renewable resources to the existing bulk transmission grid, but WWSIS did not examine intra-state transmission. 

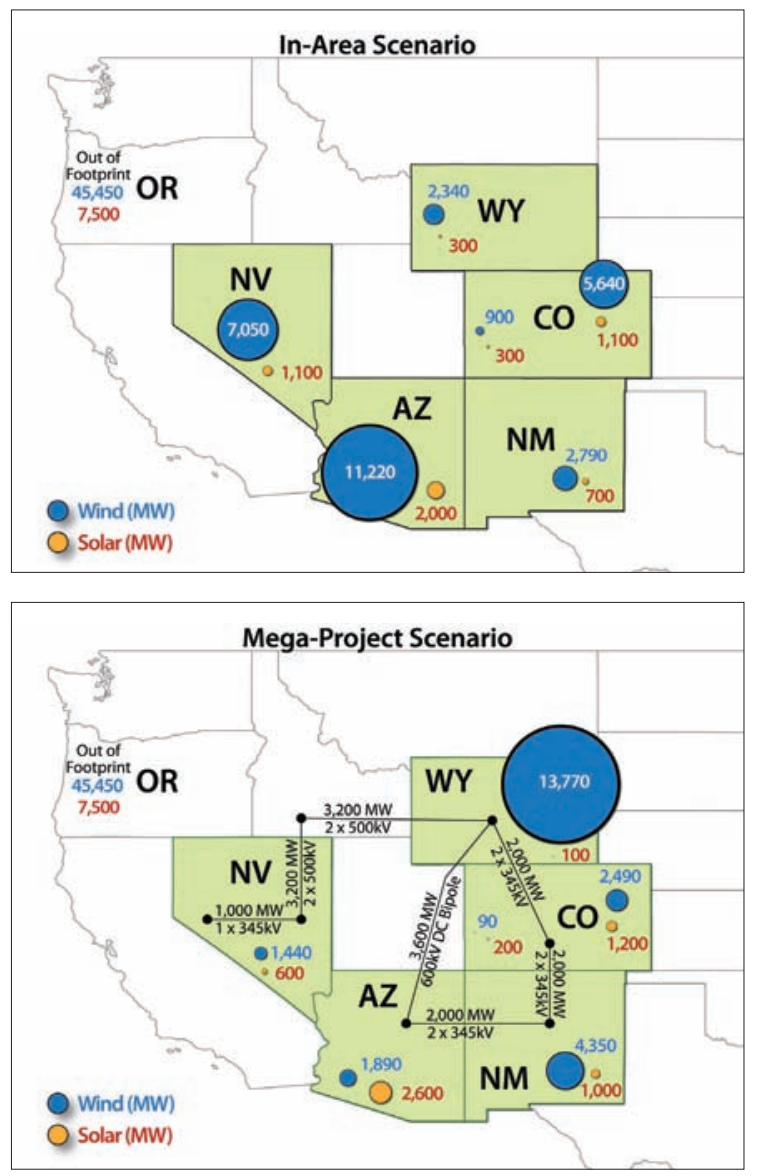

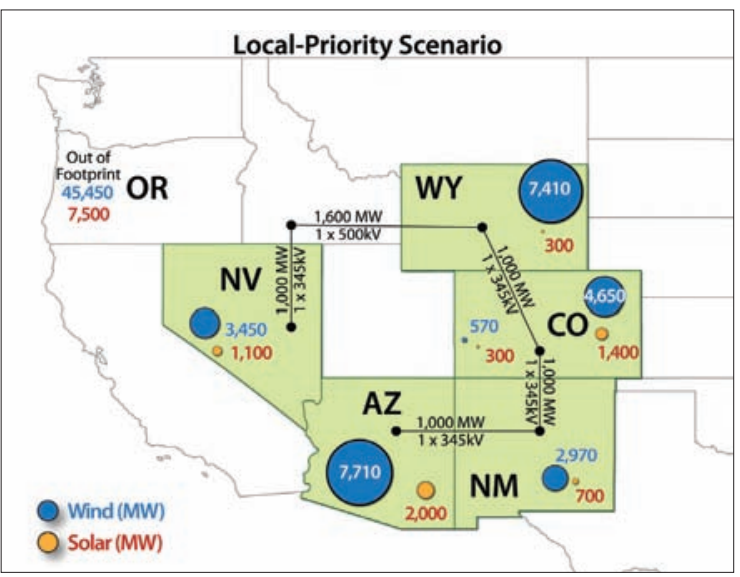

Figure 1 - Three geographic scenarios developed for siting of wind and solar plants in the $30 \%$ case, with appropriate interstate transmission included to bring resources to load.

In Area Scenario: Each state in the study footprint met its wind and solar energy targets using the best available wind and solar generation resources within its state boundary. No additional interstate transmission was added.

Local Priority Scenario: This scenario used the best wind and solar sites within the entire footprint, but included a $10 \%$ capital cost advantage to resources within each state. The result was a scenario that was about halfway between the In Area and Mega Project Scenarios. This scenario includes new interstate transmission, but not as much as the Mega Project Scenario.

Mega Project Scenario: The study footprint met its wind and solar energy targets by using the best available wind and solar resources within the study footprint. Given that many of the best wind resources are in Wyoming, this scenario includes a large penetration of wind generation in Wyoming (and other wind-rich areas), with new transmission lines to deliver the energy to load centers.

For all three of these scenarios, the rest-of-WECC scenario remains constant: each state in the rest of WECC meets its renewable energy target using the best available resources within the state boundary.

Table 2 shows a summary of the total wind and solar MW ratings by state for the three study scenarios. Table 3 summarizes the capital costs for the three study scenarios. 


\begin{tabular}{|c|c|c|c|c|c|c|c|c|}
\hline \multicolumn{9}{|l|}{ IN AREA } \\
\hline & & & $10 \%$ & $1 \%$ & $20 \%$ & $3 \%$ & $30 \%$ & $5 \%$ \\
\hline AREA & $\begin{array}{l}\text { LOAD MIN. } \\
(\mathrm{MW})\end{array}$ & $\begin{array}{c}\text { LOAD MAX. } \\
\text { (MW) }\end{array}$ & $\begin{array}{l}\text { WIND } \\
\text { (MW) }\end{array}$ & $\begin{array}{l}\text { SOLAR } \\
\text { (MW) }\end{array}$ & $\begin{array}{l}\text { WIND } \\
\text { (MW) }\end{array}$ & $\begin{array}{l}\text { SOLAR } \\
\text { (MW) }\end{array}$ & $\begin{array}{l}\text { WIND } \\
\text { (MW) }\end{array}$ & $\begin{array}{l}\text { SOLAR } \\
\text { (MW) }\end{array}$ \\
\hline ARIZONA & 6,995 & 23,051 & 3,600 & 400 & 7,350 & 1,200 & 11,220 & 2,000 \\
\hline COLORADO EAST & 4,493 & 11,589 & 2,040 & 300 & 3,780 & 800 & 5,640 & 1,400 \\
\hline COLORADO WEST & 712 & 1,526 & 300 & 0 & 600 & 200 & 900 & 300 \\
\hline NEW MEXICO & 2,571 & 5,320 & 1,080 & 200 & 1,920 & 400 & 2,790 & 700 \\
\hline NEVADA & 3,863 & 12,584 & 2,340 & 200 & 4,680 & 700 & 7,050 & 1,100 \\
\hline WYOMING & 2,369 & 4,016 & 930 & 100 & 1,620 & 100 & 2,340 & 300 \\
\hline IN FOOTPRINT & 21,249 & 58,087 & 10,290 & 1,200 & 19,950 & 3,400 & 29,940 & 5,800 \\
\hline
\end{tabular}

\begin{tabular}{|l|r|r|r|r|r|r|r|r|}
\hline \multicolumn{1}{|l|}{ LOCAL PRIORITY } & & \multicolumn{1}{|c|}{$10 \%$} & \multicolumn{1}{c|}{$1 \%$} & $20 \%$ & $3 \%$ & $30 \%$ & $5 \%$ \\
\hline AREA & $\begin{array}{c}\text { LOAD MIN. } \\
(\mathrm{MW})\end{array}$ & $\begin{array}{c}\text { LOAD MAX } \\
(\mathrm{MW})\end{array}$ & $\begin{array}{c}\text { WIND } \\
(\mathrm{MW})\end{array}$ & $\begin{array}{c}\text { SOLAR } \\
(\mathrm{MW})\end{array}$ & $\begin{array}{c}\text { WIND } \\
(\mathrm{MW})\end{array}$ & $\begin{array}{c}\text { SOLAR } \\
(\mathrm{MW})\end{array}$ & $\begin{array}{r}\text { WIND } \\
(\mathrm{MW})\end{array}$ & $\begin{array}{c}\text { SOLAR } \\
(\mathrm{MW})\end{array}$ \\
\hline ARIZONA & 6,995 & 23,051 & 2,850 & 400 & 5,2550 & 1,200 & 7,710 & 2,000 \\
\hline COLORADO EAST & 4,493 & 11,589 & 2,190 & 300 & 3,870 & 800 & 4,650 & 1,400 \\
\hline COLORADO WEST & 712 & 1,526 & 210 & 0 & 450 & 200 & 570 & 300 \\
\hline NEW MEXICO & 2,571 & 5,320 & 1,350 & 200 & 2,100 & 400 & 2,970 & 700 \\
\hline NEVADA & 3,863 & 12,584 & 1,350 & 200 & 2,490 & 700 & 3,450 & 1,100 \\
\hline WYOMING & 2,369 & 4,016 & 1,650 & 100 & 4,020 & 100 & 7,410 & 300 \\
\hline IN FOOTPRINT & $\mathbf{2 1 , 2 4 9}$ & $\mathbf{5 8 , 0 8 7}$ & $\mathbf{9 , 6 0 0}$ & $\mathbf{1 , 2 0 0}$ & $\mathbf{1 8 , 1 8 0}$ & $\mathbf{3 , 4 0 0}$ & $\mathbf{2 6 , 7 6 0}$ & $\mathbf{5 , 8 0 0}$ \\
\hline
\end{tabular}

\begin{tabular}{|c|c|c|c|c|c|c|c|c|}
\hline \multicolumn{9}{|l|}{ MEGA PROJECT } \\
\hline & & & $10 \%$ & $1 \%$ & $20 \%$ & $3 \%$ & $30 \%$ & $5 \%$ \\
\hline AREA & $\begin{array}{l}\text { LOAD MIN. } \\
\text { (MW) }\end{array}$ & $\begin{array}{l}\text { LOAD MAX. } \\
\text { (MW) }\end{array}$ & $\begin{array}{l}\text { WIND } \\
\text { (MW) }\end{array}$ & $\begin{array}{l}\text { SOLAR } \\
\text { (MW) }\end{array}$ & $\begin{array}{l}\text { WIND } \\
\text { (MW) }\end{array}$ & $\begin{array}{l}\text { SOLAR } \\
\text { (MW) }\end{array}$ & $\begin{array}{l}\text { WIND } \\
\text { (MW) }\end{array}$ & $\begin{array}{l}\text { SOLAR } \\
\text { (MW) }\end{array}$ \\
\hline ARIZONA & 6,995 & 23,051 & 810 & 400 & 1,260 & 1,200 & 1,890 & 2,600 \\
\hline COLORADO EAST & 4,493 & 11,589 & 2,010 & 300 & 2,400 & 800 & 2,490 & 1,200 \\
\hline COLORADO WEST & 712 & 1,526 & 60 & 0 & 90 & 200 & 90 & 200 \\
\hline NEW MEXICO & 2,571 & 5,320 & 1,860 & 200 & 2,700 & 400 & 4,350 & 1,000 \\
\hline NEVADA & 3,863 & 12,584 & 570 & 200 & 1,020 & 700 & 1,440 & 600 \\
\hline WYOMING & 2,369 & 4,016 & 3,390 & 100 & 8,790 & 100 & 13,770 & 100 \\
\hline \multirow[t]{2}{*}{ IN FOOTPRINT } & 21,249 & 58,087 & 8,700 & 1,200 & 16,260 & 3,400 & 24,030 & 5,700 \\
\hline & & & $10 \%$ & $1 \%$ & $20 \%$ & $3 \%$ & $30 \%$ & $5 \%$ \\
\hline $\begin{array}{l}\text { OUT OF FOOT- } \\
\text { PRINT }\end{array}$ & 46,328 & 119,696 & 22,950 & 2,500 & 22,950 & 2,500 & 45,450 & 7,500 \\
\hline
\end{tabular}

\section{TABLE 3 - CAPITAL COSTS (IN US2008\$) FOR STUDY SCENARIOS WITH 30\% WIND ENERGY AND 5\% SOLAR ENERGY} IN THE STUDY FOOTPRINT.

\begin{tabular}{|l|r|r|r|r|r|r|r|}
\hline SCENARIO & \multicolumn{1}{|c|}{$\begin{array}{l}\text { WIND } \\
\text { (MW) }\end{array}$} & $\begin{array}{c}\text { SOLAR } \\
(\mathrm{MW})\end{array}$ & $\begin{array}{c}\text { TRANSMISSION } \\
(\mathrm{GW}-\mathrm{MI})\end{array}$ & $\begin{array}{c}\text { WIND } \\
(\$ \mathrm{~B})\end{array}$ & $\begin{array}{c}\text { SOLAR } \\
(\$ \mathrm{~B})\end{array}$ & $\begin{array}{c}\text { INTERSTATE } \\
\text { TRANSMISSION (\$B) }\end{array}$ & $\begin{array}{c}\text { TOTAL } \\
(\$ \mathrm{~B})\end{array}$ \\
\hline IN-AREA & 29,940 & 5,800 & 0 & 59.9 & 23.2 & 03.1 \\
\hline LOCAL PRIORITY & 26,760 & 5,800 & 2,100 & 53.5 & 23.2 & 3.4 & 80.1 \\
\hline MEGA PROJECT & 24,030 & 5,700 & 6,900 & 48.1 & 22.8 & 11.0 & 81.9 \\
\hline
\end{tabular}


The rest of WECC includes 45,450 MW of wind ( $\$ 91$ billion), 4000 MW of PV ( $\$ 16$ billion), and 3500

MW of CSP (\$14 billion). Intrastate transmission is not included in any of these scenario costs.

\section{ANALYTICAL METHODS}

Four primary analytical methods were used to evaluate the performance of the system with high penetrations of wind and solar generation: statistical analysis, hourly production simulation analysis, sub-hourly analysis using minute-to-minute simulations, and resource adequacy analysis.

Statistical analysis was used to quantify variability due to system load, as well as wind and solar generation over multiple time frames (annual, seasonal, daily, hourly, and 10-minute). The statistical analysis quantified the grid variability due to load alone over several time scales, using the interpolated hourly load data. The changes in grid variability due to wind and solar generation were also quantified for each scenario at various levels of aggregation. The statistical analysis also examined the forecast accuracy for wind generation.

Production simulation analysis with GE's MAPS (Multi-Area Production Simulation) program was used to evaluate hour-by-hour grid operation of each scenario for 3 years with different wind, solar, and load profiles. WECC was represented as a set of 106 zones, each with its own load profile, portfolio of generating plants, and transmission capacity with neighboring areas. The zones were grouped into 20 transmission areas. The production simulation results quantified numerous impacts of additional renewable generation on grid operation including:

- Amount of flexible generation on-line during a given hour, including its available ramp-up and ramp-down capability;

- Effects of day-ahead wind forecast alternatives in unit commitment;

- Changes in conventional generation dispatch;

- Changes in emissions $\left(\mathrm{NO}_{x^{\prime}} \mathrm{SO}_{x^{\prime}}\right.$ and $\left.\mathrm{CO}_{2}\right)$ due to renewable generation;

- Changes in grid operation costs, revenues, and net cost of energy;

- Changes in transmission path loadings;

- Changes in use of hydro resources;

- Changes in use and economic value of energy storage.

Minute-to-minute simulation analysis was used to quantify grid performance trends and to investigate potential mitigation measures during challenging situations, such as large 1-hour, 3-hour and 6-hour changes in net load, high levels of wind and solar penetration, low load levels with minimal maneuverable generation on-line, and/ or high wind forecast errors. Minute-to-minute analysis simulated the operation of dispatchable generation resources as well as variable wind and solar generation in the study footprint using one-minute time steps, while enforcing constraints related to unit maximum, minimum, ramp rate, intertie flow schedule, and regional Automatic Generator Control (AGC) functions. 
Resource adequacy analysis involved loss-of-load-expectation (LOLE) calculations for the study footprint using the Multi-Area Reliability Simulation program, MARS. The analysis quantified the impact of wind and solar generation on overall reliability measures, as well as the capacity values of the wind and solar generation resources.

Impacts on system-level operating reserves were also analyzed using a variety of techniques including statistics, production simulation, and minute-to-minute simulation. This analysis quantified the effects of variability and uncertainty, and related that information to the system's increased need for operating reserves to maintain reliability and security.

The results from these analytical methods complemented each other, and provided a basis for developing observations, conclusions, and recommendations with respect to the successful integration of wind and solar generation into the WestConnect grid.

\section{OPERATIONS WITH $35 \%$ RENEWABLES}

The power system is designed to handle variability in load. With wind and solar, the power system is called on to handle variability in the net load (load minus wind minus solar), which can be considerable during certain periods of the year. Figure 2 shows the load, wind, solar, and net load profiles for the $30 \%$ case during two selected weeks in July and April.

WWSIS finds that $35 \%$ renewable energy penetration is operationally feasible provided significant changes to current operating practice are made, including balancing area cooperation and sub-hourly generation and interchange schedule.
In the July week, (top plot), the net load (blue line at bottom edge) is not significantly impacted by wind and solar variation. However, in the April week (bottom plot), the high, variable wind output dominates the net load, especially during low load

hours, leading to several hours of negative net load during the week. This week in April was the worst week in terms of operational challenges of the three years.

As an example of how the system would operate under less severe operating conditions, Figure 3 shows the generation dispatch for the same July week shown in Figure 2 for the In-Area Scenario. The left figure is without renewable generation and the right is the $30 \%$ case. Although the wind and solar generation are definitely noticeable, they primarily displace combined cycle and gas turbine generation, and have minimal impact on the steam coal units. 

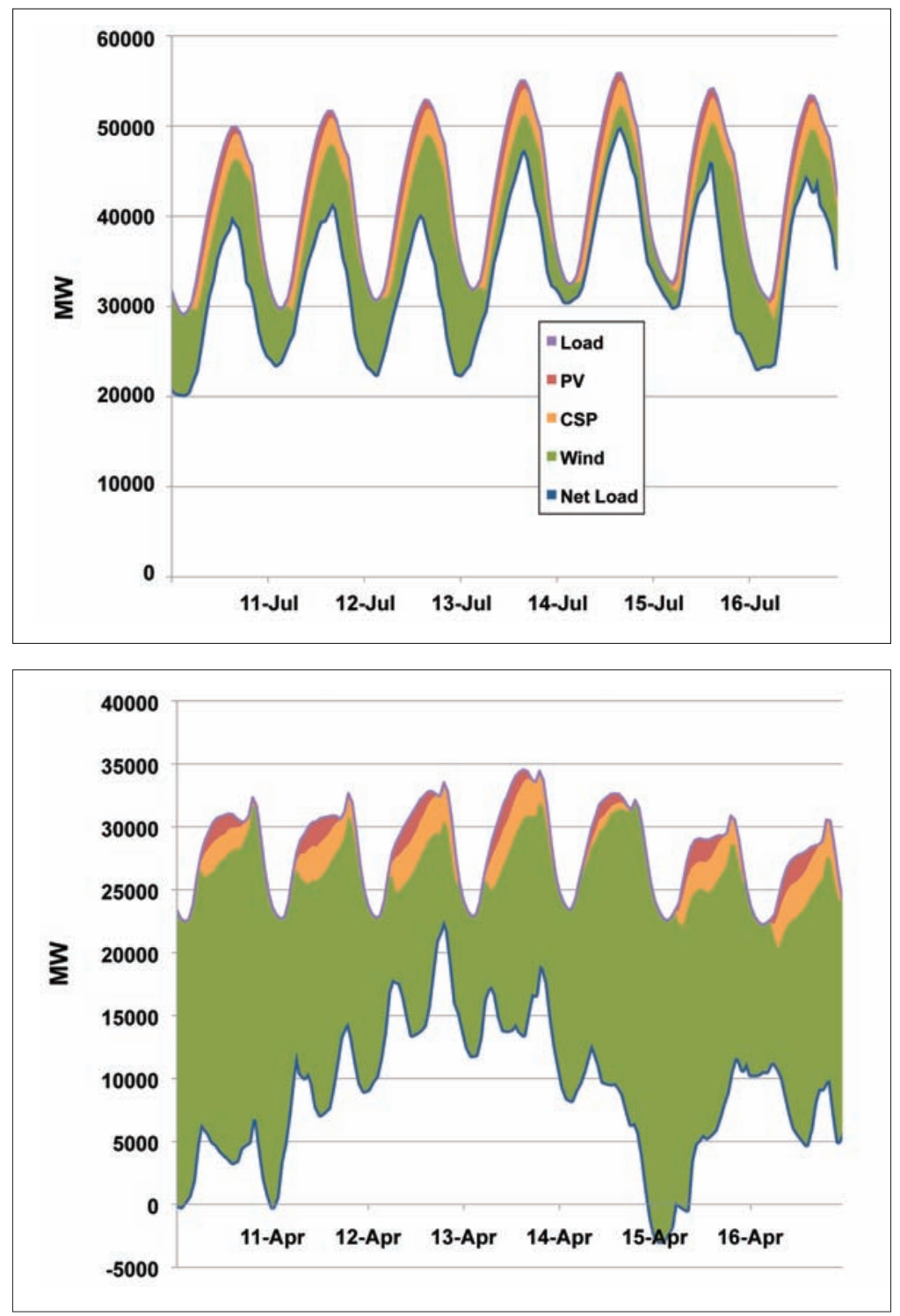

Figure 2 - With 35\% renewables, system operators must now balance generation against the net load (blue) line. This may be straightforward (top, July) or challenging (bottom, April).

Figure 4 shows similar information for the April week shown in Figure 2. Here, operating the system with renewable generation is much more challenging. The combined cycle generation has been almost completely displaced, as have significant levels of coal generation. Nonetheless, the system can operate with balancing area cooperation. Without balancing area cooperation, operations during this week would be extremely difficult, if not impossible, for individual balancing areas.

How much renewable generation can the system handle? All three geographic scenarios show significant benefits with no negative effects in the $10 \%$ case. No significant adverse impacts were observed up to the $20 \%$ case in WestConnect, given balancing area cooperation. Increased renewable generation in the rest of WECC

${ }^{3}$ WECC requires $6 \%$ of load to be held as contingency reserves, half of which is required to be spinning (i.e., synchronized to the grid) reserves. 
(20/20\% case) led to increased stress on system operations within WestConnect, with some instances of insufficient reserves ${ }^{3}$ due to wind and solar forecast error. These can be addressed, but the system has to work harder to absorb the renewables. Operations become more challenging for the $30 \%$ case in which load and contingency reserves are met only if the wind/solar forecasts are perfect. With imperfect forecasts, load is served but there are contingency reserve shortfalls. Extra spinning reserves can be held every hour of the year to meet those contingency reserve requirements, but the cost to hold enough to eliminate all contingency reserve shortfalls is very high. A more costeffective alternative is to establish a demand response program or develop strategies to more accurately predict when these shortfalls occur and schedule more reserves during those hours or add additional quick start generation where needed. In the $20 \%$ and 30\% cases, decreased flexibility of either the coal or hydro facilities made operation more difficult and increased the costs of integrating renewable generation.
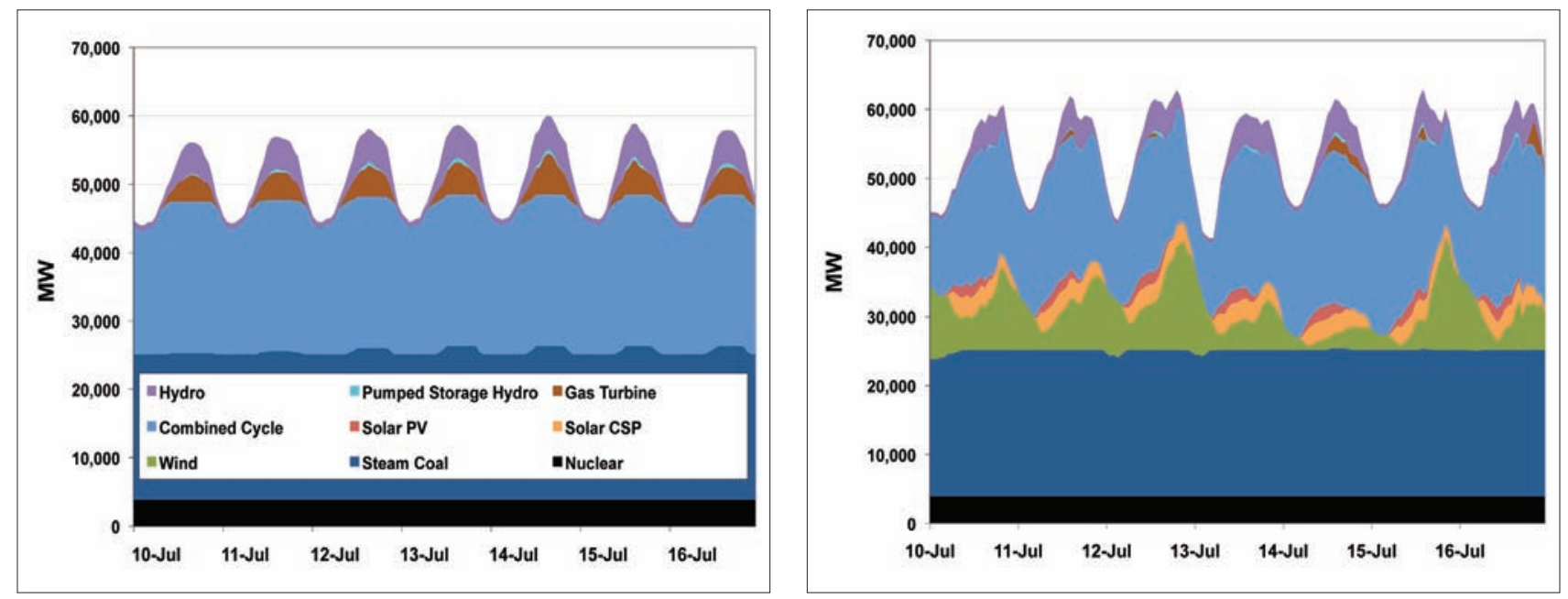

Figure $3-35 \%$ renewables have a minor impact on other generators during an easy week in July, 2006. WestConnect dispatch - no renewables (left) and 30\% case (right)
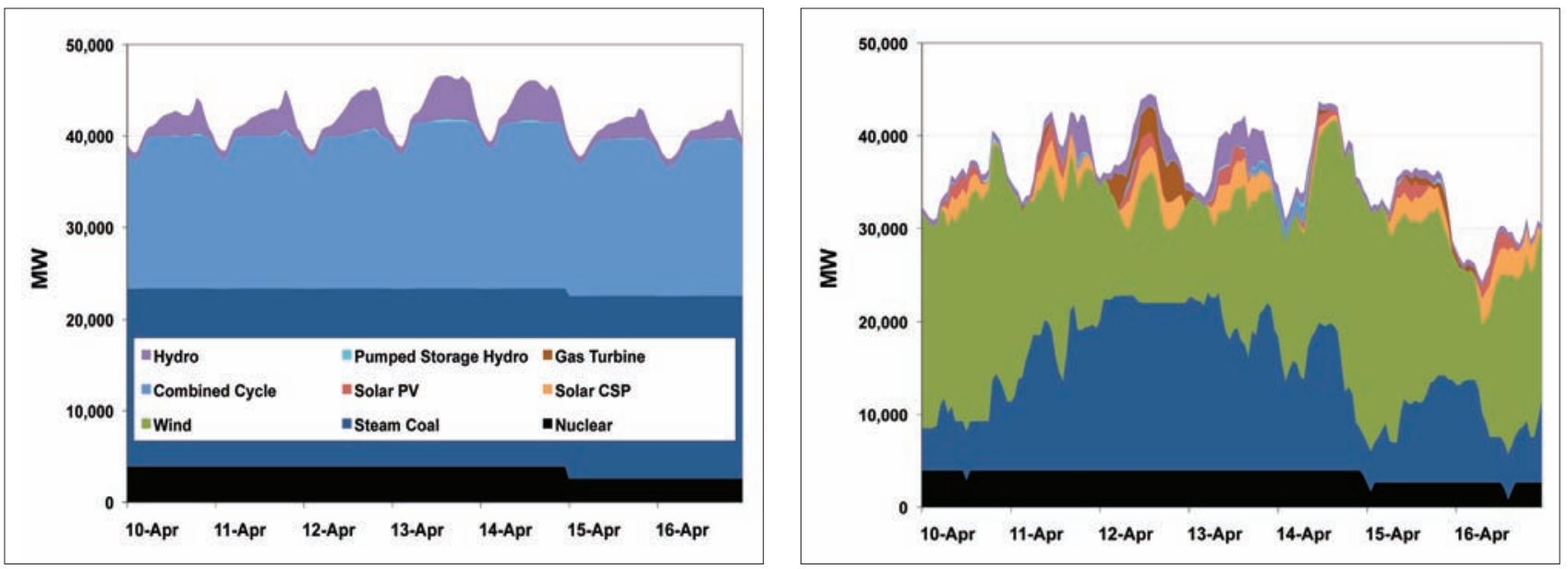

Figure $4-35 \%$ renewables have a significant impact on other generation during the hardest week of the three years (mid-April 2006). WestConnect dispatch - no renewables (left) and $30 \%$ case (right) 


\section{BENEFITS OF 35\% RENEWABLES}

Wind and solar generation primarily displace gas resources nearly all hours of the year, given the fuel prices and carbon tax assumed for this study (\$2/MBTU coal, \$9.50/ MBTU gas, $\$ 30 /$ ton $\mathrm{CO}_{2}$ ). Since gas-fired generation is typically more flexible than coal generation, the natural economic displacement of gas generation by wind and solar generation makes the balance of dispatchable generation on-line less flexible (fewer gas units, more coal units). Across WECC, operating costs drop by $\$ 20$ billion /yr ( $\$ 17$ billion/yr in 2009\$) from approx $\$ 50$ billion/yr ( $\$ 43$ billion/yr in 2009\$), resulting in a $40 \%$ savings due to offset fuel and emissions. This savings does not account for the capital or operating costs associated with the wind,

The $30 \%$ case reduced fuel and emissions costs by $40 \%$ and $\mathrm{CO}_{2}$ emissions by $25-45 \%$ across WECC.

solar, or transmission facilities, nor does it include any of the costs that would be required to implement the operational reforms needed to accommodate the renewables including balancing area cooperation or sub-hourly scheduling, although presumably some of this savings would be used to recover the capital costs of building this scenario, including payments to wind and solar generators. Figure 5 (left plot) shows the overall impact on the operating costs of WECC for the various penetration levels under the In-Area Scenario with a state-of-the-art (SOA) forecast. The 30\% case shows WECC operating cost savings of $\$ 20$ billion/yr ( $\$ 17$ billion/yr in 2009\$) due to the wind and solar generation resources. Figure 5 (right plot) divides these values by the corresponding amount of renewable energy provided. In the $30 \%$ case, this equates to $\$ 80$ / MWh ( $\$ 60 /$ MWh in 2009\$) of wind and solar energy produced. Lower penetrations of renewables showed values up to $\$ 88$ / MWh ( $\$ 75 / \mathrm{MWh}$ in 2009\$) of renewable energy produced (see Section 6.2). These operating cost savings would be applied toward the costs of the wind and solar energy, and depending on the magnitude of these costs, may or may not be sufficient to cover them.
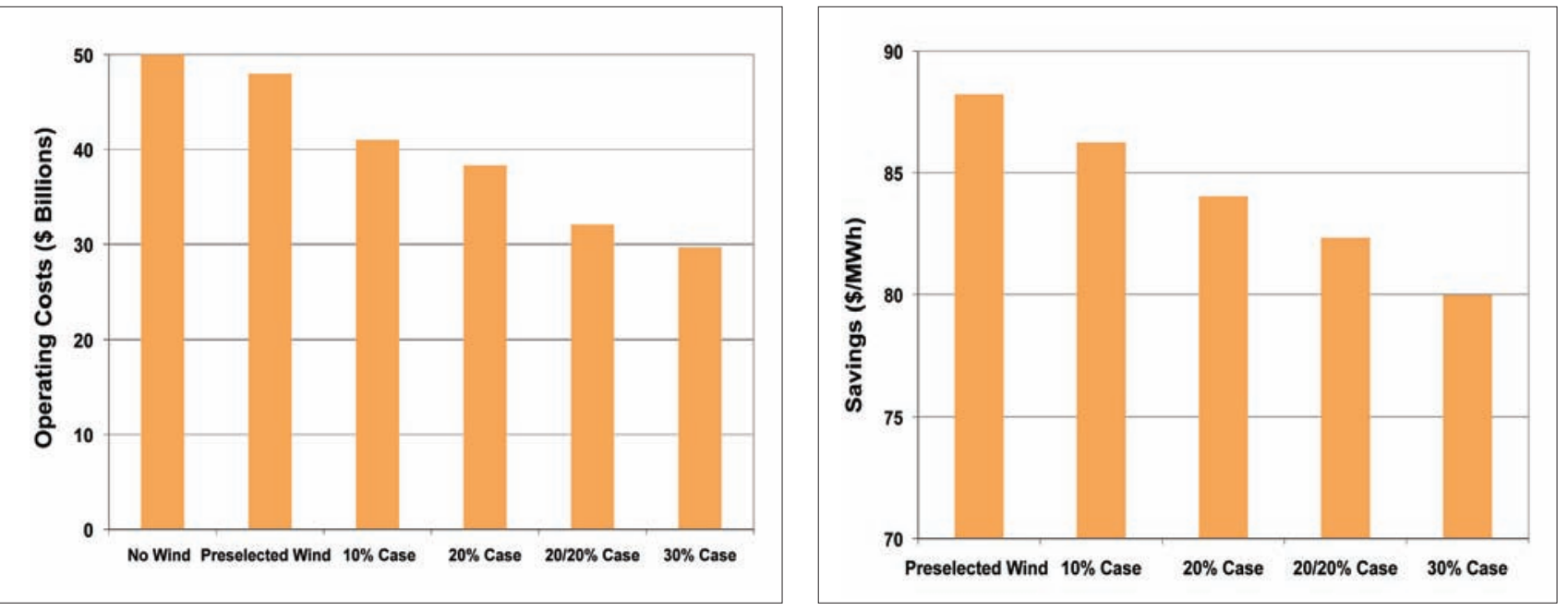

Figure 5 - WECC saves $\$ 20$ billion (\$17 billion in $2009 \$$ ), or $40 \%$, in annual operating costs in the 30\% case, which is equivalent to $\$ 80$ ( $\$ 60$ in 2009\$) per MWh of wind and solar energy produced. Note: Chart on right starts at $\$ 70 / M W h$. 
At a $\$ 3.50$ /MBTU gas price, wind and solar primarily displace coal generation, leaving the more flexible gas generation resources to operate together with the wind and solar generation. With lower gas price assumptions, operating costs are reduced by about $40 \%$, to $\$ 46 / \mathrm{MWh}$ ( $\$ 39 / \mathrm{MWh}$ in $2009 \$)$, but emissions reductions are higher.

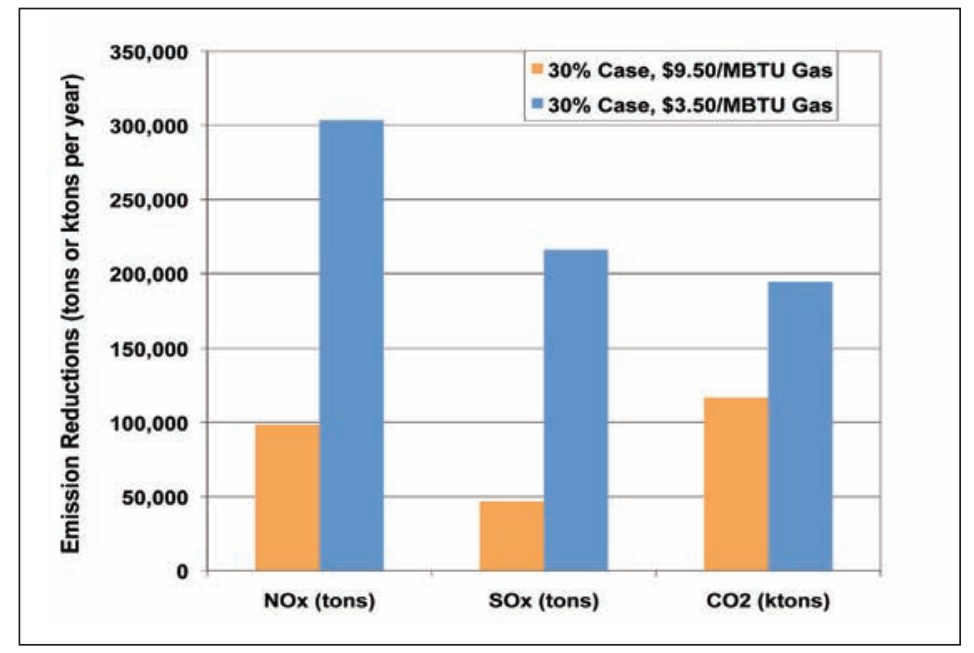

Figure 6 - Assuming \$9.50/MBTU gas, renewable energy displaces gas (orange). At lower gas prices (\$3.50/MBTU), coal is displaced instead, resulting in greater emissions reductions (blue).

Figure 6 shows the total WECC reductions in emissions for the $30 \%$ case. $\mathrm{CO}_{2}$ emissions would be reduced by nearly 120 million tons / year, or approximately $25 \%$, for the $30 \%$ case. $\mathrm{SO}_{x}$ emissions would be reduced by approximately 45,000 tons / year $(\sim 5 \%)$ and $\mathrm{NO}_{\mathrm{x}}$ would be reduced nearly 100,000 tons / year ( 15\%) (see Section 6.2.1). At a $\$ 3.50 / \mathrm{MBTU}$ gas price, $\mathrm{CO}_{2}$ emissions are reduced by nearly 200 million tons / year (45\%), and $\mathrm{NO}_{x}$ and $\mathrm{SO}_{x}$ by 300,000 tons / year (50\%) and 220,000 tons / year $(30 \%)$, respectively.

\section{BALANCING AREA COOPERATION IS ESSENTIAL}

There are three key benefits of balancing area cooperation: 1) aggregating diverse renewable resources over larger geographic areas reduces the overall variability of the renewables, 2) aggregating the load reduces the overall variability of the load, and

The technical analysis performed in this study shows that it is feasible for the WestConnect region to accommodate $30 \%$ wind and $5 \%$ solar energy penetration, but it would require extensive balancing area cooperation or consolidation, real or virtual.
3) aggregating the non-renewable balance of generation provides access to more balancing (and more flexible) resources. Figure 7 shows the reduced-variability benefit arising from aggregating smaller transmission areas into the WestConnect footprint. Variability for small areas such as Colorado-West (CO-W) or Wyoming (WY) increases significantly as renewable penetrations increase from the $10 \%$ to the $30 \%$ case This effect becomes even more extreme at a more granular level, e.g., for specific balancing areas within 
a state (see Section 7.1). However, when the balancing areas across WestConnect are aggregated, there is only a slight increase in variability with increased renewables penetrations, and even a slight decrease in variability WECC-wide.

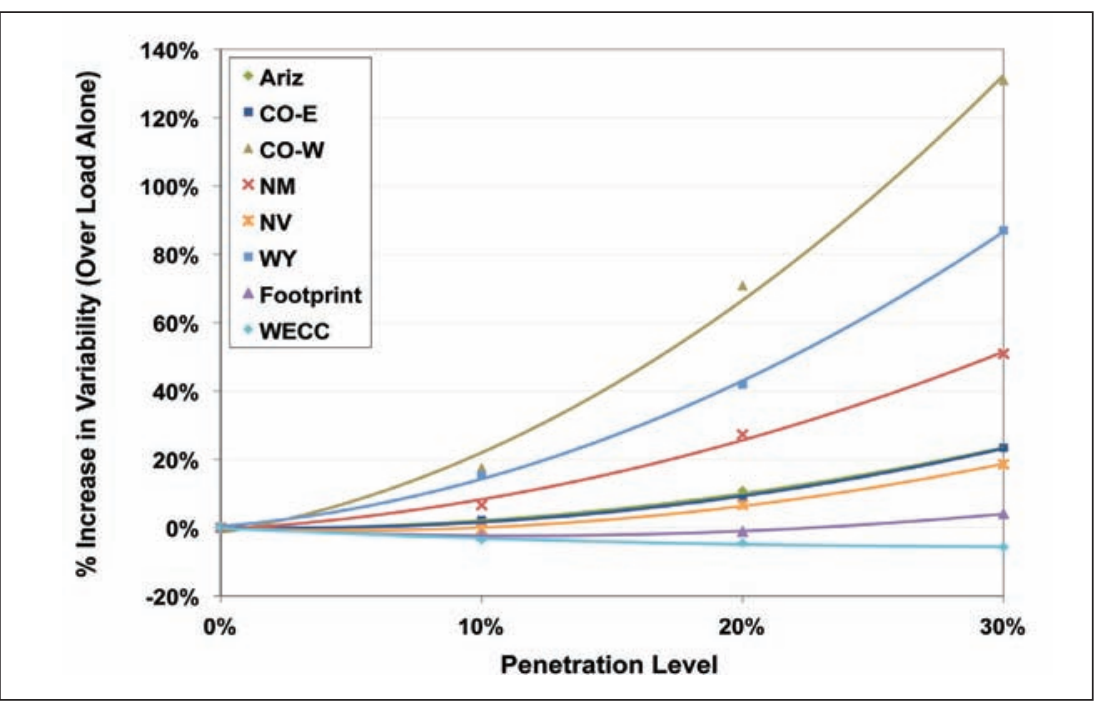

Figure 7-The variability of the net load increases with increasing renewable energy penetration. Aggregating several transmission areas over the WestConnect footprint results in reduced variability. Percent increase in the standard deviation of the hourly changes of the net load in all areas for In-Area Scenario.

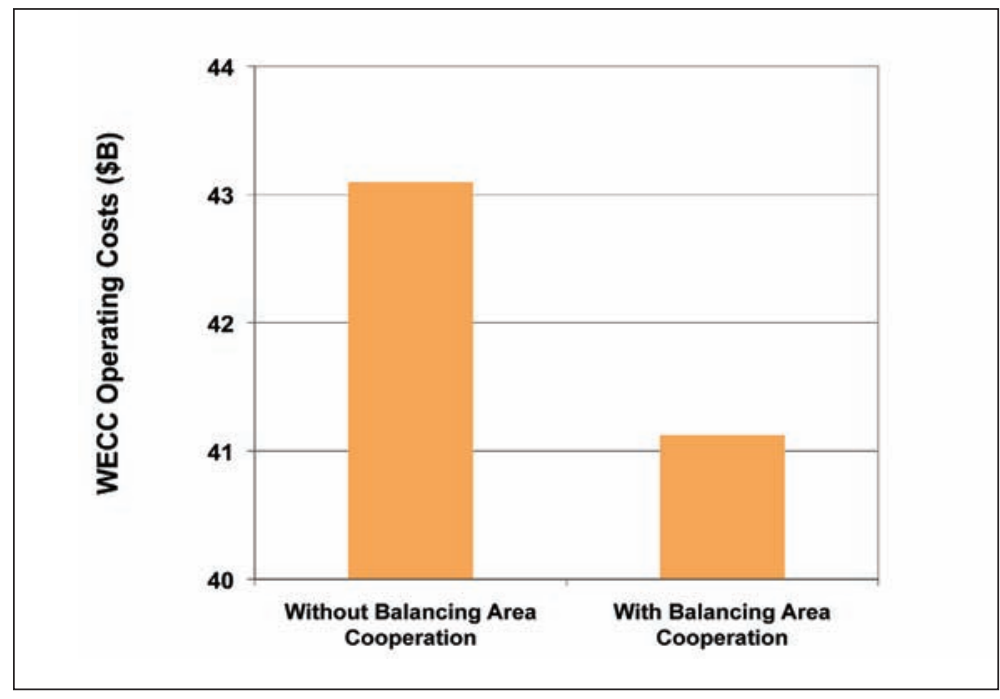

Figure 8 -WECC can save $\$ 2$ billion (\$1.7 billion in 2009\$) by holding spinning reserves as 5 large regions (right) rather than many smaller zones (left).

From an operational perspective, balancing area cooperation can lead to cost savings because reserves can be pooled. A sensitivity analysis was performed, running WECC as 106 zones (which are roughly equivalent to balancing areas in the southwest, but there are multiple zones per balancing area in the northwest) versus 5 large regions. 
Balancing area (BA) cooperation can take many forms and means different things to different people. In WWSIS, cooperation is modeled by assuming:

- All generation resources, across all BAs, are committed from a common regional generation stack on a leastcost basis

- Generation commitments assume physical transmission capability is available for import or export of power transfers between BAs

- All generation dispatches are made on a leastmarginal-cost basis

- All regional reserves are shared across BAs; i.e., the most economic resources for reserves are used

- Day-ahead generation dispatch and inter-area transmission schedules can be modified during operation to enable sharing of load-following, regulation, and reserves

Mechanisms to enable these aspects of cooperation are numerous, and include facets currently used or proposed in WECC such as the ACE diversity interchange (ADI), dynamic scheduling, an energy imbalance service, and other means of consolidating BA services. Many technical and institutional barriers will need to be addressed to achieve the level of cooperation of the work presented here.
Figure 8 shows the $\$ 2$ billion ( $\$ 1.7$ billion in 2009\$) savings in WECC operating costs in the $10 \%$ case. There are significant savings from sharing reserves over larger regions, irrespective of the renewables on the system.

\section{SUB-HOURLY SCHEDULING IS CRITICAL}

The current practice of scheduling both the generation and interstate exchange only once each hour has a significant impact on the regulation duty. At high penetration levels, such hourly schedule changes can use most, if not all, of the available regulation capability to compensate for Area Control Error (ACE) excursions during large scheduled ramps. This can leave no regulation capability for the sub-hourly variability.

The minute-to-minute simulations showed that the current practice of

hourly scheduling has a greater impact on the regulation requirements than does the wind and solar variability.

Sub-hourly scheduling can substantially reduce the maneuvering duty imposed on the units providing load following. In the $30 \%$ case, the fast maneuvering of combined cycle plants with sub-hourly

Sub-hourly scheduling will be required to successfully operate the system at high penetration levels without significantly increased regulating reserves. scheduling is about half of that with hourly scheduling, as shown in Figure 9. Sub-hourly scheduling in the $30 \%$ case is roughly equivalent to the $20 / 20 \%$ case with hourly scheduling. Improvements in plant efficiency and reductions in O\&M costs, while difficult to quantify, are expected from this smoother operation. 


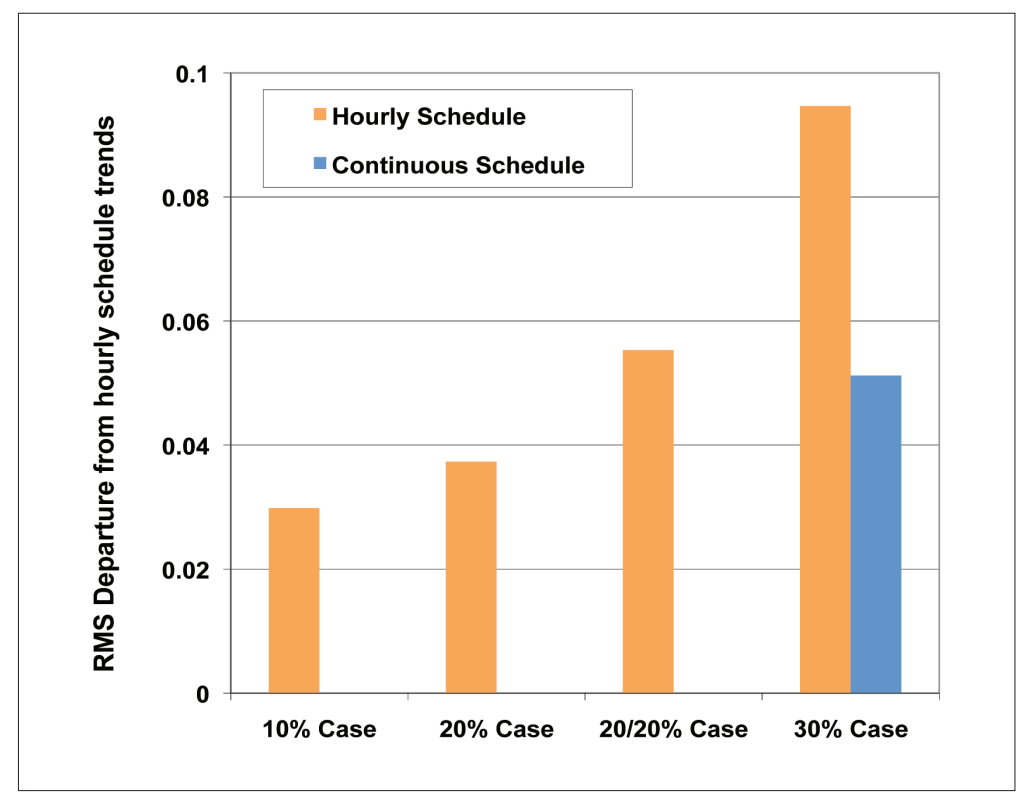

Figure 9 - Fast maneuvering duty of combined cycle units can be cut in half by moving from hourly to sub-hourly scheduling.

\section{UNCERTAINTY (FORECAST ERROR) RESULTS}

\section{IN THE BIGGEST IMPACT ON THE SYSTEM}

Integrating day-ahead wind and solar forecasts into the unit commitment process is essential to help mitigate the uncertainty of wind and solar generation. Even though SOA wind and solar forecasts are imperfect and sometimes result in reserve shortfalls due to missed forecasts, it is still beneficial to incorporate them into the day-ahead scheduling process, because this will reduce the amount of shortfalls. Over the course of the year, use of these forecasts reduces WECC operating costs by up to $14 \%$, or $\$ 5$ billion/yr ( $\$ 4$ billion/yr in $2009 \$$ ), which is $\$ 12-20 /$ MWh ( $\$ 10-$ 17/ MWh in 2009\$) of wind and solar generation. The left side of Figure

Using state-of-the-art wind and solar forecasts in day-ahead unit commitment is essential and would reduce annual WECC operating costs by up to $\$ 5$ billion (\$4 billion in 2009\$) or \$12-20/MWh (\$10-17/ MWh in 2009\$) of renewable energy, compared to ignoring renewables in the unit commitment process. Perfect forecasts would reduce annual costs by another $\mathbf{\$ 5 0 0}$ million (\$425 million in $\mathbf{2 0 0 9} \$$ ) or $\mathbf{\$ 1 - 2 /}$ MWh (\$0.9-\$1.7/MWh in 2009\$) of renewable energy. 10 shows the WECC-wide operating cost savings for using SOA forecasts compared to ignoring wind in the day-ahead commitment. The right side shows the incremental cost savings for perfect wind and solar day-ahead forecasts, which would reduce WECC operating costs by another $\$ 500$ million/yr (\$425 million/yr in 2009\$) in the 30\% case (see Section 6.2.1), or \$1-2/ MWh (\$0.9-1.7/ MWh in 2009\$) of wind and solar generation. 

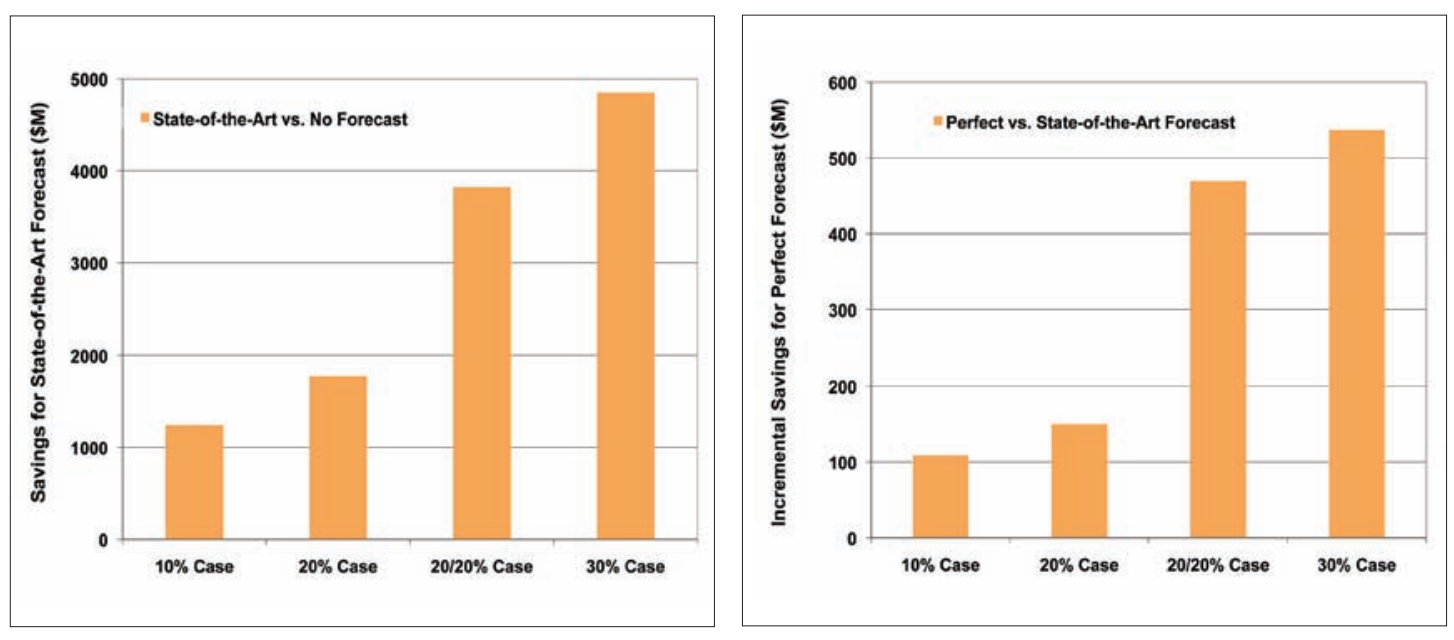

Figure 10 - WECC saves $\$ 1-5$ billion (\$1-4 billion in 2009\$) in annual operating costs just by using a SOA day-ahead forecast in the unit commitment process (left). Incremental savings for perfect forecasts are an order of magnitude less (right).

\section{THE IMPACTS OF EXTREME FORECAST ERRORS ON CONTINGENCY RESERVE SHORTFALLS}

While on average, wind forecast error is not very large (8\% mean absolute error across WestConnect), there are hours when wind forecast errors can be extreme, ranging up to over 11,000 MW of over- or under-forecast in WestConnect. Severe over-forecasts can result in contingency reserve shortfalls; severe under-forecasts can result in curtailment of wind.

Operating rules dictate that systems must carry contingency reserves to cover system events, such as tripping of a large generator. In WECC, the spinning portion of these contingency reserves is equivalent to 3\% of the system load. Applying these WECC rules, severe over-forecasts can lead to under-commitment of generation units, which can result in contingency reserve shortfalls if insufficient quick-start capacity is available.

If the forecast is perfect, there are no contingency reserve shortfalls, even in the $30 \%$ case. With a SOA forecast, Figure 11 shows that these contingency reserve shortfalls become an issue in the $30 \%$ case. It should be noted, however, that even these shortfalls represent only a tiny percentage $(\sim 0.005 \%)$ of the total load energy. 


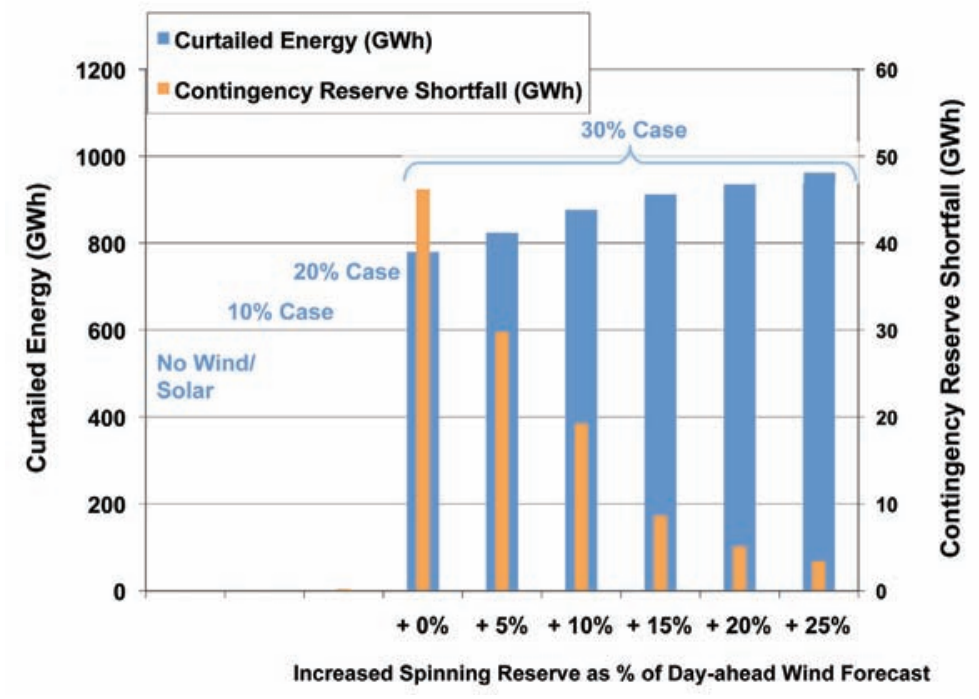

Figure 11 - Contingency reserve shortfalls start to become an issue in the $30 \%$ case. Increasing spinning reserve can reduce the shortfalls but even increasing spinning reserves by $25 \%$ of the day-ahead wind forecast does not completely eliminate reserve shortfalls. Hourly production simulation analysis shows spilled energy, or curtailment, on the left axis and contingency reserve shortfalls on the right axis for the In-Area Scenario with no wind/solar, the 10,20 , and $30 \%$ case for a SOA forecast. The five bars on the right show the effect of increasing spinning reserve by $5,10,15,20$, and $25 \%$ of the day-ahead wind forecast.

Spinning reserves can be increased to cover these contingency reserve shortfalls, but at a cost. Figure 11 shows the impact of increasing spinning reserves by 5, 10, 15, 20 and $25 \%$ of the day-ahead wind forecast. However, each additional $5 \%$ increment of committed spinning reserve is increasingly expensive, as shown in Figure 12, and even with a $25 \%$ increase in committed spinning reserves, not all contingency reserve shortfalls are eliminated.

The average cost of increasing reserves is shown in Figure 12. Increasing the committed spinning reserve by $5 \%$ of the wind forecast increases WECC operating costs by over $\$ 3,000$ per MWh $(\$ 2,550 / \mathrm{MWh}$ in 2009 ) of reduced reserve shortfall. Expressed another way, it would be comparable to pay some of the load $\$ 3,000$ / MWh (\$2,550/ MWh in 2009\$) to drop off rather than increasing the spinning reserve by $5 \%$ of the forecast. At the other extreme, if spinning

It is more cost-effective to have demand response address the 89 hours of contingency reserve shortfalls rather than increase spin for $\mathbf{8 7 6 0}$ hours of the year. Demand response can save up to $\$ 600 \mathrm{M} /$ yr (\$510M/yr in 2009\$) in operating costs versus committing additional spinning reserves. reserve is increased by $25 \%$, it would cost an average of roughly $\$ 13,600$ / MWh $(\$ 11,600 /$ MWh in $2009 \$)$ of reserve shortfall. The incremental reduction achieved by increasing the spinning reserve from $20 \%$ to $25 \%$ of the forecast would cost over $\$ 100,000 / \mathrm{MWh}(\$ 85,000 / \mathrm{MWh}$ in $2009 \$)$. It should 
be more economic to use load participation (i.e., demand response) than to increase the spinning reserves to achieve the same objectives. Using load participation instead of committing additional generation for operating reserves would save up to $\$ 600$ million (\$510 million in 2009\$) in operating costs per year (see Sections 5.4, 7.2, and 6.2.2).

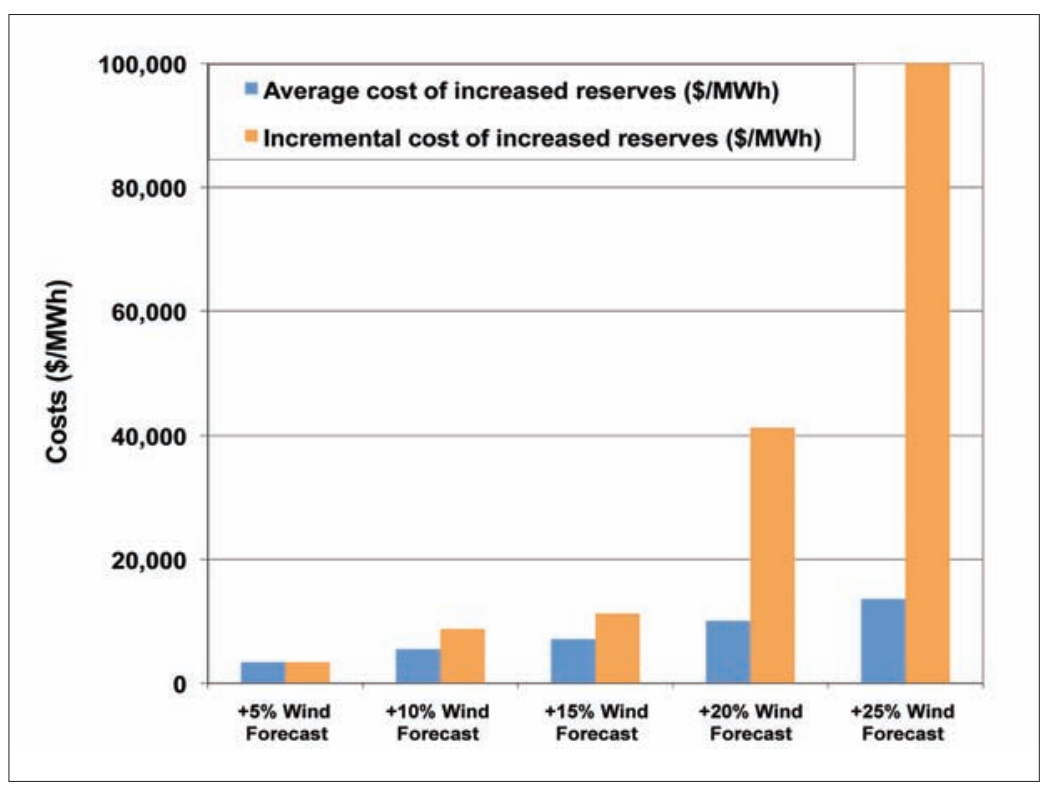

Figure 12 - The cost of increasing spinning reserves increases with higher percentages of spin. The incremental cost increases sharply at higher percentages of spin, indicating that the cost of reducing those final reserve shortfalls is prohibitively high. The five bars show the effect of increasing spinning reserve by $5,10,15,20$, and $25 \%$ of the day-ahead wind forecast.

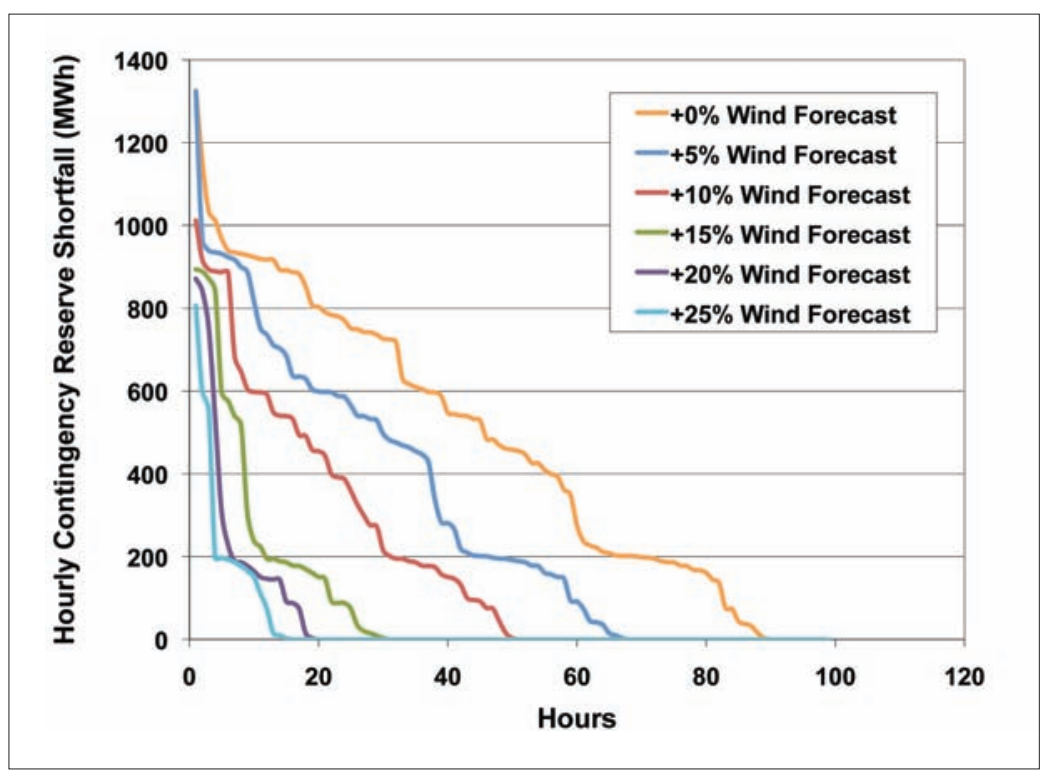

Figure 13 - A demand response program which requires load to participate in the 89 hours of the year that there are contingency reserve shortfalls is more cost-effective than increasing spin for each of the $\mathbf{8 7 6 0}$ hours of the year. Hourly contingency reserve-shortfall duration curves for the In-Area 30\% case with a SOA forecast with no additional spinning reserves, and then with spinning reserves increased by $5,10,15,20$, and $25 \%$ of the day-ahead wind forecast. 
Instead of holding additional spinning reserve for each of the 8760 hours of the year, Figure 13 shows that a demand response program could address those 89 hours of the year when there is a contingency reserve shortfall and have a total participation of approximately $1300 \mathrm{MW}$ of load. The contingency reserve shortfalls could also be met by a combination of increased spinning reserves and a smaller demand response program. An alternative to demand response or increased spinning reserve for every hour of the year could be dynamic allocation of spinning reserves based on better forecasting, improved reserve policies, and more accurate prediction of when shortfalls are likely to occur.

\section{HOW OFTEN IS WIND CURTAILED?}

Uncertainty drives both curtailment and reserve shortfalls. With a perfect forecast, no wind or solar curtailment was necessary in any of the scenarios. Even in the few hours when the renewable generation exceeded the load in WestConnect, there was sufficient flexibility within WECC to absorb all of the generation. With a SOA forecast, no curtailment occurred up through the $20 \%$ case (see Figure 11 ). The hourly production simulations showed about $800 \mathrm{GWh}$ of wind curtailment in the $30 \%$ case, representing less than $0.5 \%$ of the total wind energy production. In addition, the minute-to-minute analysis indicated that more wind curtailment may be required under some combinations of low load and high wind. Altogether, wind curtailment in the 30\% case is estimated to be on the order of $1 \%$ or less of the total wind energy. Curtailment is also affected by flexibility of the balance of generation, e.g., raising the minimum operating point of the coal units to $70 \%$ increased the wind curtailment slightly (see Sections 6.2 and 6.4.4).

\section{THE EFFECT OF VARIABILITY -}

\section{ARE ADDITIONAL RESERVES NECESSARY?}

In addition to contingency reserves, utilities are required to hold variability or load following reserves to cover 10-minute load variability $95 \%$ of the time. Typically, utilities do not commit additional variability reserves because the existing dispatchable generating fleet can adequately cover this variability reserve requirement. With wind and solar, the net load variability increases and in the $30 \%$ case, the average variability reserve requirement doubles. However, when wind and solar are added to the system, thermal units are backed down because it is sometimes more economical to back down a unit rather than to decommit it.

This results in more up-reserves available than in the case when there is no wind and solar, as shown in Figure 14. Therefore, commitment of additional reserves is not needed to cover variability in the While the need for variability reserves doubles in the $30 \%$ wind case, the backing down of conventional units results in more available up-reserves. Therefore, commitment of additional reserves is not needed to cover the increased variability.

study footprint. Figure 14 shows a duration curve of the total amount of up-reserves in the committed generation after the contingency reserve requirement is subtracted out, showing that $95 \%$ of the time, there are adequate up-reserves in the $30 \%$ Local Priority case. 


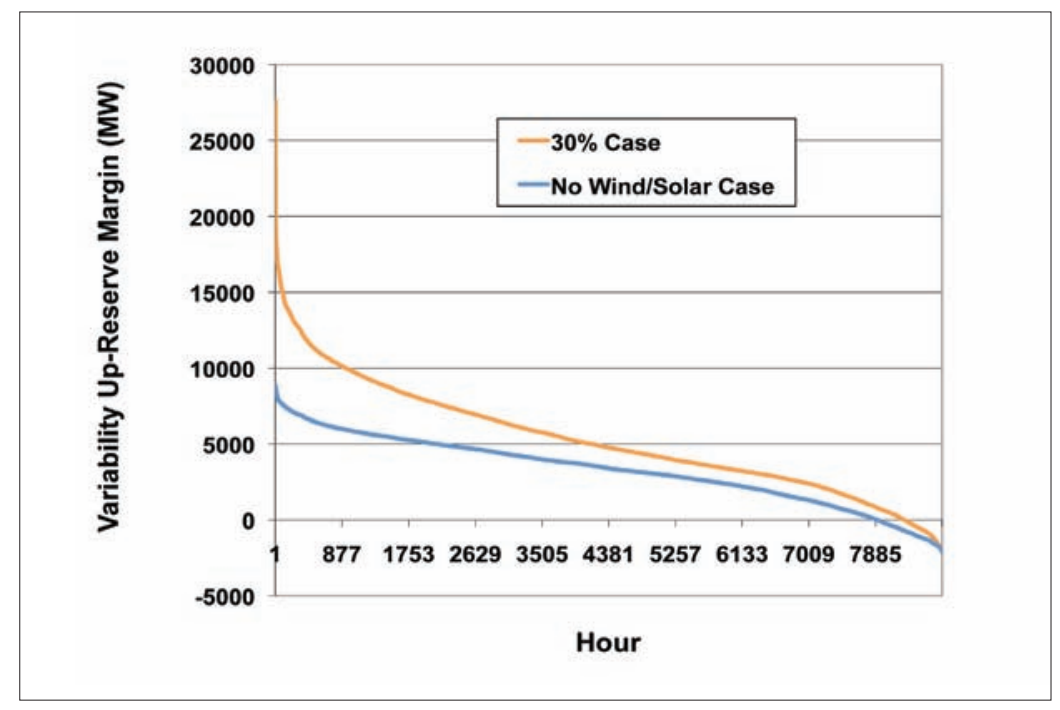

Figure 14 - There are more up-reserves available in the $30 \%$ case than in the no wind/solar case because the additional renewable energy generation causes many conventional units to be backed down. Variability Up-Reserve Margin - Local Priority 30\% vs. No Wind or Solar Case.

Regulating reserves are a subset of the fast variability requirement, but are held separately from the 10-minute variability reserves. Regulating reserves are required to be automatically controlled through AGC. While WWSIS did not evaluate which units were on AGC, the minute-to-minute analysis showed that sufficient regulating reserve capability was available in WestConnect.

Down reserves can be handled through wind curtailment when other resources are depleted. A wind plant can reduce its output very quickly in response to a command signal. Simulations in this study

Wind plants can be curtailed to provide down regulating reserves instead of moving regulating units. Even so, curtailment is estimated to be on the order of $1 \%$ or less of total wind energy in the $30 \%$ case. show that down reserves can be implemented through command signals (ACE signals) from system operators. With extensive balancing area cooperation, WestConnect can accommodate large amounts of

renewables, and curtailment of wind is expected to be on the order of $1 \%$ or less in the $30 \%$ case.

\section{WHAT IS THE EFFECT OF DIFFERENT TRANSMISSION AND GEOGRAPHIC SCENARIOS?}

The In-Area, Local Priority, and Mega Project Scenarios showed similar overall performance and economics for a given penetration level. This indicates that the specific locations of the wind and solar resources within WestConnect are not critical, provided there is adequate transmission infrastructure and access, and balancing area cooperation (see Sections 4.2.3, 5.5, 6.4.1, 6.4.6, 7.3.1). The assumption that existing transmission capacity can be fully utilized is an important change from present practice underpinning these results. 
Figure 15 shows the study footprint's monthly wind and solar energy as a percentage of load energy for all three scenarios in the 30\% case in 2006.

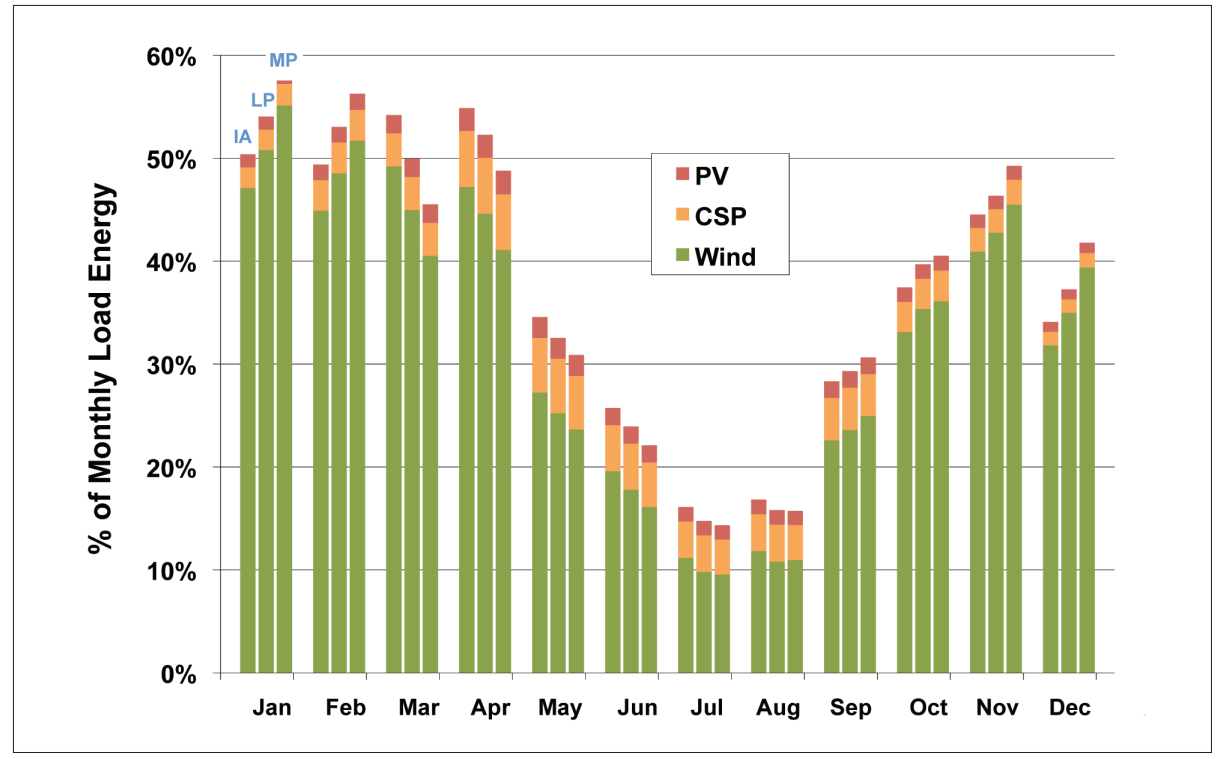

Figure 15 - The month-to-month variation of wind and solar penetration is greater than the scenario-to-scenario variation.

The plots clearly illustrate that 1) despite the month-to-month variation, there is relatively little difference among scenarios at the footprint resolution and 2) there is significant month-to-month variation in energy across the year. In fact, there is more interannual variation in each month's penetration levels than there is inter-scenario variation (see Section 4.1.1-4.1.2)

The total WECC operating cost savings per MWh of renewable energy for the different scenarios was also very similar across the three geographic scenarios, with only a slight increase in value as the wind plant locations were shifted to the higher capacity factor sites in the Local Priority and Mega Project Scenarios (see Section 6.4.1)

\section{IS NEW LONG DISTANCE TRANSMISSION NEEDED?}

Sufficient intra-area transmission within each state or transmission area for renewable energy generation to access load or bulk transmission is needed. However, the InArea Scenario, which included no additional long distance, interstate transmission, worked just as well operationally as the other scenarios. A sensitivity case examined the impact of the interstate transmission Up to $20 \%$ renewable penetration could be achieved with little or no new long distance, interstate transmission additions, assuming full utilization of existing transmission capacity. build-outs in the Local Priority and Mega Project Scenarios (which required \$3.4 and \$11 billion dollars, in 2008\$, of interstate transmission respectively). Figure 16 shows the increased annual operating 
costs for the cases in which the new interstate transmission build-outs associated with the Local Priority and Mega Project Scenarios were eliminated. These increased costs are modest because renewables have displaced other generation and freed up transmission capacity. Assuming renewables have full access to this newly opened up capacity, there is less need for new transmission.

Assuming a 15\% fixed charge rate, the 30\% Local Priority Scenario would justify about $\$ 2$ billion ( $\$ 1.7$ billion in 2009\$) in transmission investments and the Mega Project Scenario would justify a little over $\$ 10$ billion ( $\$ 8.5$ billion in $2009 \$$ ). This rough estimate suggests that the full-scale transmission build-out might be justified in the 30\% Mega Project Scenario, but not at lower penetrations in the Mega Project or for any of the other scenarios. A more limited transmission build-out may be justified for the Local Priority Scenario. Of course, these estimates do not include any reliability benefits that would be realized from adding more transmission. All scenarios could be built out to the $10 \%$ case without any new interstate transmission (see Section 6.4.6).

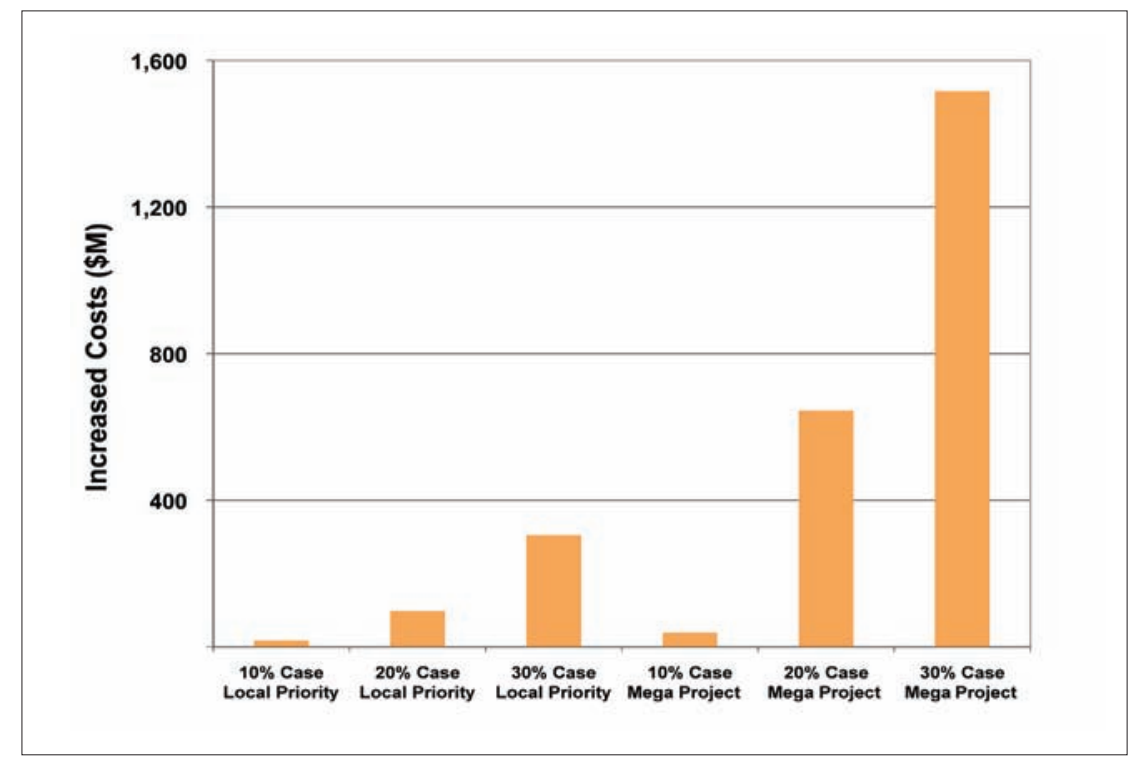

Figure 16 - Building the Local Priority and Mega Project Scenarios without the accompanying interstate transmission, increases costs at high penetrations in the Mega Project Scenario.

\section{IS ADDITIONAL STORAGE NEEDED?}

Storage can provide many benefits to the system, including price arbitrage (charging when spot prices are low and discharging when prices are high), reliability, and ancillary services. Pumped storage hydro (PSH), solar thermal storage, and plug-in hybrid electric vehicles (PHEVs) were examined in WWSIS, with the largest focus on PSH (see Chapter 8). WWSIS evaluated only the price arbitrage part of the value proposition for PSH and found it much less than sufficient to economically justify additional storage facilities. 
In the $10 \%$ and $20 \%$ wind penetration scenarios, gas generation is always on the margin (meaning that there are only small spot price variations during most days). As a result, there is no apparent opportunity to economically justify energy storage based on price arbitrage. Spot price variations increase in the $30 \%$ wind penetration scenarios, primarily due to errors in day-ahead wind energy forecasts. Occasionally, the price swings are very large. However, because this is driven by forecast uncertainty, it is not possible to strategically schedule the use of storage resources to take advantage of the price variations (and subsequently help eliminate the operational problems due to wind forecast errors).

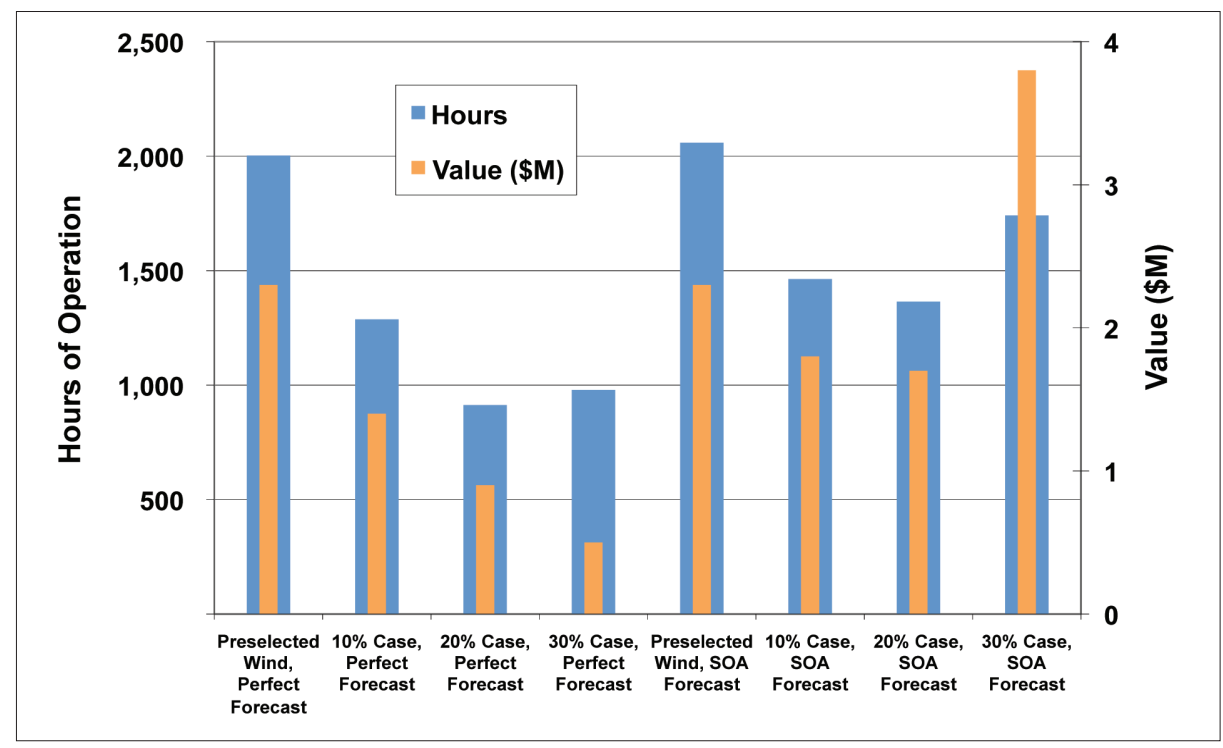

Figure 17-A new 100-MW PSH plant with perfect pricing foresight would earn approximately $\$ 4$ million/yr (\$3.4million/yr in $2009 \$$ ) from price arbitrage in the $30 \%$ case.

To examine a best-case scenario for storage, a new 100-MW PSH plant was added to the system and given perfect foresight of spot prices so that it could be dispatched to optimize revenue. The results in Figure 17 show the resulting number of operating hours and value. With no renewables, the PSH unit would run about 2200 hours (total pumping and generating time) and have an operating value of about $\$ 2.6$ million (\$2.2 million in 2009\$) for the year. With a perfect forecast, the value of the PSH unit decreased as the renewable penetration increased, due to decreased spot prices. With $30 \%$ penetration and a perfect forecast the 100-MW PSH plant only had an annual operating value of $\$ 0.5$ million ( $\$ 0.4$ million in $2009 \$$ ) which would only yield a capitalized value of about $\$ 35 / \mathrm{kW}$ ( $\$ 30 / \mathrm{kW}$ in 2009\$). With an SOA forecast, spot prices are higher due to forecast error, and the $30 \%$ case increased the $\mathrm{PSH}$ annual operating value to $\$ 3.8 \mathrm{M}$ ( $\$ 3.2 \mathrm{M}$ in $2009 \$)$. However, this is several times less than would be required to recover costs for a new PSH plant ${ }^{4}$ (see Section 8). 


\section{WHAT IS THE BENEFIT OF FLEXIBILITY IN THE REST OF THE GENERATION FLEET?}

System flexibility is the key to accommodating increased renewable generation. WWSIS finds that at higher ( $30 \%$ case) penetration levels, decreased flexibility of either the coal or hydro facilities made operation more difficult and increased the costs of integrating the renewable generation.

\section{ALLOWING HYDRO TO PROVIDE LOAD FOLLOWING FOR WIND/SOLAR VARIABILITY IS HELPFUL}

Hydro generation is capable of quick start/ stop cycling and fast ramping, which makes it a good partner for variable wind and solar generation. Sensitivity analyses were conducted to examine the effects of hydro constraints on operating costs (see Section 6.4.2).
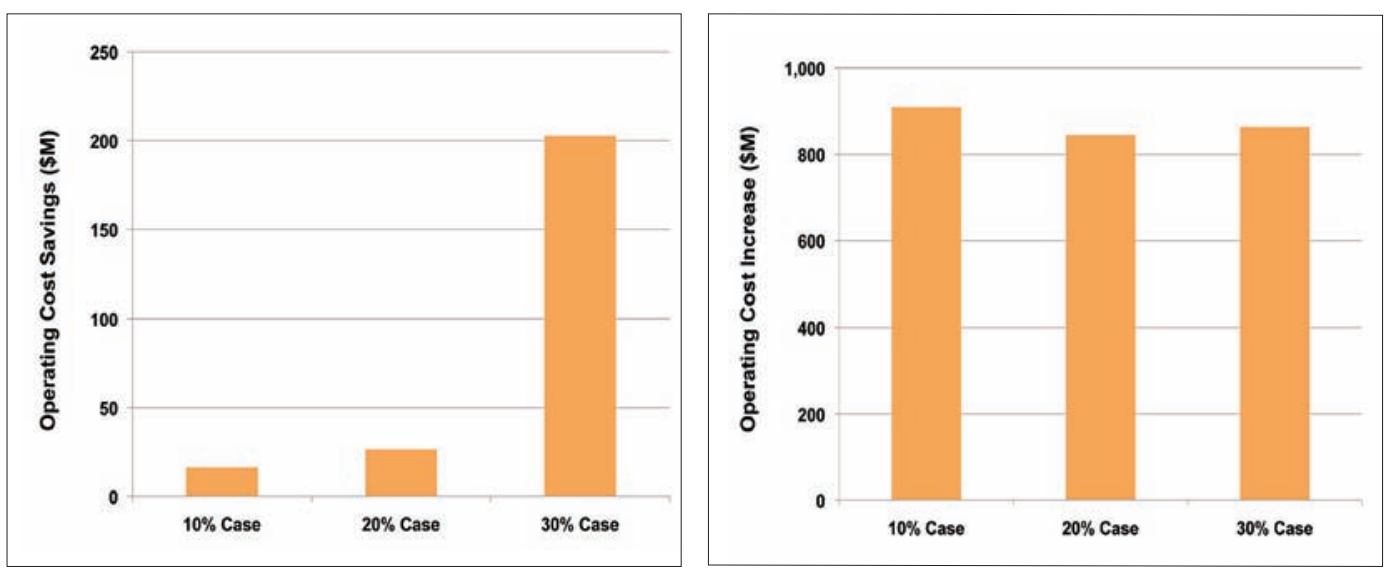

Figure 18 - Decreasing the flexibility of the hydro system increases costs. Operating cost savings for hydro dispatch to net load (left), and operating cost increase for constant output hydro operation (right), WECC.

This study assumed that hydro generation is normally committed and dispatched to serve daily peak net-load periods, while respecting the minimum operating points on the hydro units. The left side of Figure 18 shows the impact of adjusting the hydro schedules to account for the day-ahead renewable forecasts. Although the impact is relatively small at low levels of penetration, the WECC operating costs would be reduced by $\$ 200$ million/yr ( $\$ 170$ million/yr in 2009\$) at the 30\% case, increasing the value of wind and solar energy by about $\$ 1 / \mathrm{MWh}$ (\$0.9/MWh in 2009\$).

The right side of Figure 18 examines the impact if hydro operation were severely constrained, such as a requirement to maintain constant river flow. In this case, the WECC operating costs would increase by up to $\$ 1$ billion/yr ( $\$ 0.9$ billion/yr in 2009\$). Clearly it is important to maintain as much operational flexibility as possible with the hydro generation (see Section 6.4.2).

${ }^{4}$ Assuming $\$ 1200-2000 / \mathrm{kW}$ capital cost and a fixed charge rate of $15 \%$ for a new PSH, \$18-30 million annually would be needed to recover capital costs. 


\section{CONSTRAINTS ON COAL PLANTS RESULT IN HIGHER OPERATING COSTS}

In WWSIS, coal plants were assumed to be able to operate down to minimum generation levels of $40 \%$ of nameplate capacity. WWSIS finds that higher minimum generation levels result in increased operating costs.

A sensitivity case explored the impact of varying coal plant minimum loading on system operating costs. Increasing the minimum loading had minimal impact with wind penetrations less than $20 \%$. At the $30 \%$ scenario, the impact becomes more noticeable, as shown in Figure 19. If coal plants are allowed to only operate above $70 \%$ load, then WECC operating costs would increase by nearly $\$ 160$ million / yr ( $\$ 136$ million/yr in 2009\$). See Section 6.4.4.

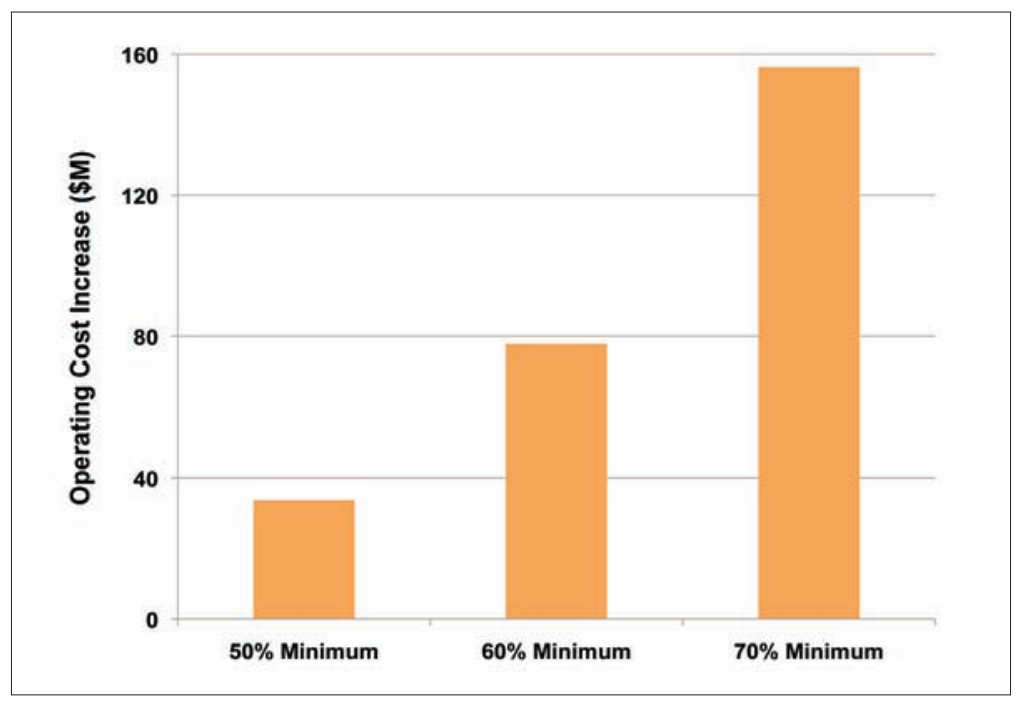

Figure 19 - Decreasing the flexibility of the coal fleet by increasing minimum generation levels on coal plants increases costs. Increased WECC operating costs over $40 \%$ minimum ratings on coal plants, $30 \%$ case.

\section{WHAT IS THE CONTRIBUTION OF RENEWABLES TO RESOURCE ADEQUACY?}

Variable resources such as wind and solar PV are primarily energy resources rather than capacity resources. However, they provide some contribution to reliability (resource adequacy). A range of capacity valuation techniques based on traditional lossof-load-expectation (LOLE) data were evaluated to consider the variability inherent with the renewable generation. This was conducted for WestConnect assuming no transmission constraints within the study footprint and no interconnections with the rest of WECC, so that the capacity value characteristics of the renewable generation could be isolated. 
Table 4 shows capacity values of wind based on daily LOLE which were typical of the overall analysis. Wind generation resources selected for this study were found to have capacity values in the range of $10 \%$ to $15 \%$. Wind plant energy output tends to be higher during winter and spring

Wind was found to have capacity values of $10-15 \%$; PV was 25-30\%; and CSP with 6 hours of thermal energy storage was $90-95 \%$. seasons, and during nighttime hours, which is contrary to system peak load periods. Hence, the capacity value is low relative to the plant rating. PV solar plants have

capacity values in the range of $25 \%$ to $30 \%$. Although PV solar produces its energy during the daytime, output tends to decline in the late afternoon and early evening when peak load hours often occur. The PV output was based on the DC rating of the system; it would be $23 \%$ higher if based on the AC rating and included inverter and other losses from the outset. Concentrating solar plants with thermal energy storage have capacity values in the range of $90 \%$ to $95 \%$, similar to thermal generating plants. Their maximum energy production tends to be during the long summer days, and the storage capability extends the energy output through the late afternoon and early evening hours, when peak loads occur (see Sections 4.2, 4.3, and 9.2 through 9.7).

\begin{tabular}{|r|r|r|r|r|}
\hline \multicolumn{1}{|c|}{ TABLE 4 - CAPACITY VALUES FOR 20042006.} \\
\hline \multicolumn{1}{c|}{ CASE } & WIND ONLY & PV ONLY & CSP ONLY & \multicolumn{1}{|c|}{ WIND+PV+CSP } \\
\hline $10 \%$ & $13.5 \%$ & $35.0 \%$ & $94.5 \%$ & $18.2 \%$ \\
\hline $20 \%$ & $12.8 \%$ & $29.3 \%$ & $94.8 \%$ & $19.7 \%$ \\
\hline $30 \%$ & $12.3 \%$ & $27.7 \%$ & $95.3 \%$ & $19.8 \%$ \\
\hline
\end{tabular}




\section{CONCLUSIONS AND NEXT STEPS}

The technical analysis performed in this study shows that it is feasible for the WestConnect region to accommodate 30\% wind and 5\% solar energy penetration. This requires key changes to current practice, including substantial balancing area cooperation, sub-hourly scheduling, and access to underutilized transmission capacity.

WWSIS finds that both variability and uncertainty of wind and solar generation impacts grid operations. However, the uncertainty (due to imperfect forecasts) leads to a greater impact on operations and results in some contingency reserve shortfalls and some curtailment, both of which are relatively small. The variability leads to a greater sub-hourly variability reserve requirement, but because conventional units are backed down, the system naturally has extra reserve margins.

This study has established both the potential and the challenges of large scale integration of wind and solar generation in WestConnect and, more broadly, in WECC. However, changes of this magnitude warrant further investigation. The project team regards the following as valuable topics for exploration:

- Characterization of the capabilities of the non-renewable generation portfolio in greater detail (e.g., minimum turndown, ramp rates, cost of additional wear and tear);

- Changes in non-renewable generation portfolio (e.g., impact of retirements, characteristics, and value of possible fleet additions or upgrades);

- Reserve requirements and strategies (e.g., off-line reserves, reserves from nongeneration resources);

- Load participation or demand response (e.g., functionality, market structures, PHEV);

- Fuel sensitivities (e.g., price, carbon taxes, gas contracts and storage, hydro constraints and strategies);

- Forecasting (e.g., calibration of forecasting using field experience, strategies for use of short-term forecasting);

- Rolling unit commitment (e.g., scheduling units more frequently than once on a day-ahead basis);

- Transmission planning and reliability analyses (e.g., transient stability, voltage stability, protection and control, intra-area constraints and challenges);

- Hydro flexibility (e.g., calibration of hydro models with plant performance). 


\section{REFERENCES}

[1] EERE (U.S. DOE Office of Energy Efficiency and Renewable Energy). July 2008. 20\% Wind Energy by 2030: Increasing Wind Energy's Contribution to U.S. Electric Supply. DOE/GO-102008-2567. Washington: EERE. www1.eere.energy. gov/windandhydro/ pdfs/41869.pdf.

[2] Enernex Corporation. January 2010. "Eastern Wind Integration and Transmission Study", NREL Report No. SR-550-47078, www.nrel.gov/wind/systemsintegration/ pdfs/2010/ewits_final_report.pdf.

[3] New York State Energy Research and Development Authority. March 2005. "The Effects of Integrating Wind Power on Transmission System Planning, Reliability, and Operations," www.nyserda.org/publications/wind_integration_report.pdf.

[4] California Energy Commission. July 2007. Intermittency Analysis Project Study. "Appendix B - Impact of Intermittent Generation on Operation of California Power Grid," www.energy.ca.gov/2007publications / CEC-500-2007-081/CEC-500-2007-081APB.PDF.

[5] Ontario Power Authority. October 2006. Independent Electricity System Operator, Canadian Wind Energy Association's "Ontario Wind Integration Study," www. powerauthority.on.ca/Storage/28/2321_OPA_Report_final.pdf.

[6] GE Energy. March 2008. Attachment A: Analysis of Wind Generation Impact on ERCOT Ancillary Services Requirements. Prepared for Electric Reliability Council of Texas. Schenectady, NY: GE Energy. http:/ / www.ercot.com/ content/ news / presentations/2008/Wind_Generation_Impact_on_Ancillary_Services_-_GE_Study.zip.

[7] Smith, C., et al. August 2007. “Utility Wind Integration and Operating Impact State of the Art", IEEE Power and Energy, Vol 22, No. 3, pp 900-908, www.nrel.gov/ wind/ systemsintegration/pdfs/2007/milligan_wind_integration.pdf.

[8] DeMeo, E., et al. Nov/ Dec 2007. "Accommodating Wind's Natural Behavior", IEEE Power \& Energy Magazine, pp 59-67 http:/ / ieeexplore.ieee.org/ iel5/8014/4382976/04383124.pdf.

[9] Corbus, D., D. Lew, G. Jordan, W. Winters, F. Van Hull, J. Manobianco, and R. Zavadil. November/ December 2009. “Up with Wind: Studying the Integration and Transmission of Higher Levels of Wind Power." IEEE Power and Energy, 7(6): 36-46.

[10] 3TIER. March 2010. Development of Regional Wind Resource and Wind Plant Output Datasets, NREL SR-550-47676, http: / / www.nrel.gov/ docs / fy10osti / 47676.pdf.

[11] NREL. Wind Integration Datasets. www.nrel.gov/wind/integrationdatasets.

[12] Wilcox, S., April 2007. “National Solar Radiation Database, 1999 - 2005: User's Manual," NREL Report TP-581-41364, http: / / www.nrel.gov/ docs/ fy07osti / 41364.pdf. 



\section{Introduction}

The Western Wind and Solar Integration Study (WWSIS) was initiated in 2007 to examine the operational impact of up to $35 \%$ energy penetration of wind generation, photovoltaics (PV), and concentrating solar power (CSP) on the power system operated by the WestConnect [1.1] group of utilities in Arizona, Colorado, Nevada, New Mexico, and Wyoming. The WestConnect geographic footprint is shown in Figure 1.1. Although WestConnect includes utilities in California, they were not included in this study because California had already completed a renewable energy integration study for the state [1.2]. This study was set up to answer questions that utilities, Public Utility Commissions (PUCs), developers, and regional planning organizations had about the impacts of integrating more renewable energy in the WestConnect region:

- Can the system operate reliably and securely with large amounts of wind and solar generation?

- How do local wind and solar resources compare to out-of-state resources?

- Can balancing area cooperation help mitigate variability?

- What is the impact of wind and solar energy on the variable cost of energy production, fuel consumption, and emissions?

- How much of the available wind and solar energy can be delivered to energy users?

- What is the role and value of energy storage?

- How would increased penetration of wind and solar generation affect reserve requirements?

- What is the benefit of wind and solar forecasting?

- Does geographic diversity of renewable energy resources help mitigate variability?

- How can hydro resources help with integration of wind and solar generation?

The study objectives, technical approach and study scenarios are presented in Chapters 1 through 3. Observations and conclusions from the various analyses are provided at the end of Chapters 4 through 9. The key findings are presented in Chapter 10. 


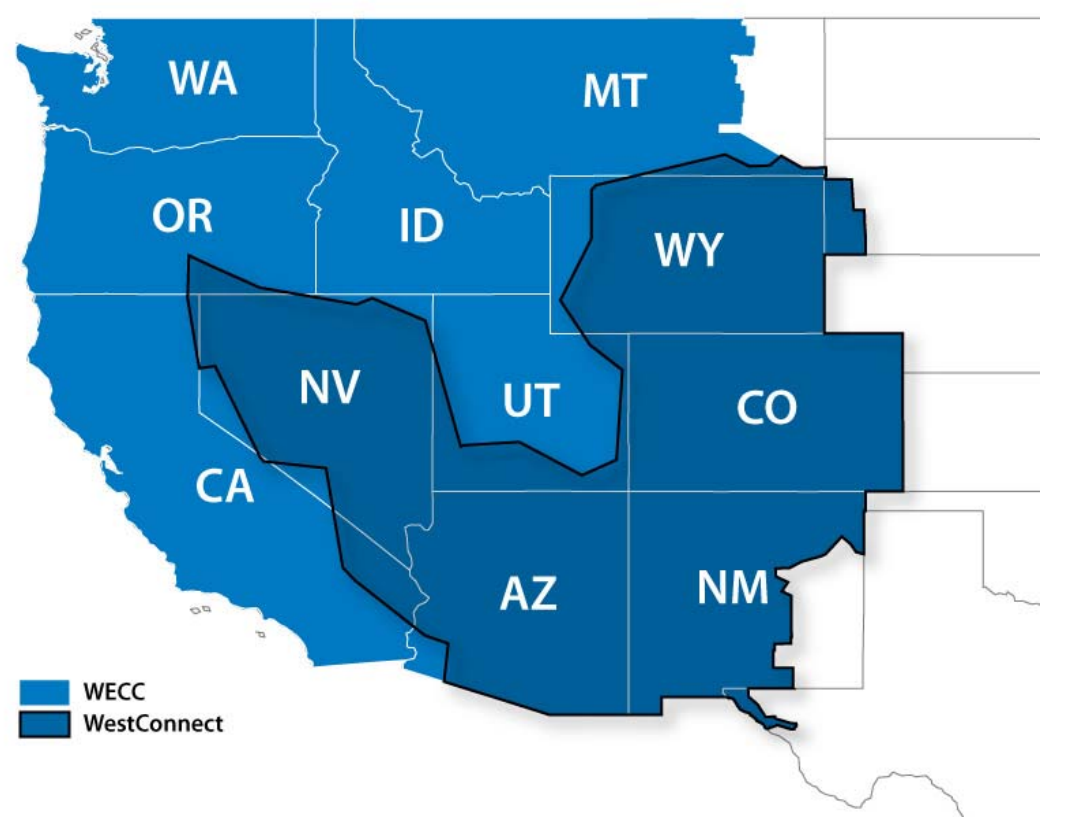

Figure 1.1 Geographic Footprint of WestConnect Utilities

The WWSIS is sponsored by the U.S. Department of Energy (DOE) and managed by the National Renewable Energy Laboratory (NREL) with WestConnect as a partner organization. The study was originally established to build upon DOE's 20\% Wind Energy by 2030 report [1.3], which did not find any technical barriers to reaching $20 \%$ wind energy in the continental United States by 2030. The WWSIS and its partner study, the Eastern Wind Integration and Transmission Study (EWITS) [1.4], performed a more in-depth operating impact analysis to see if $20 \%$ wind energy was feasible from an operational level. In DOE/NREL's analysis, the $20 \%$ wind energy target required $25 \%$ wind energy in the Western Interconnection; therefore, this study considered $20 \%$ and $30 \%$ wind energy to bracket the DOE analysis. And since solar power is rapidly growing in the west, up to $5 \%$ solar energy was also considered in this study.

WWSIS and its partner study EWITS build upon a large body of work on wind integration [1.5 through 1.10]. Previous studies examined specific utilities or states, looking at the impact of wind on operations in the regulation (seconds to minutes), load following (minutes to hours), and unit commitment (hours to days) time frames. In these studies, hypothetical wind and transmission build-outs were typically added to the existing system, which was simulated and statistically analyzed over these time frames. These studies generally consider the impact of the variability of wind (due to varying weather) and the uncertainty of wind (due to the inability to perfectly forecast the weather). Even if the weather and the wind could be perfectly forecasted, grid operators would still have to accommodate wind's variability. It is important to note that operators already manage variability and uncertainty in the load; wind and solar add to that variability and uncertainty. 
WWSIS was funded by DOE and managed by NREL. The main partner in this study was WestConnect. The project team included 3TIER Group (wind power dataset, and wind and solar forecasts), State University of New York at Albany/Clean Power Research (solar radiation dataset), Exeter Associates (data collection), Northern Arizona University (wind validation and hydro analysis), NREL (wind validation, and PV and CSP power datasets), and GE (scenarios, and main technical/economic analysis). A Technical Review Committee (TRC), composed of members of WestConnect utilities, western utility organizations, and industry and technical experts, met eight times to review technical results and progress. A broader stakeholder group, open to the public, met five times to ensure study direction and results were relevant to western grid issues. Results of this study have been extensively vetted in public forums.

The study examined grid operation for the year 2017. That is, system loads and generation expansion were projected to represent year 2017. While 35\% renewable energy penetration was not expected by 2017, this year was selected in order to start with a realistic model of the transmission grid. The study examined inter-annual operability by modeling operations for year 2017 three times, using historical load and weather patterns from years 2004, 2005, and 2006.

\subsection{References}

\section{[1.1] www.westconnect.com}

[1.2] California Energy Commission. July 2007. Intermittency Analysis Project Study. "Appendix B - Impact of Intermittent Generation on Operation of California Power Grid", www.energy.ca.gov/2007publications/CEC-500-2007-081/CEC-500-2007-081APB.PDF.

[1.3] U.S. DOE Office of Energy Efficiency and Renewable Energy. July 2008. 20\% Wind Energy by 2030: Increasing Wind Energy's Contribution to U.S. Electric Supply. DOE/GO-102008-2567. Washington: EERE. www.1.eere.energy.gov/windandhydro/pdfs/41869.pdf

[1.4] Enernex Corporation. January 2010. “Eastern Wind Integration and Transmission Study", NREL Report No. SR-550-47078, www.nrel.gov/wind/systemsintegration/pdfs/2010/ewits final report.pdf

[1.5] New York State Energy Research and Development Authority. March 2005. "The Effects of Integrating Wind Power on Transmission System Planning, Reliability, and Operations," www.nyserda.org/publications/wind integration_report.pdf 
[1.6] Ontario Power Authority. October 2006. Independent Electricity System Operator, Canadian Wind Energy Association's “Ontario Wind Integration Study," www.powerauthority.on.ca/Storage/28/2321_OPA_Report final.pdf

[1.7] GE Energy. March 2008. Attachment A: Analysis of Wind Generation Impact on ERCOT Ancillary Services Requirements. Prepared for Electric Reliability Council of Texas. Schenectady, NY: GE Energy.

http://www.ercot.com/content/news/presentations/2008/Wind_Generation_Impact on Ancillary Services - GE Study.zip

[1.8] Smith, C., et al. August 2007. “Utility Wind Integration and Operating Impact State of the Art", IEEE Power and Energy, Vol 22, No. 3, pp 900-908,

www.nrel.gov/wind/systemsintegration/pdfs/2007/milligan_wind integration.pdf

[1.9] DeMeo, E., et al. Nov/Dec 2007. “Accommodating Wind's Natural Behavior," IEEE Power \& Energy Magazine, pp 59-67

http://ieeexplore.ieee.org/iel5/8014/4382976/04383124.pdf

[1.10] Corbus, D., D. Lew, G. Jordan, W. Winters, F. Van Hull, J. Manobianco, and R. Zavadil. November/December 2009. “Up with Wind: Studying the Integration and Transmission of Higher Levels of Wind Power." IEEE Power and Energy, 7(6): 36-46. 


\section{Objectives and Technical Approach}

\subsection{Project Objectives}

The goal of the WWSIS is to understand the economic and operating impacts due to increased penetration of wind, PV, and CSP generation resources on the power grid. This is mainly an operations study and not a transmission planning study, although several scenarios include inter-regional transmission expansions to deliver power. Capital cost tradeoffs between wind and solar generation facilities and inter-regional transmission lines were considered in the development of the study scenarios. The scenarios were subsequently compared in terms of operational performance, overall operational cost savings due to avoided fuel usage, and reductions in emissions.

\subsection{Major Tasks}

In the WWSIS, the major tasks consisted of utility data collection, wind and solar dataset development, scenario development, statistical analysis, production simulation analysis, reliability analysis, quasi-steady-state analysis, and analysis of mitigation options. The WWSIS was a large team effort, with Exeter Associates responsible for data collection, 3TIER Group developing the wind dataset and the solar forecasts, State University of New York (SUNY) at Albany / Clean Power Research modeling the solar resource, NREL modeling the PV and CSP power plants, Northern Arizona University (NAU) validating the wind dataset and hydro operation, and GE developing scenarios and conducting the main technical and economic analysis. Figure 2.1 shows a flowchart of the major project tasks.

The study examined grid operation for the year 2017. That is, system loads and generation expansion were projected to represent year 2017. While 35\% renewable energy is not expected by 2017, this year was selected in order to start with a realistic model of the transmission grid. The study examined inter-annual operability by modeling operations for year 2017 three times, using historical load and weather patterns from years 2004, 2005, and 2006.

Modeling 35\% wind and solar energy penetration with the 2017 WECC system was expected to yield conservative results, because in reality, that penetration level will occur further into the future, and generation resources installed at that time may have more flexibility. Furthermore, the gas and coal fuel price assumptions for this study led to gas generation resources being displaced by wind and solar, so less flexible coal generation was left to accommodate the variability of wind and solar resources. In addition, the wind data used in the study was found to have higher variability than that measured at existing wind plants. 
The study focused on the WestConnect region with up to $35 \%$ energy penetration of wind and solar generation. And since renewable energy resources in neighboring regions can affect operations of the WestConnect region, up to 23\% energy penetration of wind and solar generation was assumed for the rest of WECC. This addressed concerns of "exporting the variability," which affected other studies that ignored significant renewable energy penetrations outside the study area.

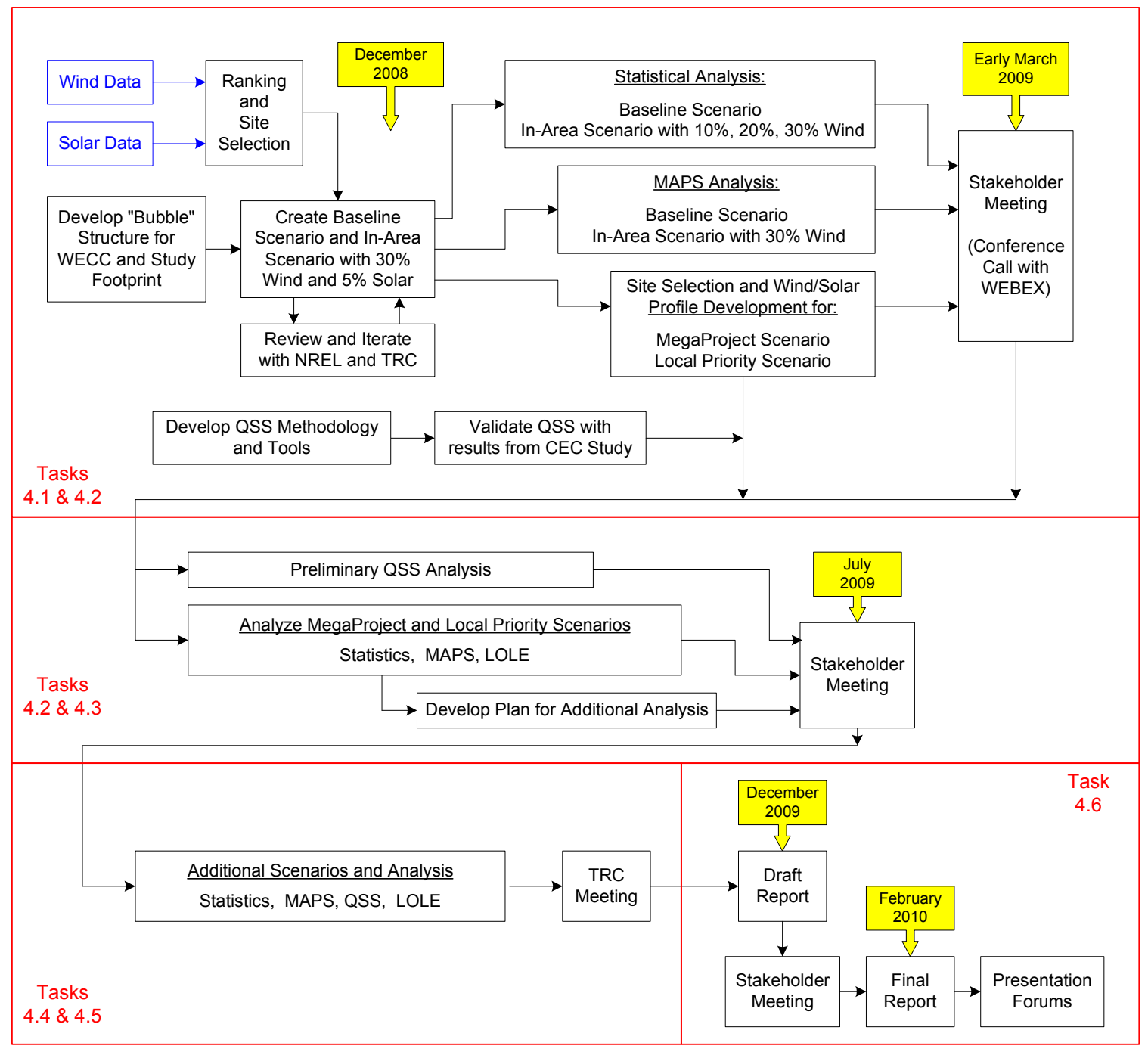

Figure 2.1 Flowchart of WWSIS Project Tasks

\subsection{Overview of Study Scenarios}

The WWSIS used a multidimensional scenario-based study approach to evaluate:

- Different levels of energy penetration for wind and solar generation, ranging from $11 \%-35 \%$

- Different geographic locations for the wind and solar resources, and 
- A wide array of sensitivities to assess issues such as fuel costs, operating reserve levels, unit commitment strategies, storage alternatives, PHEV, balancing area size, etc.

Table 2.1 shows the five levels of wind and solar energy penetration assumed for the study scenarios. The "PreSelected" case included wind and solar generation that was in operation before the end of year 2008. The "10\%" penetration level included $10 \%$ wind energy and 1\% solar energy (relative to total annual load energy) in the study footprint as well as the rest of WECC. The "20\%" penetration level included $20 \%$ wind energy and $3 \%$ solar energy in the study footprint, with $10 \%$ wind energy and $1 \%$ solar energy in the rest of WECC. The "20/20\%" penetration level included 20\% wind energy and 3\% solar energy in the study footprint as well as the rest of WECC. The "30\%" penetration level included 30\% wind energy and 5\% solar energy in the study footprint, with $20 \%$ wind energy and 3\% solar energy in the rest of WECC.

Table 2.1 Wind and Solar Energy Penetrations for WWSIS Scenarios

\begin{tabular}{|c|c|c|c|c|c|c|}
\cline { 2 - 7 } \multicolumn{1}{c|}{} & \multicolumn{3}{c|}{ In Footprint } & \multicolumn{3}{c|}{ Rest of WECC } \\
\hline Case Name & Wind + Solar & Wind & Solar & Wind + Solar & Wind & Solar \\
\hline PreSelected & $3 \%^{*}$ & $3 \%$ & $*$ & $2 \%^{*}$ & $2 \%$ & $*$ \\
\hline $10 \%$ & $11 \%$ & $10 \%$ & $1 \%$ & $11 \%$ & $10 \%$ & $1 \%$ \\
\hline $20 \%$ & $23 \%$ & $20 \%$ & $3 \%$ & $11 \%$ & $10 \%$ & $1 \%$ \\
\hline $20 / 20 \%$ & $23 \%$ & $20 \%$ & $3 \%$ & $23 \%$ & $20 \%$ & $3 \%$ \\
\hline $30 \%$ & $35 \%$ & $30 \%$ & $5 \%$ & $23 \%$ & $20 \%$ & $3 \%$ \\
\hline
\end{tabular}

* Existing solar generation embedded in load

The study evaluated four major scenarios with wind and solar resources in different geographic locations.

In-Area Scenario: Each state in the study footprint met its wind and solar energy targets using the best available wind and solar generation resources within its state boundary. Each state in the rest of WECC also met its renewable energy target using the best available resources within the state boundary. In some northern states, it was necessary to use solar resources from other states due to the lack of good solar resources. The In-Area siting was held constant for the rest of WECC, while the study footprint examined two additional scenarios.

In-Area Scenario: Each state in the study footprint met its wind and solar energy targets using the best available wind and solar generation resources within its state boundary. Each state in the rest of WECC also met its renewable energy target using the best available resources within the state boundary. In some northern states, it was necessary to use solar resources from other states due to the lack of good solar 
resources. The In-Area siting was held constant for the rest of WECC, while the study footprint examined two additional scenarios.

Mega-Project Scenario: The study footprint met its wind and solar energy targets by using the best available wind and solar resources within the study footprint to provide the least cost of delivered energy. Given that many of the best wind resources are in Wyoming, this scenario includes a large penetration of wind generation in Wyoming (and other wind-rich areas), with new transmission lines to deliver the energy to load centers.

Local-Priority Scenario: This scenario used the best wind and solar sites within the entire footprint, but the site selection algorithm included a $10 \%$ capital cost advantage to "local" resources within each state. The result was a scenario that was about halfway between the In-Area and Mega-Project Scenarios.

Table 2.2 shows a summary of the total wind and solar MW ratings by state for the study footprint. Section 3 provides additional details about the scenarios and how they were developed. It also provides data on wind and solar resources in the rest of WECC. Appendix A provides information about the site selection algorithm and also includes detailed tables showing load, wind generation, and solar generation by area for each penetration level and each scenario. 
Table 2.2 Summary of Aggregated Wind and Solar MW Ratings by State for WWSIS Scenarios

\begin{tabular}{|c|c|c|c|c|c|c|c|c|}
\hline \multirow[b]{2}{*}{ Area } & \multirow{3}{*}{$\begin{array}{l}\text { Load Minimum } \\
\text { (MW) }\end{array}$} & \multirow{3}{*}{$\begin{array}{c}\text { Load Maximum } \\
\text { (MW) }\end{array}$} & \multirow{2}{*}{\multicolumn{2}{|c|}{$\begin{array}{|cc|}10 \% & 1 \% \\
\text { Wind Rating Solar Rating } \\
\end{array}$}} & \multirow{2}{*}{\multicolumn{2}{|c|}{$\begin{array}{|cc|}20 \% & 3 \% \\
\text { Wind Rating Solar Rating }\end{array}$}} & \multicolumn{2}{|c|}{$30 \% \quad 5 \%$} \\
\hline & & & & & & & Wind Ratir & Solar Rating \\
\hline Area & & & $(M W)$ & $(M W)$ & (MW) & (MW) & $(M W)$ & (MW) \\
\hline Arizona & 6,995 & 23,051 & 3,600 & 400 & 7,350 & 1,200 & 11,220 & 2,000 \\
\hline Colorado East & 4,493 & 11,589 & 2,040 & 300 & 3,780 & 800 & 5,640 & 1,400 \\
\hline Colorado West & 712 & 1,526 & 300 & 0 & 600 & 200 & 900 & 300 \\
\hline New Mexico & 2,571 & 5,320 & 1,080 & 200 & 1,920 & 400 & 2,790 & 700 \\
\hline Nevada & 3,863 & 12,584 & 2,340 & 200 & 4,680 & 700 & 7,050 & 1,100 \\
\hline Wyoming & 2,369 & 4,016 & 930 & 100 & 1,620 & 100 & 2,340 & 300 \\
\hline In Footprint & 21,249 & 58,087 & 10,290 & 1,200 & 19,950 & 3,400 & 29,940 & 5,800 \\
\hline
\end{tabular}

\begin{tabular}{|c|c|c|c|c|c|c|c|c|}
\hline Local Priority & $\begin{array}{c}\text { Load Minimum } \\
\text { (MW) }\end{array}$ & $\begin{array}{l}\text { Load Maximum } \\
\text { (MW) }\end{array}$ & \multicolumn{2}{|c|}{\begin{tabular}{cc|}
$10 \%$ & $1 \%$ \\
Wind Rating Solar Rating
\end{tabular}} & \multicolumn{2}{|c|}{$\begin{array}{|cc|}20 \% & 3 \% \\
\text { Wind Rating Solar Rating }\end{array}$} & \multicolumn{2}{|c|}{$\begin{array}{|cc|}30 \% & 5 \% \\
\text { Wind Rating Solar Rating }\end{array}$} \\
\hline Arizona & 6,995 & 23,051 & 2,850 & 400 & 5,250 & 1,200 & 7,710 & 2,000 \\
\hline Colorado West & 712 & 1,526 & 210 & 0 & 450 & 200 & 570 & 300 \\
\hline New Mexico & 2,571 & 5,320 & 1,350 & 200 & 2,100 & 400 & 2,970 & 700 \\
\hline Nevada & 3,863 & 12,584 & 1,350 & 200 & 2,490 & 700 & 3,450 & 1,100 \\
\hline
\end{tabular}

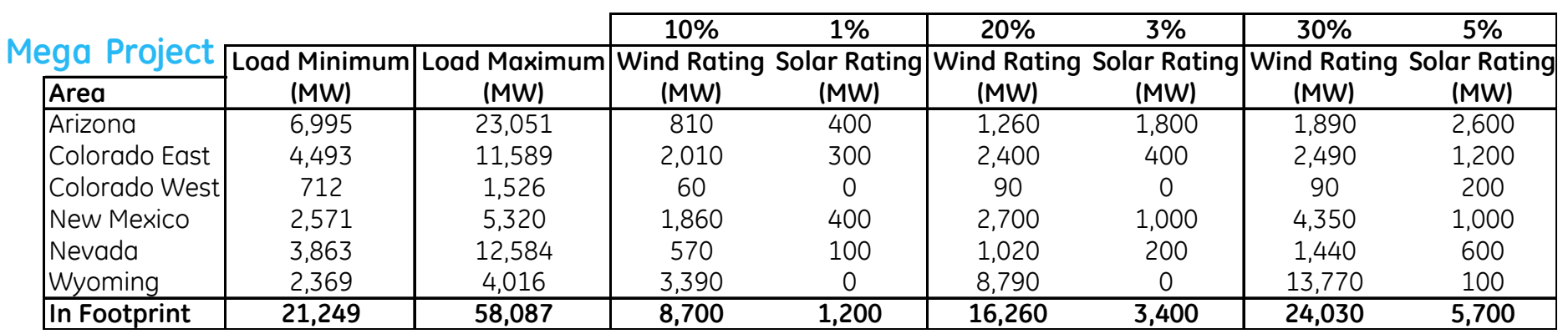




\subsection{Analytical Methods}

The primary objective of this study was to identify and quantify any system performance or operational problems with respect to load following, regulation, operation during low-load periods, etc. Four primary analytical methods were used to meet this objective; statistical analysis, hourly production simulation analysis, subhourly analysis using quasi-steady-state simulations, and reliability analysis.

Statistical analysis was used to quantify variability due to system load, as well as wind and solar generation over multiple time frames (annual, seasonal, daily, hourly, and 10minute). The power grid already has significant variability due to periodic and random changes to system load. Wind and solar generation add to that variability, and increase what must be accommodated by load following and regulation with other generation resources. The statistical analysis quantified the grid variability due to load alone over several time scales, as well as the changes in grid variability due to wind and solar generation for each scenario. The statistical analysis also characterized the forecast errors for wind generation.

Production simulation analysis with MAPS (Multi-Area Production Simulation program) was used to evaluate hour-by-hour grid operation of each scenario for 3 years with different wind and load profiles. WECC was represented as a set of 106 zones, each with its own load profile, portfolio of generating plants, and transmission capacity with neighboring areas. The zones were grouped into 20 transmission areas. The production simulation results quantified numerous impacts on grid operation including:

- Amount of maneuverable generation on-line during a given hour, including its available ramp-up and ramp-down capability to deal with grid variability due to load, wind and solar

- Effects of day-ahead wind forecast alternatives in unit commitment

- Changes in dispatch of conventional generation resources due to the addition of new renewable generation

- Changes in emissions (NOX, SOX, CO2) due to renewable generation

- Changes in costs and revenues associated with grid operation, and changes in net cost of energy

- Changes in transmission path loadings

- Changes in use of hydro resources

- Changes in use and economic value of energy storage resources

Quasi-steady-state (QSS) simulation analysis was used to quantify grid performance trends and to investigate potential mitigation measures in the minute-to-minute time 
frame. QSS analysis simulated the operation of dispatchable generation resources as well as variable wind and solar generation in the study footprint using one-minute time steps, while enforcing constraints related to unit ramp rates, ramp range, intertie flow schedules, and regional AGC functions. These time simulations enabled examination of the impact of wind and solar generation during challenging time periods, such as:

- Large 1-hour, 3-hour, and 6-hour changes in net load within the study footprint

- High levels of wind and solar penetration

- Low load levels with minimal maneuverable generation on line

- High wind forecast errors

Reliability analysis involved loss of load expectation (LOLE) calculations for the study footprint and all of WECC using the Multi-Area Reliability Simulation program, MARS. The analysis quantified the impact of wind and solar generation on overall reliability measures, as well as the capacity values of the wind and solar generation resources.

Impacts on system-level operating reserves were also analyzed using a variety of techniques including statistics, production simulation, and QSS simulation. This analysis quantified the effects of variability and uncertainty, and related that information to the system's increased need for operating reserves to maintain reliability and security.

The results from these analytical methods complemented each other, and provided a basis for developing observations, conclusions, and recommendations with respect to the successful integration of wind and solar generation into the WestConnect power grid.

\subsection{Study Assumptions}

Scenario development:

- Specific energy targets for each of three technologies: wind, PV, and CSP with storage were fixed. For example, wind sites could not be traded out for PV sites.

- A number of capital cost assumptions in 2008 dollars were used in determining the different geographic scenarios: wind at $\$ 2,000 / \mathrm{kW}, \mathrm{PV}$ at $\$ 4,000 / \mathrm{kW}, \mathrm{CSP}$ with thermal storage at $\$ 4,000 / \mathrm{kW}$, transmission at $\$ 1,600 / \mathrm{MW}$-mile, and transmission losses at 1\% per 100 miles. No tax credits are assumed or included.

- Incremental intra-state transmission build-outs were not specified in this analysis.

- The geographic scenarios considered different inter-state transmission build-outs and included these costs in the scenarios.

- Existing transmission capacity was assumed to be unavailable for new renewable energy generation only for the scenario development process. 
- New transmission was undersized: 0.7 MW of new transmission was added for each 1.0 MW of remote generation.

- Solar energy was split 70\% CSP and 30\% PV for the various scenarios.

Production simulation analysis:

- All study results were in 2017 nominal dollars with $2 \%$ escalation per year.

- \$2/MBTU coal; \$9.50/MBTU natural gas

- Carbon dioxide costs were assumed to be $\$ 30 /$ ton of $\mathrm{CO} 2$.

- Except where specified, extensive balancing area cooperation was assumed.

- The production simulation analysis assumed that all units were economically committed and dispatched while respecting existing and new transmission limits, generator cycling capabilities, and minimum turndowns.

- Existing available transmission capacity is accessible to renewable generation.

- Generation equivalent to $6 \%$ of load was held as contingency reserves - half is spinning and half is non-spinning.

- The balance of generation was not optimized for renewables. Rather, a businessas-usual capacity expansion met projected load growth in 2017. Renewable energy capacity was added to this mix, so the system analyzed was overbuilt by the amount of capacity value of the renewable plants.

- Increased O\&M of conventional generators due to increased ramping and cycling was not included due to lack of data.

- Renewable energy plant O\&M costs were not included. Wind and solar were considered price-takers.

- The hydro modeling did not reflect the specific climatic patterns of 2004, 2005, and 2006, but rather a 10-year long-term average flow per month.

The sub-hourly modeling assumed a 5-minute economic dispatch.

\subsection{Wind Data Development}

3TIER Group developed the wind dataset for the study [2.1, 2.2]. Over 75 GW of wind generation sites needed to be modeled in the study. Lacking sufficient measured data to represent this level of wind generation, it was decided to model the wind resource across the entire western United States to generate a consistent wind dataset in space and time. 3TIER Group used the Weather Research and Forecasting (WRF) mesoscale Numerical Weather Prediction Model (NWP) over the western United States at a 2-km, 10-minute resolution for years 2004-2006. In order to run this large a region at such a high resolution, it was necessary to divide it into four geographical domains that were 
run independently and then merged. The domains were run in 3-day blocks that were merged together and the seams smoothed. While the seams were smoothed so that variability did not exceed realistic limits, the days with seams unfortunately exhibited significantly more variability than the days without seams. This byproduct of the datacreation process is illustrated in Figure 2.2. In order to avoid unintended consequences during data analysis tasks, wind data from every third day (starting with day one) were eliminated from the statistical analysis of hourly and 10-minute variability. The daily energy levels were judged to be reasonable so data for all days were used for energy analysis and production simulation analysis.

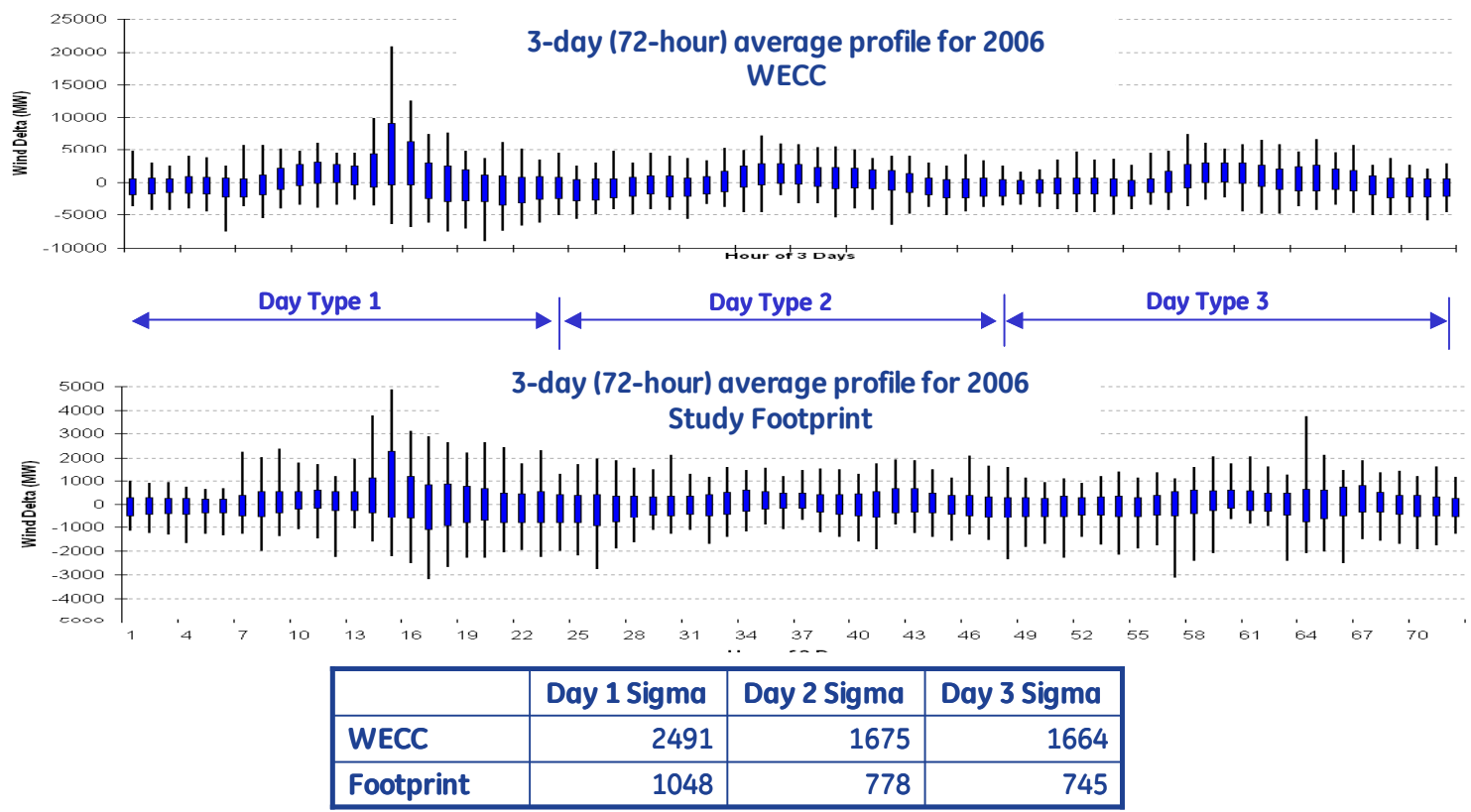

Figure 2.2 Statistical Analysis Showing Data Seam at Hour 16 of Every Third Day

3TIER Group also developed day-ahead wind forecasts for each hour. To eliminate any systematic errors that would result in the forecasts being 'too good,' a different input dataset was used for these model runs. As described above, the 'actual' wind profiles were derived from the NCAR/NCEP Reanalysis dataset with 10-minute timescale and 2$\mathrm{km}$ resolution. The 'forecast' wind profiles were derived from the Global Forecast System dataset with 1-hour timescale and 6-km resolution. One unintended consequence of this approach was that the resulting wind forecasts were biased in comparison to the 'actual' wind profiles. That is, the total annual energy of aggregated wind plant forecasts was different from the aggregated annual energy of the 'actual' wind profiles. Annual wind forecast errors by area are summarized in Figure 2.3. On average, wind forecasts in the study footprint were about $10 \%$ high and forecasts in the rest of WECC were about $20 \%$ high. Figure 2.4 shows how the wind energy forecast error varies by month. In both of these figures, unbiased forecasts would have error values of zero. 
Creating new unbiased forecasts was not feasible within the time constraints of the study. Therefore, to compensate for the bias in the forecast dataset, the hourly wind forecasts were reduced by $10 \%$ in the study footprint and $20 \%$ in the rest of WECC. This removed the annual bias in total energy, but forecast bias over shorter periods still remained.

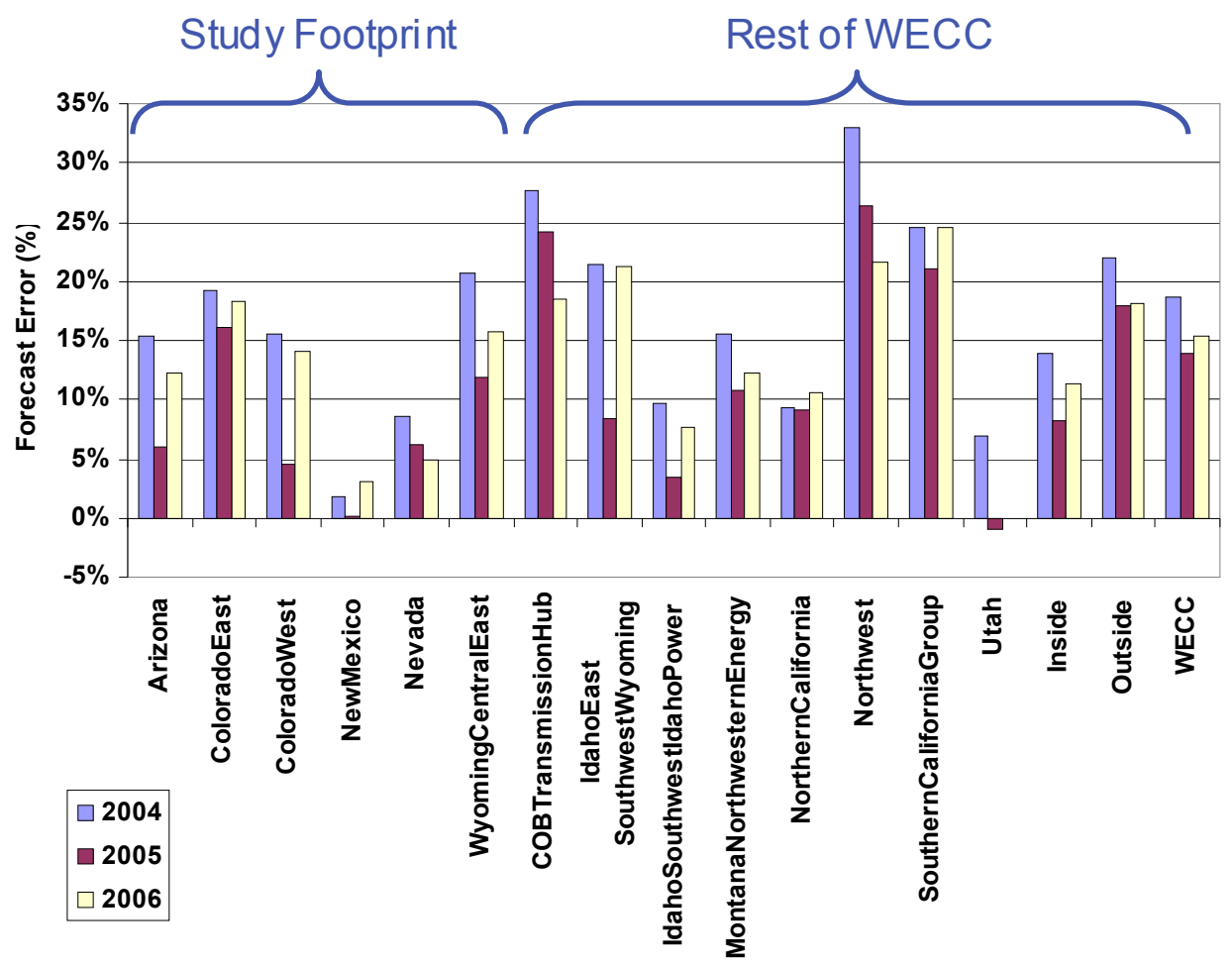

Figure 2.3 Annual Wind Energy Forecast Error by Area

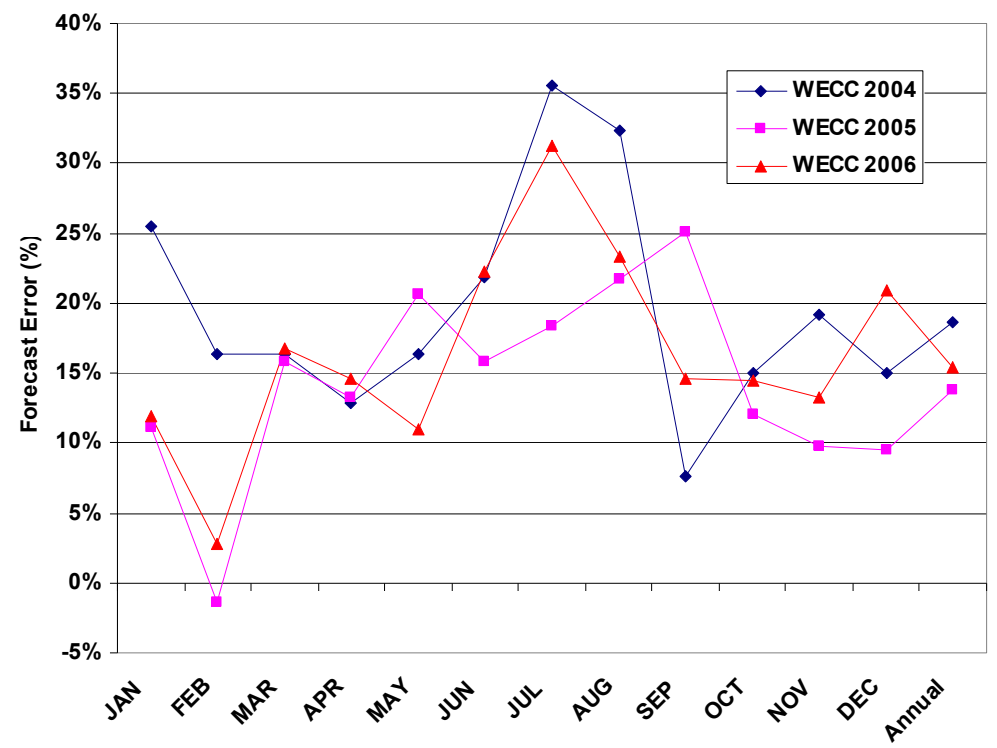

Figure 2.4 Monthly Wind Forecast Error in WECC 
Over $960 \mathrm{GW}$ of wind sites (32,000 sites of $30 \mathrm{MW}$ each) were modeled. This produced a data set that not only satisfied all the scenarios for this study, but will enable future studies of different scenarios representing different wind resources in different locations. Each 2-km x 2-km grid cell was assumed to contain 10 Vestas V90 3-MW wind turbines, yielding $30 \mathrm{MW}$ per grid cell.

Actual wind plants do not exhibit a deterministic wind plant power curve. In other words, they do not show a one-to-one correlation between wind speed and wind power. Therefore, 3TIER Group's stochastic SCORE (Statistical Correction to Output from a Record Extension) methodology was used for power conversion, instead of using the sum of ten Vestas V90 wind turbine power curves. The SCORE methodology uses measured probability density functions to reproduce the type of stochastic output that is observed in actual wind plants. The SCORE process uses observed statistical deviations from a mean value to create probability density functions of deviation from some central point. Because the process of running SCORE for each individual turbine would be very time consuming, SCORE-lite was developed. SCORE-lite models each grid point by aggregating ten individual samples from the original SCORE probability density functions. Wind speeds were converted to stochastic wind power output in $30 \mathrm{MW}$ blocks using these SCORE-lite probability density functions. SCORE-lite was validated and found to result in a realistic number of ramps while retaining the diurnal characteristics of the wind [2.1,2.2]. While array and electrical losses were not explicitly taken into account in the power conversion, the wind plant output tended to correspond very closely to a net wind plant output and was used as such. The wind dataset is publicly available [2.3].

NREL also developed 1-minute power output data based on measured data from wind plants with Vestas V90 turbines. The 10-minute data trends were removed from that measured data. The remaining fast fluctuations were sampled and overlaid on the 10minute wind dataset from 3TIER.

Validity analysis was conducted to assess the quality of the wind data. Because this was the first time such a large, high-resolution wind dataset had been created, it was critical to check the data in as many ways as possible. This included checks of the power curve, maximum and minimum output, largest ramps, average capacity factor, etc.

3TIER Group, NREL, and NAU validated the dataset against meteorological tower measurements of wind speed. In some cases, this was used to determine whether large wind ramps were real, or artifacts of the model process. NREL also validated the dataset against wind plant output for about $1 \mathrm{GW}$ of actual wind plants for which NREL could access historic data. The most critical check of a dataset for integration analysis is the accuracy of ramps, in this case, on a 10-minute and hourly timescale. A consistent overor under-production bias is less important in assessing operational impacts including regulation, ramping, and load following. 
Figure 2.5 shows validation results for the variability of the wind dataset, comparing the 10-minute wind-plant output deltas (the change from one ten-minute period to the next) or ramps of the mesoscale-modeled dataset to actual wind plant data. Two validation graphs of 10-minute ramping statistics for wind plants in two different states are shown. The top graph shows $536 \mathrm{MW}$ of wind plants in Texas from $2004-2006$. The bottom graph shows a large wind farm in the WestConnect footprint over the same time period. The dataset is considered conservative because it generally overestimates the variability of the wind plant output, as compared to actual measurements.

Seasonal and diurnal patterns of the modeled data were generally in good agreement with the actual wind plant output and capacity factors were within a few percentage points of actual wind plant output. 

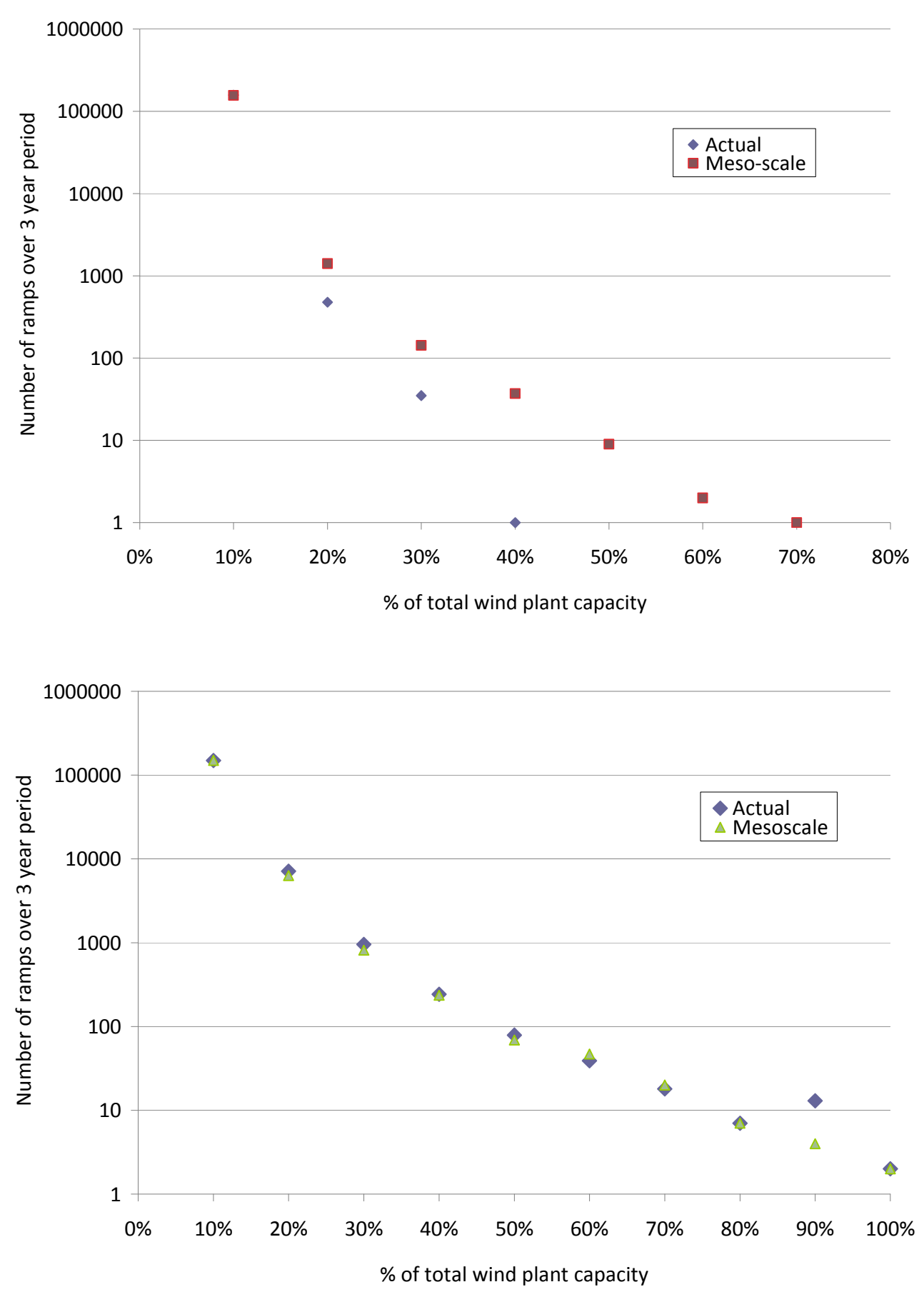

Figure 2.5 Examples from Wind Dataset Validation Analysis - Meso-Scale Model Results Versus Actual Texas Wind Plant Output (top) And Actual WestConnect Wind Plant Output (bottom)

\subsection{Solar Data Development/}

The State University of New York (SUNY) at Albany / Clean Power Research, developed the solar resource dataset (SolarAnywhere) for the study. They used a satellite cloud 
cover model to simulate the United States at a $10-\mathrm{km}$, hourly resolution [2.4]. This dataset includes global horizontal, direct normal and diffuse radiation. Hourly dayahead solar forecasts were developed by 3TIER Group [2.5].

PV was modeled in the WWSIS as distributed generation on rooftops. Modeling information for large, central station PV plants was not available at the time of the study. Preliminary data, analysis, and operating experience from the 4.6-MW Springerville Generating Station Solar System in Arizona indicated that central station PV could have significant impacts on the grid, but there was little other data to determine whether the Springerville climate was typical of other regions in the western USA. Weather stations in the western United States were modeled using PV Watts [2.6] to create PV output in block sizes of 100 MWDC. Default settings of PV Watts were used for inverter and transformer losses, soiling and other losses, and system availability, for a total derating factor of 0.77 . That means that the total AC output under standard temperature conditions was $77 \%$ of the DC rating. In order to model distributed generation from multiple, dispersed resources, PV Watts was run using 11 different system configurations of tilt, orientation, and tracking/flat-plate selection. The outputs were aggregated. The hourly PV profiles are publically available [2.7].

To refine the PV output from hourly data to a 10-minute resolution, NREL developed a model that compared the hourly average PV output to the clear sky (no clouds) PV output and added sub-hourly variability. The amount of variability added was based on measured PV output from many small PV plants in Arizona Public Service's Solar Test and Research (STAR) program, the Springerville system, and several small PV plants in Colorado.

CSP was modeled in the WWSIS as 100-MW blocks of parabolic trough plants with six hours of thermal storage. Over $200 \mathrm{GW}$ of CSP plants were included in the solar dataset and these profiles are available on the web [2.7]. The storage was initially dispatched to a typical utility load pattern (in this case, Southern California Edison). Six hours of storage requires that the solar field (solar collectors) be approximately twice as large as a system without storage. The Solar Advisor Model [2.8] was used for the power conversion using NREL's Excelergy model to represent the parabolic trough plants with thermal storage. Losses associated with the thermal storage are estimated to be minimal for storage of several hours. Because the CSP with thermal storage produces a very stable output, the 10-minute dataset was created simply by interpolating the hourly dataset.

\subsection{Load Data}

It was not possible to obtain 10-minute load data for 2004-2006 from all operating areas in the study footprint. Therefore, hourly load profile data for all operating areas in 
WECC were obtained from a Ventyx database, and 10-minute load profiles were derived by interpolating the hourly data.

\subsection{What This Study Does and Does Not Cover}

While this study undertakes detailed analysis and modeling of the power system, it was meant to be a complement to other in-depth studies:

- WWSIS is an operations study, not a transmission planning study, although different scenarios model different inter-state transmission expansion options.

- WWSIS focuses on the variable operational costs and savings due to fuel and emissions. It is not a cost-benefit analysis, even though wind and solar capital costs were incorporated in scenario development.

- WWSIS analyzed the capacity value of wind and solar to assess their contributions to resource adequacy. However, it is not a comprehensive reliability study. A full complement of planning and operational electrical studies would be required to more accurately understand and identify system impacts.

- WWSIS does not address dynamic stability issues.

- WWSIS does not optimize the balance between wind and solar resources. Wind and solar levels were fixed independently.

In 2017, it is anticipated that WestConnect and WECC will operate differently from current practice. WWSIS assumed the following changes from current operational practice:

- Production simulations of WECC grid operations assume least-cost economic dispatch in which all generation resources are shared equally and not committed to specific loads. Except for California and Alberta, WECC currently uses a bilateral contract market with long and short-term contracts in which resources are contracted out to meet specific loads.

- Other than California and Alberta, WECC currently operates as 37 separate balancing areas that use these bilateral contracts to balance their areas. Except where specified, this study assumes five regional balancing areas in WECC (Arizona-New Mexico, Rocky Mountain, Pacific Northwest, Canada, and California). WWSIS does not consider any power purchase agreements, including those for renewables.

- Except for California and Alberta, transmission in WECC is primarily contractually obligated and used. Existing available transmission capacity may be contractually obligated and not accessible to other generation. This study assumes that existing available transmission capacity is accessible to other generation on a short-term, non-firm basis. 
- Pricing developed by production cost modeling can vary widely from bilateral contract prices, and was not aligned or calibrated with current bilateral contract prices. The incremental operations and maintenance (O\&M) costs in the report do not necessarily replicate escalated current costs in the Western Interconnection.

In addition, the study results tend toward the conservative:

- WWSIS did not model a more flexible non-renewable balance of generation than what exists and is planned in WECC today. If 20-35\% variable generation were to be planned in WECC, more flexible generation would likely be planned as well, reducing the challenge that wind and solar place on operation in this study.

- This study modeled the grid for the year 2017. If WWSIS were conducted for a later year when $35 \%$ renewables would be more plausible, the power system would likely have a larger load, more flexible balance of generation, and more transmission, all of which would help accommodate the renewables.

- The wind dataset used was conservative in terms of overestimating the actual variability found in measured wind plant output.

- The base assumption of \$9.5/MBTU for gas means that gas is displaced, which leaves coal (coal, in the West, is less flexible than gas) to accommodate the variability of the renewables.

\subsection{References}

[2.1] Potter, C.W.; Lew, D.; McCaa, J.; Cheng, S.; Eichelberger, S.; Grimit, E. “Creating the Dataset for the Western Wind and Solar Integration Study (U.S.A.)" Wind Engineering, Vol 32(4), pp. 325-338, 2008.

[2.2] 3TIER, "Development of Regional Wind Resource and Wind Plant Output Datasets," NREL Report No. SR-550-47676, March 2010. http://www.nrel.gov/docs/fy10osti/47676.pdf

\section{[2.3] http://www.nrel.gov/wind/integrationdatasets}

[2.4] Wilcox, S.; Anderberg, M.; George, R.; Marion, W.; Myers, D.; Renne, D.; Lott, N.; Whitehurst, T.; Beckman, W.; Gueymard, C.; Perez, R.; Stackhouse, P.; Vignola, F. "Completing Production of the Updated National Solar Radiation Database for the United States," NREL Report No. CP-581-41511, July 2007.

[2.5] Development of Regional Wind Resource and Wind Plant Output Datasets: NREL Subcontract Report, NREL/SR-550-47676, March 2010

[2.6] http://pvwatts.org/ 
[2.7] http://mercator.nrel.gov/wwsi

[2.8] http://www.nrel.gov/analysis/sam 



\section{Study Scenarios}

This study analyzed three scenarios representing different approaches to using the available wind and solar resources in the study footprint.

In-Area: Each state within the study footprint meets its wind and solar energy penetration target using the best wind and solar resources within each state boundary.

Mega-Project: The entire study footprint meets its wind and solar energy penetration target using the best wind and solar resources within the entire study footprint, regardless of state boundaries. New transmission was added to transport renewable energy from the generation areas to the load centers.

Local-Priority: This scenario is similar to the Mega-Project Scenario; except that instate wind and solar resources have a 10\% capital cost advantage over resources in other states. This scenario falls between the In-Area and Mega-Project Scenarios.

Section 2.5 provided a high-level overview of the study scenarios, including the range of wind and solar penetration considered. This chapter provides detailed information on how the scenarios were developed. Locations of available wind and solar sites are described, and the technical algorithms used to rank the sites selected for each scenario are explained. Mathematical details of the site selection algorithms are included in Appendix A.

This study is not intended to be a transmission system design study. Renewable generation resources within each state were assumed to include the necessary local transmission facilities to deliver the renewable energy from the plant sites to each state's bulk transmission network, per traditional engineering design practices and performance criteria. New inter-area transmission lines were added for the Mega-Project and Local-Priority Scenarios to increase bulk power transfer capacities consistent with the locations of the new renewable generation and the existing load centers.

\subsection{Development of In-Area Scenario}

The In-Area Scenario assumes that each state within the study footprint meets its wind and solar energy penetration target using the best wind and solar resources within each state boundary.

\subsubsection{Objectives of the Site Selection Process}

The wind and solar datasets included far more available sites than were needed to meet the study's energy penetration objectives. Therefore, an analytical method was 
developed to select a subset of the available sites in accordance with the following objectives:

- Satisfy energy penetration targets.

- Consider expected market value of energy produced.

- Include capacity value of sites.

- Recognize that geographic diversity within areas has value.

- Include pre-selected wind plants (existing or expected to be in service by 2008).

- Independently select wind, PV, and CSP with storage per specified renewable generation mix.

\subsubsection{Available Wind and Solar Sites}

The wind database included 32,043 sites with $30 \mathrm{MW}$ of capacity each. These sites were subjected to an exclusion screen that eliminated 5,523 sites, which were in recreational areas, close to metropolitan areas, in inaccessible terrain, etc. The locations of the remaining 26,520 wind sites are shown in Figure 3.1. (Note that this includes 2004 sites that are outside of the WECC area, and were therefore excluded from this study.) The solar dataset included 275 PV sites and 501 CSP sites, each rated at $100 \mathrm{MW}$. Locations of the PV and CSP sites are shown in Figure 3.2.

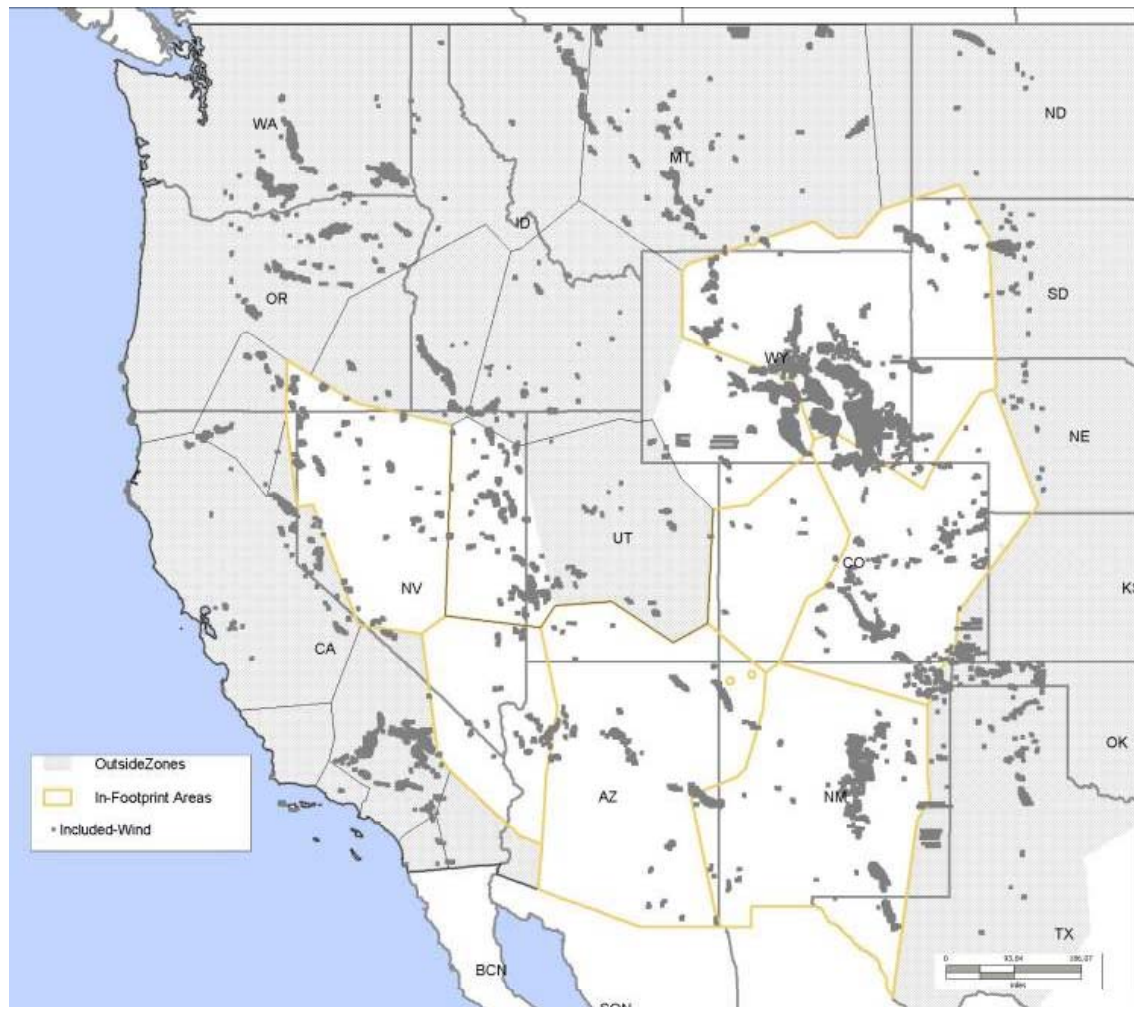

Figure 3.1 All Available Wind Sites in WECC (Each Grey Dot Represents 30 MW) 


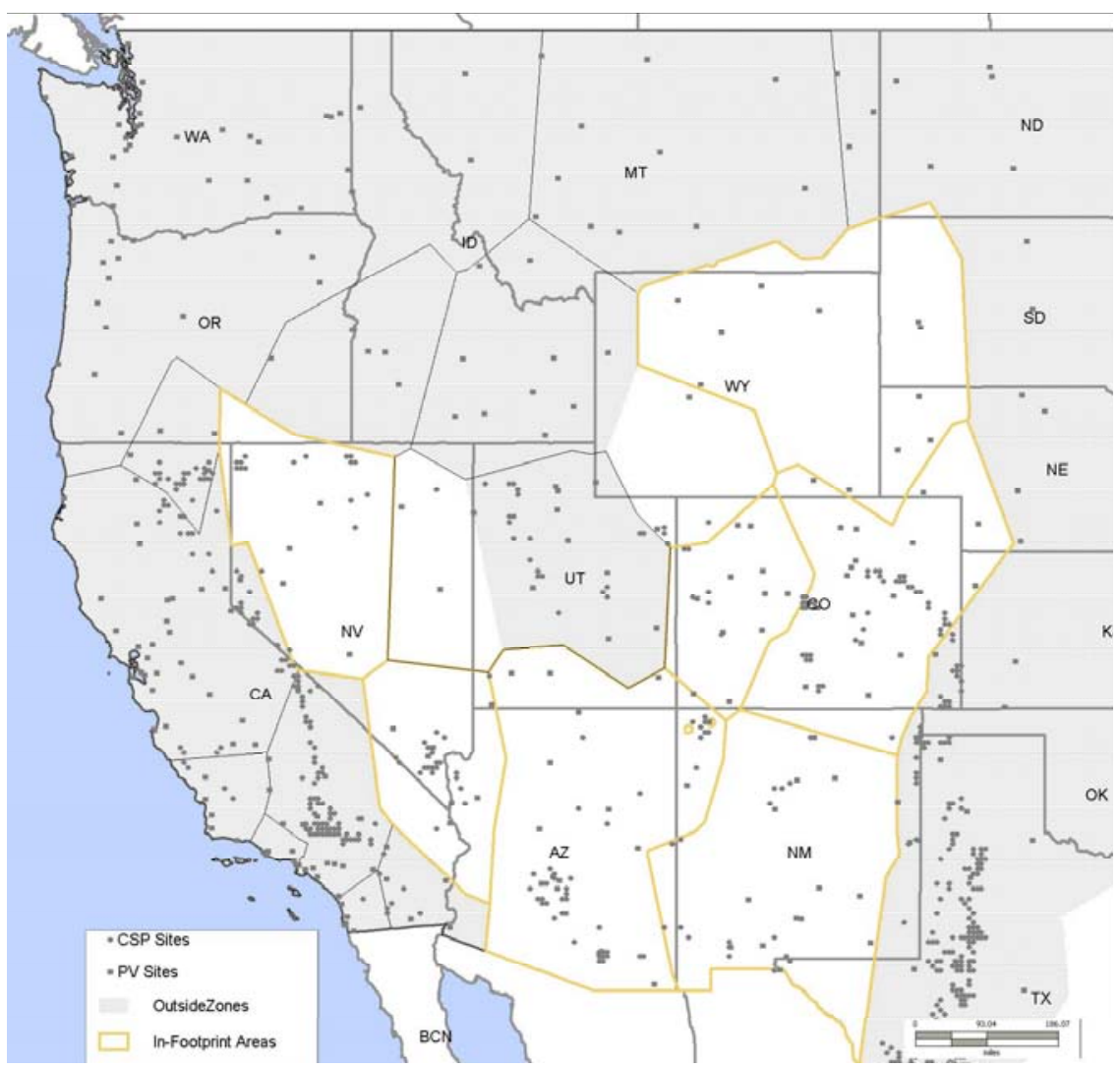

Figure 3.2 All Available Solar Sites in WECC (Each Grey Dot Represents 100 MW)

\subsubsection{Wind and Solar Site Selection}

The selection of wind sites for the In-Area Scenario followed the process described below. For each state:

- Calculate average annual energy, capacity factor, energy value, and an approximate capacity value for each site from three years of data.

- Order the sites best to worst, independently by generation type, on the basis of:

- Preselected (i.e., existing plants)

- Energy value: Using a price strip of hourly historical hub spot prices, select the hub closest to a given site and calculate its energy value

- Capacity value: Apply a $\$ 100 / \mathrm{kw}-\mathrm{yr}$ weighting factor on a capacity factor calculated between 2 p.m. and 6 p.m. for June, July, and August

- Geographic diversity as proxy for temporal diversity: Apply a 2\%/100-mile weighting factor based on the distance from center coordinates of the transmission area (latitude/longitude). East-West diversity for solar was given more weight than North-South due to the fact that sunlight migrates from East to West. North-South weighting: One for wind, zero for solar. EastWest weighting; one for wind, 1.41 for solar. 
- NOTE: Energy value was the dominant factor in the sorting algorithm, followed by capacity value. However, capacity value exceeded energy value for a few sites. Geographic diversity was found to have a secondary impact in the siting selection results.

- Starting from the highest-ranked sites, select the required number of wind and solar sites, within the energy footprint, to meet the energy target for the state (e.g., 10\%, 20\%, and 30\%). Preselected sites are included to meet the energy target for the state regardless of their energy value.

- Using a similar approach, select sites outside of the study footprint to meet wind energy targets in the rest of WECC.

A similar process was used to select the solar CSP and PV sites for the In-Area Scenario.

Using this process, wind and solar sites were selected so that their three-year average energy would match the individual annual targets for wind energy and solar energy. The same sites were used for all three load/weather years (2004, 2005, and 2006), so the total wind and solar energy varied slightly between years. Table 3.1 shows the average annual wind energy by state for the $30 \%$ scenario, as well as the values for individual years.

Figure 3.3 shows the total installed rating of wind and solar generation in the In-Area Scenario. Table 3.2 shows a summary of annual load, wind, and solar energy by area. Note that the In-footprint energy penetration is 35\% (30\% wind and $5 \%$ solar) and the out-of-footprint energy penetration is $23 \%$ (20\% wind and $3 \%$ solar). 
Table 3.1 Annual Wind Energy by State for Three Years, 30\% In-Area Scenario

\begin{tabular}{|c|c|c|c|c|c|c|c|c|c|c|c|c|}
\hline \multirow[b]{2}{*}{ WIND - $30 \%$} & \multirow[b]{2}{*}{$\begin{array}{c}\text { Sites } \\
\text { Available }\end{array}$} & \multirow[b]{2}{*}{$\begin{array}{l}\text { Sites } \\
\text { Used }\end{array}$} & \multirow[b]{2}{*}{\begin{tabular}{|c|} 
Average \\
Capacity \\
Factor (\%)
\end{tabular}} & \multirow[b]{2}{*}{$\begin{array}{l}\text { Average } \\
\text { Load } \\
\text { Energy } \\
\text { (GWh) }\end{array}$} & \multicolumn{4}{|c|}{ Annual Wind Energy (GWh) } & \multicolumn{4}{|c|}{ Annual Wind Energy (\% of load) } \\
\hline & & & & & 2004 & 2005 & 2006 & Average & 2004 & 2005 & 2006 & Average \\
\hline Arizona & 1,091 & 374 & $30.4 \%$ & 99,437 & 28,690 & 31,376 & 29,624 & 29,897 & $28.9 \%$ & $31.6 \%$ & $29.8 \%$ & $30.1 \%$ \\
\hline Colorado East & 1,673 & 188 & $37.4 \%$ & 61,372 & 18,031 & 18,577 & 18,753 & 18,453 & $29.4 \%$ & $30.3 \%$ & $30.6 \%$ & $30.1 \%$ \\
\hline Colorado West & 68 & 30 & $33.3 \%$ & 8,708 & 2,560 & 2,674 & 2,632 & 2,622 & $29.4 \%$ & $30.7 \%$ & $30.2 \%$ & $30.1 \%$ \\
\hline New Mexico & 3,062 & 93 & $38.4 \%$ & 31,260 & 9,120 & 9,472 & 9,555 & 9,382 & $29.2 \%$ & $30.3 \%$ & $30.6 \%$ & $30.0 \%$ \\
\hline Nevada & 1,591 & 235 & $28.0 \%$ & 57,505 & 16,673 & 16,601 & 18,598 & 17,290 & $29.0 \%$ & $28.9 \%$ & $32.3 \%$ & $30.1 \%$ \\
\hline Wyoming & 8,912 & 78 & $41.0 \%$ & 27,697 & 8,169 & 8,466 & 8,608 & 8,414 & $29.5 \%$ & $30.6 \%$ & $31.1 \%$ & $30.4 \%$ \\
\hline \begin{tabular}{|l|} 
In Footprint \\
\end{tabular} & 16,397 & 998 & $32.8 \%$ & 285,979 & 83,242 & 87,166 & 87,769 & 86,059 & $29.1 \%$ & $30.5 \%$ & $30.7 \%$ & $30.1 \%$ \\
\hline \multicolumn{13}{|l|}{ WIND - $20 \%$} \\
\hline $\mathrm{COB}$ & 155 & 6 & $25.0 \%$ & 1,759 & 351 & 379 & 451 & 394 & $20.0 \%$ & $21.6 \%$ & $25.6 \%$ & $22.4 \%$ \\
\hline Idaho East & 185 & 26 & $20.6 \%$ & 6,907 & 1,368 & 1,390 & 1,459 & 1,406 & $19.8 \%$ & $20.1 \%$ & $21.1 \%$ & $20.3 \%$ \\
\hline Idaho Southwest & 448 & 50 & $27.8 \%$ & 17,962 & 3,443 & 3,557 & 3,974 & 3,658 & $19.2 \%$ & $19.8 \%$ & $22.1 \%$ & $20.4 \%$ \\
\hline Montana & 1,194 & 35 & $31.2 \%$ & 14,143 & 2,805 & 2,825 & 2,990 & 2,873 & $19.8 \%$ & $20.0 \%$ & $21.1 \%$ & $20.3 \%$ \\
\hline N. California & 472 & 393 & $25.0 \%$ & 128,935 & 24,930 & 24,462 & 28,038 & 25,810 & $19.3 \%$ & $19.0 \%$ & $21.7 \%$ & $20.0 \%$ \\
\hline Northwest & 3,195 & 431 & $31.5 \%$ & 178,359 & 34,637 & 34,558 & 38,003 & 35,733 & $19.4 \%$ & $19.4 \%$ & $21.3 \%$ & $20.0 \%$ \\
\hline S.California & 1,916 & 483 & $35.4 \%$ & 224,197 & 44,447 & 46,298 & 43,924 & 44,890 & $19.8 \%$ & $20.7 \%$ & $19.6 \%$ & $20.0 \%$ \\
\hline Utah & 554 & 91 & $32.0 \%$ & 38,022 & 7,504 & 7,698 & 7,773 & 7,658 & $19.7 \%$ & $20.2 \%$ & $20.4 \%$ & $20.1 \%$ \\
\hline Out of Footprint & 8,119 & 1,515 & $30.7 \%$ & 610,284 & 119,485 & 121,167 & 126,612 & 122,421 & $19.6 \%$ & $19.9 \%$ & $20.7 \%$ & $20.1 \%$ \\
\hline Total & 24,516 & 2,513 & $31.6 \%$ & 896,263 & 202,728 & 208,333 & 214,381 & 208,480 & $22.6 \%$ & $23.2 \%$ & $23.9 \%$ & $23.3 \%$ \\
\hline
\end{tabular}

Table 3.3 shows a corresponding summary by area of maximum/minimum load MW, wind MW rating, solar MW rating, and total renewable MW rating. The maximum and minimum penetration values in this table are capacity penetrations (not energy penetrations). They are calculated as the ratio of renewable MW rating to area minimum or maximum load MW.

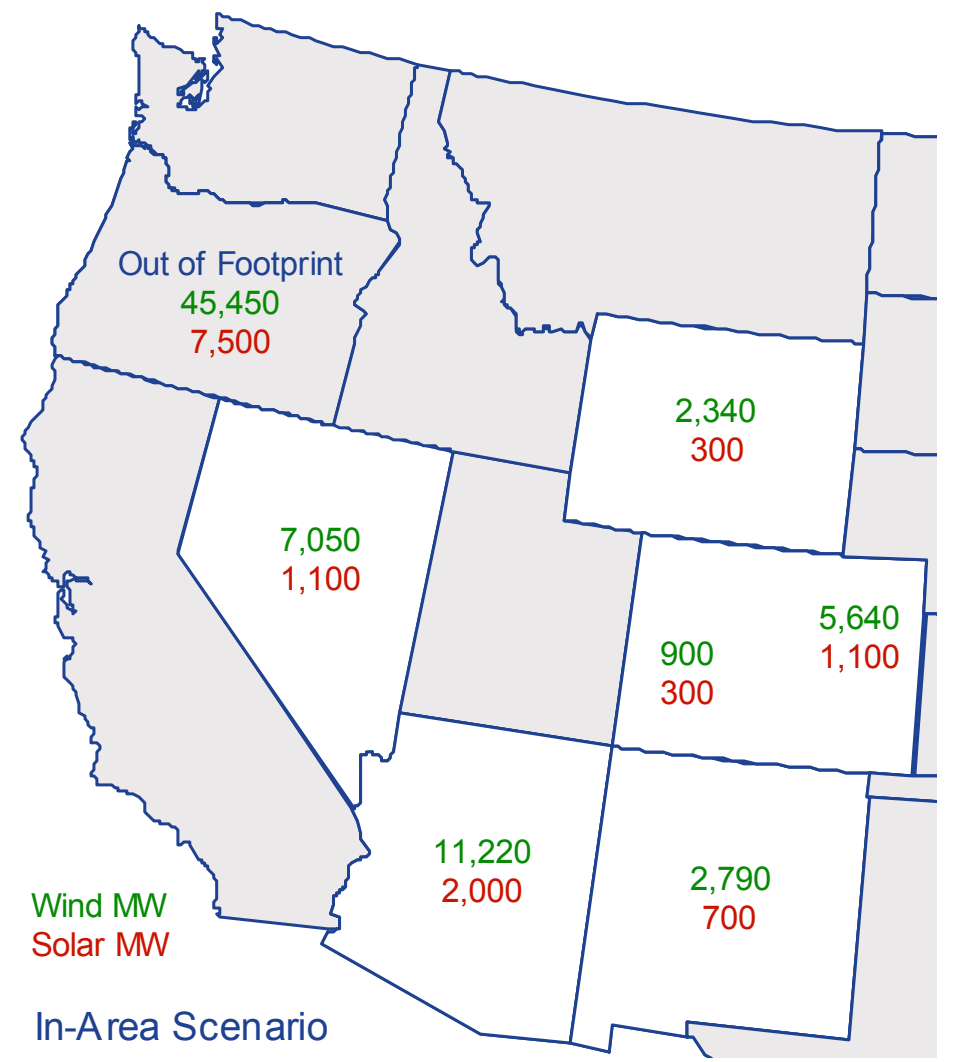

Figure 3.3 Overview of In-Area Scenario with 30\% Wind Energy and 5\% Solar Energy 
Table 3.2 Energy Summary for $30 \%$ In-Area Scenario

\begin{tabular}{|c|c|c|c|c|c|c|c|c|}
\hline In Footprint & Load & & Wind & CS & with Storage & \multirow{2}{*}{\multicolumn{3}{|c|}{\begin{tabular}{c|c} 
PV & $\begin{array}{c}\text { Total } \\
\text { Renewable }\end{array}$ \\
\# Sites & Energy \\
(Used/Available) & (GWh) \\
\end{tabular}}} \\
\hline Areas & $\begin{array}{l}\text { Energy } \\
\text { (GWh) }\end{array}$ & \begin{tabular}{|l|} 
Energy \\
(GWh)
\end{tabular} & $\begin{array}{c}\text { \# Sites } \\
\text { (Used/Available) }\end{array}$ & $\begin{array}{l}\text { Energy } \\
\text { (GWh) }\end{array}$ & $\begin{array}{c}\text { \# Sites } \\
\text { (Used/Available) }\end{array}$ & & & \\
\hline Arizona & 99,437 & 29,897 & $374 / 1091$ & 3,735 & $10 / 38$ & 1,548 & $10 / 16$ & 35,180 \\
\hline Colorado East & 61,372 & 18,453 & $188 / 1673$ & 2,252 & $7 / 58$ & 1,038 & $7 / 13$ & 21,743 \\
\hline Colorado West & 8,708 & 2,622 & $30 / 68$ & 564 & $2 / 8$ & 151 & $1 / 11$ & 3,337 \\
\hline New Mexico & 31,260 & 9,382 & $93 / 3062$ & 1,421 & $4 / 35$ & 473 & $3 / 19$ & 11,276 \\
\hline Nevada & 57,505 & 17,290 & $235 / 1591$ & 2,161 & $6 / 45$ & 773 & $5 / 10$ & 20,224 \\
\hline Wyoming & 27,697 & 8,414 & $78 / 8912$ & 0 & $0 / 0$ & 420 & $3 / 10$ & 8,834 \\
\hline In Footprint & \begin{tabular}{|l|}
285,979 \\
\end{tabular} & 86,058 & $998 / 16397$ & 10,133 & $29 / 184$ & 4,403 & $29 / 79$ & 100,594 \\
\hline
\end{tabular}

\begin{tabular}{|c|c|c|c|c|c|c|c|c|}
\hline Out of Footprint & \multirow{2}{*}{\begin{tabular}{|c|} 
Load \\
Energy \\
(GWh) \\
\end{tabular}} & \multicolumn{2}{|r|}{ Wind } & \multicolumn{2}{|c|}{ CSP with Storage } & \multicolumn{2}{|r|}{ PV } & \multirow{2}{*}{\begin{tabular}{|c|}
$\begin{array}{c}\text { Total } \\
\text { Renewable }\end{array}$ \\
Energy \\
(GWh) \\
\end{tabular}} \\
\hline Areas & & $\begin{array}{l}\text { Energy } \\
\text { (GWh) }\end{array}$ & $\begin{array}{c}\text { \# Sites } \\
\text { (Used/Available) }\end{array}$ & $\begin{array}{l}\text { Energy } \\
\text { (GWh) }\end{array}$ & $\begin{array}{c}\text { \# Sites } \\
\text { (Used/Available) }\end{array}$ & $\begin{array}{l}\text { Energy } \\
\text { (GWh) }\end{array}$ & $\begin{array}{c}\text { \# Sites } \\
\text { (Used/Available) }\end{array}$ & \\
\hline $\mathrm{COB}$ & 1,759 & 394 & $6 / 155$ & 294 & $1 / 22$ & 142 & $1 / 2$ & 830 \\
\hline Idaho East & 6,907 & 1,406 & $26 / 185$ & 0 & $0 / 0$ & 142 & $1 / 8$ & 1,548 \\
\hline Idaho Southwest & 17,962 & 3,658 & $50 / 448$ & 0 & $0 / 0$ & 132 & $1 / 5$ & 3,790 \\
\hline Montana & 14,143 & 2,873 & $35 / 1194$ & 0 & $0 / 0$ & 127 & $1 / 14$ & 3,000 \\
\hline Northern California & 128,935 & 25,812 & $393 / 472$ & 2,726 & $8 / 26$ & 1,117 & $8 / 30$ & 29,655 \\
\hline Northwest & 178,359 & 35,733 & $431 / 3195$ & 0 & $0 / 0$ & 1,645 & $13 / 54$ & 37,378 \\
\hline Southern California & 224,197 & 44,890 & $483 / 1916$ & 8,957 & $23 / 85$ & 2,050 & $13 / 39$ & 55,897 \\
\hline Utah & 38,022 & 7,658 & $91 / 554$ & 937 & $3 / 24$ & 303 & $2 / 14$ & 8,898 \\
\hline Out of Footprint & 610,284 & 122,424 & $1515 / 8119$ & 12,914 & $35 / 157$ & 5,658 & $40 / 166$ & 140,996 \\
\hline
\end{tabular}

Table 3.3 Power Summary for $30 \%$ In-Area Scenario

In Footprint

\begin{tabular}{|c|c|c|c|c|c|c|c|c|c|c|c|c|c|c|}
\hline \multirow{2}{*}{ Areas } & \multicolumn{2}{|c|}{ Load } & \multicolumn{3}{|c|}{ Wind } & \multicolumn{3}{|c|}{ CSP with Storage } & \multicolumn{3}{|c|}{ PV } & \multicolumn{3}{|c|}{ Total Renewable } \\
\hline & \begin{tabular}{|c|} 
Minimum \\
$(\mathrm{MW})$
\end{tabular} & $\begin{array}{c}\text { Maximum } \\
\text { (MW) }\end{array}$ & $\begin{array}{l}\text { Rating } \\
\text { (MW) }\end{array}$ & \multicolumn{2}{|c|}{ Penetration } & $\begin{array}{l}\text { Rating } \\
\text { (MW) }\end{array}$ & \multicolumn{2}{|c|}{ Penetration } & $\begin{array}{l}\text { Rating } \\
\text { (MW) }\end{array}$ & \multicolumn{2}{|c|}{ Penetration } & $\begin{array}{l}\text { Rating } \\
\text { (MW) }\end{array}$ & \multicolumn{2}{|c|}{ Penetration } \\
\hline Arizona & 6,995 & 23,051 & 11,220 & $160 \%$ & $49 \%$ & 1,000 & $14 \%$ & $4 \%$ & 1,000 & $14 \%$ & $4 \%$ & 13,220 & $189 \%$ & $57 \%$ \\
\hline Colorado West & 712 & 1,526 & 900 & $126 \%$ & $59 \%$ & 200 & $28 \%$ & $13 \%$ & 100 & $14 \%$ & $7 \%$ & 1,200 & $169 \%$ & $79 \%$ \\
\hline New Mexico & 2,571 & 5,320 & 2,790 & $109 \%$ & $52 \%$ & 400 & $16 \%$ & $8 \%$ & 300 & $12 \%$ & $6 \%$ & 3,490 & $136 \%$ & $66 \%$ \\
\hline Nevada & 3,863 & 12,584 & 7,050 & $183 \%$ & $56 \%$ & 600 & $16 \%$ & $5 \%$ & 500 & $13 \%$ & $4 \%$ & 8,150 & $211 \%$ & $65 \%$ \\
\hline
\end{tabular}

Out of Footprint

\begin{tabular}{|c|c|c|c|c|c|c|c|c|c|c|c|c|c|c|}
\hline \multirow[b]{3}{*}{ Areas } & \multicolumn{2}{|c|}{ Load } & \multicolumn{3}{|c|}{ Wind } & \multicolumn{3}{|c|}{ CSP with Storage } & \multicolumn{3}{|c|}{ PV } & \multicolumn{3}{|c|}{ Total Renewable } \\
\hline & \multirow{2}{*}{$\begin{array}{l}\text { Minimum } \\
\text { (MW) }\end{array}$} & \multirow{2}{*}{$\begin{array}{l}\text { Maximum } \\
(\mathrm{MW})\end{array}$} & \multirow{2}{*}{$\begin{array}{l}\text { Rating } \\
\text { (MW) }\end{array}$} & \multicolumn{2}{|c|}{ Penetration } & \multirow{2}{*}{$\begin{array}{c}\text { Rating } \\
\text { (MW) }\end{array}$} & \multicolumn{2}{|c|}{ Penetration } & \multirow{2}{*}{$\begin{array}{l}\text { Rating } \\
\text { (MW) }\end{array}$} & \multicolumn{2}{|c|}{ Penetration } & \multirow{2}{*}{$\begin{array}{c}\text { Rating } \\
\text { (MW) }\end{array}$} & \multicolumn{2}{|c|}{ Penetration } \\
\hline & & & & $\% \operatorname{Min}$ & \%Max & & $\%$ Min & $\% \operatorname{Max}$ & & $\% \operatorname{Min}$ & $\% \operatorname{Max}$ & & $\% \operatorname{Min}$ & $\% \operatorname{Max}$ \\
\hline $\mathrm{COB}$ & 138 & 294 & 180 & $131 \%$ & $61 \%$ & 100 & $73 \%$ & $34 \%$ & 100 & $73 \%$ & $34 \%$ & 380 & $276 \%$ & $129 \%$ \\
\hline & & 1,365 & 780 & & 57 & 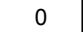 & $0^{\circ}-1+a-1-1$ & $0^{\circ}$ & 100 & $22 \%$ & $7 \%$ & 880 & & $64 \%$ \\
\hline thwect & 1188 & 3, & 1,500 & $126 \%$ & $42 \%$ & 0 & 0 & $0^{\circ}$ & 100 & $8 \%$ & $3 \%$ & 1,600 & & $45 \%$ \\
\hline & & & 1,050 & & 45 & 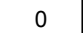 & & & 10 & & $4 \%$ & 1,150 & & $49 \%$ \\
\hline & & & 90 & 11 & 42 & 800 & & & 8 & & $3 \%$ & 13,390 & & $47 \%$ \\
\hline & & & 30 & & $42-1 \cdot 2 \cdot(-1)$ & 0 & & 0 & 0 & 9 & $4 \%$ & 14,230 & & $46 \%$ \\
\hline & & 864 & 490 & 152 & $54 \%$ & 2,300 & 24 & $9 \%$ & 1,300 & $14 \%$ & $5 \%$ & 18,090 & & $67 \%$ \\
\hline Utał & 2 & 274 & 2,730 & $121 \%$ & $38 \%$ & 300 & $13 \%$ & $4 \%$ & 200 & $9 \%$ & $3 \%$ & 3,230 & $143 \%$ & $44 \%$ \\
\hline Out of Footprint & 46,328 & 119,696 & 45,450 & $98 \%$ & $38 \%$ & 3,500 & $8 \%$ & $3 \%$ & 4,000 & $9 \%$ & $3 \%$ & 52,950 & $114 \%$ & $44 \%$ \\
\hline
\end{tabular}

\subsubsection{Transmission Zones and Areas}

Although this study focused primarily on the WestConnect footprint, the analytical models used in the study covered the entire WECC system. The WECC system was divided into 14 transmission areas. Each transmission area was further subdivided into transmission zones. Figure 3.4 shows a map of the transmission areas and zones. Table 3.4 summarizes the same information in tabular form. 
In Figure 3.5, the WECC transmission areas have been reduced to dots and the inter-area transmission paths shown with their transfer ratings (in MW). One rating is shown for each path, even though many paths have a different rating for each flow direction. Bidirectional ratings for the transmission paths within the study footprint for the In-Area Scenario are shown in Table 3.5. The WECC path ratings used in this study were derived from the Ventyx database and adjusted per discussions with the WestConnect utilities.

The Local-Priority and Mega-Project Scenarios included significant expansion to the transmission system. The incremental path ratings associated with that expansion are described in Sections 3.2 and 3.3.

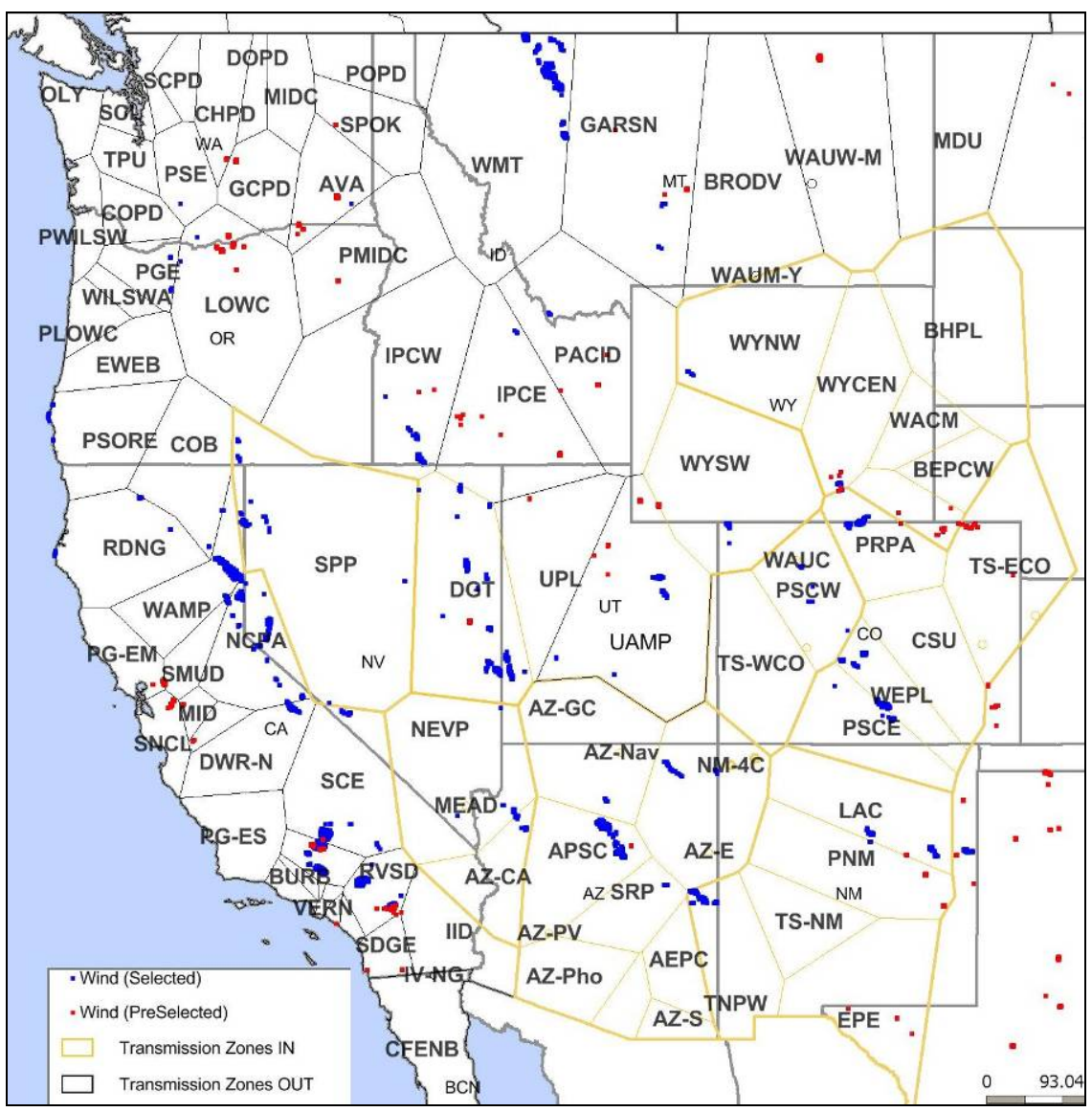

Figure 3.4 Map of Transmission Zones and Areas 
Table 3.4 Transmission Zones and Areas

\begin{tabular}{|c|c|c|c|}
\hline Transmission Area Name & Transmission Zone Name & Abbrev. & \\
\hline \multirow{6}{*}{ Arizona } & Arizona Electric Power Coop & AEPC & \multirow{29}{*}{ 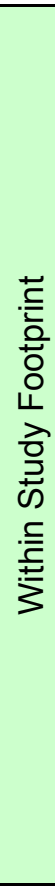 } \\
\hline & Arizona Public Service Co & APSC & \\
\hline & Glen Canyon Area & AZ-GC & \\
\hline & Salt River Project & SRP & \\
\hline & Southern Arizona & AZ-S & \\
\hline & Tucson Electric Power Co & TEP & \\
\hline \multirow{6}{*}{ Colorado East } & Sunflower Electric Power & CSUA & \\
\hline & Colorado Springs Utilities & $\mathrm{CSU}$ & \\
\hline & Platte River Power Authority & PRPA & \\
\hline & Public Service of Colorado - East & PSCE & \\
\hline & Tri-State G\&T: in East CO & TS-ECO & \\
\hline & WestPlains Energy Colorado & WEPL & \\
\hline \multirow{2}{*}{ Colorado West } & Public Service of Colorado - West & PSCW & \\
\hline & Tri-State G\&T: in West CO & TS-WCO & \\
\hline \multirow{7}{*}{ New Mexico } & Navajo Tribal Utility Authority & NAVTRUA & \\
\hline & El Paso Electric & EPE & \\
\hline & Los Alamos County & LAC & \\
\hline & Public Service Co of New Mexico & PNM & \\
\hline & Texas-New Mexico Power WECC & TNPW & \\
\hline & Tri-State G\&T: NM/Plains Electric & TS-NM & \\
\hline & Southwestern Public Service Company & FARM & \\
\hline \multirow{3}{*}{ Nevada } & Sierra Pacific Power Co & SPP & \\
\hline & Nevada Power Co & NEVP & \\
\hline & Deseret G\&T Cooperative & DGT & \\
\hline \multirow{5}{*}{ Wyoming } & PACE - Central Wyoming & WYCEN & \\
\hline & PACE - Northwest Wyoming & WYNW & \\
\hline & Tri-State G\&T: in Wyoming & TRSTWYOA & \\
\hline & WAPA - Colorado Missouri (Wyoming) & WACM & \\
\hline & PACW - Soutwest Wyoming & WYSW & \\
\hline $\mathrm{COB}$ & California-Oregon Border Area & $\mathrm{COB}$ & \multirow{41}{*}{ 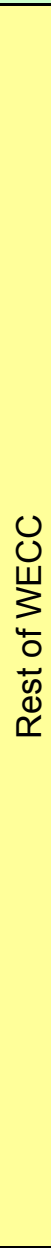 } \\
\hline \multirow{2}{*}{ Idaho East } & Idaho Power East & IPCE & \\
\hline & PACE - Idaho & PACID & \\
\hline Idaho West & Idaho Power West & IPCW & \\
\hline \multirow{3}{*}{ Montana } & Northwestern Energy - Broadview & BRODV & \\
\hline & Northwestern Energy - Garrison & GARSN & \\
\hline & WAPA - WAUW - MT North-Central & WAUW-M & \\
\hline \multirow{9}{*}{ Northern California } & Dept of Water Resources - North & DWR-N & \\
\hline & Modesto Irrigation District & MID & \\
\hline & Northern California Power Agency & NCPA & \\
\hline & Pacific Gas \& Electric - Main & PG-EM & \\
\hline & Redding Electric Dept & RDNG & \\
\hline & Sacramento Municipal Utilities District & SMUD & \\
\hline & Santa Clara Electric Dept & SNCL & \\
\hline & Turlock Irrigation District & TID & \\
\hline & WAPA - Mid Pacific (CVP) & WAMP & \\
\hline \multirow{15}{*}{ Northwest } & Avista & AVA & \\
\hline & BPA - Lower Columbia & LOWC & \\
\hline & BPA - Olympia & OLY & \\
\hline & BPA - Spokane & SPOK & \\
\hline & BPA - Western Montana & WMT & \\
\hline & Eugene Water and Electric Board & EWEB & \\
\hline & PACW - Mid Columbia & PMIDC & \\
\hline & PACW - Southern Oregon & PSORE & \\
\hline & Portland General Electric & PGE & \\
\hline & PUD No 1 of Chelan County & CHPD & \\
\hline & PUD No 1 of Cowlitz County & COPD & \\
\hline & PUD No 1 of Douglas County & DOPD & \\
\hline & PUD No 1 of Pend Oreille County & POPD & \\
\hline & PUD of Grant County & GCPD & \\
\hline & Puget Sound Energy & PSE & \\
\hline & Anaheim Public Utilities Dept. & ANHM & \\
\hline & Dept of Water Resources - South & DWR-S & \\
\hline & Riverside Utilities Dept & RVSD & \\
\hline Southern California & Southern California Edison & SCE & \\
\hline & San Diego Gas \& Electric & SDGE & \\
\hline & Imperial Irrigation District & IID & \\
\hline & Los Angeles Dept of Water and Power & LDWP & \\
\hline & PACE - Utah & UPL & \\
\hline Utah & Utah Associated Municipal Power & UAMPA & \\
\hline & Utah Municipal Power Agency & UMPAA & \\
\hline
\end{tabular}




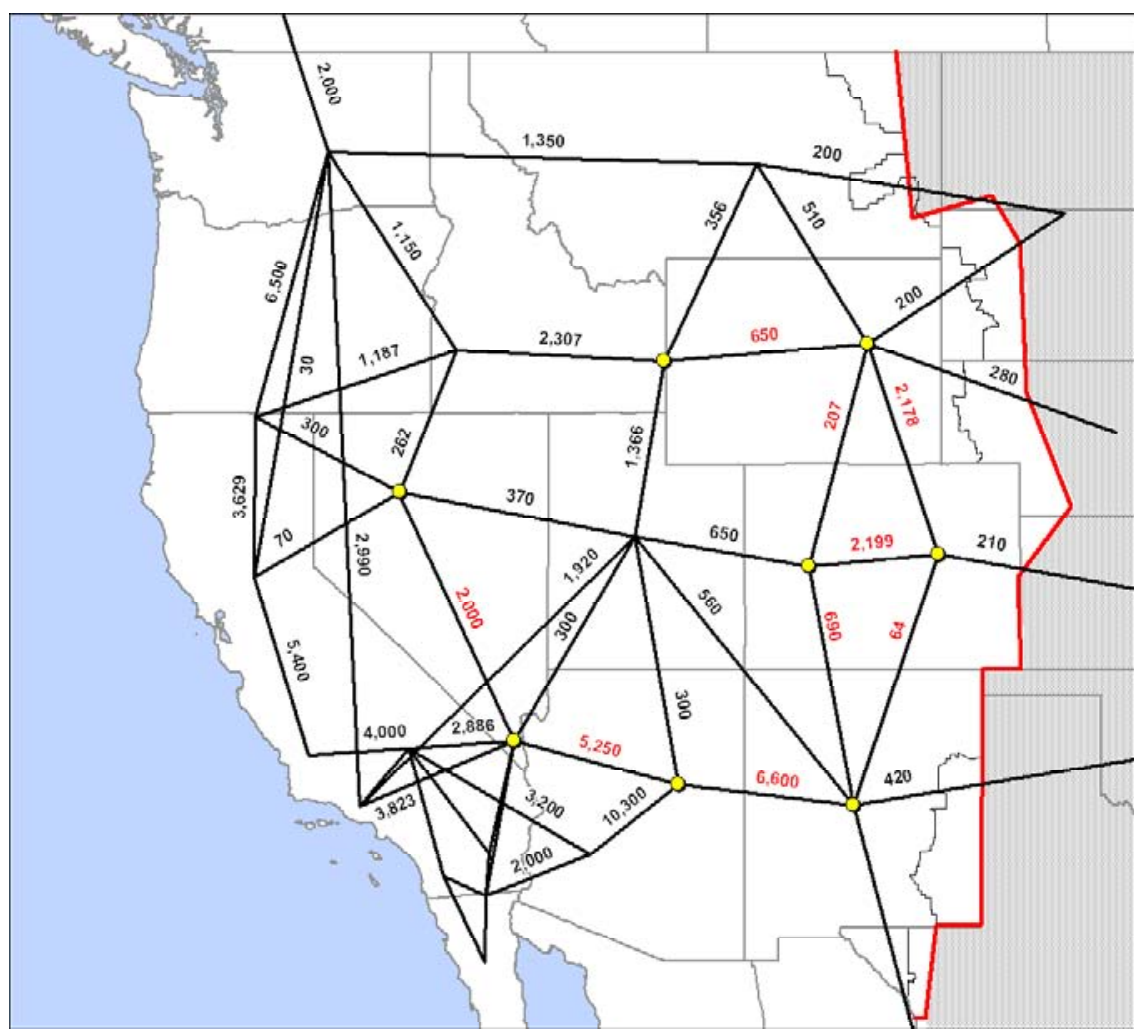

Figure 3.5 WECC Transmission Paths and Ratings (in MW)

Table 3.5 Selected WECC Transmission Path Ratings

\begin{tabular}{|l|l|l|}
\hline WECC Path & $\begin{array}{l}\text { Rating } \\
\text { (from-to, MW) }\end{array}$ & $\begin{array}{l}\text { Rating } \\
\text { (to-from, MW) }\end{array}$ \\
\hline Arizona to Southern Nevada & 5,250 & 5,250 \\
Colorado-East to New Mexico & 64 & 1 \\
Colorado-East to Colorado-West & 2,199 & 1,468 \\
Colorado-West to New Mexico & 690 & 690 \\
New Mexico to Arizona & 6,225 & 6,600 \\
Northern Nevada to Southern Nevada & 2,000 & 2,000 \\
Wyoming to Colorado-East & 1,605 & 2,178 \\
Wyoming to Colorado-West & 309 & 207 \\
\hline
\end{tabular}

\subsection{Development of Mega-Project Scenario}

The objective of the Mega-Project Scenario was to take advantage of the best wind and solar sites in the entire WestConnect footprint to meet the total renewable energy penetration targets of the region. Since many of the highest capacity factor wind sites are in Wyoming, this scenario naturally includes a high concentration of wind plants in 
Wyoming, with new transmission facilities to deliver the wind power to the large load centers in Arizona and Nevada.

The process to develop the Mega-Project Scenario started with the In-Area Scenario, which included the best wind and solar sites in each state. The concept was to swap less attractive local sites for more attractive remote sites. An algorithm was developed that displaced less attractive local sites in one area, making it a net importer, with the energy from higher-capacity sites in another area, making it a net exporter. Meanwhile, total renewable energy in the study footprint was held constant. The tradeoff of remote versus local sites accounted for the capital cost of generation equipment and transmission facilities, as well as the cost of transmission losses. The algorithm iterated towards a combination of resources that would meet the total renewable energy targets at minimum cost. An overview of the siting algorithm is presented here. Appendix A includes additional details about the methodology, data, and process.

The algorithm used the following assumptions and concepts. Capital costs are approximations in 2008 dollars.

- Capital cost for wind $=\$ 2000 / \mathrm{kW}$

- Capital cost for PV $=\$ 4000 / \mathrm{kW}$

- Capital cost for CSP plus storage $=\$ 4000 / \mathrm{kW}$

- Existing transmission would not be used for new renewable energy. New transmission would be required to accommodate wind/solar generation. Given that all remote renewable generation sites would rarely be at maximum output simultaneously, the total transmission requirement was estimated to be $70 \%$ of the maximum possible wind and solar power transfer. Therefore, $0.7 \mathrm{MW}$ of new transmission was added for each 1.0 MW of remote generation.

- New transmission was assumed to have a capital cost of $\$ 1600 / \mathrm{MW}-\mathrm{mi}$. With a $20 \%$ capital recovery factor, this translated to an annual carrying cost of \$320/MW-mi-yr.

- Transmission losses on new intra-area facilities were assumed to be $1 \%$ per 100 miles, based on the distances between the center coordinates of the transmission areas.

- Transmission cost included a proxy for the cost of inter-area transmission required to "collect" the energy from geographically diverse plants in exporting areas. A cost of $5 \%$ per 100 miles was applied to the distances from the plants to the center coordinates of the wind plants in the area.

Using this algorithm, the Mega-Project Scenario was developed to minimize the cost of generation equipment, new transmission, and losses. In addition to a list of wind and solar sites, the algorithm produced a set of inter-area transmission capacity 
requirements. These requirements were then manually adjusted to reflect practical constraints. Inter-area transfer requirements were rounded to match typical transmission line ratings; $1000 \mathrm{MW}$ for $345-\mathrm{kV}$ ac circuit, $1600 \mathrm{MW}$ for $500-\mathrm{kV}$ ac circuit, and $3600 \mathrm{MW}$ for a 600-kV HVDC bipole were assumed. Inter-area transfer requirements that fell significantly below these MW values were ignored.

Similarly, wind plants ratings were rounded off to be multiples of $30 \mathrm{MW}$ and solar plants were sized to be multiples of $100 \mathrm{MW}$. This was necessary because of how the site-swapping algorithm worked. It maintained constant wind and solar energy in the study footprint. When the algorithm removed a less attractive $30 \mathrm{MW}$ wind site in one area, it replaced it with a higher capacity factor site in another area, which meant adding a site smaller than $30 \mathrm{MW}$. Given this addition of fractional plants, it was necessary to round the final results into multiples of $30 \mathrm{MW}$ wind sites and $100 \mathrm{MW}$ solar sites.

Figure 3.6 shows a map of the Mega-Project Scenario. Table 3.6 summarizes the aggregated annual wind and solar energy for each area and Table 3.7 summarizes the aggregated wind and solar plant ratings. The red numbers on the map represent the total wind MW in each area. The change from the In-Area Scenario is shown in parentheses. Wyoming has a total of $13,770 \mathrm{MW}$ of wind generation, an increase of 11,430 MW over the In-Area Scenario. Arizona has a total of 1,890 MW of wind generation, a decrease of 9,330 MW from the In-Area Scenario. In order to meet the target of $30 \%$ wind energy, it is economically advantageous to build long-distance transmission to deliver higher capacity wind resources from Wyoming to Arizona, since the higher capacity wind resources in Wyoming can more than compensate for the costs related to transmitting the energy to Arizona. 


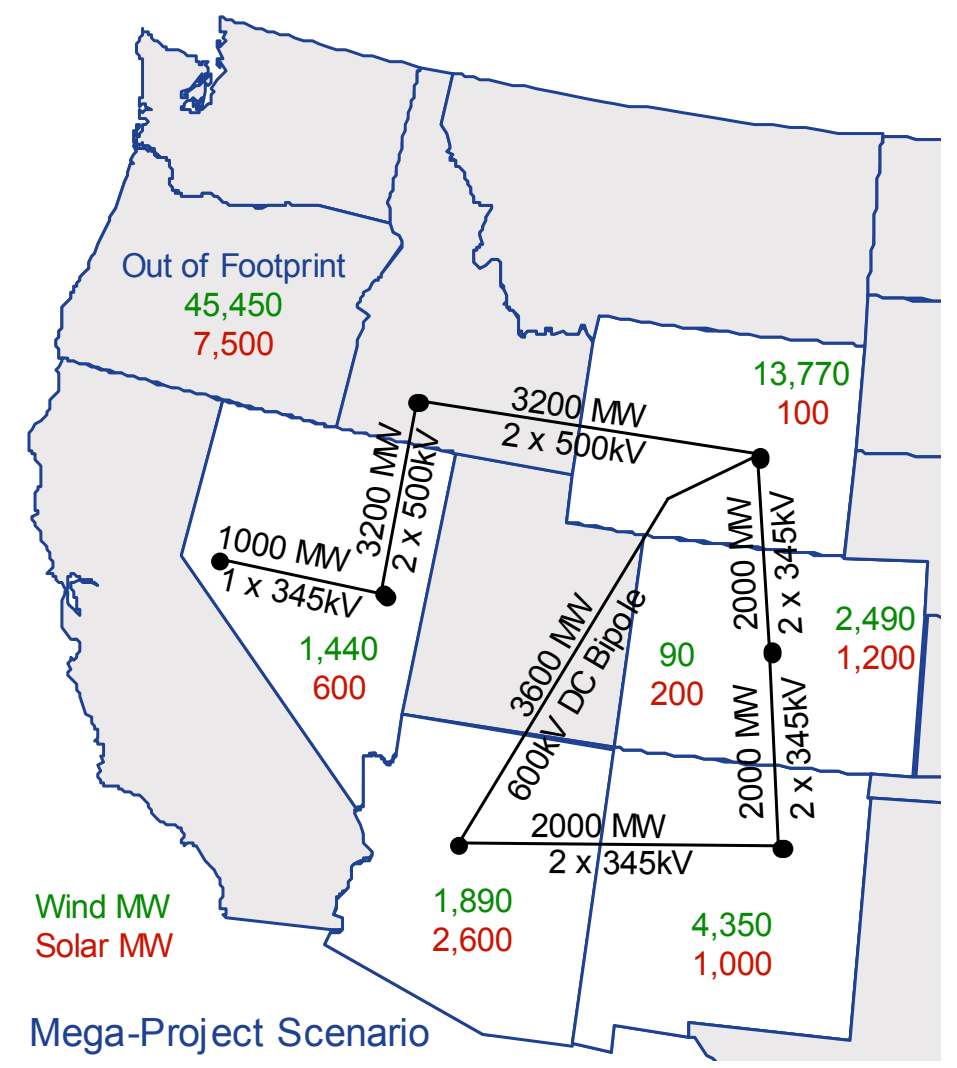

Figure 3.6 Map of Mega-Project Scenario with Transmission Additions 
Table 3.6 Energy Summary for 30\% Mega-Project Scenario

\begin{tabular}{|c|c|c|c|c|c|c|c|c|}
\hline \multirow[b]{2}{*}{ Area } & \multirow{2}{*}{$\begin{array}{c}\text { Load } \\
\text { Energy } \\
\text { (GWh) }\end{array}$} & \multicolumn{2}{|r|}{ Wind } & \multicolumn{2}{|c|}{ CSP with Storage } & \multicolumn{2}{|r|}{ PV } & \multirow{2}{*}{$\begin{array}{c}\text { Total } \\
\text { Renewable } \\
\text { Energy } \\
\text { (GWh) }\end{array}$} \\
\hline & & $\begin{array}{l}\text { Energy } \\
\text { (GWh) }\end{array}$ & \begin{tabular}{|c|} 
\# Sites \\
(Used/Available) \\
\end{tabular} & $\begin{array}{l}\text { Energy } \\
\text { (GWh) }\end{array}$ & $\begin{array}{c}\text { \# Sites } \\
\text { (Used/Available) }\end{array}$ & $\begin{array}{l}\text { Energy } \\
\text { (GWh) }\end{array}$ & \begin{tabular}{|c|} 
\# Sites \\
(Used/Available) \\
\end{tabular} & \\
\hline Arizona & 99,437 & 5,433 & 63 / 1091 & 5,503 & $15 / 38$ & 1,690 & $11 / 16$ & 12,626 \\
\hline Colorado East & 61,372 & 7,899 & $83 / 1673$ & 1,639 & $5 / 58$ & 1,035 & $7 / 13$ & 10,573 \\
\hline Colorado West & 8,708 & 293 & $3 / 68$ & 0 & $0 / 8$ & 287 & $2 / 11$ & 580 \\
\hline New Mexico & 31,260 & 14,921 & $145 / 3062$ & 1,533 & $4 / 35$ & 929 & $6 / 19$ & 17,383 \\
\hline Nevada & 57,505 & 3,755 & 48 / 1591 & 1,457 & $4 / 45$ & 320 & $2 / 10$ & 5,532 \\
\hline Wyoming & 27,697 & 53,758 & $459 / 8912$ & 0 & $0 / 0$ & 142 & $1 / 10$ & 53,900 \\
\hline In Footprint & 285,979 & 86,059 & $801 / 16397$ & 10,132 & $28 / 184$ & 4,403 & $29 / 79$ & 100,594 \\
\hline
\end{tabular}

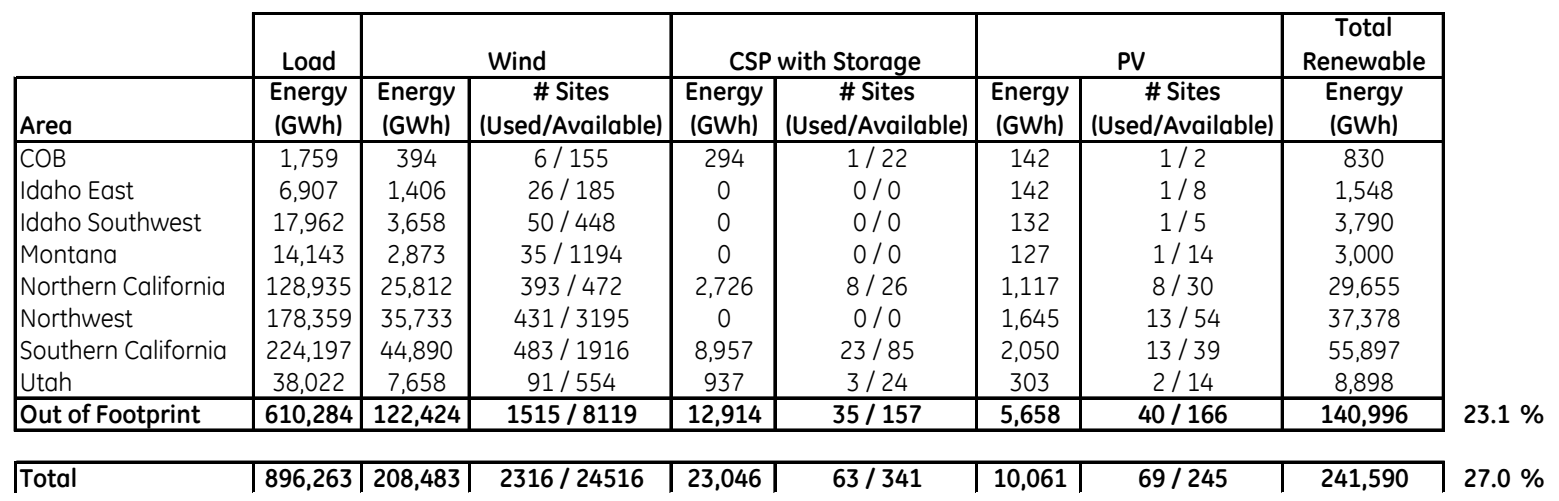

Table 3.7 Power Summary for 30\% Mega-Project Scenario

\begin{tabular}{|c|c|c|c|c|c|c|c|c|c|c|c|c|c|c|}
\hline \multirow[b]{3}{*}{ Area } & \multicolumn{2}{|c|}{ Load } & \multicolumn{3}{|c|}{ Wind } & \multicolumn{3}{|c|}{ CSP with Storage } & \multicolumn{3}{|c|}{ PV } & \multicolumn{3}{|c|}{ Total Renewable } \\
\hline & \multirow{2}{*}{\begin{tabular}{|c|} 
Minimum \\
$(\mathrm{MW})$
\end{tabular}} & \multirow{2}{*}{$\begin{array}{c}\text { Maximum } \\
(\mathrm{MW})\end{array}$} & \multirow{2}{*}{$\begin{array}{l}\text { Rating } \\
\text { (MW) }\end{array}$} & \multicolumn{2}{|c|}{ Penetration } & \multirow{2}{*}{$\begin{array}{l}\text { Rating } \\
\text { (MW) }\end{array}$} & \multicolumn{2}{|c|}{ Penetration } & \multirow{2}{*}{$\begin{array}{l}\text { Rating } \\
\text { (MW) }\end{array}$} & \multicolumn{2}{|c|}{ Penetration } & \multirow{2}{*}{$\begin{array}{l}\text { Rating } \\
\text { (MW) }\end{array}$} & \multicolumn{2}{|c|}{ Penetration } \\
\hline & & & & \% Min & \%Max & & \% Min & $\% \operatorname{Max}$ & & $\%$ Min & $\% \operatorname{Max}$ & & \% Min & $\% \operatorname{Max}$ \\
\hline Arizona & 6,995 & 23,051 & 1,890 & $27 \%$ & $8 \%$ & 1,500 & $21 \%$ & $7 \%$ & 1,100 & $16 \%$ & $5 \%$ & 4,490 & $64 \%$ & $19 \%$ \\
\hline Colorado East & 4,493 & 11,589 & 2,490 & $55 \%$ & $21 \%$ & 500 & $11 \%$ & $4 \%$ & 700 & $16 \%$ & $6 \%$ & 3,690 & $82 \%$ & $32 \%$ \\
\hline Colorado West & 712 & 1,526 & 90 & $13 \%$ & $6 \%$ & 0 & $0 \%$ & $0 \%$ & 200 & $28 \%$ & $13 \%$ & 290 & $41 \%$ & $19 \%$ \\
\hline New Mexico & 2,571 & 5,320 & 4,350 & $169 \%$ & $82 \%$ & 400 & $16 \%$ & $8 \%$ & 600 & $23 \%$ & $11 \%$ & 5,350 & $208 \%$ & $101 \%$ \\
\hline Nevada & 3,863 & 12,584 & 1,440 & $37 \%$ & $11 \%$ & 400 & $10 \%$ & $3 \%$ & 200 & $5 \%$ & $2 \%$ & 2,040 & $53 \%$ & $16 \%$ \\
\hline Wyoming & 2,369 & 4,016 & 13,770 & $581 \%$ & $343 \%$ & 0 & $0 \%$ & $0 \%$ & 100 & $4 \%$ & $2 \%$ & 13,870 & $586 \%$ & $345 \%$ \\
\hline In Footprint & 21,249 & 58,087 & 24,030 & $113 \%$ & $41 \%$ & 2,800 & $13 \%$ & $5 \%$ & 2,900 & $14 \%$ & $5 \%$ & 29,730 & $140 \%$ & $51 \%$ \\
\hline
\end{tabular}

\begin{tabular}{|c|c|c|c|c|c|c|c|c|c|c|c|c|c|c|}
\hline \multirow[b]{3}{*}{ Area } & \multicolumn{2}{|c|}{ Load } & \multicolumn{3}{|c|}{ Wind } & \multicolumn{3}{|c|}{ CSP with Storage } & \multicolumn{3}{|c|}{ PV } & \multicolumn{3}{|c|}{ Total Renewable } \\
\hline & \multirow{2}{*}{$\begin{array}{c}\text { Minimum } \\
(\mathrm{MW})\end{array}$} & \multirow{2}{*}{\begin{tabular}{|c|} 
Maximum \\
$(\mathrm{MW})$
\end{tabular}} & \multirow{2}{*}{\begin{tabular}{|l} 
Rating \\
(MW)
\end{tabular}} & \multicolumn{2}{|c|}{ Penetration } & \multirow{2}{*}{$\begin{array}{l}\text { Rating } \\
\text { (MW) }\end{array}$} & \multicolumn{2}{|c|}{ Penetration } & \multirow{2}{*}{$\begin{array}{l}\text { Rating } \\
\text { (MW) }\end{array}$} & \multicolumn{2}{|c|}{ Penetration } & \multirow{2}{*}{$\begin{array}{l}\text { Rating } \\
\text { (MW) }\end{array}$} & \multicolumn{2}{|c|}{ Penetration } \\
\hline & & & & $\%$ Min & \%Max & & $\%$ Min & $\% \operatorname{Max}$ & & $\%$ Min & $\% \operatorname{Max}$ & & $\%$ Min & $\% \operatorname{Max}$ \\
\hline$\overline{C O B}$ & 138 & 294 & 180 & $131 \%$ & $61 \%$ & 100 & $73 \%$ & $34 \%$ & 100 & $73 \%$ & $34 \%$ & 380 & $276 \%$ & $129 \%$ \\
\hline Idaho East & 460 & 1,365 & 780 & $170 \%$ & $57 \%$ & 0 & $0 \%$ & $0 \%$ & 100 & $22 \%$ & $7 \%$ & 880 & $191 \%$ & $64 \%$ \\
\hline Idaho Southwest & 1,188 & 3,592 & 1,500 & $126 \%$ & $42 \%$ & 0 & $0 \%$ & $0 \%$ & 100 & $8 \%$ & $3 \%$ & 1,600 & $135 \%$ & $45 \%$ \\
\hline Montana & 1,149 & 2,337 & 1,050 & $91 \%$ & $45 \%$ & 0 & $0 \%$ & $0 \%$ & 100 & $9 \%$ & $4 \%$ & 1,150 & $100 \%$ & $49 \%$ \\
\hline Northern California & 10,297 & 28,319 & 11,790 & $114 \%$ & $42 \%$ & 800 & $8 \%$ & $3 \%$ & 800 & $8 \%$ & $3 \%$ & 13,390 & $130 \%$ & $47 \%$ \\
\hline Northwest & 14,278 & 30,953 & 12,930 & $91 \%$ & $42 \%$ & 0 & $0 \%$ & $0 \%$ & 1,300 & $9 \%$ & $4 \%$ & 14,230 & $100 \%$ & $46 \%$ \\
\hline Southern California & 9,557 & 26,864 & 14,490 & $152 \%$ & $54 \%$ & 2,300 & $24 \%$ & $9 \%$ & 1,300 & $14 \%$ & $5 \%$ & 18,090 & $189 \%$ & $67 \%$ \\
\hline Utah & 2,263 & 7,274 & 2,730 & $121 \%$ & $38 \%$ & 300 & $13 \%$ & $4 \%$ & 200 & $9 \%$ & $3 \%$ & 3,230 & $143 \%$ & $44 \%$ \\
\hline Out of Footprint & 46,328 & 119,696 & 45,450 & $98 \%$ & $38 \%$ & 3,500 & $8 \%$ & $3 \%$ & 4,000 & $9 \%$ & $3 \%$ & 52,950 & $114 \%$ & $44 \%$ \\
\hline Total & 67.577 & 177783 & 69,480 & $103 \%$ & $39 \%$ & 6.300 & $9 \%$ & $4 \%$ & 6.900 & $10 \%$ & $4 \%$ & 82.680 & $122 \%$ & $47 \%$ \\
\hline
\end{tabular}

\subsection{Development of Local-Priority Scenario}

The Local-Priority Scenario was developed from the In-Area Scenario using the same algorithm and assumptions as for the Mega-Project Scenario. The only difference was that the capital cost of In-Area wind and solar generation was assumed to be $10 \%$ lower 
than for remote wind and solar sites in other areas. This served as a proxy for local incentive programs that would produce local social benefits such as jobs and tax revenue.

Figure 3.7 shows a map of the Local-Priority Scenario. Table 3.8 shows the total capital costs of the three scenarios at 30\% wind and 5\% solar penetration. The Local-Priority Scenario has the lowest total capital cost of the three scenarios because of the rounding off of new inter-area transmission ratings. Aggregated wind and solar generation energy and MW ratings for each area are shown in Table 3.9 and Table 3.10.

The distribution of wind and solar resources in the Local-Priority Scenario is about halfway between the In-Area Scenario and the Mega-Project Scenario. Of the three major scenarios in this study, the project team considers this the most realistic. It seems likely that states will want to produce a significant portion of their required renewable energy locally. In fact, Colorado already has a program to encourage local production of renewable energy [3.1]. However, given the extraordinary quality of the wind resources in Wyoming and a few other locations, it seems likely that some renewable energy will be exported to other states.

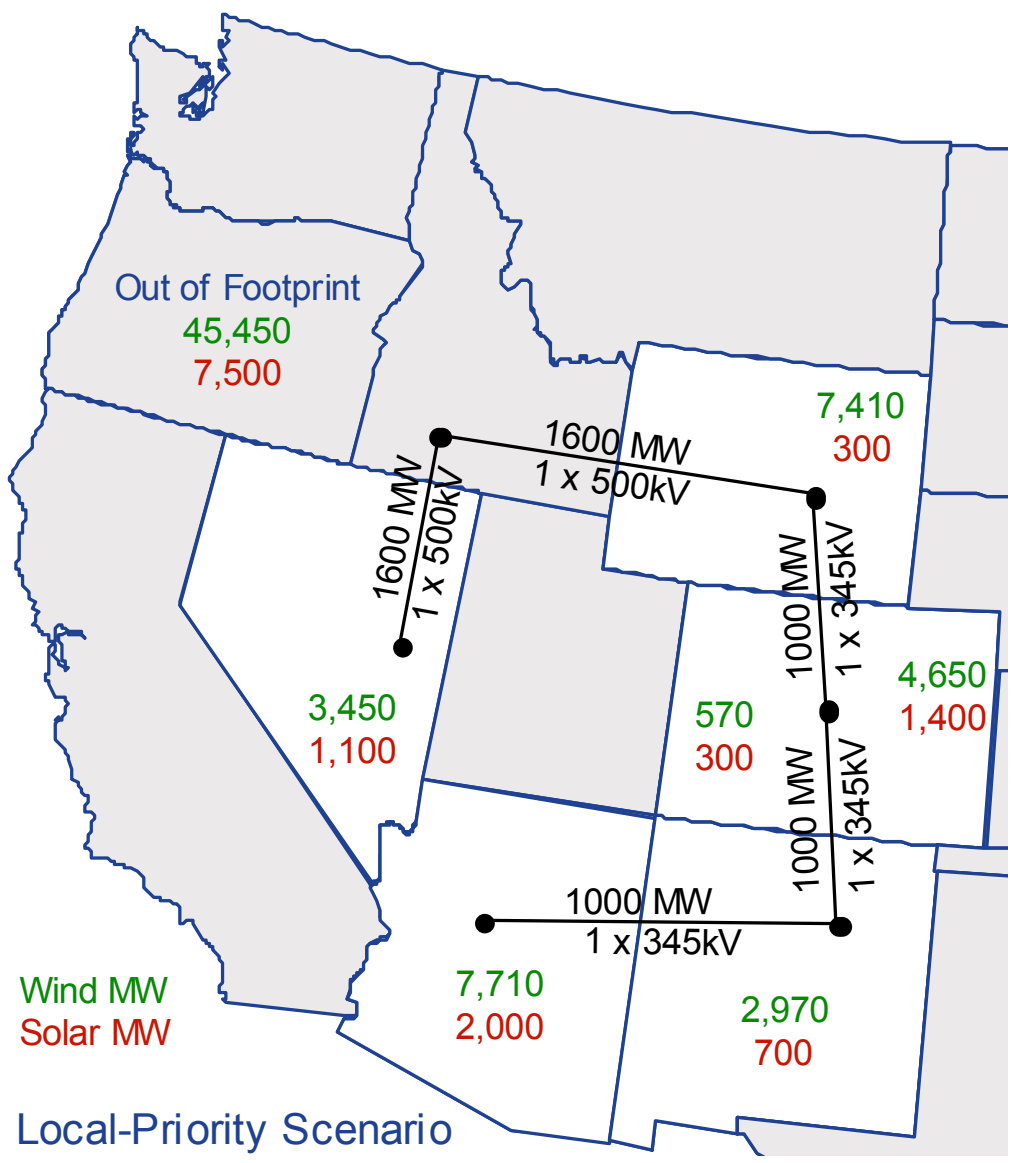

Figure 3.7 Map of Local-Priority Scenario with Transmission Additions 
Other power and energy summary tables for $10 \%$ and $20 \%$ penetration levels are included in Appendix A.

Table 3.8 Summary of Capital Costs included in Scenario Development

\begin{tabular}{|l|c|c|c|c|c|c|c|c|}
\hline Scenario & $\begin{array}{c}\text { Wind } \\
\text { (MW) }\end{array}$ & $\begin{array}{c}\text { Solar } \\
\text { (MW) }\end{array}$ & $\begin{array}{c}\text { Transmission } \\
\text { (GW-mi) }\end{array}$ & $\begin{array}{c}\text { Wind } \\
\text { Cost } \\
\text { (\$B) }\end{array}$ & $\begin{array}{c}\text { Solar } \\
\text { Cost } \\
\text { (\$B) }\end{array}$ & $\begin{array}{c}\text { Transmission } \\
\text { Cost } \\
\text { (\$B) }\end{array}$ & $\begin{array}{c}\text { Total } \\
\text { Cost } \\
\text { (\$B) }\end{array}$ & $\begin{array}{c}\Delta \\
\text { (\$B) }\end{array}$ \\
\hline In-Area & 29,940 & 5,800 & 0 & 59.9 & 23.2 & 0 & 83.1 & \\
Local-Priority & 26,760 & 5,800 & 2,100 & 53.5 & 23.2 & 3.4 & 80.1 & -3.0 \\
Mega-Project & 24,030 & 5,700 & 6,900 & 48.1 & 22.8 & 11.0 & 81.9 & -1.2 \\
\hline
\end{tabular}


Table 3.9 Energy Summary for $30 \%$ Local-Priority Scenario

\begin{tabular}{|c|c|c|c|c|c|c|c|c|}
\hline \multirow[b]{2}{*}{ Area } & \multirow{2}{*}{$\begin{array}{c}\text { Load } \\
\text { Energy } \\
\text { (GWh) }\end{array}$} & \multicolumn{2}{|r|}{ Wind } & \multicolumn{2}{|c|}{ CSP with Storage } & \multicolumn{2}{|r|}{ PV } & \multirow{2}{*}{$\begin{array}{c}\begin{array}{c}\text { Total } \\
\text { Renewable }\end{array} \\
\text { Energy } \\
\text { (GWh) }\end{array}$} \\
\hline & & $\begin{array}{l}\text { Energy } \\
\text { (GWh) }\end{array}$ & $\begin{array}{c}\text { \# Sites } \\
\text { (Used/Available) }\end{array}$ & $\begin{array}{l}\text { Energy } \\
\text { (GWh) }\end{array}$ & $\begin{array}{c}\text { \# Sites } \\
\text { (Used/Available) }\end{array}$ & $\begin{array}{l}\text { Energy } \\
\text { (GWh) }\end{array}$ & $\begin{array}{c}\text { \# Sites } \\
\text { (Used/Available) }\end{array}$ & \\
\hline Arizona & 99,437 & 21,138 & $257 / 1091$ & 3,735 & $10 / 38$ & 1,548 & $10 / 16$ & 26,421 \\
\hline Colorado East & 61,372 & 15,320 & $155 / 1673$ & 2,252 & $7 / 58$ & 1,038 & $7 / 13$ & 18,610 \\
\hline Colorado West & 8,708 & 1,736 & $19 / 68$ & 564 & $2 / 8$ & 151 & $1 / 11$ & 2,451 \\
\hline New Mexico & 31,260 & 10,047 & $99 / 3062$ & 1,421 & $4 / 35$ & 473 & $3 / 19$ & 11,941 \\
\hline Nevada & 57,505 & 9,101 & 115 / 1591 & 2,161 & $6 / 45$ & 773 & $5 / 10$ & 12,035 \\
\hline Wyoming & 27,697 & 28,716 & 247 / 8912 & 0 & $0 / 0$ & 420 & $3 / 10$ & 29,136 \\
\hline In Footprint & 285,979 & 86,058 & 892 / 16397 & 10,133 & $29 / 184$ & 4,403 & $29 / 79$ & 100,594 \\
\hline
\end{tabular}

\begin{tabular}{|c|c|c|c|c|c|c|c|c|}
\hline \multirow[b]{2}{*}{ Area } & Load & \multicolumn{2}{|r|}{ Wind } & \multicolumn{2}{|c|}{ CSP with Storage } & \multicolumn{2}{|r|}{ PV } & \multirow{2}{*}{$\begin{array}{c}\text { Total } \\
\text { Renewable } \\
\text { Energy } \\
\text { (GWh) }\end{array}$} \\
\hline & $\begin{array}{l}\text { Energy } \\
\text { (GWh) }\end{array}$ & $\begin{array}{l}\text { Energy } \\
\text { (GWh) }\end{array}$ & $\begin{array}{c}\text { \# Sites } \\
\text { (Used/Available) }\end{array}$ & $\begin{array}{l}\text { Energy } \\
\text { (GWh) }\end{array}$ & $\begin{array}{c}\text { \# Sites } \\
\text { (Used/Available) }\end{array}$ & $\begin{array}{l}\text { Energy } \\
\text { (GWh) }\end{array}$ & $\begin{array}{c}\text { \# Sites } \\
\text { (Used/Available) }\end{array}$ & \\
\hline $\mathrm{COB}$ & 1,759 & 394 & $6 / 155$ & 294 & $1 / 22$ & 142 & $1 / 2$ & 830 \\
\hline Idaho East & 6,907 & 1,406 & $26 / 185$ & 0 & $0 / 0$ & 142 & $1 / 8$ & 1,548 \\
\hline Idaho Southwest & 17,962 & 3,658 & $50 / 448$ & 0 & $0 / 0$ & 132 & $1 / 5$ & 3,790 \\
\hline Montana & 14,143 & 2,873 & $35 / 1194$ & 0 & $0 / 0$ & 127 & $1 / 14$ & 3,000 \\
\hline Northern California & 128,935 & 25,812 & $393 / 472$ & 2,726 & $8 / 26$ & 1,117 & $8 / 30$ & 29,655 \\
\hline Northwest & 178,359 & 35,733 & $431 / 3195$ & 0 & $0 / 0$ & 1,645 & $13 / 54$ & 37,378 \\
\hline Southern California & 224,197 & 44,890 & $483 / 1916$ & 8,957 & $23 / 85$ & 2,050 & $13 / 39$ & 55,897 \\
\hline Utah & 38,022 & 7,658 & $91 / 554$ & 937 & $3 / 24$ & 303 & $2 / 14$ & 8,898 \\
\hline Out of Footprint & 610,284 & 122,424 & $1515 / 8119$ & 12,914 & $35 / 157$ & 5,658 & $40 / 166$ & 140,996 \\
\hline
\end{tabular}

Table 3.10 Power Summary for 30\% Local-Priority Scenario

\begin{tabular}{|c|c|c|c|c|c|c|c|c|c|c|c|c|c|c|}
\hline \multirow[b]{3}{*}{ Area } & \multicolumn{2}{|c|}{ Load } & \multicolumn{3}{|c|}{ Wind } & \multicolumn{3}{|c|}{ CSP with Storage } & \multicolumn{3}{|c|}{ PV } & \multicolumn{3}{|c|}{ Total Renewable } \\
\hline & \multirow{2}{*}{$\begin{array}{l}\text { Minimum } \\
\text { (MW) }\end{array}$} & \multirow{2}{*}{$\begin{array}{l}\text { Maximum } \\
(\mathrm{MW})\end{array}$} & \multirow{2}{*}{$\begin{array}{l}\text { Rating } \\
\text { (MW) }\end{array}$} & \multicolumn{2}{|c|}{ Penetration } & \multirow{2}{*}{$\begin{array}{l}\text { Rating } \\
\text { (MW) }\end{array}$} & \multicolumn{2}{|c|}{ Penetration } & \multirow{2}{*}{$\begin{array}{l}\text { Rating } \\
\text { (MW) }\end{array}$} & \multicolumn{2}{|c|}{ Penetration } & \multirow{2}{*}{$\begin{array}{l}\text { Rating } \\
\text { (MW) }\end{array}$} & \multicolumn{2}{|c|}{ Penetration } \\
\hline & & & & $\%$ Min & \%Max & & $\%$ Min & $\% \operatorname{Max}$ & & $\% \operatorname{Min}$ & $\%$ Max & & $\%$ Min & $\% \operatorname{Max}$ \\
\hline Arizona & 6,995 & 23,051 & 7,710 & $110 \%$ & $33 \%$ & 1,000 & $14 \%$ & $4 \%$ & 1,000 & $14 \%$ & $4 \%$ & 9,710 & $139 \%$ & $42 \%$ \\
\hline Colorado East & 4,493 & 11,589 & 4,650 & $103 \%$ & $40 \%$ & 700 & $16 \%$ & $6 \%$ & 700 & $16 \%$ & $6 \%$ & 6,050 & $135 \%$ & $52 \%$ \\
\hline Colorado West & 712 & 1,526 & 570 & $80 \%$ & $37 \%$ & 200 & $28 \%$ & $13 \%$ & 100 & $14 \%$ & $7 \%$ & 870 & $122 \%$ & $57 \%$ \\
\hline New Mexico & 2,571 & 5,320 & 2,970 & $116 \%$ & $56 \%$ & 400 & $16 \%$ & $8 \%$ & 300 & $12 \%$ & $6 \%$ & 3,670 & $143 \%$ & $69 \%$ \\
\hline Nevada & 3,863 & 12,584 & 3,450 & $89 \%$ & $27 \%$ & 600 & $16 \%$ & $5 \%$ & 500 & $13 \%$ & $4 \%$ & 4,550 & $118 \%$ & $36 \%$ \\
\hline Wyoming & 2,369 & 4,016 & 7,410 & $313 \%$ & $184 \%$ & 0 & $0 \%$ & $0 \%$ & 300 & $13 \%$ & $7 \%$ & 7,710 & $325 \%$ & $192 \%$ \\
\hline \multirow[t]{3}{*}{ In Footprint } & 21,249 & 58,087 & 26,760 & $126 \%$ & $46 \%$ & 2,900 & $14 \%$ & $5 \%$ & 2,900 & $14 \%$ & $5 \%$ & 32,560 & $153 \%$ & $56 \%$ \\
\hline & \multicolumn{2}{|c|}{ Load } & \multicolumn{3}{|c|}{ Wind } & \multicolumn{3}{|c|}{ CSP with Storage } & \multicolumn{3}{|c|}{ PV } & \multicolumn{3}{|c|}{ Total Renewable } \\
\hline & \multirow{2}{*}{$\begin{array}{c}\text { Minimum } \\
(\mathrm{MW})\end{array}$} & \multirow{2}{*}{$\begin{array}{l}\text { Maximum } \\
\text { (MW) }\end{array}$} & \multirow{2}{*}{$\begin{array}{l}\text { Rating } \\
\text { (MW) }\end{array}$} & \multicolumn{2}{|c|}{ Penetration } & \multirow{2}{*}{\begin{tabular}{|l|} 
Rating \\
(MW)
\end{tabular}} & \multicolumn{2}{|c|}{ Penetration } & \multirow{2}{*}{$\begin{array}{l}\text { Rating } \\
(\mathrm{MW})\end{array}$} & \multicolumn{2}{|c|}{ Penetration } & \multirow{2}{*}{$\begin{array}{l}\text { Rating } \\
\text { (MW) }\end{array}$} & Penet & ration \\
\hline Area & & & & $\%$ Min & \%Max & & \% Min & $\% \operatorname{Max}$ & & $\% \operatorname{Min}$ & $\% \operatorname{Max}$ & & \% Min & $\% \operatorname{Max}$ \\
\hline $\mathrm{COB}$ & 138 & 294 & 180 & $131 \%$ & $61 \%$ & 100 & $73 \%$ & $34 \%$ & 100 & $73 \%$ & $34 \%$ & 380 & $276 \%$ & $129 \%$ \\
\hline Idaho East & 460 & 1,365 & 780 & $170 \%$ & $57 \%$ & 0 & $0 \%$ & $0 \%$ & 100 & $22 \%$ & $7 \%$ & 880 & $191 \%$ & $64 \%$ \\
\hline Idaho Southwest & 1,188 & 3,592 & 1,500 & $126 \%$ & $42 \%$ & 0 & $0 \%$ & $0 \%$ & 100 & $8 \%$ & $3 \%$ & 1,600 & $135 \%$ & $45 \%$ \\
\hline Montana & 1,149 & 2,337 & 1,050 & $91 \%$ & $45 \%$ & 0 & $0 \%$ & $0 \%$ & 100 & $9 \%$ & $4 \%$ & 1,150 & $100 \%$ & $49 \%$ \\
\hline Northern California & 10,297 & 28,319 & 11,790 & $114 \%$ & $42 \%$ & 800 & $8 \%$ & $3 \%$ & 800 & $8 \%$ & $3 \%$ & 13,390 & $130 \%$ & $47 \%$ \\
\hline Northwest & 14,278 & 30,953 & 12,930 & $91 \%$ & $42 \%$ & 0 & $0 \%$ & $0 \%$ & 1,300 & $9 \%$ & $4 \%$ & 14,230 & $100 \%$ & $46 \%$ \\
\hline Southern California & 9,557 & 26,864 & 14,490 & $152 \%$ & $54 \%$ & 2,300 & $24 \%$ & $9 \%$ & 1,300 & $14 \%$ & $5 \%$ & 18,090 & $189 \%$ & $67 \%$ \\
\hline Utah & 2,263 & 7,274 & 2,730 & $121 \%$ & $38 \%$ & 300 & $13 \%$ & $4 \%$ & 200 & $9 \%$ & $3 \%$ & 3,230 & $143 \%$ & $44 \%$ \\
\hline Out of Footprint & 46,328 & 119,696 & 45,450 & $98 \%$ & $38 \%$ & 3,500 & $8 \%$ & $3 \%$ & 4,000 & $9 \%$ & $3 \%$ & 52,950 & $114 \%$ & $44 \%$ \\
\hline
\end{tabular}

\subsection{References}

[3.1] Excerpt from Colorado Renewable Energy Standard, "Eligible electricity generated in Colorado is favored. Each kilowatt-hour ( $k W h$ ) of eligible electricity generated in-state, other than retail DG, receives $125 \%$ credit for RPS-compliance purposes."

http://www.dsireusa.org/incentives/incentive.cfm?Incentive Code=CO24R. 


\section{Statistical Analysis: Seasonal, Monthly, and Daily Trends}

One undeniable constant of power systems operation is that load is always changing second-to-second, minute-to-minute, hourly, seasonally, and yearly. The variability of load (or the degree to which it changes) is dependent on a wide range of factors, not the least among them being human behavior. The key goal of power systems operation is to maintain the crucial balance between system load and dispatchable generation.

As the amount of variable resources like wind and solar generation increases on the system, the balancing act becomes more intricate. Because they are not considered "firm" generation, wind, and solar output contribute to the "net load"1 seen by the balance of generation. As such, they increase the variability of the system load and consequently the ramp and range requirements for units committed to meet the load.

The goal of this chapter (and the next) is to statistically characterize the hourly variability of the load, wind, solar, and net load, in individual states/transmission areas, the study footprint, and WECC. The analysis will begin with an overall examination of broad trends in Chapter 4, and progressively delve into the nuances and implications of period-to-period changes in Chapter 5.

As discussed earlier in Chapter 2, there is a tremendous amount of data available for analysis. The breakdown from a statistical point-of-view is as follows

- Three data years: 2004, 2005, and 2006

- 14 transmission areas (comprising 108 load zones): 6 in study footprint, 8 outside

- Four penetration levels: baseline, $10 \%, 20 \%$, and $30 \%$

- Three study scenarios: In-Area (IA), Local-Priority (LP), and Mega-Project (MP)

These cases taken together lead to 504 unique combinations that could potentially be analyzed. This is shown graphically in Figure 4.1 below. For example, a plot of the average daily load, wind, and solar profiles for year 2006 in Arizona for the 30\% penetration level in the Local-Priority Scenario may be interesting, but is only one of 504 such plots that convey similar information for other years, states, penetration levels, and scenarios. Since it would be prohibitive to present this level of detail, the chapter focuses on meaningful representative samples of the analyses. Most often (though not always), the statistical characterization is illustrated using 2006 data, the Local-Priority Scenario,

\footnotetext{
${ }^{1}$ Net load is defined as the instantaneous system load minus the generation output of nondispatchable wind and solar generation (Load-Wind-Solar), i.e., net load is the amount of generation required from dispatchable units.
} 
$30 \%$ penetration level and the In-footprint states (blue path in Figure 4.1). The reasons for this are discussed throughout the chapter. Other illustrative charts and data are included in the appendices. However, even the appendix material is selected to give a broad overview of the work, as opposed to exhaustive detail of every chart and graph produced in the study.

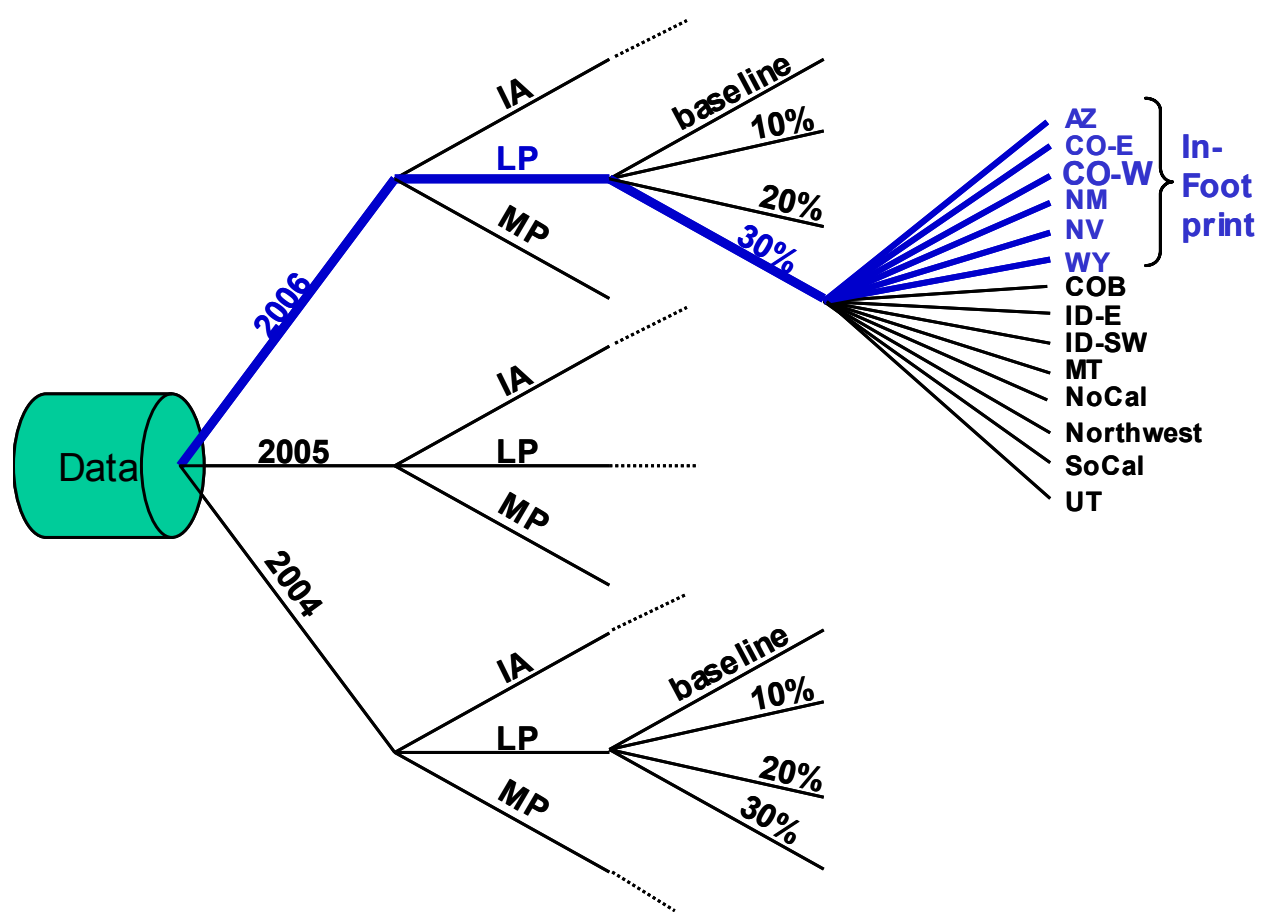

Figure 4.1 Graphical Representation of Statistical Study Permutations and Most Often Used Path

\subsection{Monthly Energy from Wind and Solar}

As discussed in Chapter 3, the wind and solar sites were selected within the WECC region to produce the $10 \%, 20 \%$, and $30 \%$ penetration levels under the 3 scenarios InArea, Local-Priority, and Mega-Project. Earlier in Table 2.1, the wind and solar combinations for the penetration levels were described. For the $30 \%$ penetration level under the In-Area Scenario, wind sites are selected such that the aggregate nameplate wind can supply on average $30 \%$ of the annual load energy inside the footprint and $20 \%$ of the annual load energy outside the footprint. Similarly, $\mathrm{CSP}^{2}$ and PV sites are chosen to supply $5 \%$ of the annual load energy inside the footprint and $3 \%$ outside.

However, due to the variable nature of the wind and solar resources, one would not expect that they would meet $35 \%$ of the load energy at all times. A key question examined in is this section is, what is the monthly/seasonal and yearly variation of wind and solar energy within footprint and across states/areas, and how closely does it correspond to the expected penetration outlined earlier in Table 2.1.

${ }^{2}$ In many figures and charts, CSP is listed as CSPws where "ws" denotes "with storage." 


\subsubsection{Year-to-Year Comparison}

Earlier in Chapter 3, wind and solar site selection was discussed, and Table 3.1 listed the annual wind energy by state in 2004, 2005, and 2006 for the 30\% In-Area Scenario. At the footprint level, the total annual wind and solar energy only varies slightly between years, but more variation is expected at higher spatial and temporal resolutions.

Figure 4.2 shows the study footprint monthly energy from wind and solar for 2004, 2005, and 2006 wind shapes in GWh. Figure 4.3 plots the monthly energy as a percentage of load energy in the month. In both plots, the green sections of each bar indicate monthly energy from wind, the orange sections indicate monthly energy from CSP, and the magenta sections indicate monthly energy from PV. The height of the bar gives the total renewable energy for each month of the year. For illustration, the $30 \%$ penetration level for the In-Area Scenario is shown. Similar plots for the other penetration levels and scenarios are included in Appendix B.

The data in the plots and Table 4.1 clearly indicate two salient points (1) there is significant year-to-year variation in the total monthly energies, and (2) $30 \%$ is not always $30 \%$ ! These points are discussed further below.

On the first point, overall, there is 5\% more wind and solar energy in 2006 than 2004, but the seasonal differences are even more evident. During winter and early spring there is over 30\% more energy from 2006 wind and solar than for the same period in 2004.

Conversely, in the late spring to summer period there is over $20 \%$ more wind and solar energy in 2004 than 2006. The highest monthly total energy $(11,651 \mathrm{GWh})$ is recorded in March 2006, and the lowest (5,022 GWh) occurs in August 2006. The 2006 data exhibits much more dynamic range and deviation than other years, which makes 2006 a good year to illustrate the statistical variability.

On the second point, Figure 4.3 clearly shows the seasonal characteristic of the wind and solar energy and highlights its relationship to the load variation (this will be explored further in Section 4.3). Over each year, wind energy is 30\% of the load, and solar energy is $5 \%$, but the monthly penetration varies significantly from season to season regardless of the year. For example, in March and April 2006, wind and solar combine to meet 55\% of the load, but in July and August 2006, they combine to meet just $17 \%$. This is partially because the study footprint load is higher in summer than in the fall, but also the wind energy content tends to be more in the spring months and less in the summer (reverse for solar). Later sections in this chapter will delve deeper into the load-wind coincidence and the potential operational issues, particularly during low-load, high-wind hours. 


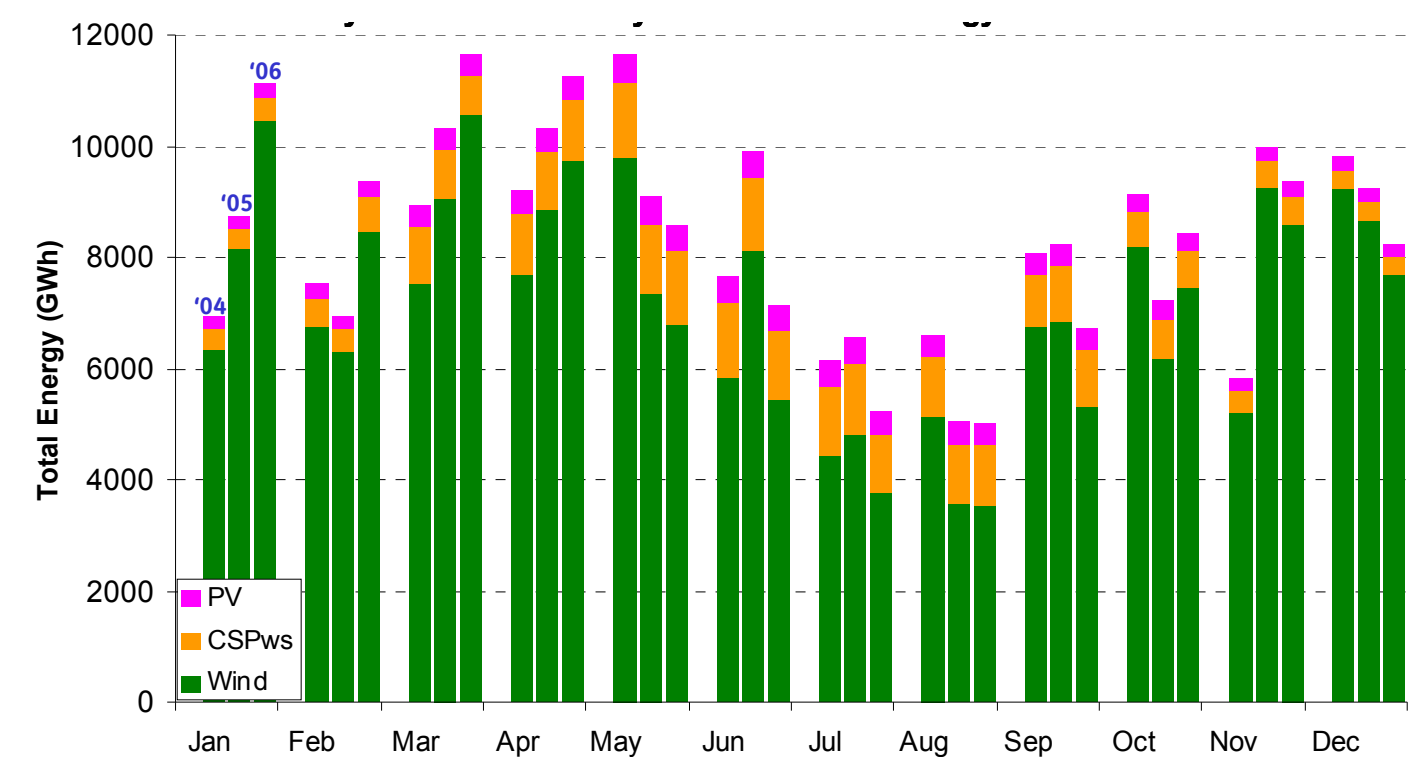

Figure 4.2 Total In-Footprint Monthly Energy from Wind and Solar for 2004 - 2006 (30\% In-Area Scenario)

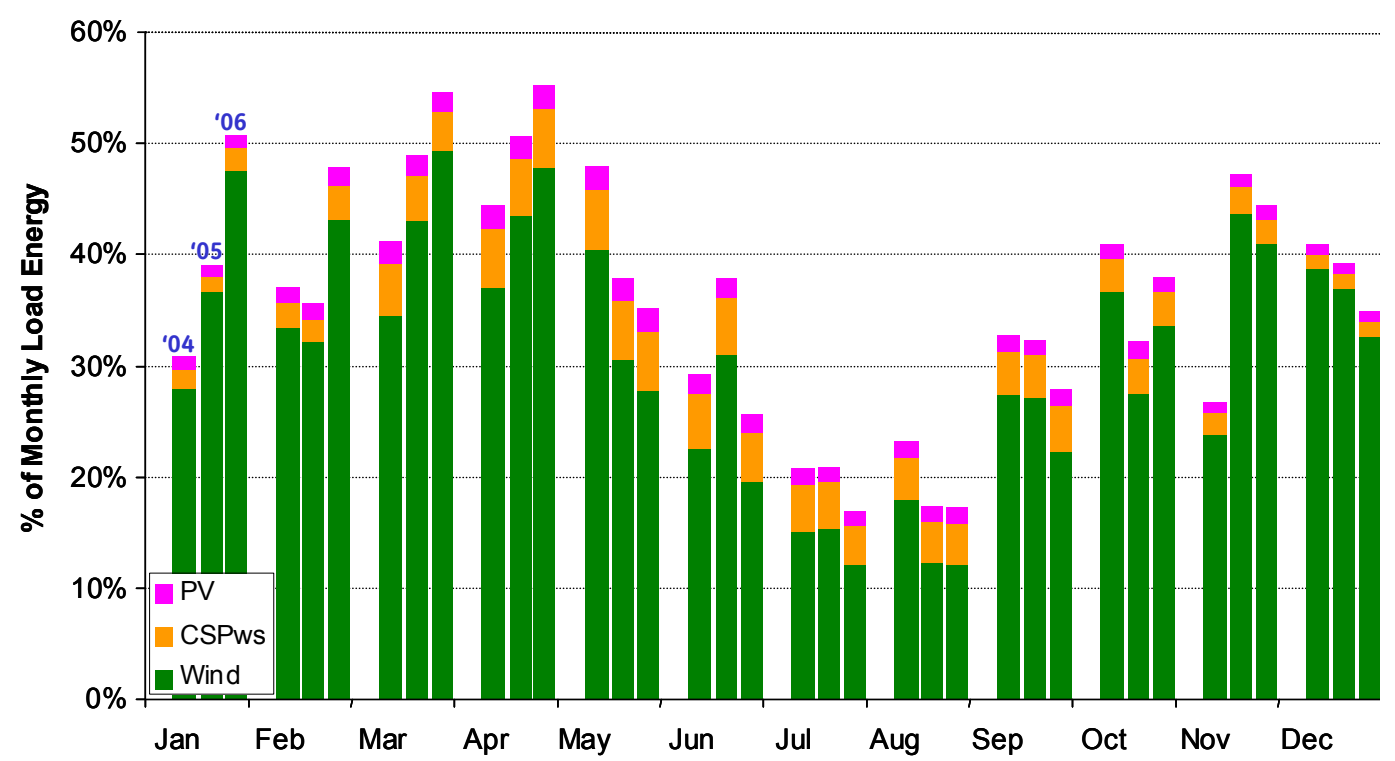

Figure 4.3 Total In-Footprint Monthly Energy from Wind and Solar for $2004-2006$ (30\% In-Area Scenario)

Table 4.1 summarizes the monthly energies for individual states in the study footprint and the total footprint. These data support the two salient points made earlier. Within each area, there is significant year-to-year variation in the total monthly energies, and in some months, the energy penetration in certain states is well over $30 \%$. The second point is discussed further in Section 4.1.3. 
Table 4.1 Monthly Wind and Solar Energy Summary for 2004, 2005, and 2006, 30\% In-Area Scenario

\begin{tabular}{|c|c|c|c|c|c|c|c|c|c|c|c|c|}
\hline \multicolumn{13}{|c|}{$2004 \mathrm{GWh}$} \\
\hline & Jan & Feb & Mar & Apr & May & Jun & Jul & Aug & Sep & Oct & Nov & Dec \\
\hline$A Z$ & 1,779 & 2,681 & 2,637 & 3,638 & 4,273 & 2,812 & 2,020 & 2,207 & 3,023 & 3,519 & 2,356 & 2,890 \\
\hline CE & 1,891 & 1,590 & 2,194 & 1,708 & 2,489 & 1,510 & 1,284 & 1,401 & 1,781 & 1,850 & 1,199 & 2,413 \\
\hline CW & 227 & 208 & 338 & 284 & 433 & 280 & 236 & 231 & 278 & 283 & 132 & 328 \\
\hline NMI & 888 & 895 & 1,061 & 1,011 & 1,191 & 829 & 654 & 773 & 978 & 963 & 668 & 1,058 \\
\hline NV & 1,204 & 1,570 & 1,767 & 1,945 & 2,302 & 1,601 & 1,404 & 1,496 & 1,430 & 1,876 & 986 & 2,046 \\
\hline WY & 981 & 602 & 953 & 637 & 955 & 609 & 528 & 513 & 592 & 640 & 485 & 1,072 \\
\hline FP & 6,970 & 7,546 & 8,951 & 9,222 & 11,643 & 7,641 & 6,127 & 6,620 & 8,082 & 9,131 & 5,827 & 9,807 \\
\hline \multicolumn{13}{|c|}{2005 GWh } \\
\hline & Jan & Feb & Mar & Apr & May & Jun & Jul & Aug & Sep & Oct & Nov & Dec \\
\hline$A Z$ & 2,977 & 2,810 & 4,055 & 4,083 & 3,510 & 3,810 & 2,441 & 1,574 & 3,080 & 2,642 & 3,268 & 2,376 \\
\hline CE & 1,938 & 1,512 & 2,002 & 2,085 & 1,873 & 1,901 & 1,294 & 1,112 & 1,654 & 1,505 & 2,414 & 2,569 \\
\hline CW & 250 & 217 & 307 & 321 & 329 & 339 & 229 & 196 & 301 & 228 & 325 & 351 \\
\hline NM & 1,103 & 754 & 1,127 & 1,139 & 956 & 1,017 & 788 & 586 & 926 & 884 & 1,099 & 999 \\
\hline NV & 1,556 & 1,083 & 1,995 & 2,036 & 1,748 & 2,188 & 1,345 & 1,069 & 1,656 & 1,359 & 1,724 & 1,751 \\
\hline WY & 914 & 589 & 853 & 683 & 673 & 663 & 477 & 500 & 601 & 590 & 1,168 & 1,186 \\
\hline FP & 8,737 & 6,964 & 10,339 & 10,347 & 9,088 & 9,917 & 6,574 & 5,037 & 8,217 & 7,208 & 9,997 & 9,232 \\
\hline \multicolumn{13}{|c|}{2006 GWh } \\
\hline & Jan & Feb & Mar & Apr & May & Jun & Jul & Aug & Sep & Oct & Nov & Dec \\
\hline$A Z$ & 3,657 & 2,890 & 4,629 & 4,213 & 3,261 & 2,440 & 1,723 & 1,568 & 2,234 & 2,988 & 2,816 & 2,430 \\
\hline CE & 2,491 & 2,401 & 2,208 & 2,236 & 1,761 & 1,500 & 1,047 & 823 & 1,514 & 1,917 & 2,083 & 2,018 \\
\hline CW & 340 & 340 & 323 & 396 & 288 & 273 & 167 & 166 & 225 & 271 & 311 & 255 \\
\hline NM & 1,156 & 994 & 1,269 & 1,198 & 853 & 802 & 721 & 566 & 860 & 1,023 & 1,052 & 933 \\
\hline NV & 2,325 & 1,657 & 2,485 & 2,461 & 1,739 & 1,602 & 1,237 & 1,528 & 1,279 & 1,415 & 2,099 & 1,670 \\
\hline WY & 1,167 & 1,087 & 736 & 765 & 690 & 544 & 358 & 371 & 588 & 814 & 991 & 916 \\
\hline FP & 11,135 & 9,370 & 11,651 & 11,269 & 8,592 & 7,162 & 5,253 & 5,022 & 6,700 & 8,429 & 9,352 & 8,222 \\
\hline
\end{tabular}

\subsubsection{Scenario Comparison}

For the three study scenarios, suitable wind and solar sites were selected based on a number of factors, as discussed in Chapter 3. The distribution of the sites, capacity factor, and diversity are distinct enough to affect the overall energy profile. Figure 4.4 and Figure 4.5 plot the footprint total and percent monthly energy from wind and solar for the three scenarios, in 2006 for $30 \%$ penetration.

From a total and percent energy viewpoint, there is a much greater difference from month to month than from scenario to scenario. The Mega-Project Scenario exhibits slightly more dynamic range over the year than the other two scenarios, with $58 \%$ energy from wind and solar in January, and 14\% in July.

The large amount of temporal (month-to-month) variation is expected since the energy content of the sites is so different from season to season. However, the degree to which the scenarios conform was not altogether expected, though in retrospect it is not surprising. The difference in geographic diversity among scenarios suggests that state monthly energies would be very different in each scenario. However, at the footprint level the implicit assumption of sufficient transmission to move energy to where it is needed tends to obscure local iniquities. In fact, it is safe to say that there is relatively 
little difference among scenarios at this resolution. This observation is reinforced in subsequent analyses.

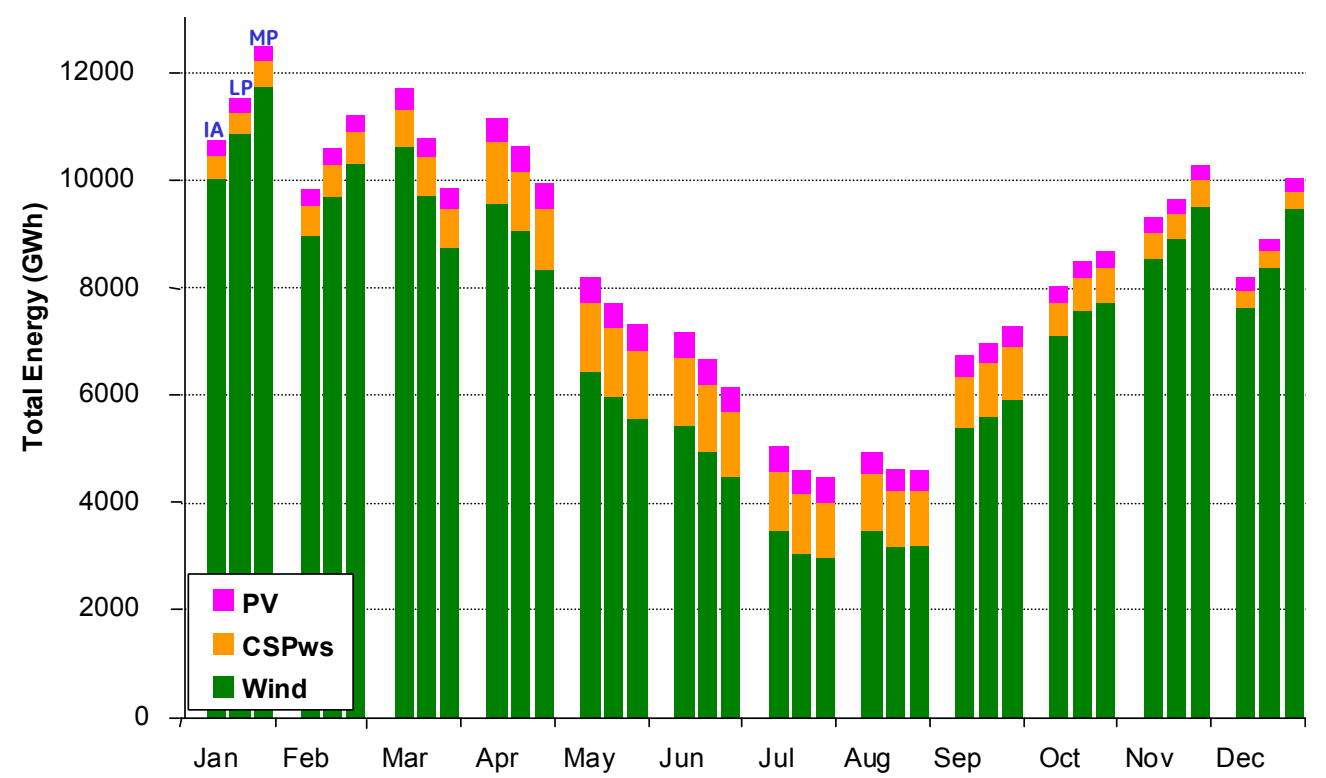

Figure 4.4 2006 Total In-Footprint Monthly Energy from Wind and Solar for All Scenarios (30\% Penetration)

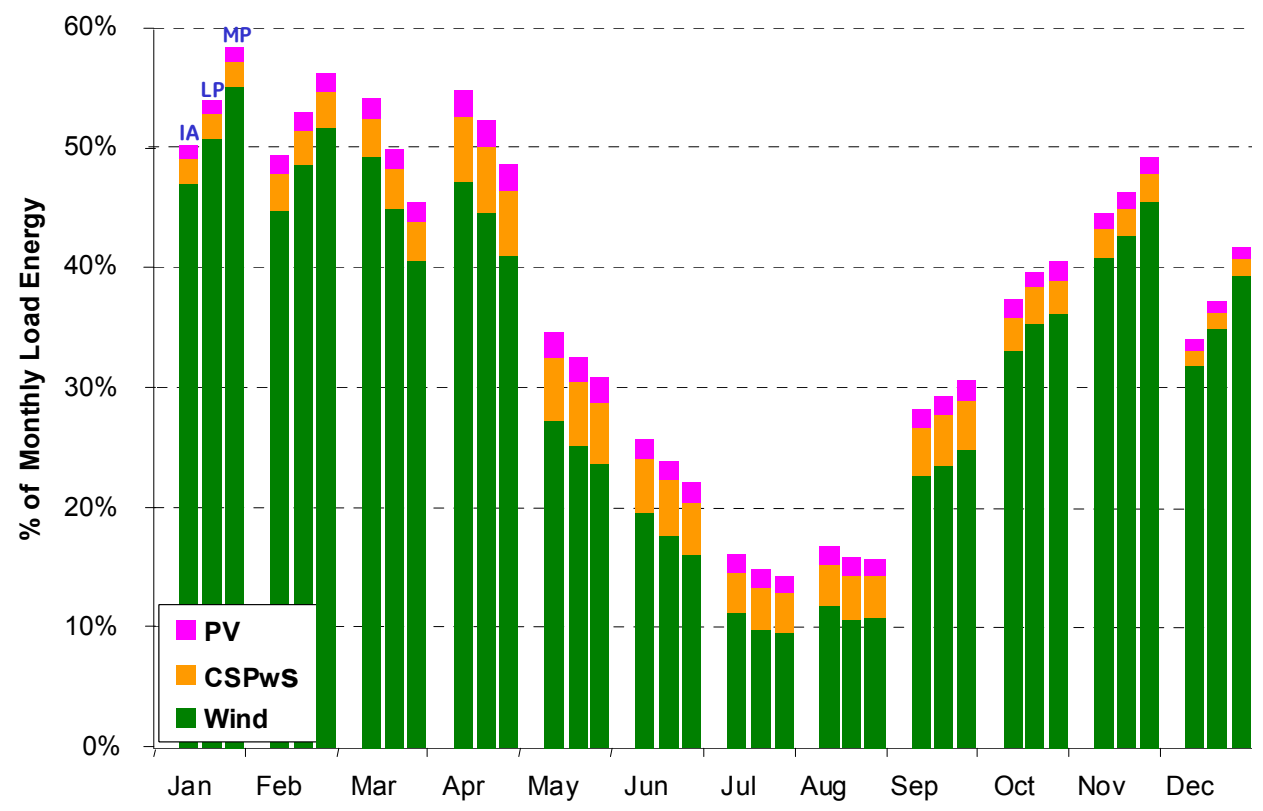

Figure 4.5 2006 Percent In-Footprint Monthly Energy from Wind and Solar for All Scenarios (30\% Penetration)

\subsubsection{State-by-State Comparison}

The monthly energy at the footprint level tends to obscure some interesting differences among the states, due to size variances. Chapter 3 summarized the wind and solar energy in each state for the three scenarios. Figure 4.6 and Figure 4.7 plot the total monthly energy in Arizona and Wyoming for the 30\% Local-Priority Scenario. The 
numbers above the bars indicate the renewable energy as a percent of the state load energy.

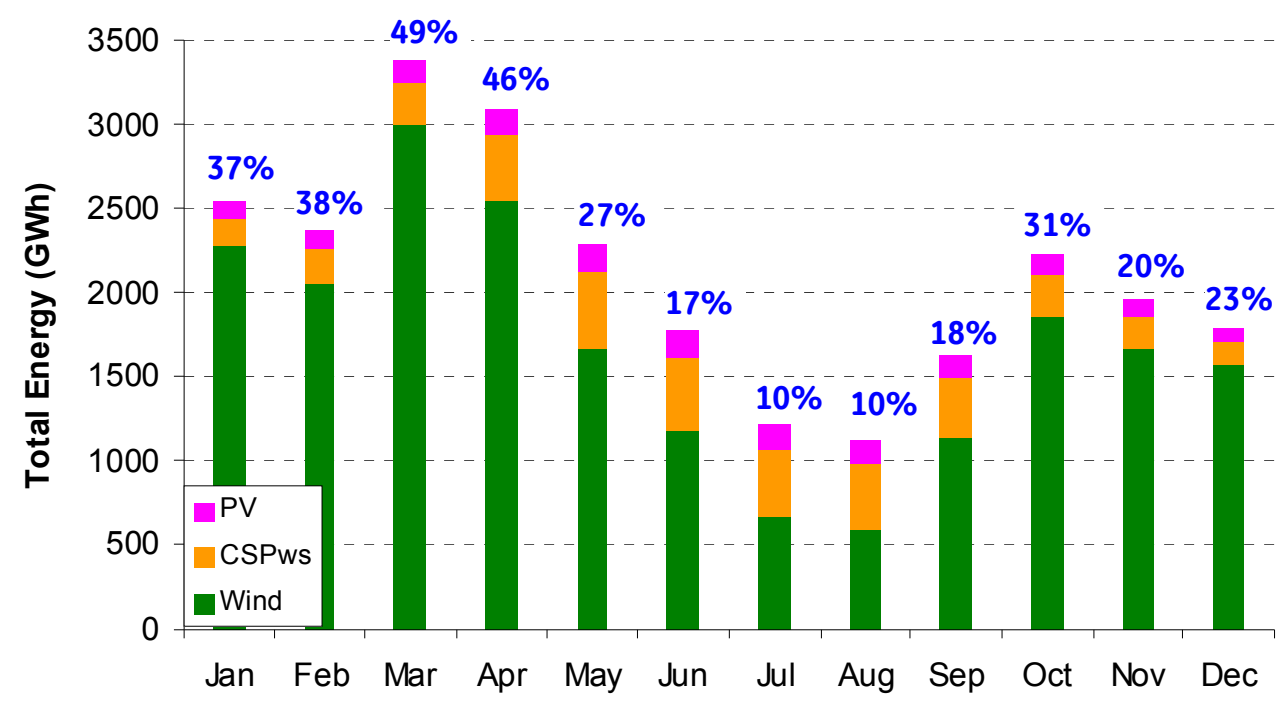

Figure 4.6 2006 Arizona Total and Percent Monthly Energy from Wind and Solar (30\% Local-Priority Scenario)

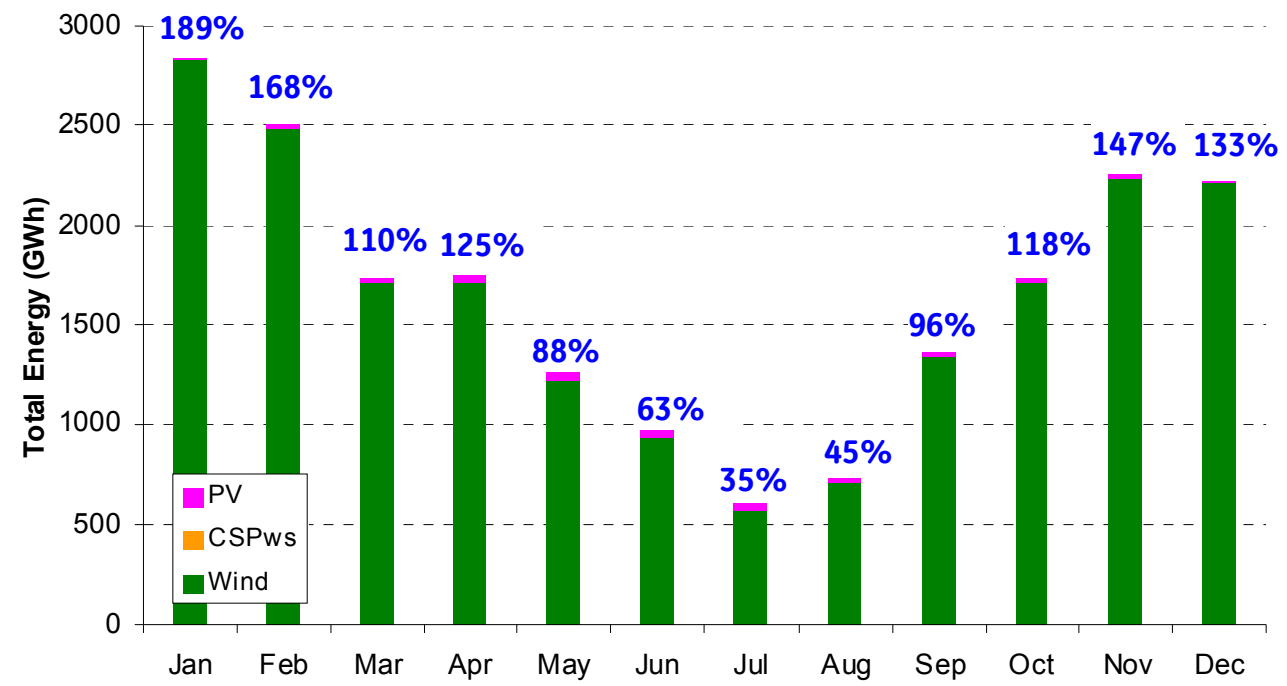

Figure 4.7 2006 Wyoming Total and Percent Monthly Energy from Wind and Solar (30\% Local-Priority Scenario)

These two states are chosen for illustration because they have just about the same amount of installed wind capacity but are polar opposites with regard to the size of the load and the quality of wind and solar resources. As expected, the seasonal patterns observed at the footprint level are evident at the state level, but there is a dramatic difference in the monthly energy penetration. During the fall and winter months, wind and solar energy is well over $100 \%$ of Wyoming's load energy, reaching almost $200 \%$ in January. Similar wind capacity in Arizona gives a high penetration value of less than $50 \%$ in March. This underscores the need for export capability and transmission capacity within the footprint and to areas outside. This point is emphasized even more clearly by 
the data in Table 4.2. The table summarizes the percent monthly energy from wind and solar for individual states in the study footprint, the total study footprint (FP), and all of WECC. The areas are arranged in order of increasing size (load) from left to right.

Table 4.2 Summary of 2006 Percent Monthly Energy from Wind and Solar (30\% Wind Energy in Footprint)

\begin{tabular}{|c|c|c|c|c|c|c|c|c|}
\hline & CO-W & WY & NM & CO-E & NV & Ariz & FP & WECC \\
\hline Jan & $47 \%$ & $52 \%$ & $45 \%$ & $50 \%$ & $54 \%$ & $50 \%$ & $50 \%$ & $37 \%$ \\
\hline Feb & $53 \%$ & $50 \%$ & $44 \%$ & $51 \%$ & $48 \%$ & $50 \%$ & $49 \%$ & $34 \%$ \\
\hline Mar & $47 \%$ & $33 \%$ & $53 \%$ & $45 \%$ & $60 \%$ & $66 \%$ & $54 \%$ & $37 \%$ \\
\hline Apr & $62 \%$ & $37 \%$ & $51 \%$ & $51 \%$ & $60 \%$ & $61 \%$ & $55 \%$ & $37 \%$ \\
\hline May & $40 \%$ & $31 \%$ & $33 \%$ & $35 \%$ & $34 \%$ & $36 \%$ & $35 \%$ & $29 \%$ \\
\hline Jun & $36 \%$ & $24 \%$ & $27 \%$ & $27 \%$ & $30 \%$ & $22 \%$ & $26 \%$ & $22 \%$ \\
\hline Jul & $18 \%$ & $14 \%$ & $24 \%$ & $17 \%$ & $17 \%$ & $13 \%$ & $16 \%$ & $16 \%$ \\
\hline Aug & $20 \%$ & $16 \%$ & $20 \%$ & $14 \%$ & $24 \%$ & $13 \%$ & $17 \%$ & $17 \%$ \\
\hline Sep & $35 \%$ & $30 \%$ & $35 \%$ & $34 \%$ & $27 \%$ & $23 \%$ & $28 \%$ & $22 \%$ \\
\hline Oct & $40 \%$ & $34 \%$ & $42 \%$ & $38 \%$ & $31 \%$ & $40 \%$ & $37 \%$ & $27 \%$ \\
\hline Nov & $47 \%$ & $43 \%$ & $41 \%$ & $43 \%$ & $57 \%$ & $40 \%$ & $45 \%$ & $32 \%$ \\
\hline Dec & $33 \%$ & $36 \%$ & $34 \%$ & $36 \%$ & $35 \%$ & $31 \%$ & $34 \%$ & $25 \%$ \\
\hline
\end{tabular}

\begin{tabular}{|c|c|c|c|c|c|c|c|c|}
\hline & CO-W & WY & NM & CO-E & NV & Ariz & FP & WECC \\
\hline Jan & $34 \%$ & $189 \%$ & $48 \%$ & $42 \%$ & $30 \%$ & $37 \%$ & $54 \%$ & $38 \%$ \\
\hline Feb & $38 \%$ & $168 \%$ & $47 \%$ & $44 \%$ & $28 \%$ & $38 \%$ & $53 \%$ & $35 \%$ \\
\hline Mar & $33 \%$ & $110 \%$ & $56 \%$ & $38 \%$ & $31 \%$ & $49 \%$ & $50 \%$ & $35 \%$ \\
\hline Apr & $45 \%$ & $125 \%$ & $54 \%$ & $43 \%$ & $35 \%$ & $46 \%$ & $52 \%$ & $36 \%$ \\
\hline May & $30 \%$ & $88 \%$ & $35 \%$ & $30 \%$ & $21 \%$ & $27 \%$ & $33 \%$ & $28 \%$ \\
\hline Jun & $28 \%$ & $63 \%$ & $28 \%$ & $24 \%$ & $19 \%$ & $17 \%$ & $24 \%$ & $22 \%$ \\
\hline Jul & $14 \%$ & $35 \%$ & $25 \%$ & $15 \%$ & $10 \%$ & $10 \%$ & $15 \%$ & $15 \%$ \\
\hline Aug & $16 \%$ & $45 \%$ & $21 \%$ & $13 \%$ & $14 \%$ & $10 \%$ & $16 \%$ & $16 \%$ \\
\hline Sep & $27 \%$ & $96 \%$ & $37 \%$ & $29 \%$ & $17 \%$ & $18 \%$ & $29 \%$ & $22 \%$ \\
\hline Oct & $29 \%$ & $118 \%$ & $45 \%$ & $33 \%$ & $21 \%$ & $31 \%$ & $40 \%$ & $27 \%$ \\
\hline Nov & $35 \%$ & $147 \%$ & $44 \%$ & $37 \%$ & $32 \%$ & $30 \%$ & $46 \%$ & $33 \%$ \\
\hline Dec & $23 \%$ & $133 \%$ & $36 \%$ & $30 \%$ & $20 \%$ & $23 \%$ & $37 \%$ & $26 \%$ \\
\hline
\end{tabular}

\begin{tabular}{|c|c|c|c|c|c|c|c|c|}
\hline & CO-W & WY & NM & CO-E & NV & Ariz & FP & WECC \\
\hline Jan & $8 \%$ & $481 \%$ & $73 \%$ & $23 \%$ & $12 \%$ & $14 \%$ & $59 \%$ & $39 \%$ \\
\hline Feb & $9 \%$ & $433 \%$ & $71 \%$ & $24 \%$ & $13 \%$ & $16 \%$ & $56 \%$ & $36 \%$ \\
\hline Mar & $8 \%$ & $410 \%$ & $82 \%$ & $21 \%$ & $13 \%$ & $19 \%$ & $46 \%$ & $34 \%$ \\
\hline Apr & $11 \%$ & $433 \%$ & $78 \%$ & $24 \%$ & $16 \%$ & $21 \%$ & $49 \%$ & $35 \%$ \\
\hline May & $8 \%$ & $280 \%$ & $49 \%$ & $17 \%$ & $10 \%$ & $15 \%$ & $31 \%$ & $28 \%$ \\
\hline Jun & $7 \%$ & $216 \%$ & $39 \%$ & $14 \%$ & $9 \%$ & $11 \%$ & $22 \%$ & $21 \%$ \\
\hline Jul & $4 \%$ & $121 \%$ & $33 \%$ & $10 \%$ & $5 \%$ & $8 \%$ & $14 \%$ & $15 \%$ \\
\hline Aug & $4 \%$ & $131 \%$ & $29 \%$ & $9 \%$ & $7 \%$ & $8 \%$ & $16 \%$ & $16 \%$ \\
\hline Sep & $7 \%$ & $265 \%$ & $53 \%$ & $18 \%$ & $8 \%$ & $11 \%$ & $31 \%$ & $23 \%$ \\
\hline Oct & $7 \%$ & $345 \%$ & $64 \%$ & $19 \%$ & $10 \%$ & $13 \%$ & $41 \%$ & $27 \%$ \\
\hline Nov & $8 \%$ & $388 \%$ & $67 \%$ & $19 \%$ & $13 \%$ & $13 \%$ & $49 \%$ & $34 \%$ \\
\hline Dec & $6 \%$ & $334 \%$ & $60 \%$ & $17 \%$ & $8 \%$ & $9 \%$ & $42 \%$ & $28 \%$ \\
\hline
\end{tabular}

In the Mega-Project Scenario, most of the wind capacity inside the study footprint is installed in Wyoming and New Mexico (13,770 MW and 4,350 MW respectively). This leads to large penetration levels in the winter and spring months, as shown in the bottom section of Table 4.2. In January, wind and solar energy is $481 \%$ of Wyoming's load energy. However, the penetration on a footprint-wide basis is $59 \%$ and $39 \%$ on a WECC-wide basis. Again, this reinforces the need for export capability and transmission capacity inside the footprint, and to loads outside the footprint. The next section uses duration plots to more closely examine some of these high-wind, low-load periods. 


\subsection{Yearly Duration Trends}

The ability of the system to accommodate net load variations is in large part a function of the absolute net load level. Since system maneuverability tends to increase with the generation level, and vice versa, the generation mix is more flexible at high load hours and less flexible at low-load hours. Past studies have shown that during the hours when load is lowest, there tends to be more wind energy, which could lead to minimum load issues at high-wind penetration levels. This section will examine the duration of wind and solar over the year and the correlation of net load variability with load levels throughout the year. For clarity and brevity, most of the charts and discussion will focus on year 2006, the $30 \%$ penetration level, the study footprint, and a few selected states. Information on other years, penetration levels, and states are included in Appendix B.

\subsubsection{Wind Duration}

Figure 4.8 below shows the 2006 wind duration plots for all three scenarios at the $30 \%$ penetration level. Each division on the x-axis represents deciles, or $10 \%$ of the yearly hours (876 hours). The highest instantaneous penetration achieved in the year is listed in the inset box.

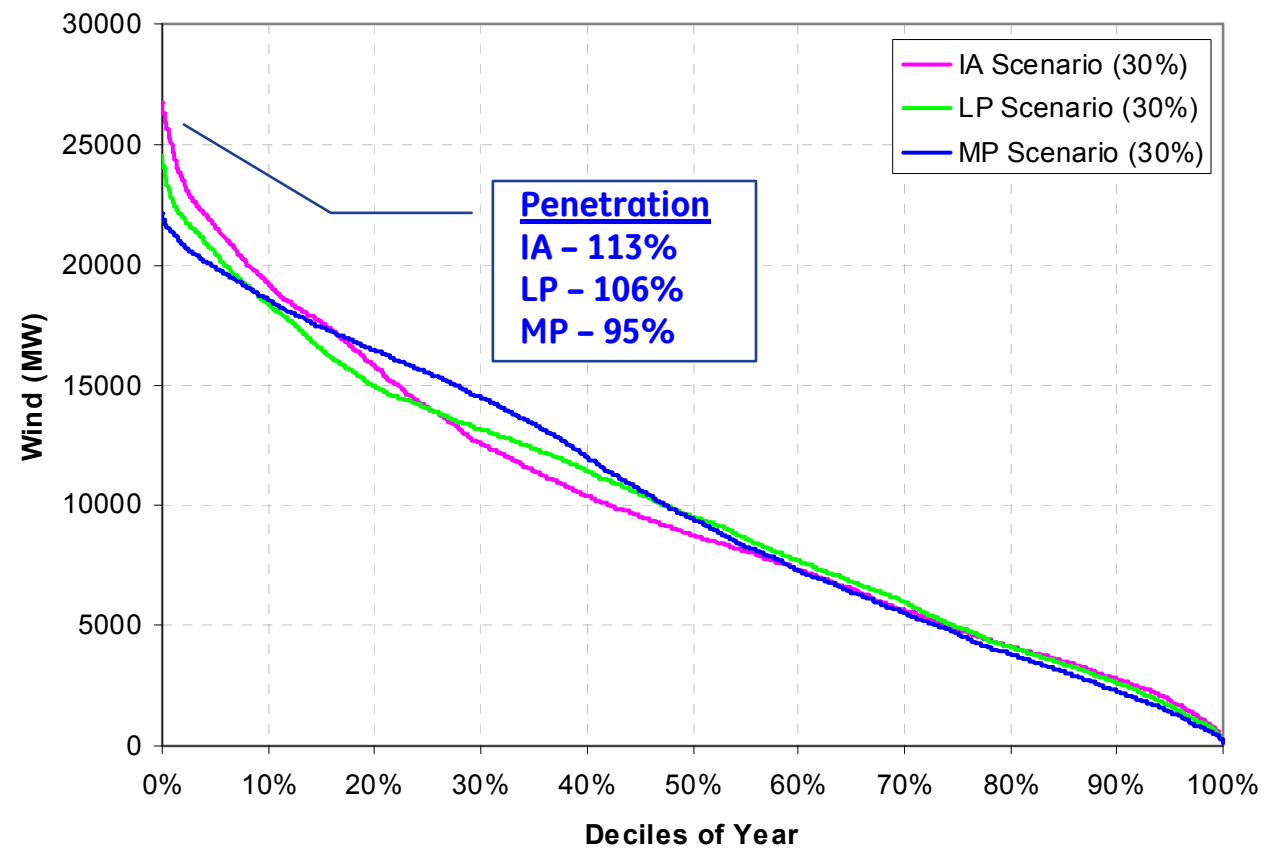

Figure 4.8 2006 Study Footprint Wind Duration Plots for All Three Scenarios (30\% Penetration)

Not surprisingly, of the three, the In-Area Scenario has the highest instantaneous penetration. There are 24 hours with over 100\% instantaneous penetration, versus 5 hours for the Local-Priority Scenario and none for the Mega-Project. This is because the In-Area Scenario has more installed capacity than the other scenarios. However, it tends 
to drop more steeply than the others do because the overall capacity value of the wind plants in the In-Area Scenario is not as good as in other scenarios.

This is more clearly shown in Figure 4.9, which plots the same traces normalized as a percent of nameplate wind. It is clear that there is a distinct separation of traces due to difference in quality of wind resources. The In-Area Scenario trace drops quite a bit faster than the Mega-Project trace, because there are more hours in the year when the wind output is high in the Mega-Project Scenario. The Mega-Project wind is at or above $50 \%$ of nameplate capacity for $40 \%$ of the year, or 3504 hours. By comparison, the InArea wind is at or above $50 \%$ of nameplate capacity for only $22 \%$ of the year, or 1927 hours. The Local-Priority Scenario, as expected, is somewhere in between the In-Area and Mega-Project. On the flipside, all three scenarios exhibit similar characteristics at the low end. In-Area, Local-Priority, and Mega-Project wind are less than 10\% of nameplate capacity for approximately 10\% of the yearly hours. Both Figure 4.8 and Figure 4.9 highlight the fact that the real difference between the three wind scenarios is at the midrange to high-wind levels.

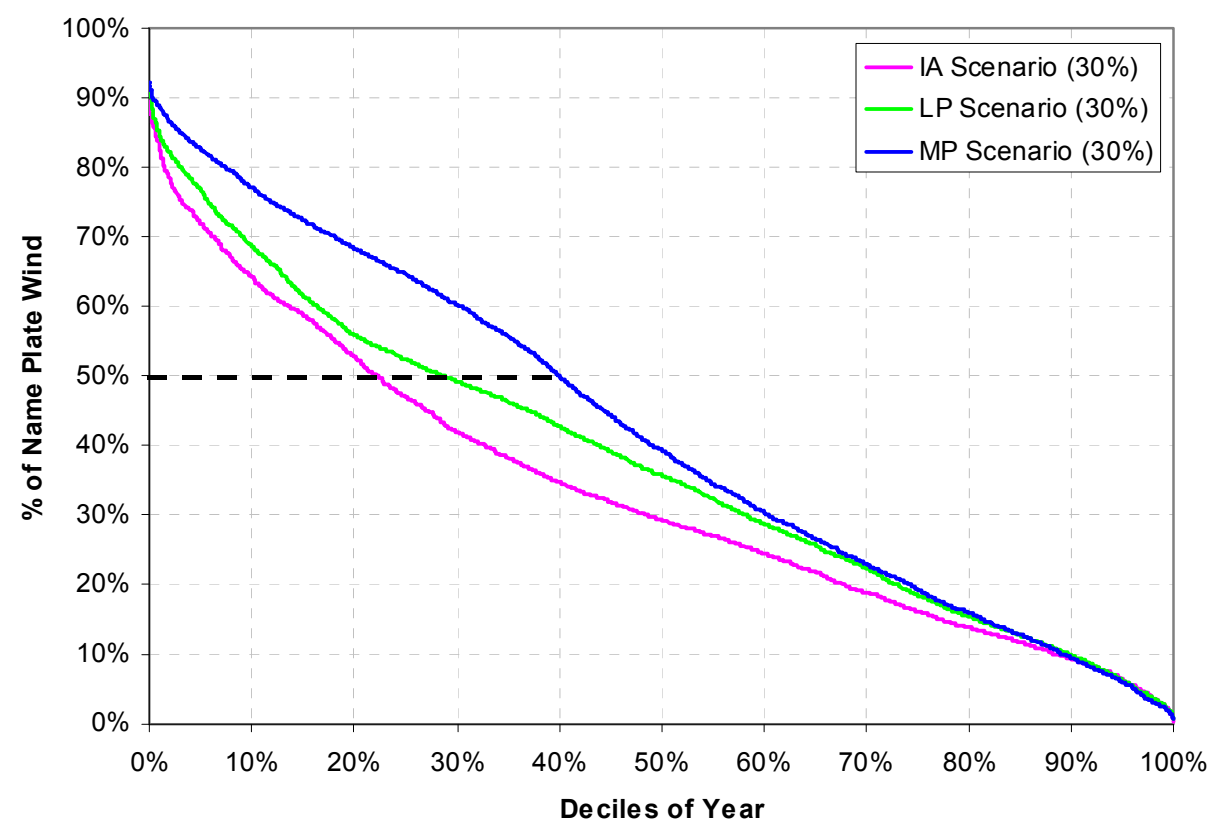

Figure 4.9 2006 Study Footprint Wind Duration Plots Normalized as \% of Nameplate Wind

As pointed out in Chapter 3, the wind profiles for the $20 \%$ and $30 \%$ penetration levels were created by adding new sites to the $10 \%$ profile. For each of the three scenarios, the $10 \%$ profiles generally contain the best available sites in terms of energy content. For the $20 \%$ and $30 \%$ profiles, the next best sites are added, subject to the rules of the site selection algorithm for the particular scenario.

Yearly duration plots in Figure 4.10 show the 10\%, 20\%, and 30\% penetration profiles for the Local-Priority Scenario. Each division on the x-axis represents deciles, or $10 \%$ of the yearly hours (876 hours). 


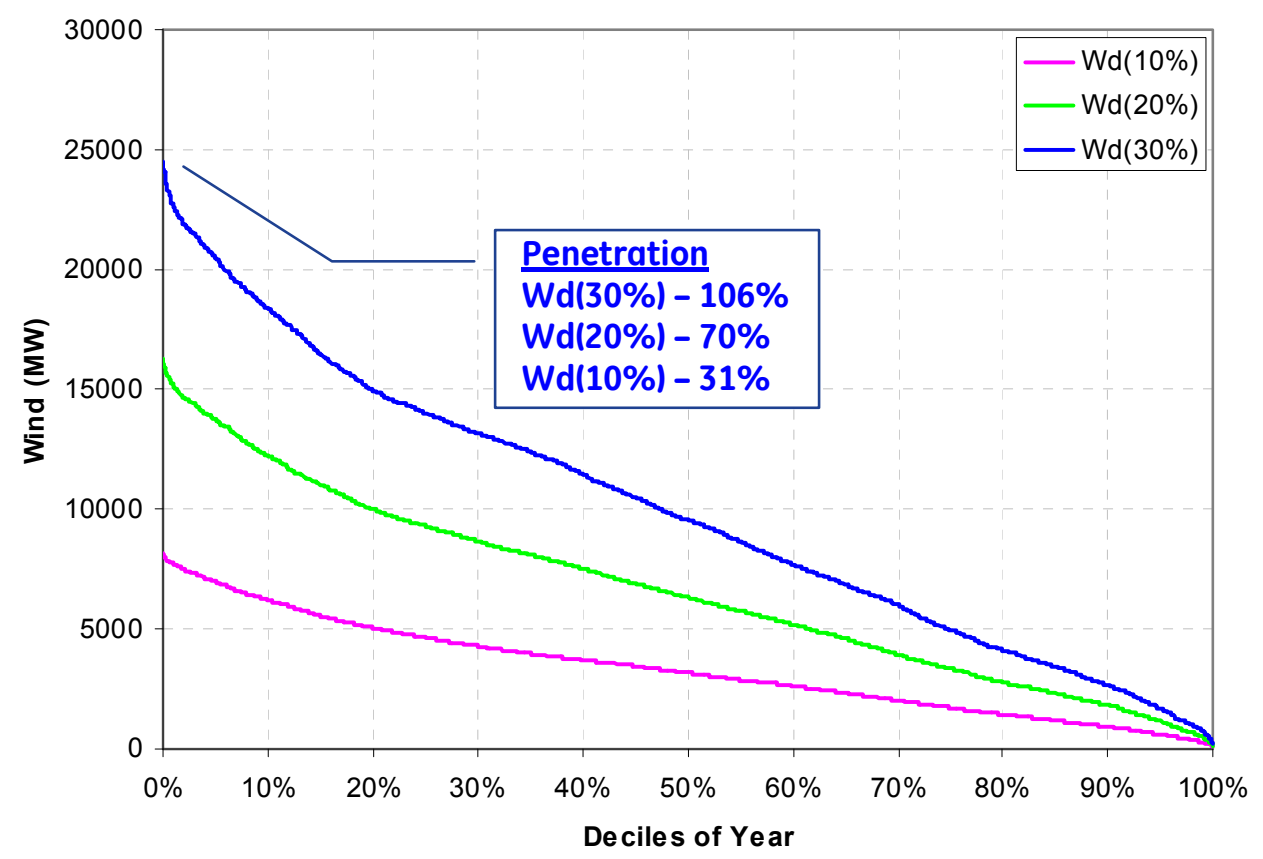

Figure 4.10 2006 Study Footprint Wind Duration Plots for All Three Penetration Levels (Local-Priority Scenario)

The data underscore the fact that high instantaneous wind penetrations can be achieved during the year even for the lowest energy penetration level. Figure 4.11 shows the duration plots normalized as a percent of nameplate wind capacity. One unexpected observation is the $10 \%$ profile has a lower overall capacity value than the $20 \%$ and $30 \%$, which appears to contradict the fact that the best available sites were chosen for the $10 \%$ penetration level. However, recall that about $3 \%$ of the sites in the first $10 \%$ are preselected (existing) sites, which disproportionately affects the overall capacity value.

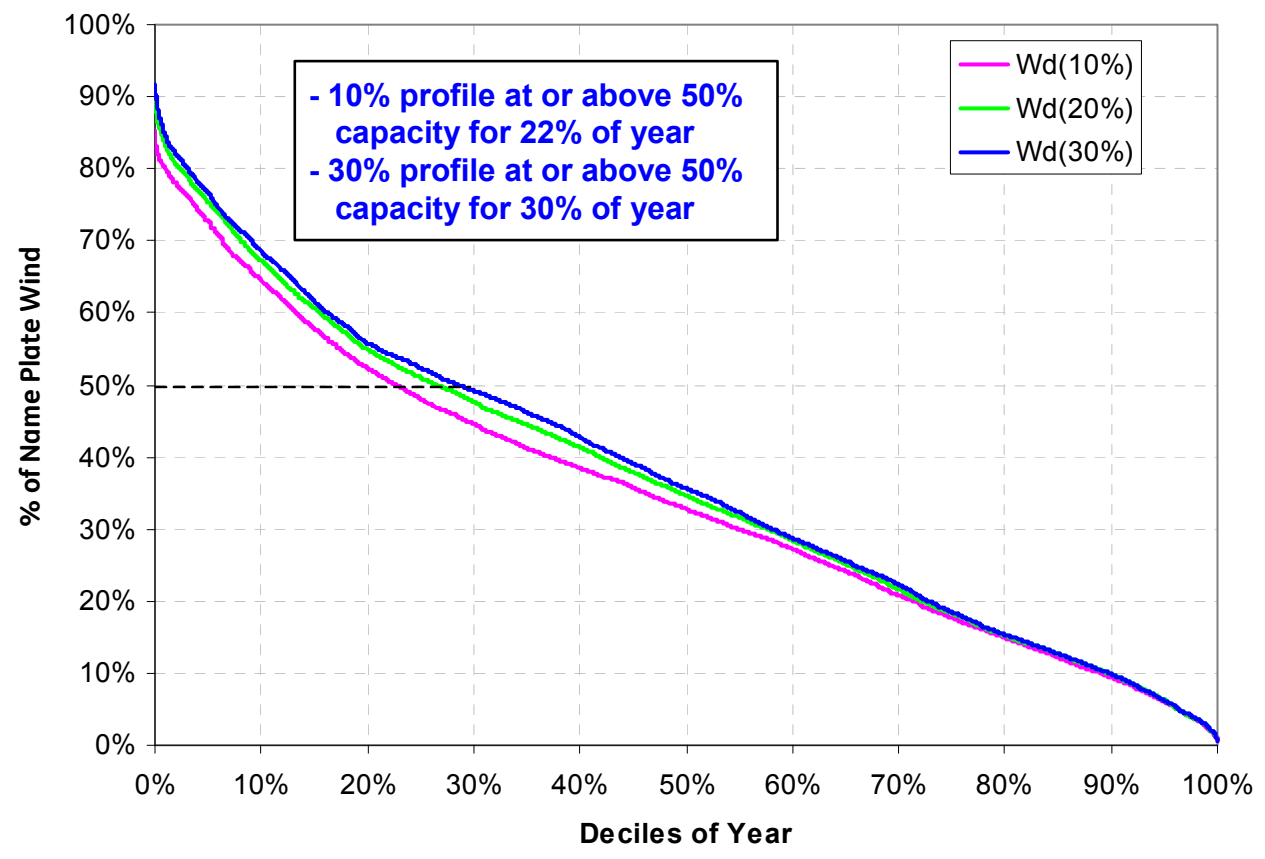

Figure 4.11 2006 Study Footprint Wind Duration Plots Normalized as \% of Nameplate Wind 
At the state/area level, one would expect the difference between individual wind profiles to be significant. Figure 4.12 plots the 2006 wind duration curves for all states/areas in the footprint for the 30\% Local-Priority Scenario. Figure 4.13 shows the same traces normalized as a percentage of the installed wind capacity in the area. The highest instantaneous penetration achieved in the year is listed in the inset box on Figure 4.12 .

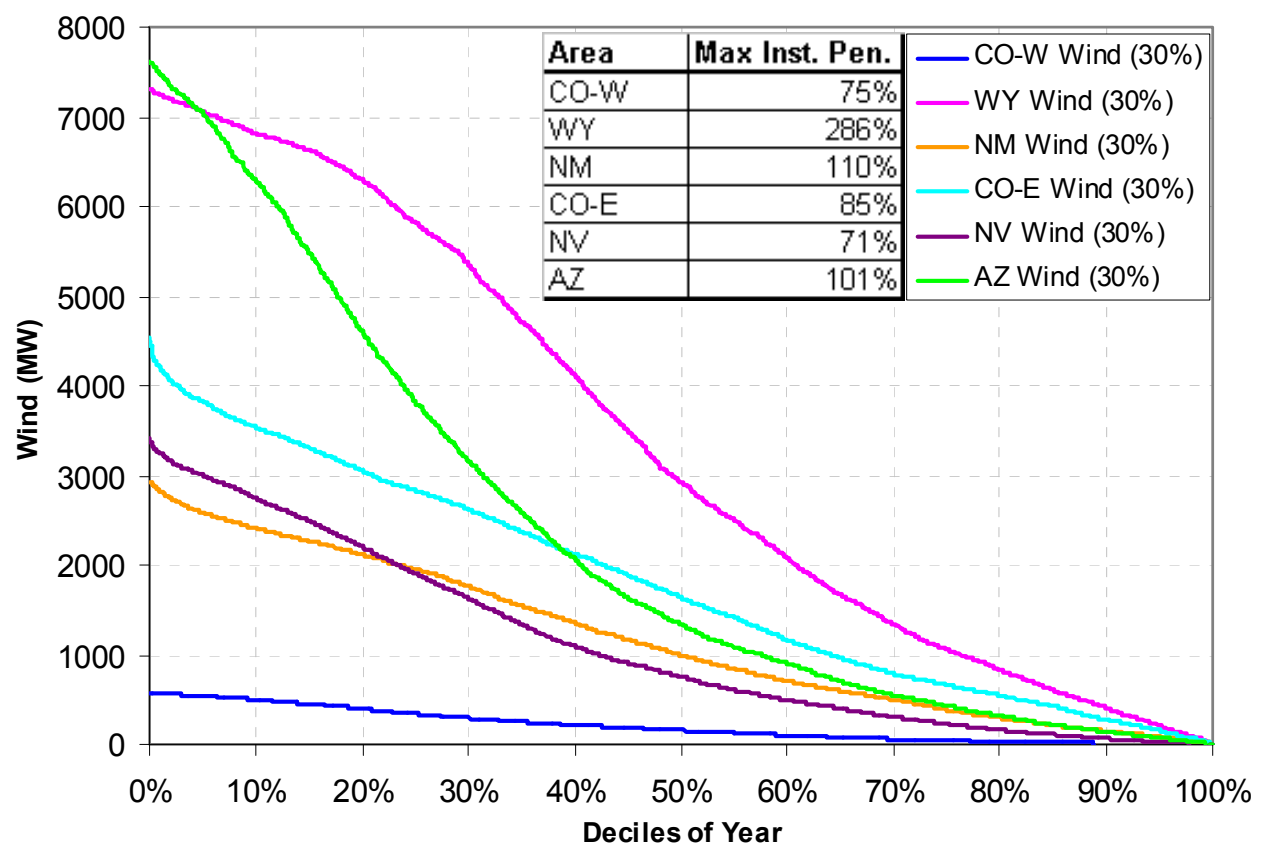

Figure 4.12 2006 In-Footprint Area Wind Duration Plots for the 30\% Local-Priority Scenario

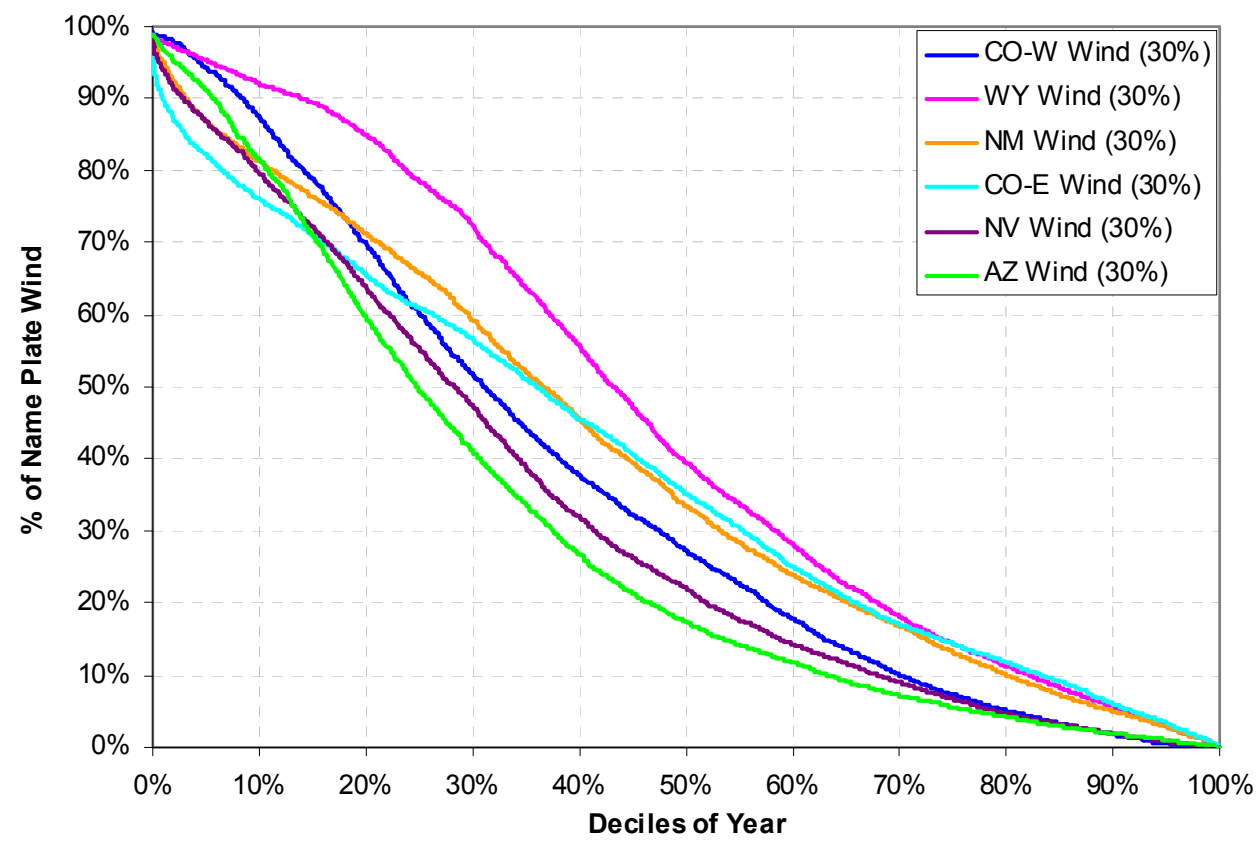

Figure 4.13 2006 In-Footprint Area Wind Duration Plots Normalized as \% of Nameplate Wind 
Recall that for the Local-Priority Scenario, Arizona and Wyoming have the largest installed base of wind, while Colorado-West and New Mexico have the least. On Figure 4.12 , the states/areas are arranged by load size (smallest to largest) in the legend. It is obvious why Wyoming (with big wind and small load) would have very high instantaneous wind penetration (286\%) during the year, while Arizona (big wind and big load) does not rise to this penetration level-though it is still very high at $101 \%$. On the other hand, Colorado-West, despite the relatively small installed wind capacity, still has a high instantaneous penetration due to the small load in the area.

The slope of the traces, especially in Figure 4.13, gives some insight into the quality of installed wind in each area for the Local-Priority Scenario. Generally, the flatter the trace, the higher quality the wind resource is. In Figure 4.13, Arizona has the steepest trace, while Wyoming has the flattest, confirming what we already know about the quality of the wind in these states for the Local-Priority Scenario. The most curious trace is Colorado-East, which is steep in the first decile, but flattens out considerably for other deciles - meaning wind in Colorado-East spends more time in $20 \%-80 \%$ of nameplate range than wind in other areas.

\subsubsection{Solar Duration}

Figure 4.14 shows the 2006 yearly duration and instantaneous penetration for the total CSP output in the study footprint under the 30\% Local-Priority Scenario. The orange trace is the CSP output plotted against the left axis, and the blue trace is the instantaneous penetration plotted against the right axis.

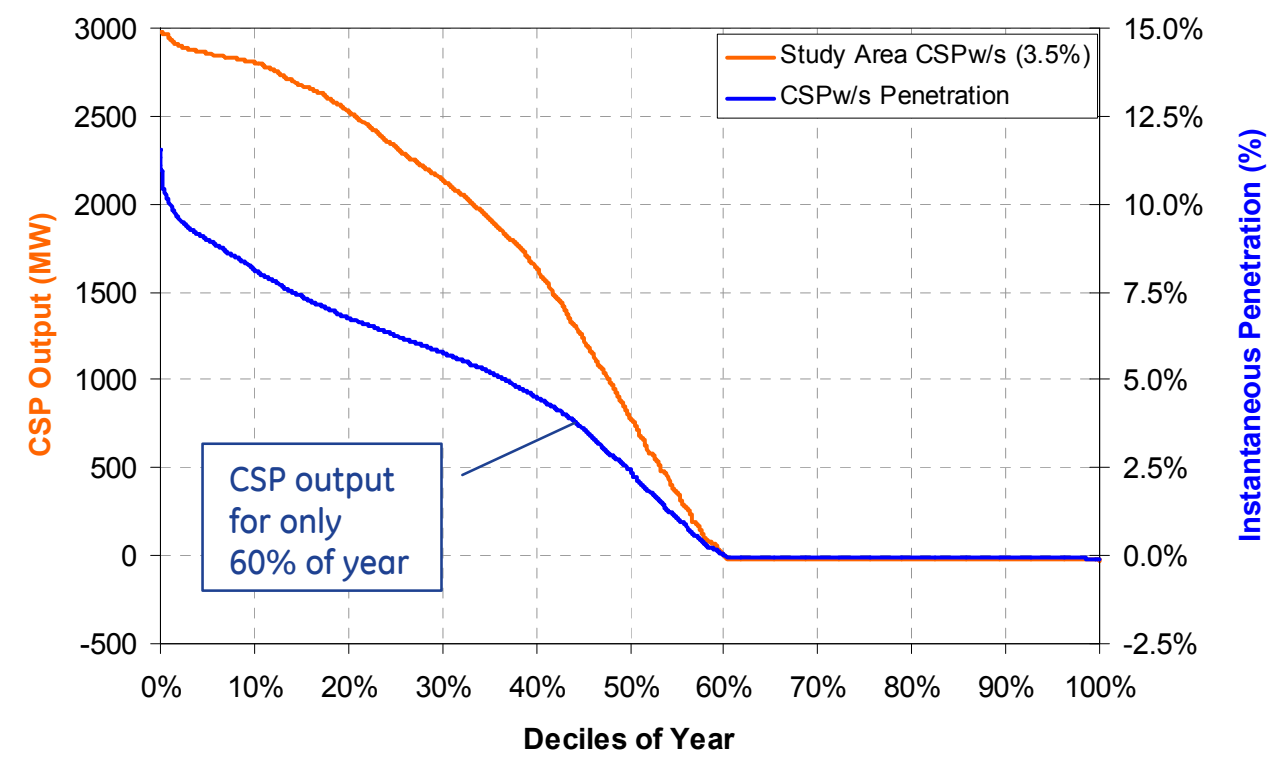

Figure 4.14 2006 Study Footprint CSP Duration and Penetration for the 30\% Local-Priority Scenario

For the Local-Priority 30\% scenario, sufficient CSP capacity is installed to supply only $3.5 \%$ of the load energy. However, over the course of the year, the instantaneous CSP 
penetration reaches $11.5 \%$, and is in fact greater than or equal to $5 \%$ for about $35 \%$ of the year. The CSP duration curve is strongly influenced by the built-in six hours of storage. This is evidenced by the fact that the orange trace is convex-shaped, so that it persists longer at its peak and rolls off quickly thereafter. With 2,900 MW of installed CSP (with storage) capacity, the output is at or above $2,500 \mathrm{MW}$ for $20 \%$ of the year. However, this rolls off very quickly, such that CSP output is at or above $500 \mathrm{MW}$ only $54 \%$ of the year. There is no output for $40 \%$ of the year, the hours when it is dark and storage is exhausted.

Figure 4.15 shows the 2006 yearly duration and instantaneous penetration for the study footprint PV under the 30\% Local-Priority Scenario. The purple trace is the PV output plotted against the left axis, and the blue trace is the instantaneous penetration plotted against the right axis.

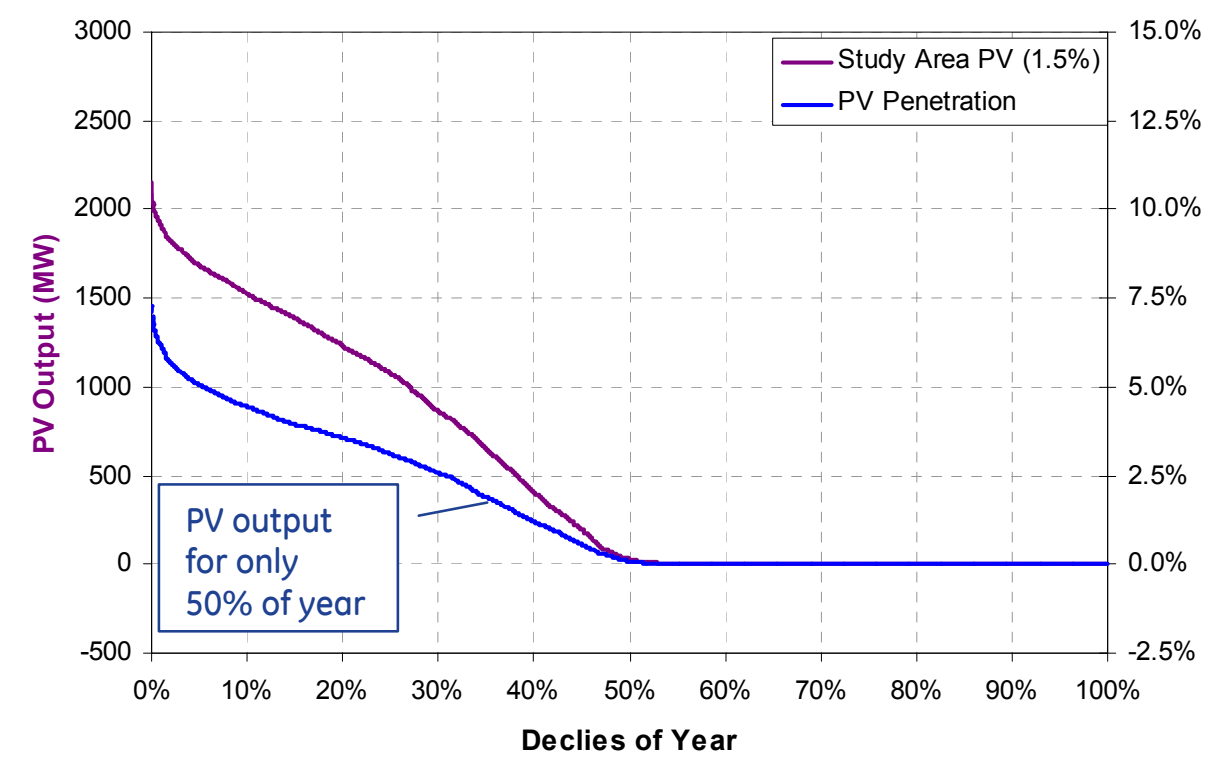

Figure 4.15 2006 Study Footprint PV Duration and Penetration for the 30\% Local-Priority Scenario

PV resources are installed to supply $1.5 \%$ of load energy, but like the CSP output, the PV output also reaches a relatively high penetration of $7.3 \%$ during the year. A key difference between the two, however, is that PV has no storage so it tends to have a sharper peak than CSP (this will discussed further in the next section) and a more gentle roll-off. The purple trace in Figure 4.15 tends to drop very steeply during the peak output hours, but then ramp more gradually toward zero output. With 2,900 MW of installed PV capacity, the output is at or above 1,250 MW for $20 \%$ of the year, and at or above $500 \mathrm{MW}$ only $38 \%$ of the year. There is no PV output for $50 \%$ of the year, $10 \%$ more zero output hours than CSP, which is extended by the storage capacity. 


\subsubsection{Net Load Duration}

The wind and solar duration in isolation may be interesting and may give some insight into the quality of the resources, but the main goal is to determine their impact on load variability and, by extension, generation requirements. The net load duration is more useful for looking at the trend in the coincident combinations of load, wind, and solar over the year. As discussed earlier, net load is determined by subtracting the hourly chronological wind and solar generation from the corresponding hourly load. The data are then sorted to produce the net load duration curve for the year.

Figure 4.16 shows the 2006 study footprint net load duration curves for the In-Area Scenario. The magenta trace is for the baseline or existing wind and solar, and the others, green, orange and blue, are for the $10 \%, 20 \%$, and $30 \%$ penetration levels respectively. The inset boxes list the maximum and minimum net load observed during the year for each penetration level. The horizontal black trace indicates the minimum load point (without existing wind) for the year.

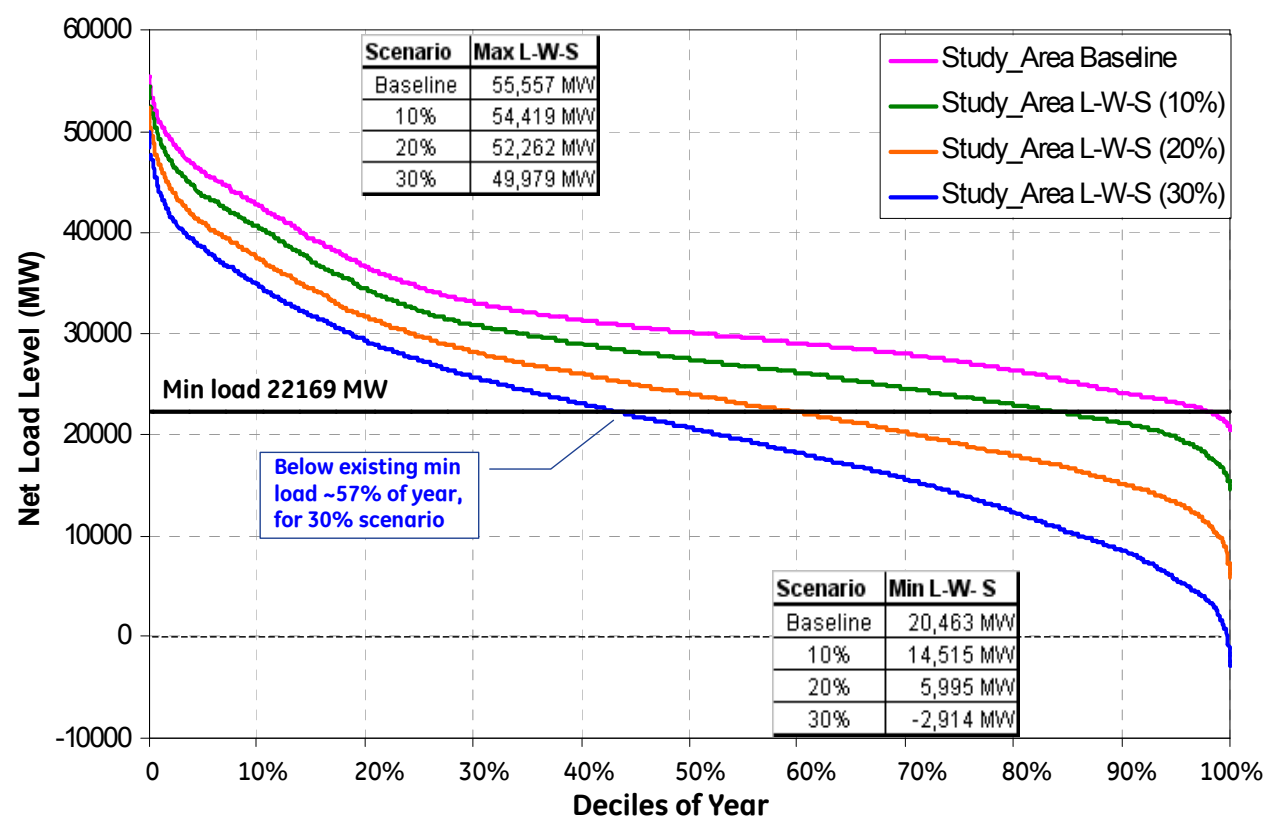

Figure 4.16 Study Footprint 2006 Net Load Duration for the In-Area Scenario

The duration curves illustrate the impact of increasing amounts of wind and solar generation on net load, especially during low-load hours (the tenth decile). Over the year, regardless of wind penetration, there are several hours when net load dips below the minimum load point $(22,169 \mathrm{MW})$. For the baseline, this is $1.68 \%$ of the year or 147 hours, but for $30 \%$ penetration, it is $57 \%$ of the year or almost 5000 hours. Now, there is nothing inherently critical about this minimum load threshold. The system may be able to operate well below this load level, but it simply serves as a reference point for illustration. During the absolute minimum net load hour for $30 \%$ penetration, the wind and solar generation push the net load down to -2,914 MW. In other words, during that 
hour there is 2,914 MW more renewable generation alone than load on the system footprint! Clearly, the footprint system must either export to the rest of WECC or spill renewable generation. This is an "interesting" operating problem to have, and it gets even more "interesting" at the state/area and zone level, especially if each area or zone has to provide ancillary services to integrate these resources. Chapter 6, Operational Analysis, will demonstrate the system impact on this day and other challenging days, and discuss strategies to improve operations.

As we have seen earlier, the statistical character of the net load at the footprint level is not altogether dissimilar for the three scenarios (differences become starker at the state level, as will be shown later). Figure 4.17 plots the 2006 study area net load duration curves for the three scenarios at $30 \%$ penetration on one chart. The figure also includes call-outs with the top and bottom ten percent of load hours, as well as the median load hours.

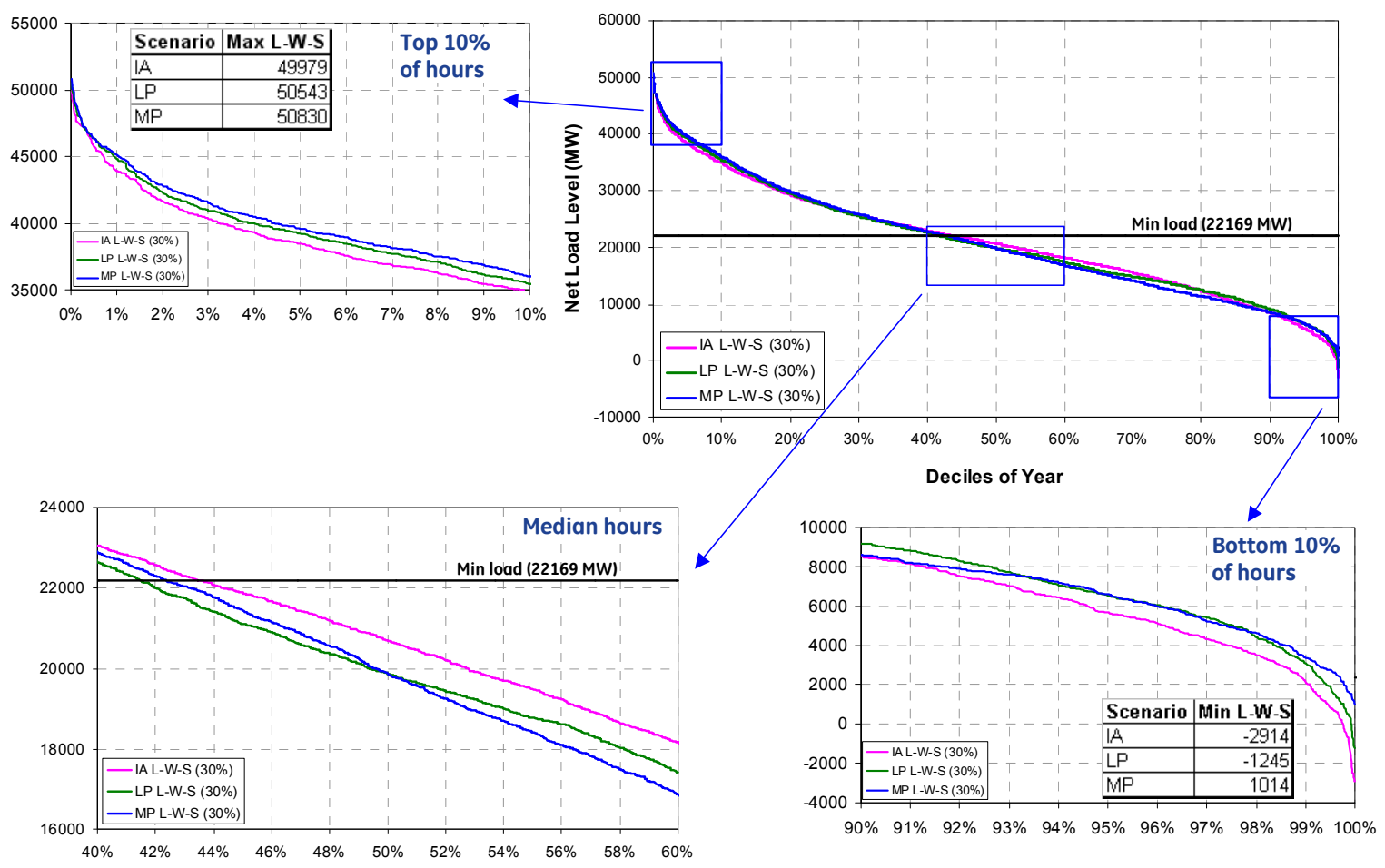

Figure 4.17 Study Footprint 2006 Net Load Duration for All Scenarios (30\% Penetration)

The charts confirm that there is no significant differentiation between the three scenarios at the footprint level. During the low-load hours, all three scenarios present a similar challenge. For the minimum load hour, the Local-Priority net load is $-1,245 \mathrm{MW}$ and the Mega-Project net load is 1,014 MW - not that much different qualitatively from the InArea net load in that hour. The number of hours that net load is below the minimum load point is basically the same in all three scenarios, as is the maximum net load observed during the year. 
The next few charts plot the 2006 net load duration curves for the three different scenarios in three selected states: In-Area in Arizona, Local-Priority in New Mexico, and Mega-Project in Wyoming. This combination of states and scenarios is selected to give a flavor of the stress imposed at the area level by the various scenarios.

In each of these states, the wind and solar output has an increasingly dramatic impact on the net load. In Arizona, there is enough wind to supply $30 \%$ of Arizona's load. Since Arizona is a large state and the wind resources are not particularly great (in terms of capacity factor), this leads to a large installation base and potential operation difficulties when the load is low. In Figure 4.18, for 30\% penetration, Arizona's net load is below the observed state minimum load $45 \%$ of the year, and the minimum net load is almost $-5,000 \mathrm{MW}$ (wind and solar output is 5,000 MW more than load). In New Mexico, under the Local-Priority Scenario, the installed wind and solar capacity pushes down the minimum load point considerably, as shown in Figure 4.19 . For $30 \%$ penetration, New Mexico's net load is below the state minimum load 60\% of the year. In Wyoming under the Mega-Project Scenario, most of the wind resources are installed to supply load elsewhere in the footprint. With this large base of installed wind on a small load footprint, Figure 4.20 shows the resulting net load duration curves. The load in Wyoming is completely dominated by wind and solar generation even at the $10 \%$ penetration level. For 30\% penetration, the net load is below the Wyoming minimum load $90 \%$ of the time, and is in fact negative for $65 \%$ of the year. The minimum net load observed under this scenario is about -10,400 MW. For Wyoming to balance this wind output with generation resources in Wyoming alone is impossible. Chapter 6, Operational Analysis, will demonstrate how the system can be successfully operated with wide-area balancing.

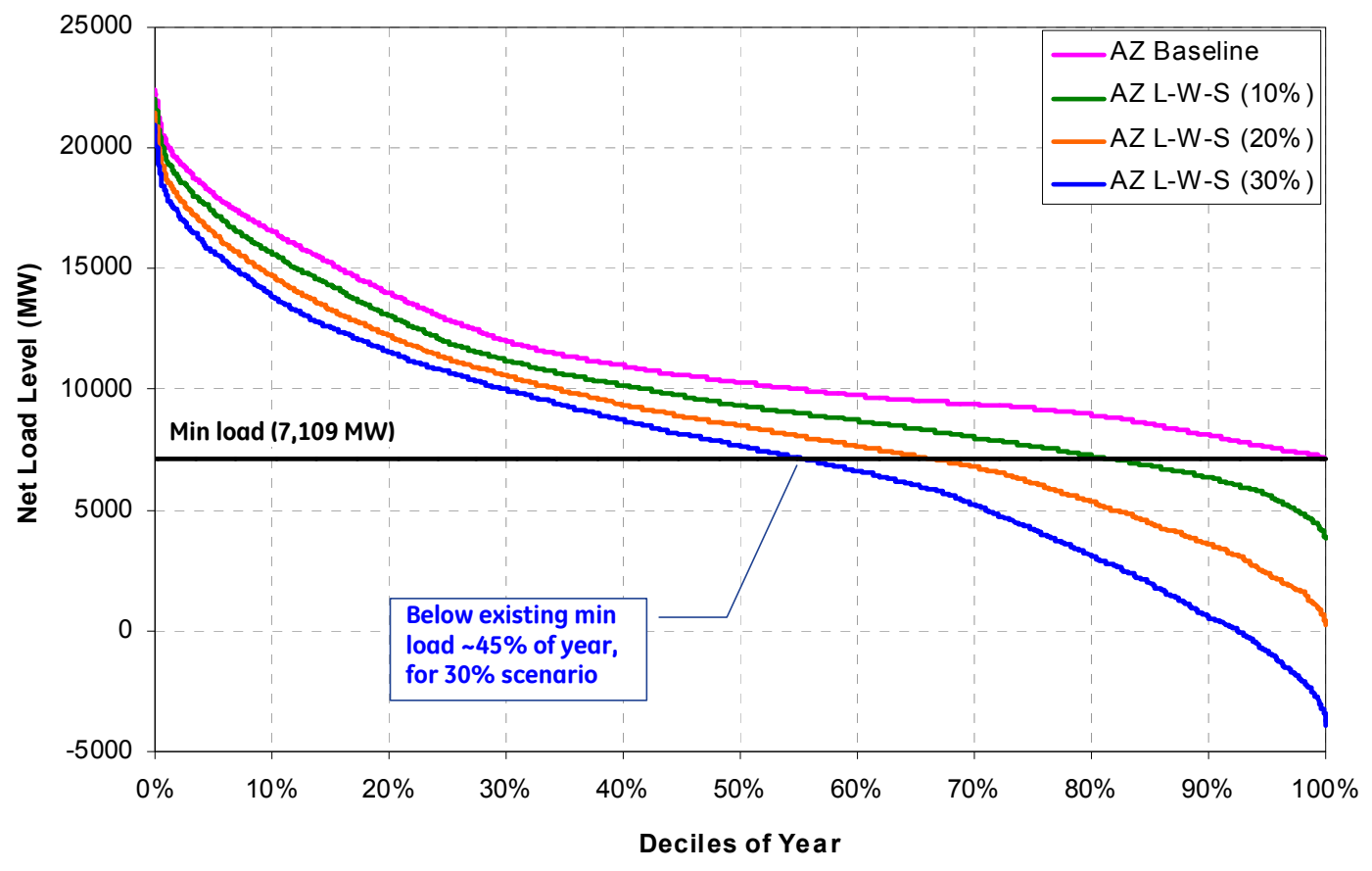

Figure 4.18 Arizona 2006 Net Load Duration for the In-Area Scenario 


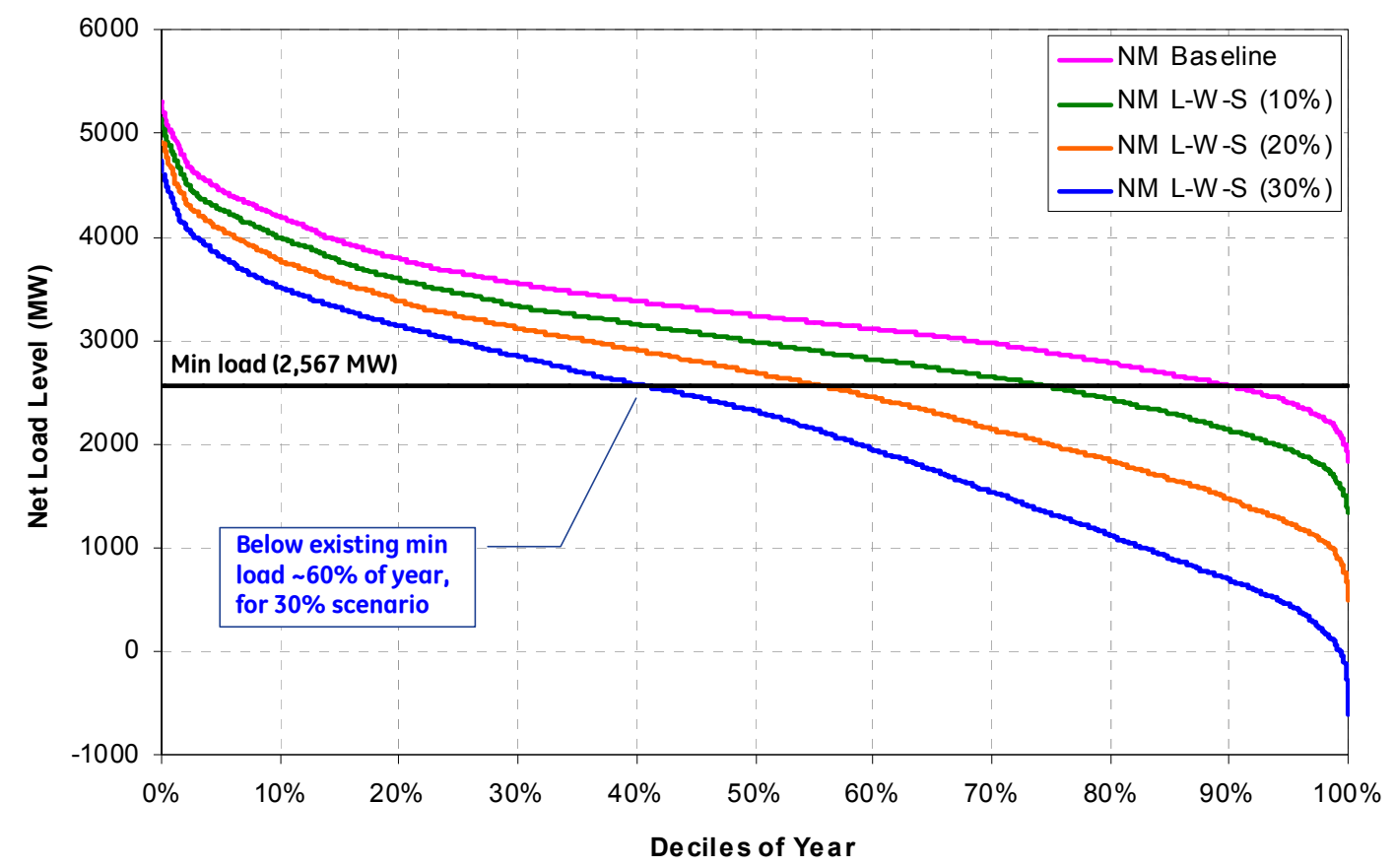

Figure 4.19 New Mexico 2006 Net Load Duration for the Local-Priority Scenario

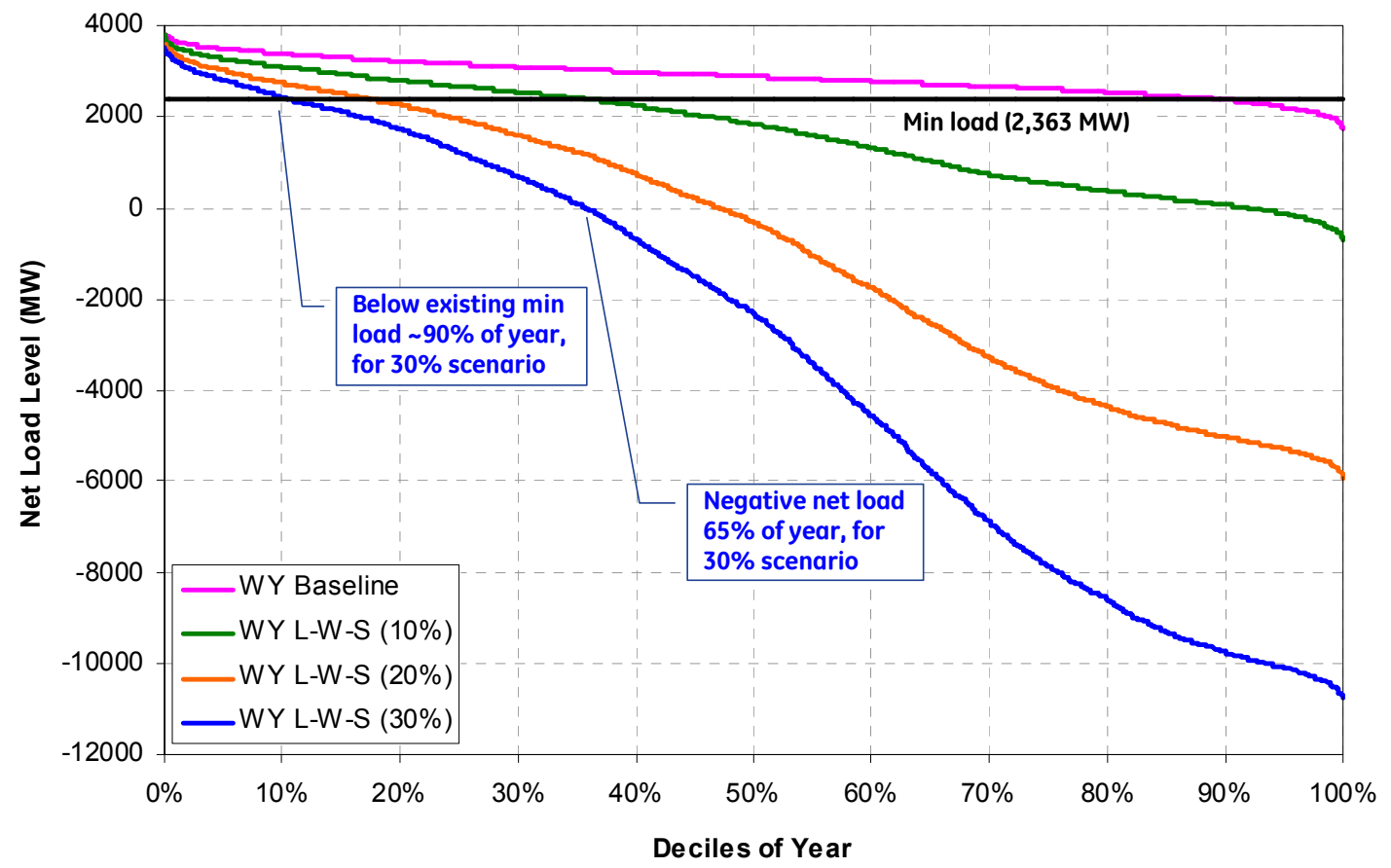

Figure 4.20 Wyoming 2006 Net Load Duration for the Mega-Project Scenario

The next section digs a little deeper into the net load characteristics dictated by load, wind and solar coincidence by looking at average daily profiles on a seasonal/monthly basis, typical day profiles, and time series profiles for selected periods. 


\subsection{Wind, Load, and Solar Coincident Relationships}

The temporal relationship between wind and solar output, and load is the key driver for resulting net load variability. Depending on the nature of wind and solar, when load is rising or falling, the net variability could be better or worse than load alone. In some cases, wind and solar may exaggerate or curtail net load peaks and valleys, increase or decrease ramp rates (period-to-period changes), shift time-of-peak, and generally aggravate or mitigate operationally challenging periods. All of these are important factors when considering the impact of wind and solar on operational requirements such as regulation and operating reserves. Some of the key issues this section highlights are coincidence of load, wind, and solar; impacts on net load peaks and valleys; and increases on ramp rates during the daily cycle.

\subsubsection{Average Daily Profiles}

Figure 4.21 plots the 2006 average daily profiles of load, wind and solar for the total study footprint under the 30\% Local-Priority Scenario. Profiles are shown for four seasonally representative months: January, April, July, and October. Plots for other scenarios and penetration levels are included in Appendix B. Load is plotted against the left scale and wind and solar against the right, amplified by three relative to the load.

The average daily profiles reveal several general trends with regard to the relationship between load, wind, and solar output:

- At the footprint level, wind output is generally falling during the early-morning hours when load is rising most rapidly and tends to recover or flatten later in the morning. However, this inverse-phase relationship is not as strong during the summer as in other seasons.

- In the late evening to early morning hours when load is decreasing or at its minimum, the wind output is still high across the seasons. This may exacerbate the down-ramp requirements during traditionally low-load hours.

- The solar output complements the load as expected. It is either rising or at its peak around noon when the load is high, and rolls off in the late afternoon.

- The impact of the CSP thermal storage can be seen in the shape of the profiles. CSP tends to persist a lot longer in the day than PV, even until midnight in April and July. While this is great for load support in most cases, it sometimes creates problems where it rolls off very quickly just as the load is ramping up (as in January). This could presumably be managed by smarter dispatch of thermal storage (see Section 8.3).

These observations have implications for net-load variability (and operational requirements) at different times of the day and during the various seasons of the year, as discussed later in this section. 

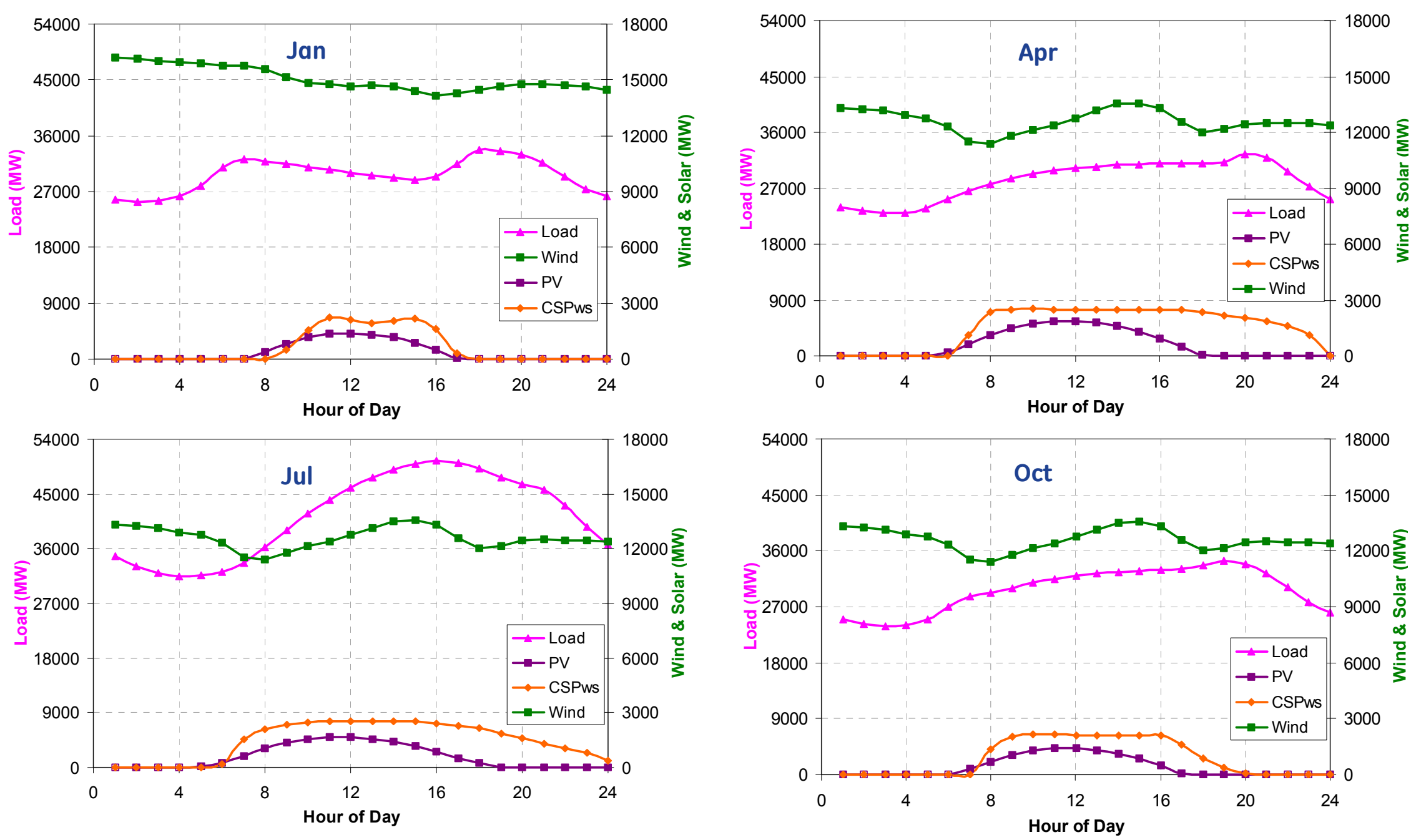

Figure 4.21 2006 Study Footprint Load, Wind, and Solar Average Daily Profiles by Seasonal Month for the 30\% Local-Priority Scenario 
The January and July average daily profiles in Arizona, Nevada, and Wyoming for the 30\% Local-Priority Scenario are shown in Figure 4.22 - Figure 4.24. What is clear from these figures is that the broad observations at the footprint level are even more pronounced at the state level. Note that different scales and axes are used for load, wind and solar in each figure.
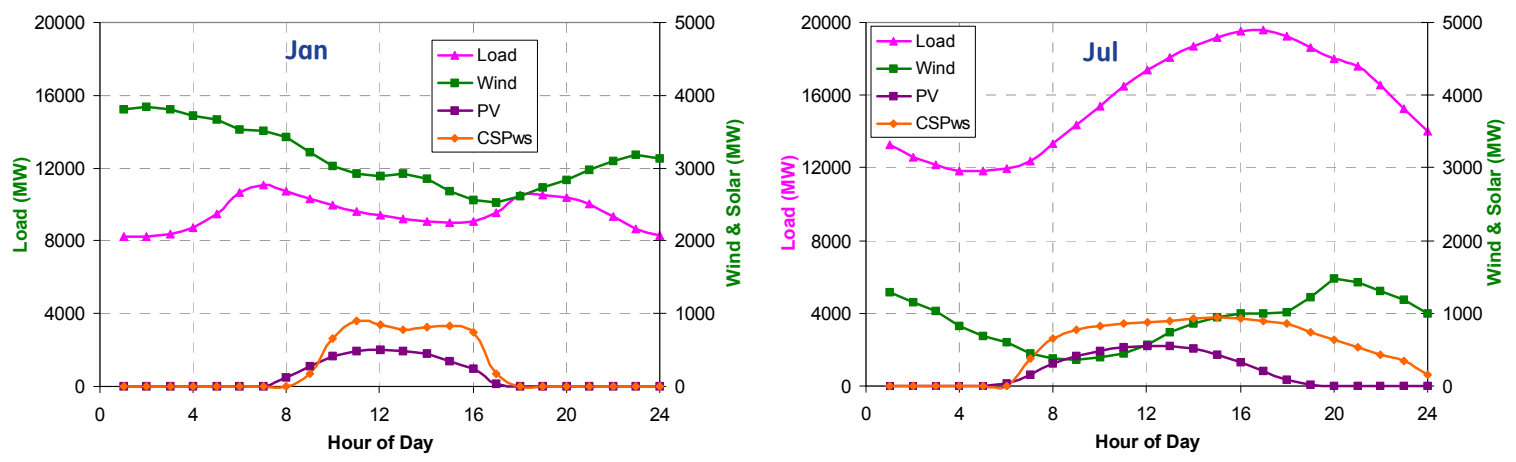

Figure 4.22 Arizona Load, Wind, and Solar Average Daily Profiles for January and July, 30\% LP Scenario
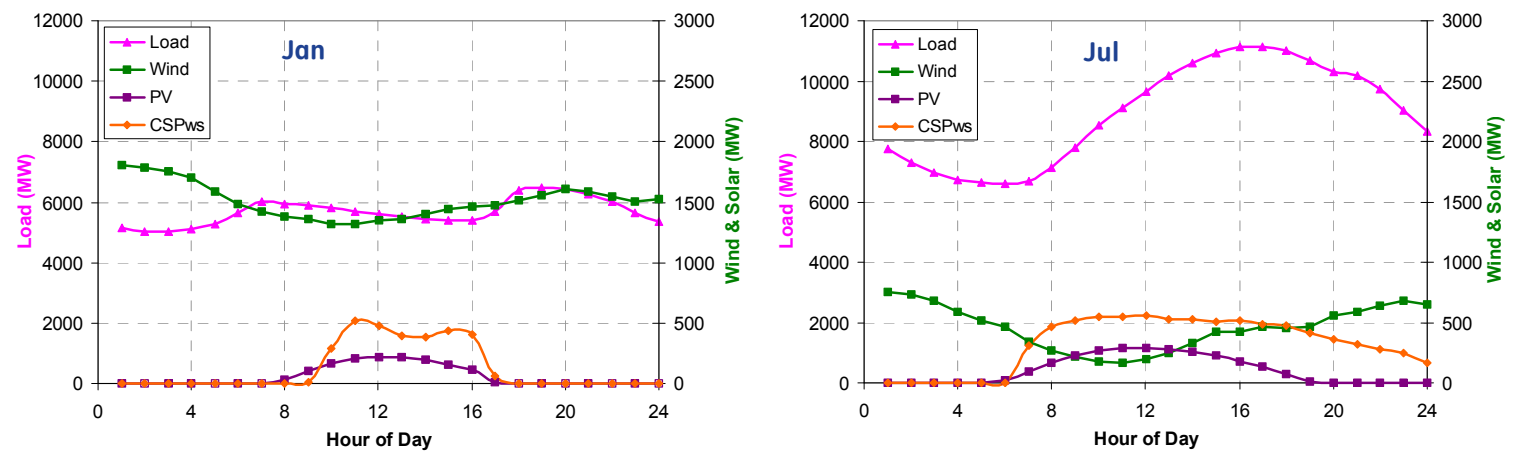

Figure 4.23 Nevada Load, Wind, and Solar Average Daily Profiles for January and July, 30\% LP Scenario
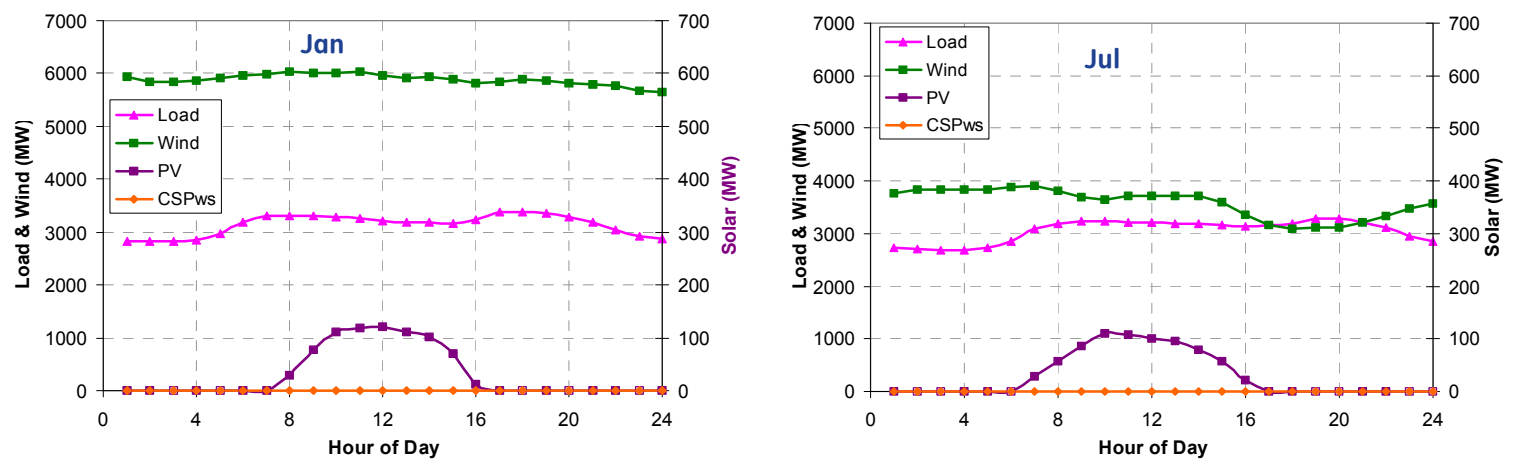

Figure 4.24 Wyoming Load, Wind, and Solar Average Daily Profiles for January and July, 30\% LP Scenario

The general observations regarding average daily profiles at the footprint level become even more pointed when actual time series (as opposed to averages) are examined, especially at the state/area level. At this resolution, it is easier to see how wind and solar combine to impact the net load. 


\subsubsection{Weekly Time Series}

Figure 4.25 and Figure 4.26 show the footprint actual load, wind, and solar series and the net load for the 30\% Local-Priority Scenario during a week in July 2006.

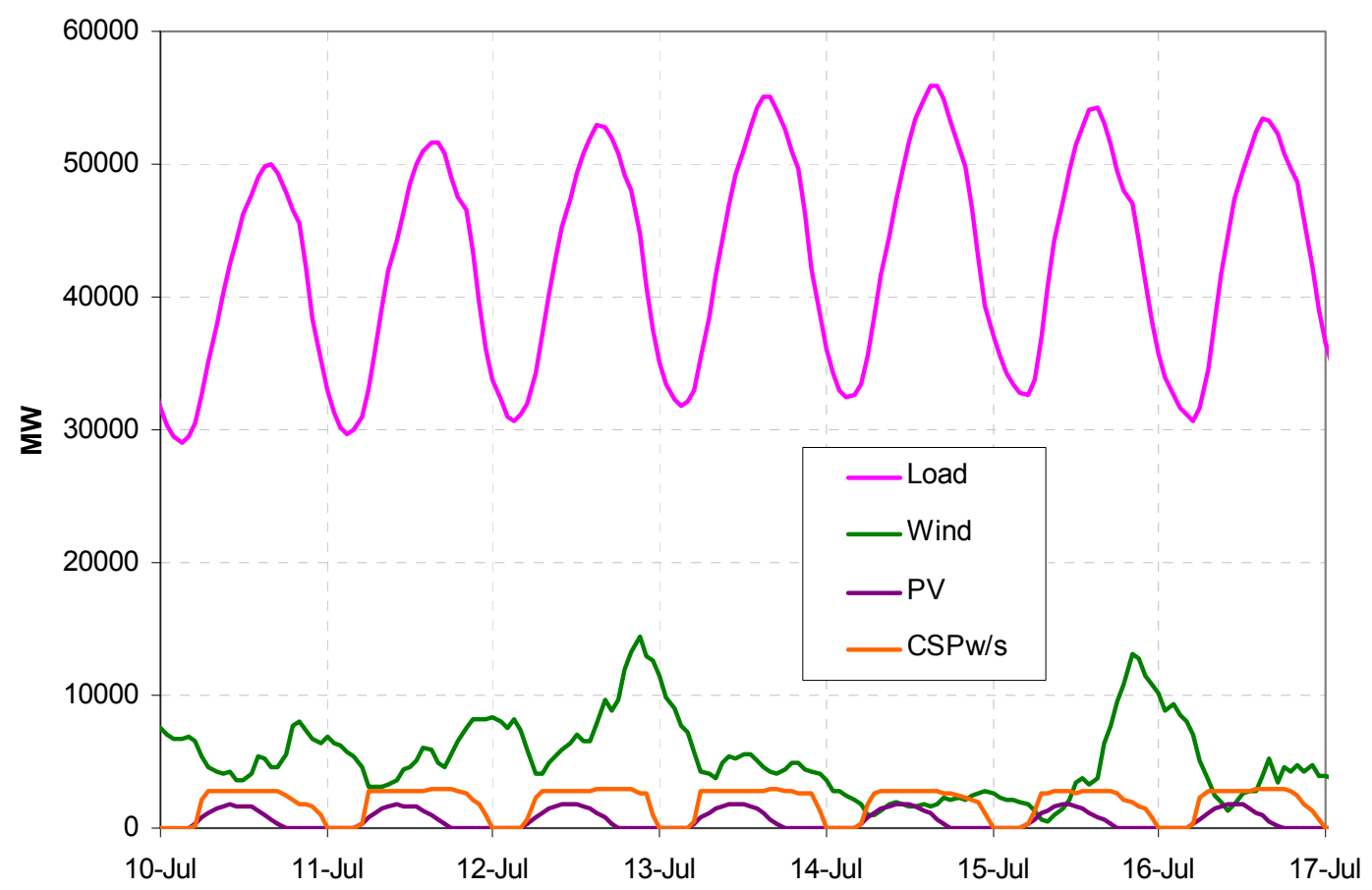

Figure 4.25 Study Footprint Total Load, Wind, and Solar for a Week in July 2006 30\% Local-Priority Scenario

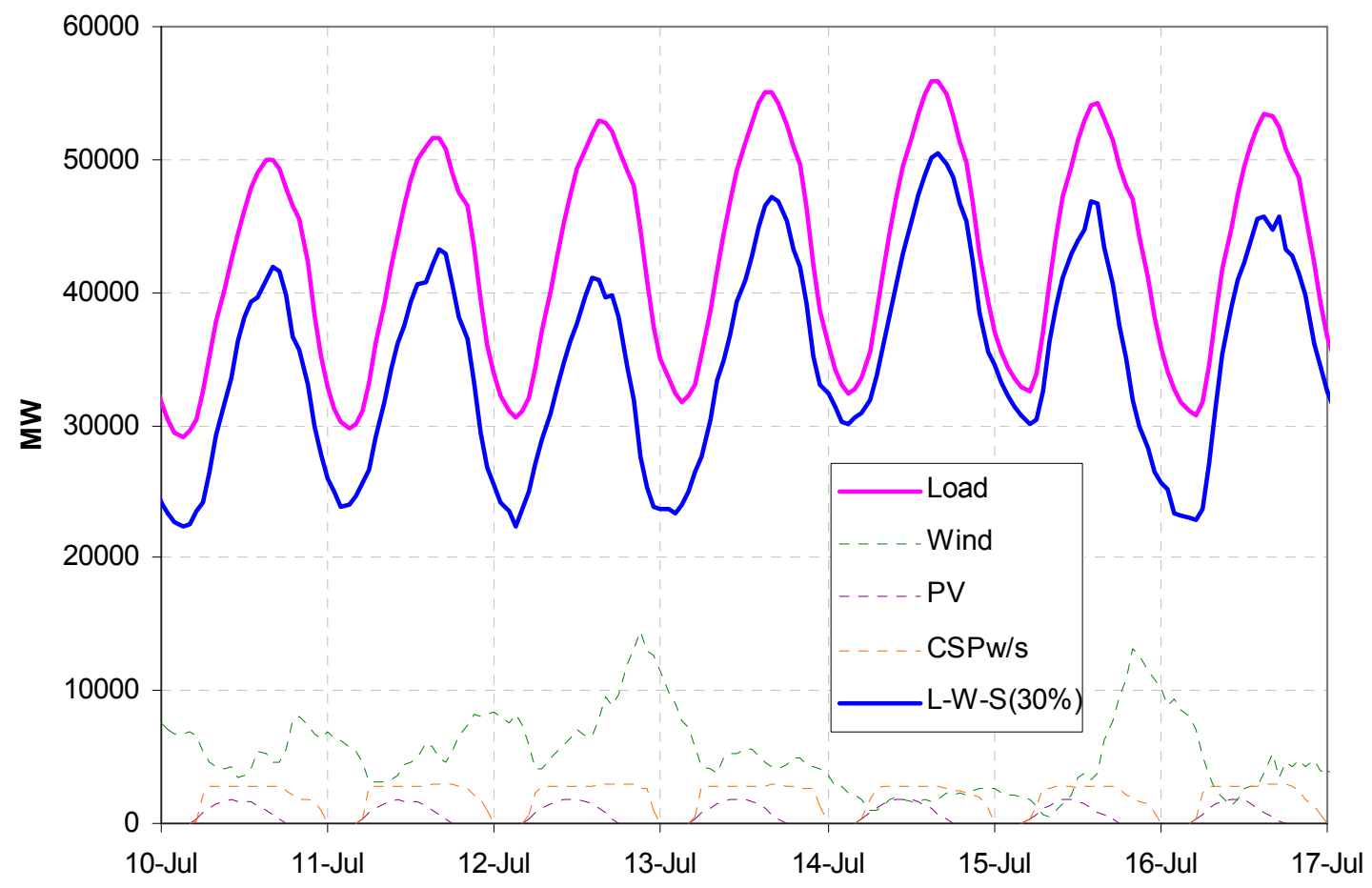

Figure 4.26 Study Footprint Total Load, Net Load for a Week in July 2006 30\% Local-Priority Scenario 
Figure 4.25 illustrates that over the course of the week, load, wind, and solar output vary considerably from day to day and throughout the day. There is a very strong diurnal load and solar cycle, as expected but the wind output also displays an observable, though weaker, periodic characteristic. Overall, the wind output tends to be anticorrelated with the load, i.e., wind generation peaks tend to coincide with load valleys. This is a key driver for the wind, PV, and CSP capacity values observed in Section 9.2.

Figure 4.26 shows the net load resulting from the Load-Wind-Solar combination. In the summer months, we have seen that the load usually dominates the wind (see Figure 4.21) so it is not surprising that the net load shape is similar to the load, with some nuances due to the variability of wind. Wind and solar output produce an offset in the net load that varies in magnitude. The greatest offset is in the late evening to early morning hours when wind output is highest and the load is falling. The impact of the strong summer solar cycle is seen particularly in the mornings. At times when the wind is falling or at minimum, the solar output is rising, which mitigates the increase in net load up-ramp. In the evenings, the CSP roll-off tends to coincide with the load decline and wind drops. Consequently, there is not much apparent difference between loadalone and net load ramping requirements. Figure 4.27 plots the net load for each of the three penetration levels, $10 \%, 20 \%$, and $30 \%$.

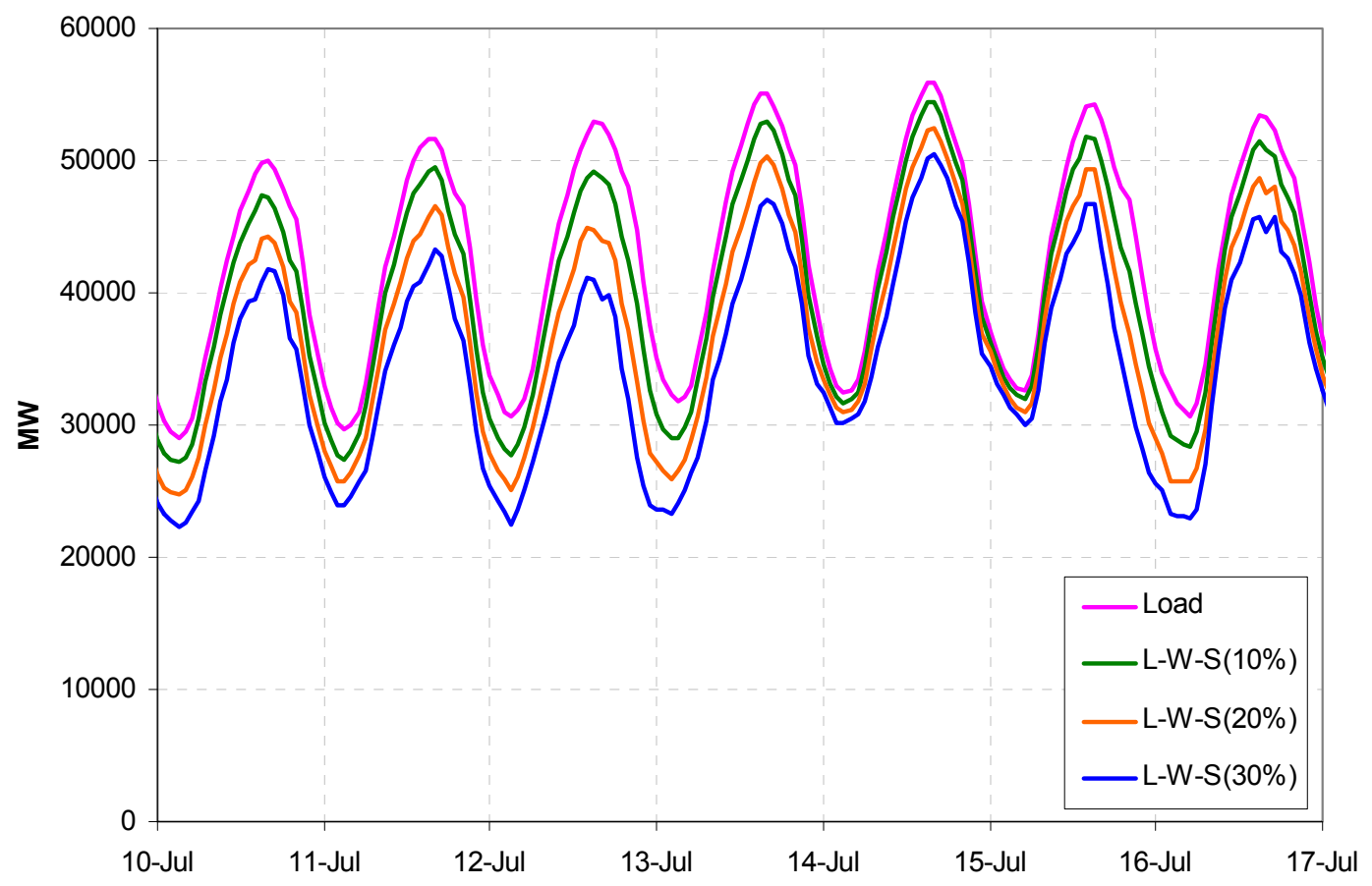

Figure 4.27 Study Footprint Net Load for a Week in July 2006 for 10\%, 20\% and 30\% Local-Priority Scenario

The plot is almost remarkable in its homogeneity. At the $10 \%$ penetration level wind and solar has a small impact on the net load (green trace), causing some deeper valleys at low-load and smaller peaks. As the penetration increases, the impact is more discernable, but even at the $30 \%$ level it does not overwhelmingly change the profile. 
The July week is in stark contrast to the April 2006 week shown in Figure 4.28 and Figure 4.29 below. In this week, the wind output is higher and more volatile, so load is not as dominant. The data shown is for the total footprint, $30 \%$ Local-Priority Scenario.

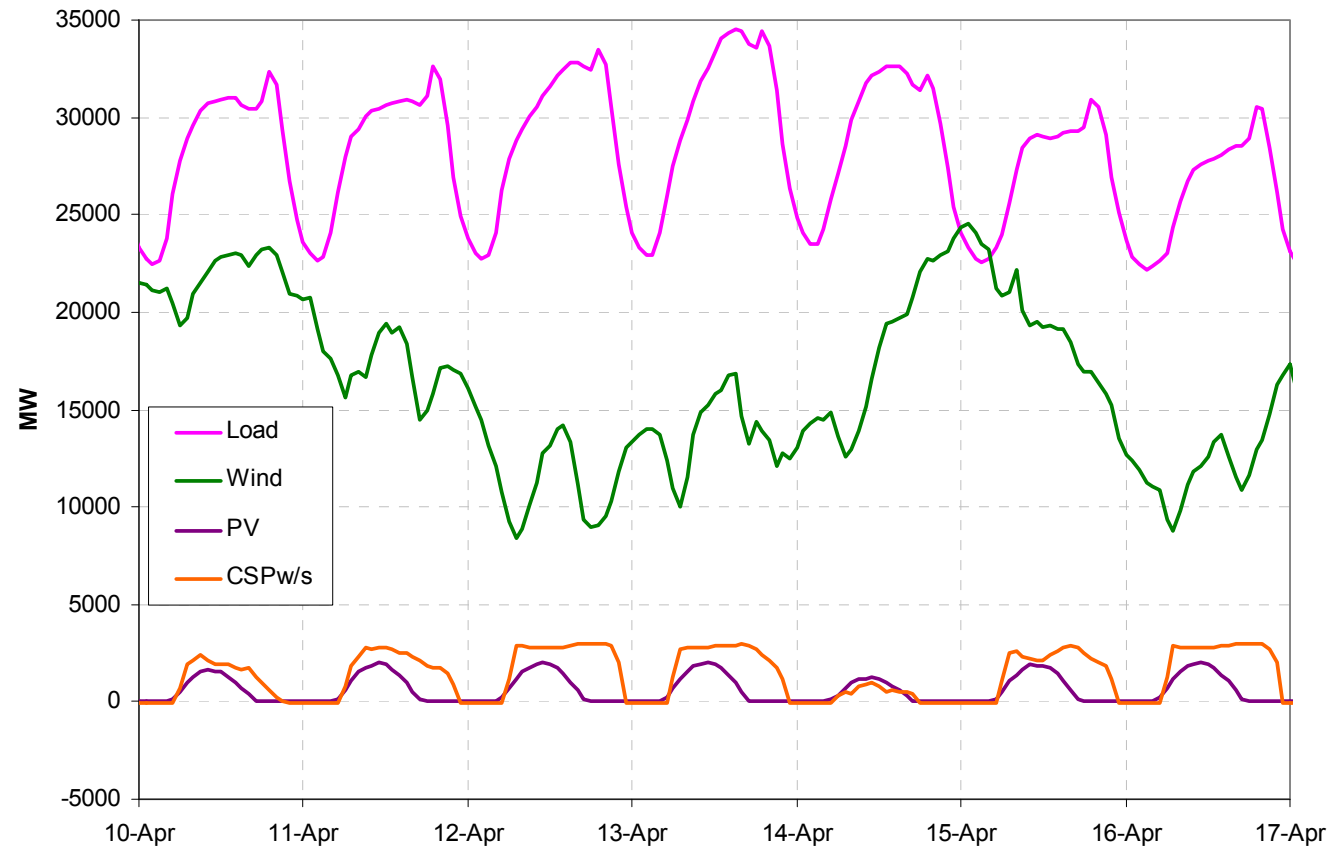

Figure 4.28 Study Footprint Total Load, Wind, and Solar for a Week in April 2006 30\% Local-Priority Scenario

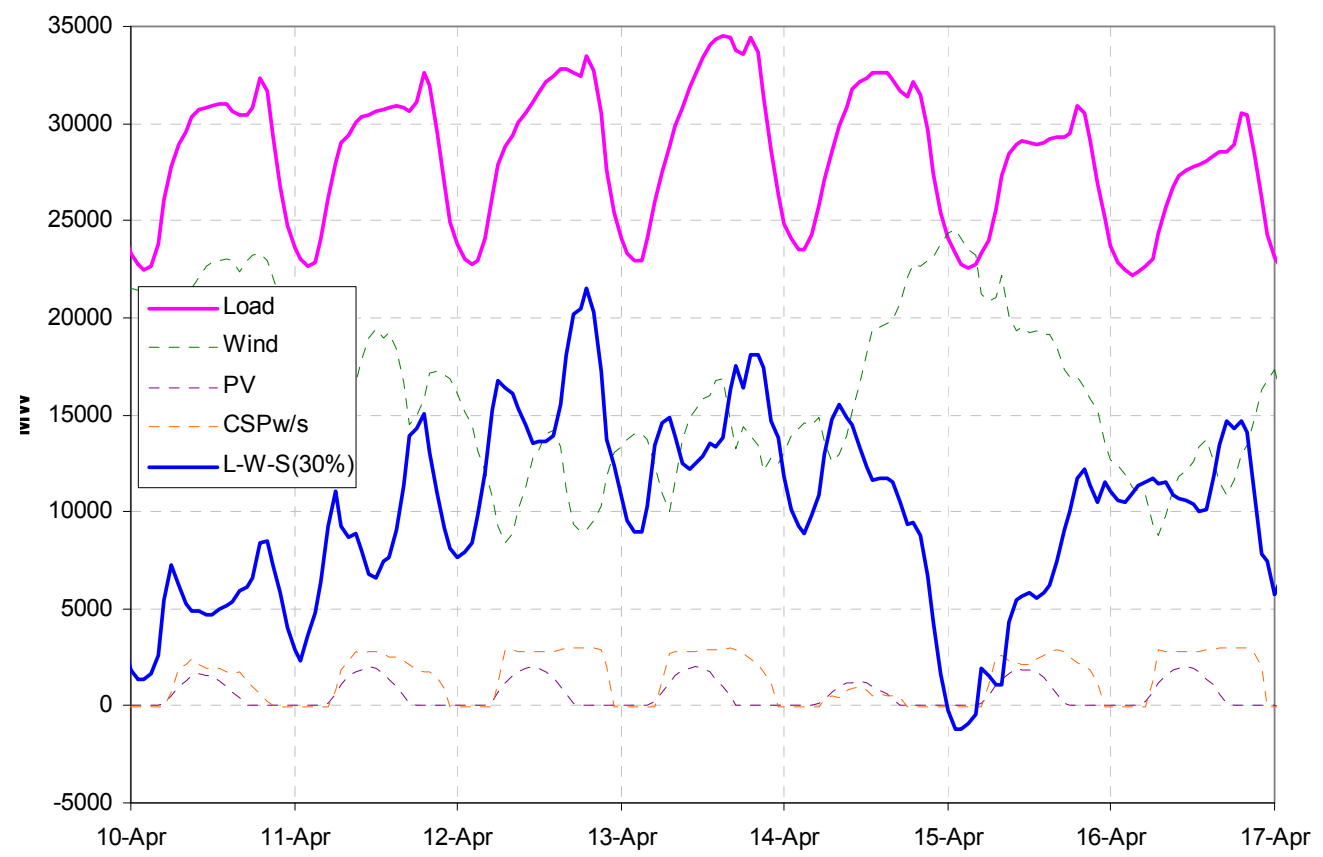

Figure 4.29 Study Footprint Total Load, Net Load for a Week in April 2006 30\% Local-Priority Scenario

As in the July week, there is a strong diurnal cycle for load and solar, but now there is no recognizable pattern to the wind output in this week. At the beginning of the week, 
early in the morning of the $10^{\text {th }}$, wind generation is almost equal to the load, and later, on the $15^{\text {th }}$, actually exceeds the load for a few hours. The wind ramps up and down several times during the week with sustained ramps in one direction sometimes spanning an entire day. The impact of this "unusual" wind output is seen in the net load trace in Figure 4.29. The net load no longer resembles the load in any way, but has taken on variability characteristics of the wind output. Over the course of the week, there are days with extremely large and sustained net load ramps, the likes of which did not exist in the original load, and instances of rapid swings in ramping direction over a few hours. On three days the net load drops below 5,000 MW, including going negative on the $15^{\text {th }}$, and is less than $10,000 \mathrm{MW}$ on every day of the week. Clearly, this week might present some challenges to a generation portfolio accustomed to dealing with the usual April load profile. Results of the hourly and sub-hourly operation during this April week (and the July week for that matter) are detailed in Section 6.3 and Section 7.2.

Figure 4.30 plots the net load for the April week for each of the three penetration levels, $10 \%, 20 \%$, and $30 \%$. The progressively disruptive impact of wind and solar on net load is seen in the net load traces. For $10 \%$ penetration, the net load is distorted, but there is still some semblance of a diurnal cycle. With $20 \%$ penetration, the load cycle is less evident, and at $30 \%$ there are many more rapid swings in net load and steep sustained ramps.

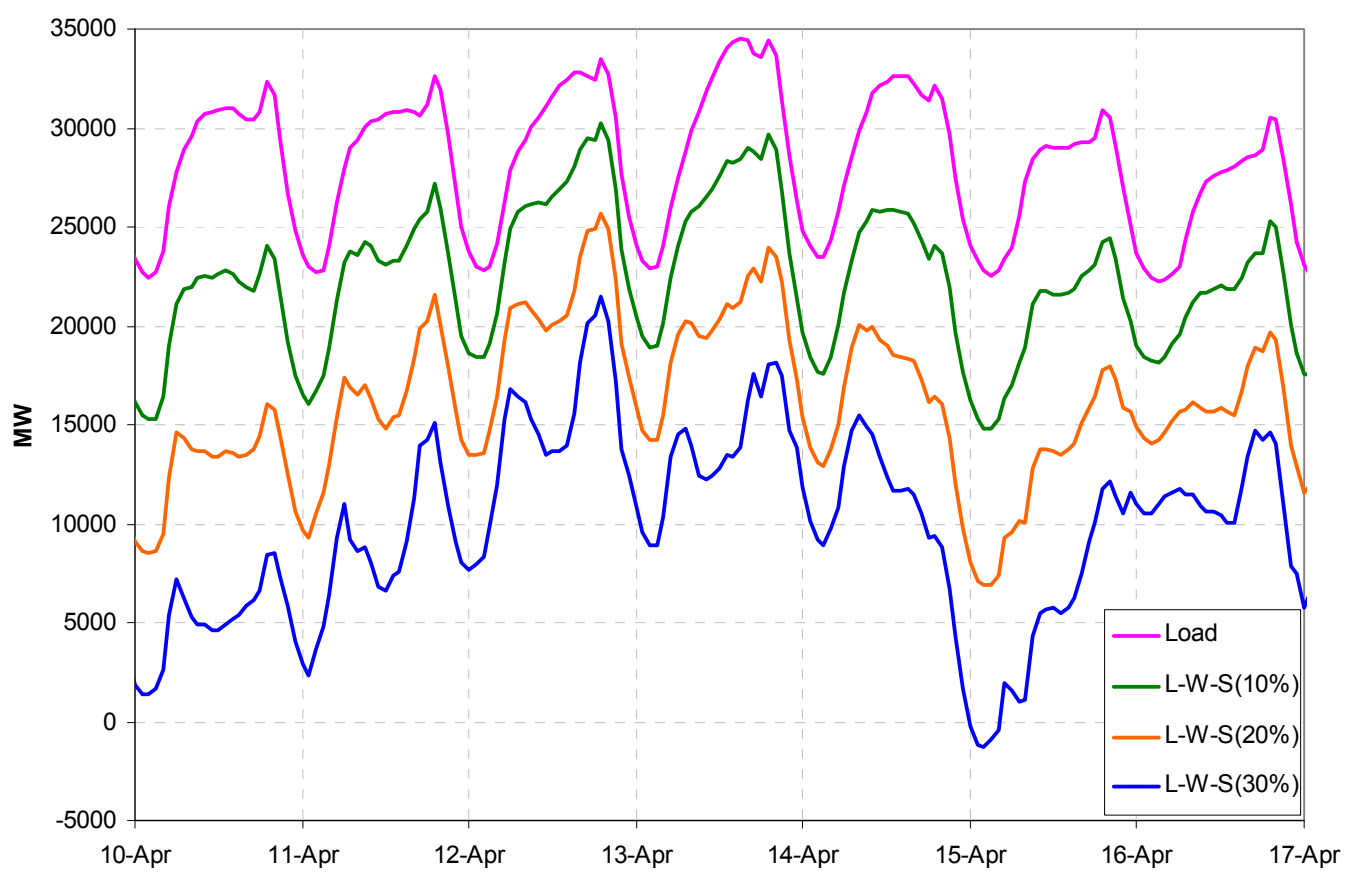

Figure 4.30 Study Footprint Net Load for a Week in April 2006 for 10\%, 20\%, and 30\% Local-Priority Scenario

The net load traces above, especially at 30\% penetration, clearly indicate the need for flexible generation capacity. This was not as evident from the July traces in Figure 4.27. However, note that the July peak net load for the $30 \%$ penetration case in Figure 4.27 is almost 30,000 MW more than the corresponding April peak net load. This indicates that no additional dispatchable generation capacity may be needed for the April week, but 
that the capacity available to meet the planning reserve margin needs to be flexible enough to accommodate this large penetration (see Chapter 6 for further discussion).

\subsubsection{Selected Periods}

Load, wind, solar, and net load for selected days are shown in the plots below. The impact of wind and solar on net load ramp and range are highlighted on the plots.

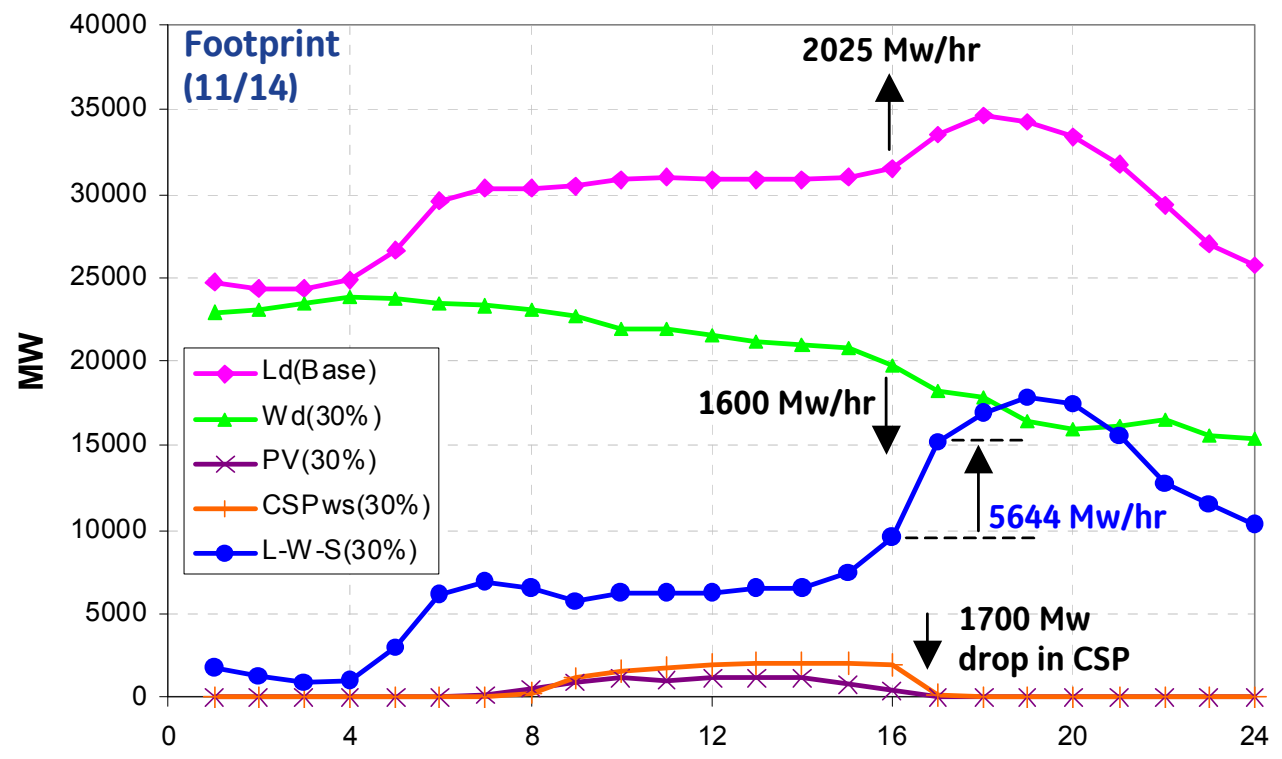

Figure 4.31 Study Footprint Net Load for November $14^{\text {th }}, 2006,30 \%$ Local-Priority Scenario

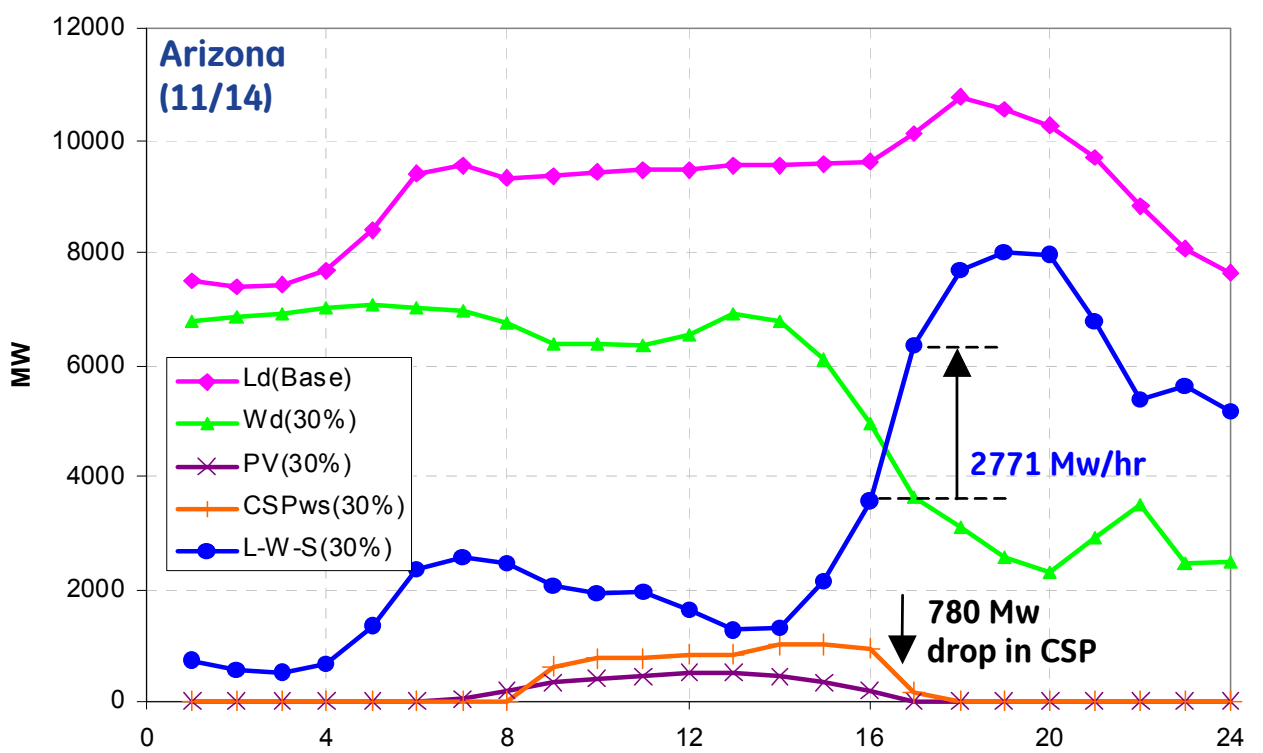

Figure 4.32 Arizona Net Load for November $14^{\text {th }}, 2006,30 \%$ Local-Priority Scenario

On November $14^{\text {th }}$, there is about a $180 \%$ increase in the footprint net load up-ramp during the late afternoon, around 4 p.m. As the details on Figure 4.31 show, this is due to a simultaneous rise in load and a drop in wind and solar. Figure 4.32 shows that the 
main driver for this footprint event is the wind and solar in Arizona. During the 4 p.m. hour in Arizona, wind output drops by $1316 \mathrm{MW}$ and CSP drops by $780 \mathrm{MW}$, causing a $2771 \mathrm{MW} / \mathrm{hr}$ up-ramp in Arizona net load. This is much more aggressive than the upramp without wind and solar, and lasts a lot longer. In fact, the sustained up-ramp in net load spans 5 hours, ( 2 p.m. -7 p.m.) whereas the original load ramp was only 2 hours long. This sort of event might increase the ramp and range requirements for the balance of generation in Arizona or the study footprint.

Figure 4.33 and Figure 4.34 show the load, wind, solar, and net load traces for another large ramping event at the footprint and state/area level.

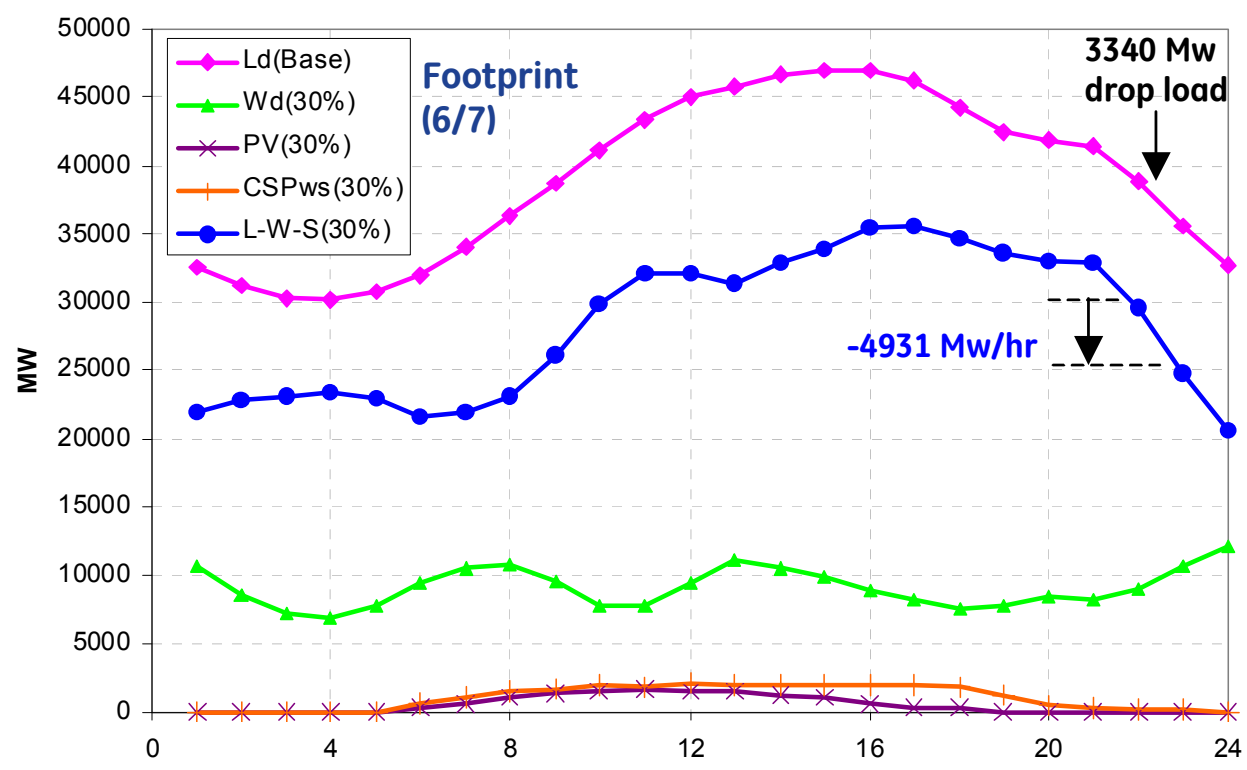

Figure 4.33 Study Footprint Net Load for June ${ }^{\text {th }}, 2006,30 \%$ Local-Priority Scenario

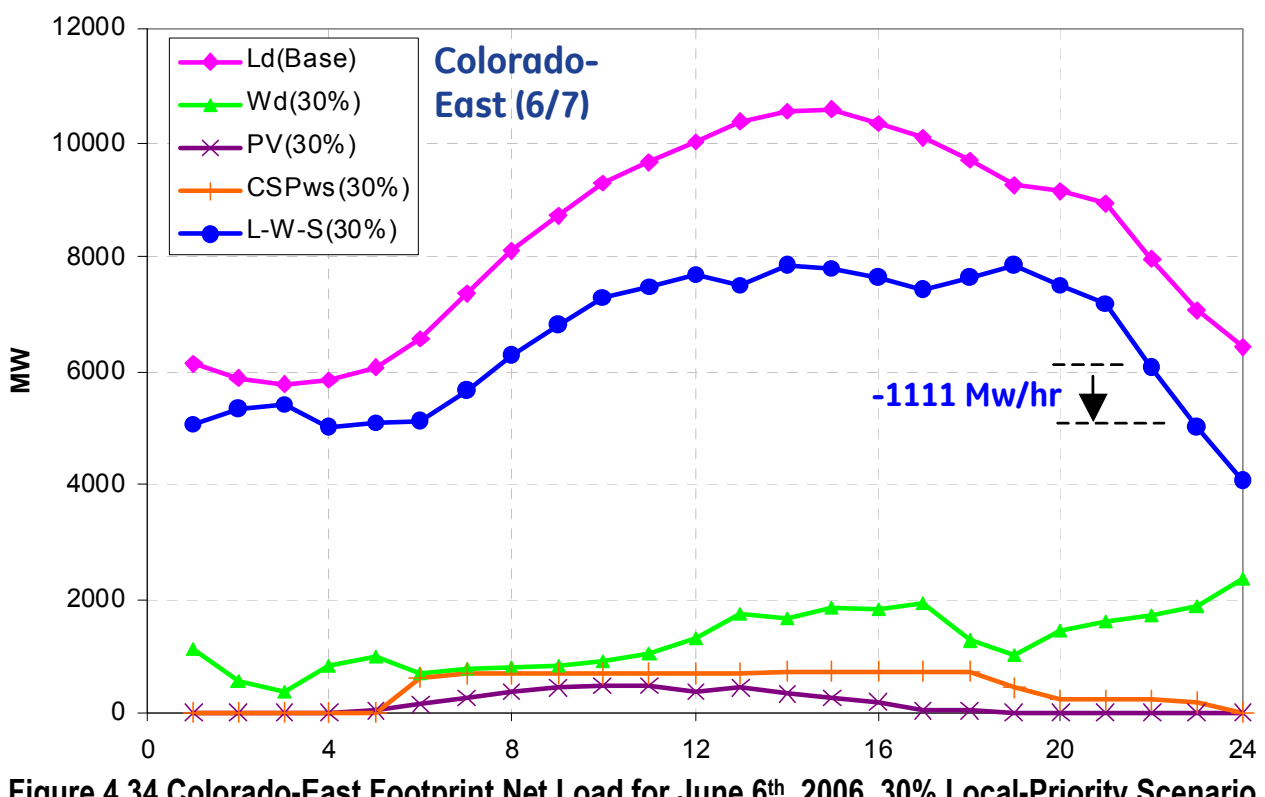

Figure 4.34 Colorado-East Footprint Net Load for June 6 $6^{\text {th }}, 2006,30 \%$ Local-Priority Scenario 
On June $6^{\text {th }}, 2006$, the net load in the study footprint drops precipitously around 9 p.m. in the evening due to a roll-off in load while the wind is picking up. At the state level, one of the key contributors is Colorado-East wind, which is ramping up aggressively in that hour, while solar is flat. These are but a few examples of the potentially challenging periods throughout the year. Other interesting days where wind and solar aggravate the net load are plotted in Appendix B.

Finally, as a lead-in to a discussion of period-to-period variability in the next chapter, Figure 4.35 shows the time series for the entire month of April at the footprint level for the 30\% Local-Priority Scenario. Recall that a week from this month was used earlier to illustrate the impact of wind and solar on net load.

Over the course of the month, rapid excursions can be seen in the net load as it goes through large changes from hour to hour. The number and size of the period-to-period changes can statistically characterize the obvious visual variability of this series. The next chapter will describe the variability of net load by analyzing the hour-to-hour changes (called deltas) that are driven by wind and solar.

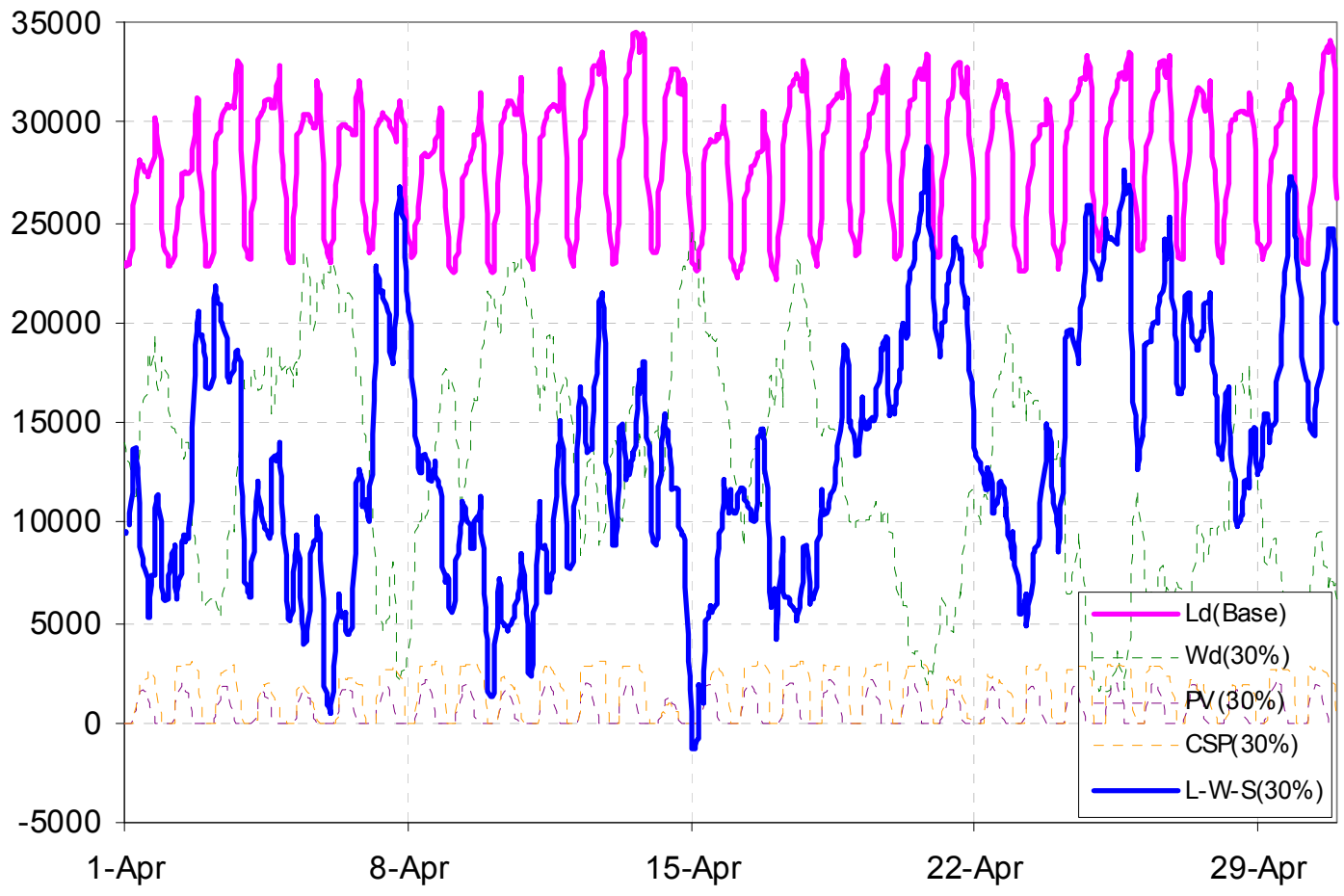

Figure 4.35 Study Footprint Total Load, Net Load for April 2006, 30\% Local-Priority Scenario

\subsection{Observations and Conclusions}

This chapter has examined broad trends in wind and solar generation monthly energy, yearly duration, and daily profiles. The hourly coincident relationship between load, 
wind and solar has been examined and the impact on net load at the footprint level and within individual states has been discussed.

For $30 \%$ wind penetration, at the footprint level sufficient wind capacity is installed to supply $30 \%$ of the load energy in the footprint. However, an examination of the monthly energy output shows that $30 \%$ is not always $30 \%$ ! For each of the scenarios, in a given month more than half the energy in the footprint may be derived from wind and solar especially in winter and spring months. At the state area level, the percent penetration can far exceed $100 \%$, depending on the state and scenario.

The impact of wind and solar is a lot less dramatic at the aggregate footprint and WECC levels than at the individual state level, regardless of the scenario. In fact, in most cases there is relatively little observable difference among scenarios at the footprint level, despite the fact that there are significant differences in plant number and locations.

The analysis showed that area size (load and geography) is a key determinant of a state's ability to absorb the variability inherent in high-wind and solar penetration. Large amounts of wind in a small area may lead to challenging operational issues, but larger balancing areas can better accommodate the variability from high-wind and solar penetration.

Within each state and at the footprint level, the temporal relationship between wind and solar output and load is the key driver for resulting net load variability. Depending on the coincidence with load, wind and solar might exaggerate or curtail net load peaks and valleys, increase or decrease ramp rates (period-to-period changes), shift time-ofpeak, and generally aggravate or mitigate operationally challenging periods. Some pertinent observations from the seasonal average daily profiles are:

- Wind output is generally falling during the early-morning hours when load is rising most rapidly

- In the late evening to early morning hours when load is decreasing or at its minimum, the wind output is still fairly strong across the seasons

- Solar output complements the load as expected, but CSP output tends to roll off very quickly in late afternoons and evenings when storage is depleted. In months where the load is ramping up in late afternoons, this could increase balance of portfolio generation ramping requirements. Note that this outcome is driven by the study assumption of continuous use of the six hours storage. Section 8.3 includes a sensitivity case where CSP storage is used optimally.

These general observations regarding average daily profiles at the footprint level become even more pointed when actual times series (as opposed to averages) are examined and when the state/area level variability is considered. 



\section{Statistical Analysis: Hourly Variability and Day-Ahead Predictability}

The variability of net load in different time frames influences various aspects of bulk power-system operation. Implications for regulation requirements, ramp and range considerations, operating reserves, and unit commitment issues can be drawn from an analysis of net load variability in various time frames, depending on the ancillary service definitions and market rules. This chapter will focus on the statistical analysis of load and net load variability in the hourly time frame and reserve a fuller discussion of operation impacts and requirements for later chapters.

In this chapter, several terms are used to characterize the load and net load variability. They include:

- Delta $(\Delta)$-the difference between successive data points in a series, or period-toperiod ramp rate

- Positive delta is a rise or up-ramp

- Negative delta is a drop or down-ramp

- Mean $(\mu)$ of the deltas - the average of the deltas (typically zero within a diurnal cycle)

- Sigma $(\sigma)$ of the deltas - the standard deviation of the deltas; measures spread of the deltas about the mean; this is also referred to here as "delta sigma" or $\Delta \sigma$.

The standard deviation of the deltas is a good indication of how much the series changes from period-to-period, therefore sigma of the deltas is used as the main measure of variability in this study. If the deltas are normally distributed, then delta sigma relates to the proportion of deltas within a certain distance of the mean, $\mu$ (as shown in Figure 5.1).

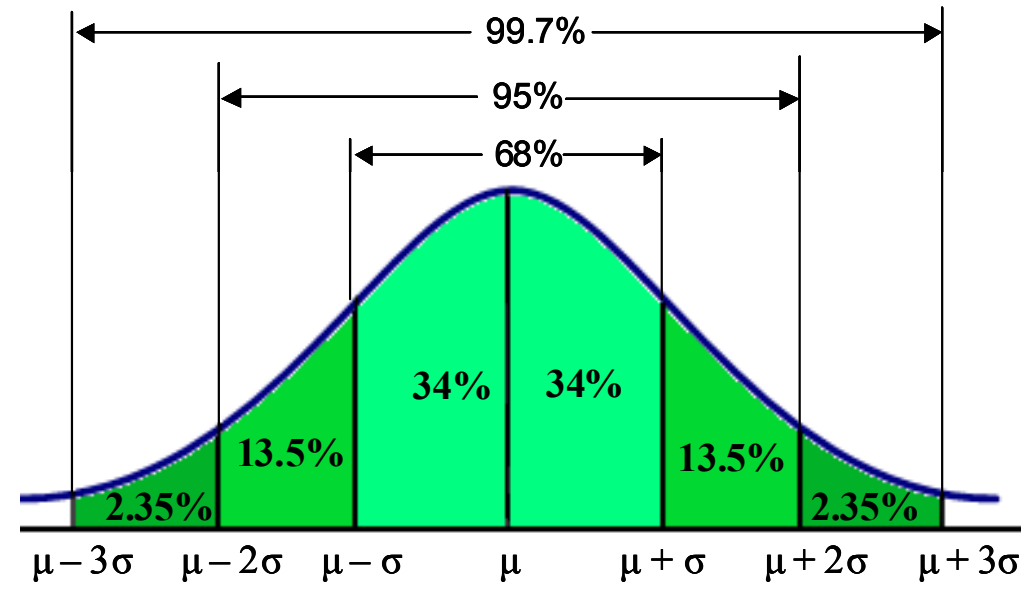

Figure 5.1 Relationship Between Standard Deviation and Area Under the Standard Normal Curve 


\subsection{Seasonal Trends in Variability}

As seen earlier in Figure 4.21, load, wind, and solar have distinct seasonal characteristics that influence the variability of net load at different times of year. The majority of net load variability is driven by the wind, (since solar is relatively small), so it is important to understand the interaction with load. Figure 5.2 is a scatter plot of load and wind deltas that attempts to show when load and wind combine to exacerbate the net load.

On the chart, each square represents a simultaneous pair of load and wind deltas. Orange squares are fall load and wind deltas, blue triangles are winter deltas, green diamonds are spring, and pink circles are summer. For example, the pink circle in the top left quadrant (Q2) labeled $(-3339,1611)$ indicates that during the summer months of 2006, (June, July, August) there is an hour when load drops by 3,339 MW while at the same time, wind output rises by 1,611 MW. The combination of these two events (ignoring solar) creates a net load down-ramp of 4,950 MW in that hour. In Q2 and Q4, load and wind combine to increase net load ramps, (down and up respectively) while in Q1 and Q3, they offset each other to reduce the resulting ramping requirements. The diagonal lines in Q2 and Q4 indicate boundaries of equal net load delta in the load-wind space, i.e. any load-wind pair along the line will combine to give the same net load value. The red diagonal in Q2 is at the maximum load-alone down-ramp (-4250 MW), but is also close to the $99.7 \%$ percentile line for load-wind deltas in Q2 (roughly $-4,400 \mathrm{MW}$ ).

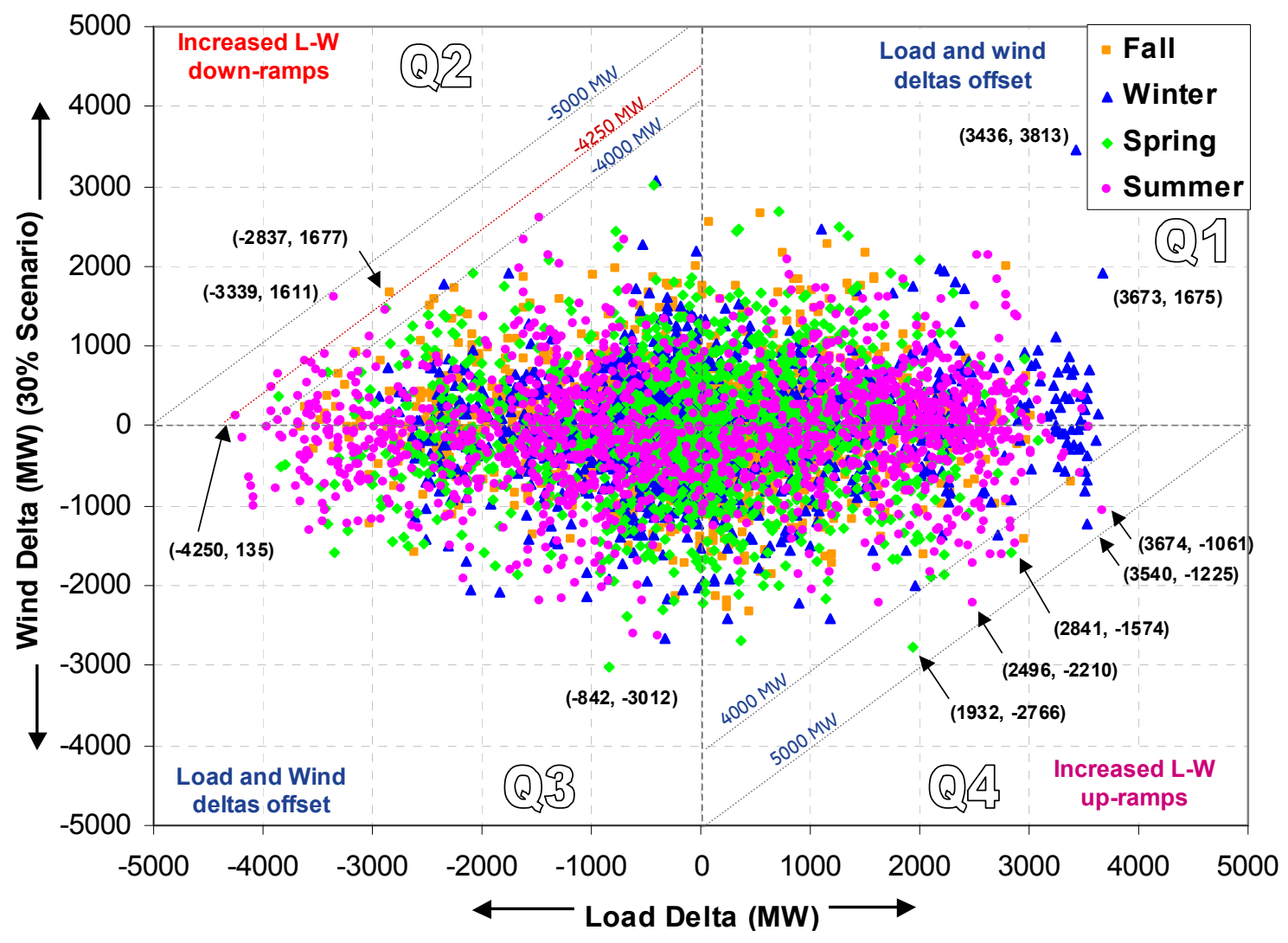

Figure 5.2 2006 Study Footprint Wind Deltas vs. Load Deltas by Season for 30\% Local-Priority Scenario 
The first noticeable thing about the scatter plot in Figure 5.2 is its shape. There is no obvious trend in the data, which seems to indicate that over the year, load and wind deltas are not highly correlated at the footprint level. Moreover, the points are stretched along the x-axis, which implies that large load deltas are not usually accompanied by similarly large wind deltas, i.e., the risk of simultaneous delta reinforcement is small. A closer examination of the extreme load and wind deltas confirm that there are relatively few cases where simultaneous load and wind deltas create extreme net load deltas. In Q2, the quadrant where load and wind deltas combine to increase net load down-ramps, there are only a few instances where net load down-ramps are greater than the largest load-alone down-ramp of 4,250 MW (as shown by the red diagonal line in Q2 on Figure 5.2). These all occur in the summer and fall, and are mostly driven by load changes. These events do not represent a great operational challenge as the wind can always be curtailed. In Q4, the quadrant where load and wind deltas combine to increase net load up-ramps, there are many more hours where net load up-ramps are greater than the largest load-alone up-ramp (3674 MW). These events are scattered over all seasons, and some are driven by large wind drops, which can create a significant operational challenge, especially if the generation mix at the time is not flexible.

Figure 5.3 is a similar scatter plot of load and wind deltas that includes all three scenarios at the $30 \%$ penetration level.

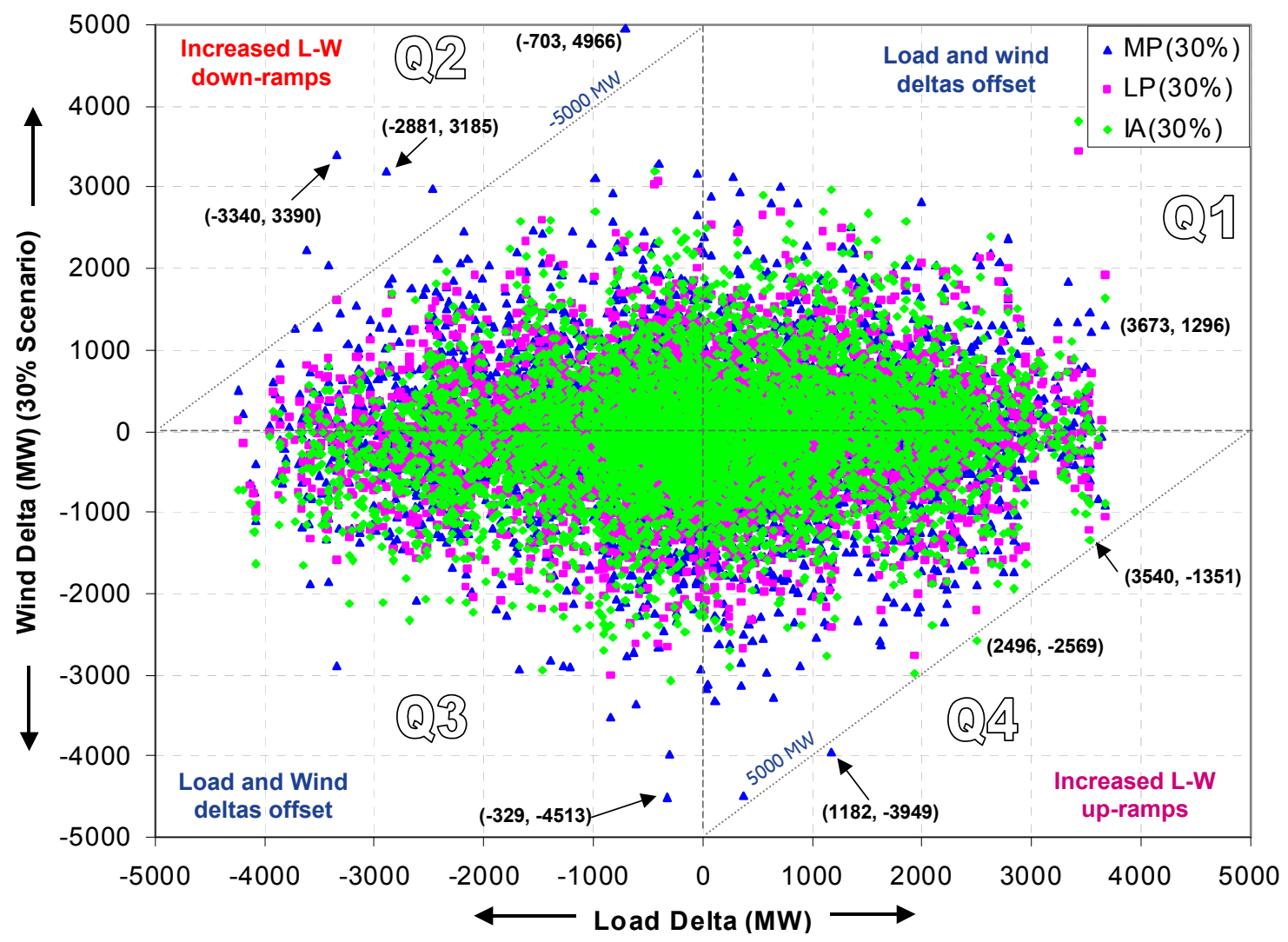

Figure 5.3 2006 Study Footprint Wind Deltas vs. Load Deltas by Scenario for 30\% Penetration 
In the scatter plot, most of the extreme deltas in Q1 and Q2 are from the Mega-Project Scenario and the In-Area Scenario. A few of the more troubling ones in Q4 are driven by extremely large drops in wind output (4,000 MW or more) under the Mega-Project Scenario. However, none of these coincides with similarly large load rises, probably because they occur in low-load hours. Also in Q4, there are a couple of instances under the In-Area Scenario, where moderately large wind drops combine with moderately large load rises, to create large net load up-ramps. There are also a number of extreme Mega-Project instances in Q1 driven by wind pickup, but these can always be mitigated by curtailment if necessary.

\subsection{Variability by Hour-of-Day}

Earlier sections have discussed broad trends in variability across the year and during different months and seasons. From an operation point of view, broad trends are not nearly as important as variability during particularly challenging periods. One such period is the minimum load period, which was discussed in Section 4.2.3. This section will discuss this and other challenging operating periods during the day created by large swings in net load.

In order to examine the variability by time of day, average daily profiles of load and net load are overlaid with the hourly variability. Figure 5.4 shows this plot at the footprint level for the $10 \%$ Local-Priority Scenario. The dashed magenta and blue curves are average daily load profiles, plotted against the right scale. The box and whisker plot captures the variability for each hour of the day, across the year. The length of the rectangular boxes represents a spread of one standard deviation $(\sigma)$ around the mean of the hourly change for a particular hour of the day. The whiskers show the maximum 1hour deltas over the year for a particular hour of the day.

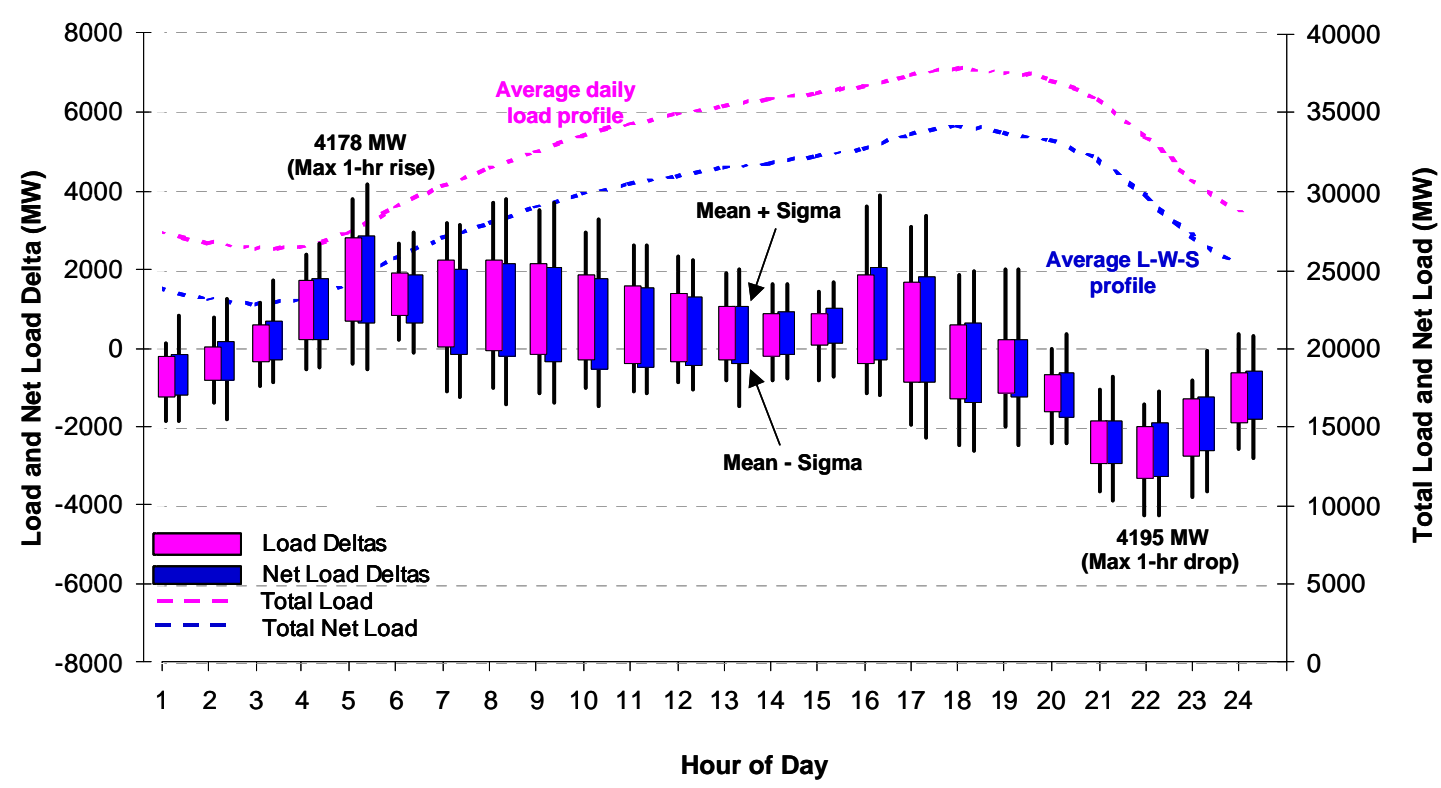

Figure 5.4 Study Footprint Average Daily Profile of Deltas Over Year 2006, 10\% Local-Priority Scenario 
Considering the relative length of the boxes in Figure 5.4, wind and solar generation do not increase the net load variability much over the baseline at $10 \%$ penetration, other than a slight increase in the morning and evening hours. In addition, the extreme deltas (whiskers) across the day are mostly due to load. In the morning hours when load is ramping up, the maximum 1-hour net-load rise is $4,178 \mathrm{MW}, 90 \%$ of which is due to the load. In the late evening when load is ramping down, the maximum 1-hour drop is 4,195 MW. At this hour, the baseline ramp is actually greater than the net load ramp.

For $20 \%$ and $30 \%$ penetration, the impact of wind and solar at various hours of the day is more dramatic, as shown in Figure 5.5 and Figure 5.6 below.

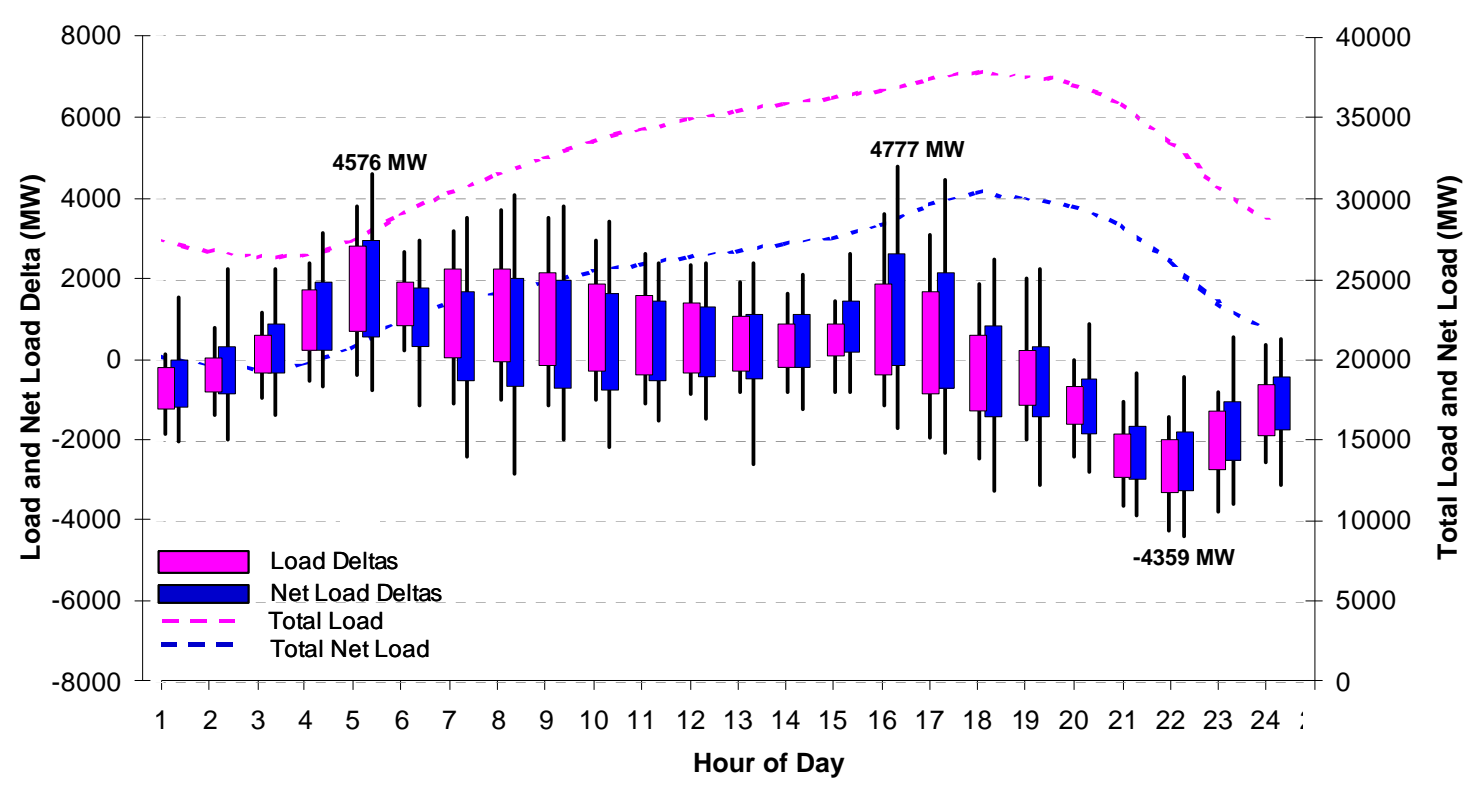

Figure 5.5 Study Footprint Average Daily Profile of Deltas Over Year 2006, 20\% Local-Priority Scenario

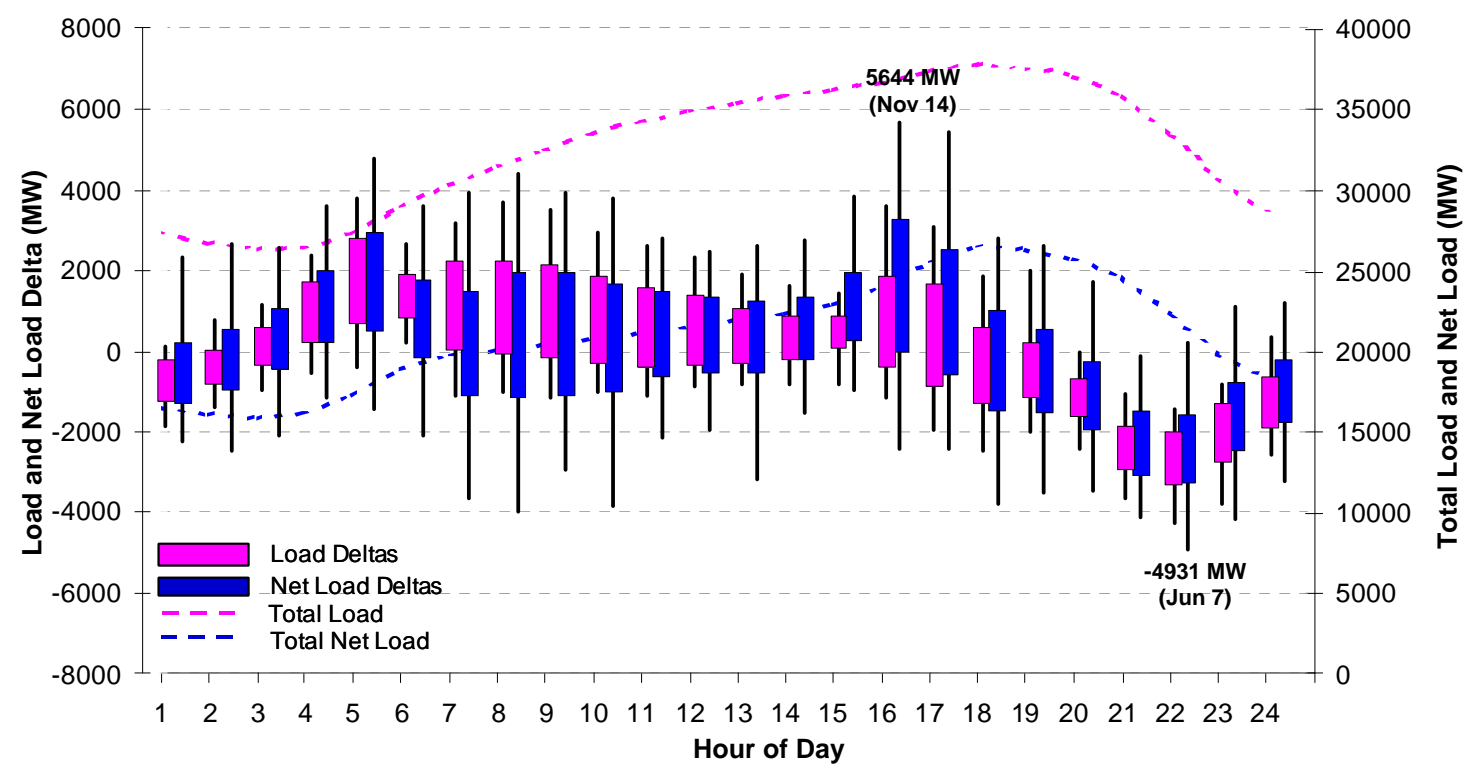

Figure 5.6 Study Footprint Average Daily Profile of Deltas Over Year 2006, 30\% Local-Priority Scenario 
These plots illustrate how wind and solar progressively affect the net load at larger penetration levels. At this temporal and spatial resolution, the impact on the variability (sigma of the deltas) is not as dramatic as the impact on the extreme values. This is due to natural averaging over the year and the footprint. Nevertheless, the blue rectangles (net load delta sigma) grow larger than the magenta ones (baseline delta sigma), especially in the mid-morning and early afternoon hours. Considering the average daily profiles shown earlier in Figure 4.21, these periods roughly correspond with times when the wind and solar are either ramping up or down.

At this resolution, the impact on extreme deltas is more apparent because they are not impacted by averaging. However, note that the extreme delta is only the largest delta observed at that hour over the year, but it may not be representative of all deltas in that hour. Regardless, the extreme delta represents an event during the year that the system must get through, operationally. For 30\% penetration, the extreme net load deltas are far greater than the baseline deltas. The maximum 1-hour net load rise is 5,644 MW in the early afternoon, and the maximum 1-hour net load drop is 4,931 MW in the late evening. Both have a significant wind and solar component superimposed on load.

Figure 5.7 shows the profile of load and net load deltas in April at the footprint level. Comparing this plot to Figure 5.6, one can see how the differences in variability are more pronounced when shorter time periods are considered. The month of April was previously shown (Figure 4.35) to have a tremendous amount of net load variability. This shows up as difference in the heights of the magenta and blue bars across the daily hours of April. The difference is more evident in the early morning and late evening hours, which is when wind is undergoing the most drastic changes. As discussed, higher spatial resolution will also reveal much about the time-of-day variability. The plots in Figure 5.8 show the profiles of deltas for several selected states.

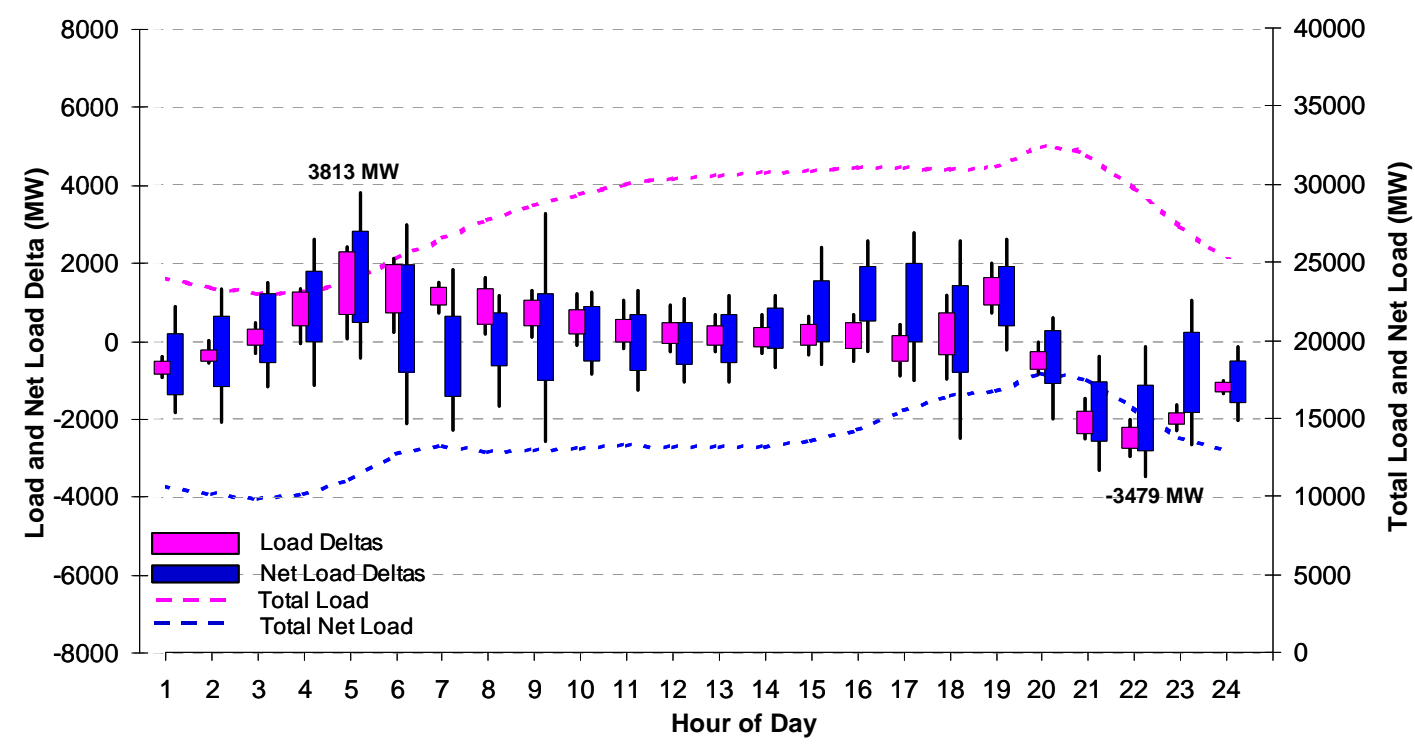

Figure 5.7 Study Footprint Average Daily Profile of Deltas Over April 2006, 30\% Local-Priority Scenario 

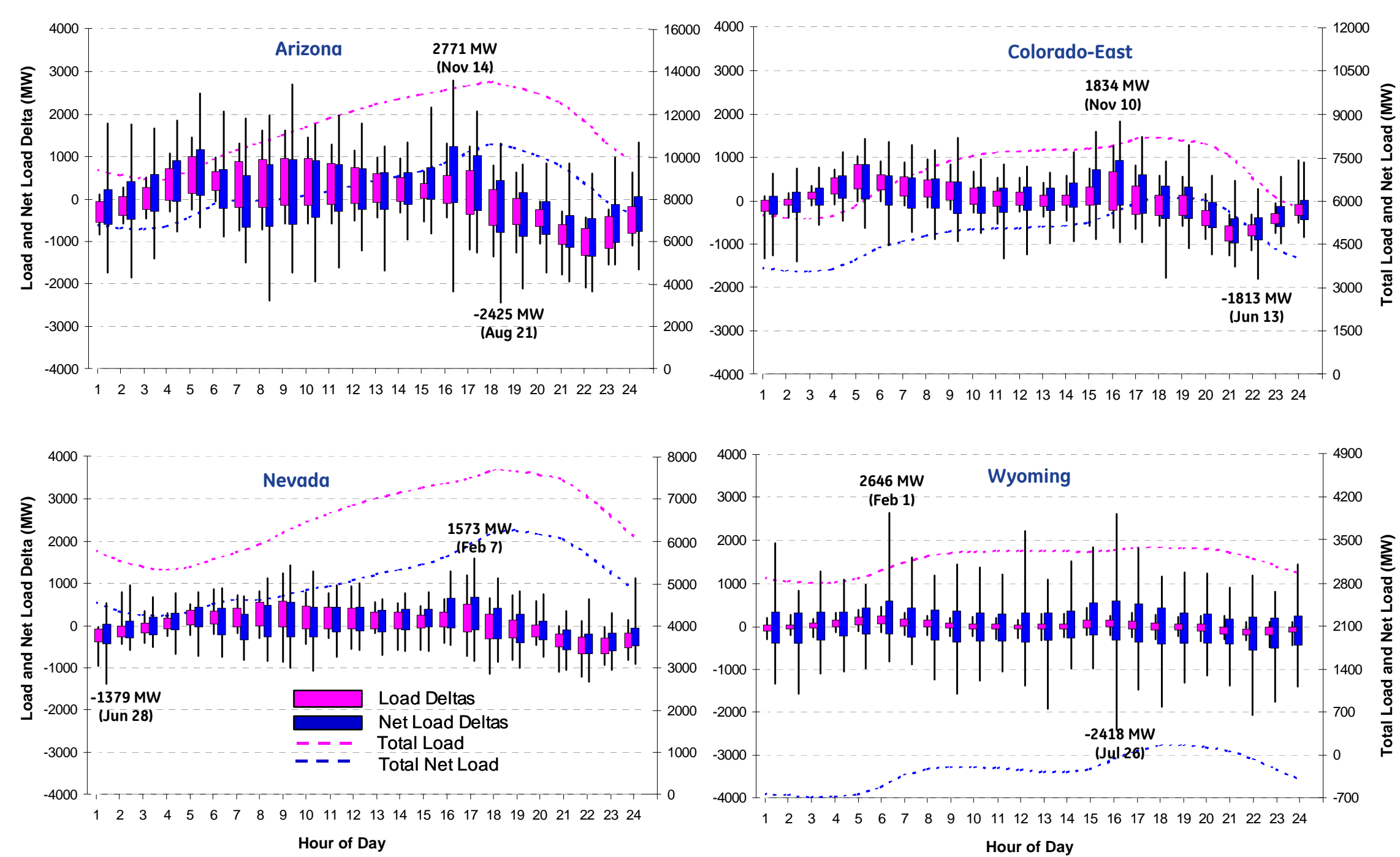

Figure 5.8 Average Daily Profile of Load and Net Load Deltas Over 2006 for Selected Areas, 30\% Local-Priority Scenario 
For the Local-Priority Scenario, each of the states in Figure 5.8 has a fairly large amount of wind, (as detailed in Section 2.5), but only Wyoming has an overwhelming amount compared to the load in the state. The delta profiles show that in each hour of the year, the Wyoming load variability is insignificant compared to the net load variability. The same can be said of the net load extreme deltas compared to the load deltas. The maximum 1-hour net load rise in Wyoming, 2,646 MW, occurs on the morning of February $1^{\text {st }}$, and the maximum 1-hour drop of 2,418 MW, occurs in the afternoon of July $26^{\text {th }}$. In the other states shown, the ratio of installed wind to load is much smaller, so some patterns emerge. There is more net load variability in the early morning hours, afternoon, and late evening, all consistent with observations at the footprint level. As before, it is clear that even though the overall net load variability has increased, the largest (and most important) impact is on the extreme deltas, which increase dramatically in all hours, but especially during the mornings and afternoons. These are events that may place additional requirements on the ramp and range capability of the balance of generation.

Earlier in Section 4.3.3, the load, wind, solar, and net load profiles for selected extreme delta days were examined to understand the drivers behind the magnitude and timing of large deltas seen at the footprint level. The profile of another such day, January $8^{\text {th }}$, 2006 is shown in Figure 5.9 below. The left plot reveals that the large afternoon ramp in the net load is due to the aggressive drop in wind and solar output around this time. The right plot indicates that a large portion of the wind roll-off is in Wyoming. This creates a sustained afternoon net load ramp event in Wyoming that might be a challenge for Wyoming generation alone to follow - even at that time of day, i.e., during the winter afternoon load rise period when sufficient generation would normally be committed to meet the customary load ramping requirements.
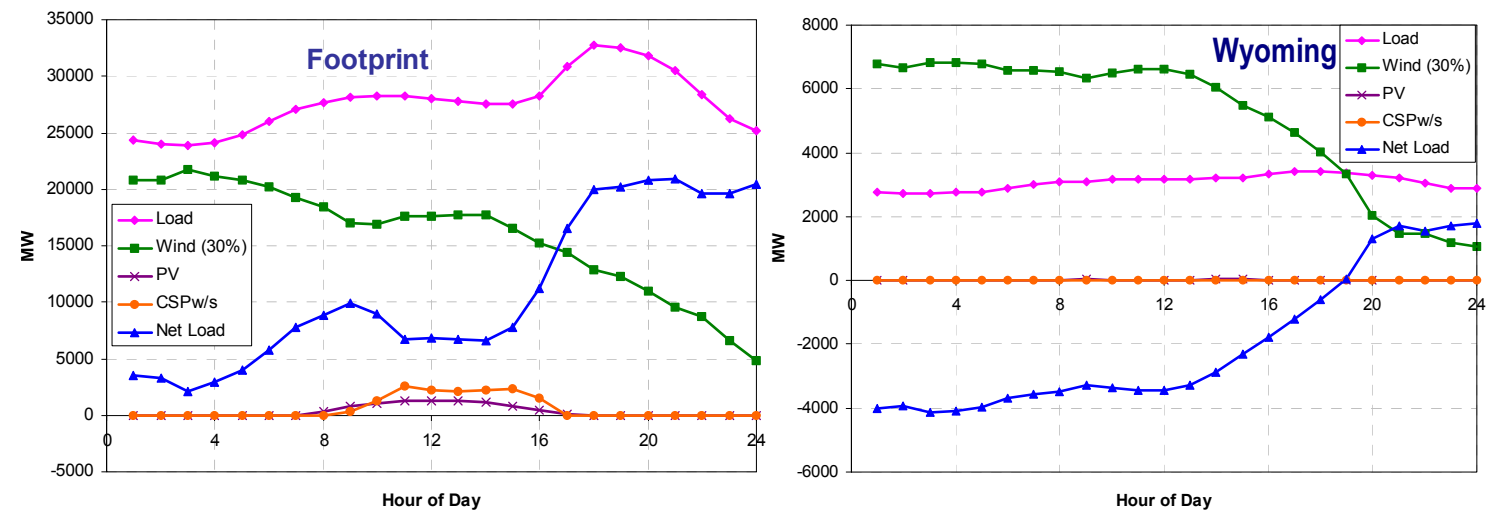

Figure 5.9 Study Footprint and Wyoming Net Load for January $8^{\text {th }}, 2006,30 \%$ Local-Priority Scenario

\subsection{Variability by Time-of-Year}

The timing of the net load deltas (both time-of-day and time-of-year) is just as important as the size of the deltas. A large net load up-ramp in the mid-morning may not be as 
challenging as a large up-ramp in the early morning hours. This is because there is a more flexible mix of generation committed during the morning load-rise hours than during the early morning hours when load is typically low. An examination of when large deltas occur should reveal some of the challenges presented by large penetration of wind and solar during specific times of the year.

Figure 5.10 shows the timing of the extreme positive load deltas in the footprint for 2006. The extreme deltas are presented in $24 \times 12$ surface plots using color intensity to represent the magnitude of the deltas. According to the legend, the areas of the plot that are shaded grey represent daily hours in particular months where the load is ramping down. Areas shaded dark yellow represent daily hours in particular months where the load increases by $3000-4000 \mathrm{MW}$ in an hour.

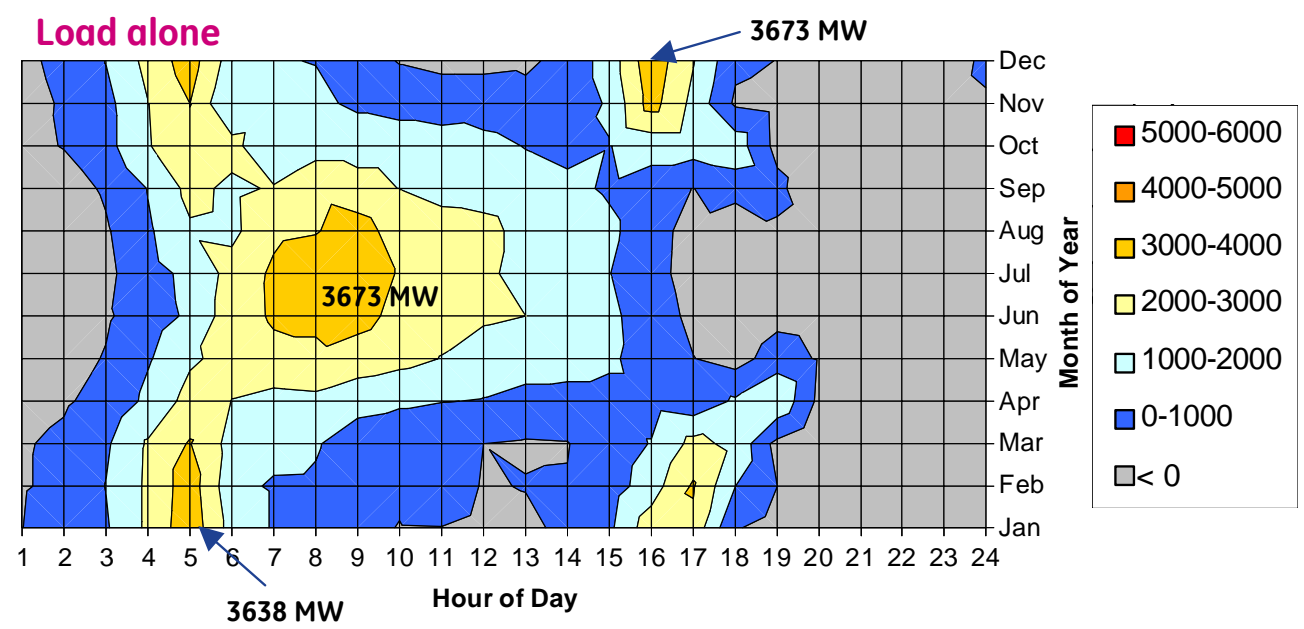

Figure 5.10 Timing of Extreme Load-Alone Positive Deltas in Study Footprint for 2006

This surface plot forms a basis for the comparison of net load deltas in the $10 \%, 20 \%$, and $30 \%$ penetration scenarios. With no additional wind or solar on the system (beyond the baseline), the largest 1-hour load rises, at the footprint level, tend to occur during the summer morning and winter early morning and winter afternoon hours. The largest such ramps, 3,673 MW, are observed in the 8-9 a.m. time frame in July and the 4-5 p.m. hour in December. Across the year, the footprint load is ramping down from about 8 p.m. -1 a.m. and there are practically no load up-ramps greater than 1,000 MW from about 7 p.m. -3 a.m.

Figure 5.11, Figure 5.12, and Figure 5.13, on the next page demonstrate how increasing amounts of wind and solar change the timing and magnitude of the extreme positive deltas across the year. The legend is kept the same as Figure 5.10 for all plots, so the color progression is a good indicator of incremental impact. 


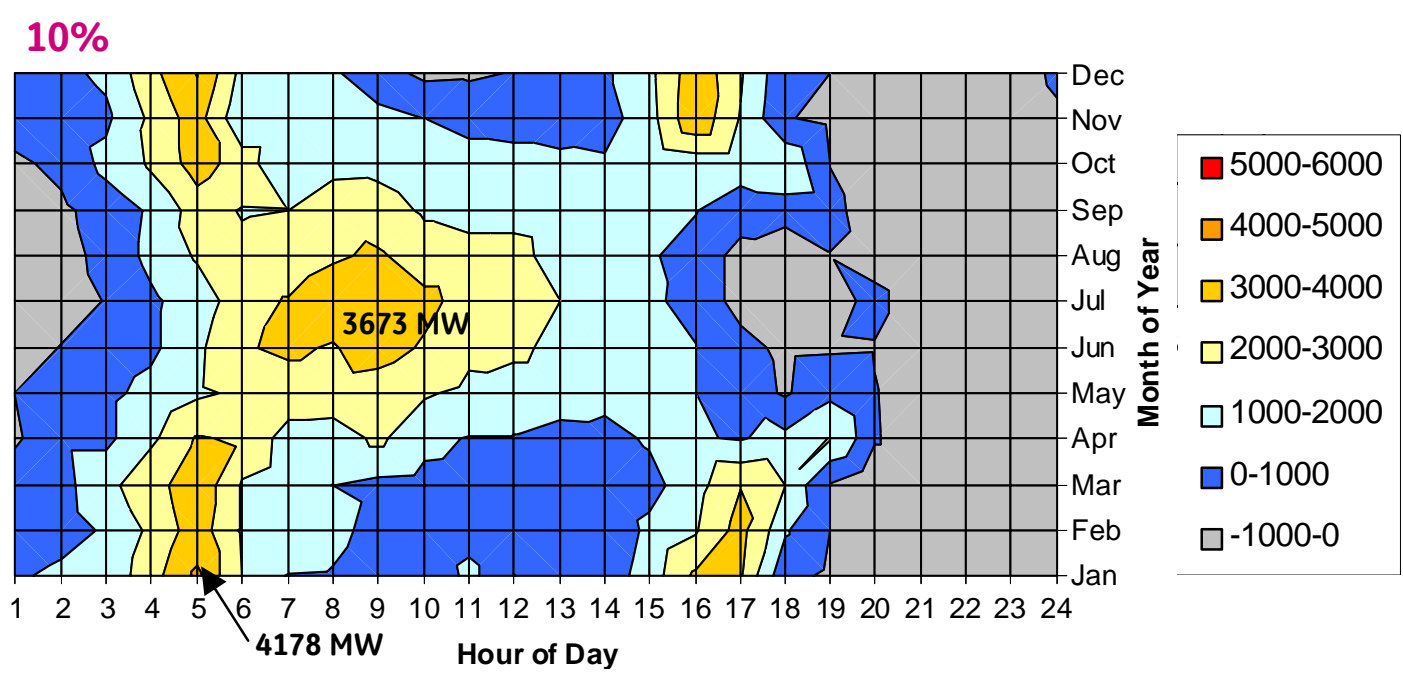

Figure 5.11 Timing of Extreme Net Load Positive Deltas in Study Footprint for 2006, 10\% LP Scenario

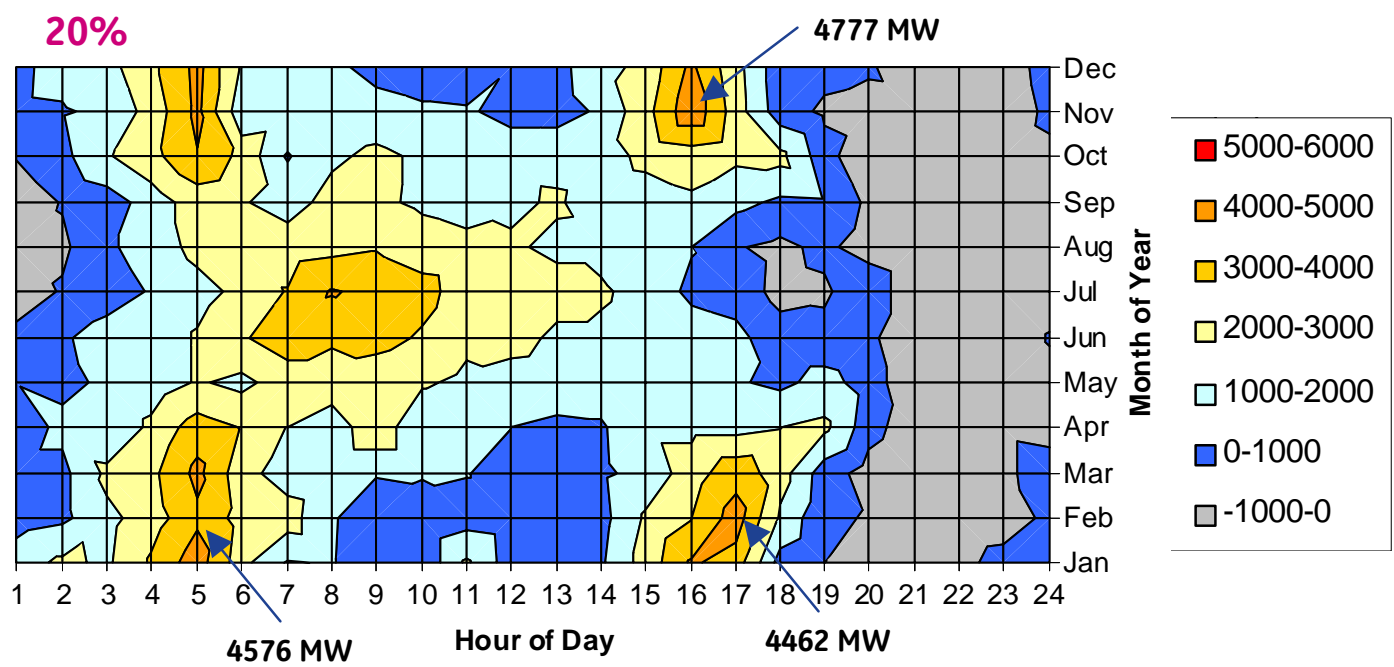

Figure 5.12 Timing of Extreme Net Load Positive Deltas in Study Footprint for 2006, 20\% LP Scenario

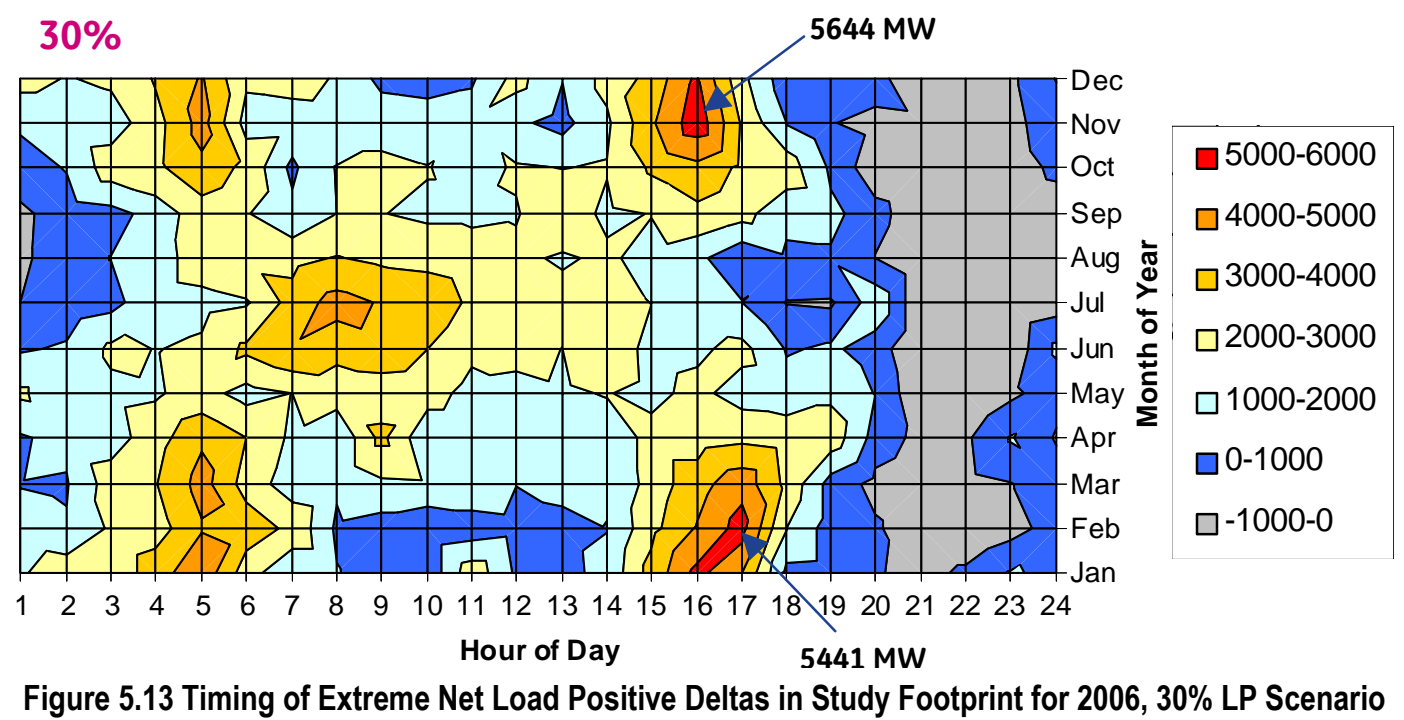


As wind and solar penetration increases within the footprint, there is an overall shift in the timing and number and size of extreme net load deltas. At 30\%, large net load ramps show up in all months, but they tend to be more severe in the winter afternoon. This is consistent with the fact that wind and CSP drop-offs often coincide with the winter afternoon load rise, as shown earlier in Figure 4.32. Another observation is that the grey area, which indicates the down-ramp times, is squeezed down from about 5 hours in the baseline to about 3 hours between 9 p.m. and midnight. At $30 \%$, there is now a greater requirement for ramping up capability during the minimum load hours, (less grey between 1-4 a.m.), than was present with no-wind and solar output.

The surface plots below show the timing of the extreme negative load and net load deltas in the footprint for 2006. Admittedly, down-ramps are less of an operational concern as large wind up-ramps can always be curtailed. Nevertheless, the data shows that even with load alone, there are substantial negative deltas in the late evenings, especially in summer months, driven by the customary load decline. With increasing wind and solar penetration, these extreme down-ramps progressively show up all across the year in the evening hours, and even in the late afternoons. However, even at the $30 \%$ penetration level, the worst net load down-ramp is only $16 \%$ worse that the load-alone down-ramp at that hour. This is entirely consistent with the earlier observations from Figure 5.2 that where there are only a few instances where wind and solar increases the net-load down-ramps beyond the ramps with load alone. Surface plots for other scenarios and areas are included in Appendix C.
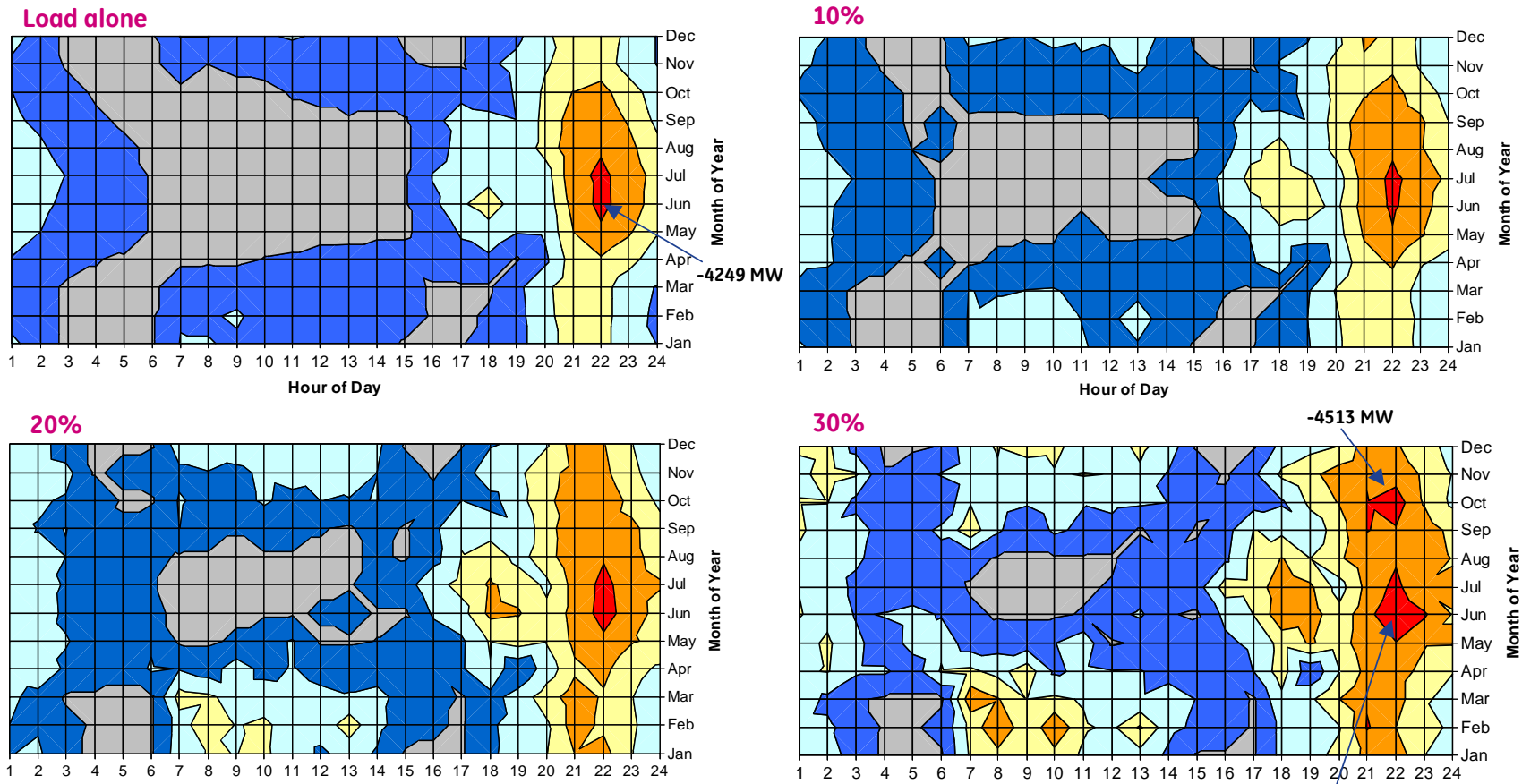

$$
\text { Hour of Day }
$$

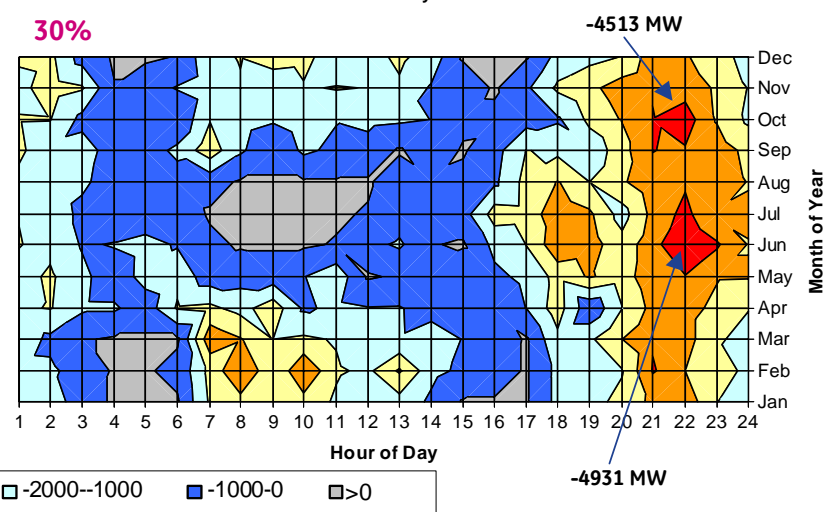

Figure 5.14 Timing of Extreme Net Load Negative Deltas in Study Footprint for 2006 Local-Priority Scenario 


\subsection{Analysis of Extreme Deltas}

Figure 5.15 shows the distribution of 1-hour deltas for the baseline (existing), and net load at the footprint level for the 10\%, 20\%, and 30\% Local-Priority Scenario. The heights of the magenta bars represent the number of hours during 2006 when baseline deltas are in the range given by the bin on the x-axis. Similarly, the dark blue bars represent the number of hours during 2006 when net load deltas in the $30 \%$ case are in the range given by the bin on the x-axis. The top half of the plot shows the overall distribution of net load deltas, and the bottom half focuses on the tails of the distribution so the extreme deltas can be clearly seen. Table 5.1 is a summary of the footprint net load variability.

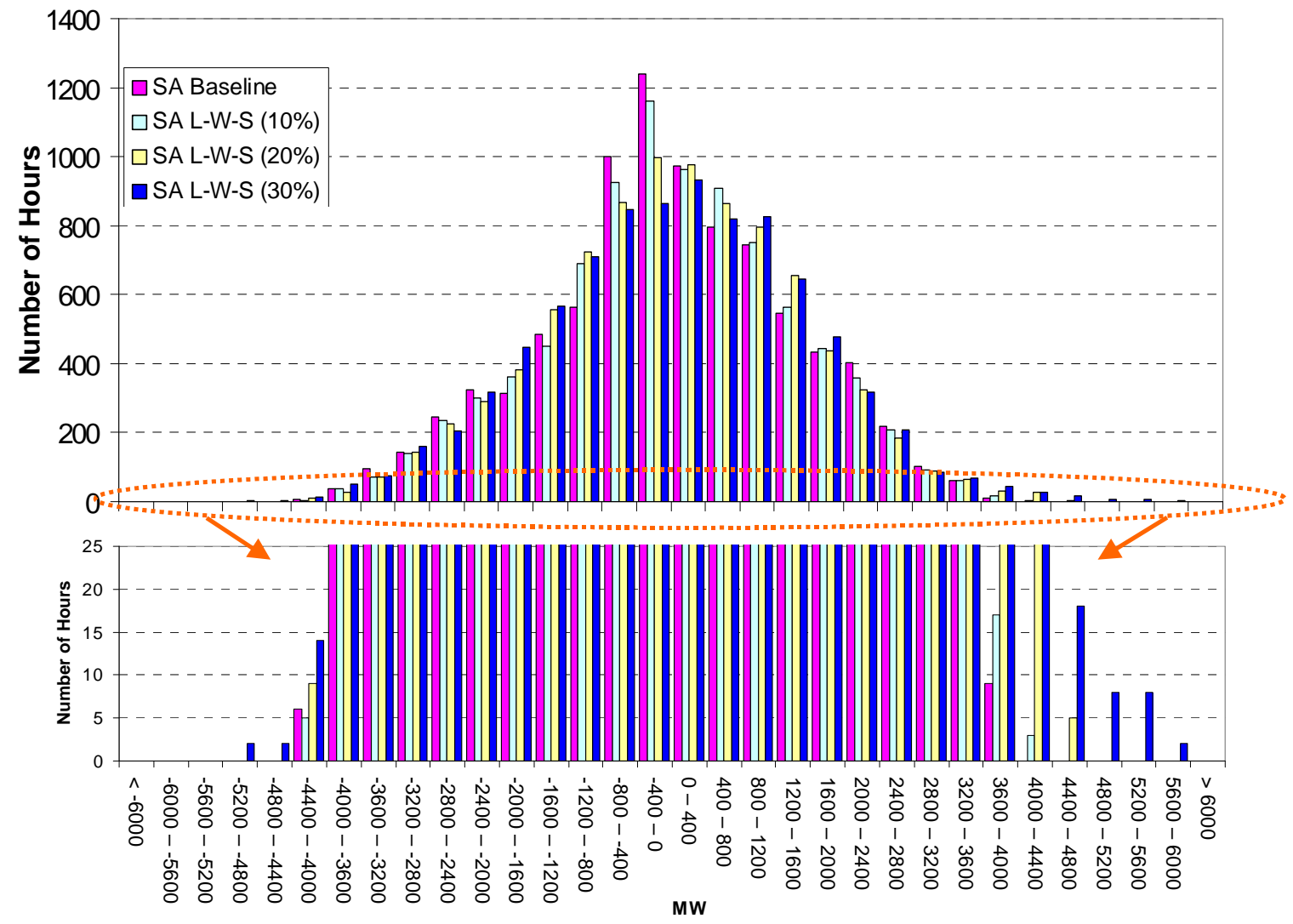

Figure 5.15 2006 Study Area (SA) or Footprint Distribution of Net Load Deltas for Local-Priority Scenario)

Table 5.1 Summary of 2006 Footprint Net Load Variability for the Local-Priority Scenario

\begin{tabular}{|c|c|c|c|c|c|}
\hline Scenario & $\begin{array}{c}\text { Sigma }(\sigma) \text { of Net } \\
\text { Load Deltas } \\
\text { (MW/hr) }\end{array}$ & $\begin{array}{c}\text { Max. Negative Net } \\
\text { Load Delta } \\
\text { (MW/hr) }\end{array}$ & $\begin{array}{l}\text { Max. Positive Net } \\
\text { Load Delta } \\
\text { (MW/hr) }\end{array}$ & $\begin{array}{c}\text { No. Deltas > } \\
3^{*}(\text { load } \sigma) \\
(-/+)\end{array}$ & $\begin{array}{c}\sigma \% \\
\text { Increase with } \\
\text { Wind \& Solar }\end{array}$ \\
\hline Baseline & 1,429 & $-4,282$ & 3,793 & $0 / 0$ & -- \\
\hline $10 \%$ LP Scenario & 1,414 & $-4,195$ & 4,178 & $0 / 0$ & $-1.0 \%$ \\
\hline $20 \%$ LP Scenario & 1,437 & $-4,359$ & 4,777 & $1 / 2$ & $0.6 \%$ \\
\hline $30 \%$ LP Scenario & 1,523 & $-4,931$ & 5,644 & $3 / 28$ & $6.6 \%$ \\
\hline
\end{tabular}


For a particular penetration level, the shape of the overall distribution appears to be approximately normal. For a normal distribution, one would expect $99.7 \%$ of the deltas to be within 3 sigma of the mean. For the $30 \%$ penetration case, $3 \sigma=4,569 \mathrm{MW}$, and from the bottom half of Figure 5.15, one can see that approximately $99 \%$ of the deltas are within 4,400 MW of the mean. The bottom (blown-up) section of Figure 5.15 confirms that there are significantly more extreme net-load deltas beyond the largest baseline delta, especially up-ramps (positive deltas). There are about nine baseline extreme deltas of 3,600 MW or greater, but several times more extreme deltas for the $30 \%$ Local-Priority Scenario-the largest being more than 5,600 MW.

The spread in the distribution of deltas going from the baseline case to $30 \%$ indicates that the net load variability increases with higher wind and solar penetration. The data in Table 5.1 bears this out. However, at the footprint level, the overall increase in variability is not dramatic. The sigma of the deltas (or the measure of variability) actually decreases from the baseline to $10 \%$ penetration, and is $6.6 \%$ greater than the baseline at $30 \%$ penetration. The difference in variability between $10 \%$ and $20 \%$ penetration is significantly less than between $20 \%$ and $30 \%$ penetration.

Power-system operations are predominately focused on managing the extreme events rather than the average system behavior. Table 5.1 also shows that the increase in the number and size of extreme deltas at the footprint level (as penetration goes from baseline to $30 \%$ ) is much more impressive than the increase in overall sigma. At $30 \%$ penetration, the biggest net load up-ramp $(5,644 \mathrm{MW})$ is almost $50 \%$ larger than at the baseline (3,793 MW), and there are many more of these on the tails of the distribution. To better illustrate this point, Figure 5.16 plots the cumulative distribution of the deltas on the tails of the distribution. For a point on the curves, the y-axis gives the number of 1-hour extreme drops or rises in net load that are equal to or larger than the corresponding value on the x-axis. The examples on the plot illustrate this more clearly.

Figure 5.16 makes a great case for load participation in managing the impact of extreme positive delta on operations. Consider the right half of the figure where wind and solar drops increase net load up-ramps. In the baseline case, there are nine up-ramps of 3,400 $\mathrm{MW} / \mathrm{hr}$ or more. Contrast this to the $30 \%$ penetration case, where there are 108 up-ramps of $3400 \mathrm{MW} / \mathrm{hr}$ or more. As you move along the blue trace, the size of the extreme deltas increase, but the frequency also decreases. Instead of carrying reserves to cover all large infrequent events, demand-side participation could alleviate the need for additional resources and ensure reliable and economic power system operation. Further analyses in Chapter 6 and Chapter 7 underscore this point. 


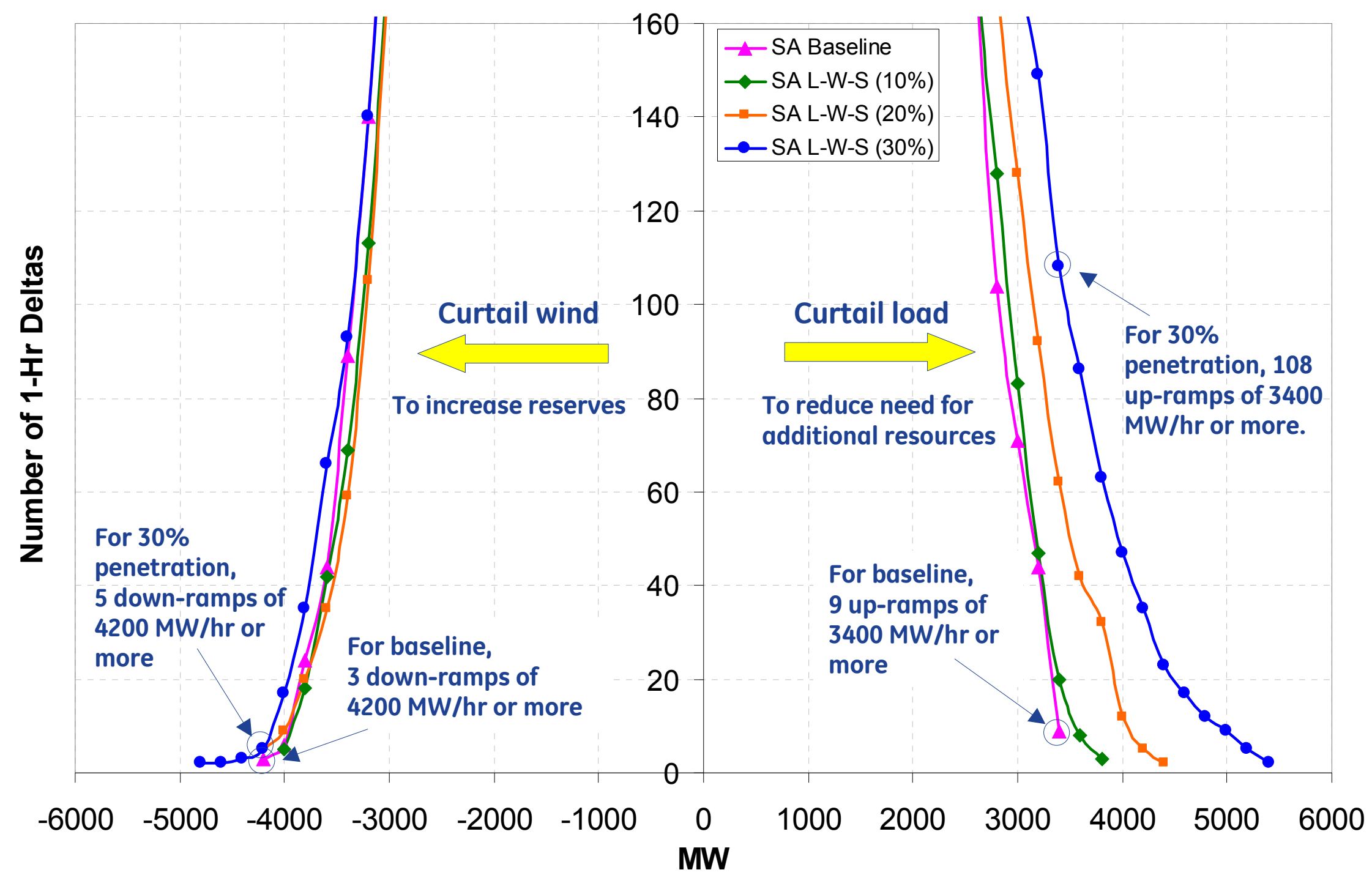

Figure 5.16 Study Area (SA) or Footprint Distribution of Extreme Hourly Net Load Deltas 2006 Local-Priority Scenario 


\subsection{Area-to-Area Variability}

Up to this point, most of the discussion of deltas has focused on the total footprint wind, under the assumption that balancing on a wider area has certain operational merits. This section discusses variability within individual states for the three scenarios and contrasts the experiences at this level with the experiences at the footprint and WECC level. Table 5.2 summarizes the net load statistical variability measures for the states in the study footprint, the total footprint, and WECC-wide for all scenarios.

The first section of Table 5.2 shows sigma of the 1-hour deltas for increasing levels of wind and solar. As discussed in the previous section, variability increases with wind and solar penetration regardless of area or scenario. This is not surprising. What is interesting, however, is the rate at which the variability increases relative to the size of the area and the amount of renewables it contains. Figure 5.17 shows the percent increase in net load variability (over the load-alone case) for the In-Area Scenario.

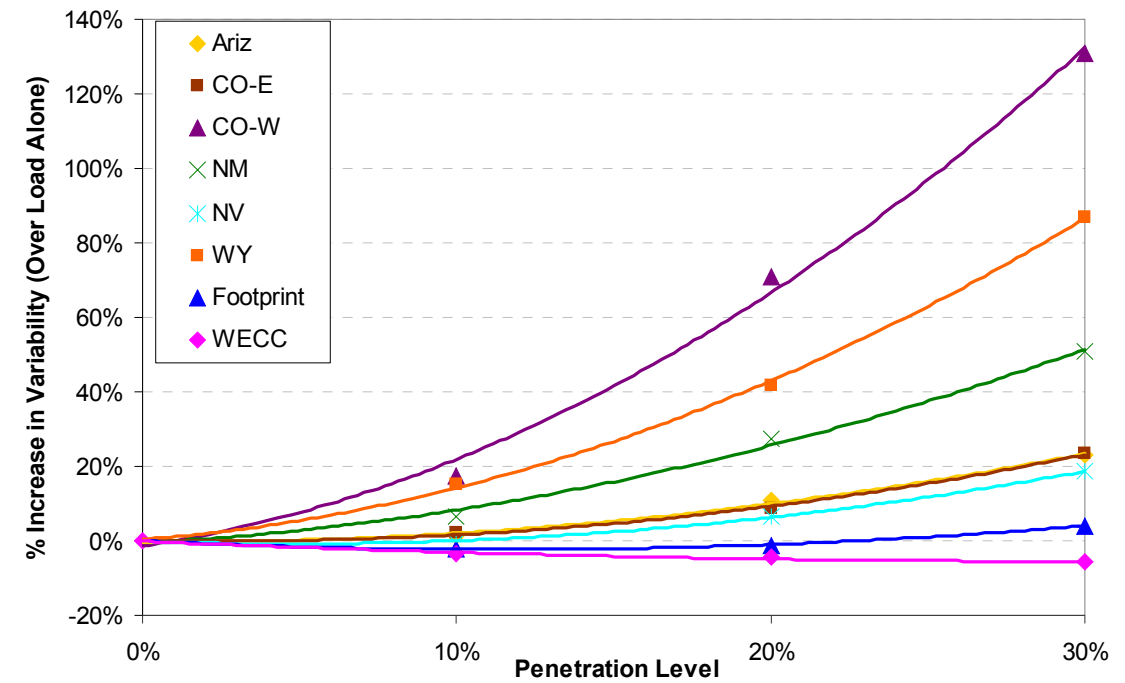

Figure 5.17 Percent Increase in Net Load Delta Sigma in All Areas for In-Area Scenario

The three smallest areas by load (and wind in the In-Area Scenario), Colorado-West, Wyoming and New Mexico, show the largest relative increase in variability with penetration. At the 30\% penetration level, sigma of the 1-hour deltas in Wyoming is $87 \%$ larger than with load-alone. As the area size becomes larger, the variability grows a lot slower. At the footprint level, sigma at $30 \%$ penetration is only $4 \%$ higher than sigma with load-alone. WECC-wide, the variability at $30 \%$ penetration is actually less than the variability with load alone. This suggests that the aggregate load and wind are slightly more in phase WECC-wide than within the footprint alone.

The fact that the net load variability at the footprint and WECC level does not significantly increase with penetration speaks volumes about the impact of temporal averaging, geographic diversity and wide-area aggregation on variability. 
Table 5.2 Summary Statistics for In-Footprint States, Total Footprint and WECC for All Scenarios in 2006

\begin{tabular}{|c|c|c|c|c|c|c|c|c|c|c|c|c|c|c|c|c|c|c|c|c|c|c|c|c|}
\hline \multirow{2}{*}{$\begin{array}{r}\text { Area: } \\
\text { Scenario } \\
\end{array}$} & \multicolumn{3}{|c|}{ Arizona } & \multicolumn{3}{|c|}{ Colorado East } & \multicolumn{3}{|c|}{ Colorado West } & \multicolumn{3}{|c|}{ New Mexico } & \multicolumn{3}{|c|}{ Nevada } & \multicolumn{3}{|c|}{ Wyoming } & \multicolumn{3}{|c|}{ Footprint } & \multicolumn{3}{|c|}{ WECC } \\
\hline & IA & LP & MP & IA & LP & MP & IA & LP & MP & IA & $\mathrm{LP}$ & MIP & IA & LP & MP & IA & LP & MP & IA & LP & MP & IA & LP & MP \\
\hline \multicolumn{25}{|l|}{ Sigma (MW) } \\
\hline Load-alone & 584 & 584 & 584 & 367 & 367 & 367 & 37 & 37 & 37 & 152 & 152 & 152 & 312 & 312 & 312 & 87 & 87 & 87 & 1433 & 1433 & 1433 & 4384 & 4384 & 4384 \\
\hline Baseline (Existing) & 583 & 583 & 583 & 374 & 374 & 374 & 37 & 37 & 37 & 158 & 158 & 158 & 311 & 311 & 311 & 101 & 101 & 101 & 1429 & 1429 & 1429 & 4226 & 4392 & 4392 \\
\hline $10 \%$ In FP Scenario & 591 & 587 & 573 & 375 & 377 & 376 & 44 & 41 & 38 & 162 & 170 & 192 & 311 & 315 & 311 & 101 & 118 & 188 & 1401 & 1414 & 1426 & 4229 & 4240 & 4248 \\
\hline $20 \%$ In FP Scenario & 647 & 613 & 587 & 399 & 401 & 379 & 64 & 57 & 38 & 194 & 203 & 228 & 333 & 331 & 313 & 124 & 212 & 434 & 1417 & 1437 & 1480 & 4187 & 4209 & 4230 \\
\hline \multirow[t]{2}{*}{$30 \%$ In FP Scenario } & 719 & 657 & 616 & 453 & 427 & 380 & 86 & 73 & 40 & 230 & 240 & 288 & 370 & 352 & 317 & 164 & 366 & 658 & 1491 & 1523 & 1594 & 4134 & 4153 & 4182 \\
\hline & \multicolumn{3}{|c|}{ Ariz } & \multicolumn{3}{|c|}{$\mathrm{CO}-\mathrm{E}$} & \multicolumn{3}{|c|}{$\mathrm{CO}-\mathrm{W}$} & \multicolumn{3}{|c|}{ NM } & \multicolumn{3}{|c|}{ NV } & \multicolumn{3}{|c|}{ WY } & \multicolumn{3}{|c|}{ In Foot Print } & \multicolumn{3}{|c|}{ WECC } \\
\hline Max Neg Delta (MW) & IA & $\mathrm{LP}$ & $\mathrm{MP}$ & IA & $\mathrm{LP}$ & $\mathrm{MP}$ & IA & $\mathrm{LP}$ & $\mathrm{MP}$ & IA & LP & $\mathrm{MP}$ & IA & $\mathrm{LP}$ & MP & IA & $L P$ & $\mathrm{MP}$ & IA & LP & $\mathrm{MP}$ & IA & LP & MP \\
\hline Load-alone & -2088 & -2088 & -2088 & -1340 & -1340 & -1340 & -120 & -120 & -120 & -515 & -515 & -515 & -1201 & -1201 & -1201 & -316 & -316 & -316 & -4250 & -4250 & -4250 & -13435 & -13435 & -13435 \\
\hline Baseline (Existing) & -2087 & -2087 & -2087 & -1337 & -1337 & -1337 & -120 & -120 & -120 & -522 & -522 & -522 & -1201 & -1201 & -1201 & -529 & -492 & -492 & -4282 & -4282 & -4282 & -13385 & -13385 & -13385 \\
\hline $10 \%$ In FP Scenario & -2184 & -1886 & -1941 & -1321 & -1317 & -1323 & -199 & -151 & -128 & -514 & -571 & -874 & -1660 & -1342 & -1156 & -508 & -547 & -1359 & -4053 & -4195 & -4434 & -12709 & -12861 & -13157 \\
\hline $20 \%$ In FP Scenario & -2492 & -2115 & -1867 & -1624 & -1627 & -1306 & -312 & -255 & -135 & -869 & -982 & -1186 & -1252 & -1368 & -1260 & -650 & -1412 & -3204 & -4168 & -4359 & -5511 & -12386 & -12396 & -12840 \\
\hline $30 \%$ In FP Scenario & -3043 & -2425 & -2354 & -1913 & -1813 & -1298 & -460 & -404 & -135 & -1146 & -1242 & -1427 & -1407 & -1379 & -1327 & -1086 & -2418 & -4798 & -4534 & -4931 & -6729 & -12260 & -12532 & -12552 \\
\hline & & Ariz & & & CO-E & & & $\mathrm{CO}-\mathrm{W}$ & & & NM & & & NV & & & WY & & & Foot Pri & & & WECC & \\
\hline Max Pos Delta (MW) & $\mathrm{A}$ & LP & $\mathrm{MP}$ & $\mathrm{A}$ & $\mathrm{LP}$ & $\mathrm{MP}$ & $\mathrm{IA}$ & $\mathrm{LP}$ & $\mathrm{MP}$ & $\mathrm{A}$ & $\mathrm{LP}$ & MP & $\mathrm{A}$ & $\mathrm{LP}$ & $\mathrm{MP}$ & IA & $\mathrm{LP}$ & $\mathrm{MP}$ & $\mathrm{A}$ & $\mathrm{LP}$ & $\mathrm{MP}$ & $\mathrm{A}$ & LP & MP \\
\hline Load-alone & 1601 & 1601 & 1601 & 1172 & 1172 & 1172 & 130 & 130 & 130 & 636 & 636 & 636 & 1234 & 1234 & 1234 & 505 & 505 & 505 & 3674 & 3674 & 3674 & 10715 & 10715 & 10715 \\
\hline Baseline (Existing) & 1600 & 1600 & 1600 & 1692 & 1257 & 1257 & 130 & 130 & 130 & 605 & 605 & 605 & 1229 & 1229 & 1229 & 435 & 435 & 435 & 3793 & 3793 & 3793 & 10896 & 10896 & 10896 \\
\hline $10 \%$ In FP Scenario & 1858 & 1852 & 1642 & 1479 & 1280 & 1266 & 202 & 157 & 131 & 616 & 626 & 887 & 1185 & 1170 & 1200 & 429 & 539 & 1290 & 4178 & 4178 & 3898 & 11299 & 10959 & 10983 \\
\hline $20 \%$ In FP Scenario & 2732 & 2295 & 2165 & 1416 & 1445 & 1281 & 351 & 281 & 131 & 875 & 957 & 1120 & 1492 & 1394 & 1176 & 506 & 1537 & 3085 & 4853 & 4777 & 4499 & 11661 & 11366 & 12202 \\
\hline $30 \%$ In FP Scenario & 3483 & 2771 & 2564 & 2097 & 1834 & 1364 & 512 & 373 & 152 & 1047 & 1129 & 1355 & 1711 & 1573 & 1383 & 809 & 2646 & 4487 & 5820 & 5644 & 5864 & 15259 & 14383 & 14318 \\
\hline & & Ariz & & & CO-E & & & $\mathrm{CO}-\mathrm{W}$ & & & $\mathrm{NM}$ & & & NV & & & WY & & & Foot Pri & & & WECC & \\
\hline No. Drops $>3^{*}$ Ld Sigma & IA & $\mathrm{LP}$ & $\mathrm{MP}$ & IA & $\mathrm{LP}$ & MP & IA & $\mathrm{LP}$ & $\mathrm{MP}$ & $\mathrm{IA}_{\mathrm{A}}$ & $\mathrm{LP}$ & MP & IA & $\mathrm{LP}$ & $\mathrm{MP}$ & IA & $\mathrm{LP}$ & $\mathrm{MP}$ & IA & LP & $\mathrm{MP}$ & IA & LP & MP \\
\hline Load-alone & 2 & 2 & 2 & 4 & 4 & 4 & 10 & 10 & 10 & 5 & 5 & 5 & 6 & 6 & 6 & 5 & 5 & 5 & 0 & 0 & 0 & 1 & 1 & \\
\hline Baseline (Existing) & 2 & 2 & 2 & 5 & 5 & 5 & 10 & 10 & 10 & 5 & 5 & 5 & 6 & 6 & 6 & 39 & 38 & 38 & 0 & 0 & 0 & 1 & 0 & 1 \\
\hline $10 \%$ In FP Scenario & 3 & 2 & 1 & 11 & 13 & 13 & 38 & 24 & 10 & 15 & 27 & 73 & 8 & 8 & 6 & 35 & 98 & 367 & 0 & 0 & 1 & 0 & 0 & 0 \\
\hline $20 \%$ In FP Scenario & 23 & 7 & 3 & 24 & 25 & 14 & 218 & 133 & 11 & 72 & 90 & 143 & 17 & 12 & 6 & 112 & 469 & 1164 & 0 & 1 & 9 & 0 & 0 & 0 \\
\hline $30 \%$ In FP Scenario & 52 & 20 & 13 & 45 & 31 & 13 & 485 & 310 & 11 & 144 & 176 & 300 & 34 & 16 & 6 & 278 & 1009 & 1492 & 3 & 3 & 15 & 0 & 0 & 0 \\
\hline & & Ariz & & & $\mathrm{CO}-\mathrm{E}$ & & & $\mathrm{CO}-\mathrm{W}$ & & & $\mathrm{NM}$ & & & NV & & & WYCE & & & Foot Pri & & & WECC & \\
\hline No. Rises $>3^{*}$ Ld Sigma & IA & LP & $\mathrm{MP}$ & $\mathrm{A}$ & $\mathrm{LP}$ & $\mathrm{MP}$ & $\mathrm{IA}$ & $\mathrm{LP}$ & $\mathrm{MP}$ & $\mathrm{A}$ & $\mathrm{LP}$ & MP & $\mathrm{A}$ & LP & $\mathrm{MP}$ & IA & LP & $\mathrm{MP}$ & $\mathrm{A}$ & LP & $\mathrm{MP}$ & IA & LP & MP \\
\hline Load-alone & 0 & 0 & 0 & 4 & 4 & 4 & 8 & 8 & 8 & 11 & 11 & 11 & 3 & 3 & 3 & 45 & 45 & 45 & 0 & 0 & 0 & 0 & 0 & 0 \\
\hline Baseline (Existing) & 0 & 0 & 0 & 3 & 2 & 2 & 8 & 8 & 8 & 13 & 13 & 13 & 3 & 3 & 3 & 65 & 65 & 65 & 0 & 0 & 0 & 0 & 0 & 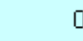 \\
\hline $10 \%$ In FP Scenario & 3 & 3 & 0 & 2 & 3 & 2 & 38 & 26 & 10 & 26 & 37 & 83 & 7 & 8 & 6 & 69 & 118 & 417 & 0 & 0 & 0 & 0 & 0 & 0 \\
\hline $20 \%$ In FP Scenario & 30 & 12 & 7 & 22 & 21 & 7 & 249 & 178 & 11 & 84 & 100 & 162 & 22 & 22 & 7 & 140 & 530 & 1198 & 10 & 2 & 5 & 0 & 0 & \\
\hline $30 \%$ In FP Scenario & 63 & 32 & 32 & 81 & 56 & 12 & 491 & 340 & 35 & 168 & 187 & 328 & 67 & 43 & 10 & 326 & 1061 & 1555 & 29 & 28 & 26 & 4 & 3 & \\
\hline
\end{tabular}


Firstly, note that the plot in Figure 5.17 covers an entire year's worth of operation. Variability that might manifest itself over a shorter period, such as the summer morning load rise, is masked by the temporal averaging over the 8,760 data points. Secondly, geographic diversity in load and renewable resources ensures that the aggregate footprint and WECC-wide variability is much less than individual smaller areas. Thirdly, even with temporal and spatial averaging, little or no increase in overall variability does not eliminate the extreme deltas on the tails of the distribution, which are the hours that tend to create the most operational challenges.

For the Local-Priority and Mega-Project Scenarios, the impact of wide-area aggregation is even clearer for Wyoming. For Local-Priority and Mega-Project at the $30 \%$ penetration level, sigma of the 1-hour deltas in Wyoming is $366 \mathrm{MW}$ and $658 \mathrm{MW}$ respectively. These are $318 \%$ and $652 \%$ larger than with load-alone (87 MW). However, at the footprint level, the growth is only $6 \%$ and $11 \%$ respectively.

The second and third sections of Table 5.2 list the largest negative and positive deltas in each area for all scenarios. A similar observation can be made about these extreme deltas. As the penetration of wind and solar increases, the magnitude of the extreme delta grows much faster in small areas than in large areas and on an aggregated basis. Figure 5.18 shows the percent increase in net load extreme deltas (over the load-alone case) for the In-Area Scenario.
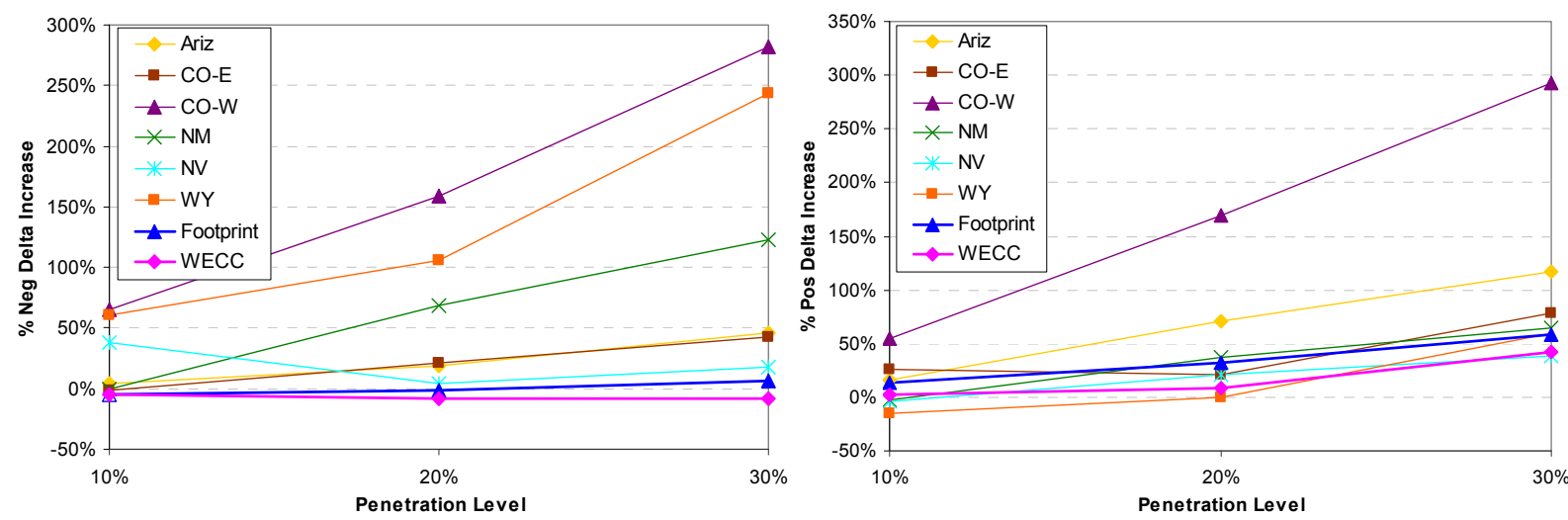

Figure 5.18 Percent Increase in Net Load Negative and Positive Deltas in All Areas for In-Area Scenario

In general, the smaller the area, the more the extreme deltas increase with larger amounts of wind and solar on the system. In Colorado-West for example, the largest drop in net load with $30 \%$ penetration is $460 \mathrm{MW}, 282 \%$ higher than with load alone (120 MW). By contrast, in Arizona, the largest drop in net load with 30\% penetration (3043 MW) is $46 \%$ higher than with load alone (2088 MW). At the aggregate footprint level, the increase in the largest net load drop at $30 \%$ penetration is only $7 \%$. 
Drops in net load are caused by sudden increases in wind and solar. What is more operationally challenging are sudden drops in wind and solar that lead to increases in net load or positive deltas. The second chart in Figure 5.18 shows that large increases in net load are also mitigated over wide areas, though not as much as large drops. Previous analysis has demonstrated that there are many more extreme positive deltas than negative ones, and that they tend to be larger. This chart also suggests that large wind and solar drops tend to be more coincident across wide areas than large rises. This is consistent with earlier observations that wind and CSP drop-offs often coincide with the winter late afternoon load rise, to create large net load up-ramps in those hours.

Therefore, in a large area and at the footprint level, the reduction in the growth of the largest positive delta is not as dramatic. In Colorado-West, the largest rise in net load with $30 \%$ penetration ( $512 \mathrm{MW}$ ) is $292 \%$ higher than with load alone (130 MW).

However, at the aggregate footprint level, the increase in the largest net load rise at $30 \%$ penetration is still a robust $58 \%$. It is interesting to note that in this case, the largest area, Arizona, has the second largest rate of increase in positive net load deltas. This is due to the relatively large amount of CSP with storage in Arizona that contributes to increased positive net load deltas when it rolls off in the late afternoon. Nevertheless, data from this scenario and the other two confirm the fact that extreme drops and rises in net load are mitigated over wide areas.

The last two sections of Table 5.2 list the number of negative and positive deltas larger than a certain value in each area for all scenarios. In this case, the value is defined as three times the sigma of the load delta ( $3^{*}$ load sigma). Since the load is the same for each penetration level, $3^{*}$ load sigma defines the default level of variability, and serves as a yardstick to measure how much wind and solar increase the number of extreme deltas. Once again, the data highlights the fact that smaller areas have more extreme deltas than larger ones, and as penetration increases, the number grows faster. For the In-Area Scenario, Colorado-West has 491 large net load up-ramps at 30\% penetration, but at the footprint level, there are only 29 instances where wind and solar increase the net load up-ramps beyond those experienced with load alone, and near zero at the WECC-wide level.

Finally, to summarize the case for wide-area aggregation, Figure 5.19 plots the duration of all the net load deltas for the 30\% Local-Priority Scenario. The chart includes a blowup of the area around the largest positive deltas and the largest negative deltas. The top chart in Figure 5.19 shows the overall duration of the deltas for the year. At the footprint level, the 1-hour net load rises are at least 2,000 MW for 10\% of the year and the 1-hour drops are 2,000 MW or more for $10 \%$ of the year. The plot appears to roughly symmetrical, but with slightly more positive deltas than negative ones (i.e., crossover is not at $50 \%$ ).

The bottom left inset shows the top $1 \%$ of positive deltas in each area. At the footprint level, net load positive deltas are $4,800 \mathrm{MW}$ or more for $0.2 \%$ of the year (175 hours), 
whereas in Arizona, net load positive deltas are 2,100 MW or more for $0.2 \%$ of the year. The key observation, however, is that the maximum positive deltas in each area do not occur simultaneously across the footprint. If they did, the maximum 1-hour rise in aggregate net load would be 10,300 MW. Even though more than half the wind and solar in this scenario is in Arizona and Wyoming, geographic diversity in the output ensures that the observed maximum 1-hour rise at the footprint level is 5,644 MW, 45\% less than the maximum possible simultaneous 1-hour rise. The bottom right inset tells a similar story for the negative deltas. At the footprint level, the largest 1-hour net load drop is 4,931 MW. If all the largest drops in each area across the footprint coincided, the aggregate 1-hour drop would be 9,682 MW. Geographic diversity over the footprint ensures that this is never the case in the 2006 data. These observations are consistent with earlier observations in this chapter and analysis in Chapter 6, Operational Analysis, which shows that balancing over a wider area leads to greater diversity, less relative variability and improved operations.

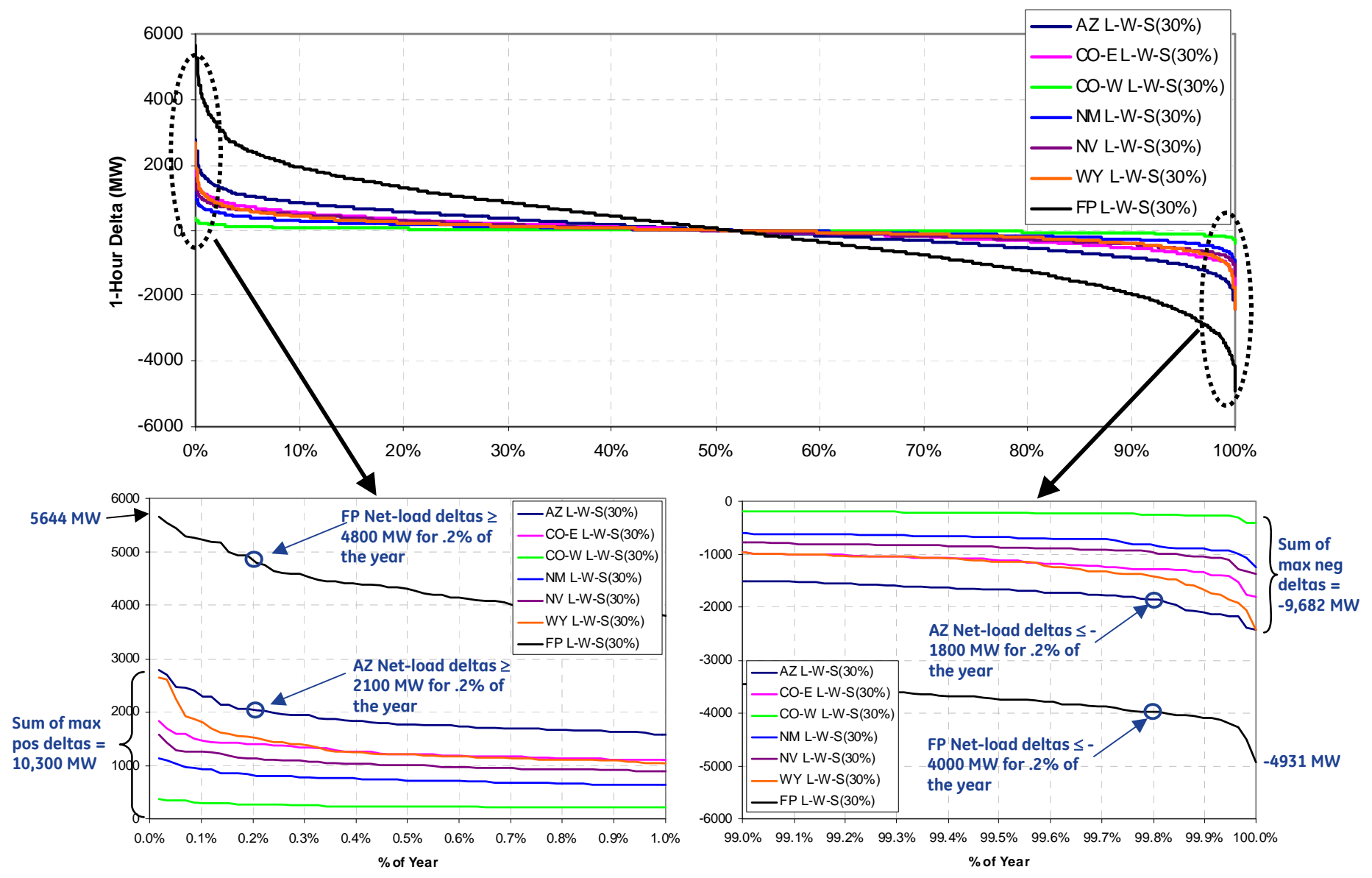

Figure 5.19 2006 Duration of Extreme Hourly Net Load Deltas in All Areas for 30\% Wind Local-Priority Scenario 


\subsection{Wind Forecast Error Analysis}

Day-ahead predictability of net load is important to the unit commitment process. Inaccuracies (large forecast errors) may compromise reliability, increase operating costs, and require greater ancillary service procurement.

For the purposes of this study, "forecast error" is defined as day-ahead forecasted wind generation minus actual wind generation. Therefore, positive forecast error (forecasted quantity greater than actual) is defined as over-forecast and negative forecast error (forecasted quantity less than actual) is defined as under-forecast. Over-forecasting load may lead to more generation being committed than needed (which has potential economic consequences), while under-forecasting load may lead to under-commitment, which is a potential reliability problem. From a system operation point of view, net load under-forecasting is a more serious issue. Net load under-forecast errors are driven by wind over-forecast errors. Therefore, positive wind forecast errors (times when wind is expected to show up and it does not) are more critical in this study.

Two statistical measures are normally used to characterize forecast accuracy. They are:

- Mean Absolute Error (MAE) - measures the average magnitude of the forecast errors; mean of the absolute value of the errors.

- Root Mean Square Error (RMSE) - characterizes forecast error by measuring the "average" of the square of the deviations. RMSE will always be larger than MAE will because it penalizes large deviations more.

Figure 5.20 shows the distribution of wind generation forecast errors for the total footprint during 2006, for the 10\%, 20\%, and 30\% Local-Priority (LP) Scenarios.

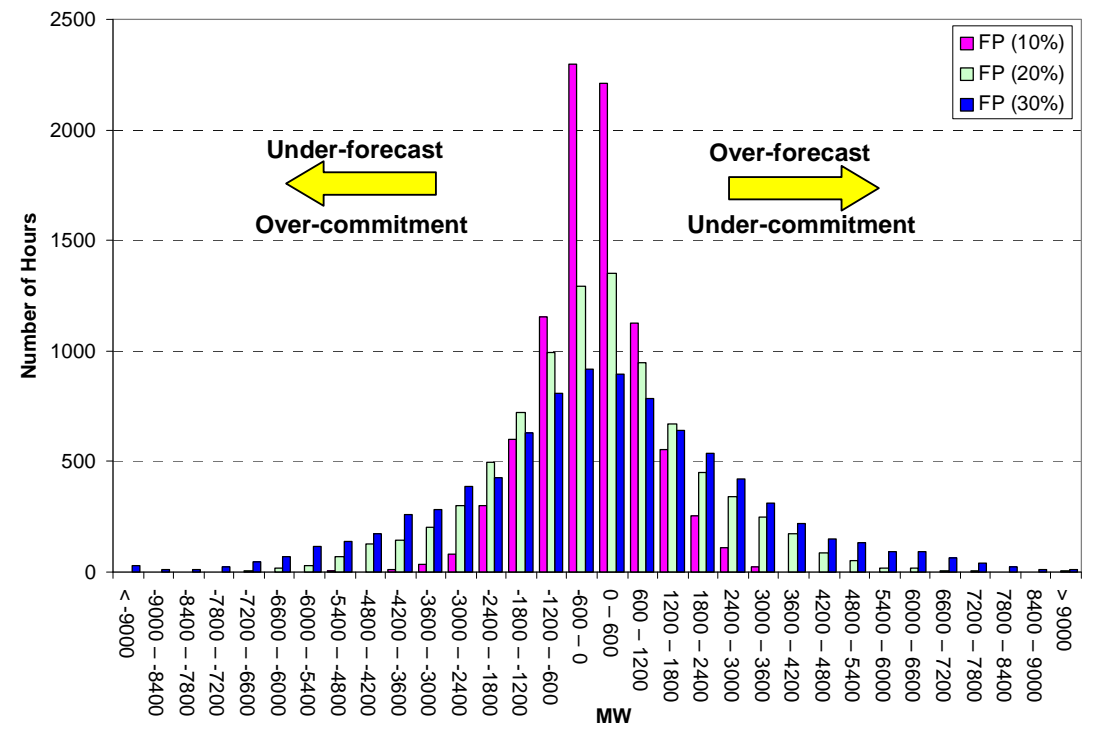

Figure 5.20 Distribution of Footprint Day-Ahead Wind Forecast Errors for 2006 10\%, 20\%, 30\% LP Scenario 
The distribution of forecast errors appears to quite symmetric, meaning that in the given dataset there is a general tendency to over-forecast as much as under-forecast wind output. As wind penetration increases, the number and size of large forecast errors also increase. On both tails, there is a significant increase in large errors going from $10 \%$ penetration to $30 \%$ penetration. At $10 \%$ penetration, there are no over-forecast errors greater than 4,300 MW ( $3^{*}$ load sigma), but at 30\% penetration there are 587 such errors. The largest over-forecast error at $30 \%$ penetration is $11,771 \mathrm{MW}$, compared with 3,603 MW at $10 \%$.

To place these forecast errors in context, Figure 5.21 plots the wind forecast errors versus load level within the study footprint for the 30\% Local-Priority Scenario. The largest forecast error is about $38 \%$ of the actual load in that hour. Similarly, Figure 5.22 is a scatter plot of wind forecast errors versus wind, and wind forecast errors versus net load for the study footprint 30\% Local-Priority Scenario.

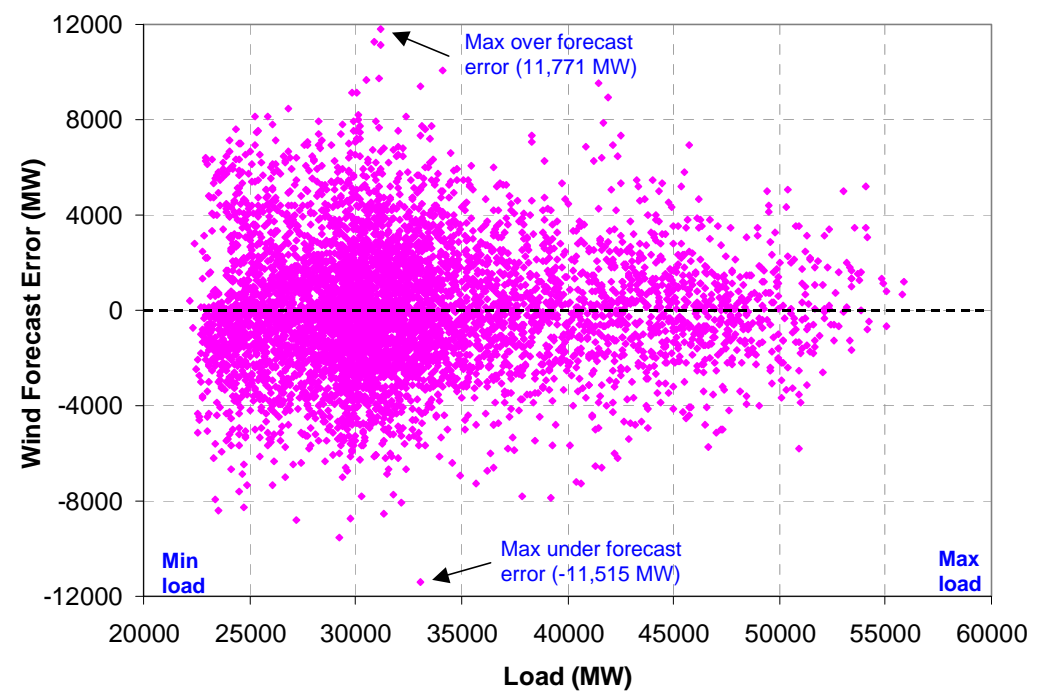

Figure 5.21 Scatter Plot of Wind Forecast Errors vs. Load for Study Footprint 30\% LP Scenario 

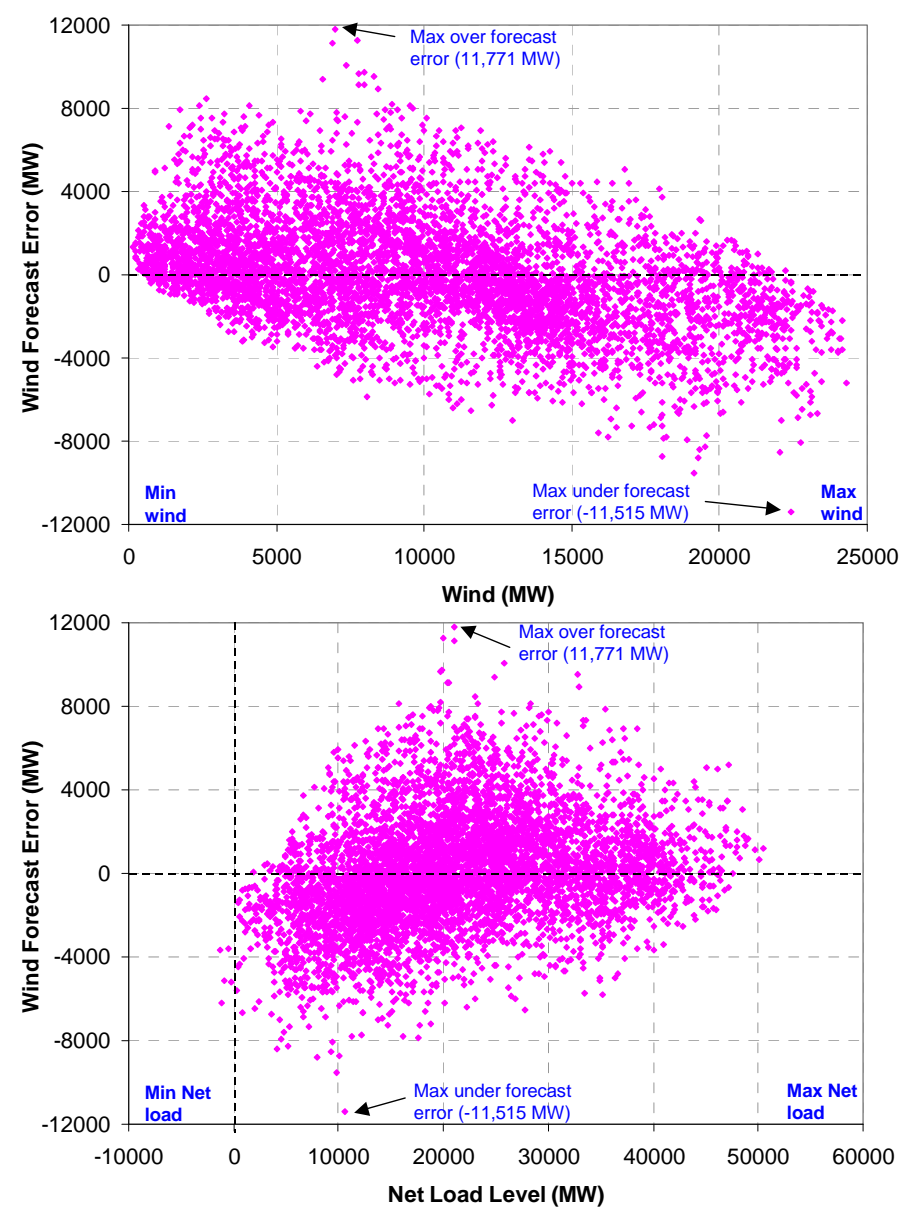

Figure 5.22 Scatter Plot of Wind Forecast Errors vs. Wind and Net Load for Study Footprint 30\% LP Scenario

The scatter plots confirm that the wind forecast error is generally greater in the midrange of the wind output, which is when the wind output is most volatile. Wind underforecast errors are highest when net load is low (such as early morning hours) and overforecast errors are highest in the net load mid-range.

As we saw in the variability analysis, the ability of an area to absorb large forecast errors improves as the area size gets larger. Figure 5.23 plots the duration of wind forecast errors for each individual area in the study footprint and for the total footprint for the $30 \%$ Local-Priority Scenario. Table 5.3 shows a summary of the wind forecast errors in each area, at the footprint level, and all of WECC for the 30\% Local-Priority Scenario. 


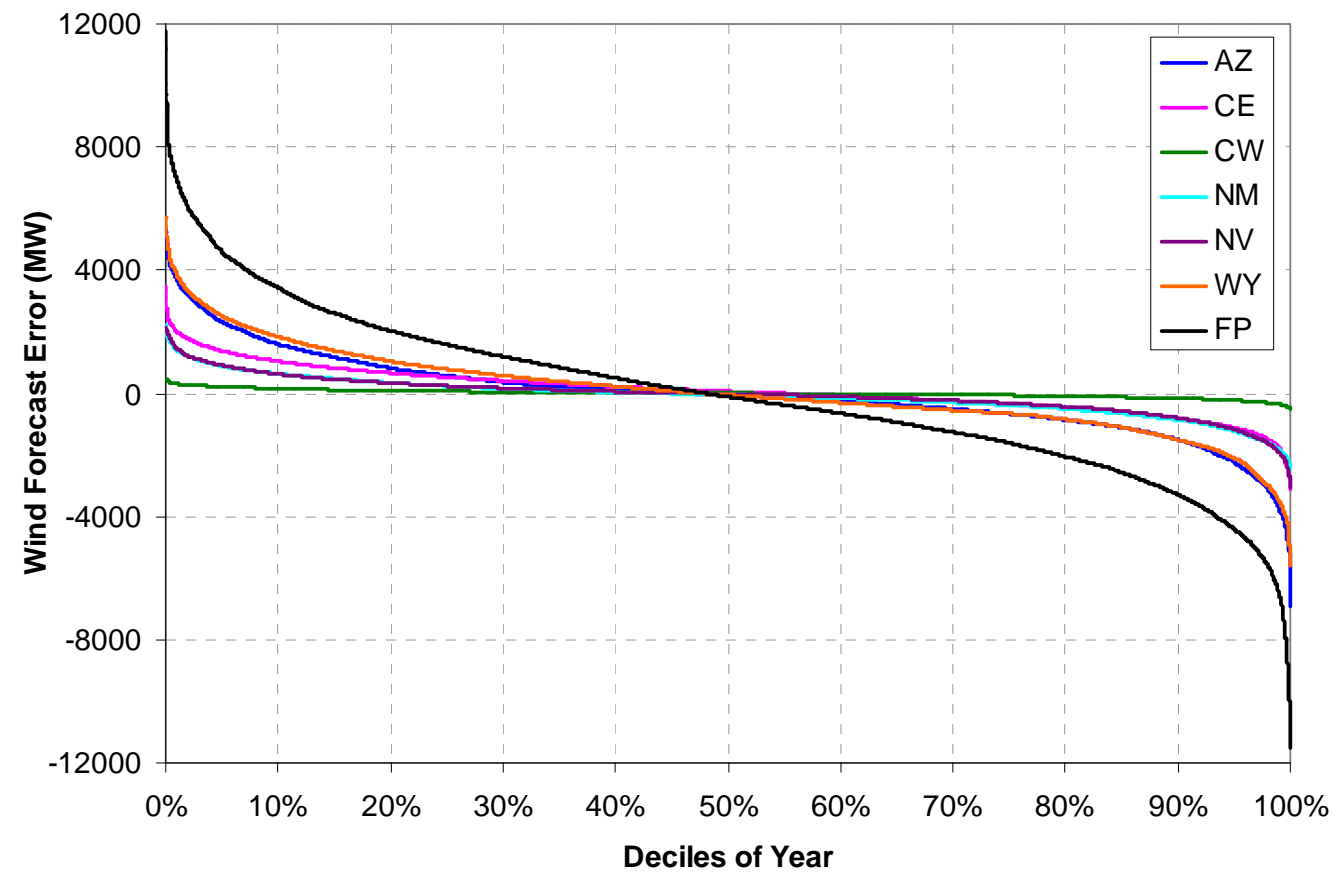

Figure 5.23 Duration of Day-Ahead Wind Forecast Errors for Areas and Total Footprint, 2006 30\% LP Scenario

Table 5.3 Summary of Forecast Errors by Area, 30\% Local-Priority Scenario

\begin{tabular}{|c|c|c|c|c|c|c|c|c|c|}
\hline & $\begin{array}{c}\text { Installed } \\
\text { MWW }\end{array}$ & $\begin{array}{l}\text { MAE } \\
\text { MW }\end{array}$ & $\begin{array}{c}\text { MAE } \\
\%\end{array}$ & $\begin{array}{c}\text { RHSE } \\
\text { MW }\end{array}$ & $\begin{array}{c}\text { RMSE } \\
\%\end{array}$ & $\begin{array}{c}\text { Max Neg Err } \\
\text { MW }\end{array}$ & $\begin{array}{c}\text { Max Neg Err } \\
\%\end{array}$ & $\begin{array}{c}\text { Max Pos Err } \\
\text { MW }\end{array}$ & $\begin{array}{c}\text { Max Pos Err } \\
\%\end{array}$ \\
\hline$A Z$ & 7,710 & 934 & $12.1 \%$ & 1,333 & $17.3 \%$ & $-6,909$ & $-89.6 \%$ & 5,357 & $69.5 \%$ \\
\hline CE & 4,650 & 570 & $12.3 \%$ & 756 & $16.3 \%$ & $-3,102$ & $-66.7 \%$ & 3,471 & $74.6 \%$ \\
\hline CW & 570 & 92 & $16.2 \%$ & 128 & $22.5 \%$ & 489 & $-85.8 \%$ & 475 & $83.4 \%$ \\
\hline Nhy & 2,970 & 450 & $15.1 \%$ & 620 & $20.9 \%$ & $-2,450$ & $-82.5 \%$ & 2,233 & $75.2 \%$ \\
\hline NV & 3,450 & 426 & $12.4 \%$ & 612 & $17.7 \%$ & $-3,048$ & $-88.4 \%$ & 2,144 & $62.1 \%$ \\
\hline WY & 7,410 & 1,018 & $13.7 \%$ & 1,380 & $18.6 \%$ & $-5,591$ & $-75.5 \%$ & 5,707 & $77.0 \%$ \\
\hline FP & 26,760 & 2,059 & $7.7 \%$ & 2,694 & $10.1 \%$ & $-11,515$ & $-43.0 \%$ & 11,771 & $44.0 \%$ \\
\hline WECC & 72,210 & 4,667 & $6.5 \%$ & 6,012 & $8.3 \%$ & $-30,934$ & $-42.8 \%$ & 19,337 & $26.8 \%$ \\
\hline
\end{tabular}

The data confirm that as we move from state to footprint to WECC, the absolute size of the forecast errors become larger, but the error as a percent of the installed wind base becomes progressively smaller.

Figure 5.24 shows a scatter plot of the study footprint forecast errors and the wind forecast as a percent of the maximum forecast. For each $10 \%$ bin of wind forecasts, the blue, magenta, and green lines show the $50^{\text {th }}, 80^{\text {th }}$, and $90^{\text {th }}$ percentile forecast error. The shape of the plot suggests that forecast error is not a linear function of the wind forecast. Chapter 6 discusses the implications of increasing the spinning reserves by some fraction of the forecast. 


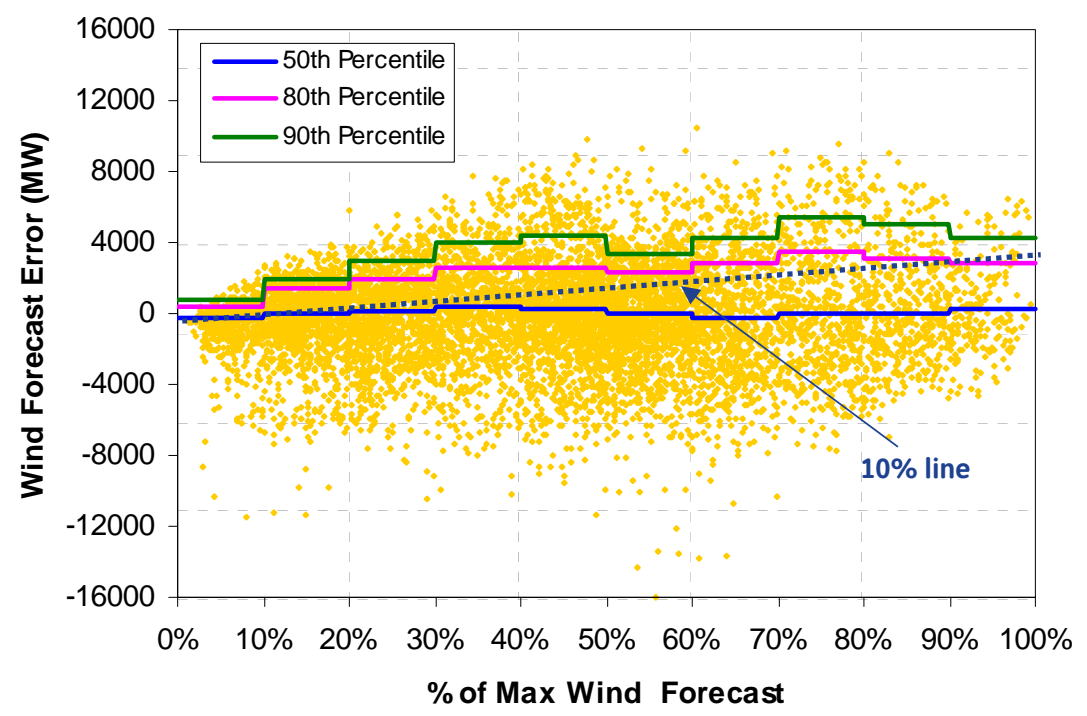

Figure 5.24 Study Footprint Next Day Wind Forecast Errors vs. Forecast, 30\% Local-Priority Scenario

\subsection{Observations and Conclusions}

The variability of net load in the 1-hour time frame impacts various aspects of bulk power system operation including ramp and range requirements, operating reserves, and unit commitment issues. Consequently, this chapter focused on the statistical character of load and net load variability in the hourly time frame. Deltas or hour-tohour differences are used to determine ramping requirements, and the standard deviation of the deltas is used as a measure of hourly variability.

Considering the wind and load deltas over several years of data, the study concluded that load and wind deltas are not highly correlated at the footprint level, and large load deltas are not usually accompanied by similarly large wind deltas, i.e., the risk of simultaneous delta reinforcement is relatively small. Nevertheless, there are a few hours during the year when load and wind deltas combine to increase net load down-ramps, and many more hours where net load up-ramps are greater than the largest load-alone up-ramp. The latter events are driven by large wind drops and are more of an operational concern than the former.

At footprint and state level, more and larger extreme net load ramps tend to occur (1) during fall and winter late afternoons due to simultaneous load rise, and wind and solar drops, and (2) during summer and early fall evenings driven by mostly evening load roll-off.

The size and number of extreme ramp events increase dramatically with wind and solar penetration. At $30 \%$ penetration, the largest net load up-ramp is almost $50 \%$ larger than at the baseline, and there are many more of these on the tails of the distribution. There is 
a good case to be made for load participation to reduce ramping requirements and alleviate the need for large amounts of reserves to cover extremely infrequent events.

Due to geographic diversity, extreme events and overall variability are mitigated when wind and solar generation is aggregated over a wide area. In each scenario, the variability more than doubles for certain states, from baseline to $30 \%$ penetration. However, at the footprint and WECC level, the increase in variability is relatively modest. The study concluded that wide area balancing leads to greater diversity, less relative variability, and reduced ramping requirements.

Day-ahead predictability of net load is important to the unit commitment process. Inaccuracies (large forecast errors) may compromise reliability, increase operating costs, and require greater ancillary service procurement. At the footprint level, the distribution of forecast errors is symmetric, and the size and number of large errors increase as wind penetration increases. As with variability, the ability of an area to absorb large forecast errors improves, as the area size gets larger. Forecast error is not a linear function of the wind forecast. 



\section{Operational Analysis}

The purpose of the operational analysis is to evaluate the operational feasibility of integrating large amounts of intermittent renewable generation into the study area footprint and WECC. A range of renewable penetrations was considered as well as various system sensitivities such as fuel prices, hydro flexibility, and non-renewable generation expansion plans. The analysis was performed using the GE Multi Area Production Simulation program, MAPS, which performs a day-ahead unit commitment and an hourly dispatch recognizing transmission constraints within the system and individual unit operating characteristics. Except where noted, day-ahead wind and solar forecasts were used in the commitment process. As a by-product of the analysis, the cost and emission impact of renewables were also determined. While that information is useful and of interest to many, it is important to recognize that it is not the intent of this study to economically justify renewable generation. This study seeks to determine the overall feasibility of incorporating large amounts of wind and solar generation into the operation of WECC, what operational challenges might arise, and what changes might be required to facilitate this integration.

\subsection{Operational Analysis Assumptions}

The operation of WECC was simulated for the year 2017 with the renewable scenarios described in Chapter 3. The basic WECC system was developed from publicly available generation and data from the Velocity Suite database of Ventyx along with transmission interface data from WECC and load forecasts based on NERC peak load projections. The generator data included full and part load heat rates and emission rates along with minimum operating points and other operating characteristics. Ten-year historical monthly energies were used for the hydro generation. An additional $24 \mathrm{GW}$ of thermal capacity was added to the roughly $200 \mathrm{GW}$ of existing generation to cover the load growth through 2017. Over half of this was specific units that are currently under construction or in advanced development stages. Additional combined-cycle and peaking generation was added to maintain historical regional reserve margins. No changes were made to the base expansion plan when renewable generation was added in order to avoid clouding the results with secondary changes. A sensitivity case was examined which excluded the additional unit additions to match the capacity value of the renewable units based on the reliability analysis discussed in Chapter 9. A topic for future study would be the tailoring of future non-renewable generation to specifically facilitate the integration of the wind and solar generation. If anything, this should increase the value of the renewables and help to offset any negative impacts.

The key 2017 fuel prices assumed coal at roughly \$2/Mbtu and natural gas at \$9.5/Mbtu, with some seasonal and regional variations as appropriate. An initial carbon tax of $\$ 30 /$ ton was assumed. Gas prices have dropped since the start of the study, so a 
sensitivity case examined the operation of the system if gas prices drop enough to make the operating costs of combined-cycle units less than coal generation with a carbon tax. The WECC system was modeled as over 100 separate load areas with transfer limits between the areas. These areas were grouped into five regions for operations and summary purposes. The base operating reserve was assumed to be $6 \%$ of the hourly load, with at least half of that synchronized. The base-cases did not change the spinning reserve as renewables were added to the system. Sensitivity cases examined the impact of increasing the spinning reserve requirement based on renewable penetration. The system was committed and dispatched in an economically rational manner for all of WECC, recognizing the limitations of the transmission systems (see Figure 3.5) and the cycling capability of the individual generators. The WECC system was divided into 106 load zones that were assigned to 20 transmission areas. Interface constraints were defined between neighboring transmission areas. The load zones were also grouped into five regions for operating reserves. As the statistical analysis showed, incorporating large amounts of intermittent renewable generation without consolidation of the smaller balancing areas in either a real or virtual sense could be difficult.

The important assumptions in this analysis surrounded the treatment of the wind and solar generation. Hourly generation profiles were generated for each of the sites based on historical weather data for the study years 2004, 2005, and 2006. These were then time -synchronized with historical load profiles from the same years to capture any correlation between the load and the wind and solar generation. The load profiles were escalated to the 2017 peak and energy projections as mentioned above. Of prime importance is that a second set of wind and solar profiles was also created for each site that specified the day-ahead forecast of the renewable generation based on the dayahead weather forecast. The MAPS model uses a separate renewable generator forecast profile for the day-ahead commitment decision as compared to the actual hourly dispatch.

The various penetration cases were examined using these State-of-the-Art forecasts as well as with perfect forecasts to evaluate the impact of the variability independent of the forecast accuracy. Sensitivity cases were also examined that considered zero forecasts in the day-ahead operations.

The bulk of the cases and sensitivities were run using the 2006 shape and the In-Area site selection scenario. Results for the other annual shapes and siting are shown as additional sensitivities.

\subsubsection{Case-Naming Conventions}

For the summary results, it was necessary to develop a shorthand naming convention to describe the various cases. The convention is: Scenario-Penetration-Forecast-Sensitivity. 
The allowable options for the first three categories are shown in Table 6.1. The sensitivities will be explained as they are introduced.

Table 6.1 Case-Naming Conventions

\begin{tabular}{|l|l|l|}
\hline Scenario & Penetration & Forecast \\
\hline I- In-Area & Pre - Preselected Sites & P-Perfect Forecast \\
\hline M- Mega-Project & $10 \%-10 \%$ Case & R-State of the Art Forecast \\
\hline L- Local-Priority & $20 \%-20 \%$ Case & N-No-Forecast \\
\hline & $20 / 20 \%-20 / 20 \%$ Case & \\
\hline & $30 \%-30 \%$ Case & \\
\hline
\end{tabular}

As an example, a case labeled I10P would refer to the In-Area Scenario, 10\% penetration with a perfect forecast. In addition, a "no-wind" case which removed even the existing wind generation was run for comparison purposes.

\subsection{Annual Operational Impacts}

A variety of metrics are presented to address the question, "What happens to the operation of the system with high levels of intermittent renewable generation?" These include annual generation displacement by type, system operating costs, and spilled energy. An important measure is the hourly marginal cost of energy, or spot price. In a deregulated market, this is the price paid for energy each hour, but it is useful in a regulated market as an hourly measure of the value of the energy. When transmission constraints are present, these values will vary across the system for any given hour, but they can be weighted by the hourly load in the area to produce a system spot price. These were calculated chronologically for each hour of each year for each case. They were then sorted for easy comparison of the overall impacts.

Figure 6.1 shows these annual system spot prices for five levels of renewable penetration for the In-Area Scenario assuming perfect day-ahead forecasting of the wind and solar generation. 


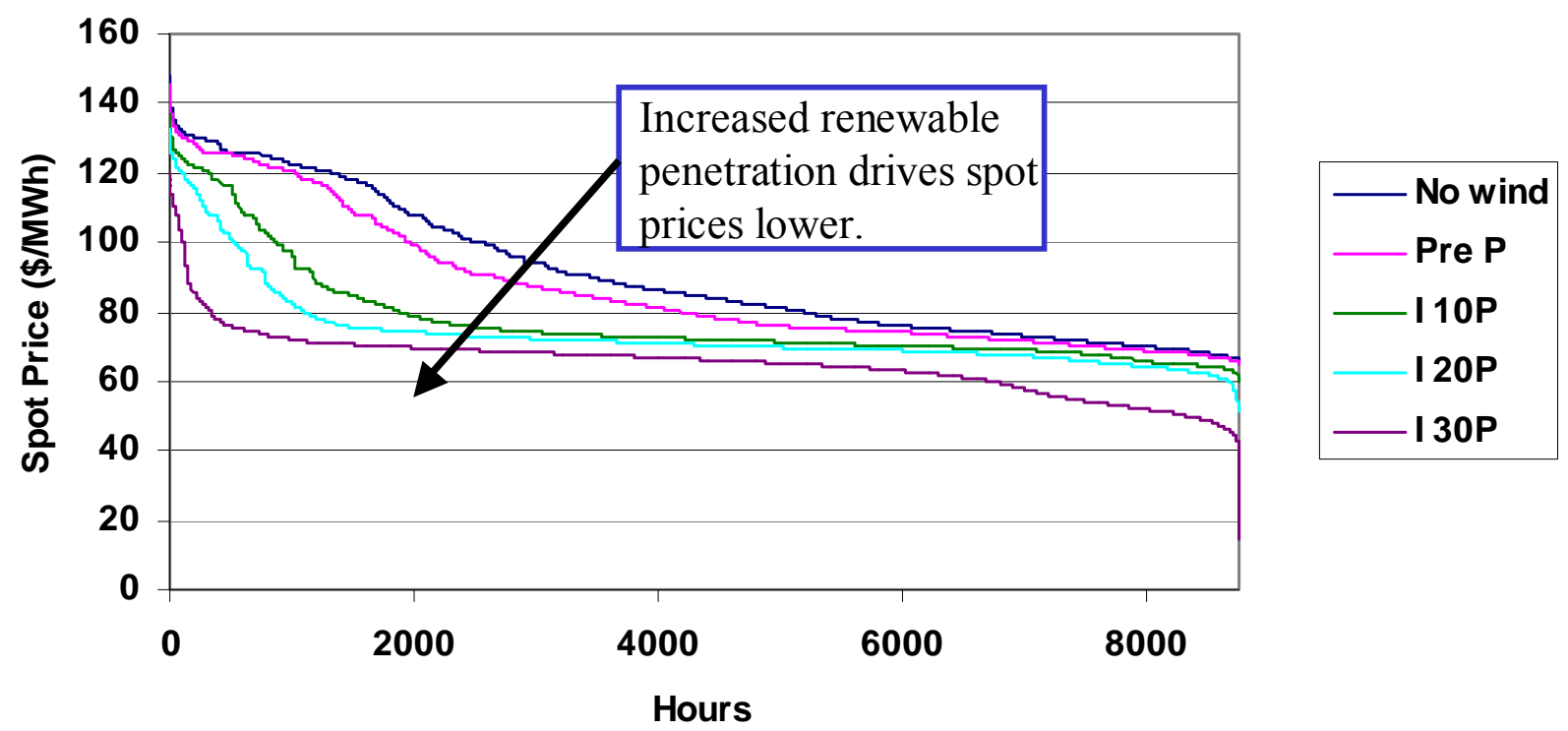

Figure 6.1 Annual WECC Spot Price Duration Curve, Perfect Forecast, In-Area Scenarios

With no renewable generation on the system, the spot price ranges from a high of roughly $\$ 150 / \mathrm{MWh}$ to a low of about $\$ 65 / \mathrm{MWh}$. The Preselected case (Pre) caused a slight drop in prices because the highest cost units in the system are being displaced. As the penetration levels increase, the spot prices are driven down even further as the more expensive generation is eliminated from the hourly dispatch. At the $30 \%$ penetration level, the highest cost is about $\$ 120 / \mathrm{MWh}$ and the lowest is about $\$ 40 / \mathrm{MWh}$, with the exception of a few hours going as low as $\$ 15 / \mathrm{MWh}$.

Figure 6.2 shows the same cases when an unbiased State-of-the-Art (S-o-A) forecast is assumed. The initial trend is the same for low levels of penetration because the forecast errors are less than the spinning reserve and therefore can be covered without stressing the system. But as the penetration levels increase, the forecast error starts to have an impact. When the wind and solar forecast is too high, peaking generation must come on to cover the shortfall, and in some hours, there may be unserved energy or contingency reserve shortfalls because there is not enough local peaking generation available. At the other extreme, when the wind and solar forecast is too low, then too many generators will be committed that must then be backed down to minimum operating levels and some spillage of the wind generation may occur during off-peak periods when the loads are low. The $30 \%$ case has over 100 hours when the average system spot price has dropped below $\$ 20 / \mathrm{MWh}$. Prices in some locations dropped to zero. 


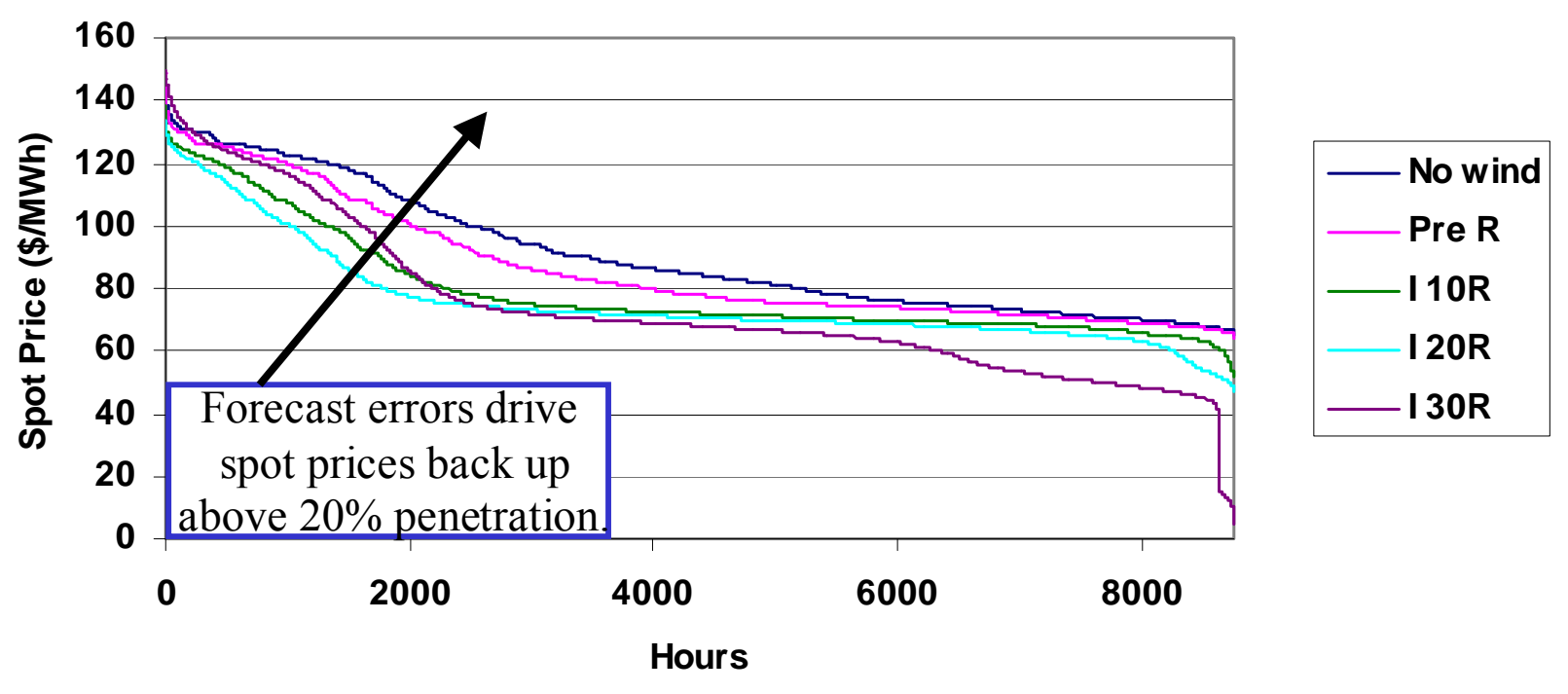

Figure 6.2 Annual WECC Spot Price Duration Curve, S-o-A Forecast, In-Area Scenarios

Figure 6.3 shows the total generation by type for all of WECC for various In-Area cases. The bulk of the energy that is displaced by the renewable generation is coming from the combined-cycle (CC) units with some slight variations in the simple-cycle gas turbines (GT), pumped storage hydro (PSH), and steam oil and gas units (ST). The steam coal units (ST-COAL) are only affected at the higher penetrations. The hydro generation units (HY) did not change their annual energy output, but their hourly output patterns were shifted, as will be examined later. Figure 6.4 shows that the results are similar for the study footprint.

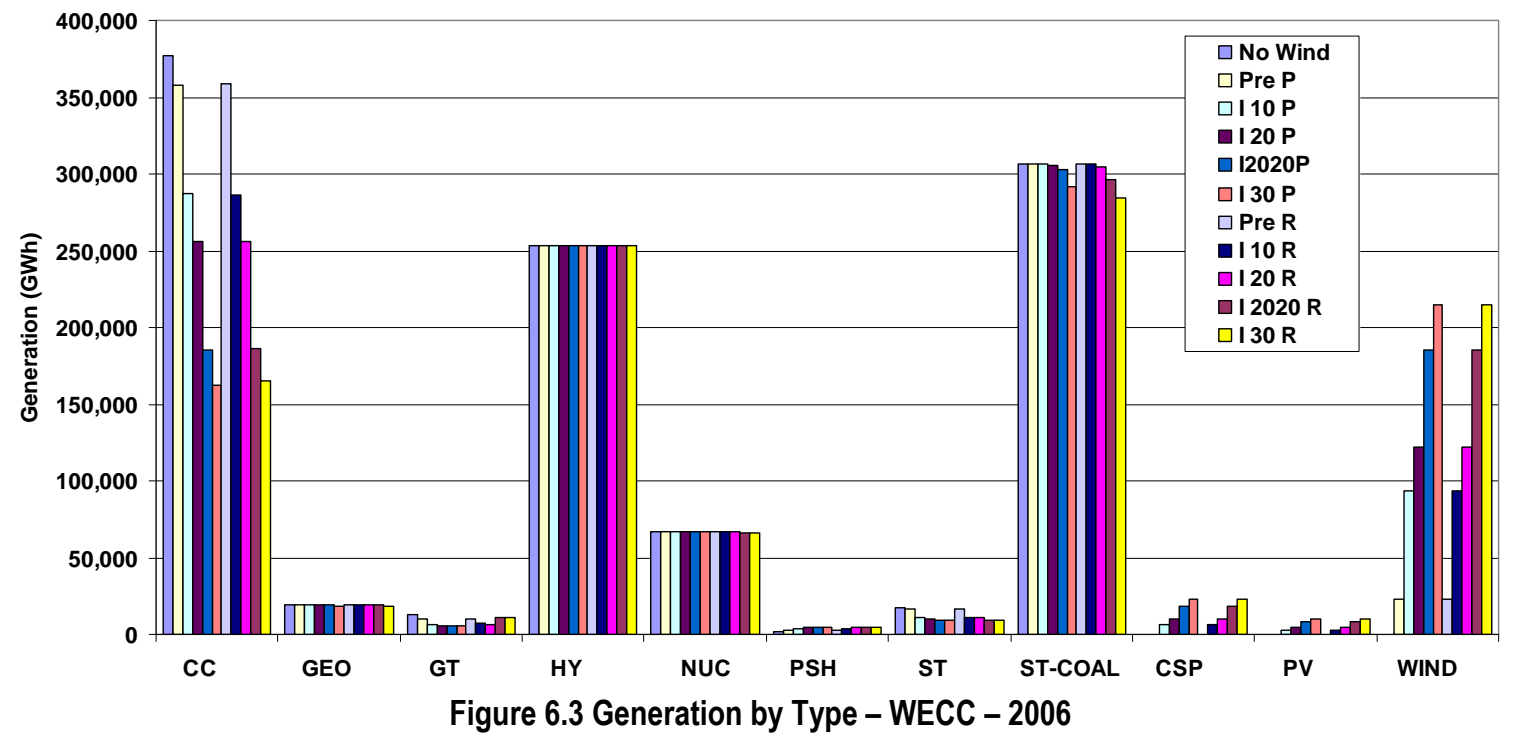




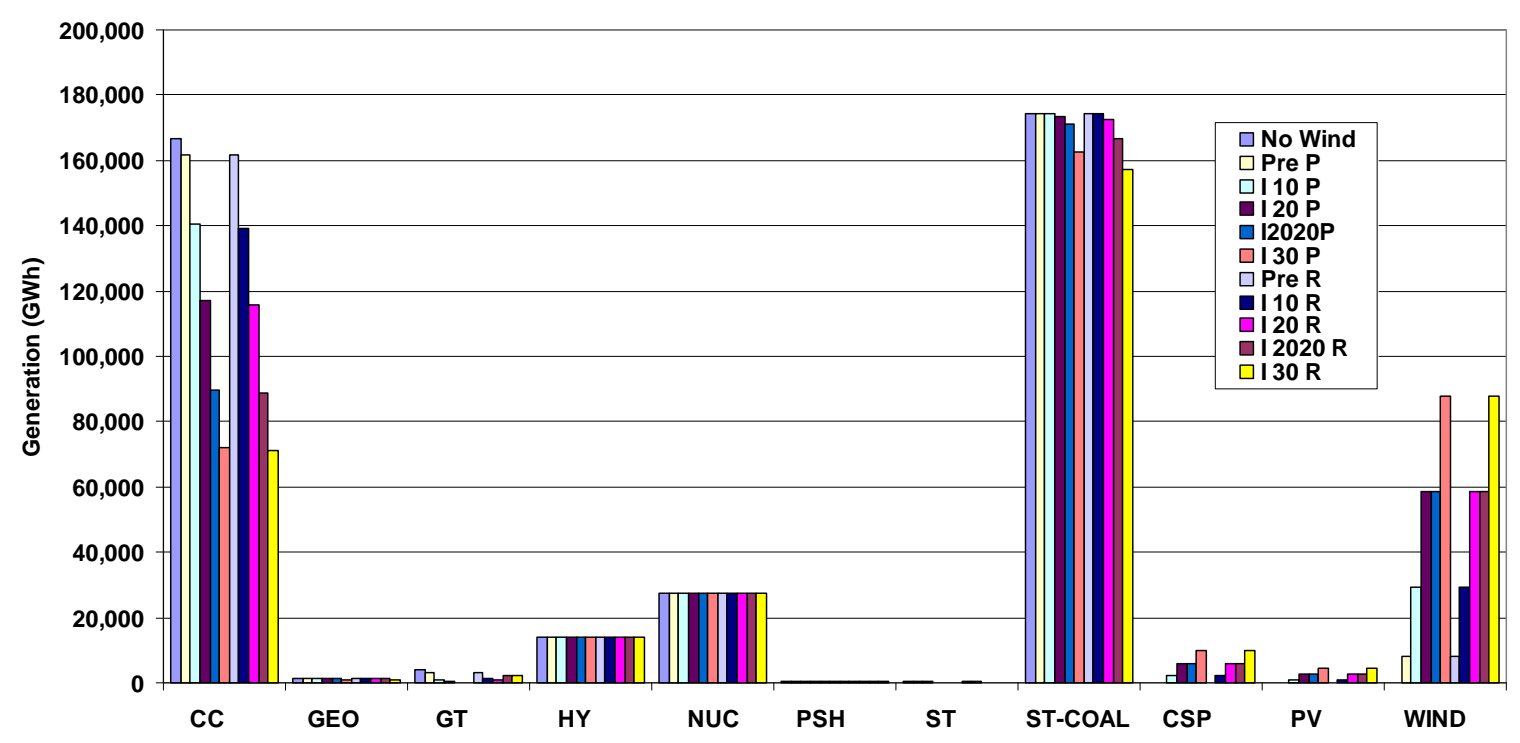

Figure 6.4 Generation by Type - Study Area - 2006

Figure 6.5 shows the total WECC operating costs for the various cases. Figure 6.6 shows the WECC-wide operational cost savings, that is, the reduction in fuel, variable O\&M and startup costs from the "No-wind" case for the various cases.

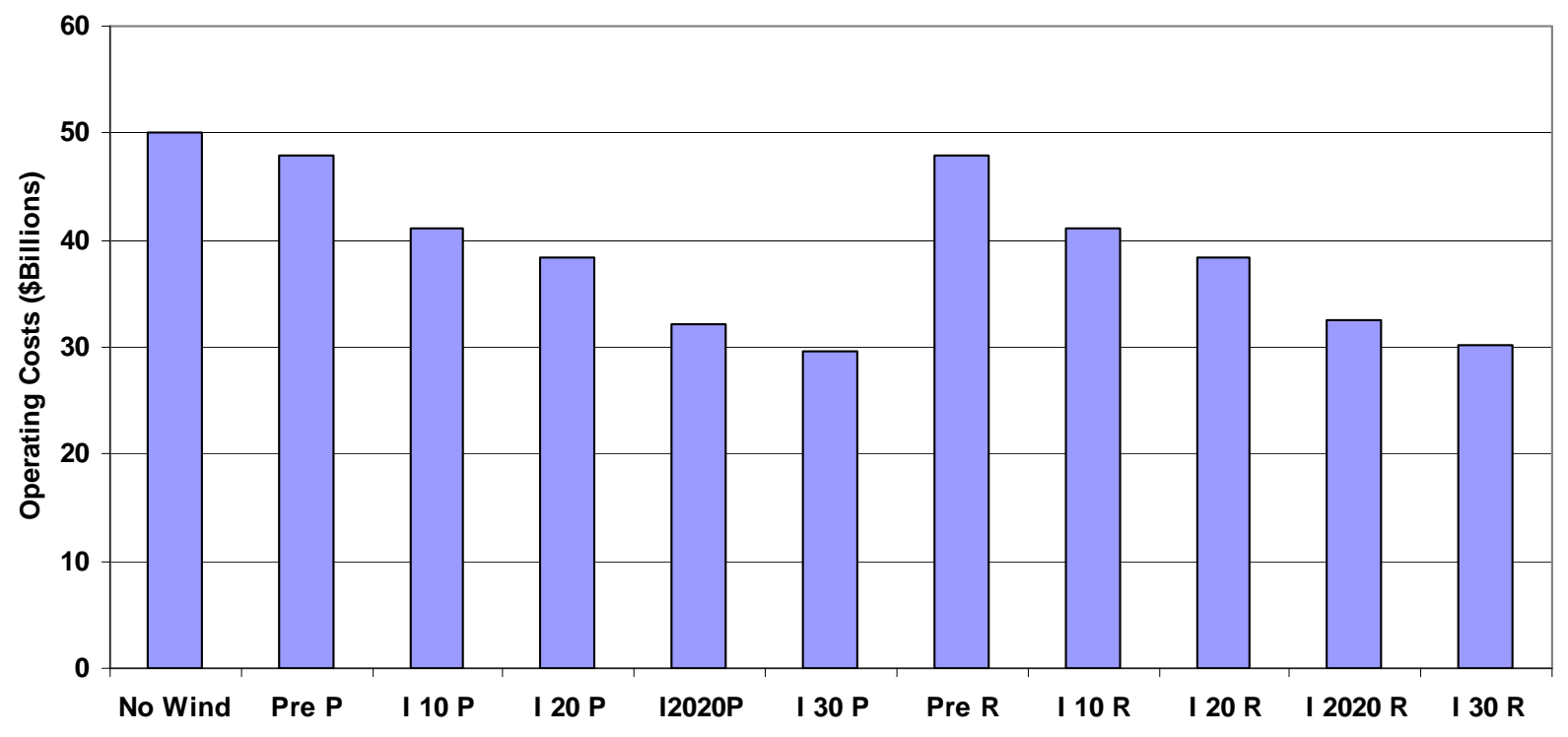

Figure 6.5 Total WECC Operating Costs (\$B) - 2006 


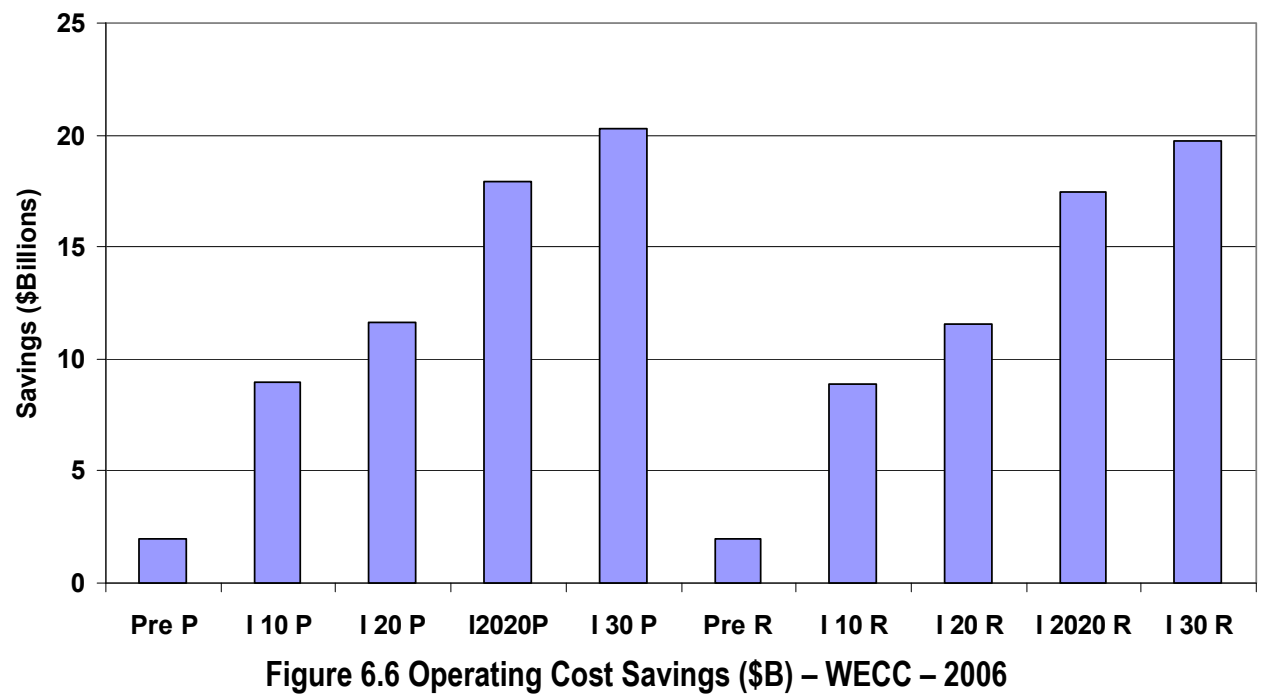

If we divide the savings in this figure by the amount of renewable energy in each case, then we get the results shown in Figure 6.7. This shows the average value of the renewable energy in each case. As might be expected, as the amount of renewable energy is increased, its average value decreases slightly. It is also not surprising that the cases with a perfect day-ahead forecast show slightly higher value than the corresponding cases using the S-o-A forecast.

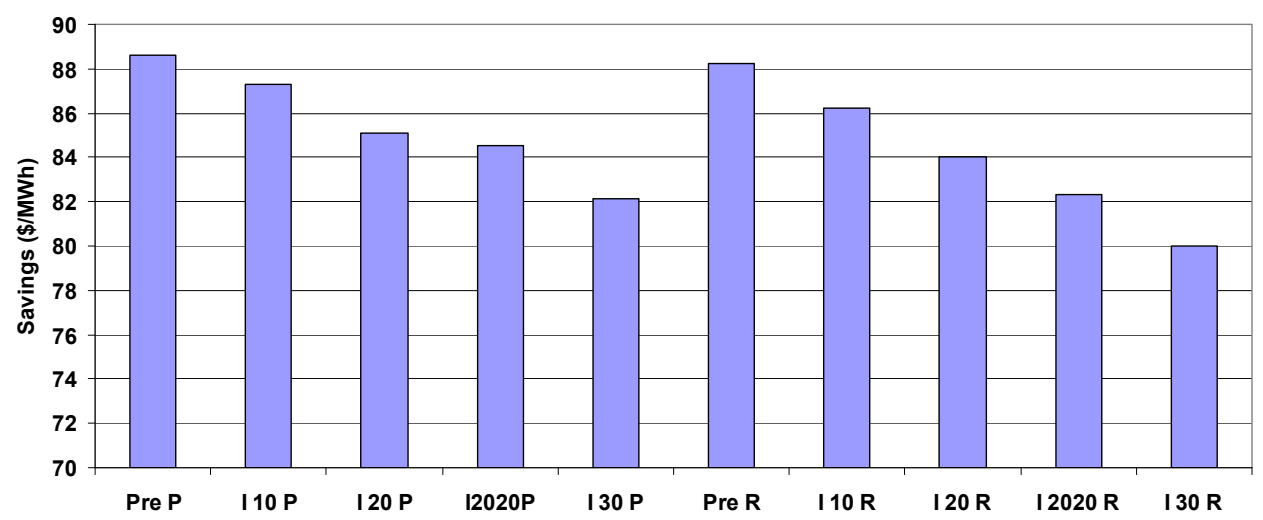

Figure 6.7 Operating Cost Savings Per MWh of Renewable Energy, WECC - 2006

Figure 6.8 looks at the incremental savings between each case divided by the incremental renewable energy. So while the previous figure was relative to the no-wind case, these results are relative to the preceding case. The incremental values for the $30 \%$ case relative to the $20 / 20 \%$ case have dropped significantly. 


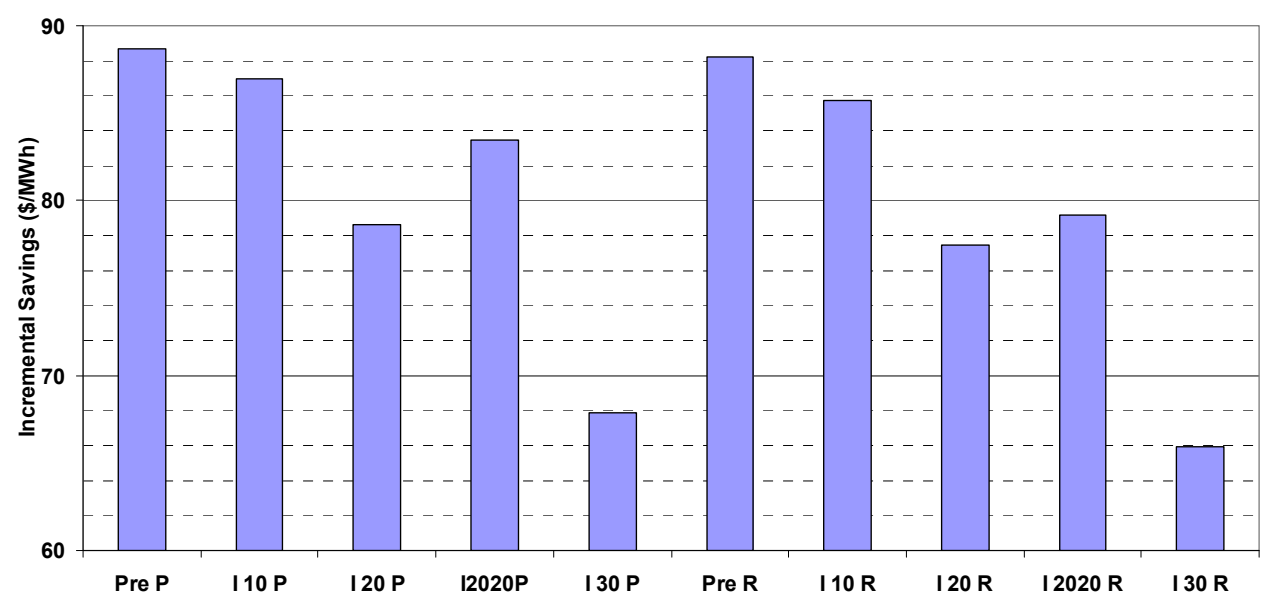

Figure 6.8 Incremental Savings per Incremental Renewable Energy (\$/MWh). WECC - 2006

Many previous renewable energy integration studies have concentrated on adding renewable generation to the area of interest without much concern for the neighboring regions. This often allowed the neighbors to provide a large buffer to either absorb the excess generation in times of high wind, or to provide additional backup if the wind suddenly drops off or falls significantly short of the forecast. That is why it was so important in this study to not only examine the WestConnect footprint as a part of WECC, but also to recognize that the rest of WECC would be adding significant amounts of wind and solar generation. In the initial case matrix, there was an I20R case that had $20 \%$ wind penetration in the study footprint and $10 \%$ penetration in the rest of WECC. The next level was the I30R case that increased the wind penetration to $30 \%$ in the footprint and 20\% penetration in the rest of WECC. When examining the changes between the two cases, it was difficult to separate the changes due to the additional $10 \%$ in the footprint from those due to the additional 10\% in the rest of WECC. To address that concern, the I2020R case was developed which had $20 \%$ penetration throughout all of WECC. Comparing the I20R and the I2020R cases in Figure 6.9 shows the impact on footprint exports of only increasing the wind penetration outside the study footprint and comparing the I2020R with the I30R case shows the impact of only increasing the wind penetration inside the study footprint.

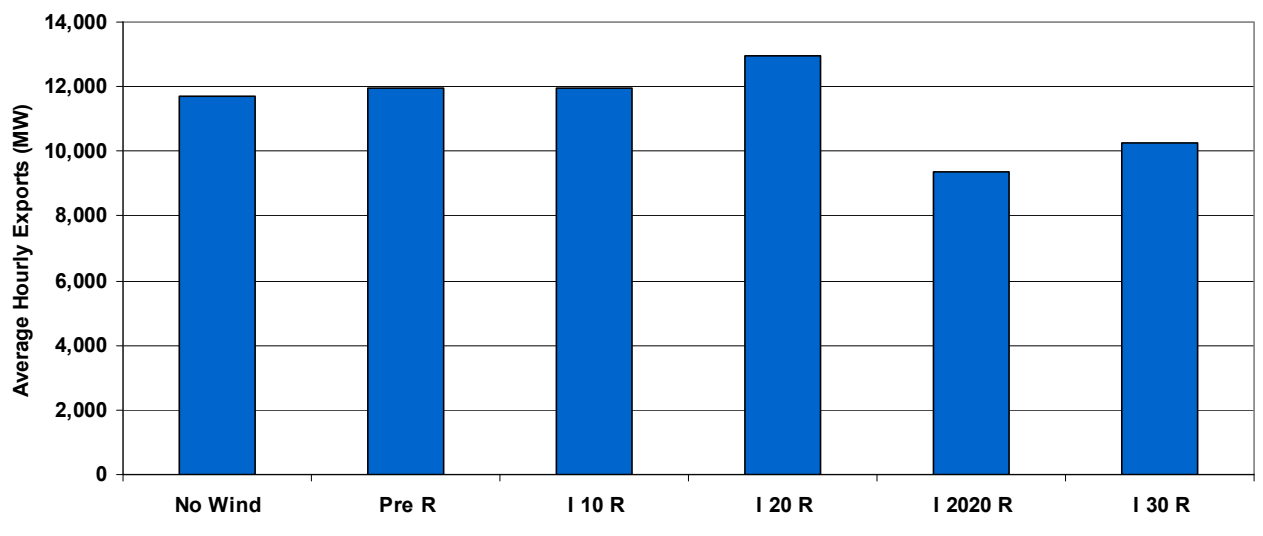

Figure 6.9 Average Hourly Exports From Study Footprint 
The day-ahead forecast and renewable penetration levels have operational impacts beyond just the cost. As the penetration levels increase, the system can become "overloaded" with generation, at times requiring the system to curtail, or "spill" some of the excess wind energy. In addition, the changes in commitment due to the renewable generation may sometimes result in shortfalls in generation and cause either some energy to be unserved in certain hours, or cause the system operator to dip into the contingency reserves to make sure load is served. Figure 6.10 shows the change in curtailed energy and contingency reserve shortfalls for these cases. Most of the growth in reserve shortfalls from the I20R to the I30R cases occurred due to the growth in renewable generation outside the study footprint. To put these values in perspective, the $800 \mathrm{GWh}$ of curtailed energy in the $30 \%$ case is less than $0.4 \%$ of the total wind energy generated in WECC. The $50 \mathrm{GWh}$ of reserve shortfall energy is less than $0.005 \%$ of the total WECC load energy.

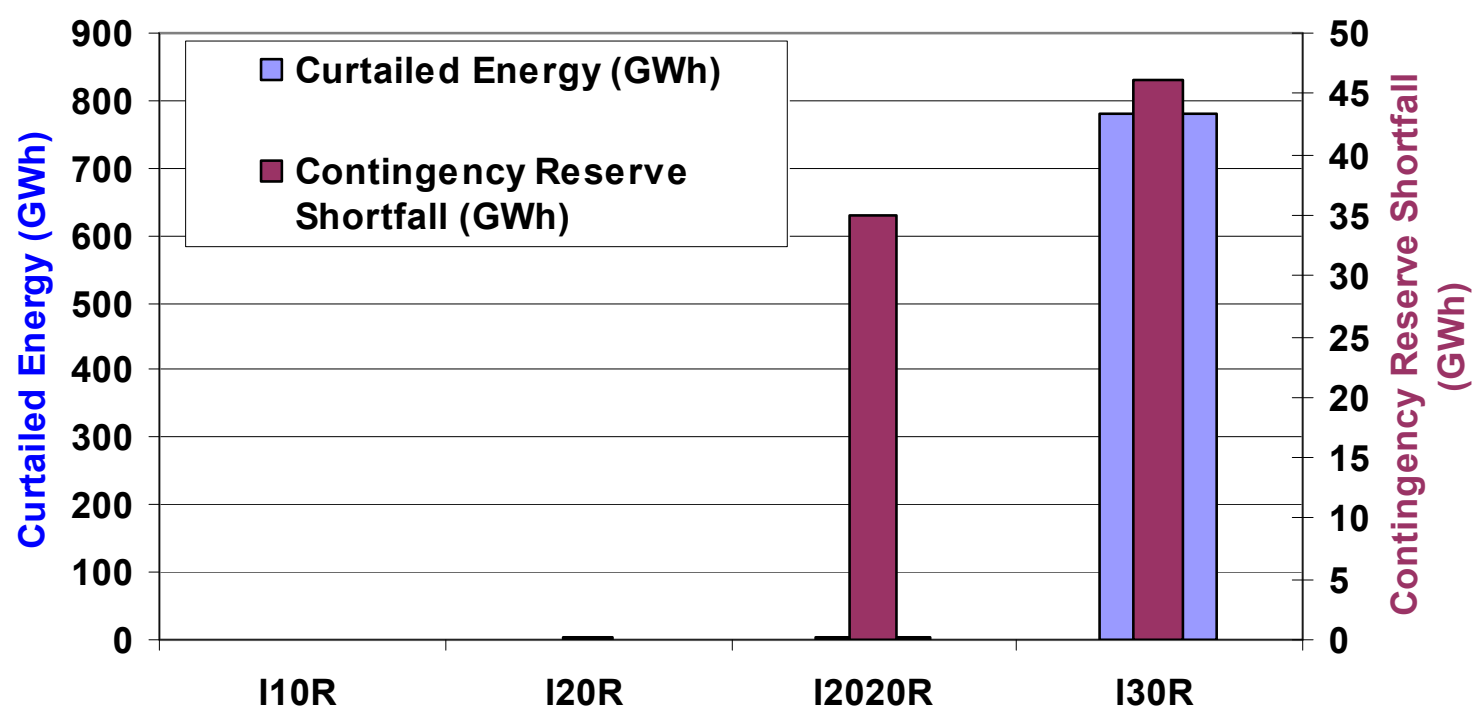

Figure 6.10 Impact of Renewables in Neighboring Areas, all of WECC

Figure 6.11 and Figure 6.12 show additional statistics that were being tracked for the various cases. The coal units maintained a fairly constant number of starts, hours on line, and capacity factor out through the $20 \%$ penetration case, although the revenue was dropping due to decreased spot prices. Note, revenue was determined by multiplying the hourly unit output times the corresponding spot price. At the $30 \%$ level, the number of starts increases significantly, particularly for the smaller units. Also, the capacity factor and hours on line begin to drop. In contrast, the operating hours and capacity factors of combined-cycle units dropped almost immediately as the renewable energy penetration began to increase. 


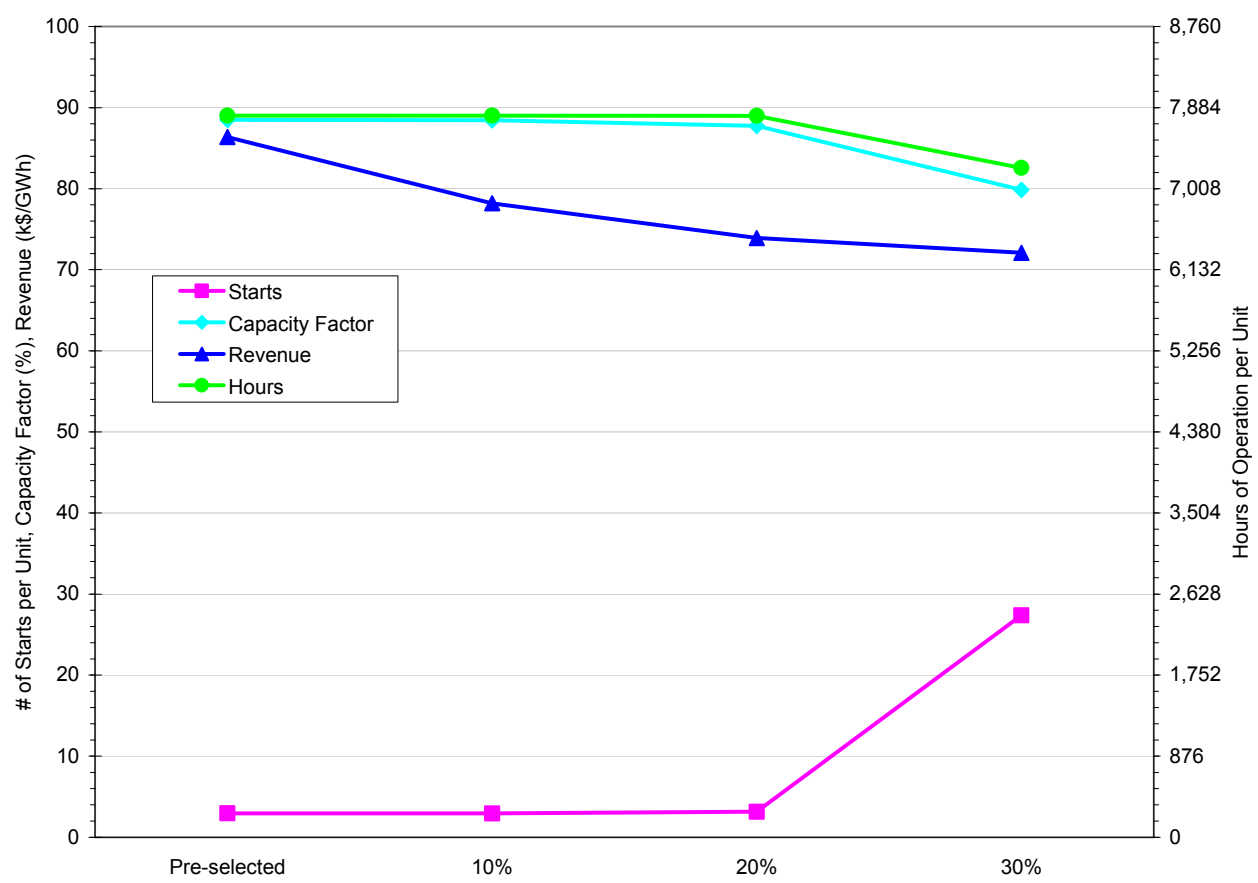

Figure 6.11 Coal Plant Statistics, Local-Priority Scenario

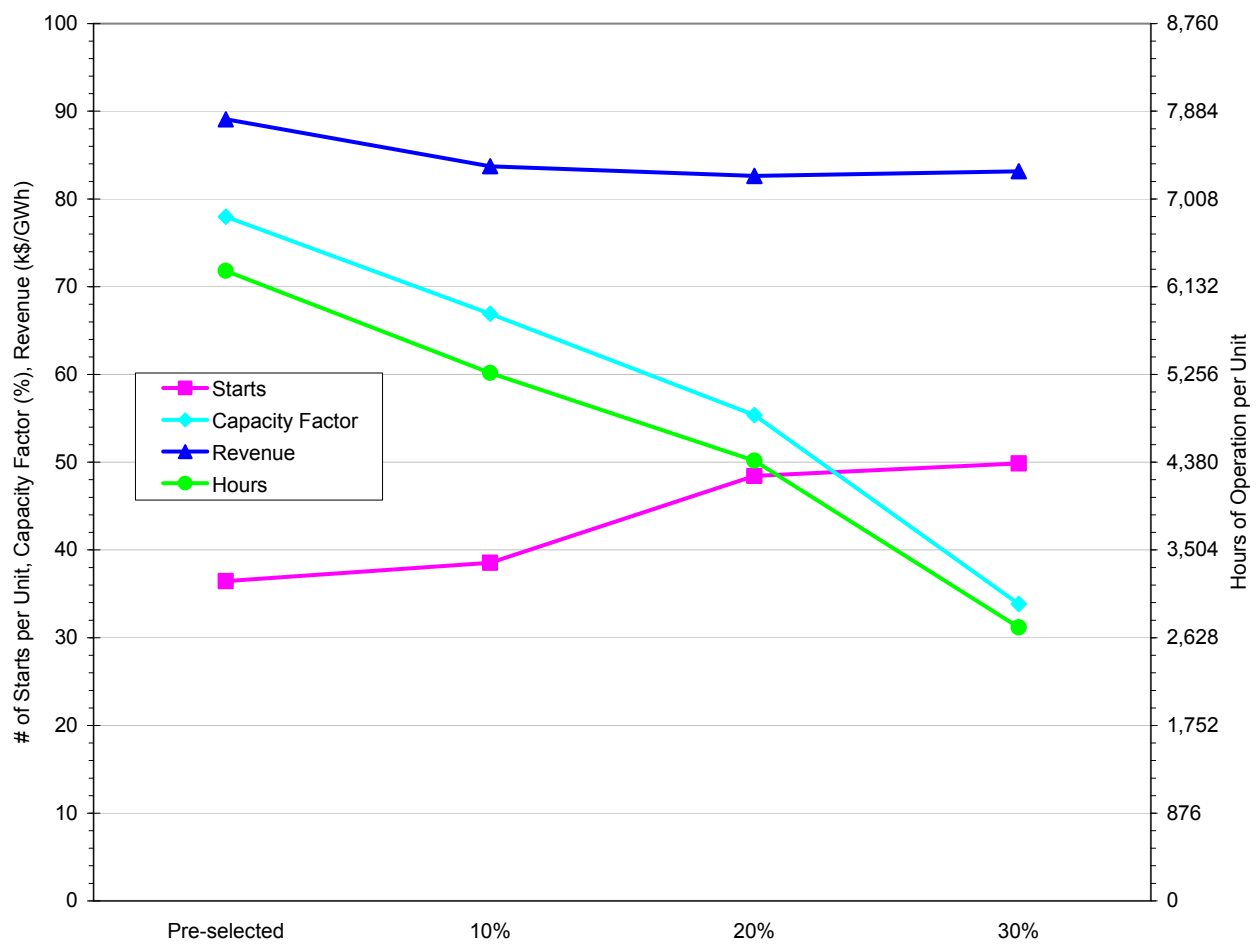

Figure 6.12 Combined-Cycle Plant Statistics, Local-Priority Scenario

Figure 6.13 is an example of the transmission flows being tracked for the key interfaces. This figure shows the flow duration curves for the Wyoming to Colorado interface. The base flow limit was seldom reached on any of the In-Area Scenarios. Flows, as well as ratings, increased with the Local-Priority and Mega-Project Scenarios, but were 
essentially only limiting with the $30 \%$ cases. Flat spots on the beginning of the curves are hours with the interface at saturation. Not surprisingly, Wyoming heavily exports most of the time in the L30 and M30 scenarios. Table 6.2 lists key statistics for each of the cases. More of these summaries for other interfaces are included in Appendix D.

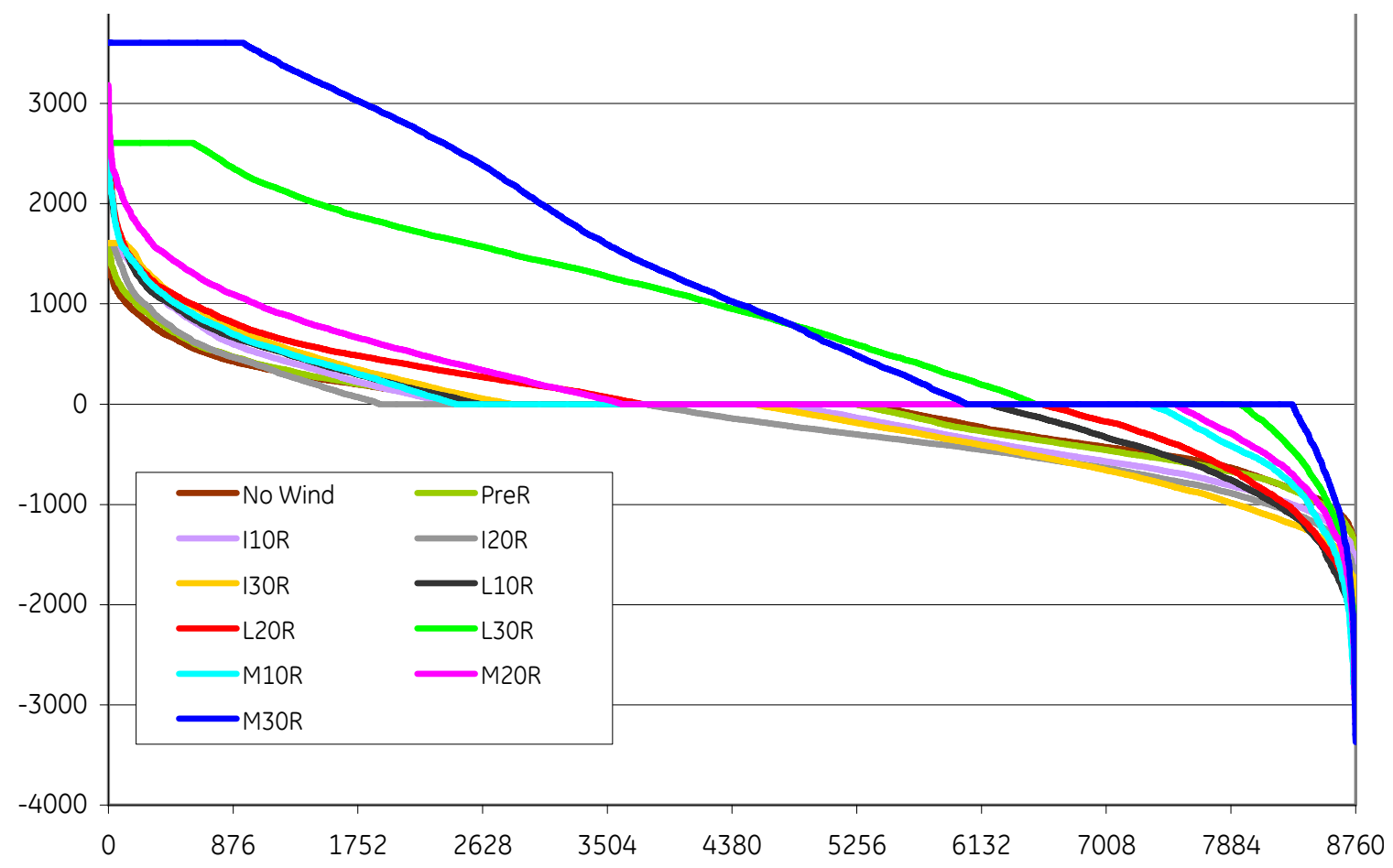

Figure 6.13 Wyoming to Colorado Interface Flows

Table 6.2 Wyoming to Colorado Interface Flow Statistics

\begin{tabular}{|c|c|c|c|c|c|c|c|c|c|c|c|}
\hline & No Wind & PreR & I10R & I20R & I30R & L10R & L20R & L30R & M10R & M20R & M30R \\
\hline Max & 1605 & 1605 & 1605 & 1605 & 1605 & 2605 & 2605 & 2605 & 3605 & 3605 & 3605 \\
\hline \# hrs at Max & 0 & 4 & 58 & 29 & 120 & 1 & 5 & 598 & 0 & 0 & 950 \\
\hline \# hrs Pos & 2599 & 2674 & 2451 & 1903 & 2827 & 2608 & 3747 & 6506 & 2438 & 3604 & 6026 \\
\hline \# hrs Neg & 3432 & 3518 & 3965 & 4987 & 4231 & 2573 & 2205 & 814 & 1451 & 1270 & 446 \\
\hline CF Abs & 18 & 20 & 25 & 26 & 30 & 14 & 15 & 41 & 8 & 11 & 40 \\
\hline CF & -5 & -5 & -6 & -12 & -7 & -1 & 3 & 37 & 2 & 6 & 37 \\
\hline Avg & -72 & -74 & -96 & -196 & -105 & -21 & 79 & 953 & 62 & 218 & 1351 \\
\hline
\end{tabular}

\subsubsection{Value of Forecasts}

The next two figures, Figure 6.14 and Figure 6.15, examine the value of the wind and solar forecasts. The first figure shows the difference in WECC operating costs between a case with a perfect day-ahead forecast and the corresponding case using a State-of-theArt forecast. The second figure then divides these savings by the amount of renewable energy in each case. Not surprisingly, the importance of the forecast increases at higher penetration levels. 

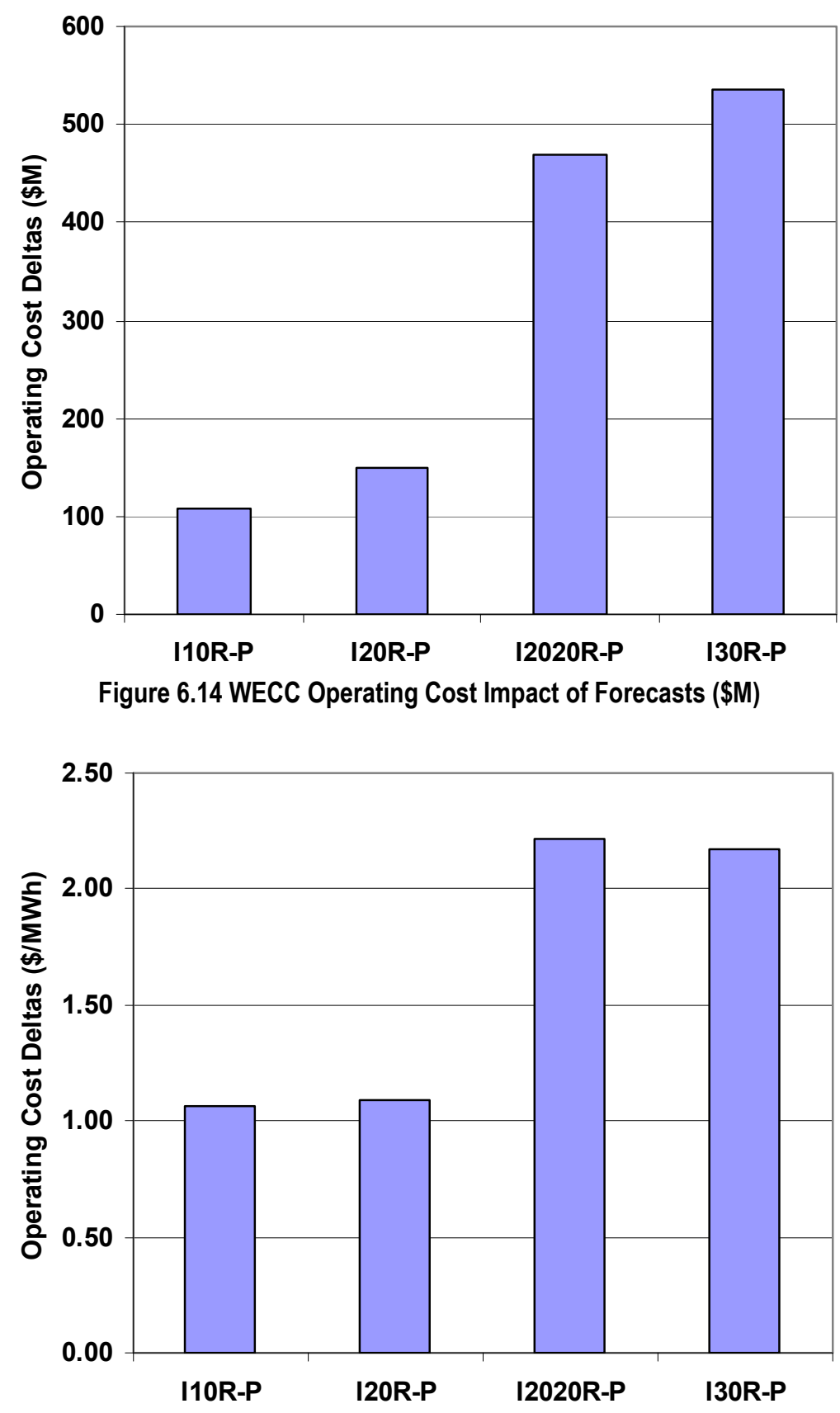

Figure 6.15 Operating Cost Impact of Forecast (\$/MWh)

Figure 6.16 presents a summary of the curtailed and reserve shortfall energy values for the perfect and S-o-A forecasts. In cases where there are insufficient contingency reserves available, this may result in some level of unserved energy. As can be seen, there are essentially no reserve shortfalls with the perfect forecast. Over-forecasting the day-ahead renewable generation causes less thermal generation to be committed, which can result in more reserve shortfalls at higher penetrations. Note that the contingency reserve shortfalls will only occur after available imports and quick-start generation have been exhausted. As noted before, these values are still a very small percentage $(\sim 0.005 \%)$ 
of the total load energy. The curtailed energy occurs in both of the $30 \%$ cases. When the day-ahead forecast is low then additional thermal generation will be committed, which causes the curtailed energy to be slightly larger.

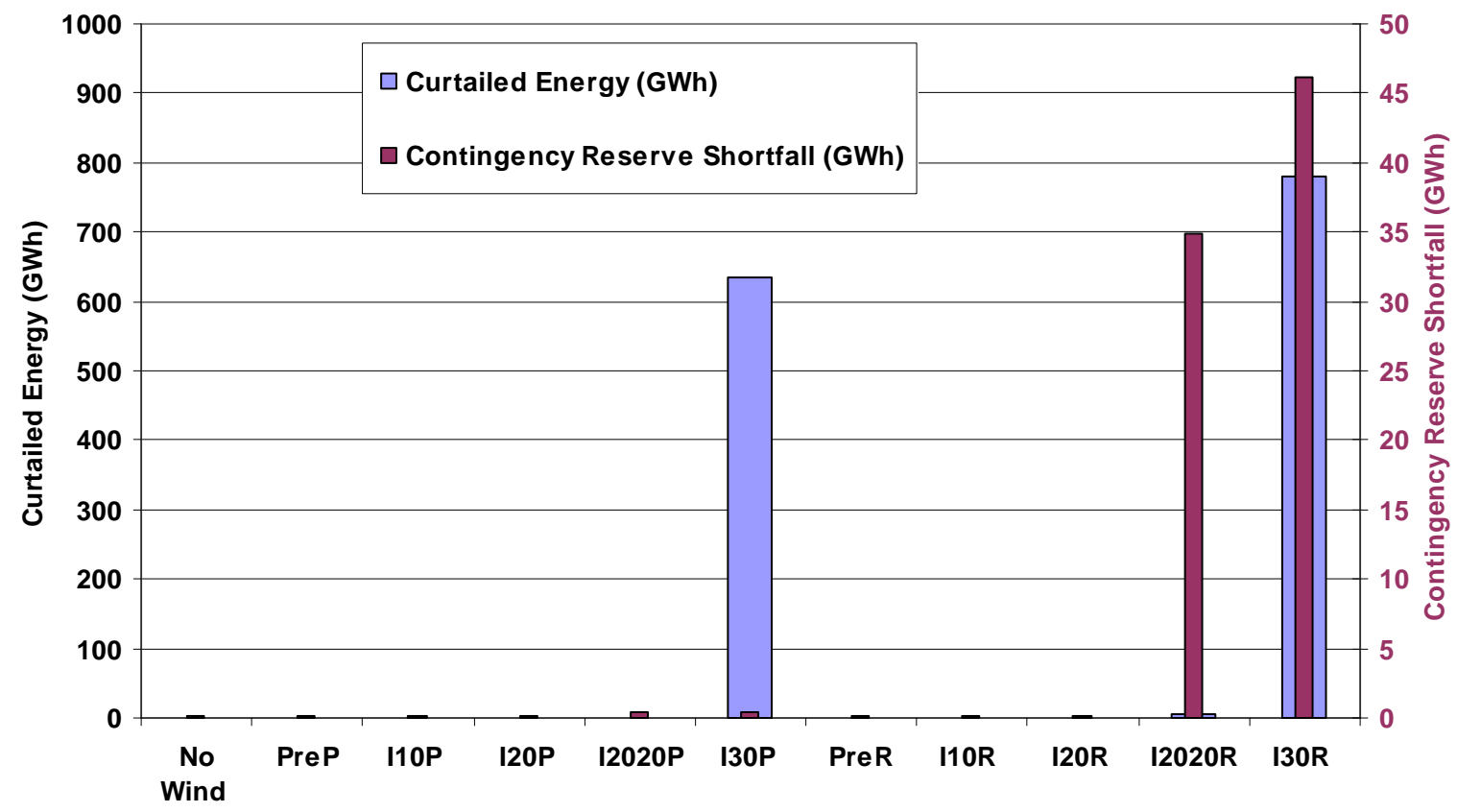

Figure 6.16 Impact of Forecast on Curtailed Energy and Contingency Reserve Shortfalls

Figure 6.17 shows a comparison between the operating costs for the No-Forecast and So-A Forecast cases. Again, there is a jump at the higher penetrations. It is also important to note that the values are an order of magnitude greater than the differences between the S-o-A and perfect forecasts.

Often, the system operators will ignore renewables, particularly wind, in the day-ahead operation due to the lack of confidence in the forecast. Figure 6.18 shows this impact per MWh of renewable energy. As the chart shows, recognizing the day-ahead forecast, even with its inherent errors, can add $\$ 12 / \mathrm{MWh}-\$ 20 / \mathrm{MWh}$ to the value of the renewable energy. 


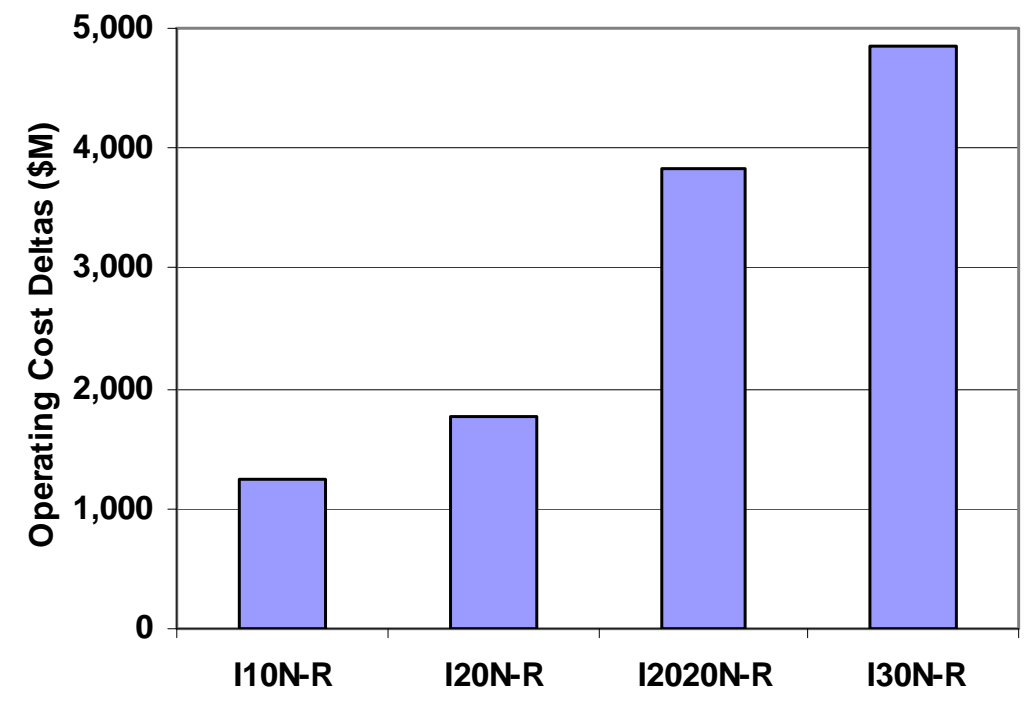

Figure 6.17 WECC Operating Cost Impact of No Forecasts versus a S-o-A Forecast (\$M)

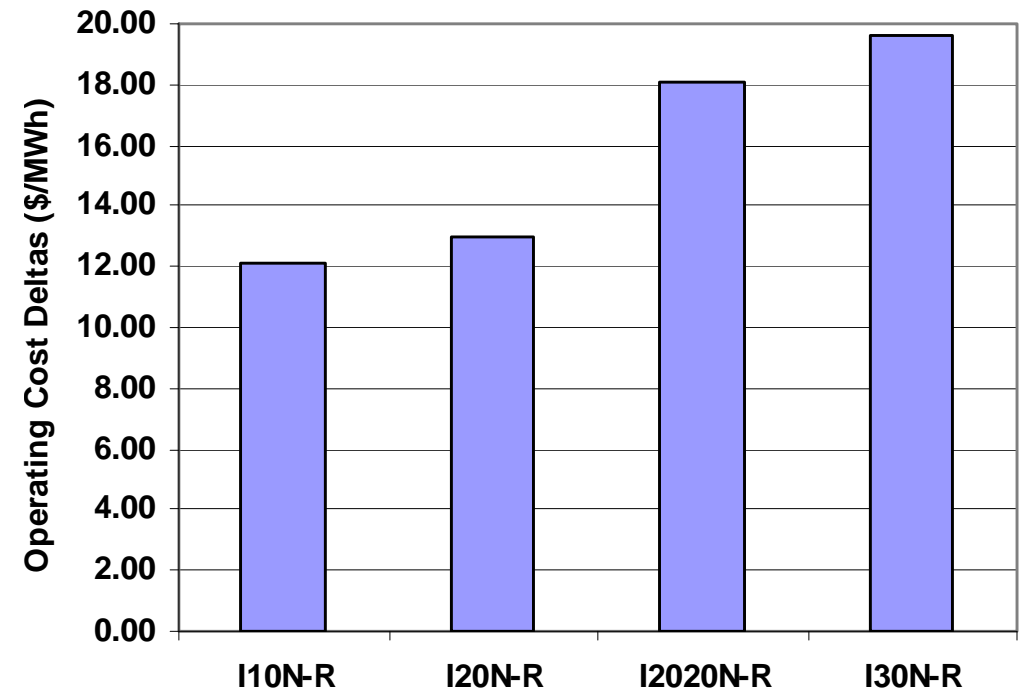

Figure 6.18 WECC Operating Cost Impact of No Forecasts versus a S-o-A Forecast (\$/MWh)

Figure 6.19 shows "the rest of the story." By ignoring the wind forecast, the system has avoided the almost $50 \mathrm{GWh}$ of unserved energy due to over forecasts in the $30 \%$ case. However, it has done that at the expense of greatly increasing the amount of spilled wind energy. Note that the scale on the left is 200 times greater than the one on the right. Increased flexibility of the committed generation would help to mitigate this. Being able to cycle the units to lower minimums or turn them off for short periods of time could avoid the necessity of spilling the wind energy during low load periods. 


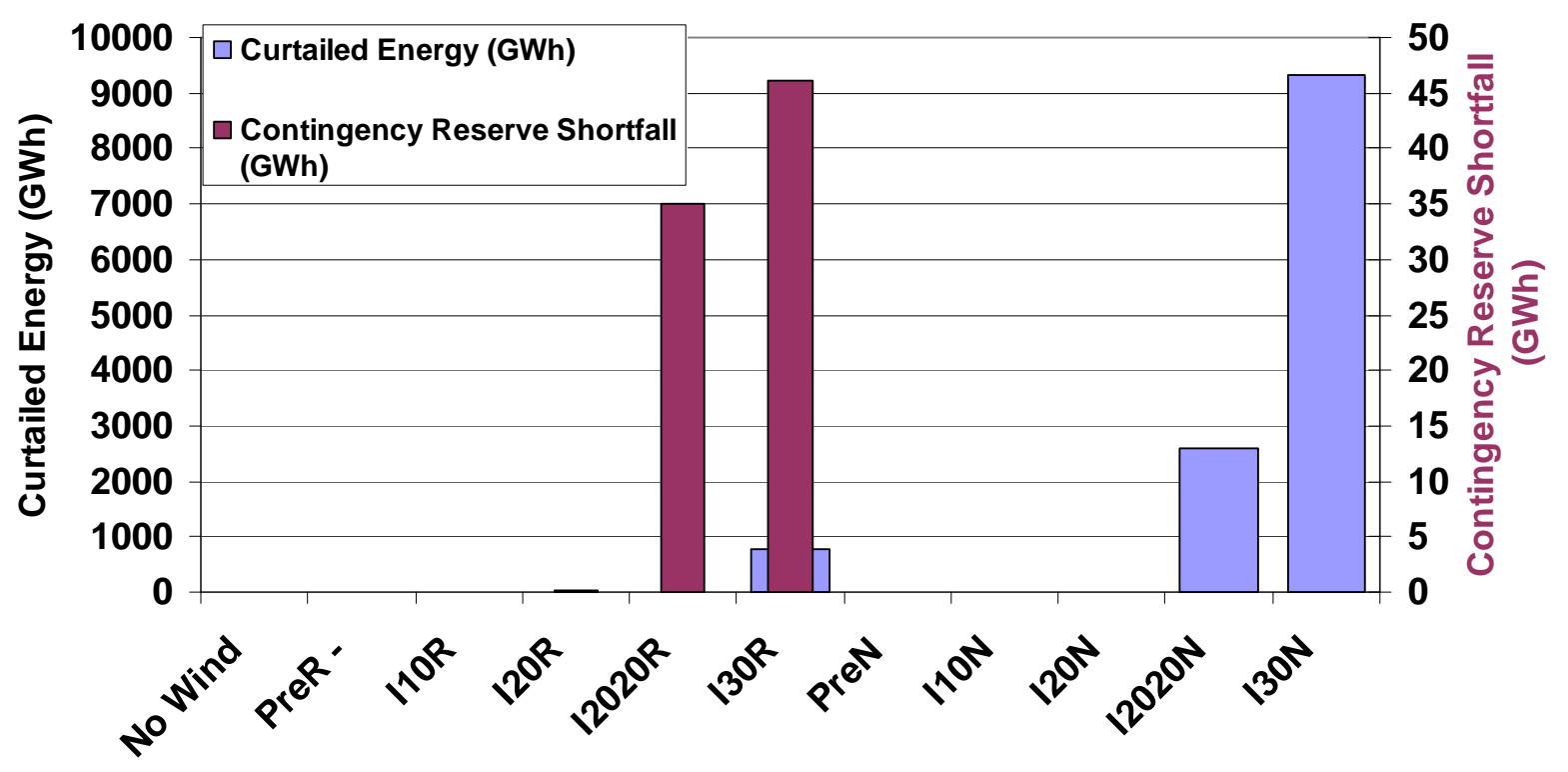

Figure 6.19 WECC Impact of No Forecast on Spilled and Unserved Energy

Figure 6.20 shows the total generation in the study area for renewable and nonrenewable generators. In both the perfect and S-o-A forecasts, the non-renewable generation drops when going from the $20 \%$ to the $20 / 20 \%$ cases due to the increase in renewable generation outside the study footprint. Overall, the total generation within the study area remains relatively constant across all of the cases. Variations in the total generation were due to changes in the net exports from the study area. This can be seen most clearly in the difference between the " 20 " and "2020" cases.

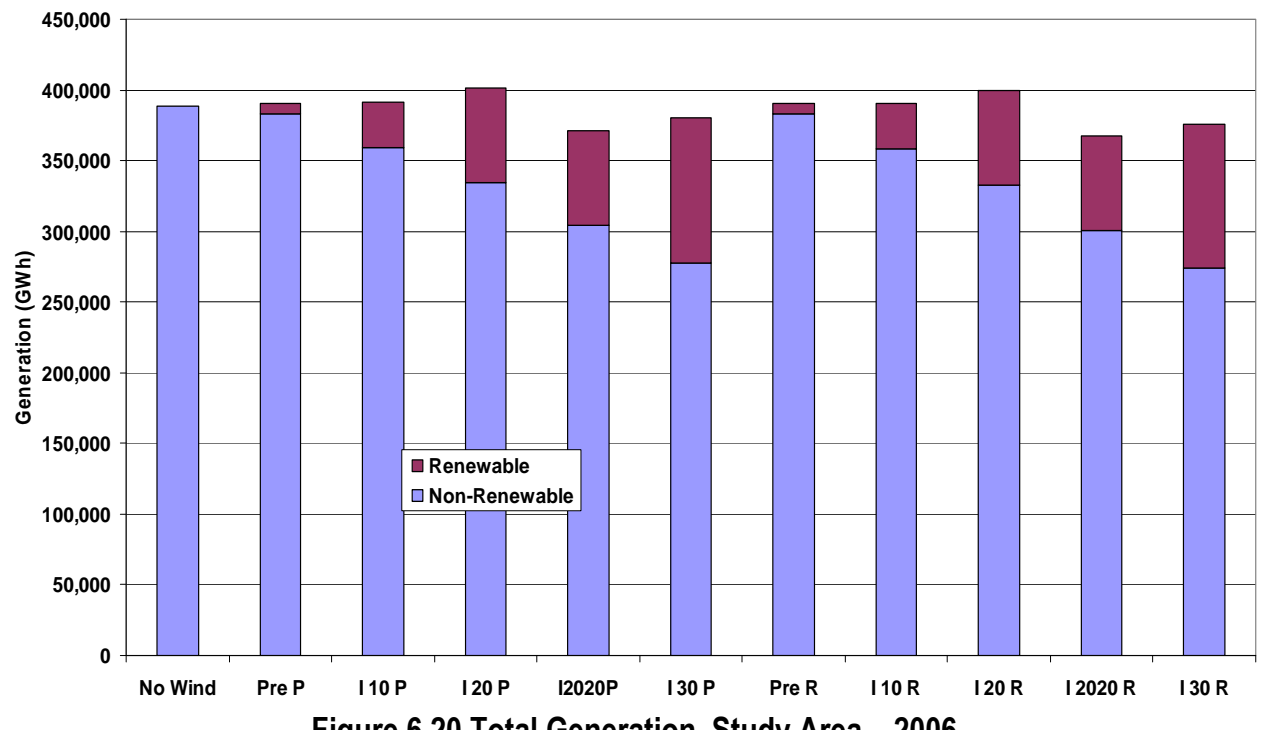

Figure 6.20 Total Generation, Study Area - 2006

Figure 6.21 shows the generator revenue for the study area. The revenue is calculated by multiplying the hourly generation times the hourly spot price at each location and then 
summing this product over the entire year. Although the total generation stayed roughly constant, the revenue drops as renewable penetration increases because the spot prices are decreasing.

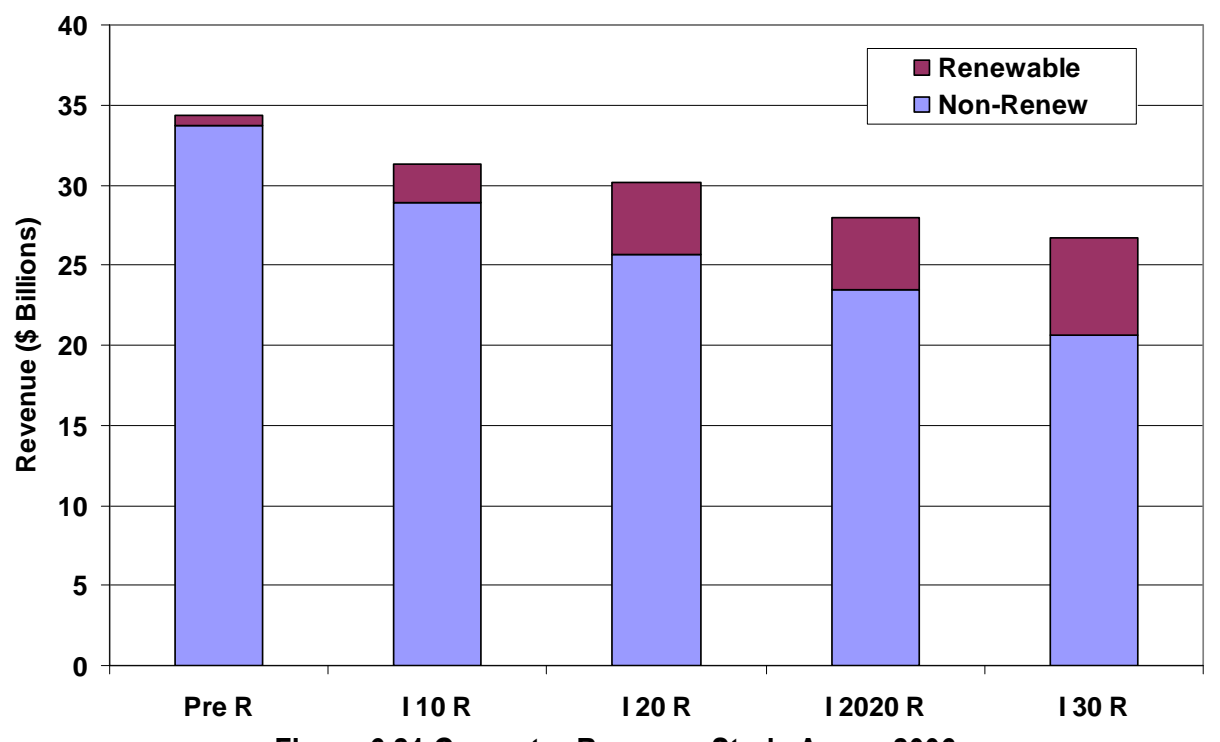

Figure 6.21 Generator Revenue, Study Area - 2006

Earlier figures looked at the value of the renewable generation based on the change in overall operating costs. We can also look at the value based on the hourly spot price of the generation. This also allows differentiation between the different types of renewable generation since each plant has its own spot price and hourly dispatch pattern. Figure 6.22 shows the change in revenue per the change in renewable energy between the penetration levels. These are similar to the values shown in Figure 6.8, but now they are being calculated on a spot price basis rather than displaced operating costs. Note that the wind has a lower value than solar because much of the wind energy occurs in the nighttime when spot prices are lower. The low spot prices in the $30 \%$ penetration case drive down the value of all of the renewables so that the incremental value drops particularly low. Figure 6.23 shows similar results for all of WECC. 


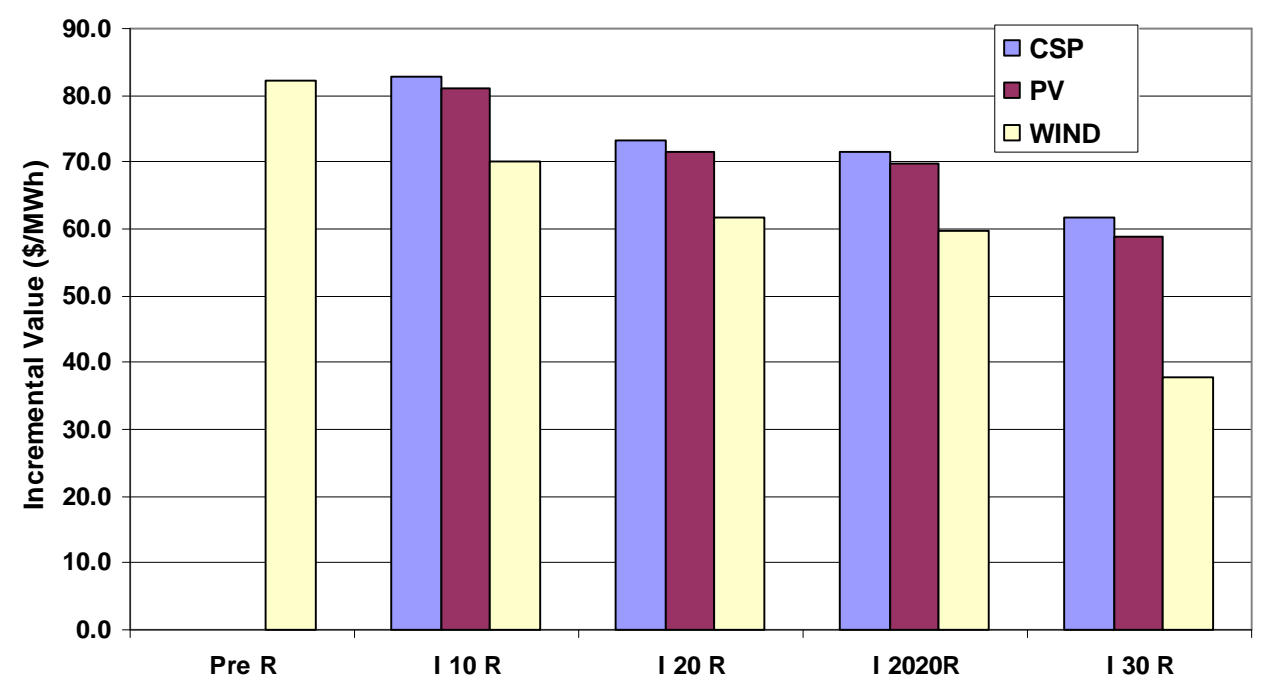

Figure 6.22 Incremental Value of Renewable Energy Based on Spot Price Revenue, Study Area

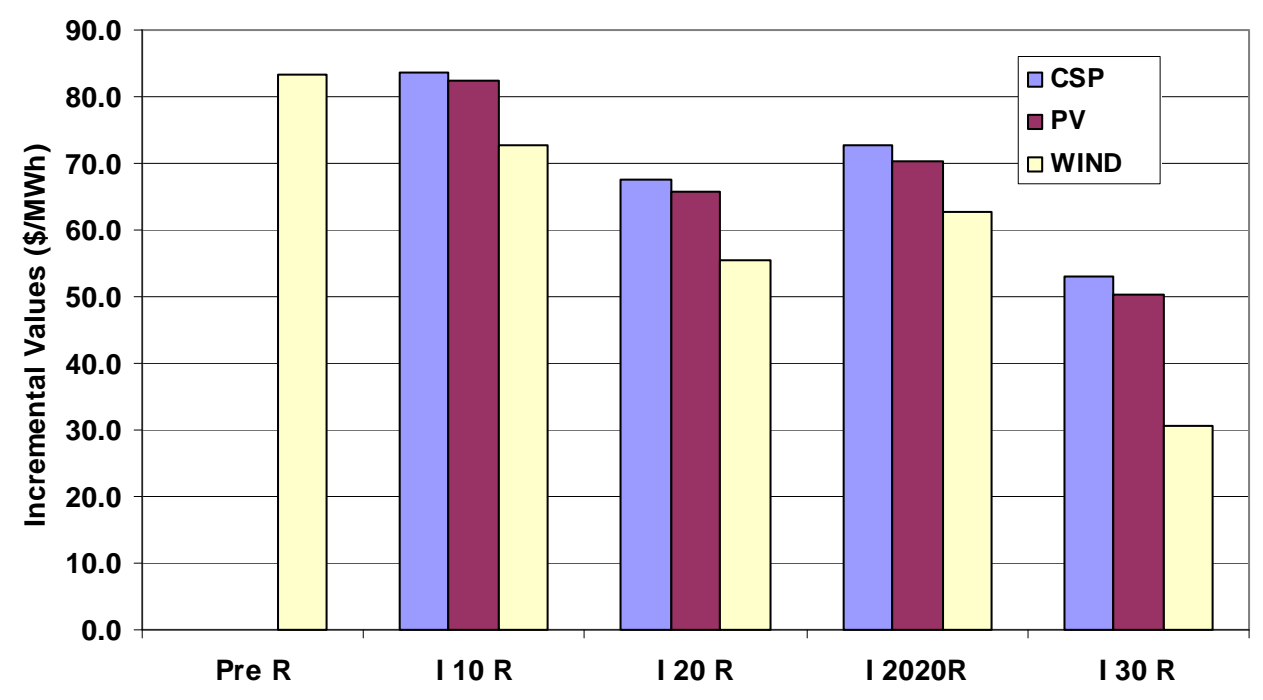

Figure 6.23 Incremental Value of Renewable Energy Based on Spot Price Revenue, WECC

Figure 6.24 shows the total WECC emission reductions as the renewable penetration is increased. These correspond to the reductions in generation shown in Figure 6.3. The SOX emission reductions only occur in the higher penetrations when coal generation is displaced. To put these values in perspective, the emission reductions in the In-Area, $30 \%$ case for NOX, SOX and CO2 represent reductions of $14 \%, 6 \%$ and $23 \%$ respectively from the base-case. Figure 6.25 expresses the emission reductions per MWh of renewable generation. 


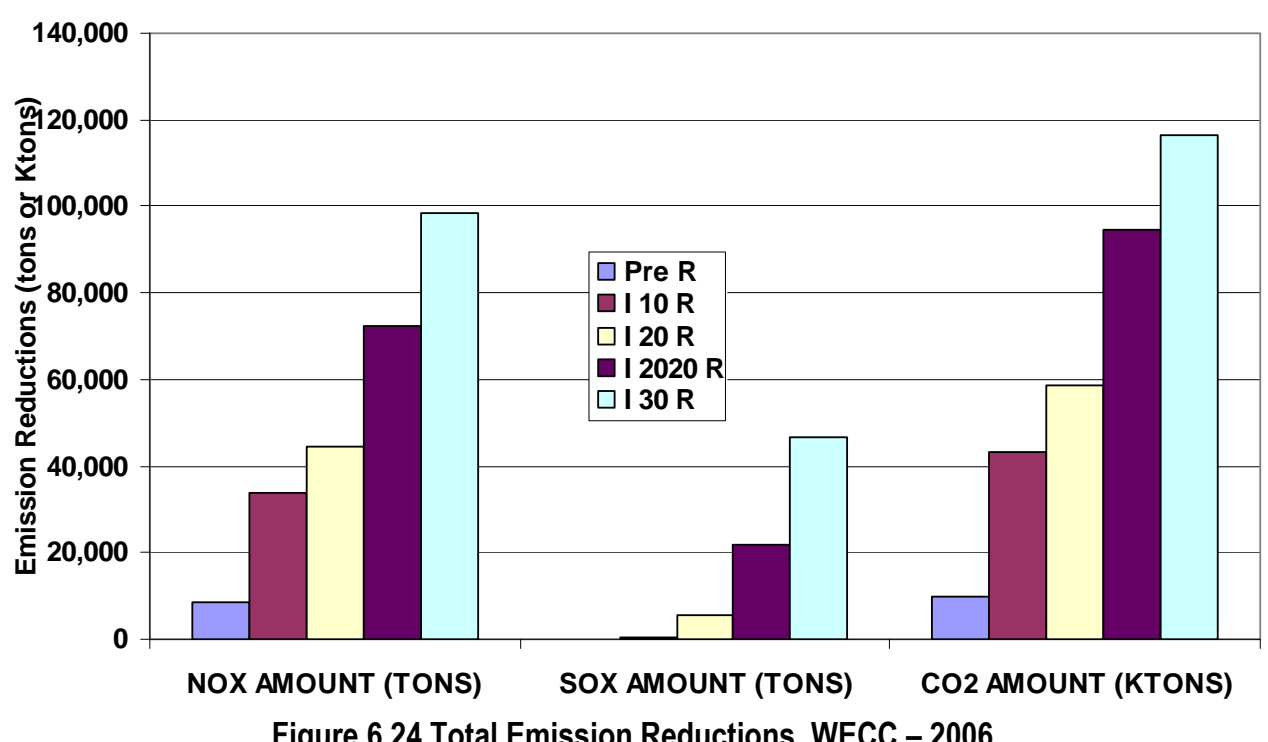

Figure 6.24 Total Emission Reductions, WECC - 2006.

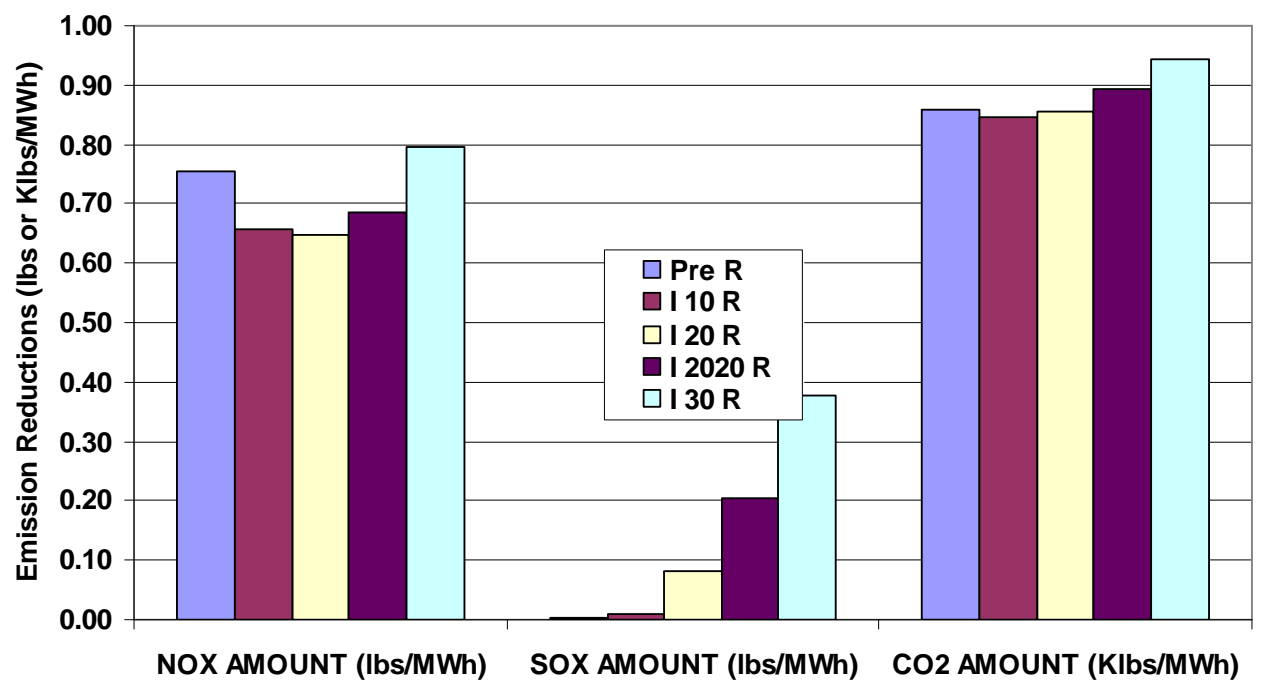

Figure 6.25 Total Emission Reductions Per MWh of Renewable Generation, WECC - 2006

\subsubsection{Spinning Reserve}

Figure 6.26 shows a distribution of the annual wind forecast error for the study footprint and all of WECC. Although the averages were near zero, the extremes were rather large. When the wind is over-forecasted, insufficient thermal generation may be committed to meet the load. The base-cases held the regional spinning reserve requirements constant at $3 \%$ of the load, regardless of the amount of renewable generation. Spinning reserves and quick-start generation could respond to cover the shortfall, but at times, they might not be sufficient. Conversely, when the wind is under-forecasted, then excess thermal generation may be committed, which could lead to minimum operating constraints and possibly the need to spill or export the excess wind energy. Although there was some forecast error associated with the solar generation as well, the bulk of the error was due to the wind. 


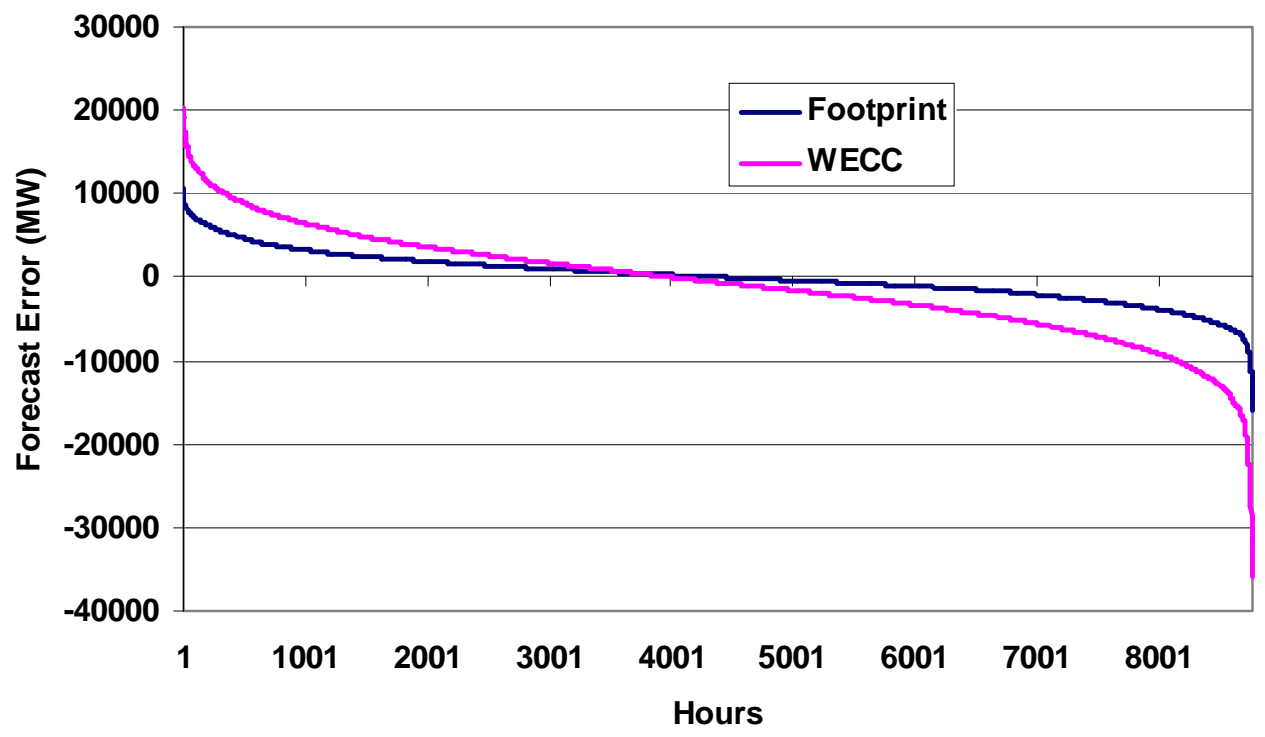

Figure 6.26 Adjusted Wind Forecast Error, I30R

One way to reduce the shortages due to the over-forecasting of the wind would be to increase the amount of spinning reserve planned in the day-ahead unit commitment. The spinning reserve was increased proportional to the day-ahead wind forecast. At the $25 \%$ level, this would increase the total WECC spinning reserve by over $13,000 \mathrm{MW}$ in some high wind hours. Figure 6.27 shows the resulting impact on curtailed energy and contingency reserve shortfalls for the $30 \%$ case. Increasing the spinning reserve had the desired effect of reducing the reserve shortfalls by roughly an order of magnitude. Figure 6.28 shows a duration curve of how these outages were distributed throughout the year. In the base I30R case, the maximum shortfall was roughly $1300 \mathrm{MW}$ and the maximum number of hours in the year during which there was any reserve shortfall was about 90 hours. These curves can be viewed as the requirements for a demand response program or the operating requirements for additional quick-start, non-spinning reserve. If the area under the curve is divided by the maximum shortfall, we can determine the expected number of hours that each demand response participant would be called upon. For the base system with $1300 \mathrm{MW}$ of load participating in demand response, this represents about 35 hours/year for each participant. If more participants are signed up for the program then the number of hours for each participant will decrease. If fewer participants are signed up, then there will still be some remaining contingency reserve shortfall and the number of hours required per participant will increase. This relationship is depicted in Figure 6.29. 


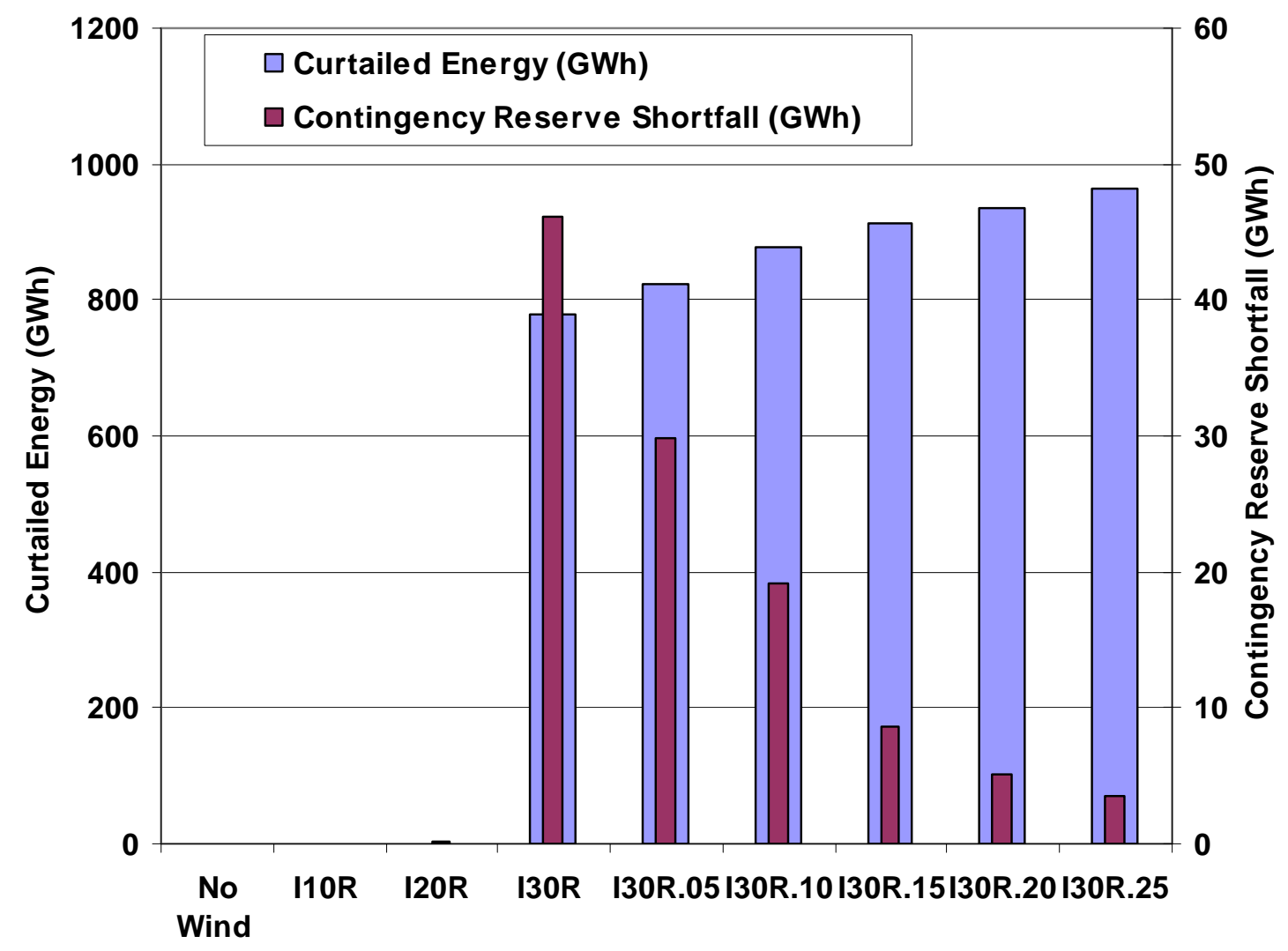

Figure 6.27 Curtailed Energy and Contingency Reserve Shortfall When Discounting Forecast

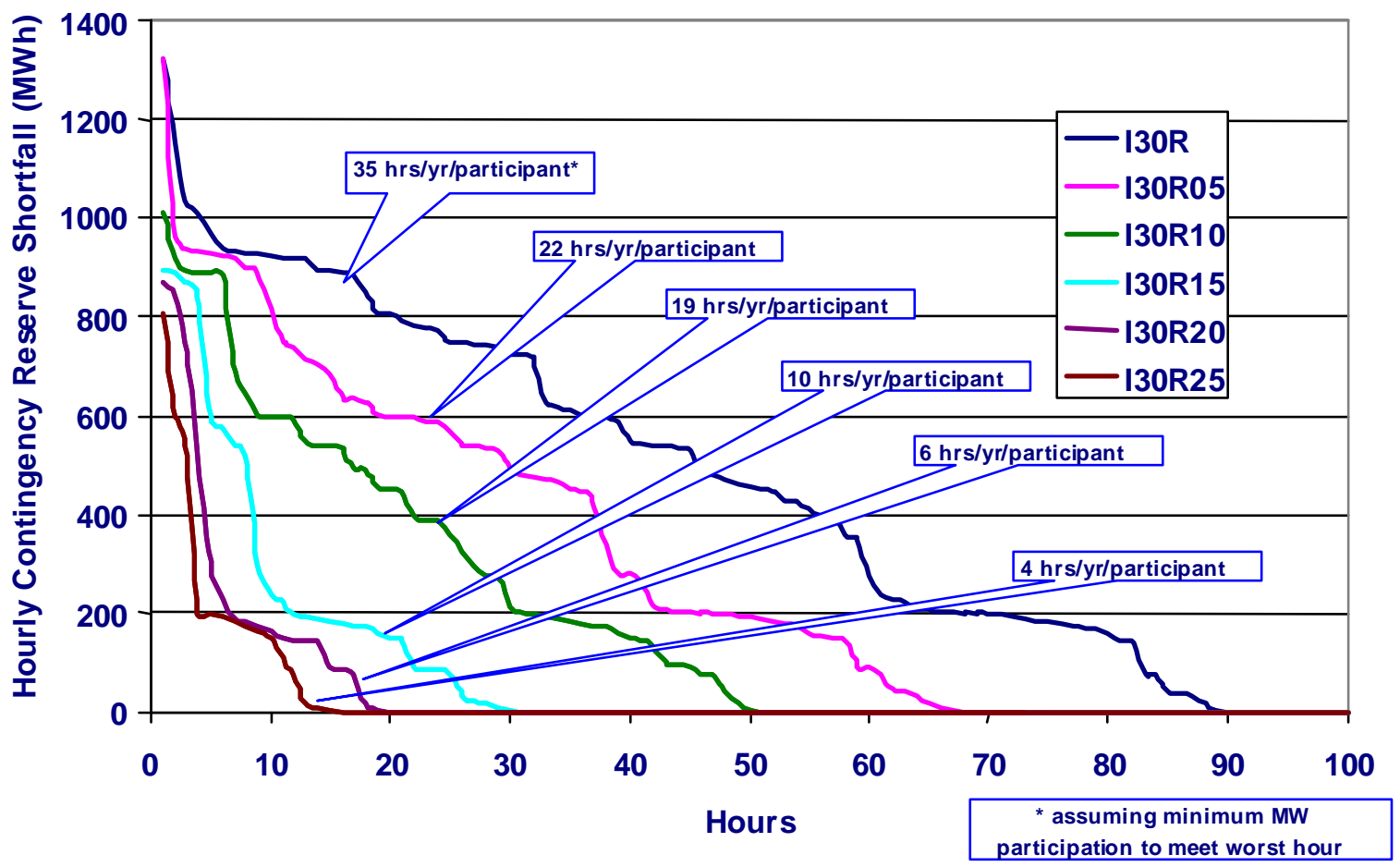

Figure 6.28 Distribution of Contingency Reserve Shortfall vs. Discounting of Wind Forecasts 


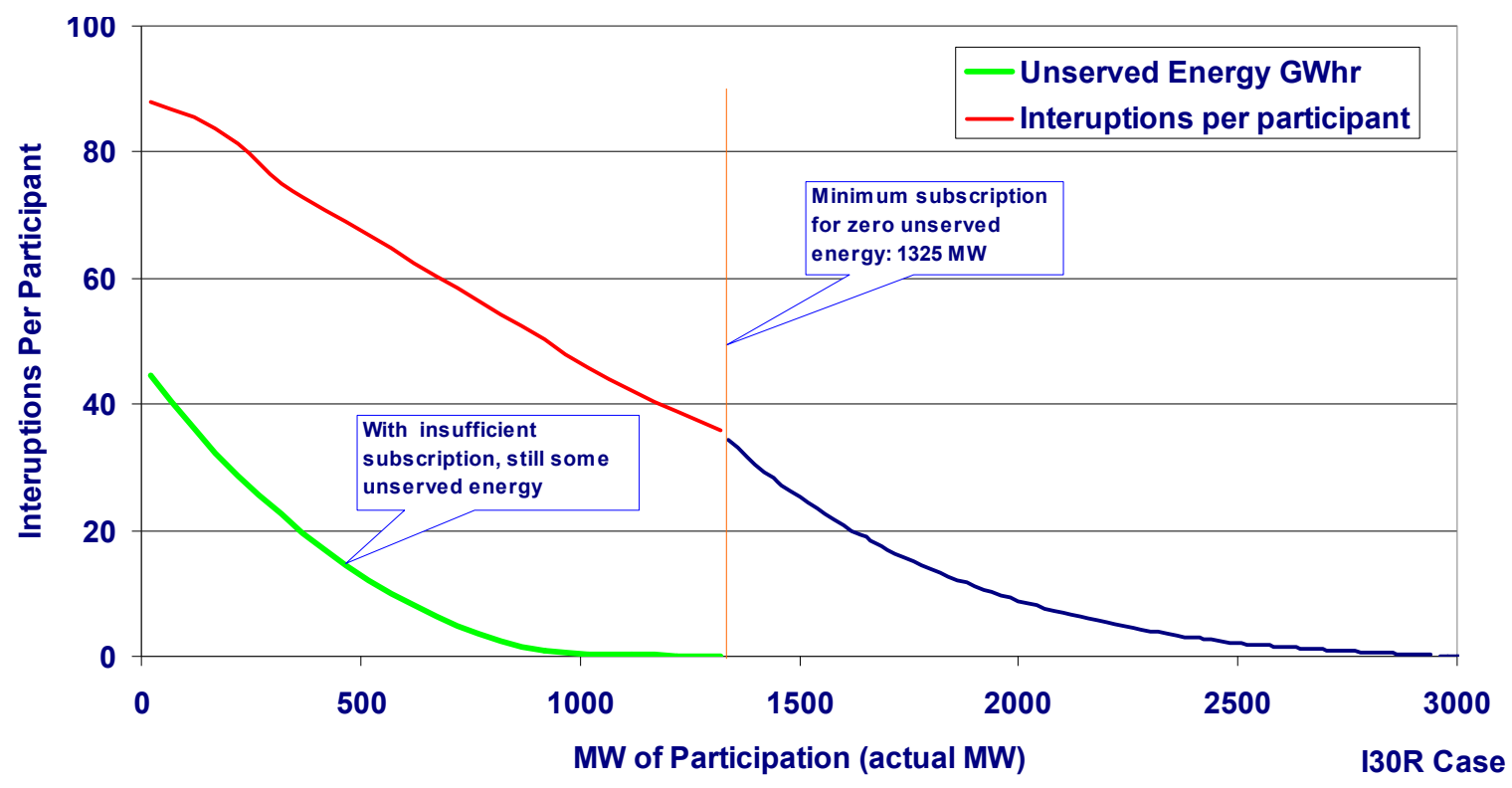

Figure 6.29 Hours of Interruption vs. MW of Demand Response Participation

Figure 6.30 shows the resulting impact on the hourly spot price duration curve for the $30 \%$ case. The impact of increasing the spinning reserve as a function of the forecasted wind is to consistently commit more capacity for the same total load, which will therefore drive the spot prices down and total operating costs up.

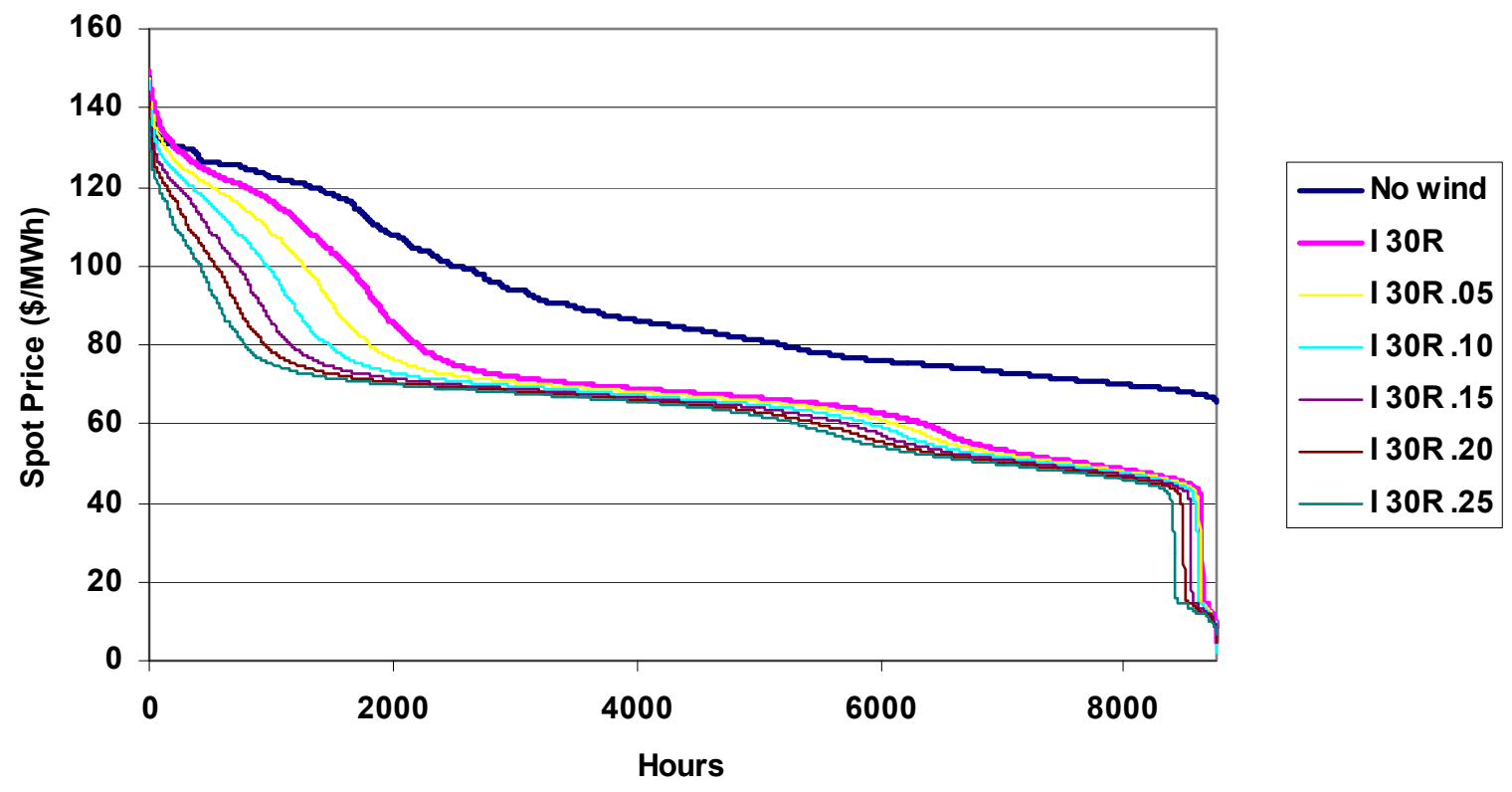

Figure 6.30 Annual Spot Price Duration Curve - I30R - Impact of Discounting Forecast

Figure 6.31 shows the total WECC operating costs for a range of cases with discounted wind forecasts and increased reserves. Although the impact looks relatively small on the scale of the total WECC costs, there is a cost increase for committing to additional spinning reserve. 


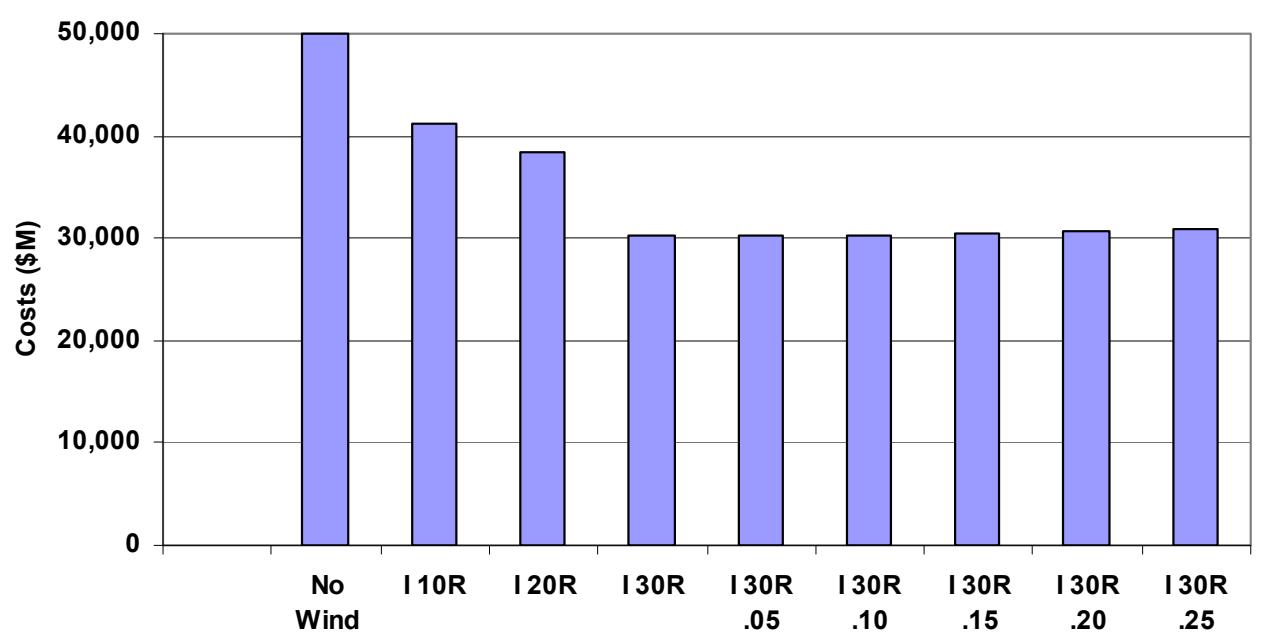

Figure 6.31 Total WECC Operating Costs as a Function of Discounting the Wind Forecast (\$M)

The operating cost increases from Figure 6.31 are combined with the unserved energy reductions in Figure 6.27 to produce Figure 6.32. Increasing the spinning reserve in the commitment by $5 \%$ of the wind forecast increases the total operating costs by about $\$ 50$ million for the year and reduces the unserved energy by about 17 GWh annually.

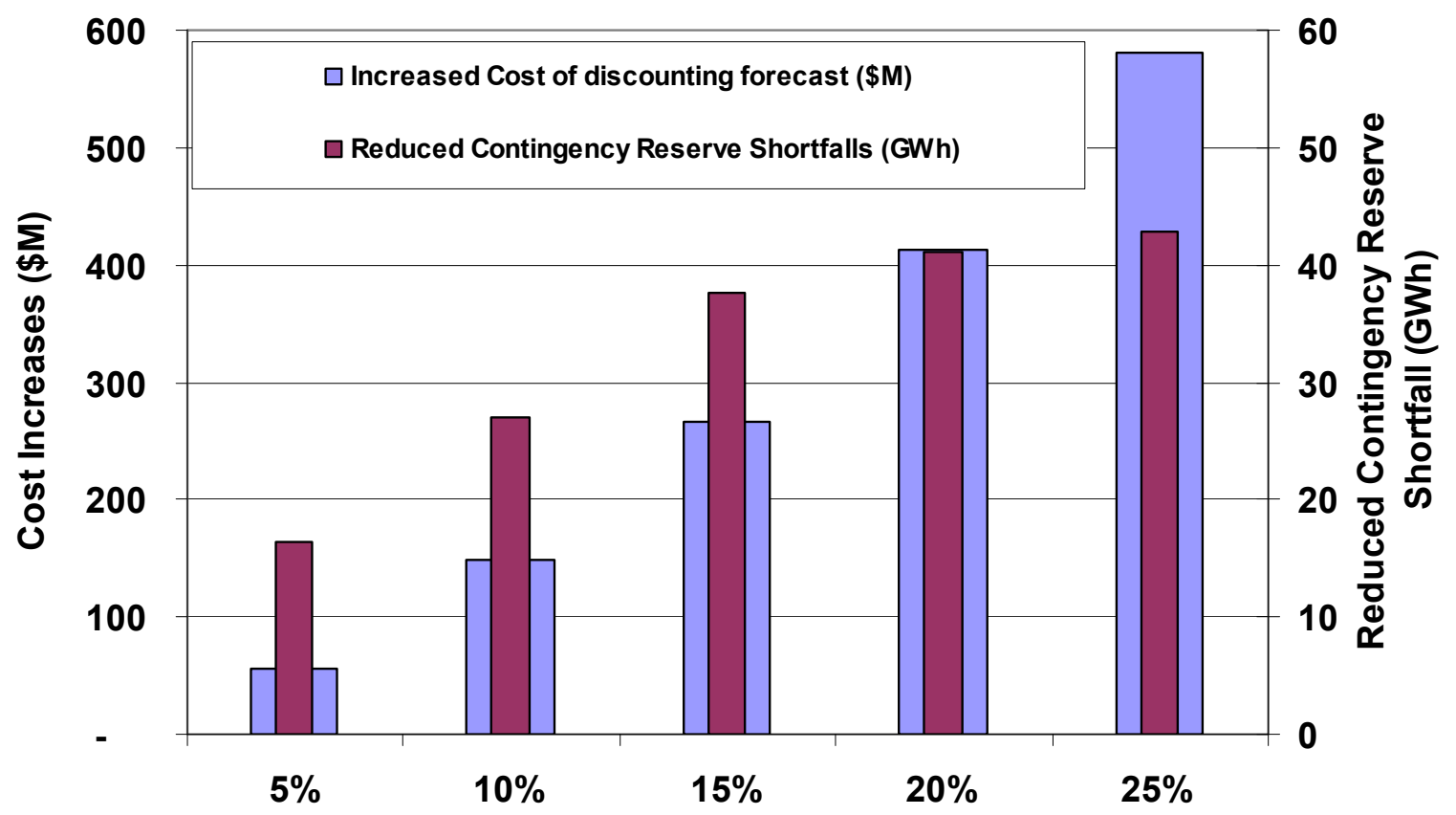

Figure 6.32 Reduced Unserved Energy with Increased Reserves Committed for Wind Generation

Dividing one curve by the other produces the average cost of reducing the contingency reserve shortfall shown in Figure 6.33. Increasing the committed spinning reserve by $5 \%$ of the wind forecast increases the WECC operating costs by over $\$ 3,000$ per MWh of reduced reserve shortfall. This is because the spinning reserve would be increased for all 
of the hours in the year while there were only about 90 hours in which contingency reserve shortfalls occurred. Unfortunately, the 90 hours of shortfall are not known ahead of time. Expressed another way, it would be comparable to pay some of the load $\$ 3,000 / \mathrm{MWh}$ to drop off rather than to increase the spinning reserve by $5 \%$ of the forecast. At the other extreme, to eliminate as much reserve shortfall as could be achieved by increasing the spinning reserve by $25 \%$ would cost an average of roughly $\$ 13,600 / \mathrm{MWh}$ of shortfall. The incremental reduction in contingency reserve shortfall achieved by increasing the spinning reserve from $20 \%-25 \%$ of the forecast would cost over $\$ 100,000 / \mathrm{MWh}$. Clearly, it would be more economically attractive to utilize demand response than to increase the spinning reserves to achieve the same objectives.

Alternatives to increased spinning reserves beyond demand response include increases in quick-start, non-spinning capacity, which would incur a capacity charge but have a minimal operating impact; performing unit commitment 4-6 hours ahead, which could be beneficial because of the lower forecast error in that time frame; and moving to a smarter unit commitment algorithm that learned when these reserve shortfalls were most likely to occur and committed more flexible units.

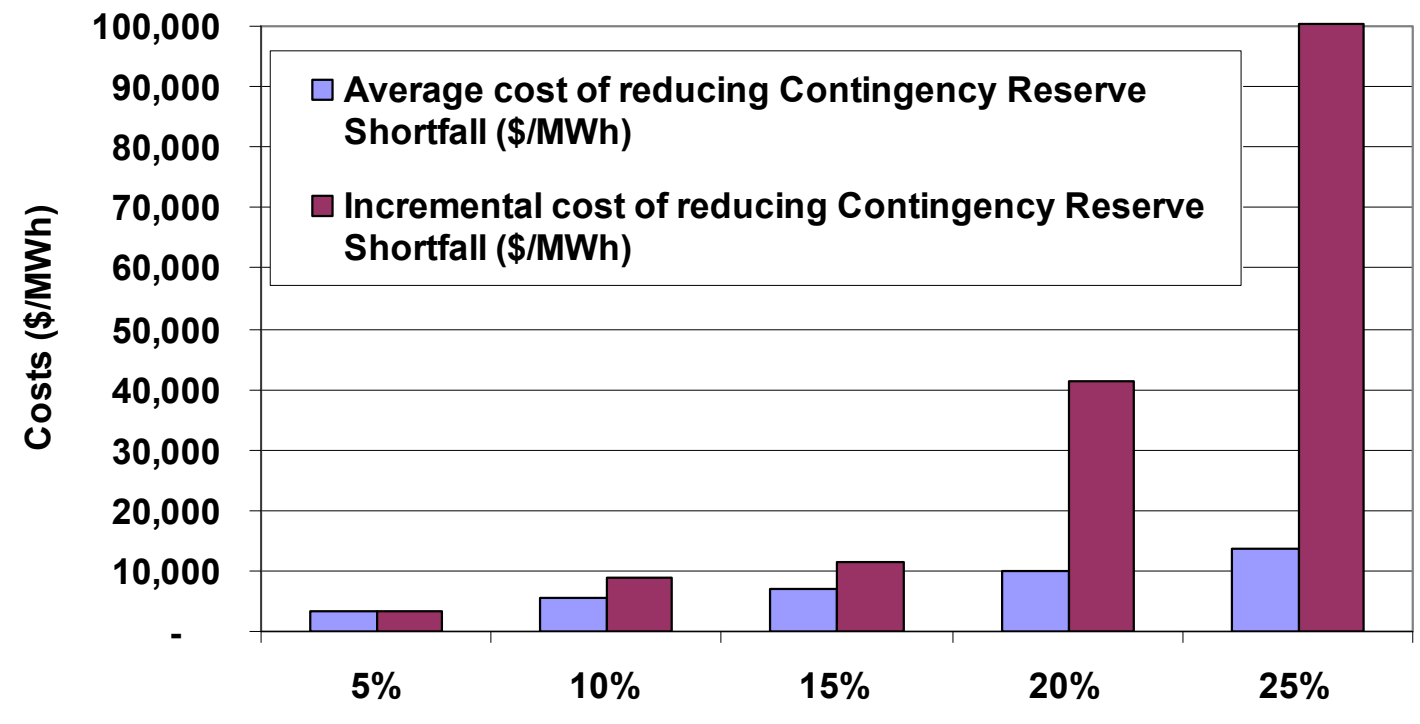

Figure 6.33 Cost of Reducing Contingency Reserve Shortfall by Increasing Reserve Commitment for Renewables.

Another way of looking at the cost increase is to divide the dollars in Figure 6.32 by the amount of wind energy that is provided. This result is shown in Figure 6.34. This could be viewed as a subset of the "integration cost" that is often calculated for renewable energy. For example, if it is determined that spinning reserves need to be increased by $10 \%$ of the forecasted wind, then that would result in a cost of about 70 cents per MWh. This would reduce the savings shown in Figure 6.7 by less than 1\%. Chapter 7 examines intra-hour operation and the possible need for increases in the spinning reserve requirements to address the volatility and uncertainty of renewable energy. 


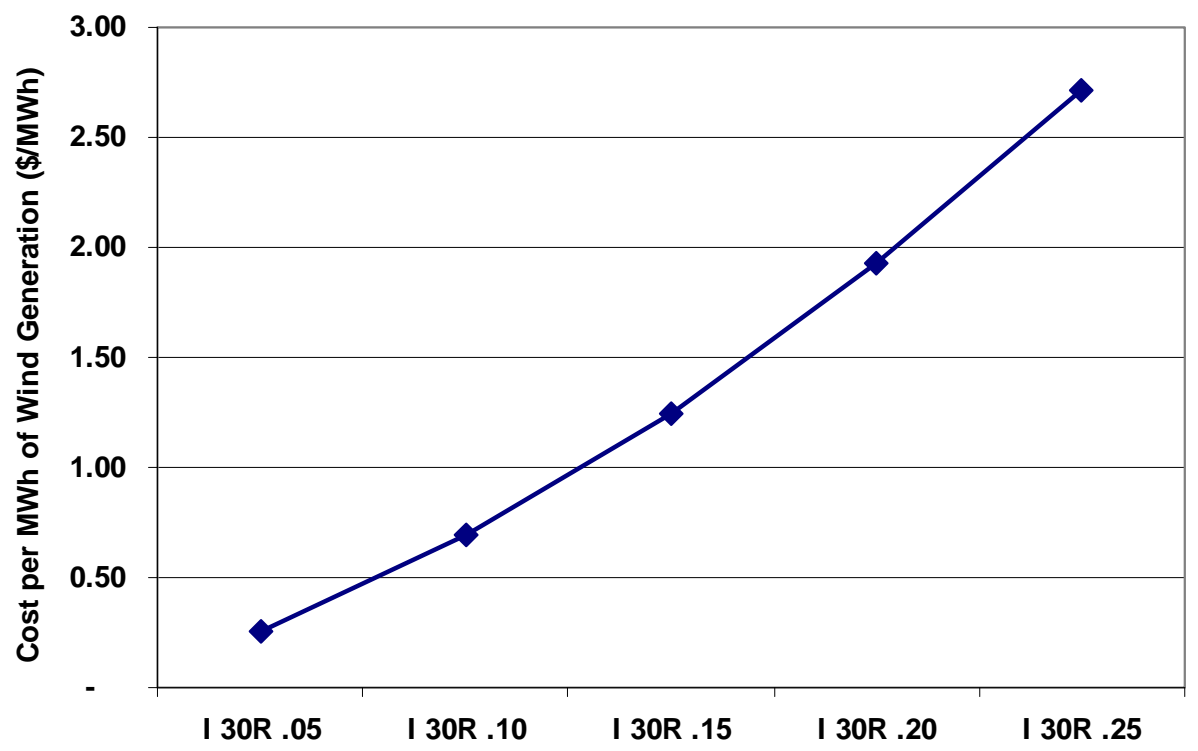

Figure 6.34 Cost of Increasing Committed Spinning Reserve Per MWh of Wind Generation (\$/MWh)

\subsubsection{Annual Profile Sensitivities}

Most of the analysis was performed using the load, wind, and solar profiles from 2006. All of the primary cases were also run using the shapes from 2004 and 2005. Figure 6.35 shows WECC generation by type for the no-wind, and 10, 20, and 30 percent penetration levels with S-o-A forecasts for the 3-shape years (key: last digit of case name refers to the year). The slight variation between the cases seems to be driven by the variation in wind energy for the three years. This annual variation in renewable generation was discussed in Chapter 4, but is repeated here to explain the variations in system operation. Figure 6.36 shows the generation by renewable type for the $30 \%$ case. Both types of solar generation were fairly constant over the three weather years. The wind generation varied about $+/-3 \%$ over the three-year period. The same nameplate capacities were used for all three years.

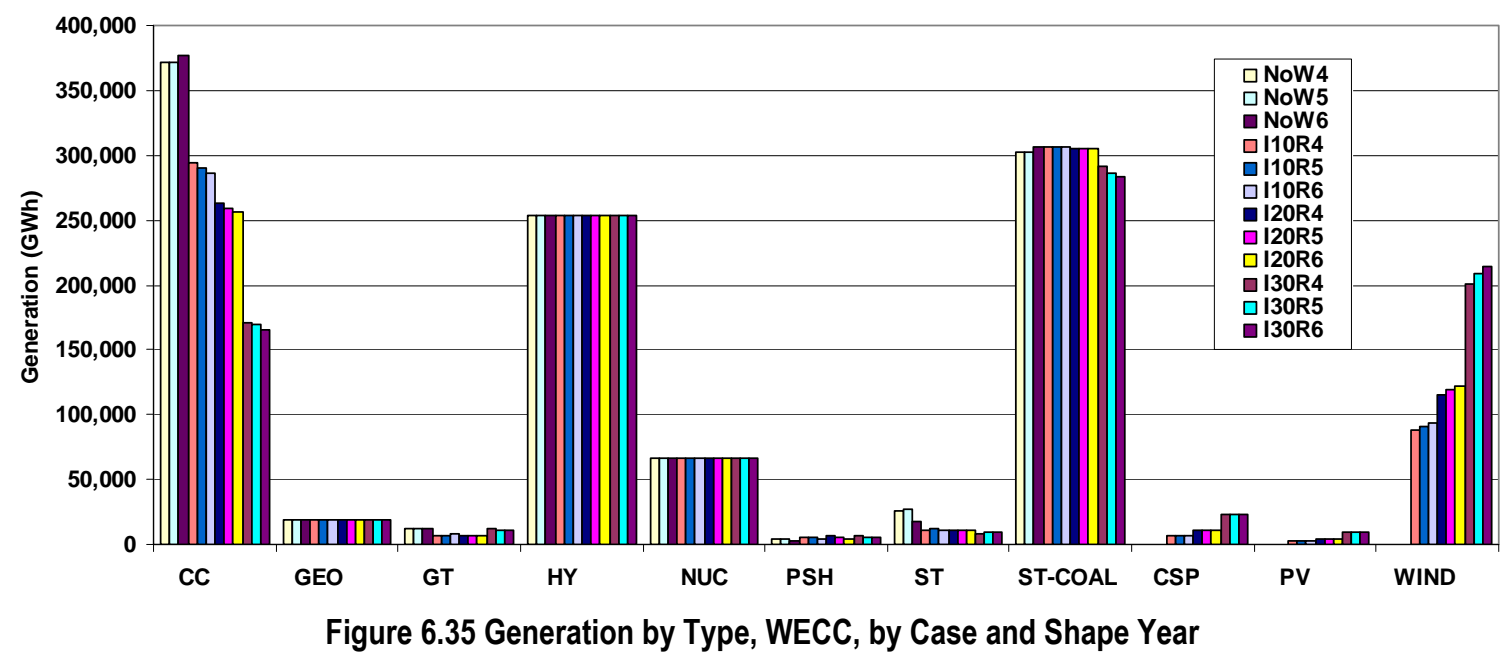




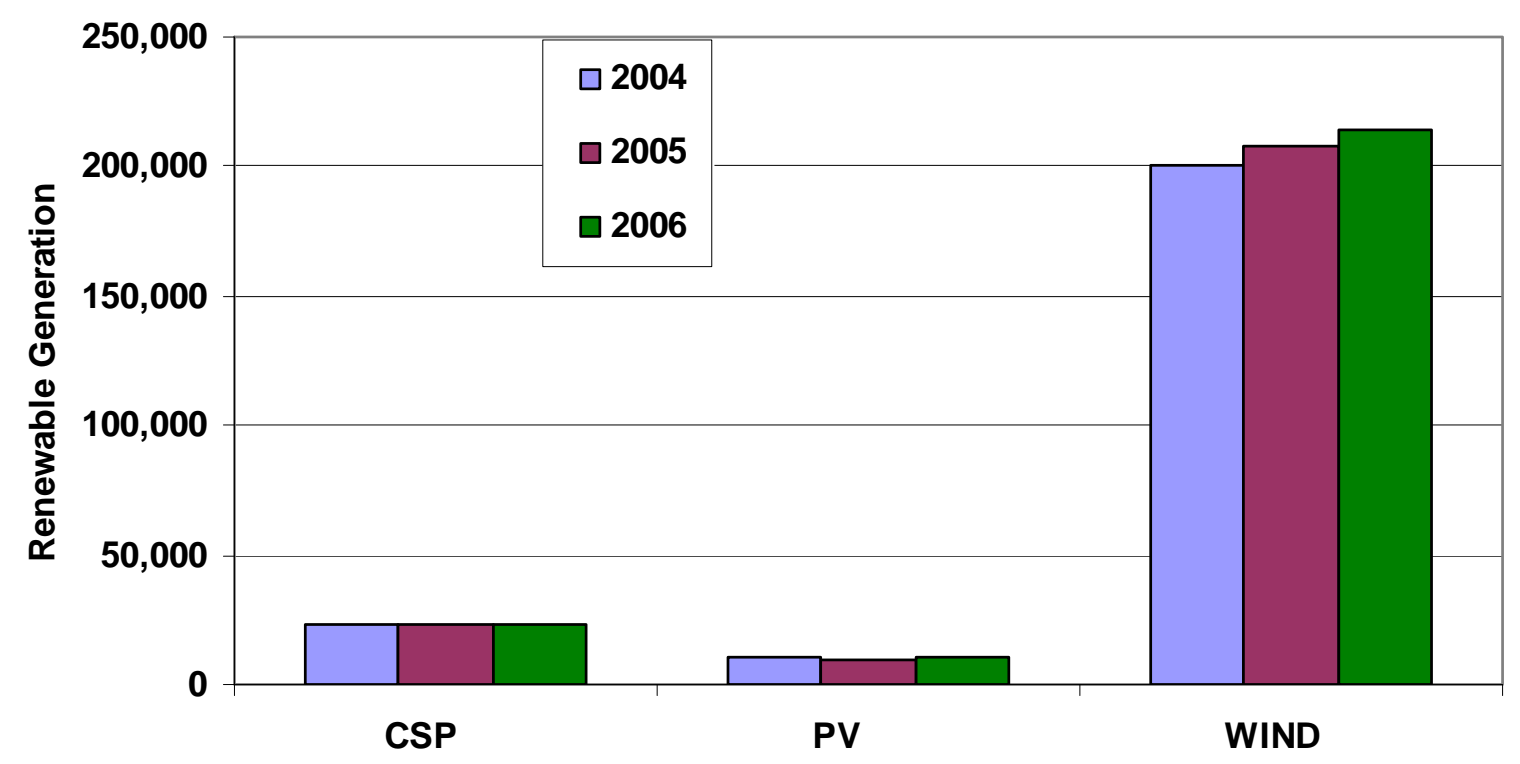

Figure 6.36 Variation in Renewable Generation by Weather Year for 30\% Case

Figure 6.37 shows the variation in the incremental value of the renewable generation as a function of penetration and shape year. The annual variations are within a few dollars per MWh.

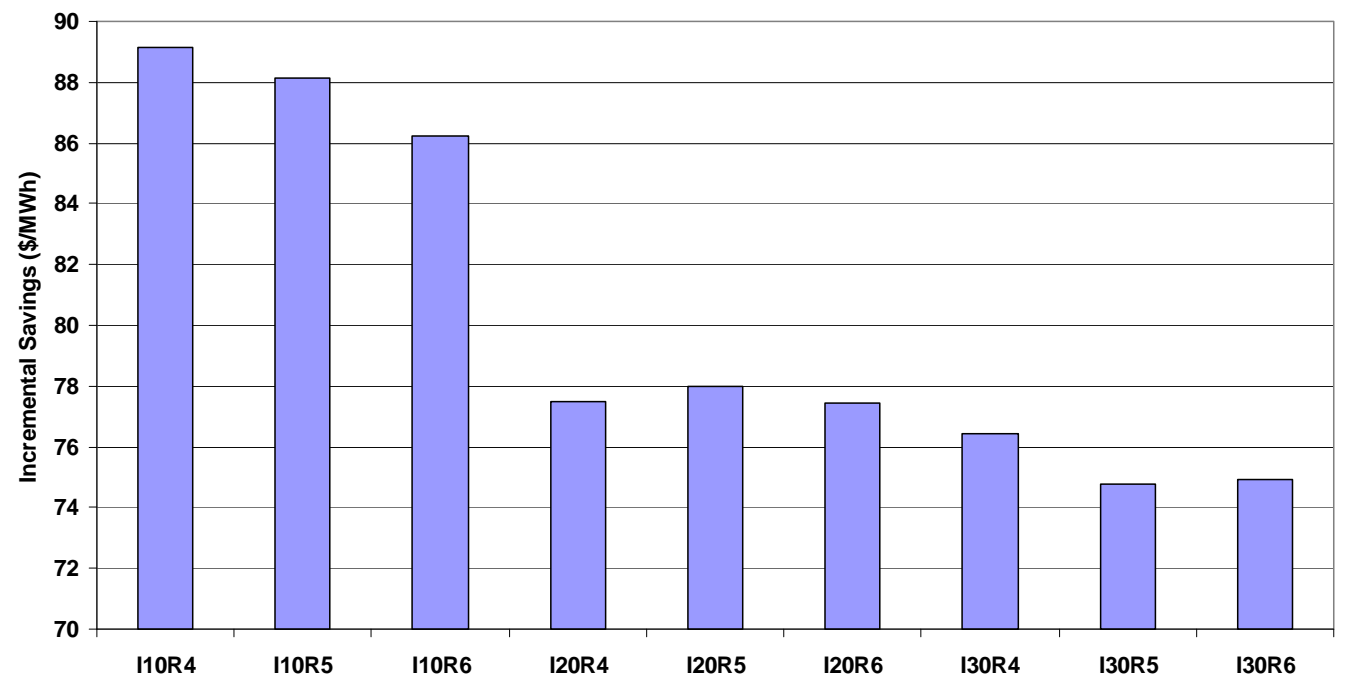

Figure 6.37 Incremental Savings Per Incremental Renewable Energy, WECC

Figure 6.38 shows the total study area generation for the three shape years. Although the renewable generation varied somewhat, the total generation changed by less than $0.1 \%$ from the average value over the three years. 


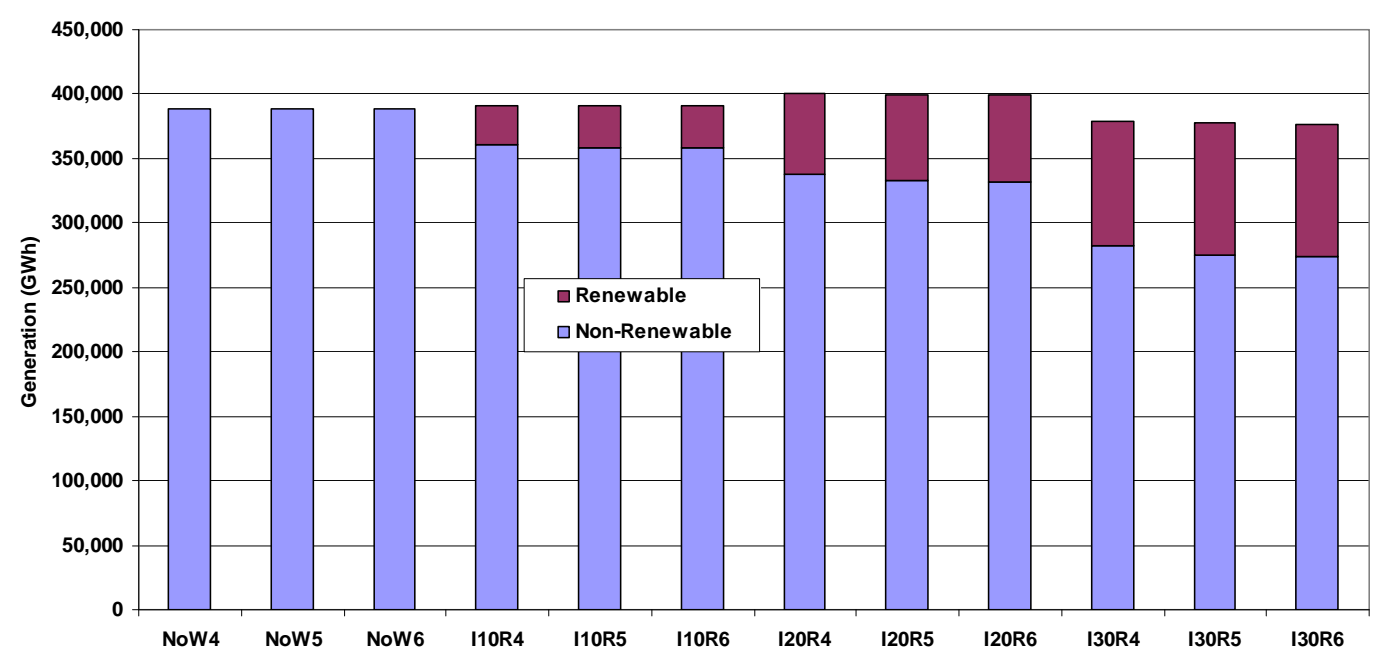

Figure 6.38 Total Generation, Study Area, for Different Annual Shapes

Figure 6.39 shows the change in emissions over the three shape years. The emission reductions tended to be slightly higher in the years with higher renewable generation, as would be expected.

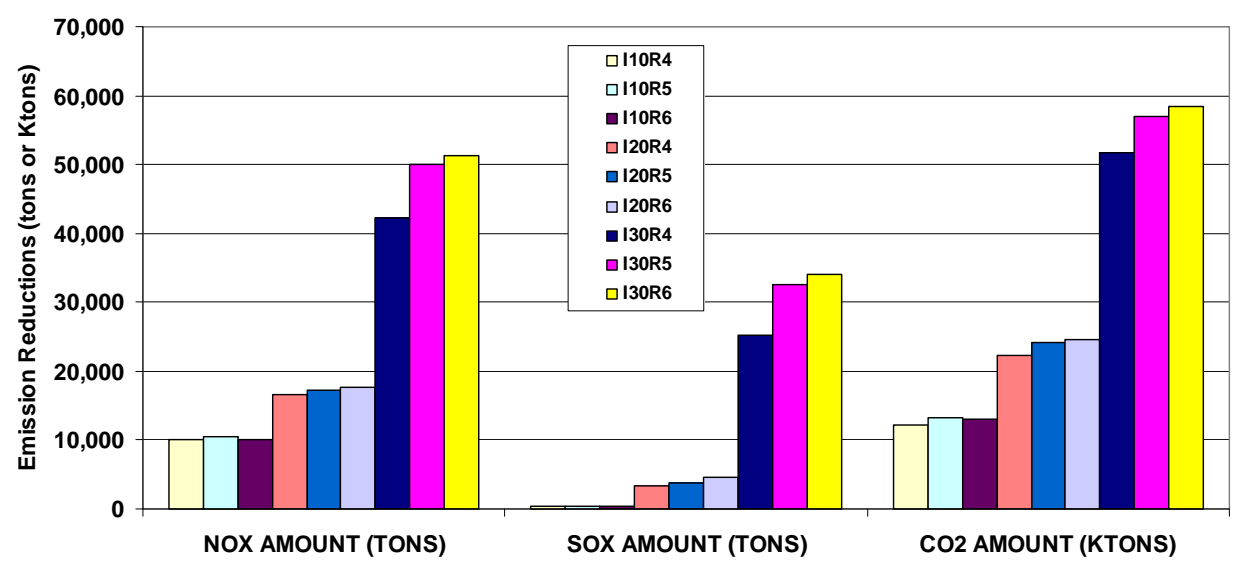

Figure 6.39 Emission Reductions, Study Area, for Different Annual Shapes

\subsection{Weekly Operational Analysis}

The previous section examined the operational impacts of renewable generation from an annual basis. This section examines a spring and summer week to look the changes in operation more closely. The dark blue line in the center of Figure 6.40 shows the hourly study area load for the week of April 10 ${ }^{\text {th }}$. The green line on the bottom is the sum of the Wind, CSP and PV generation inside the study area for each hour for the $10 \%$ penetration level. The teal line $(\mathrm{Load}-\mathrm{W} / \mathrm{C} / \mathrm{P})$ between the two is the hourly "load minus renewables" or net load that must be served. If the study area were not interconnected to the rest of WECC, this is the profile that the rest of the generation in the study footprint would be required to follow. The remaining two curves show the load and net load including the net hourly exports from the study area. Figure 6.41 
shows the operation of the generation by type within the study area when there is no renewable generation present. The top of the curve represents the total load plus exports for the study area. The nuclear generation was flat except for the start of planned maintenance on Saturday. The coal generation was relatively constant, the hydro provided the bulk of the peaking operation and the combined-cycle filled in the intermediate operation.

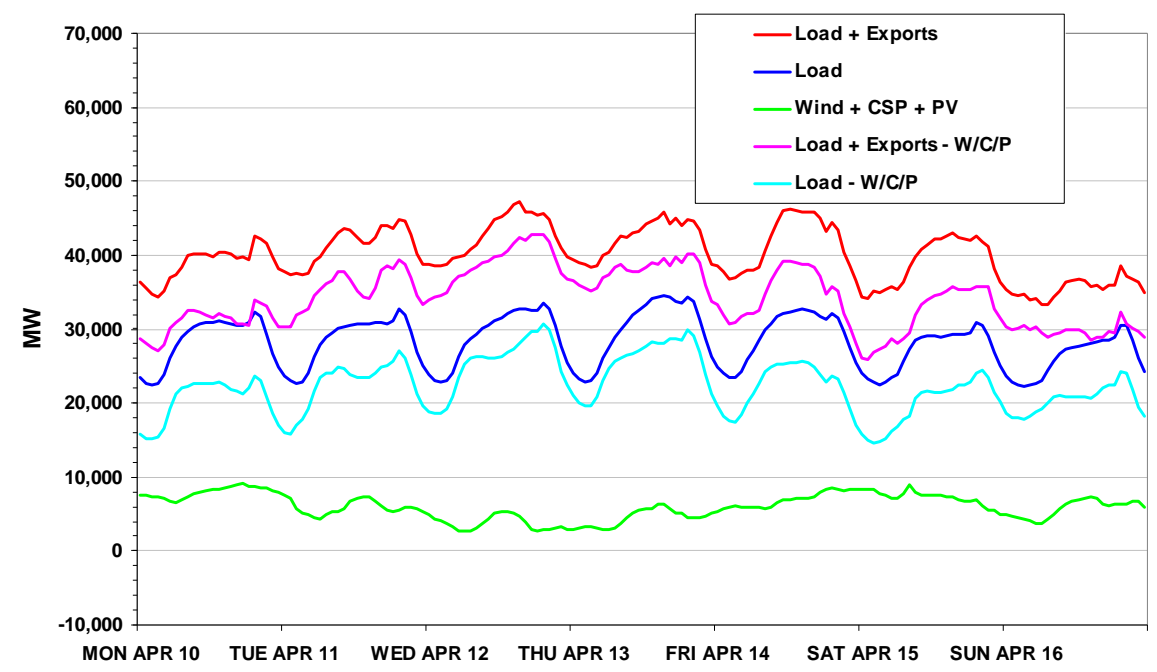

Figure 6.40 Week of April 10th - Hourly Load, Renewables and Exports for $10 \%$ Case

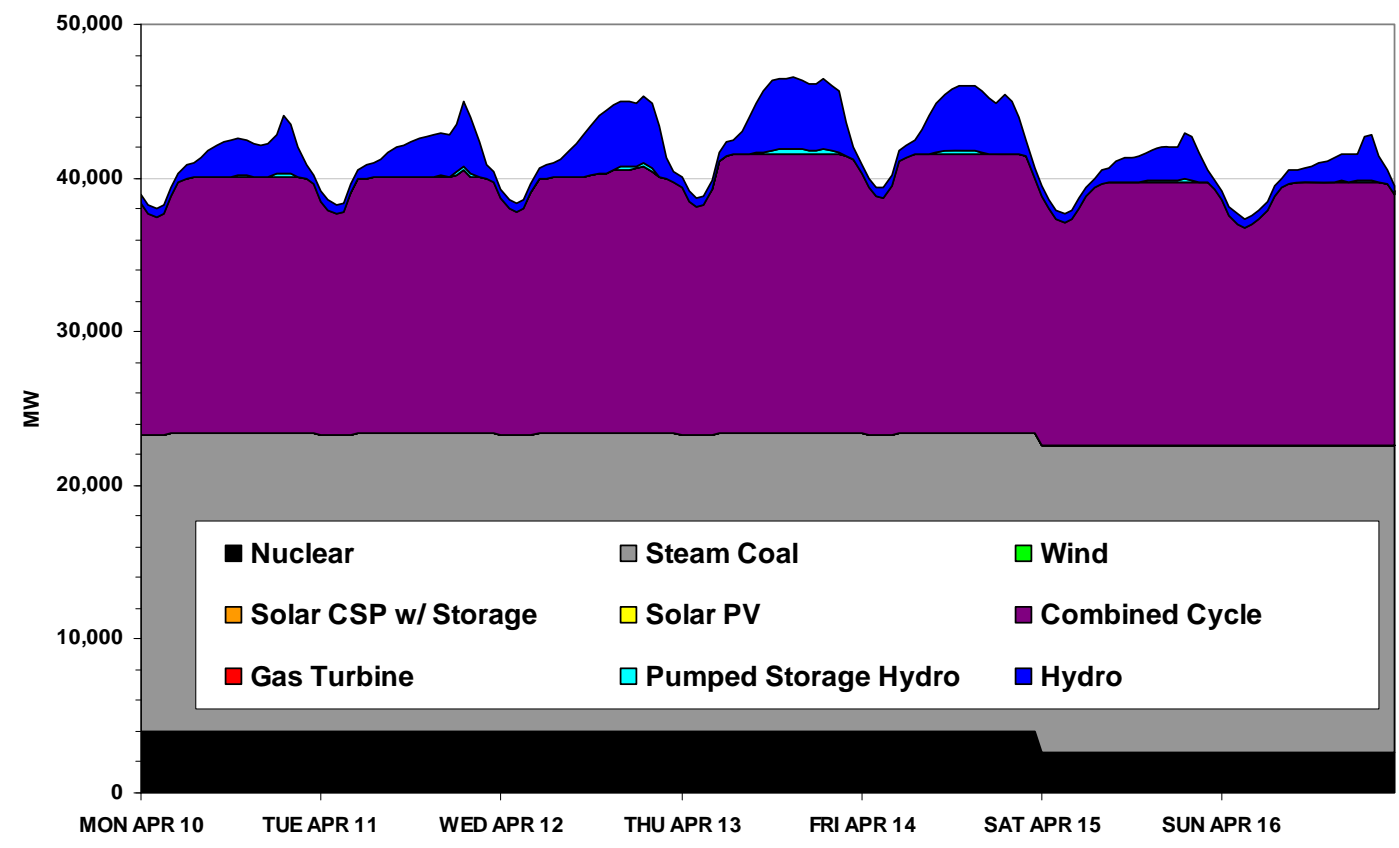

Figure 6.41 Study Area Dispatch - Week of April 10th - No Renewables

Figure 6.42 shows how this operation changed for the $10 \%$ penetration level. Note that the top of the curve changes slightly from the previous figure because the study area exports have changed. The nuclear generation is unchanged and the coal is still mostly 
constant but the dispatch of the other generation has begun to change. The most noticeable shift is the introduction of the green band, which represents the wind generation. Thin orange and yellow bands can also be seen for the solar generation. The hydro generation has shifted slightly. Each hydro plant was scheduled to meet specific monthly energy targets. Introduction of renewable generation could cause the hydro to shift the hourly schedule but the monthly energy production would remain constant. More consideration of the hydro will be discussed in Section 6.4.2. The bulk of the displacement came from the combined-cycle units, which is consistent with what was seen on an annual basis. Also, it is interesting to note the small patch of red introduced on Wednesday. This is simple-cycle gas turbine operation caused by an over-forecast of the wind generation. When the expected wind generation did not materialize some of the imbalance was provided by generation on units already committed. However, it was necessary to turn on some quick-start peaking generators to balance the mismatch. As shown earlier, more combined-cycle units could have been committed in those hours in anticipation of an over-forecast. This would have lowered the fuel costs in this particular hour but would be less economic overall due to the over commitment in other hours. Also note that the total generation each hour changed slightly from the previous figure. This was because the exports changed when the renewable generation was added.

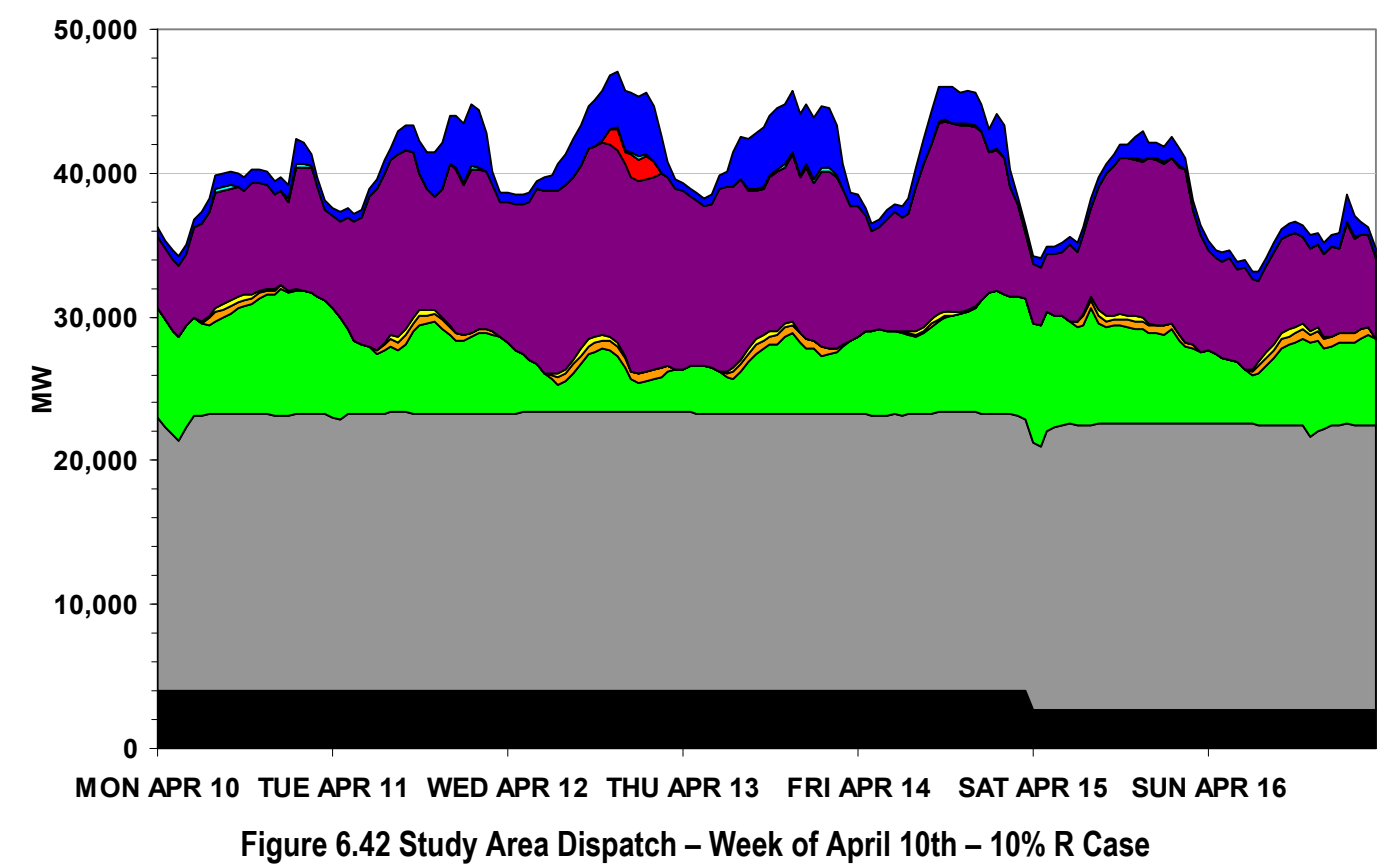

Figure 6.43 compares the hourly load profile for the week to the sum of the renewable generation in the study area for the $10 \%, 20 \%$, and $30 \%$ cases. Note how the $30 \%$ wind + solar profile crosses over the load profile on Saturday, and nearly crosses over on Tuesday. Figure 6.44 subtracts the renewable generation from the load to present the load and net loads for the various penetrations. Finally, Figure 6.45 adds the regional exports to the net loads. This is the total generation needed from the non-renewable 
generators in the study area. Although this net demand dips lower as the renewable generation increases, it never goes negative.

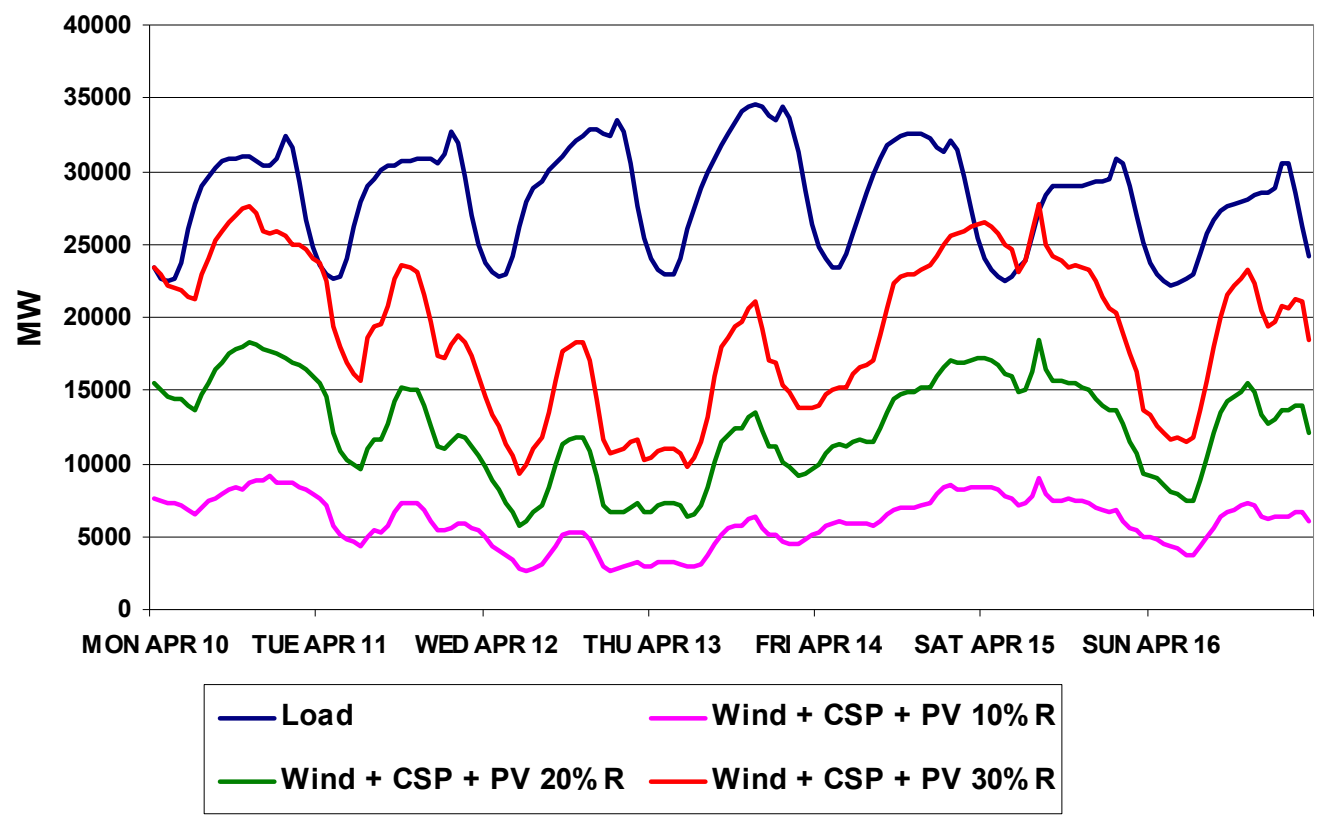

Figure 6.43 Study Area - Week of April 10th - Load and Renewables by Penetration

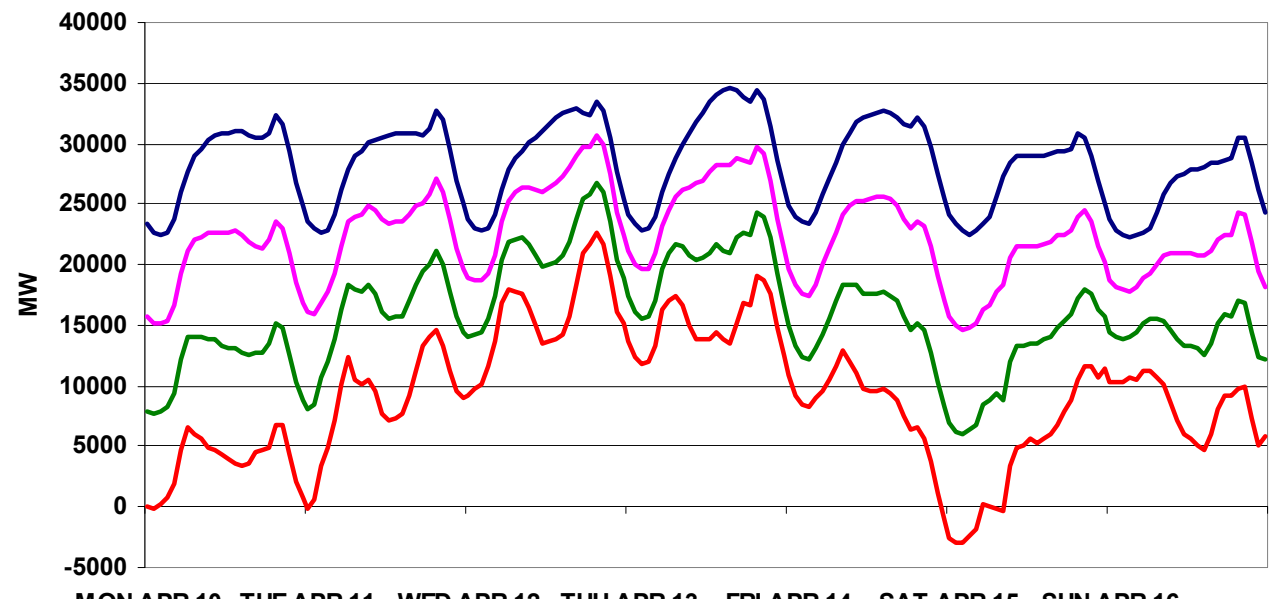

MON APR 10 TUEAPR 11 WED APR 12 THUAPR 13 FRI APR 14 SAT APR 15 SUNAPR 16

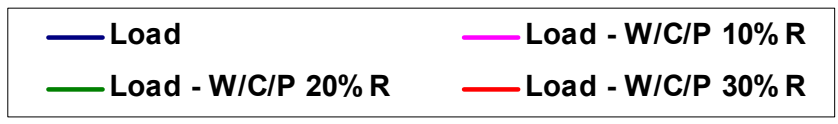

Figure 6.44 Study Area - Week of April 10th - Net Load by Penetration 


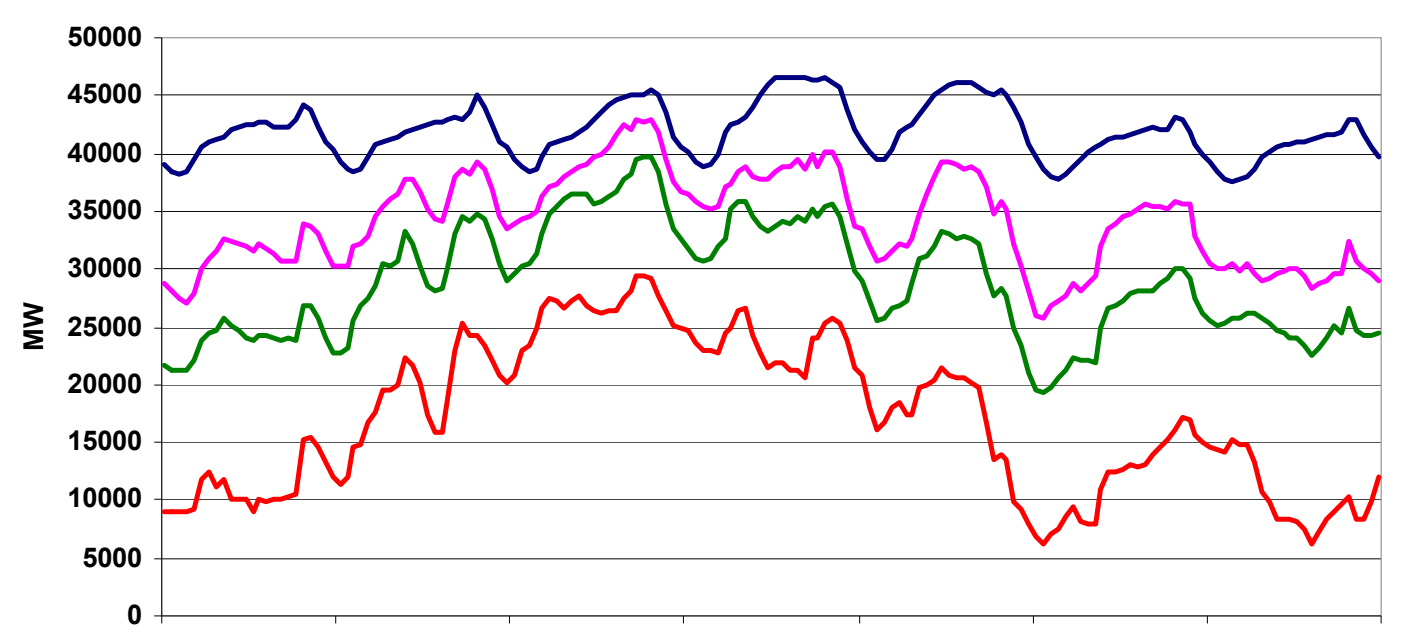

MON APR 10 TUE APR 11 WED APR 12 THU APR 13 FRI APR 14 SAT APR 15 SUN APR 16

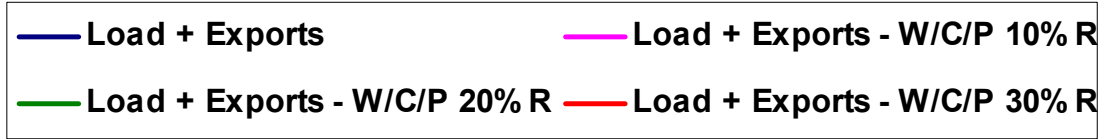

Figure 6.45 Study Area - Week of April 10th - Net Loads Plus Exports by Penetration

Figure 6.46 shows how the generation by type shifts for the $20 \%$ case. The coal is now cycling in the top $10 \%-20 \%$ of its operating range. Not only has the wind generation grown significantly, but the solar generation is now much more noticeable. The combined-cycle operation is now about a third of its production with no renewables on the system.

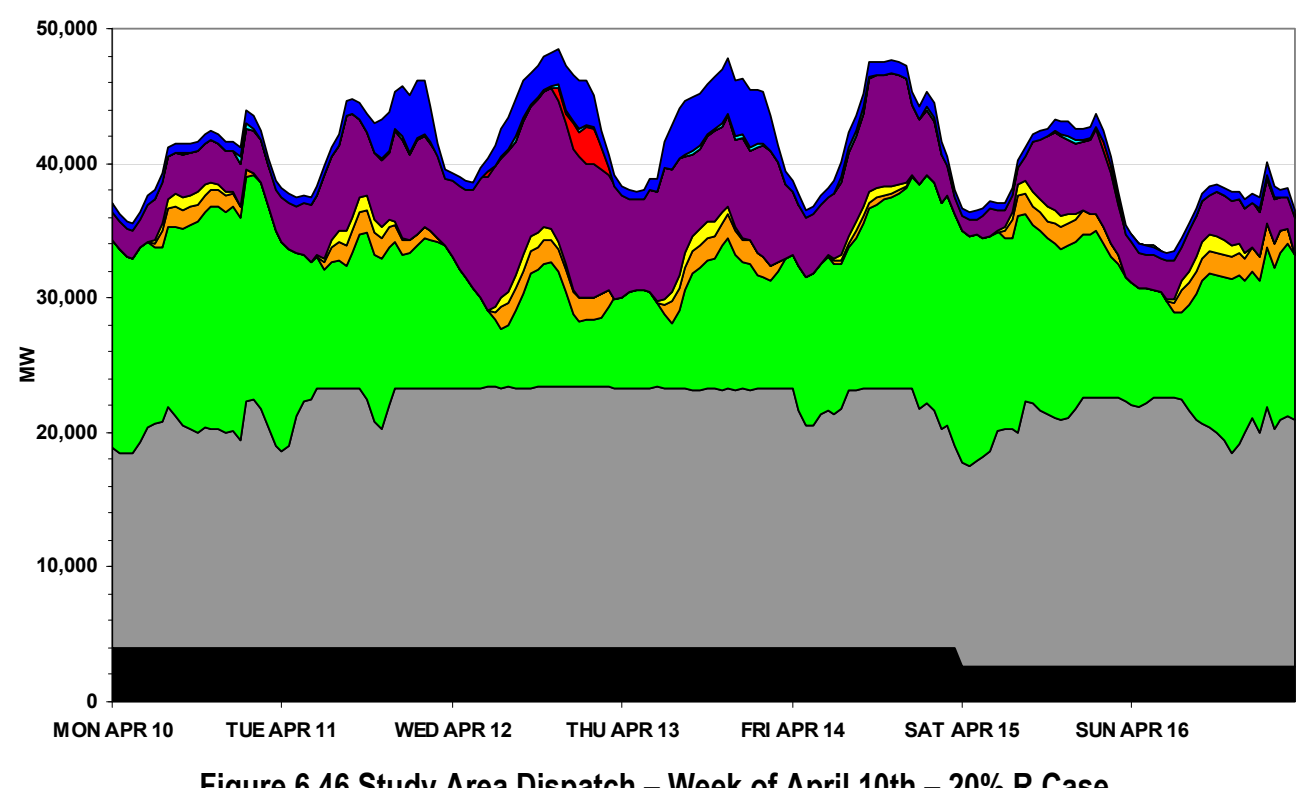

Figure 6.46 Study Area Dispatch - Week of April 10th - 20\% R Case

Figure 6.47 shows the operation under the $30 \%$ penetration case. Combined-Cycle units are now down to about $1 \%$ of their original output and coal generation has dropped 
$40 \%$. In addition to much of the coal being decommitted for the week, the remaining units are required to do a lot more cycling. This may have a significant impact on the life of the generating units and cost of operations. Figure 6.48 shows how the combinedcycle and coal units have changed for this week of operations compared to the case with no-wind or solar generation.
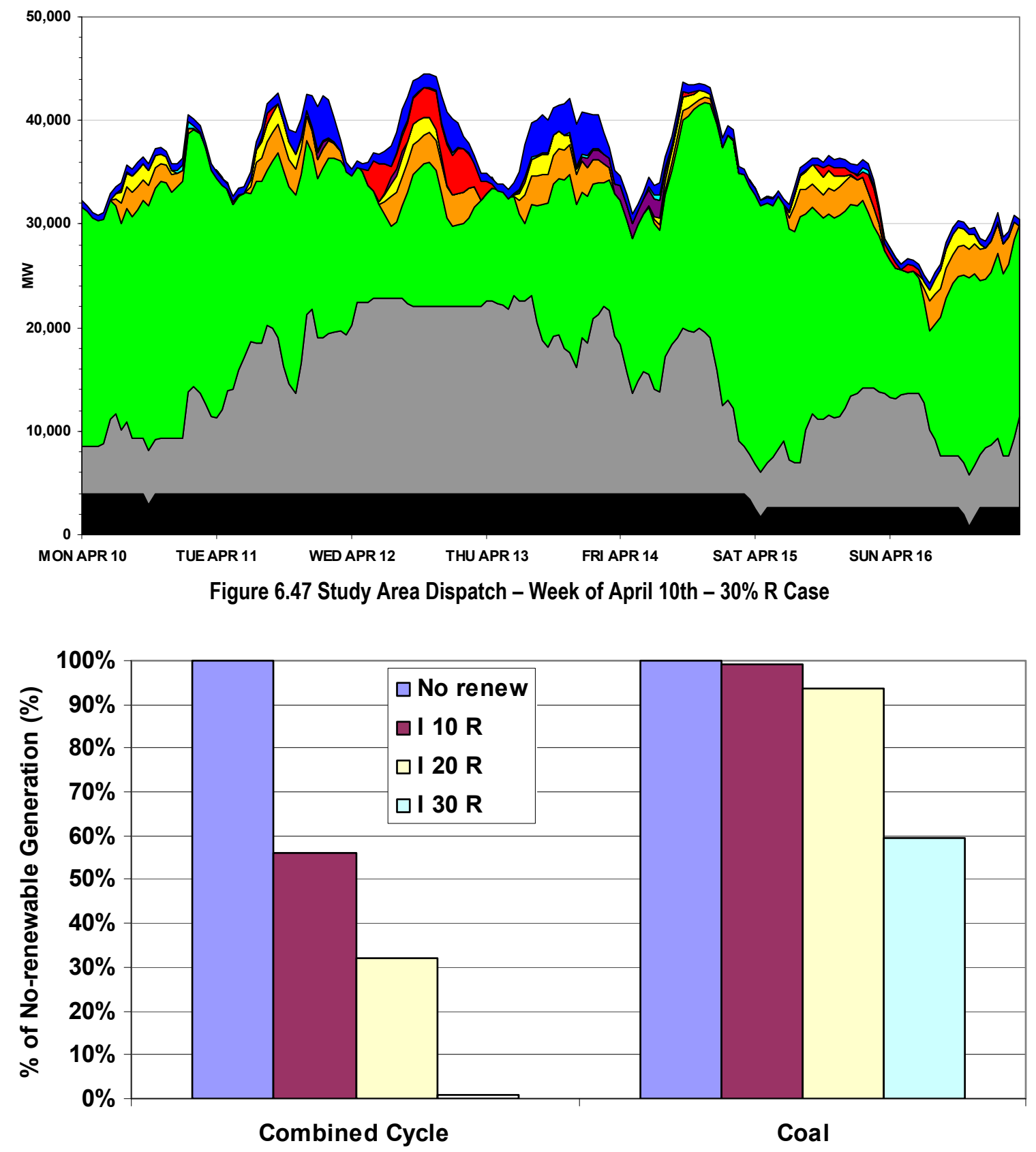

Figure 6.48 Change in Operation - Week of April 10th

The graphs in Figure 6.49, Figure 6.50 and Figure 6.51 show a similar set of renewable operation, net load and net load plus exports for the week of July $10^{\text {th }}$. In this month the 
variation between penetration levels is far less, because load is higher and wind generation is lower.

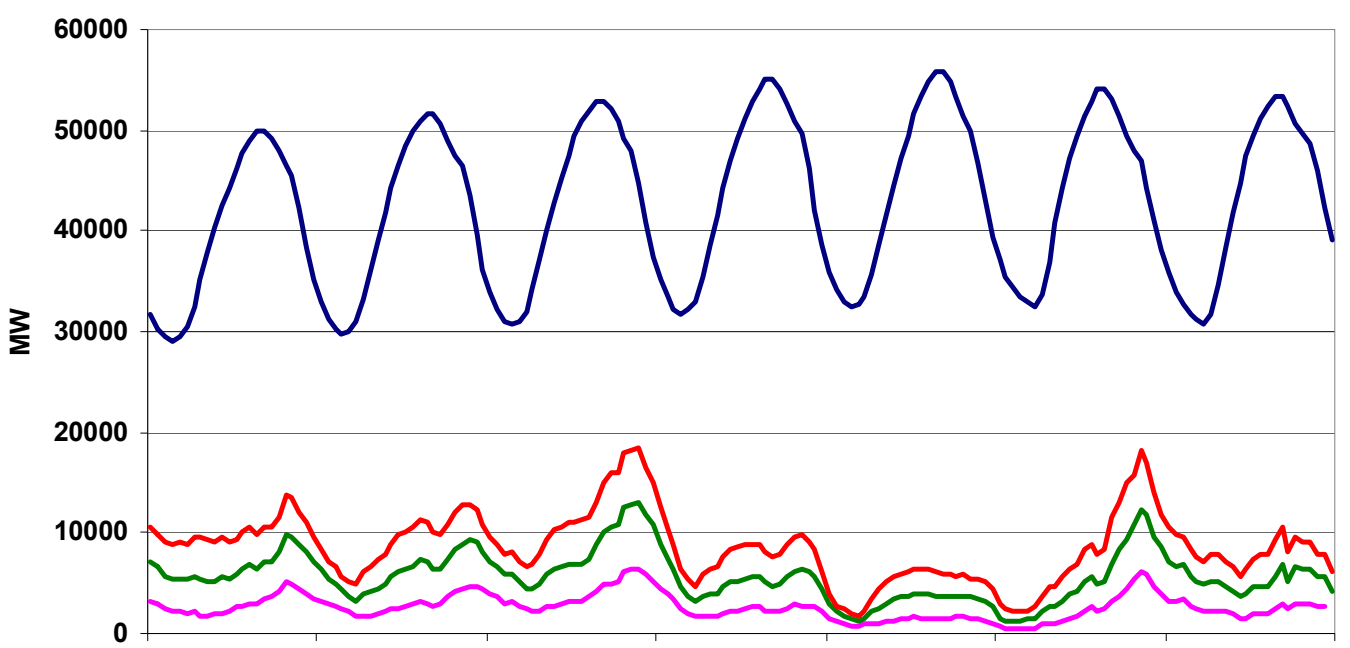

MON JUL 10 TUE JUL 11 WED JUL 12 THU JUL 13 FRI JUL 14 SAT JUL 15 SUN JUL 16

$\begin{array}{ll}\text { Load } & \text { Wind + CSP + PV 10\%R } \\ \text { Wind + CSP + PV 20\%R } & - \text { Wind + CSP + PV } 30 \% R\end{array}$

Figure 6.49 Study Area - Week of July 10th - Renewables by Penetration

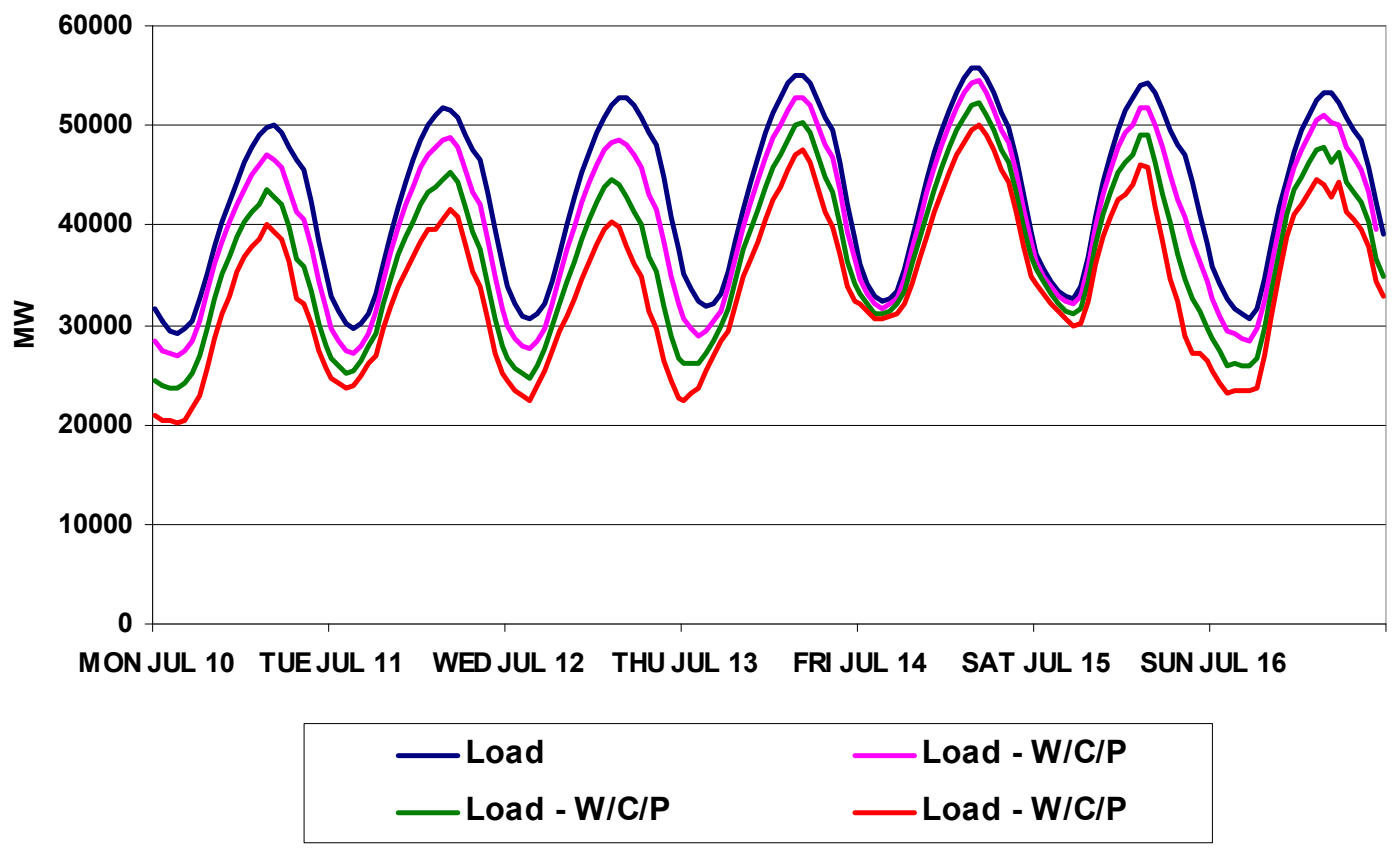

Figure 6.50 Study Area - Week of April 10th - Net Load by Penetration 


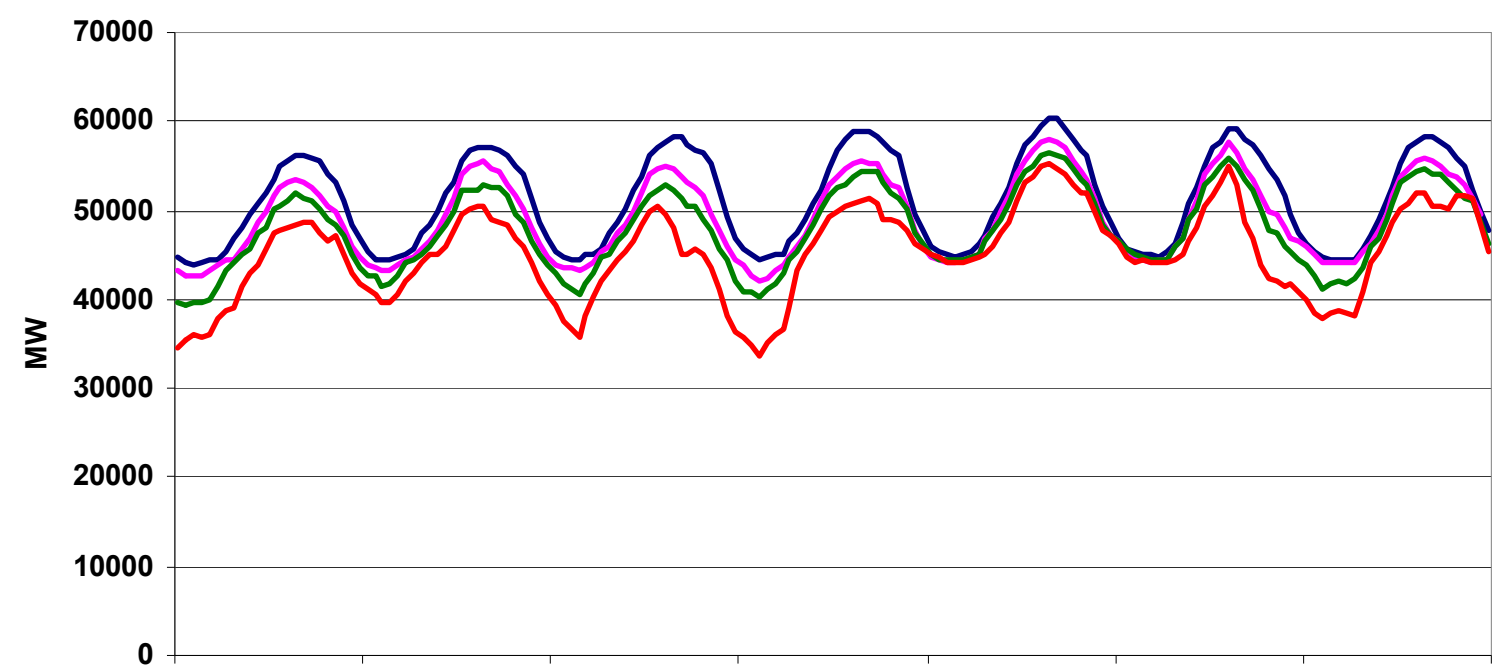

MON JUL 10 TUE JUL 11 WED JUL 12 THU JUL $13 \quad$ FRI JUL 14 SAT JUL 15 SUN JUL 16

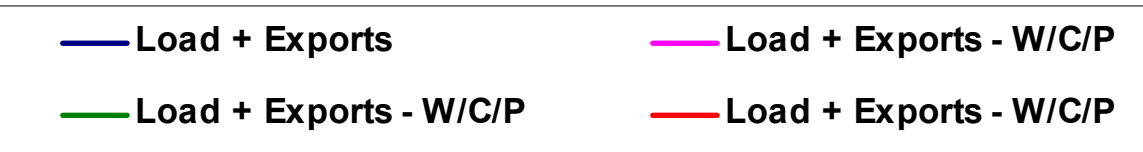

Figure 6.51 Study Area - Week of April 10th - Net Load Plus Exports by Penetration

The graphs in Figure 6.52 through Figure 6.55 show the hourly operation by generation type for this week in July. Even at the $30 \%$ penetration level there is very little change in the coal plant operation. Most notable is the decrease in the gas turbine generation needed for peaking operation. It has been largely displaced by the wind and solar generation.

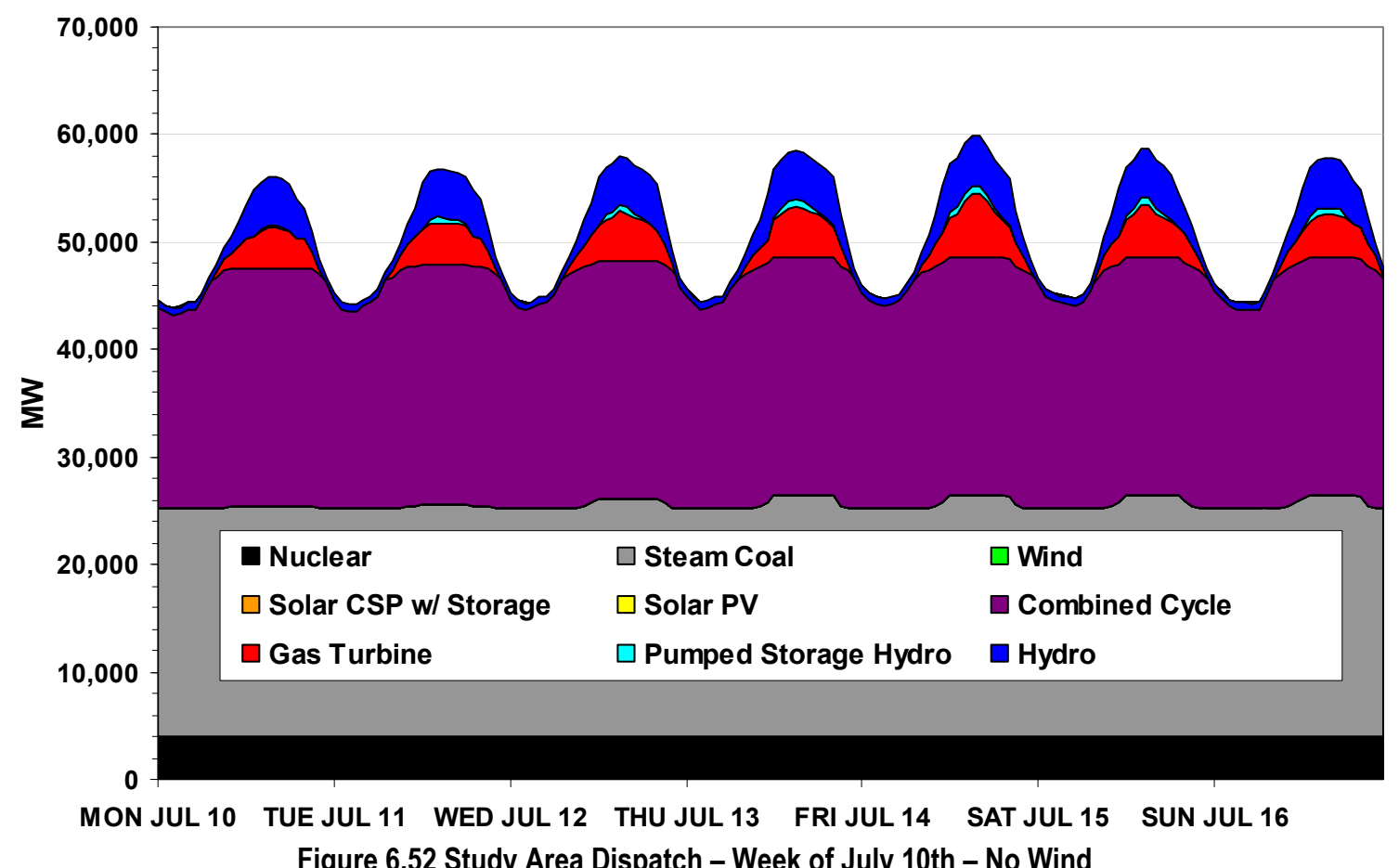

Figure 6.52 Study Area Dispatch - Week of July 10th - No Wind 


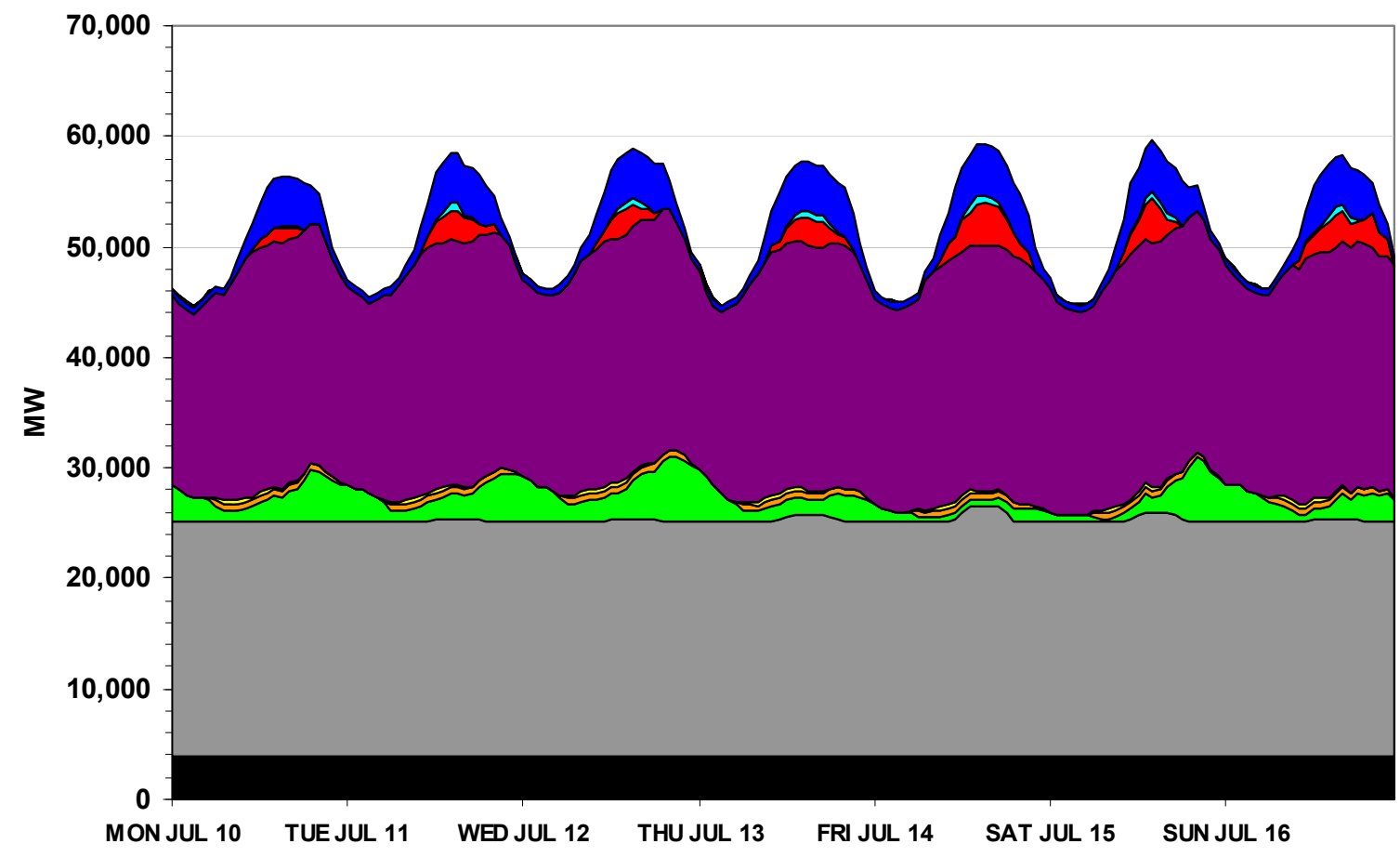

Figure 6.53 Study Area Dispatch - Week of July 10th - 10\% R Case

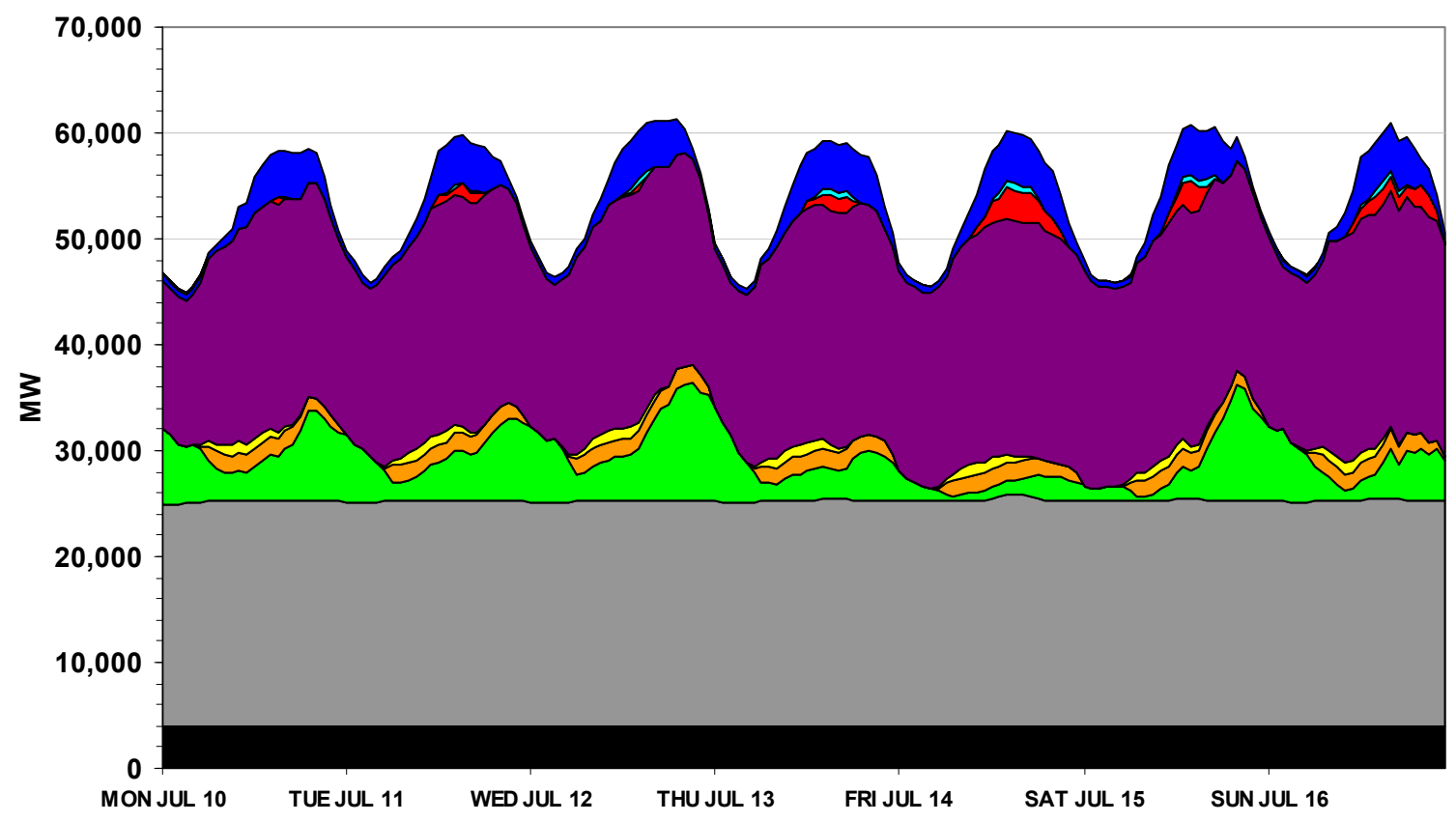

Figure 6.54 Study Area Dispatch - Week of July 10th - 20\% R Case 


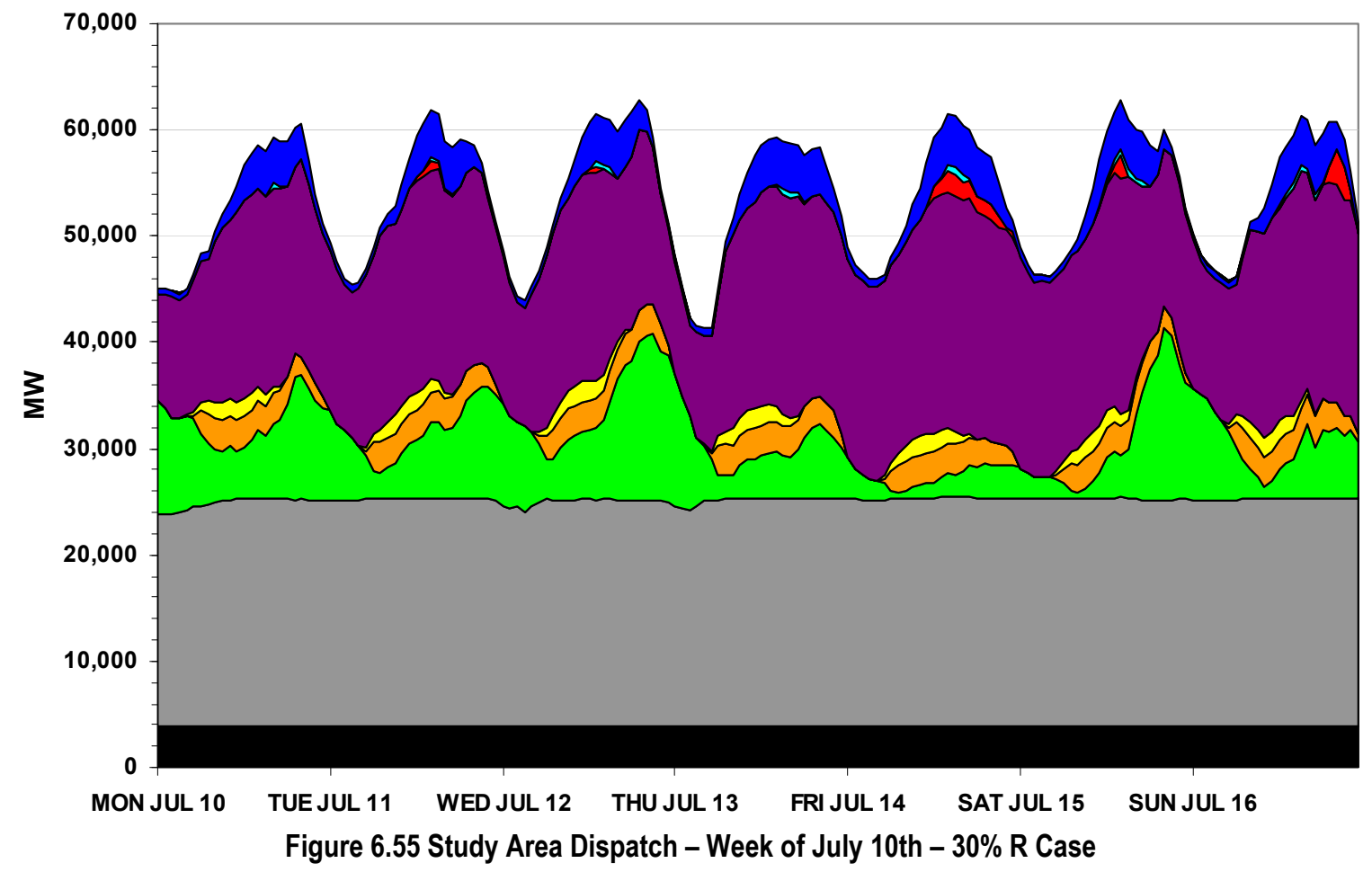

Figure 6.56 highlights the shift in combined-cycle and coal plant generation. The coal plant output is nearly constant and the combined-cycle energy output has dropped by less than $20 \%$. A comparison of the series of figures for the April and July weeks shows that while high penetration of renewables may cause significant disruptions at certain times of the year, their impacts at other times will be much less severe. It may be that at low-load/high-wind times of the year more of the base load generation should be taken out of service to allow generators that are better able to cycle to provide the balance of the energy.

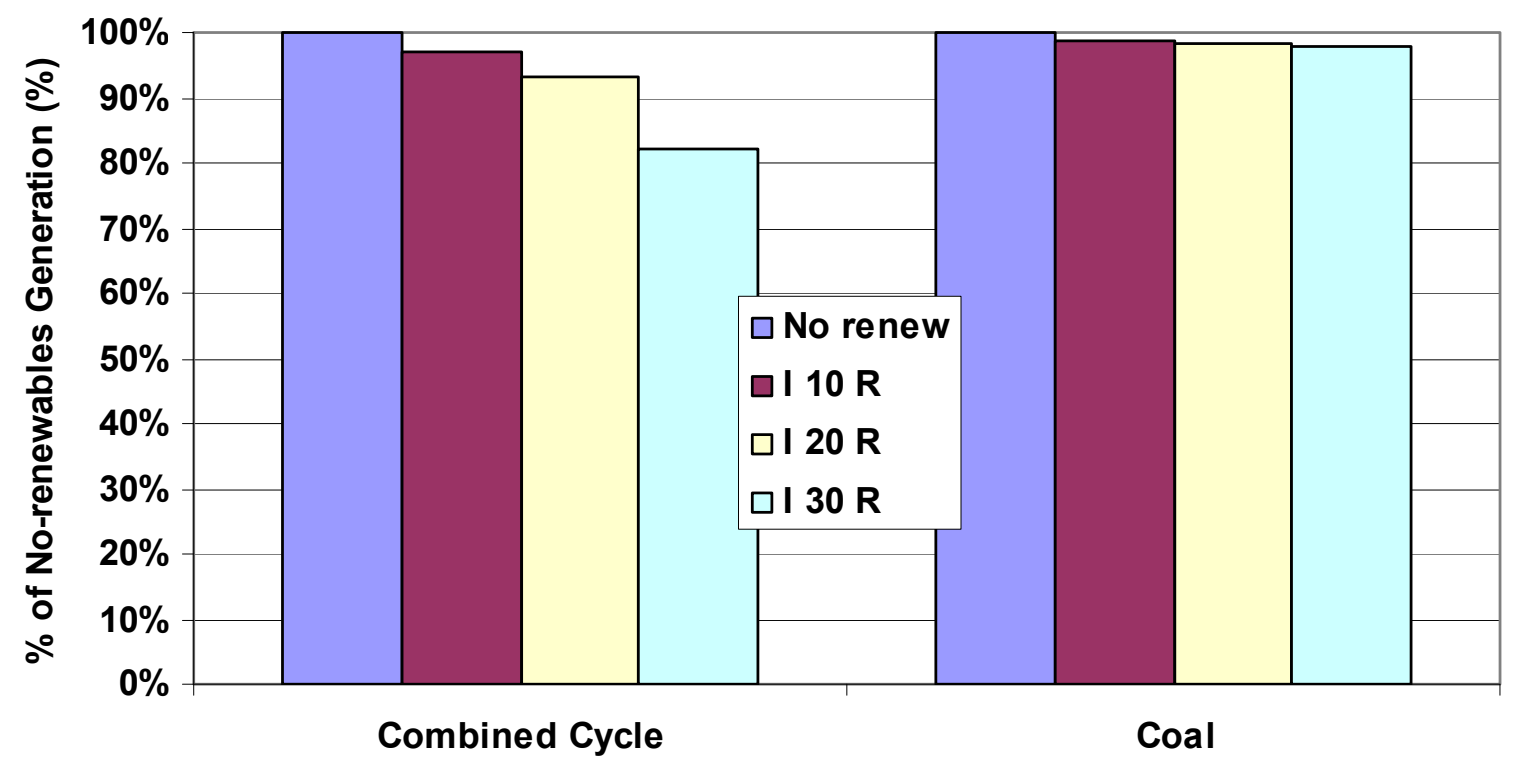

Figure 6.56 Change in Operation - Week of July 10th 


\subsection{Additional Operational Sensitivities}

This section examines several additional sensitivities including variations in siting of the renewable units; sensitivity of the results to hydro and coal unit cycling capabilities; variations of the gas and coal unit priority positions; and changes in the transmission and generation expansions.

\subsubsection{Variations in Renewable Unit Siting}

The bulk of the results shown so far were for the In-Area Scenario. The various penetration levels were also examined for the Local-Priority and Mega-Project Scenarios. Several comparative results will be shown over the next few pages. Only the sites within the study footprint were changed. The renewable sites in the rest of WECC were unchanged. Although the number of wind sites was changed significantly, the annual energy target remained constant. The solar sites within the study footprint were mostly unchanged in the various scenarios.

Figure 6.57 shows the generation in the study area by type for the various scenarios and penetration levels. Although there were some small variations the results were fairly consistent across the scenarios. This is not surprising in light of the fact that sufficient transmission was added to allow the wind generation to be delivered to the same load areas as in the In-Area Scenario.

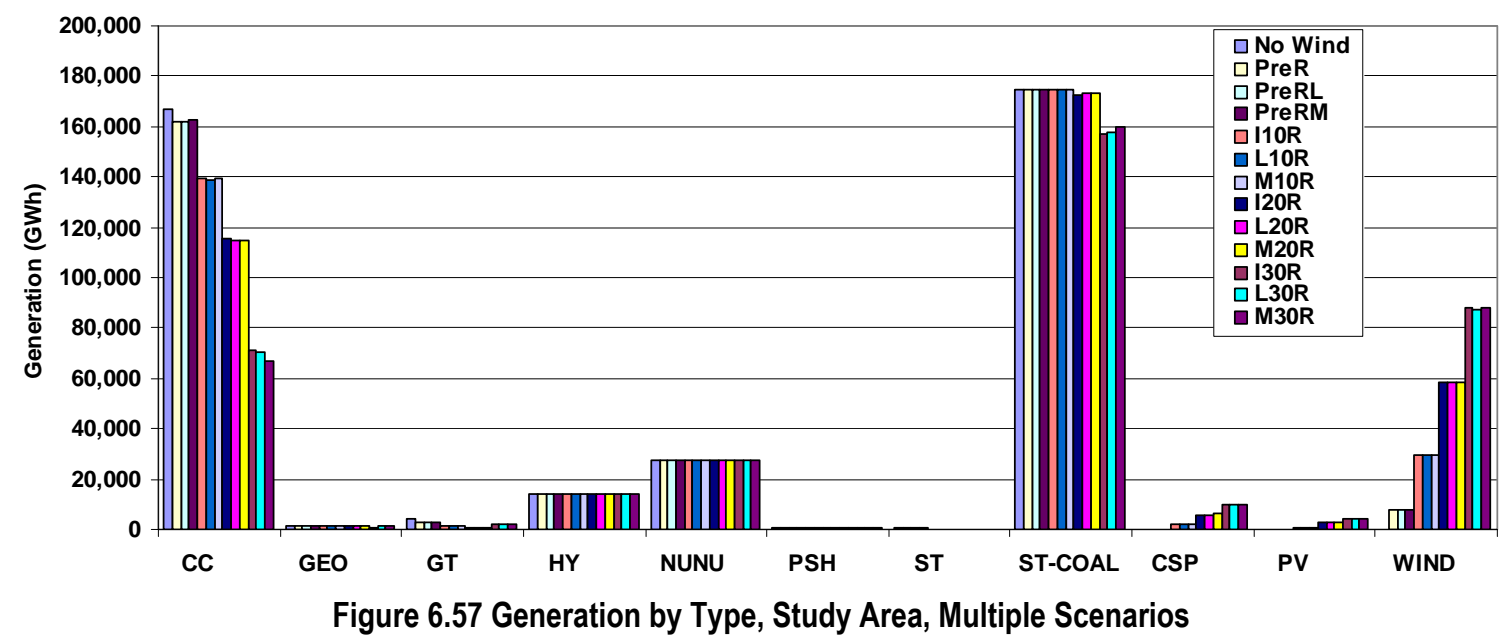

Figure 6.58, Figure 6.59 and Figure 6.60 show the spot price duration curves by penetration level for the In-Area, Local-Priority and Mega-Project Scenarios respectively. The set of curves are very similar. Figure 6.61 and Figure 6.62 compare the curves across the various scenarios for the $30 \%$ penetration cases. Only slight differences exist between the three curves. 


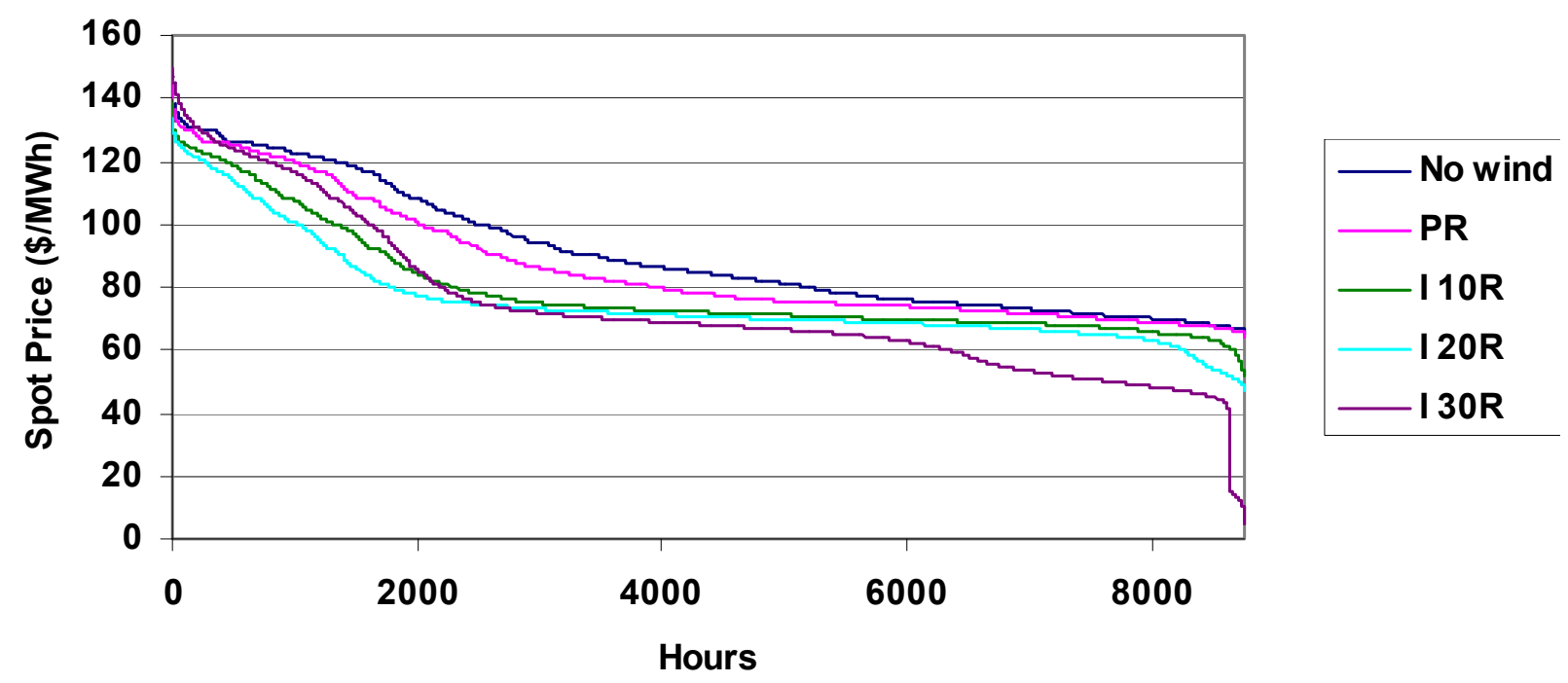

Figure 6.58 Annual Spot Price Duration Curve - S-o-A Forecast, In-Area Scenario

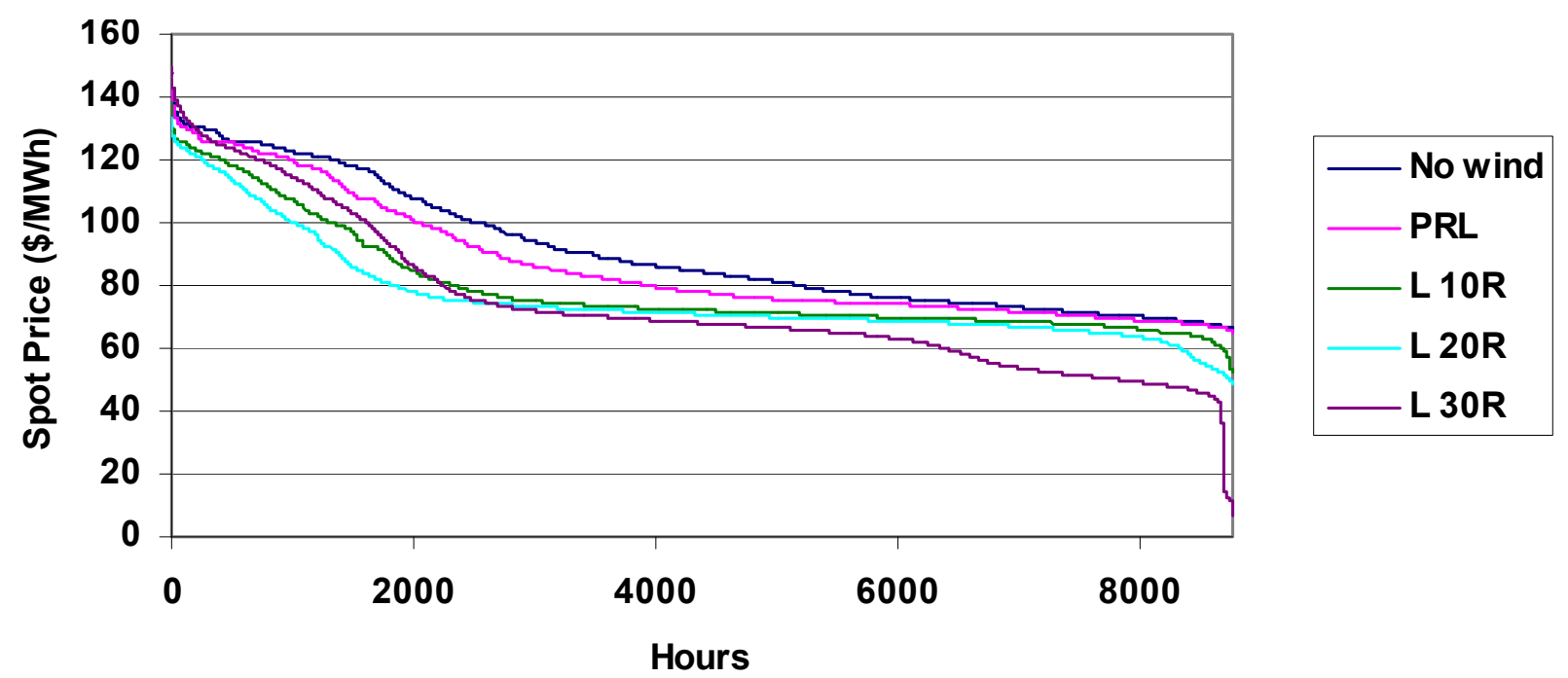

Figure 6.59 Annual Spot Price Duration Curve - S-o-A Forecast, Local-Priority Scenario 


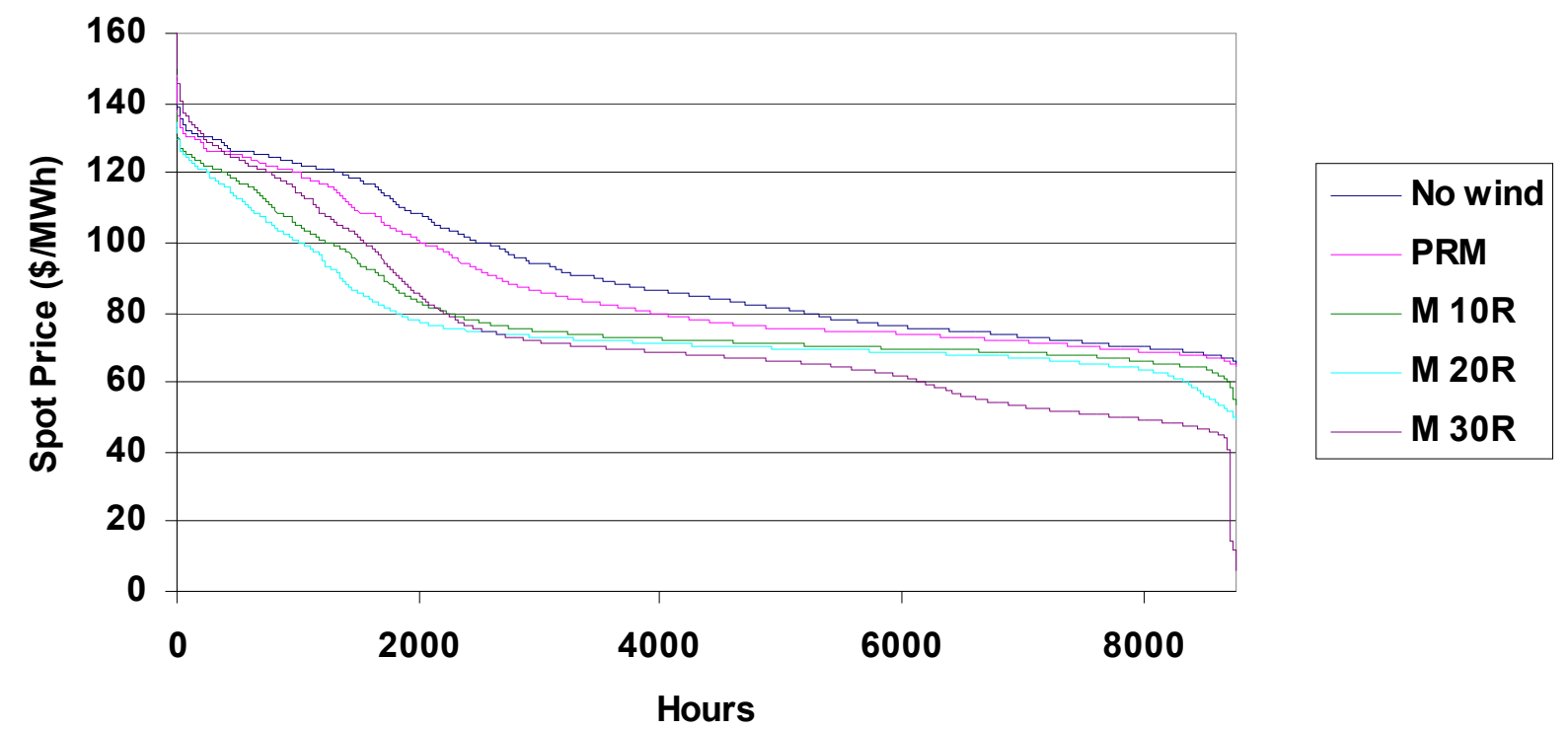

Figure 6.60 Annual Spot Price Duration Curve - S-o-A Forecast, Mega-Project Scenario

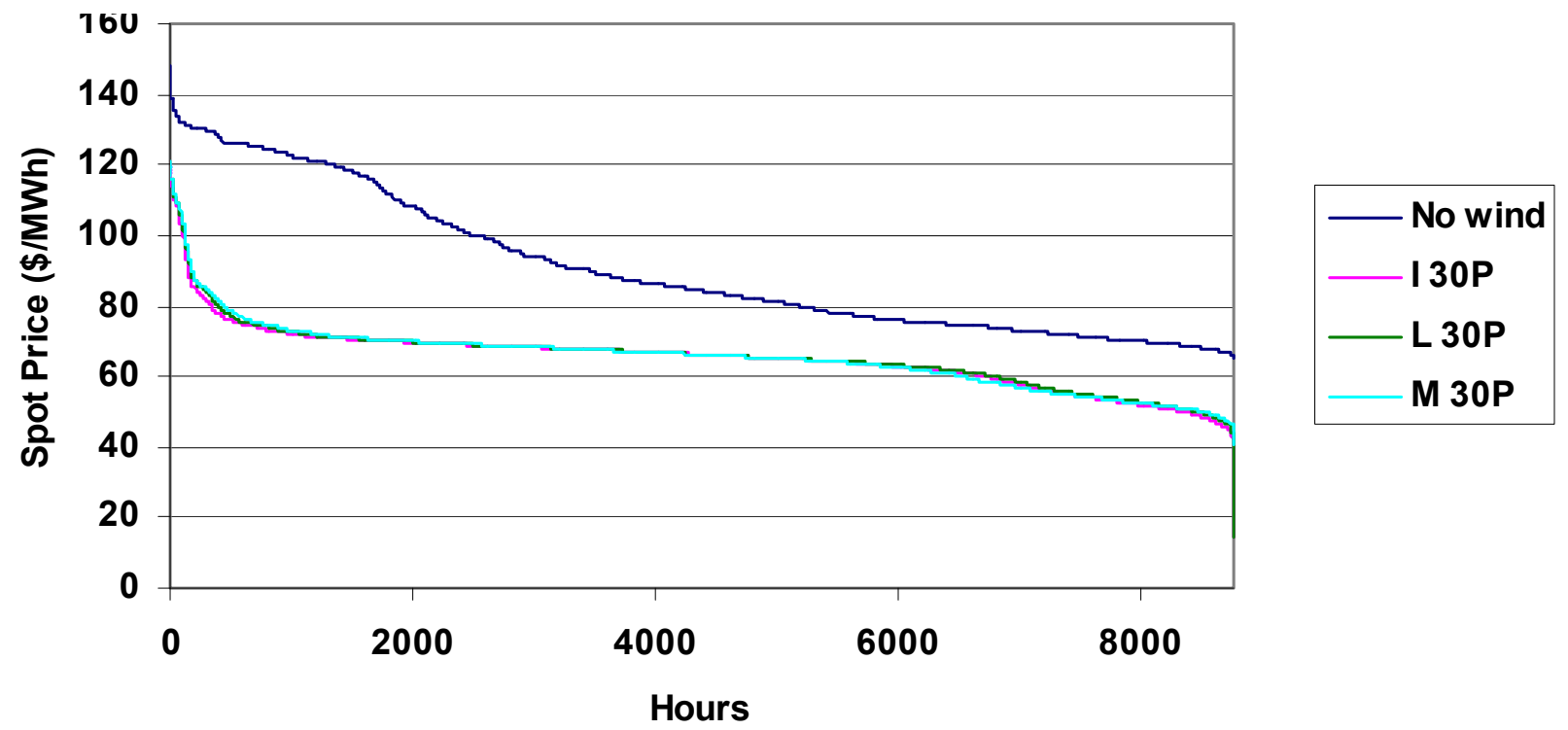

Figure 6.61 Annual Spot Price Duration Curve, Perfect Forecast, 30\% Scenarios 


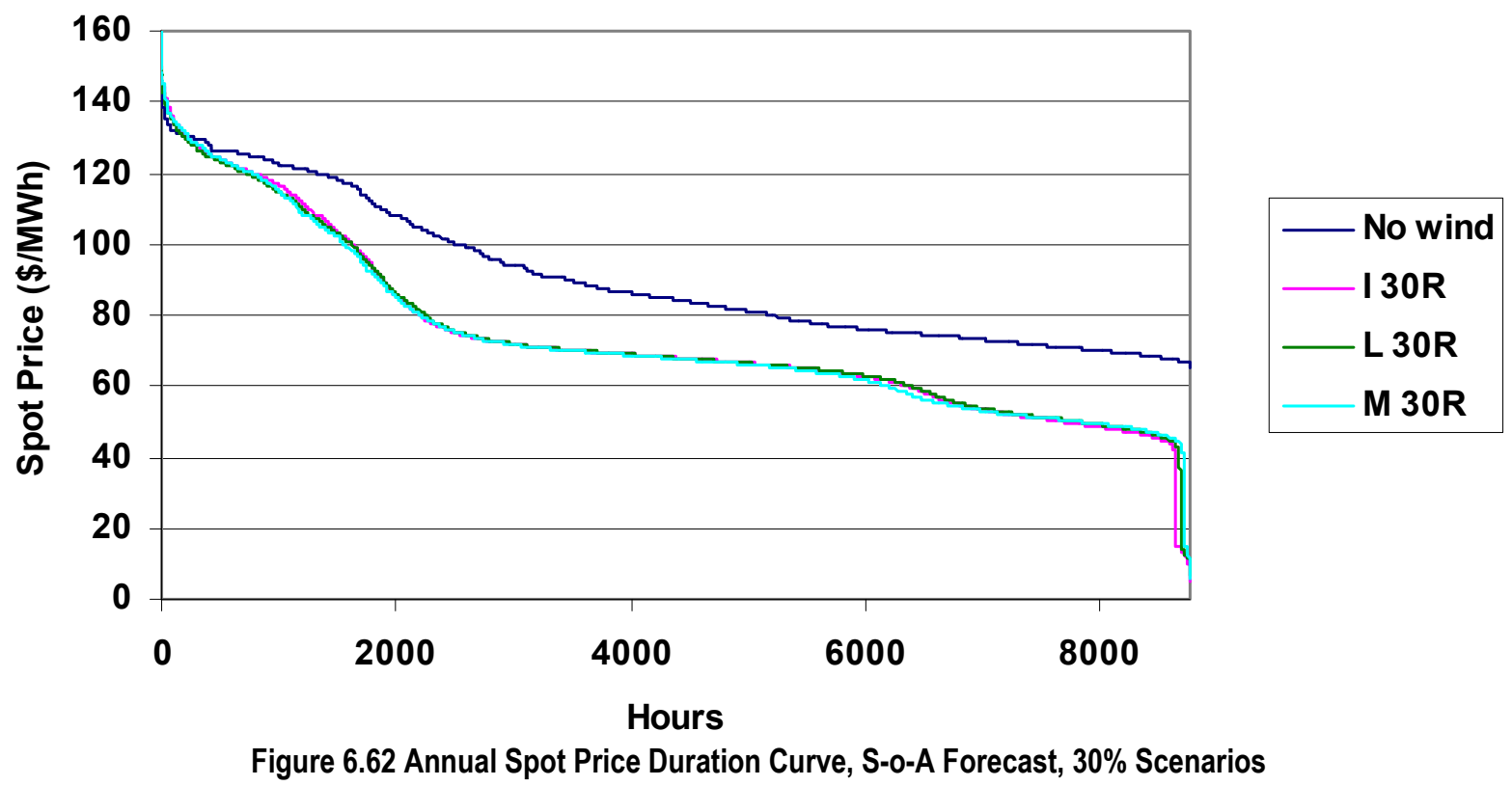

Figure 6.63 shows that the total generation in the study area is largely unchanged for a given penetration level regardless of where the wind and solar resources were located. Figure 6.64 shows the total WECC operating cost savings per MWh of renewable energy for the different cases. There was a slight increase in value as the wind plant locations were shifted to the higher capacity factor sites in the Local-Priority and Mega-Project Scenarios. This trend is also visible in Figure 6.65 that shows the incremental value per incremental renewable MWh. Although all of the scenarios had the same annual energy for renewables, the monthly patterns were slightly different, as was shown in Chapter 4 . This may explain some of the variation in the operational benefits.

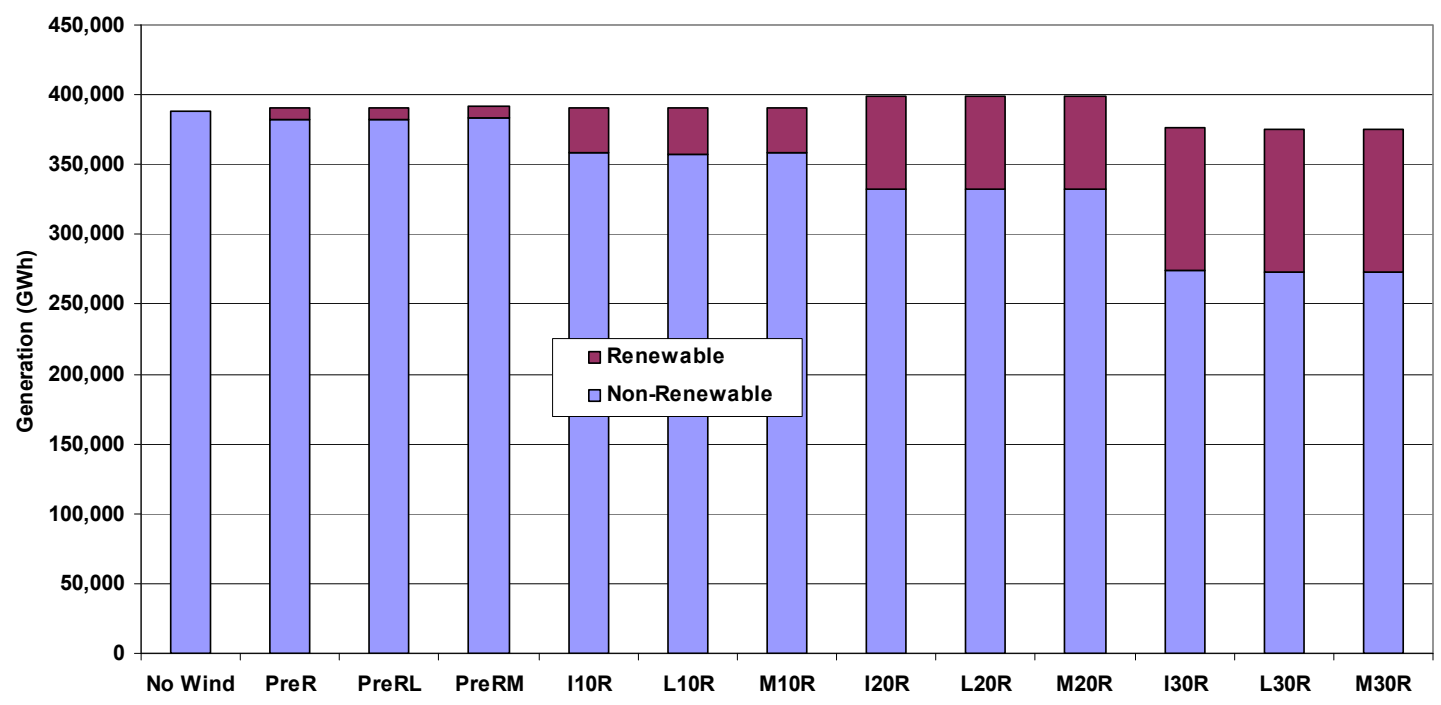

Figure 6.63 Total Generation, Study Area, Multiple Scenarios 

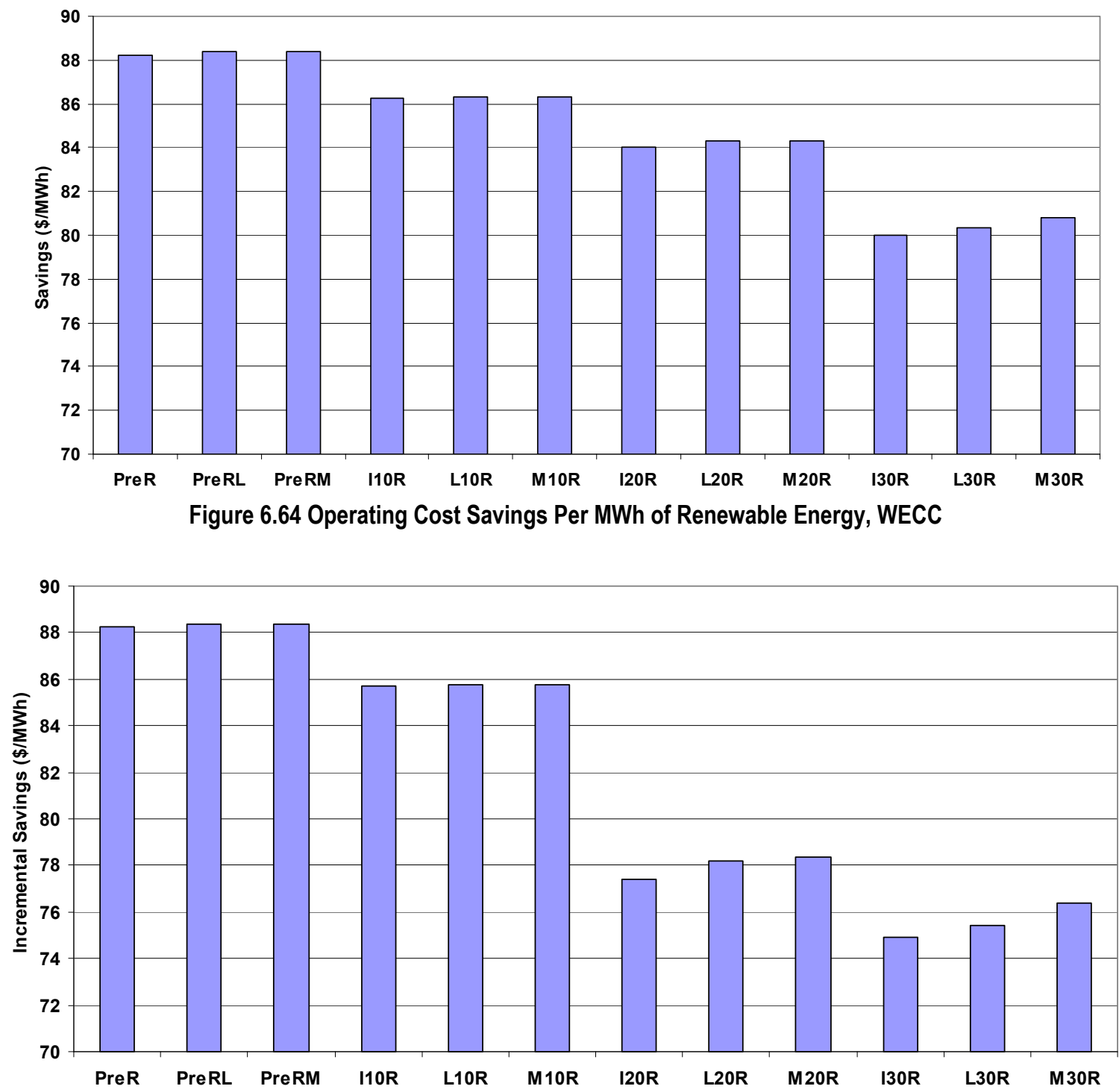

Figure 6.65 Incremental Savings Per Incremental Renewable Energy, WECC

Figure 6.66, Figure 6.67 and Figure 6.68 show the incremental savings per incremental renewable MWh by penetration level for the three shape years for the three different scenarios. Although there is a slight difference between the years, the differences in incremental savings with increasing penetration are consistent with what was seen for the 2006 shapes. 


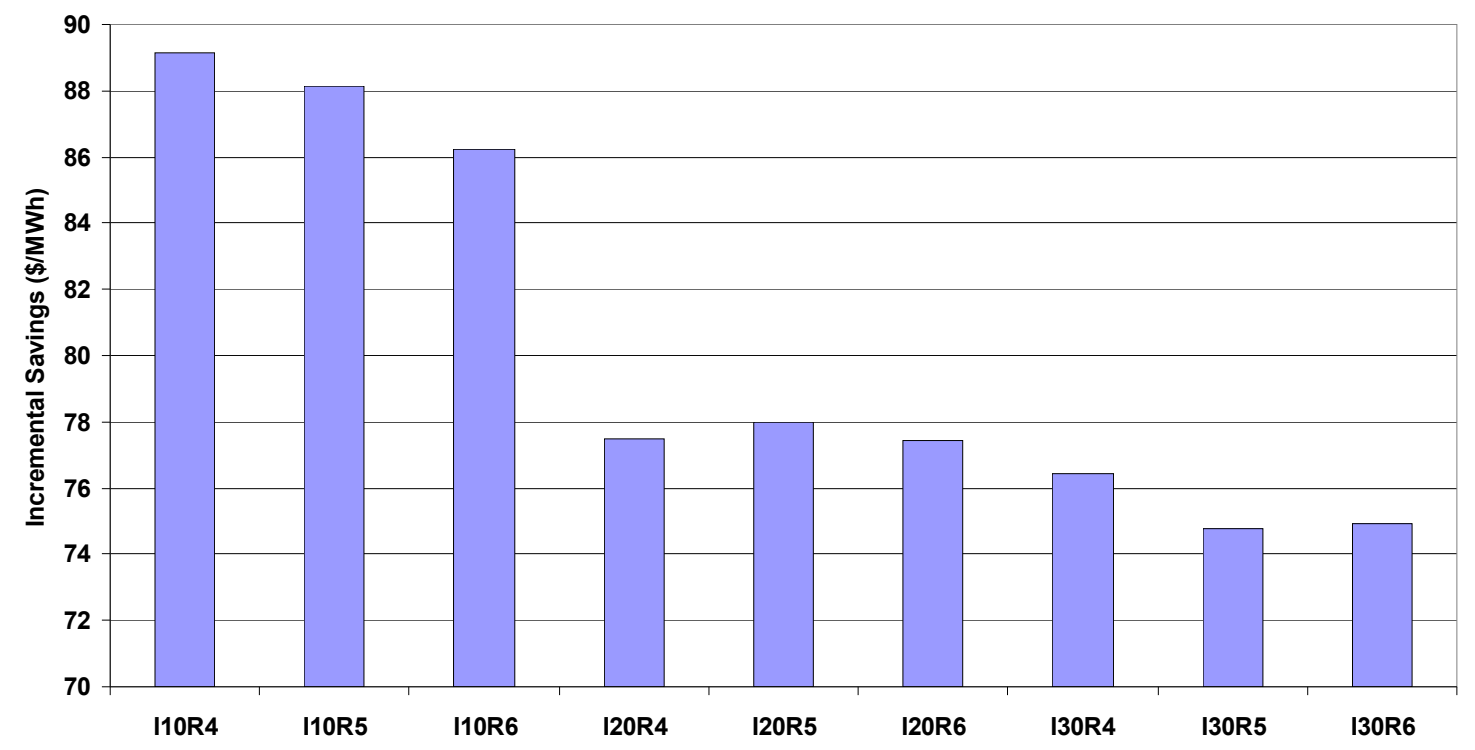

Figure 6.66 Incremental Savings Per Incremental Renewable Energy, WECC, In-Area Scenario

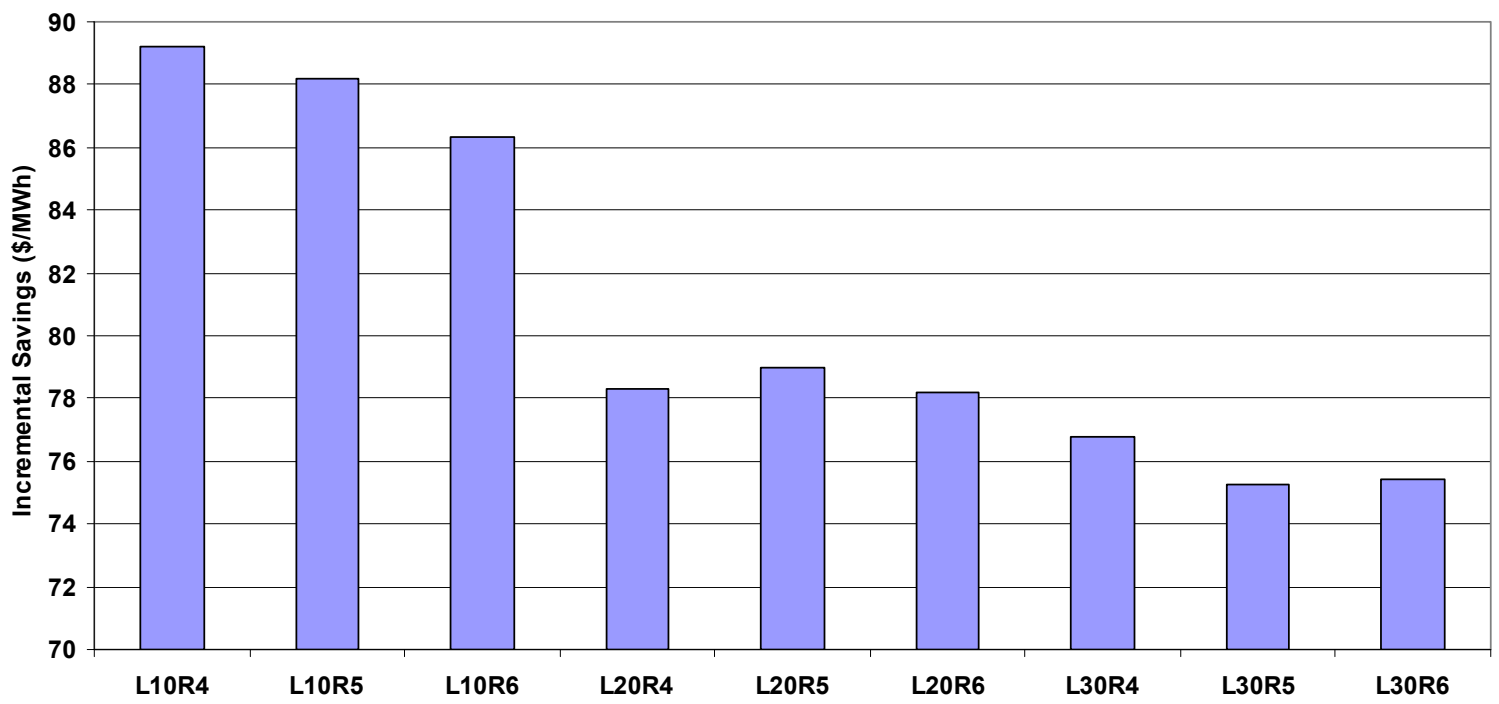

Figure 6.67 Incremental Savings Per Incremental Renewable Energy, WECC, Local-Priority Scenario 


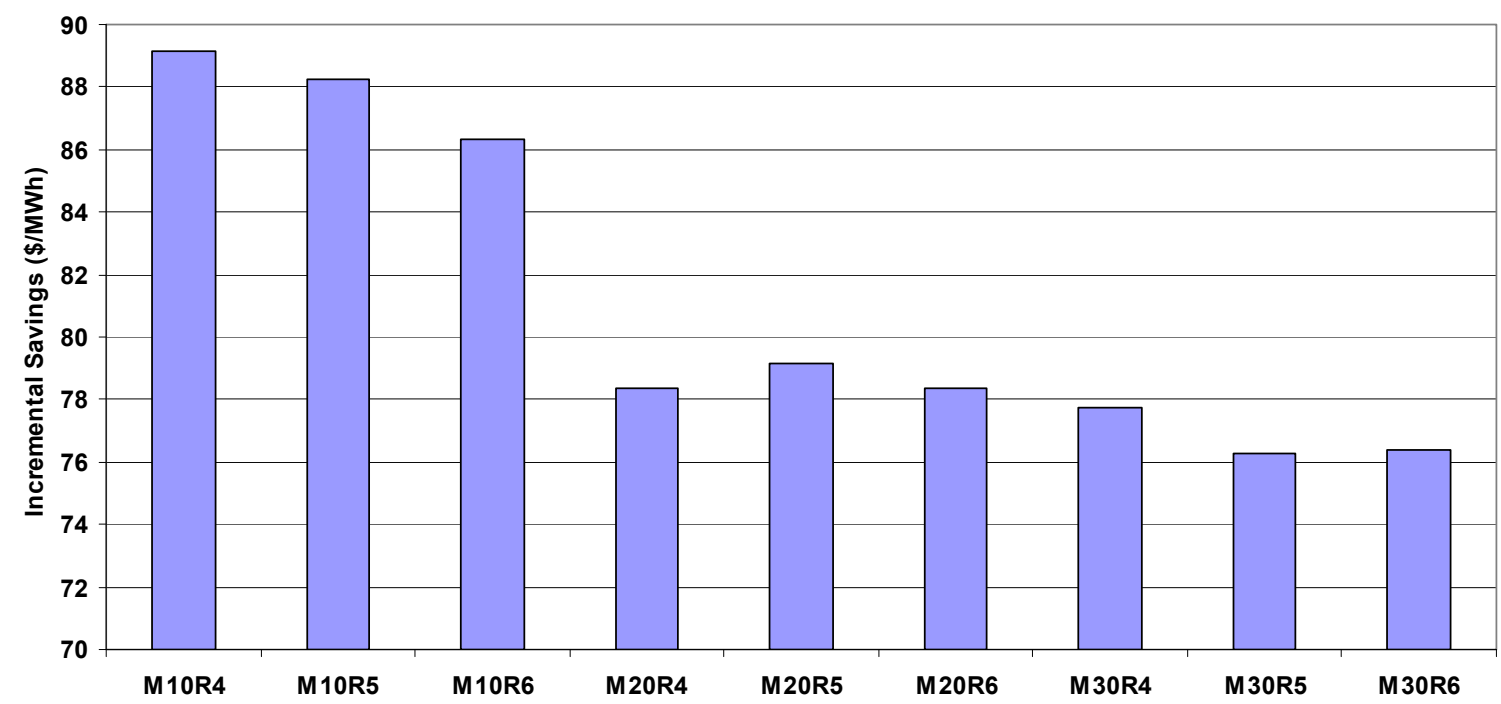

Figure 6.68 Incremental Savings Per Incremental Renewable Energy, WECC, Mega-Project Scenario

\subsubsection{Hydro Operation}

This section examines the operation of the hydro plants in the base-case (no wind and solar) and how it changes with the introduction of wind and solar renewable generation. Comparisons are made to historical operation, and the impacts of restrictions on the hydro operation are examined. Figure 6.69 shows the total hourly operation of the WECC hydro plants for the week of April $10^{\text {th }}$ as a function of renewable penetration. These cases assumed that the hydro was scheduled after the wind forecast. Therefore, as more wind was added the hourly schedule of the hydro changed but the total monthly energy remained constant, as well as the monthly minimum and maximum power output levels. Although the patterns shifted somewhat, particularly at the $30 \%$ penetration level, there did not appear to be major changes in the hydro operation. Figure 6.70 shows a similar comparison for a July week. 


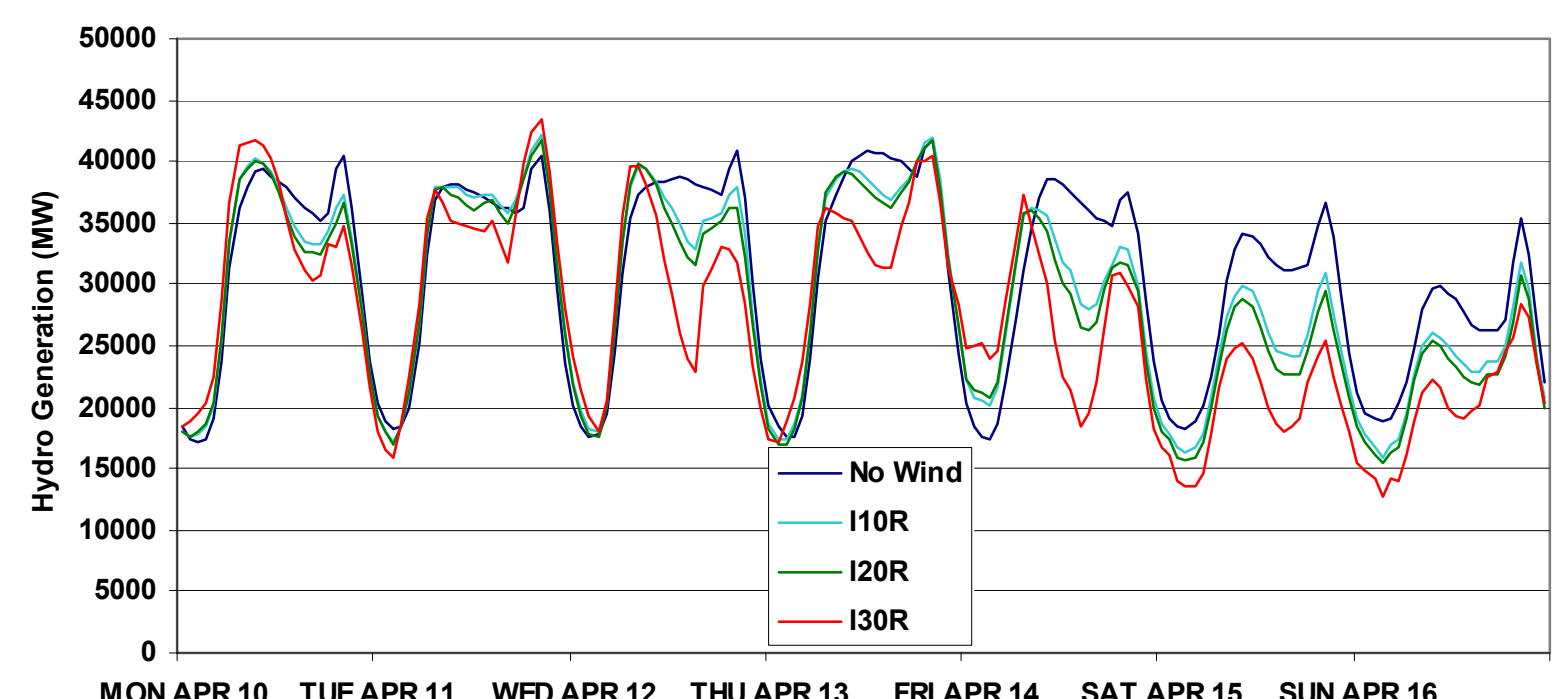

Figure 6.69 Hydro Operation - Week of April $10^{\text {th }}$

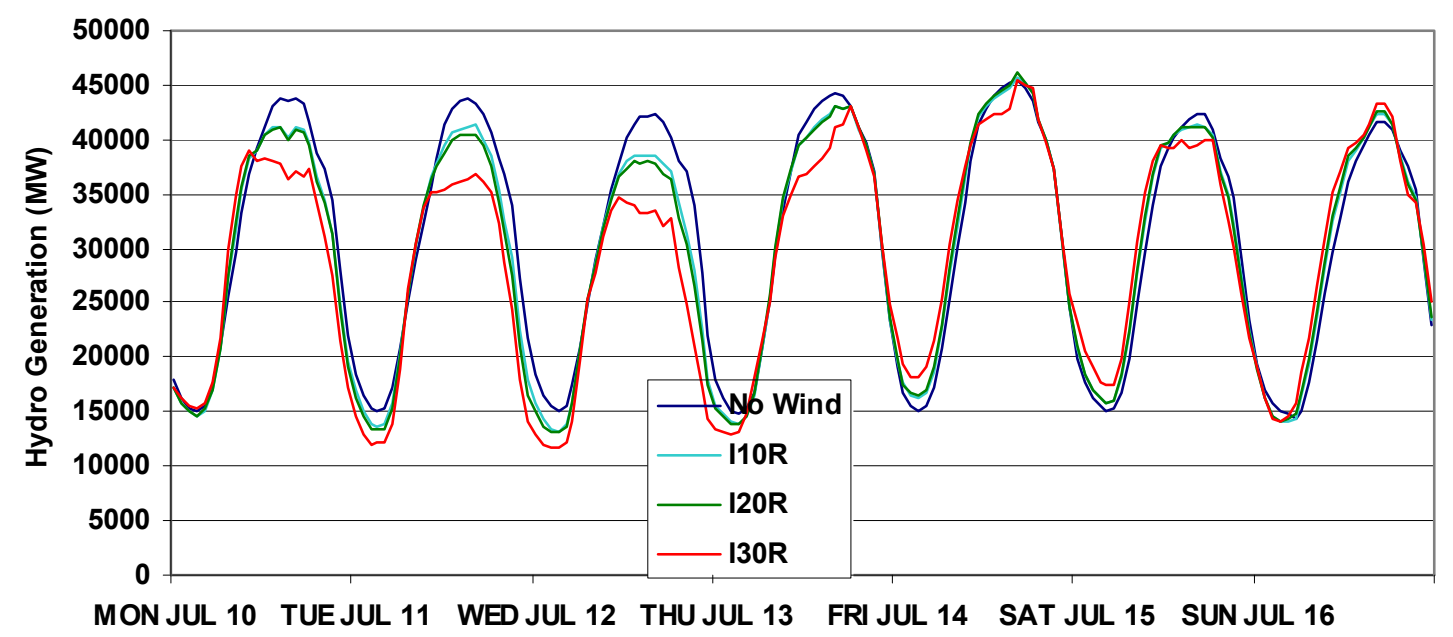

Figure 6.70 Hydro Operation - Week of July 10th

Figure 6.71 shows an annual duration curve for the hydro operation for the same set of cases. Again, from a macro level, there does not appear to be any significant shifts in the operation of the hydro. Figure 6.72 shows the hourly delta for the hydro generation from the chronological curves, sorted and plotted as duration curves. It shows that the wind generation did not seem to be pushing the hydro into any significant up or down ramps that were different from the no-wind case. 


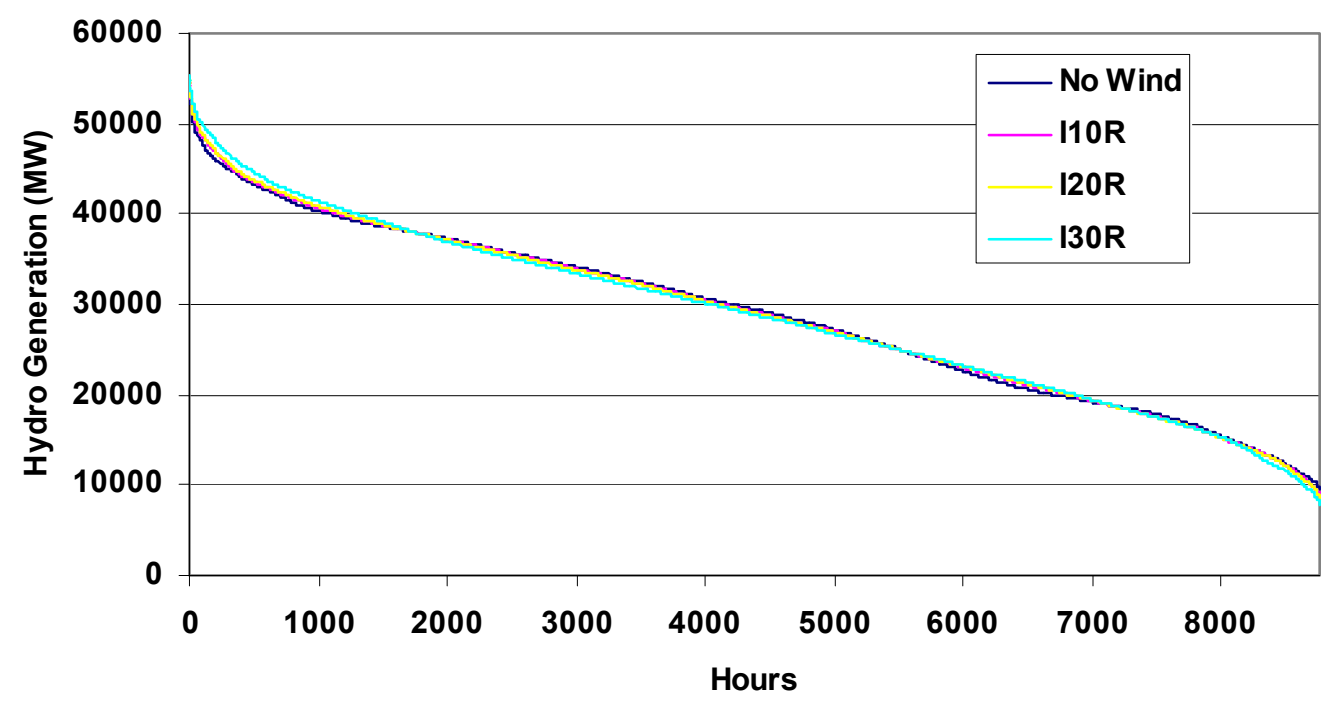

Figure 6.71 Annual Hydro Generation Duration Curve, WECC

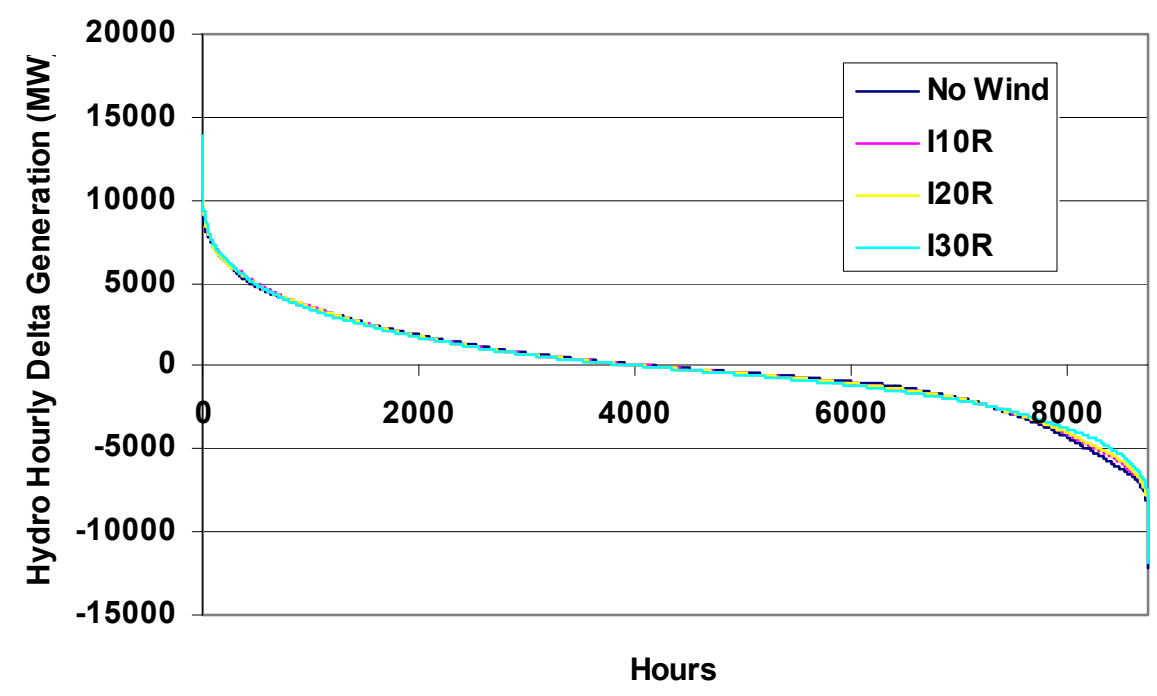

Figure 6.72 Annual Hydro Hourly Delta Duration Curve, WECC

The next step was to examine the assumption that the hydro was scheduled based on the forecasted wind. Historically, the hydro generation ramps up and down, based on the forecasted load demand. Therefore, it seemed reasonable to assume that hydro would try to follow the net load. Figure 6.73 shows spot price duration curves for the various penetration levels for the base assumptions, when the hydro is scheduled on load alone (noted as "- $\mathrm{H}^{\prime \prime}$ ). In these cases, the hourly hydro generation remains unchanged 
between the cases. As can be seen, there is not much of a shift between any pair of cases at the same penetration.

Figure 6.74 shows the operating cost increases when hydro is scheduled before the renewables rather than after. Although the values are relatively small for low renewable penetrations they exceed $\$ 200$ million for the $30 \%$ case.

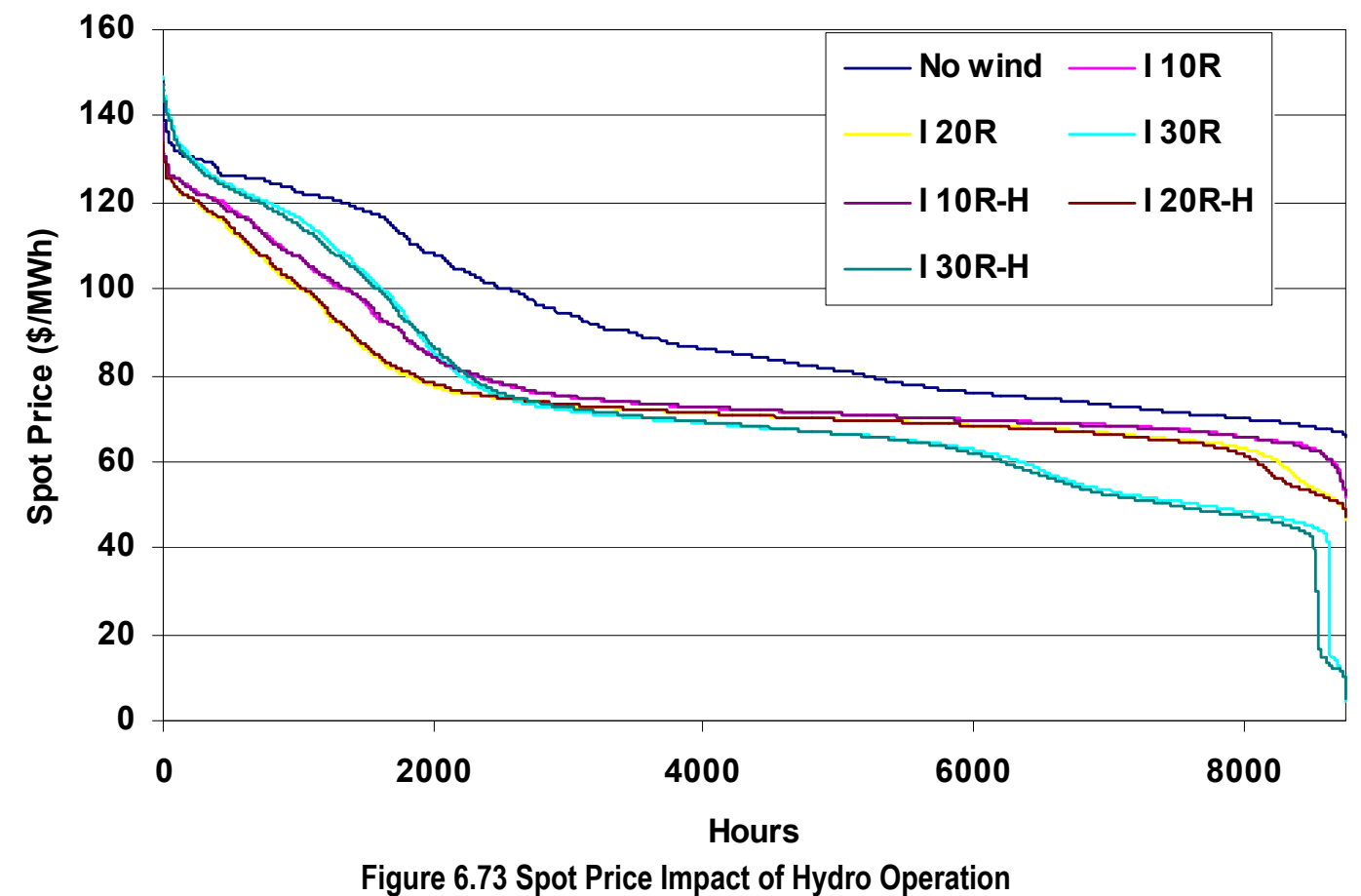

Figure 6.73 Spot Price Impact of Hydro Operation 


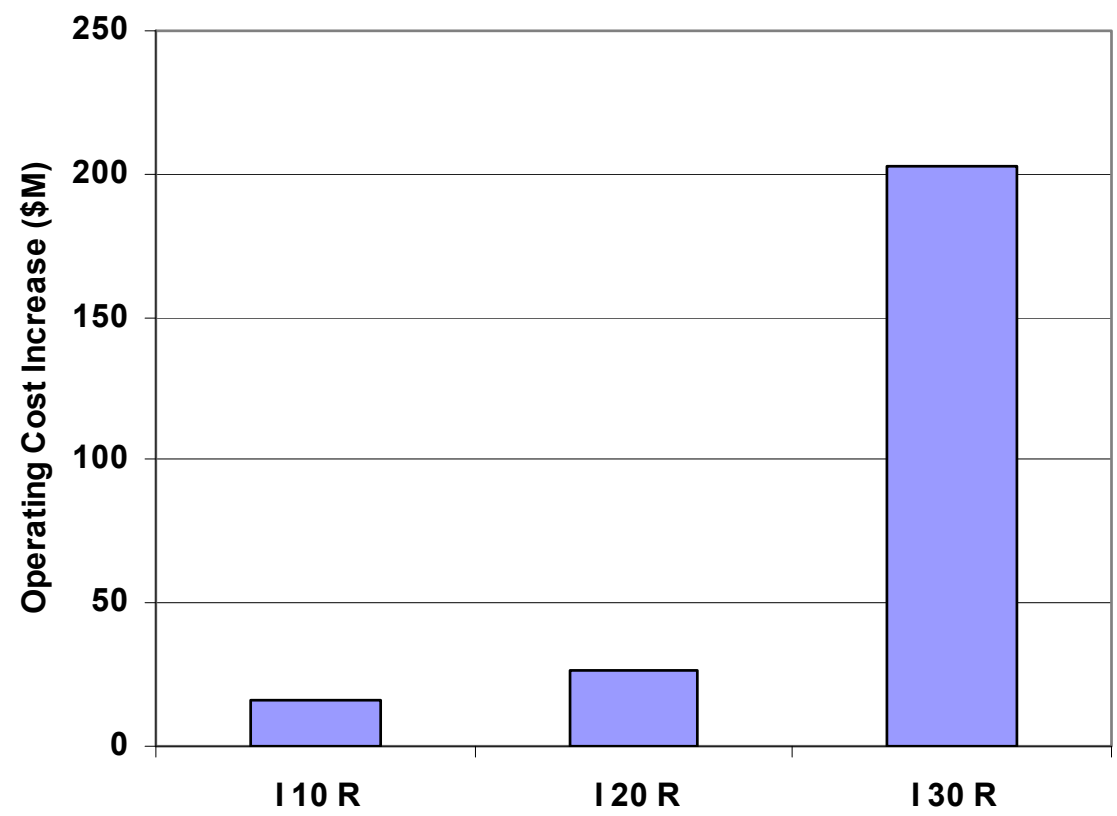

Figure 6.74 Operating Cost Increase for Dispatching Hydro on Load Alone vs. Net Load, WECC

The results above address the aggregate influence of the hydro generation, but do not look at the operation of any individual facilities in specific detail. The next section compares the model output to the historical operation at Glen Canyon and extrapolates the observations to a few additional sensitivities. A more in depth analysis of this subject is being studied jointly with Northern Arizona University and will be published separately. The next few charts examine the historical flexibility of hydro generation, particularly in low water periods. Figure 6.75 shows the variation in the capacity at the Glen Canyon plant as a function of the elevation of water behind the dam. When the water level drops, not only is the amount of energy that the plant is capable of producing severely limited, but the capacity at which the unit can operate is significantly reduced. Although the nameplate rating is over $1300 \mathrm{MW}$ there is less than half of that capacity available at low head elevations. Figure 6.76 shows the historical water levels at Lake Powell that feeds the Glen Canyon plant. From these two charts it can be seen that available capacity over the last several years is down significantly from its maximum value. Figure 6.77 shows that the monthly generation at the plant over the past several years is less than half of the high values seen in 1996. 


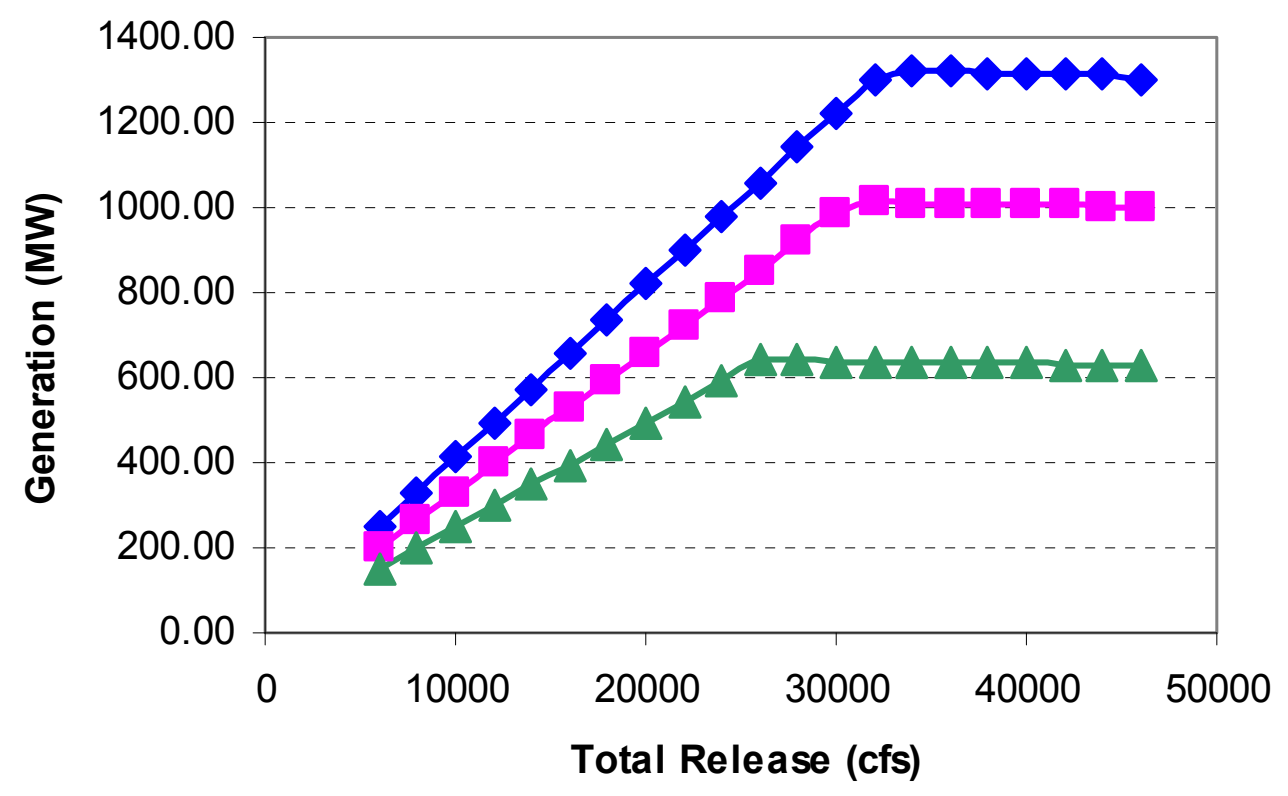

$\multimap$ elev=3700 —elev=3600 $\multimap$ elev=3500

Figure 6.75 Glen Canyon Capacity vs. Elevation

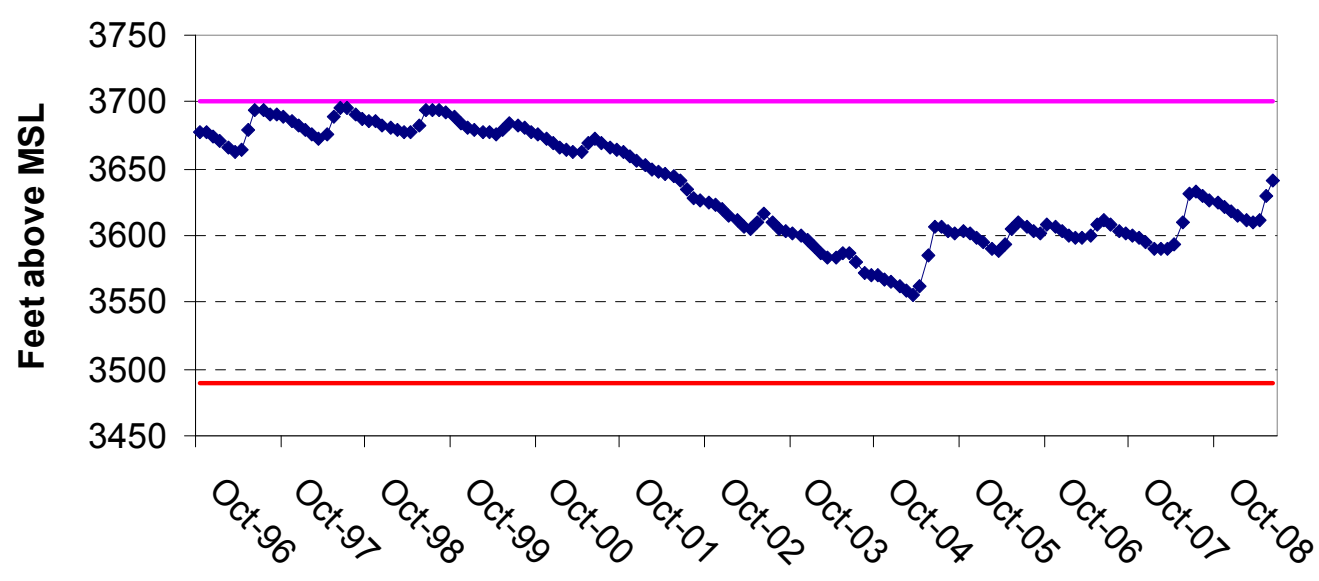

Figure 6.76 Historical Elevation of Lake Powell 


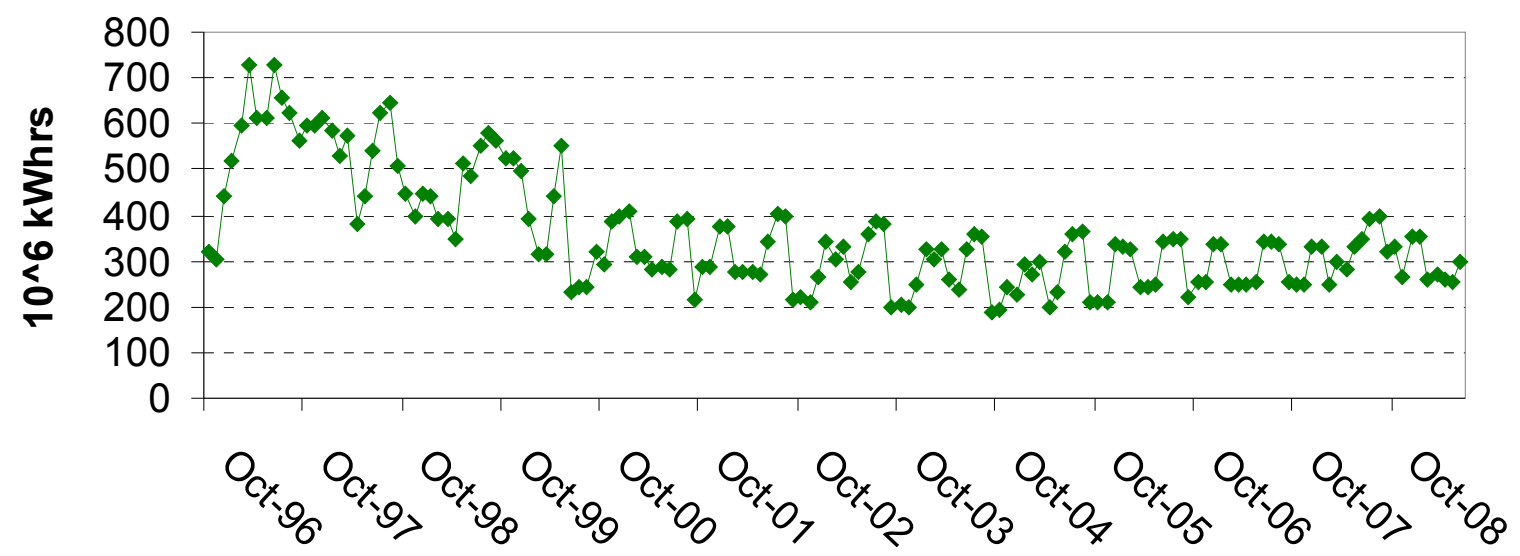

Figure 6.77 Net Monthly Generation at Glen Canyon

Although the energy values used in the simulation reflected the averages over the last several years the capacity values were not adjusted to reflect the drop in elevation.

Figure 6.78 shows the daily maximum, minimum, and average outputs from the plant. The model assumed that the plant could swing from its maximum to minimum value within 24 hours. Figure 6.79 shows the simulation values on a monthly basis. Figure 6.80 adds in the historical maximum monthly values from 2005 and 2006, which fall roughly in line with the average values used in the simulation. Figure 6.81 adds in the historical average values. Because the simulation used 10-year historical average monthly energy values for the hydro they are slightly higher than the 2005 and 2006 numbers.

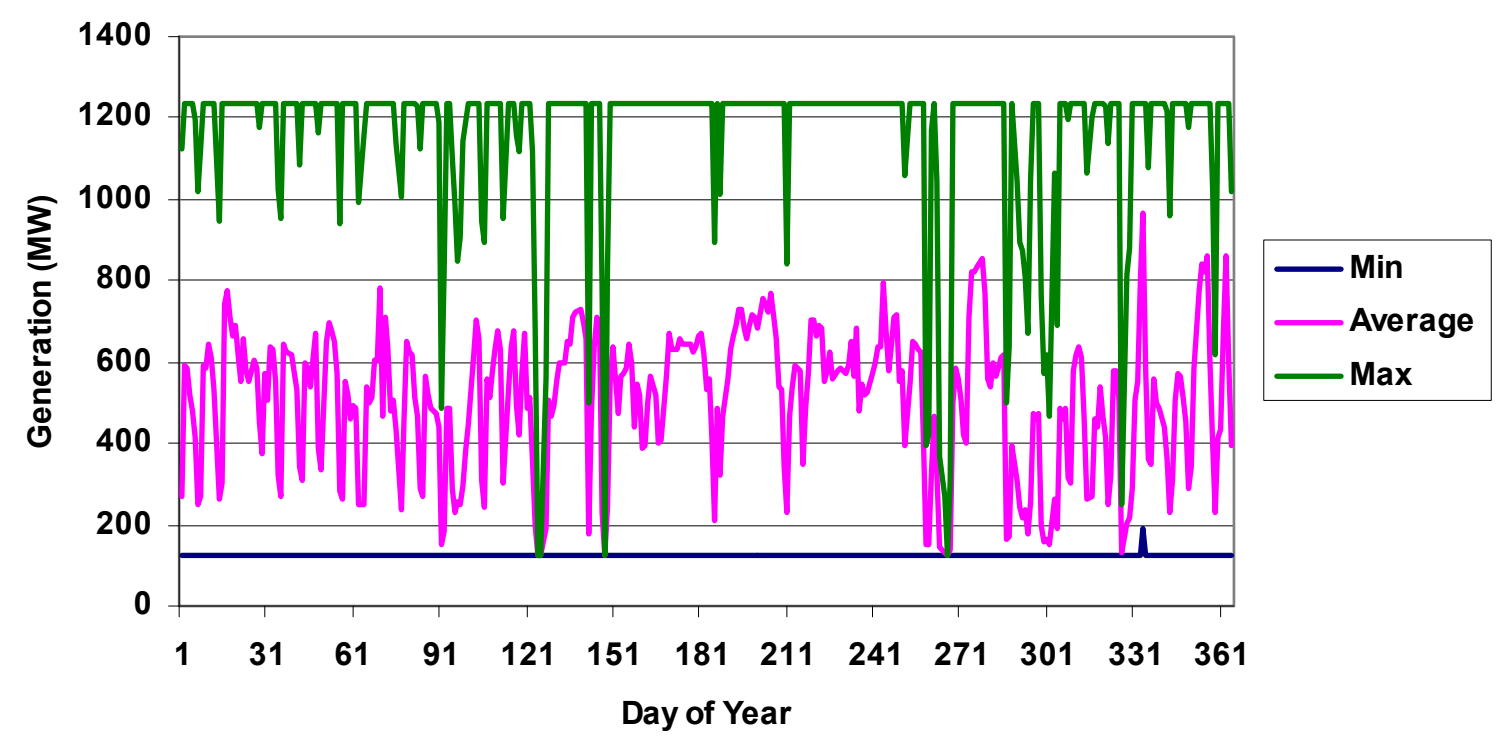

Figure 6.78 MAPS Daily Simulation of Glen Canyon with No Wind 


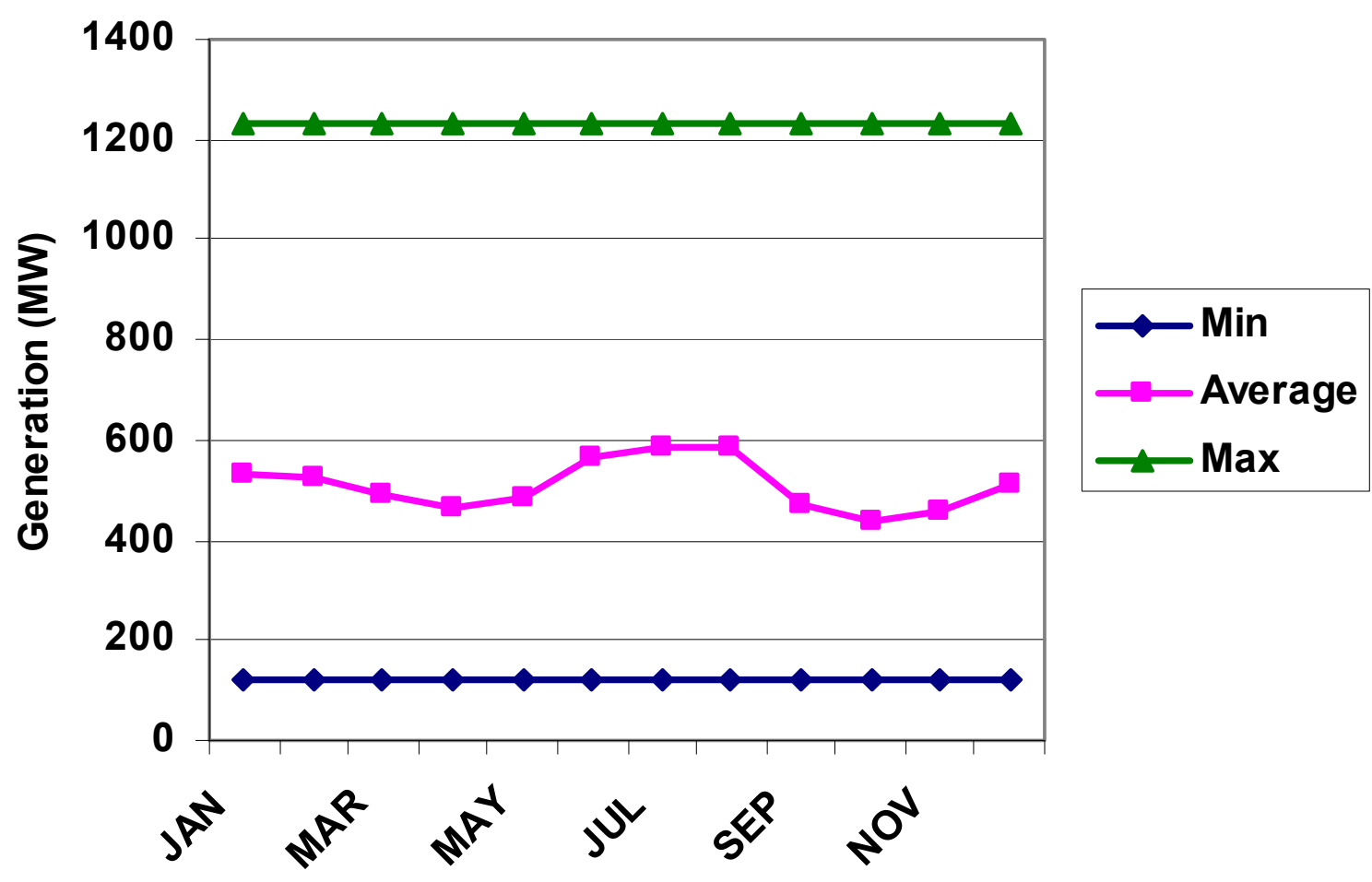

Figure 6.79 MAPS Monthly Simulation of Glen Canyon with No Wind

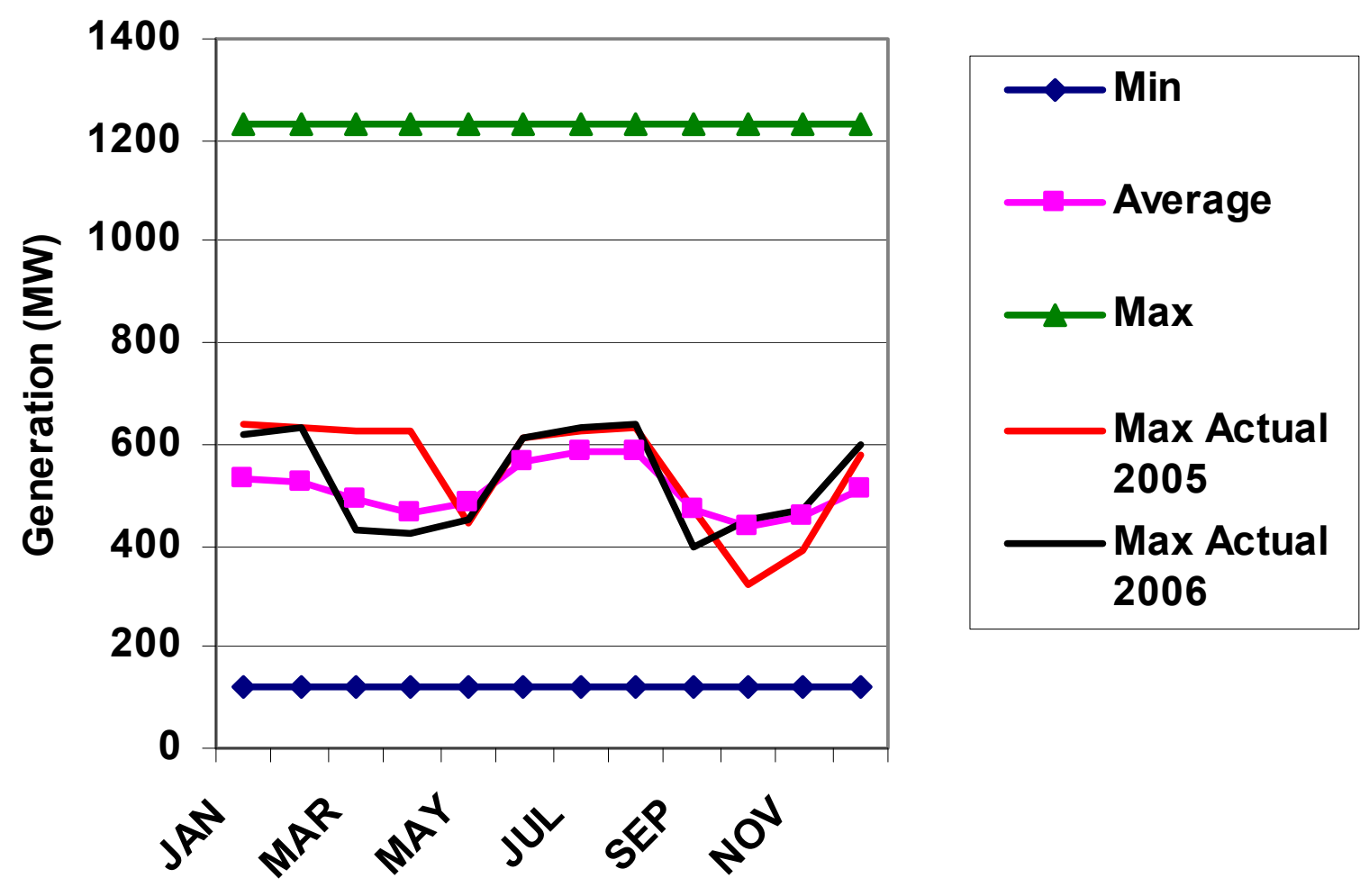

Figure 6.80 Historical Maximum Operation of Glen Canyon 


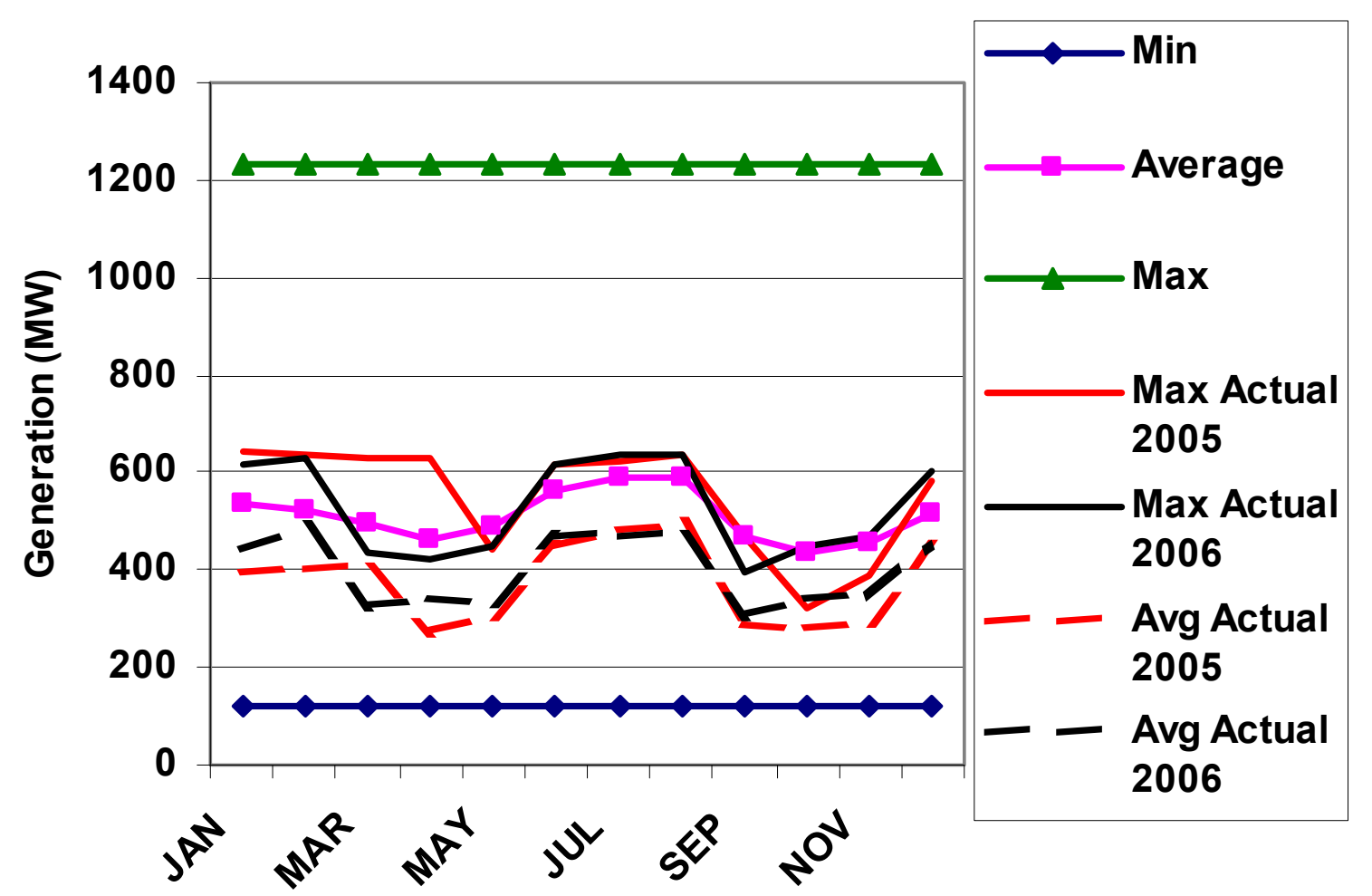

Figure 6.81 Historical Maximum and Average Operation of Glen Canyon

Fluctuations in monthly available energy do not affect all hydro plants as much as was seen at Glen Canyon. However, in order to assess the impact of reduced capacity and flexibility, all of the individual hydro plants were modeled as a flat block with a constant output each month set to utilize the same monthly energy as the base-case. The capacities were reduced to this average value so that the hydro plants did not provide any spinning reserve support. Figure 6.82 shows a comparison of the WECC operating costs for the various cases for the base assumptions and the corresponding case with flat hydro (cases ending in Hf). Figure 6.83 shows the delta impact on the WECC operating costs of the flat hydro. Restricting the hydro flexibility to fit with the reduced water levels adds $\$ 800 \mathrm{M}-\$ 1200 \mathrm{M}$ to the total cost of WECC operations. Restricting the flexibility of the hydro actually increases the value of the renewables by a few $\$ / \mathrm{MWh}$, because the no-wind case with flat hydro experiences shortages and much higher usage of the peaking units. The addition of the wind and solar generation alleviates these shortages. Additional sensitivities which modeled historical energies and capacities at hydro plants throughout the study footprint are discussed in the separate Northern Arizona University report. 


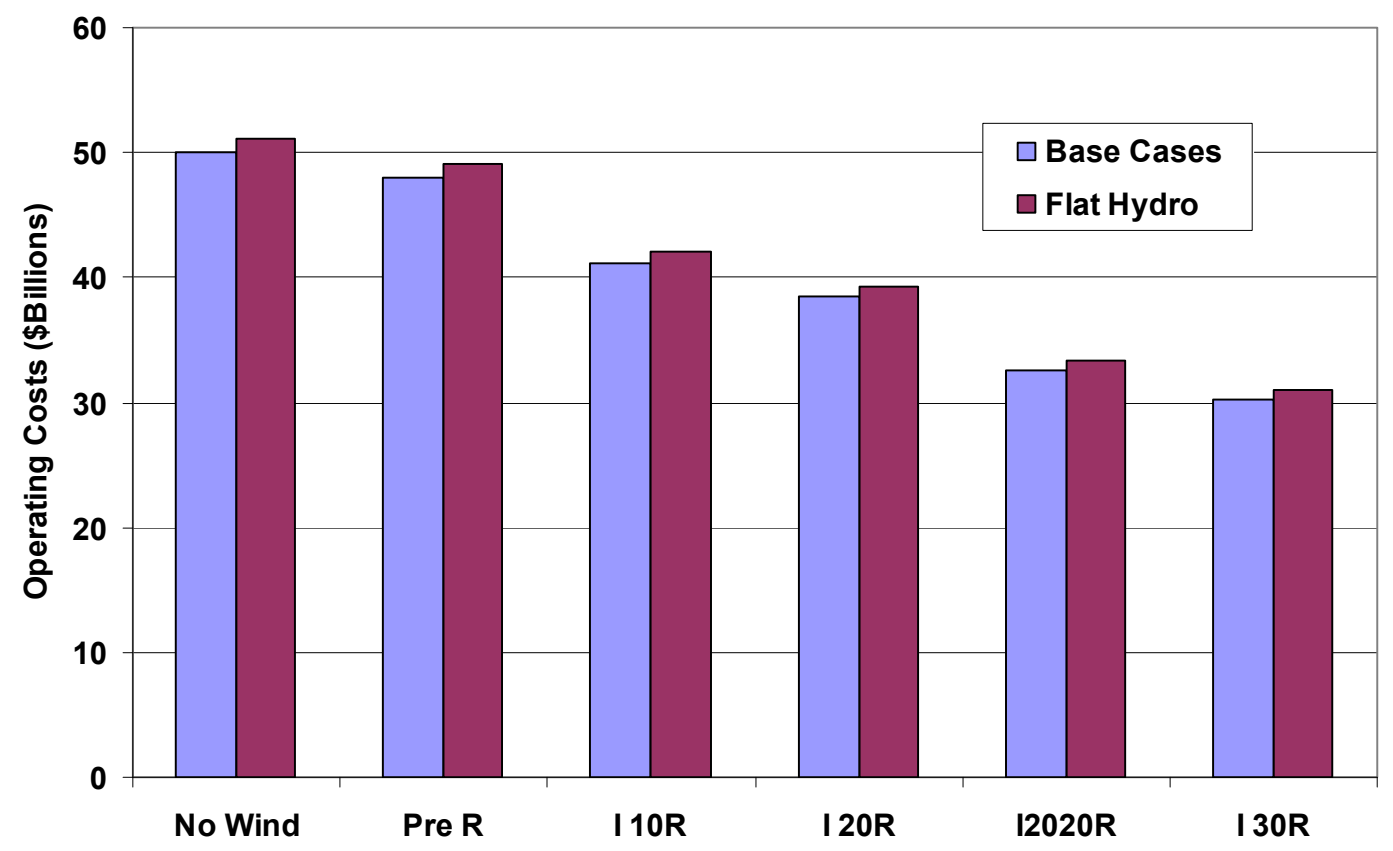

Figure 6.82 Impact of Flat Hydro Operation on WECC Operating Costs

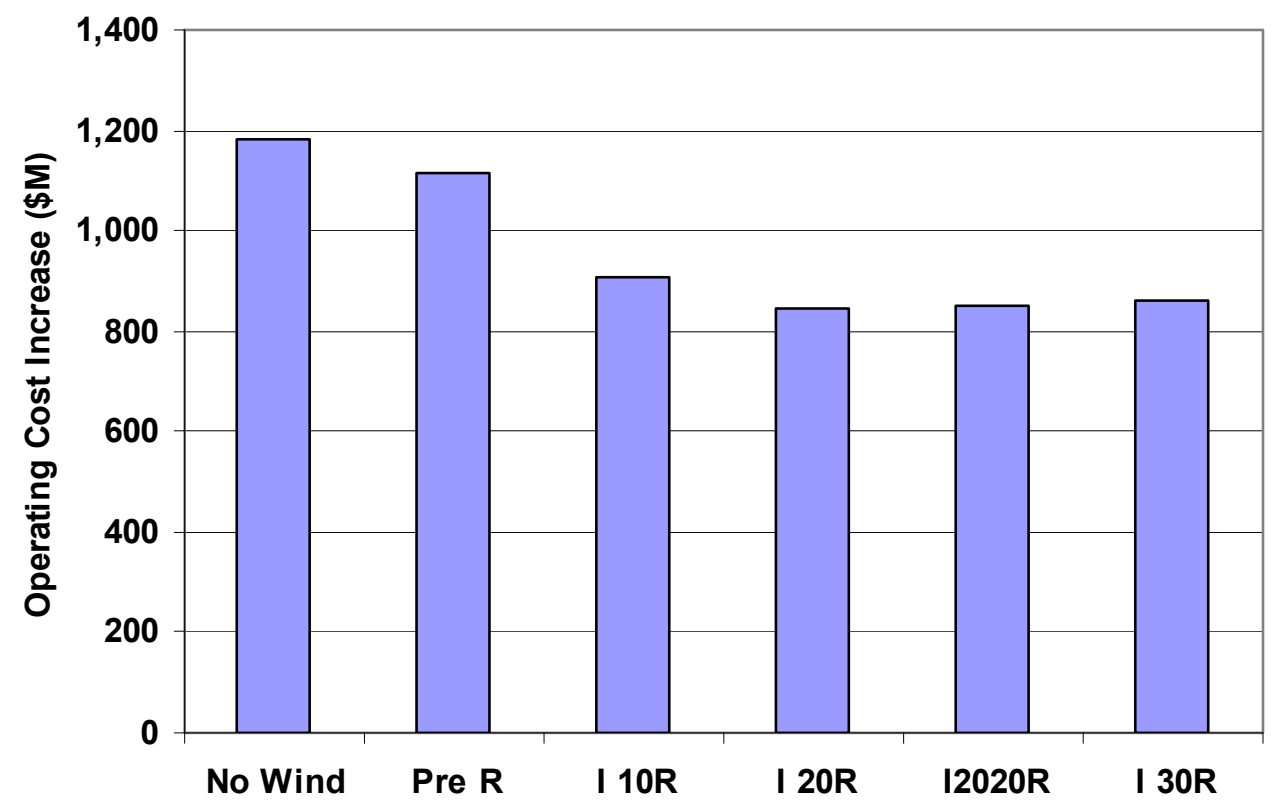

Figure 6.83 Incremental Operating Cost Impact of Flat Hydro Operation at Various Wind Penetrations

Figure 6.84 compares the spot price duration curves for the no-wind and $30 \%$ cases for the original hydro schedules and flat hydro schedules. With no renewables the higher spot prices go up significantly since the hydro is not allowed to do any "peak shaving". The low end drops slightly due to more hydro being dispatched off-peak. With $30 \%$ 
penetration, the high-end costs are mostly unchanged while the low-end cost drop slightly. Figure 6.85 shows the spot price curves for the "flat hydro" schedules for various renewable penetrations.

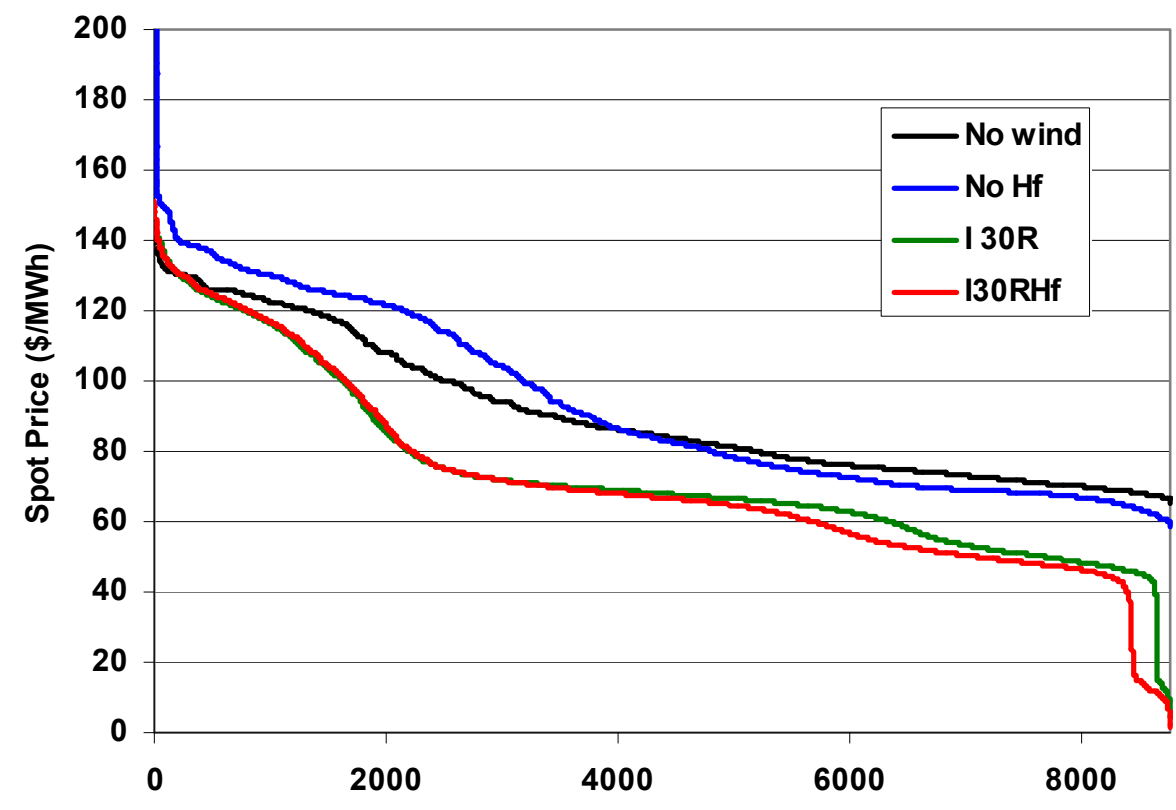

Figure 6.84 Spot Price Impact of Flat Hydro Operation

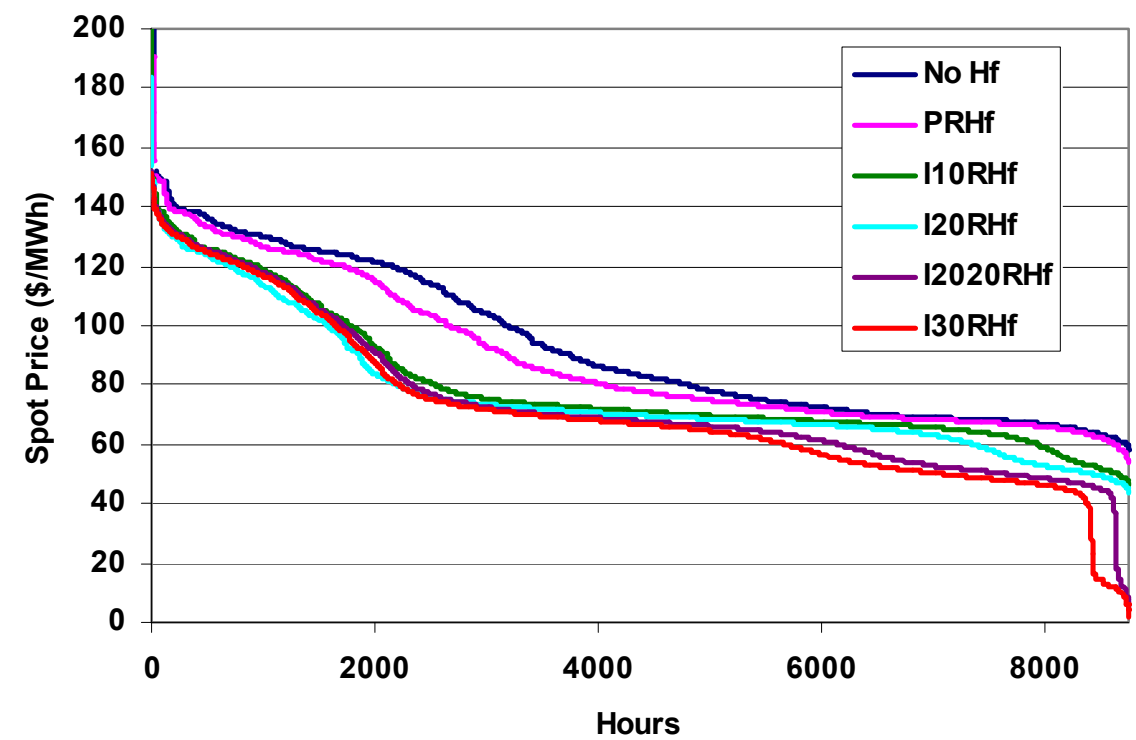

Figure 6.85 Spot Price Impact of Flat Hydro Operation by Penetration

As has been stressed previously, increased intermittent renewable penetrations will require increased flexibility from the balance of the system. Because there are no thermal 
cycling issues involved, hydro generation offers a potentially critical source for both inter-hour and intra-hour flexibility. Limits to this flexibility should be assessed at all of the hydro plants to further refine their role in renewable integration.

\subsubsection{Coal and Gas Pricing Sensitivity}

As stated earlier, the 2017 natural gas price assumed for this study was roughly $\$ 9.5 / \mathrm{MBTu}$. However, as seen in Figure 6.86, gas prices can be volatile. Future gas prices may be high or may drop significantly. Figure 6.87 shows a comparison of the typical cost of energy from a steam coal plant and a gas-fired, combined-cycle unit as a function of gas price. When the gas price is low enough the combined-cycle units are more economically attractive to operate than the coal units. This reversal in the priority order of these two technologies can also take place due to increases in the carbon tax. All of the initial cases assumed a $\$ 30 /$ ton tax on $\mathrm{CO} 2$ emissions. Each $\$ 1 /$ ton increase in this tax will increase the cost of coal generation by about $\$ 1 / \mathrm{MWh}$ but will only add $\$ 0.6 / \mathrm{MWh}$ to the cost of gas-fired, combined-cycle generation.

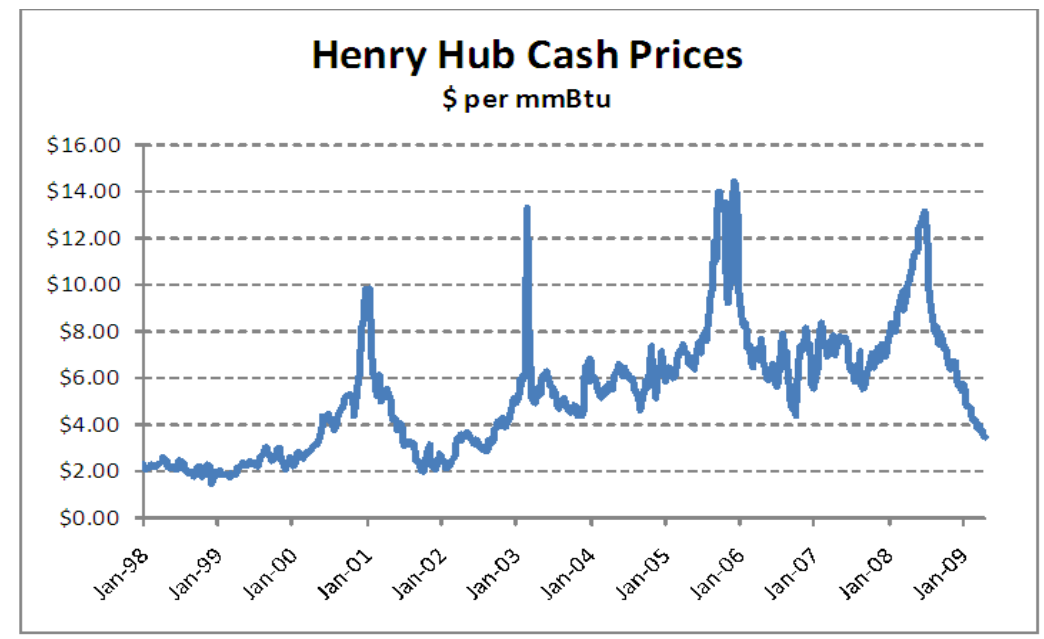

$\bigoplus$ Energy Ventures Analysis, Inc.

Figure 6.86 Historical Henry Hub Natural Gas Prices 


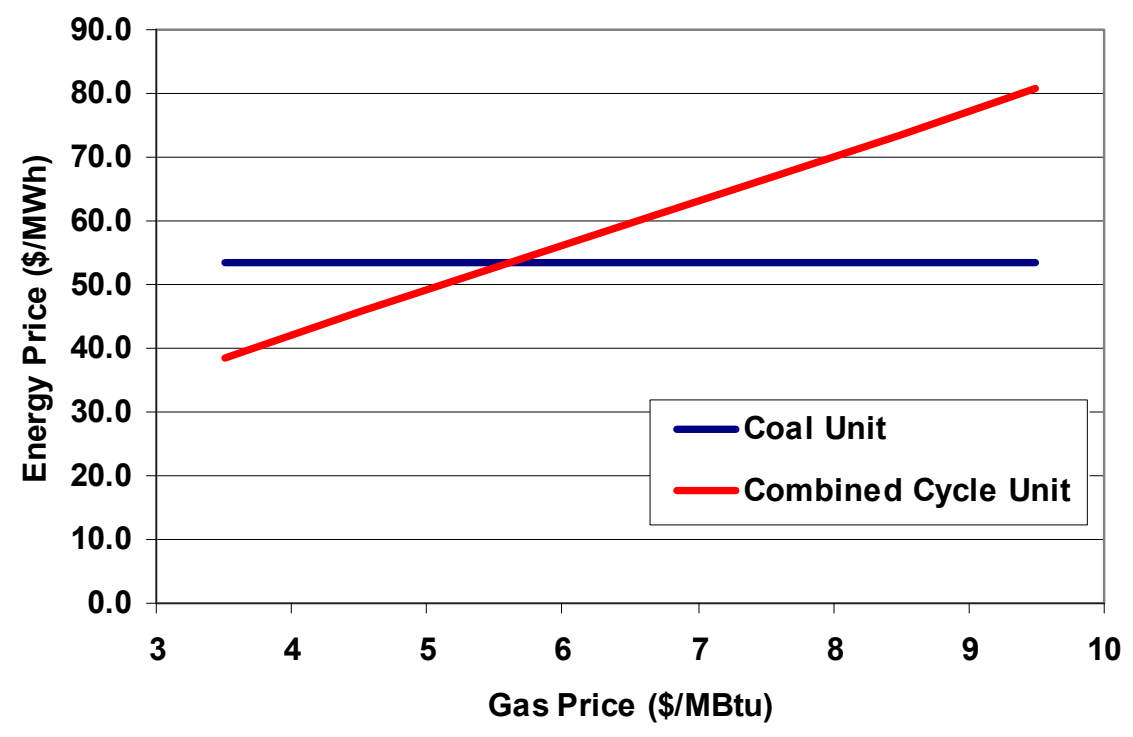

Figure 6.87 Coal vs. Combined-Cycle Cost Comparison with \$30/ton CO2 Tax

In order to evaluate the impact, cases were run with the carbon tax held at $\$ 30 /$ ton but with the gas price reduced to $\$ 3.50 / \mathrm{Mbtu}$, thereby making the combined-cycle plants more attractive to operate than the coal plants. Figure 6.88 shows the generation by type for the no-wind and 30\% case for the base-case assumptions and the low gas price cases. In the new "no-wind" case, the combined-cycle generation increases and steam coal generation drops off compared to the original case. In the new $30 \%$ case, it is now the coal plants that pick up two thirds of the displacement and combined-cycle units only carry about a third of the change. Figure 6.89 shows the impact on the total emissions and Figure 6.90 shows the incremental change in WECC emissions due to the reduced gas price. The emission reductions due to the renewable generation have jumped significantly now that coal plants are being displaced rather than gas plants. 


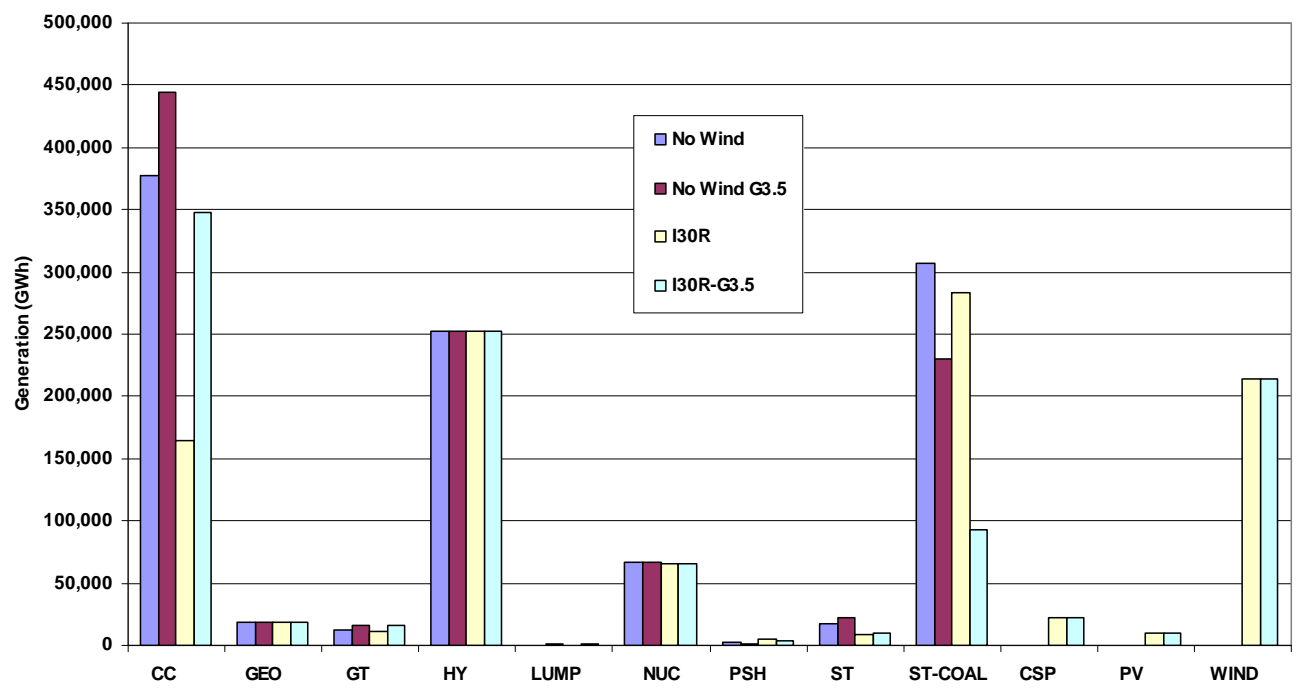

Figure 6.88 Generation by Type with Gas Price Reductions, WECC

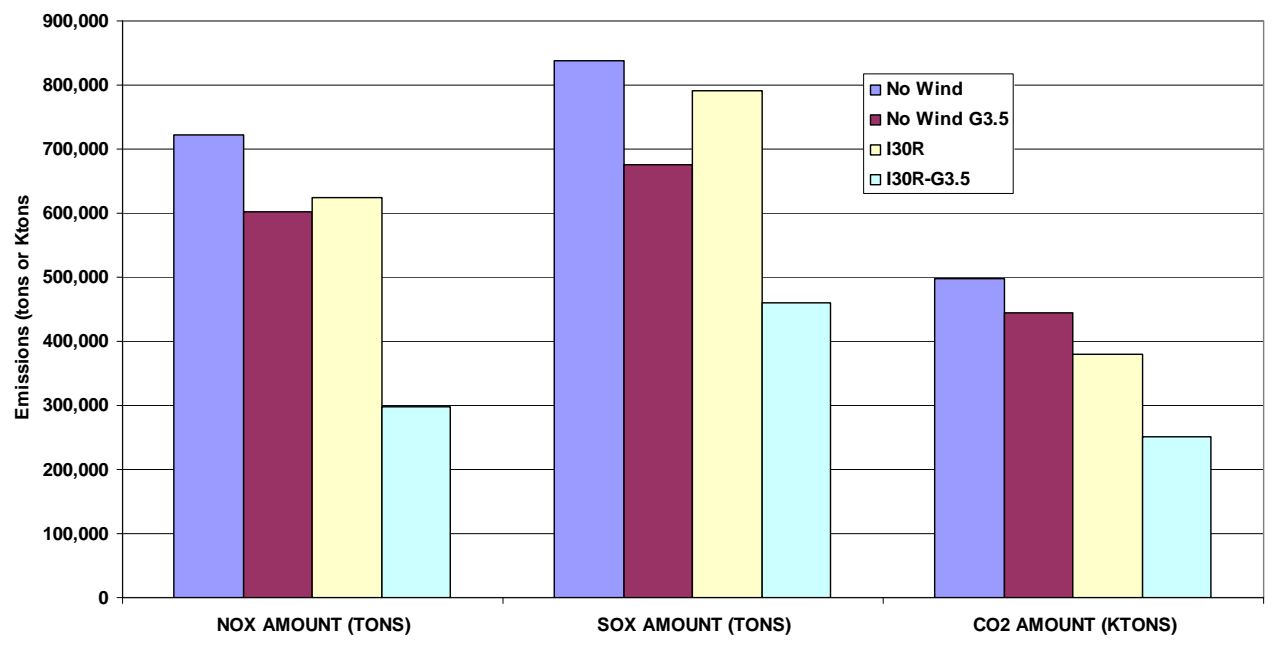

Figure 6.89 Emissions with Gas Price Reductions, WECC 


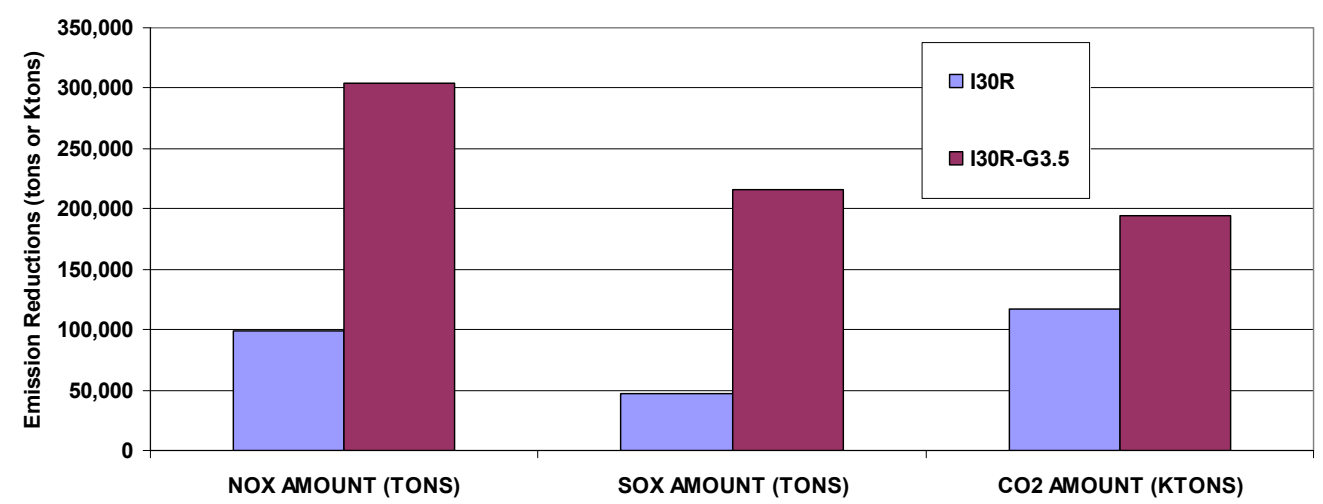

Figure 6.90 Emission Reductions with Gas Price Reductions

Figure 6.91 shows the change in savings due to the renewable generation in both millions of dollars and in dollars per megawatt hour of renewable generation. Because gas prices have dropped so much, the value of the renewable generation has been almost cut in half. If the priority reversal in the economic dispatch had been accomplished by increasing the coal price or the carbon tax then the reduction in coal generation and corresponding emissions would have been achieved without dropping the value of the renewables. In fact, the value of the renewables would have increased. One significant advantage of the reduced gas price cases is that the less flexible coal generation is being turned off and the more flexible gas-fired generation is remaining on line. The gas-fired units are more able to respond to the variability needs of the system with high renewable penetrations. Flexibility and the ability to start up and shut down generation quickly will be important as renewable penetration levels increase.
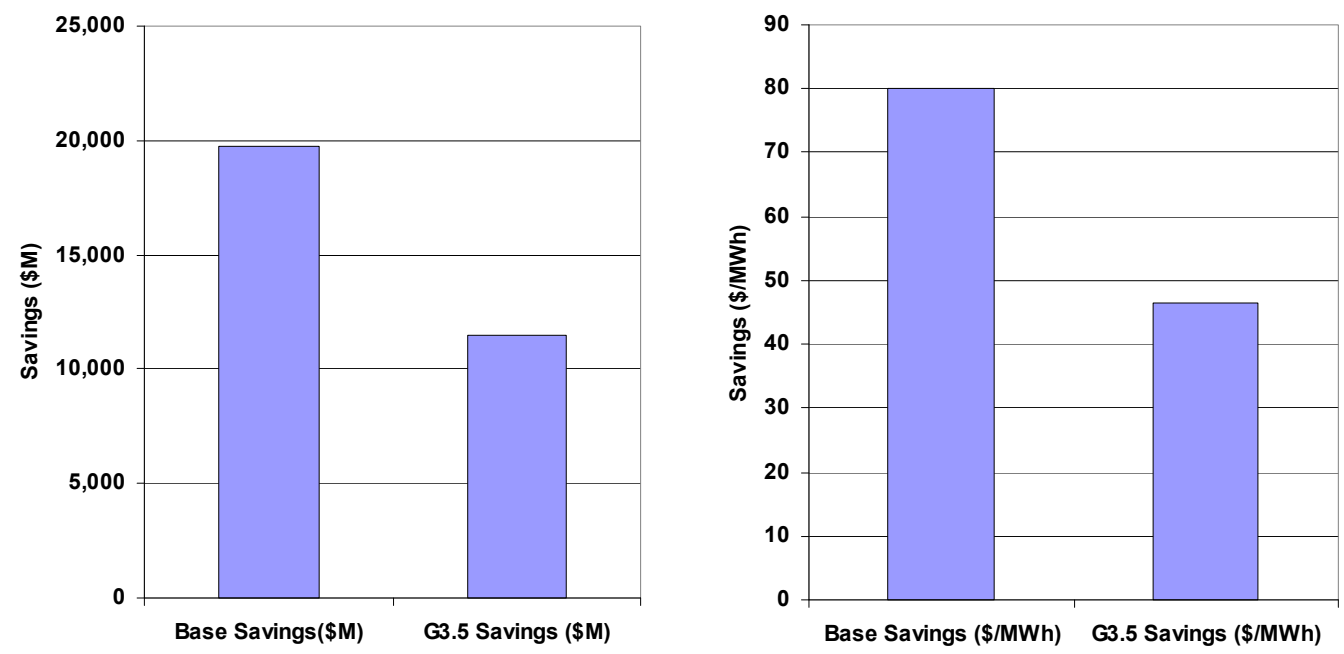

Figure 6.91 Impact of Gas Price Reductions on Operations Savings, I30 Case 


\subsubsection{Coal Plant Operation}

One concern that has been raised is the amount of flexibility that has been assumed for the fleet of coal-fired generation. The simulation assumed that coal units could cycle down to $40 \%$ of their maximum ratings when needed. The simulations also did not increase the variable O\&M costs for units that needed to increase their cycling. This will likely be the case but there is a lack of good industry data available to quantify these values. Figure 6.92 shows some actual economic and emergency minimum operating points for a number of coal plants in the WECC system. While the values assumed in the simulation appear quite reasonable compared to the emergency minimums, it was pointed out by one system operator that this is not an operating point that can be achieved several times a week. The amount and depth of the cycling will vary by plant based on the unit design. For some plants, frequent cycling to emergency minimums can lead to tube leaks and increased maintenance cost on the units. The "Economic Minimum" was described as the operating point where the units could be dispatched to without significant increases in costs.

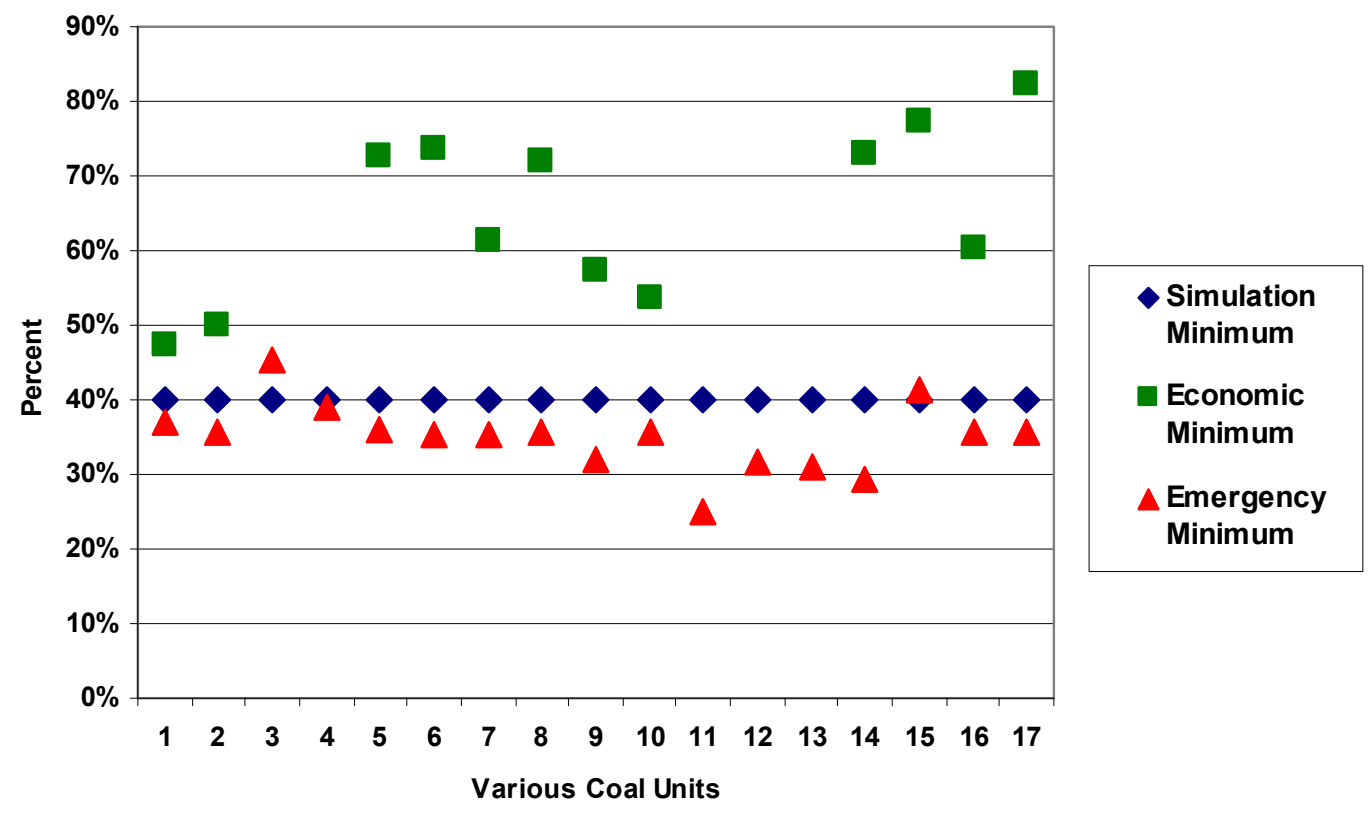

Figure 6.92 Typical Minimum Operating Ratings of Coal Units as a Percent of Capacity

Figure 6.93 shows the number of times a sample coal unit was cycled below a particular operating point and then back up again in the simulated cases. For example, the $70 \%$ bar represents the number of times that a unit's generation dropped below $70 \%$ of its rating and then came back up above $70 \%$. From this figure it can be seen that up to a $10 \%$ penetration of renewables resulted in essentially no cycling of the unit. The $20 \%$ case resulted in about 20 cycles per year below the $60 \%$ level. But most of the movement was just within the top $20 \%$ of the unit's capacity. It wasn't until the $30 \%$ case that significant 
deep cycling occurred on this unit. Figure 6.94 and Figure 6.95 show similar plots for $350-\mathrm{MW}$ and 500-MW units. For the larger units, minimal cycling occurs before the $30 \%$ penetration level, and even then is primarily limited to the top $10 \%-20 \%$ of their range.

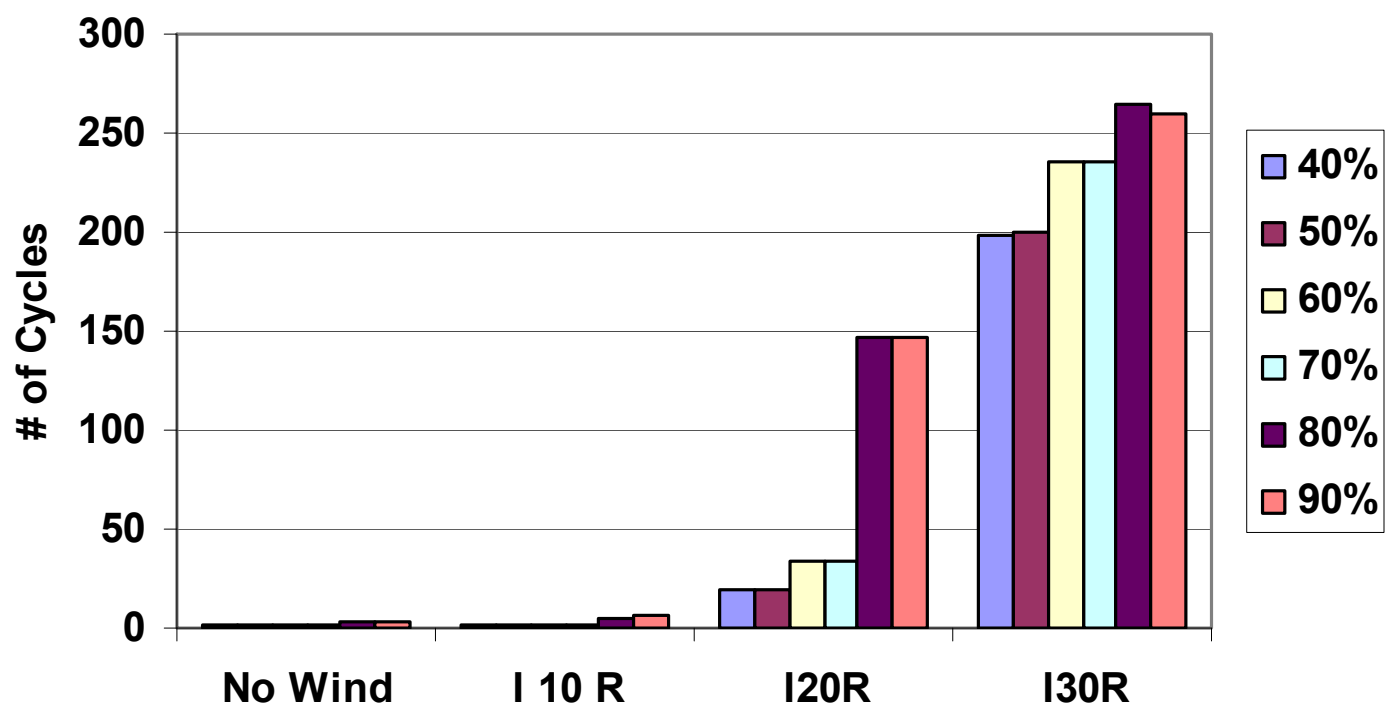

Figure 6.93 Number of Cycles Below Operating Levels - 100-MW Coal Unit

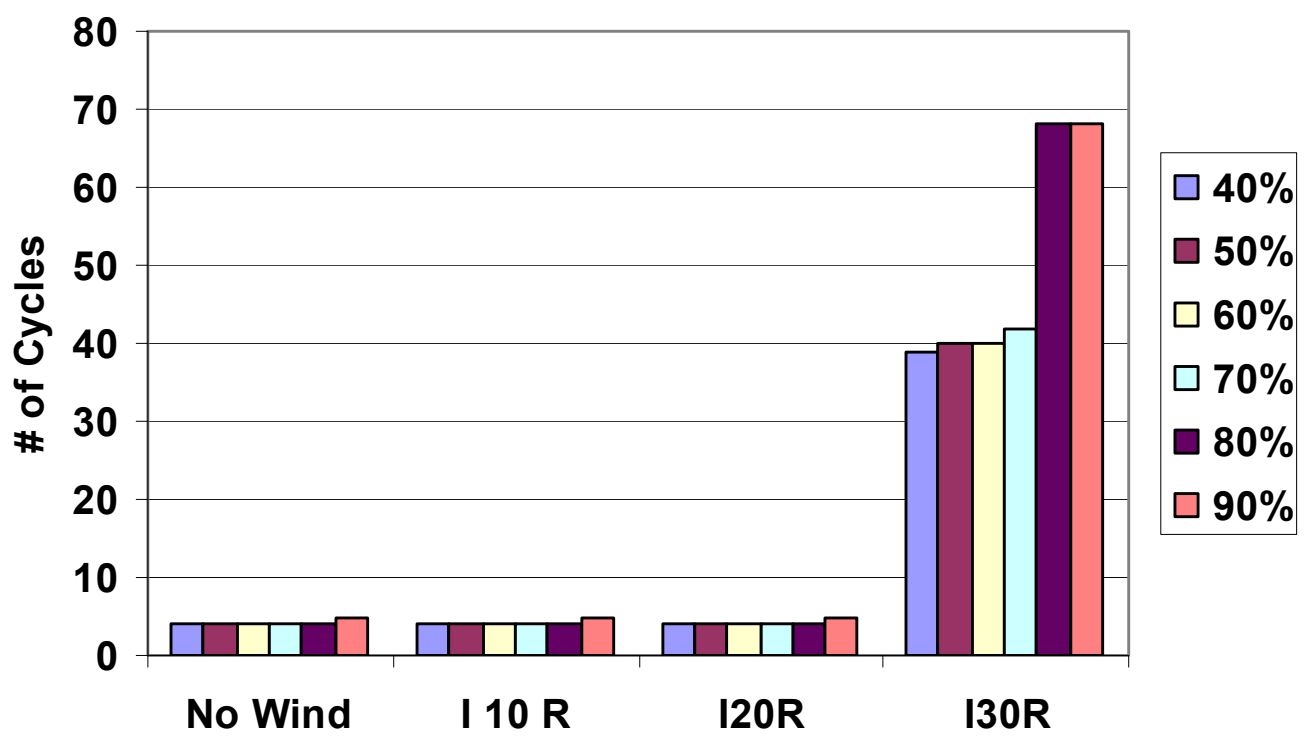

Figure 6.94 Number of Cycles Below Operating Levels - 350-MW Coal Unit

Figure 6.96 shows the higher level cycling, (the number of times that the unit dropped below $80 \%$ or $90 \%$ of its rating,) for the 500-MW unit on a monthly basis for the $30 \%$ case. The bulk of the cycling appears to occur in just a few months. Coal plants will 
typically schedule their maintenance in the off-peak months. It may be appropriate to shut down additional coal plants in some of the higher wind months, and allow more flexible gas-fired generation to be operated if these cycling levels are too severe for the coal plants to handle. However, doing this will reduce the economic attractiveness of the renewable generation. Figure 6.97 shows the operation for the week of April $10^{\text {th }}$. This high-wind, low-load week caused the unit to cycle more than the operators would likely care to, but this was fairly rare even at the $30 \%$ penetration level.

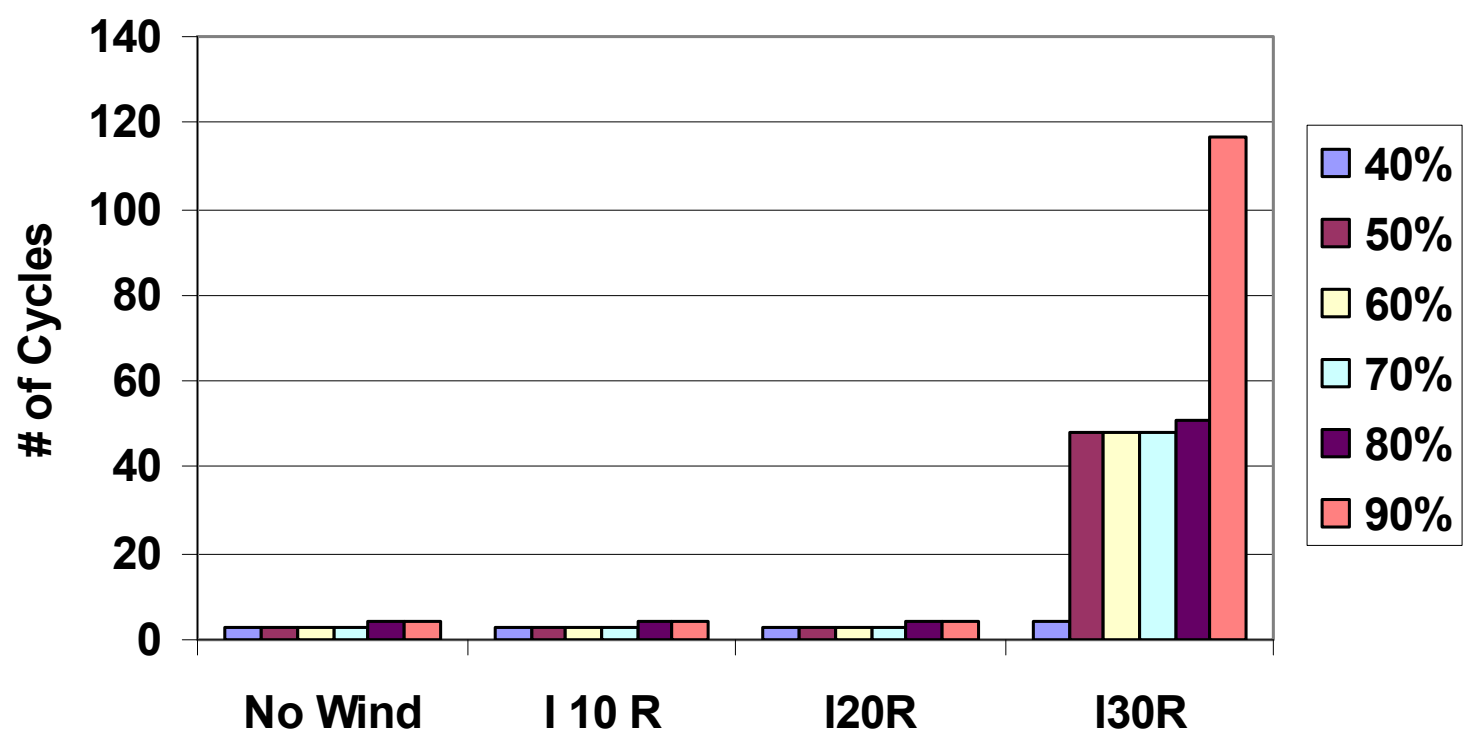

Figure 6.95 Number of Cycles Below Operating Levels - 500-MW Coal Unit

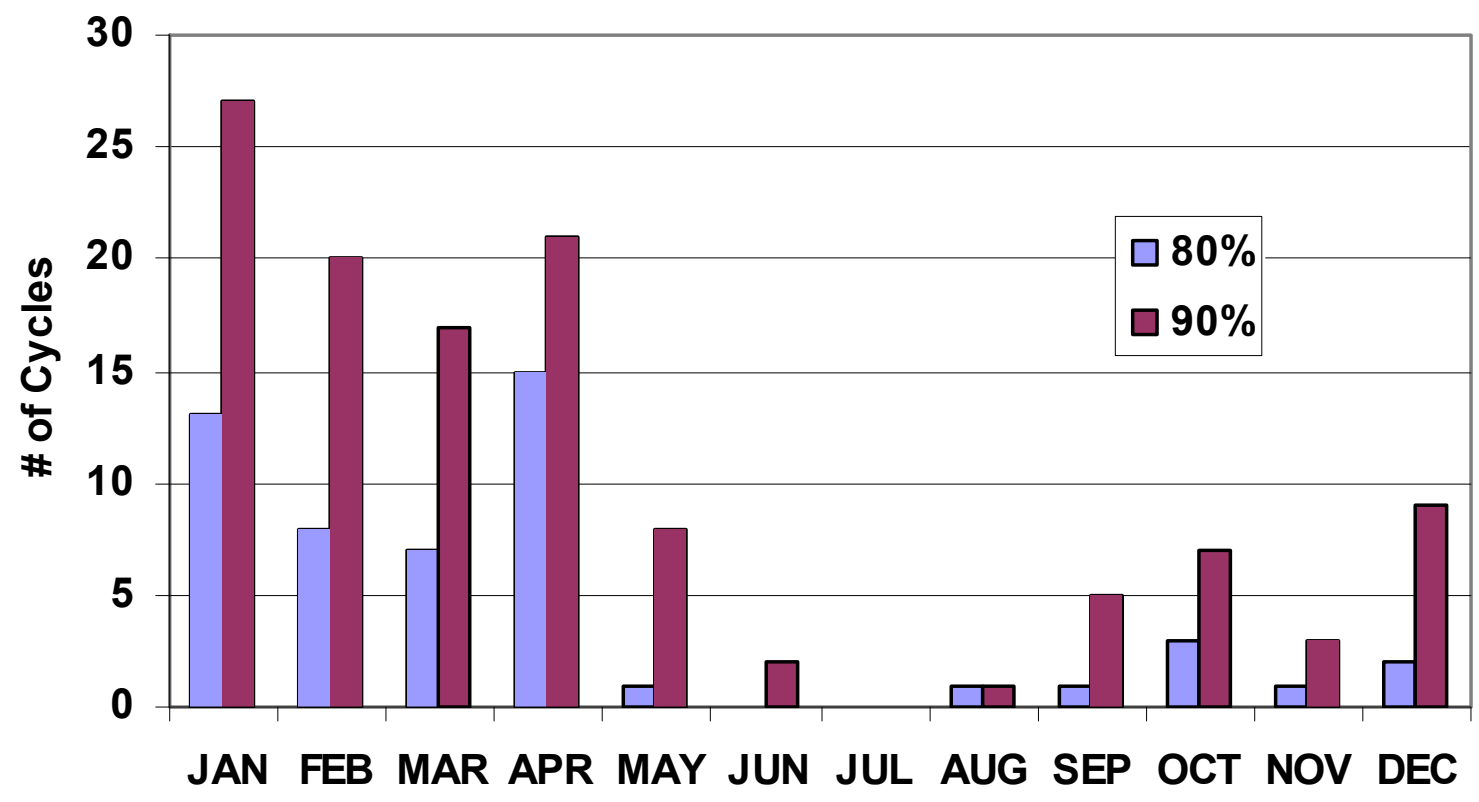

Figure 6.96 Cycles Per Month Below $80 \%$ or $90 \%$ of Rating for $500-M W$ Coal Unit at $30 \%$ Penetration 


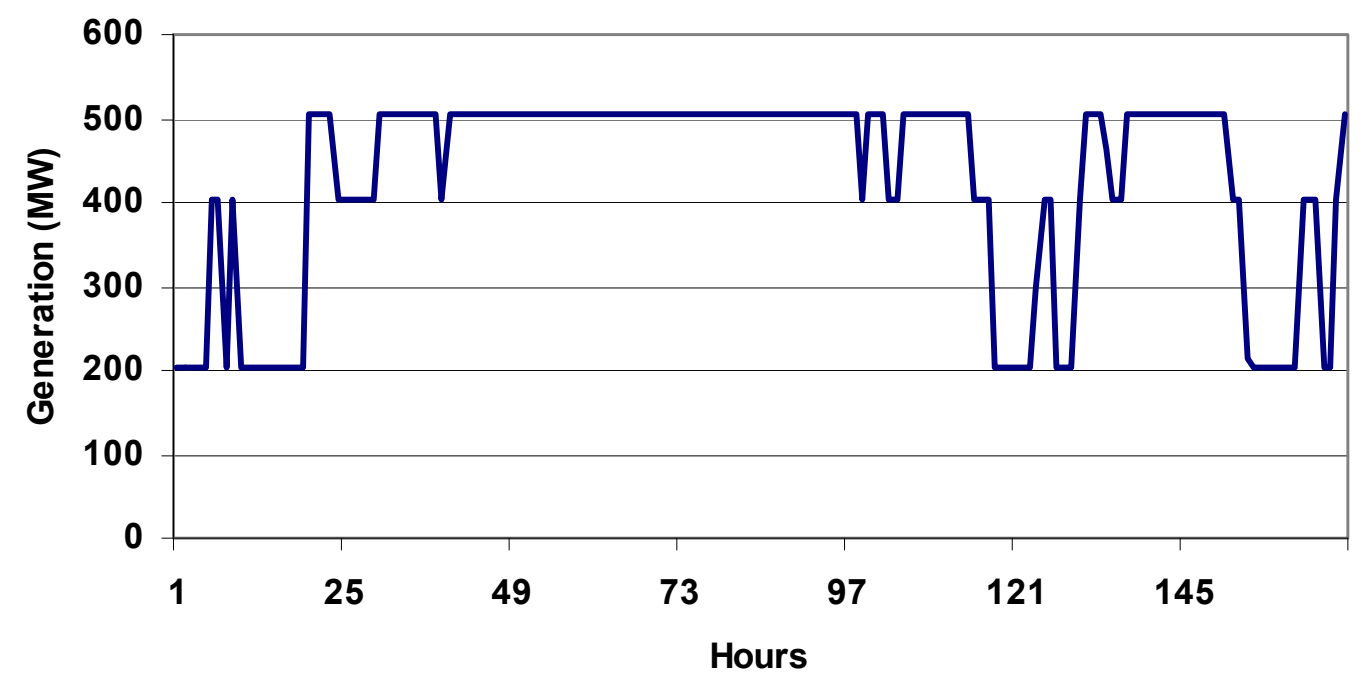

Figure 6.97 Operation of 500-MW Coal Unit Week of April 10th at 30\% Penetration, In-Area Scenario

Several additional cases were simulated with the minimum operating points of all of the coal plants in WECC restricted to $50 \%, 60 \%$, and $70 \%$ of the full rating. In terms of spilled energy, Figure 6.98 shows that even in the 20/20 case with minimums at $60 \%$ there is not any significant increase in spilled energy. (In the following figures, a case ending in "C60" refers to the coal unit minimums set to $60 \%$ of their nameplate rating.) At the $30 \%$ penetration level, the spilled energy starts increasing at the $50 \%$ minimum level and is nearly doubled when the minimums are raised to $70 \%$, but this is still less than $1 \%$ of the total wind generation. Figure 6.99 shows that there is minimal impact on the unserved energy when the coal minimums are increased above $40 \%$.

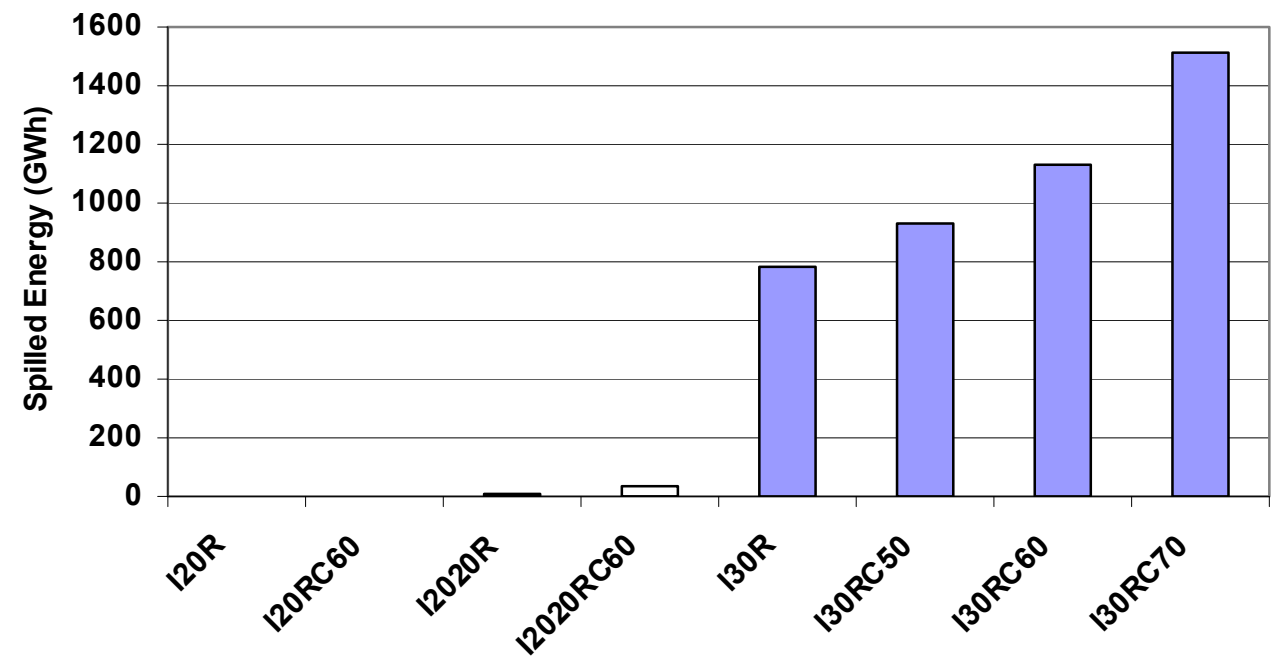

Figure 6.98 Spilled Energy as a Function of Coal Minimum Operating Points 


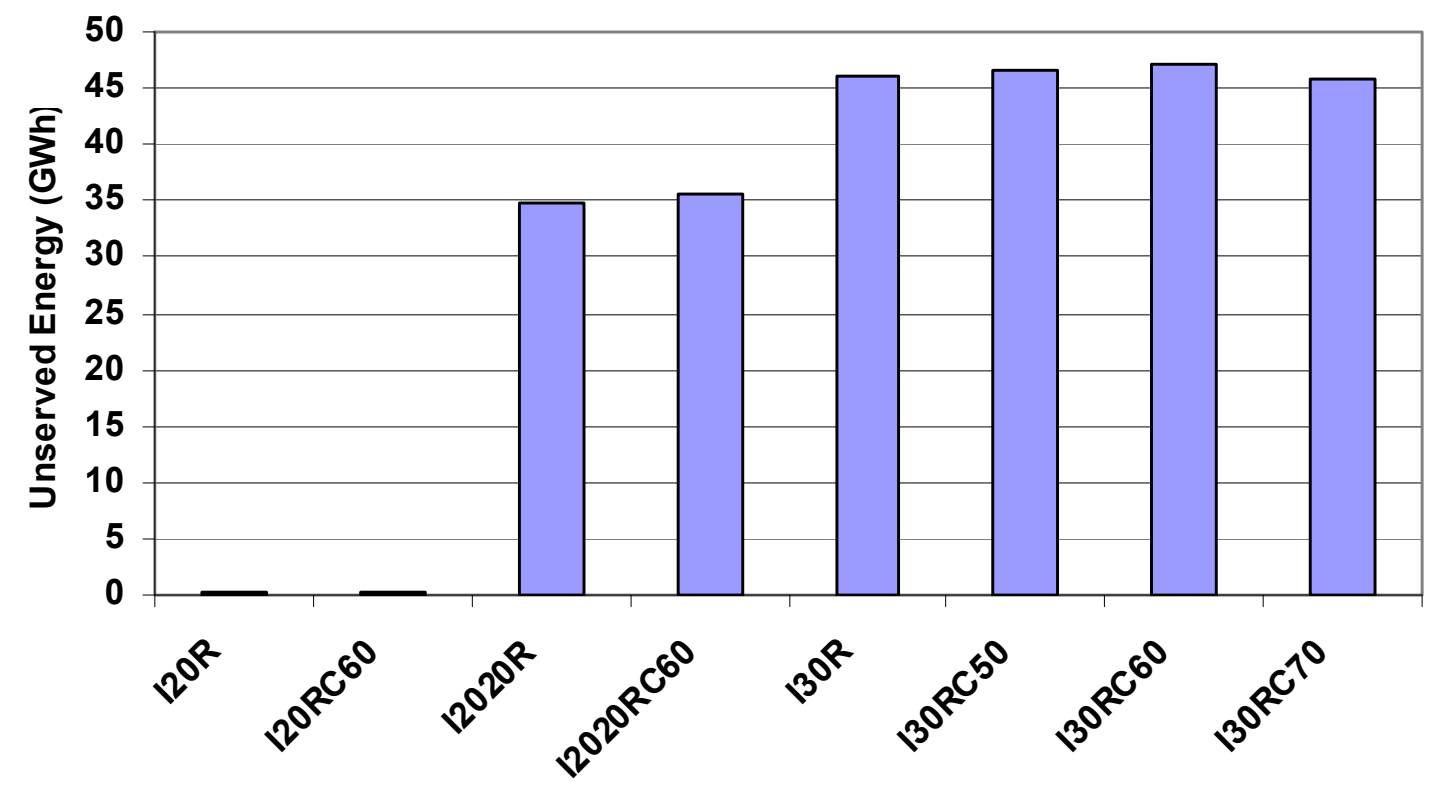

Figure 6.99 Unserved Energy as a Function of Coal Minimum Operating Points

Figure 6.100 shows the impact of coal minimums on the average system spot price. Reducing the flexibility of the coal plants keeps more generation on line and forces the spot prices to drop slightly.

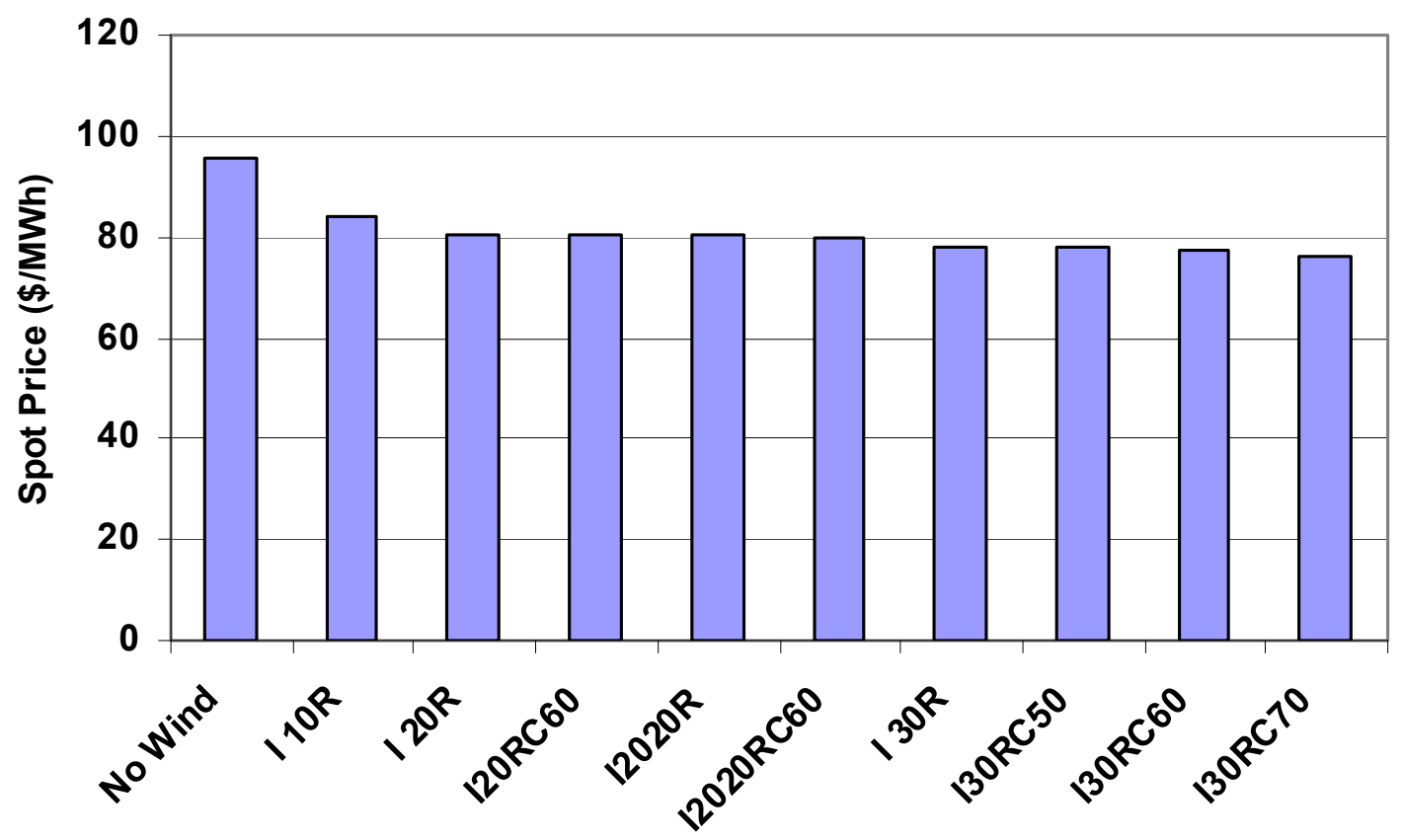

Figure 6.100 Average System Spot Price vs. Coal Minimums 
The bars in Figure 6.101 show the total WECC operating costs. The differences appear fairly small. However, as Figure 6.102 demonstrates, increasing the coal minimums from $40 \%-70 \%$ can increase system operating costs by almost $\$ 160$ million, which is not insignificant.

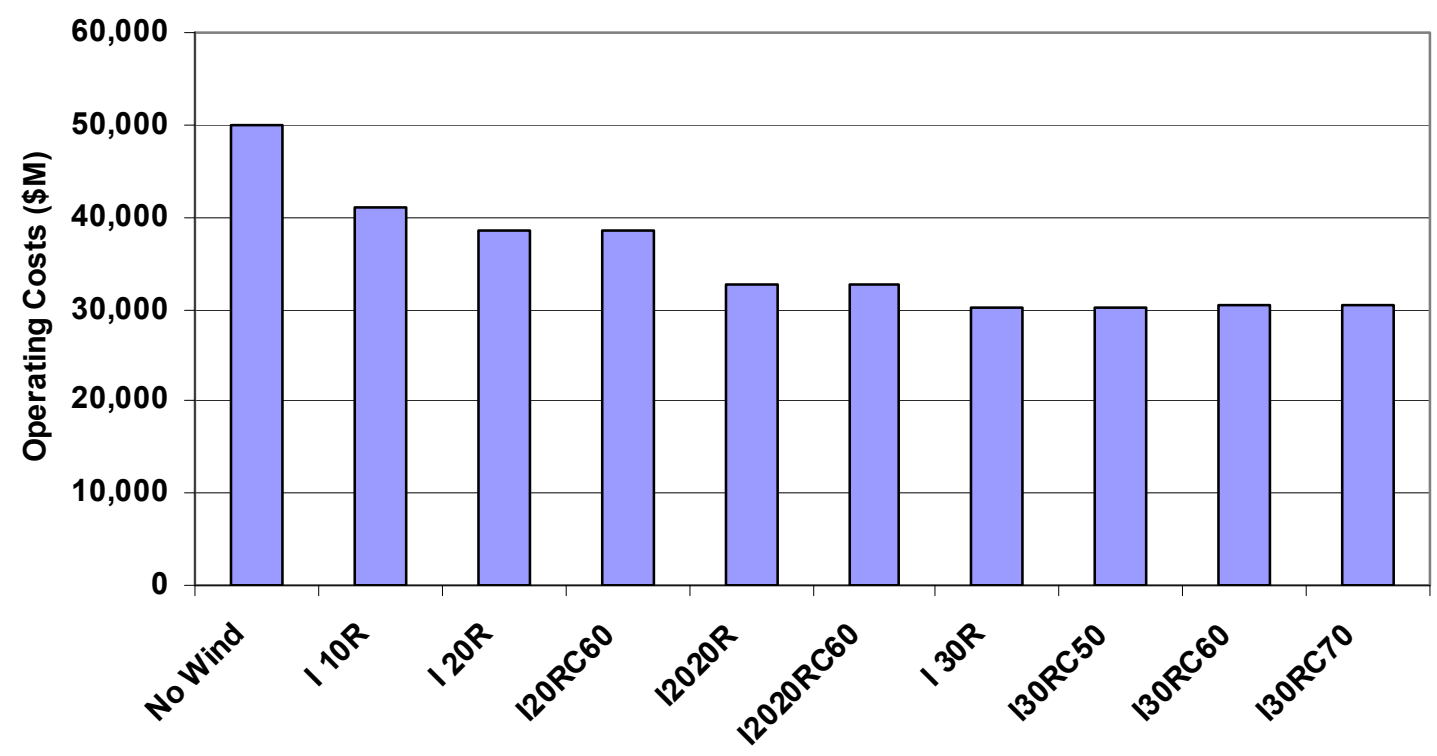

Figure 6.101 WECC Operating Costs vs. Coal Minimums

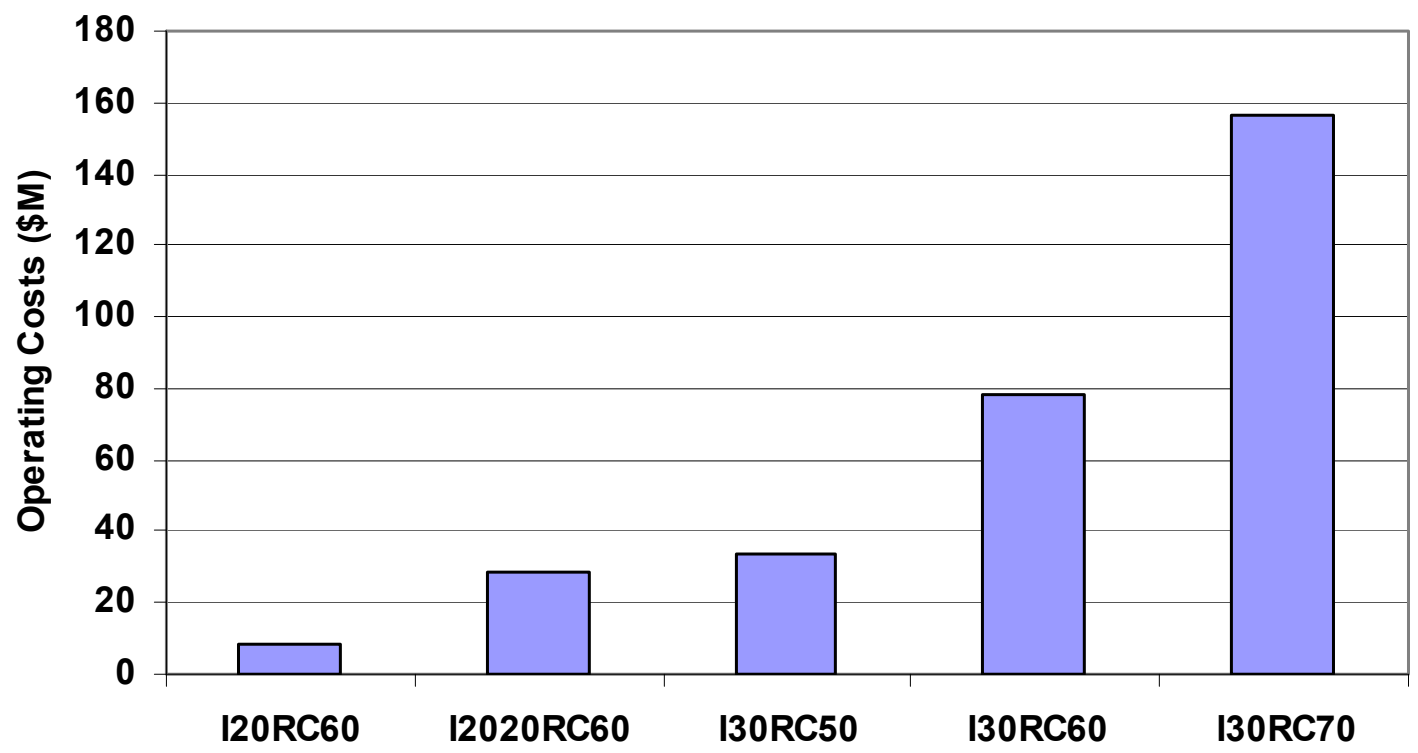

Figure 6.102 Increased WECC Operating Costs Over Coal at 40\% Minimum Rating 
Figure 6.103 shows an interesting impact on the coal units themselves. Increasing the minimums on the coal plants makes them unavailable to cycle down in off-peak periods so that their energy production also increases. However, their total revenue, as measured by their generation multiplied by the hourly spot price, actually decreases. This implies that while their energy production has increased, their actual value to the system has diminished.

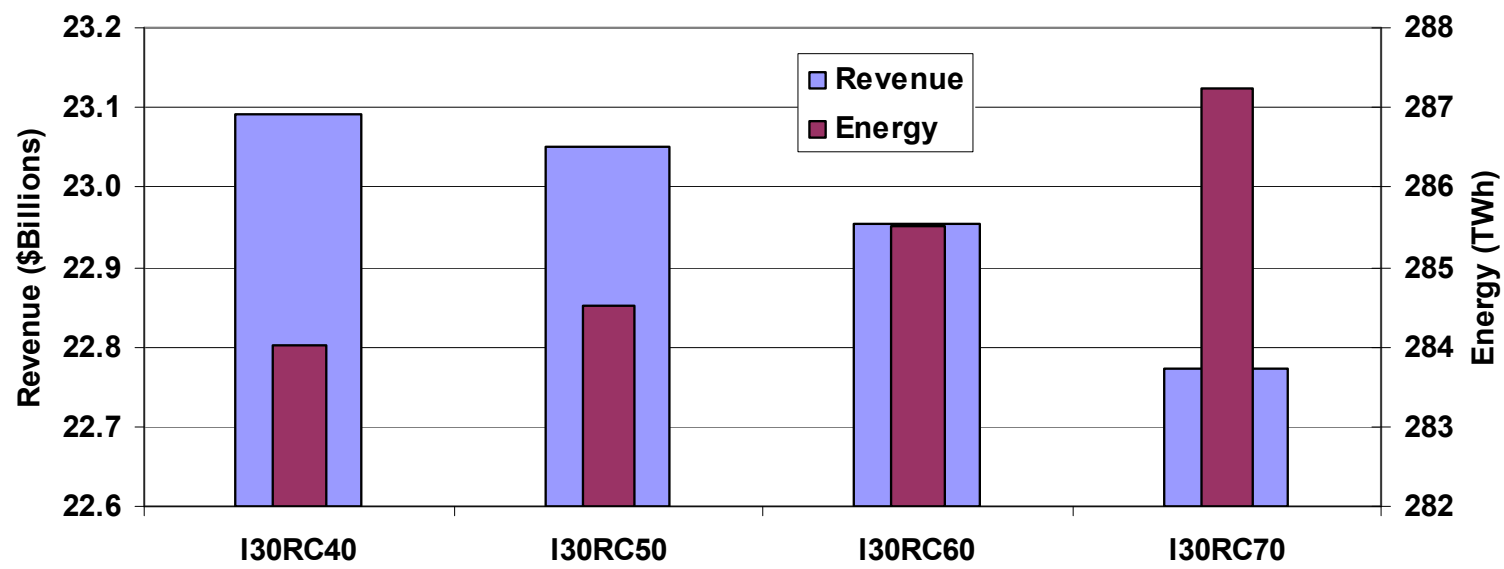

Figure 6.103 WECC Coal Plant Revenue versus Coal Unit Minimum Operating Points

Figure 6.104 shows that increasing the coal minimums increases the total emissions since the coal plants can no longer be displaced as much by the cleaner renewable energy, but the changes are slight.

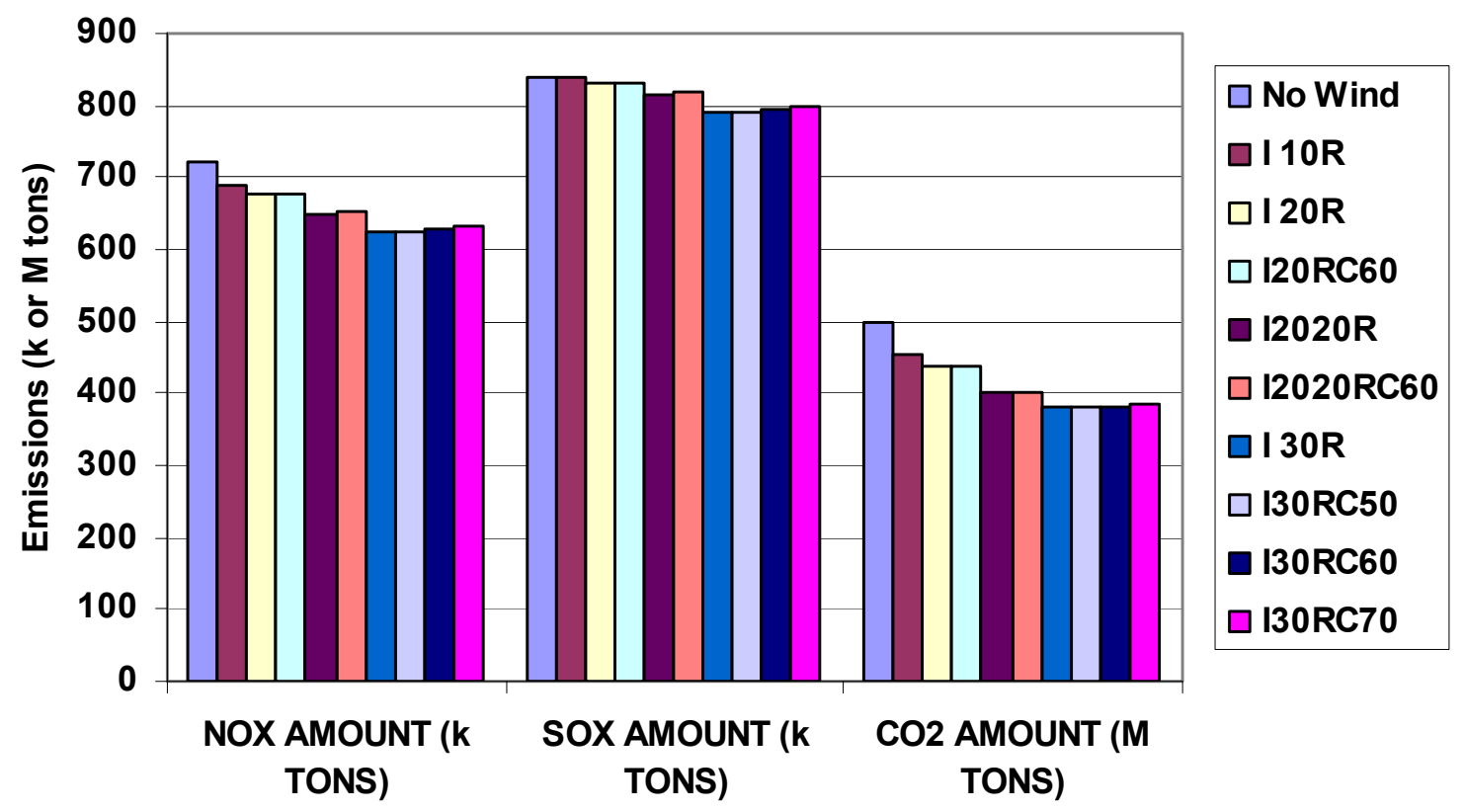

Figure 6.104 Impact of Coal Minimums on WECC Emissions 


\subsubsection{Balancing Area Consolidation}

The statistical analysis discussed the need for consolidation (either real or virtual) of the small balancing areas when trying to accommodate high penetrations of renewable generation. A test case was run to see the operational impact for the In-Area, 10\% penetration case. The base-case modeled spinning reserve as $6 \%$ of load on a regional basis (i.e. Arizona/New Mexico, Rocky Mountain, etc) with at least half of that required to be spinning. For the change case, the system still held spinning reserve at $6 \%$ of the load, but on a zonal basis. For comparison, WECC was divided into 5 regions but 106 zones. These zones are roughly equivalent to the existing system balancing areas in the Southwest, but there are multiple zones per balancing area in the Northwest. Figure 6.105 compares the annual spot price duration curve between the two $10 \%$ penetration cases and with no wind. (The case ending in ZSR refers to "zonal spinning reserves.") When the spinning reserve is held on a zonal basis, it requires a lot more generation to be committed, which in turn drives down the system spot prices and the value of renewable generation, and increases total operating costs.

With spinning reserves determined on a zonal basis, WECC simulated operating costs were about \$2 Billion higher than with the reserves shared over larger regions for the $10 \%$ In-Area case. This is expected to increase with higher penetration levels. In this example, the total system spinning reserve was held constant. It was simply allocated over multiple zones. As the statistical analysis showed, the volatility and uncertainty are much higher for the smaller balancing areas, which mean that even more spinning reserve would be required to accommodate renewable generation. This would drive costs up even more. Because of the significant operating benefits of balancing area cooperation, this may be a fertile area for further investigation in another study.

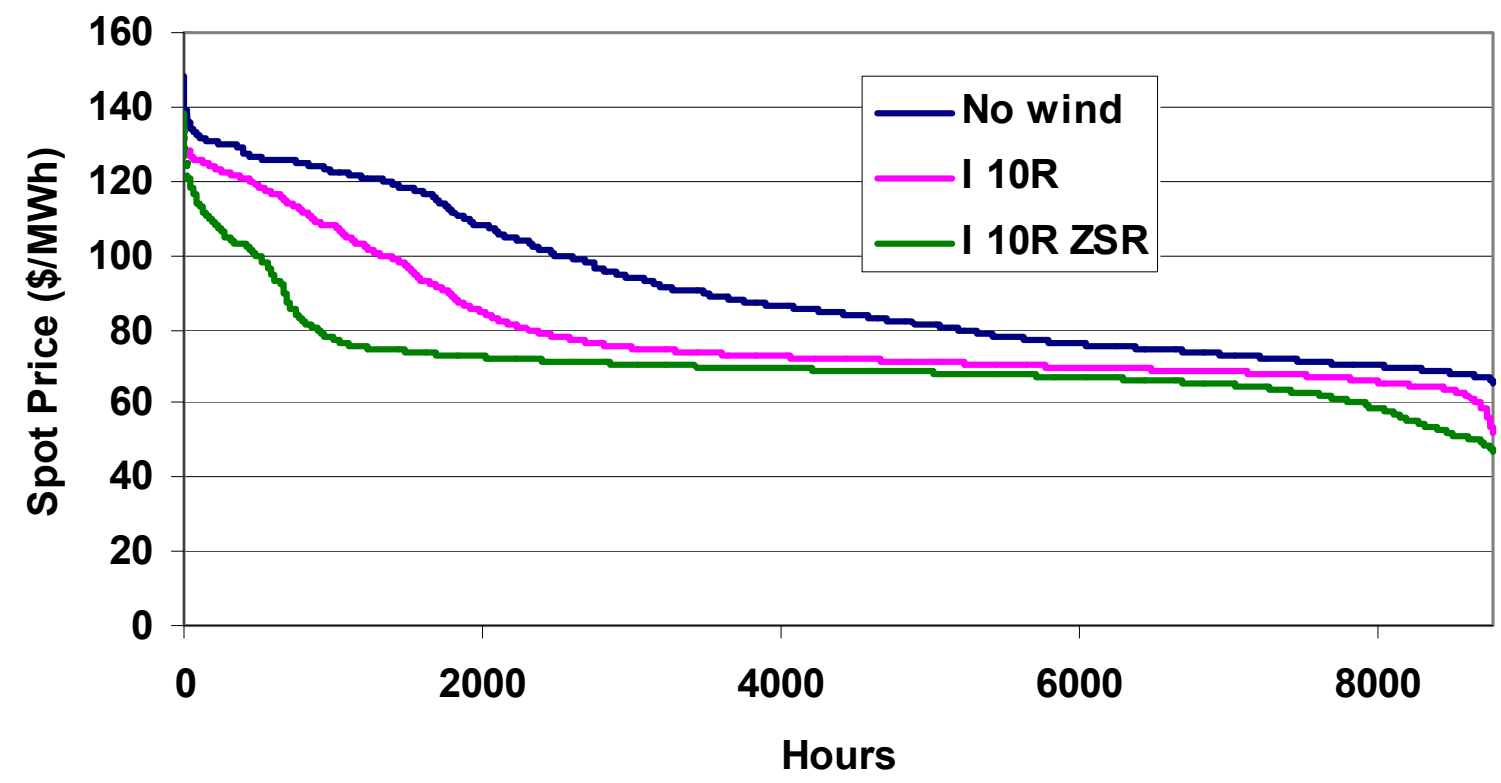

Figure 6.105 Spot Price Impact of Balancing Area Consolidation, 10\% Penetration. 


\subsubsection{Transmission Expansion Sensitivity}

The Local-Priority and Mega-Project Scenarios utilized wind sites with higher capacity factors, but consequently needed to include additional transmission facilities in order to deliver the more remote wind energy. Sensitivities were run to see the impact of building the more remote wind sites without reinforcing the transmission capability between the regions. It was assumed that all existing transmission was available to all of the generators to compete on an economic basis. Figure 6.106 shows the impact on system curtailed energy and contingency reserve shortfalls. The cases ending in " $\mathrm{t}$ " assumed no new transmission build-out. The reserve shortfalls did not change significantly between the cases, which is not surprising, because when the new renewable resources were constrained there were sufficient existing resources in the region to serve the load. Curtailed energy was not evident in either case at the $10 \%$ penetration level. This suggests that initial expansions down these paths could be accomplished without new long distance transmission enhancements. Even at the higher penetrations, the Local-Priority Scenario did not experience significant growth in curtailed energy. Beyond the 10\% level, however, the Mega-Project Scenario began curtailing more wind energy, and by the 30\% penetration level, it was dropping about $8 \%$ of the total WECC wind generation. Recognizing that only the study area wind sites were changed between scenarios, the curtailed energy in the 30\% Mega-Project Scenario without transmission reinforcement, represents $20 \%$ of the study area total wind generation, which is clearly significant. Figure 6.107 shows the reduction in annual operating savings when the transmission expansion is ignored. For the Local-Priority Scenario, the reductions are about $\$ 100$ million per year, suggesting that some targeted transmission expansion might be viable at that point, but not the full build-out. The Mega-Project Scenario clearly has issues at the $20 \%$ level that would require some level of transmission expansion. At the $30 \%$ level, both scenarios require transmission expansion. Depending on the cost of capital, these values are in the right ballpark to cover the capital cost for the construction of the additional transmission. 


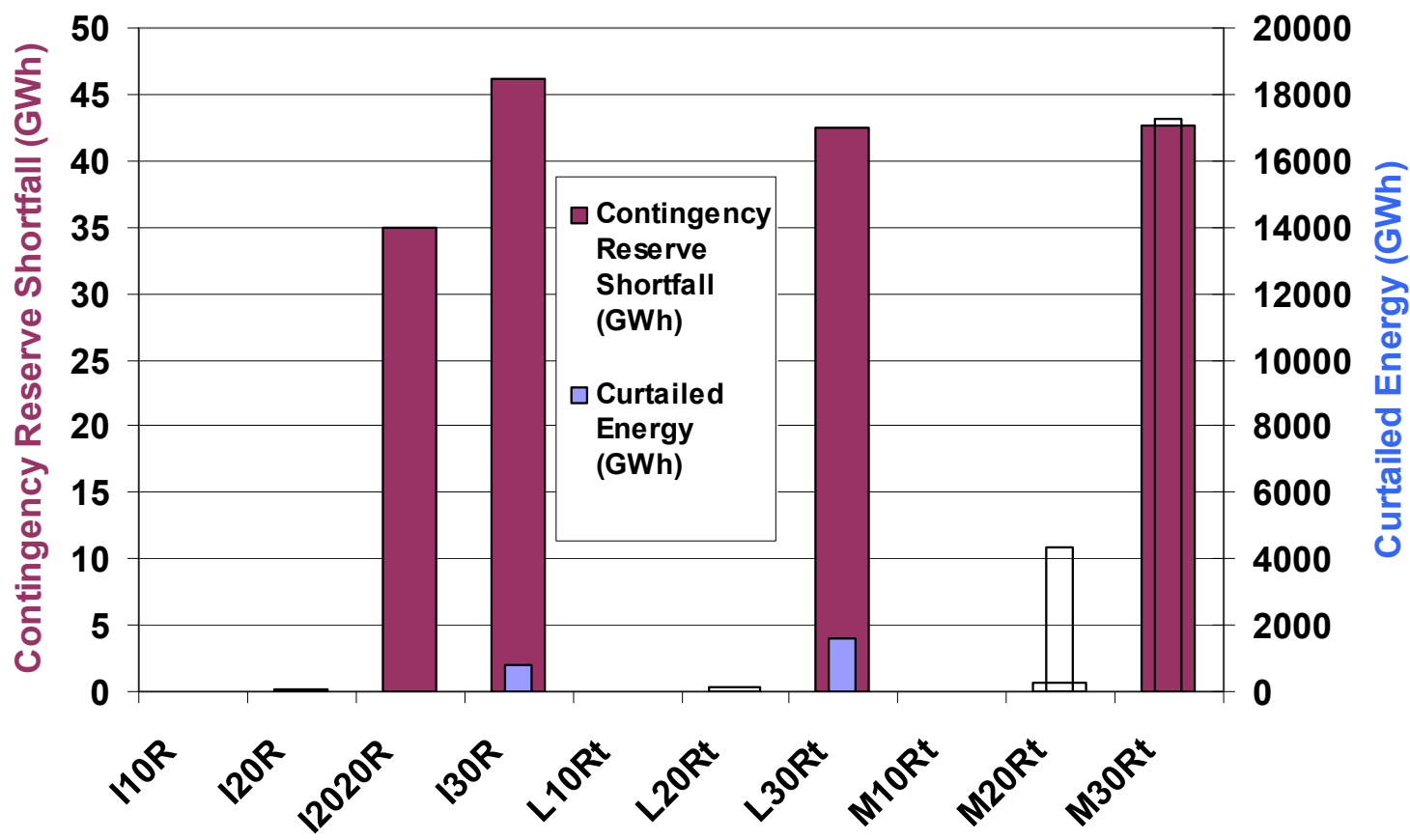

Figure 6.106 Curtailed Energy and Contingency Reserve Shortfall w/o Transmission Expansion

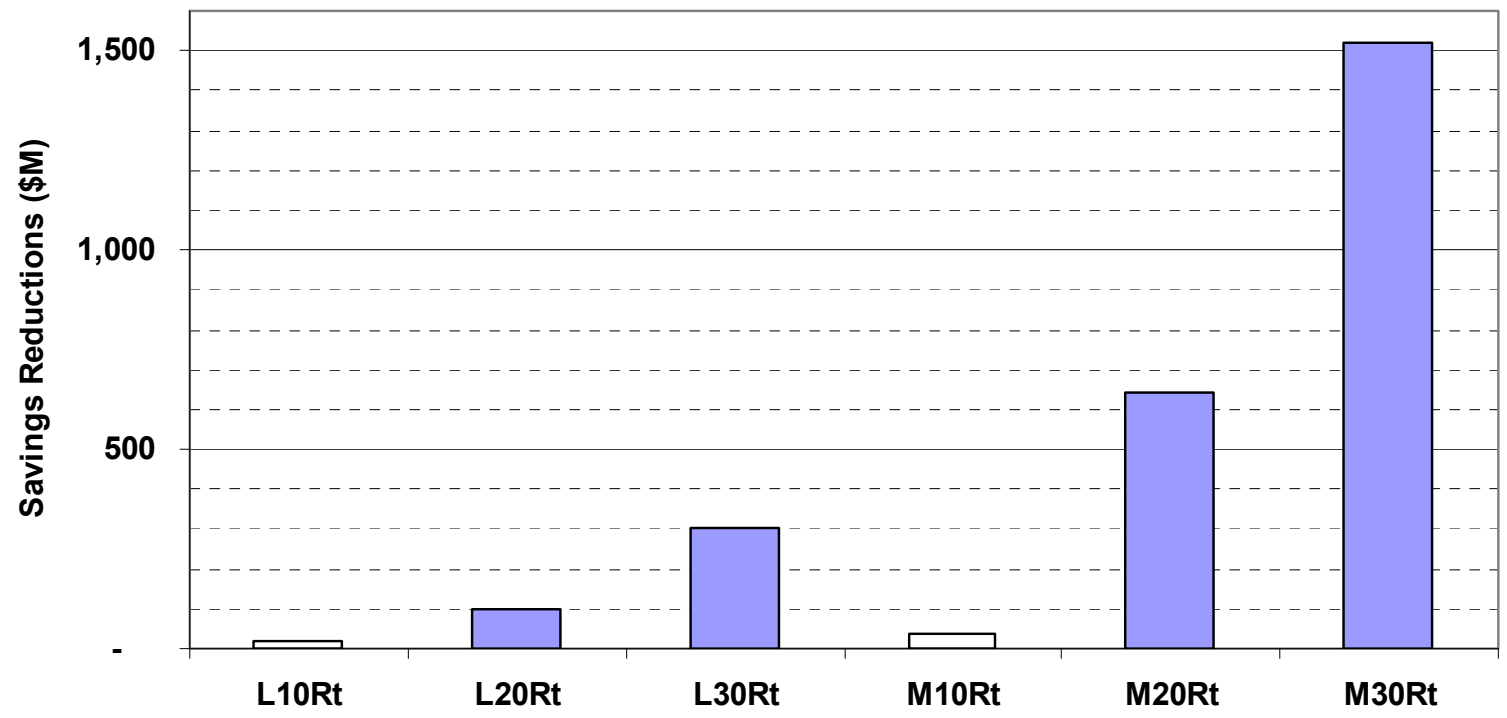

Figure 6.107 Savings Reductions with No Transmission Expansion

\subsubsection{Generation Expansion Sensitivity}

The base generation expansion plan for year 2017 was developed in the absence of renewable generation. As wind and solar generation is added to the system, it may be possible to delay or cancel some of this new conventional generation and still maintain required reserve margins. Based on results presented in Chapter 9, the capacity value of 
the renewable generation in the study area for $30 \%$ penetration is about $6600 \mathrm{MW}$. This is roughly equal to the $6450 \mathrm{MW}$ of gas-fired generation that was added after 2012 in the base expansion. A sensitivity case was run that deleted this new generation so that the reliability would be roughly equal between the systems, with and without renewable generation. Figure 6.108 shows a comparison of the system emissions for the base $30 \%$ case and the one with the reduced capacity expansion. They are almost identical. This is due to the fact that the renewable generation reduced the need for much of the gas-fired generation in the system. The new units would have been slightly more efficient than the existing generation, but not enough to make a noticeable difference in the total results. Figure 6.109 shows similar results for the total variable costs and the generator revenues. There would likely be more differences if some of the existing, older, coalfired generation had been retired, but that was not examined.

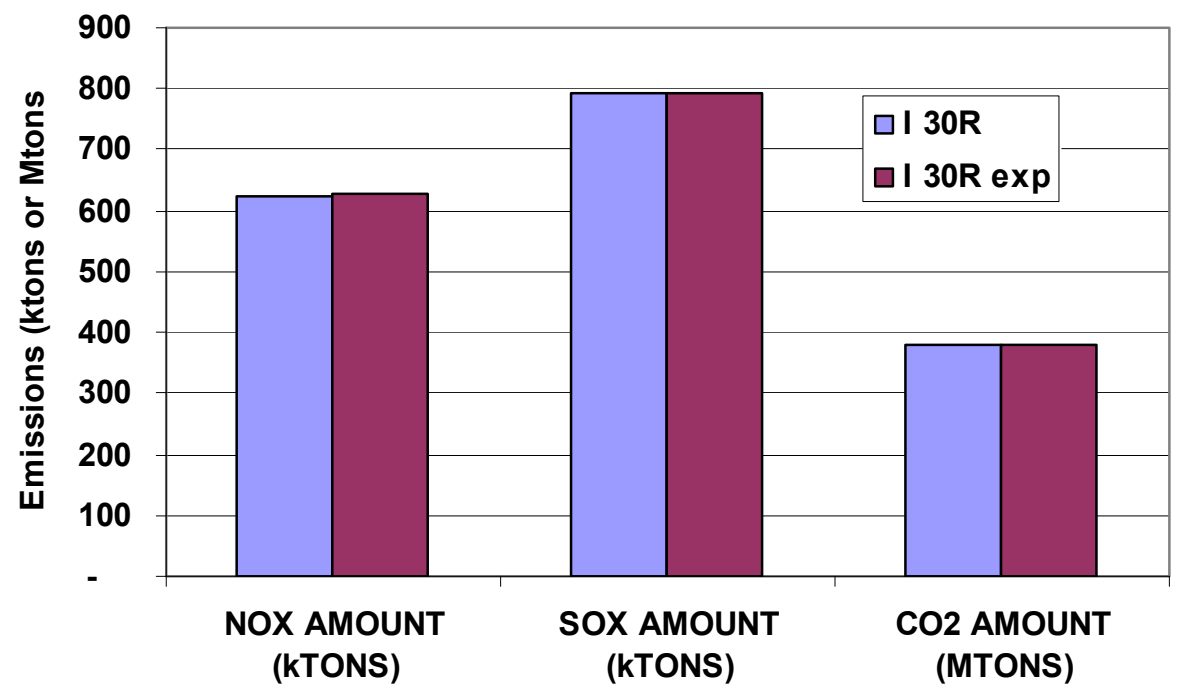

Figure 6.108 Emission Impact of Reduced Capacity Additions

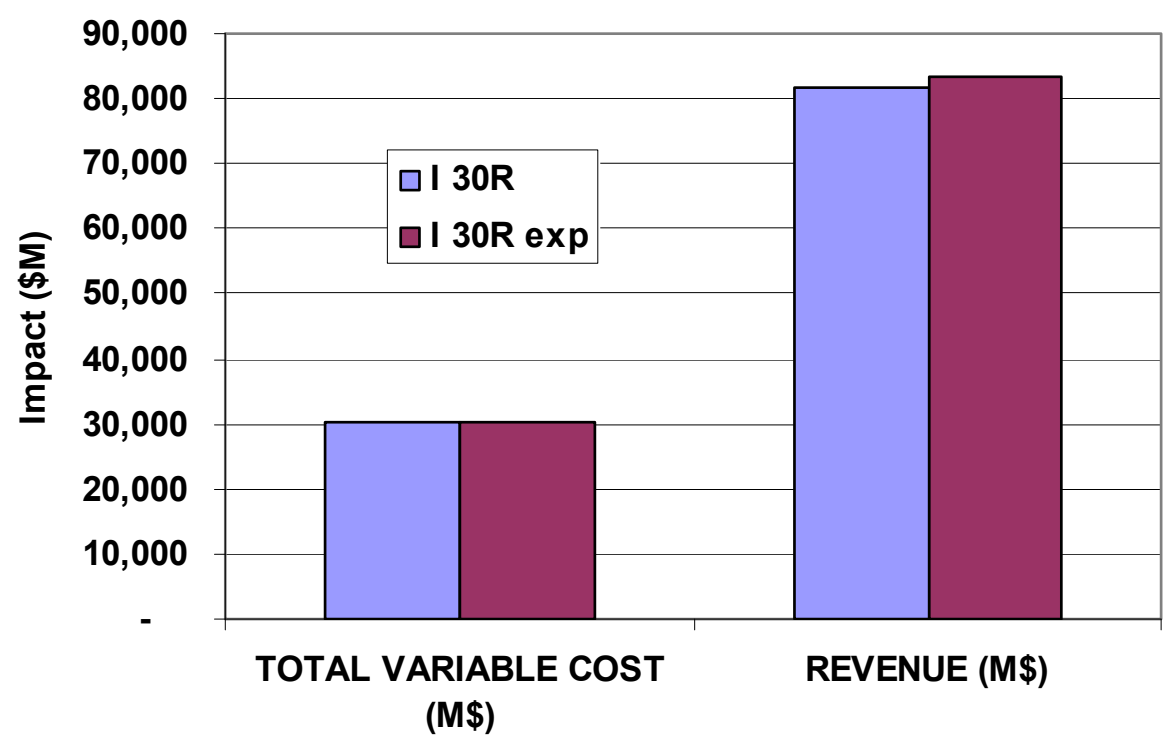

Figure 6.109 Operating Cost Impact of Reduced Capacity Additions 
The capital cost savings of the $6450 \mathrm{MW}$ of generation not added could offset some of the cost of the renewable generation. The amount of spilled energy was essentially unchanged with the reduced expansion plan. However, the unserved energy, or contingency reserve reductions, within the study footprint tripled. This indicates that rather than deferring or deleting other expansion capacity, it may be better to shift it to a more flexible type. This would be a good area for additional analysis.

\subsection{Operational Analysis Observations and Conclusions}

From an hourly operational analysis viewpoint, integration of high levels of renewables into the WestConnect and surrounding regions is feasible and produces energy values in the range of $\$ 80-\$ 90$ per megawatt hour of renewable energy generated. The study examined up to $30 \%$ of the study area's annual energy needs being met by wind generation with another $5 \%$ supplied by solar. The balance of WECC had $20 \%$ energy from wind and 3\% from solar. This high renewables case resulted in a $40 \%$ reduction of the fuel and emission costs across WECC. Variations in siting appeared to have little impact. Improved capacity factors resulted in less nameplate capacity of renewables required, but generally needed new transmission to facilitate the energy transfer. The economic value of the renewable energy was roughly the same. Remote, high capacity factor wind generation that can take advantage of existing transmission is attractive. Major build-outs of remote wind and long distance transmission do not appear to have any significant operational advantages or disadvantages, and need to be judged on the specific economics involved.

There appears to be minimal stress on system operations at up to $20 \%$ wind penetration. Beyond that point, the system's operational flexibility is stretched, particularly if the rest of WECC is also aggressively pursuing renewables. Minimum operating points on coal plants and the cycling capabilities of hydro generation become more important beyond the $20 \%$ penetration level, although neither is an absolute show stopper.

Incorporation of the wind and solar forecast in the day-ahead unit commitment decisions is very important. Even though there may be times that the renewable generation is over-forecasted, resulting in the operation of higher priced quick-start generation, ignoring the forecasts reduces the value of renewables by up to $\$ 20 / \mathrm{MWh}$. Improving the current level of forecasting accuracy could add an additional \$2/MWh. Increasing the spinning reserve in all hours to address the variability and uncertainty of the renewable generation appears to be an expensive option. It may be better to adjust the day-ahead commitments on a shorter-term basis $(4-6$ hours ahead as has been discussed in ERCOT and California), provide additional quick-start generation capacity, and have the load play a more active role in operations. The latter was examined in more detail. A demand response program in which utilities paid $1300 \mathrm{MW}$ of load to shut off when needed would be a more cost-effective way to deal with the 
approximately 90 hours of contingency reserve shortfall than increasing the spinning reserves for 8760 hours of the year.

Base economic assumptions in the report favor coal generation over gas-fired units. As a result, renewable generation tends to displace gas-fired generation that is generally more operationally flexible and cleaner, and leaves the less flexible, higher emission coal-fired units still running. This makes higher penetrations of renewable generation more difficult. Even so, emissions in WECC were seen to decrease by 5\% for SOX, 15\% for NOX, and up to $25 \%$ for CO2. Decreased gas prices and/or increased carbon taxes will reverse the relative operational attractiveness of coal and gas generation in the dispatch stack. This would lead to more displacement of the coal generation, significantly improved emission reductions, and enhanced operational flexibility of the balance of the generation fleet. All of this would help smooth the introduction of greater amounts of intermittent renewable generation such as wind and solar. Assuming a natural gas price of $\$ 3.5 / \mathrm{MBTU}$, renewables offset coal instead of gas, resulting in emissions reductions across WECC of $50 \%$ for NOX, 30\% for SOX and $45 \%$ for CO2. 



\section{Sub-hourly Operational Analysis}

This chapter focuses on multiple aspects of sub-hourly variability, specifically 10-minute variability. Section 7.1 describes the 10-minute statistical analysis, which provides a broad overview of the impact of significant wind and solar generation on system performance and sets the stage for the subsequent detailed exploration of sub-hourly behavior. In Section 7.2, the quasi-steady-state (QSS) analysis, which consists of detailed time simulations of specific 24-hour periods, is presented. It is designed to illustrate key aspects of system performance and potential mitigation measures within the broader context of the statistical and production simulation analyses. The relationship between sub-hourly variability and dynamic reserve requirements is explored in Section 7.3. The focus is primarily on understanding how much maneuvering room is required of on-line generation to handle the changes that occur within each hour, i.e., variability rather than uncertainty. Section 7.4 explores possible ways in which system operations could translate the reserve requirements into simple usable operating rules or guidelines. Section 7.5 further examines selected MAPS and QSS simulations in the context of the requirements identified. Discussion of possible demand participation and energy storage in providing these sub-hourly reserves is included.

\subsection{Statistical Analysis}

Extensive statistical analysis of hourly wind, solar and load variability was presented in Chapter 5. In this section, we present additional statistical analysis of variability that occurs in the 10-minute time frame. There are two major reasons for focusing on 10minute variability. First, we have extensive 10-minute data. All the wind data was generated at 10-minute time resolution, with the exception of selected windows used in QSS analysis discussed in the next section. But equally important, 10 minutes is a good divider between running reserves and cold, or standby reserves. The latter reserves may be brought on-line quickly, but do not contribute to managing variability until committed. The basic concept behind this analysis, and the results presented in subsequent sections of this chapter, is that the sub-hourly variability drives dynamic reserve requirements.

In the results presented here, the focus is on the Local-Priority Scenario. Similar plots and data for the other scenarios are included in Appendix E. The results presented here have a similar structure to those presented in Chapter 5. That chapter provides background and context for the variability statistics and analytical methods. The discussion in this section is limited to examining the details of 10-minute variability that are relevant to reserve requirements.

In Table 7.1, statistics on 10-minute variability by area, study footprint and WECC are presented. These data are presented in the same way as the hourly variability presented 
in Chapter 5. At the study footprint level, the overall 10-minute variability increases from the load-alone case, measured by the standard deviation ("sigma" or $\sigma$ ) of the deltas ("delta sigma" or $\Delta \sigma$ ), is relatively small, even for the $30 \%$ Mega-Project case. The increase is on the order of $20 \%$.

However, the extrema at the foot print level can double or triple. At individual transmission area/state resolution, the differences become more pronounced. The Arizona increase is not great. But in Wyoming, there is an order of magnitude increase in the standard deviation, a factor of 20 increase in the maximum positive delta, and a factor of 40 increase in extreme net load drops. The difference between the In-Area, Local-Priority and Mega-Project Scenarios for Wyoming are consistent with the dramatic increase in installed wind across the scenarios.

Table 7.1 10-minute Net Load Variability by Area, Footprint and WECC

\begin{tabular}{|c|c|c|c|c|c|c|c|c|c|c|c|c|c|c|c|c|c|c|c|c|c|c|c|c|}
\hline & \multicolumn{3}{|c|}{ Ariz } & \multicolumn{3}{|c|}{ CO-E } & \multicolumn{3}{|c|}{ CO-W } & \multicolumn{3}{|c|}{ NM } & \multicolumn{3}{|c|}{ NV } & \multicolumn{3}{|c|}{ WY } & \multicolumn{3}{|c|}{ In Foot Print } & \multicolumn{3}{|c|}{ WECC } \\
\hline & IA & LP & MP & IA & LP & MP & IA & LP & MP & IA & LP & MP & IA & LP & MP & IA & LP & MP & IA & LP & MP & IA & LP & MP \\
\hline \multicolumn{25}{|c|}{ Sigma (MW) } \\
\hline Load-alone & 97 & 97 & 97 & 61 & 61 & 61 & 6 & 6 & 6 & 25 & 25 & 25 & 52 & 52 & 52 & 15 & 15 & 15 & 239 & 239 & 239 & 731 & 731 & 731 \\
\hline Baseline (Existing) & 97 & 97 & 97 & 65 & 65 & 65 & 6 & 6 & 6 & 31 & 31 & 31 & 52 & 52 & 52 & 23 & 23 & 23 & 240 & 240 & 240 & 734 & 734 & 734 \\
\hline $10 \%$ In FP Scenario & 107 & 105 & 97 & 69 & 69 & 69 & 11 & 10 & 7 & 33 & 36 & 43 & 57 & 56 & 53 & 24 & 31 & 49 & 243 & 245 & 246 & 717 & 718 & 719 \\
\hline $20 \%$ In FP Scenario & 128 & 116 & 100 & 80 & 81 & 71 & 19 & 16 & 7 & 43 & 46 & 54 & 68 & 63 & 55 & 34 & 56 & 108 & 259 & 260 & 268 & 716 & 718 & 722 \\
\hline $30 \%$ In FP Scenario & 146 & 128 & 106 & 98 & 89 & 72 & 25 & 20 & 10 & 54 & 56 & 70 & 79 & 69 & 57 & 46 & 93 & 157 & 284 & 286 & 301 & 728 & 728 & 734 \\
\hline \multicolumn{25}{|l|}{ Max Neg Delt } \\
\hline Load-alone & -348 & -348 & -348 & -223 & -223 & -223 & -20 & -20 & -20 & -86 & -86 & -86 & -200 & -200 & -200 & -53 & -53 & -53 & -708 & -708 & -708 & -2239 & -2239 & -2239 \\
\hline Baseline (Existing) & -352 & -352 & -352 & -412 & -412 & -412 & -20 & -20 & -20 & -260 & -260 & -260 & -200 & -200 & -200 & -334 & -334 & -334 & -810 & -810 & -810 & -2279 & -2279 & -2279 \\
\hline $10 \%$ In FP Scenario & -901 & -772 & -406 & -412 & -420 & -409 & -134 & -109 & -41 & -252 & -240 & -354 & -367 & -340 & -250 & -327 & -340 & -903 & -843 & -867 & -991 & -3744 & -3734 & -3706 \\
\hline $20 \%$ In FP Scenario & -1367 & -1052 & -367 & -825 & -829 & -490 & -234 & -205 & -60 & -394 & -408 & -532 & -568 & -565 & -305 & -401 & -962 & -1725 & -1360 & -1216 & -1821 & -3656 & -3672 & -3713 \\
\hline $30 \%$ In FP Scenario & -1616 & -1169 & -418 & -1082 & -956 & -559 & -288 & -239 & -91 & -516 & -565 & -708 & -767 & -633 & -386 & -501 & -1476 & -2274 & -1576 & -1641 & -2325 & -4266 & -4270 & -4308 \\
\hline \multicolumn{25}{|l|}{ Max Pos Del } \\
\hline Load-alone & 267 & 267 & 267 & 195 & 195 & 195 & 22 & 22 & 22 & 106 & 106 & 106 & 206 & 206 & 206 & 84 & 84 & 84 & 612 & 612 & 612 & 1786 & 1786 & 1786 \\
\hline Baseline (Existing) & 269 & 269 & 269 & 306 & 306 & 306 & 22 & 22 & 22 & 147 & 147 & 147 & 207 & 207 & 207 & 228 & 228 & 228 & 710 & 710 & 710 & 1865 & 1865 & 1865 \\
\hline $10 \%$ In FP Scenario & 711 & 590 & 289 & 334 & 336 & 364 & 160 & 93 & 30 & 166 & 201 & 320 & 319 & 311 & 209 & 233 & 290 & 567 & 899 & 873 & 770 & 2084 & 2093 & 2118 \\
\hline $20 \%$ In FP Scenario & 917 & 784 & 887 & 548 & 555 & 360 & 211 & 197 & 51 & 323 & 358 & 448 & 455 & 449 & 233 & 459 & 630 & 1069 & 1193 & 1075 & 1219 & 2211 & 2172 & 2203 \\
\hline $30 \%$ In FP Scenario & 1169 & 1041 & 1207 & 1010 & 804 & 426 & 296 & 221 & 93 & 462 & 466 & 543 & 582 & 519 & 293 & 649 & 994 & 1805 & 1430 & 1416 & 1542 & 2728 & 2681 & 2677 \\
\hline \multicolumn{25}{|l|}{ No. Drops $>3^{\star}$ Ld } \\
\hline Load-alone & 18 & 18 & 18 & 36 & 36 & 36 & 90 & 90 & 90 & 45 & 45 & 45 & 54 & 54 & 54 & 45 & 45 & 45 & 0 & 0 & 이 & 9 & 9 & g \\
\hline Baseline (Existin & 18 & 18 & 18 & 155 & 155 & 155 & 90 & 90 & 90 & 617 & 617 & 617 & 54 & 54 & 54 & 1458 & 1458 & 1458 & 12 & 12 & 12 & 9 & 9 & 9 \\
\hline $10 \%$ In FP Scenario & 203 & 158 & 21 & 296 & 323 & 302 & 2163 & 1367 & 294 & 822 & 1121 & 1938 & 219 & 185 & 59 & 1649 & 2979 & 6399 & 44 & 42 & 62 & 6 & 6 & 11 \\
\hline $20 \%$ In FP Scenario & 672 & 350 & 47 & 669 & 687 & 362 & 5426 & 4298 & 476 & 1977 & 2262 & 3188 & 680 & 464 & 117 & 3638 & 7836 & 12968 & 125 & 140 & 224 & 6 & 5 & 11 \\
\hline $30 \%$ In FP Scenario & 1172 & 632 & 159 & 1388 & 995 & 344 & 7884 & 6074 & 1415 & 3156 & 3452 & 5262 & 1317 & 684 & 156 & 5993 & 12185 & 15905 & 240 & 284 & 468 & 17 & 17 & 42 \\
\hline \multicolumn{25}{|l|}{ No. Rises $>3^{\star}$ Ld S } \\
\hline Load-alone & 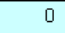 & 0 & & 36 & 36 & 36 & 72 & 72 & 72 & 107 & 107 & 107 & 27 & 27 & 27 & 405 & 405 & 405 & 0 & 0 & & 0 & 0 & \\
\hline Baseline (Existing) & 0 & 0 & 미 & 66 & 66 & 66 & 72 & 72 & 72 & 449 & 449 & 449 & 35 & 35 & 35 & 1563 & 1563 & 1563 & 0 & 0 & 이 & 0 & 0 & \\
\hline $10 \%$ In FP Scenario & 165 & 123 & 0 & 194 & 236 & 204 & 1956 & 1194 & 236 & 636 & 951 & 1940 & 198 & 138 & 45 & 1779 & 3144 & 6681 & 15 & 15 & 12 & 0 & 0 & 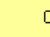 \\
\hline $20 \%$ In FP Scenario & 722 & 419 & 132 & 738 & 764 & 303 & 5663 & 4521 & 386 & 2043 & 2367 & 3611 & 714 & 468 & 74 & 3801 & 8039 & 13319 & 203 & 171 & 212 & 2 & 0 & 2 \\
\hline $30 \%$ In FP Scenario & 1284 & 782 & 357 & 1656 & 1209 & 435 & 7983 & 6146 & 1908 & 3497 & 3864 & 5850 & 1350 & 824 & 191 & 6288 & 12513 & 16353 & 521 & 476 & 599 & 63 & 48 & 50 \\
\hline
\end{tabular}

In Table 7.2, the variation within small balancing areas is illustrated. The blue cells in this table are for the 7 zones that make up the Wyoming area. Some of these zones, TRSTWYOA (Tri-States G\&T Wyoming, other labels are decoded in Table 3.4), for example, show a 50-fold increase in net load 10-minute $\Delta \sigma$. The single most extreme net load rise in 10 minutes is 100 times as great as with load-alone. Clearly, managing incremental variability due to additional wind power is infeasible for these small balancing areas with high wind penetration. 
Table 7.2 Summary Statistics for 10-Minute Delta for Wyoming

\begin{tabular}{|c|c|c|c|c|c|c|c|c|c|c|}
\hline & BEPCWA & BHPLA & WYCENA & WYNWA & WYSWA & TRSTWYOA & WACMA & WY & In FootPrint & WECC \\
\hline \multicolumn{11}{|c|}{ Sigma (MW) } \\
\hline Load-alone & 2 & 2 & 2 & 3 & 3 & 1 & 2 & 15 & 239 & 731 \\
\hline Baseline (Existing) & 2 & 2 & 8 & 3 & 17 & 2 & 2 & 23 & 240 & 734 \\
\hline $10 \%$ In FP Scenario & 2 & 2 & 15 & 4 & 17 & 3 & 14 & 31 & 245 & 718 \\
\hline $20 \%$ In FP Scenario & 2 & 2 & 33 & 8 & 17 & 18 & 27 & 56 & 260 & 718 \\
\hline $30 \%$ In FP Scenario & 2 & 2 & 47] & 9 & 18 & 53 & 36 & 93 & 286 & 728 \\
\hline \multicolumn{11}{|l|}{\begin{tabular}{|l|} 
Max Neg Delta (MW) \\
\end{tabular}} \\
\hline Load-alone & -7 & -8 & -10 & -10 & -11 & -3 & -8 & -53 & -708 & -2239 \\
\hline Baseline (Existing) & -7 & -8 & -91 & -10 & -335 & -29 & -8 & -334 & -810 & -2279 \\
\hline $10 \%$ In FP Scenario & -7 & -8 & -162 & -56 & -335 & -35 & -369 & -340 & -867 & -3734 \\
\hline $20 \%$ In FP Scenario & -7 & -8 & -409 & -170 & -335 & -439 & -860 & -962 & -1216 & -3672 \\
\hline $30 \%$ In FP Scenario & -7 & -8 & -590 & -170 & -308 & -809 & -1128 & -1476 & -1641 & -4270 \\
\hline \multicolumn{11}{|l|}{\begin{tabular}{|c|} 
Max Pos Delta (MW) \\
\end{tabular}} \\
\hline Load-alone & 11 & 13 & 16 & 17 & 19 & 6 & 7 & 84 & 612 & 1786 \\
\hline Baseline (Existing) & 11 & 13 & 90 & 17 & 227 & 27 & 7 & 228 & 710 & 1865 \\
\hline $10 \%$ In FP Scenario & 11 & 13 & 168 & 50 & 227 & 31 & 264 & 290 & 873 & 2093 \\
\hline $20 \%$ In FP Scenario & 11 & 13 & 443 & 134 & 227 & 276 & 548 & 630 & 1075 & 2172 \\
\hline $30 \%$ In FP Scenario & 11 & 13 & 618 & 134 & 227 & 620 & 716 & 994 & 1416 & 2681 \\
\hline
\end{tabular}

The temporal coincidence of load and wind changes is shown in Figure 7.1. These plots are similar to those presented in Figure 5.3. The results have a broadly similar character to the hourly variability. However, there is some increase in the relative amplitude of extreme wind changes compared to load. This is most noticeable in the summer data. Interestingly, the outlier wind changes do not coincide with load change outliers, which may be understated because of the linearization of the one-hour data.
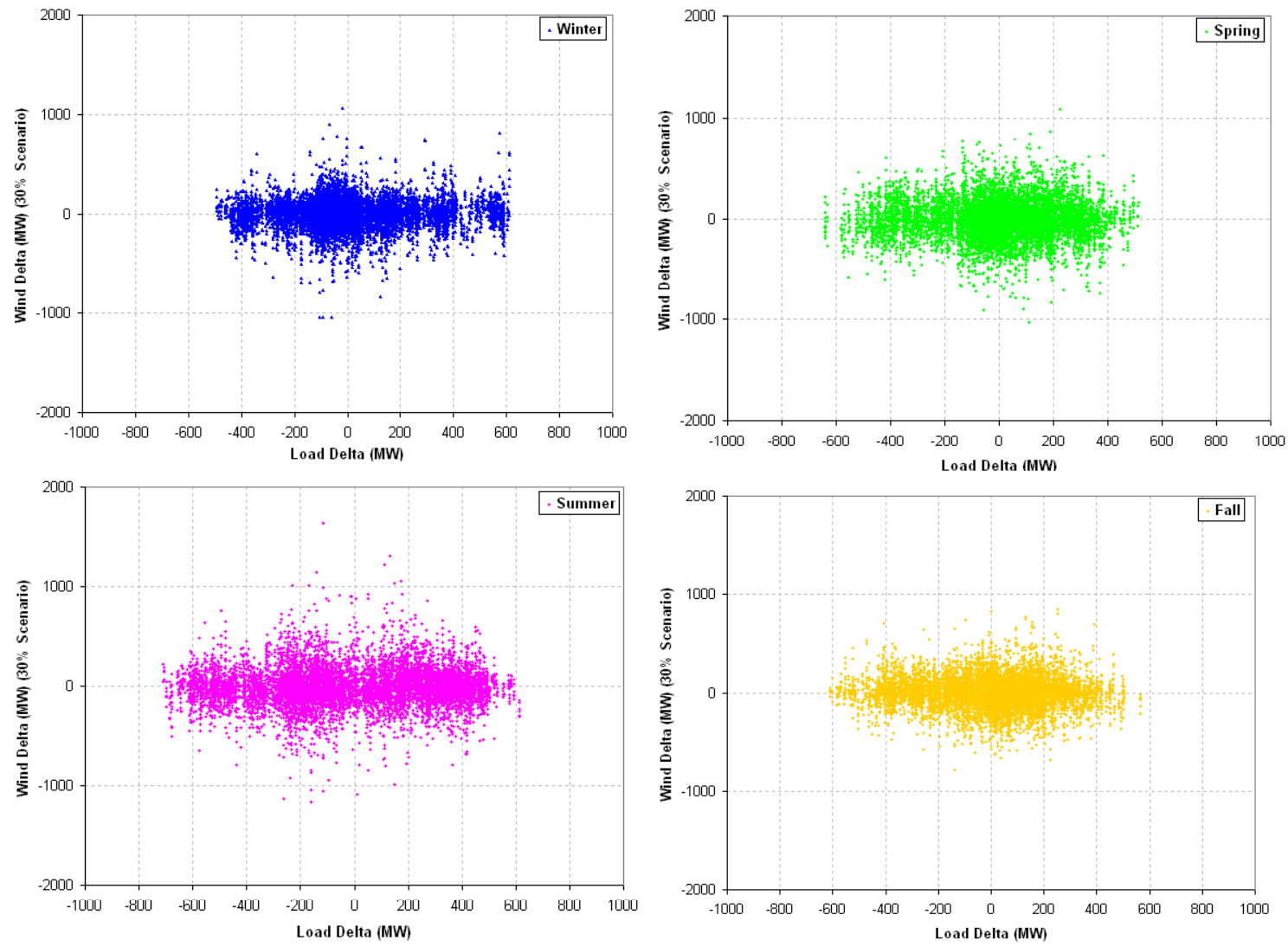

Figure 7.1 10-minute Wind Deltas vs. Load Deltas by Season for Study Area (2006, 30\%, Local-Priority Scenario) 
The timing of extreme 10-minute net load increases is shown in Figure 7.2. This figure presents the same type of information as presented in Figures 5.10 to 5.14. However, the carpet plots in Figure 7.2 look quite different. There are 6 times as many 10-minute periods in a day as hours, so the plots are stretched horizontally. As expected, periods of rapid net load rise tend to occur in summer mornings and early evenings, and winter late evenings. The increases in the evenings from October to April are substantial, with the largest occurring at 16:20 in December.

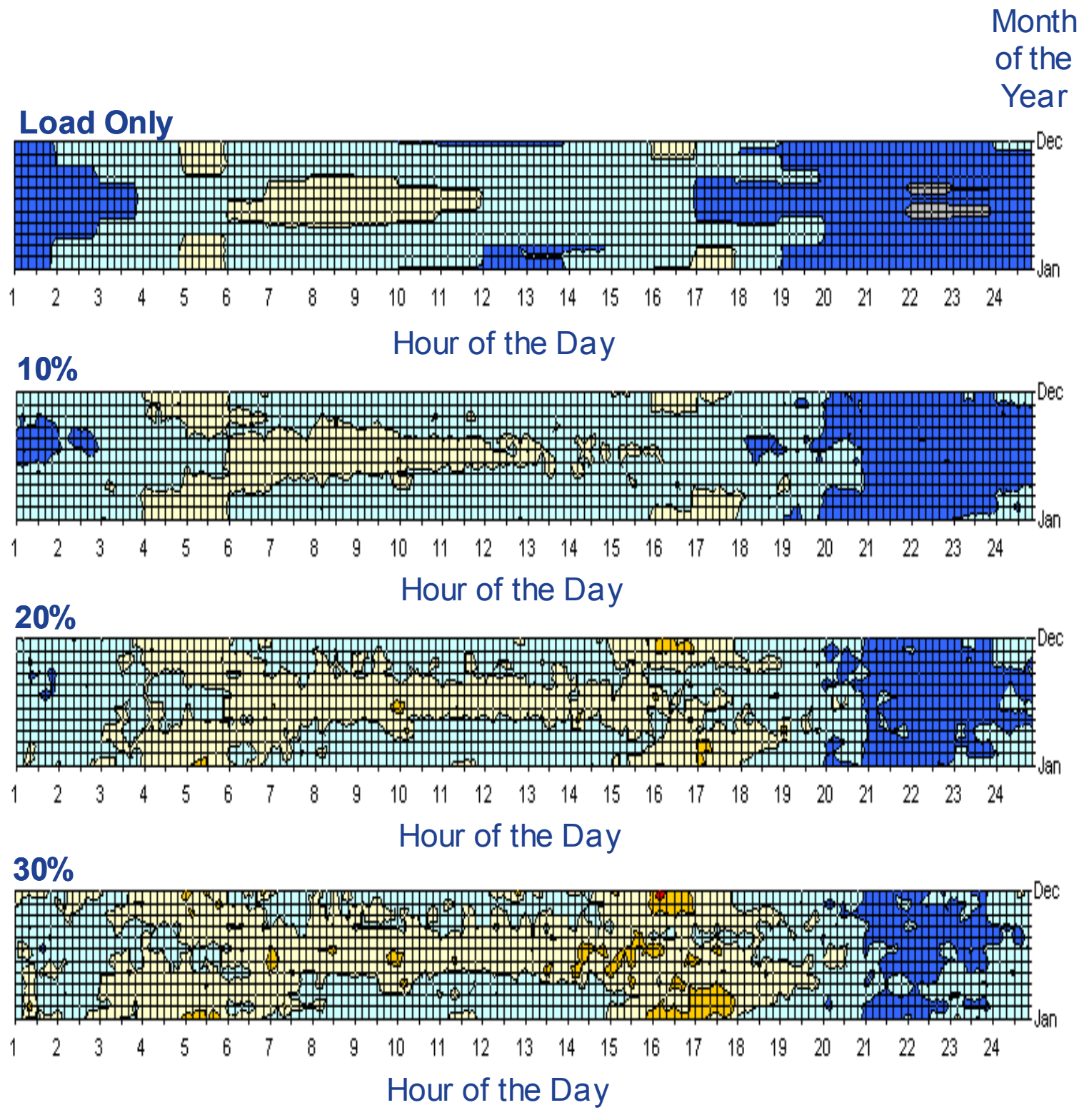

$$
\begin{array}{llllll}
0-800-400 & \mathbf{0}-400-0 & \mathbf{0} 0-400 & \mathbf{0} 400-800 & \mathbf{0} 800-1200 & \mathbf{0 1 2 0 0 - 1 6 0 0}
\end{array}
$$

Figure 7.2 Timing of Maximum Daily 10-Min Positive Deltas for Study Area (2006, Local-Priority Scenario) 
Figure 7.3 shows the timing of extreme net load drops. Just as in Figure 5.14, the biggest net load drops occur in the early night, especially in the summer. Mid-morning numbers in this figure can be positive, showing the smallest increase for any hour in that cell.

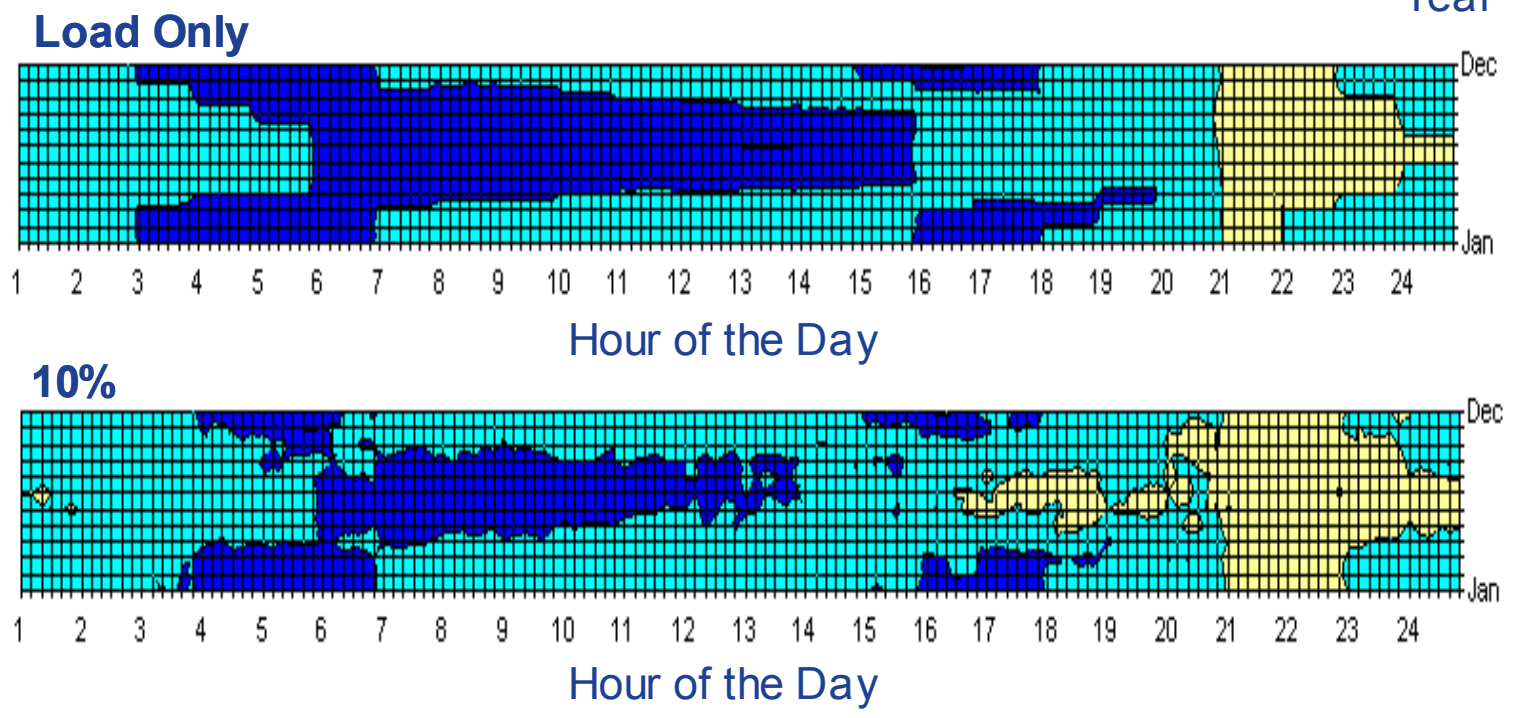

Month

of the

Year

\section{$20 \%$}

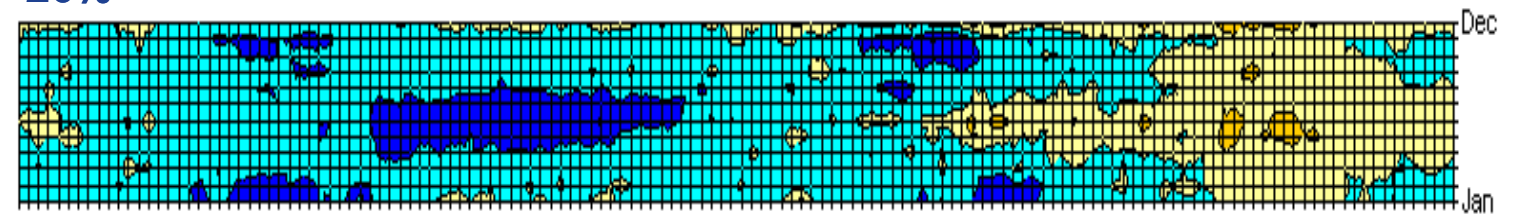
$\begin{array}{llllllllllllllllllllllll}1 & 2 & 3 & 4 & 5 & 6 & 7 & 8 & 9 & 10 & 11 & 12 & 13 & 14 & 15 & 16 & 17 & 18 & 19 & 20 & 21 & 22 & 23 & 24\end{array}$

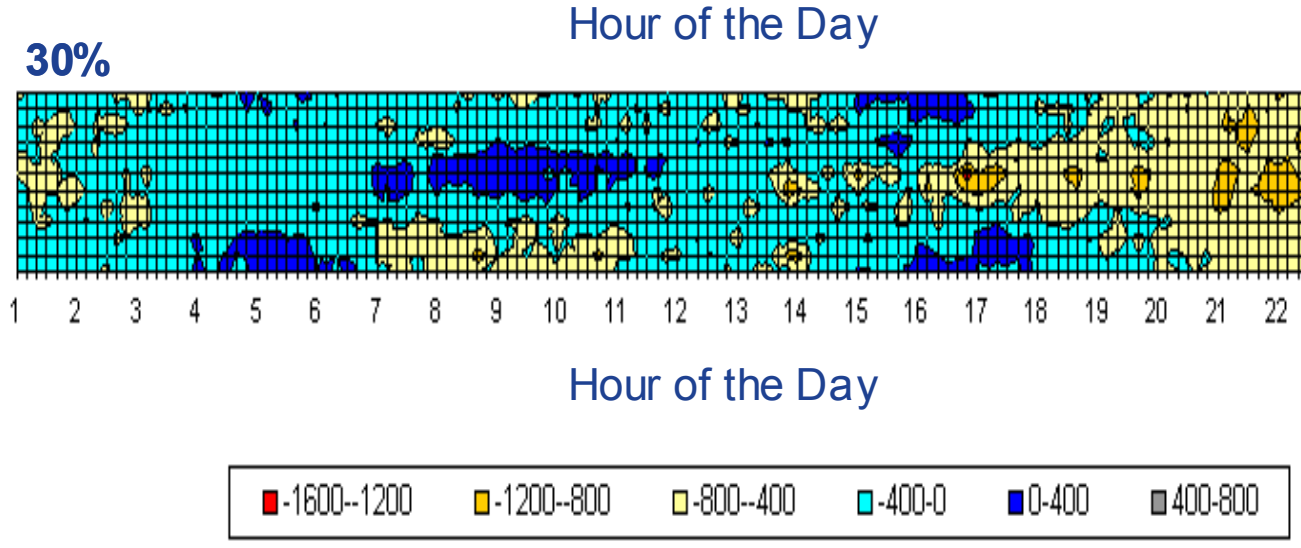

Figure 7.3 Timing of Maximum Daily 10-Min Negative Deltas for Study Area (2006, Local-Priority Scenario)

A histogram of the study footprint 10-minute net load changes is shown in Figure 7.4. As with Figure 5.15, the distribution widens and appears to become slightly less normal with increasing renewable penetration. The bottom part of the figure shows that there 
are occasions of substantial net load rise and drop that exceed the maximum observed with load-alone.

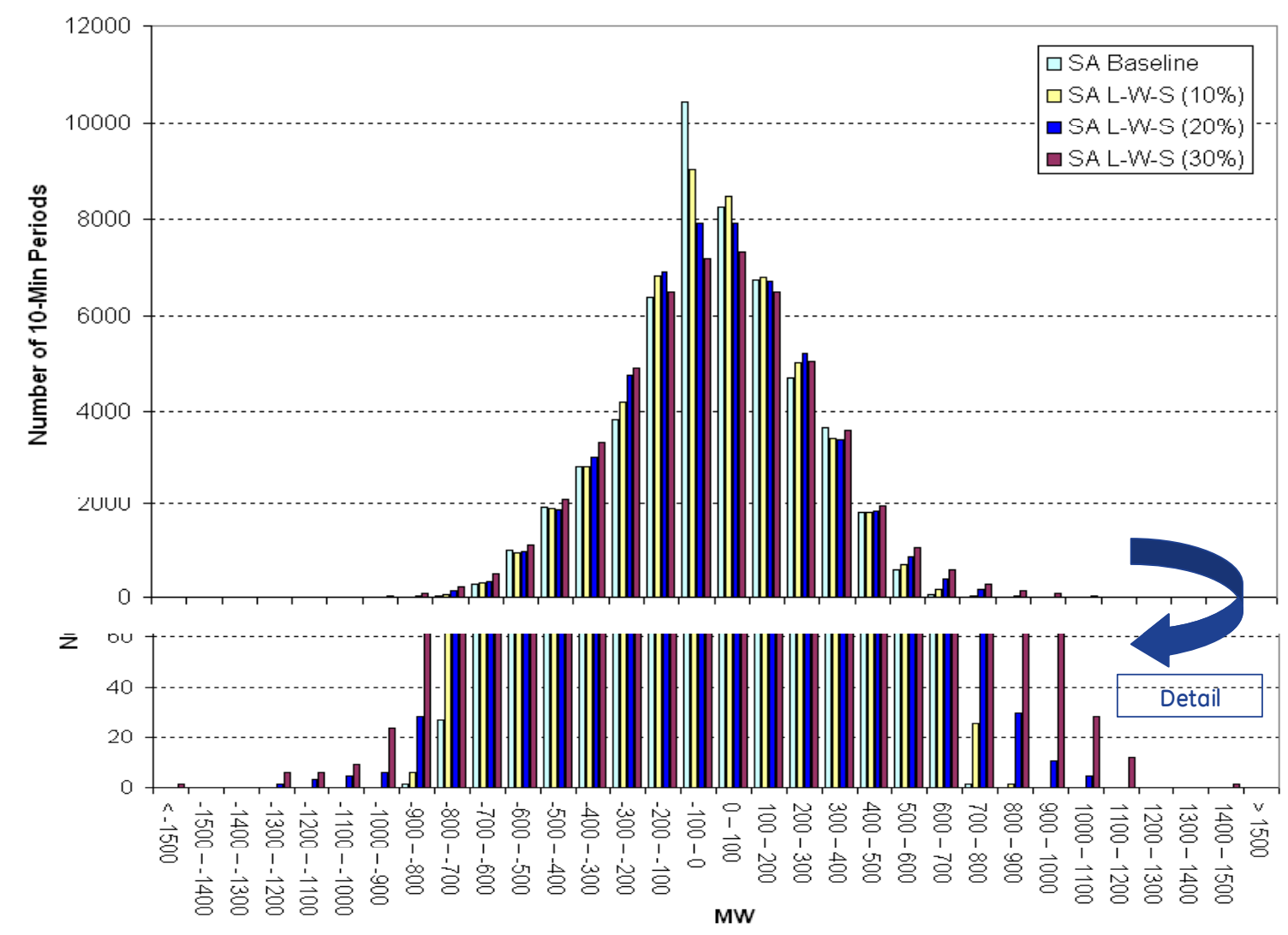

Figure 7.4 Distribution of Net Load Deltas for Study Area (2006, Local-Priority Scenario)

The extremes of net load rise and drop are shown in a cumulative fashion in Figure 7.5. Again, this figure contains similar information to that presented in Figure 5.16. The tails of these distributions are consistent with the extremes summarized in Section 5.4.

Overall, at both the study footprint and transmission area/state level, the increases in 10minute variability with the addition of wind and solar generation are incremental, not monumental. Strategies for addressing these increases are also incremental. The increase in variability is likely insurmountable for smaller balancing areas without substantial changes in cooperation. 


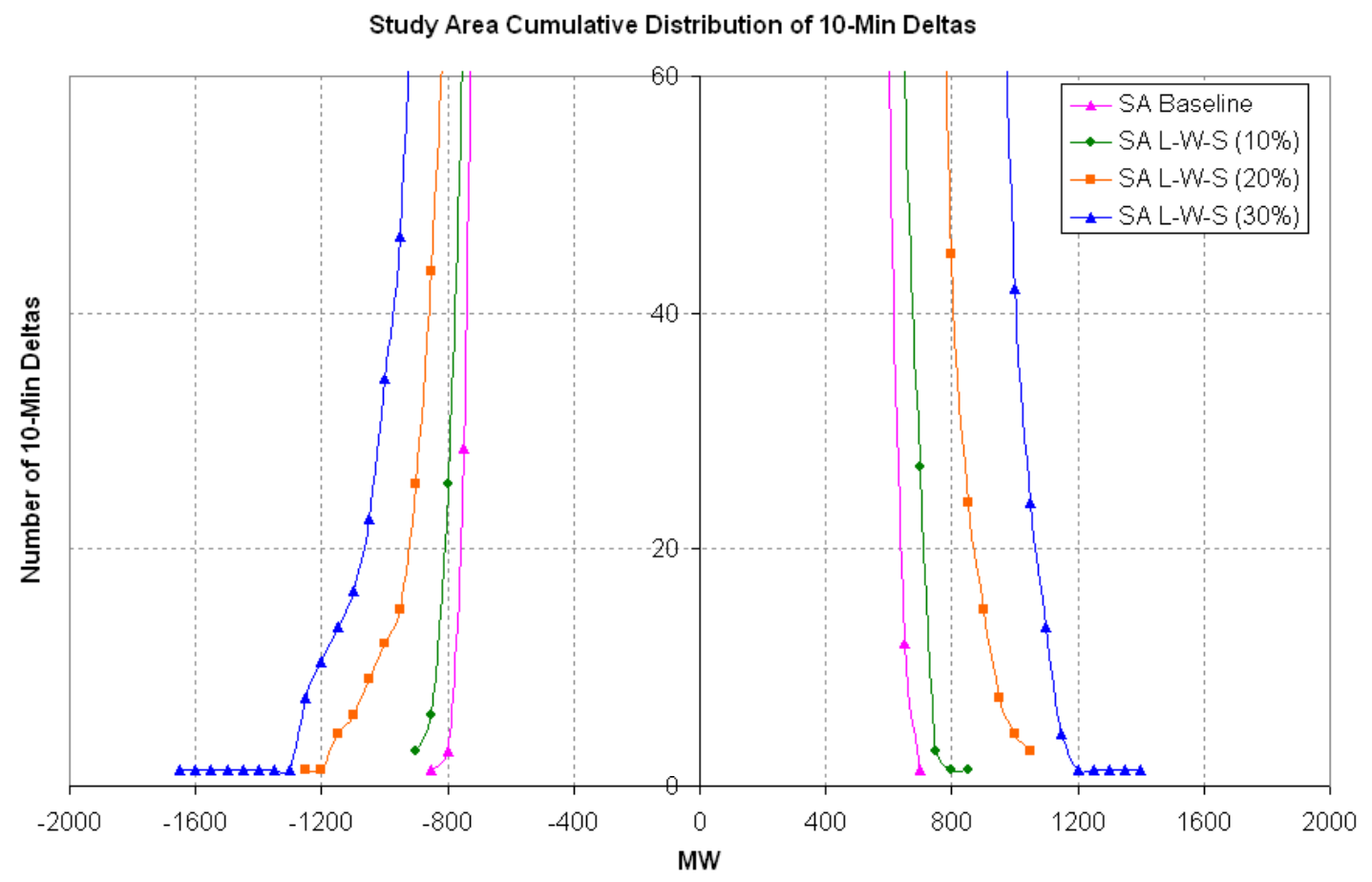

Figure 7.5 Distribution of Extreme Hourly Wind Deltas for 2006 (2006, Local-Priority Scenario)

\subsection{Quasi-Steady State (QSS) Analysis}

The statistical and production simulation analyses provided a broad view of the impact of significant wind and solar generation on system performance. They covered a range of time scales, from sub-hourly to hourly as well as daily, seasonal and annual. In contrast, the quasi-steady-state (QSS) analysis consists of detailed time simulations of specific 24-hour periods. It is designed to illustrate key aspects of system performance and potential mitigation measures within the broader context of the statistical and production simulation analyses. As such, the QSS analysis is tightly linked to both of these analyses. For example, the QSS study scenarios were selected on the basis of the statistical analysis, and the QSS boundary conditions were set by the production simulation analysis.

The data, methods, assumptions, study scenarios, and results for the QSS analysis are described in the following sections. All QSS analysis was performed using MATLAB.

\subsubsection{Overview of Algorithm}

The primary objectives of the QSS analysis were to evaluate the impact of significant wind and solar generation on load following (5-minute time scale) and regulation (1minute time scale) requirements within the study footprint. 
The study footprint was represented by seven, separate transmission areas - Arizona, New Mexico, Colorado-East, Colorado-West, Wyoming, Northern Nevada, and Southern Nevada. Each area was represented by aggregate profiles of all the individual loads, generation sources, and inter-area transmission path flows (i.e., exports). One profile represented all loads in a given area and another profile represented the total export schedule from a given area. However, generation profiles were aggregated by type. Thus, there was one profile for wind; one profile for solar, one for hydro, one for combined-cycle, etc.

Up to four types of generating units (e.g., hydro, combined-cycle, steam, gas turbine) were identified as participants in load following via the 5-minute economic dispatch function. Each unit type was assigned a priority number. If the priority \#1 unit type exhausted its capability, the priority \#2 unit type made up the difference. If priority \#2 unit type reached its limit, the priority $\# 3$ unit type began to participate. When the net load and therefore, economic dispatch commands reversed direction, the lowest priority unit type reversed direction first. Thus, the priority \#3 unit type would return to its nominal dispatch, followed by the priority \#2 unit type, leaving the primary load following responsibility to the priority \#1 unit type. Maxima, minima and ramp rate limits were respected for each unit type.

Up to four types of generating units were also identified as providers of regulation. Each unit type was assigned both a priority number and a specific amount of regulation (e.g., + and/or - MW) capability. Each unit type's participation in regulation followed a similar priority procedure as described above for the economic dispatch unit types. The maxima and minima of the assigned regulation capability are respected. Regulation units were assumed to have sufficient ramp rate capability, so no ramp rate limits were applied.

The remaining generating unit types were treated as un-dispatchable generation subject only to an hourly schedule.

The load, wind, and solar profiles varied on a minute-by-minute basis. The export and balance of portfolio (BOP) generation schedules changed on an hourly basis in accordance with the production simulation results. The hourly schedule changes were implemented as a ramp +/-10 minutes around the hour, unless otherwise noted.

The difference between total generation and load plus exports was calculated at 1minute intervals, and assigned to the regulation units. At 5-minute intervals, a simplified economic dispatch was performed to redistribute the power from the regulation units onto the designated load following units over the next 5 minutes. Ramp rate limits (MW/minute) and absolute power limits (maximum and minimum MW) were respected on each unit type. 
If the economic dispatch units in a particular transmission area lacked sufficient capability, the regulation units picked up the excess load following. If the regulation units lacked sufficient capability, an area control error (ACE) signal was generated, and the export to other transmission areas was affected. The ACE signal was compared to an approximate L10 limit to identify potential Control Performance Standard 2 (CPS2) violations [7.1].

Additional details on the study periods and input data, as well as regulation and economic dispatch unit selection from the boundary conditions are presented in Sections 7.2.2 through 7.2.4.

The results of selected 24-hour QSS simulations are described in Sections 7.2.5 and 7.2.6.

\subsubsection{Input Data}

Several types of data were used in the QSS analysis: wind generation profiles, solar generation profiles, load profiles, unit type ramp rate capabilities, transmission area regulation requirements, and L10 limits for CPS2.

NREL provided 1-minute wind generation profiles for all wind plants included in a given scenario (e.g., Local-Priority) for each QSS study period. These individual 30-MW plant profiles were created by superimposing a 1-minute variation onto the 10-minute data described in Chapter 2. The 1-minute variations were statistically generated from the spectral density of measured wind plant output from existing wind plants using the same Vestas V90 3-MW turbine. These profiles were then aggregated by transmission area.

Limited high resolution solar and load data were available. Therefore, the necessary 1minute solar profiles were created with a cubic spline interpolation of the 10-minute data. The necessary 1-minute load profiles were created with a cubic spline interpolation of the 1-hour data. Additional information on the data used in this study is provided in Chapter 2.

Since appropriate WECC data were not available, generic ramp rate data (\%/minute) were used for each unit type. These data were drawn from the CEC Intermittency Analysis Project [7.2], and are shown in Table 7.3. Actual unit ramp rates will vary. Some WestConnect units report much lower ramp rate capabilities. Therefore, future work may include a sensitivity analysis to evaluate the impact of using actual rather than generic ramp rate data. 
Table 7.3. Generic Ramp Rate Data by Unit Type

\begin{tabular}{|l|c|}
\cline { 2 - 2 } \multicolumn{1}{c|}{} & $\begin{array}{c}\text { Ramp Rate } \\
\text { (\% of Nameplate/Minute) }\end{array}$ \\
\hline Combined-Cycle & 3.8 \\
Combustion Turbine & 13.5 \\
Hydroelectric & 22.3 \\
Steam & 3.1 \\
\hline
\end{tabular}

The regulation requirement for each transmission area was estimated as $1 \%$ of peak load for both positive and negative regulation. This assumption is consistent with the actual regulation requirements observed in other studies. The regulation requirement for each transmission area is shown in Table 7.4.

Table 7.4. Regulation Requirement by Transmission Area

\begin{tabular}{|l|c|}
\cline { 2 - 2 } \multicolumn{1}{l|}{} & $\begin{array}{c}\text { Regulation } \\
\text { (+/- MW })\end{array}$ \\
\hline Arizona & 231 \\
Colorado - East & 116 \\
Colorado - West & 15 \\
Northern Nevada & 29 \\
Southern Nevada & 97 \\
New Mexico & 53 \\
Wyoming & 40 \\
\hline In Footprint & 581 \\
Out of Footprint & 1200 \\
\hline
\end{tabular}

CPS2 requires that the average ACE for each of the six 10-minute periods during an hour must be within specific limits. These limits are called L10, and vary with each operating area. Approximate L10 values were used in the QSS analysis to identify potential CPS2 violations. These values were based on the NERC “2007 CPS2 Bounds Report" [7.3], aggregated to match the transmission areas under study, and shown in Table 7.5. The L10 limits were constant across all scenarios and all penetration levels. 
Table 7.5 Approximate CPS2 L10 Limits by Transmission Area

\begin{tabular}{|l|c|}
\cline { 2 - 2 } \multicolumn{1}{c|}{} & $\begin{array}{c}\text { L10 } \\
\text { (MW) }\end{array}$ \\
\hline Arizona & 187 \\
Colorado - East & 83 \\
Colorado - West & 15 \\
Northern Nevada & 25 \\
Southern Nevada & 85 \\
New Mexico & 63 \\
Wyoming & 48 \\
\hline
\end{tabular}

\subsubsection{Boundary Conditions}

The export schedule for each transmission area changed on an hourly basis in accordance with the production simulation results. These schedules were based on the dispatch, not the day-ahead commitment with its imperfect renewable generation forecast, and as such have taken the day-ahead forecast error into account.

The production simulation results were also used to identify up to four types of generating units (e.g., hydro, combined-cycle, steam, gas turbine) as participants in load following via the 5-minute economic dispatch function. The unit types that moved the most across the study period were selected as participants with an appropriate priority number. For example, if the MAPS analysis showed the combined-cycle dispatch moved between 1,000 MW and 2,500 MW across a study period, and the hydro dispatch moved between $50 \mathrm{MW}$ and $200 \mathrm{MW}$, then the QSS economic dispatch priority would be combined-cycle \#1 and hydro \#2.

The production simulation results were also used to identify up to four types of generating units as providers of regulation. First, a priority number was assigned to each regulation unit type-similar to that described above for the economic dispatch unit types. Next, the available up range (unit type maximum minus unit type dispatch) or down range (unit type dispatch minus unit type minimum) was used to assign a specific percentage of the desired regulation capability to each unit type. For example, if both the combined-cycle and hydro units had significant available up range, then about $91 \%$ (i.e., $\left.[1,500 /(1,500+150)]^{*} 100\right)$ of the required up regulation capability would be assigned to the combined-cycle units and about $9 \%$ of the required up regulation capability would be assigned to the hydro units. For situations with limited available range on one particular unit type, the required regulation would be assigned to a unit type with available range. 
The maximum and minimum limits of the unit types on economic dispatch were adjusted (i.e., decreased and increased, respectively) to accommodate the desired regulation capability.

The remaining generating unit types were treated as non-dispatchable generation subject only to the hourly schedule from the production simulation results.

Changes in commitment observed in the MAPS results were implemented in the QSS algorithm as hourly changes to the maximum and/or minimum of each unit type. The ability to incorporate such commitment changes allowed for a longer (i.e., 24-hour) QSS study period than that used in previous studies (i.e., 3-hour) [7.2].

\subsubsection{Study Periods}

The QSS study periods were selected based upon the hourly statistical analysis. The primary objective was to identify challenging, but credible, system conditions.

Therefore, the hourly statistical analysis was used to identify study periods with any of the following characteristics:

- Large 1-hour, 3-hour, and 6-hour changes in net footprint load (e.g., load minus wind minus solar)

- High levels of wind and solar penetration

- Low load levels

- High wind forecast errors

Two primary study periods were chosen - an April day with low load and high wind penetration, and an October day with a high wind forecast error. The focus of the analysis was on the Local-Priority Scenario with 30\% wind penetration. Selected other scenarios and penetrations were analyzed as needed.

An overview of the hourly system characteristics for the April QSS period with 30\% wind penetration in the Local-Priority Scenario is shown in Figure 7.6. The total load in the study footprint is shown in dark blue, total wind generation in green, total PV generation in red, total CSP generation with 6 hours of storage in gold, and net load in light blue. Note that net load is negative during the nighttime minimum load period: It is the hour with the most negative load in the study footprint in 3 years of data. This period is also discussed in Chapters 4 and 6 . 


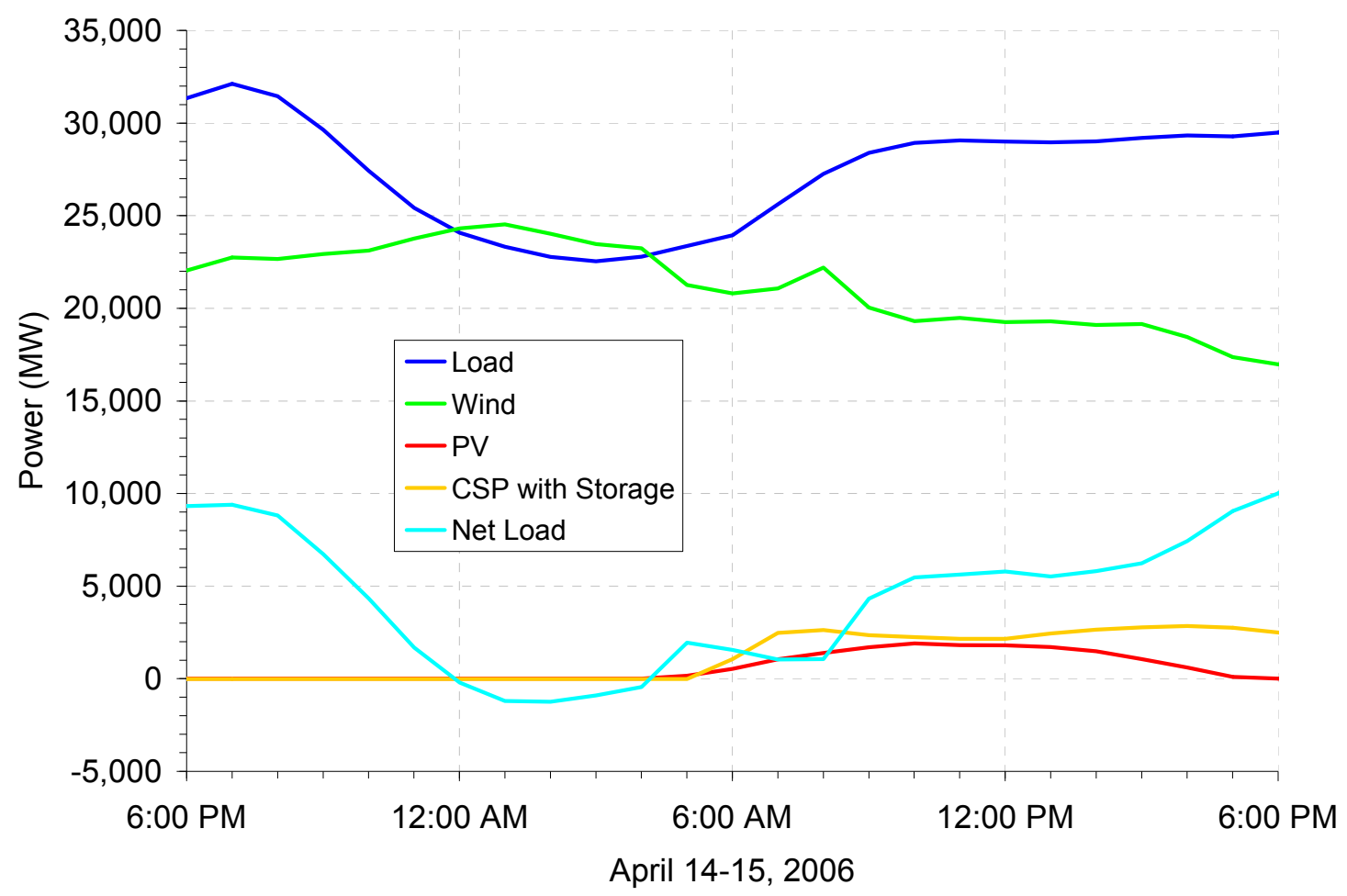

Figure 7.6 Study Footprint Characteristics for April QSS Period, Local-Priority, 30\% Wind Penetration

An overview of the hourly system characteristics for the October day is shown in Figure 7.7. The same color-coding is used for the traces, with one addition. The dashed green line represents the total day-ahead hourly wind forecast for the study footprint. Note that the maximum forecast error (i.e., forecast minus actual for a given hour) in the study footprint reached almost 12,000 MW, the single worst forecast error in 2006. The maximum forecast error in each transmission area was about 2,600 MW in Arizona, 3,500 MW in Colorado-East, $300 \mathrm{MW}$ in Colorado-West, $700 \mathrm{MW}$ in Nevada, 2,000 MW in New Mexico, and 4,700 MW in Wyoming. Due to geographic diversity, the total study footprint forecast error is less than the sum of the individual transmission area forecast errors. 


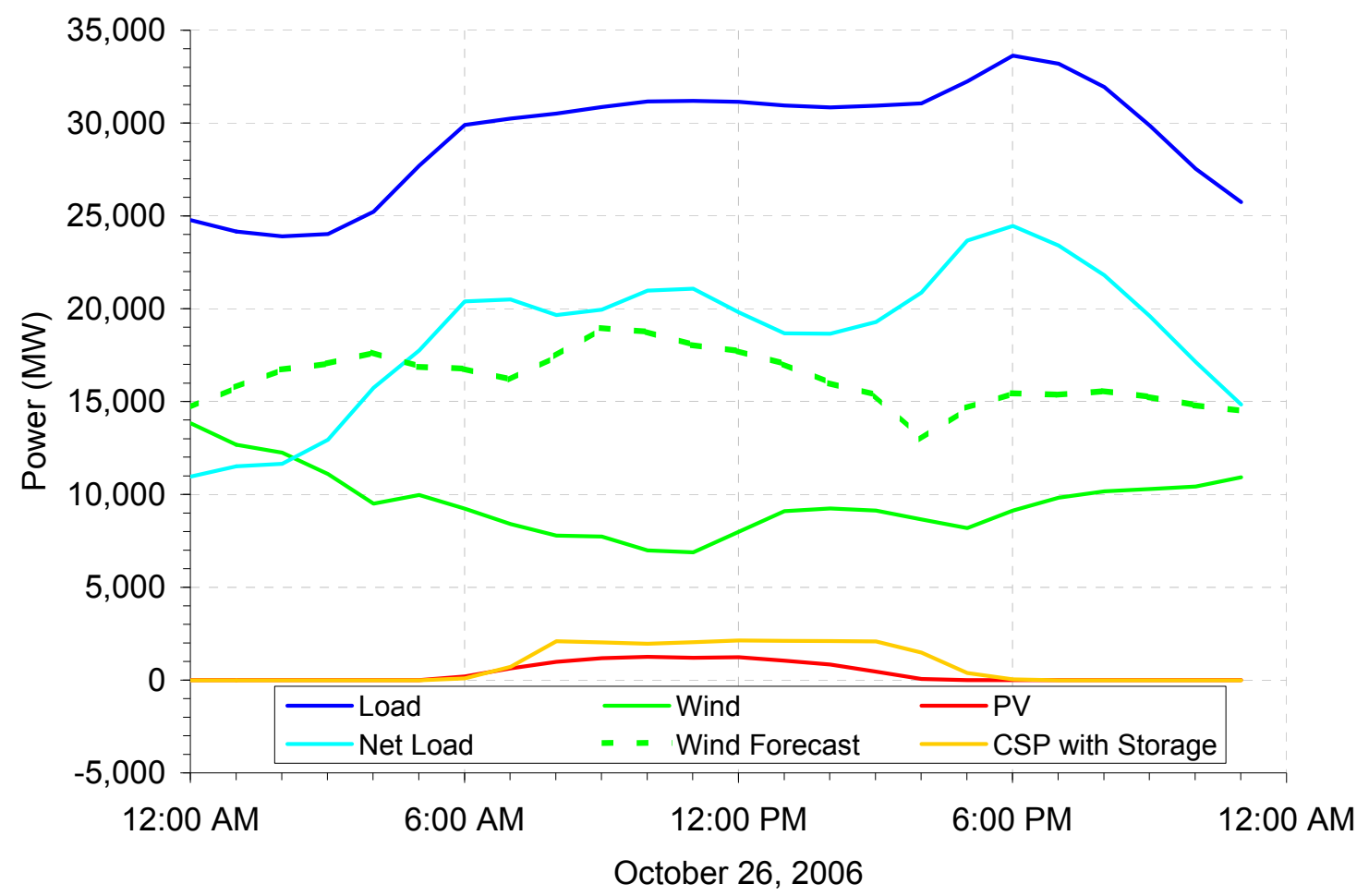

Figure 7.7 Study Footprint Characteristics for October QSS Period, Local-Priority, 30\% Wind Penetration

\subsubsection{April 15, 2006 Example}

The QSS simulation results for the April day with low load and high wind penetration are presented in this section. The Local-Priority Scenario was analyzed for four penetration levels $-10 \%, 20 \%, 20 / 20 \%$, and $30 \%$. See Chapter 3 for additional description of the scenario development and site selection. The focus is on the Arizona transmission area, with other areas brought in for comparison and contrast as needed. Additional QSS results are provided in Appendix F.

The Arizona wind and load 1-minute input profiles are shown in Figure 7.8. The gray line represents the total Arizona load, the pink line represents the total wind plant output with $30 \%$ energy penetration, the green line represents the total wind plant output with $20 \%$ penetration, and the dark blue line represents the total wind plant output with $10 \%$ penetration.

As shown in Table 2.2, 2,850 MW of wind projects were installed to achieved 10\% penetration. For $20 \%$ penetration, 5,250 MW of wind projects were installed, and for $30 \%$ penetration, 7,710 MW of wind project were installed. At the beginning of this April study period, the wind projects are at almost $100 \%$ rated output. At about 4 a.m., the wind output for the $30 \%$ penetration level exceeds the load. 


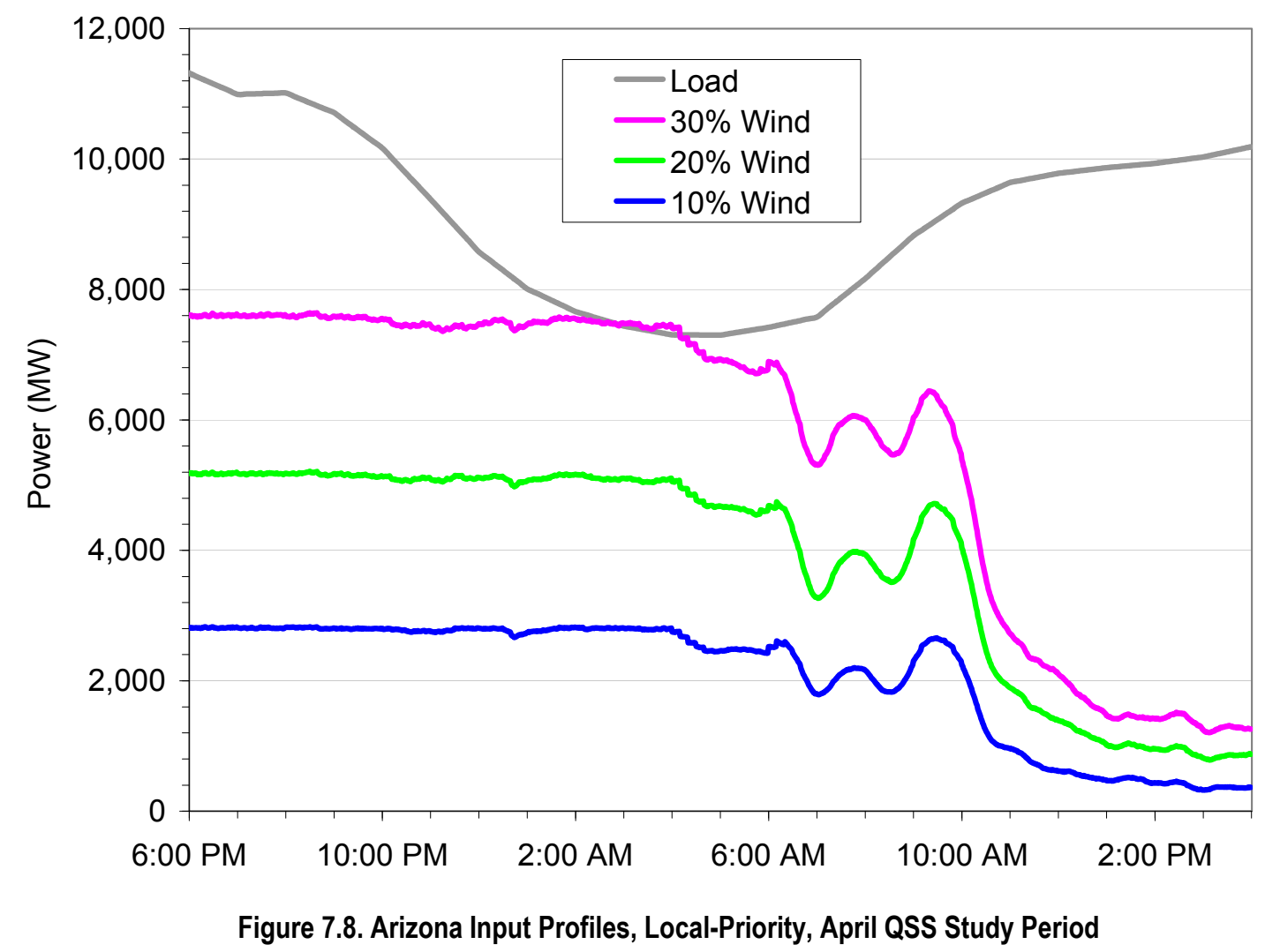

The boundary conditions from the production simulation analysis are shown in Figure 7.9 and Figure 7.10. Figure 7.9 shows the hourly schedule for the BOP generation at $10 \%$, $20 \%, 20 / 20 \%$, and $30 \%$ wind penetration. As the amount of wind generation increases, the amount of conventional generation decreases.

Figure 7.10 shows the hourly export schedule at $10 \%, 20 \%, 20 / 20 \%$, and $30 \%$ wind penetration. As the amount of wind generation increases from $10 \%-20 / 20 \%$, the Arizona exports decrease. This indicates that Arizona conventional generation is displaced by wind generation both inside and outside Arizona as penetration increases. As the wind penetration increases from $20 / 20 \%-30 \%$, the export schedule increases. This indicates that the additional Arizona wind is displacing conventional generation outside Arizona.

The difference between the $20 \%$ and $20 / 20 \%$ cases (dark blue and light blue, respectively) is entirely due to additional renewable generation outside the study footprint. The generation mix of the rest of WECC has a significant impact on the performance of the study footprint.

The generation boundary conditions were separated into unit types and used to identify the participants in load following via the 5-minute economic dispatch function, and the providers of regulation. The economic dispatch unit types, and their priority, are shown 
in Table 7.6 for each penetration level. The regulation unit types, their priority and capability, are shown in Table 7.7.

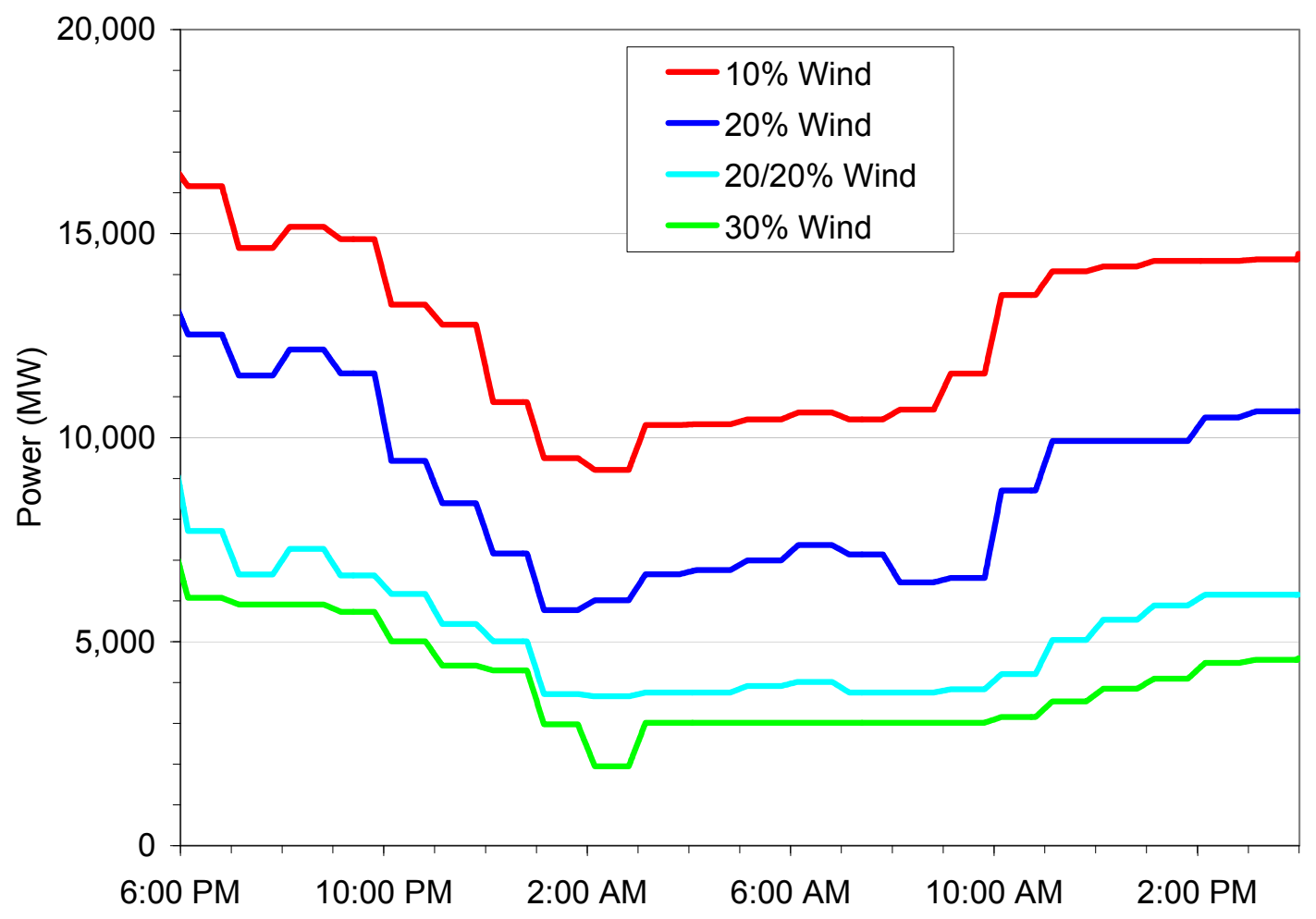

Figure 7.9 Arizona Conventional Generation Hourly Schedule, Local-Priority, April QSS Study Period 


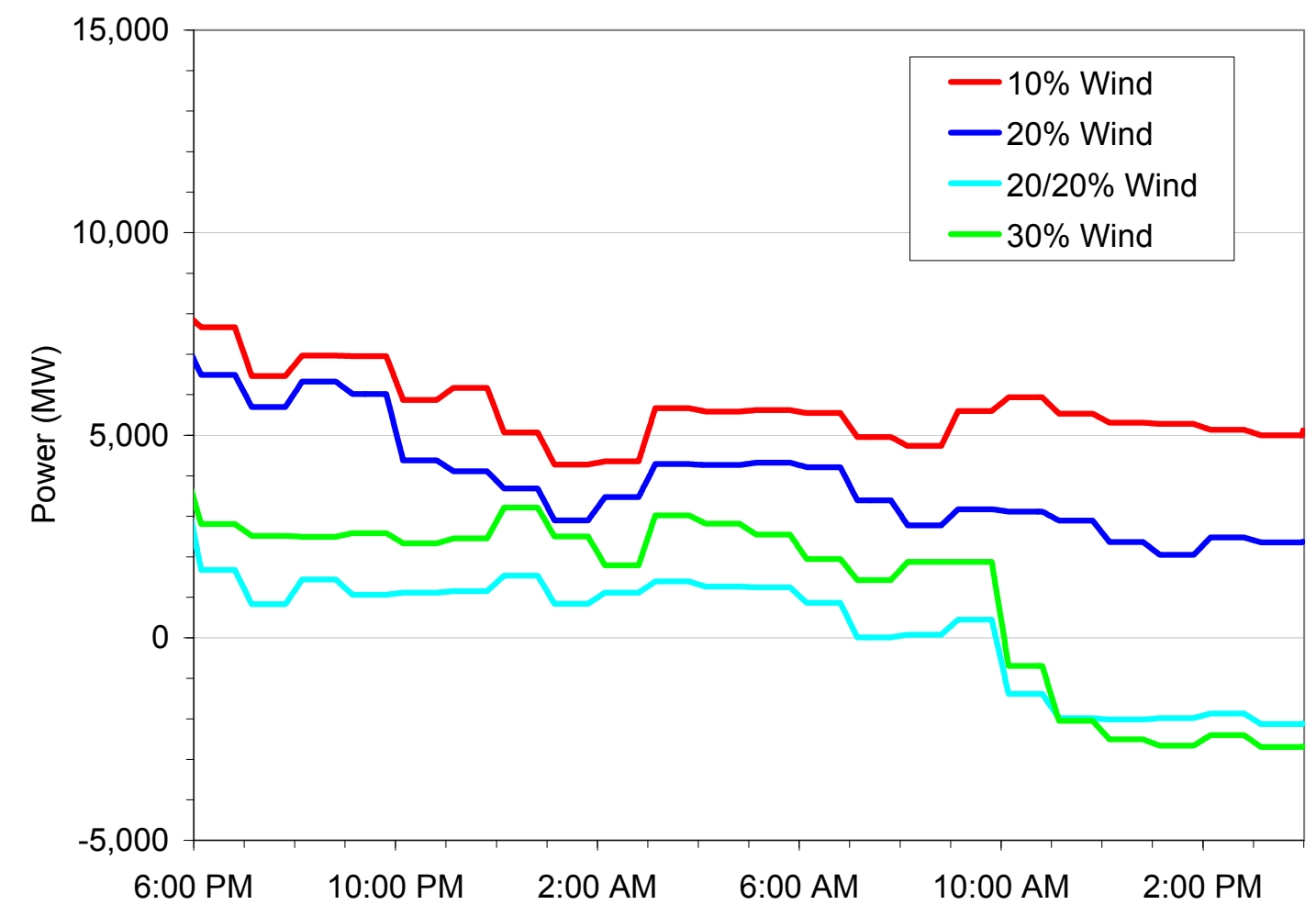

Figure 7.10 Arizona Export Schedule, Local-Priority, April QSS Study Period.

Table 7.6 Economic Dispatch Priority for Arizona during April QSS Study Period.

\begin{tabular}{|l|c|c|c|c|}
\cline { 2 - 5 } \multicolumn{1}{c|}{} & $\mathbf{1 0 \%}$ Wind & $\mathbf{2 0} \%$ Wind & $\mathbf{2 0 / 2 0 \%}$ Wind & $\mathbf{3 0} \%$ Wind \\
\hline Hydro & 3 & 3 & 4 & 3 \\
Combined-Cycle & 1 & 1 & 3 & 4 \\
Gas Turbine & 4 & 4 & 2 & 2 \\
Steam & 2 & 2 & 1 & 1 \\
\hline
\end{tabular}

Table 7.7 Regulation Priority and Capability for Arizona During April QSS Study Period.

\begin{tabular}{|c|c|c|c|c|c|c|c|c|c|c|c|c|}
\hline & \multicolumn{3}{|c|}{$10 \%$ Wind } & \multicolumn{3}{|c|}{$20 \%$ Wind } & \multicolumn{3}{|c|}{$20 / 20 \%$ Wind } & \multicolumn{3}{|c|}{$30 \%$ Wind } \\
\hline & Priority & $+M W$ & $-M W$ & Priority & $+M W$ & $-M W$ & Priority & $+M W$ & $-M W$ & Priority & $+M W$ & $-M W$ \\
\hline Hydro & 3 & 83 & -23 & 3 & 47 & -6 & 4 & 119 & -7 & 3 & 62 & 0 \\
\hline Combined-Cycle & 1 & 124 & -131 & 1 & 138 & -91 & 3 & 12 & -17 & 4 & 0 & 0 \\
\hline Gas Turbine & 4 & 0 & 0 & 4 & 0 & 0 & 2 & 14 & -14 & 2 & 11 & -11 \\
\hline Steam & 2 & 24 & -76 & 2 & 45 & -134 & 1 & 86 & -193 & 1 & 158 & -220 \\
\hline
\end{tabular}

The results of the April QSS analysis with the above input data and boundary conditions are presented in Figure 7.11 through Figure 7.25. 
The Arizona dispatch with 10\% wind penetration for the Local-Priority Scenario is shown in Figure 7.11. As shown in Table 7.6, the economic dispatch priority for this case is combined-cycle (green), steam (light blue), hydro (dark blue), and gas turbine (pink). The heavyweight lines represent the unit type power output, and the lightweight lines represent the maxima and minima of the committed units. Changes in commitment can be observed in the changes to the maxima and minima.

Over the course of this simulation, the top three unit types (e.g., combined-cycle, steam, and hydro) respond to the system economic dispatch, and follow load down, and then up again. The bulk of the load following is performed by the combined-cycle units, with these units operating at minimum for several hours over night when the load is at its lowest level.

The amount of regulation provided is shown in Figure 7.12, as the dark blue line. The conventional generation hourly schedule (pink) and the hourly export schedule (gold) are also shown. The right vertical scale applies to regulation; the left vertical scale applies to the hourly schedules.

As shown in Table 7.4, +/-231 MW of regulation capability is available in Arizona during the simulation. The regulation that is actually provided is largely driven by the hourly schedule changes, not by the wind and solar variability. Each time the provided regulation approaches or hits a capability limit (i.e., +/-231 MW), it is due to an hourly schedule change. 


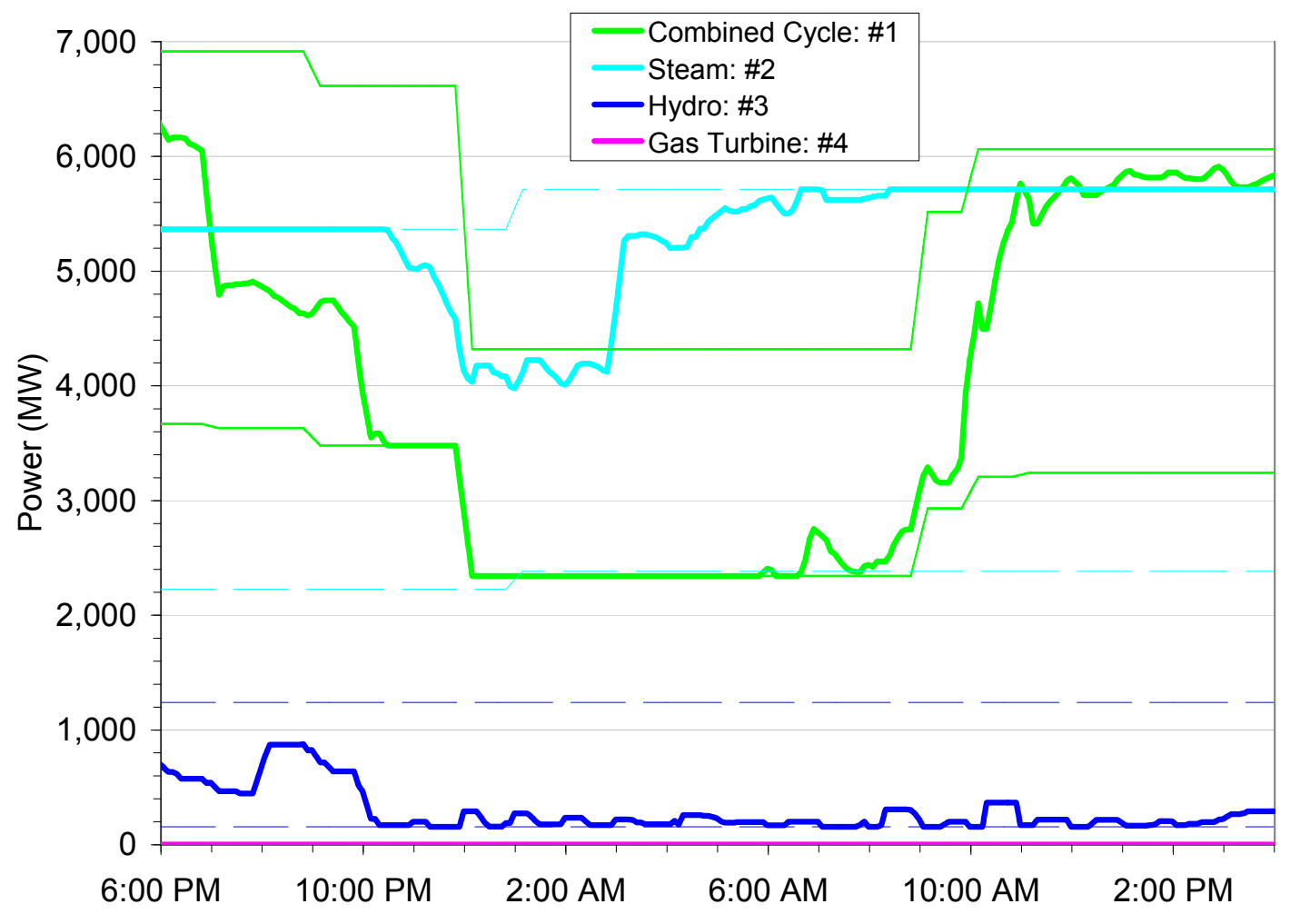

Figure 7.11 Arizona Dispatch, 10\% Wind, Local-Priority, April QSS Study Period.

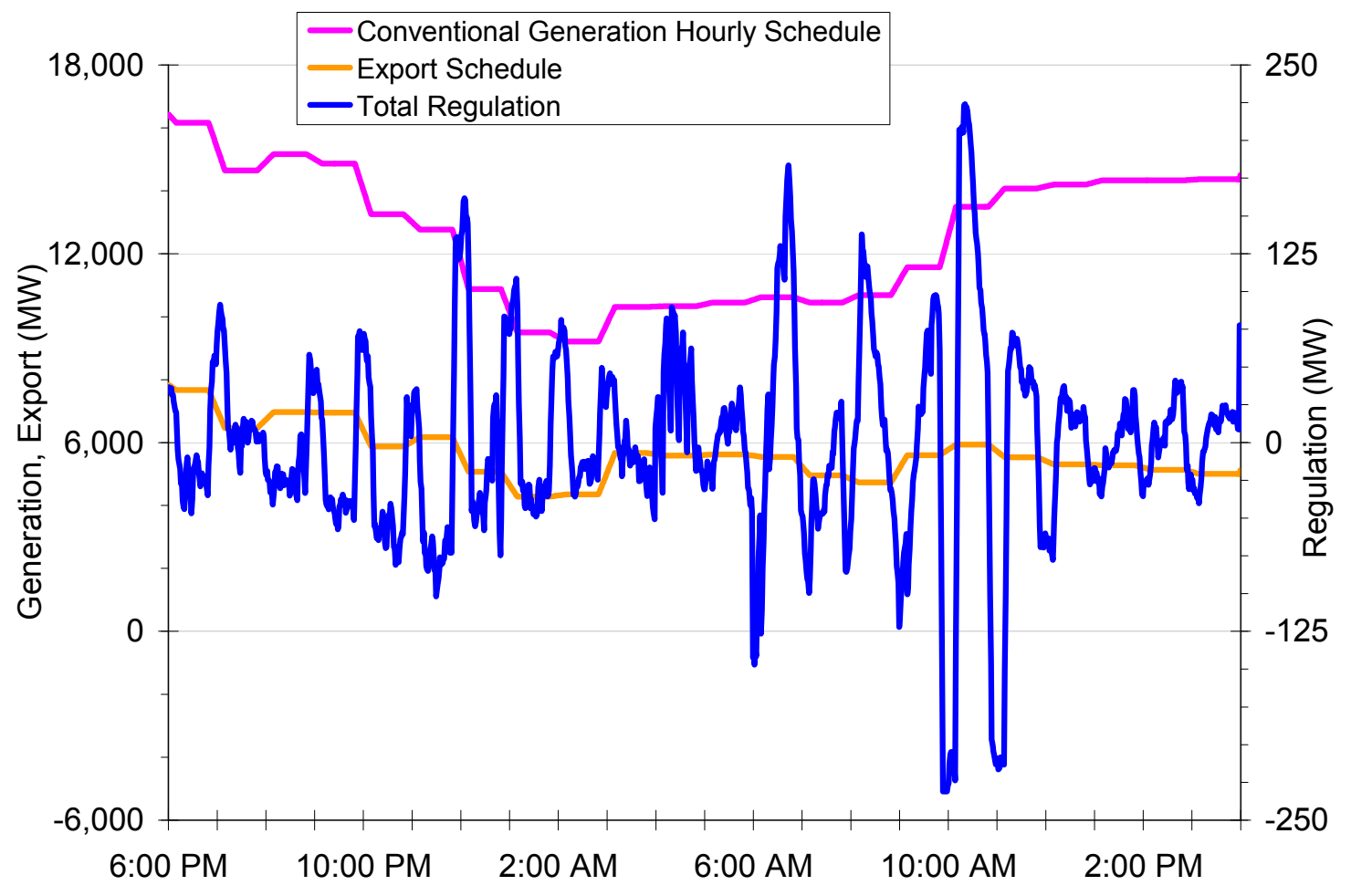

Figure 7.12. Arizona Regulation, 10\% Wind, Local-Priority, April QSS Study Period. 
The overall performance of the Arizona system with $10 \%$ wind penetration is shown in Figure 7.13. The dark blue line represents the Total Generation, or all generation in the Arizona transmission area including wind and solar. The pink line represents the Conventional Generation Hourly Schedule or the hourly boundary conditions from the production simulation analysis. The gray line (generally hidden behind the light blue line) represents the Conventional Generation Total, which includes all generation responding to economic dispatch, regulation, and hourly schedules-effectively, all generation except wind and solar. The light blue line represents the Load-WindSolar+Export or load minus wind minus solar plus the hourly export schedule. The green line represents Load-Wind-Solar or load minus wind minus solar, and the gold line represents the hourly Export Schedule. Both the Conventional Generation Hourly Schedule and the Export Schedule contain hourly information only. The other traces change minute-by-minute.

The key comparison in this plot is between the light blue line (Load-Wind-Solar+Export) and the gray line (Conventional Generation Total). When the Arizona generation and net load are in balance, then these two traces will be indistinguishable. When the generation-load balance is not maintained, then the gray line (Conventional Generation Total) will no longer be hidden by the light blue line (Load-Wind-Solar+Export). A minor generation-load imbalance occurs about 10 a.m. This imbalance is small and difficult to identify given the plot scale. Therefore, it is indicated by the black circle. At this time, the units on economic dispatch and the units providing regulation have insufficient capability to meet the net changes in load, wind, solar, and hourly export.

This will result in an ACE, as shown by the red line in Figure 7.14. The amount of regulation provided is represented by the blue line, and matches that shown in Figure 7.12. A positive ACE signal means that conventional generation exceeds net load, and the actual export will exceed the export schedule. A negative ACE signal means that load exceeds generation, and the actual export will fail to meet the export schedule. As with the regulation itself, the ACE is largely driven by the hourly generation and export schedule changes, not by the wind and solar variability. The two black lines, shown in Figure 7.14, represent the approximate L10 limits. A potential CPS2 violation occurs when the ten-minute average ACE exceeds L10 [7.1]. Whether an actual violation occurs depends on the number of times the ten-minute ACE exceeds L10 in a given month. Each balancing area is not required to keep the ten-minute ACE below L10 all of the time, but must do so $90 \%$ of the time. 


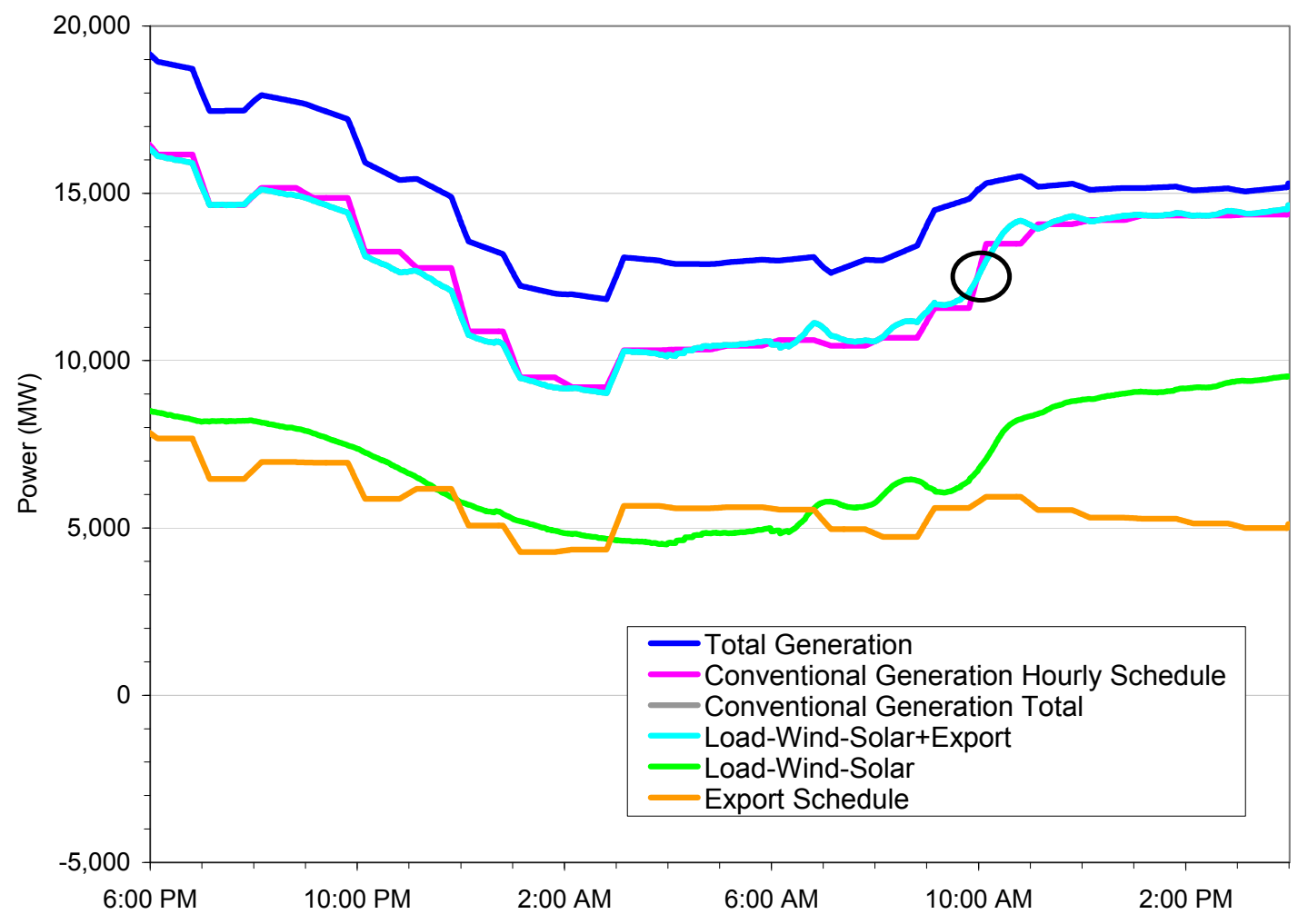

Figure 7.13 Arizona Totals, 10\% Wind, Local-Priority, April QSS Study Period.

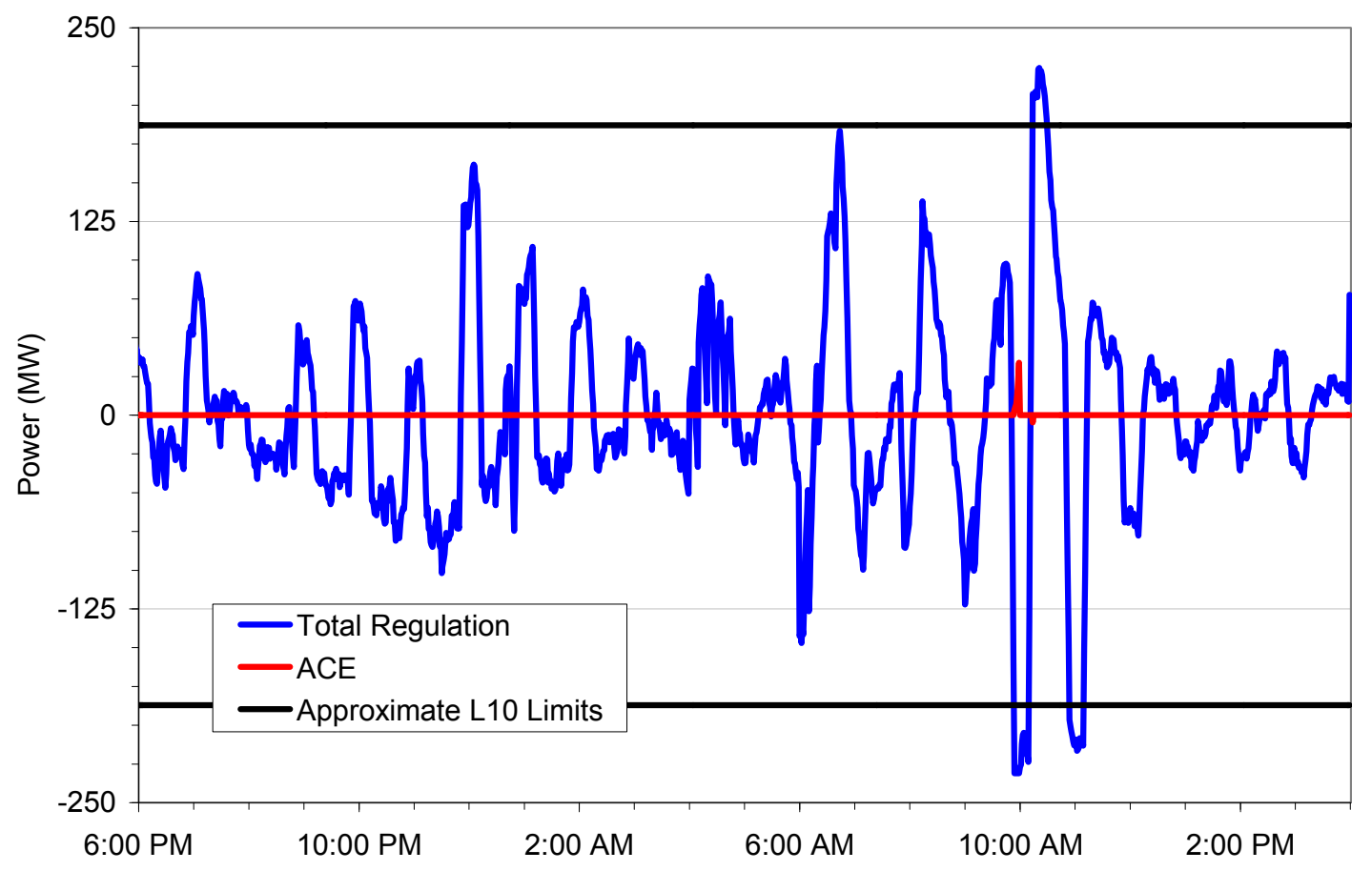

Figure 7.14 Arizona Regulation and ACE, 10\% Wind, Local-Priority, April QSS Study Period. 
The impact of increasing levels of wind and solar penetration, and therefore increasing variability, was also evaluated.

Figure 7.15 - Figure 7.17 show the Arizona dispatch for the Local-Priority Scenario with $20 \%, 20 / 20 \%$, and $30 \%$ wind penetration. The $20 / 20 \%$ case has the same wind penetration within Arizona as the $20 \%$ case. However, the penetration outside the study footprint has increased from $10 \%-20 \%$.

Several effects were observed as penetration increased from the $10 \%$ penetration level shown in Figure 7.11. First, the combined-cycle commitment was significantly reduced. This is consistent with the results reported in Chapter 6, which showed that wind generation displaces the conventional generation with the highest marginal cost. Second, the economic dispatch priority order changed. At $10 \%$ penetration, the priority was combined-cycle, steam, hydro, and gas turbine. At 30\% penetration, the priority was steam, gas turbine, hydro, and combined-cycle. This reflects the changing BOP generation mix as wind and solar displace combined-cycle units. Finally, the amount of wind and solar generation in neighboring systems had a significant impact. At $20 \%$ penetration, the bulk of the Arizona load following was performed by combined-cycle and steam units. With the increased wind and solar penetration outside the study footprint in the $20 / 20 \%$ case, the bulk of the load following was performed by steam units. The additional out-of-footprint wind and solar effectively reduced the opportunity for Arizona to export power.

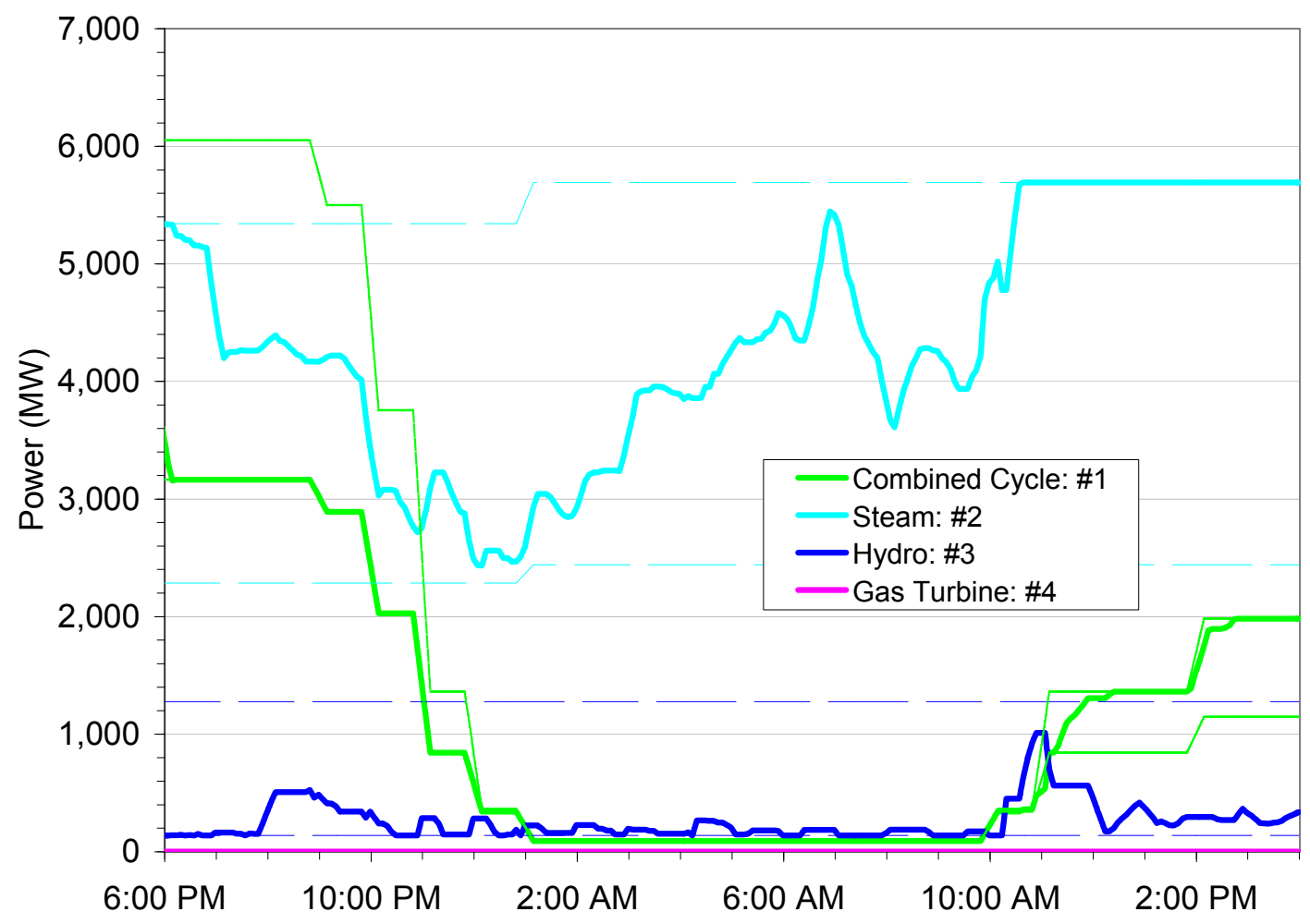

Figure 7.15 Arizona Dispatch, 20\% Wind, Local-Priority, April QSS Study Period. 


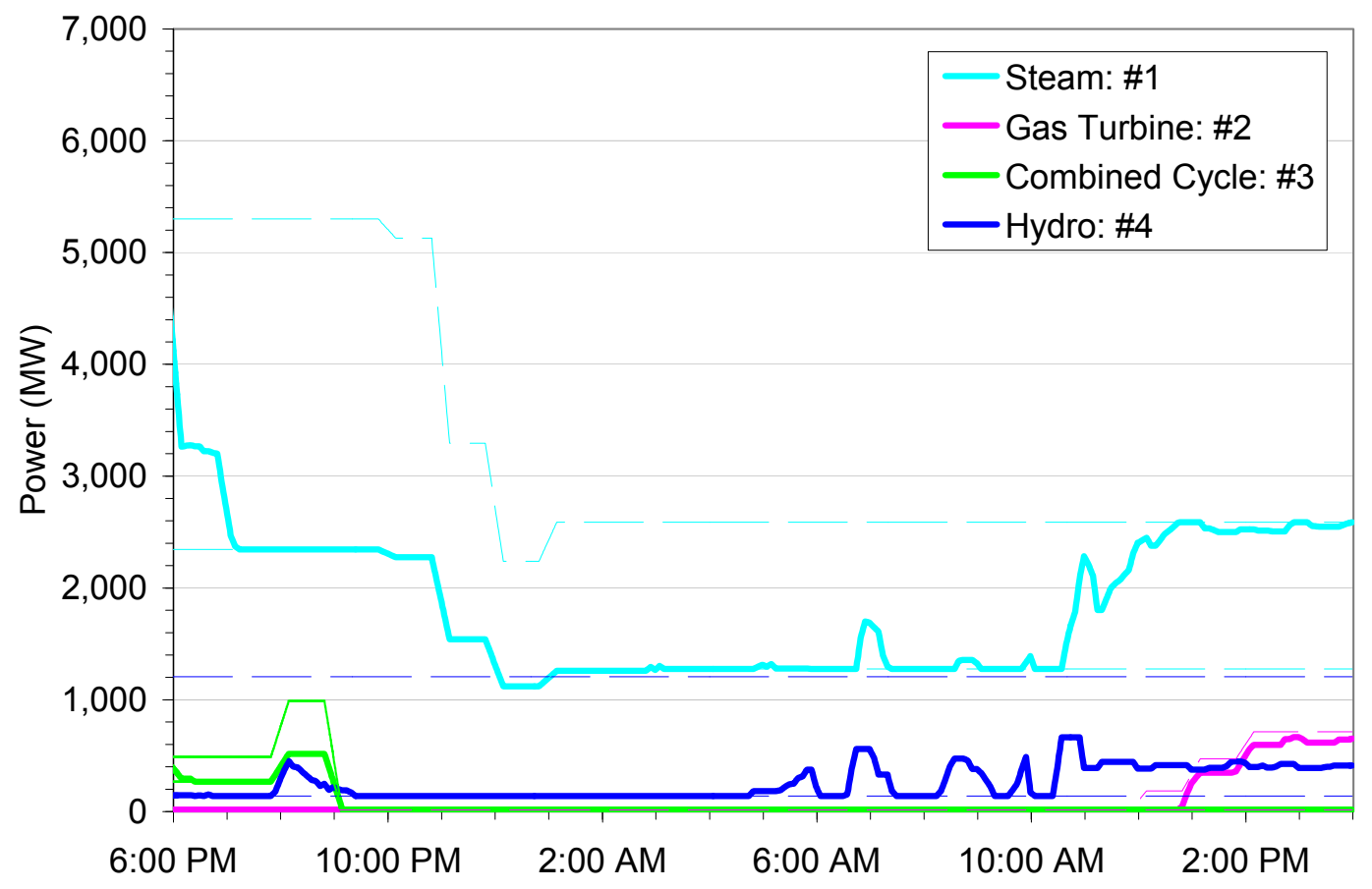

Figure 7.16 Arizona Dispatch, 20/20\% Wind, Local-Priority, April QSS Study Period

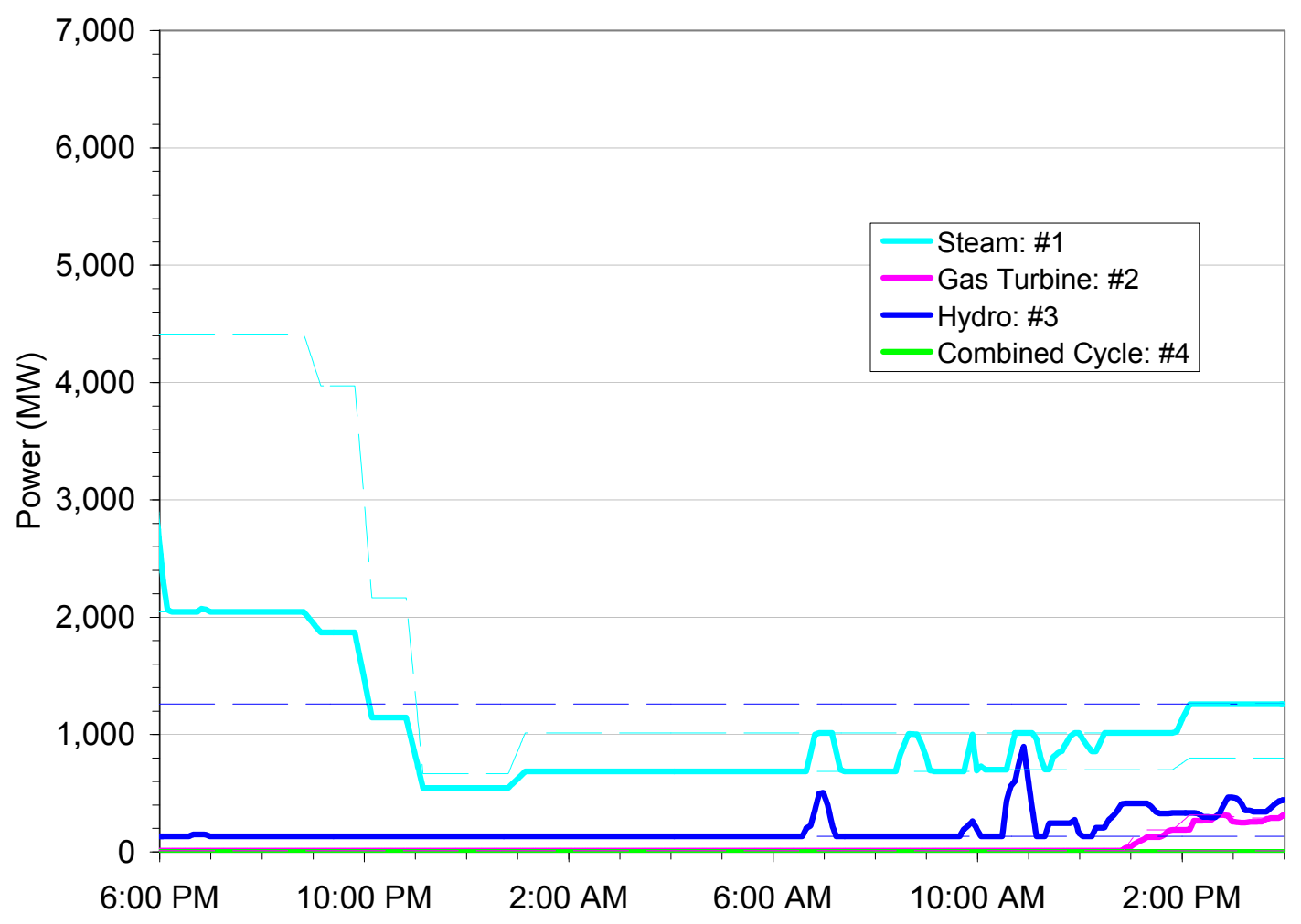

Figure 7.17 Arizona Dispatch, 30\% Wind, Local-Priority, April QSS Study Period 
Figure 7.18 - Figure 7.20 show the Arizona regulation and hourly schedules for the Local-Priority Scenario with 20\%, 20/20\%, and 30\% wind penetration.

The export schedule decreased significantly as penetration increased from the $10 \%$ penetration level shown in Figure 7.12. At $10 \%$ and $20 \%$ penetration, the Arizona system begins with an export schedule in the range of 7,000-8,000 MW, and it is always exporting. With the increased wind and solar penetration outside the study footprint in the $20 / 20 \%$ case, the initial export schedule is reduced to about 2,000 MW and the system ends up as an importer; a similar pattern is observed for the $30 \%$ case.

At $20 \%$ penetration, the impact of wind and solar variability on regulation was greater than that observed at $10 \%$ penetration. However, the largest regulation swings were still driven by the hourly schedule changes.

At $20 / 20 \%$ and $30 \%$ penetration, the lack of sufficient down regulation during the lowload period overnight exceeded the impact of variability. The high level of wind generation within Arizona, the reduced exports from Arizona due to high wind penetration outside of the study footprint, and the resulting reduction in the commitment and dispatch of the remainder of Arizona's generation fleet make minimum load operation more difficult.

These results are pessimistic because the hourly boundary conditions consistently lag the load decrease. This is inherent to MAPS with its hourly-integrated loads and "hour ending" convention, and can be observed in Figure 7.13. In this figure, the net load (light blue line) consistently leads the conventional generation hourly schedule (pink line). In reality, such an hour-after-hour lag would not be allowed. 


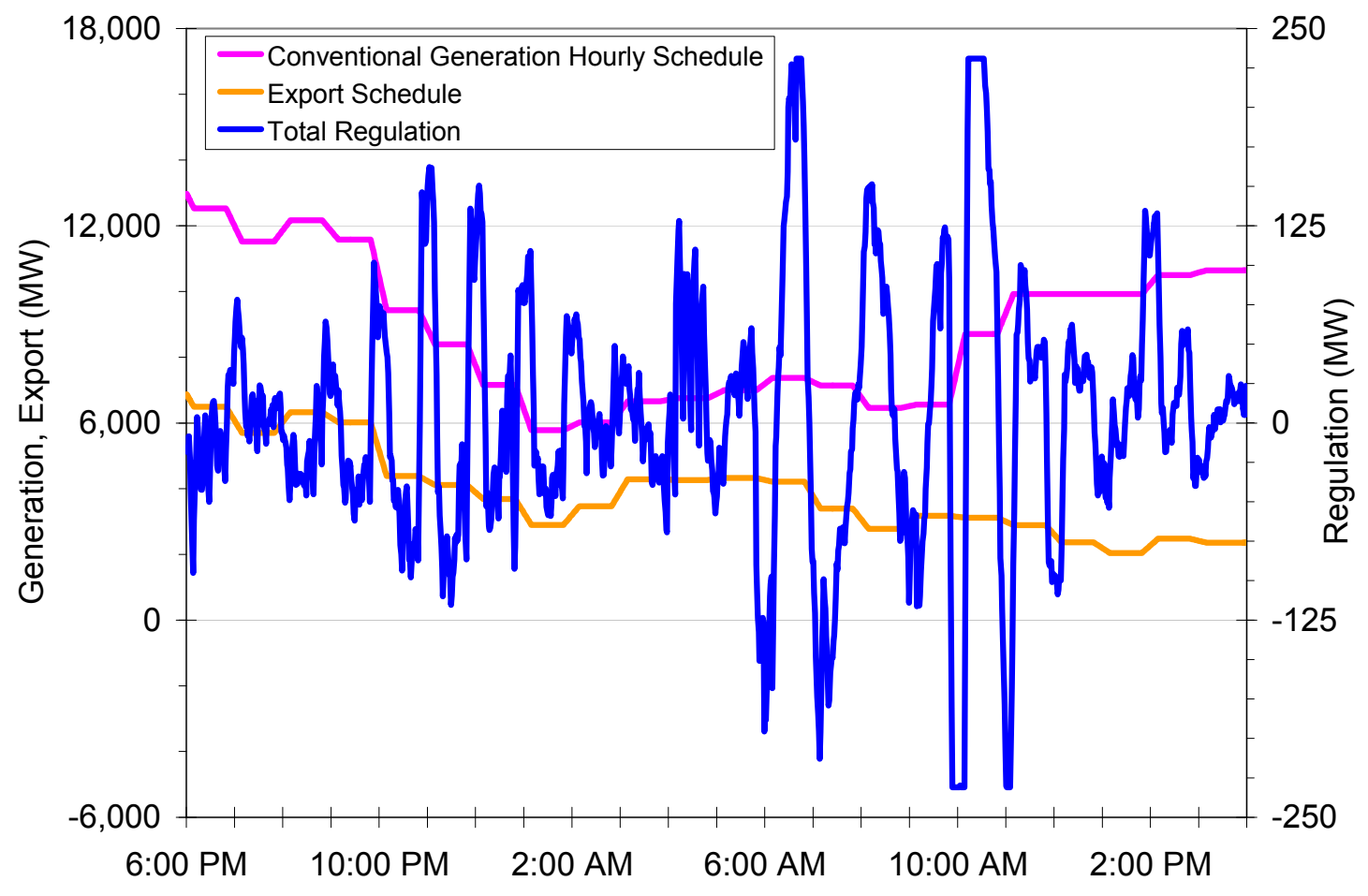

Figure 7.18 Arizona Regulation, 20\% Wind, Local-Priority, April QSS Study Period.

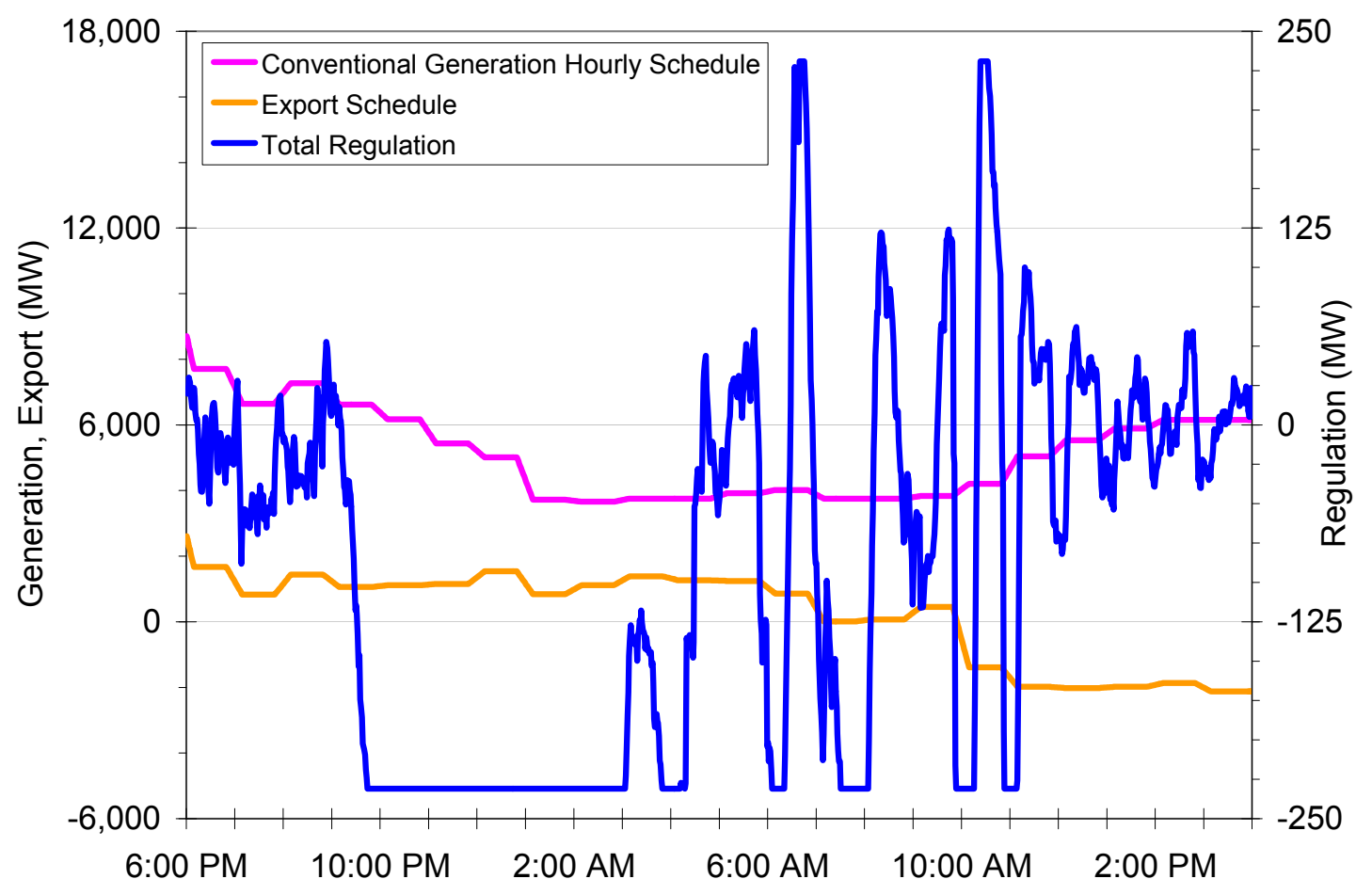

Figure 7.19 Arizona Regulation, 20/20\% Wind, Local-Priority, April QSS Study Period. 


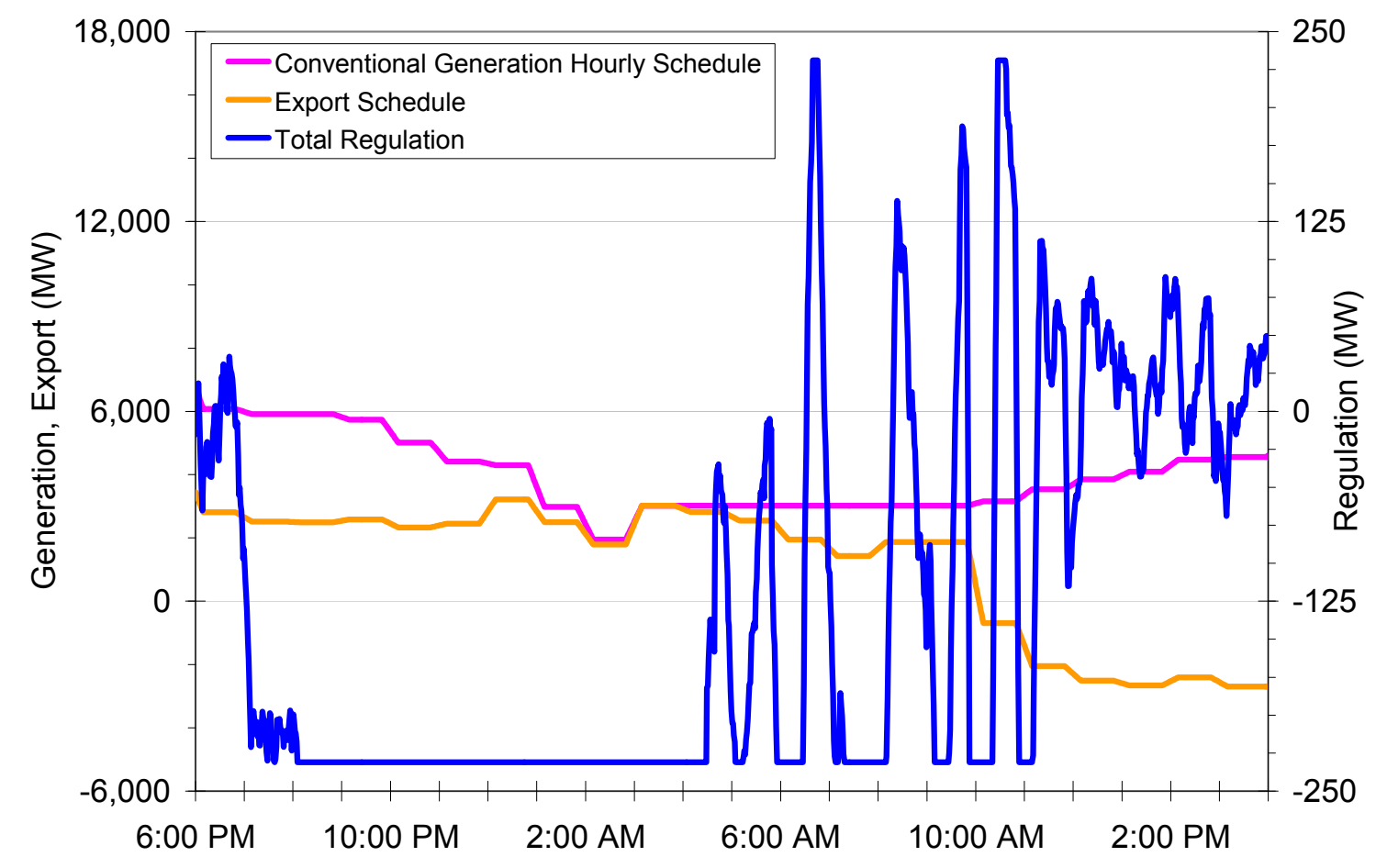

Figure 7.20 Arizona Regulation, 30\% Wind, Local-Priority, April QSS Study Period.

An ACE will occur when there is insufficient regulating capability to meet the net changes in load, wind, solar, and hourly schedules. Figure 7.21 shows the Arizona ACE for the Local-Priority Scenario with various levels of wind penetration. The red line represents $10 \%$ wind penetration, the green line represents $20 \%$ wind, the light blue line represents $20 / 20 \%$ wind, the dark blue line represents $30 \%$ wind, and the black lines represent the approximate L10 limits. These L10 values were used to identify potential CPS2 violations.

Several effects are observed as wind penetration increased. First, the Arizona ACE increased. Second, the performance of the $10 \%$ and $20 \%$ cases were similar, and the performance of the $20 / 20 \%$ and $30 \%$ cases were similar. The breakpoint between the $20 \%$ case and the $20 / 20 \%$ case illustrates that the amount of wind and solar generation in neighboring systems has a significant impact on the performance of the study footprint. Third, the $20 / 20 \%$ and $30 \%$ cases lack sufficient down regulation during the low load period overnight and extending into the morning load rise. This resulted in extended ACE excursions over the L10 limit and likely CPS2 violations. 


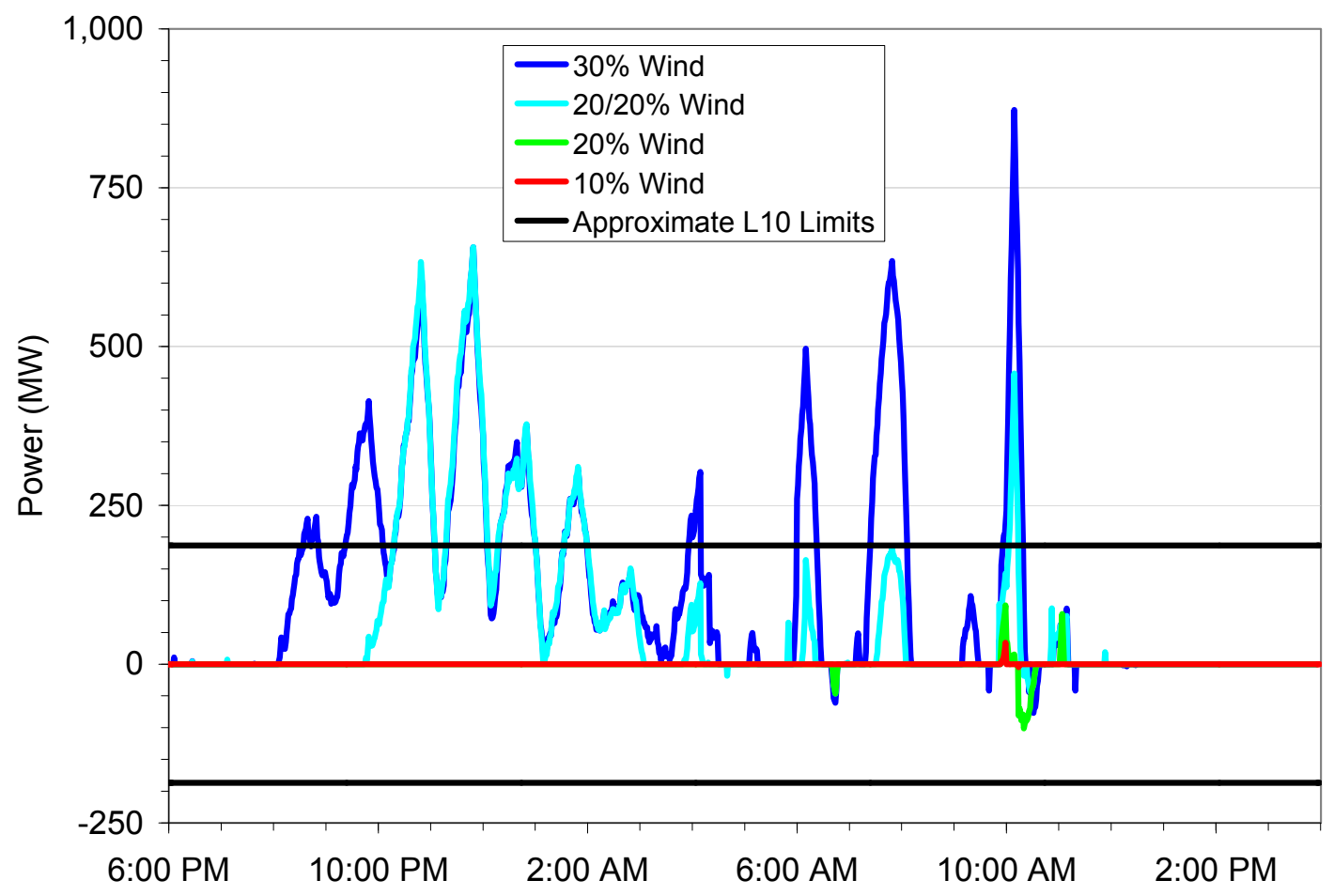

Figure 7.21 Arizona ACE, All Penetration Levels, Local-Priority, April QSS Study Period.

Wind curtailment could be an effective response to such ACE excursions under lowload, high-wind conditions. Wind curtailment addresses positive ACE signals, which occur when generation is greater than load and results in the actual export exceeding the scheduled export. An example curtailment is shown in Figure 7.22, with the original aggregate wind profile for Arizona in pink and the curtailed profile in green. Total load is shown in dark blue. Since curtailment of wind generation contributes to system down reserve, the curtailment was calculated using the simplified variability reserve requirement that will be described in Section 7.4. Specifically, the original wind profile was reduced by $2.2 \%$ of load and $5.6 \%$ of actual wind, up to $36 \%$ of rated wind. At 10 a.m., the curtailment was removed.

Figure 7.23 shows the Arizona regulation (blue line) and ACE (red line) for 30\% wind penetration with curtailment. Again, the black lines represent the approximate L10 limits. Both the regulation and the ACE were significantly reduced from that shown in Figure 7.20 and Figure 7.21 for $30 \%$ wind penetration without curtailment. The number of potential CPS2 violations were also significantly reduced. This was achieved by curtailing about $5 \%$ of the wind energy available during this day. 


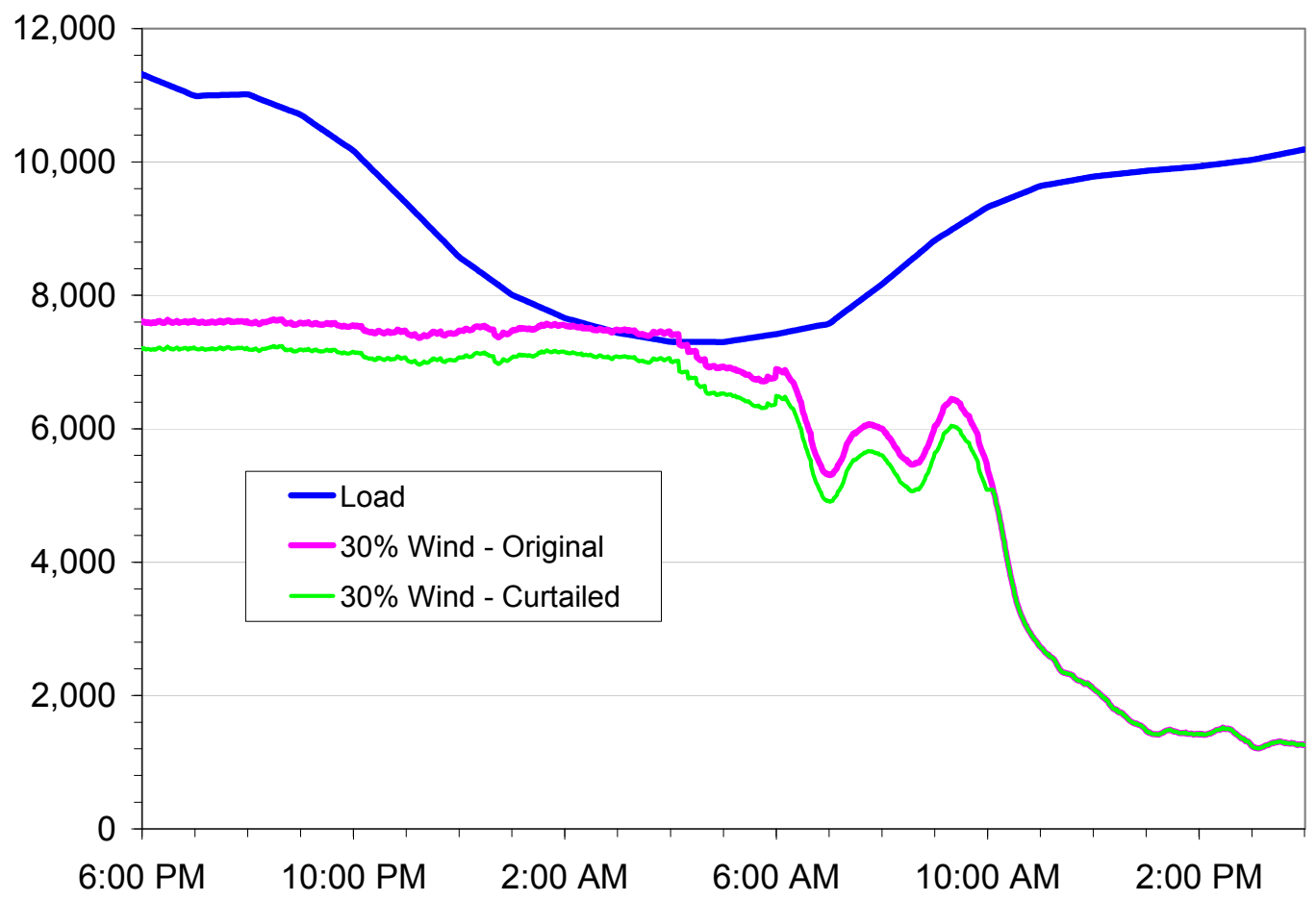

Figure 7.22 Arizona Wind Curtailment, April QSS Study Period.

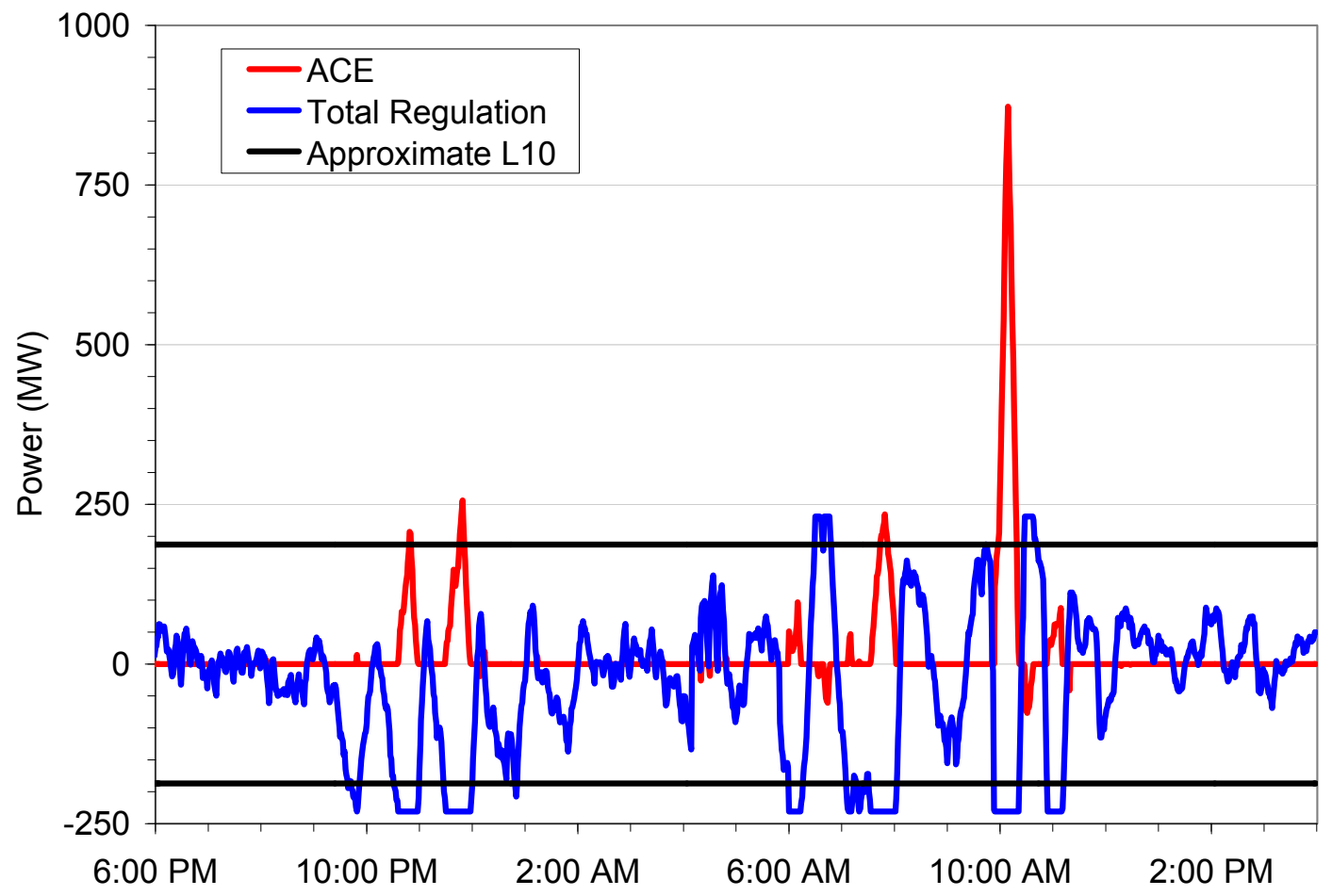

Figure 7.23 Arizona Regulation and ACE, 30\% Wind with Curtailment, Local-Priority, April QSS Study Period. 
Extensive balancing area cooperation or consolidation can also improve system operation by sharing load following, regulation and reserve responsibilities. Figure 7.24 and Figure 7.25 show the impact of such coordination on the Local-Priority Scenario with $30 \%$ wind penetration and no curtailment.

Figure 7.24 shows the individual ACEs for each transmission area in the study footprint. Figure 7.25 shows the entire study footprint ACE with two types of area cooperation or consolidation. The dark blue line is the sum of the individual ACEs shown in Figure 7.24, or the footprint ACE with some cooperation between areas. This is similar to ACE diversity interchange (ADI) [7.4], which pools individual ACEs to take advantage of their sign differences to reduce generator movement and improve CPS performance. The green line represents the ACE with significantly more cooperation or consolidation-all seven transmission areas are operated as one to share sub-hourly reserves. Under many conditions, the ACE has been eliminated and the total energy under the ACE curve was reduced by $30 \%$. Overnight, the ACE is unchanged, indicating the need for additional measures (e.g., wind curtailment) during such low load, high wind conditions.

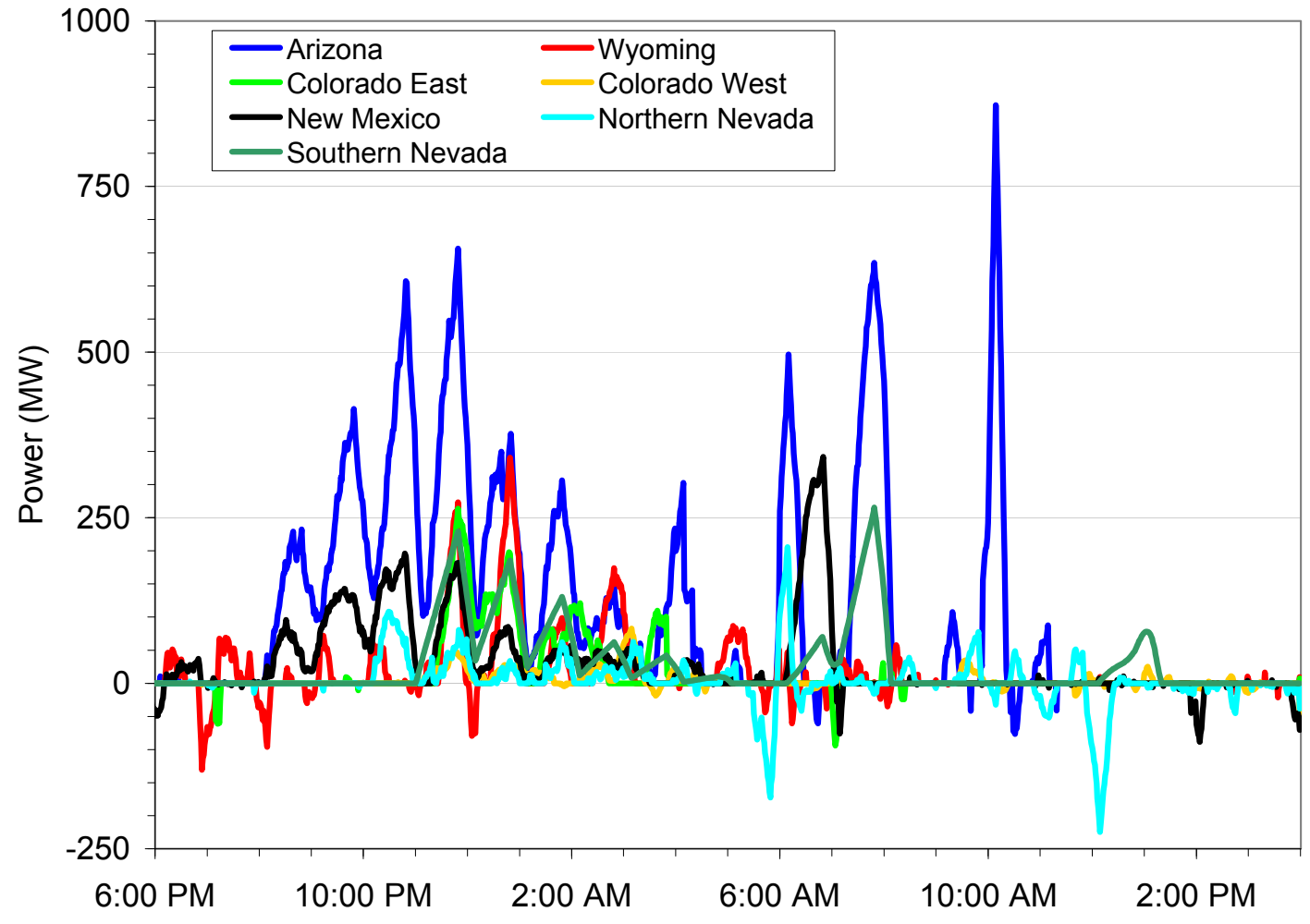

Figure 7.24 Transmission Area ACEs, 30\% Wind, Local-Priority, April QSS Study Period. 


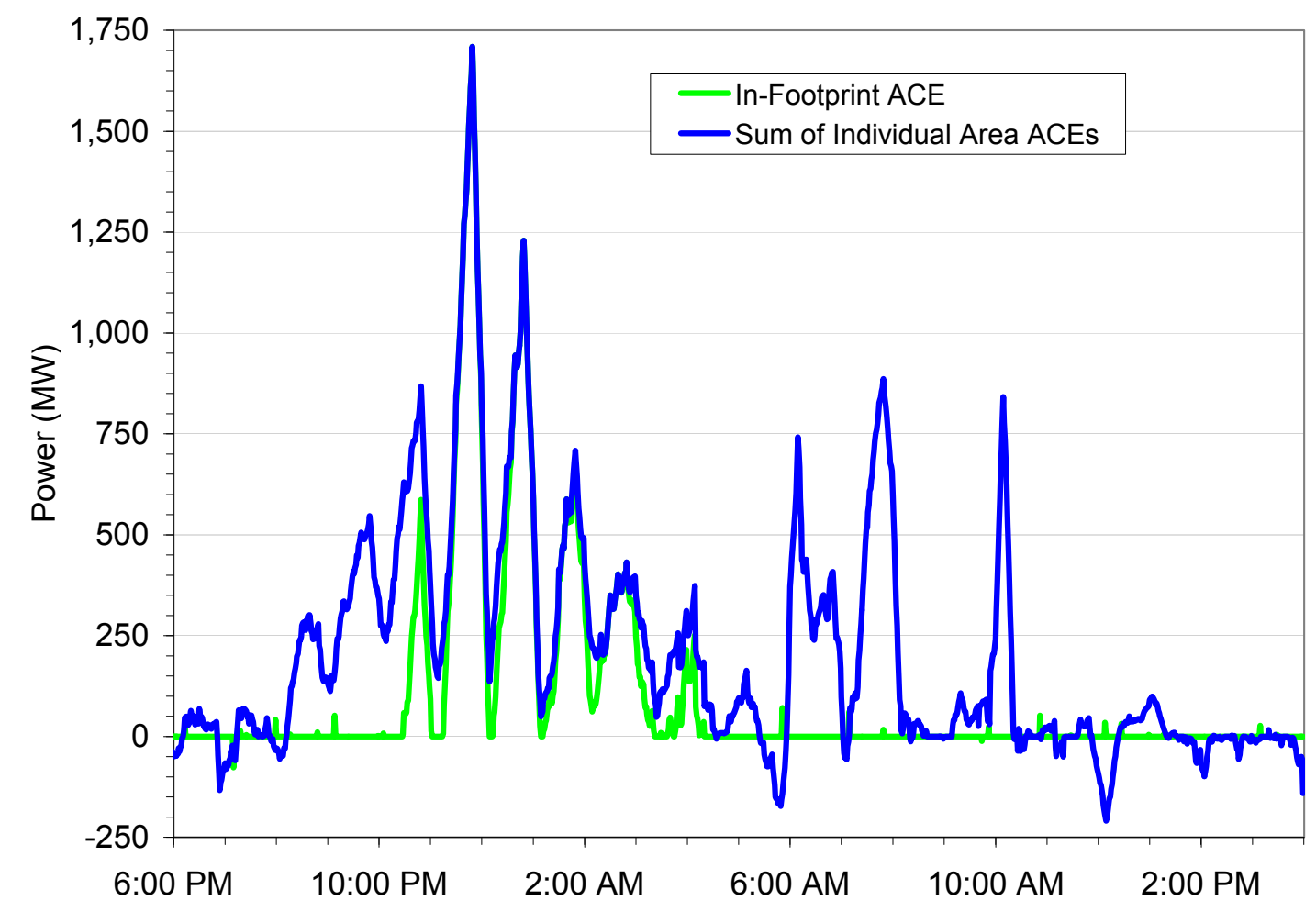

Figure 7.25 Footprint ACEs with Cooperation and Consolidation, 30\% Wind, Local-Priority, April QSS Study Period.

\subsubsection{October 26, 2006 Example}

The QSS simulation results for the October day with a high wind forecast error are presented in this section. The Local-Priority Scenario was analyzed for four penetration levels $-10 \%, 20 \%, 20 / 20 \%$, and $30 \%$. See Chapter 3 for additional description of the scenario development and site selection. The focus is on the Colorado-East transmission area, with other areas brought in for comparison and contrast as needed. Additional QSS results are provided in Appendix F.

The Colorado-East wind and load input profiles are shown in Figure 7.26. The gray line represents the total Colorado-East load, the solid pink line represents the total wind plant output with 30\% energy penetration, and the dashed pink line represents the 30\% wind forecast. Similarly, the green lines represent the total wind plant output (solid) and forecast (dashed) with $20 \%$ penetration, and the dark blue lines represent the total wind plant output (solid) and forecast (dashed) with $10 \%$ penetration.

As shown in Table 2.2, 2,190 MW of wind projects were installed to achieved 10\% penetration. For $20 \%$ penetration, 3,870 MW of wind projects were installed, and for $30 \%$ penetration, 4,650 MW of wind project were installed. During this October study period, the maximum output for all penetration levels is about $45 \%$ of rated capacity. As 
discussed in Chapter 5, the maximum forecast error, about 3,500 MW, occurred near 10 a.m. with $30 \%$ wind penetration.

The boundary conditions from the production simulation analysis are shown in Figure 7.27 and Figure 7.28. Figure 7.27 shows the hourly schedule for the BOP generation at $10 \%, 20 \%, 20 / 20 \%$, and $30 \%$ wind penetration. Figure 7.28 shows the hourly export schedule at each wind penetration level. As the amount of wind generation increases, the amount of conventional generation decreases. Similarly, as the amount of wind generation increases, the Colorado-East exports decrease.

The generation boundary conditions were separated into unit types and used to identify the participants in load following via the 5-minute economic dispatch function, and the providers of regulation. The economic dispatch unit types, and their priority, are shown in Table 7.8 for each penetration level. The regulation unit types, their priority and capability, are shown in Table 7.9.

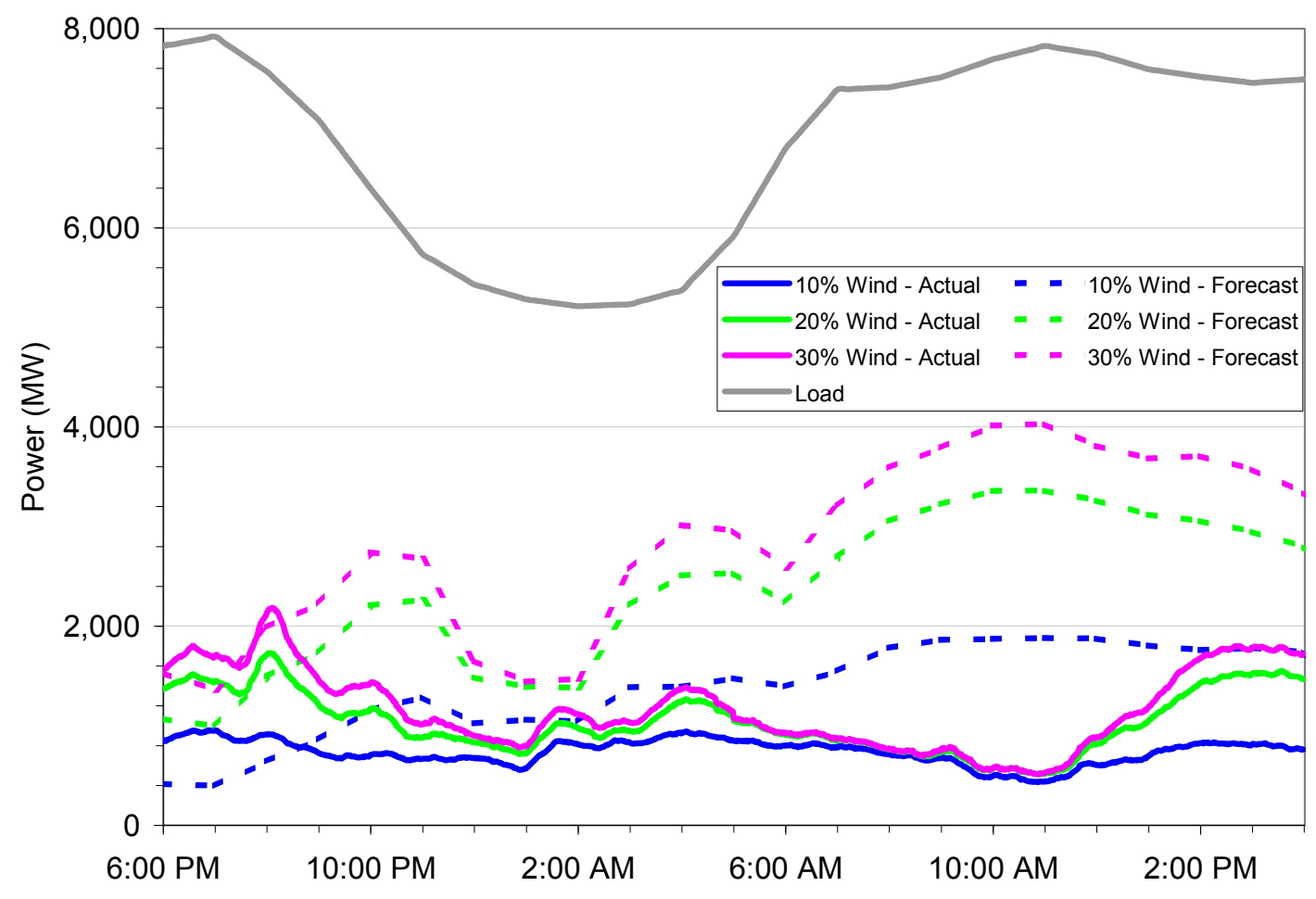

Figure 7.26 Colorado-East Input Profiles, Local-Priority, October QSS Study Period. 


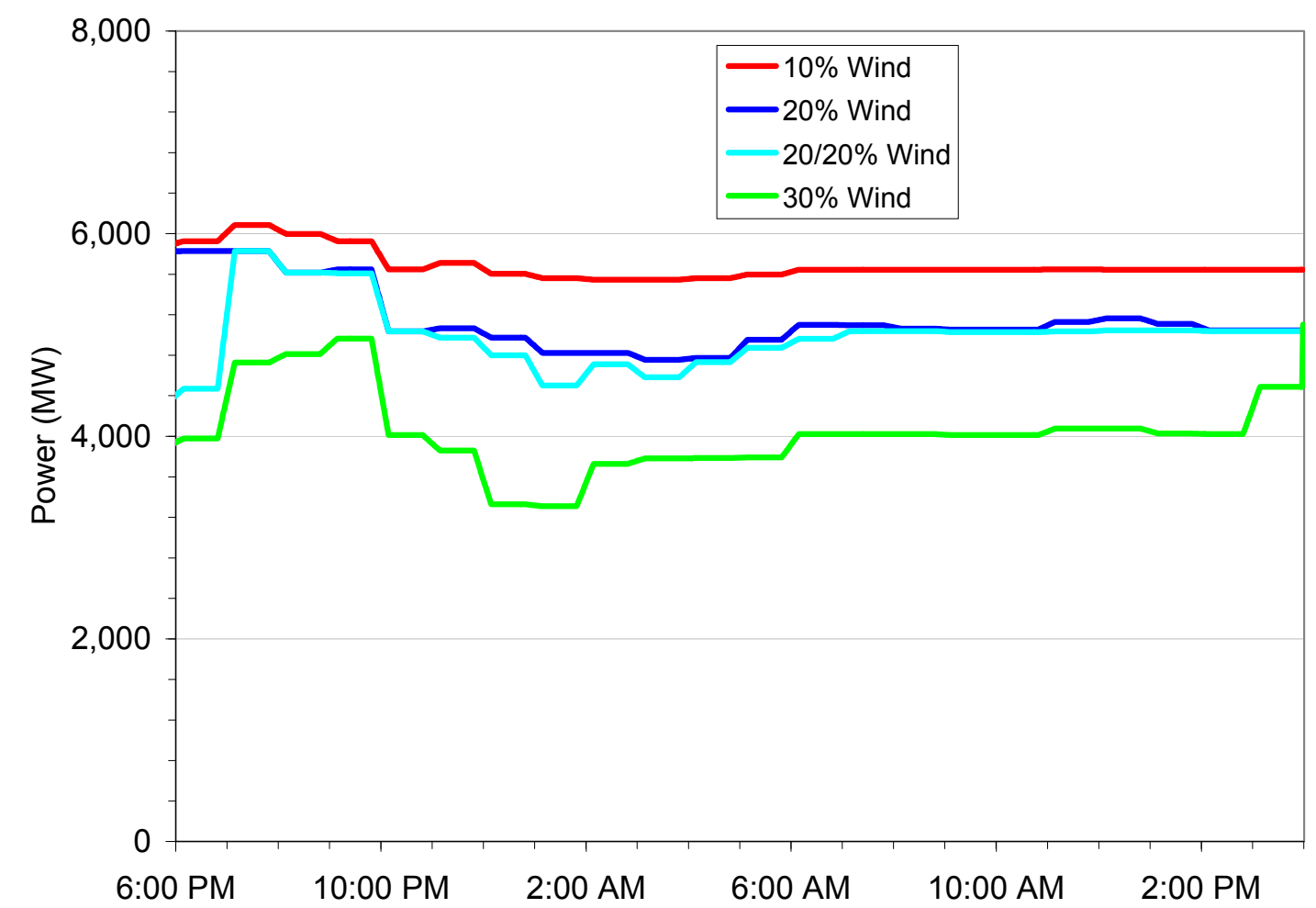

Figure 7.27 Colorado-East Conventional Generation Hourly Schedule, Local-Priority, October QSS Study Period.

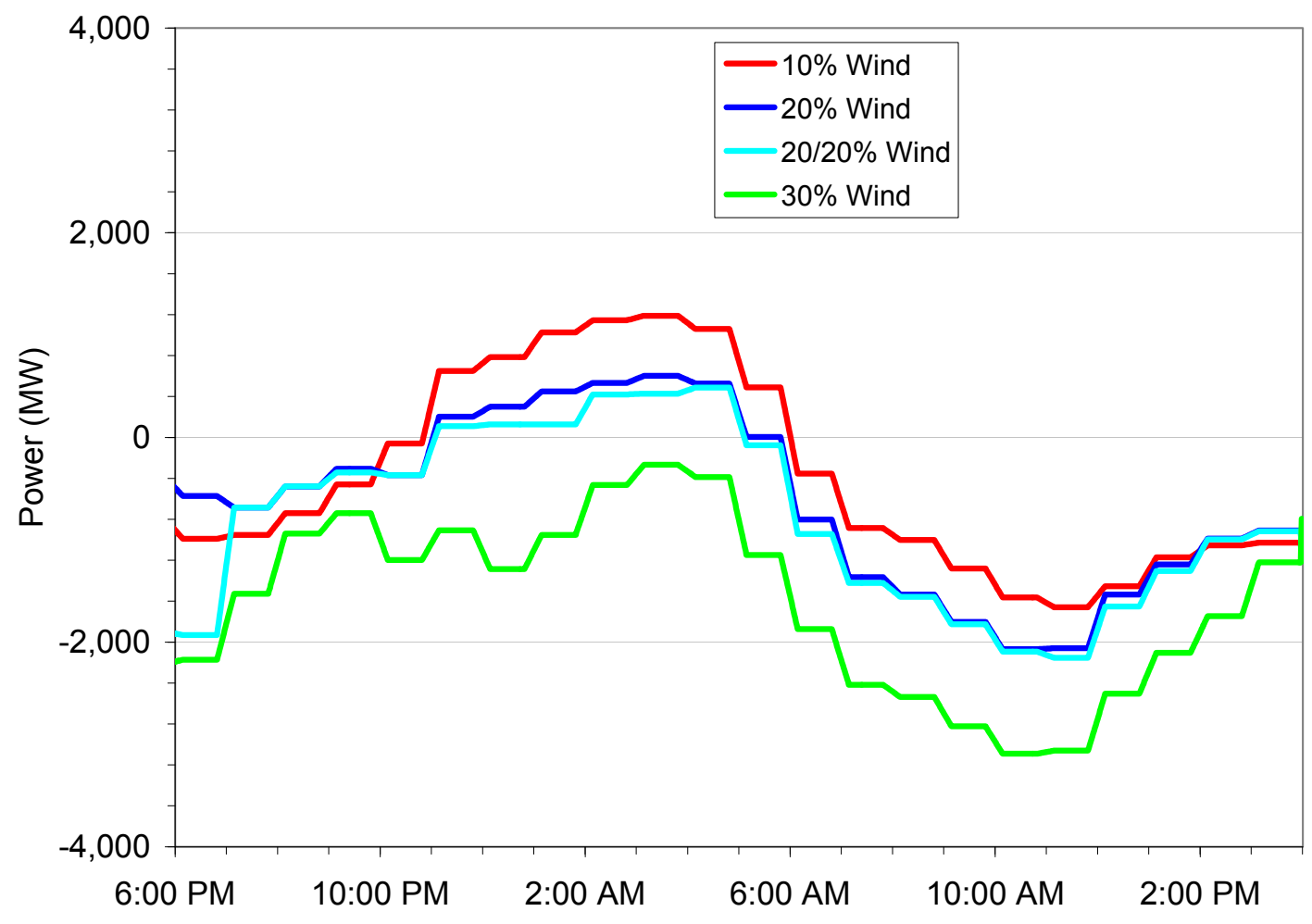

Figure 7.28 Colorado-East Export Schedule, Local-Priority, October QSS Study Period. 
Table 7.8 Economic Dispatch Priority for Colorado-East during October QSS Study Period.

\begin{tabular}{|l|c|c|c|c|}
\cline { 2 - 5 } \multicolumn{1}{c|}{} & $\mathbf{1 0} \%$ Wind & $\mathbf{2 0} \%$ Wind & $\mathbf{2 0 / 2 0} \%$ Wind & $\mathbf{3 0} \%$ Wind \\
\hline Hydro & 2 & 2 & 3 & 3 \\
Combined-Cycle & 1 & 1 & 1 & 1 \\
Gas Turbine & 4 & 4 & 4 & 4 \\
Steam & 3 & 3 & 2 & 2 \\
\hline
\end{tabular}

Table 7.9 Regulation Priority and Capability for Colorado-East during October QSS Study Period.

\begin{tabular}{|c|c|c|c|c|c|c|c|c|c|c|c|c|}
\hline & \multicolumn{3}{|c|}{$10 \%$ Wind } & \multicolumn{3}{|c|}{$20 \%$ Wind } & \multicolumn{3}{|c|}{$20 / 20 \%$ Wind } & \multicolumn{3}{|c|}{$30 \%$ Wind } \\
\hline & Priority & $+M W$ & $-M W$ & Priority & $+M W$ & $-M W$ & Priority & $+M W$ & $-M W$ & Priority & $+M W$ & $-M W$ \\
\hline Hydro & 2 & 33 & -12 & 2 & 25 & -2 & 3 & 7 & -10 & 3 & 8 & -1 \\
\hline $\begin{array}{l}\text { Combined- } \\
\text { Cycle }\end{array}$ & 1 & 82 & -75 & 1 & 91 & -84 & 1 & 105 & -54 & 1 & 96 & 52 \\
\hline Gas Turbine & 4 & 0 & 0 & 4 & 0 & 0 & 4 & 0 & 0 & 4 & 0 & 0 \\
\hline Steam & 3 & 0 & -29 & 3 & 0 & -30 & 2 & 5 & -60 & 2 & 12 & -63 \\
\hline
\end{tabular}

The results of the October QSS analysis with the above input data and boundary conditions are presented in Figure 7.29 through Figure 7.45.

Figure 7.29 - Figure 7.32 show the Colorado-East dispatch for the Local-Priority Scenario with $10 \%, 20 \%, 20 / 20 \%$, and 30\% wind penetration, respectively. As shown in Table 7.8, the economic dispatch priority for this case is combined-cycle (green), hydro (dark blue), steam (light blue), and gas turbine (pink). The heavyweight lines represent the unit type power output, and the lightweight lines represent the maxima and minima of the committed units. Changes in commitment can be observed in the changes to the maxima and minima.

Over the course of this simulation, the top three unit types (e.g., combined-cycle, steam, and hydro) respond to the economic dispatch system and follow load down and then up again. The bulk of the load following is performed by the combined-cycle units. During the morning load rise, all units spend a significant amount of time at their maxima.

As penetration increases, the combined-cycle commitment was significantly reduced. This is consistent with the results reported in Chapter 6, which showed that wind generation displaces the conventional generation with the highest marginal cost. In addition, the economic dispatch priority order changed. As the combined-cycle generation was reduced, the steam generation moved up from third to second priority for the $20 / 20 \%$ wind penetration case. 


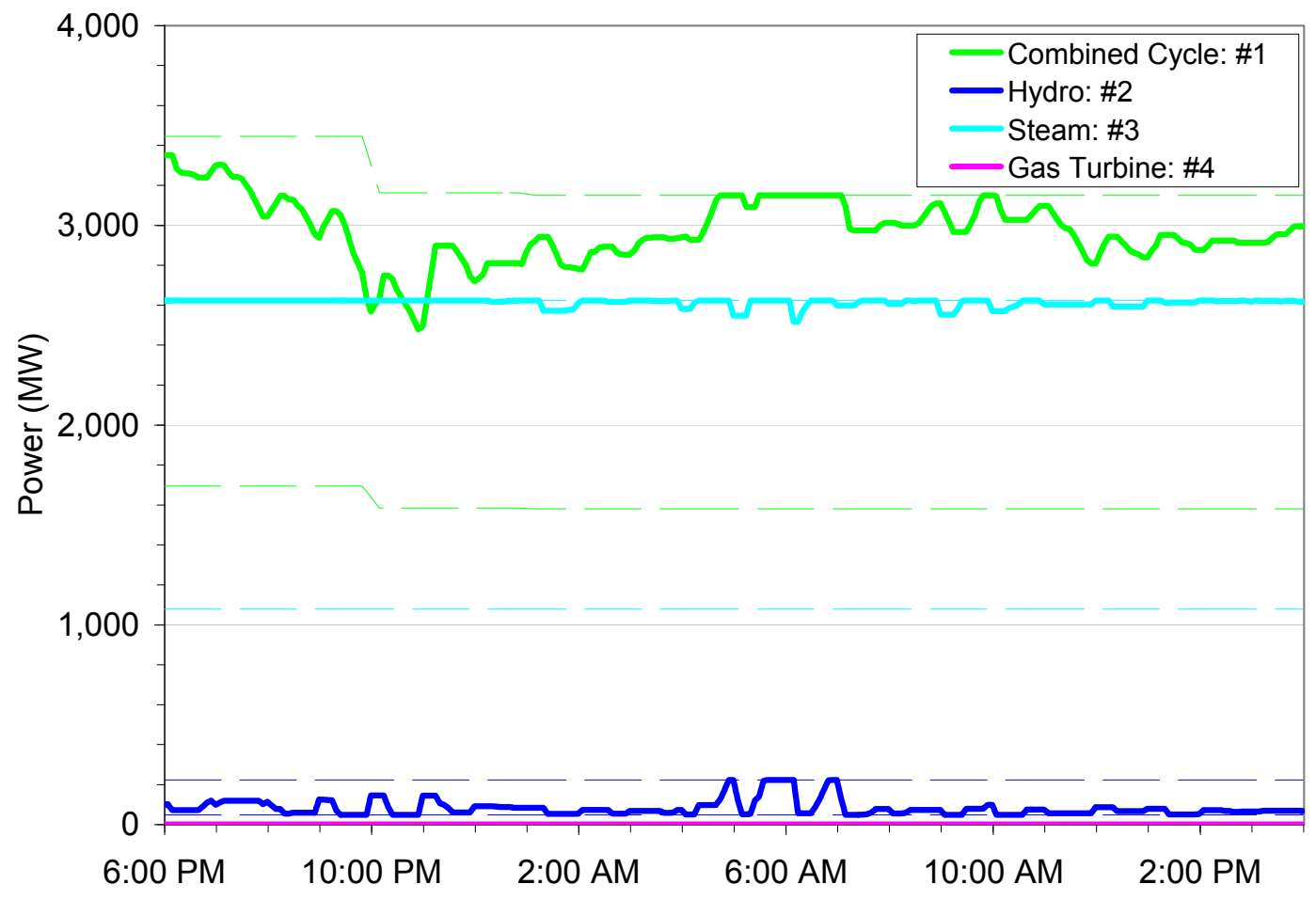

Figure 7.29 Colorado-East Dispatch, 10\% Wind, Local-Priority, October QSS Study Period.

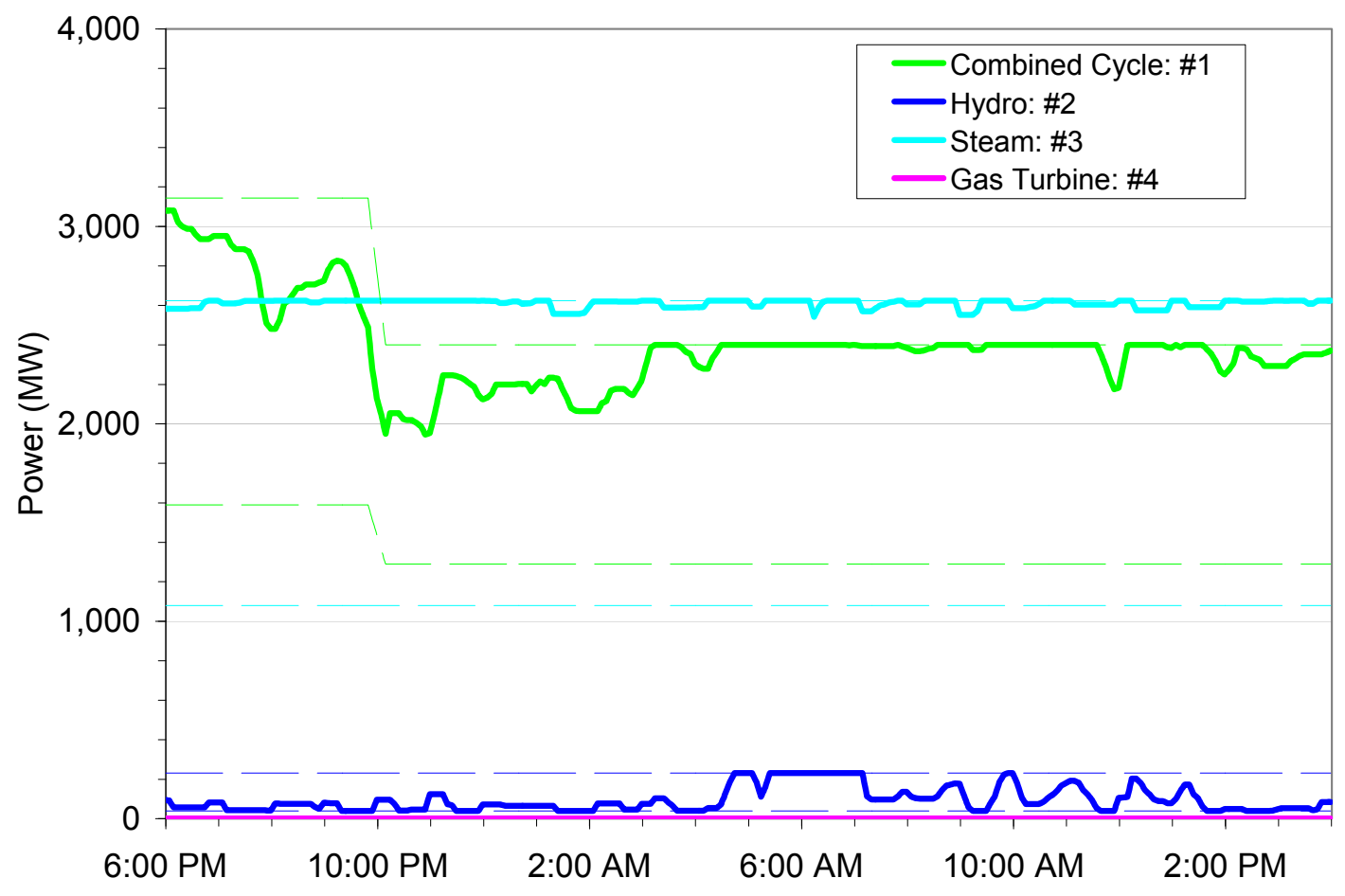

Figure 7.30 Colorado-East Dispatch, 20\% Wind, Local-Priority, October QSS Study Period. 


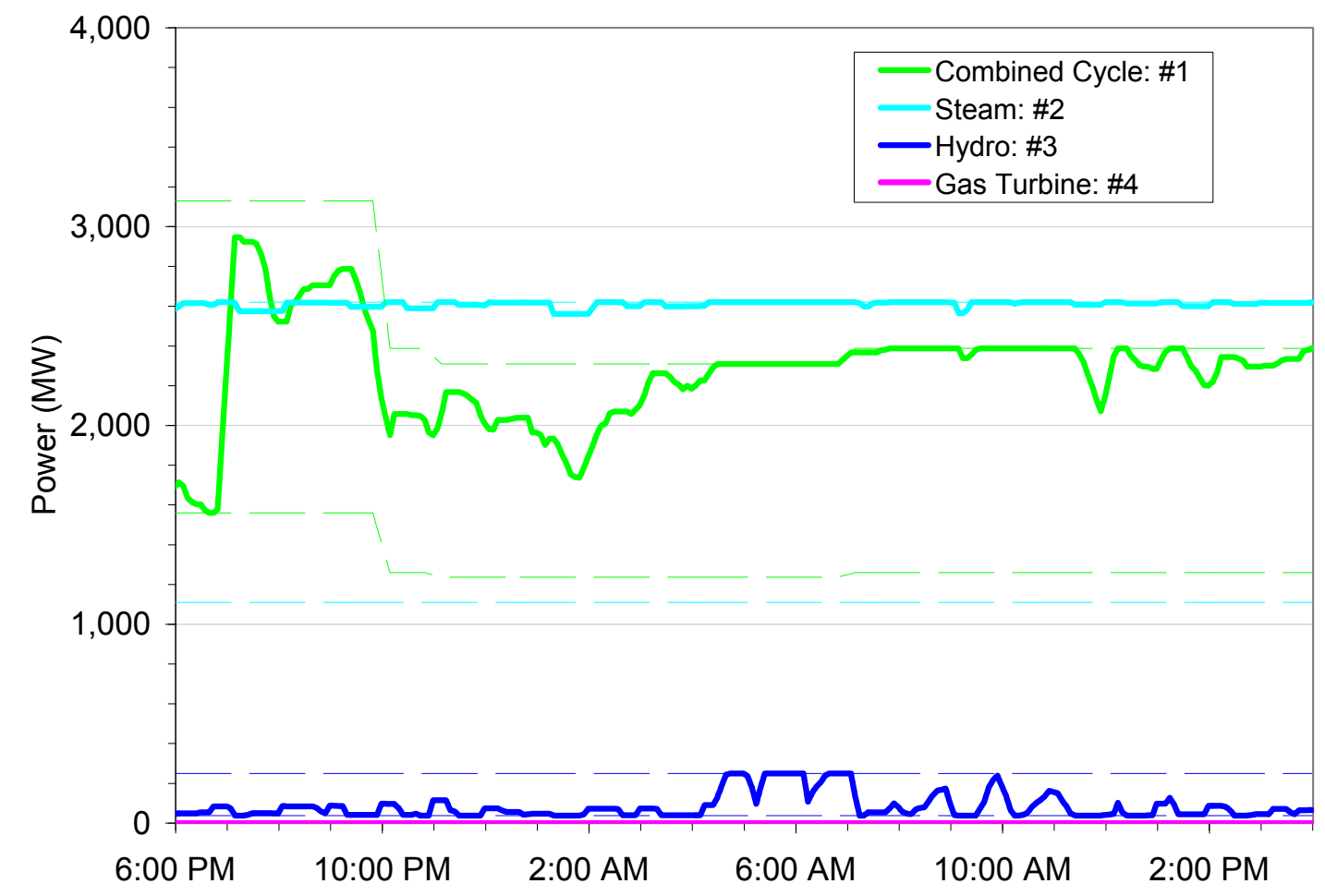

Figure 7.31. Colorado-East Dispatch, 20/20\% Wind, Local-Priority, October QSS Study Period.

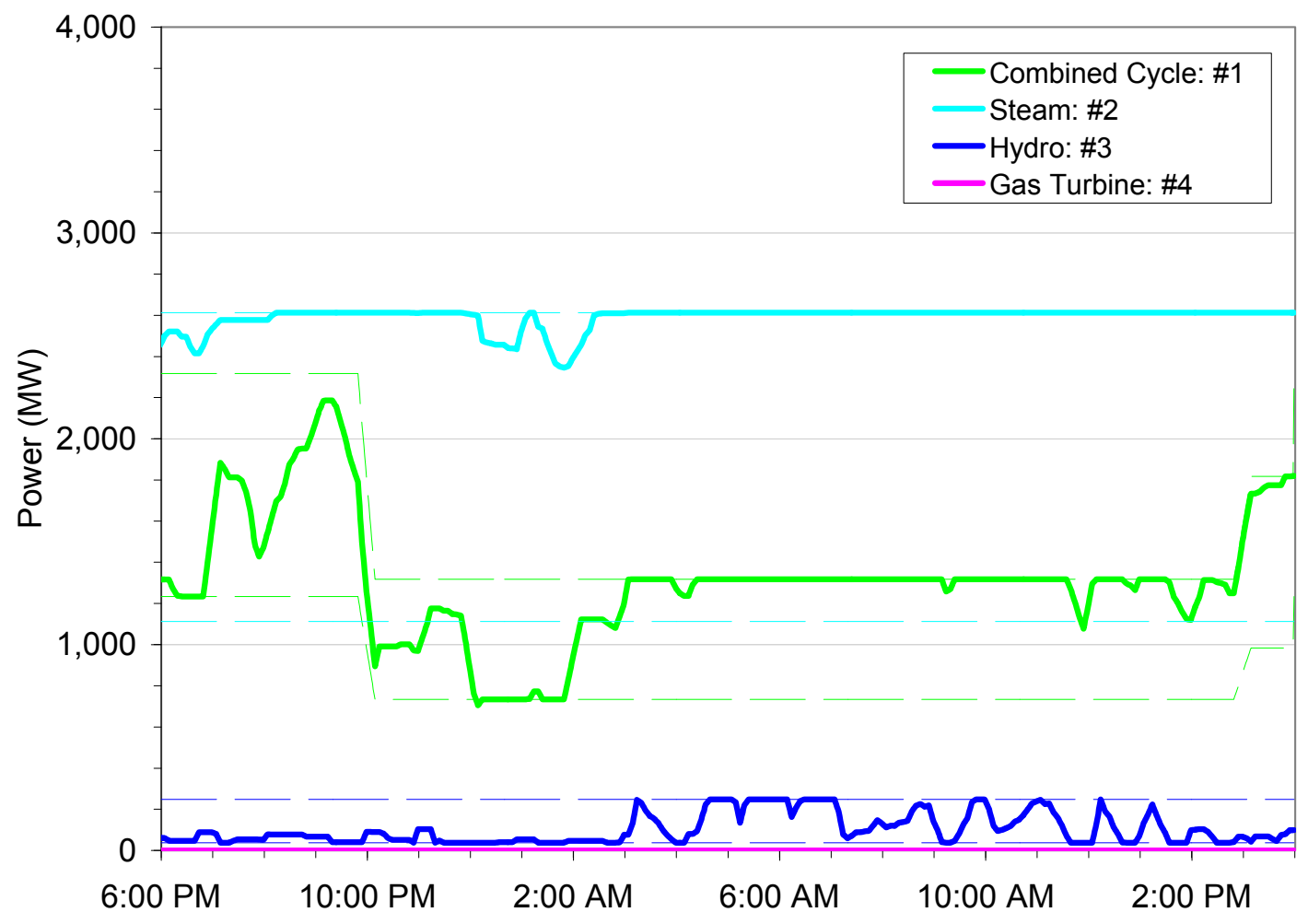

Figure 7.32 Colorado-East Dispatch, 30\% Wind, Local-Priority, October QSS Study Period. 
The amount of regulation provided for each penetration level is shown in Figure 7.33 Figure 7.36. In each figure, regulation is the dark blue line. The conventional generation hourly schedule (pink) and the hourly export schedule (gold) are also shown. The right vertical scale applies to regulation; the left vertical scale applies to the hourly schedules.

As shown in Table 7.4,+/-116 MW of regulation capability is available in Colorado-East during the simulation. For all penetration levels, the regulation that is actually provided is largely driven by the hourly schedule changes, not by the wind and solar variability. As the penetration increases, the schedule changes tend to increase as more flexibility is required of fewer units. In general, when the provided regulation hits a capability limit (i.e., +/-116 MW), it is due to an hourly schedule change.

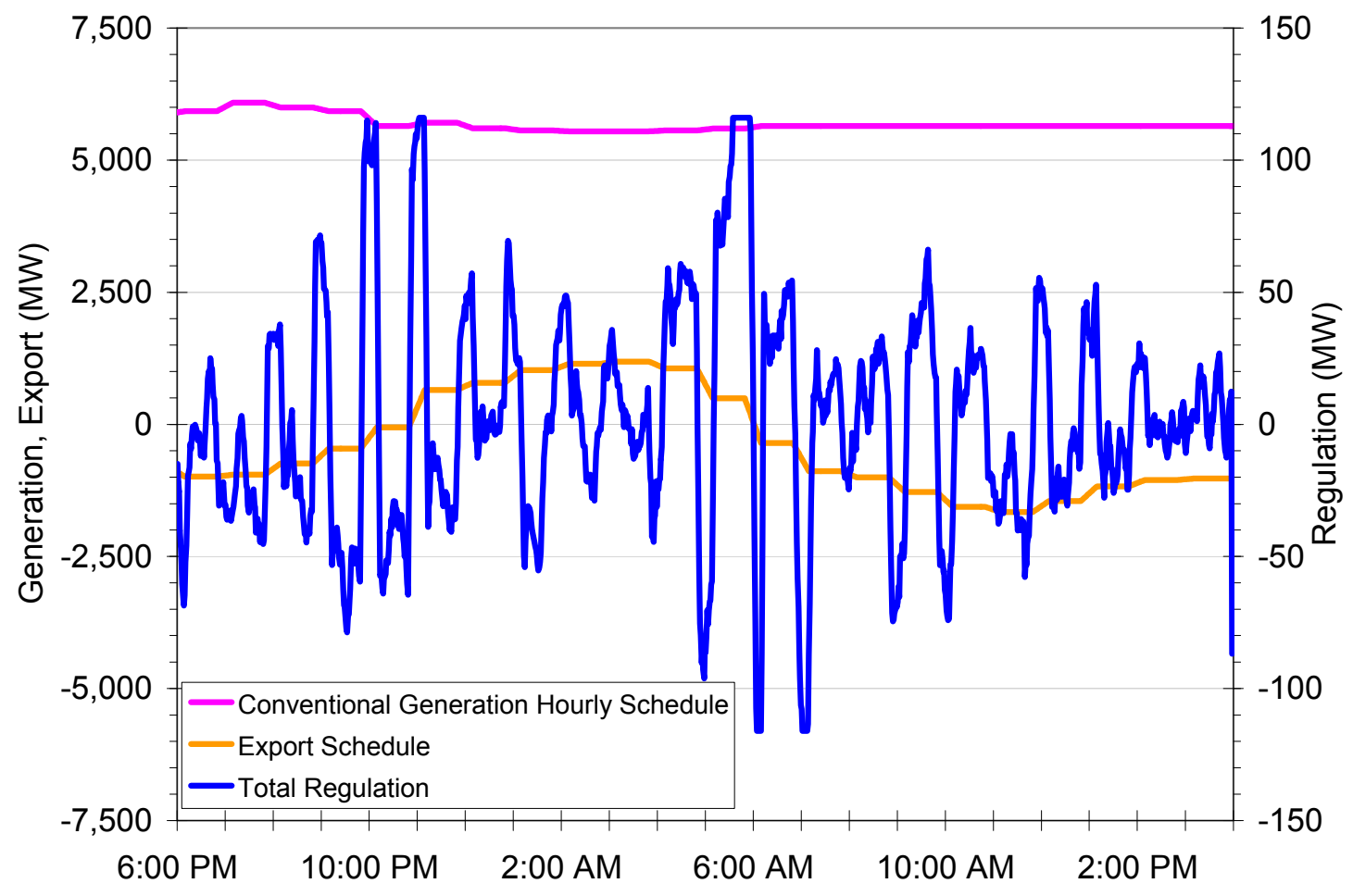

Figure 7.33 Colorado-East Regulation, 10\% Wind, Local-Priority, October QSS Study Period. 


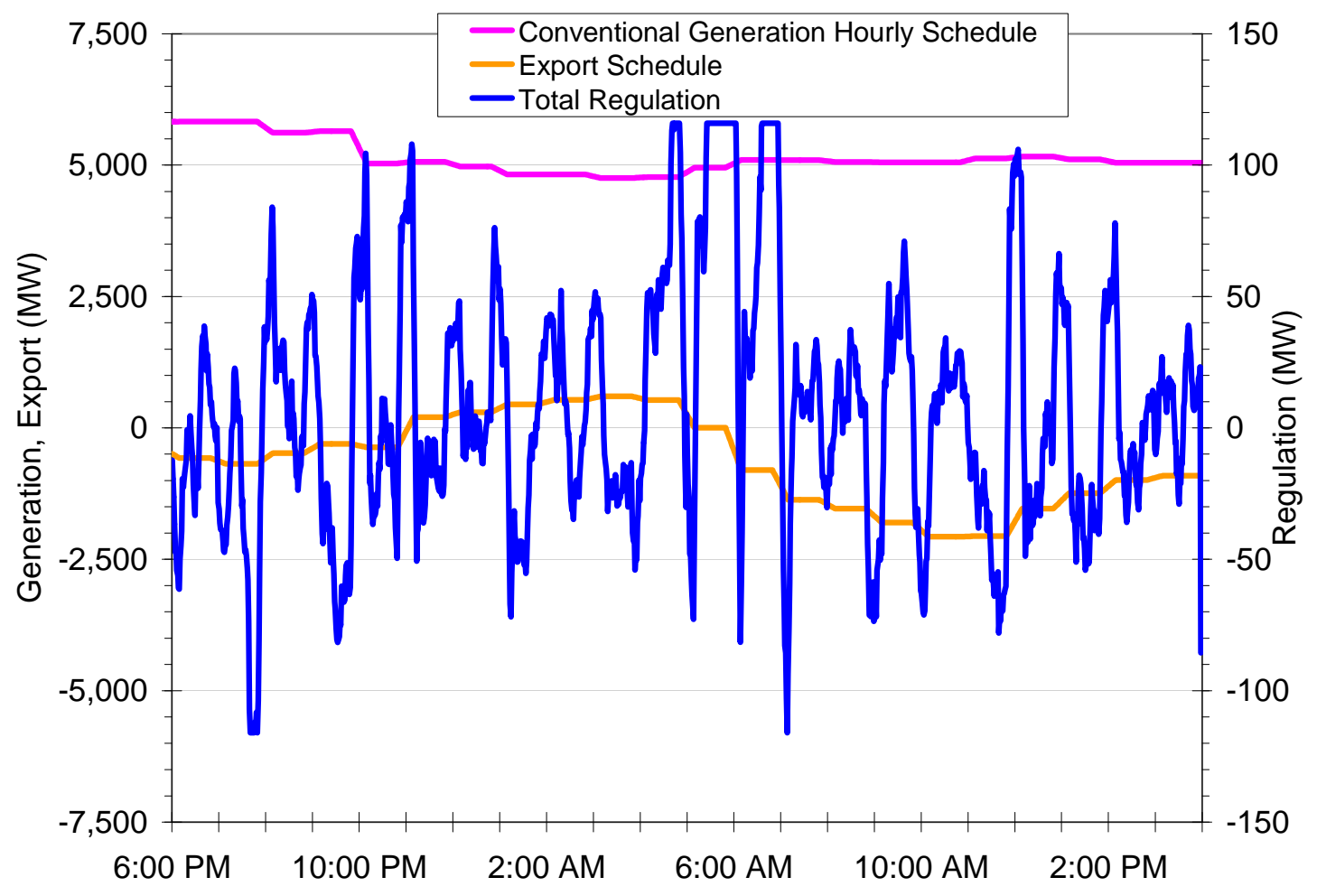

Figure 7.34 Colorado-East Regulation, 20\% Wind, Local-Priority, October QSS Study Period.

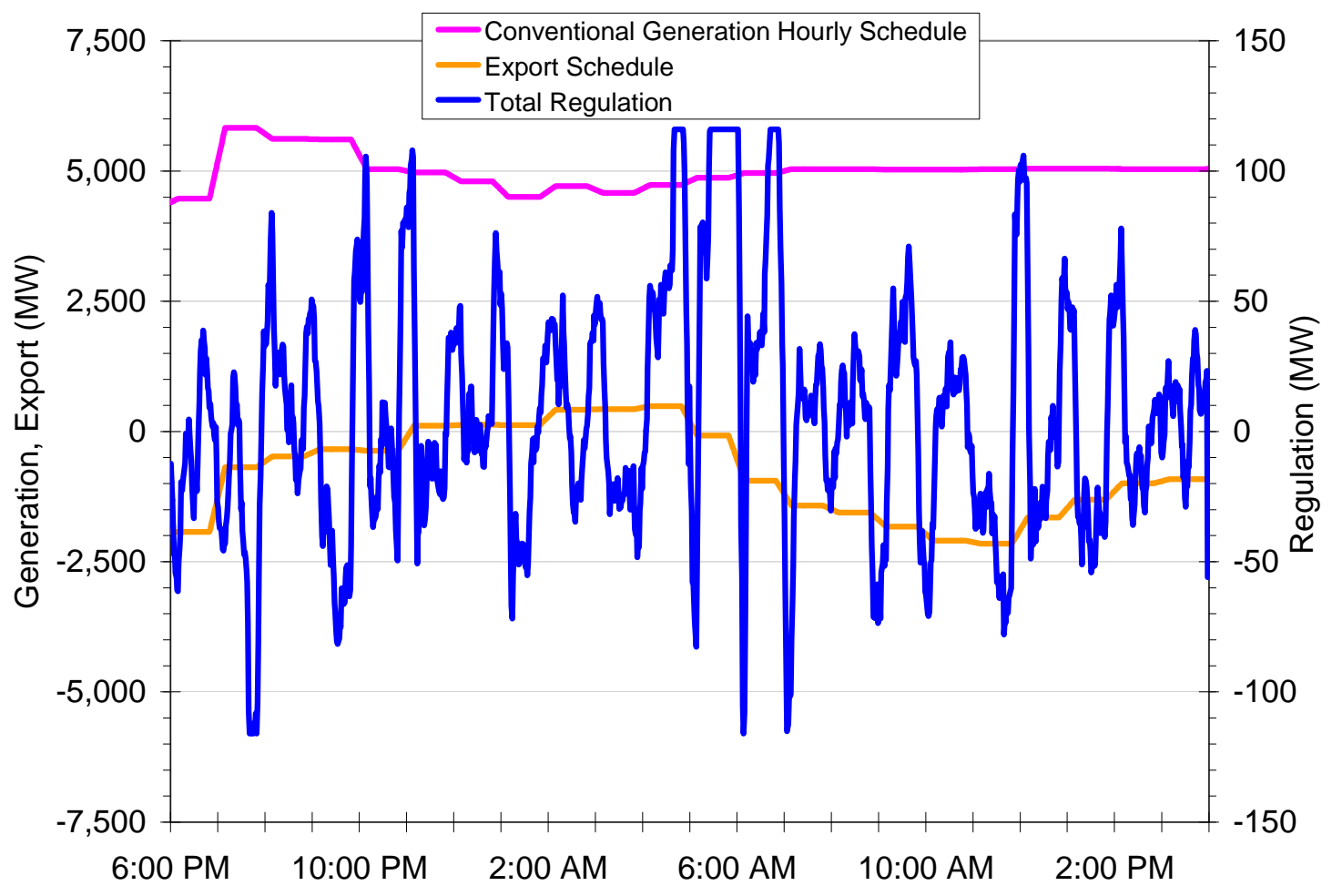

Figure 7.35. Colorado-East Regulation, 20/20\% Wind, Local-Priority, October QSS Study Period. 


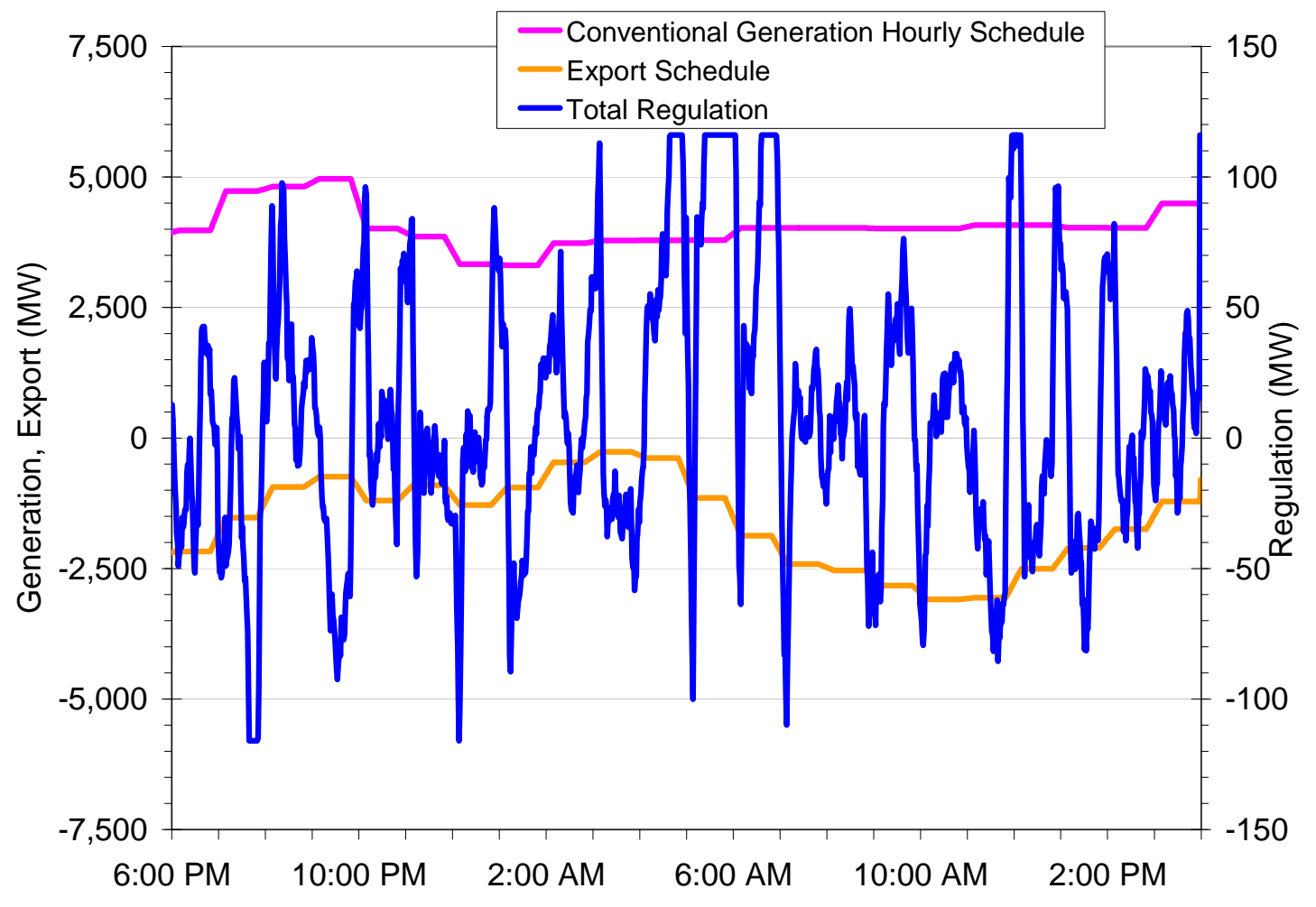

Figure 7.36 Colorado-East Regulation, 30\% Wind, Local-Priority, October QSS Study Period.

The number of minutes that the Colorado-East regulation is at its maximum or minimum, for each level of wind penetration, is shown in Table 7.10. The overall trend is for the amount of time at the maximum or minimum to increase with penetration.

Table 7.10. Colorado-East Regulation at Capability Limits.

\begin{tabular}{|l|c|c|}
\cline { 2 - 3 } \multicolumn{1}{c|}{} & $\begin{array}{c}\text { Minutes } \\
\text { at Maximum }\end{array}$ & $\begin{array}{c}\text { Minutes } \\
\text { at Minimum }\end{array}$ \\
\hline $10 \%$ Wind & 34 & 13 \\
$20 \%$ Wind & 73 & 8 \\
$20 / 20 \%$ Wind & 64 & 8 \\
$30 \%$ Wind & 88 & 12 \\
\hline
\end{tabular}

Some of these events result in ACEs, as shown in Figure 7.37. This figure shows the Colorado-East ACE for the Local-Priority Scenario with various levels of wind penetration. The red line represents $10 \%$ wind penetration, the green line represents $20 \%$ wind, the light blue line represents $20 / 20 \%$ wind, the dark blue line represents $30 \%$ wind, and the black lines represent the approximate L10 limits. These L10 values were used to identify potential CPS2 violations. 
A positive ACE signal means that conventional generation exceeds net load, and the actual export will exceed the export schedule. A negative ACE signal means that net load exceeds generation, and the actual export will fail to meet the export schedule. The two black lines represent the approximate L10 limits (Table 7.5) that indicate a potential CPS2 violation. As with the regulation itself, the ACE is largely driven by the hourly generation and export schedule changes, not by the wind and solar variability.

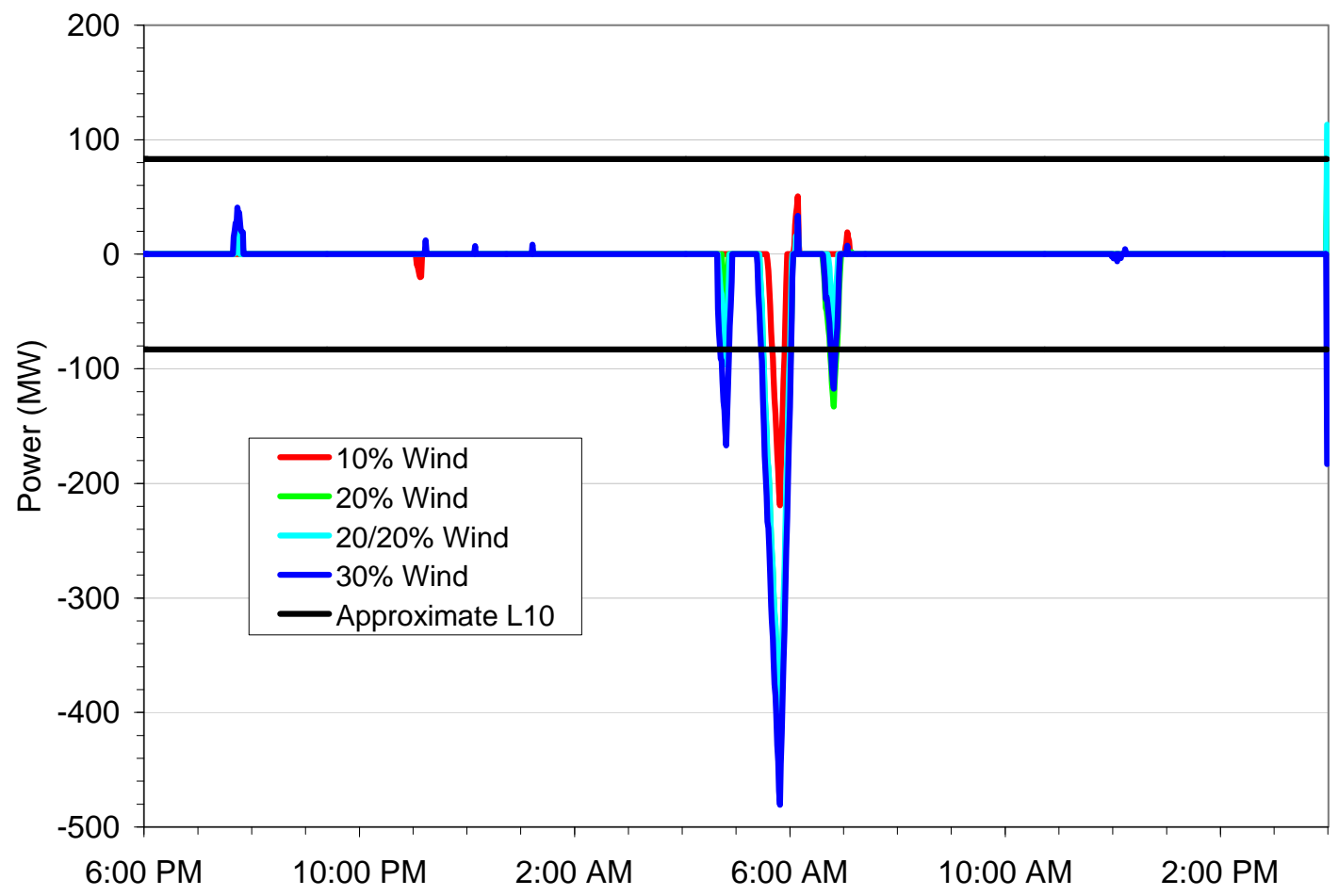

Figure 7.37 Colorado-East ACEs, All Penetration Levels, Local-Priority, October QSS Study Period.

The impact of an imperfect wind forecast and the hourly schedule on both the duty imposed on the economic dispatch units and the required regulation was also investigated.

Two additional QSS simulations were performed, both with $30 \%$ wind penetration and the Local-Priority Scenario. The first QSS simulation implemented the hourly generation and export schedules using a +/-30-minute ramp around the hour. These smoother schedules approximate a continuous schedule. Note that discontinuities remain at 30 minutes after the hour.

All of the QSS simulations described above used the MAPS boundary conditions for cases with the state of the art (S-o-A) forecast (e.g., L30R). The second, exploratory QSS simulation used the MAPS boundary conditions from the perfect forecast, or L30P, production simulation case of Chapter 6. 
Figure 7.38 shows the Colorado-East dispatch with the continuous generation and export schedules, and 30\% wind penetration. In comparison to Figure 7.32, the continuous schedule requires less movement from the economic dispatch units.

Figure 7.39 shows the Colorado-East regulation with a continuous schedule, and $30 \%$ wind penetration. In comparison to Figure 7.36, the regulation swings on the hour and the average regulation are significantly reduced. The extension of the hourly schedule ramp, however, does not eliminate the exhaustion of regulation capability at about 6 a.m. A more sophisticated schedule algorithm would be required to address that.

Figure 7.40 shows the Colorado-East dispatch with the perfect forecast, and 30\% wind penetration. Since the actual wind was much less than the forecasted wind, the perfect forecast resulted in more combined-cycle units committed across the board in comparison to Figure 7.32.

Figure 7.41 shows the Colorado-East regulation with the perfect forecast, and $30 \%$ wind penetration. In comparison to Figure 7.36, the regulation swings on the hour are about the same-both simulations include an hourly schedule. However, the number of minutes that the Colorado-East regulation is at its maximum or minimum is significantly reduced.

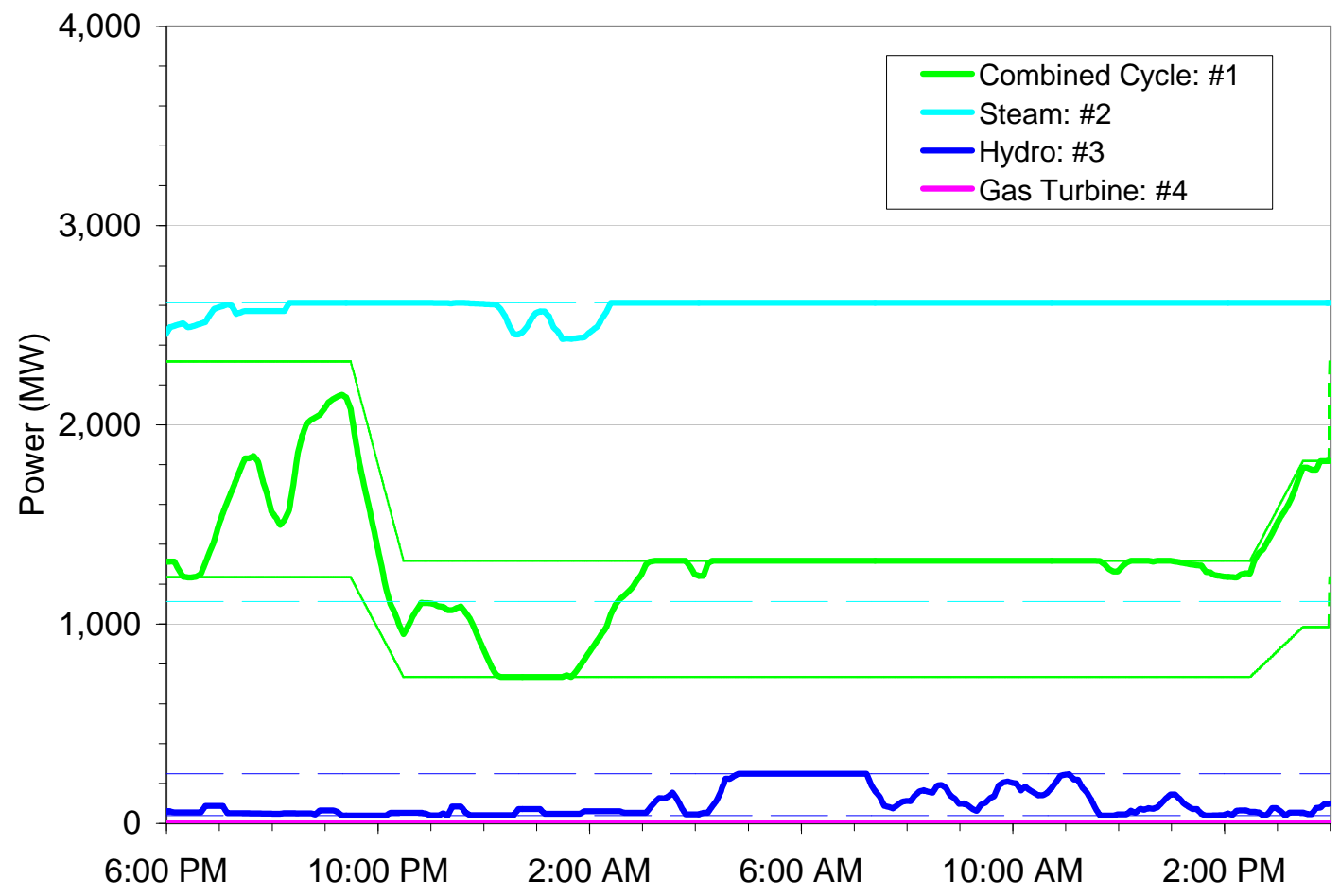

Figure 7.38 Colorado-East Dispatch, Continuous Schedule, 30\% Wind, Local-Priority, October QSS Study Period. 


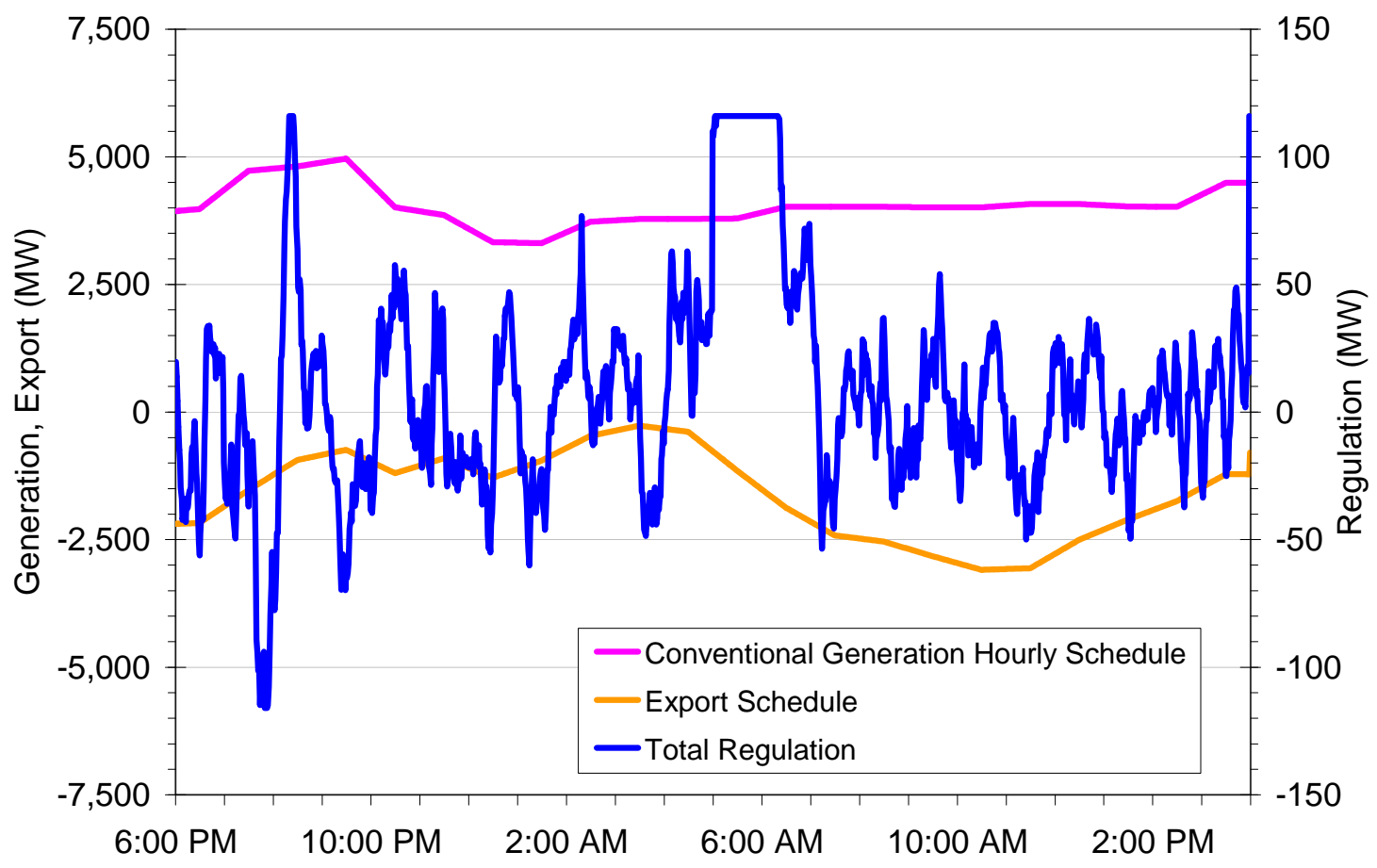

Figure 7.39 Colorado-East Regulation, Continuous Schedule, 30\% Wind, Local-Priority, October QSS Study Period.

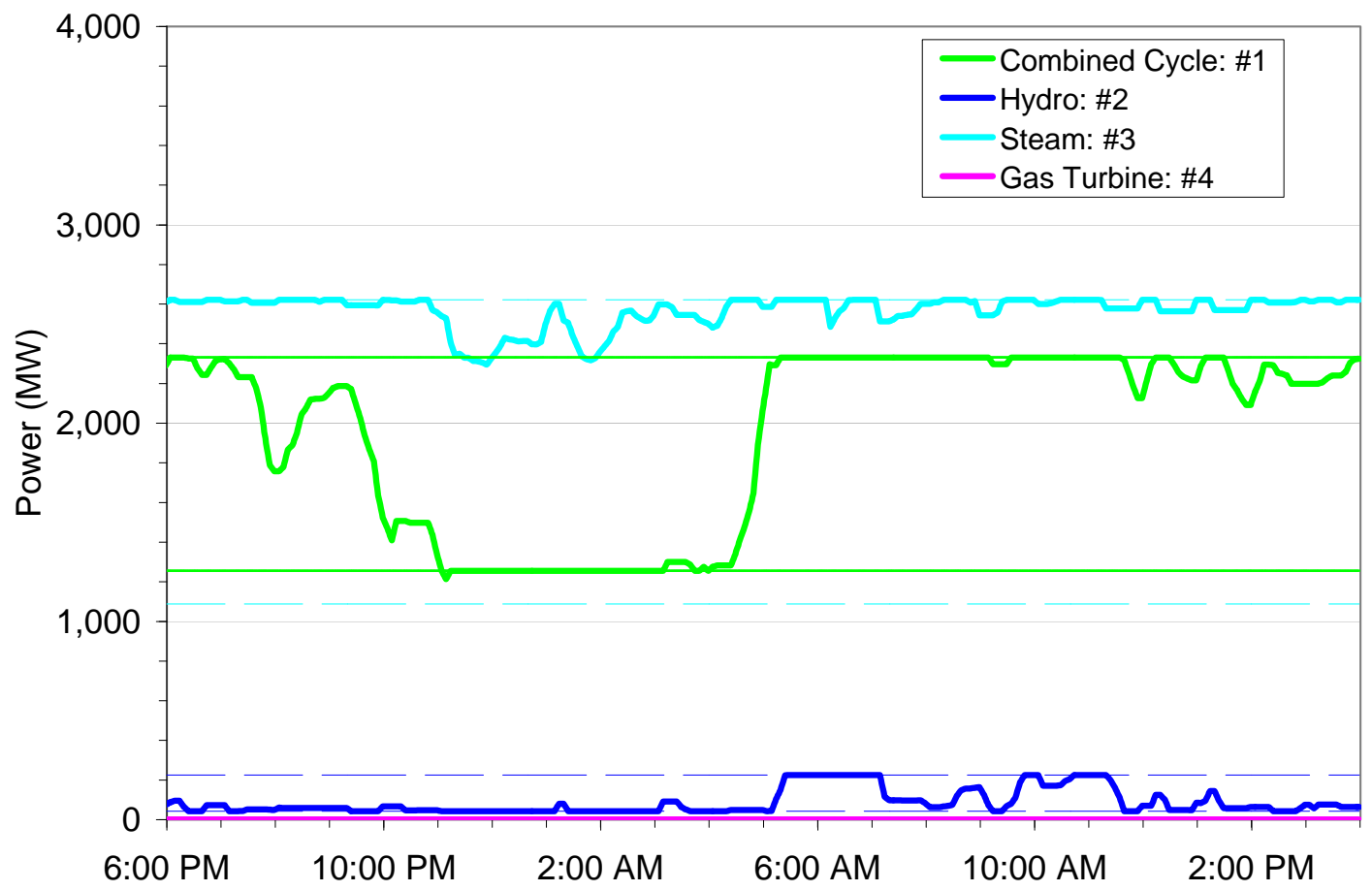

Figure 7.40. Colorado-East Dispatch, Perfect Forecast, 30\% Wind, Local-Priority, October QSS Study Period. 


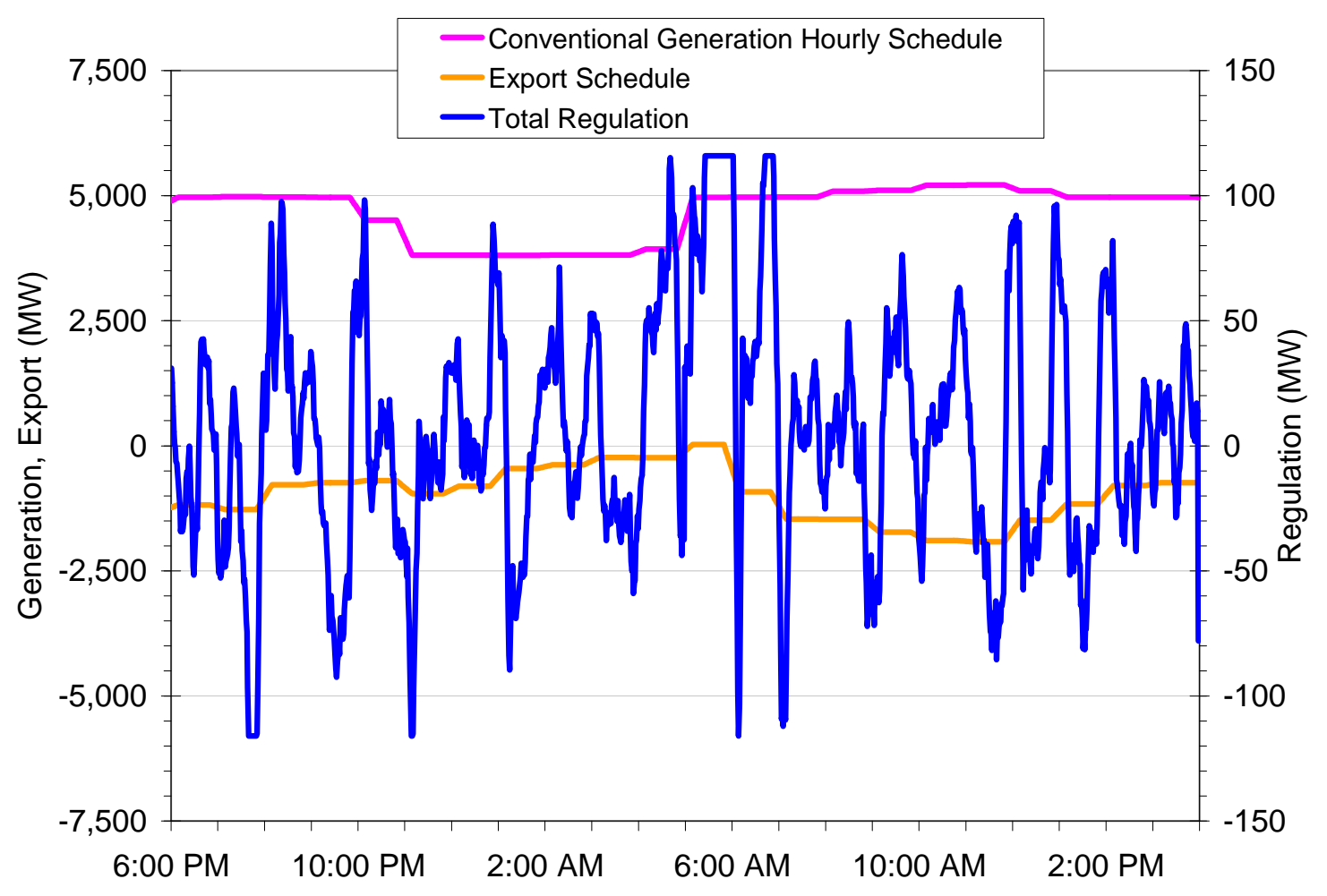

Figure 7.41. Colorado-East Regulation, Perfect Forecast, 30\% Wind, Local-Priority, October QSS Study Period.

The duty applied to the economic dispatch units under various scenarios was also explored. Figure 7.42 shows the combined-cycle unit output (dark blue line) on economic dispatch for the $30 \%$ wind penetration, Local-Priority Scenario with the S-o-A forecast. The red line represents a 1-hour rolling average of the combined-cycle generation. The difference between the actual output and the rolling average output is represented by the green line. This difference is a measure of the amount of the subhourly duty, MW, applied to the combined-cycle units. However, the same sub-hourly duty applied to a lot of units is less challenging than when it is applied to a few units. In this simulation, the combined-cycle commitment decreased by about 1,000 MW between 6 p.m. and midnight, but the sub-hourly MW duty remained about the same. Therefore, this duty is per unitized on the committed range (i.e., maximum output minus minimum output) for the subsequent figures.

Figure 7.43 shows this per unit usage metric for all penetration levels, with the S-o-A forecast. The dark blue line represents $10 \%$ wind, the red line represents $20 \%$ wind, the light blue line represents $20 / 20 \%$ wind, and the green line represents $30 \%$ wind. As penetration increases, the duty required of the BOP generation increases.

Figure 7.44 shows this per unit usage metric for the two sensitivity cases, as well as the original 30\% wind penetration case. The green line represents the original 30\% wind penetration case with the S-o-A forecast and hourly schedule changes. The dark blue 
line represents the sensitivity case with the perfect forecast, and the red line represents the sensitivity case with the continuous schedule. Both sensitivity cases apply less duty to the economic dispatch units.

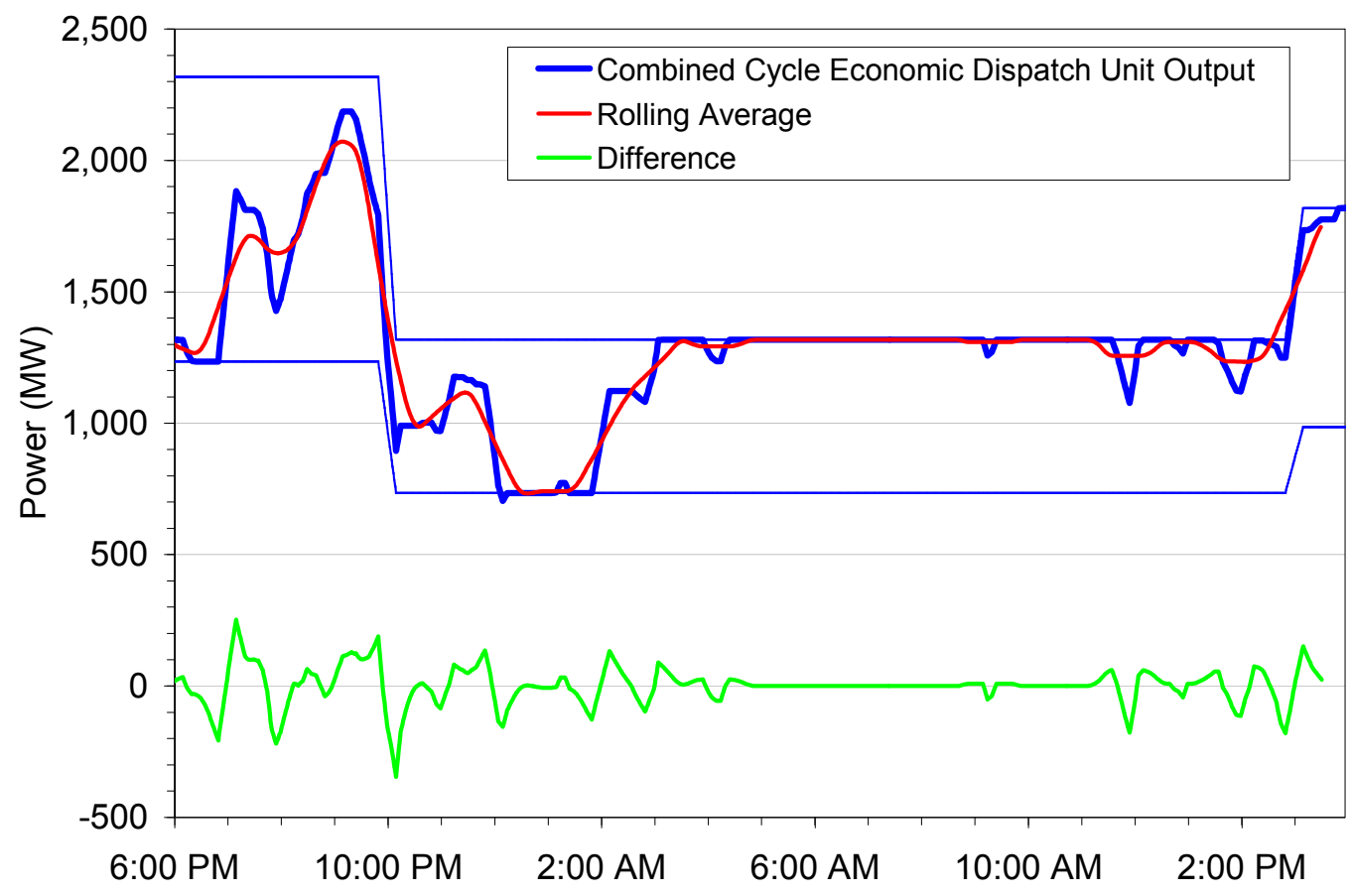

Figure 7.42. Colorado-East Combined-Cycle Usage, 30\% Wind, Local-Priority, October QSS Study Period. 


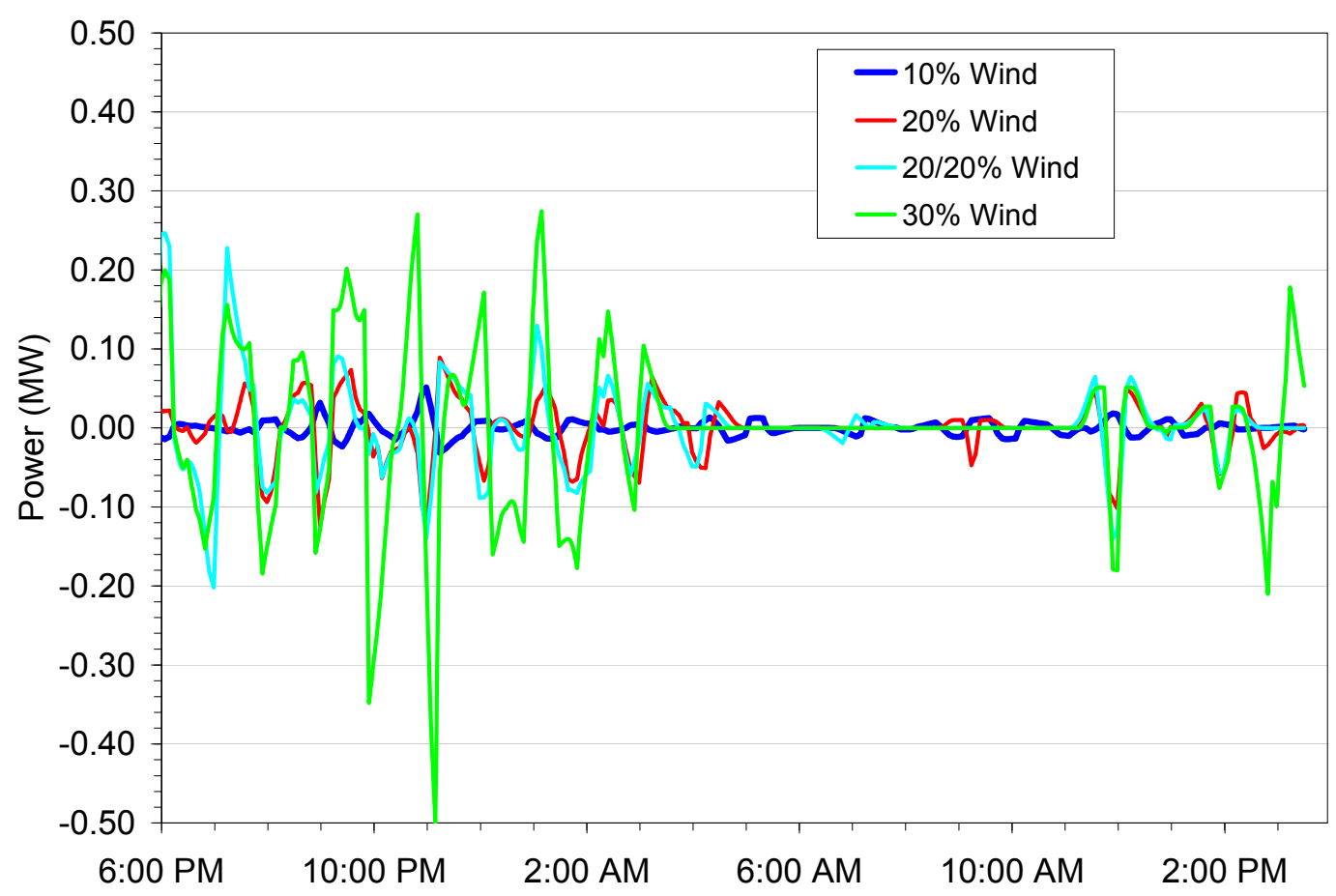

Figure 7.43 Colorado-East Combined-Cycle Usage, All Penetration Levels, Local-Priority, October QSS Study Period.

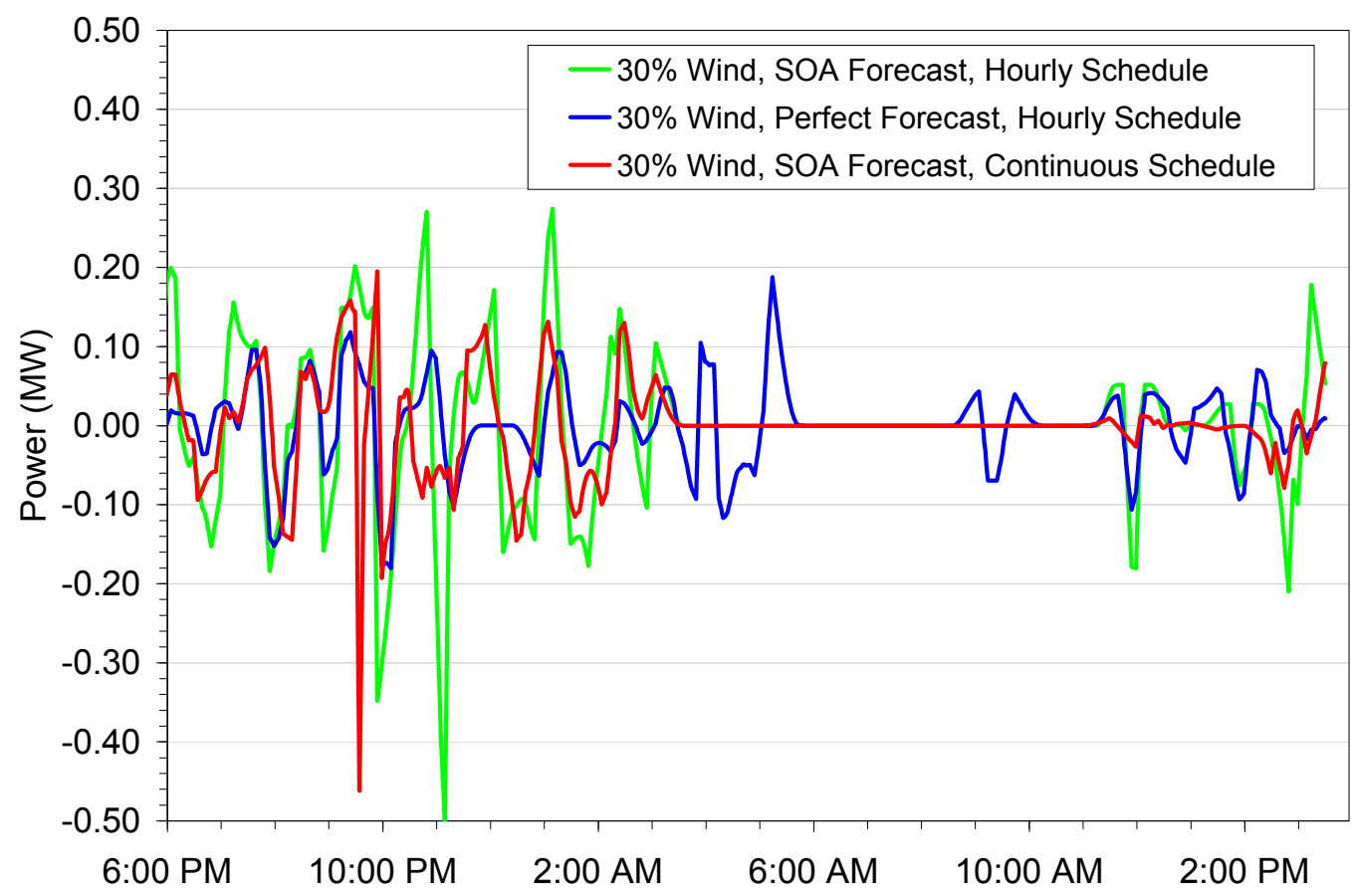

Figure 7.44. Colorado-East Combined-Cycle Usage, Forecast and Schedule Variations, 30\% Wind, LocalPriority, October QSS Study Period.

The root-mean-square (RMS) of this combined-cycle usage index was calculated for each example case and is shown in Table 7.11. This usage index is also plotted in Figure 7.45. 
The combined-cycle usage index at 30\% wind penetration with either a continuous schedule or perfect forecast is about one-half that of $30 \%$ wind penetration with an hourly schedule and S-o-A forecast. In other words, the usage index for 30\% penetration has been reduced to about the same as for $20 / 20 \%$ penetration.

Table 7.11. Combined-Cycle RMS Usage Index.

\begin{tabular}{|l|c|}
\cline { 2 - 2 } \multicolumn{1}{c|}{} & $\begin{array}{c}\text { Usage } \\
\text { (pu, RMS) }\end{array}$ \\
\hline $10 \%$ Wind, S-o-A Forecast, Hourly Schedule & 0.03 \\
$20 \%$ Wind, S-o-A Forecast, Hourly Schedule & 0.037 \\
$20 / 20 \%$ Wind, S-o-A Forecast, Hourly Schedule & 0.055 \\
$30 \%$ Wind, S-o-A Forecast, Hourly Schedule & 0.095 \\
$30 \%$ Wind, S-o-A Forecast, Continuous Schedule & 0.051 \\
$30 \%$ Wind, Perfect Forecast, Hourly Schedule & 0.049 \\
\hline
\end{tabular}

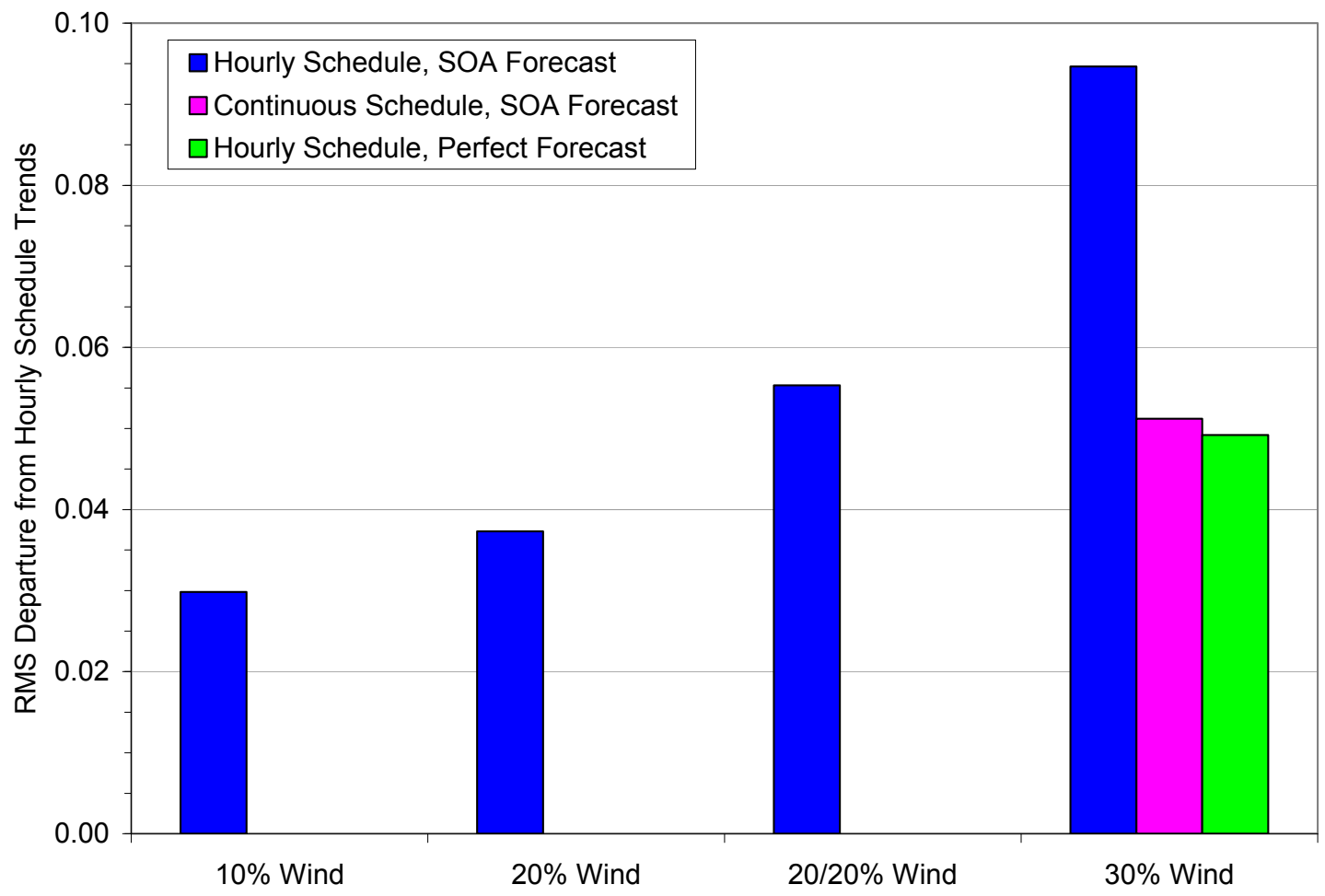

Figure 7.45. Colorado-East Combined-Cycle RMS Usage Index, Forecast and Schedule Variations, $30 \%$ Wind, Local-Priority, October QSS Study Period.

This time period was chosen because of the high wind forecast error at mid morning. However, as observed above, the challenging period of operation turned out to be several hours earlier in the morning load rise. 


\subsubsection{Mitigation}

The QSS simulations showed varying ACE levels, some of which exceeded the approximate L10 limits and indicated a potential CPS2 violation. Mitigation of the ACE could be achieved by a combination of wind energy curtailment and load participation (or demand side management, DSM). Wind curtailment can be an effective response to a positive ACE signal, which occurs when generation is greater than load and results in the actual export exceeding the scheduled export. Curtailment of wind generation in response to an AGC command makes wind generation a contributor to system down reserve. Load participation addresses a negative ACE signal, which occurs when load exceeds generation and results in the actual export failing to meet the export schedule.

The amount of wind curtailment required to reduce the positive Arizona ACE to zero was calculated for the April QSS cases with 10\%, 20\%, 20/20\%, and 30\% wind penetration (Local-Priority Scenario, S-o-A wind forecast, hourly schedule changes). This defines the maximum wind curtailment for the given study period. It was calculated as the energy, in MWh, under the positive ACE signal. In addition, the maximum ramp down required was calculated as the largest 1-minute change in ACE. The maximum ramp down capability of the wind plants was assumed to be $10 \% /$ minute on the MW rating. These results are summarized in Table 7.12.

The wind curtailment for this single worst event in 3 years of data constituted about $0.01 \%$ of Arizona's annual wind energy production for all penetration levels. The maximum ramp down required was within the wind plants' capability.

Table 7.12. Maximum Arizona Wind Curtailment in Single Worst Event for Various Penetration Levels, LocalPriority, April QSS Study Period.

\begin{tabular}{|l|c|c|c|c|}
\cline { 2 - 5 } \multicolumn{1}{c|}{} & $\mathbf{1 0 \%}$ & $\mathbf{2 0 \%}$ & $\mathbf{2 0 / 2 0 \%}$ & $\mathbf{3 0 \%}$ \\
\hline Arizona Wind Energy Curtailed in Single Worst Event, GWh & 0.001 & 0.01 & 1.4 & 2.4 \\
\hline Annual Arizona Wind Energy, GWh & 8,048 & 14,569 & 14,569 & 21,138 \\
\hline Maximum Wind Curtailment Ramp Down, MW/min & 16 & 36 & 94 & 153 \\
Total Arizona Wind Plant Rating, MW & 2,850 & 5,250 & 5,250 & 7,710 \\
Maximum Ramp Down Capability, MW/min & 285 & 525 & 525 & 771 \\
\hline
\end{tabular}

The amount of load participation required to bring the negative Colorado-East ACE to zero was calculated for the October QSS cases with 10\%, 20\%, 20/20\%, and 30\% wind penetration (Local-Priority Scenario, S-o-A wind forecast, hourly schedule changes). This defines the maximum load participation for the given study period. It was calculated as the energy, in MWh, under the negative ACE signal. These results are summarized in Table 7.13. In contrast to curtailment of wind generation, response of load to an AGC command makes the load response a contributor to system up reserve. 
The load participation required for the single worst forecast error in 2006 constituted less than $0.001 \%$ of Colorado-East's annual load energy for all penetration levels.

Table 7.13 Maximum Colorado-East Load Participation for Single Worst Forecast Error for Various Penetration Levels, Local-Priority, October QSS Study Period.

\begin{tabular}{|l|c|c|c|c|}
\cline { 2 - 5 } \multicolumn{1}{c|}{} & $\mathbf{1 0 \%}$ & $\mathbf{2 0} \%$ & $\mathbf{2 0 / 2 0 \%}$ & $\mathbf{3 0 \%}$ \\
\hline $\begin{array}{l}\text { Colorado-East Load Energy Participation for Single } \\
\text { Worst Forecast Error, GWh }\end{array}$ & 0.05 & 0.17 & 0.15 & 0.22 \\
\hline \begin{tabular}{l} 
Annual Colorado-East Load Energy, GWh \\
\hline
\end{tabular} & 61,372 & 61,372 & 61,372 & 61,372 \\
\hline
\end{tabular}

\subsubsection{Summary}

In general, the $10 \%$ and $20 \%$ wind penetration cases exhibit similar characteristics, and the $20 / 20 \%$ and $30 \%$ wind penetration cases exhibit similar characteristics. Thus, the impact of increasing wind penetration has two aspects - wind penetration in the study footprint, and wind penetration outside the study footprint. With low levels of wind and solar penetration outside the study footprint, exports from the study footprint could increase. With higher levels of penetration outside the footprint, exports were constrained.

The hourly generation and export schedules drive the regulation requirements and AGC performance violations more than the wind and solar variability. As penetration increases, the magnitude and frequency of the hourly schedule changes also increases. Therefore, the regulation requirements will increase at higher levels of penetration. However, a more frequent or even continuous generation and export schedule would improve performance for units on both regulation and economic dispatch.

Extensive control area cooperation or consolidation significantly improves the system's ability to respond to hourly schedule changes and sub-hourly variability. As a result, high levels of wind and solar penetration have less impact on larger control areas.

Uncertainty due to forecast error has an impact on both commitment and dispatch. As such, improved forecasting can reduce the maneuvering required of the balance of portfolio generation to follow net load and to provide regulation.

Mitigation of the area control errors can be achieved with a combination of wind curtailment when generation exceeds load, and load participation when load exceeds generation. This approach makes both wind and load contributors to system reserves. Neither appears to have significant technical or economic hurdles.

In short, the scheduled hourly generator changes had a bigger impact on regulation duty than the variability of wind and solar. In the $30 \%$ case, the movement from an hourly to 
an approximately continuous schedule reduced the fast maneuvering duty on combined cycle units by about half. Wind plants can also provide down regulation, which may also reduce CPS2 violations.

\subsection{Variability Impacts on Reserves}

In the following four sections, the relationship between variability of load and wind, and how that variability affects system reserve requirements will be examined. The discussion is aimed primarily at understanding how much maneuvering room is required of on-line generation to handle the changes that occur within each hour. It is therefore focused on variability, rather than uncertainty. However, these two concerns are interrelated aspects of incorporating variable renewables. Specifically, day-ahead forecast error is the major contributor to uncertainty, and day-ahead forecasts are included in unit commitment decisions. Section 6.2.1 is devoted to exploring strategies for managing uncertainty, as manifested in wind forecast errors. Ultimately, the difference between forecasted and actual wind and load, and the unit commitment strategies used to deal with those differences, will have a major impact on the resources available in real-time to respond to variability. This section is dedicated to building understanding of the requirements for sub-hourly reserves and to developing useable rules for operation.

Initially, the key rules and guidelines that govern reserves and practice within WECC will be described. Then, load and wind variability in the 10-minute time frame is examined, and its relationship to the net load variability that must be balanced by the rest of the system. Load variability alone imposes requirements for reserves or additional committed generation, which have the ability to respond to either AGC or short-term (within 10-minute) load following dispatch commands. In broad, study area terms, the addition of wind variability will roughly double the amount of the reserves required to meet net load variability. A fraction of the reserves, amounting to about 1 standard deviation of the expected 10-minute variability, should be allocated to the fastest acting reserves, i.e., regulation. Then, in Section 7.4, simple rules, such as carrying reserves to cover variability based on a fraction of load PLUS wind production (not installed MW), are presented.

In Section 7.5, the MAPS simulations of Chapter 6 and QSS time simulations presented in Section 7.2 are revisited in the context of reserves. Means to account for wind variability in the allocation of system reserves during operation are explored. Results will show how the economic commitment and dispatch of the system are generally in harmony with the reserve needs for high wind and solar penetration.

\subsubsection{Existing Reserve Rules and Practices}

Grid operations throughout WECC are governed by a number of rules and guidelines. The WECC standard on operating reserves is the "Regional Reliability Standard to 
address the Operating Reserve requirements of the Western Interconnection" [7.5]. This standard addresses contingency and regulating reserves.

This standard dictates that contingency reserves, "at least half of which must be Spinning Reserve", will be (at least):

"(b) The sum of five percent of the load responsibility served by hydro generation and seven percent of the load responsibility served by thermal generation."

The intent of this quantitative reserve requirement is to make the system compliant with the NERC Disturbance Control Standard BAL-002-0. In practice, this clause has been simplified to an equivalent spinning reserve requirement equal to $3 \%$ of load, as discussed in Section 6.1 and used throughout the MAPS runs. There is an additional clause accounting for the worst contingency. However, this tends to be less than $3 \%$ of load in the large areas examined in this study.

The standard dictates that regulating reserves must be carried as well:

"Sufficient Spinning Reserve, immediately responsive to Automatic Generation Control (AGC) to provide sufficient regulating margin to allow the Balancing Authority to meet NERC's Control Performance Criteria (see BAL-001-0)."

Unlike the contingency reserve definition, this requirement is not translated into formulaic rules for the specific amount of regulating reserves that must be carried. The amount of regulation procured is variable, and the practice for identifying that amount is largely experience-based as operators get feedback via system performance. An approximate regulation requirement was developed in Section 7.2, and is used for reference in this section. That regulation requirement was approximated as $1 \%$ of peak load, assigned to committed units.

Thus, unlike the specific quantitative rules that dictate minimum spinning reserve levels, there is no equivalent rule for maintaining reserves for handling load, or net load, variability. Rather, there are guidelines and rules covering system performance measures for which the reserves are required. NERC's Control Performance Criteria, BAL-001-0, [7.1] requires a certain adherence to scheduled interchange and frequency. Area Control Error (ACE), which is a function of deviation from scheduled exchange and deviation from nominal frequency, must generally be within bounds $\left(\mathrm{L}_{10}\right)$ that are themselves a function of balancing area size. In simplified terms, ACE must be within these bounds a minimum of $90 \%$ of the time to meet CPS2 criteria. However, most balancing areas target somewhat higher CPS2 levels - 95\% is representative. The $95 \%$ target is reinforced by other operating guidelines. For example, a WECC operating guideline [7.6] states that "Ten (10) minute area load variation" is: 
"The anticipated load variation (increase or decrease) the control area expects in the next 10 minutes. Allowance shall be made for uncertainty in forecasting the load variation. A confidence factor should be used such that the uncertainty can be limited to no more than 5\% error. Another way to arrive at this confidence factor is to track the performance of the load forecast error variance. Two times the standard deviation results in a 95\% expected area load variation about the mean load forecast. Errors in excess of the 5\% uncertainty could be considered as a legitimate reason to use the contingency reserve portion of operating reserve."

So, while specific reserves are not normally allocated to variability, system operation must meet expected load variability most (i.e. 95\%) of the time. In the next sections, the fundamental notion that sub-hourly reserves have historically been used, but not formally procured, to cover most expected load variability is extended to net load variability. These reserves will be called "variability reserves", to broaden the concept from just regulating reserves and to distinguish them from contingency reserves.

\subsubsection{Load and Net Load Variability}

In Section 7.1, 10-minute load and net load variations were presented. Since spinning reserve rules are based on load level (the $3 \%$ of load rule), it is useful to examine the reserves necessary to handle system variability in the same way. In Figure 7.46, the variability of wind power in the study footprint is shown as a function of wind power. The three traces in the figure each include ten data points. The data for each of these points are from statistical analyses of $1 / 10^{\text {th }}$ of the wind data for the year -876 hours or 5,256 10-minute periods. Consequently, each point is the standard deviation of the 10minute deltas for all the hours in that bucket (y value) plotted versus the average wind power of all the wind data in that bucket ( $x$ value). The callout on the plot indicates one example. The data are not, therefore, equidistant on the $x$ axis. Further, individual 10minute samples exist with wind power less than the left-most point, or greater than the right-most point. They are reflected in the average data for their respective decile points. Superimposed on the curve are dotted orange lines that roughly capture the $30 \%$ wind variability curve in two linear segments. The slope of the first part shows an increase in wind variability equal to about $3.3 \%$ of wind output. The second, flat section begins at about $8000 \mathrm{MW}$, or about $30 \%$ of nominal maximum wind. This piecewise curve fit will be revisited later in this chapter. 


\section{Wind LP 2006}

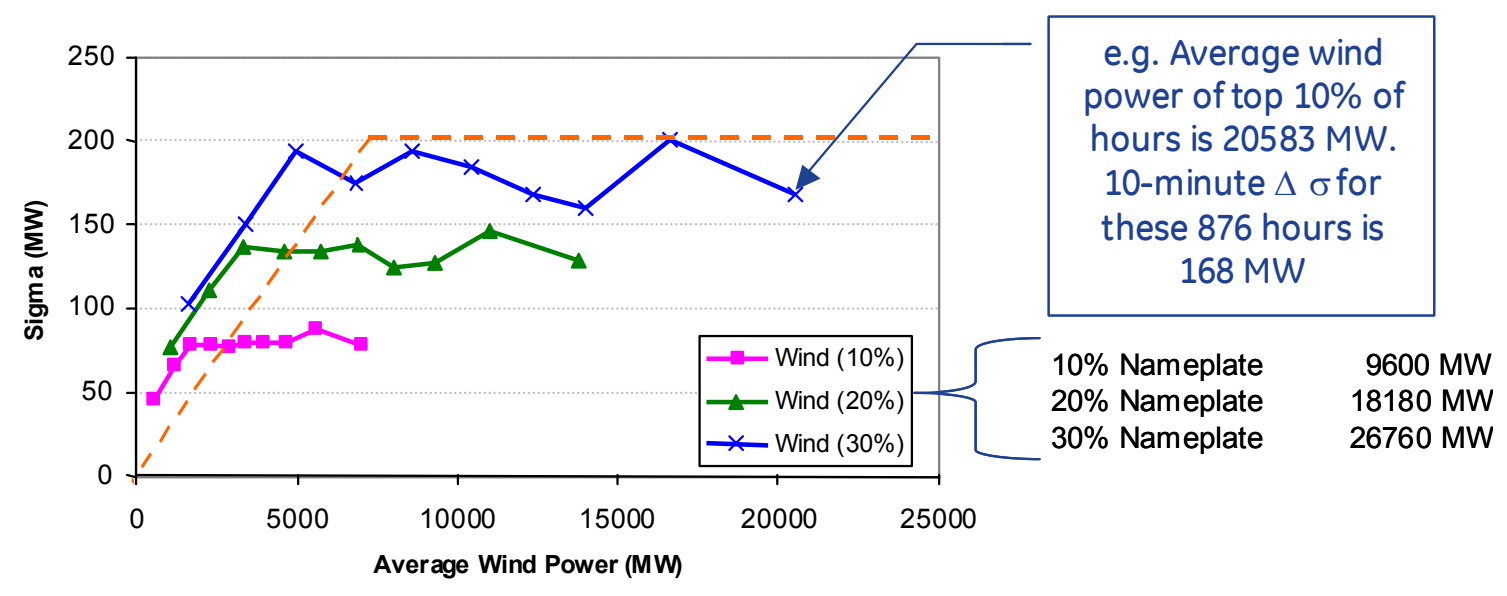

Figure 7.46 Local Priority Case - 10-Minute Wind Variability for 2006

While this result is of interest to understanding system needs, the variability of wind power is only part of the problem. Preceding sections have shown that the challenge for the system is to operate to the net load. Figure 7.47 shows the net load ${ }^{3}$ variability of the study area for three penetration levels with the Local-Priority Scenario. The figure also shows the variability of the load alone, as the orange trace with circular symbols. As with Figure 7.46, the data are analyzed in 10 buckets of $876\left(1 / 10^{\text {th }}\right.$ of the year) hours. Thus, for example, the average net load power in the ninth decile for the L30 case is $34,821 \mathrm{MW}$, as indicated by the arrow. The standard deviation, $\sigma$, of the 10-minute delta for these 876 hours is $374 \mathrm{MW}$.

As expected, the variability increases with increased penetration. In particular, periods of low net load tend to have considerably more variability. These tend to coincide with periods of higher wind. Indeed, for the $30 \%$ case, roughly half of the year has net load less than the system minimum load, as indicated with the vertical orange line. This is consistent with the load duration curve of Figure 4.16.

${ }^{3}$ For simplicity the contribution of solar to variability was neglected throughout the balance of this section. Net load here refers to load minus wind power. The concepts presented conceptually extend to variability from solar and other variable generation resources, which for this work represent a relatively small contribution to variability. 


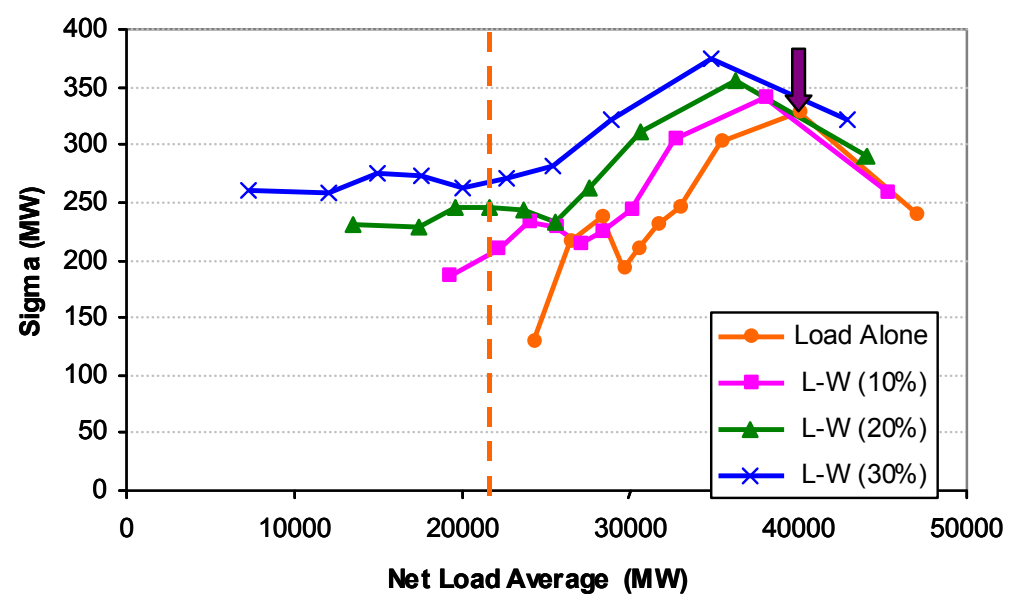

Figure 7.47 Local Priority 10-Minute Net Load Variability (10-minute $\Delta \sigma$ in MW) for 2006

Clearly, variability at low levels of net load is greater than the variability at just low system load. And, this higher level of variability must be accommodated by the total non-renewable generation on-line to serve system net load.

The sensitivity of net load variability and wind variability to scenarios is shown in Figure 7.48. In this figure, the wind variability shapes vary with spatial diversity. This is most evident in the wind variability for the Mega-Project Scenario. The Mega-Project profile, with clear relative maxima at mid power range, is similar to that observed in a single plant. 
IA, 2006
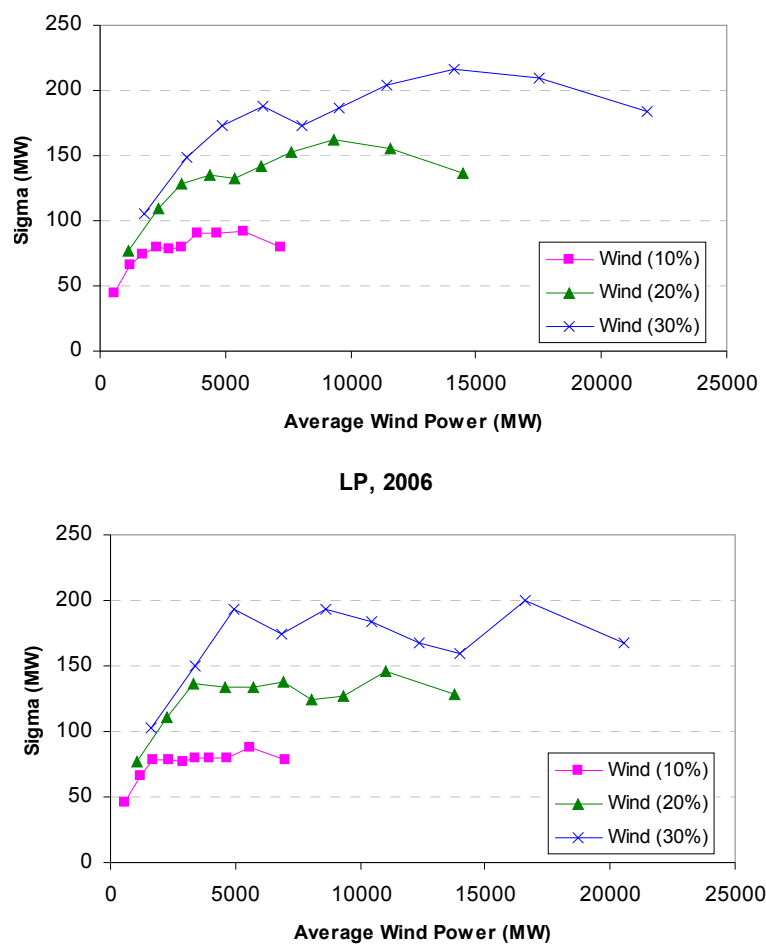

MP, 2006

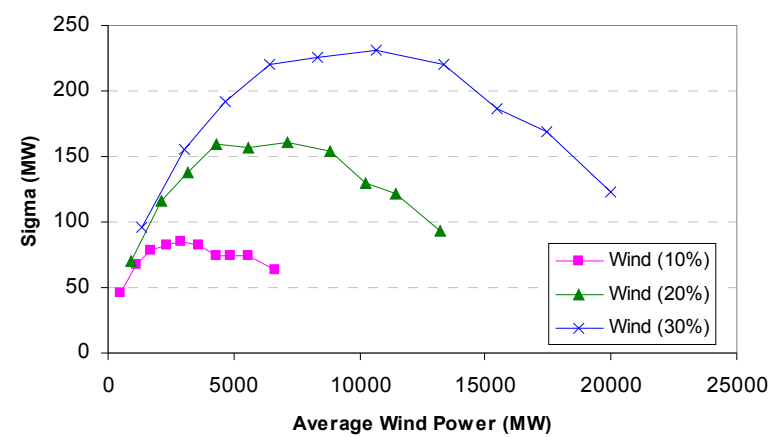

IA, 2006

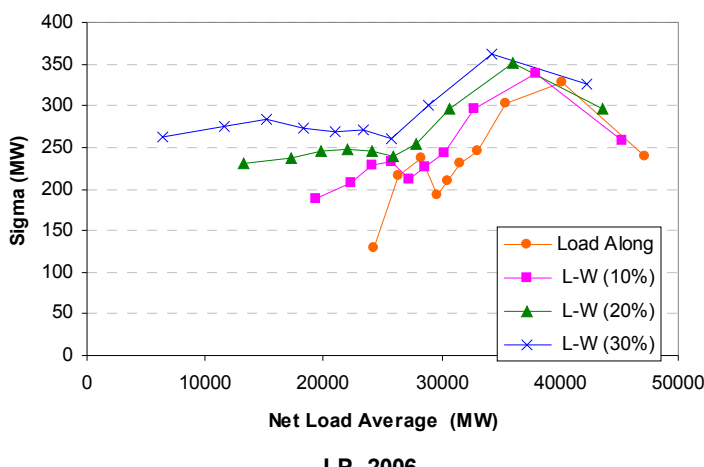

LP, 2006
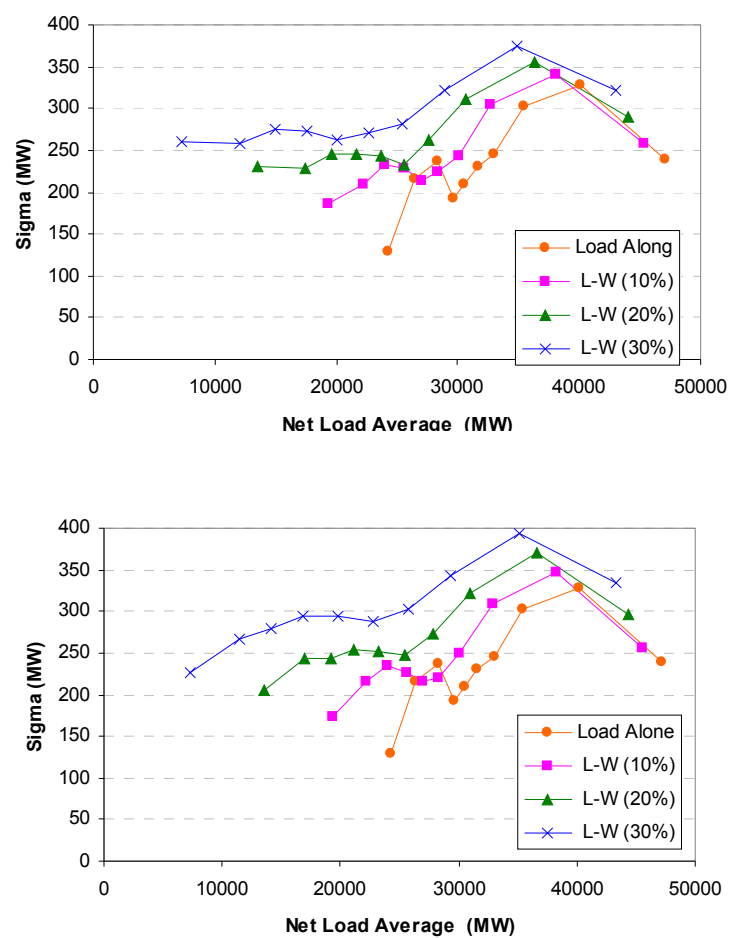

Figure 7.48 10-Minute Variability: Wind and Net Load - 3 Scenarios

Figure 7.49 shows the same information as the left side of the preceding figure. On the right hand side, the variability, $\Delta \sigma$, is normalized by the installed nameplate MW of wind. These nameplate ratings are summarized in the embedded tables. Note that, the normalized variability drops with increased penetration for the In-Area and LocalPriority Scenarios. This is a consequence of increased spatial diversity as more wind plants are added. This effect is least observable in the Mega-Project Scenario, because the wind generation is added in essentially the same relatively small geographic area as penetration increases. Broadly, these curves show that (a) there is less wind variability in a normalized sense at higher penetration, and (b) the overall wind variability is less dependent on wind level (i.e. the curves get flatter) for wider spatial distribution. The 
roughly parabolic shape of the wind only variability curves is examined further in Section 7.4.7.
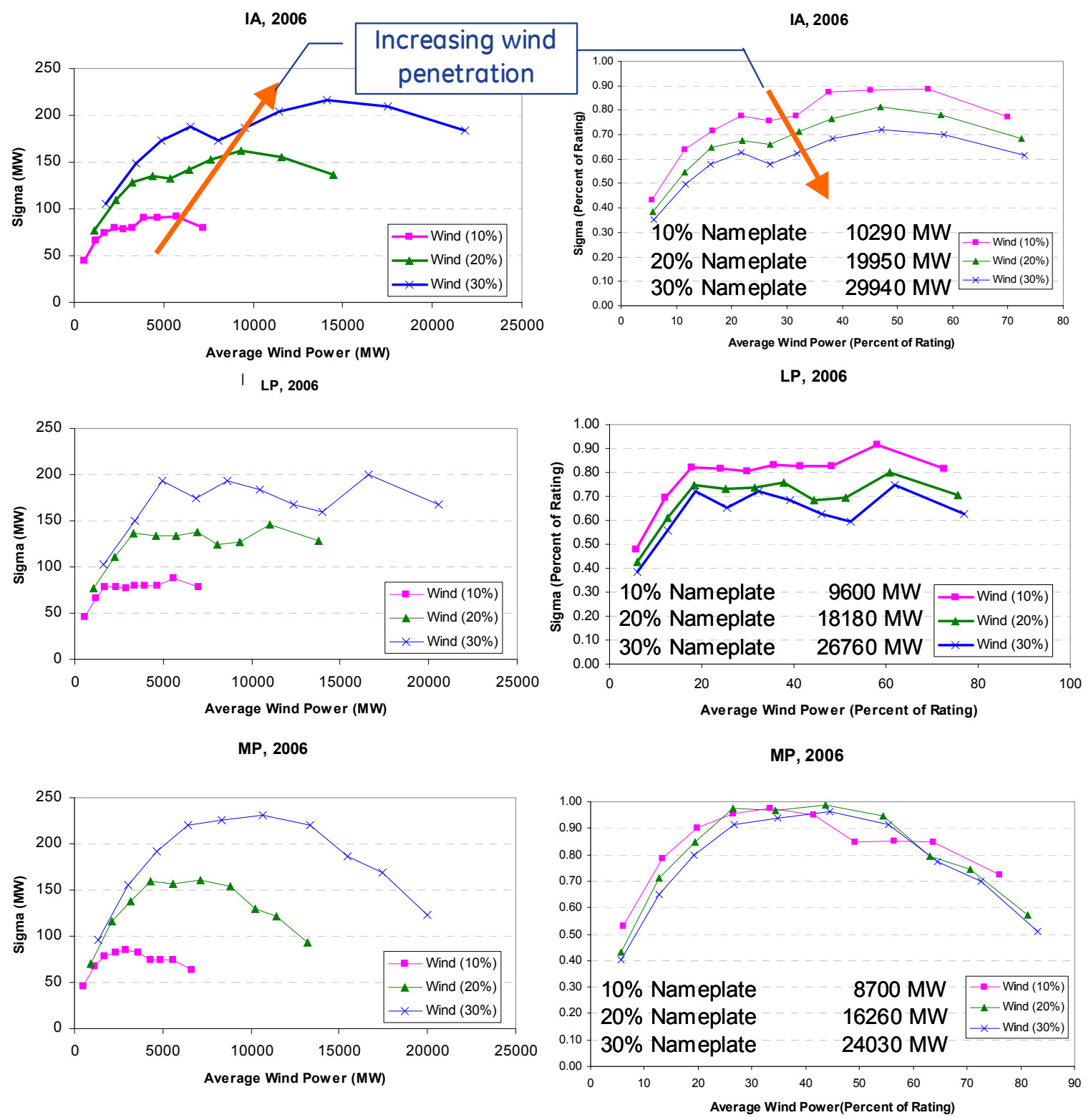

Figure 7.49 10-Minute Wind Variability: Normalized to Installed Rating

The behavior becomes more interesting as individual areas are examined. The wind and net load variability of Arizona and Wyoming are shown in Figure 7.50. Consistent with observations from the hourly analysis, the wind impact is more observable at the area or state level. In a very high wind system like Wyoming, wind dominates. The Wyoming net load variability curves are essentially mirrored and shifted from the wind curves. 

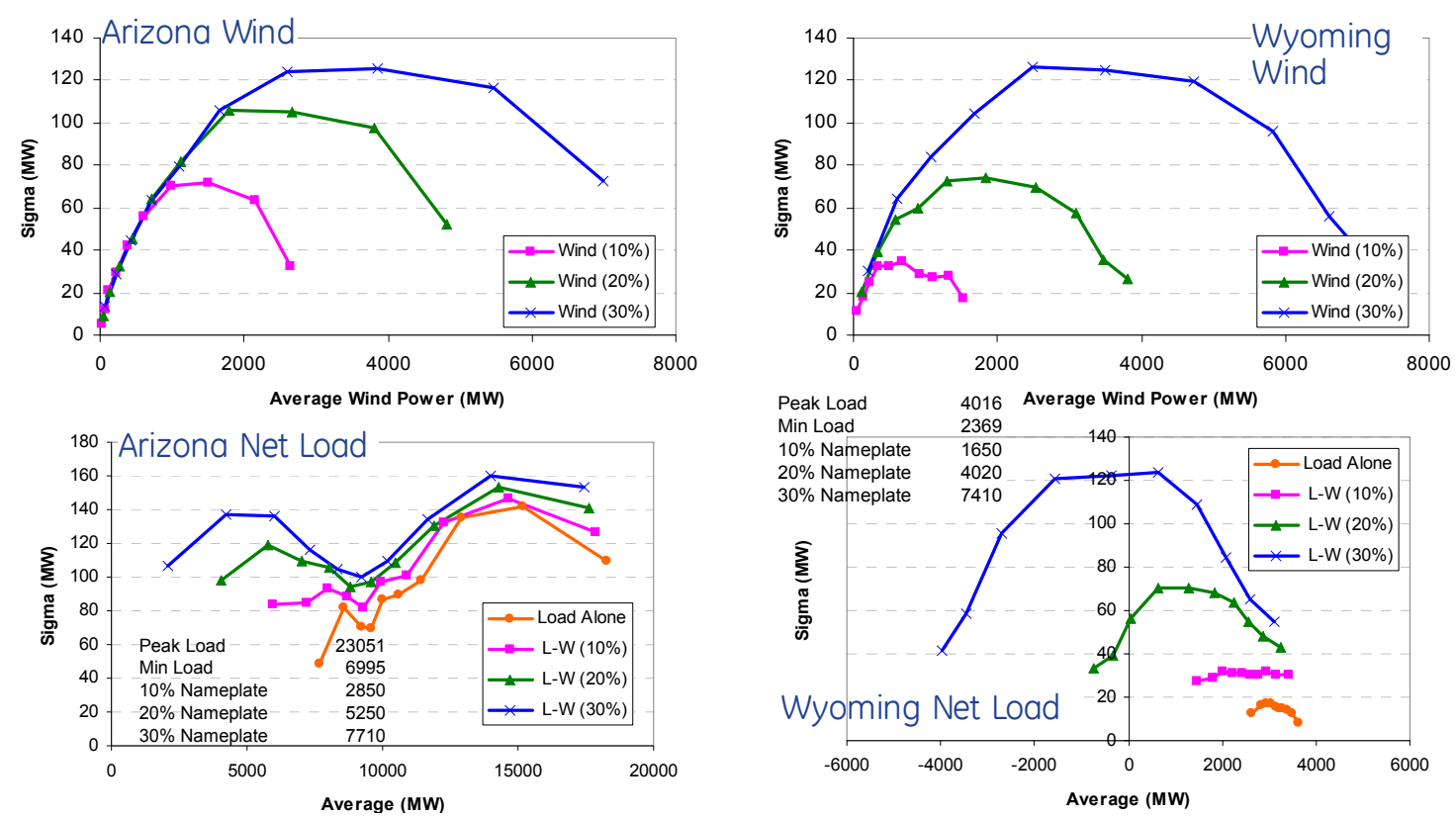

Figure 7.50 Arizona and Wyoming: Average (MW) vs. Sigma of 10-Minute Delta (10-minute $\Delta \sigma$ in MW)

\subsubsection{Relationship to Existing Reserve Rules}

The fact that system variability increases with load is no surprise to system operators. The development of reserve rules that are a function of system load is partially a reflection of that knowledge.

In Figure 7.47 the increase in system variability, as measured by the 10 -minute $\Delta \sigma$, is apparent in the "Load-alone" trace. On closer inspection, the relationship between variability and load is roughly linear for most load levels. The exception is at, and near, system peak loads. In Figure 7.51, a simple straight line has been overlaid on the loadalone trace for the study footprint and for Arizona. Note that the slope of this line is about $1 \%$. This means that the statistical expectation of load variability that must be met by increased generation maneuvering is about $1 \%$ of load. The analysis throughout this study has shown that load variability is statistical, and roughly normal. Therefore, a rule that covers most variability is needed. 

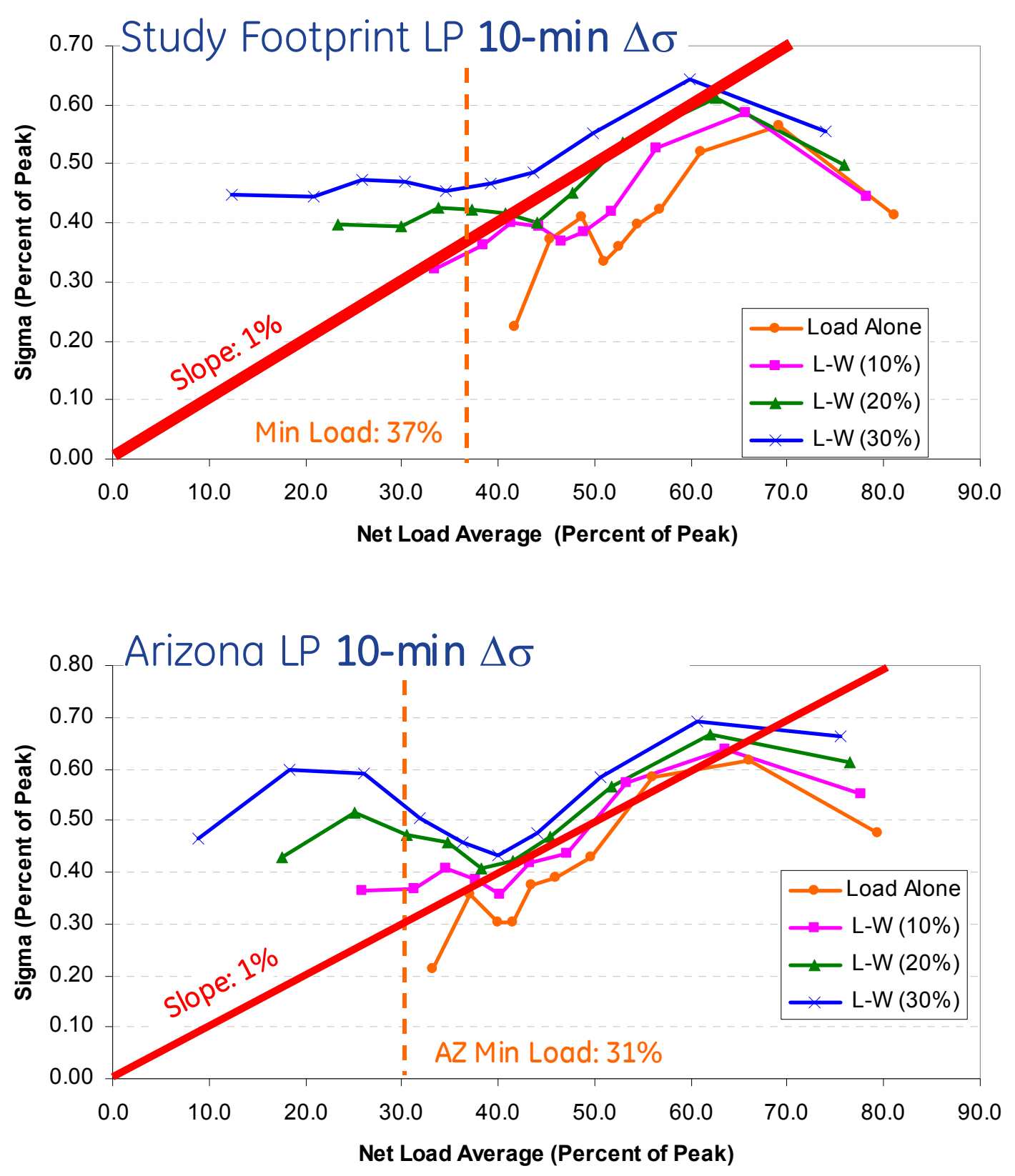

Figure 7.51 10-Minute Variability (10-minute $\Delta \sigma$ in percent of peak load)

The $1 \%$ line in Figure 7.51 is only one standard deviation $-68 \%$ of events in a normal distribution. It is necessary to address more possible outcomes than this. Using 3 standard deviations will cover the vast majority (99.7\%) of expected variability. This simple observation suggests an implied quantitative rule to cover variability, namely that 3 times the standard deviation of 10 -minute delta $(3 \times 10$-minute $\Delta \sigma)$ should be carried. This implied " $3 \Delta \sigma$ " rule will be examined over the next several sections. 
For the load only situation, this $3 \times \sigma$ of the 10-minute $\Delta$ is approximately $3 \%$ of load. Coincidentally, this is equal to the $3 \%$ of load dispatched for spinning reserve.

\subsubsection{Net Load Variability Relationship to Load and Wind Levels}

With the addition of wind (and solar) generation, system variability is driven by two separate, but not necessarily independent phenomena. As shown repeatedly in Chapter 6 , system reserves must be provided by resources available under each load and wind condition. This includes contingency reserves, which are formally procured or allocated and expected to be available all the time, and variability reserves, which must be adequate most of the time.

Section 7.3.2 showed the change in variability with net load. Here, the impact of load and wind on that net load variability are separated. In order to do so, some simple relationships between load and wind need to be established.

In Figure 7.52, a load and wind space is presented. The x-axis corresponds to system load. Load varies between a minimum and maximum. At the foot print level, the minimum load is $21,249 \mathrm{MW}$ and the maximum is $58,087 \mathrm{MW}$ (as noted on the figure). Similarly, the wind power varies between zero and a maximum. The maximum for the $30 \%$ penetration, Local-Priority Scenario is $26,760 \mathrm{MW}$ - the total installed nameplate of wind generation in the study footprint. The range of load and wind are each divided into 10 equal sized buckets. Consequently, there are 100 combinations of load and wind. The colors indicate net load level, i.e. load minus wind, as listed in the legend. The color at each intersection is the net load for that combination. 


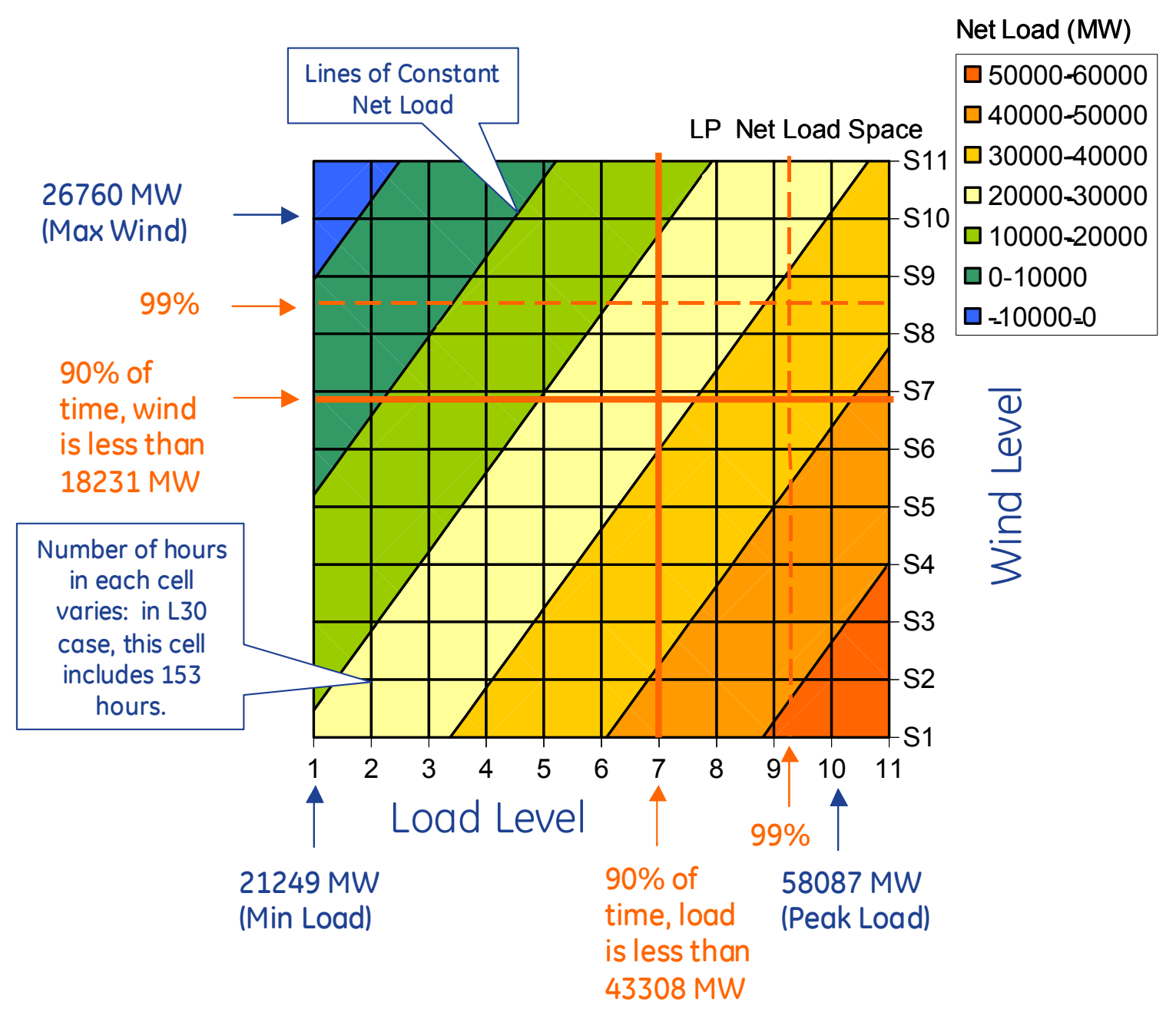

Figure 7.52 Load-Wind-Net Load Relationship for L30 System

This space represents 100 evenly distributed load and wind "cells." They are evenly distributed, in that load and wind levels are divided into 10 equal sized groups from minimum to maximum. It is critical to recognize, however, that the system does not reside in each of the 100 cells for the same amount of time. Indeed some cells corresponding to coincident high wind and high load conditions (upper right hand corner) are never met, at least not on an hourly basis. Thus, it is useful to examine the orange lines on the figure. The solid orange lines represent the $90 \%$ upper boundaries for load and wind. That is, the system load and wind are to the left of, and below, respectively, these lines $90 \%$ of the time. Similarly, the dotted lines are the $99 \%$ boundaries. These results are, at first glance, surprising. The reasons for this become more obvious by examining the familiar net load duration curves presented in Figure 7.53. In this net load duration curve from Section 4, heavy weight lines showing the minimum and maximum system load are drawn. It is this load range that is divided into 10 equal parts for the $\mathrm{x}$-axis in the load and wind space of Figure 7.52. The lighter orange lines show that system load is greater than $70 \%$ of the range from minimum to maximum 
about $10 \%$ of the time. This is consistent with the solid vertical orange line in the preceeding figure.

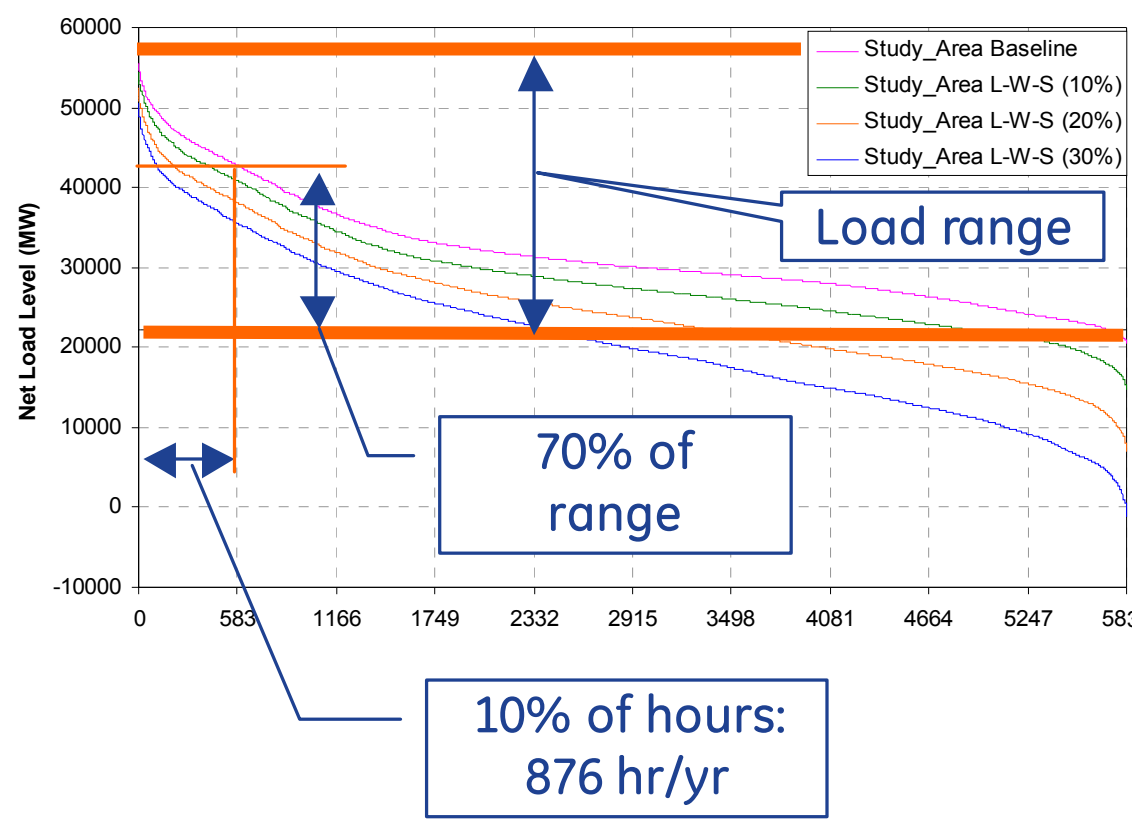

Figure 7.53 Relationship Between Load Duration and Load Range for Local-Priority Scenario

The total time spent in each cell, in hours per year, for each load and wind combination is shown in Figure 7.54. The 8760 hours, and 52,560 10-minute periods, in a year are distributed non-uniformly across the surface. The light gray "never" cells are wind and load combinations that never occur. The figure shows that the system spends the majority of time at moderate load, and wind levels in the neighborhood of 20 to $40 \%$ of nameplate. This is consistent with wind generation with capacity factors of 30 to $40 \%$. 


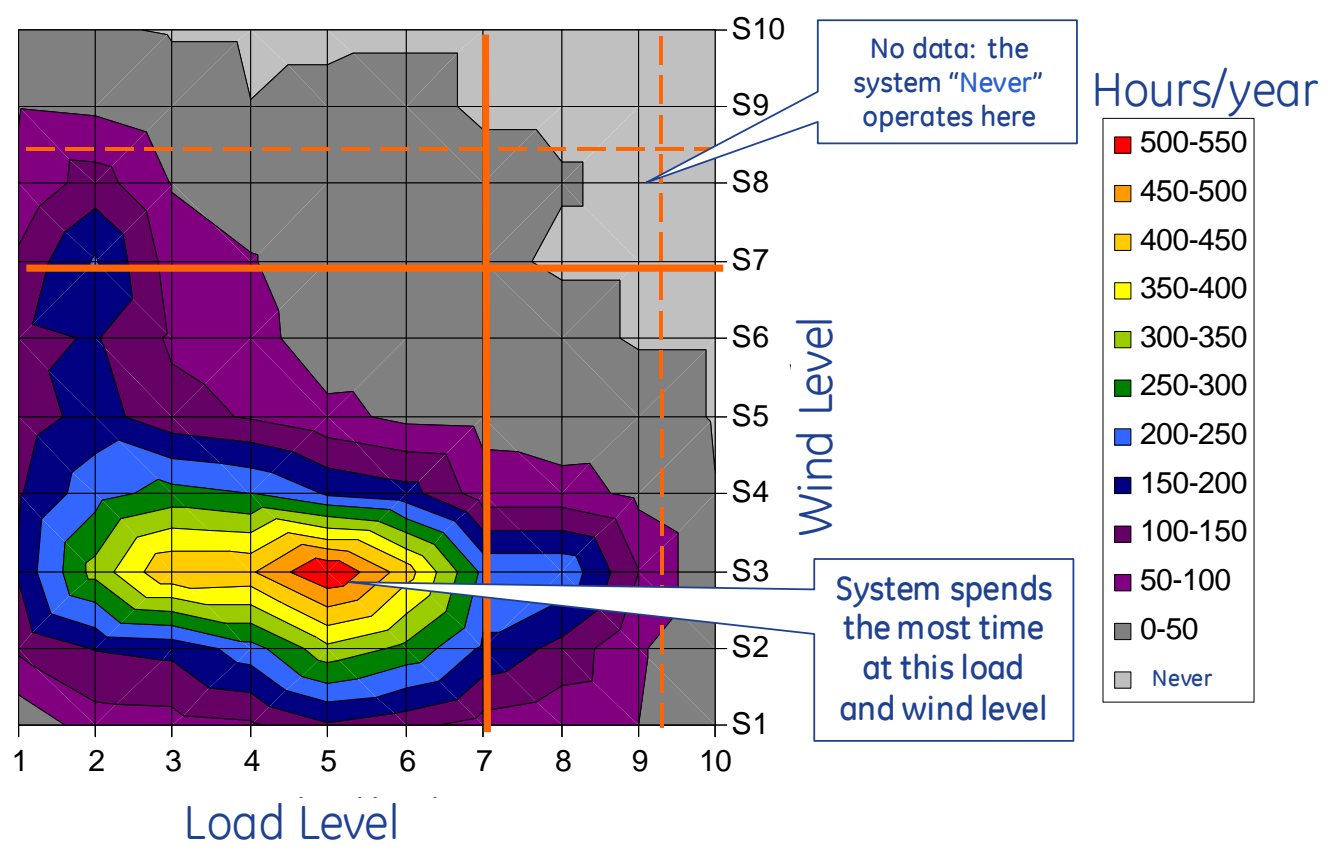

Figure 7.54 Operations L30: Hours of Load and Wind Combinations

Wind, load and net load variation are investigated in this load and wind space. In Figure 7.55 , the variability of wind is shown. The value associated with each cell corresponds to the standard deviation of the 10-minute wind deltas for all of the data samples associated with that cell. Some cells have a large number of data samples, and some have few or none ("never"). Note that these figures have additional cells around the outer maxima to facilitate plotting the edges. All the surfaces presented in this section show steep gradients at the edges abutting "never" cells. This is an artifact of the gradient between actual non-zero data and zero values associated with "never" cells, which are empty of data.

Two trends are observed in this surface. First, as expected, wind variability increases with increasing wind up to roughly the mid-range of wind power. This is observable by moving from bottom to top on the surface. What is less obvious, and something of a surprise at first, is that there is a significant correlation between load level and wind variability. Moving from left to right across the surface, it is clear that the wind is more variable at higher system load levels. This apparent incongruity is probably due to the atmospheric conditions during the day, which are more variable around these times of high load than at night. Thus, the wind is more variable during periods of high load.

Figure 7.56 shows the variability of load in this load and wind space. As expected, and consistent with the orange trace in Figure 7.47, load variability tends to increase with load to moderately high levels of load, and then it drops off again near peak load. The load variability is dominated by the diurnal cycles. The calculation of 10-minute load 
variability is based on linear interpolation of hourly data; therefore these calculations may understate the load-only variability.

Figure 7.57 shows the variability of net load. In general, the values of sigma are somewhat higher across the entire space. Further, two areas of relative maxima are apparent: one at mid-load (around 7) and moderate wind (around 5), and another at high load (around 9) and moderate wind (around 3). This surface is roughly the convolution, not the arithmetic sum, of the wind and load variability surfaces. The bimodal character is consistent with the blue (30\% penetration) trace in Figure 7.47.

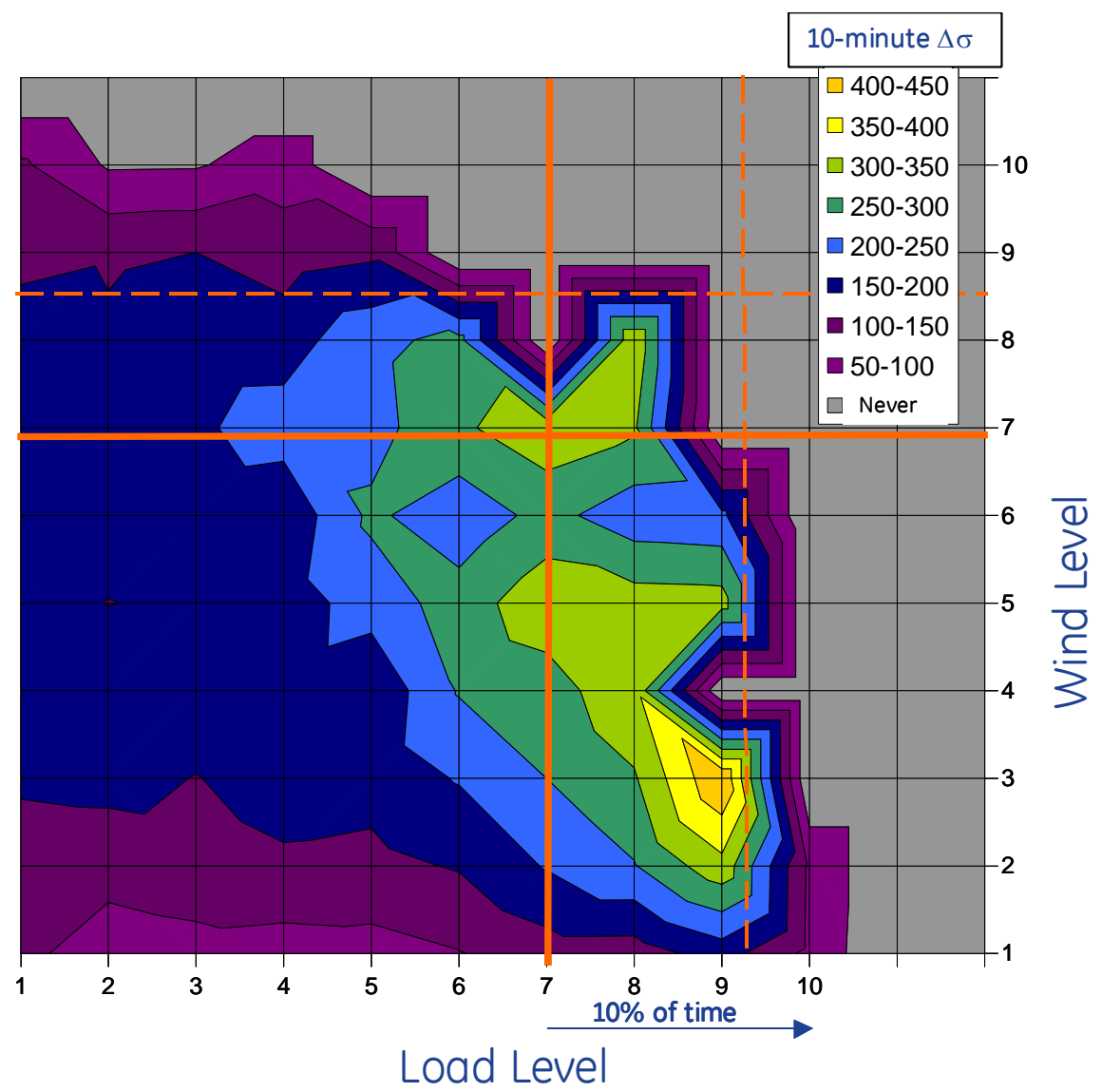

Figure 7.55 Wind Only Variability L30 


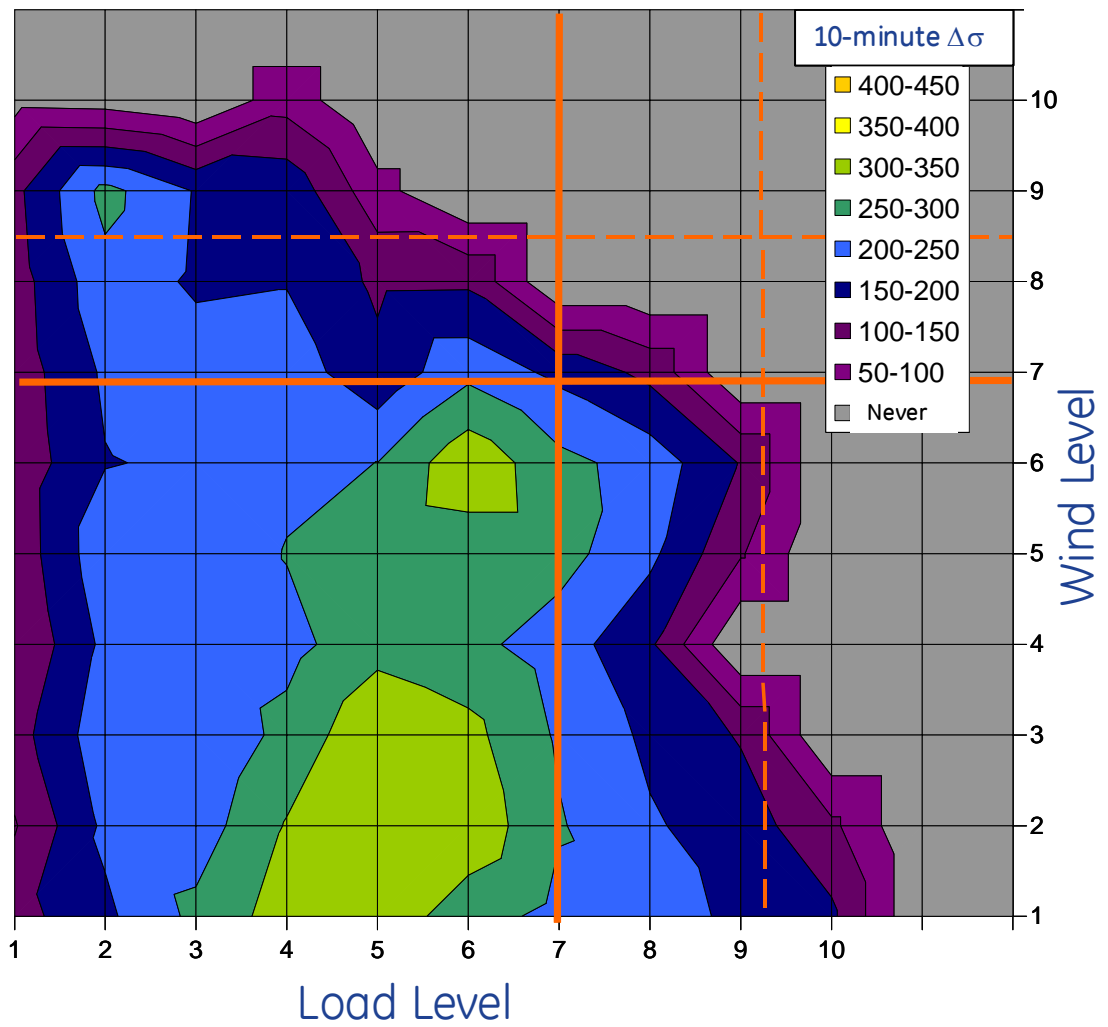

Figure 7.56 Load-Only Variability L30 


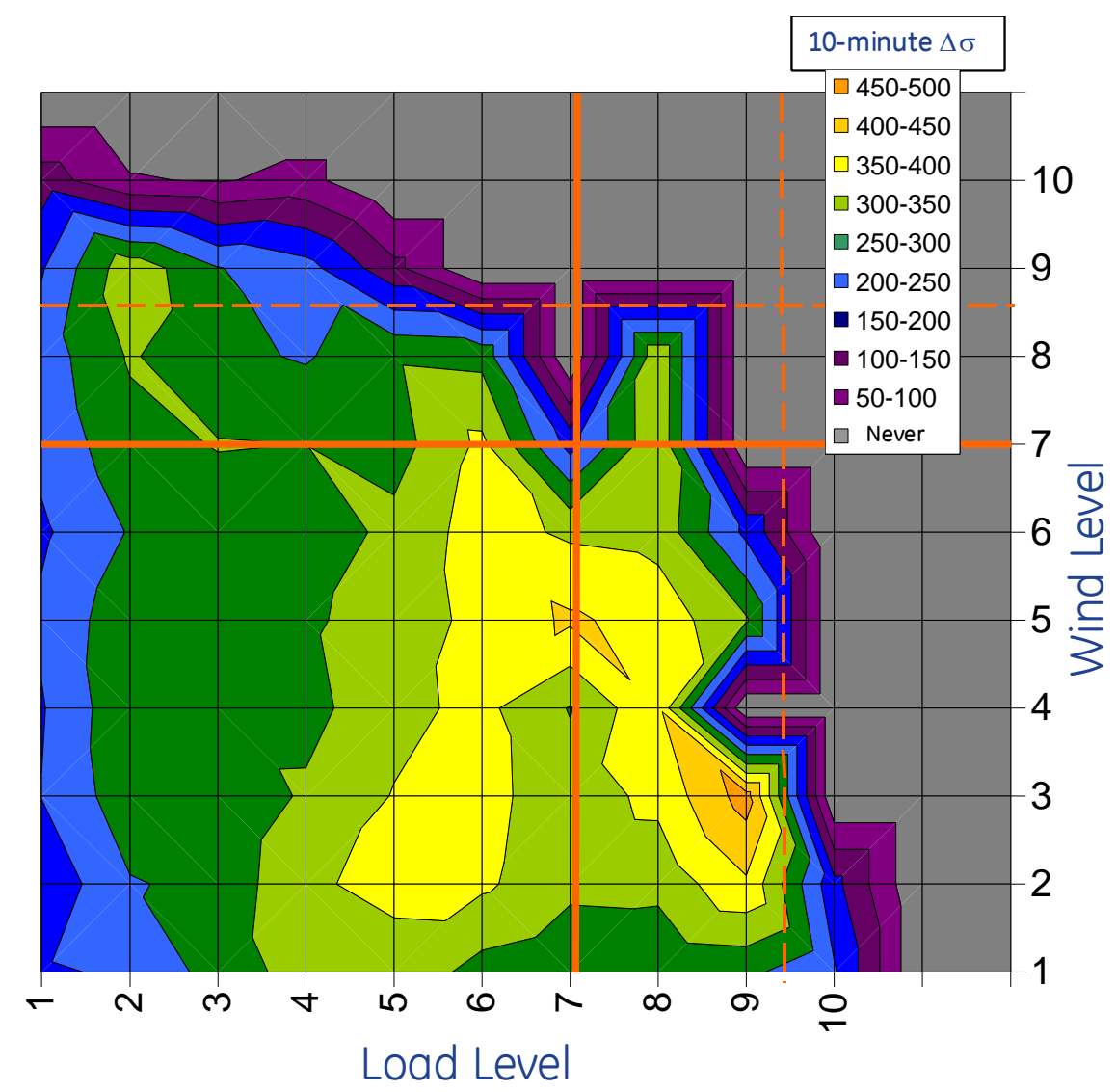

Figure 7.57 Net Load Variability L30

The net load variability increases with wind and load, reaching maxima somewhat below peak load and below peak wind levels. In the preceding section, the concept was introduced that a logical extension of the present practice for reserves is to require 3 times the standard deviation of expected 10-minute variability. Thus, for this load and wind space, the implied reserve requirement is $3 \Delta \sigma$. It is a function of both load level and wind level.

This sequence of figures provides some useful insights into the variability of the entire study foot print. The variability within each individual area is important to understanding the requirements and allocation of reserves across the system. The concept of net load variability in the load and wind space is now examined by transmission area or state.

The Arizona system has a peak load of 23,051 MW, a minimum load of 6995MW, and 7,710 MW of wind generation installed in this case. Therefore, the boundaries of the Arizona load and wind space are adjusted accordingly. Figure 7.58 shows the load-alone variability for Arizona. The Arizona load variability is relatively unaffected by wind level, and reaches a relative maximum at moderate levels of load. 
In contrast, the Arizona wind variability, as shown in Figure 7.59, is highly correlated to load level: Again, the wind is clearly more variable during the day, i.e. coincident with high load levels.

The Arizona net load variability, as shown in Figure 7.60, is a mix of wind and load impacts, consistent with the traces on the left side of Figure 7.50.

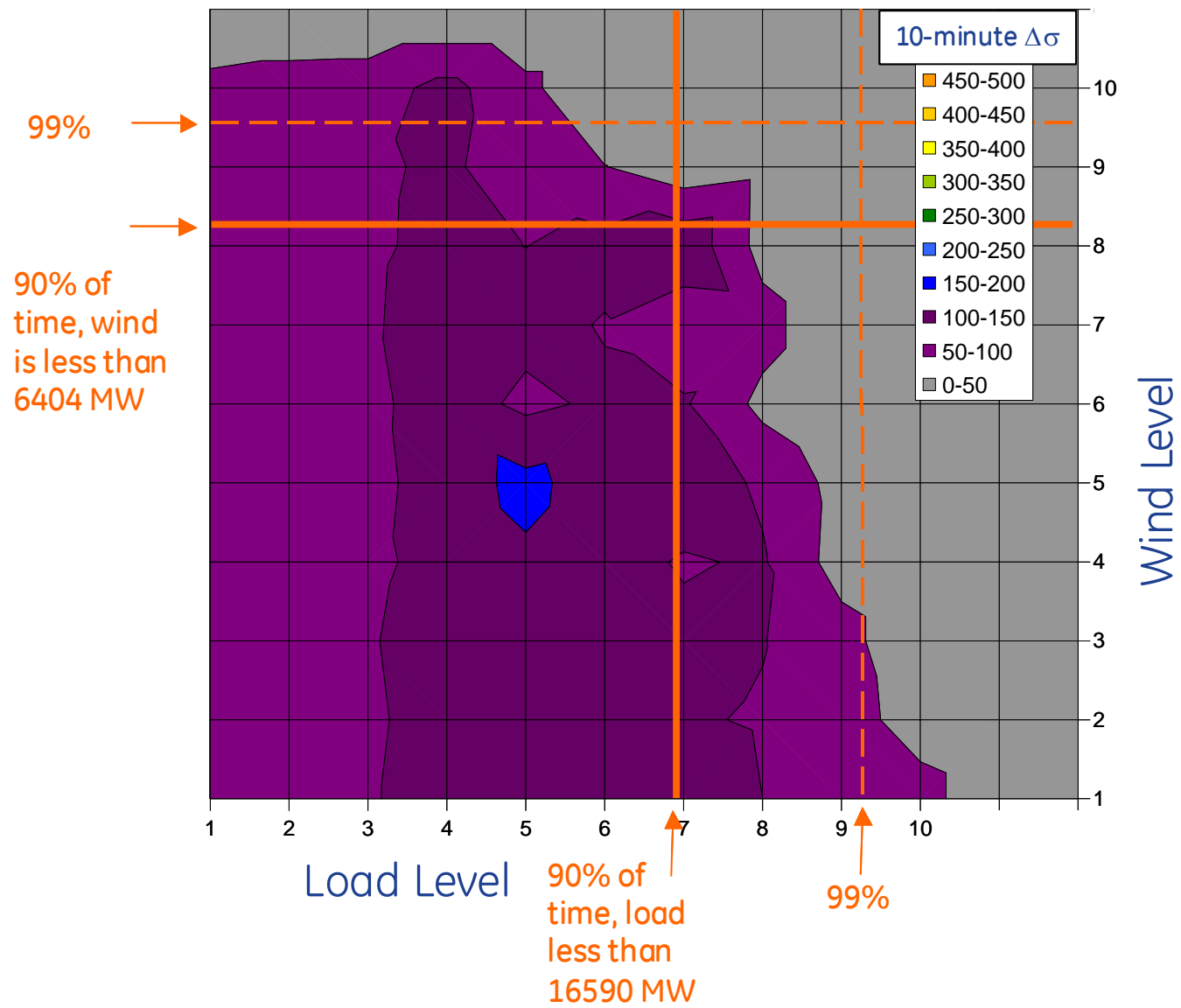

Figure 7.58 Arizona L30 Load Variability 


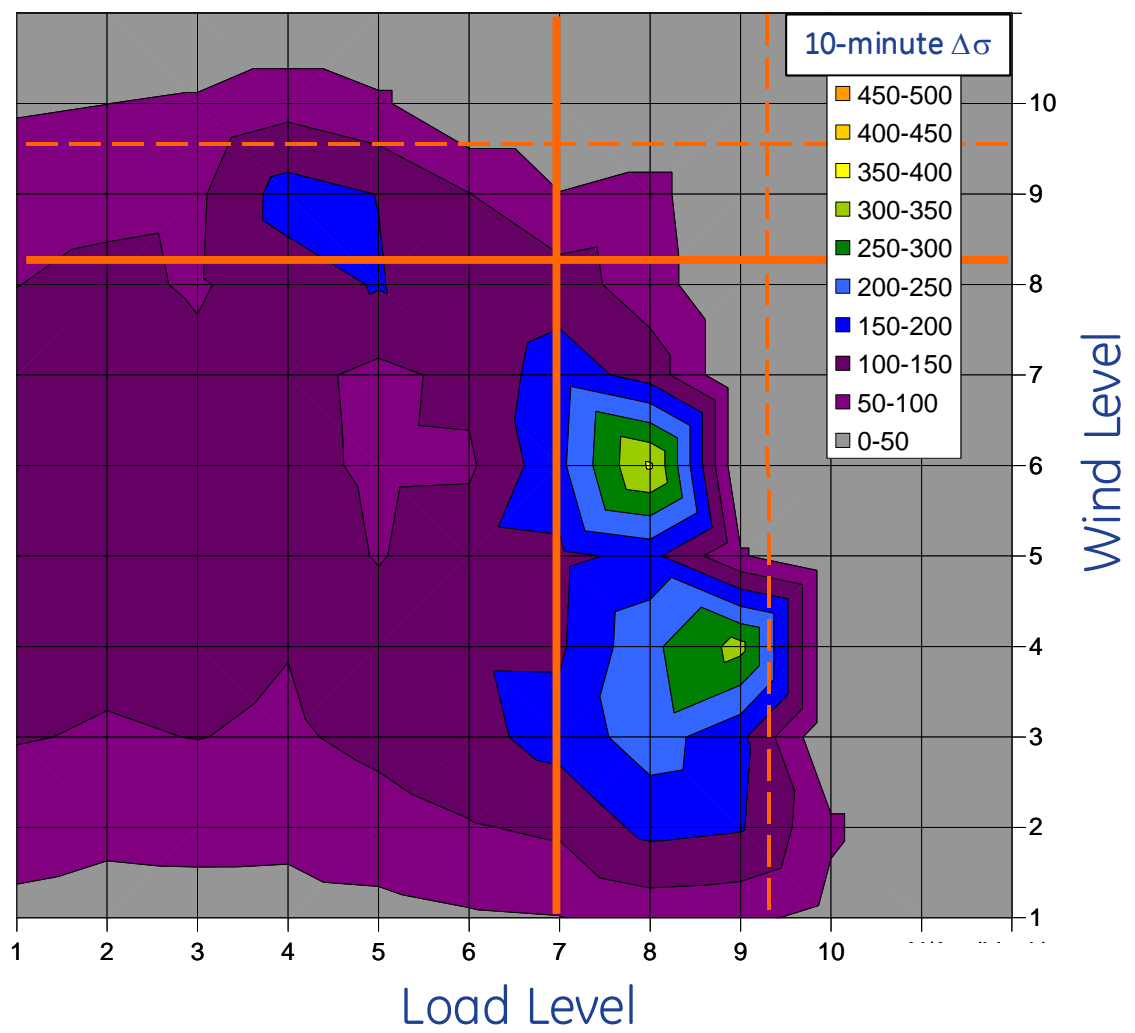

Figure 7.59 Arizona L30 Wind Variability

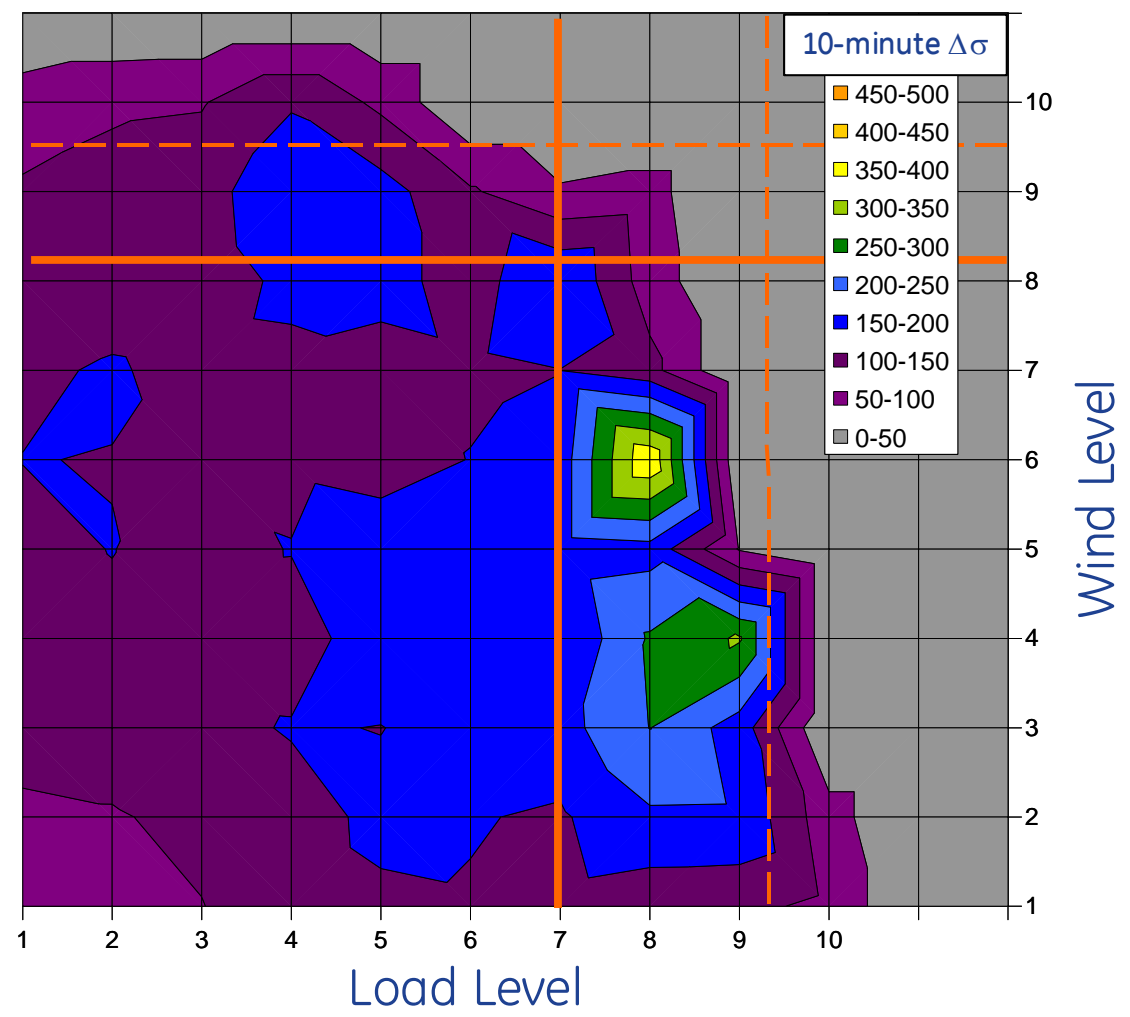

Figure 7.60 Arizona L30 Net Load Variability 
Wyoming has a peak load of 4,016 MW, a minimum load of 2,360 MW, and 7,410 MW of wind generation installed in this case. The wind power penetration is $185 \%$. Figure 7.61 shows wind only variability for Wyoming. Again, the wind variability shows a slight correlation with load level. There is a very small sample of data points at very high load and moderate wind. Consequently, the high variability shown may not be statistically significant. Overall, the high variability in the middle of the wind power range is an expected characteristic.

Figure 7.62 shows load-only variability. Note the scale in the figure. Load variability is insignificant compared to wind variability - load $\Delta \sigma$ is an order of magnitude smaller than wind variability.

Finally, Figure 7.63 shows net load variability. The net load variability is totally dominated by wind variability, which is consistent with the right side traces of Figure 7.50 .

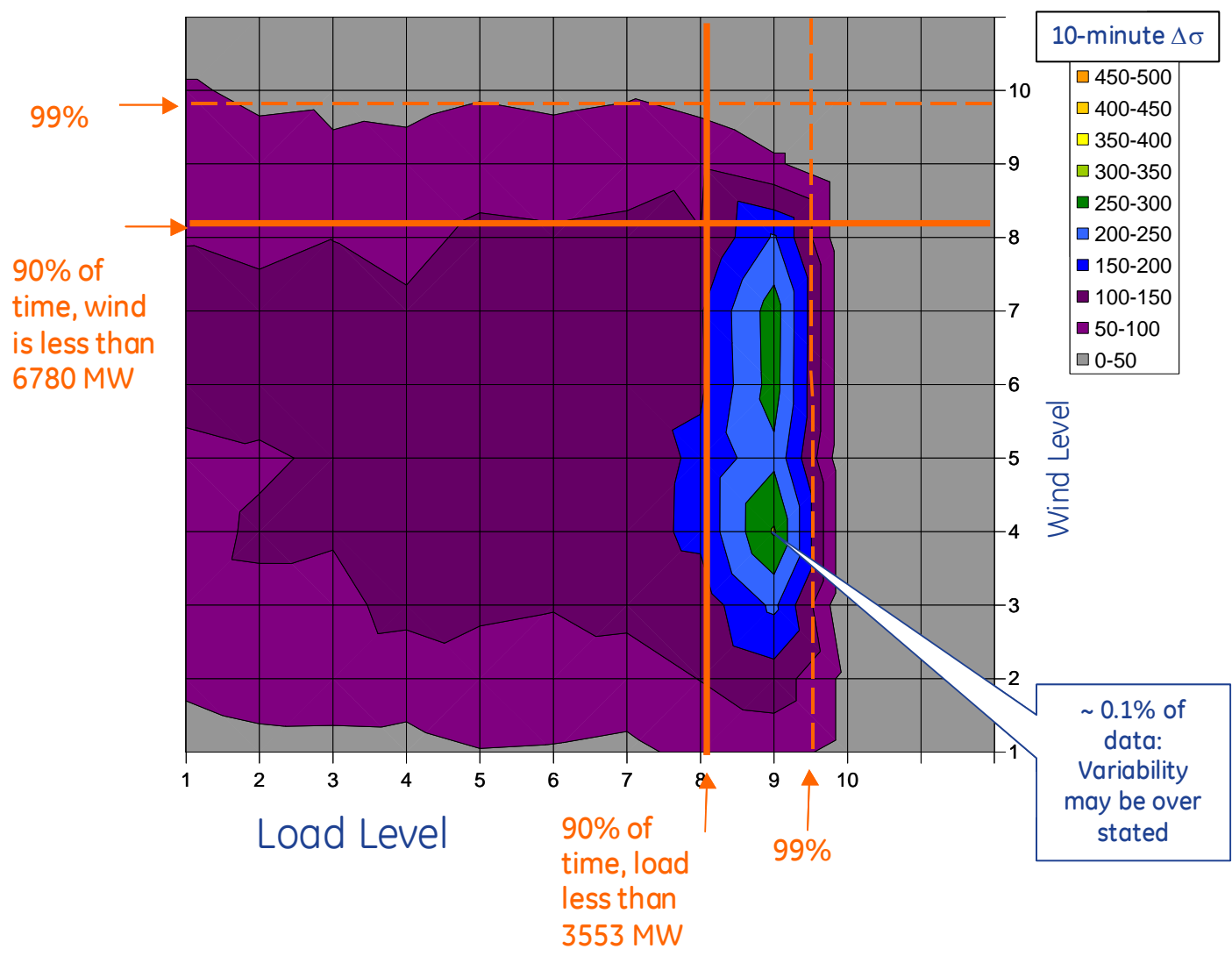

Figure 7.61 Wyoming L30 Wind Variability 


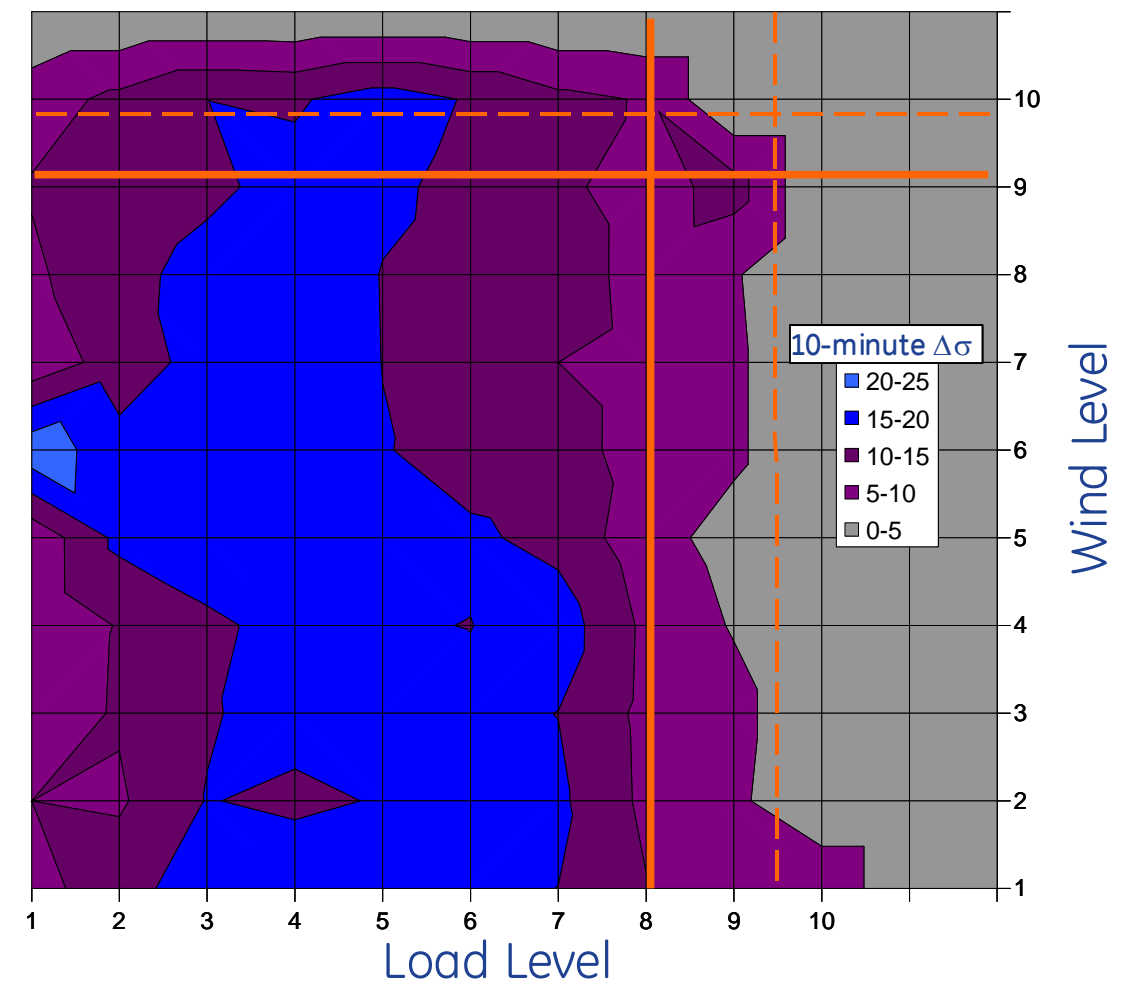

Figure 7.62 Wyoming L30 Load Variability

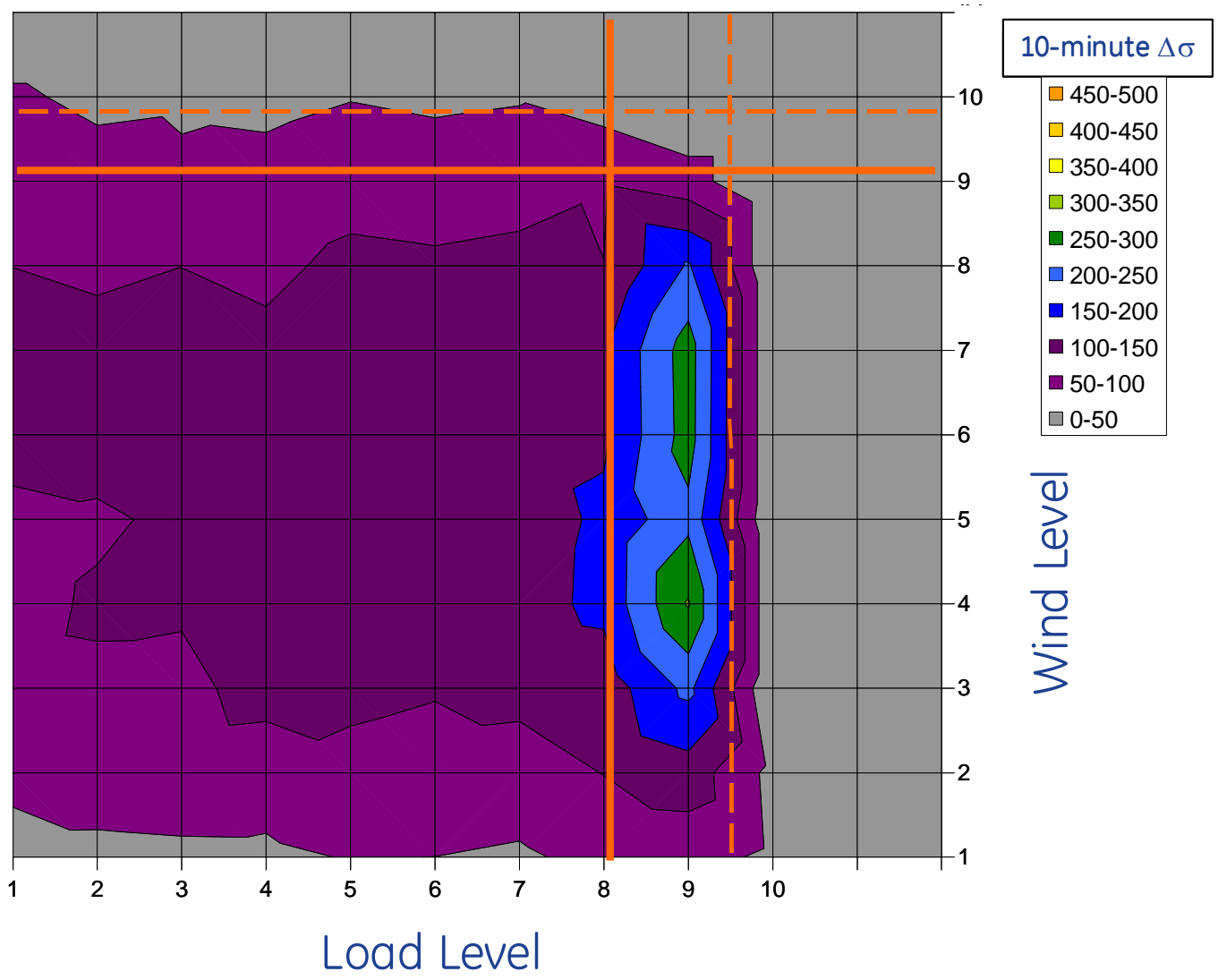

Figure 7.63 Wyoming L30 Net Load Variability 
The time that the Wyoming system spends in the various cells provides some insight into the Wyoming wind resource. In Figure 7.64, it is clear that Wyoming wind is bimodal: it has relatively light wind or relatively strong wind. This can also be seen in the duration curves of Figures 4.12 and 4.13. Figure 7.63 shows that the periods of highest implied reserve requirements occur at high load and moderate wind. From Figure 7.64 it is clear that these conditions are relatively uncommon.

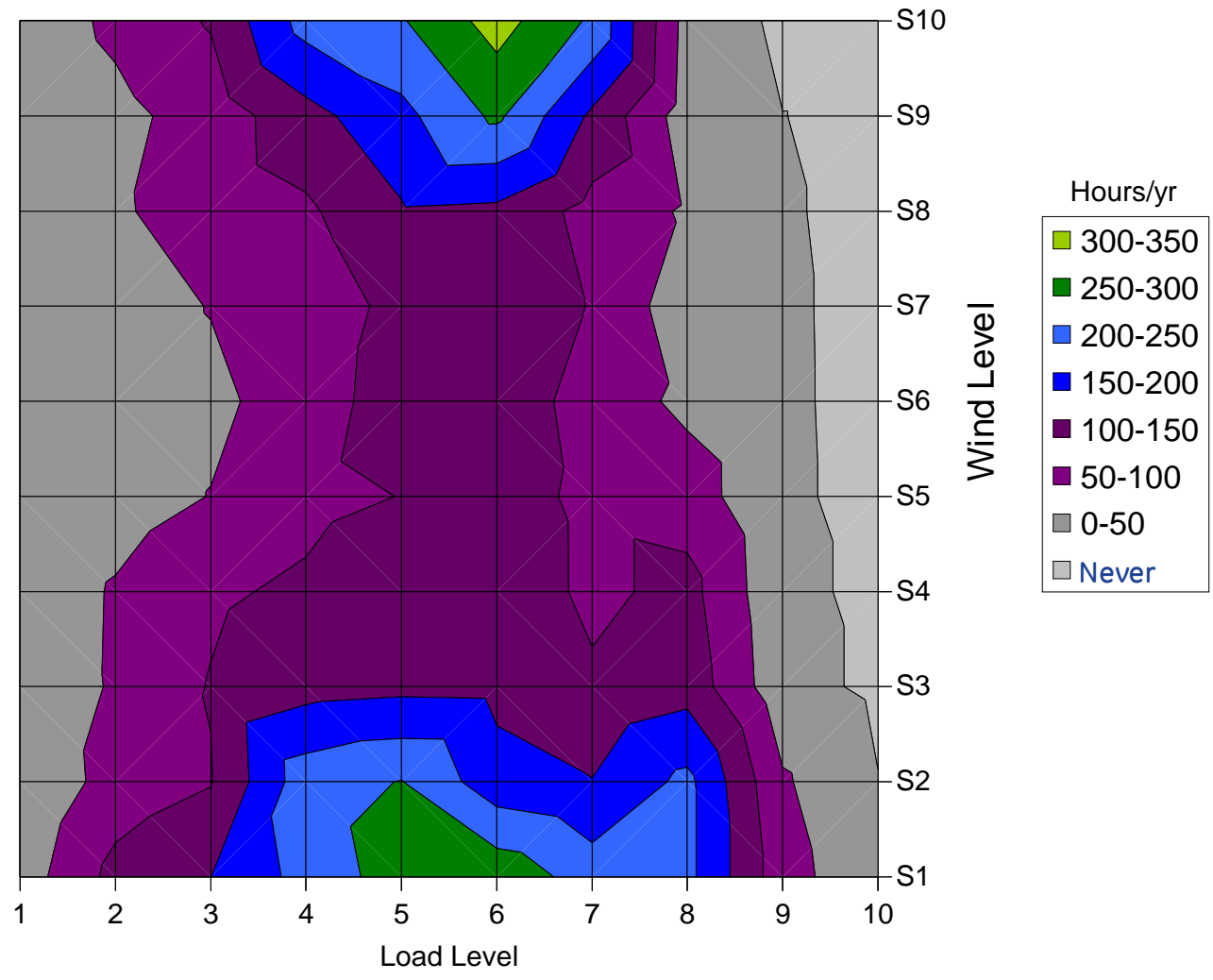

Figure 7.64 Wyoming L30 Time Space

The dominance of wind in the Wyoming system raises some interesting questions. For the calculation of reserves, is it reasonable to consider all net load variations? The primary concern for reserves is for rises in net load. Down reserves, which will be examined in the next section, are of less concern. Figure 7.65 shows the results of an exercise in which only the frequency and amplitude of increases in net load, which are dominated by drops in wind, are considered. The lower figure is the mean value of all net load rises, $\mu$, plus three times the standard deviation of the distribution of rises, $3 \Delta \sigma-$ rise. The upper plot shows the $3 \Delta \sigma$ implied reserve.

This result is consistent with other wind variability statistics. Over the majority of operating conditions, the expectation of wind variability is symmetric. Adding distinction between up and down to assessment of the required reserve does not appear to significantly improve the information. 


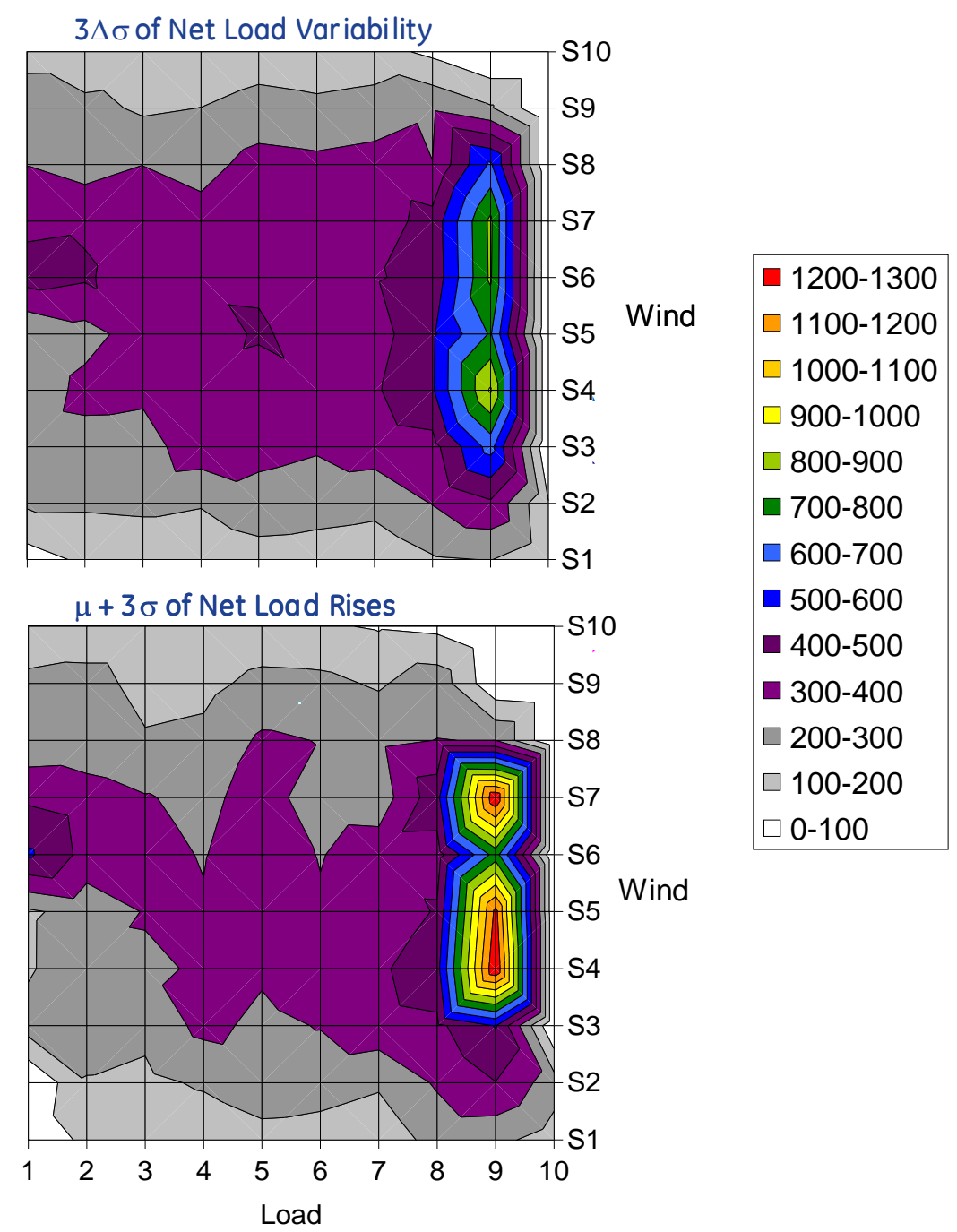

Figure 7.65 Wyoming L30 Net Load Variability: $3 \Delta \sigma$ vs. $\mu+3 \Delta \sigma-R i s e$

Figures for all the individual areas present a somewhat confusing picture of the reserve requirements. However, when viewed together, the trends become more understandable. A summary of the implied $3 \Delta \sigma$ requirements for the individual areas is shown in Figure 7.66. All the points in this figure are for average values over the year. The average reserve required to meet $3 \Delta \sigma$ of load-only is indicated with blue diamonds. The net load averages are shown as circles. For comparison, the average reserve required using the $3 \%$ of load spinning reserve rule are shown as red triangles. The insert gives the study footprint wide summation of the averages: $979 \mathrm{MW}, 730 \mathrm{MW}$ and 1,393 MW, for 3\% spinning reserve rule, load-only, and net load, respectively. The $1393 \mathrm{MW}$ average requirement represents a $90 \%$ increase over $730 \mathrm{MW}$, or roughly double. Thus, in order to meet $3 \Delta \sigma$ performance objectives with reserves carried by area, the reserve requirement increases about $90 \%$ from that carried today for load variability. 


\section{Reserve Requirements (by Area)}

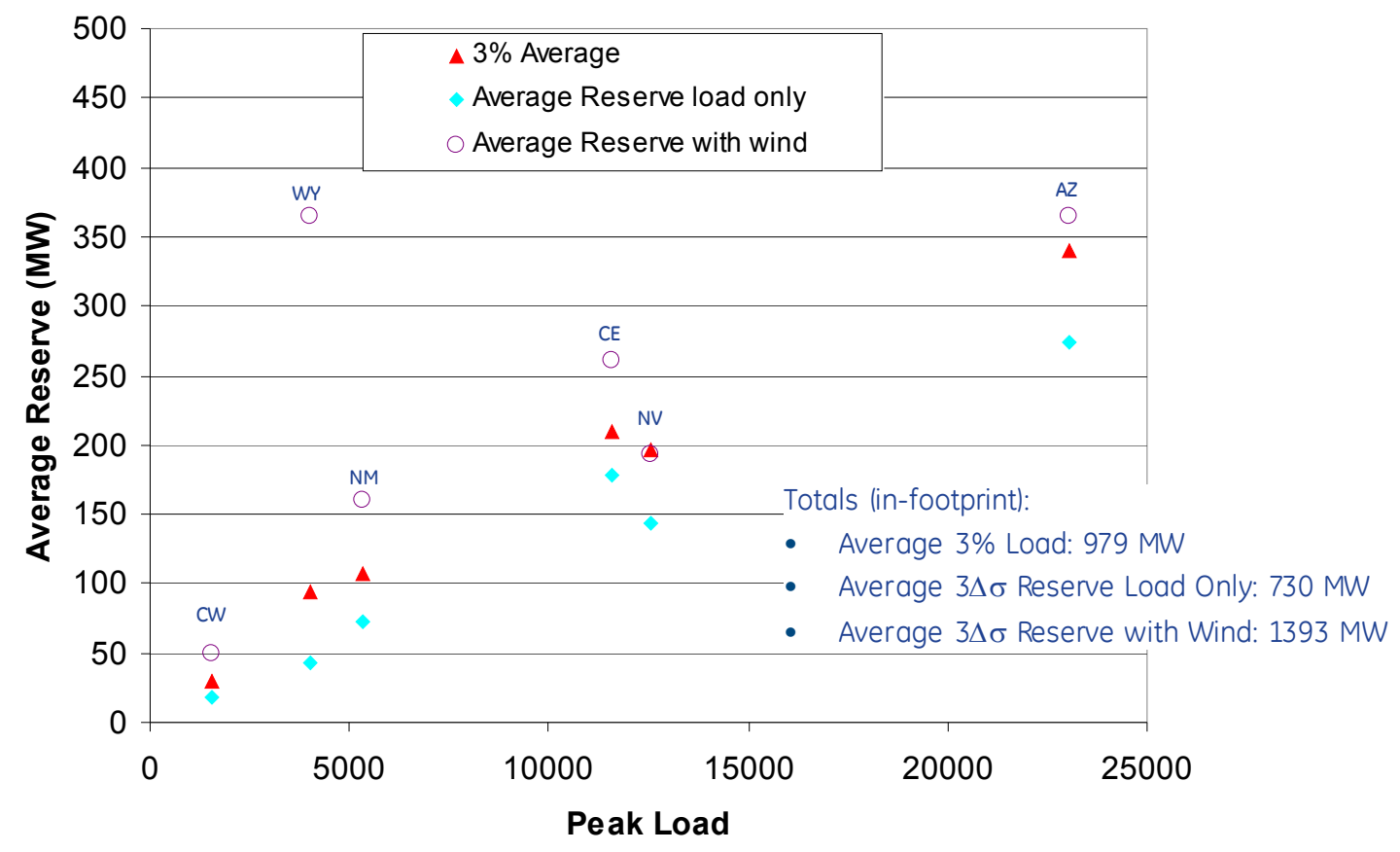

Figure 7.66 Average Reserve Requirements for Local Priority $30 \%$ Scenario

\subsubsection{Reserve Time Frames and Other Reserve Drivers}

The statistical analysis presented so far in the section is focused on 10-minute variability. As noted, the availability of sub-hourly reserves is driven in significant part by the unit commitment strategies employed in the day ahead. The hour-to-hour changes, examined in Section 5, impact the system hourly dispatch.

For successful operation of the system, some resources must be allocated that can respond rapidly to signals from system AGC. These regulation reserves are, for the purpose of this analysis, a subset of the sub-hourly variability reserves and not the spinning reserves. Since the regulating reserves are the primary mechanism by which the system maintains balance in the 5-minute intervals between economic redispatch, there must be enough regulating reserves to cover most changes that occur within those 5 minutes.

Five-minute data was unavailable for this study, but past study experience with system variability shows common characteristics. Figure 7.67 shows trend lines for a number of statistical analyses performed by GE on data from other regions of North America. The variability of net load over 5 minutes is statistically about $1 / 2$ that for 10 minutes. 
This provides some guidance for allocating regulation reserves. Since regulating reserves are backed up by longer time frame on-line reserves, the need for perfect balancing in the sub-economic dispatch time frame is less critical. The operational expectation that balancing within the 10-minute time frame will not always be perfect is also reflected in the NERC rules governing CPS2. The expectation is that systems will be within L10, i.e. will be successful in balancing in the regulation time frame, at least $90 \%$ of the time.

Since the 5 -minute variability is about $1 / 2$ of the 10 -minute variability, and there is an expectation of at least $90 \%$ success implied by CPS 2 rules, allocation of one 10 -minute $\Delta \sigma$ to regulation results in approximately $2 \sigma$, or $95 \%$, of 5 -minute variability being covered by regulation. This relatively simple rule then fits nicely with the $3 \Delta \sigma$ rule for overall sub-hourly on-line reserves.

This also relates well to the $1 \%$ of peak load regulation rule used in the QSS simulations as broadly representative of utility practice. In Figure 7.68 , the $1 \%$ of peak load rule (red triangles) is compared with the average requirement resulting from the $1 \Delta \sigma$ of $10-$ minute variability rule for all areas. The results are on the same order of magnitude.

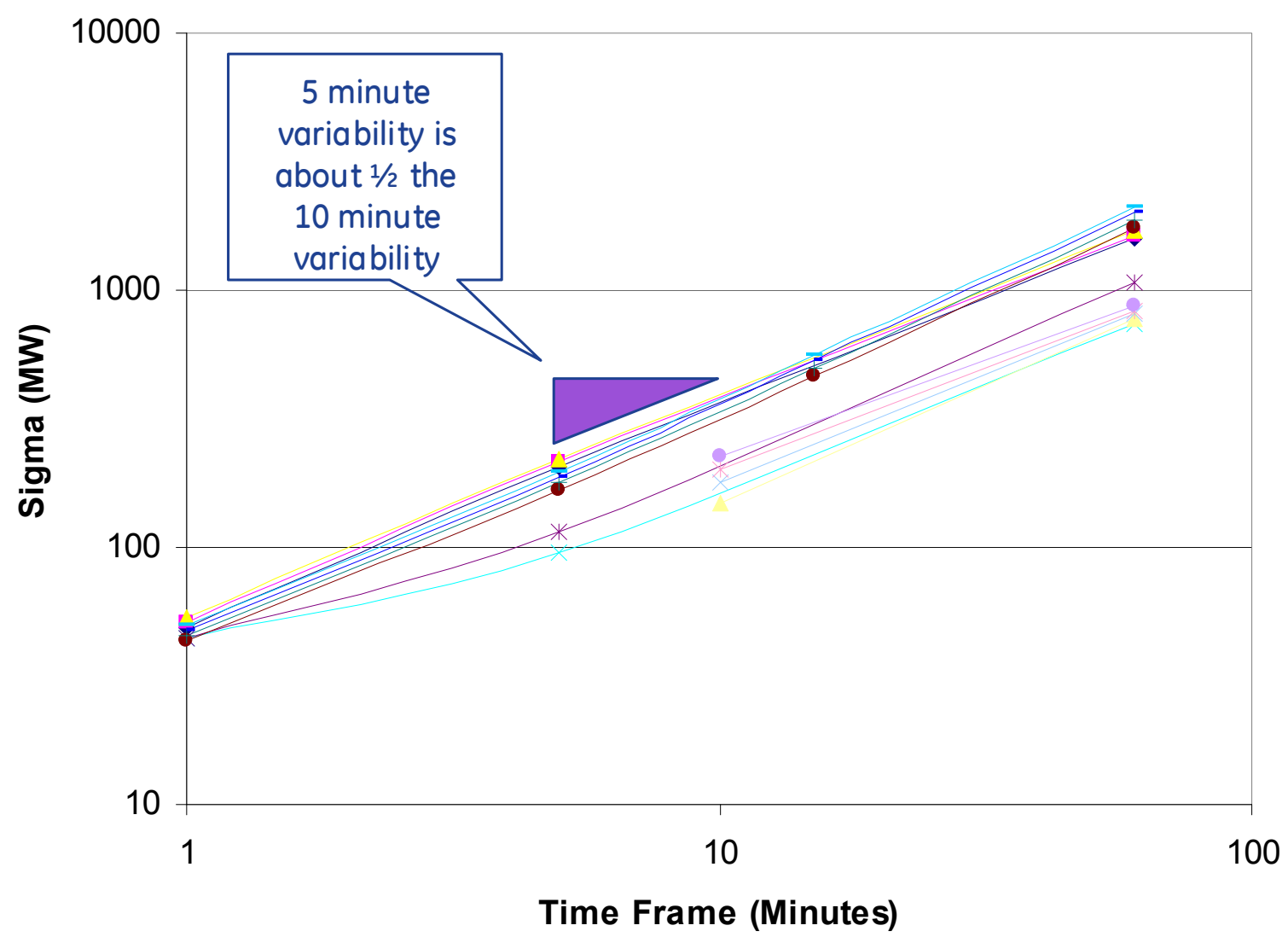

Figure 7.67 Net Load Variability in Different Time Frames - Various GE Studies. 


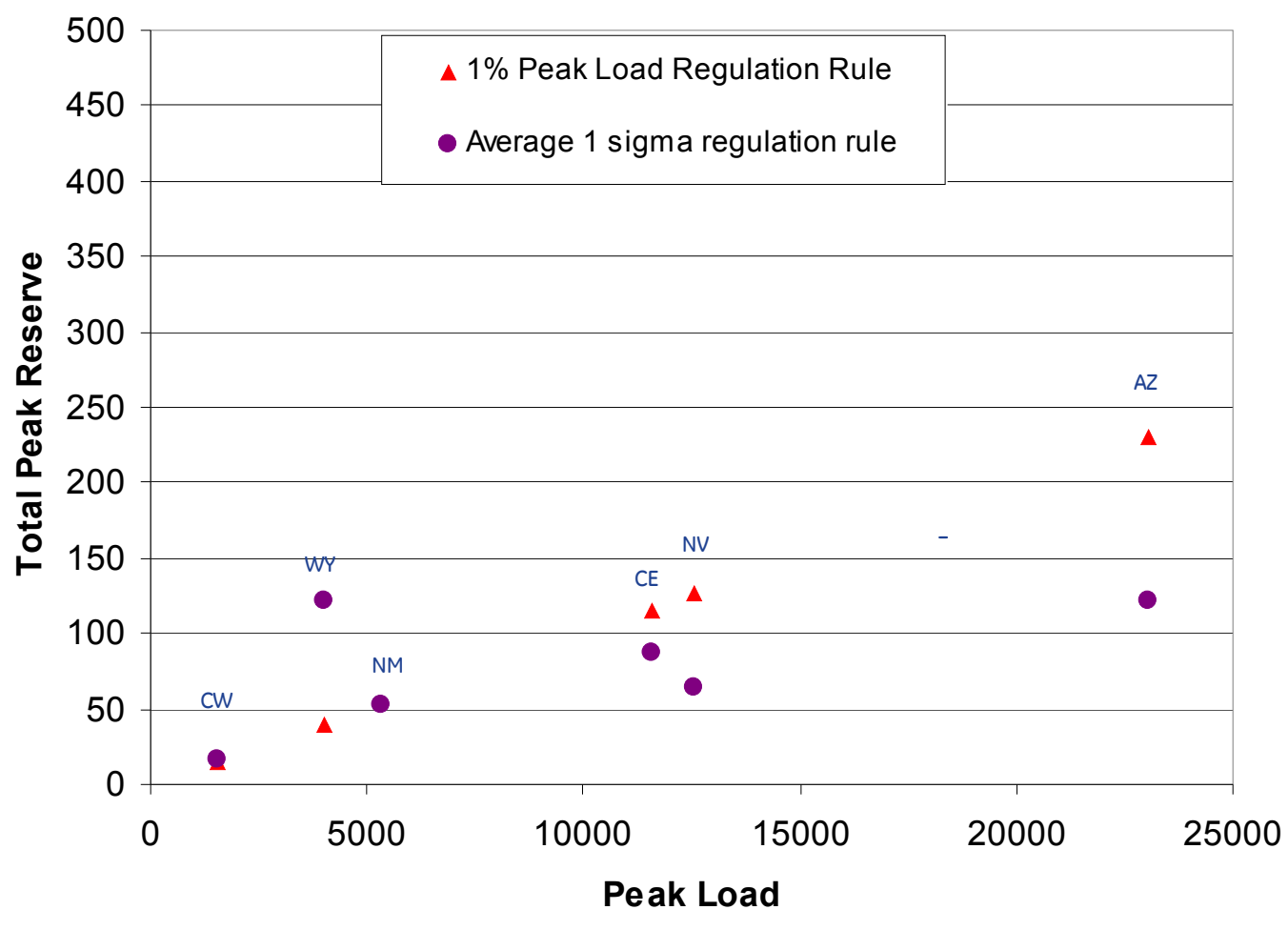

Figure 7.68 Regulation Reserve Requirements (by Area)

There are considerations beyond variability that drive system reserves. Most notably, the system must carry sufficient contingency reserves to handle the worst single system contingency (e.g. loss of the biggest plant) [7.5]. Thus industry practice is actually for $3 \%$ of load or for $50 \%$ of the worst contingency, which ever is greater. Further, systems are required to carry non-spinning reserves - off-line resources that can provide power within a specified time, such as 10 minutes, 15 minutes, and/or 30 minutes.

At this time, most systems consider these reserves to be additive. That is, separate reserves are typically carried to handle substantial load variation and contingency events. Continuation of this practice is implicit in the results and recommendations provided here.

\subsection{Concepts for Simple Reserve Rules}

The requirements for reserves, as they relate to system variability, have been presented in the preceding two sections. The relatively complex variability surfaces reflect the needs of the system. However, these surfaces in themselves do not present particularly usable information for system operations.

This section will explore concepts for reserve rules that could be used by system operators to allocate the reserves necessary to handle variability. These rules should be relatively simple and understandable; they should be based on the physical reality of the system, and they should be reasonably accurate. 


\subsubsection{Load and Wind Level Based Rules}

In the following subsections different concepts, and how they relate to the different areas, are presented. Graphical analysis for this work covered each of the areas. However, only a subset of the results are used to illustrate each of the potential rules. Results for all areas are presented in Appendix G. In each successive section of the appendix, the area results are reported in the sequence Arizona, Wyoming, Colorado East, Nevada, New Mexico, and Colorado West. This is the descending order of installed wind power in the six areas for $30 \%$ penetration in the Local-Priority Scenario.

\subsubsection{3+5 Rule}

In this section, a simple and somewhat conservative heuristic rule is explored: $3 \%$ of load (as suggested by the discussion in Section 7.3.3 ) plus 5\% of the wind. Note that this is $5 \%$ of actual or short-term forecasted wind, and not $5 \%$ of installed nameplate MW. This is a " $3+5$ rule" that governs the commitment of additional reserves equal to $5 \%$ of the wind forecast, as discussed in Section 6.2.2.

Figure 7.69 shows the $3+5$ rule for the study footprint. The figure presents three surfaces. The top surface is the implied $3 \Delta \sigma$ of net load requirement for the study footprint. This is exactly the same information presented in Figure 7.57, except that variability has been multiplied by three to get $3 \Delta \sigma$. The middle surface is the amount of reserves that would be carried to meet the $3+5$ rule. The bottom surface is the margin, or difference, between the $3+5$ rule and the $3 \Delta \sigma$ reserve requirement. Violations occur when this margin is negative. For the study footprint there are no cells, and therefore, no conditions for which the $3+5$ rule results in insufficient reserves to meet the implied $3 \Delta \sigma$ requirement for net load variability. For most conditions, considerably more reserves are carried than are needed to meet the $3 \Delta \sigma$ requirement. 


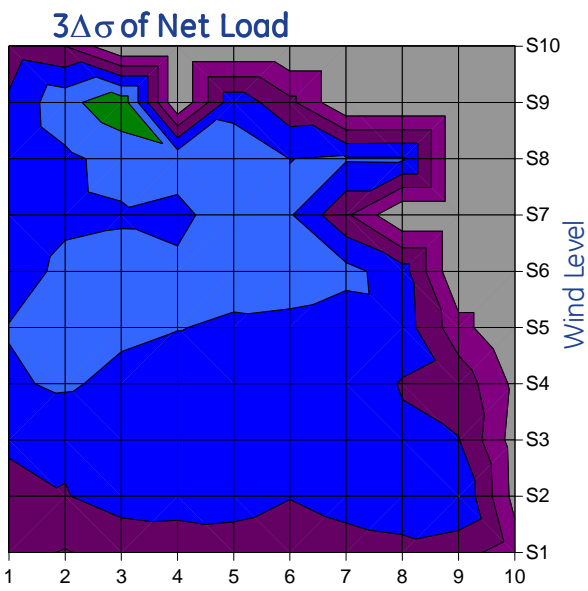

$3 \%$ Load and $5 \%$ Wind Rule

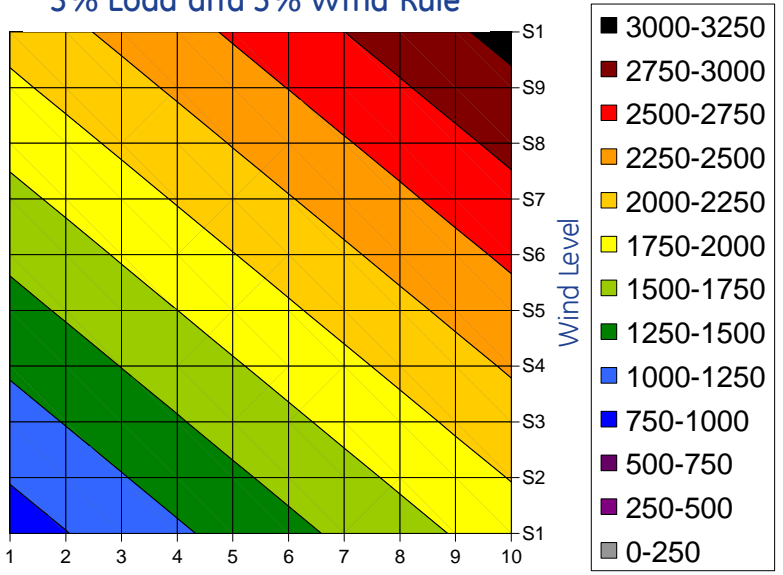

3+5 Rule Margins

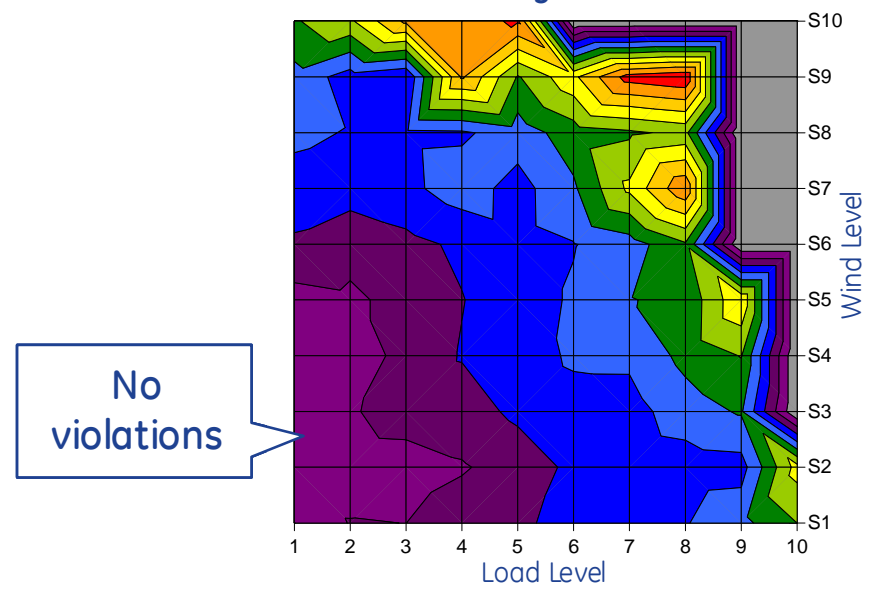

Figure 7.69 Study Footprint L30 3\% Load Plus 5\% Wind Rule

The performance varies by individual area. The $3+5$ rule performance for Arizona is shown in Figure 7.70. There are generally positive margins, although pockets of violations occur at some rare periods of high load and moderate wind (load 8 and wind 6). Similar graphics for the other areas are included in Appendix G. 


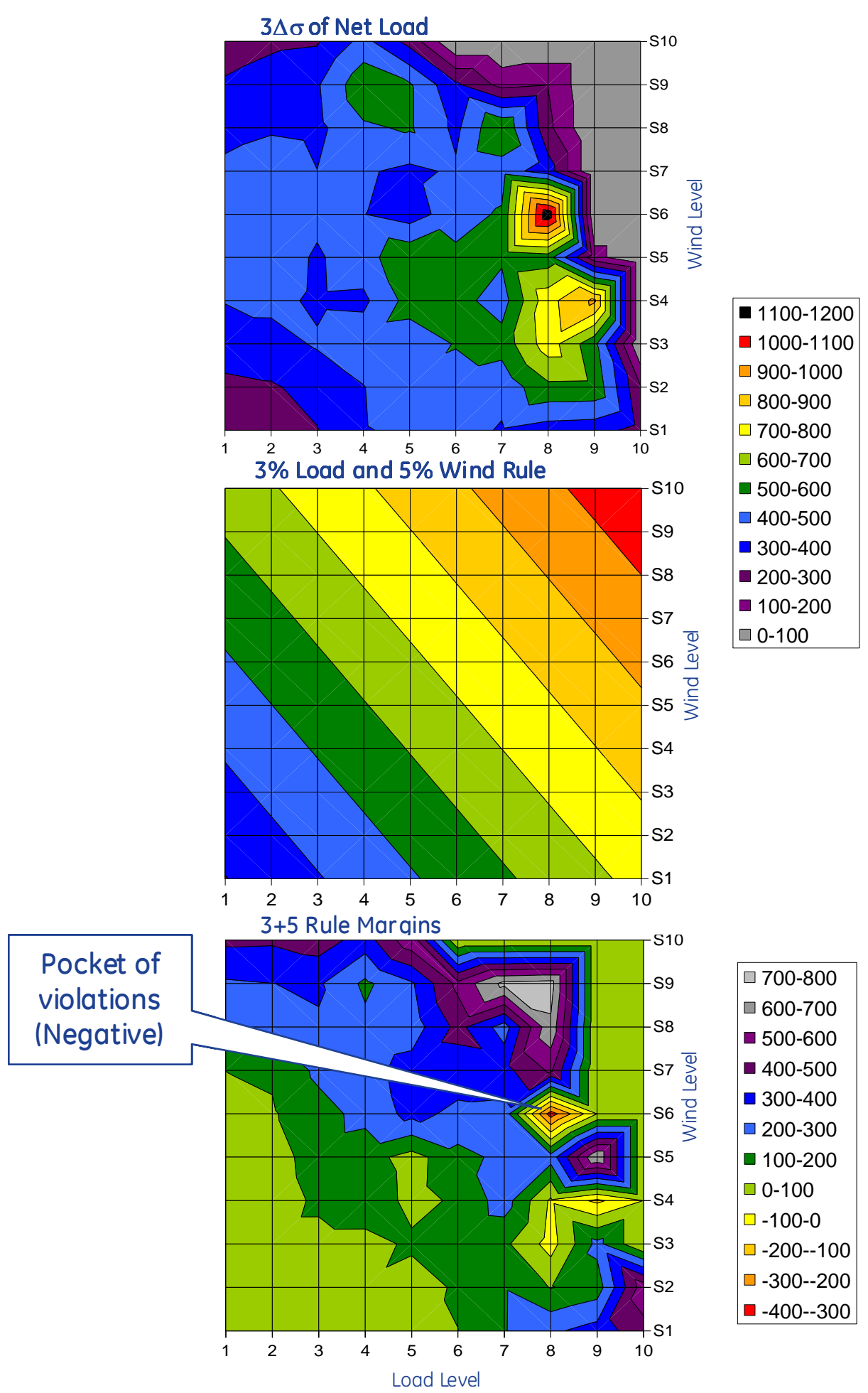

Figure 7.70 Arizona L30 3\% Load Plus 5\% Wind Rule

The $3+5$ rule performance for $W y o m i n g$ showed that reserves were generally short of the requirements. Colorado East had pockets of violations at high wind and moderately light load. Colorado East shows higher volatility at high wind than is observed in other 
areas. Nevada had violations at moderate wind and light load. New Mexico had small violations at unusually high wind and light load conditions. Colorado West's reserves were almost entirely inadequate across the entire load and wind space.

In summary, the $3+5$ rule usually works. It is conservative at the study footprint level, i.e. assuming control areas cooperate for intra-hour balancing. The performance of individual areas ranges from good coverage of the $3 \Delta \sigma$ reserve requirements to frequent violations. This suggests that customized reserve rules for individual areas may be beneficial.

\subsubsection{General Load + Wind Rules}

The results shown in the preceding section suggest that simple rules, like the $3+5$ rule hold promise. As noted above, the $3+5$ rule is a logical extension of the linear fit to the load only variation of Figure 7.51. While that fitted line depended only on load, the $3+5$ rule is a plane in the load and wind space. However, the $3+5$ rule was chosen heuristically, i.e. it was an educated guess. In this section, a more rigorous mapping from the complexity of the $3 \Delta \sigma$ requirement surfaces to a "best" planar fit rule is introduced.

The idea of a fit is relatively simple. Single scalar values of $X$ (a "load coefficient") and $Y$ ( $a$ "wind coefficient") are determined, such that the error, given by:

$$
\text { Equation } 7.1 \text { error }=\iint \beta(\lambda, \omega) *(3 \Delta \sigma(\lambda, \omega)-(X \lambda+\mathrm{Y} \omega)) 2 \mathrm{~d} \lambda \mathrm{d} \omega
$$

is minimized, where

$\lambda$ is a particular load level

$\omega$ is a particular wind level

$\beta(\lambda, \omega)$ is the probability of being at a particular load $\lambda$ and wind power $\omega$

$\Delta \sigma(\lambda, \omega)$ is the 10 -minute delta sigma of the net load

The equation is integrated over the entire load and wind space, as previously described. The net result is an "RMS" best fit.

To find the minimum error, the equation is solved using a simple gradient descent algorithm. The resulting optimum $X$ (the load coefficient) and optimum $Y$ (the wind coefficient) provide a new rule of the same character as the $3+5$ rule, but with a best fit. For the subsequent discussion, these are called " $X+Y$ " rules.

For the study foot print, the $X+Y$ rule is shown in the left surface of Figure 7.71. The load and wind coefficients are 1.3 and 3.6, respectively. The right surface plot shows the $3 \Delta \sigma$ requirement for which the $X+Y$ surface is the best fit. By comparison, the $X+Y$ rule 
performance for Arizona is shown in Figure 7.72. The load and wind coefficients are 2.5 and 2.0, respectively. The fit is broadly consistent with the complex $3 \Delta \sigma$ surface. Each of the areas (for which similar plots are provided in Appendix $\mathrm{G}$ ) result in a range of load and wind coefficients. For example, the Wyoming load and wind coefficients are $5.6 \%$ Load $+2.1 \%$ Wind. This particular result is difficult to accept, since it apparently provides too little reserve, especially at lower wind and load levels. As observed in Figure 7.63, the net load variability is dominated by wind, which has a roughly quadratic shape. And a planar fit is not well-suited to such a surface. Additional experiments with Wyoming are presented in the appendix. Colorado East has load and wind coefficients of $2 \%$ Load $+5.5 \%$ Wind. As noted above, Colorado East seems to have uncharacteristically high variability at high wind and moderate load. This might reflect something particular to the terrain or wind regime at high wind conditions. This reinforces the idea of customized reserve requirements for each area. Nevada has load and wind coefficients of $1.3 \%$ Load $+7.8 \%$ Wind, for an acceptable fit. New Mexico's coefficients of $3 \%$ Load $+3.6 \%$ Wind provide a relatively good fit. The reserves are somewhat generous at high load, moderate wind and a little tight in the highest wind conditions. And finally, Colorado West has load and wind coefficients of 3.1\% Load + $7.3 \%$ Wind. The high wind co-efficient is likely a consequence of the relative lack of geographic diversity for the wind plants in this area.

The results provided in this section do a reasonable job of matching the implied $3 \Delta \sigma$ requirements for net load. Nevertheless, they tend to exceed the requirements at high load and wind conditions, and to be short at lower load and wind conditions. This suggests that further refinement may be desirable. One possible refinement is presented in the next section.
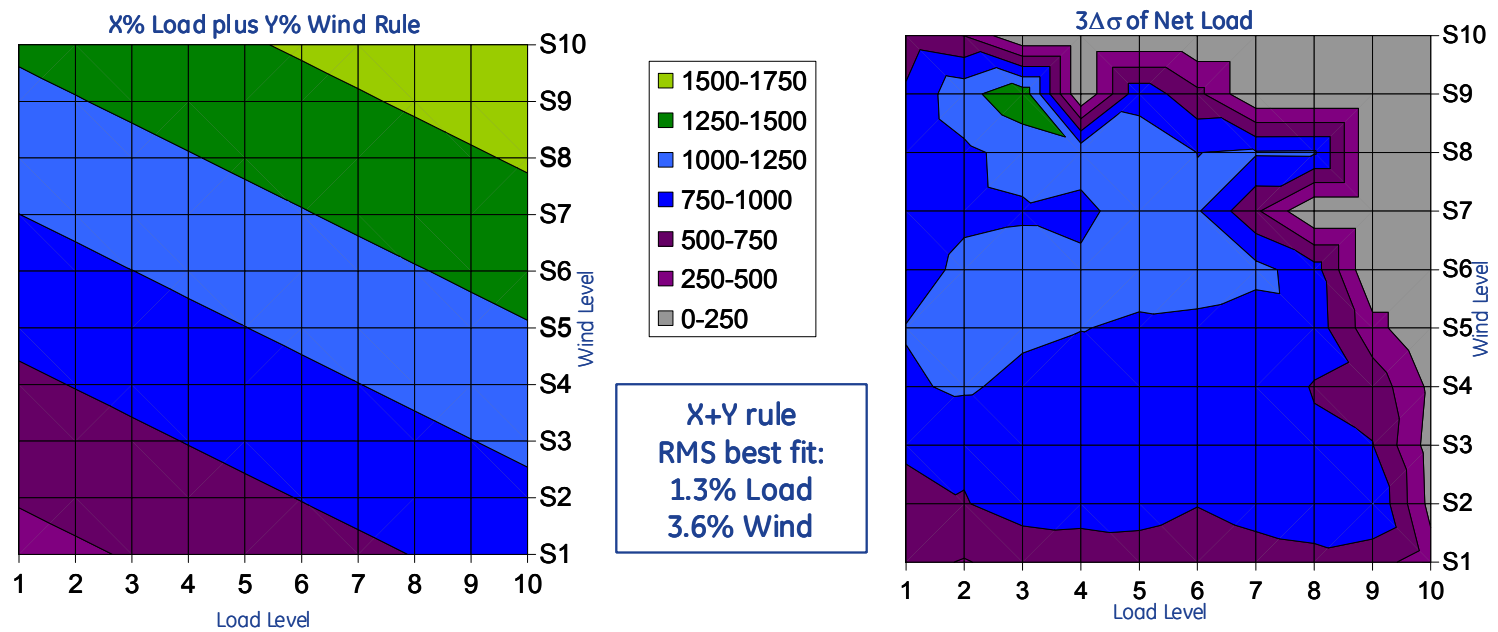

Figure 7.71 Study Footprint L30 Best RMS Fit X\% Load Plus Y\% Wind Rule 

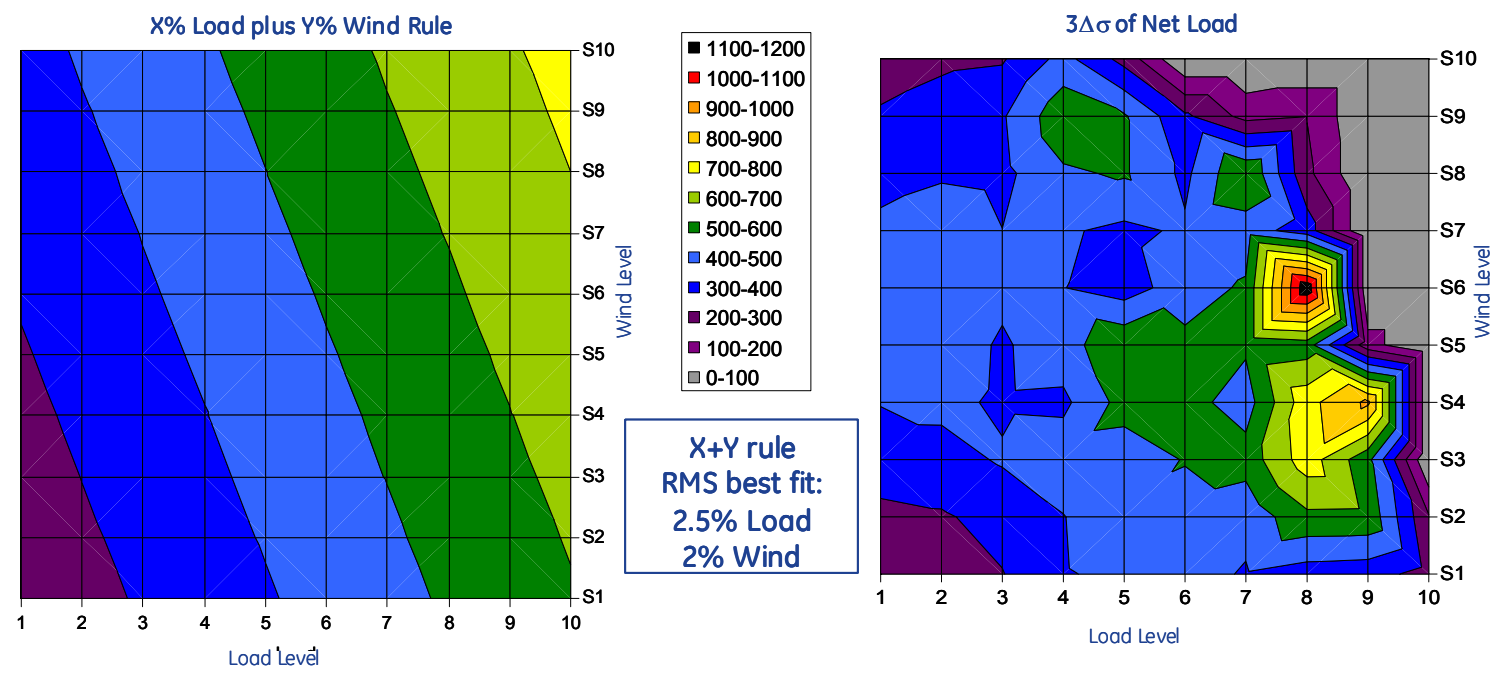

Figure 7.72 Arizona L30 Best RMS Fit X\% Load Plus Y\% Wind Rule

\subsubsection{Conditional Load + Wind Rules}

The variability due to wind, as shown in Figure 7.49, tends to reach a relative maximum at part power. As a result, there is no reason to increase reserves above a certain level of wind. In this section, conditional Load plus Wind rules are examined. Specifically, a wind power level is identified above which no additional reserves are added.

The error minimization of Equation 7.1 is used again, but with an extra degree of freedom. Therefore, the optimization problem has three degrees of freedom: the load coefficient $(X)$, the wind coefficient $(Y)$, and now, a specific wind level, above which the reserves no longer increase in proportion to the wind power. With this approach, the RMS fit to the $3 \Delta \sigma$ requirements surface can be greatly improved. In each case, the minimization of Equation 7.1 includes a conditional maximum, resulting in a load coefficient $(X)$, a wind coefficient $(Y)$ and a percentage of nameplate wind.

Representative results of this " $\mathrm{X}+\mathrm{Y}$ conditional" rule are presented here, with additional supporting graphics in Appendix G.

The study footprint $X+Y$ conditional rule is shown in Figure 7.73. The results for the $X+Y$ conditional rule best fit are: $1.1 \%$ load $+5.0 \%$ wind for up to $47 \%$ of nameplate wind. That is, variability reserves are equal to $1.1 \%$ of load plus $5.0 \%$ of the wind production up to a maximum of $47 \%$ of the installed nameplate of wind. For this case, the installed nameplate is $26,760 \mathrm{MW}$, so $47 \%$ of that is $12,577 \mathrm{MW}$, and $5.0 \%$ of that is $629 \mathrm{MW}$. Therefore, the wind term never exceeds $629 \mathrm{MW}$ for the footprint. This fit is generally satisfactory. The conditionality is closely related to the simple piecewise linear curve fit of Figure 7.46. The $3 \Delta \sigma$ slope fit for that figure is $10 \%$ wind up to $30 \%$ wind nameplate. This is somewhat different because of the presence of the load term in the fit and because of the RMS optimization. 
The $\mathrm{X}+\mathrm{Y}$ conditional rule performance for Arizona is shown in Figure 7.74. The results for the $X+Y$ conditional rule best fit are: $2.2 \%$ load $+5.6 \%$ wind up to $36 \%$ wind nameplate. With the added conditionality, the fit is generally satisfactory. The results for the Wyoming $\mathrm{X}+\mathrm{Y}$ conditional rule best fit are: $2.2 \%$ load $+8.7 \%$ wind up to $33 \%$ wind nameplate. This fit is better. This approach still carries too much reserve at high wind, based on the $3 \Delta \sigma$ requirement. Further improvements seem desirable. The concepts outlined in Section 7.4.7 may be appropriate for very high wind penetration systems. Additional experimental reserve rules for Wyoming are shown in Appendix $\mathrm{G}$ as well. The Colorado East results for the $X+Y$ conditional rule best fit are: $2.0 \%$ load $+5.7 \%$ wind up to $68 \%$ wind nameplate. In this case, the conditionality is not so beneficial for the high volatility, high wind, and moderate load condition. The Nevada $\mathrm{X}+\mathrm{Y}$ conditional rule performance for best fit are: $1.0 \%$ load $+10.7 \%$ wind up to $54 \%$ wind nameplate. This fit is generally satisfactory. The heavy weight on wind is somewhat at odds with the results from the other fits. However, convergence of the gradient descent optimization for this area was very sensitive. Therefore, the optimum locus must be relatively flat in load and wind space. This suggests that a different set of load + wind coefficients could give similar results. The New Mexico best fit $X+Y$ conditional rule coefficients are: $3 \%$ Load $+3.5 \%$ Wind up to $70 \%$ wind nameplate. Inspection suggested that the result was no better than that with just a simple $X+Y$ rule. Additional experiments are included in the appendix. Interestingly, the $X+Y$ conditional rule performance for Colorado West is the same as the simple $X+Y$ rule. That is, the wind term increases linearly at a rate of $7.3 \%$ of wind up to maximum wind. This is somewhat surprising, but may be a consequence of the relative lack of spatial diversity in Colorado West wind sites.

In summary, the $X+Y$ conditional rules appear to strike a good compromise between simplicity and fidelity of fit to the $3 \Delta \sigma$ requirements of load and wind variability.
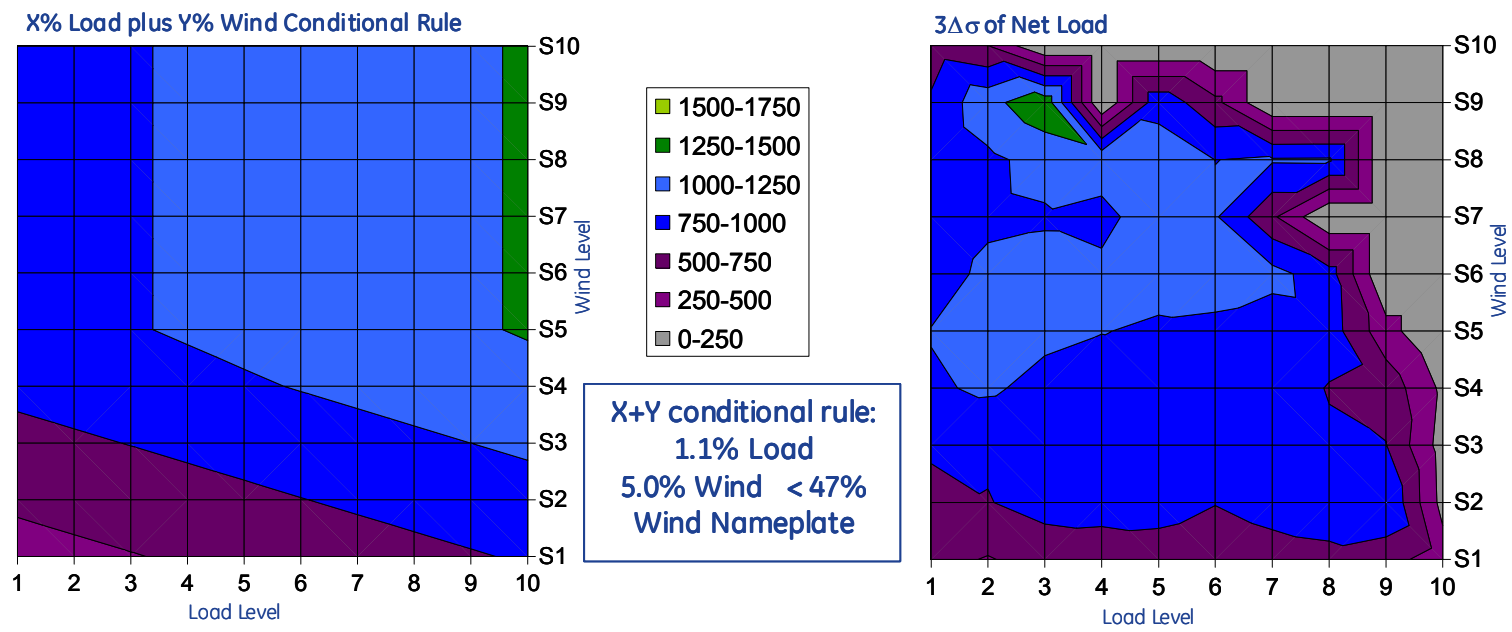

Figure 7.73 Study Foot Print L30 X\% Load Plus Y\% Conditional Wind Rule 


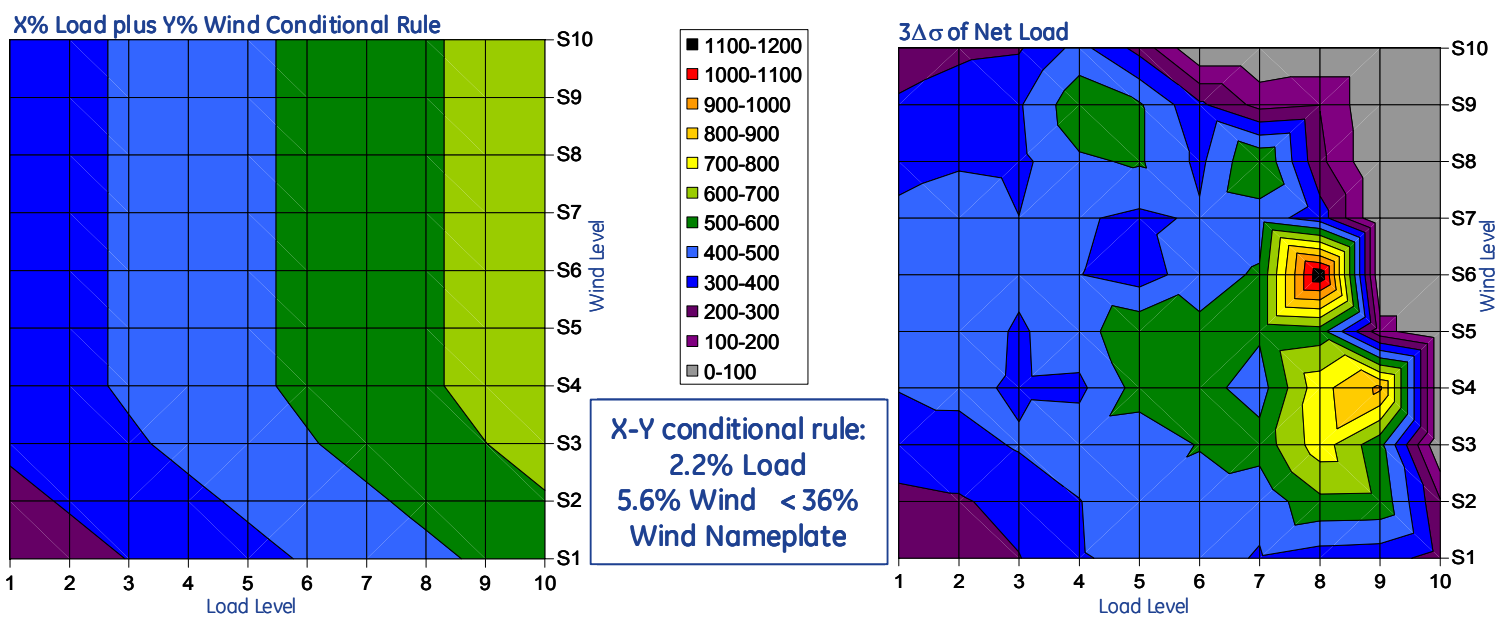

Figure 7.74 Arizona L30 X\% Load Plus Y\% Conditional Wind Rule

\subsubsection{Load-Only Rules}

The results presented throughout this section raise some questions about the existing practice for determining load variability reserves. The "need" for incremental reserves is based on the presumption that without wind, the "right" reserves are already carried. However, as noted in Section 7.3.1, present practice does not dictate specific levels of reserve, only performance targets. In the preceding section, rules based on wind and load levels that "fit" the implied $3 \Delta \sigma$ requirements were developed. For comparison, it is useful to calculate a mathematical equivalent for load-only variability.

Therefore, load-only variability is tested against a simple 3\% rule, and then against rules similar to the $\mathrm{X}+\mathrm{Y}$ and $\mathrm{X}+\mathrm{Y}$ conditional rules for wind and load variability. The intent is to provide a consistent basis for comparison between the load only variability rules and those with wind. Results are presented here for Arizona.

The $3+0$ rule for performance for Arizona is shown in Figure 7.75. The load variability surface is on the left. The center surface is the $3 \%$ of load rule, which is simple since it lacks dependence on wind. On the right, the difference between the $3 \%$ of load rule and the $3 \Delta \sigma$ of load-only is shown. There is one small area of violation, but overall the $3 \%$ of load is excessive throughout the load range, especially at high load levels.

Some experiments on load-only rules for Arizona are shown in Figure 7.76. The best RMS fit to the $3 \Delta \sigma$ of load-only, is $2.2 \%$ of load. The surface for this rule is shown in the upper right hand part of the figure. Like the rules for wind, and as was observed in Figure 7.51 , the $3 \%$ rule results in excess reserves at high load. This holds true for this $2.2 \%$ best fit as well. This suggests that a rule with a maximum condition would fit better. The best conditional fit for load-only is shown in the lower left surface. For this rule, $2.4 \%$ of load up to $50 \%$ of the load range (NOT 50\% of peak load!) gives a better fit. The relationship between the two fits is sketched onto the net load variable curves (from 
Figure 7.51) for illustration. The orange line is $2.2 \%$ of load. The blue line shows the steeper $2.4 \%$ slope reaching a maximum at $50 \%$ of the load range. Similar results for the other five areas are included in Appendix G.

These load-only results for each area are summarized in Figure 7.77. The average reserves carried to meet the 3\% of load for spinning reserves rule are shown with a red triangle. The average for the entire footprint is $979 \mathrm{MW}$. For comparison, the average reserve needed to meet $3 \Delta \sigma$ of load-only is shown in blue diamonds. The average for the study footprint is $730 \mathrm{MW}$. The maximum of the load-only conditional rule is $880 \mathrm{MW}$.

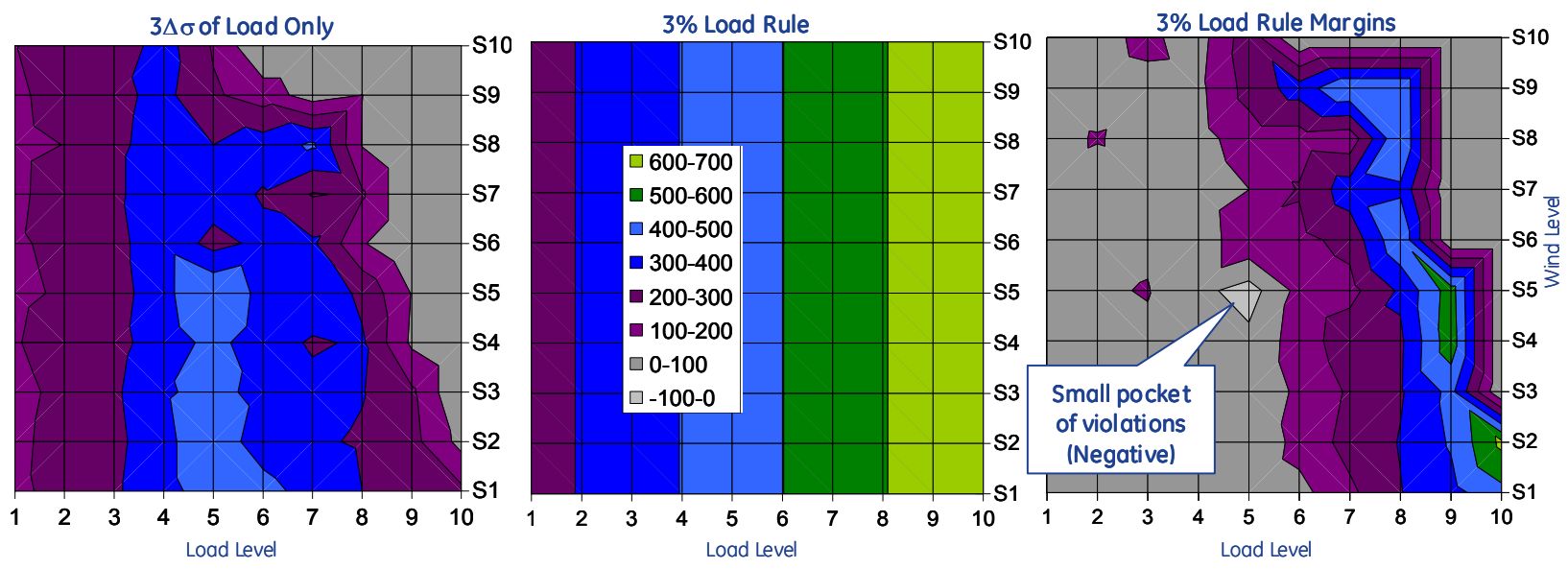

Figure 7.75 Arizona L30 Load-Only Variability 

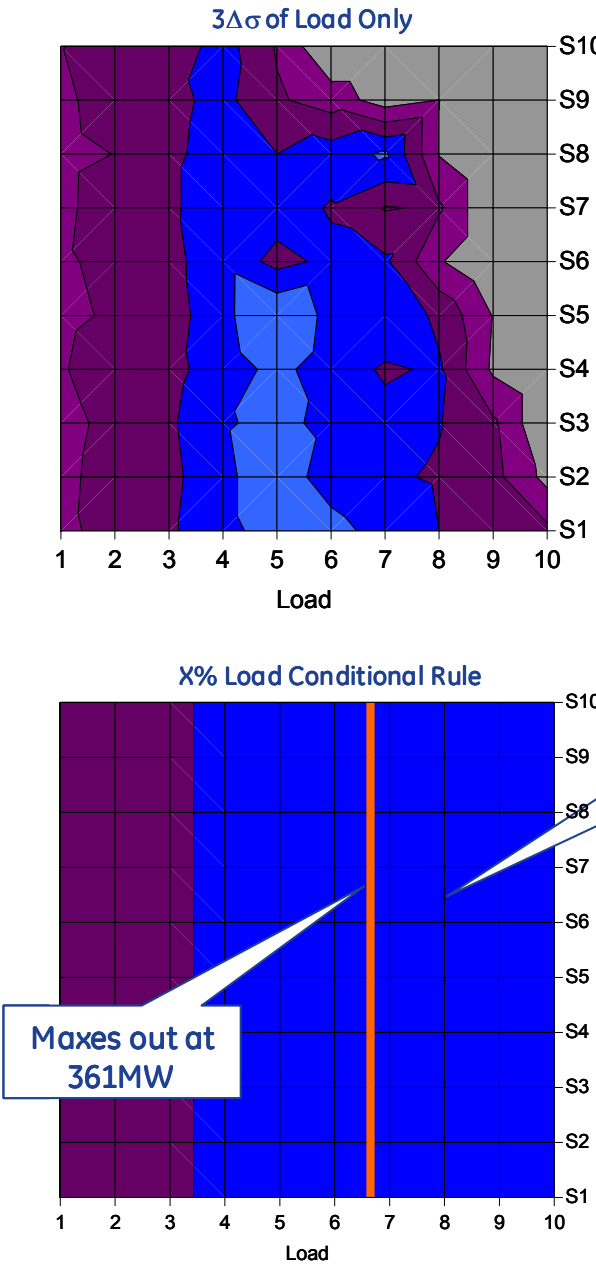

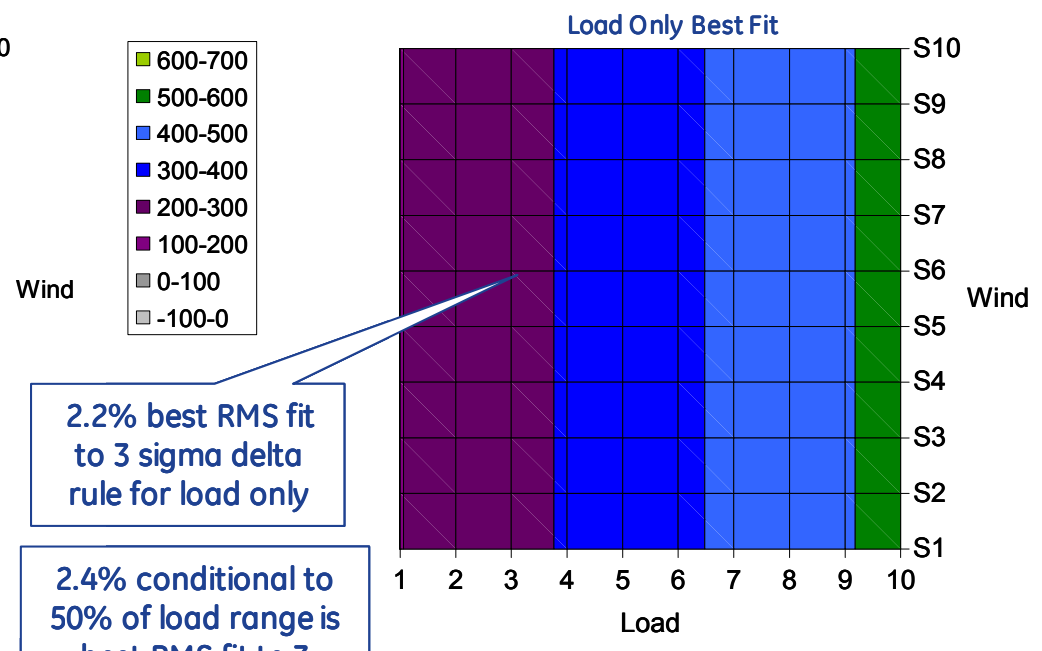

best RMS fit to 3

sigma delta rule for load only

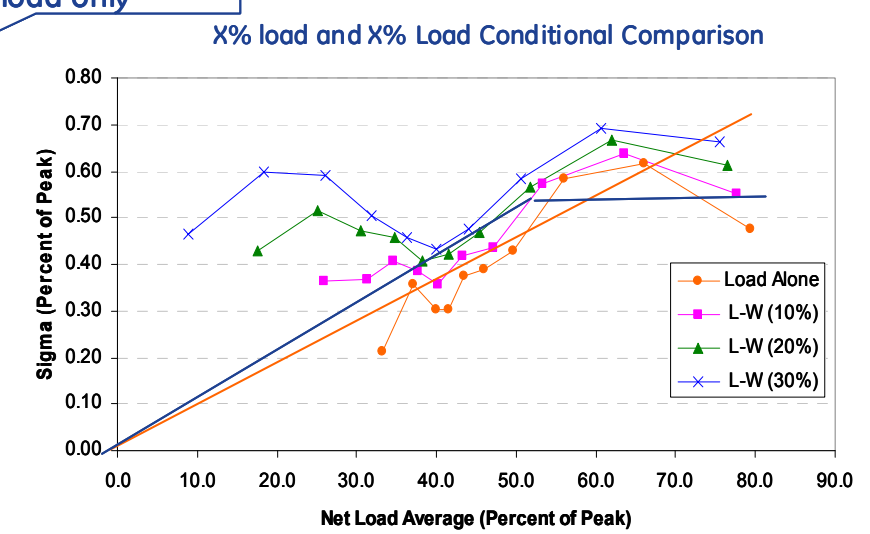

Figure 7.76 Arizona L30 Scenario Load-Only Variability 


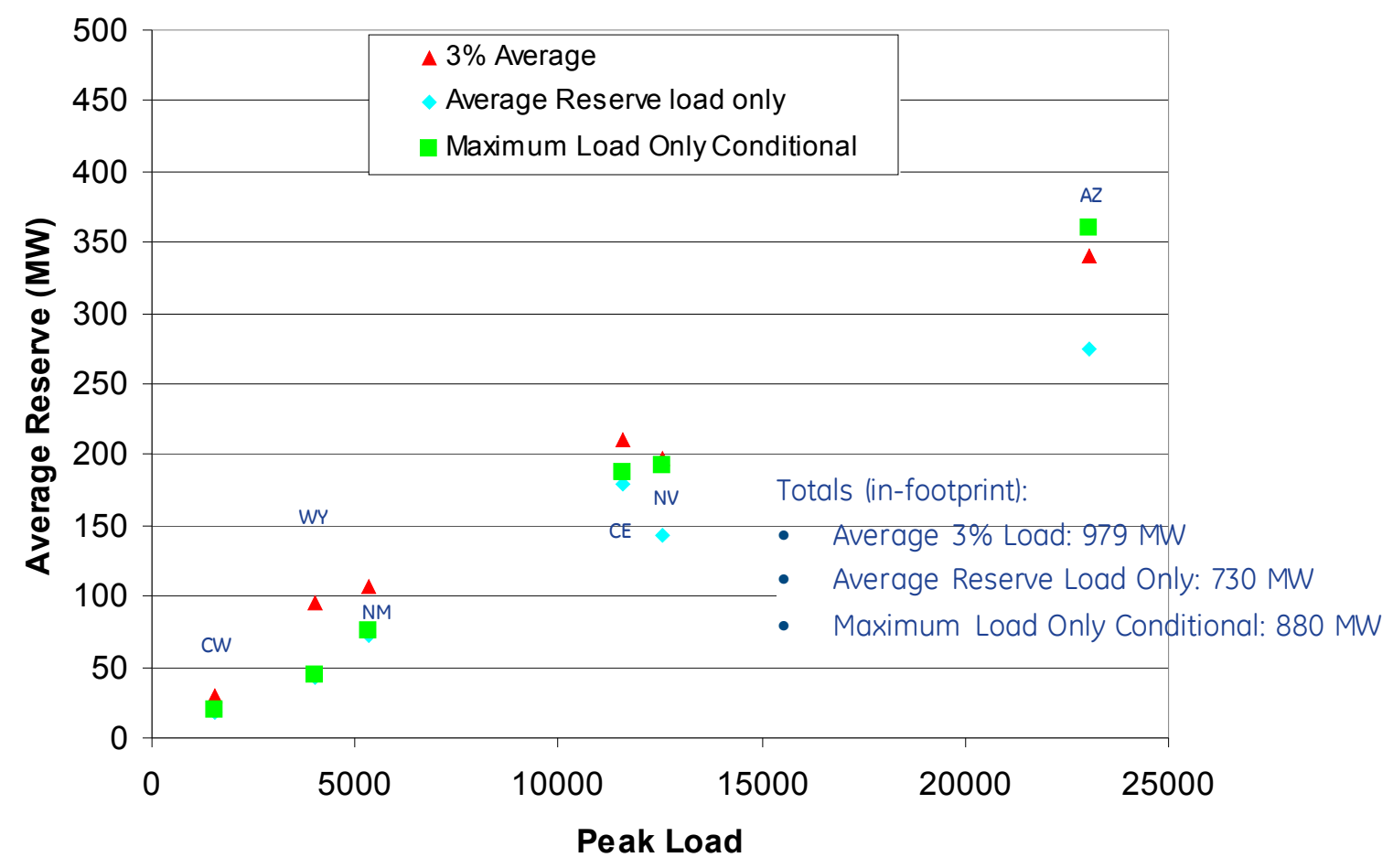

Figure 7.77 Regulation Reserve Requirements for Load-Only (by Area)

\subsubsection{Summary and Trends for Simplified Reserve Rules}

Overall, the investigations presented in this section show promise for the creation of simple rules that can be used by system operators to allocate subhourly reserves. The conditional $\mathrm{X}+\mathrm{Y}$ rules summarized in Table 7.14 are a good step forward.

Table 7.14 Summary of Variability Reserve Rules

\begin{tabular}{|l|c|c|cc|}
\hline \multirow{2}{*}{} & Load Only & \multicolumn{3}{|c|}{$30 \%$ LP Scenario } \\
\cline { 3 - 5 } & (\% of load) & $\begin{array}{c}\text { Load Term } \\
\text { (\% of load) }\end{array}$ & $\begin{array}{c}\text { Wind Term } \\
\text { (\% of wind } \\
\text { production) }\end{array}$ & $\begin{array}{c}\text { up to } \\
\text { (\% of wind } \\
\text { nameplate) }\end{array}$ \\
\hline \hline Footprint & 1.3 & 1.1 & 5 & 47 \\
\hline Arizona & 2.2 & 2.2 & 5.6 & 36 \\
Nevada & 2.1 & 1 & 10.7 & 54 \\
Colorado East & 2.4 & 2 & 5.7 & 68 \\
New Mexico & 2 & 3.1 & 3.5 & 70 \\
Wyoming & 1.3 & 2.7 & 8.7 & 33 \\
Colorado West & 1.8 & 3.1 & 7.3 & 100 \\
\hline
\end{tabular}

The coefficients of Table 7.14 are plotted in Figure 7.78, with a line showing the 3\% spinning reserve rule added for reference. These reserves are translated into MW levels, as shown in Figure 7.80. The values are the maximum reserve corresponding to peak load and/or peak wind. These figures show that (a) about twice the variability reserves are needed in the high wind case, (b) these reserves are of a similar order of magnitude 
to spinning reserve requirements, and (c) some refinements, based on the particular characteristics of the individual areas, will tend to redistribute those reserves.

There is substantial diversity between areas in the amount of incremental reserves required as a fraction of wind power. The relationships between area size, wind penetration, and incremental reserve requirements are not straightforward.

In Figure 7.78, the load and wind coefficients ( $X$ and $Y$, respectively) are shown relative to the total MW of installed wind. There is a roughly negative correlation between amount of wind installed and the required reserves on a per unit basis. This is as expected, and is a manifestation of the benefits of diversity.

The correlation is also somewhat negative from the perspective of power penetration (MW wind installed/MW peak load) in the top plot of Figure 7.79, and area size as measured by peak load in the bottom plot. All of these are driven by spatial diversity. The highest amount of reserves, on a per unit basis, is required in Colorado West. This is not surprising; as Colorado West has relatively few wind plants (a total of $570 \mathrm{MW}$ ), that are all in rather close geographic proximity. The spatial diversity here is relatively poor, and the incremental reserve requirement is higher. The diversity of reserve requirements is also apparent in Figure 7.79. That different areas have different reserve needs is not surprising, nor does it imply that those reserves must necessarily be provided within the particular area. Cooperation between neighboring areas is needed. Wyoming, for example, is a major exporter in the Local-Priority Scenario with 30\% penetration. Providing the reserves necessary to ensure exports can be maintained is suboptimal, and maybe even nonsensical. Higher reserves in the receiving systems may make sense. Indeed, there is an overall increase in the need for reserves due to wind generation.

Specifying the exact increase in reserve requirement is a matter of perspective. In Figure 7.66, the foot print wide average requirements for reserves were shown to be $730 \mathrm{MW}$ for average load-only variability, $1393 \mathrm{MW}$ for net load variability, and $979 \mathrm{MW}$ for the $3 \%$ of load spinning reserve rule. The increase from the load-alone requirement to the net load requirement is $90 \%$. For practical rules, such as the $\mathrm{X}+\mathrm{Y}$ and conditional load rules outlined in this section, the maximum requirements also increase. The maxima, listed in the call out of Figure 7.80, show a 57\% increase in the peak-required reserve for the conditional $\mathrm{X}+\mathrm{Y}$ rule (1946 MW vs. $1236 \mathrm{MW})$. However, when viewed against the load-only conditional maximum of $880 \mathrm{MW}$ (from Figure 7.77), the increase in reserve requirement is $121 \%$ (1946 MW vs. $880 \mathrm{MW}$ ). Ultimately, great precision in this discussion is not warranted: the reserve requirements roughly double. 

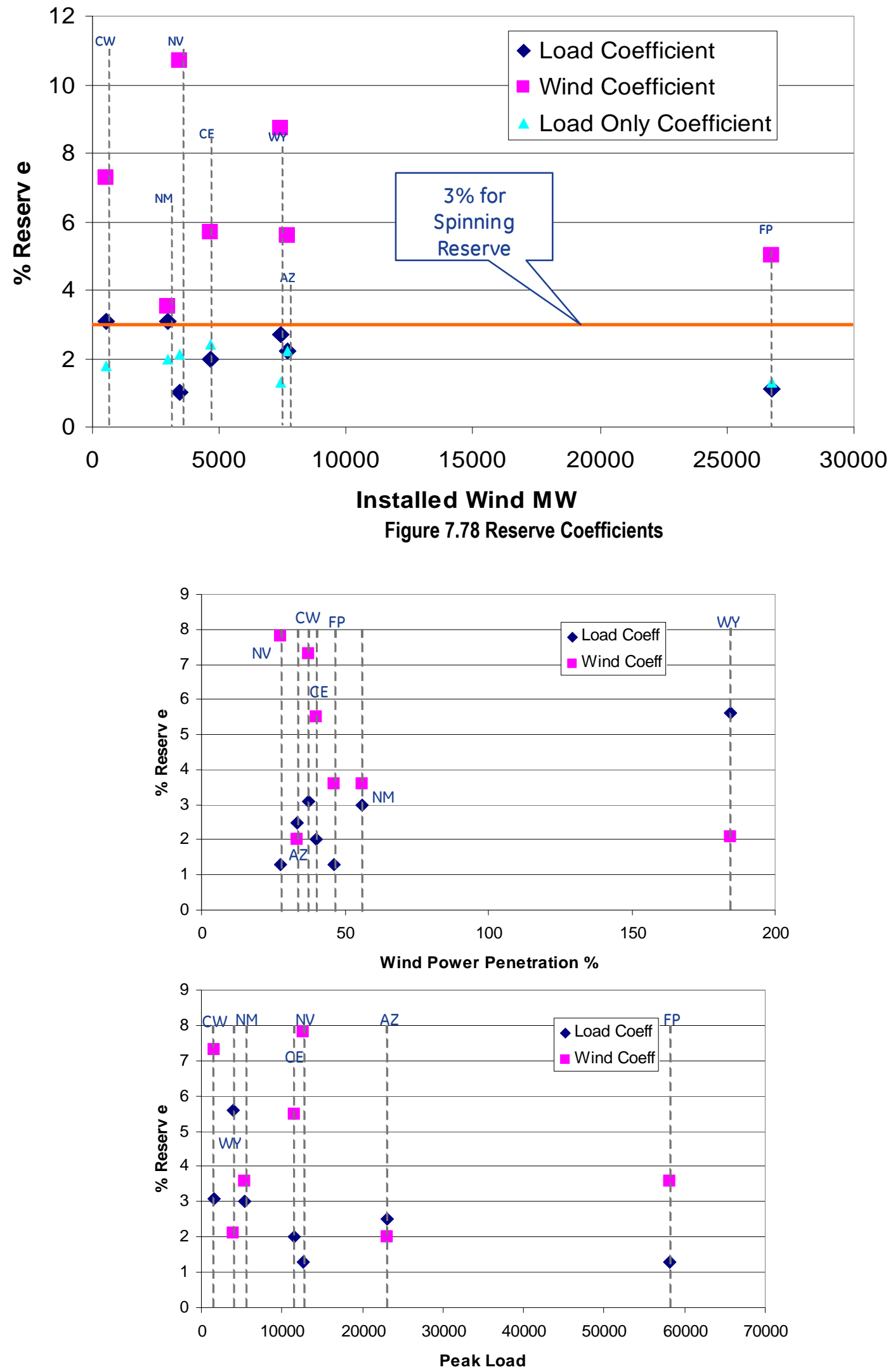

Figure 7.79 X\% Load and Y\% Wind Reserve Rule - Relationships to Penetration 


\section{Comparison of Peak Reserve Requirements Rules (by Area)}

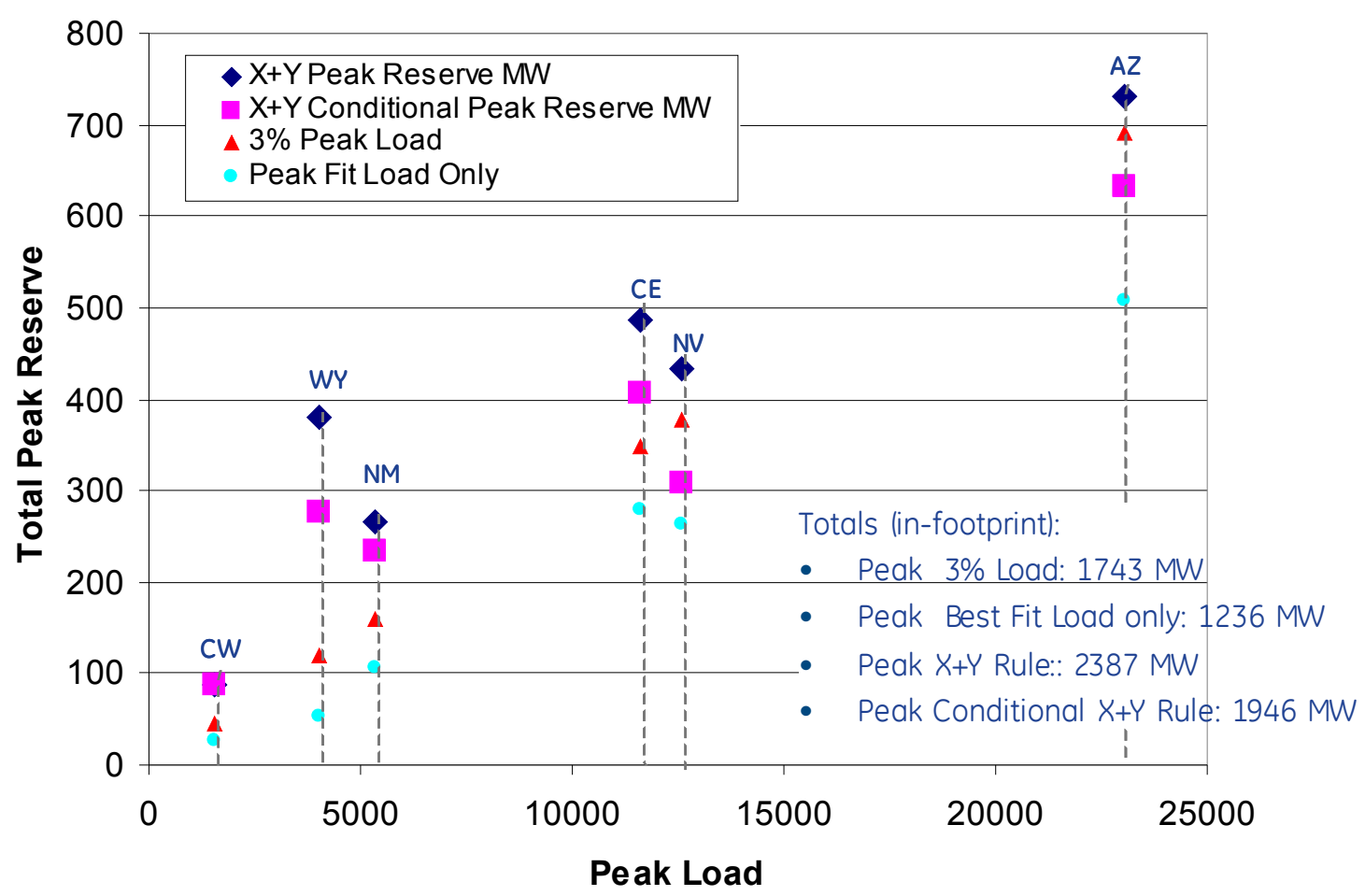

Figure 7.80 Comparison of Peak Reserve Requirement Rules (by Area)

\subsubsection{Other Concepts for Rules}

All of the results of Sections 7.4.2 through 7.4.5 are based on simplified linear rules. As the statistical variations show (including Figure 7.49 and Figure 7.50), the relationships between load and wind variability are not especially linear. In this section, possible improved "fits" to the system requirements using more complex rules are explored.

Figure 7.81 shows quadratic fits to the net load variability curves of Figure 7.47. This approach does not seem to hold much promise, as the distinct drivers of load and wind are netted out in this curve fit. However, this fitting approach does suggest that the wind term, which was made piece-wise linear in Sections 7.4.3 and 7.4.4, might be made more complex.

Figure 7.82 shows the variability of wind as a function of average wind. These are the same traces that were shown on the right side of Figure 7.49, but they have been overlaid with quadratic best-fit equations. The equations are color coded on the figure. The curvature of the In-Area curves - and especially of the Mega-Project curves - are more obviously quadratic than for the Local Priority curves. Similarly, the net load 
variability curves of Figure 7.48 show that the quadratic relationship with net load is much more distinct for the Mega-Project Scenarios. This suggests that it may be beneficial, in terms of matching reserves to actual requirements driven by variability, to develop more complex, non-linear reserve rules for some areas. For example, the Wyoming results (7.4.3 and 7.4.4) are marginal, and might benefit from a more sophisticated relationship.

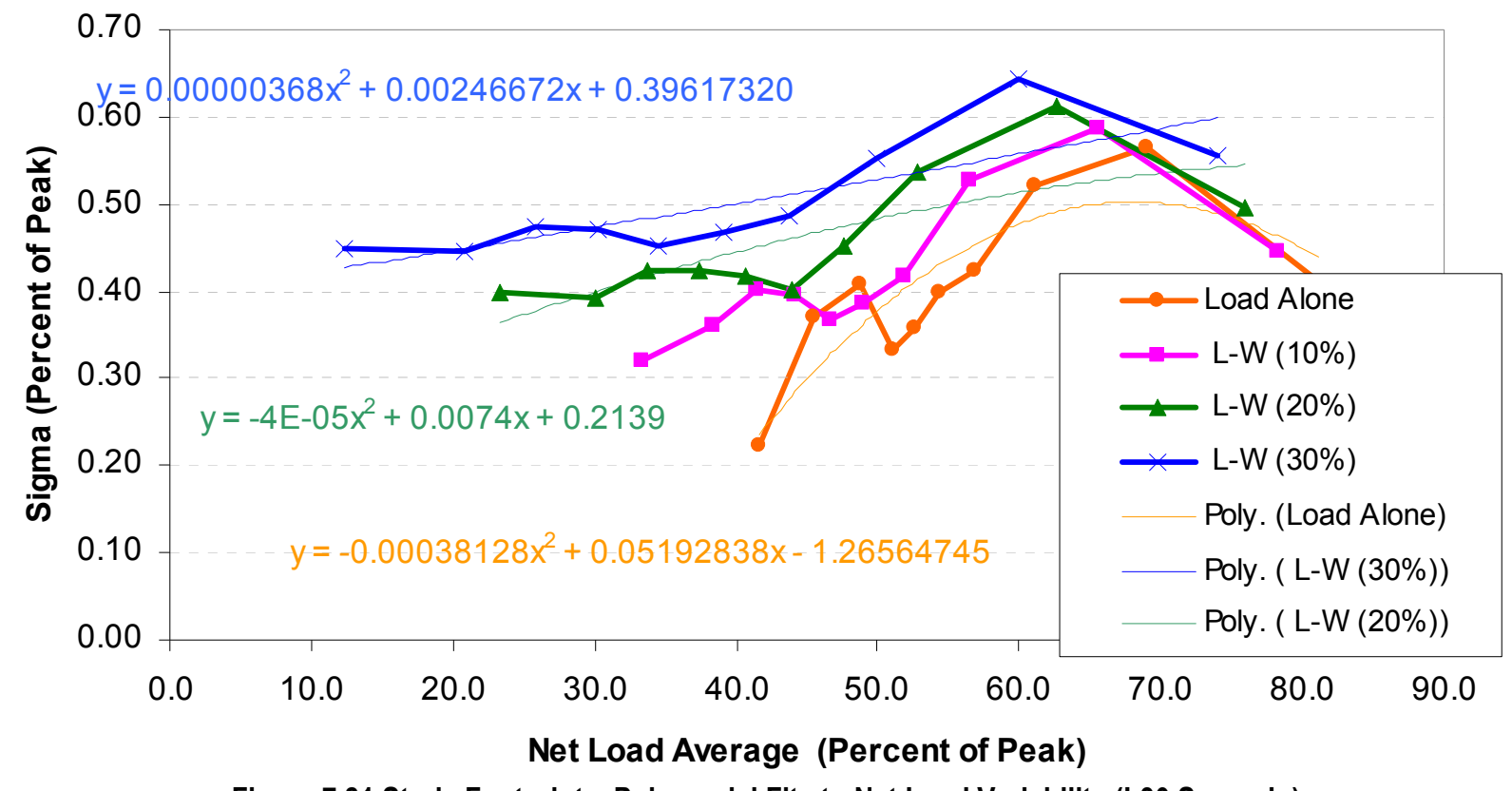

Figure 7.81 Study Footprint - Polynomial Fits to Net Load Variability (L30 Scenario) 


\section{In Area 2006}
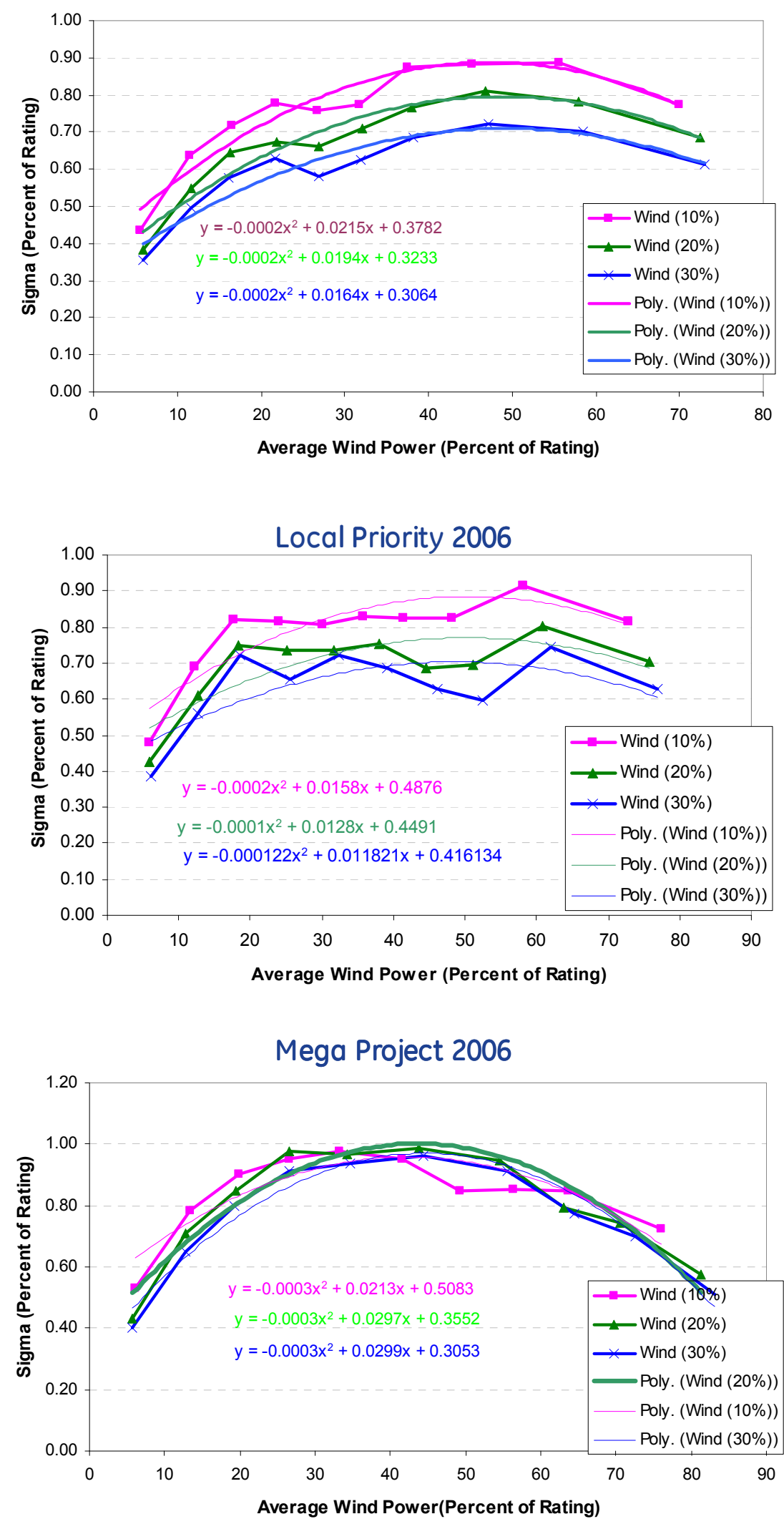

Figure 7.82 Study Footprint - Polynomial Fits to Wind Variability 


\subsection{Reserve Performance}

This section will show selected examples from MAPS and QSS simulations to illustrate the relationship between the concepts reported in the previous section and the behavior of the system as simulated.

\subsubsection{Hourly Reserves at Dispatch}

In this section, the behavior of the system in the MAPS runs, presented in Chapter 6, will be compared to the requirements implied by the $3 \Delta \sigma$ reserve rules. Unlike the spinning reserve, which requires $3 \%$ of load at all times, the variability reserve requirements implied by the surfaces shown in Section 7.3.4 are not explicitly recognized in the MAPS simulations. However, the day-ahead unit commitment and the real-time (hourly) dispatch result in some level of reserve in each hour. These on-line reserves will, in general, be greater than or equal to the $3 \%$ of load spinning reserve requirement across the MAPS regions.

In this section, the on-line reserves from the Local Priority, 30\% penetration, S-o-A wind forecast (L30R) MAPS simulation, are compared to the implied $3 \Delta \sigma$ reserve requirements.

The ability of committed generation to provide reserves requires that it have the ability to increase its power output above the actual dispatch. It must, in short, have "range" to move up. There are other constraints as well, most notably, it must be able to move at a rate sufficient to meet the system needs. In Figure 7.83, the up range from the Local Priority, $30 \%$ penetration, state of the art wind forecasting case is examined. The top trace, in blue, is the duration curve of up range for all committed generation in the study footprint. The total for the study footprint is always positive, and it exceeds $5000 \mathrm{MW}$ for about $70 \%$ of all hours. A portion of this range, corresponding to $3 \%$ of load, is dedicated to providing the spinning portion of contingency reserve. The green trace represents additional reserves beyond those needed for spin, i.e., the up range minus the spinning reserve requirement. There are 233 hours during which this is negative, which suggests that the spinning reserve rules have been violated. In fact, per the discussion in Section 6.2.2, there are 89 hours during which this occurs. The other hours are a consequence of a mis-alignment of the MAPS spinning reserve regions and the study footprint areas.

Per the discussion in Section 7.3.1, WECC guidelines expect that this reserve will be available and untouched by net load variation most of the time. Therefore, the reserves needed to handle normal load variation are subtracted. This corresponds to $1.3 \%$ of load, as shown in Table 7.14. The reserve after subtracting this component are represented by the red trace, which is negative for 423 hours. And finally, the term related to wind 
variability (5\% of wind up to $47 \%$ of wind nameplate) is subtracted to get the light blue trace, which is negative for 492 hours.

On the first look, it would appear that these 492 hours of insufficient reserves are problematic. However, the WECC guidelines and NERC CPS rules set an expectation that performance will be on target $95 \%$ of the time. The 492 hours represents about $5 \%$ of hours, and therefore at the foot print level the reserves available without actively committing additional resources for the explicit purpose of providing variability reserves are sufficient to meet performance objectives. This is a significant result.

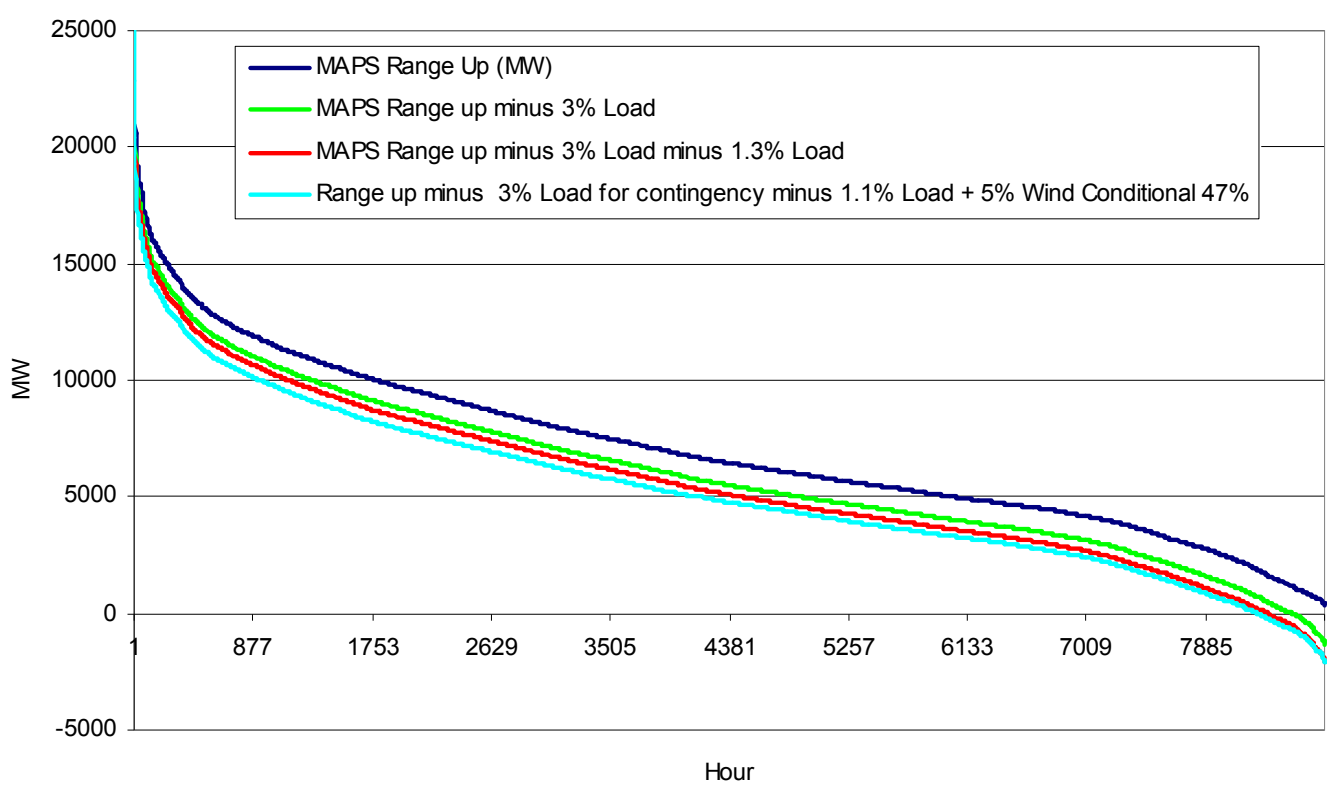

Figure 7.83 L30R Reserve Duration (Best Fit X+Y Conditional rules)

A comparison of this result with the behavior of the system with no wind or solar is shown in Figure 7.84. In this figure, the total up range and total contingency margins are plotted for the two cases. The no wind and solar case has adequate reserves only about $90 \%$ of time. Even though variability requirements increase with wind and solar, the economic operation of the system means that the displacement of other resources tends to release up reserves. Overall, the Local Priority 30\% case consistently has more reserves and more reserve margin. Without explicitly requiring (or committing for) additional reserves for variability, the system economics provide sufficient reserves about $95 \%$ of the time, at the study footprint level.

The MAPS cases, described in Section 6.2.2, showed that committing units to provide $5 \%$ of wind for reserve reduced the value of wind power by $\sim \$ 0.25$ per MWhr. These results suggest this is not necessary. However, should this more conservative approach be adopted, the cost impact is less than $1 \%$ of the value of the wind and solar generation. 


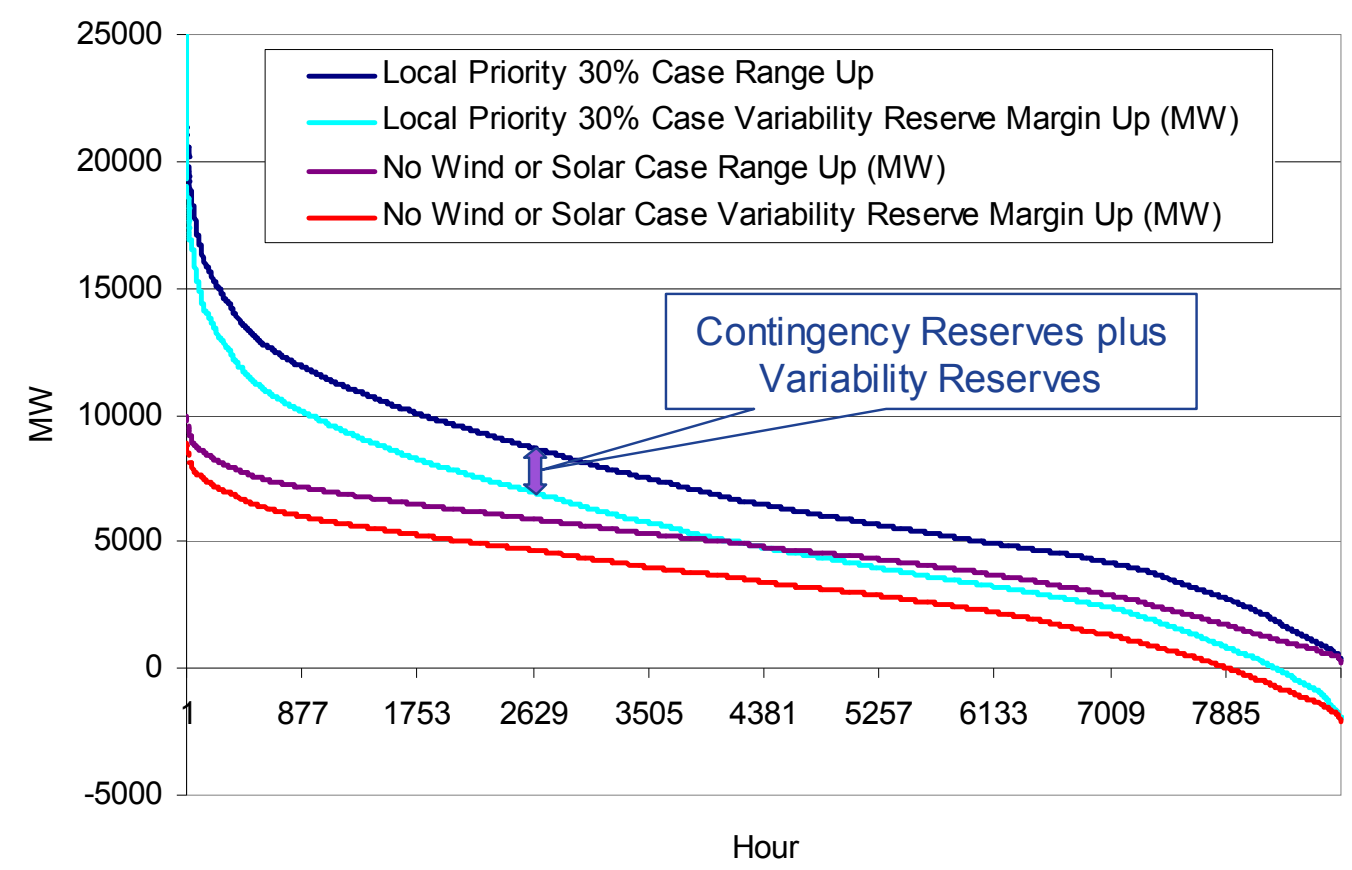

Figure 7.84 Reserve Margins: Comparison to No Wind and Solar

The tendency for wind to release reserves from other resources is shown further in Figure 7.85. This shows the variability reserve margin for every hour as a function of system load and as a function of wind production. The blue points and trend line are for the Local Priority 30\% case vs. System Load. There is considerable spread in the reserve margins. As expected, up reserves tend to be released as system load drops. This is the net result of (a) backing down committed generation which increases reserves and (b) decommitting units which decreases reserves. The trend line shows that, on average, about $1 \mathrm{MW}$ of reserve is released for each $4 \mathrm{MW}$ of load drop. The green points and trend line are for the no wind or solar case. The average trend shows roughly the same 1:4 ratio, but on average the system carries about $2000 \mathrm{MW}$ less reserve than with $30 \%$ wind and solar. The other point of interest is that the dispersal or spread of the green (no wind or solar) points in much less. This is a reflection of the fact that there is considerably more uncertainty during commitment with wind and solar. The pink dots and trend line show the up reserve margin as a function of wind power. In this case, the trend is positive with a slope of 1:3, meaning that a 3-MW increase in wind production tends to release about $1 \mathrm{MW}$ of variability reserve. 


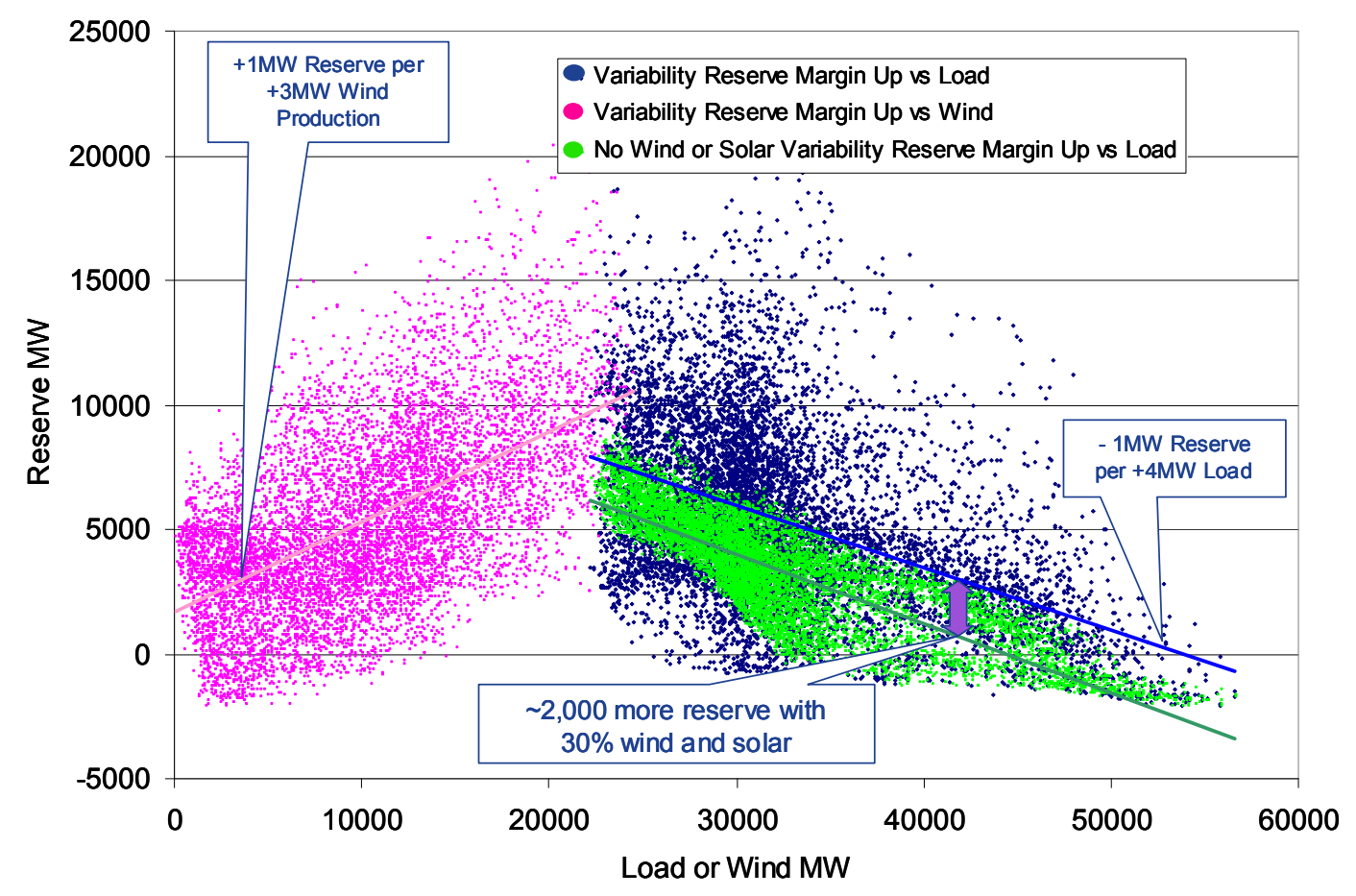

Figure 7.85 Impact of Load and Wind Levels on Variability Reserve Margins

The annual duration curves give a sense of the level of variability reserve margin that is produced "naturally" by economic operation of the system. They do not, however, illuminate how the reserve margins relate to wind and load levels. In the plots below, the hourly load and wind are segregated in exactly the same fashion as described in Section 7.3.2. For each hour in a load and wind cell, the actual dispatch is compared to the available up range for the committed units. This is the total sub-hourly reserve for that hour.

In Figure 7.86, the average system reserves for the study footprint are shown in the load and wind space. Reserves here mean the difference between maximum power and dispatched power of committed units. Overall, the system has substantial reserves. Two trends are obvious: system reserves tend to be lower at higher load levels, and system reserves tend to be higher at higher wind levels. Both of these trends make physical sense, since higher load levels will tend to load up committed generation, and higher wind levels will tend to unload committed generation.

Of course, average performance is only a broad measure of behavior. The system needs to be secure at each hour. In Figure 7.87, the implied reserves based on the $3 \Delta \sigma$ rule are compared for each hour. Specifically, the margin is calculated as the MAPS reserves minus the implied reserves, i.e. 3 times the values from Figure 7.57 plus 3\% of load for spinning reserve. Negative values are violations, i.e. the MAPS simulation ended up with fewer reserves than the $3 \Delta \sigma$ rule implies are necessary. A violation here does not mean that load was unserved, but rather that the system was short of the required 
reserves. The upper plot in Figure 7.87 shows the amplitude of the single worst violation of implied reserves for each cell in the load-wind space. The color key is that blue areas represent load-wind combinations that do not ever have reserve violations. The gray "never" cells are, again, load-wind combinations that never occur. The lower plot is the count of hours with any violations. The total count of violations in the year is 525 , about $5 \%$ of all hours. This is consistent with Figure 7.83, although the count of hours is slightly different. This calculation is based on actual $3 \Delta \sigma$, rather than the simple best fit rule used in the duration curves. This suggests that the rules used with the MAPS runs are largely correct in that they produce adequate reserves. However, these results also suggest that some refinement in the reserve rules would be beneficial.

Violations tend to occur at relatively high load and low wind power. Note that the increased reserve requirement associated with modest amounts of wind combines with the high load levels to make deficiencies in reserve most acute at high load and low, but not very low, wind. This is also a reflection of the impact of forecast error. When wind is under-forecasted, the committed units use up-range to serve load, depleting reserve margins. Scarcity of reserves at very high load is aggravated by increased reserve requirement due to wind variability. A violation means that the hour has insufficient reserves to meet the implied requirement. Load is still served. Further, the existing resources can provide the necessary reserves, but need to be properly committed and dispatched. There is no indication that new resources are required.

The deficiency of up reserves at low wind power corresponds to hours for which the wind forecast was higher than the actual wind. In Figure 7.88, the actual reserves are plotted against the wind forecast error for the full year of the L30R case. There is some trend towards lower reserves with higher forecast error, but clearly there are other factors involved as well. In Figure 7.89, details of three cells from Figure 7.87 (marked in that figure with color coded symbols) are plotted. The color-coded lines correspond to $3 \Delta \sigma$, from Figure 7.57, plus $3 \%$ of load for spinning reserve for the respective cells. Overall the up-range or reserves for the two cells (green triangle and blue diamond) that showed violations tend to be much lower. Again, there is some correlation to wind forecast error, but other factors contribute to the availability of reserves as well.

For down reserves, the issue is less troublesome. As shown in Section 7.2, wind can contribute to down reserves when necessary. The pair of plots in Figure 7.90 conveys similar information to Figure 7.87, except for down reserves. There are no negative (down) spinning reserve requirements, so down variability reserves need not be added to the spin requirement. Down reserves are abundant at high load and low wind levels, and there are no violations. Conversely, at high wind and low load, there are eight hours during which down reserves are below the implied requirements. These results suggest that down reserves are unlikely to be a limiting or substantial economic consideration. 


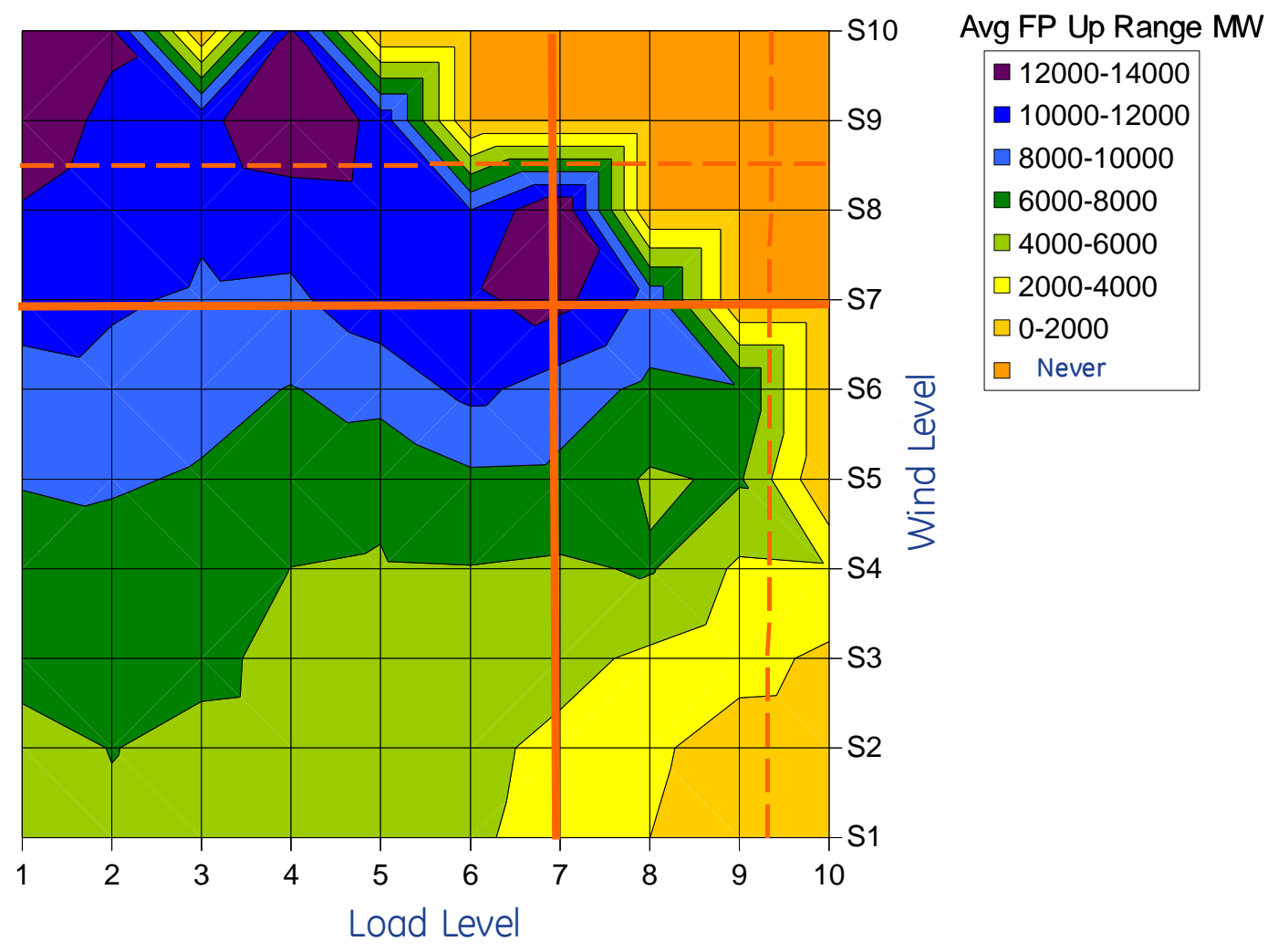

Figure 7.86 Average Up Range L30R Scenario 


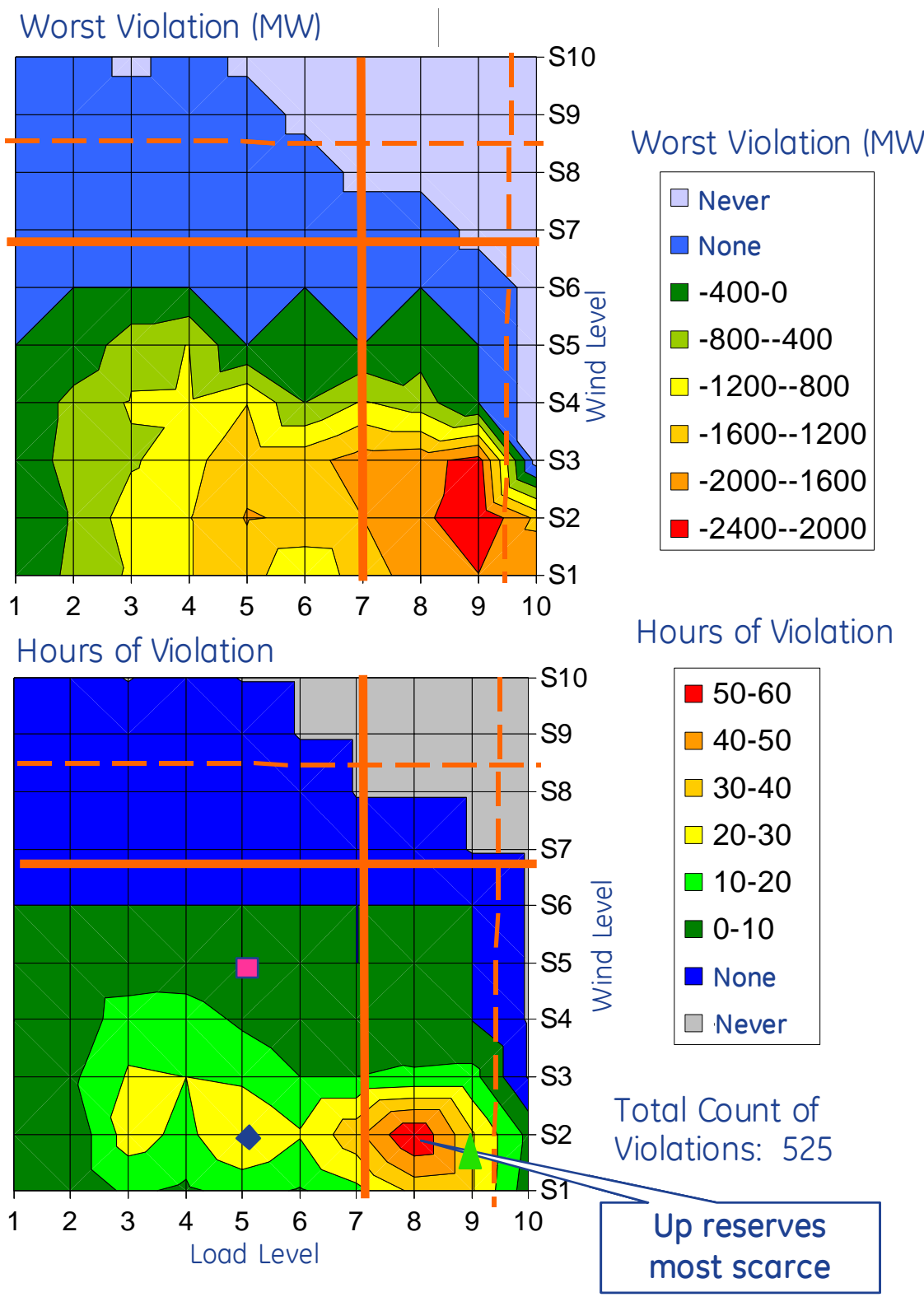

Figure 7.87 L30R Scenario Up Reserve Violations 
Up Range vs Forecast Error L30R Full Year

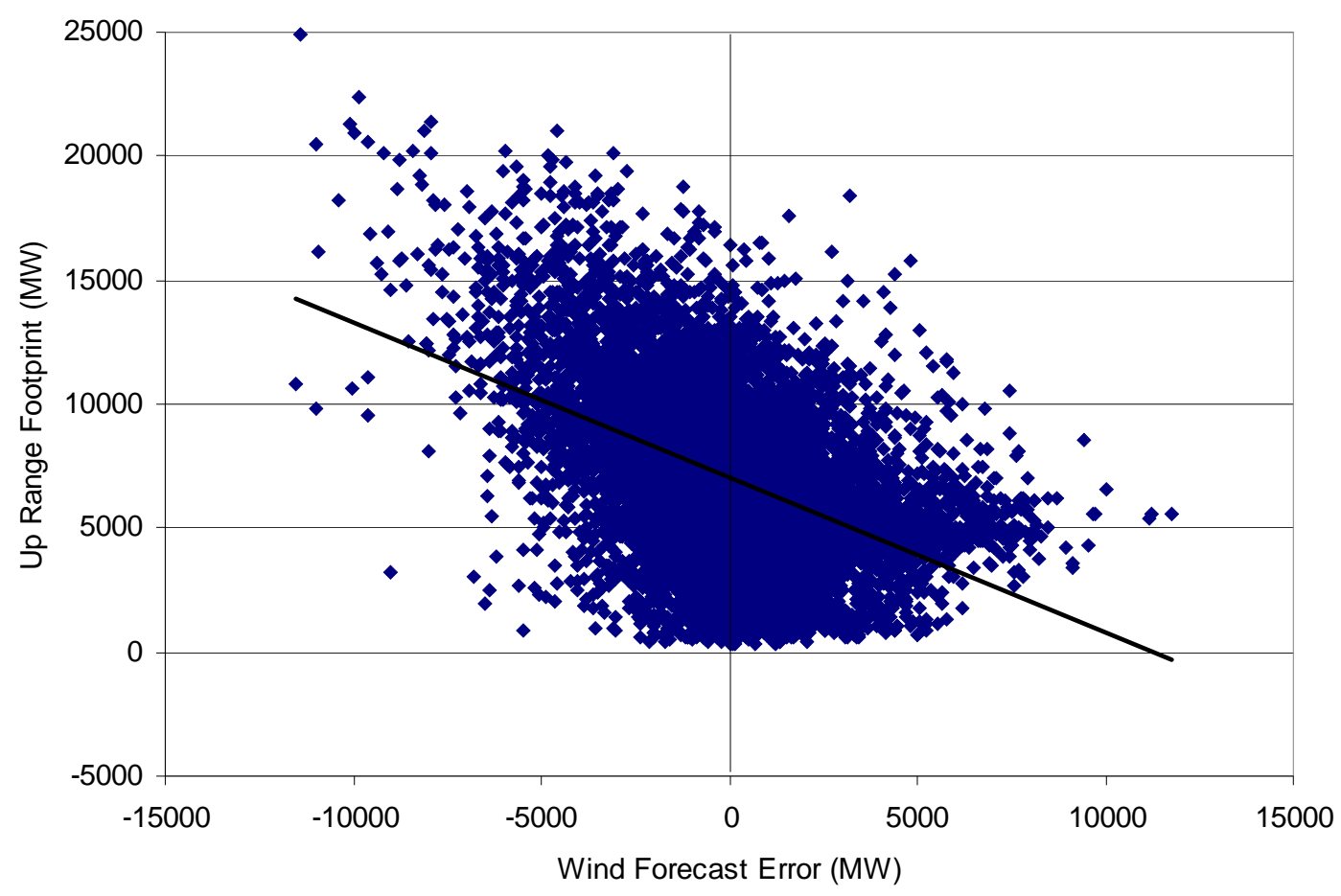

Figure 7.88 Up Reserves vs. Forecast Error (Full Year)

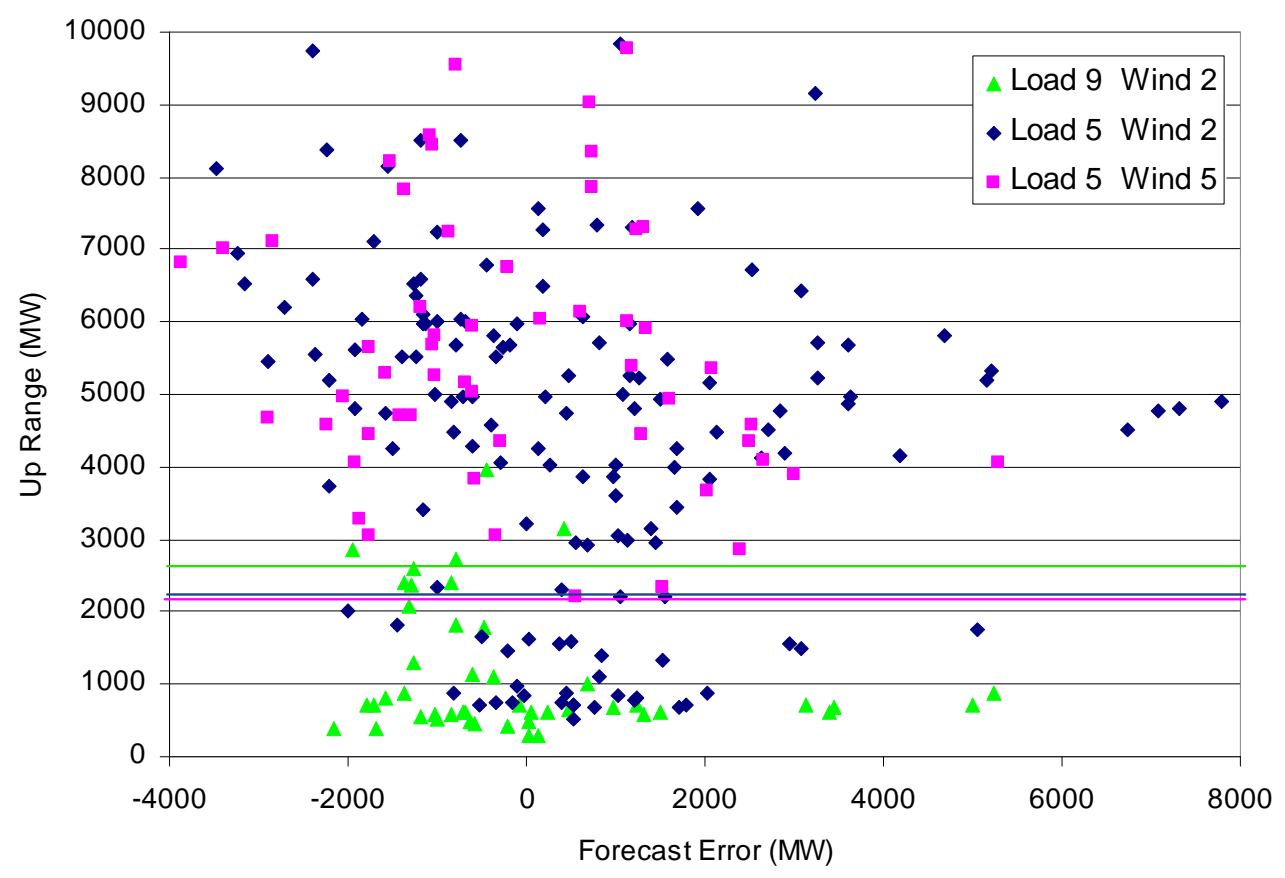

Figure 7.89 Up Reserves vs. Wind Forecast Error (Selected Load - Wind Pairs) 


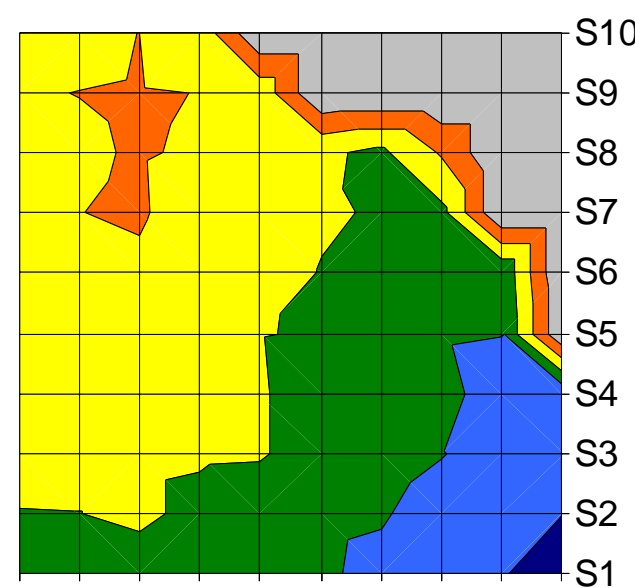

$\begin{array}{llllllllll}1 & 2 & 3 & 4 & 5 & 6 & 7 & 8 & 9 & 10\end{array}$

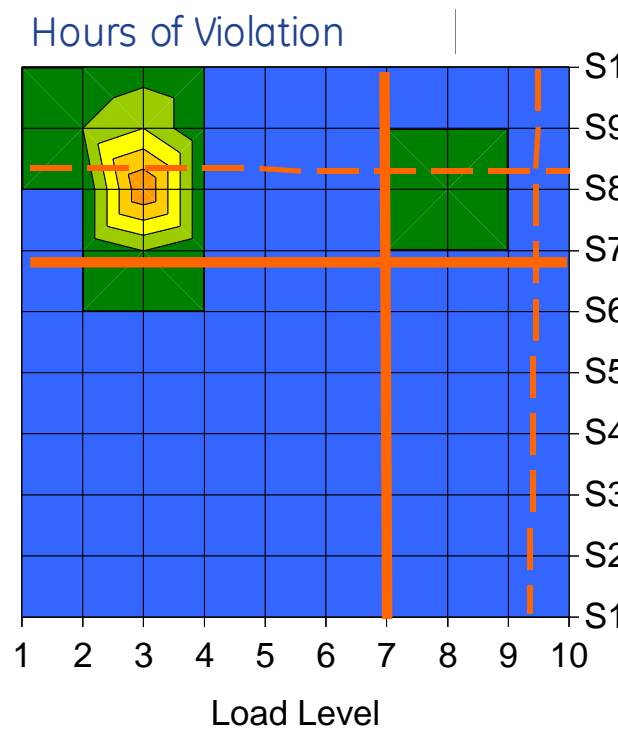

Figure 7.90 L30R Scenario Down Reserve Violations

The performance for individual areas is more complex. The total up reserve, spinning reserve margin and total reserve margin for Arizona is shown in Figure 7.91. Since spinning reserve is not allocated by area in these cases, the area has negative spinning reserve margin for about $10 \%$ of hours, and negative total reserve margin about $15 \%$ of the time. Both of these results are consistent with the notion that inter-area cooperation is needed.

Looking more closely, the up reserve violations for Arizona are shown in Figure 7.92. The two plots convey the same information as Figure 7.87, but for Arizona only. In Arizona, with $7710 \mathrm{MW}$ of wind and a peak load of $23 \mathrm{GW}$, the system has a total of 1354 hours of violations - about $15 \%$ of hours. There is a tendency to be short of reserves at low wind conditions, with the worst violations occurring at high load and moderately 
low wind. Again, the shortage of wind generation tends to deplete the available up range.

The behavior of Wyoming as shown in Figure 7.93 is much worse, and consistent with the results shown in Figure 7.64. As shown in Section 7.3.4 and Figure 7.66, committing and dispatching for spinning reserve on a regional basis will tend to result in significantly less reserves than the implied reserve rules for Wyoming. With a wind power penetration of nearly $200 \%$, the shortage of reserves in the area is substantial greatly in excess of the target of meeting variability requirements $95 \%$ of the time. In the figure, 3312 hours, about $1 / 3$ of the year, are in violation. The implication is that either Wyoming must carry substantially more reserves, or the neighboring (i.e. importing) systems must contribute to the reserve requirements. The implications of these trade-offs will be explored further in the next section.

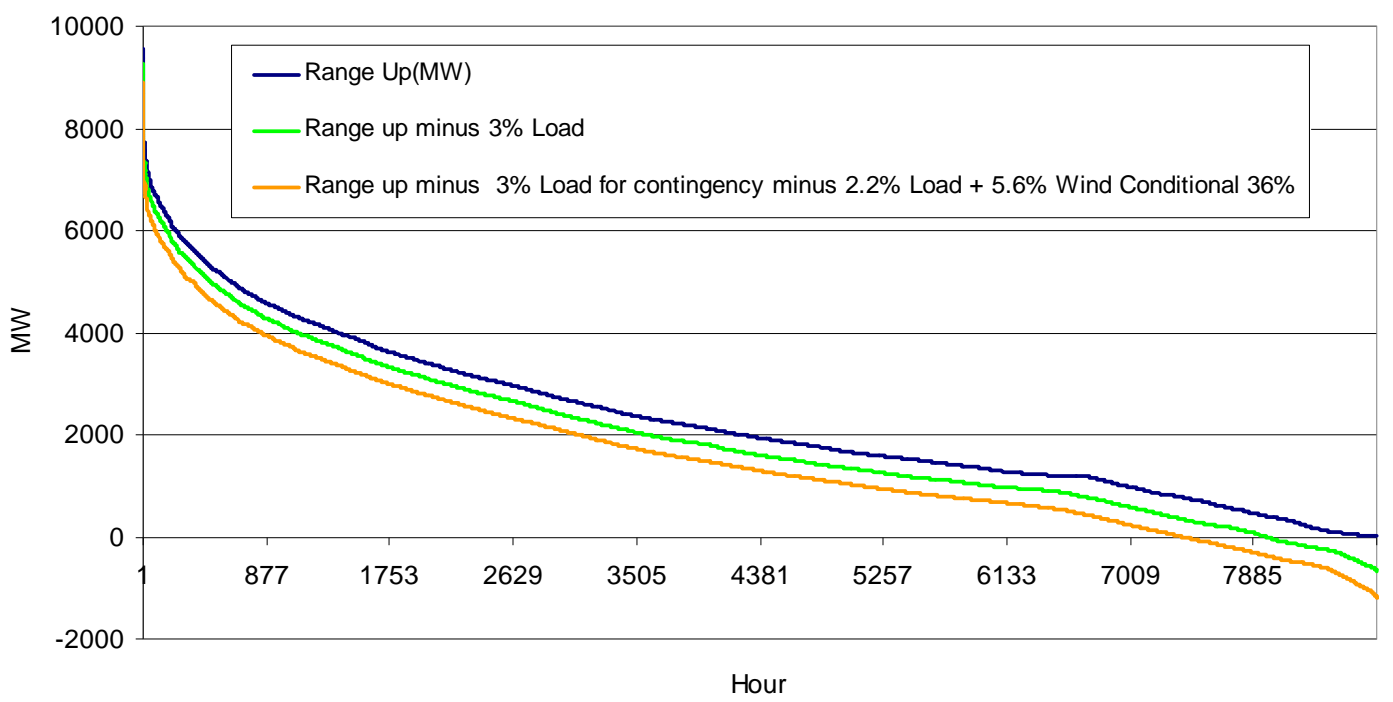

Figure 7.91 L30R Arizona Reserve Duration - Best fit rules 


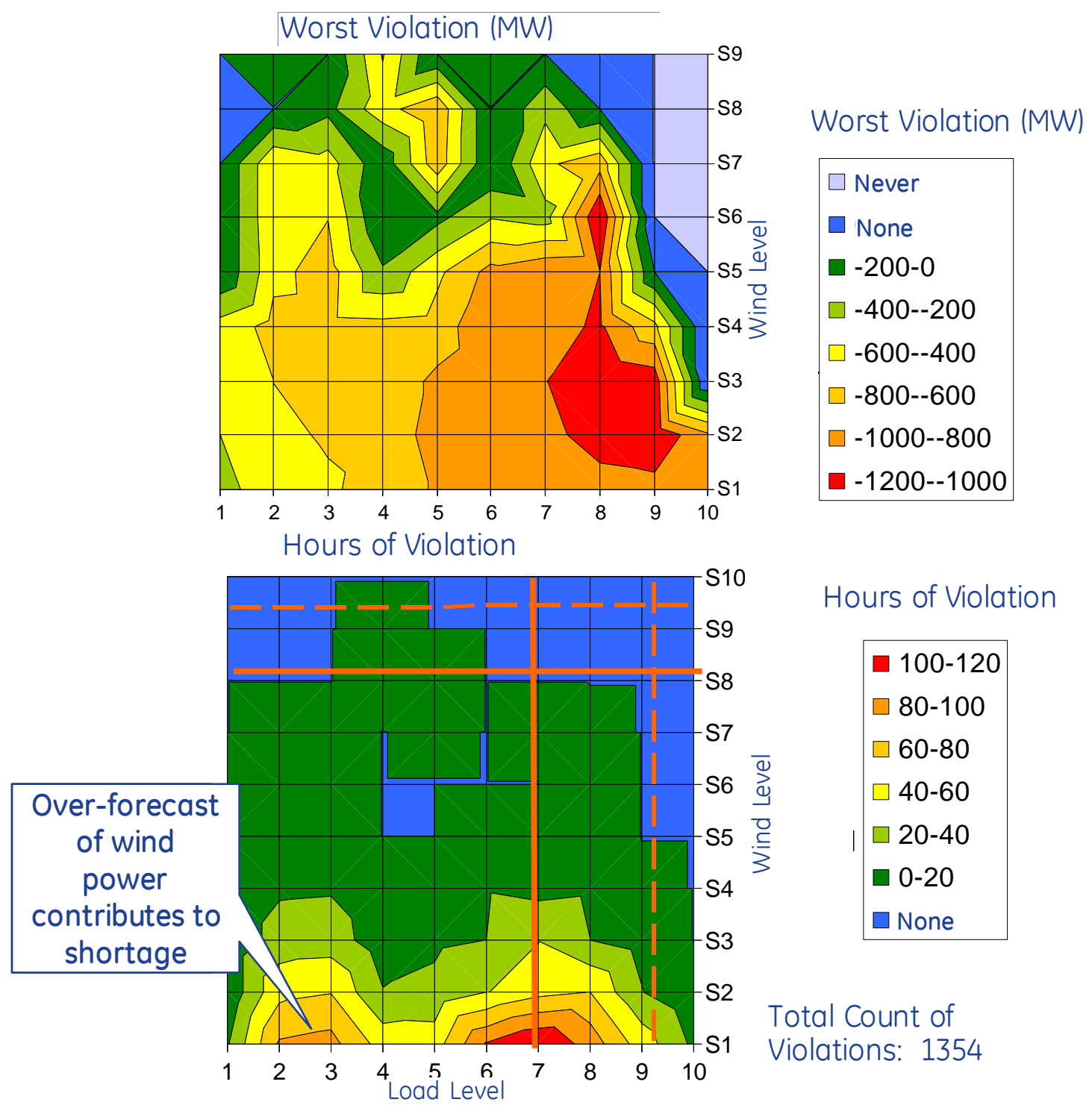

Figure 7.92 Arizona L30R Scenario Reserve Violations 


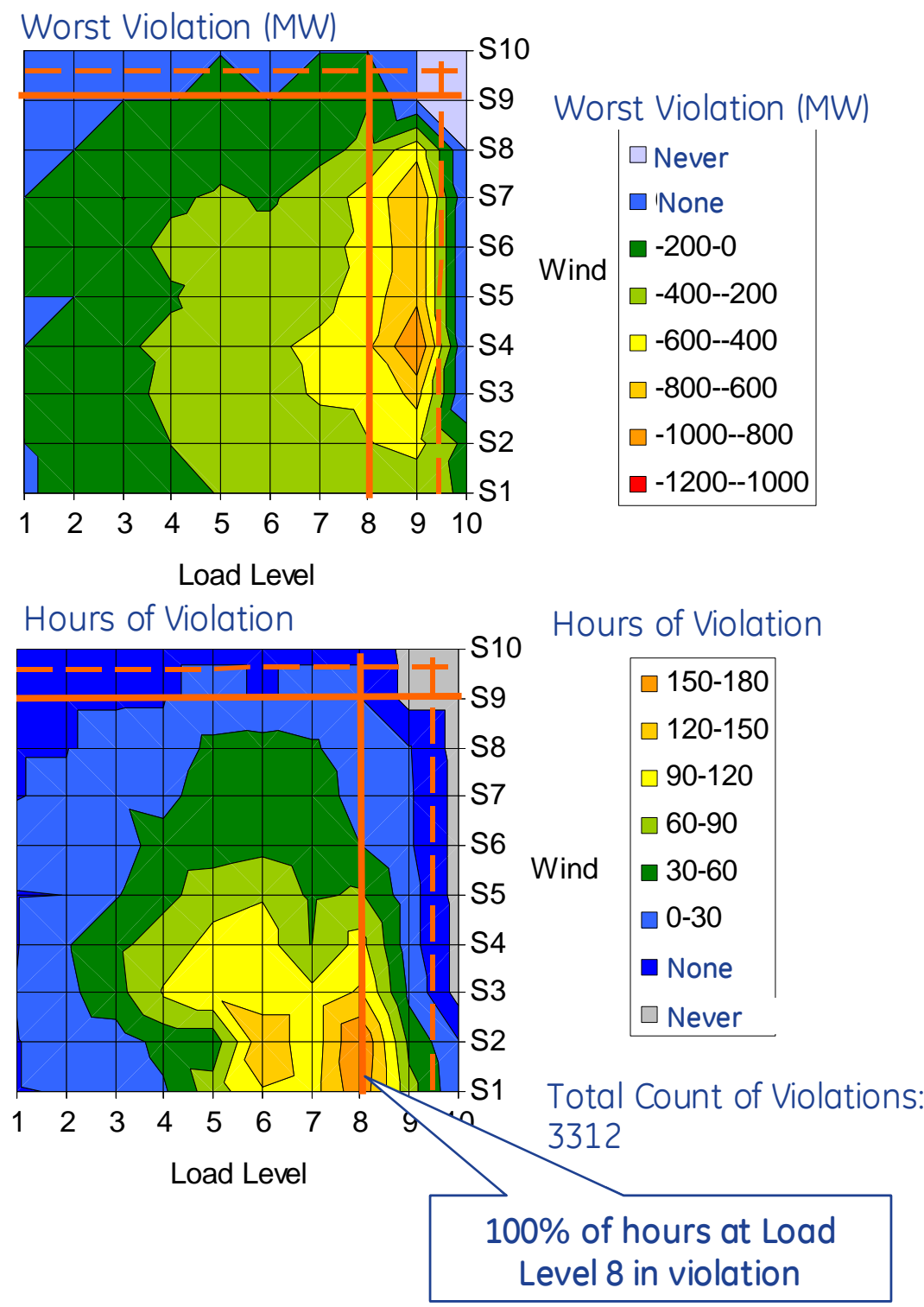

Figure 7.93 Wyoming L30R Scenario Up Reserve Violations

\subsubsection{Intrahour Performance}

This section re-examines the QSS simulations presented in Section 7.2 with the intent of exploring the dynamic balance of the system, including intrahour behavior against the concepts of $3 \Delta \sigma$ reserve rules.

In Figure 7.94, the Arizona L30R $\Delta \sigma$ surface is reproduced from Figure 7.60. Overlaid on that surface is the load-wind trajectory of the April 15, 2006 QSS simulation. The minuteby-minute trajectory is shown in blue, with dots added at one-hour intervals. The arrow shows the direction of the progression in time. This particular day was chosen because of the simultaneous high wind, low load condition. Recall that at one point in this particular day, the instantaneous wind penetration exceeded $100 \%$ for the entire study 
footprint. In the figure, it is apparent that the reserve requirements are, as expected, varying but modest, with $\Delta \sigma$ in the range of 50 to $150 \mathrm{MW}$, compared to $\Delta \sigma$ at high load and moderate wind.

In Figure 7.95, the actual and implied reserves for the day are plotted against time. As committed generation that is available for maneuvering is economically re-dispatched, the available reserves constantly change. Early in the simulation $(6-10 \mathrm{pm})$, there is a considerable amount of reserve. In the middle of the night, units are decommitted and the reserve level drops. The blue curve with pink dots is the $3 \Delta \sigma+3 \%$ of load for spin reserve requirement approximated from the trajectory on Figure 7.94. Note that the factor of 3 (in $3 \Delta \sigma$ ) is taken into account in this trace. The regulation deficiency from the QSS simulation is shown in green. Non-zero points correspond to times when the regulation (not economic dispatch) was unable to keep up with the net load and schedule changes. As was shown in Section 7.2, hourly schedule changes tend to dominate ACE. There is a rough correlation between the drops in range and reserve depletion. The depletions tend to lead the relative minima of range. This is an indication that the capability of the units dedicated to regulation has been exceeded, but not that the overall reserves are deficient.

In Figure 7.96, the behavior of Arizona for the October 26, 2006 QSS simulation is shown on the Arizona L30R $\Delta \sigma$ surface. Again there is substantial wind, but then as the day progresses, the wind power drops. This drop was poorly forecast, so system reserves, as seen in Figure 7.97, around 6 a.m. are relatively low. Nevertheless, the actual reserves still meet the spin plus implied $3 \Delta \sigma$ reserve requirements, and the only regulation deficits are associated with hourly schedule changes.

The behavior of the Wyoming system is, as suggested by the results presented in the previous section, substantially different. The April 15, 2006 trajectory is shown in Figure 7.98. The wind rises to very high levels during low load conditions. As shown in Figure 7.93 , the proportionately high variability of the wind tends to drive a significant number of regulation reserve deficits. However, the trend in Wyoming is for the other generation (including a significant coal fleet) to stay on-line even at light load and high wind. Thus, the total up reserve is proportionally quite large. In Figure 7.99, the upper left-hand plot shows that up reserves are about $2000 \mathrm{MW}$ for most of the day, with implied reserve requirements being in the neighborhood of 200-400 MW. Nevertheless, the detail on the lower left shows frequent regulating-reserve deficiencies.

Using $1 \%$ of peak load for regulation in Wyoming only places about $40 \mathrm{MW}$ of generation on regulation. With wind power penetration of nearly $200 \%$, this is inadequate. The pair of plots on the right is for exactly the same commitment and dispatch rules, but with $100 \mathrm{MW}$ of the available reserves dedicated to regulation. As discussed in Section 7.3.5, Figure 7.68, this level of regulation corresponds approximately to one $\Delta \sigma$ of the 10-minute variability. The regulation performance is 
greatly improved. Overall, this reinforces the conclusion that roughly $1 / 3$ of the minimum implied sub-hourly variability reserves should be allocated to regulation. For most of the study system, this represents roughly $1 \%$ of peak-load. For this very high wind penetration system, which is otherwise relatively small electrically, the required regulation is about $2 \frac{1}{2} 2$ times greater.

In the October 26, 2006 case for Wyoming, as shown in Figure 7.100, the poor wind forecast results in heavily dispatched thermal generation to cover the wind shortfall. The resulting sub-hourly reserve is much less than was shown in the April 15 case. On this day, the trend in sub-hourly reserve is roughly coincident with the implied requirements. The substantial hourly schedule changes cause fluctuation around that trend line. Deficits in regulation, as shown in Figure 7.101, are coincident with periods during which reserves are drawn down rapidly. Again, this is partly due to an insufficient allocation of variability reserves to regulation, and partly due to the generally lower levels of reserves due to a large wind forecast error. The hourly periodicity of reserve swings is further evidence of the consequence of hourly scheduling. Sub-hourly scheduling strategies would undoubtedly decrease these reserve swings, and the resulting in depletion of regulation.

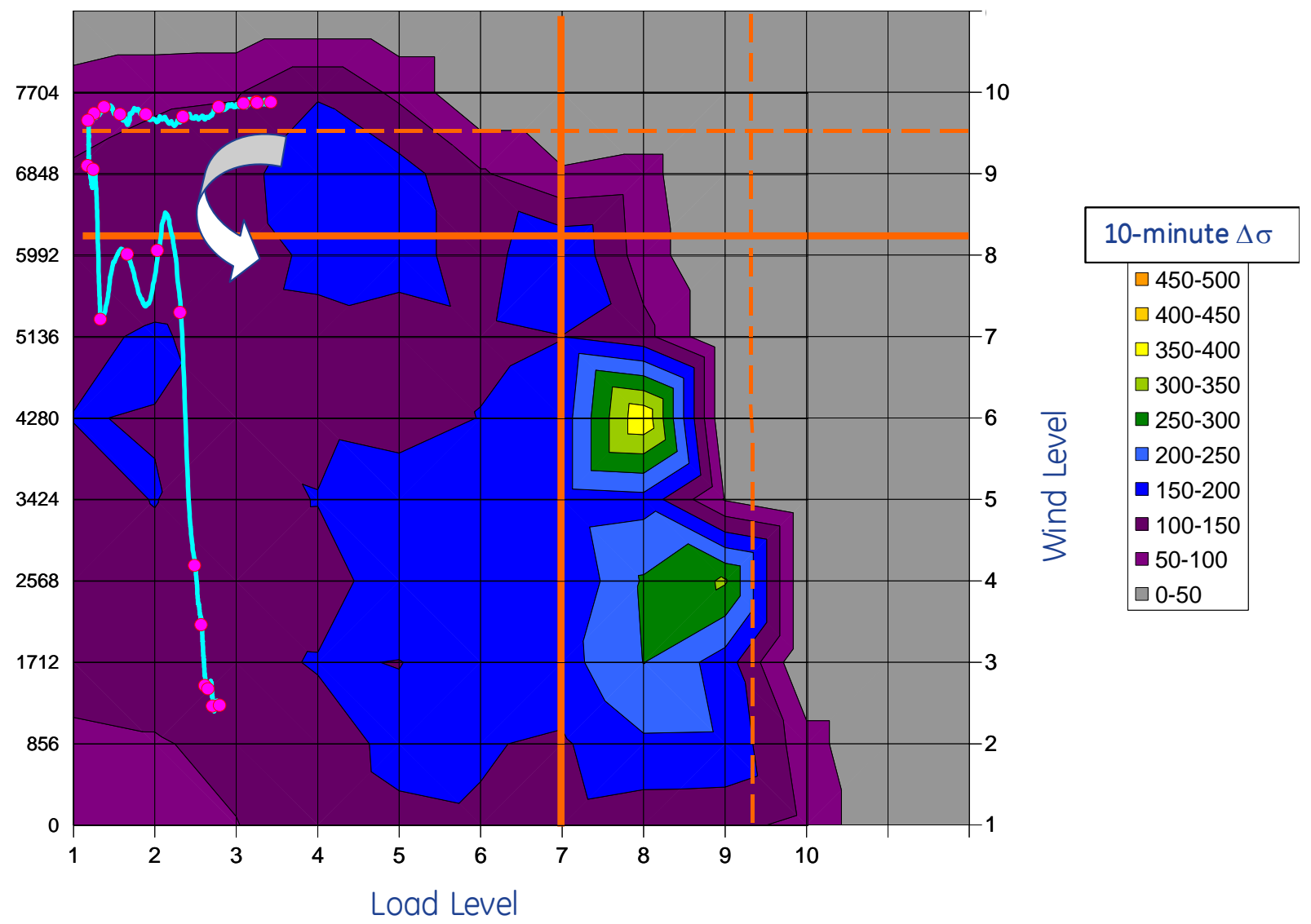

Figure 7.94 Arizona L30R Scenario April 15, 2009 Trajectory 


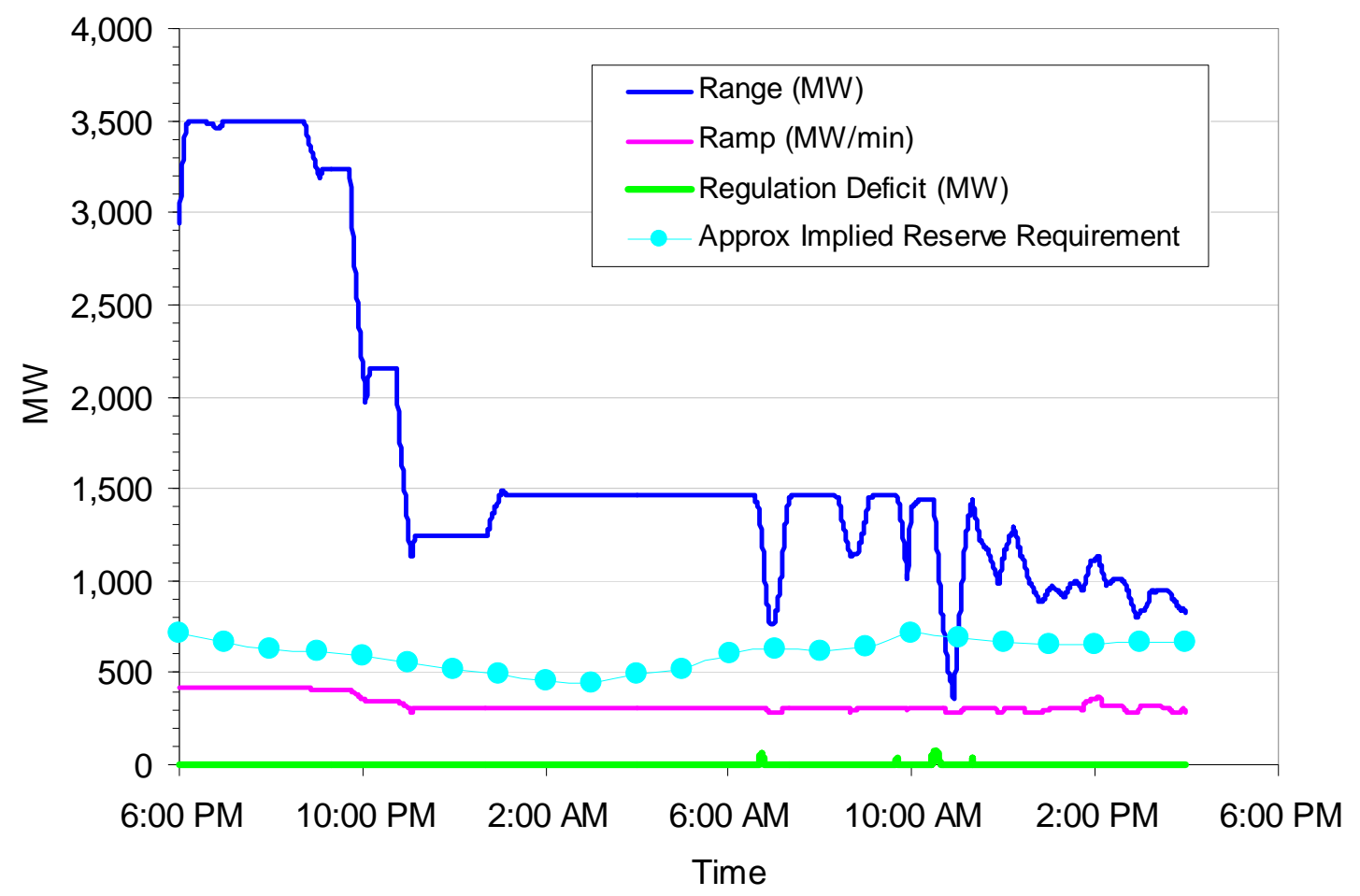

Figure 7.95 Arizona L30R Scenario Results April 152006

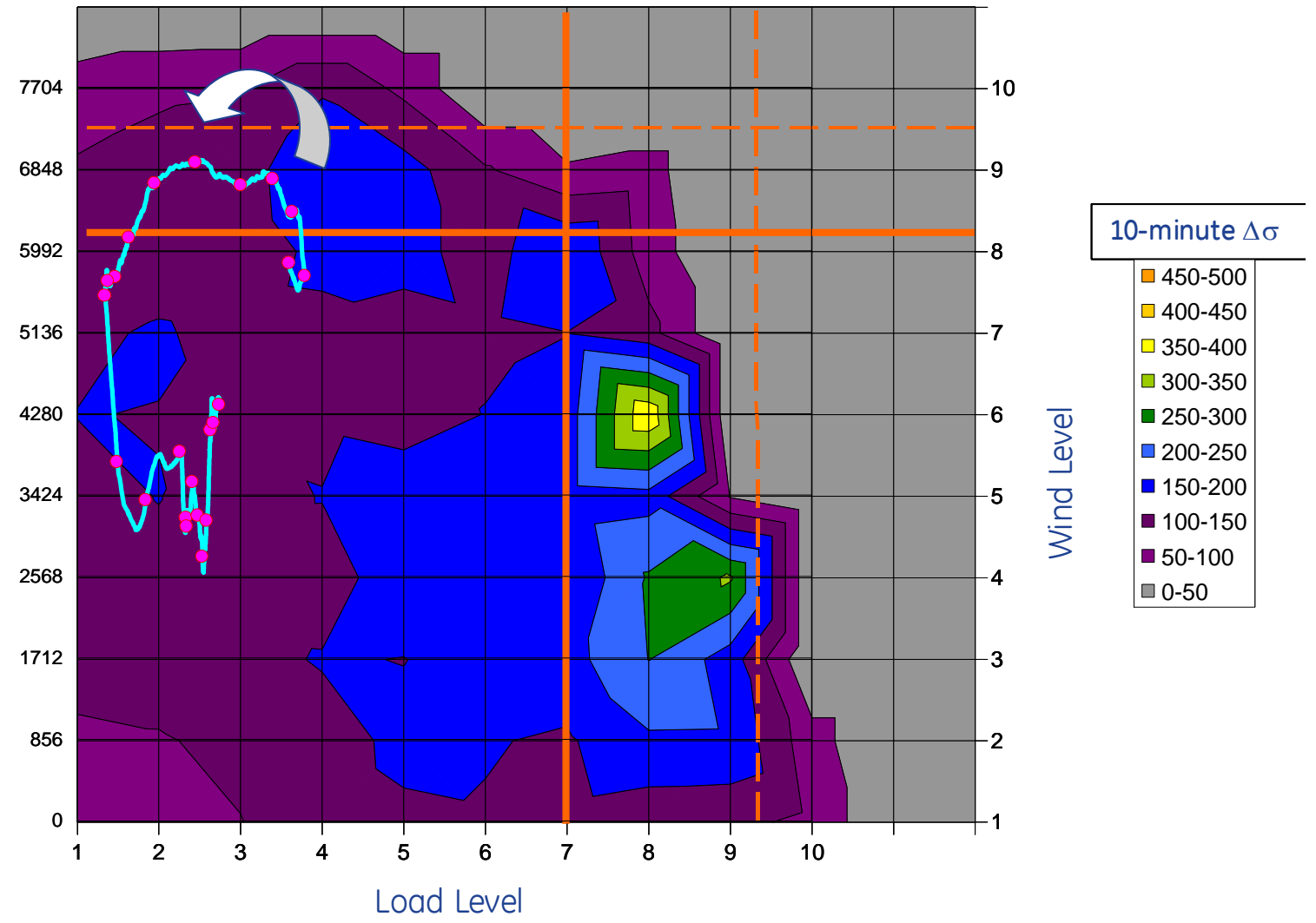

Figure 7.96 Arizona L30R Scenario October 262006 Trajectory 


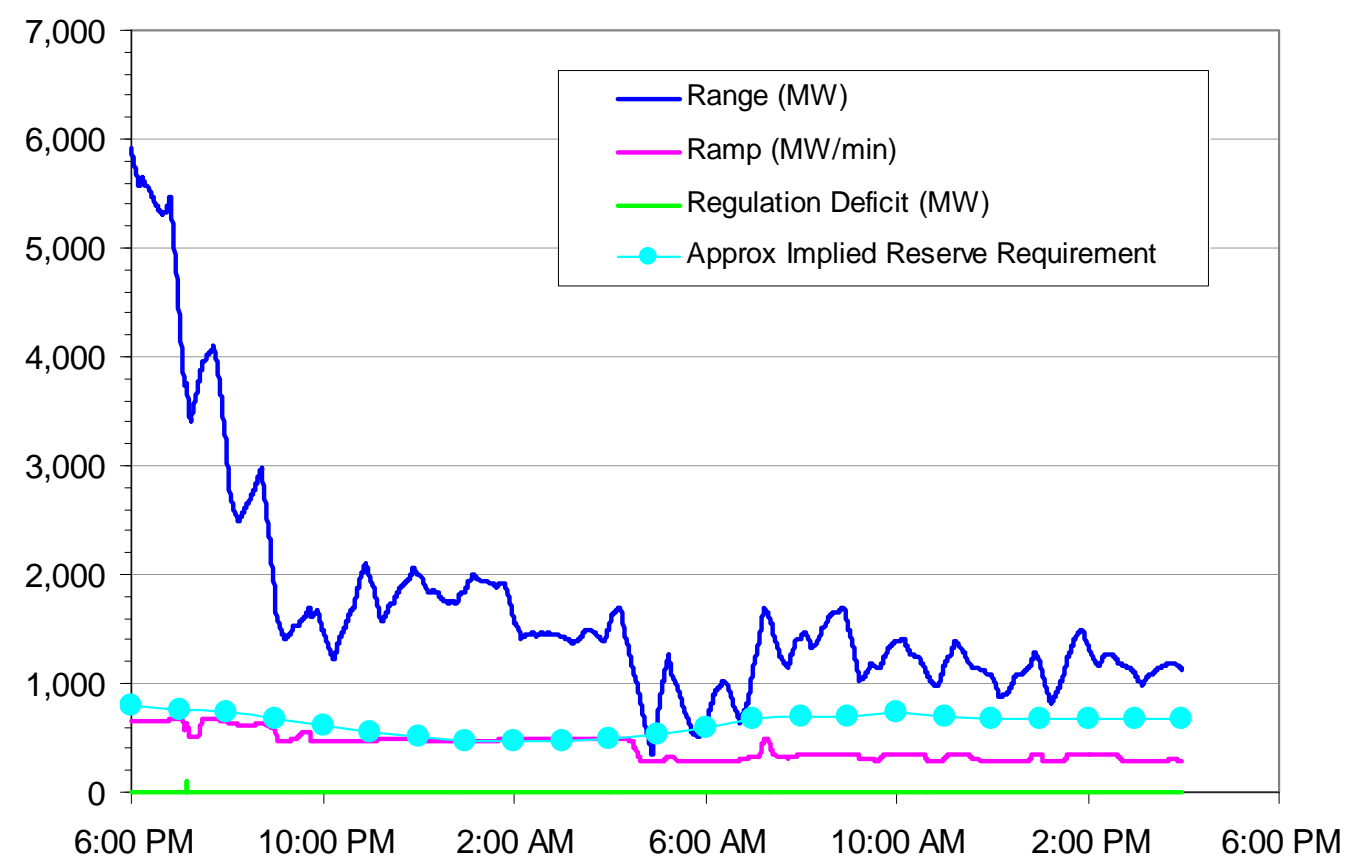

Figure 7.97 Arizona L30R Scenario Results October 262006

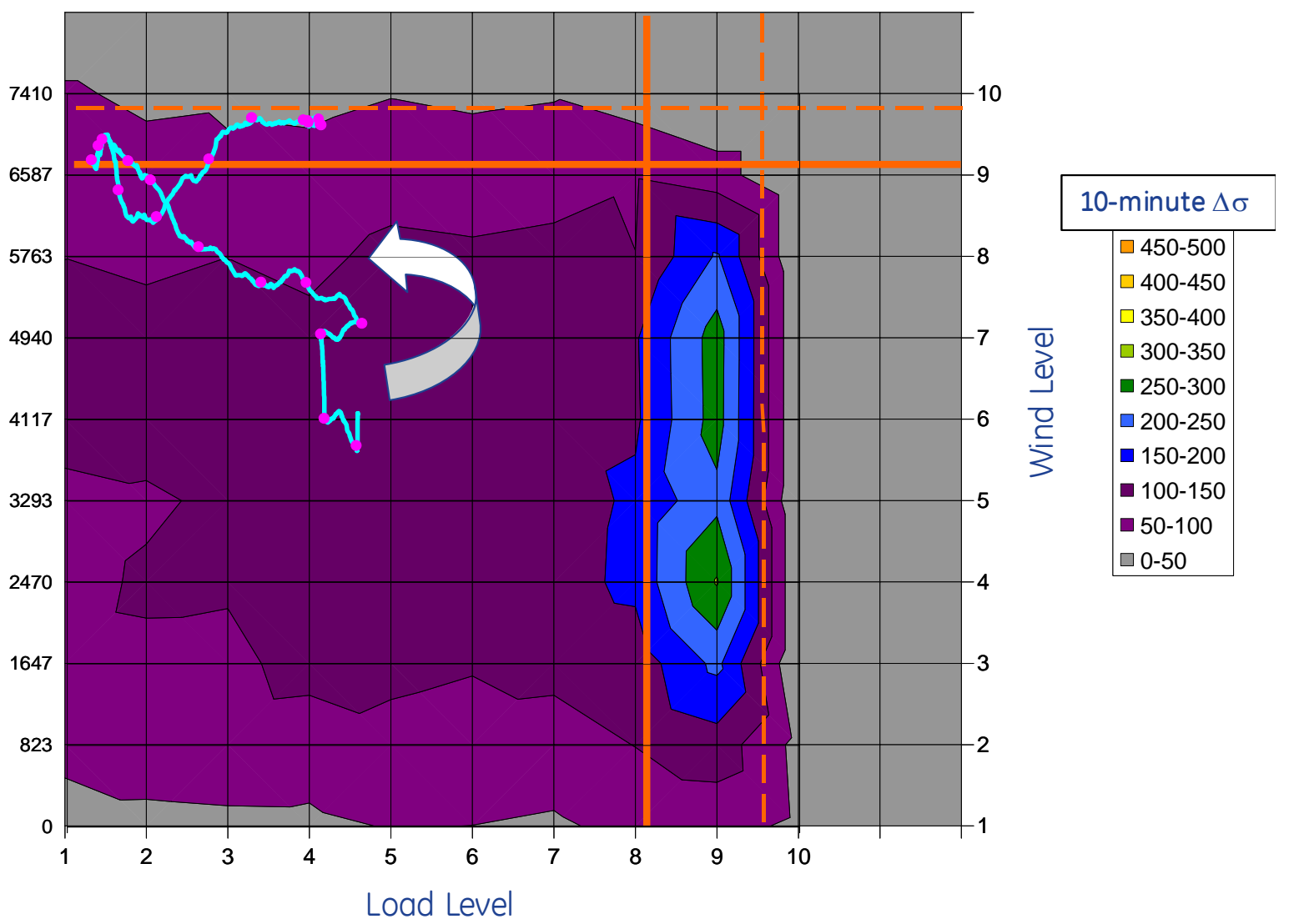

Figure 7.98 Wyoming L30R Scenario April 15, 2006 Trajectory 

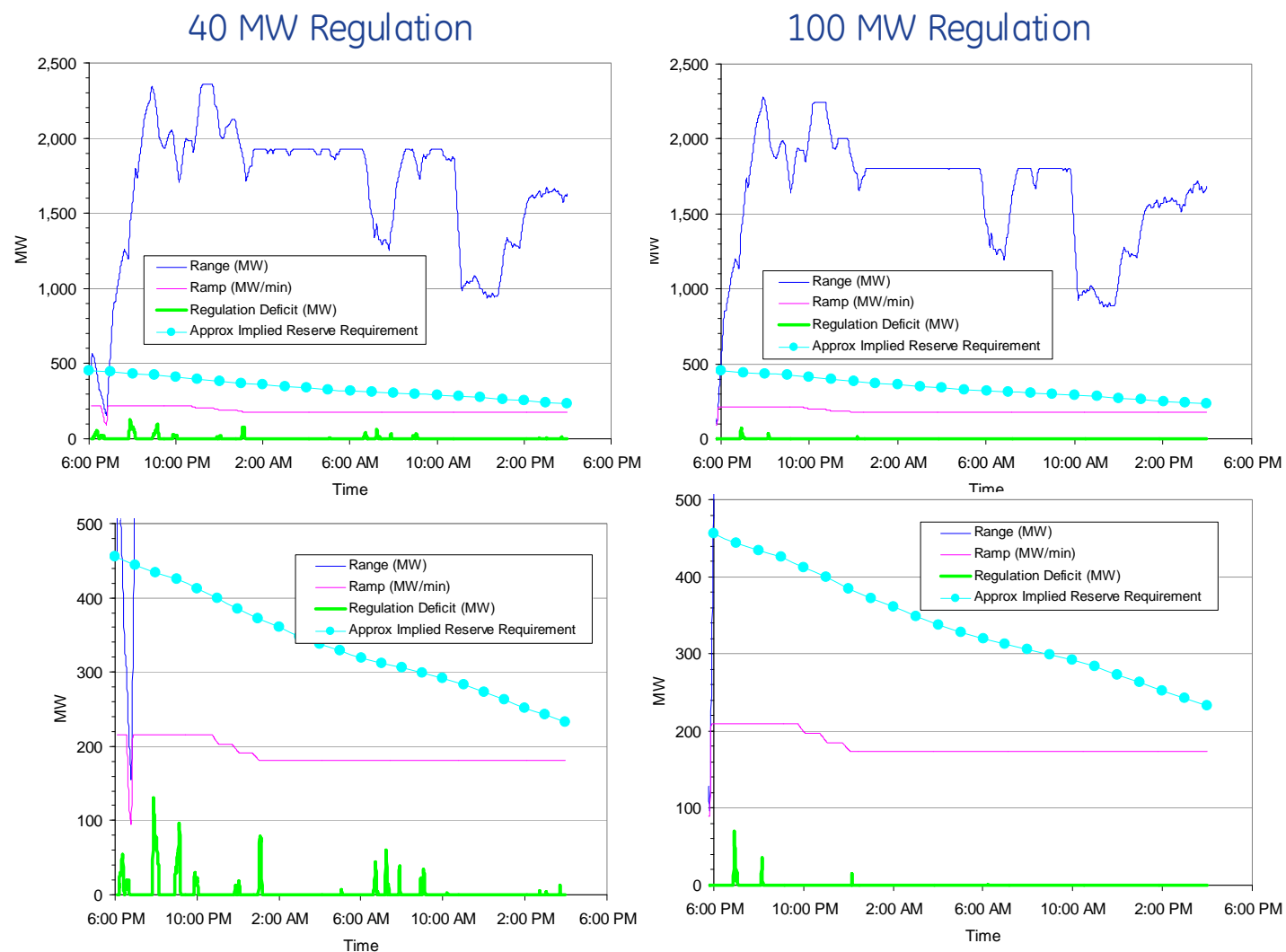

Figure 7.99 Wyoming L30R Scenario - Regulation Allocation Detail - April 15 Results

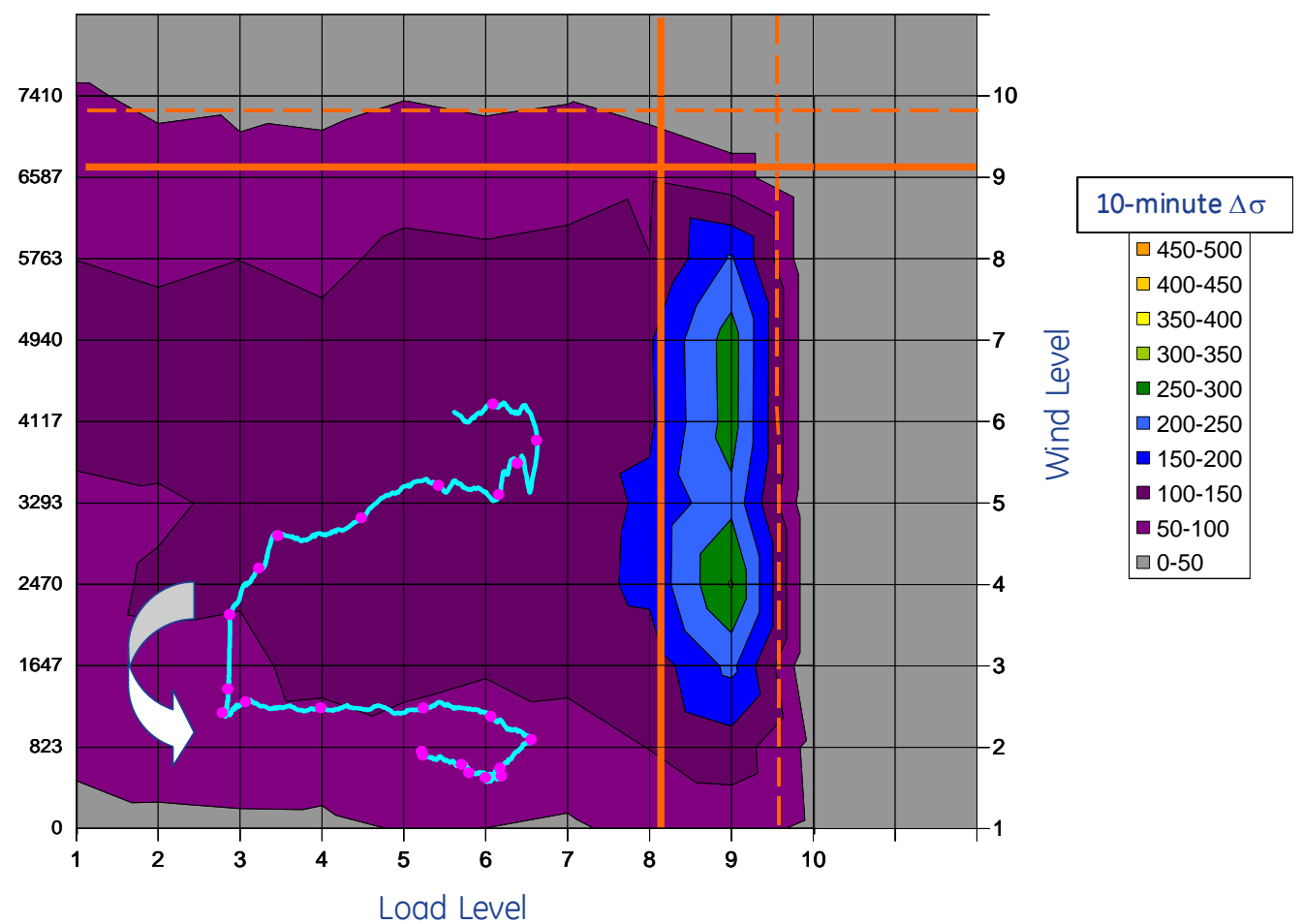

Figure 7.100 Wyoming L30R Net Load Variability October 262006 Trajectory 


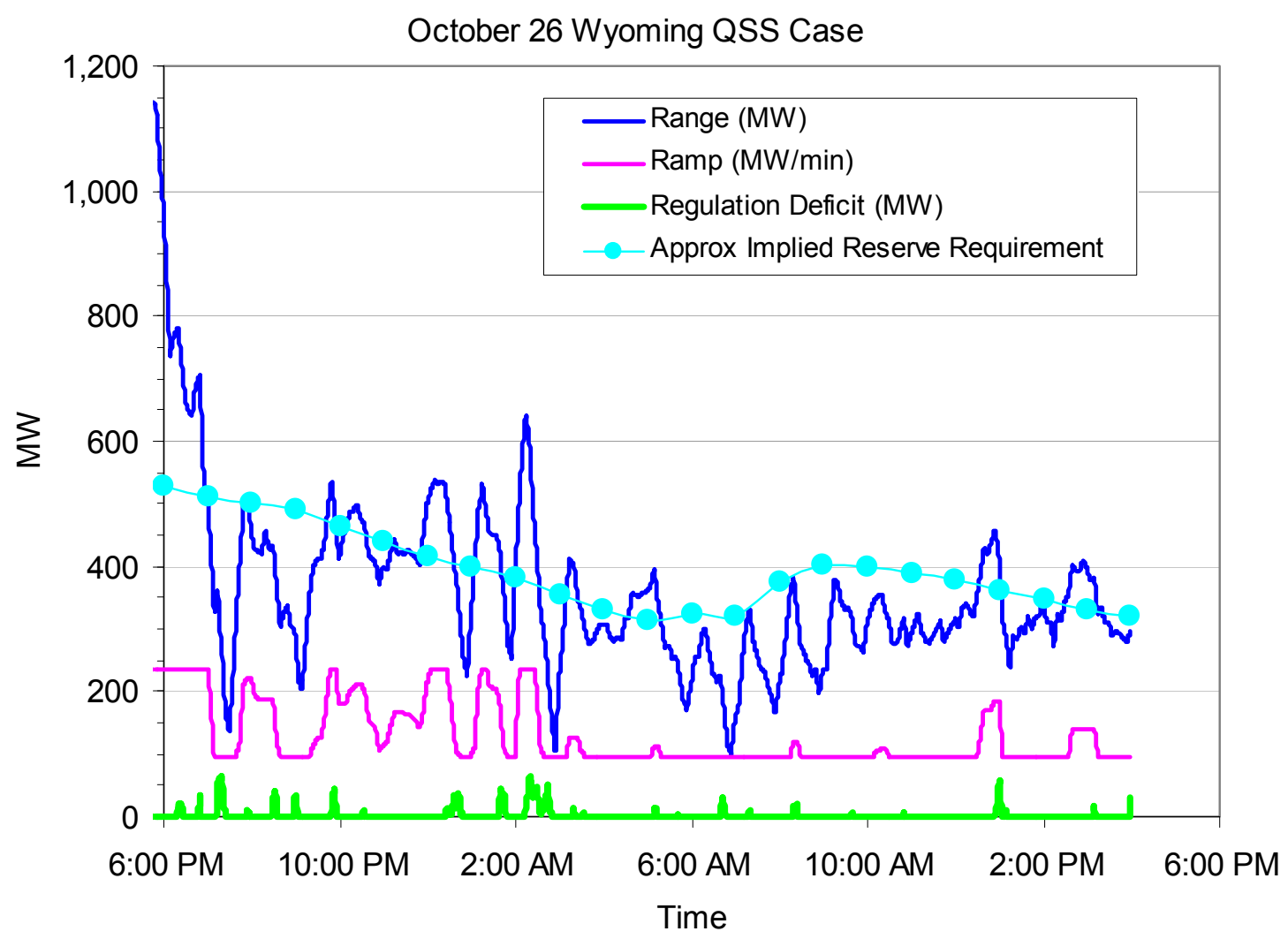

Figure 7.101 Wyoming L30R Scenario October 26, 2006 Results

\subsubsection{Non-Spinning Reserve Performance}

Throughout the discussion on variability reserves, there is an explicit expectation that system operators, when armed with guidelines, will act to position the system to maintain sufficient variability reserves to meet most changes in the shorter time frame (i.e., 10-minute). This simple statement implies that when those variability reserves are used and the level of variability reserves drops below the guidelines, e.g. the simplified rules based on $3 \Delta \sigma$ of 10 -minute variability, the system operators will replenish those reserves by starting new generation.

There are multiple time frames to consider:

Section 6 showed that the day-ahead commitment for reserves could be increased by $5 \%$ of the wind forecast, an amount that matches the wind term for 10-minute variability. This is the first and longest line of defense for the system operator. Obviously, the farther in advance that system operator "sees" their reserves being depleted, the more (and cheaper) options exist. By an hour ahead, the operator can only rely on quick-starts. Within the hour, the analysis assumes that nothing can be started faster than 10-minutes, 
so anything that happens in that window needs to be hot and synchronized, i.e. spinning - but not just "spinning reserves."

In the above results, the study footprint had enough up range from synchronized generation to cover spin plus variability reserves $95 \%$ of the time. For the discussion here, the term "quick start reserve" will be used. It will not be called "non-spinning reserve." "Spinning reserves", i.e., $1 / 2$ of the contingency reserves, are being distinguished from variability reserves, i.e., synchronized reserves that specifically aren't for the spinning component of contingency reserves.

In the local priority $30 \%$ case, the system generally has a large amount of quick start reserve available in the study footprint. Figure 7.102 shows duration curves of quickstart reserve for the $30 \%$ case and the no wind or solar case. Only uncommitted simple cycle combustion turbines were counted as quick-start. The no wind and solar case used quick start generation more frequently. Hence, these units did not count toward reserves when dispatched. The energy from the quick-starts in the no wind or solar case ( 4000GWh) is about double that in the Local Priority 30\% case ( 2000GWh). This duration curve makes sense, but gives no relationship between the quick-start reserves and the availability, or lack thereof, of variability reserves.

Figure 7.103 shows the variability reserve duration curves, and the variability reserve PLUS quick-start reserves for a total reserve. Two key observations can be made. First, in both cases the total reserve is always positive. Second, the reserve with wind and solar is generally higher.

Next, the relationships between quick start reserves, changes in wind power, and wind power level are explored. Figure 7.104 shows quick-start reserves vs. wind power at every hour (blue dots) and the wind drop (as a positive number) for every hour for which the wind dropped (orange dots). The basic premise is to test for a connection between quick-start reserve and wind power. And yes, higher wind tends to release quick-start reserve just as it does variability reserve. The hourly variability of wind is also tested for a connection to quick-start reserve. The relative bulge in the wind deltas (note the convention: a positive number means the wind power dropped and presumably depleted reserves) is at middle levels of wind power, as expected, not high. So at moderate wind levels, the quick-start reserves have some relative minima at wind levels with relatively high deltas. Further investigation shows that there is some correlation between lower reserves and a drop in wind power during the preceding hour. However, that correlation is relatively weak, indicating that many other operational factors besides wind variability drive the use of peakers. 
LP30 vs Base Case in Footprint - Quick Start Reserves

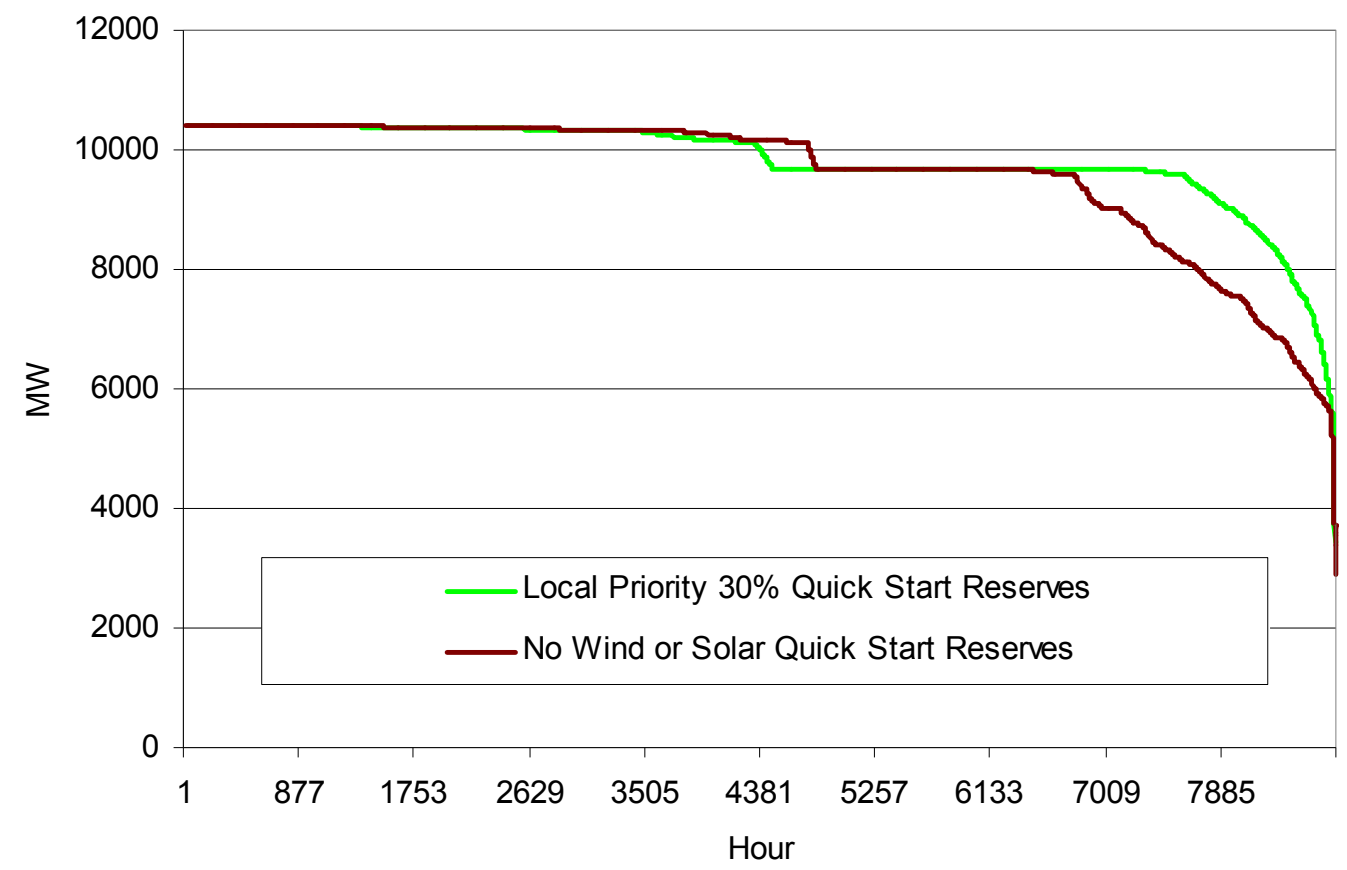

Figure 7.102 Comparison of Quick Start Reserves

LP30 vs Base Case in Footprint - Variability Margins ( $X+Y$ Rules) and Quick Start Reserves

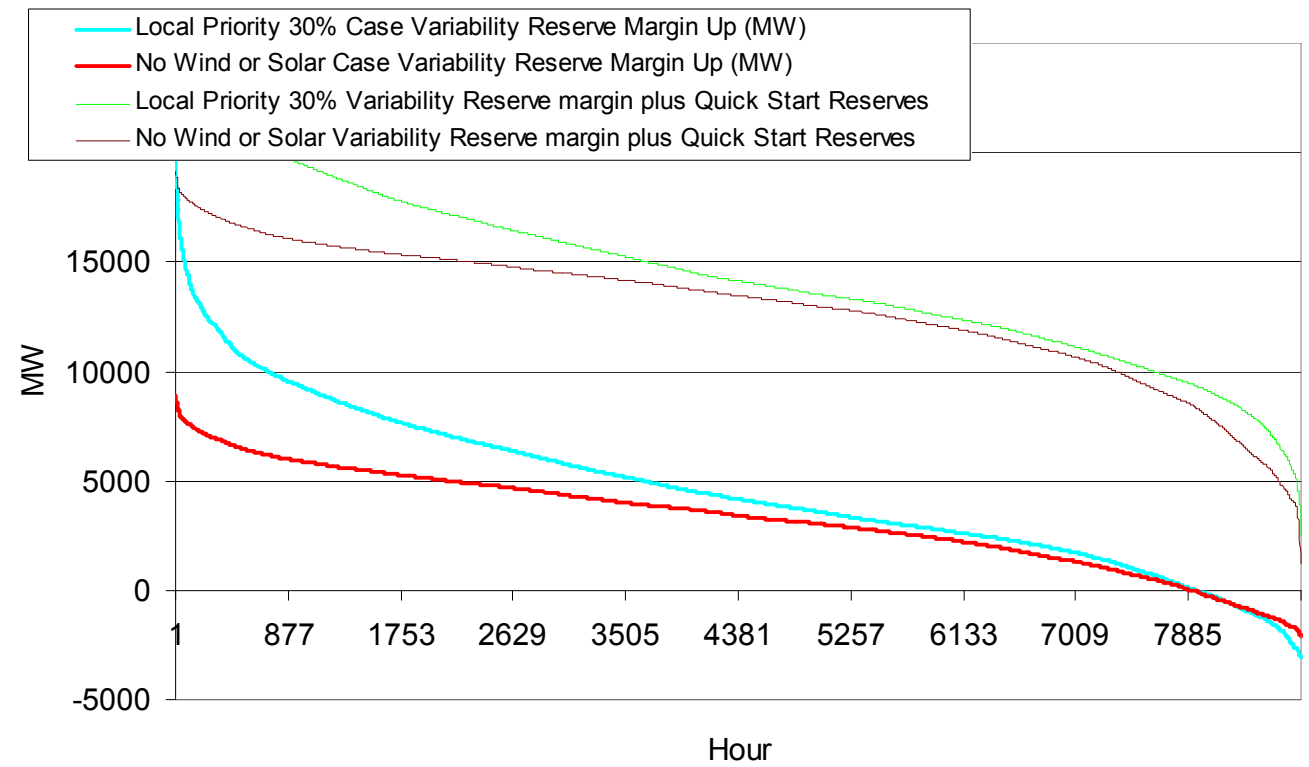

Figure 7.103 Comparison of Combined Reserve Margins 


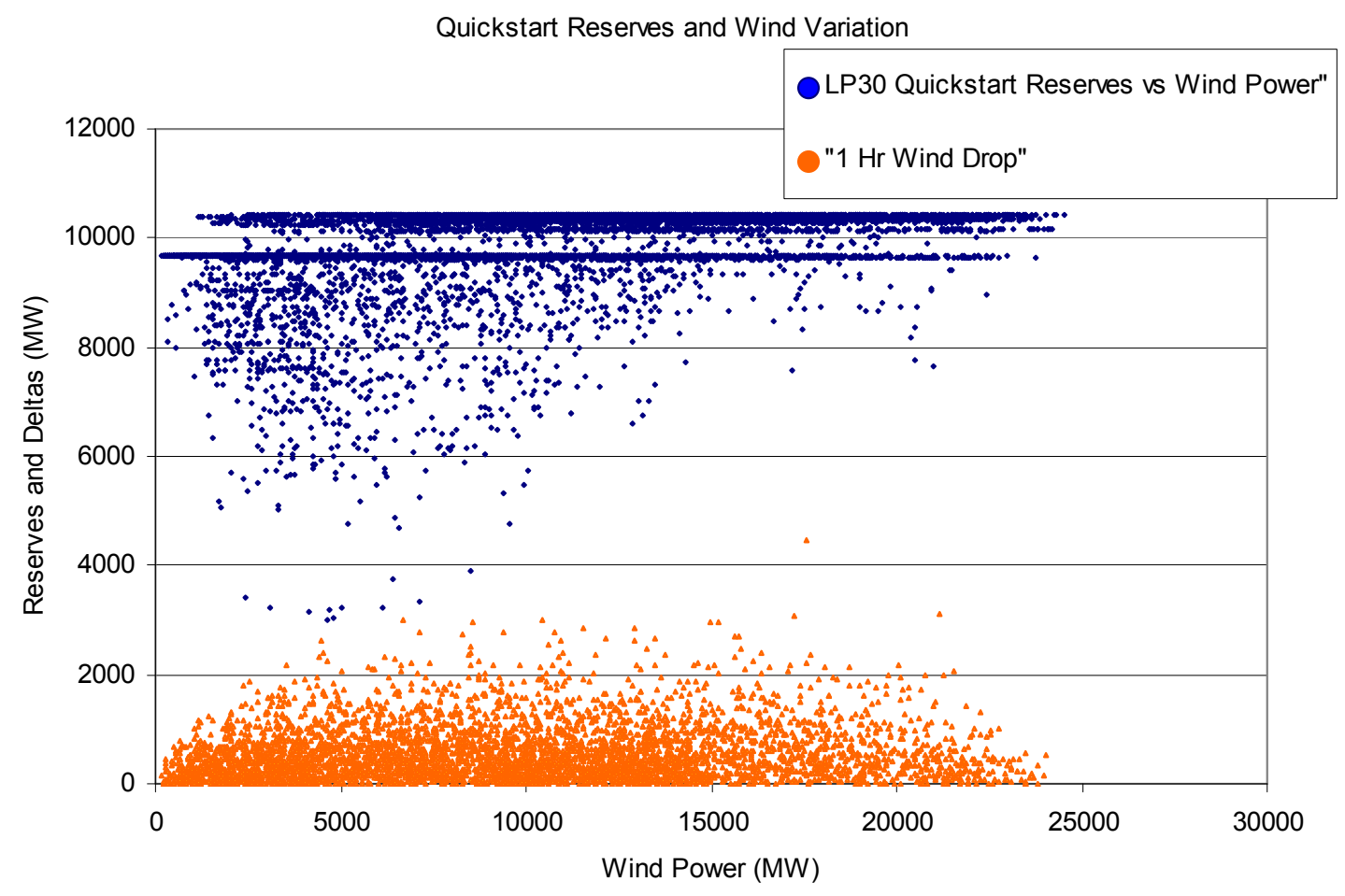

Figure 7.104 Relationship of Wind Drops and Quick-start Reserves to Wind Power Level

In summary, the use of quick-start generation is needed to maintain variability reserves under some conditions. When variability reserves are reduced, it is expected that system operators will then commit new units, specifically quick starts if they are the only resource that meets the time constraints. The availability of quick-start reserves has some positive correlation to wind power production, which is good. However, the correlation between quick-start reserves and wind power deltas is largely masked by other operational variability. When quick-start reserve levels drop, they tend to correspond to wind power levels with relatively higher levels of variability.

At the study footprint level, the system tends to naturally carry sufficient reserves for short-term, unexpected wind variability. The allocation and trade-offs between quickstart and other types of synchronized and non-synchronized reserves needs further investigation.

\subsubsection{Demand Participation in Reserves}

The reserves discussed throughout this section are required in response to net load fluctuations. In order to have those reserves, resources must be available. Under some conditions (e.g., over forecasting wind or under forecasting load), uncertainty will consume reserves needed to address variability. These mismatches in reserve must be addressed as they occur. 
In the end, the most likely and realistic use of demand participation covers the reserve shortfalls due to uncertainty. The results of Section 6.2.2 show significant economic benefits to this approach. Load demand is reduced, so the range on committed generation is freed up to provide variability reserves. Some systems (e.g. ERCOT) use load participation to provide spinning reserve today.

A more ambitious approach is for the demand resources to actually provide the reserve by responding to short-term changes in economic dispatch or even regulation signals. Such functionality is clearly possible, and could ultimately prove to be economical.

\subsubsection{Energy Storage Contribution to Reserves}

The QSS traces of Section 7.2 and the reserve requirements described in 7.3.2, show substantial reserve requirements during periods when relatively limited amounts of flexible generation are on-line. Unlike energy storage for arbitrage, energy storage technologies that can provide reserve services, both regulation and sub-hourly spinning reserve, may be economically attractive. The economics of energy storage for these services must compete with the costs, both operational and opportunity, of providing these services with conventional generation. This is grounds for further investigation. Costs to conventional generation, in terms of increased operation and maintenance, heat-rate penalty, and loss of life are not quantified for the higher variability environment of substantial wind and solar penetration.

\subsection{Conclusions on Sub-hourly Reserves}

1. Reserve requirements for resources needed to meet system variability increase with wind penetration. The required reserves are a function of the expected variability for the load and wind (and solar) power at a given operating condition.

2. These on-line reserves must be provided in addition to the spinning reserve portion of contingency reserves, so that most variability can be handled without impacting spinning reserve.

3. The incremental variability is a function of system size, wind penetration, and wind site diversity. At 30\% energy, overall sub-hourly reserve requirements roughly double over those necessary for load variability alone.

4. Reserves available within the study area, when the system is both committed and dispatched to maintain a minimum of $3 \%$ of load for spinning reserve, are generally sufficient to meet variability needs.

5. Uncertainty can cause resources (i.e. control range for reserves) needed for variability to be depleted. Unit commitment strategies are required to maintain reserve range. These strategies include, adding reserves in the day-ahead unit commitment to cover forecast uncertainty, changing unit commitment in time 
frames shorter than day-ahead to take advantage of higher fidelity forecasts, and using non-generation resources to free up reserves.

6. Operational analysis in Chapter 6 showed demand side participation (DSM) to be highly effective in managing uncertainty. DSM could be a key contributor to assuring that sufficient sub-hourly reserves are available to manage variability by reducing system load in response to operator dispatch commands at critical times.

7. Sub-hourly reserves need to be allocated between regulation and load following range (i.e. resources responding to frequent subhourly economic dispatch).

Carrying $1 \%$ of peak load for regulation appears to be adequate for modest wind penetrations. Allocating regulation equal to the standard deviation of 10-minute net load changes, i.e. $1 \times \Delta \sigma$, is analytically rational and was worked well under some extreme conditions.

8. Sub-hourly reserves, both regulation and load-following, can be consumed by hourly schedule changes. Therefore, scheduling strategies (including more frequent or continuous interchange and generation schedules) that avoid unnecessary consumption of sub-hourly reserves will improve system performance. Further investigation into economic aspects of various dispatch strategies for maintaining sub-hourly reserves is warranted.

9. Operating reserve rules based on load and wind power levels will allocate maneuverable reserves to match times and conditions of load and wind variability. Relatively simple linear rules, such as carrying $1-2 \%$ of load, and about $5 \%$ of wind power appear to work well. Some high penetration subsystems will need more reserves, if reserve sharing is not realized.

10. Energy storage may be a cost effective alternative to generation options for providing regulation and load following. Detailed subhourly analysis of storage was not performed, but could warrant further investigation.

11. Resources that can be started quickly provide an important mechanism to maintain on-line variability reserves. Such quick-start reserves appear to be adequate at the study footprint level, but may need to be increased by individual area. Further investigation of the requirements and relationships between on-line and off-line reserves is warranted.

12. Control area cooperation is essential. Small control areas will not be able to balance large amounts of wind. State-size areas will benefit, in terms of reduced reserve requirements, by sharing reserve burdens with other areas.

13. If each individual (state-size) area is required to provide its own sub-hourly reserves, the total amount of reserves required increases. To meet reserve requirements on an area-by-area basis will require, on average, about double the reserves required across the entire study footprint. 


\subsection{References}

[7.1] Standard BAL-001-0.1a - Real Power Balancing Control Performance, http://www.nerc.com/files/BAL-001-0_1a.pdf

[7.2] California Energy Commission's Intermittency Analysis Project Study “Appendix B-Impact of Intermittent Generation on Operation of California Power Grid" http://www.energy.ca.gov/2007publications/CEC-500-2007-081/CEC-500-2007-081-

$\underline{\text { APB.PDF }}$

[7.3] NERC 2007 CPS2 Bounds Report, January 31, 2007, http://www.nerc.com/docs/oc/opman/CPS2Bounds 2007.pdf

[7.4] "Developmental and Staffing Reliability Functional Agreement-Final Report on 2007 Studies," Columbia Grid.

[7.5] WECC Standard BAL-STD-002-0 - Operating Reserves

[7.6] WSCC Operating Reserve White Paper, July 16, 1998 version 1.0 


\section{Impact of Energy Storage and PHEV}

This chapter is divided into two main topics. The first part looks at the interaction of energy storage devices and variable renewable energy. The second part looks at the impact of a high penetration of Plug-in Hybrid Electric Vehicles (PHEV) on the value of renewable generation.

This analysis only considered the application of energy storage devices such as pumped storage hydro (PSH), Compressed Air Energy Storage (CAES), and batteries for the hourly arbitrage of energy throughout the day or week. These are used to provide generation during the high-priced hours of the week and replace the energy when it can be provided by much lower cost coal and nuclear generation. These devices typically have $6-12$ hours of storage and roundtrip efficiency on the order of $75 \%$. Storage can be used to compensate for wind forecast uncertainty and thereby allow wind to bid into the day-ahead markets for commitment. However, this study indicates that it may make more economic sense, from a system standpoint, to commit the system generation based on the State-of-the-Art forecast and cover the shortfalls with the operating reserve, quick-start generation and demand response. Storage devices also have capacity value and can be used to provide spinning reserve and voltage support but that was not evaluated in this study.

\subsection{Base Pumped Storage Operation}

Figure 8.1 shows annual duration curves for all of the WECC pumped storage hydro units for a range of renewable penetrations. The individual plants in WECC were modeled separately and summed chronologically before being sorted for this graph. As can be seen, increased renewables increases the utilization of the storage slightly but does not push the usage to a point where more storage would seem to be required. This is indicated by flat portions at either extreme of the curve, which indicate saturation. A plot of all of the storage within the study footprint would show similar results. Although some of the smaller, individual sites showed some increased saturation, the overall footprint did not. 


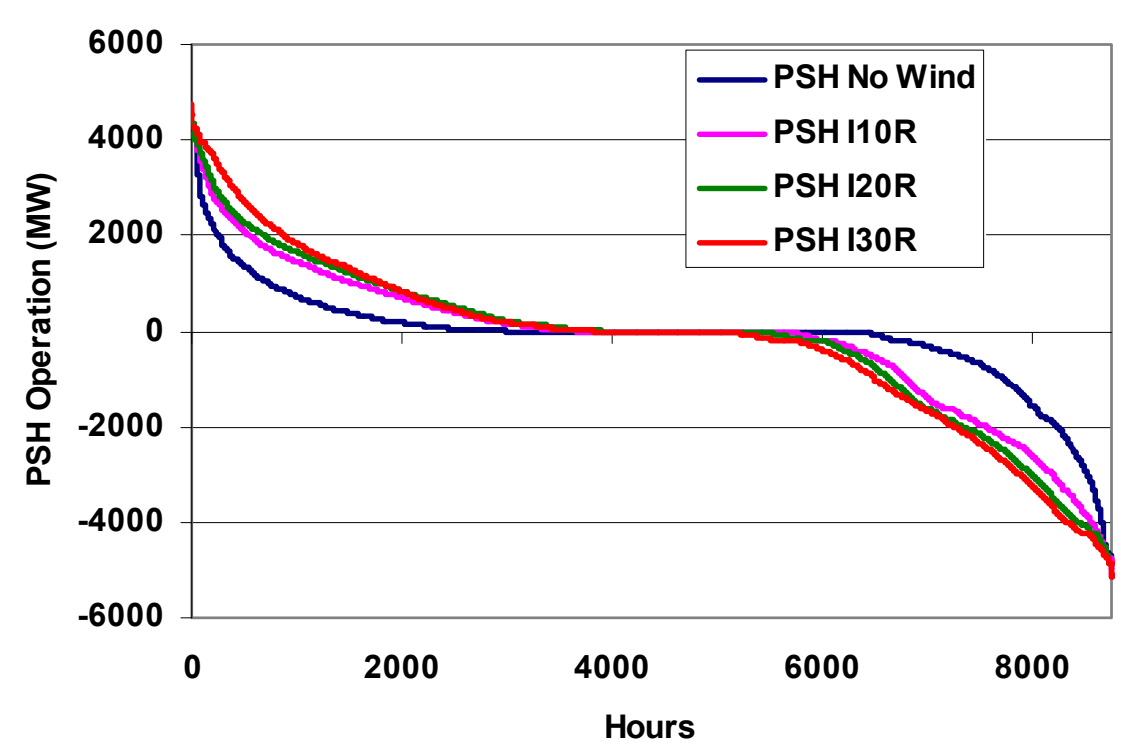

Figure 8.1 PSH Annual Duration Curve

The storage schedule is planned based on the assumed cost of pumping versus the assumed value of the displaced generation for the coming days and week. In order to verify that these assumptions were not being overly conservative, simulations were made with the cost of pumping multiplied by either .75 or .50 before being compared to the generation savings. For example, in the base-case it would make economic sense to displace $\$ 500$ of generation on-peak with off-peak pumping energy that cost $\$ 400$ to produce. However, if the pumping energy costs $\$ 600$ then the action wouldn't take place. In the ".75" case the pumping cost would be multiplied by 0.75 so that the "effective pumping costs" would appear to only be $\$ 450$ and the action would occur. These factors are used only for PSH decision purposes; they do not effect the actual costs of operations. The resulting utilization curves are shown in Figure 8.2. Clearly the PSH operation has increased as the assumed cost of pumping has decreased.

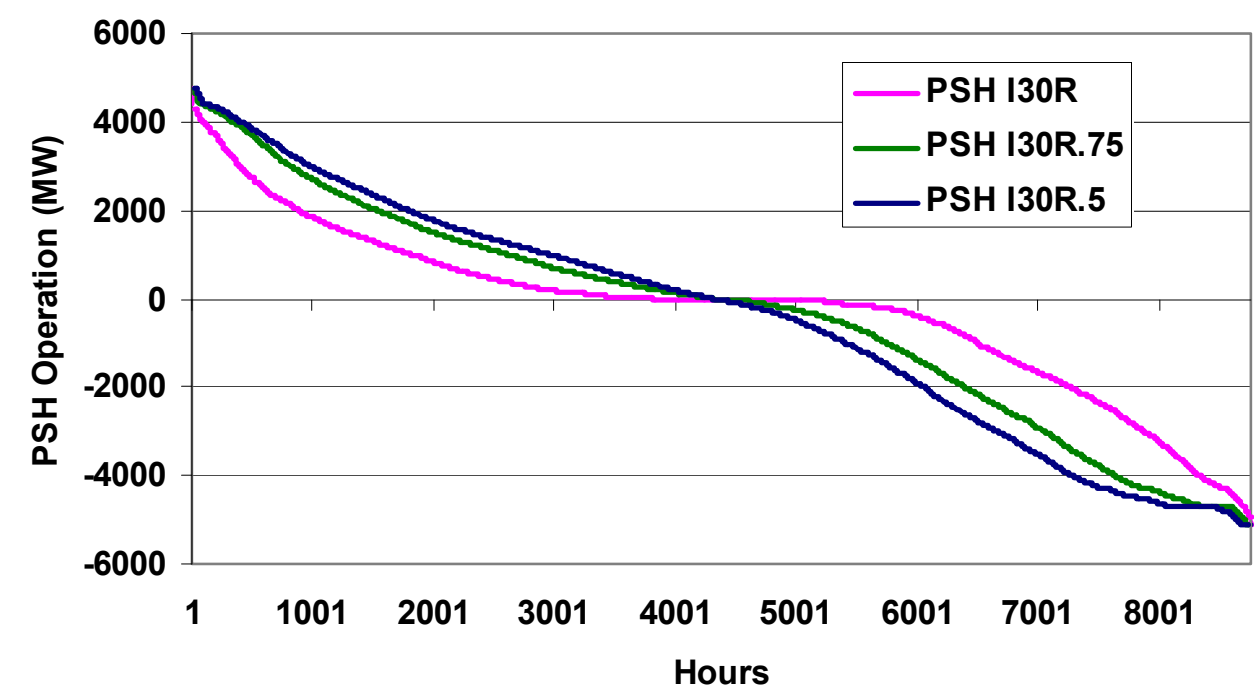

Figure 8.2 PSH Operation with Discounted Pumping Costs 
Figure 8.3 shows the impact on the WECC operating costs. Forcing the PSH to operate more than the model felt was necessary did indeed increase the annual total cost of operations by $\$ 60 \mathrm{M}-\$ 120 \mathrm{M}$. This confirms that the base-case assumptions on the PSH operation were valid. The next section digs deeper into how storage could be optimized to improve the integration of renewable energy.

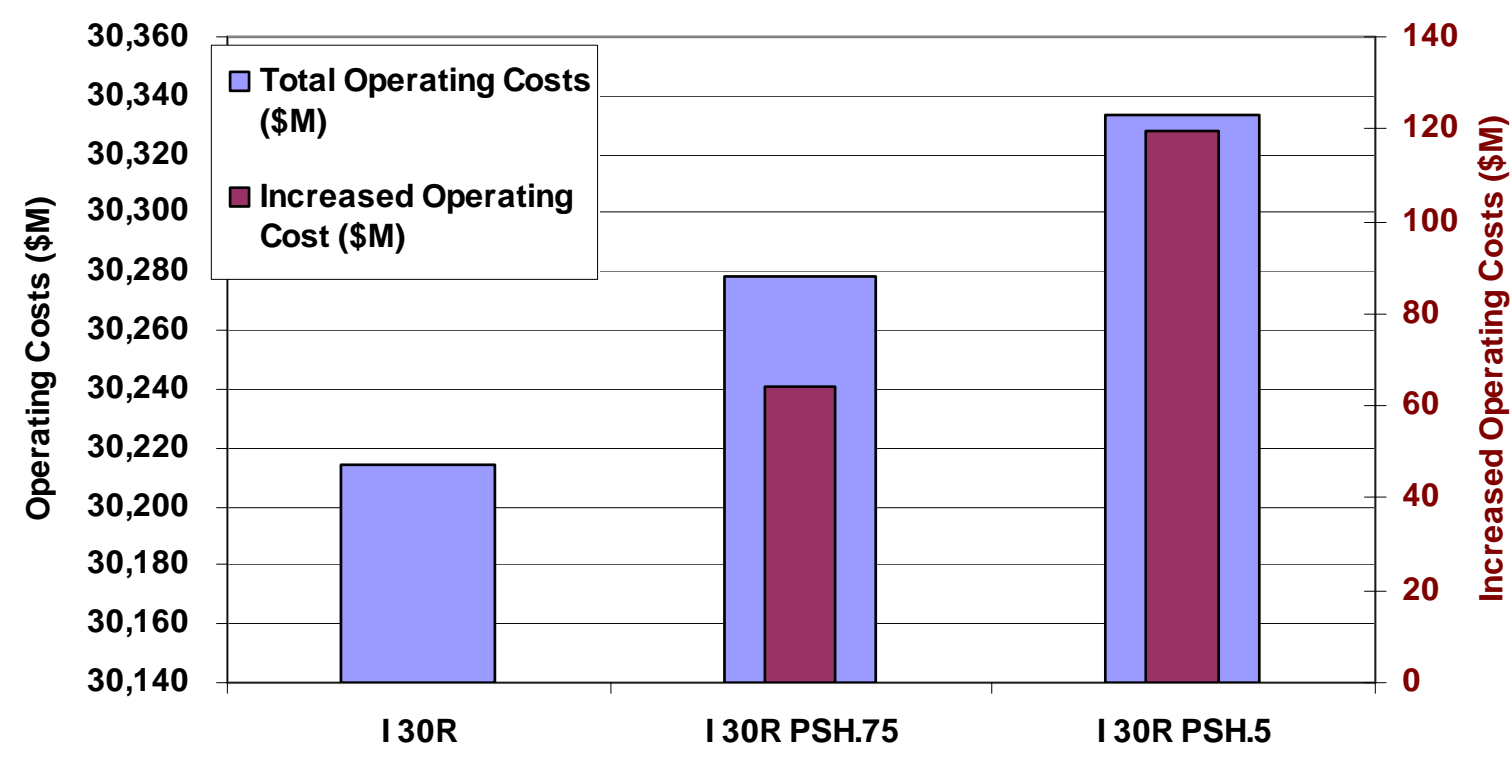

Figure 8.3 Operational Impact of Forcing PSH Operation

\subsection{Pumped Storage Optimization}

In order to examine the phenomenon of storage operation a little closer, additional analysis of a spot price curve for the Arizona/New Mexico portion of the system was performed. Figure 8.4 shows the hourly load (against the right hand axis) and the hourly spot price for a range of wind and solar penetrations for the week of April 10 $10^{\text {th }}$ assuming a perfect forecast. The prices were relatively low since this is a relatively low load week. More importantly, the price variation across the week was small. This is consistent with what was seen in the dispatch curve in Figure 6.41. The marginal cost is coming pretty consistently from combined-cycle generation and so the variation is due to heat rate differences on similar units. As more renewable generation is introduced with a perfect forecast, it simply depresses the spot price across the week. The prices dip significantly on Saturday when the marginal cost is being set by coal plants, but otherwise there does not appear to be sufficient price variation across the day to overcome the $25 \%$ round trip losses inherent in a conventional storage plant. 


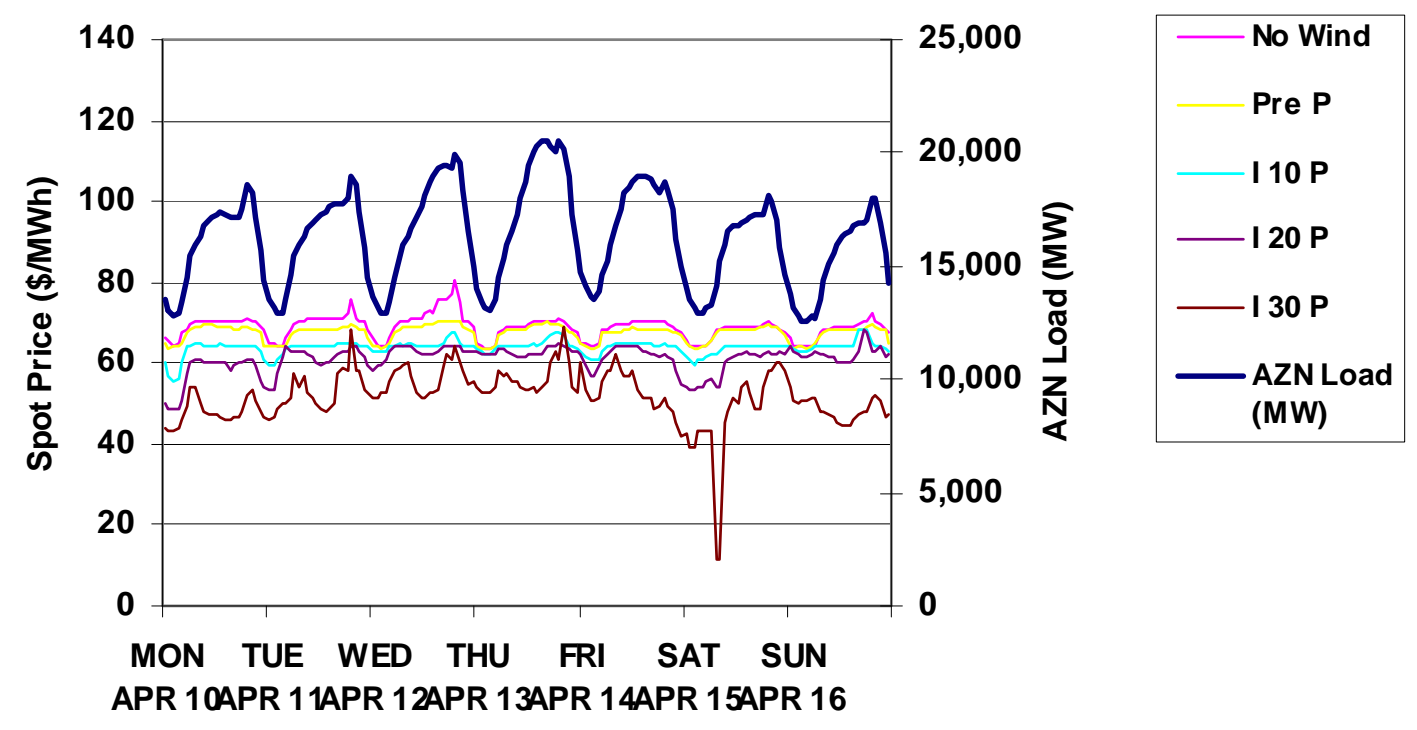

Figure 8.4 Arizona Spot Prices, Week of April 10th, Perfect Forecasting

This changes rather significantly in Figure 8.5, which assumes a State-of-the-Art forecast. The no-wind curve is the same as above and even the low penetration curves are similar. At the higher penetrations, particularly the $20 \%$ and $30 \%$ cases, the impact of the imperfect forecast can be seen. Under-forecasts at the beginning of the week result in over commitment of generation and drive the spot prices down significantly. This is then followed closely by an over-forecast that forces the system to turn on peaking units and drives the spot prices up. This pattern continues throughout the week.

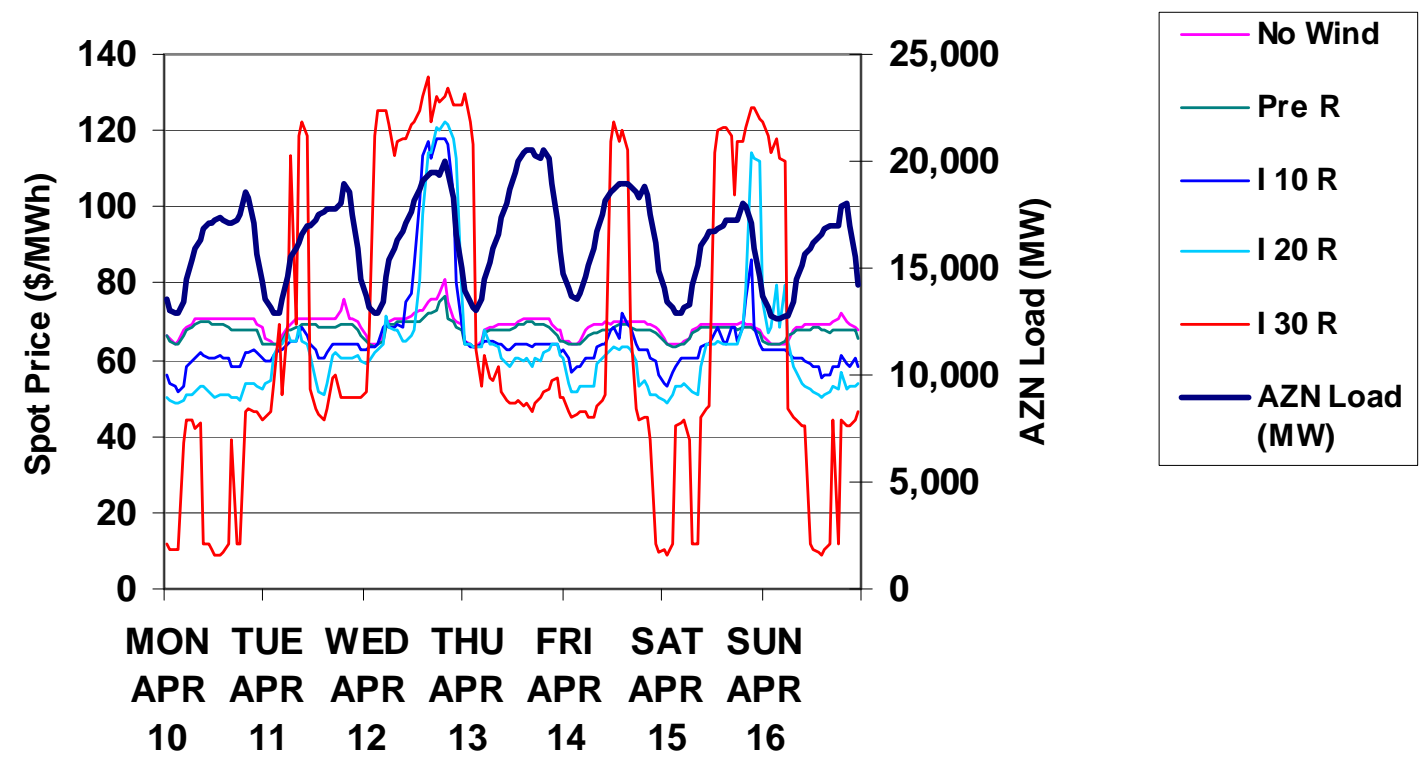

Figure 8.5 Arizona Spot Prices, Week of April 10th, S-0-A Forecasting 
It would be very desirable to schedule the storage against these curves. Unfortunately, this would require perfect foreknowledge of when the wind was being over-forecasted and when it was being under-forecasted. If this were known in advance then the forecasts could be adjusted to bring the results back to the perfect forecast as shown in Figure 8.4. Again, this is only looking at the inter-hour application of the storage. Intra-hour applications may have benefits.

But let's assume that a new PSH plant had a tool that could predict when the spot price would be high and low. A separate model was used to dispatch a 100-MW PSH unit against the spot price curves shown in Figure 8.4 and Figure 8.5 for the entire year. The results in Figure 8.6 show the number of hours and the operating value resulting from this simulation. With no renewables, the PSH unit would run about 2200 hours (total pumping and generating time) and have an operating value of about \$2.6 $\mathrm{M}$ for the year. With a $10 \%$ fixed charge rate this would result in a capitalized value of roughly $\$ 260 / \mathrm{KW}$. This is far less than the $\$ 2500 / \mathrm{KW}$ estimated cost for a new storage facility (LEAPS project, a 500 MW PSH in California). Even though a PSH plant would likely receive a full capacity credit of $\$ 600-\$ 1000 / \mathrm{kW}$ there is still a shortfall. As expected, with a perfect forecast the value of the PSH unit decreased as the renewable penetration increased. With $30 \%$ penetration and a perfect forecast the 100-MW PSH plant had an annual operating value of $\$ 0.5$ million, which would yield a capitalized value of about $\$ 50 / \mathrm{KW}$.

With the State-of-the-Art forecast, the values initially decreased, but not as much as with the perfect forecast. At the $30 \%$ penetration level, the value jumps up significantly, but is still only $\$ 3.8$ million/year of operating value. This translates to roughly $\$ 380 / \mathrm{KW}$ which, even with a generous capacity value, is still more than $\$ 1000 / \mathrm{kW}$ below the cost of a new facility. Even perfect foreknowledge of when the prices will spike and drop does not seem to provide sufficient value to justify adding any new storage facilities. 


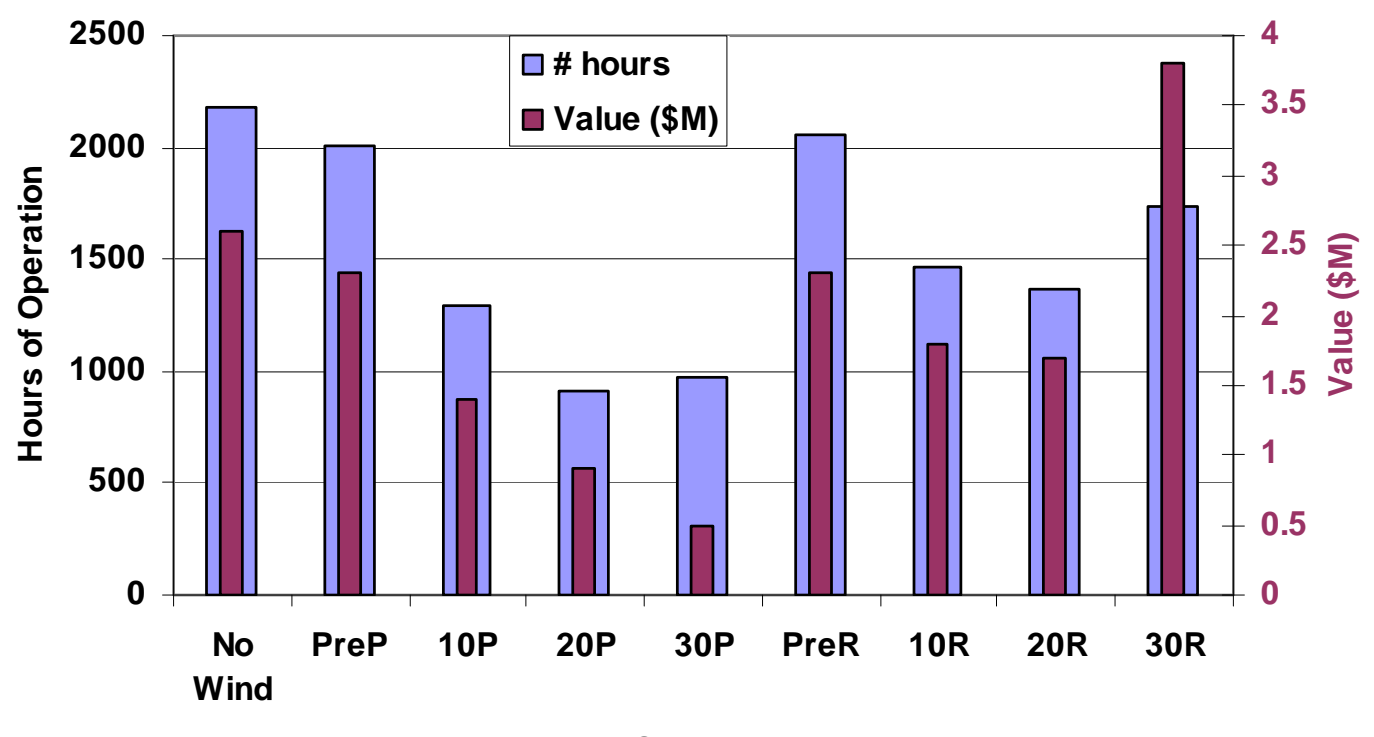

Scenario

Figure 8.6 Incremental Value of a 100-MW PSH Plant

\subsection{CSP Operation Optimization}

The Concentrating Solar Plants, CSP, simulated in this analysis were designed with six hours of thermal storage included. The collector field was over-sized relative to the steam turbine and generator, and the additional heat was stored to allow the unit to continue producing electricity after the sun went down. The question was posed, "Will a different schedule generate more revenue?" The curves in Figure 8.7 examine the operation of a single plant for one day. The blue curve is the schedule as operated. The purple curve is the hourly spot price. The green curve is the operation, which maximizes the daily revenue while recognizing the six-hour storage limit, and specifying that the energy must be used up within the day. Rather than using the energy as it became available at 7 a.m., the energy was stored until prices spiked at 10 a.m., and then stored again until after noon. Output was curtailed when the spot price dipped at 7 p.m. and then run at rated power for the balance of the day. The daily revenue (= hourly spot price times hourly generation) for the base operation was $\$ 657 \mathrm{~K}$, while the revised revenue was $\$ 772 \mathrm{~K}$, an increase of $18 \%$. Although there may be ramping limits that were not considered in this simple analysis, and this approach assumes perfect foreknowledge of the price curve, this analysis provides an operational bookend, and gives an insight into potential rescheduling benefits. 


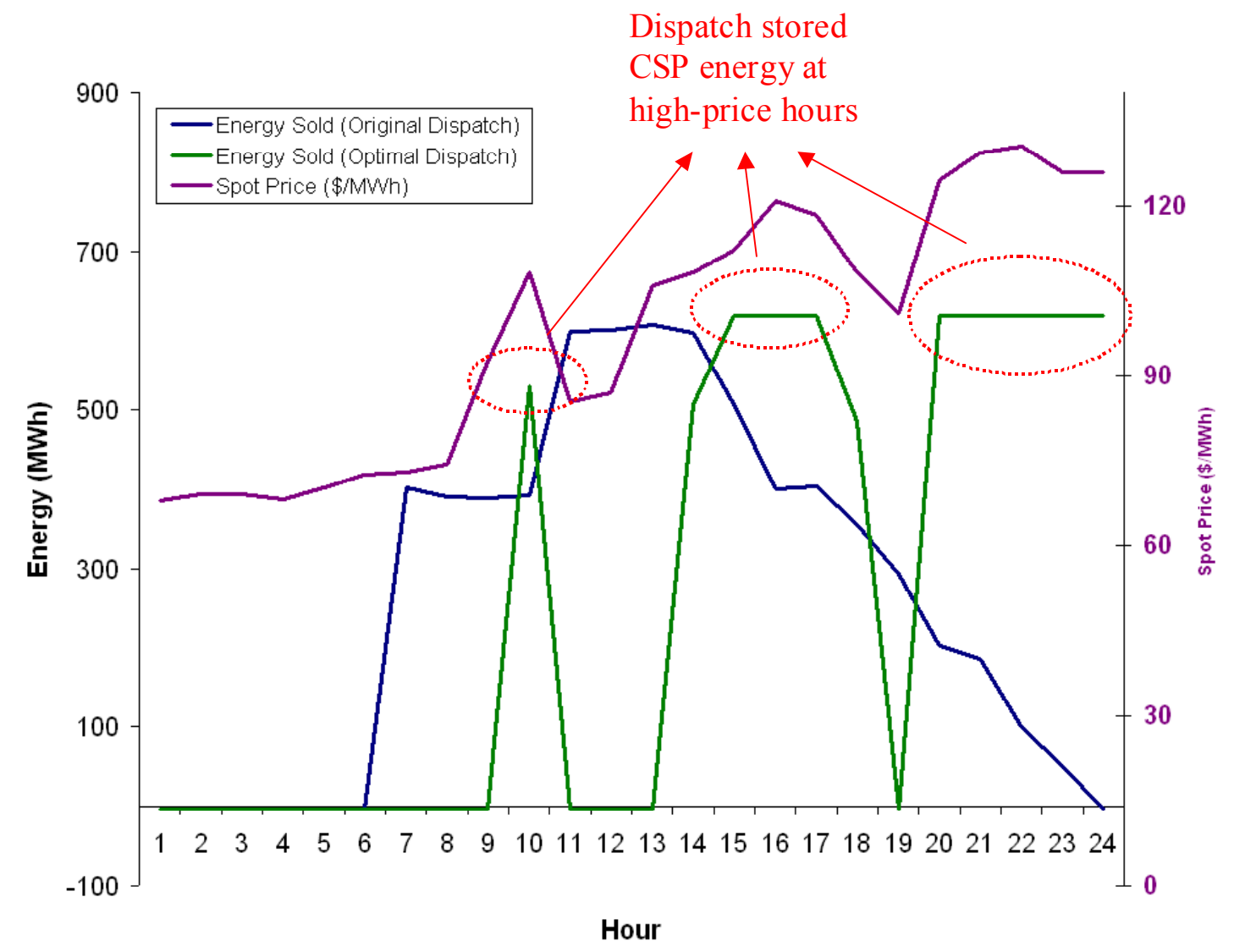

Figure 8.7 Sample Energy Shift for CSP "Optimal" Dispatch

The curves in Figure 8.8 show the monthly energy production for all of the CSP plants.

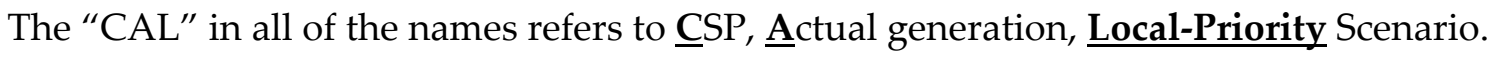
The remainder of the name refers to the region of the system, as described in Chapter 3. As might be expected, the energy is highest in May through October and drops off for the rest of the year. This actually corresponds well with the load profiles for the system, as discussed in Chapter 4. Figure 8.9 shows the total annual energy by zone. 


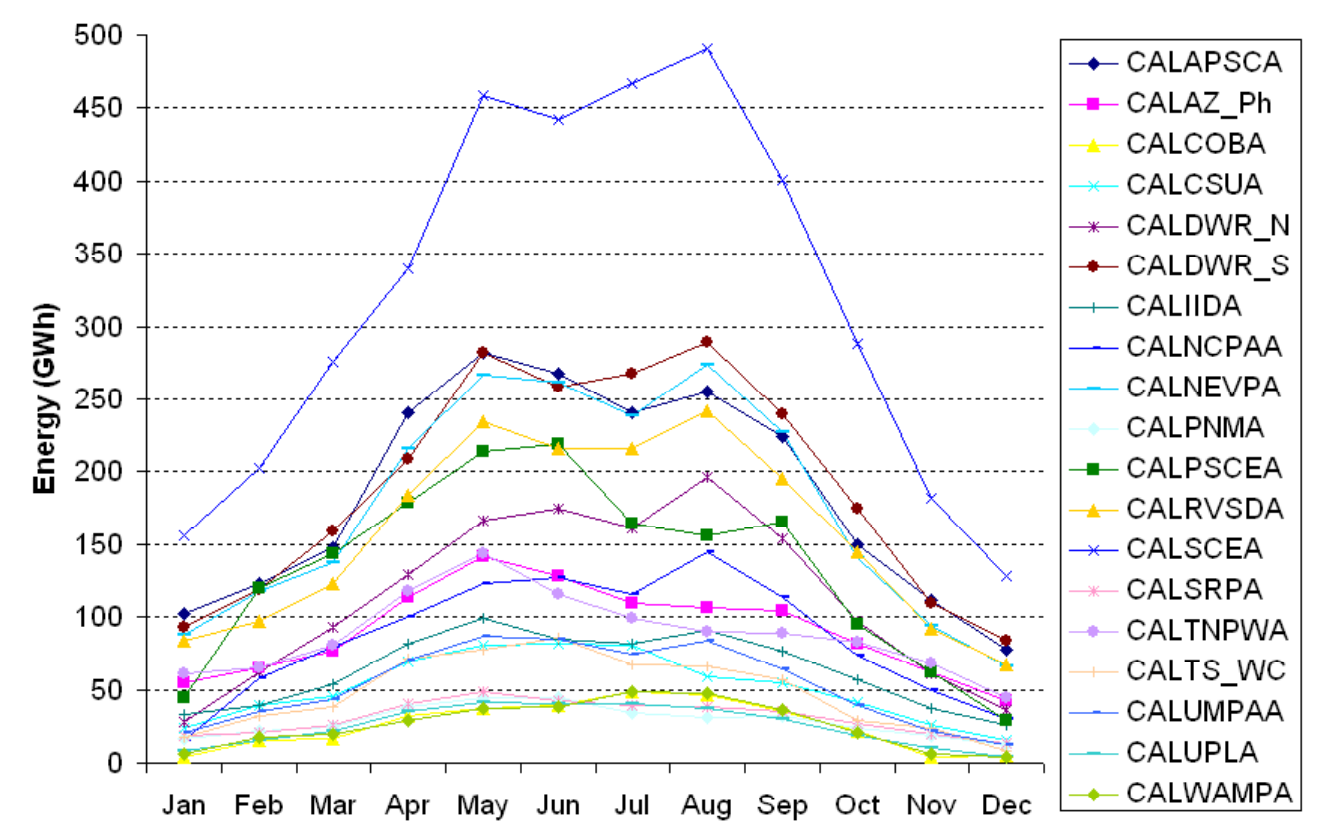

Figure 8.8 Monthly CSP Energy by Zone

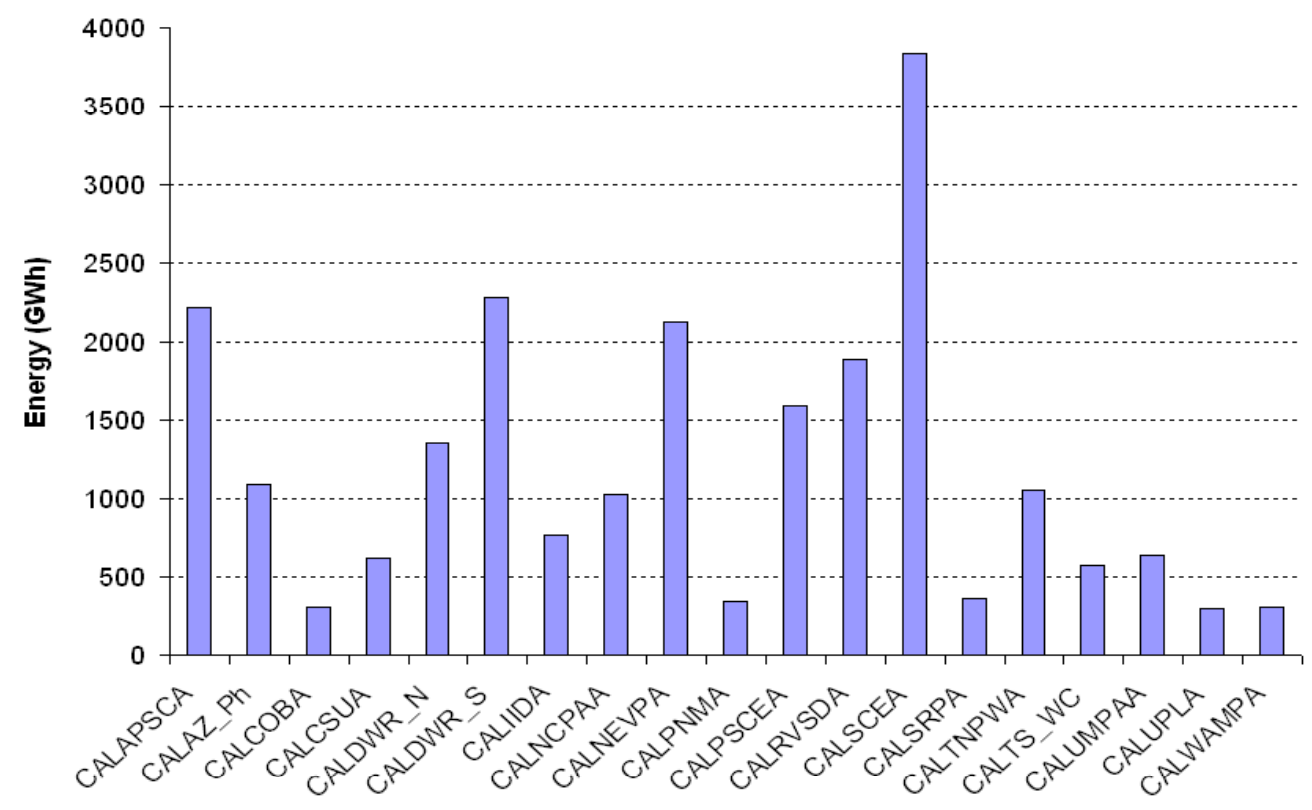

Figure 8.9 Annual CSP Energy by Zone

As could be seen in Figure 8.7, having more storage capability would allow more energy to be saved for hours with higher spot prices. Conversely, having less storage would reduce the value. Figure 8.10 shows the range in increased value for each of the plants as a function of the hours of storage. Six hours seems to be a reasonable saturation point. 


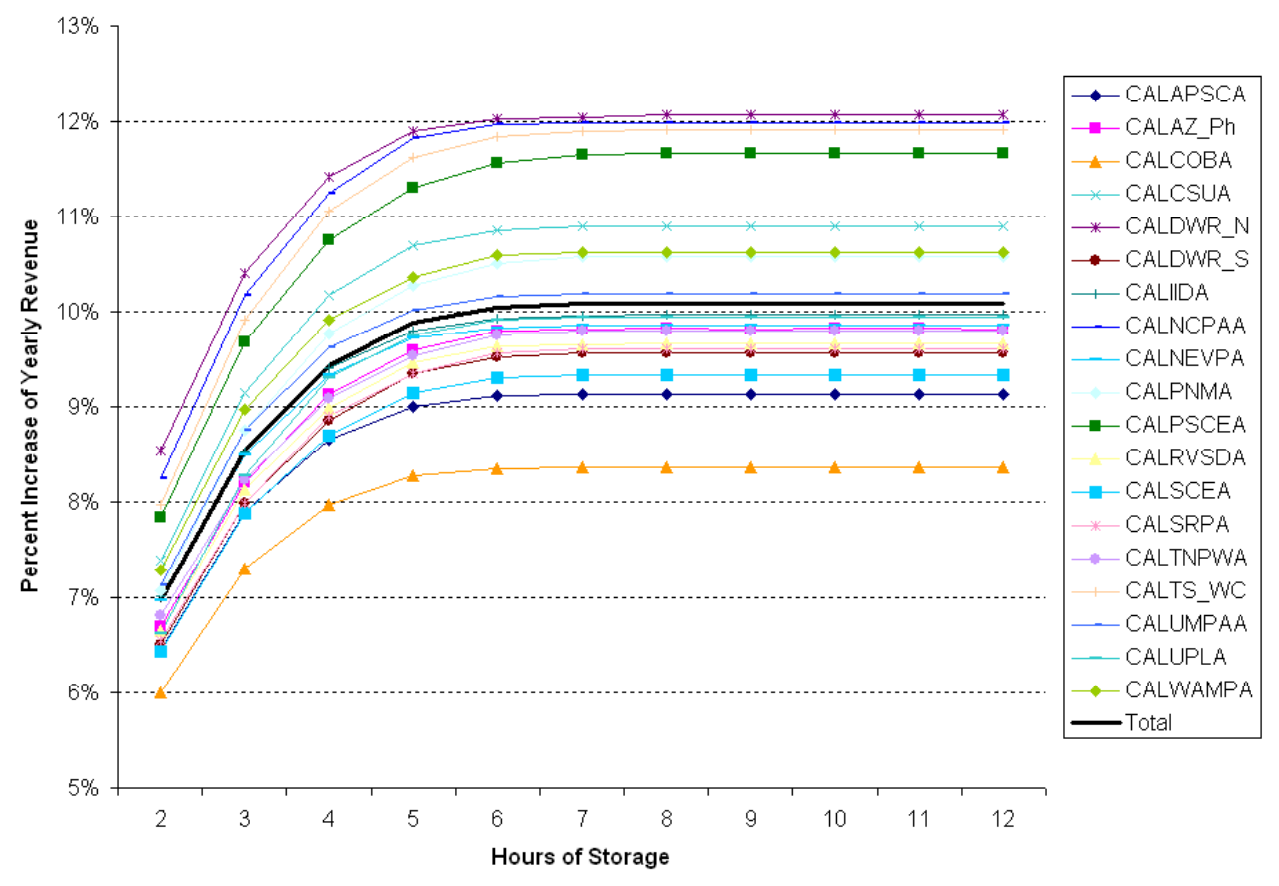

Figure 8.10 Annual CSP Revenue Increase vs. Hours of Storage

Figure 8.11 shows the annual increase in revenue over the original operating schedule for all of the sites assuming six hours of storage. There is roughly a $10 \%-12 \%$ improvement for all of the locations. 


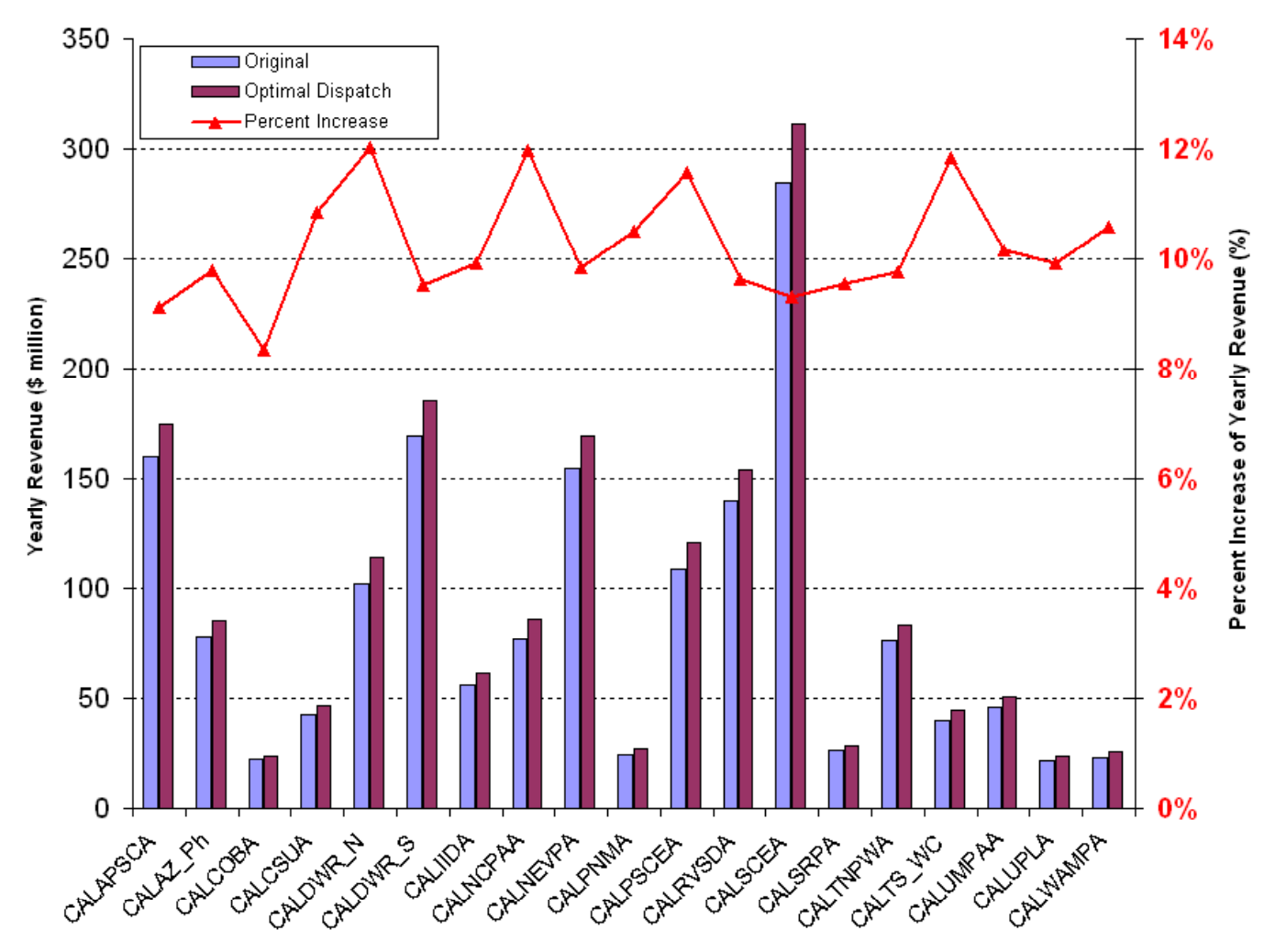

Figure 8.11 Annual CSP Revenue Increase by Zone Due to Optimal Dispatch.

Figure 8.12 depicts the increases by plant on a monthly basis. The on-peak periods of the year, which had the greatest amount of energy, had a modest $5 \%-10 \%$ increase. This is because the six hours of storage inherent in the base operation was largely saturated in these months so there was little room for improvement. The off-peak months, which had less energy available, showed that there was significant room for improvement by storing more of the energy during the day for use at night when the spot prices were higher. The base operation only stored energy during the day that was in excess of the system's capability to utilize directly. Figure 8.13 shows the monthly results for the total system. 


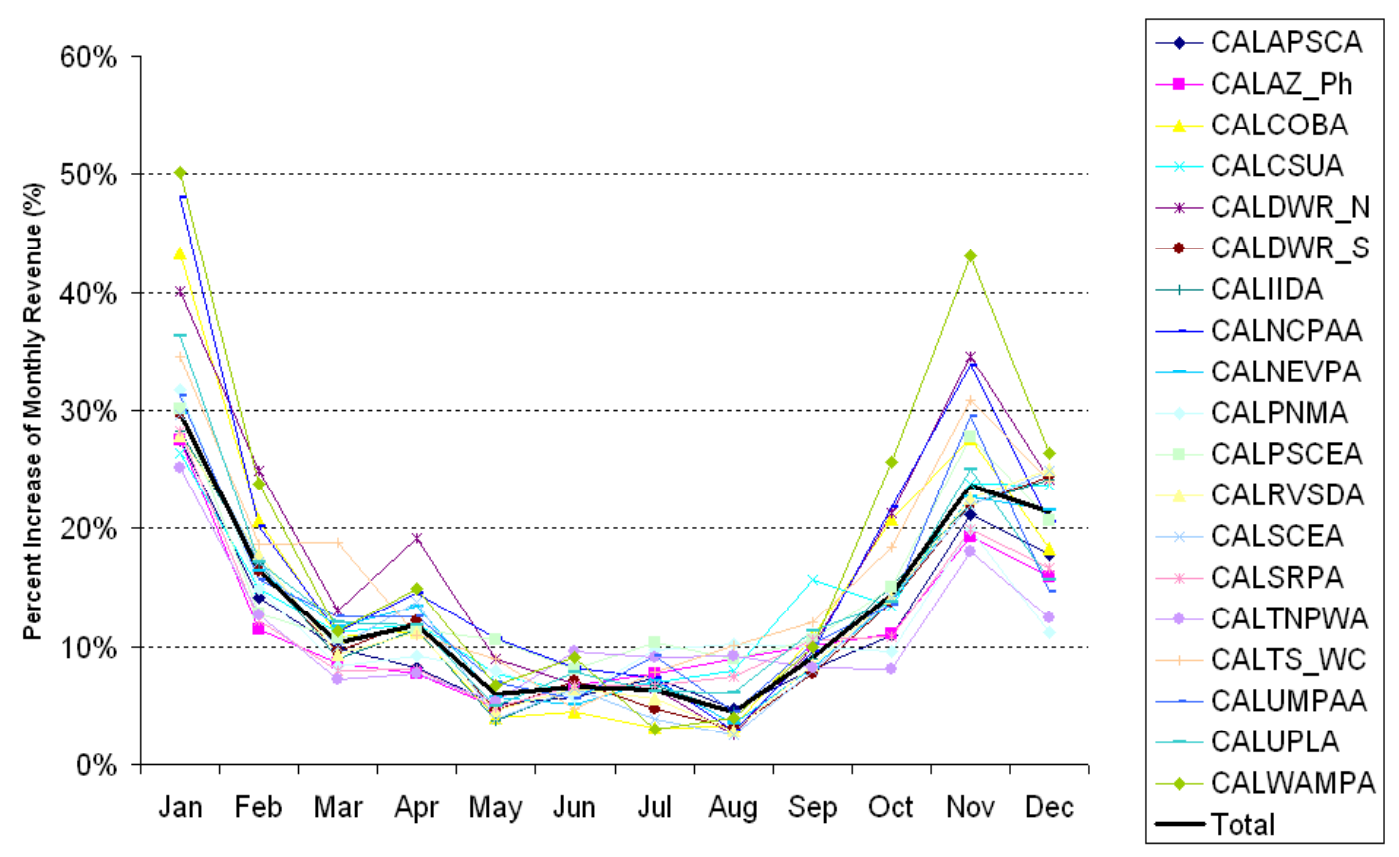

Figure 8.12 Monthly CSP \% Revenue Increase by Zone Due to Optimal Dispatch

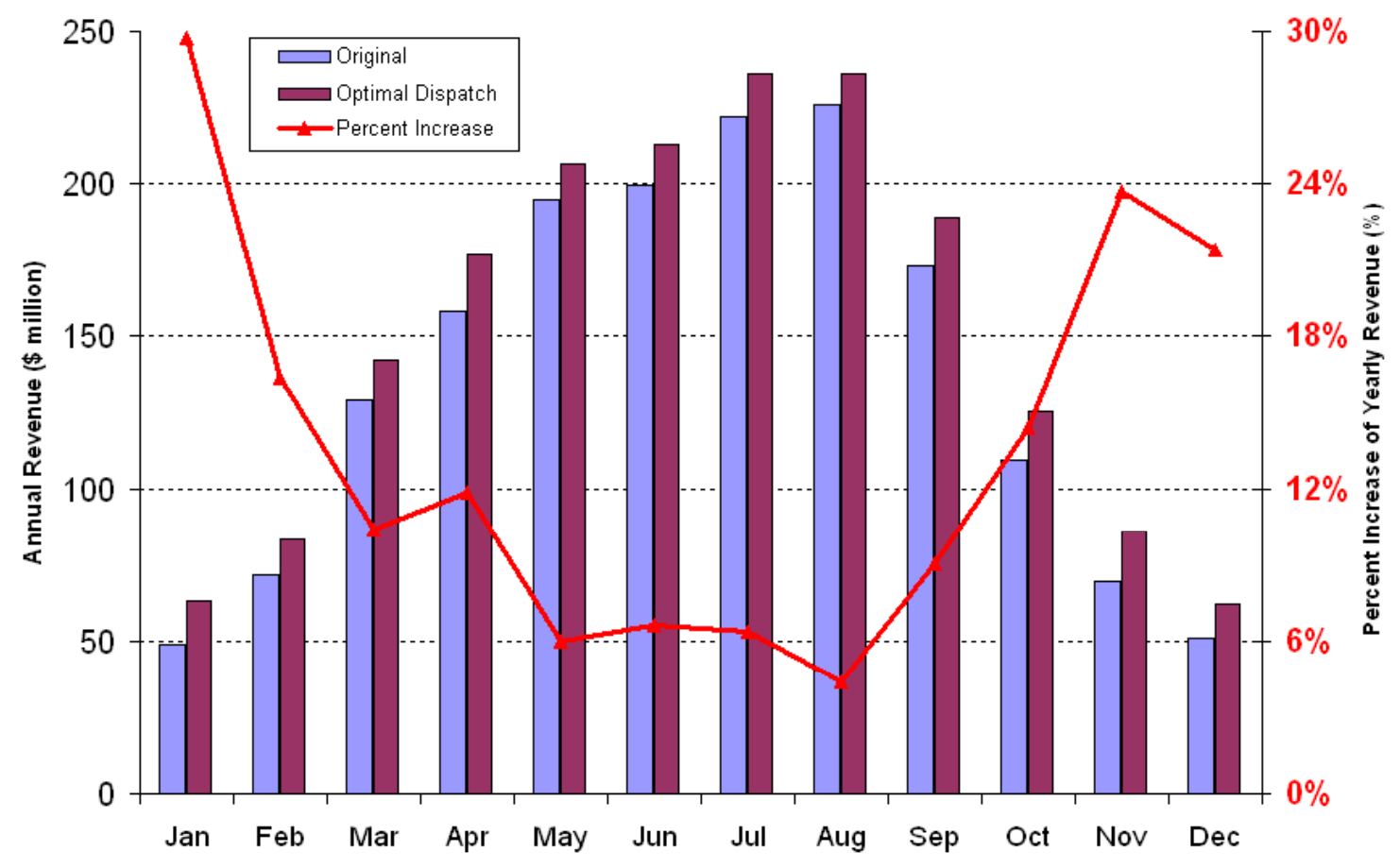

Figure 8.13 Total Monthly CSP Revenue Increase 


\subsection{Plug-in Hybrid Electric Vehicles, PHEV}

Some of the difficulties that arise with large amounts of wind generation on the system are due to the fact that much of the wind generation occurs at night when the loads are lower. One potential solution would be to increase the off-peak loads with the charging demands of plug-in hybrid electric vehicles, PHEV. Table 8.1 lists the potential daily and annual load increase by state if $15 \%$ of all light-duty vehicles and $3 \%$ of heavy-duty vehicles were converted to PHEVs. On an annual basis, this would represent about a $1.5 \%$ increase in the total WECC load.

Table 8.1 Potential Load Increase from PHEV by State

\begin{tabular}{|l|r|r|}
\hline State & \multicolumn{1}{|c|}{$\begin{array}{l}\text { Daily Load } \\
\text { (MWh) }\end{array}$} & \multicolumn{1}{l|}{$\begin{array}{l}\text { Annual Load } \\
\text { (GWH) }\end{array}$} \\
\hline Arizona & 2,558 & 934 \\
\hline California & 21,734 & 7,933 \\
\hline Colorado & 1,051 & 384 \\
\hline Idaho & 704 & 257 \\
\hline Montana & 585 & 213 \\
\hline Nevada & 812 & 296 \\
\hline New Mexico & 885 & 323 \\
\hline Oregon & 1,734 & 633 \\
\hline Utah & 1,312 & 479 \\
\hline Washington & 3,547 & 1,295 \\
\hline Wyoming & 327 & 119 \\
\hline WECC Total & 35,248 & 12,865 \\
\hline
\end{tabular}

The curve in Figure 8.14 shows the assumed chronological distribution of the PHEV charging load in each area. 5\% was assigned to 11 p.m., $10 \%$ to 12 p.m., $15 \%$ from 1 a.m. -5 a.m. and $10 \%$ at 6 a.m. On a WECC-wide basis, this represented about a 5,000-MW load increase in the middle of the night. 


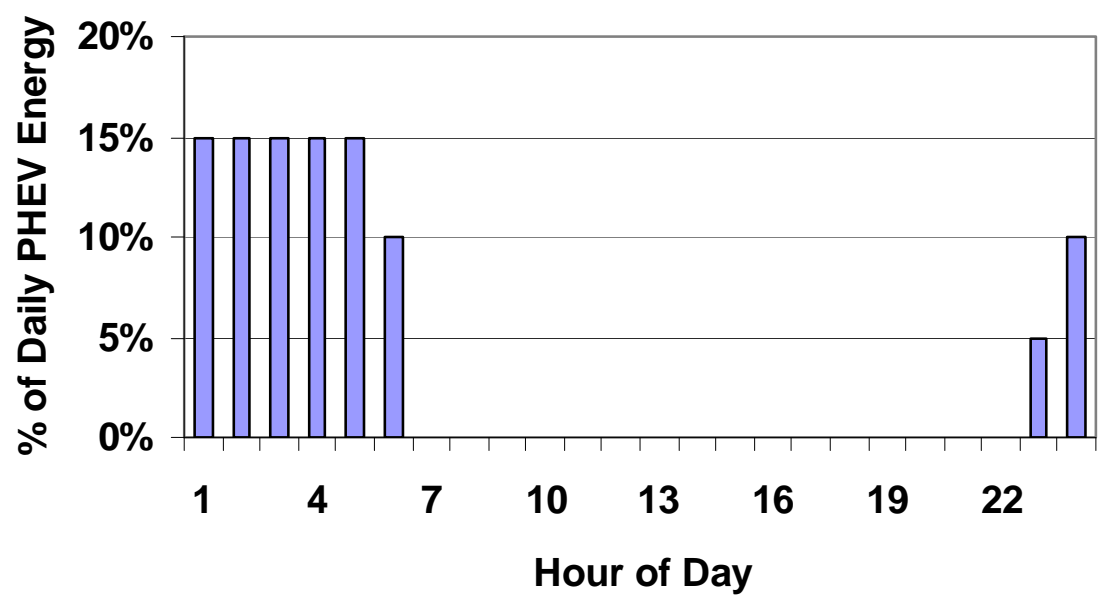

Figure 8.14 Distribution of PHEV Charging Energy

Figure 8.15 shows the operational cost impact of adding the PHEV loads to the system for the range of renewable penetrations. The left-hand side shows the increase in millions of dollars, and the right-hand scale shows the costs in \$/MWh of PHEV load. Figure 8.16 shows that adding PHEVs increased the value of the renewable energy by about 50 cents per MWh. Adding the PHEV demand did not significantly change either the unserved or the spilled energy.

However, it should be noted that this PHEV load could be used to implement the demand side management, or load participation, that was shown to be so valuable in Section 6.6.2, and will be addressed further in Section 7.5.

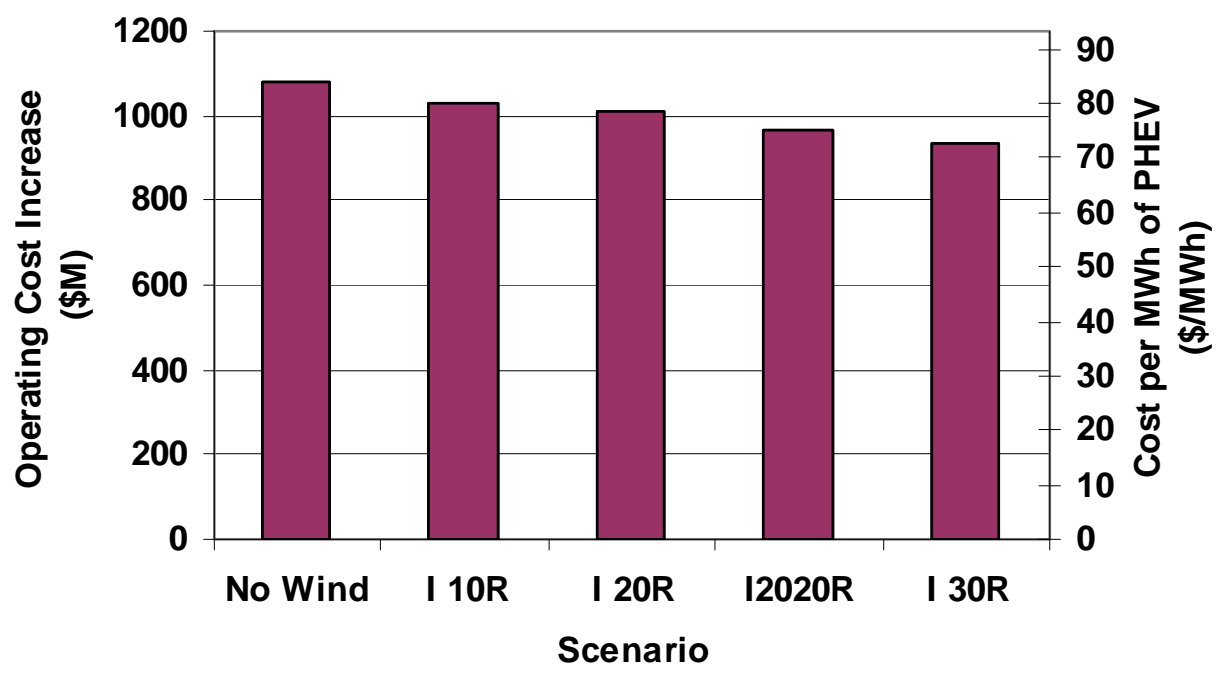

Figure 8.15 Cost Impact of Adding PHEV Load 


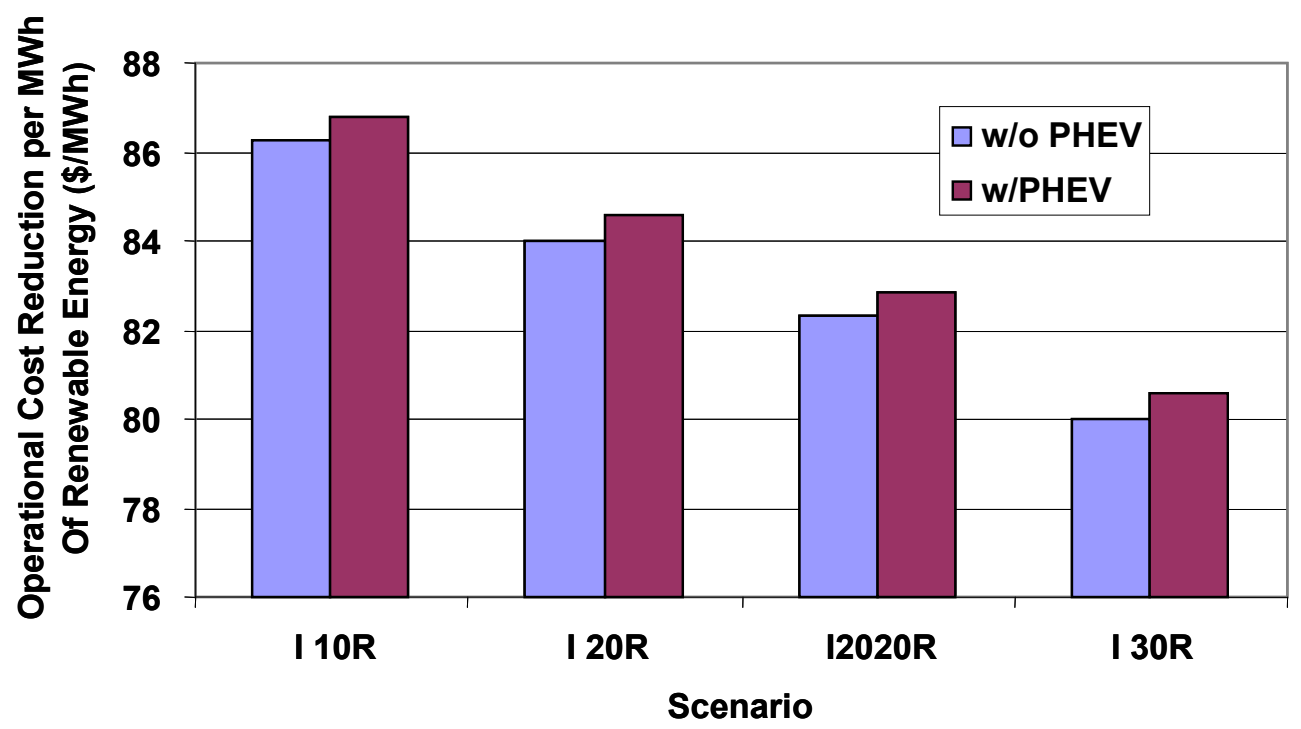

Figure 8.16 Operational Impact of Renewable Energy with PHEV

\subsection{Storage and PHEV Observations}

The best way to integrate wind and solar generation into the system is to make full use of the capabilities of all of the generating units in the system. Although the flexible operation of storage is attractive, when gas fired generation in on the margin both on peak and off peak there is little economic room for using the storage in an "energy arbitrage" manner due to the $25 \%$ losses associated with pumped storage hydro or batteries. As long as balancing area issues don't interfere, utilizing the ramping capabilities of system's dispatchable generation is generally more efficient. With perfect forecasts of the renewable generation, the spot prices drop throughout the day and generally reduce the price ranges needed throughout the day to make storage attractive. Broader price ranges may exist with state-of-the-art forecasts and high levels of renewables but even then, the value appears to fall significantly short of what is needed to make new storage economically attractive.

If thermal storage is added to Concentrating Solar Plants, CSP, it may be possible to improve the revenue stream beyond the operating mode of simply using the storage to handle excess thermal energy. Storing all of the energy early in the day when energy prices are low and releasing it later in the evening when prices are higher was shown to increase revenues in some months by $30 \%$. This study did not look at the cost of the additional collector fields and storage facilities.

Wind generation and plug-in hybrid electric vehicles are synergistic. Adding PHEVs to a system makes it more attractive to wind generation and adding high penetrations of wind reduces the cost of charging PHEV. More analysis on the emissions and other benefits of PHEVs still needs to be done. The presumed ability of PHEVs to participate in DSM for maintaining reserve margins increases their attractiveness. 


\section{Capacity Value Analysis}

Chapter 6 of this report examined the economic and operating impact of intermittent renewable generation. This chapter uses a generation reliability analysis, also known as a Loss of Load Probability (LOLP) analysis, of the system to determine the capacity value of the wind and solar generation. The GE Multi-Area Reliability Simulation program, MARS, was used in this analysis.

\subsection{Introduction}

A Loss of Load Probability analysis is typically performed on a system to determine the amount of capacity that needs to be installed to meet the desired reliability target, generally one day in ten years, commonly expressed as 0.1 days/year. This calculation involves convolving the load profiles and the scheduled generator outages with the probability of generator forced outages to determine the expected number of days in the year when a shortage might occur. Because the result is actually an expected value, the index is often referred to as Loss of Load Expectation (LOLE), but the historical terminology is LOLP. Both terms are valid, and they are often used interchangeably. It is important to note that the historical measure was interested in "the number of days of shortage," rather than the total outage time. Since generator outages tended to last for several days, if a shortage was to occur, it would happen at the time of the daily peak load. Therefore, the calculations were only done for the peak hour of each day. (Note: the historical calculation method was developed long before computers.)

This historical focus on the peak hour is very important to the treatment of intermittent resources. If a particular resource produces $100 \mathrm{MW}$ of generation for 23 hours of the day but only generates $10 \mathrm{MW}$ at the hour of the daily peak then the calculation will see it as just $10 \mathrm{MW}$. It will have no greater capacity value than a generator that puts out 10 MW for every hour of the day. This explains why the capacity value of wind is often much lower than traditional thermal generation. Likewise, a device that can consistently generate $100 \mathrm{MW}$ at the daily peak but zero MW the rest of the day will have the same capacity value as a unit that produces $100 \mathrm{MW}$ all day long.

One shortfall of this methodology is that with the capacity output changing hourly, it is possible to have capacity shortages at times other than the peak hour. This could occur if a resource was generating $100 \mathrm{MW}$ in the peak hour but only $10 \mathrm{MW}$ in the next hour when the load only dropped $30 \mathrm{MW}$. In order to capture this effect the model was adjusted to look at all 24 hours in the day. In addition to calculating the number of hours that the system might be short, which is a measure used in some regions, the model counted up the number of days in which an outage occurred at any time of the day. In this manner, all shortages are captured regardless of the time of day and all capacity levels are also considered. This method also captures the synergy between the capacity 
impacts of different types of intermittent renewable generation. PV and wind generation tend to occur more during off-peak periods which reduces their capacity value. CSP with storage, on the other hand, can be shifted to reduce the peak loads. This then pushes the relative peaks into the shoulder hours, allowing the PV and wind to have more of an impact. This will be shown in the results.

In addition to the daily and hourly indices, the program also determined the magnitude of any shortages so that total energy shortfalls could be calculated. This value will differ from the value calculated in the production simulation analysis. A reliability analysis assumes that all capacity not on outage is available to serve load. Most of the shortages - or unserved energy - in the production modeling were due to forecast errors that caused units to not be committed and available for dispatch.

\subsubsection{Location is Critical}

One aspect of capacity value is where the unit is located. A perfectly reliable generator located behind a transmission constraint may not add any capacity value to the system. In this analysis, we wanted to capture the capacity value of the renewable generation based on their generation profiles, the area load profiles, and the characteristics of the rest of the generators in the study area. In order to do that it was assumed that there were no transmission constraints within the study area for the reliability analysis, often referred to as a "copper sheet" analysis. In this way, the capacity values will not be penalized due to transmission constraints.

The study area has thousands of megawatts of interconnections to the rest of WECC. In order to calculate non-zero reliability indices these ties were set to zero. This resulted in an LOLE index that ranged from 1.8 - 6.1 days/year for the single-area, isolated study footprint, depending on the shape of the load data. This provided a good starting point for the capacity value calculations and still resulted in non-zero values of LOLE for the $30 \%$ renewables scenario. Variation of the starting point would not significantly change the results.

\subsection{Capacity Value}

The question "How much capacity is a wind plant worth?" can be answered in a few different ways. It could be compared to the number of gas turbines or coal plants needed to get the same reliability impact. Or it could be a measure of the amount of peak load increase allowed while still maintaining the original reliability level. Or a third measure would be how much "perfect capacity" would be needed to achieve the same level of reliability. All of these measures produce similar results. This analysis used the "perfect capacity" measure, and it will relate the results to the other methods in a later section. An advantage of perfect capacity is that it is independent of forced outage rate, unit size and load profiles which affect the other measures. 
Figure 9.1 shows how the daily LOLP of the study footprint improved with the addition of a series of 500-MW blocks of perfect capacity. All of the initial results are based on the 2006 load shape. Similar analyses were performed for the 2004 and 2005 load shapes and the results will be presented later in this section. It is important to note that the scale on the $y$-axis is logarithmic. Figure 9.2 shows the results of multiple simulations with various combinations of renewable generation added, and then plotted on the same curve as the previous figure. Each of the three types of generators was examined separately as well as combined with the others for different levels of penetration. For example, the three red triangles represent the impact of wind generation alone at the $10 \%, 20 \%$, and $30 \%$ penetration levels.

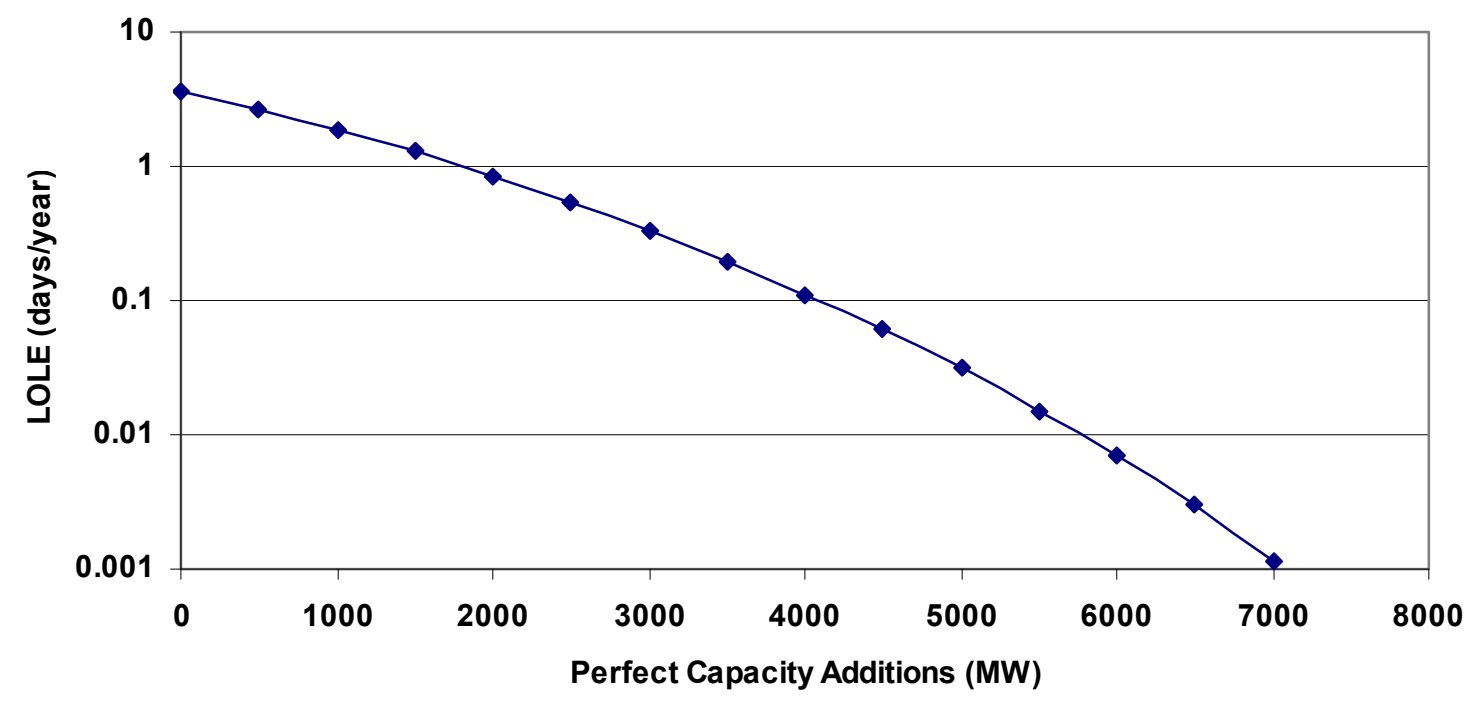

Figure 9.1 Study Area Risk vs. Capacity Additions

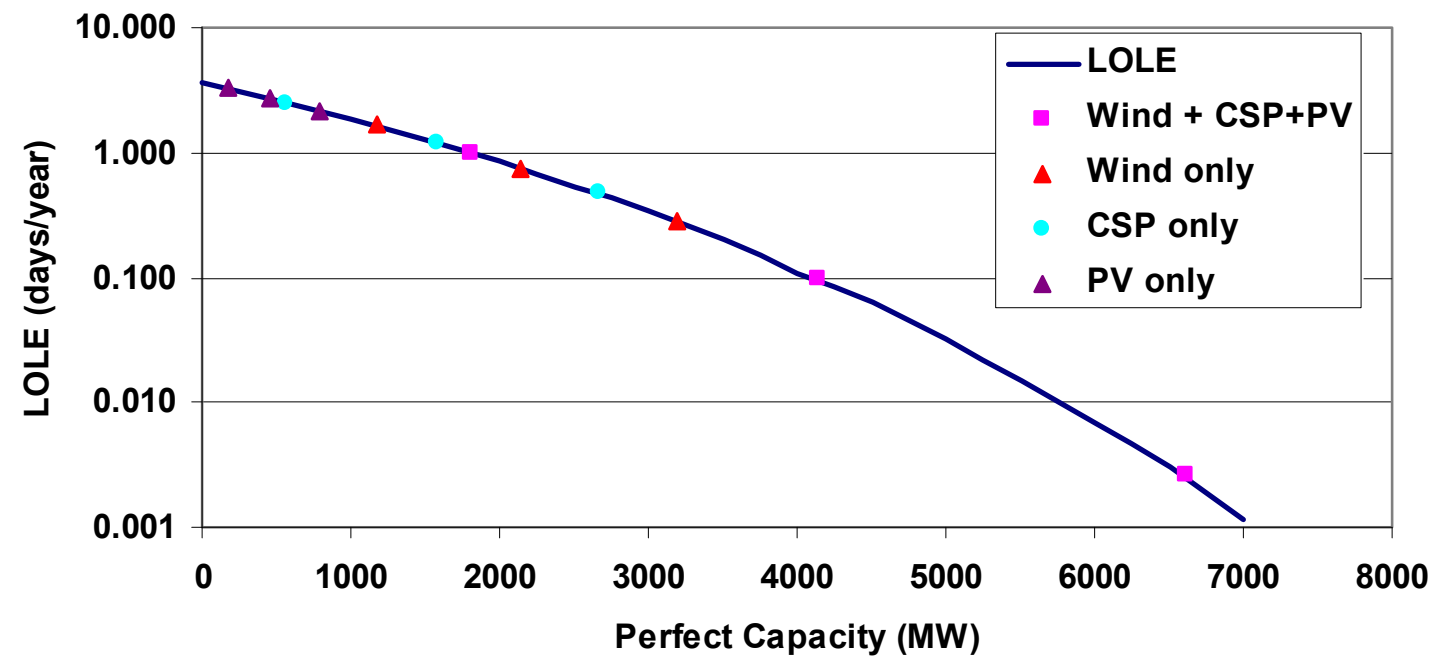

Figure 9.2 Study Area Risk with Renewable Additions

Table 9.1 shows the nameplate capacity of the wind, CSP, and PV generation added in the three levels of penetration. Table 9.2 shows how the capacity values derived from 
Figure 9.2 compare to their nameplate ratings on a percentage basis. It is clear that there is significant variation in capacity value among the different types of renewable generation.

Table 9.1 Renewable Capacities By Type

\begin{tabular}{|l|c|c|c|c|}
\hline Penetration & $\begin{array}{c}\text { Total Renewables } \\
\text { (MW) }\end{array}$ & $\begin{array}{c}\text { Wind Capacity } \\
\text { (MW) }\end{array}$ & $\begin{array}{c}\text { CSP Capacity } \\
\text { (MW) }\end{array}$ & $\begin{array}{c}\text { PV Capacity } \\
\text { (MW) }\end{array}$ \\
\hline $10 \%$ wind, $1 \%$ solar & 11,490 & 10,290 & 600 & 600 \\
\hline $20 \%$ wind, 3\% solar & 23,350 & 19,950 & 1,700 & 1,700 \\
\hline $30 \%$ wind, 5\% solar & 35,740 & 29,940 & 2,900 & 2,900 \\
\hline
\end{tabular}

Table 9.2 Renewable Capacity Values By Type, 2006 Shapes, Perfect Capacity, Daily LOLE.

\begin{tabular}{|l|c|c|c|c|}
\hline Penetration & Wind + CSP + PV & Wind only & CSP only & PV only \\
\hline $10 \%$ wind, $1 \%$ solar & $15.8 \%$ & $11.4 \%$ & $92.6 \%$ & $28.6 \%$ \\
\hline $20 \%$ wind, $3 \%$ solar & $17.7 \%$ & $10.8 \%$ & $93.3 \%$ & $26.9 \%$ \\
\hline $30 \%$ wind, $5 \%$ solar & $18.5 \%$ & $10.7 \%$ & $92.2 \%$ & $26.9 \%$ \\
\hline
\end{tabular}

\subsubsection{Timing is Everything}

Figure 9.3 shows the average daily profile of the study-area load and wind generation for the $30 \%$ scenario in the peak month of July. Although the 30\% In-Area Scenario includes 30,000 MW of wind plants, their total output is less than 6,000 MW at the peak hour.

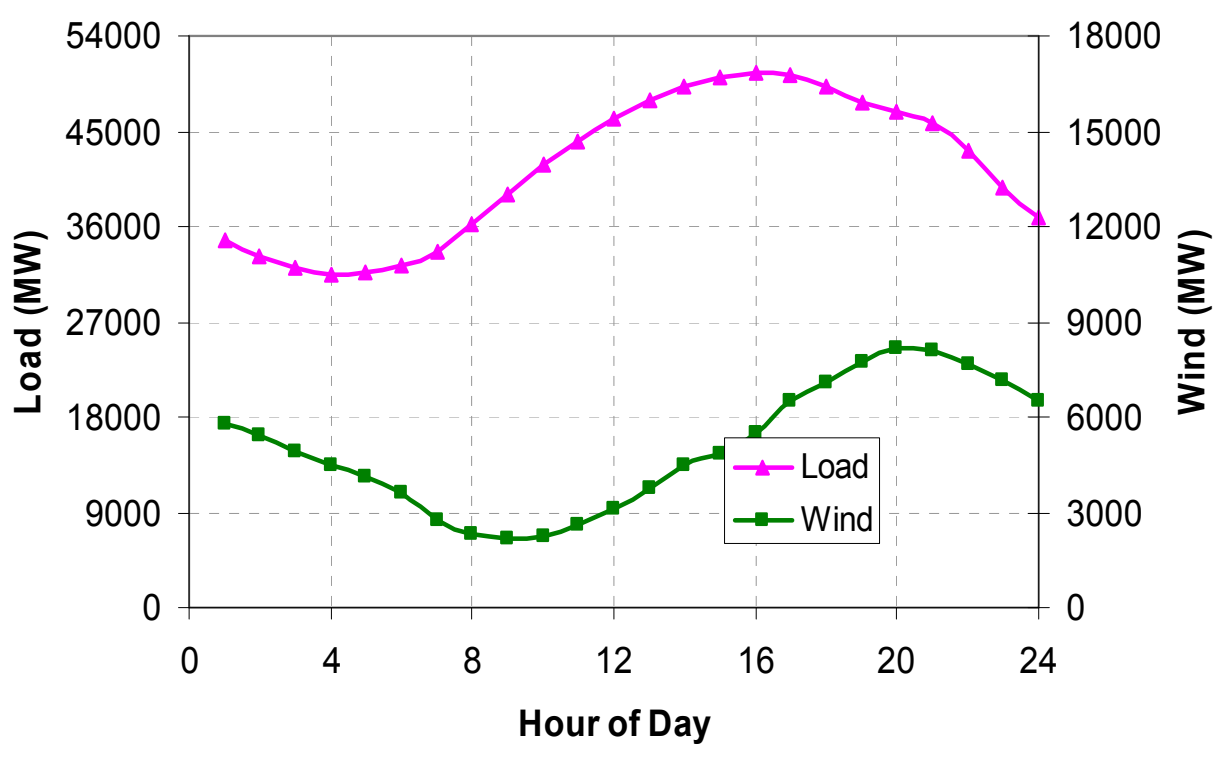

Figure 9.3 Hourly Average Wind and Load Shape 
This can be compared to the curves in Figure 9.4 that shows the average CSP and PV outputs. The CSP (with storage) had an average output of about 2,400 MW and the PV was about $800 \mathrm{MW}$ at the peak load hour. Both of them had an installed capacity of 2,900 MW. Figure 9.5 shows the wind and solar energy production by month for the 2006 shapes. When the daily and monthly profiles are compared to the load, it is not surprising that the wind capacity value is low. The PV value is limited by the fact that the solar energy peaks at noon and has dropped significantly by the time that the load reaches its peak in late afternoon. The PV was also somewhat penalized because the nameplate rating was based on the DC rating under ideal conditions. Various factors, including inverter losses, soiling, and orientation reduced the net output by $23 \%$, so that the maximum output from a $100 \mathrm{MW}$ PV site was only $77 \mathrm{MW}$. The CSP, on the other hand, had over-sized collector fields to provide additional energy for storage. Its rating was based on the net output of the generator. In addition, the storage on the CSP allows the output to be held near its full rating later in the day and this is what contributes to its high capacity values.

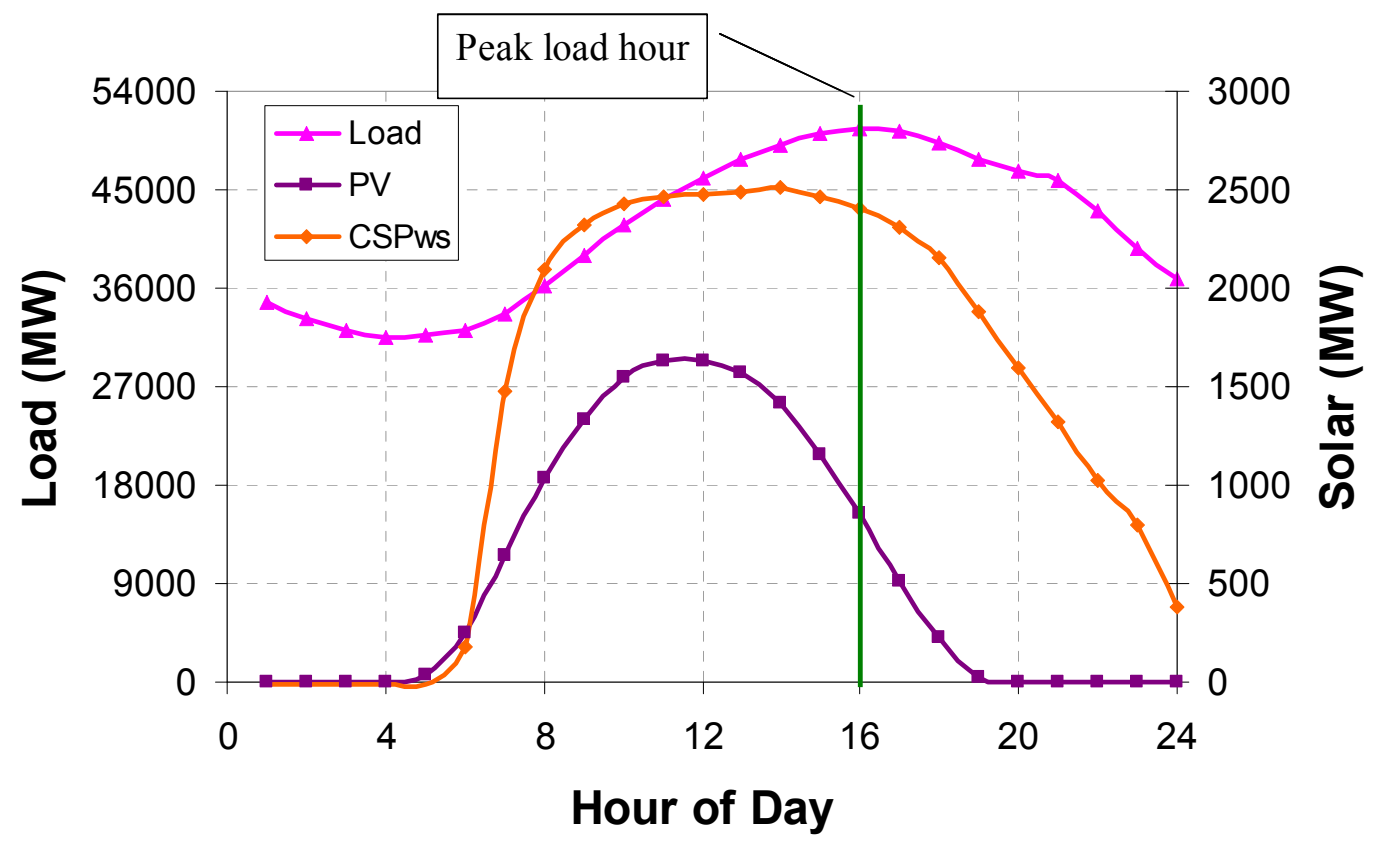

Figure 9.4 Average Solar and Load Shapes 


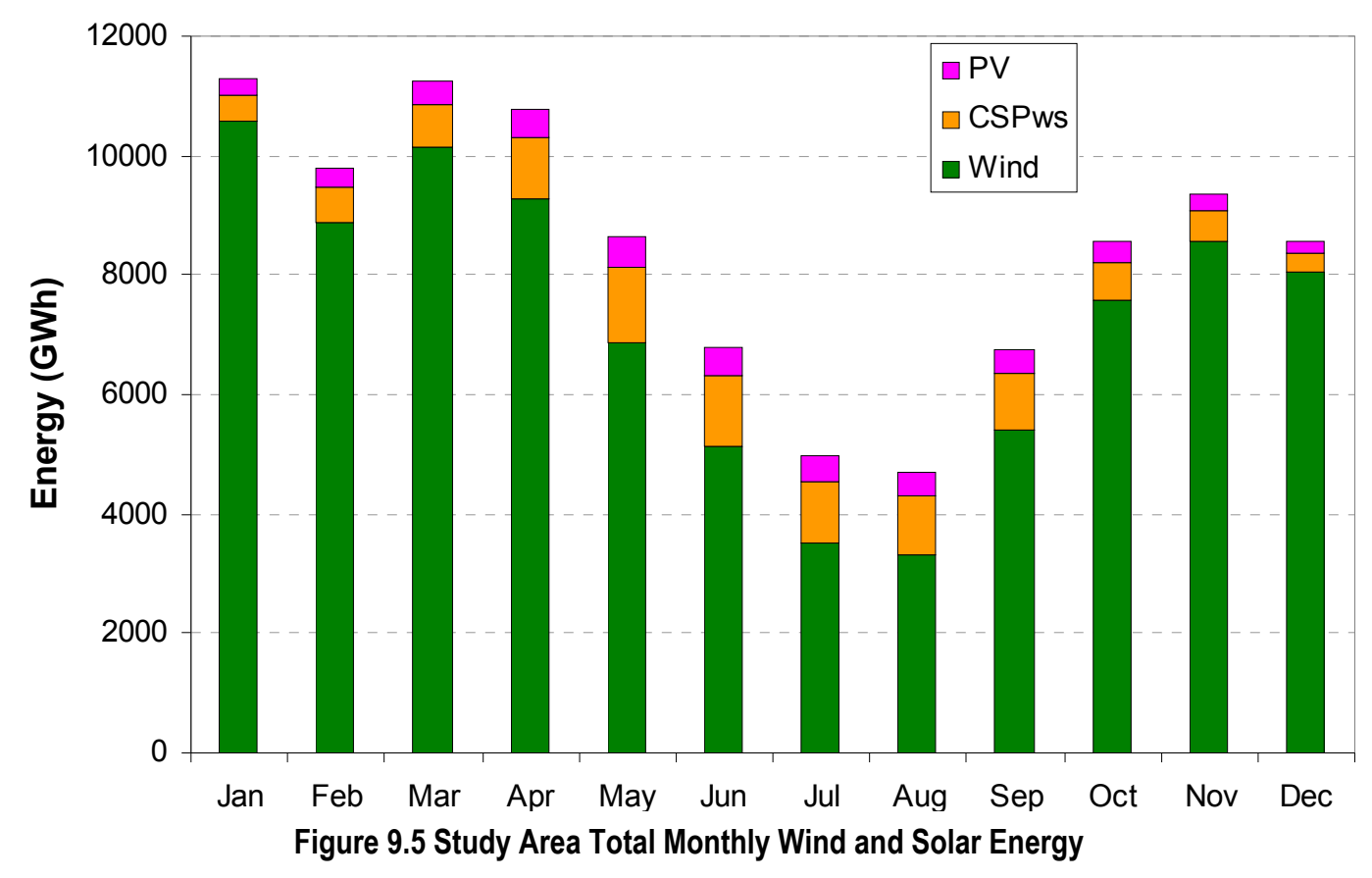

\subsubsection{Hourly and Unserved Energy Measures}

The same type of analysis can be done using the hourly LOLP index and the unserved energy. Just as the daily LOLP analysis calculated the expected number of days of shortage, applying the same calculations to all of the hours of the day can calculate the expected number of hours of shortage. If each hour of shortage is combined with the corresponding magnitude of the shortage then the expected unserved energy for the year can be determined. Wind and solar generation can then be added to the system to determine the equivalent amount of perfect capacity required to have the same impact on the hourly and unserved energy indices. Figure 9.6 and Figure 9.7 show the curves corresponding to these calculations. Table 9.3 and Table 9.4 are the companion capacity values. 


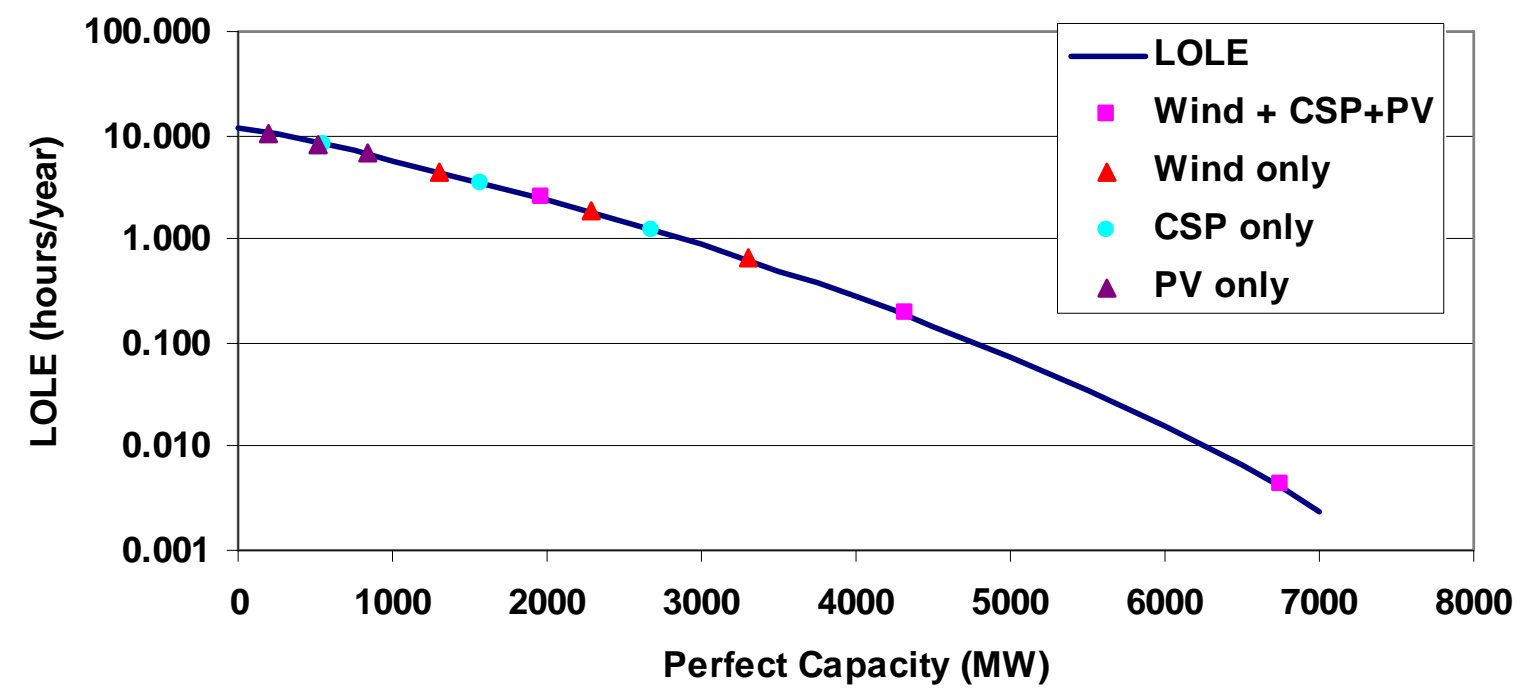

Figure 9.6 Study Area Risk in Hours/Year

Table 9.3 Renewable Capacity Values By Type, 2006 Shapes, Perfect Capacity, Hourly LOLE.

\begin{tabular}{|l|c|c|c|c|}
\hline Penetration & Wind + CSP + PV & Wind only & CSP only & PV only \\
\hline $10 \%$ wind, $1 \%$ solar & $17.1 \%$ & $12.6 \%$ & $90.8 \%$ & $32.1 \%$ \\
\hline $20 \%$ wind, 3\% solar & $18.5 \%$ & $11.5 \%$ & $92.7 \%$ & $30.3 \%$ \\
\hline $30 \%$ wind, 5\% solar & $18.9 \%$ & $11.0 \%$ & $92.6 \%$ & $29.0 \%$ \\
\hline
\end{tabular}

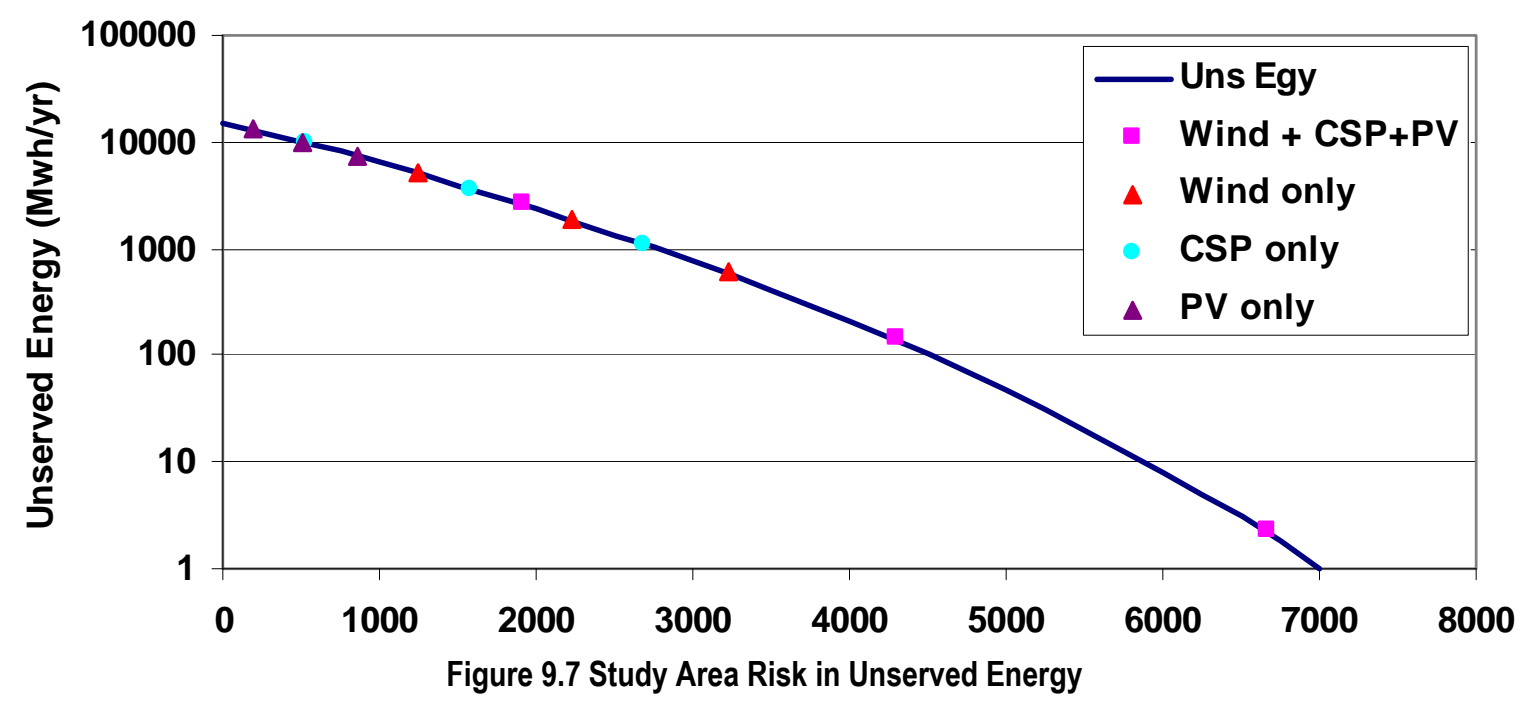


Table 9.4 Renewable Capacity Values By Type, 2006 Shapes, Perfect Capacity

\begin{tabular}{|l|c|c|c|c|}
\hline Penetration & Wind + CSP + PV & Wind only & CSP only & PV only \\
\hline $10 \%$ wind, $1 \%$ solar & $16.6 \%$ & $12.1 \%$ & $88.5 \%$ & $33.2 \%$ \\
\hline $20 \%$ wind, 3\% solar & $18.4 \%$ & $11.2 \%$ & $92.6 \%$ & $30.0 \%$ \\
\hline $30 \%$ wind, $5 \%$ solar & $18.6 \%$ & $10.8 \%$ & $92.6 \%$ & $29.3 \%$ \\
\hline
\end{tabular}

Table 9.5 Renewable Capacity Values By Type, 2006 Shapes, Perfect Capacity, Average Across Indices

\begin{tabular}{|l|c|c|c|c|}
\hline Penetration & Wind + CSP + PV & Wind only & CSP only & PV only \\
\hline $10 \%$ wind, $1 \%$ solar & $17 \%$ & $12 \%$ & $91 \%$ & $31 \%$ \\
\hline $20 \%$ wind, $3 \%$ solar & $18 \%$ & $11 \%$ & $93 \%$ & $29 \%$ \\
\hline $30 \%$ wind, $5 \%$ solar & $19 \%$ & $11 \%$ & $92 \%$ & $28 \%$ \\
\hline
\end{tabular}

\subsection{Capacity Value Variation by Scenario}

The 2006 analysis was repeated for the three different siting scenarios. Although the megawatts in each area, and in total, changed between the scenarios, particularly for the wind generation, there was very little change in the capacity value as shown in Table 9.6. The results are shown graphically in Figure 9.8, Figure 9.9, and Figure 9.10. 
Table 9.6 Renewable Capacity Values By Type, Perfect Capacity, Daily LOLE, By Scenario.

\begin{tabular}{|c|c|c|c|c|}
\hline Penetration & Wind + CSP + PV & Wind only & CSP only & PV only \\
\hline \multicolumn{5}{|l|}{ In-Area } \\
\hline $10 \%$ wind, $1 \%$ solar & $15.8 \%$ & $11.4 \%$ & $92.6 \%$ & $28.6 \%$ \\
\hline $20 \%$ wind, $3 \%$ solar & $17.7 \%$ & $10.8 \%$ & $93.3 \%$ & $26.9 \%$ \\
\hline $30 \%$ wind, $5 \%$ solar & $18.5 \%$ & $10.7 \%$ & $92.2 \%$ & $26.9 \%$ \\
\hline \multicolumn{5}{|l|}{ Local-Priority } \\
\hline $10 \%$ wind, $1 \%$ solar & $16.5 \%$ & $11.4 \%$ & $92.6 \%$ & $28.6 \%$ \\
\hline $20 \%$ wind, $3 \%$ solar & $18.7 \%$ & $11.3 \%$ & $93.3 \%$ & $26.9 \%$ \\
\hline $30 \%$ wind, $5 \%$ solar & $18.8 \%$ & $10.5 \%$ & $92.2 \%$ & $26.9 \%$ \\
\hline \multicolumn{5}{|l|}{ Mega-Project } \\
\hline $10 \%$ wind, $1 \%$ solar & $18.5 \%$ & $13.0 \%$ & $91.6 \%$ & $25.8 \%$ \\
\hline $20 \%$ wind, $3 \%$ solar & $19.0 \%$ & $11.9 \%$ & $94.1 \%$ & $24.7 \%$ \\
\hline $30 \%$ wind, $5 \%$ solar & $19.3 \%$ & $10.0 \%$ & $92.8 \%$ & $24.8 \%$ \\
\hline
\end{tabular}

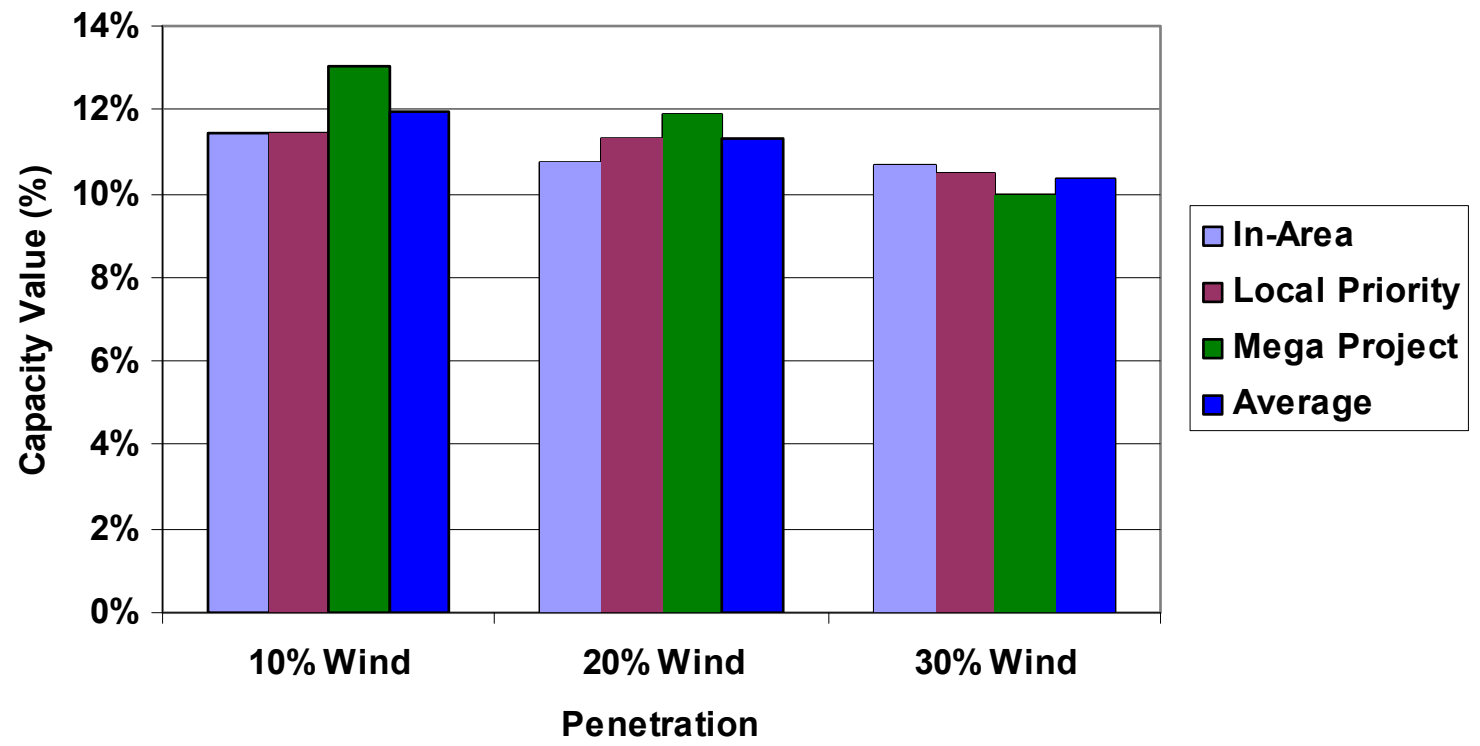

Figure 9.8 Wind Capacity Values by Scenario, 2006 Shapes, Perfect Capacity 


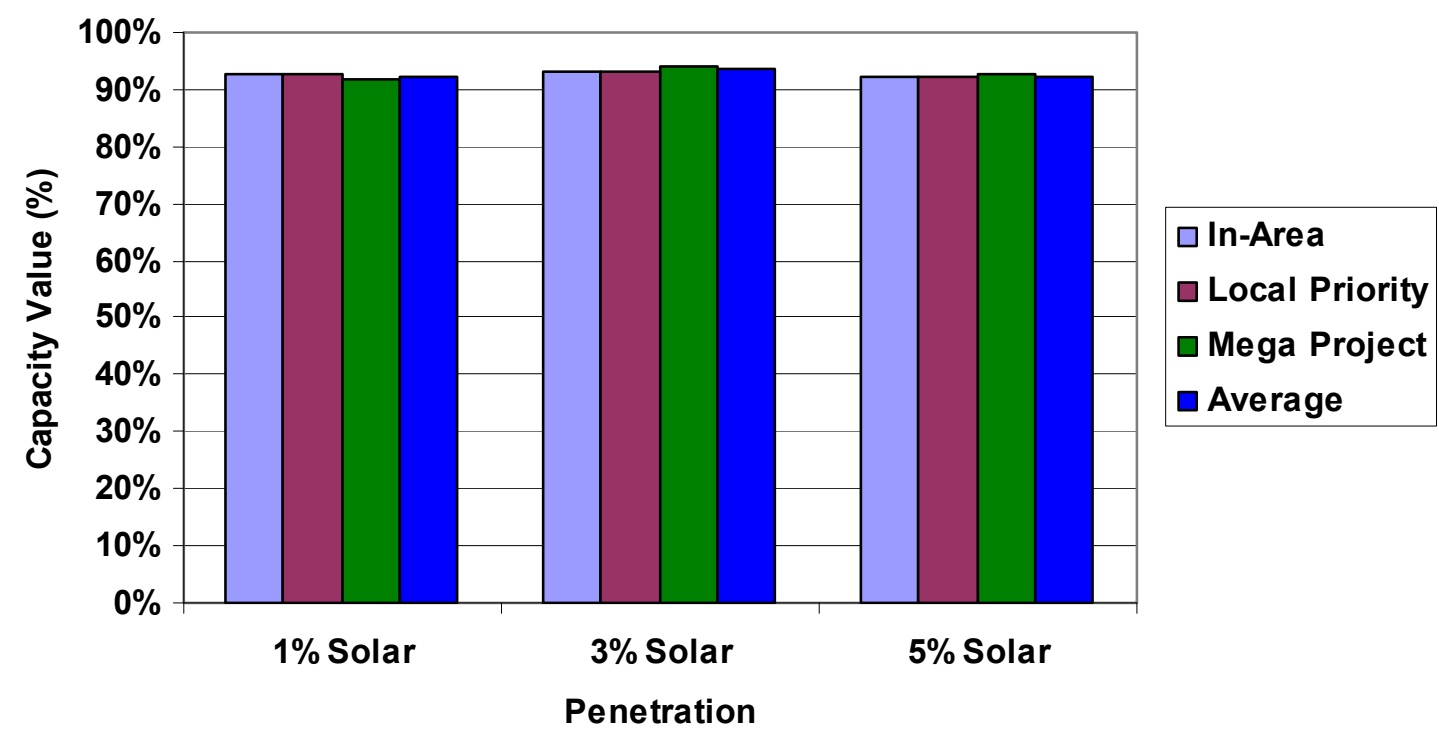

Figure 9.9 CSP with Storage Capacity Values by Scenario, 2006 Shapes, Perfect Capacity

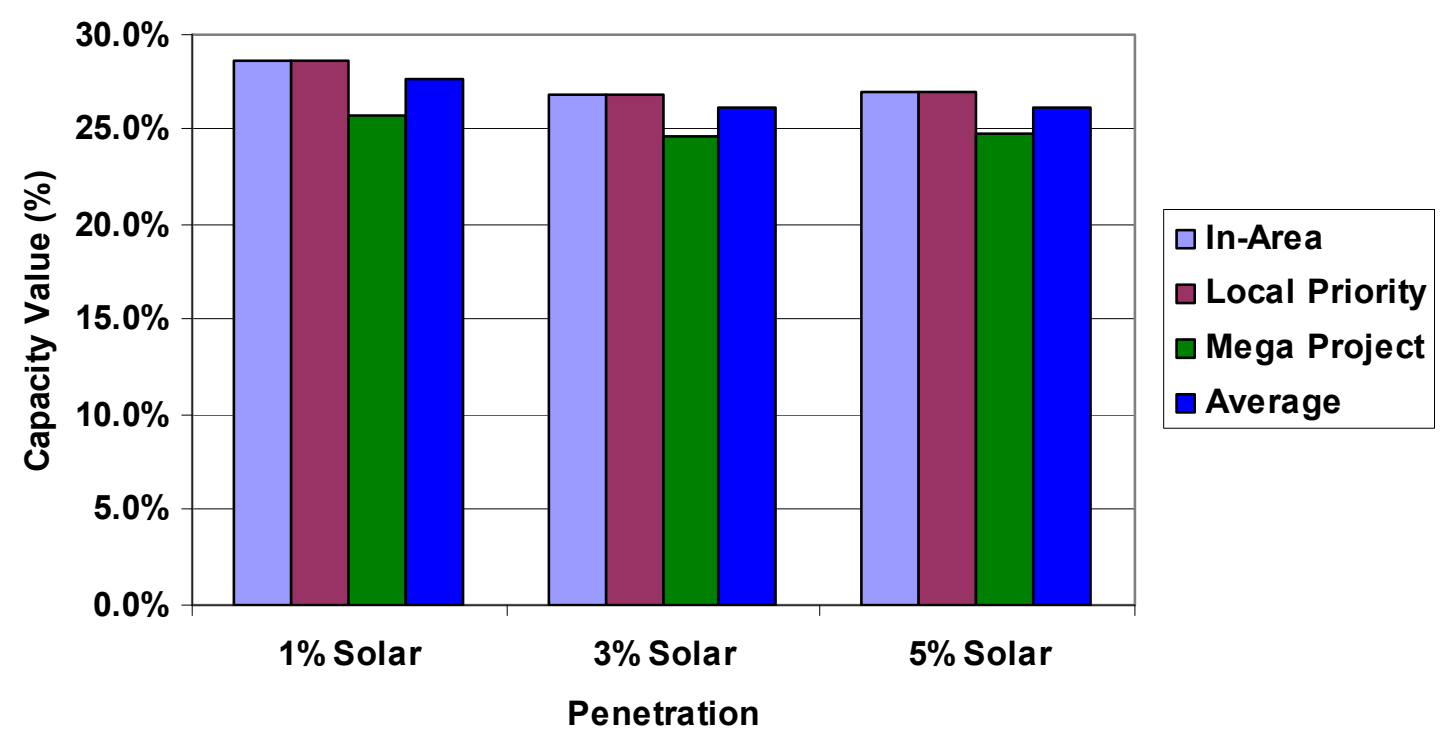

Figure 9.10 PV Capacity Values by Scenario, 2006 Shapes, Perfect Capacity

\subsection{Capacity Value Variation by Shape Year}

The results shown so far were based on the 2006 load and weather shapes. The In-Area analysis was also done using the shapes from 2004 and 2005. Figure 9.11 shows the monthly energy variation by type for the three years for the In-Area Scenario. The green bar indicates the wind energy, the orange is for the CSP, and the pink is for the PV plants. Figure 9.12, Figure 9.13, Figure 9.14, and Figure 9.15 show the variations in 
capacity value for the individual generation types as well as the combined total for the three shape-years. The individual renewable types tend to decrease in value with increased penetration. The combined values show an increase because of the high value of the CSP plants in the mix. There is some year-to-year variation but it does not appear to be significant.

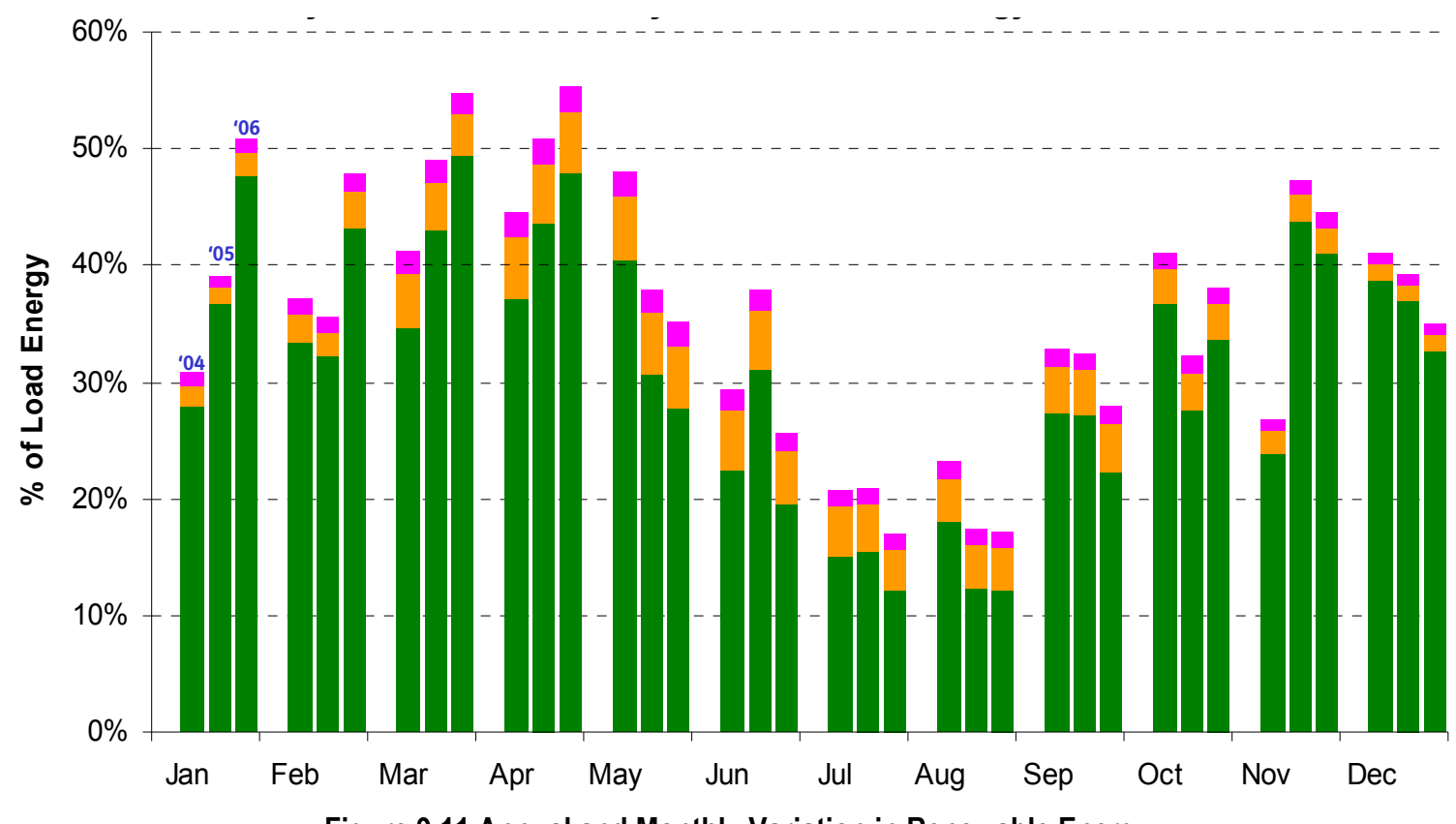

Figure 9.11 Annual and Monthly Variation in Renewable Energy

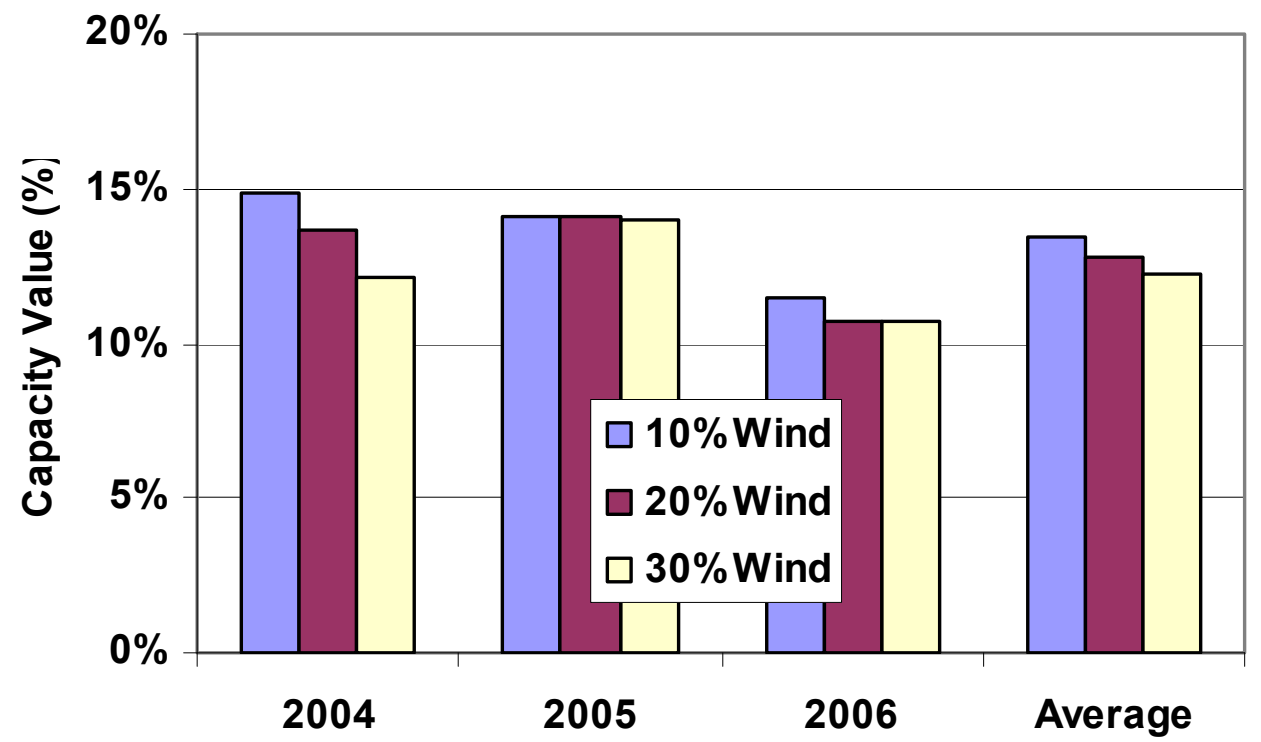

Figure 9.12 Capacity Value for Wind, Perfect Capacity, Daily LOLE, All Years 


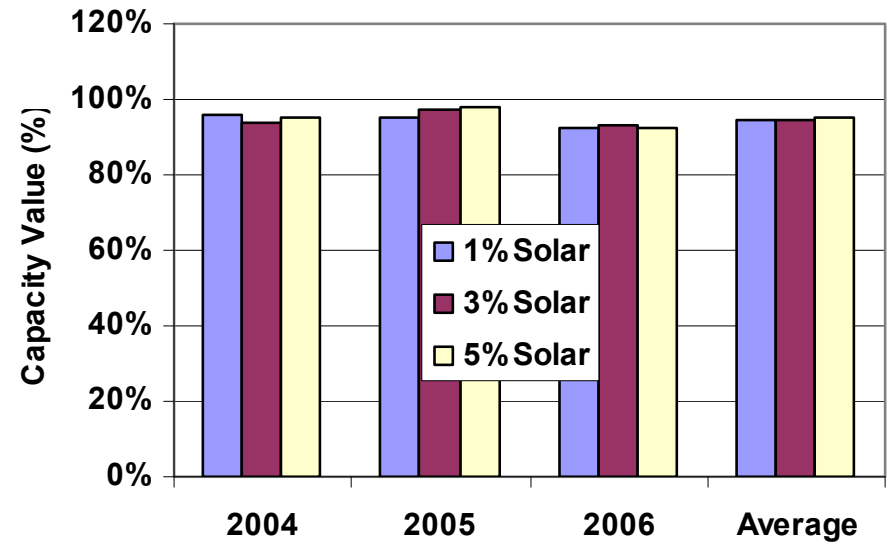

Figure 9.13 Capacity Value for CSP with 6 Hours of Storage, Perfect Capacity, Daily LOLE, All Years

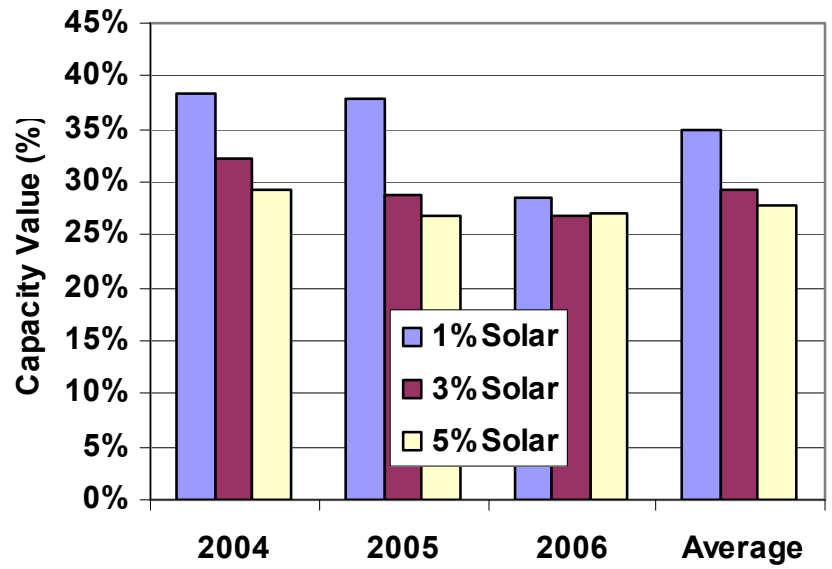

Figure 9.14 Capacity Value for PV, Perfect Capacity, Daily LOLE, All Years

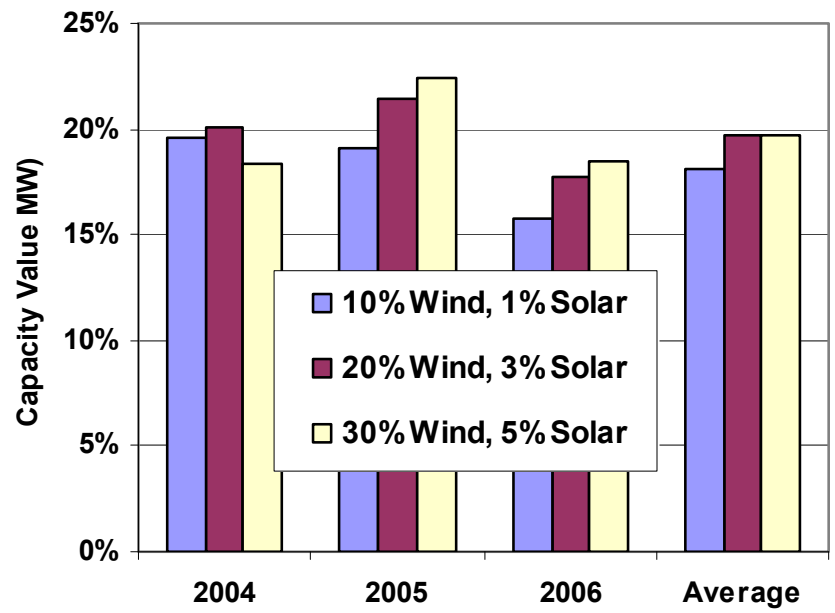

Figure 9.15 Combined Capacity Value for Wind, CSP and PV, Perfect Capacity, Daily LOLE, All Years 


\subsection{Comparison to Other Measures}

This analysis has equated the renewable generation to the amount of perfect capacity that would produce the same result. Other studies have considered the amount of equivalent generators or increased load that could be carried. This section will compare the measures.

If a unit is large relative to the size of the system then this will distort its capacity value. However, if the unit's capacity is small relative to the system size then its effective capacity is typically estimated as its nameplate capacity times one minus the forced outage rate. For example, a $100-\mathrm{MW}$ gas turbine with a $5 \%$ forced outage rate would have an effective capacity of $95 \mathrm{MW}$. Therefore, to convert the perfect capacity values to an equivalent capacity of gas turbines with $5 \%$ forced outage rates you would divide the previous values by 0.95 . Similarly, to convert the perfect capacity to equivalent units with a $10 \%$ forced outage rate you would divide by 0.90 . Referring back to the "wind only" value for the 30\% scenario in Table 9.2, $100 \mathrm{MW}$ of nameplate wind generation would have a value of $10.7 \mathrm{MW}$ of "perfect" capacity. This would correspond to 11.3 MW of capacity when compared to gas turbines or 11.8 MW of capacity when compared to a unit with a $10 \%$ force outage rate.

Another method that is used in the industry is the effective load carrying capability or ELCC. In this case, after a generator is added to the system, the peak load is increased until the risk is back to its original value. When the peak load is increased, the other loads are also increased proportionately. So if the annual peak load is $1000 \mathrm{MW}$ and another day has a peak of $900 \mathrm{MW}$, when the peak is increased by $100 \mathrm{MW}$, or $10 \%$, the other day is only increased by $90 \mathrm{MW}$. This has the effect of increasing the value over the perfect capacity method since $100 \mathrm{MW}$ of perfect capacity is worth $100 \mathrm{MW}$ in every other hour. For comparison purposes, the case with 2006 shapes, 30\% In-Area Scenario was examined. The perfect capacity value for all of the renewables was $6610 \mathrm{MW}$ or $18.5 \%$. The ELCC method shows that increasing the peak load by $7260 \mathrm{MW}$ returns the system with all of the renewables back to its original daily LOLE value. Therefore the ELCC produces a value of $20.3 \%$, roughly $10 \%$ higher $(=7260 / 6610)$ than the perfect capacity method.

All of these methods are roughly equivalent within the general level of accuracy. Switching from the perfect capacity measure to effective capacity or effective load carrying capability increases the values by 5 to $10 \%$. But as seen in Figure 9.15, the perfect capacity values change by $+/-10 \%$ when looking at three different shape years. Similar variations were seen for a given shape when varying the penetration levels and the siting scenarios. The important aspect is the relative capacity value of the different types of renewable generation compared to more conventional generation. Thermal generators typically have capacity values in the $90 \%-95 \%$ range. For this system, wind generation has capacity values in the $10 \%-15 \%$ range. Photovoltaic generation is in the 
$25 \%-30 \%$ range and concentrating solar plants with six hours of storage had values in the $90 \%-95 \%$ range. It is this relative capacity value that is important.

\subsubsection{ELCC in a Multi-Area Environment}

The ELCC method works very well in a single-area environment. Let's say that a single area system, "Area A," adds $1000 \mathrm{MW}$ of wind generation and then finds that it can increase the load by $150 \mathrm{MW}$ in order to return to its original reliability level. The capacity value of the wind in Area A is then 15\%. Lets further assume that Area A is tied to another system, "Area B", and that the addition of the $1000 \mathrm{MW}$ of wind in Area A also provides some benefit to Area B. In addition to adding $150 \mathrm{MW}$ to Area A's load we can also add $50 \mathrm{MW}$ to the load in Area B in order to return both areas to their original reliability levels. In one sense the capacity value of the wind is now $200 \mathrm{MW}(=150+$ $50)$, or $20 \%$.

However, the capacity market in Area A only sees the $150 \mathrm{MW}$ of value and therefore will only pay for the $150 \mathrm{MW}$. Area B sees that the value of the tie line to Area A has increased by $50 \mathrm{MW}$. This may allow them to decrease their reserve margin required to maintain their desired risk, but this is not a capacity that they would have to pay for. This is simply the reliability value of interconnected systems.

Let's extend the example and assume that Area B also adds $1000 \mathrm{MW}$ of wind generation that allows Area B to increase its load by $150 \mathrm{MW}$ and Area A can increase its load by 50 MW. Area A and Area B now each see a total of $200 \mathrm{MW}$ of load increase from the total additions of wind generation, $150 \mathrm{MW}$ of value from the wind in their area and $50 \mathrm{MW}$ of value from the wind in their neighbor's area. This makes the value of the wind to appear to be $20 \%$ in each area. At some level this is true. However, each area will only pay for $150 \mathrm{MW}$ of capacity value from its wind plant and will receive $50 \mathrm{MW}$ of increased tie value from their neighbor that they will not pay for.

It appears to be misleading to add wind to all of the areas and then calculate the ELCC in each area. The wind generation should be added into each area one at a time. The correct ELCC is the load increase in the corresponding area and should not include the tie benefits to neighboring systems. The capacity values calculated in the EWITS analysis for the isolated areas were similar to the values determined in this analysis. When transmission was introduced, however, they were taking advantage of these "crossarea" benefits and the capacity values were much larger. It is not clear if the capacity value should be based on the unit's pure reliability value or the actual value (in dollars) that the unit would be paid in a capacity market.

\subsection{Yet Another Calculation Method}

Much care has been taken to keep the load data and weather data coincident. The 2004 load shapes are used with the 2004 weather patterns to ensure that any correlation 
between load and wind and solar generation is captured. From the statistical analysis in Chapter 4, it is clear that loads, wind generation, and solar generation have a clear seasonal and diurnal pattern. However, the variations in reliability value from year to year imply more randomness in the daily correlation. If it is windy or sunny on the peak day, the capacity value is high. Alternatively, if the wind dies down or a cloud passes by at the time of peak, the capacity value is lower.

The reliability model was adjusted to randomly pick a day of weather from within the month. In this way, when the risk for a day in July is being evaluated, it will be matched up with a wind and solar generation pattern from a randomly selected day in July. Two a.m. will still occur at two a.m., and July generation will still occur in July but the specific line up of good days and bad days will be shuffled within the month. The Monte Carlo analysis will match up the loads from every day in the month with every day of the wind and solar generation in the month. In this simulation, 1500 Monte Carlo iterations were made to capture all combinations of loads and wind shapes. It is hoped that this will produce a more robust result.

The results of this analysis are shown for the individual technologies in Figure 9.16, Figure 9.17, and Figure 9.18. Figure 9.19 shows the combined results for all renewables.

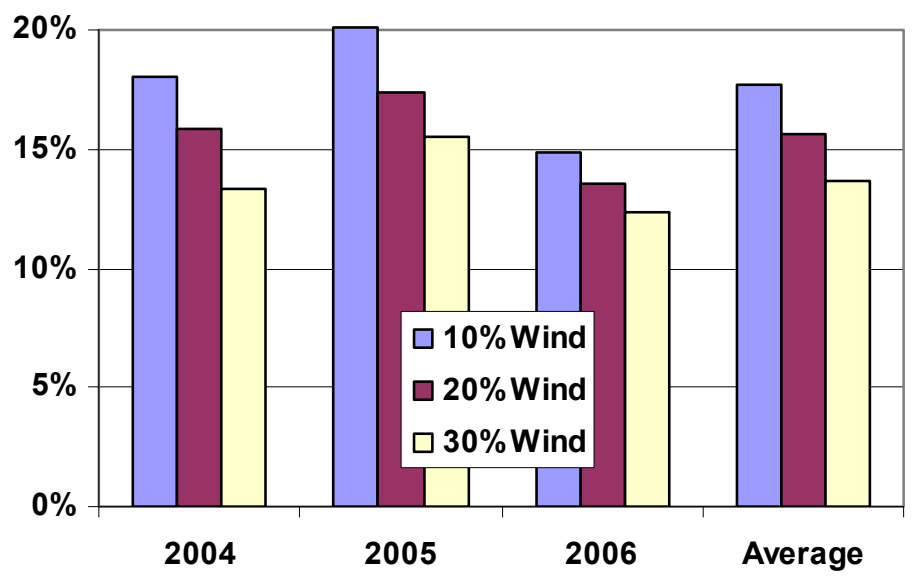

Figure 9.16 Capacity Value for Wind Only, 2006 Load Shapes, Random Wind Shapes 


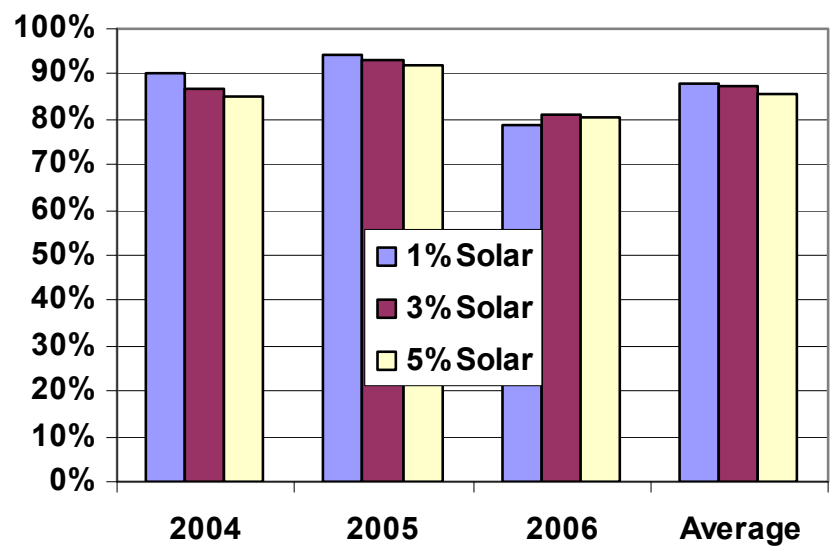

Figure 9.17 Capacity Value for CSP with Storage, 2006 Load Shapes, Random Solar Shapes

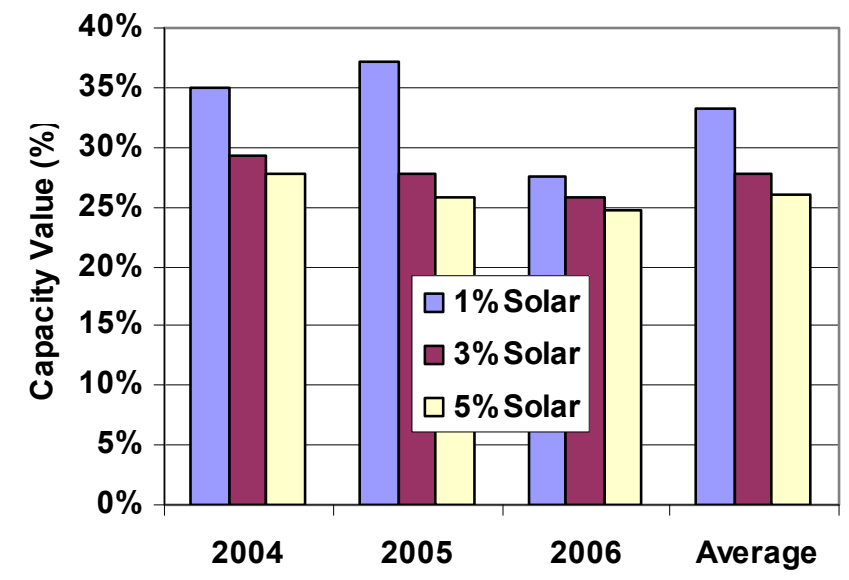

Figure 9.18 Capacity Value for PV Only, 2006 Load Shapes, Random Solar Shapes

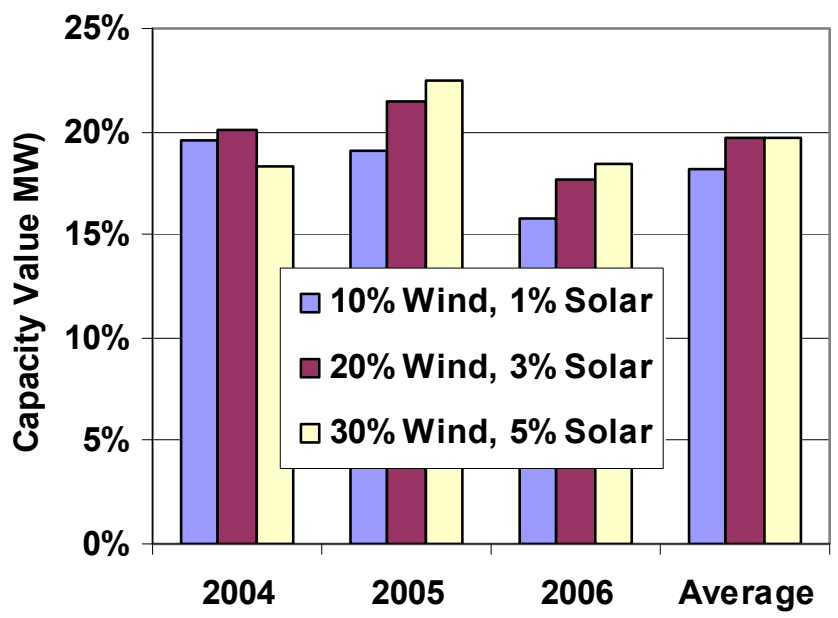

Figure 9.19 Capacity Value for Wind, CSP and PV, 2006 Load Shapes, Random Wind and Solar Shapes 
Figure 9.20, Figure 9.21, and Figure 9.22 show the average capacity values for the three years by type and penetration level. The results appear to be relatively consistent between the two approaches. The advantage of this approach is that it is not subject to the possibly random hit or miss of good generation days at the time of peak load. The disadvantage is that it will miss whatever correlation may exist within the month between the loads and wind and solar generation.

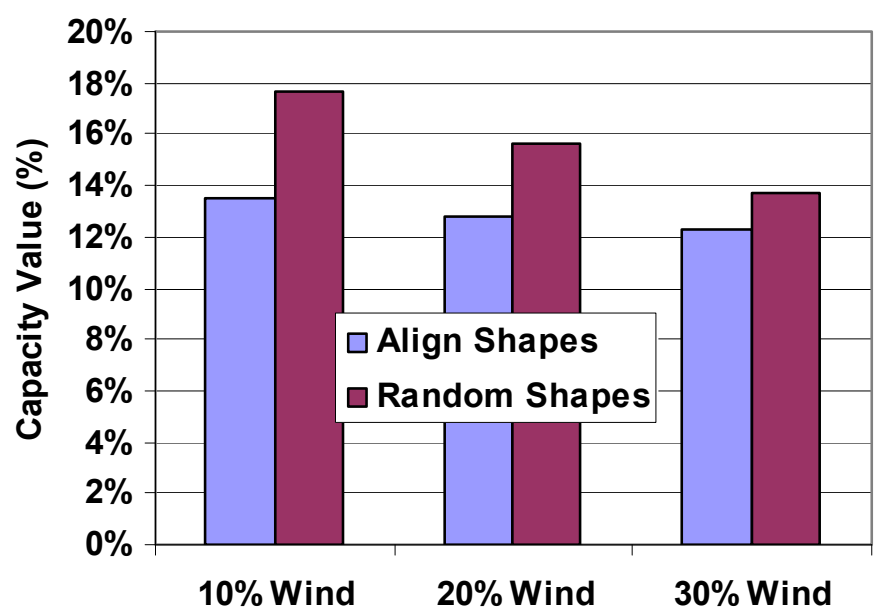

Figure 9.20 Comparison of Wind Capacity Values, Random and Aligned Shapes

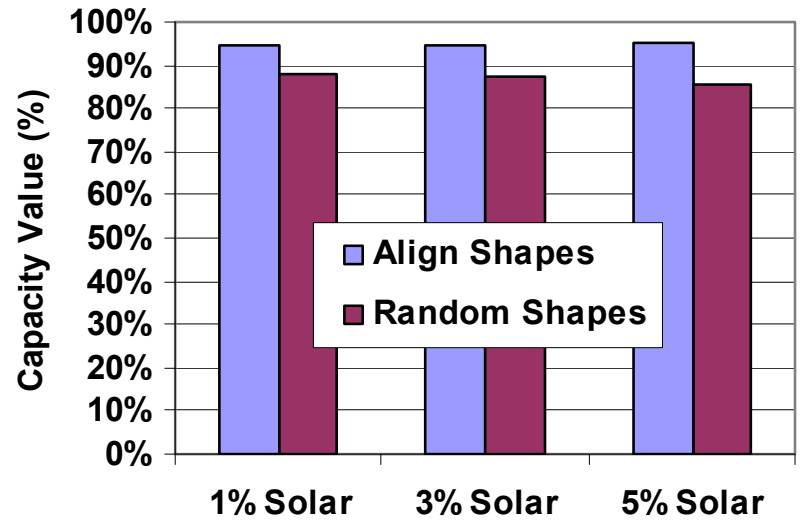

Figure 9.21 Comparison of CSP Capacity Values, Random and Aligned Shapes 


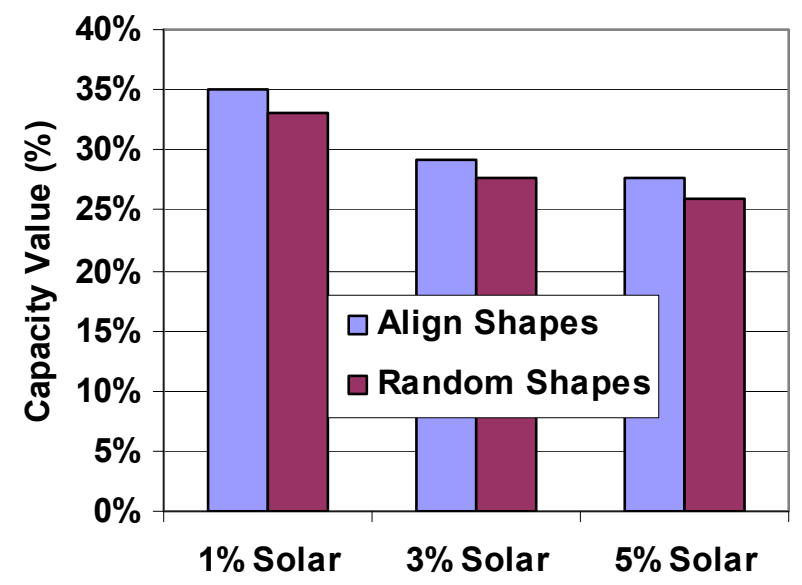

Figure 9.22 Comparison of PV Capacity Values, Random and Aligned Shapes

\subsection{Capacity Value Observations}

Wind generation is added to a system for its energy value, not its capacity value; having said that, its capacity value is not zero, but tends to fall in the $10 \%-15 \%$ of nameplate range as compared to thermal units that are in the $90 \%-95 \%$ range. These results reflect the fact that the summer peak load months tend to have lower values of wind generation than the low load spring and fall months. Also, within the day, wind generation tends to be higher in the middle of the night rather than during the day.

Photovoltaic generation has capacity values in the $25 \%-30 \%$ range. The generation comes, naturally, during the day rather than at night, which gives it a better capacity value than wind. Also, PV tends to do well in the summer peak load months. The relatively low value is due to the peak loads tending to come later in the day when the solar energy has begun to wane, and the use of the DC rating rather than the AC rating.

Concentrating solar plants would normally tend to suffer the same capacity value fate as the PV. However, by their very nature the CSPs lend themselves well to storage. The collector field can be oversized and the collection medium can store the thermal energy without the large collection losses inherent with battery or pumped storage hydro. Because of this, the CSP with six hours of storage was seen to have capacity values in the $90 \%-95 \%$ range that is on par with conventional thermal generation.

Different methods can be used to determine capacity value, including daily LOLE, hourly LOLE, and unserved energy. All of the measures tend to produce results within the same range. 


\section{Key Findings}

The technical analysis performed in this study shows that it is operationally feasible for WestConnect to accommodate $30 \%$ wind and 5\% solar energy penetration, assuming the following changes to current practice are made over time:

- Substantially increase balancing area cooperation or consolidation, either real or virtual

- Increase the use of sub-hourly scheduling for generation and interchanges

- Increase utilization of existing transmission

- Enable coordinated commitment and economic dispatch of generation over wider regions

- Incorporate state of the art (S-o-A) wind and solar forecasts in unit commitment and grid operations

- Increase the flexibility of dispatchable generation where appropriate (e.g., reduce minimum generation levels, increase ramp rates, reduce start/stop costs or minimum down time)

- Commit additional operating reserves as appropriate

- Build transmission as appropriate to accommodate renewable energy expansion

- Target new or existing demand response or load participation programs to accommodate increased variability and uncertainty

- Require wind plants to provide down reserves

In addition to the key findings listed here, there are observations and conclusions at the end of Chapters 4 through 9.

\subsection{Operations with $35 \%$ Renewables}

WWSIS finds that 35\% renewable energy penetration is operationally feasible provided significant changes to current operating practice are made, including balancing area cooperation and sub-hourly scheduling.

The power system is designed to handle variability in load. With wind and solar, the power system is called on to handle variability in the net load (load minus wind minus solar), which can be considerable during certain periods of the year. Study results presented in Chapter 6 illustrate the impact of 30\% wind and 5\% solar energy penetration during two representative weeks; one in July and one in April. In the July week, wind and solar generation do not significantly affect net load. Although the wind and solar generation are definitely noticeable, they primarily displace combined-cycle 
and gas turbine generation, and have minimal impact on the steam coal units. In the April week, however, the high, variable wind output dominates the net load, especially during low load hours, leading to several hours of negative net load during the week. Combined-Cycle generation is almost completely displaced, and significant levels of coal generation are displaced by wind and solar generation. Nonetheless, the system can operate through this challenging week with balancing area cooperation. Without balancing area cooperation, operations during this week would be extremely difficult, if not impossible, for individual balancing areas.

\subsection{Benefits of $35 \%$ Renewables}

Wind and solar generation primarily displace gas resources nearly all hours of the year, given the fuel prices and carbon tax assumed for this study (\$2/MBTU coal, \$9.50/MBTU gas, $\$ 30 /$ ton CO2). Since gas-fired generation is typically more flexible than coal generation, the natural economic displacement of gas generation by wind and solar generation makes the balance of dispatchable generation on-line less flexible (fewer gas units, more coal units). Across WECC, operating costs drop by $\$ 20$ billion/yr ( $\$ 17$ billion/yr 2009\$) from approximately $\$ 50$ billion/yr ( $\$ 43$ billion/yr in 2009\$), resulting in a $40 \%$ savings due to offset fuel and emissions. This savings does not account for the capital or operating costs associated with the wind, solar, or transmission facilities, nor does it include any of the costs that would be required to implement the operational reforms needed to accommodate the renewables including balancing area cooperation or subhourly scheduling. Presumably some of this savings would be used to recover the capital costs of building this scenario, including payments to wind and solar generators.

If the price of natural gas was \$3.5/MBTU (instead of \$9.50/MBTU), study results show that wind and solar generation primarily displace coal generation, leaving the more flexible gas generation resources to operate together with the wind and solar generation. With lower gas price assumptions, operating cost savings are $40 \%$ lower, or $\$ 46 / \mathrm{MWh}$ (\$39/MWh in 2009\$), but emissions reductions are higher.

With the base-case fuel price assumptions and 30\% wind penetration, CO2 emissions would be reduced by nearly 120 million tons per year, or approximately $25 \%$. SOx emissions would be reduced by approximately 45,000 tons ( $5 \%)$ and NOx would be reduced nearly 100,000 tons per year ( 15\%) (see Section 6.2.1). At a $\$ 3.5 / \mathrm{MBTU}$ gas price, CO2 emissions would be reduced by nearly 200 million tons/year (45\%), and NOx and SOx by 300,000 tons/year (50\%) and 220,000 tons/year (30\%), respectively.

\subsection{What Changes are Needed to Integrate 35\% Renewables?}

\subsubsection{Balancing Area Cooperation is Essential}

There are three key benefits of balancing area cooperation: 
- Aggregating diverse renewable resources over larger geographic areas reduces the overall variability of the renewables,

- Aggregating the load reduces the overall variability of the load, and

- Aggregating the non-renewable balance of generation provides access to more balancing and more flexible resources.

Figure 5-17 shows how overall variability is reduced when balancing is performed over a larger area. Variability for small areas such as Colorado-West (CO-W) or Wyoming (WY) increases significantly as renewable penetrations increase from the $10 \%$ to the $30 \%$ case. This effect becomes even more extreme at a more granular level, e.g., for specific balancing areas within a state (see Section 7.1). However, when the balancing areas across WestConnect are aggregated, there is only a slight increase in variability with increased renewables penetrations.

From an operational perspective, balancing area cooperation can lead to cost savings because reserves can be pooled. A sensitivity analysis was performed, running WECC as 106 zones versus 5 large regions. The zones are roughly equivalent to balancing areas in the southwest, but there are multiple zones per balancing area in the northwest. The results show $\$ 2$ billion ( $\$ 1.7$ billion in $2009 \$$ ) savings in WECC operating costs in the $10 \%$ case (see Section 6.4.5). There are significant savings from sharing reserves over larger regions, irrespective of the renewables on the system.

\subsubsection{Sub-Hourly Scheduling is Critical}

The current practice of scheduling both the generation and inter-state exchange only once each hour has a significant impact on the regulation duty. At high penetration levels, such hourly schedule changes can use most, if not all, of the available regulation capability to compensate for Area Control Error (ACE) excursions during large scheduled ramps. This can leave no regulation capability for the sub-hourly variability.

The minute-to-minute simulations showed that the current practice of hourly scheduling has a greater impact on the regulation requirements than does the wind and solar variability.

Sub-hourly scheduling can substantially reduce the maneuvering duty imposed on the units providing load following. In the $30 \%$ case, the maneuvering of combined-cycle plants with sub-hourly scheduling is about half of that with hourly scheduling, as shown in Figure 7.45. Improvements in plant efficiency and reductions in O\&M costs, while difficult to quantify, are expected from this smoother operation. 


\subsection{What is the Impact of Uncertainty and Variability?}

\subsubsection{Using Forecasts Results in the Biggest Impact on the System}

Integrating day-ahead wind and solar forecasts into the unit commitment process is essential to help mitigate the uncertainty of wind and solar generation. Even though S-o-A wind and solar forecasts are imperfect and sometimes result in reserve shortfalls due to missed forecasts, it is still beneficial to incorporate them into the day-ahead scheduling process. Over the course of the year, use of S-o-A wind and solar forecasts reduces WECC operating costs by up to $14 \%$, or $\$ 5$ billion/yr ( $\$ 4$ billion/yr in 2009\$), as compared to not using wind or solar forecasts for day-ahead unit commitment. This corresponds to operating cost savings of $\$ 12-20 / \mathrm{MWh}(\$ 10-17 / \mathrm{MWh}$ in $2009 \$$ ) of wind and solar generation (see Section 6.2.1). WECC operating costs could be reduced by an additional $\$ 500$ million/yr ( $\$ 425$ million/yr 2009\$) in the 30\% case if wind and solar forecasts were perfect (i.e., no forecast error). This is an incentive to improve the accuracy of S-o-A wind and solar forecasting technology.

\subsubsection{Extreme Forecast Errors and Contingency Reserve Shortfalls}

While on average, wind forecast error is not very large $(8 \%$ mean absolute error across WestConnect), there are hours when wind forecast errors can be extreme, ranging up to 11,000 MW of over- or under-forecast in WestConnect. Severe over-forecasts can result in contingency reserve shortfalls; severe under-forecasts can result in curtailment of wind.

Operating rules dictate that systems must carry contingency reserves to cover system events, such as tripping of a large generator. In WECC, the spinning portion of these contingency reserves is equivalent to $3 \%$ of the system load. Applying these WECC rules, severe over-forecasts can lead to under-commitment of generation units, which can result in contingency reserve shortfalls if insufficient quick-start capacity is available. Spinning reserves can be increased to cover these contingency reserve shortfalls, but at a cost.

Increasing the committed spinning reserve by $5 \%$ of the wind forecast increases WECC operating costs by over $\$ 3,000$ per MWh $(\$ 2,550 / \mathrm{MWh}$ in $2009 \$)$ of reduced reserve shortfall. This would be equivalent to paying some of the load $\$ 3,000 / \mathrm{MWh}$ $(\$ 2,550 / \mathrm{MWh}$ in $2009 \$)$ to drop off rather than increase the spinning reserve by $5 \%$ of the forecast. Increasing spinning reserve by $25 \%$ would cost an average of roughly $\$ 13,600 / \mathrm{MWh}$ (\$11,600/MWh in 2009\$) of reserve shortfall. Given the diminishing returns from increased spinning reserves, marginal operating costs for reducing reserve shortfalls would exceed $\$ 100,000 / \mathrm{MWh}$.

It should be more economic to use load participation (i.e., demand response) than to increase the spinning reserves to achieve the same objectives. Using load participation 
instead of committing additional generation for operating reserves would save up to $\$ 600$ million (\$510 million in 2009\$) in operating costs per year (see Sections 5.4, 7.2, and 6.2.2).

\subsubsection{How Often is Wind Curtailed?}

Uncertainty drives both curtailment and reserve shortfalls. With a perfect forecast, no wind or solar curtailment was necessary in any of the scenarios. Even in the few hours when the renewable generation exceeded the load in WestConnect, there was sufficient flexibility within WECC to absorb all of the generation. With a S-o-A forecast, no curtailment occurred up through the $20 \%$ case (see Figure 6.27 ). The hourly production simulations showed about 800 GWh of wind curtailment in the $30 \%$ case, representing less than $0.5 \%$ of the total wind energy production. In addition, the minute-to-minute analysis indicated that more wind curtailment may be required under some combinations of low load and high wind. Altogether, wind curtailment in the $30 \%$ case is estimated to be on the order of $1 \%$ or less of the total wind energy. Curtailment is also affected by the flexibility of the balance of generation, e.g., raising the minimum operating point of the coal units to $70 \%$ increased the wind curtailment slightly (See Sections 6.2 and 6.4.4).

\subsubsection{Are Additional Reserves Necessary to Cover Variability?}

In addition to contingency reserves, utilities are required to hold variability or load following reserves to cover 10 -minute load variability $95 \%$ of the time. Typically, utilities do not commit additional variability reserves because the existing dispatchable generating fleet can adequately cover this variability reserve requirement. With wind and solar, the net load variability increases and in the $30 \%$ case, the average variability reserve requirement doubles. However, when wind and solar are added to the system, thermal units are backed down because it is more economical to do so than to decommit them. This results in more available up-reserves than in the case when there is no wind and solar (see Section 7.5.1). Therefore, commitment of additional reserves is not needed to cover variability in the study footprint.

Down reserves can be handled through wind curtailment when other resources are depleted. A wind plant can reduce its output very quickly in response to a command signal. Simulations in this study show that down reserves can be implemented through command signals (ACE signals) from system operators. With extensive balancing area cooperation, WestConnect can accommodate large amounts of renewables, and curtailment of wind is expected to be on the order of $1 \%$ or less in the $30 \%$ case.

\subsection{What are the Major Differences Between the Scenarios?}

The In-Area, Local-Priority, and Mega-Project Scenarios showed similar overall performance and economics for a given penetration level. This indicates that the specific 
locations of the wind and solar resources within WestConnect are not critical, provided there is adequate transmission infrastructure and access, and balancing area cooperation (see Sections 4.2.3, 5.5, 6.4.1, 6.4.6). The assumption that existing transmission capacity can be fully used is an important change from present practice underpinning these results.

Analysis of seasonal wind and solar patterns in the WestConnect footprint show significant difference between seasons. For the 30\% case, wind and solar energy would serve as much as $50 \%$ of the load in the winter months but only about $15 \%$ in the summer months. Despite the month-to-month variation in energy penetration, there is relatively little difference among the three geographic scenarios at the footprint resolution (see Section 4.1.1-4.1.2)

The total WECC operating cost savings per MWh of renewable energy for the different scenarios was also very similar across the three geographic scenarios, with only a slight increase in value as the wind plant locations were shifted to the higher capacity factor sites in the Local-Priority and Mega-Project Scenarios (see Section 6.4.1)

\subsubsection{Is New Long Distance Transmission Needed?}

The In-Area Scenario uses in-state wind and solar resources, so no new interregional transmission would be required. For the Local-Priority and Mega-Project Scenarios, up to $20 \%$ renewable penetration could be achieved with little or no new long distance, inter-state transmission additions, assuming full use of existing transmission capacity.

Assuming a $15 \%$ fixed charge rate, the 30\% Local-Priority Scenario would justify about $\$ 2$ billion ( $\$ 1.7$ billion in 2009\$) in transmission investments and the Mega-Project Scenario would justify a little over $\$ 10$ billion ( $\$ 8.5$ billion in $2009 \$$ ). This rough estimate suggests that the full-scale transmission build-out might be justified in the $30 \%$ MegaProject Scenario, but not at lower penetrations in the Mega-Project or for any of the other scenarios. A more limited transmission build-out may be justified for the Local-Priority Scenario. Of course, these estimates do not include any reliability benefits that would be realized from adding more transmission. All scenarios could accommodate the $10 \%$ case without any new inter-state transmission (see Section 6.4.6).

\subsection{Is Additional Energy Storage Needed?}

Storage can provide many benefits to the system, including price arbitrage (charging when spot prices are low and discharging when prices are high), reliability, and ancillary services. Pumped storage hydro (PSH), solar thermal storage, and Plug-in Hybrid Electric Vehicles (PHEVs) were examined in WWSIS, with the largest focus on PSH (see Chapter 8). WWSIS evaluated only the price arbitrage part of the value proposition for PSH and found it much less than sufficient to economically justify additional storage facilities. 
In the $10 \%$ and $20 \%$ wind penetration scenarios, gas generation is always on the margin (meaning that there are only small spot price variations during most days). As a result, there is no apparent opportunity to economically justify energy storage based on price arbitrage. Spot price variations increase in the $30 \%$ wind penetration scenarios, primarily due to errors in day-ahead wind energy forecasts. Occasionally, the price swings are very large. However, because this is driven by forecast uncertainty, it is not possible to strategically schedule the use of storage resources to take advantage of the price variations (and subsequently help eliminate the operational problems due to wind forecast errors).

\subsection{What is the Benefit of Flexibility in the Generation Fleet?}

System flexibility is the key to accommodating increased renewable generation. WWSIS finds that at higher penetration levels (30\% case), decreased flexibility of either the coal or hydro facilities made operation more difficult and increased the costs of integrating the renewable generation.

\subsubsection{Load Following by Hydro Plants is Helpful}

Hydro generation is capable of quick-start/stop cycling and fast ramping, which makes it a good partner for variable wind and solar generation. Sensitivity analyses were conducted to examine the effects of hydro constraints on operating costs (Section 6.4.2).

This study assumed that hydro generation is normally committed and dispatched to serve daily peak net-load periods, while respecting the minimum operating points on the hydro units. Adjusting hydro schedules to account for the day-ahead renewable forecasts was evaluated as an alternative to the normal practice. Although the impact of adjusting the hydro dispatch schedule is relatively small at low levels of penetration, the WECC operating costs would be reduced by $\$ 200$ million/yr ( $\$ 170$ million/yr in 2009\$) for the $30 \%$ case. This increases the value of wind and solar energy by about $\$ 1 / \mathrm{MWh}$ (\$0.9/MWh in 2009\$).

On the other hand, if hydro operation were severely constrained, such as a requirement to maintain constant river flow, WECC operating costs would increase by up to $\$ 1$ billion/yr ( $\$ 0.9$ billion/yr in 2009\$). Flexibility in the scheduling of hydro generation is clearly beneficial to the integration of wind and solar resources (see Section 6.4.2).

\subsubsection{Constraints on Coal Plants Result in Higher Operating Costs}

Coal plants were assumed to be able to operate down to minimum generation levels of $40 \%$ of nameplate capacity. WECC operating costs would increase if coal plant

minimum load levels were unable or unwilling to operate down to this level. 
A sensitivity case explored the impact of varying coal plant minimum load on system operating costs. Increasing the minimum load had minimal impact with wind penetrations less than $20 \%$. If coal plants only operate between $70 \%$ and $100 \%$ load, then WECC operating costs would increase by about $\$ 160$ million/yr ( $\$ 136$ million/yr in $2009 \$$ ) for the $30 \%$ case.

\subsection{How do Renewables Contribute to Resource Adequacy?}

Variable resources such as wind and solar PV are primarily energy resources rather than capacity resources. However, they provide some contribution to resource adequacy. Several capacity valuation techniques based on traditional Loss of Load Expectation (LOLE) data were used to consider the variability inherent with the renewable generation. This analysis was conducted for WestConnect assuming no transmission constraints within the study footprint and no interconnections with the rest of WECC, so that the capacity value characteristics of the renewable generation could be isolated.

Wind generation resources selected for this study were found to have capacity values in the range of $10 \%-15 \%$. Wind plant energy output tends to be higher during winter and spring seasons, and during nighttime hours, which is contrary to system peak load periods. Hence, the capacity value is low relative to the plant rating.

PV solar plants have capacity values in the range of $25 \%-30 \%$. Although PV solar produces its energy during the daytime, output tends to decline in the late afternoon and early evening when peak load hours often occur. In addition, the PV data represented the gross power rating at the DC bus, not a net power rating at the AC interface to the grid. As such, it did not account for the significant inverter and other losses (typically 23\%). If the capacity value of the PV plants were calculated on the AC rating, it would be higher.

Concentrating solar plants with thermal energy storage have capacity values in the range of $90 \%-95 \%$, similar to thermal generating plants. Their maximum energy production tends to be during the long summer days, and the storage capability extends the energy output through the late afternoon and early evening hours, when peak loads occur (see Sections 4.2, 4.3, 9.2 through 9.7).

\subsection{Conclusions and Next Steps}

The technical analysis performed in this study shows that it is feasible for the WestConnect region to accommodate $30 \%$ wind and 5\% solar energy penetration. This requires key changes to current practice, including substantial balancing area cooperation, sub-hourly scheduling, and access to under used transmission capacity. 
WWSIS finds that both the variability and uncertainty of wind and solar generation impacts grid operations. However, the uncertainty (due to imperfect forecasts) leads to a greater impact on operations and results in some contingency reserve shortfalls and some curtailment, both of which are relatively small. The variability leads to a greater sub-hourly variability reserve requirement, but because conventional units are backed down, the system naturally has extra reserve margins.

This study has established both the potential and the challenges of large-scale integration of wind and solar generation in WestConnect and, more broadly, in WECC. However, changes of this magnitude warrant further investigation. The project team regards the following as valuable topics for exploration:

- Characterization of the capabilities of the non-renewable generation portfolio in greater detail (minimum turndown, ramp rates, cost of additional wear and tear)

- Changes in non-renewable generation portfolio (e.g., impact of retirements, characteristics, and value of possible fleet additions or upgrades)

- Reserve requirements and strategies (e.g., strategies and requirements for off-line reserves, reserves from non-generation resources, shared reserves across balancing areas)

- Load participation or demand response (e.g., functionality, market structures, PHEV)

- Fuel sensitivities (e.g., price, carbon taxes, gas contracts and storage, hydro constraints and strategies)

- Forecasting (e.g., calibration of forecasting using field experience, strategies for use of short-term forecasting)

- Rolling unit commitment (e.g., scheduling units more frequently than once on a day-ahead basis)

- Transmission planning and reliability analyses (e.g., transient stability, voltage stability, protection and control, intra-area constraints and challenges)

- Hydro flexibility (calibration of hydro models with plant performance) 



\section{Appendix A Tables of Wind and Solar by State; Description of Site Selection Algorithm}

This appendix contains the following information:

- Tables showing load, wind generation and solar generation by area for each penetration level of each scenario, and

- A set of PowerPoint slides that explain the algorithm used to sort/select wind and solar generation sites for the three study scenarios. 
Table A- 1 In-Area Scenario, 10\% Wind In Footprint - Energy Summary

\begin{tabular}{|c|c|c|c|c|c|c|c|c|}
\hline \multirow[b]{2}{*}{ Areas } & \multirow{2}{*}{\begin{tabular}{|c|} 
Load \\
Energy \\
(GWh)
\end{tabular}} & \multicolumn{2}{|r|}{ Wind } & \multicolumn{2}{|c|}{ CSP with Storage } & \multicolumn{2}{|r|}{ PV } & \multirow{2}{*}{$\begin{array}{c}\begin{array}{c}\text { Total } \\
\text { Renewable }\end{array} \\
\text { Energy } \\
\text { (GWh) }\end{array}$} \\
\hline & & \begin{tabular}{|l|} 
Energy \\
(GWh) \\
\end{tabular} & $\begin{array}{c}\text { \# Sites } \\
\text { (Used/Available) }\end{array}$ & $\begin{array}{l}\text { Energy } \\
\text { (GWh) }\end{array}$ & $\begin{array}{c}\text { \# Sites } \\
\text { (Used/Available) }\end{array}$ & $\begin{array}{l}\text { Energy } \\
\text { (GWh) }\end{array}$ & $\begin{array}{c}\text { \# Sites } \\
\text { (Used/Available) }\end{array}$ & \\
\hline Arizona & \begin{tabular}{|l|}
99,437 \\
\end{tabular} & 9,996 & $120 / 1091$ & 760 & $2 / 38$ & 318 & $2 / 16$ & 11,074 \\
\hline Colorado East & 61,372 & 6,230 & $68 / 1673$ & 670 & $2 / 58$ & 160 & $1 / 13$ & 7,060 \\
\hline Colorado West & 8,708 & 932 & $10 / 68$ & 0 & $0 / 8$ & 0 & $0 / 11$ & 932 \\
\hline New Mexico & 31,260 & 3,143 & 36 / 3062 & 359 & $1 / 35$ & 155 & $1 / 19$ & 3,657 \\
\hline Nevada & 57,505 & 5,814 & 78 / 1591 & 370 & $1 / 45$ & 160 & $1 / 10$ & 6,344 \\
\hline Wyoming & 27,697 & 2,810 & $31 / 8912$ & 0 & $0 / 0$ & 142 & $1 / 10$ & 2,952 \\
\hline In Footprint & 285,979 & \begin{tabular}{|l|}
28,925 \\
\end{tabular} & $343 / 16397$ & 2,159 & $6 / 184$ & 935 & $6 / 79$ & 32,019 \\
\hline
\end{tabular}

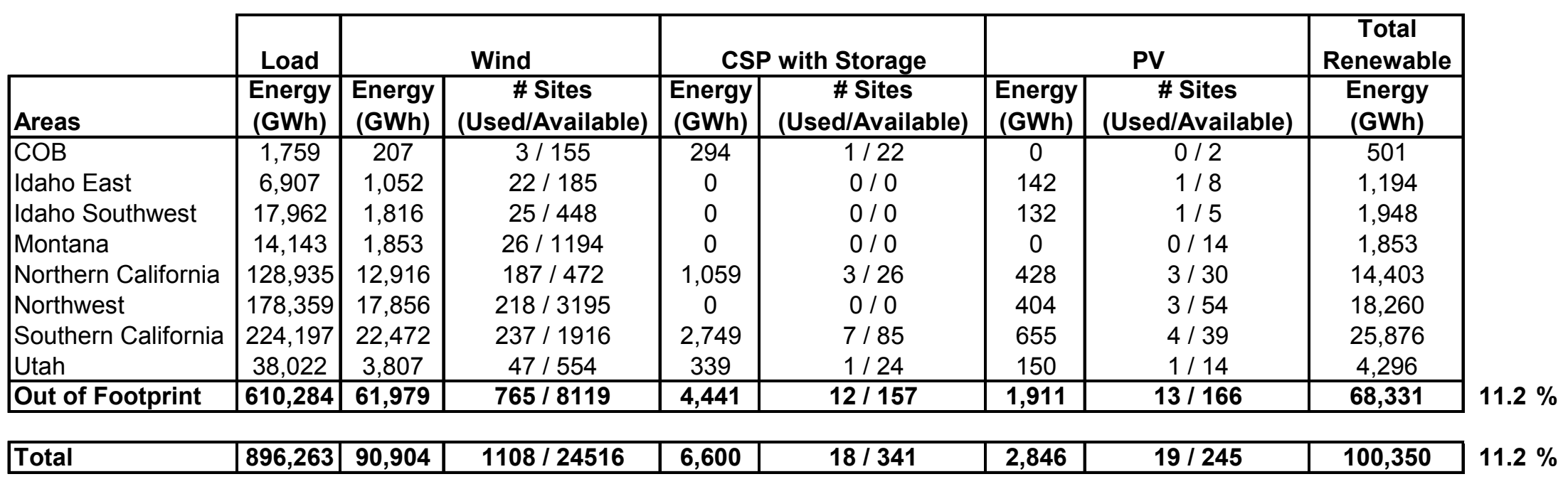


Table A- 2 In-Area Scenario, 10\% Wind In Footprint - Power Summary

\begin{tabular}{|c|c|c|c|c|c|c|c|c|c|c|c|c|c|c|}
\hline \multirow[b]{3}{*}{ Area } & \multicolumn{2}{|c|}{ Load } & \multicolumn{3}{|c|}{ Wind } & \multicolumn{3}{|c|}{ CSP with Storage } & \multicolumn{3}{|c|}{$\overline{\mathbf{P V}}$} & \multicolumn{3}{|c|}{ Total Renewable } \\
\hline & \multirow{2}{*}{$\begin{array}{l}\text { Minimum } \\
\text { (MW) }\end{array}$} & \multirow{2}{*}{$\begin{array}{c}\text { Maximum } \\
(\mathrm{MW})\end{array}$} & \multirow{2}{*}{$\begin{array}{l}\text { Rating } \\
\text { (MW) }\end{array}$} & \multicolumn{2}{|c|}{ Penetration } & \multirow{2}{*}{$\begin{array}{l}\text { Rating } \\
\text { (MW) }\end{array}$} & \multicolumn{2}{|c|}{ Penetration } & \multirow{2}{*}{$\begin{array}{l}\text { Rating } \\
\text { (MW) }\end{array}$} & \multicolumn{2}{|c|}{ Penetration } & \multirow{2}{*}{$\begin{array}{l}\text { Rating } \\
\text { (MW) }\end{array}$} & \multicolumn{2}{|c|}{ Penetration } \\
\hline & & & & $\% \operatorname{Min}$ & \%Max & & $\%$ Min & $\% \operatorname{Max}$ & & $\% \operatorname{Min}$ & $\%$ Mar & & $\% \operatorname{Min}$ & $\% \operatorname{Max}$ \\
\hline Arizona & 6,995 & 23,051 & 3,600 & $51 \%$ & $16 \%$ & 200 & $3 \%$ & $1 \%$ & 200 & $3 \%$ & $1 \%$ & 4,000 & $57 \%$ & $17 \%$ \\
\hline ast & 4,493 & 11, & 2,040 & $45 \%$ & 18 & 200 & $4 \%$ & $2 \%$ & 100 & $2 \%$ & $7 \%$ & 2,340 & $52 \%$ & $20 \%$ \\
\hline do West & 712 & 1,526 & 300 & $42 \%$ & $20 \%$ & 0 & $0 \%$ & $0 \%$ & 0 & $0 \%$ & $0 \%$ & 300 & $\%$ & $20 \%$ \\
\hline exico & 2,571 & 5,320 & 1,080 & $42 \%$ & $20 \%$ & 100 & $4 \%$ & $2 \%$ & 100 & $4 \%$ & $2 \%$ & 1,280 & $50 \%$ & $24 \%$ \\
\hline Nevada & 363 & 12,584 & 2,340 & $61 \%$ & $19 \%$ & 100 & $3 \%$ & $1 \%$ & 100 & $3 \%$ & $1 \%$ & 2,540 & $66 \%$ & $20 \%$ \\
\hline & 9 & 4,016 & 930 & $39 \%$ & $23 \%$ & 0 & $\%$ & $0 \%$ & 100 & $4 \%$ & $2 \%$ & 1,030 & $43 \%$ & $26 \%$ \\
\hline In Footprint & 21,249 & 58,087 & 10,290 & $48 \%$ & $18 \%$ & 600 & $3 \%$ & $1 \%$ & 600 & $3 \%$ & $1 \%$ & 11,490 & $54 \%$ & $20 \%$ \\
\hline
\end{tabular}

\begin{tabular}{|c|c|c|c|c|c|c|c|c|c|c|c|c|c|c|}
\hline \multirow[b]{3}{*}{ Area } & \multicolumn{2}{|c|}{ Load } & \multicolumn{3}{|c|}{ Wind } & \multicolumn{3}{|c|}{ CSP with Storage } & \multicolumn{3}{|c|}{ PV } & \multicolumn{3}{|c|}{ Total Renewable } \\
\hline & \multirow{2}{*}{$\begin{array}{c}\text { Minimum } \\
(\mathrm{MW})\end{array}$} & \multirow{2}{*}{$\begin{array}{c}\text { Maximum } \\
(\mathrm{MW})\end{array}$} & \multirow{2}{*}{\begin{tabular}{|c|} 
Rating \\
(MW)
\end{tabular}} & \multicolumn{2}{|c|}{ Penetration } & \multirow{2}{*}{$\begin{array}{c}\text { Rating } \\
\text { (MW) }\end{array}$} & \multicolumn{2}{|c|}{ Penetration } & \multirow{2}{*}{$\begin{array}{c}\text { Rating } \\
\text { (MW) }\end{array}$} & \multicolumn{2}{|c|}{ Penetration } & \multirow{2}{*}{$\begin{array}{c}\text { Rating } \\
\text { (MW) }\end{array}$} & \multicolumn{2}{|c|}{ Penetration } \\
\hline & & & & $\%$ Min & \%Max & & $\% \operatorname{Min}$ & $\% \operatorname{Max}$ & & \begin{tabular}{|l|}
$\% \operatorname{Min}$ \\
\end{tabular} & $\% \operatorname{Max}$ & & $\% \operatorname{Min}$ & $\% \operatorname{Max}$ \\
\hline COB & 138 & 294 & 90 & $65 \%$ & $31 \%$ & 100 & $73 \%$ & $34 \%$ & 0 & $0 \%$ & $0 \%$ & 190 & \begin{tabular}{|l|}
$138 \%$ \\
\end{tabular} & $65 \%$ \\
\hline Idaho East & 460 & 1,365 & 660 & $144 \%$ & $48 \%$ & 0 & $0 \%$ & $0 \%$ & 100 & $22 \%$ & $7 \%$ & 760 & $\%$ & $56 \%$ \\
\hline Idaho Southwest & 1,188 & 3,592 & 750 & $63 \%$ & $21 \%$ & 0 & $0 \%$ & $0 \%$ & 100 & $8 \%$ & $3 \%$ & 850 & $72 \%$ & $24 \%$ \\
\hline Montana & 1,149 & 2,337 & 780 & $68 \%$ & $33 \%$ & 0 & $0 \%$ & $0 \%$ & 0 & $0 \%$ & $0 \%$ & 780 & $68 \%$ & $33 \%$ \\
\hline Northern California & 10,297 & 28,319 & 5,610 & $54 \%$ & $20 \%$ & 300 & $3 \%$ & $1 \%$ & 300 & $3 \%$ & $1 \%$ & 6,210 & $60 \%$ & $22 \%$ \\
\hline No & 14,278 & 953 & 6,540 & $46 \%$ & $21 \%$ & 0 & 0 & $0 \%$ & 300 & $2 \%$ & $1 \%$ & 6,840 & $48 \%$ & $22 \%$ \\
\hline Southe & 9,557 & 26,864 & 110 & $\%$ & $26 \%$ & 700 & 7 & $3 \%$ & 400 & 4 & $1 \%$ & 8,210 & 86 & $31 \%$ \\
\hline Uta & & 274 & 1,410 & $62 \%$ & $19 \%$ & 100 & $4 \%$ & $1 \%$ & 100 & $4 \%$ & $1 \%$ & 1,610 & $71 \%$ & $22 \%$ \\
\hline Out of $F$ & 46,328 & 119,696 & 22,950 & $50 \%$ & $19 \%$ & 1,200 & $3 \%$ & $1 \%$ & 1,300 & $3 \%$ & $1 \%$ & 25,450 & $55 \%$ & $21 \%$ \\
\hline otal & 67,577 & 177,783 & 33,240 & $49 \%$ & $19 \%$ & 1,800 & $3 \%$ & $1 \%$ & 1,900 & $3 \%$ & $1 \%$ & 36,940 & $55 \%$ & $21 \%$ \\
\hline
\end{tabular}


Table A- 3 In-Area Scenario, 20\% Wind In Footprint - Energy Summary

\begin{tabular}{|c|c|c|c|c|c|c|c|c|}
\hline \multirow[b]{2}{*}{ Areas } & \multirow{2}{*}{\begin{tabular}{|c|} 
Load \\
Energy \\
(GWh)
\end{tabular}} & \multicolumn{2}{|r|}{ Wind } & \multicolumn{2}{|c|}{ CSP with Storage } & \multicolumn{2}{|r|}{ PV } & \multirow{2}{*}{$\begin{array}{c}\begin{array}{c}\text { Total } \\
\text { Renewable }\end{array} \\
\text { Energy } \\
(\text { GWh })\end{array}$} \\
\hline & & $\begin{array}{l}\text { Energy } \\
\text { (GWh) }\end{array}$ & $\begin{array}{c}\text { \# Sites } \\
\text { (Used/Available) }\end{array}$ & $\begin{array}{l}\text { Energy } \\
\text { (GWh) }\end{array}$ & $\begin{array}{c}\text { \# Sites } \\
\text { (Used/Available) }\end{array}$ & $\begin{array}{l}\text { Energy } \\
\text { (GWh) }\end{array}$ & $\begin{array}{c}\text { \# Sites } \\
\text { (Used/Available) }\end{array}$ & \\
\hline Arizona & 99,437 & 19,906 & $245 / 1091$ & 2,254 & $6 / 38$ & 933 & $6 / 16$ & 23,093 \\
\hline Colorado East & 61,372 & 12,342 & $126 / 1673$ & 1,304 & $4 / 58$ & 597 & $4 / 13$ & 14,243 \\
\hline Colorado West & 8,708 & 1,806 & $20 / 68$ & 283 & $1 / 8$ & 151 & $1 / 11$ & 2,240 \\
\hline New Mexico & 31,260 & 6,257 & 64 / 3062 & 714 & $2 / 35$ & 315 & $2 / 19$ & 7,286 \\
\hline Nevada & 57,505 & 11,555 & $156 / 1591$ & 1,457 & $4 / 45$ & 468 & $3 / 10$ & 13,480 \\
\hline Wyoming & 27,697 & 5,572 & $54 / 8912$ & 0 & $0 / 0$ & 142 & $1 / 10$ & 5,714 \\
\hline In Footprint & 285,979 & 57,438 & $665 / 16397$ & 6,012 & $17 / 184$ & 2,606 & $17 / 79$ & 66,056 \\
\hline
\end{tabular}

\begin{tabular}{|c|c|c|c|c|c|c|c|c|}
\hline \multirow[b]{2}{*}{ Areas } & \multirow{2}{*}{\begin{tabular}{|c|} 
Load \\
Energy \\
(GWh) \\
\end{tabular}} & \multicolumn{2}{|r|}{ Wind } & \multicolumn{2}{|c|}{ CSP with Storage } & \multicolumn{2}{|r|}{ PV } & \multirow{2}{*}{$\begin{array}{c}\text { Total } \\
\text { Renewable } \\
\text { Energy } \\
(\text { GWh })\end{array}$} \\
\hline & & \begin{tabular}{|l|} 
Energy \\
(GWh)
\end{tabular} & $\begin{array}{c}\text { \# Sites } \\
\text { (Used/Available) }\end{array}$ & \begin{tabular}{|l|} 
Energy \\
(GWh)
\end{tabular} & $\begin{array}{c}\text { \# Sites } \\
\text { (Used/Available) }\end{array}$ & \begin{tabular}{|l|} 
Energy \\
(GWh)
\end{tabular} & $\begin{array}{c}\text { \# Sites } \\
\text { (Used/Available) }\end{array}$ & \\
\hline $\mathrm{COB}$ & 1,759 & 207 & $3 / 155$ & 294 & $1 / 22$ & 0 & $0 / 2$ & 501 \\
\hline Idaho East & 6,907 & 1,052 & $22 / 185$ & 0 & $0 / 0$ & 142 & $1 / 8$ & 1,194 \\
\hline Idaho Southwest & 17,962 & 1,816 & $25 / 448$ & 0 & $0 / 0$ & 132 & $1 / 5$ & 1,948 \\
\hline Montana & 14,143 & 1,853 & $26 / 1194$ & 0 & $0 / 0$ & 0 & $0 / 14$ & 1,853 \\
\hline Northern California & 128,935 & 12,916 & $187 / 472$ & 1,059 & $3 / 26$ & 428 & $3 / 30$ & 14,403 \\
\hline Northwest & 178,359 & 17,856 & $218 / 3195$ & 0 & $0 / 0$ & 404 & $3 / 54$ & 18,260 \\
\hline Southern California & 224,197 & 22,472 & $237 / 1916$ & 2,749 & $7 / 85$ & 655 & $4 / 39$ & 25,876 \\
\hline Utah & 38,022 & 3,807 & 47 / 554 & 339 & $1 / 24$ & 150 & $1 / 14$ & 4,296 \\
\hline Out of Footprint & 610,284 & 61,979 & $765 / 8119$ & 4,441 & $12 / 157$ & 1,911 & $13 / 166$ & 68,331 \\
\hline Total & 896,263 & 119,417 & $1430 / 24516$ & 10,453 & $29 / 341$ & 4,517 & $30 / 245$ & 134,387 \\
\hline
\end{tabular}


Table A- 4 In-Area Scenario, 20\% Wind In Footprint - Power Summary

\begin{tabular}{|c|c|c|c|c|c|c|c|c|c|c|c|c|c|c|}
\hline \multirow[b]{3}{*}{ Area } & \multicolumn{2}{|c|}{ Load } & \multicolumn{3}{|c|}{ Wind } & \multicolumn{3}{|c|}{ CSP with Storage } & \multicolumn{3}{|c|}{$\overline{\mathbf{P V}}$} & \multicolumn{3}{|c|}{ Total Renewable } \\
\hline & \multirow{2}{*}{$\begin{array}{l}\text { Minimum } \\
\text { (MW) }\end{array}$} & \multirow{2}{*}{$\begin{array}{c}\text { Maximum } \\
(\mathrm{MW})\end{array}$} & \multirow{2}{*}{$\begin{array}{l}\text { Rating } \\
\text { (MW) }\end{array}$} & \multicolumn{2}{|c|}{ Penetration } & \multirow{2}{*}{$\begin{array}{l}\text { Rating } \\
\text { (MW) }\end{array}$} & \multicolumn{2}{|c|}{ Penetration } & \multirow{2}{*}{$\begin{array}{l}\text { Rating } \\
\text { (MW) }\end{array}$} & \multicolumn{2}{|c|}{ Penetration } & \multirow{2}{*}{$\begin{array}{l}\text { Rating } \\
\text { (MW) }\end{array}$} & \multicolumn{2}{|c|}{ Penetration } \\
\hline & & & & $\% \operatorname{Min}$ & \%Max & & $\%$ Min & $\% \operatorname{Max}$ & & $\%$ Min & $\% \operatorname{Max}$ & & $\% \operatorname{Min}$ & $\% \operatorname{Max}$ \\
\hline Arizon & 6,995 & 23,051 & 7,350 & $105 \%$ & $32 \%$ & 600 & $9 \%$ & $3 \%$ & 600 & $9 \%$ & $3 \%$ & 8,550 & $122 \%$ & $37 \%$ \\
\hline do East & 4,493 & 11, & 3,780 & 84 & 33 & 400 & 9 & $3 \%$ & 400 & y & 3 & 4,580 & $\%$ & $40 \%$ \\
\hline est & 712 & 1,526 & 600 & $84 \%$ & $39 \%$ & 100 & $14 \%$ & $7 \%$ & 100 & $14 \%$ & $7 \%$ & 800 & $112 \%$ & $52 \%$ \\
\hline exico & 2,571 & 320 & 1,920 & $75 \%$ & $36 \%$ & 200 & $8 \%$ & $4 \%$ & 200 & $8 \%$ & $4 \%$ & 2,320 & $90 \%$ & $44 \%$ \\
\hline $\mathrm{Ne}$ & 363 & 12,584 & 4,680 & $121 \%$ & $37 \%$ & 400 & $10 \%$ & $3 \%$ & 300 & $8 \%$ & $2 \%$ & 5,380 & $139 \%$ & $43 \%$ \\
\hline & 9 & 16 & 1,620 & $68 \%$ & $40 \%$ & 0 & $0 \%$ & $0 \%$ & 100 & $4 \%$ & $2 \%$ & 1,720 & $73 \%$ & $43 \%$ \\
\hline In Footprint & 21,249 & 58,087 & 19,950 & $94 \%$ & $34 \%$ & 1,700 & $8 \%$ & $3 \%$ & 1,700 & $8 \%$ & $3 \%$ & 23,350 & $110 \%$ & $40 \%$ \\
\hline
\end{tabular}

\begin{tabular}{|c|c|c|c|c|c|c|c|c|c|c|c|c|c|c|}
\hline \multirow[b]{3}{*}{ Area } & \multicolumn{2}{|c|}{ Load } & \multicolumn{3}{|c|}{ Wind } & \multicolumn{3}{|c|}{ CSP with Storage } & \multicolumn{3}{|c|}{ PV } & \multicolumn{3}{|c|}{ Total Renewable } \\
\hline & \multirow{2}{*}{$\begin{array}{c}\text { Minimum } \\
\text { (MW) }\end{array}$} & \multirow{2}{*}{$\begin{array}{c}\text { Maximum } \\
(\mathrm{MW})\end{array}$} & \multirow{2}{*}{$\begin{array}{l}\text { Rating } \\
\text { (MW) }\end{array}$} & \multicolumn{2}{|c|}{ Penetration } & \multirow{2}{*}{\begin{tabular}{|c|} 
Rating \\
(MW)
\end{tabular}} & \multicolumn{2}{|c|}{ Penetration } & \multirow{2}{*}{$\begin{array}{c}\text { Rating } \\
\text { (MW) }\end{array}$} & \multicolumn{2}{|c|}{ Penetration } & \multirow{2}{*}{$\begin{array}{l}\text { Rating } \\
\text { (MW) }\end{array}$} & \multicolumn{2}{|c|}{ Penetration } \\
\hline & & & & $\%$ Min & $\% \operatorname{Max}$ & & $\%$ Min & $\% \operatorname{Max}$ & & $\%$ Min & $\% \operatorname{Max}$ & & $\%$ Min & $\% \operatorname{Max}$ \\
\hline COB & 138 & 294 & 90 & $65 \%$ & $31 \%$ & 100 & $73 \%$ & $34 \%$ & 0 & $0 \%$ & $0 \%$ & 190 & $138 \%$ & $65 \%$ \\
\hline Eas & 460 & 1,365 & 660 & $144 \%$ & $48 \%$ & 0 & $0 \%$ & $0 \%$ & 100 & $22 \%$ & $7 \%$ & 760 & $\%$ & $56 \%$ \\
\hline Id & 1,188 & & 750 & $63 \%$ & $21 \%$ & 0 & & & 100 & & 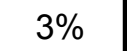 & 850 & & $24 \%$ \\
\hline & 1 & & 780 & $68 \%$ & 33 & 0 & & $0 \%$ & 0 & & $0 \%$ & 780 & & $33 \%$ \\
\hline nia & 7 & 2 & 0 & $54 \%$ & $20 \%$ & 300 & & $1 \%$ & 300 & $3 \%$ & $1 \%$ & 6 & $\%$ & $22 \%$ \\
\hline $\mathrm{Nc}$ & 1 & & 0 & $46 \%$ & $21 \%$ & 0 & $0^{\circ}$ & $0^{c}$ & 30 & $2 \%$ & $1 \%$ & 6 , & $48 \%$ & $22 \%$ \\
\hline Calif & 9 & 2 & 0 & $74 \%$ & $26 \%$ & 700 & 79 & $3 \%$ & 40 & $4^{c}$ & $1 \%$ & 8,210 & $86 \%$ & $31 \%$ \\
\hline Ut: & 263 & & 0 & $62 \%$ & $19 \%$ & 100 & $\%$ & $1 \%$ & 10 & $4 \%$ & $1 \%$ & 1,610 & $\%$ & $22 \%$ \\
\hline Out of Footprint & 46,328 & 119,696 & 22,950 & $50 \%$ & $19 \%$ & 1,200 & $3 \%$ & $1 \%$ & 1,300 & $3 \%$ & $1 \%$ & 25,450 & $55 \%$ & $21 \%$ \\
\hline Total & 67,577 & 177,783 & 42,900 & $63 \%$ & $24 \%$ & 2,900 & $4 \%$ & $2 \%$ & 3,000 & $4 \%$ & $2 \%$ & 48,800 & $72 \%$ & $27 \%$ \\
\hline
\end{tabular}


Table A- 5 In-Area Scenario, 30\% Wind In Footprint - Energy Summary

\begin{tabular}{|c|c|c|c|c|c|c|c|c|}
\hline \multirow[b]{2}{*}{ Area } & \multirow{2}{*}{\begin{tabular}{|c|} 
Load \\
Energy \\
(GWh) \\
\end{tabular}} & \multicolumn{2}{|r|}{ Wind } & \multicolumn{2}{|c|}{ CSP with Storage } & \multicolumn{2}{|r|}{ PV } & \multirow{2}{*}{$\begin{array}{c}\begin{array}{c}\text { Total } \\
\text { Renewable }\end{array} \\
\text { Energy } \\
(\text { GWh })\end{array}$} \\
\hline & & \begin{tabular}{|l|} 
Energy \\
(GWh)
\end{tabular} & $\begin{array}{c}\text { \# Sites } \\
\text { (Used/Available) }\end{array}$ & $\begin{array}{l}\text { Energy } \\
\text { (GWh) }\end{array}$ & $\begin{array}{c}\text { \# Sites } \\
\text { (Used/Available) }\end{array}$ & $\begin{array}{l}\text { Energy } \\
\text { (GWh) }\end{array}$ & $\begin{array}{c}\text { \# Sites } \\
\text { (Used/Available) }\end{array}$ & \\
\hline Arizona & 99,437 & 29,897 & $374 / 1091$ & 3,735 & $10 / 38$ & 1,548 & $10 / 16$ & 35,180 \\
\hline Colorado East & 61,372 & 18,453 & $188 / 1673$ & 2,252 & $7 / 58$ & 1,038 & $7 / 13$ & 21,743 \\
\hline Colorado West & 8,708 & 2,622 & $30 / 68$ & 564 & $2 / 8$ & 151 & $1 / 11$ & 3,337 \\
\hline New Mexico & 31,260 & 9,382 & 93 / 3062 & 1,421 & $4 / 35$ & 473 & $3 / 19$ & 11,276 \\
\hline Nevada & 57,505 & 17,290 & $235 / 1591$ & 2,161 & $6 / 45$ & 773 & $5 / 10$ & 20,224 \\
\hline Wyoming & 27,697 & 8,414 & 78 / 8912 & 0 & $0 / 0$ & 420 & $3 / 10$ & 8,834 \\
\hline In Footprint & 285,979 & 86,058 & $998 / 16397$ & 10,133 & $29 / 184$ & 4,403 & $29 / 79$ & 100,594 \\
\hline
\end{tabular}

\begin{tabular}{|c|c|c|c|c|c|c|c|c|}
\hline \multirow[b]{2}{*}{ Area } & \multirow{2}{*}{\begin{tabular}{|c|} 
Load \\
Energy \\
(GWh) \\
\end{tabular}} & \multicolumn{2}{|r|}{ Wind } & \multicolumn{2}{|c|}{ CSP with Storage } & \multicolumn{2}{|r|}{ PV } & \multirow{2}{*}{$\begin{array}{c}\begin{array}{c}\text { Total } \\
\text { Renewable }\end{array} \\
\text { Energy } \\
(\text { GWh })\end{array}$} \\
\hline & & \begin{tabular}{|l|} 
Energy \\
(GWh)
\end{tabular} & $\begin{array}{c}\text { \# Sites } \\
\text { (Used/Available) }\end{array}$ & \begin{tabular}{|c|} 
Energy \\
(GWh)
\end{tabular} & $\begin{array}{c}\text { \# Sites } \\
\text { (Used/Available) }\end{array}$ & \begin{tabular}{|c|} 
Energy \\
(GWh)
\end{tabular} & $\begin{array}{c}\text { \# Sites } \\
\text { (Used/Available) }\end{array}$ & \\
\hline $\mathrm{COB}$ & 1,759 & 394 & $6 / 155$ & 294 & $1 / 22$ & 142 & $1 / 2$ & 830 \\
\hline Idaho East & 6,907 & 1,406 & $26 / 185$ & 0 & $0 / 0$ & 142 & $1 / 8$ & 1,548 \\
\hline Idaho Southwest & 17,962 & 3,658 & $50 / 448$ & 0 & $0 / 0$ & 132 & $1 / 5$ & 3,790 \\
\hline Montana & 14,143 & 2,873 & $35 / 1194$ & 0 & $0 / 0$ & 127 & $1 / 14$ & 3,000 \\
\hline Northern California & 128,935 & 25,812 & $393 / 472$ & 2,726 & $8 / 26$ & 1,117 & $8 / 30$ & 29,655 \\
\hline Northwest & 178,359 & 35,733 & $431 / 3195$ & 0 & $0 / 0$ & 1,645 & $13 / 54$ & 37,378 \\
\hline Southern California & 224,197 & 44,890 & 483 / 1916 & 8,957 & $23 / 85$ & 2,050 & $13 / 39$ & 55,897 \\
\hline Utah & 38,022 & 7,658 & $91 / 554$ & 937 & $3 / 24$ & 303 & $2 / 14$ & 8,898 \\
\hline Out of Footprint & 610,284 & 122,424 & $1515 / 8119$ & \begin{tabular}{|l|}
12,914 \\
\end{tabular} & $35 / 157$ & 5,658 & $40 / 166$ & 140,996 \\
\hline Total & \begin{tabular}{|l|}
896,263 \\
\end{tabular} & \begin{tabular}{|l|}
208,482 \\
\end{tabular} & $2513 / 24516$ & \begin{tabular}{|l|}
23,047 \\
\end{tabular} & 64 / 341 & 10,061 & $69 / 245$ & 241,590 \\
\hline
\end{tabular}


Table A- 6 In-Area Scenario, 30\% Wind In Footprint - Power Summary

\begin{tabular}{|c|c|c|c|c|c|c|c|c|c|c|c|c|c|c|}
\hline \multirow[b]{3}{*}{ Area } & \multicolumn{2}{|c|}{ Load } & \multicolumn{3}{|c|}{ Wind } & \multicolumn{3}{|c|}{ CSP with Storage } & \multicolumn{3}{|c|}{ PV } & \multicolumn{3}{|c|}{ Total Renewable } \\
\hline & \multirow{2}{*}{$\begin{array}{c}\text { Minimum } \\
\text { (MW) }\end{array}$} & \multirow{2}{*}{$\begin{array}{l}\text { Maximum } \\
\text { (MW) }\end{array}$} & \multirow{2}{*}{$\begin{array}{l}\text { Rating } \\
\text { (MW) }\end{array}$} & \multicolumn{2}{|c|}{ Penetration } & \multirow{2}{*}{$\begin{array}{l}\text { Rating } \\
\text { (MW) }\end{array}$} & \multicolumn{2}{|c|}{ Penetration } & \multirow{2}{*}{$\begin{array}{l}\text { Rating } \\
\text { (MW) }\end{array}$} & \multicolumn{2}{|c|}{ Penetration } & \multirow{2}{*}{$\begin{array}{l}\text { Rating } \\
\text { (MW) }\end{array}$} & \multicolumn{2}{|c|}{ Penetration } \\
\hline & & & & $\% \operatorname{Min}$ & \%Max & & $\% \operatorname{Min}$ & $\%$ Mas & & $\% \operatorname{Min}$ & $\%$ Ma> & & $\% \operatorname{Min}$ & $\% \operatorname{Max}$ \\
\hline Arizor & 6,995 & 23,051 & 11,220 & $160 \%$ & $49 \%$ & 1,000 & $14 \%$ & $4 \%$ & 1,000 & $14 \%$ & $4 \%$ & 13,220 & $189 \%$ & $57 \%$ \\
\hline $\mathrm{C}$ & 4,493 & 11 & 5,640 & 12 & 49 & 70 & 1 & 6 & 70 & $16 \%$ & $6 \%$ & 10 & $\%$ & $61 \%$ \\
\hline $\mathrm{Cc}$ & 712 & 26 & 900 & $126 \%$ & $59 \%$ & 200 & 28 & $13^{\circ}$ & 100 & 14 & 7 & 0 & $\%$ & $79 \%$ \\
\hline exico & 2,571 & ,320 & 2,790 & $109 \%$ & $52 \%$ & 400 & $16 \%$ & $8 \%$ & 300 & $12 \%$ & $6 \%$ & 3,490 & $136 \%$ & $66 \%$ \\
\hline$N$ & 863 & 12,584 & 7,050 & $183 \%$ & $56 \%$ & 600 & $16 \%$ & $5 \%$ & 500 & $13 \%$ & $4 \%$ & 8,150 & $211 \%$ & $65 \%$ \\
\hline & 369 & 16 & 2,340 & $99 \%$ & $58 \%$ & 0 & $0 \%$ & $0 \%$ & 300 & $13 \%$ & $7 \%$ & 2,640 & $111 \%$ & $66 \%$ \\
\hline In Footprint & 21,249 & 58,087 & 29,940 & $141 \%$ & $52 \%$ & 2,900 & $14 \%$ & $5 \%$ & 2,900 & $14 \%$ & $5 \%$ & 35,740 & $168 \%$ & $62 \%$ \\
\hline
\end{tabular}

\begin{tabular}{|c|c|c|c|c|c|c|c|c|c|c|c|c|c|c|}
\hline \multirow[b]{3}{*}{ Area } & \multicolumn{2}{|c|}{ Load } & \multicolumn{3}{|c|}{ Wind } & \multicolumn{3}{|c|}{ CSP with Storage } & \multicolumn{3}{|c|}{ PV } & \multicolumn{3}{|c|}{ Total Renewable } \\
\hline & \multirow{2}{*}{$\begin{array}{l}\text { Minimum } \\
\text { (MW) }\end{array}$} & \multirow{2}{*}{\begin{tabular}{|c|} 
Maximum \\
(MW)
\end{tabular}} & \multirow{2}{*}{$\begin{array}{l}\text { Rating } \\
\text { (MW) }\end{array}$} & \multicolumn{2}{|c|}{ Penetration } & \multirow{2}{*}{$\begin{array}{l}\text { Rating } \\
\text { (MW) }\end{array}$} & \multicolumn{2}{|c|}{ Penetration } & \multirow{2}{*}{$\begin{array}{l}\text { Rating } \\
\text { (MW) }\end{array}$} & \multicolumn{2}{|c|}{ Penetration } & \multirow{2}{*}{$\begin{array}{c}\text { Rating } \\
\text { (MW) }\end{array}$} & \multicolumn{2}{|c|}{ Penetration } \\
\hline & & & & $\%$ Min & \%Max & & $\%$ Min & $\% \operatorname{Max}$ & & $\%$ Min & $\% \operatorname{Max}$ & & \% Min & $\% \operatorname{Max}$ \\
\hline $\mathrm{COB}$ & 138 & 294 & 180 & $131 \%$ & $61 \%$ & 100 & $73 \%$ & $34 \%$ & 100 & $73 \%$ & $34 \%$ & 380 & $276 \%$ & $129 \%$ \\
\hline o Eas & 460 & 5 & 780 & & 5 & 0 & $0 \%$ & $0 \%$ & 10 & $22 \%$ & 7 & 880 & $\%$ & $64 \%$ \\
\hline thwest & 88 & 2 & 500 & 12 & $42 \%$ & 0 & 0 & $0 \%$ & 10 & $8 \%$ & $3 \%$ & 1,600 & $\%$ & $45 \%$ \\
\hline Mor & 49 & 2,337 & 1,050 & $91 \%$ & $45 \%$ & 0 & 0 & $0 \%$ & 100 & $9 \%$ & $4 \%$ & 1,150 & $100 \%$ & $49 \%$ \\
\hline California & 10,297 & 28,319 & ,790 & $114 \%$ & $42 \%$ & 800 & $8 \%$ & $3 \%$ & 800 & $8 \%$ & $3 \%$ & 13,390 & $130 \%$ & $47 \%$ \\
\hline Nor & 14,278 & 30,953 & 12,930 & $91 \%$ & $42 \%$ & 0 & $0 \%$ & $0 \%$ & 1,300 & $9 \%$ & $4 \%$ & 14,230 & $100 \%$ & $46 \%$ \\
\hline Southern California & 9,557 & 26,864 & 14,490 & $152 \%$ & $54 \%$ & 2,300 & $24 \%$ & $9 \%$ & 1,300 & $14 \%$ & $5 \%$ & 18,090 & $189 \%$ & $67 \%$ \\
\hline Utah & 263 & 274 & 2,730 & $121 \%$ & $38 \%$ & 300 & $13 \%$ & $4 \%$ & 200 & $9 \%$ & $3 \%$ & 3,230 & $143 \%$ & $44 \%$ \\
\hline Out of Foo & 46,328 & 119,696 & 45,450 & $98 \%$ & $38 \%$ & 3,500 & $8 \%$ & $3 \%$ & 4,000 & $9 \%$ & $3 \%$ & 52,950 & $114 \%$ & $44 \%$ \\
\hline Total & 67,577 & 177,783 & 75,390 & $112 \%$ & $42 \%$ & 6,400 & $9 \%$ & $4 \%$ & 6,900 & $10 \%$ & $4 \%$ & 88,690 & $131 \%$ & $50 \%$ \\
\hline
\end{tabular}


Table A- 7 Local Priority Scenario, 10\% Wind In Footprint - Energy Summary

\begin{tabular}{|c|c|c|c|c|c|c|c|c|}
\hline \multirow[b]{2}{*}{ Areas } & \multirow{2}{*}{\begin{tabular}{|c|} 
Load \\
Energy \\
(GWh) \\
\end{tabular}} & \multicolumn{2}{|r|}{ Wind } & \multicolumn{2}{|c|}{ CSP with Storage } & \multicolumn{2}{|r|}{ PV } & \multirow{2}{*}{$\begin{array}{c}\begin{array}{c}\text { Total } \\
\text { Renewable }\end{array} \\
\begin{array}{c}\text { Energy } \\
\text { (GWh) }\end{array}\end{array}$} \\
\hline & & $\begin{array}{l}\text { Energy } \\
\text { (GWh) } \\
\end{array}$ & $\begin{array}{c}\text { \# Sites } \\
\text { (Used/Available) }\end{array}$ & $\begin{array}{l}\text { Energy } \\
\text { (GWh) }\end{array}$ & $\begin{array}{c}\text { \# Sites } \\
\text { (Used/Available) }\end{array}$ & $\begin{array}{l}\text { Energy } \\
\text { (GWh) }\end{array}$ & $\begin{array}{c}\text { \# Sites } \\
\text { (Used/Available) }\end{array}$ & \\
\hline Arizona & 99,437 & 8,048 & $95 / 1091$ & 760 & $2 / 38$ & 318 & $2 / 16$ & 9,126 \\
\hline Colorado East & 61,372 & 6,801 & $73 / 1673$ & 670 & $2 / 58$ & 160 & $1 / 13$ & 7,631 \\
\hline Colorado West & 8,708 & 665 & $7 / 68$ & 0 & $0 / 8$ & 0 & $0 / 11$ & 665 \\
\hline New Mexico & 31,260 & 4,162 & 45 / 3062 & 359 & $1 / 35$ & 155 & $1 / 19$ & 4,676 \\
\hline Nevada & 57,505 & 3,497 & $45 / 1591$ & 370 & $1 / 45$ & 160 & $1 / 10$ & 4,027 \\
\hline Wyoming & 27,697 & 5,753 & 55 / 8912 & 0 & $0 / 0$ & 142 & $1 / 10$ & 5,895 \\
\hline In Footprint & 285,979 & 28,926 & $320 / 16397$ & 2,159 & $6 / 184$ & 935 & $6 / 79$ & 32,020 \\
\hline
\end{tabular}

\begin{tabular}{|l|c|c|c|c|c|c|c|c|}
\cline { 2 - 9 } \multicolumn{1}{c|}{} & Load & \multicolumn{2}{|c|}{ Wind } & \multicolumn{2}{c|}{ CSP with Storage } & \multicolumn{2}{c|}{ PV } & $\begin{array}{c}\text { Total } \\
\text { Renewable }\end{array}$ \\
\hline & $\begin{array}{c}\text { Energy } \\
\text { (GWh) }\end{array}$ & $\begin{array}{c}\text { Energy } \\
\text { (GWh) }\end{array}$ & $\begin{array}{c}\text { \# Sites } \\
\text { (Used/Available) }\end{array}$ & $\begin{array}{c}\text { Energy } \\
\text { (GWh) }\end{array}$ & $\begin{array}{c}\text { \# Sites } \\
\text { (Used/Available) })\end{array}$ & $\begin{array}{c}\text { Energy } \\
\text { (GWh) }\end{array}$ & $\begin{array}{c}\text { \# Sites } \\
\text { (Used/Available) }\end{array}$ & $\begin{array}{c}\text { Energy } \\
\text { (GWh) }\end{array}$ \\
\hline COB & 1,759 & 207 & $3 / 155$ & 294 & $1 / 22$ & 0 & $0 / 2$ & 501 \\
Idaho East & 6,907 & 1,052 & $22 / 185$ & 0 & $0 / 0$ & 142 & $1 / 8$ & 1,194 \\
Idaho Southwest & 17,962 & 1,816 & $25 / 448$ & 0 & $0 / 0$ & 132 & $1 / 5$ & 1,948 \\
Montana & 14,143 & 1,853 & $26 / 1194$ & 0 & $0 / 0$ & 0 & $0 / 14$ & 1,853 \\
Northern California & 128,935 & 12,916 & $187 / 472$ & 1,059 & $3 / 26$ & 428 & $3 / 30$ & 14,403 \\
Northwest & 178,359 & 17,856 & $218 / 3195$ & 0 & $0 / 0$ & 404 & $3 / 54$ & 18,260 \\
Southern California & 224,197 & 22,472 & $237 / 1916$ & 2,749 & $7 / 85$ & 655 & $4 / 39$ & 25,876 \\
Utah & 38,022 & 3,807 & $47 / 554$ & 339 & $1 / 24$ & 150 & $1 / 14$ & 4,296 \\
\hline Out of Footprint & $\mathbf{6 1 0 , 2 8 4}$ & $\mathbf{6 1 , 9 7 9}$ & $\mathbf{7 6 5 / 8 1 1 9}$ & $\mathbf{4 , 4 4 1}$ & $\mathbf{1 2 / 1 5 7}$ & $\mathbf{1 , 9 1 1}$ & $\mathbf{1 3 / 1 6 6}$ & $\mathbf{6 8 , 3 3 1}$ \\
$\mathbf{1 1 . 2} \%$
\end{tabular}


Table A- 8 Local Priority Scenario, 10\% Wind In Footprint - Power Summary

\begin{tabular}{|c|c|c|c|c|c|c|c|c|c|c|c|c|c|c|}
\hline \multirow[b]{3}{*}{ Areas } & \multicolumn{2}{|c|}{ Load } & \multicolumn{3}{|c|}{ Wind } & \multicolumn{3}{|c|}{ CSP with Storage } & \multicolumn{3}{|c|}{ PV } & \multicolumn{3}{|c|}{ Total Renewable } \\
\hline & \multirow{2}{*}{\begin{tabular}{|c|}
$\begin{array}{c}\text { Minimum } \\
(\mathrm{MW})\end{array}$ \\
\end{tabular}} & \multirow{2}{*}{$\begin{array}{c}\text { Maximum } \\
(\mathrm{MW})\end{array}$} & \multirow{2}{*}{$\begin{array}{l}\text { Rating } \\
\text { (MW) }\end{array}$} & \multicolumn{2}{|c|}{$\begin{array}{l}\text { Penetration } \\
\end{array}$} & \multirow{2}{*}{$\begin{array}{l}\text { Rating } \\
\text { (MW) }\end{array}$} & \multicolumn{2}{|c|}{ Penetration } & \multirow{2}{*}{$\begin{array}{l}\text { Rating } \\
\text { (MW) }\end{array}$} & \multicolumn{2}{|c|}{ Penetration } & \multirow{2}{*}{$\begin{array}{l}\text { Rating } \\
\text { (MW) }\end{array}$} & \multicolumn{2}{|c|}{ Penetration } \\
\hline & & & & $\%$ Min & \%Max & & $\%$ Min & $\% \operatorname{Max}$ & & $\%$ Min & $\% \operatorname{Max}$ & & $\% \operatorname{Min}$ & $\% \operatorname{Max}$ \\
\hline Arizona & 6,995 & 23,051 & 2,850 & $41 \%$ & $12 \%$ & 200 & $3 \%$ & $1 \%$ & 200 & $3 \%$ & $1 \%$ & 3,250 & $46 \%$ & $14 \%$ \\
\hline Colorado East & 4,493 & 11,589 & 2,190 & $49 \%$ & $19 \%$ & 200 & $4 \%$ & $2 \%$ & 100 & $2 \%$ & $1 \%$ & 2,490 & $55 \%$ & $21 \%$ \\
\hline Colorado West & 712 & 1,526 & 210 & $30 \%$ & $14 \%$ & 0 & $0 \%$ & $0 \%$ & 0 & $0 \%$ & $0 \%$ & 210 & $30 \%$ & $14 \%$ \\
\hline New Mexico & 2,571 & 5,320 & 1,350 & $53 \%$ & $25 \%$ & 100 & $4 \%$ & $2 \%$ & 100 & $4 \%$ & $2 \%$ & 1,550 & $60 \%$ & $29 \%$ \\
\hline Nevada & 3,863 & 12,584 & 1,350 & $35 \%$ & $11 \%$ & 100 & $3 \%$ & $1 \%$ & 100 & $3 \%$ & $1 \%$ & 1,550 & $40 \%$ & $12 \%$ \\
\hline Wyoming & 2,369 & 4,016 & 1,650 & $70 \%$ & $41 \%$ & 0 & $0 \%$ & $0 \%$ & 100 & $4 \%$ & $2 \%$ & 1,750 & $74 \%$ & $44 \%$ \\
\hline In Footprint & 21,249 & 58,087 & 9,600 & $45 \%$ & $17 \%$ & 600 & $3 \%$ & $1 \%$ & 600 & $3 \%$ & $1 \%$ & 10,800 & $51 \%$ & $19 \%$ \\
\hline
\end{tabular}

\begin{tabular}{|c|c|c|c|c|c|c|c|c|c|c|c|c|c|c|}
\hline \multirow[b]{3}{*}{ Areas } & \multicolumn{2}{|c|}{ Load } & \multicolumn{3}{|c|}{ Wind } & \multicolumn{3}{|c|}{ CSP with Storage } & \multicolumn{3}{|c|}{ PV } & \multicolumn{3}{|c|}{ Total Renewable } \\
\hline & \multirow{2}{*}{$\begin{array}{l}\text { Minimum } \\
\text { (MW) }\end{array}$} & \multirow{2}{*}{$\begin{array}{l}\text { Maximum } \\
\text { (MW) }\end{array}$} & \multirow{2}{*}{$\begin{array}{l}\text { Rating } \\
\text { (MW) }\end{array}$} & \multicolumn{2}{|c|}{ Penetration } & \multirow{2}{*}{$\begin{array}{l}\text { Rating } \\
\text { (MW) }\end{array}$} & \multicolumn{2}{|c|}{ Penetration } & \multirow{2}{*}{\begin{tabular}{|l|} 
Rating \\
(MW)
\end{tabular}} & \multicolumn{2}{|c|}{ Penetration } & \multirow{2}{*}{$\begin{array}{l}\text { Rating } \\
\text { (MW) }\end{array}$} & \multicolumn{2}{|c|}{ Penetration } \\
\hline & & & & $\%$ Min & \%Max & & $\%$ Min & $\% \operatorname{Max}$ & & $\% \operatorname{Min}$ & $\% \operatorname{Max}$ & & $\%$ Min & $\% \operatorname{Max}$ \\
\hline $\mathrm{COB}$ & 138 & 294 & 90 & $65 \%$ & $31 \%$ & 100 & $73 \%$ & $34 \%$ & 0 & $0 \%$ & $0 \%$ & 190 & $138 \%$ & $65 \%$ \\
\hline Idaho East & 460 & 1,365 & 660 & $144 \%$ & $48 \%$ & 0 & $0 \%$ & $0 \%$ & 100 & $22 \%$ & $7 \%$ & 760 & $\%$ & $56 \%$ \\
\hline Idaho Southwest & 1,188 & 3,592 & 750 & $63 \%$ & $21 \%$ & 0 & $0 \%$ & $0 \%$ & 100 & $8 \%$ & $3 \%$ & 850 & $\%$ & $24 \%$ \\
\hline Montana & 49 & 2,337 & 780 & $68 \%$ & $33 \%$ & 0 & $0 \%$ & $0 \%$ & 0 & $0 \%$ & $0 \%$ & 780 & $68 \%$ & $33 \%$ \\
\hline Northern California & 10,297 & 28,319 & 5,610 & $54 \%$ & $20 \%$ & 300 & $3 \%$ & $1 \%$ & 300 & $3 \%$ & $1 \%$ & 6,210 & $60 \%$ & $22 \%$ \\
\hline North & 14,278 & 30,953 & 6,540 & $46 \%$ & $21 \%$ & 0 & $0 \%$ & $0 \%$ & 300 & $2 \%$ & $1 \%$ & 6,840 & $48 \%$ & $22 \%$ \\
\hline Southern California & 9,557 & 26,864 & 7,110 & $74 \%$ & $26 \%$ & 700 & $7 \%$ & $3 \%$ & 400 & $4 \%$ & $1 \%$ & 8,210 & $86 \%$ & $31 \%$ \\
\hline Utah & 2,263 & 7,274 & 1,410 & $62 \%$ & $19 \%$ & 100 & $4 \%$ & $1 \%$ & 100 & $4 \%$ & $1 \%$ & 1,610 & $71 \%$ & $22 \%$ \\
\hline Out of Footprint & 46,328 & 119,696 & 22,950 & $50 \%$ & $19 \%$ & 1,200 & $3 \%$ & $1 \%$ & 1,300 & $3 \%$ & $1 \%$ & 25,450 & $55 \%$ & $21 \%$ \\
\hline
\end{tabular}


Table A- 9 Local Priority Scenario, 20\% Wind In Footprint - Energy Summary

\begin{tabular}{|c|c|c|c|c|c|c|c|c|}
\hline \multirow[b]{2}{*}{ Areas } & \multirow{2}{*}{$\begin{array}{l}\text { Load } \\
\text { Energy } \\
\text { (GWh) }\end{array}$} & \multicolumn{2}{|r|}{ Wind } & \multicolumn{2}{|c|}{ CSP with Storage } & \multicolumn{2}{|r|}{ PV } & \multirow{2}{*}{$\begin{array}{c}\begin{array}{c}\text { Total } \\
\text { Renewable }\end{array} \\
\begin{array}{c}\text { Energy } \\
\text { (GWh) }\end{array}\end{array}$} \\
\hline & & $\begin{array}{l}\text { Energy } \\
\text { (GWh) }\end{array}$ & $\begin{array}{c}\text { \# Sites } \\
\text { (Used/Available) }\end{array}$ & $\begin{array}{l}\text { Energy } \\
\text { (GWh) }\end{array}$ & $\begin{array}{c}\text { \# Sites } \\
\text { (Used/Available) }\end{array}$ & $\begin{array}{l}\text { Energy } \\
\text { (GWh) }\end{array}$ & $\begin{array}{c}\text { \# Sites } \\
\text { (Used/Available) }\end{array}$ & \\
\hline Arizona & \begin{tabular}{|l|}
99,437 \\
\end{tabular} & 14,569 & $175 / 1091$ & 2,254 & $6 / 38$ & 933 & $6 / 16$ & 17,756 \\
\hline Colorado East & 61,372 & 12,683 & $129 / 1673$ & 1,304 & $4 / 58$ & 597 & $4 / 13$ & 14,584 \\
\hline Colorado West & 8,708 & 1,386 & $15 / 68$ & 283 & $1 / 8$ & 151 & $1 / 11$ & 1,820 \\
\hline New Mexico & 31,260 & 6,942 & $70 / 3062$ & 714 & $2 / 35$ & 315 & $2 / 19$ & 7,971 \\
\hline Nevada & 57,505 & 6,580 & $83 / 1591$ & 1,457 & $4 / 45$ & 468 & $3 / 10$ & 8,505 \\
\hline Wyoming & 27,697 & 15,278 & 134 / 8912 & 0 & $0 / 0$ & 142 & $1 / 10$ & 15,420 \\
\hline In Footprint & 285,979 & 57,438 & $606 / 16397$ & 6,012 & $17 / 184$ & 2,606 & $17 / 79$ & 66,056 \\
\hline
\end{tabular}

$23.1 \%$

\begin{tabular}{|l|c|c|c|c|c|c|c|c|}
\cline { 2 - 9 } \multicolumn{1}{c|}{} & Load & \multicolumn{2}{|c|}{ Wind } & \multicolumn{2}{c|}{ CSP with Storage } & \multicolumn{2}{c|}{ Total } & $\begin{array}{c}\text { Tot } \\
\text { Renewable }\end{array}$ \\
\hline & $\begin{array}{c}\text { Energy } \\
\text { (GWh) }\end{array}$ & $\begin{array}{c}\text { Energy } \\
\text { (GWh) }\end{array}$ & $\begin{array}{c}\text { \# Sites } \\
\text { (Used/Available) }\end{array}$ & $\begin{array}{c}\text { Energy } \\
\text { (GWh) }\end{array}$ & $\begin{array}{c}\text { \# Sites } \\
\text { (Used/Available) }\end{array}$ & $\begin{array}{c}\text { Energy } \\
\text { (GWh) }\end{array}$ & $\begin{array}{c}\text { \# Sites } \\
\text { (Used/Available) }\end{array}$ & $\begin{array}{c}\text { Energy } \\
\text { (GWh) }\end{array}$ \\
\hline Areas & 1,759 & 207 & $3 / 155$ & 294 & $1 / 22$ & 0 & $0 / 2$ & 501 \\
Idaho East & 6,907 & 1,052 & $22 / 185$ & 0 & $0 / 0$ & 142 & $1 / 8$ & 1,194 \\
Idaho Southwest & 17,962 & 1,816 & $25 / 448$ & 0 & $0 / 0$ & 132 & $1 / 5$ & 1,948 \\
Montana & 14,143 & 1,853 & $26 / 1194$ & 0 & $0 / 0$ & 0 & $0 / 14$ & 1,853 \\
Northern California & 128,935 & 12,916 & $187 / 472$ & 1,059 & $3 / 26$ & 428 & $3 / 30$ & 14,403 \\
Northwest & 178,359 & 17,856 & $218 / 3195$ & 0 & $0 / 0$ & 404 & $3 / 54$ & 18,260 \\
Southern California & 224,197 & 22,472 & $237 / 1916$ & 2,749 & $7 / 85$ & 655 & $4 / 39$ & 25,876 \\
Utah & $\mathbf{G}, 022$ & 3,807 & $47 / 554$ & 339 & $1 / 24$ & 150 & $1 / 14$ & 4,296 \\
\hline Out of Footprint & $\mathbf{6 1 0 , 2 8 4}$ & $\mathbf{6 1 , 9 7 9}$ & $\mathbf{7 6 5 / 8 1 1 9}$ & $\mathbf{4 , 4 4 1}$ & $\mathbf{1 2 / 1 5 7}$ & $\mathbf{1 , 9 1 1}$ & $\mathbf{1 3 / 1 6 6}$ & $\mathbf{6 8 , 3 3 1}$ \\
\hline
\end{tabular}


Table A- 10 Local Priority Scenario, 20\% Wind In Footprint - Power Summary

\begin{tabular}{|c|c|c|c|c|c|c|c|c|c|c|c|c|c|c|}
\hline \multirow[b]{3}{*}{ Areas } & \multicolumn{2}{|c|}{ Load } & \multicolumn{3}{|c|}{ Wind } & \multicolumn{3}{|c|}{ CSP with Storage } & \multicolumn{3}{|c|}{ PV } & \multicolumn{3}{|c|}{ Total Renewable } \\
\hline & \multirow{2}{*}{$\begin{array}{c}\text { Minimum } \\
(\mathrm{MW})\end{array}$} & \multirow{2}{*}{$\begin{array}{c}\text { Maximum } \\
\text { (MW) }\end{array}$} & \multirow{2}{*}{$\begin{array}{c}\text { Rating } \\
\text { (MW) }\end{array}$} & \multicolumn{2}{|c|}{ Penetration } & \multirow{2}{*}{$\begin{array}{c}\text { Rating } \\
\text { (MW) }\end{array}$} & \multicolumn{2}{|c|}{ Penetration } & \multirow{2}{*}{$\begin{array}{c}\text { Rating } \\
\text { (MW) }\end{array}$} & \multicolumn{2}{|c|}{ Penetration } & \multirow{2}{*}{$\begin{array}{c}\text { Rating } \\
\text { (MW) }\end{array}$} & \multicolumn{2}{|c|}{ Penetration } \\
\hline & & & & $\%$ Min & \%Max & & $\%$ Min & $\%$ Max & & $\%$ Min & $\%$ Max & & $\%$ Min & $\%$ Max \\
\hline Arizona & 6,995 & 23,051 & 5,250 & $75 \%$ & $23 \%$ & 600 & $9 \%$ & $3 \%$ & 600 & $9 \%$ & $3 \%$ & 6,450 & $92 \%$ & $28 \%$ \\
\hline Colorado & 4,493 & 11,589 & 3,870 & $86 \%$ & $33 \%$ & 400 & $9 \%$ & $3 \%$ & 400 & $9 \%$ & $3 \%$ & 4,670 & $104 \%$ & $40 \%$ \\
\hline Colc & 712 & & 450 & $63 \%$ & $29 \%$ & 100 & $14 \%$ & $7 \%$ & 100 & $14 \%$ & $7 \%$ & 650 & $91 \%$ & $43 \%$ \\
\hline New Mexico & 2,571 & 5,320 & 2,100 & $82 \%$ & $39 \%$ & 200 & $8 \%$ & $4 \%$ & 200 & $8 \%$ & $4 \%$ & 2,500 & $97 \%$ & $47 \%$ \\
\hline $\mathrm{Ne}$ & 3,863 & 12,584 & 2,490 & $64 \%$ & $20 \%$ & 400 & $10 \%$ & $3 \%$ & 300 & $8 \%$ & $2 \%$ & 3,190 & $83 \%$ & $25 \%$ \\
\hline Wyoming & 2,369 & 4,016 & 4,020 & $170 \%$ & $100 \%$ & 0 & $0 \%$ & $0 \%$ & 100 & $4 \%$ & $2 \%$ & 4,120 & $174 \%$ & $103 \%$ \\
\hline In Footprint & 21,249 & 58,087 & 18,180 & $86 \%$ & $31 \%$ & 1,700 & $8 \%$ & $3 \%$ & 1,700 & $8 \%$ & $3 \%$ & 21,580 & $102 \%$ & $37 \%$ \\
\hline
\end{tabular}

\begin{tabular}{|c|c|c|c|c|c|c|c|c|c|c|c|c|c|c|}
\hline \multirow[b]{3}{*}{ Areas } & \multicolumn{2}{|c|}{ Load } & \multicolumn{3}{|c|}{ Wind } & \multicolumn{3}{|c|}{ CSP with Storage } & \multicolumn{3}{|c|}{ PV } & \multicolumn{3}{|c|}{ Total Renewable } \\
\hline & \multirow{2}{*}{$\begin{array}{c}\text { Minimum } \\
(\mathrm{MW})\end{array}$} & \multirow{2}{*}{$\begin{array}{c}\text { Maximum } \\
\text { (MW) }\end{array}$} & \multirow{2}{*}{$\begin{array}{c}\text { Rating } \\
\text { (MW) }\end{array}$} & \multicolumn{2}{|c|}{ Penetration } & \multirow{2}{*}{$\begin{array}{c}\text { Rating } \\
\text { (MW) }\end{array}$} & \multicolumn{2}{|c|}{ Penetration } & \multirow{2}{*}{$\begin{array}{c}\text { Rating } \\
\text { (MW) }\end{array}$} & \multicolumn{2}{|c|}{ Penetration } & \multirow{2}{*}{$\begin{array}{c}\text { Rating } \\
\text { (MW) }\end{array}$} & \multicolumn{2}{|c|}{ Penetration } \\
\hline & & & & $\%$ Min & \%Max & & $\%$ Min & $\% \operatorname{Max}$ & & $\%$ Min & $\% \operatorname{Max}$ & & $\%$ Min & $\% \operatorname{Max}$ \\
\hline $\mathrm{COB}$ & 138 & 294 & 90 & $65 \%$ & $31 \%$ & 100 & $73 \%$ & $34 \%$ & 0 & $0 \%$ & $0 \%$ & 190 & $138 \%$ & $65 \%$ \\
\hline Idaho & 460 & 1,365 & 660 & $144 \%$ & $48 \%$ & 0 & $0 \%$ & $0 \%$ & 100 & $22 \%$ & $7 \%$ & 760 & $165 \%$ & $56 \%$ \\
\hline Southwest & & & 750 & $63 \%$ & $21 \%$ & 0 & $0 \%$ & $0 \%$ & 100 & $8 \%$ & $3 \%$ & 850 & $\%$ & $24 \%$ \\
\hline Montana & 1,149 & 2,337 & 780 & $68 \%$ & $33 \%$ & 0 & $0 \%$ & $0 \%$ & 0 & $0 \%$ & $0 \%$ & 780 & $68 \%$ & $33 \%$ \\
\hline Northern California & 10,297 & 28,319 & 5,610 & $54 \%$ & $20 \%$ & 300 & $3 \%$ & $1 \%$ & 300 & $3 \%$ & $1 \%$ & 6,210 & $60 \%$ & $22 \%$ \\
\hline Northwest & 14,278 & 30,953 & 6,540 & $46 \%$ & $21 \%$ & 0 & $0 \%$ & $0 \%$ & 300 & $2 \%$ & $1 \%$ & 6,840 & $48 \%$ & $22 \%$ \\
\hline Southern California & 9,557 & 26,864 & 7,110 & $74 \%$ & $26 \%$ & 700 & $7 \%$ & $3 \%$ & 400 & $4 \%$ & $1 \%$ & 8,210 & $86 \%$ & $31 \%$ \\
\hline Utah & 2,263 & 7,274 & 1,410 & $62 \%$ & $19 \%$ & 100 & $4 \%$ & $1 \%$ & 100 & $4 \%$ & $1 \%$ & 1,610 & $71 \%$ & $22 \%$ \\
\hline Out of Footprint & 46,328 & 119,696 & 22,950 & $50 \%$ & $19 \%$ & 1,200 & $3 \%$ & $1 \%$ & 1,300 & $3 \%$ & $1 \%$ & 25,450 & $55 \%$ & $21 \%$ \\
\hline
\end{tabular}


Table A- 11 Local Priority Scenario, 30\% Wind In Footprint - Energy Summary

\begin{tabular}{|c|c|c|c|c|c|c|c|c|}
\hline \multirow[b]{2}{*}{ Area } & \multirow{2}{*}{$\begin{array}{c}\text { Load } \\
\text { Energy } \\
\text { (GWh) }\end{array}$} & \multicolumn{2}{|r|}{ Wind } & \multicolumn{2}{|c|}{ CSP with Storage } & \multicolumn{2}{|r|}{ PV } & \multirow{2}{*}{$\begin{array}{c}\text { Total } \\
\text { Renewable } \\
\text { Energy } \\
\text { (GWh) }\end{array}$} \\
\hline & & $\begin{array}{l}\text { Energy } \\
\text { (GWh) }\end{array}$ & $\begin{array}{c}\text { \# Sites } \\
\text { (Used/Available) }\end{array}$ & $\begin{array}{l}\text { Energy } \\
\text { (GWh) }\end{array}$ & $\begin{array}{c}\text { \# Sites } \\
\text { (Used/Available) }\end{array}$ & $\begin{array}{l}\text { Energy } \\
\text { (GWh) }\end{array}$ & $\begin{array}{c}\text { \# Sites } \\
\text { (Used/Available) }\end{array}$ & \\
\hline Arizona & 99,437 & 21,138 & 257 / 1091 & 3,735 & $10 / 38$ & 1,548 & $10 / 16$ & 26,421 \\
\hline Colorado East & 61,372 & 15,320 & $155 / 1673$ & 2,252 & $7 / 58$ & 1,038 & $7 / 13$ & 18,610 \\
\hline Colorado West & 8,708 & 1,736 & 19 / 68 & 564 & $2 / 8$ & 151 & $1 / 11$ & 2,451 \\
\hline New Mexico & 31,260 & 10,047 & $99 / 3062$ & 1,421 & $4 / 35$ & 473 & $3 / 19$ & 11,941 \\
\hline Nevada & 57,505 & 9,101 & 115 / 1591 & 2,161 & $6 / 45$ & 773 & $5 / 10$ & 12,035 \\
\hline Wyoming & 27,697 & 28,716 & 247 / 8912 & 0 & $0 / 0$ & 420 & $3 / 10$ & 29,136 \\
\hline In Footprint & 285,979 & 86,058 & $892 / 16397$ & 10,133 & $29 / 184$ & 4,403 & $29 / 79$ & 100,594 \\
\hline
\end{tabular}

\begin{tabular}{|c|c|c|c|c|c|c|c|c|}
\hline \multirow[b]{2}{*}{ Area } & \multirow{2}{*}{$\begin{array}{c}\text { Load } \\
\text { Energy } \\
\text { (GWh) }\end{array}$} & \multicolumn{2}{|r|}{ Wind } & \multicolumn{2}{|c|}{ CSP with Storage } & \multicolumn{2}{|r|}{ PV } & \multirow{2}{*}{$\begin{array}{c}\text { Total } \\
\text { Renewable } \\
\text { Energy } \\
\text { (GWh) }\end{array}$} \\
\hline & & $\begin{array}{l}\text { Energy } \\
\text { (GWh) }\end{array}$ & $\begin{array}{c}\text { \# Sites } \\
\text { (Used/Available) }\end{array}$ & $\begin{array}{l}\text { Energy } \\
\text { (GWh) }\end{array}$ & $\begin{array}{c}\text { \# Sites } \\
\text { (Used/Available) }\end{array}$ & $\begin{array}{l}\text { Energy } \\
\text { (GWh) }\end{array}$ & $\begin{array}{c}\text { \# Sites } \\
\text { (Used/Available) }\end{array}$ & \\
\hline $\mathrm{COB}$ & 1,759 & 394 & $6 / 155$ & 294 & $1 / 22$ & 142 & $1 / 2$ & 830 \\
\hline Idaho East & 6,907 & 1,406 & $26 / 185$ & 0 & $0 / 0$ & 142 & $1 / 8$ & 1,548 \\
\hline Idaho Southwest & 17,962 & 3,658 & $50 / 448$ & 0 & $0 / 0$ & 132 & $1 / 5$ & 3,790 \\
\hline Montana & 14,143 & 2,873 & $35 / 1194$ & 0 & $0 / 0$ & 127 & $1 / 14$ & 3,000 \\
\hline Northern California & 128,935 & 25,812 & $393 / 472$ & 2,726 & $8 / 26$ & 1,117 & $8 / 30$ & 29,655 \\
\hline Northwest & 178,359 & 35,733 & $431 / 3195$ & 0 & $0 / 0$ & 1,645 & $13 / 54$ & 37,378 \\
\hline Southern California & 224,197 & 44,890 & 483 / 1916 & 8,957 & $23 / 85$ & 2,050 & $13 / 39$ & 55,897 \\
\hline Utah & 38,022 & 7,658 & $91 / 554$ & 937 & $3 / 24$ & 303 & $2 / 14$ & 8,898 \\
\hline Out of Footprint & 610,284 & 122,424 & $1515 / 8119$ & 12,914 & $35 / 157$ & 5,658 & $40 / 166$ & 140,996 \\
\hline
\end{tabular}


Table A- 12 Local Priority Scenario, 30\% Wind In Footprint - Power Summary

\begin{tabular}{|c|c|c|c|c|c|c|c|c|c|c|c|c|c|c|}
\hline \multirow[b]{3}{*}{ Area } & \multicolumn{2}{|c|}{ Load } & \multicolumn{3}{|c|}{ Wind } & \multicolumn{3}{|c|}{ CSP with Storage } & \multicolumn{3}{|c|}{ PV } & \multicolumn{3}{|c|}{ Total Renewable } \\
\hline & \multirow{2}{*}{$\begin{array}{l}\text { Minimum } \\
\text { (MW) }\end{array}$} & \multirow{2}{*}{$\begin{array}{l}\text { Maximum } \\
\text { (MW) }\end{array}$} & \multirow{2}{*}{$\begin{array}{l}\text { Rating } \\
\text { (MW) }\end{array}$} & \multicolumn{2}{|c|}{ Penetration } & \multirow{2}{*}{$\begin{array}{l}\text { Rating } \\
\text { (MW) }\end{array}$} & \multicolumn{2}{|c|}{ Penetration } & \multirow{2}{*}{$\begin{array}{l}\text { Rating } \\
\text { (MW) }\end{array}$} & \multicolumn{2}{|c|}{ Penetration } & \multirow{2}{*}{$\begin{array}{l}\text { Rating } \\
\text { (MW) }\end{array}$} & \multicolumn{2}{|c|}{ Penetration } \\
\hline & & & & $\% \operatorname{Min}$ & \%Max & & $\% \operatorname{Min}$ & $\% \operatorname{Max}$ & & $\% \operatorname{Min}$ & $\% \operatorname{Max}$ & & $\% \operatorname{Min}$ & $\% \operatorname{Max}$ \\
\hline Arizona & 6,995 & 23,051 & 7,710 & $110 \%$ & $33 \%$ & 1,000 & $14 \%$ & $4 \%$ & 1,000 & $14 \%$ & $4 \%$ & 9,710 & $139 \%$ & $42 \%$ \\
\hline & 93 & 9 & 4,650 & $103 \%$ & $40 \%$ & 700 & $16 \%$ & $6 \%$ & 70 & $16 \%$ & $6 \%$ & 6,050 & 13 & $52 \%$ \\
\hline 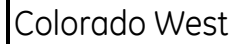 & 712 & 1 & 570 & $80 \%$ & $37 \%$ & 200 & $28 \%$ & $13 \%$ & 100 & $14 \%$ & $7 \%$ & 870 & $122 \%$ & $57 \%$ \\
\hline xico & 2,571 & 5,320 & 2,970 & $116 \%$ & $56 \%$ & 400 & $16 \%$ & $8 \%$ & 300 & $12 \%$ & $6 \%$ & 3,670 & $143 \%$ & $69 \%$ \\
\hline & 3,863 & 12,584 & 3,450 & $89 \%$ & $27 \%$ & 600 & $16 \%$ & $5 \%$ & 500 & $13 \%$ & $4 \%$ & 4,550 & $118 \%$ & $36 \%$ \\
\hline Wyoming & 69 & 4,016 & 7,410 & $313 \%$ & $184 \%$ & 0 & $0 \%$ & $0 \%$ & 300 & $13 \%$ & $7 \%$ & 7,710 & $325 \%$ & $192 \%$ \\
\hline In Footprint & 21,249 & 58,087 & 26,760 & $126 \%$ & $46 \%$ & 2,900 & $14 \%$ & $5 \%$ & 2,900 & $14 \%$ & $5 \%$ & 32,560 & $153 \%$ & $56 \%$ \\
\hline
\end{tabular}

\begin{tabular}{|c|c|c|c|c|c|c|c|c|c|c|c|c|c|c|}
\hline \multirow[b]{3}{*}{ Area } & \multicolumn{2}{|c|}{ Load } & \multicolumn{3}{|c|}{ Wind } & \multicolumn{3}{|c|}{ CSP with Storage } & \multicolumn{3}{|c|}{ PV } & \multicolumn{3}{|c|}{ Total Renewable } \\
\hline & \multirow{2}{*}{$\begin{array}{l}\text { Minimum } \\
\text { (MW) }\end{array}$} & \multirow{2}{*}{$\begin{array}{l}\text { Maximum } \\
\text { (MW) }\end{array}$} & \multirow{2}{*}{$\begin{array}{l}\text { Rating } \\
\text { (MW) }\end{array}$} & \multicolumn{2}{|c|}{ Penetration } & \multirow{2}{*}{$\begin{array}{c}\text { Rating } \\
\text { (MW) }\end{array}$} & \multicolumn{2}{|c|}{ Penetration } & \multirow{2}{*}{$\begin{array}{l}\text { Rating } \\
\text { (MW) }\end{array}$} & \multicolumn{2}{|c|}{ Penetration } & \multirow{2}{*}{$\begin{array}{l}\text { Rating } \\
\text { (MW) }\end{array}$} & \multicolumn{2}{|c|}{ Penetration } \\
\hline & & & & $\% \operatorname{Min}$ & \%Max & & $\%$ Min & $\% \operatorname{Max}$ & & $\% \operatorname{Min}$ & $\% \operatorname{Max}$ & & $\% \operatorname{Min}$ & $\% \operatorname{Max}$ \\
\hline $\mathrm{COB}$ & 138 & 294 & 180 & $131 \%$ & $61 \%$ & 100 & $73 \%$ & $34 \%$ & 100 & $73 \%$ & $34 \%$ & 380 & $276 \%$ & $129 \%$ \\
\hline Idaho East & 460 & 1,365 & 780 & $170 \%$ & $57 \%$ & 0 & $0 \%$ & $0 \%$ & 100 & $22 \%$ & $7 \%$ & 880 & $191 \%$ & $64 \%$ \\
\hline Idaho Southwest & 1,188 & 3,592 & 1,500 & $126 \%$ & $42 \%$ & 0 & $0 \%$ & $0 \%$ & 100 & $8 \%$ & $3 \%$ & 1,600 & $135 \%$ & $45 \%$ \\
\hline Montana & 1,149 & 2,337 & 1,050 & $91 \%$ & $45 \%$ & 0 & $0 \%$ & $0 \%$ & 100 & $9 \%$ & $4 \%$ & 1,150 & $100 \%$ & $49 \%$ \\
\hline Northern California & 10,297 & 28,319 & 11,790 & $114 \%$ & $42 \%$ & 800 & $8 \%$ & $3 \%$ & 800 & $8 \%$ & $3 \%$ & 13,390 & $130 \%$ & $47 \%$ \\
\hline Northwest & 14,278 & 30,953 & 12,930 & $91 \%$ & $42 \%$ & 0 & $0 \%$ & $0 \%$ & 1,300 & $9 \%$ & $4 \%$ & 14,230 & $100 \%$ & $46 \%$ \\
\hline Southern California & 9,557 & 26,864 & 14,490 & $152 \%$ & $54 \%$ & 2,300 & $24 \%$ & $9 \%$ & 1,300 & $14 \%$ & $5 \%$ & 18,090 & $189 \%$ & $67 \%$ \\
\hline Utah & 2,263 & 7,274 & 2,730 & $121 \%$ & $38 \%$ & 300 & $13 \%$ & $4 \%$ & 200 & $9 \%$ & $3 \%$ & 3,230 & $143 \%$ & $44 \%$ \\
\hline Out of Footprint & 46,328 & 119,696 & 45,450 & $98 \%$ & $38 \%$ & 3,500 & $8 \%$ & $3 \%$ & 4,000 & $9 \%$ & $3 \%$ & 52,950 & $114 \%$ & $44 \%$ \\
\hline
\end{tabular}


Table A- 13 Mega-Project Scenario, 10\% Wind In Footprint - Energy Summary

\begin{tabular}{|l|c|c|c|c|c|c|c|c|}
\cline { 2 - 9 } \multicolumn{1}{c|}{} & Load & \multicolumn{2}{|c|}{ Wind } & \multicolumn{2}{c|}{ CSP with Storage } & \multicolumn{2}{c|}{ Total } \\
Renewable
\end{tabular}

\begin{tabular}{|c|c|c|c|c|c|c|c|c|}
\hline \multirow[b]{2}{*}{ Areas } & \multirow{2}{*}{$\begin{array}{l}\text { Load } \\
\text { Energy } \\
\text { (GWh) }\end{array}$} & \multicolumn{2}{|r|}{ Wind } & \multicolumn{2}{|c|}{ CSP with Storage } & \multicolumn{2}{|r|}{ PV } & \multirow{2}{*}{$\begin{array}{c}\begin{array}{c}\text { Total } \\
\text { Renewable }\end{array} \\
\begin{array}{c}\text { Energy } \\
\text { (GWh) }\end{array}\end{array}$} \\
\hline & & $\begin{array}{l}\text { Energy } \\
\text { (GWh) }\end{array}$ & $\begin{array}{c}\text { \# Sites } \\
\text { (Used/Available) }\end{array}$ & $\begin{array}{l}\text { Energy } \\
\text { (GWh) }\end{array}$ & $\begin{array}{c}\text { \# Sites } \\
\text { (Used/Available) }\end{array}$ & $\begin{array}{l}\text { Energy } \\
\text { (GWh) }\end{array}$ & $\begin{array}{c}\text { \# Sites } \\
\text { (Used/Available) }\end{array}$ & \\
\hline $\mathrm{COB}$ & 1,759 & 207 & $3 / 155$ & 294 & $1 / 22$ & 0 & $0 / 2$ & 501 \\
\hline Idaho East & 6,907 & 1,052 & $22 / 185$ & 0 & $0 / 0$ & 142 & $1 / 8$ & 1,194 \\
\hline Idaho Southwest & 17,962 & 1,816 & $25 / 448$ & 0 & $0 / 0$ & 132 & $1 / 5$ & 1,948 \\
\hline Montana & 14,143 & 1,853 & $26 / 1194$ & 0 & $0 / 0$ & 0 & $0 / 14$ & 1,853 \\
\hline Northern California & 128,935 & 12,916 & 187 / 472 & 1,059 & $3 / 26$ & 428 & $3 / 30$ & 14,403 \\
\hline Northwest & 178,359 & 17,856 & $218 / 3195$ & 0 & $0 / 0$ & 404 & $3 / 54$ & 18,260 \\
\hline Southern California & 224,197 & 22,472 & $237 / 1916$ & 2,749 & $7 / 85$ & 655 & $4 / 39$ & 25,876 \\
\hline Utah & 38,022 & 3,807 & $47 / 554$ & 339 & $1 / 24$ & 150 & $1 / 14$ & 4,296 \\
\hline Out of Footprint & 610,284 & 61,979 & $765 / 8119$ & 4,441 & $12 / 157$ & 1,911 & $13 / 166$ & 68,331 \\
\hline
\end{tabular}


Table A- 14 Mega-Project Scenario, 10\% Wind In Footprint - Power Summary

\begin{tabular}{|c|c|c|c|c|c|c|c|c|c|c|c|c|c|c|}
\hline \multirow[b]{3}{*}{ Areas } & \multicolumn{2}{|c|}{ Load } & \multicolumn{3}{|c|}{ Wind } & \multicolumn{3}{|c|}{ CSP with Storage } & \multicolumn{3}{|c|}{$\mathbf{P V}$} & \multicolumn{3}{|c|}{ Total Renewable } \\
\hline & \multirow{2}{*}{$\begin{array}{c}\text { Minimum } \\
(\mathrm{MW})\end{array}$} & \multirow{2}{*}{$\begin{array}{l}\text { Maximum } \\
(\mathrm{MW})\end{array}$} & \multirow{2}{*}{\begin{tabular}{|l|} 
Rating \\
(MW)
\end{tabular}} & \multicolumn{2}{|c|}{ Penetration } & \multirow{2}{*}{$\begin{array}{l}\text { Rating } \\
\text { (MW) }\end{array}$} & \multicolumn{2}{|c|}{ Penetration } & \multirow{2}{*}{\begin{tabular}{|l|} 
Rating \\
(MW)
\end{tabular}} & \multicolumn{2}{|c|}{ Penetration } & \multirow{2}{*}{$\begin{array}{l}\text { Rating } \\
\text { (MW) }\end{array}$} & \multicolumn{2}{|c|}{ Penetration } \\
\hline & & & & $\% \operatorname{Min}$ & \%Max & & $\% \operatorname{Min}$ & $\% \operatorname{Max}$ & & $\% \operatorname{Min}$ & $\% \operatorname{Max}$ & & $\% \operatorname{Min}$ & $\% \operatorname{Max}$ \\
\hline Arizona & 6,995 & 23,051 & 810 & $12 \%$ & $4 \%$ & 300 & $4 \%$ & $1 \%$ & 100 & $1 \%$ & $0 \%$ & 1,210 & $17 \%$ & $5 \%$ \\
\hline Colorado East & 4,493 & 11,589 & 2,010 & $45 \%$ & $17 \%$ & 100 & $2 \%$ & $1 \%$ & 200 & $4 \%$ & $2 \%$ & 2,310 & $51 \%$ & $20 \%$ \\
\hline Colorado West & 712 & 1,526 & 60 & $8 \%$ & $4 \%$ & 0 & $0 \%$ & $0 \%$ & 0 & $0 \%$ & $0 \%$ & 60 & $8 \%$ & $4 \%$ \\
\hline New Mexico & 2,571 & 5,320 & 1,860 & $72 \%$ & $35 \%$ & 200 & $8 \%$ & $4 \%$ & 200 & $8 \%$ & $4 \%$ & 2,260 & $88 \%$ & $42 \%$ \\
\hline Nevada & 3,863 & 12,584 & 570 & $15 \%$ & $5 \%$ & 0 & $0 \%$ & $0 \%$ & 100 & $3 \%$ & $1 \%$ & 670 & $17 \%$ & $5 \%$ \\
\hline Wyoming & 2,369 & 4,016 & 3,390 & $143 \%$ & $84 \%$ & 0 & $0 \%$ & $0 \%$ & 0 & $0 \%$ & $0 \%$ & 3,390 & $143 \%$ & $84 \%$ \\
\hline In Footprint & 21,249 & 58,087 & 8,700 & $41 \%$ & $15 \%$ & 600 & $3 \%$ & $1 \%$ & 600 & $3 \%$ & $1 \%$ & 9,900 & $47 \%$ & $17 \%$ \\
\hline
\end{tabular}

\begin{tabular}{|c|c|c|c|c|c|c|c|c|c|c|c|c|c|c|}
\hline \multirow[b]{3}{*}{ Areas } & \multicolumn{2}{|c|}{ Load } & \multicolumn{3}{|c|}{ Wind } & \multicolumn{3}{|c|}{ CSP with Storage } & \multicolumn{3}{|c|}{ PV } & \multicolumn{3}{|c|}{ Total Renewable } \\
\hline & \multirow{2}{*}{$\begin{array}{l}\text { Minimum } \\
\text { (MW) }\end{array}$} & \multirow{2}{*}{$\begin{array}{l}\text { Maximum } \\
\text { (MW) }\end{array}$} & \multirow{2}{*}{$\begin{array}{l}\text { Rating } \\
\text { (MW) }\end{array}$} & \multicolumn{2}{|c|}{ Penetration } & \multirow{2}{*}{$\begin{array}{l}\text { Rating } \\
\text { (MW) }\end{array}$} & \multicolumn{2}{|c|}{ Penetration } & \multirow{2}{*}{$\begin{array}{l}\text { Rating } \\
\text { (MW) }\end{array}$} & \multicolumn{2}{|c|}{ Penetration } & \multirow{2}{*}{$\begin{array}{l}\text { Rating } \\
\text { (MW) }\end{array}$} & \multicolumn{2}{|c|}{ Penetration } \\
\hline & & & & $\%$ Min & \%Max & & $\% \operatorname{Min}$ & $\% \operatorname{Max}$ & & $\% \operatorname{Min}$ & $\% \operatorname{Max}$ & & $\% \operatorname{Min}$ & $\% \operatorname{Max}$ \\
\hline $\mathrm{COB}$ & 138 & 294 & 90 & $65 \%$ & $31 \%$ & 100 & $73 \%$ & $34 \%$ & 0 & $0 \%$ & $0 \%$ & 190 & $138 \%$ & $65 \%$ \\
\hline Idaho East & 460 & 1,365 & 660 & $144 \%$ & $48 \%$ & 0 & $0 \%$ & $0 \%$ & 100 & $22 \%$ & $7 \%$ & 760 & $165 \%$ & $56 \%$ \\
\hline Idaho Southwest & 1,188 & 3,592 & 750 & $63 \%$ & $21 \%$ & 0 & $0 \%$ & $0 \%$ & 100 & $8 \%$ & $3 \%$ & 850 & $72 \%$ & $24 \%$ \\
\hline Montana & 1,149 & 2,337 & 780 & $68 \%$ & $33 \%$ & 0 & $0 \%$ & $0 \%$ & 0 & $0 \%$ & $0 \%$ & 780 & $68 \%$ & $33 \%$ \\
\hline Northern California & 10,297 & 28,319 & 5,610 & $54 \%$ & $20 \%$ & 300 & $3 \%$ & $1 \%$ & 300 & $3 \%$ & $1 \%$ & 6,210 & $60 \%$ & $22 \%$ \\
\hline Northwest & 14,278 & 30,953 & 6,540 & $46 \%$ & $21 \%$ & 0 & $0 \%$ & $0 \%$ & 300 & $2 \%$ & $1 \%$ & 6,840 & $48 \%$ & $22 \%$ \\
\hline Southern California & 9,557 & 26,864 & 7,110 & $74 \%$ & $26 \%$ & 700 & $7 \%$ & $3 \%$ & 400 & $4 \%$ & $1 \%$ & 8,210 & $86 \%$ & $31 \%$ \\
\hline Utah & 2,263 & 7,274 & 1,410 & $62 \%$ & $19 \%$ & 100 & $4 \%$ & $1 \%$ & 100 & $4 \%$ & $1 \%$ & 1,610 & $71 \%$ & $22 \%$ \\
\hline Out of Footprint & 46,328 & 119,696 & 22,950 & $50 \%$ & $19 \%$ & 1,200 & $3 \%$ & $1 \%$ & 1,300 & $3 \%$ & $1 \%$ & 25,450 & $55 \%$ & $21 \%$ \\
\hline
\end{tabular}


Table A- 15 Mega-Project Scenario, 20\% Wind In Footprint - Energy Summary

\begin{tabular}{|l|c|c|c|c|c|c|c|c|}
\cline { 2 - 9 } \multicolumn{1}{c|}{} & Load & \multicolumn{2}{|c|}{ Wind } & \multicolumn{2}{c|}{ CSP with Storage } & \multicolumn{2}{c|}{ PV } & $\begin{array}{c}\text { Total } \\
\text { Renewable }\end{array}$ \\
\hline & $\begin{array}{c}\text { Energy } \\
\text { (GWh) }\end{array}$ & $\begin{array}{c}\text { Energy } \\
\text { (GWh) }\end{array}$ & $\begin{array}{c}\text { \# Sites } \\
\text { (Used/Available) }\end{array}$ & $\begin{array}{c}\text { Energy } \\
\text { (GWh) }\end{array}$ & $\begin{array}{c}\text { \# Sites } \\
\text { (Used/Available) }\end{array}$ & $\begin{array}{c}\text { Energy } \\
\text { (GWh) }\end{array}$ & $\begin{array}{c}\text { \# Sites } \\
\text { (Used/Available) }\end{array}$ & $\begin{array}{c}\text { Energy } \\
\text { (GWh) }\end{array}$ \\
\hline Areas & 99,437 & 3,645 & $42 / 1091$ & 4,065 & $11 / 38$ & 1,081 & $7 / 16$ & 8,791 \\
Arizona & 61,372 & 7,574 & $80 / 1673$ & 996 & $3 / 58$ & 160 & $1 / 13$ & 8,730 \\
Colorado East & 8,708 & 293 & $3 / 68$ & 0 & $0 / 8$ & 0 & $0 / 11$ & 293 \\
Nelorado West & 31,260 & 9,076 & $90 / 3062$ & 950 & $3 / 35$ & 1,046 & $7 / 19$ & 11,072 \\
Nevada & 57,505 & 2,594 & $34 / 1591$ & 0 & $0 / 45$ & 320 & $2 / 10$ & 2,914 \\
Wyoming & 27,697 & 34,256 & $293 / 8912$ & 0 & $0 / 0$ & 0 & $0 / 10$ & 34,256 \\
\hline In Footprint & $\mathbf{2 8 5 , 9 7 9}$ & $\mathbf{5 7 , 4 3 8}$ & $\mathbf{5 4 2 / 1 6 3 9 7}$ & $\mathbf{6 , 0 1 1}$ & $\mathbf{1 7 / 1 8 4}$ & $\mathbf{2 , 6 0 7}$ & $\mathbf{1 7 / 7 9}$ & $\mathbf{6 6 , 0 5 6}$ \\
\hline
\end{tabular}

$23.1 \%$

\begin{tabular}{|l|c|c|c|c|c|c|c|c|}
\cline { 2 - 9 } \multicolumn{1}{c|}{} & Load & \multicolumn{2}{|c|}{ Wind } & \multicolumn{2}{c|}{ CSP with Storage } & \multicolumn{2}{c|}{ PV } & $\begin{array}{c}\text { Total } \\
\text { Renewable }\end{array}$ \\
\hline & $\begin{array}{c}\text { Energy } \\
\text { (GWh) }\end{array}$ & $\begin{array}{c}\text { Energy } \\
\text { (GWh) }\end{array}$ & $\begin{array}{c}\text { \# Sites } \\
\text { (Used/Available) }\end{array}$ & $\begin{array}{c}\text { Energy } \\
\text { (GWh) }\end{array}$ & $\begin{array}{c}\text { \# Sites } \\
\text { (Used/Available) })\end{array}$ & $\begin{array}{c}\text { Energy } \\
\text { (GWh) }\end{array}$ & $\begin{array}{c}\text { \# Sites } \\
\text { (Used/Available) }\end{array}$ & $\begin{array}{c}\text { Energy } \\
\text { (GWh) }\end{array}$ \\
\hline Areas & 1,759 & 207 & $3 / 155$ & 294 & $1 / 22$ & 0 & $0 / 2$ & 501 \\
Idaho East & 6,907 & 1,052 & $22 / 185$ & 0 & $0 / 0$ & 142 & $1 / 8$ & 1,194 \\
Idaho Southwest & 17,962 & 1,816 & $25 / 448$ & 0 & $0 / 0$ & 132 & $1 / 5$ & 1,948 \\
Montana & 14,143 & 1,853 & $26 / 1194$ & 0 & $0 / 0$ & 0 & $0 / 14$ & 1,853 \\
Northern California & 128,935 & 12,916 & $187 / 472$ & 1,059 & $3 / 26$ & 428 & $3 / 30$ & 14,403 \\
Northwest & 178,359 & 17,856 & $218 / 3195$ & 0 & $0 / 0$ & 404 & $3 / 54$ & 18,260 \\
Southern California & 224,197 & 22,472 & $237 / 1916$ & 2,749 & $7 / 85$ & 655 & $4 / 39$ & 25,876 \\
Utah & 38,022 & 3,807 & $47 / 554$ & 339 & $1 / 24$ & 150 & $1 / 14$ & 4,296 \\
\hline Out of Footprint & $\mathbf{6 1 0 , 2 8 4}$ & $\mathbf{6 1 , 9 7 9}$ & $\mathbf{7 6 5 / 8 1 1 9}$ & $\mathbf{4 , 4 4 1}$ & $\mathbf{1 2 / 1 5 7}$ & $\mathbf{1 , 9 1 1}$ & $\mathbf{1 3 / 1 6 6}$ & $\mathbf{6 8 , 3 3 1}$ \\
\hline
\end{tabular}


Table A- 16 Mega-Project Scenario, 20\% Wind In Footprint - Power Summary

\begin{tabular}{|c|c|c|c|c|c|c|c|c|c|c|c|c|c|c|}
\hline \multirow[b]{3}{*}{ Areas } & \multicolumn{2}{|c|}{ Load } & \multicolumn{3}{|c|}{ Wind } & \multicolumn{3}{|c|}{ CSP with Storage } & \multicolumn{3}{|c|}{ PV } & \multicolumn{3}{|c|}{ Total Renewable } \\
\hline & \multirow{2}{*}{$\begin{array}{c}\text { Minimum } \\
\text { (MW) }\end{array}$} & \multirow{2}{*}{$\begin{array}{l}\text { Maximum } \\
(\mathrm{MW})\end{array}$} & \multirow{2}{*}{$\begin{array}{l}\text { Rating } \\
\text { (MW) }\end{array}$} & \multicolumn{2}{|c|}{ Penetration } & \multirow{2}{*}{$\begin{array}{l}\text { Rating } \\
\text { (MW) }\end{array}$} & \multicolumn{2}{|c|}{ Penetration } & \multirow{2}{*}{\begin{tabular}{|l|} 
Rating \\
(MW)
\end{tabular}} & \multicolumn{2}{|c|}{ Penetration } & \multirow{2}{*}{$\begin{array}{l}\text { Rating } \\
\text { (MW) }\end{array}$} & \multicolumn{2}{|c|}{ Penetration } \\
\hline & & & & \% Min & \%Max & & $\%$ Min & $\% \operatorname{Max}$ & & \% Min & $\% \operatorname{Max}$ & & $\%$ Min & $\% \operatorname{Max}$ \\
\hline Arizon & 6,995 & 23,051 & 1,260 & $18 \%$ & $5 \%$ & 1,100 & $16 \%$ & $5 \%$ & 700 & $10 \%$ & $3 \%$ & 3,060 & $44 \%$ & $13 \%$ \\
\hline C & 3 & 11,589 & 2,400 & 53 & 21 & 300 & 7 & $3 \%$ & 100 & 2 & 1 & 2,800 & $62 \%$ & $24 \%$ \\
\hline C & 712 & 6 & 90 & 13 & 6 & 0 & $0 \%$ & $0 \%$ & 0 & $0 \%$ & $0 \%$ & 90 & $13 \%$ & $6 \%$ \\
\hline xico & 2,571 & 0 & 2,700 & $105 \%$ & $51 \%$ & 300 & $12 \%$ & $6 \%$ & 700 & $27 \%$ & $13 \%$ & 3,700 & $144 \%$ & $70 \%$ \\
\hline $\mathrm{Nev}$ & 3,863 & 12,584 & 1,020 & $26 \%$ & $8 \%$ & 0 & $0 \%$ & $0 \%$ & 200 & $5 \%$ & $2 \%$ & 1,220 & $32 \%$ & $10 \%$ \\
\hline & 2,369 & 4,016 & 8,790 & $371 \%$ & $219 \%$ & 0 & $0 \%$ & $0 \%$ & 0 & $0 \%$ & $0 \%$ & 8,790 & $371 \%$ & $219 \%$ \\
\hline In Footprint & 21,249 & 58,087 & 16,260 & $77 \%$ & $28 \%$ & 1,700 & $8 \%$ & $3 \%$ & 1,700 & $8 \%$ & $3 \%$ & 19,660 & $93 \%$ & $34 \%$ \\
\hline
\end{tabular}

\begin{tabular}{|c|c|c|c|c|c|c|c|c|c|c|c|c|c|c|}
\hline \multirow[b]{3}{*}{ Areas } & \multicolumn{2}{|c|}{ Load } & \multicolumn{3}{|c|}{ Wind } & \multicolumn{3}{|c|}{ CSP with Storage } & \multicolumn{3}{|c|}{ PV } & \multicolumn{3}{|c|}{ Total Renewable } \\
\hline & \multirow{2}{*}{$\begin{array}{l}\text { Minimum } \\
\text { (MW) }\end{array}$} & \multirow{2}{*}{\begin{tabular}{|c|} 
Maximum \\
(MW)
\end{tabular}} & \multirow{2}{*}{$\begin{array}{l}\text { Rating } \\
\text { (MW) }\end{array}$} & \multicolumn{2}{|c|}{ Penetration } & \multirow{2}{*}{$\begin{array}{l}\text { Rating } \\
\text { (MW) }\end{array}$} & \multicolumn{2}{|c|}{ Penetration } & \multirow{2}{*}{\begin{tabular}{|l|} 
Rating \\
(MW)
\end{tabular}} & \multicolumn{2}{|c|}{ Penetration } & \multirow{2}{*}{$\begin{array}{l}\text { Rating } \\
\text { (MW) }\end{array}$} & \multicolumn{2}{|c|}{ Penetration } \\
\hline & & & & $\%$ Min & \%Max & & $\%$ Min & $\% \operatorname{Max}$ & & $\%$ Min & $\% \operatorname{Max}$ & & $\% \operatorname{Min}$ & $\% \operatorname{Max}$ \\
\hline COB & 138 & 294 & 90 & $65 \%$ & $31 \%$ & 100 & $73 \%$ & $34 \%$ & 0 & $0 \%$ & $0 \%$ & 190 & $138 \%$ & $65 \%$ \\
\hline Idaho East & 460 & 1,365 & 660 & $144 \%$ & $48 \%$ & 0 & 0 & $0 \%$ & 100 & $22 \%$ & $7 \%$ & 760 & $165 \%$ & $56 \%$ \\
\hline Idaho Southwest & 1,188 & 3,592 & 750 & $63 \%$ & $21 \%$ & 0 & $0 \%$ & $0 \%$ & 100 & $8 \%$ & $3 \%$ & 850 & $72 \%$ & $24 \%$ \\
\hline Montana & 1,149 & 2,337 & 780 & $68 \%$ & $33 \%$ & 0 & $0 \%$ & $0 \%$ & 0 & $0 \%$ & $0 \%$ & 780 & $68 \%$ & $33 \%$ \\
\hline Northern California & 10,297 & 28,319 & 5,610 & $54 \%$ & $20 \%$ & 300 & $3 \%$ & $1 \%$ & 300 & $3 \%$ & $1 \%$ & 6,210 & $60 \%$ & $22 \%$ \\
\hline Northwest & 14,278 & 30,953 & 6,540 & $46 \%$ & $21 \%$ & 0 & $0 \%$ & $0 \%$ & 300 & $2 \%$ & $1 \%$ & 6,840 & $48 \%$ & $22 \%$ \\
\hline Southern California & 9,557 & 26,864 & 7,110 & $74 \%$ & $26 \%$ & 700 & $7 \%$ & $3 \%$ & 400 & $4 \%$ & $1 \%$ & 8,210 & $86 \%$ & $31 \%$ \\
\hline Utah & 2,263 & 7,274 & 1,410 & $62 \%$ & $19 \%$ & 100 & $4 \%$ & $1 \%$ & 100 & $4 \%$ & $1 \%$ & 1,610 & $71 \%$ & $22 \%$ \\
\hline Out of Footprint & 46,328 & 119,696 & 22,950 & $50 \%$ & $19 \%$ & 1,200 & $3 \%$ & $1 \%$ & 1,300 & $3 \%$ & $1 \%$ & 25,450 & $55 \%$ & $21 \%$ \\
\hline
\end{tabular}


Table A- 17 Mega-Project Scenario, 30\% Wind In Footprint - Energy Summary

\begin{tabular}{|c|c|c|c|c|c|c|c|c|}
\hline \multirow[b]{2}{*}{ Area } & Load & \multicolumn{2}{|r|}{ Wind } & \multicolumn{2}{|c|}{ CSP with Storage } & \multicolumn{2}{|r|}{ PV } & \multirow{2}{*}{$\begin{array}{c}\text { Total } \\
\text { Renewable } \\
\text { Energy } \\
\text { (GWh) }\end{array}$} \\
\hline & $\begin{array}{l}\text { Energy } \\
\text { (GWh) }\end{array}$ & $\begin{array}{l}\text { Energy } \\
\text { (GWh) }\end{array}$ & $\begin{array}{c}\text { \# Sites } \\
\text { (Used/Available) }\end{array}$ & $\begin{array}{l}\text { Energy } \\
\text { (GWh) }\end{array}$ & $\begin{array}{c}\text { \# Sites } \\
\text { (Used/Available) }\end{array}$ & $\begin{array}{l}\text { Energy } \\
\text { (GWh) }\end{array}$ & $\begin{array}{c}\text { \# Sites } \\
\text { (Used/Available) }\end{array}$ & \\
\hline Arizona & 99,437 & 5,433 & $63 / 1091$ & 5,503 & $15 / 38$ & 1,690 & $11 / 16$ & 12,626 \\
\hline Colorado East & 61,372 & 7,899 & $83 / 1673$ & 1,639 & $5 / 58$ & 1,035 & $7 / 13$ & 10,573 \\
\hline Colorado West & 8,708 & 293 & $3 / 68$ & 0 & $0 / 8$ & 287 & $2 / 11$ & 580 \\
\hline New Mexico & 31,260 & 14,921 & $145 / 3062$ & 1,533 & $4 / 35$ & 929 & $6 / 19$ & 17,383 \\
\hline Nevada & 57,505 & 3,755 & 48 / 1591 & 1,457 & $4 / 45$ & 320 & $2 / 10$ & 5,532 \\
\hline Wyoming & 27,697 & 53,758 & 459 / 8912 & 0 & $0 / 0$ & 142 & $1 / 10$ & 53,900 \\
\hline In Footprint & 285,979 & 86,059 & $801 / 16397$ & 10,132 & $28 / 184$ & 4,403 & $29 / 79$ & 100,594 \\
\hline
\end{tabular}

\begin{tabular}{|c|c|c|c|c|c|c|c|c|}
\hline \multirow[b]{2}{*}{ Area } & \multirow{2}{*}{$\begin{array}{c}\text { Load } \\
\text { Energy } \\
\text { (GWh) }\end{array}$} & \multicolumn{2}{|r|}{ Wind } & \multicolumn{2}{|c|}{ CSP with Storage } & \multicolumn{2}{|r|}{ PV } & \multirow{2}{*}{$\begin{array}{c}\text { Total } \\
\text { Renewable } \\
\text { Energy } \\
\text { (GWh) }\end{array}$} \\
\hline & & $\begin{array}{l}\text { Energy } \\
\text { (GWh) }\end{array}$ & $\begin{array}{c}\text { \# Sites } \\
\text { (Used/Available) }\end{array}$ & $\begin{array}{l}\text { Energy } \\
\text { (GWh) }\end{array}$ & $\begin{array}{c}\text { \# Sites } \\
\text { (Used/Available) }\end{array}$ & $\begin{array}{l}\text { Energy } \\
\text { (GWh) }\end{array}$ & $\begin{array}{c}\text { \# Sites } \\
\text { (Used/Available) }\end{array}$ & \\
\hline $\mathrm{COB}$ & 1,759 & 394 & $6 / 155$ & 294 & $1 / 22$ & 142 & $1 / 2$ & 830 \\
\hline Idaho East & 6,907 & 1,406 & $26 / 185$ & 0 & $0 / 0$ & 142 & $1 / 8$ & 1,548 \\
\hline Idaho Southwest & 17,962 & 3,658 & $50 / 448$ & 0 & $0 / 0$ & 132 & $1 / 5$ & 3,790 \\
\hline Montana & 14,143 & 2,873 & $35 / 1194$ & 0 & $0 / 0$ & 127 & $1 / 14$ & 3,000 \\
\hline Northern California & 128,935 & 25,812 & $393 / 472$ & 2,726 & $8 / 26$ & 1,117 & $8 / 30$ & 29,655 \\
\hline Northwest & 178,359 & 35,733 & $431 / 3195$ & 0 & $0 / 0$ & 1,645 & $13 / 54$ & 37,378 \\
\hline Southern California & 224,197 & 44,890 & 483 / 1916 & 8,957 & $23 / 85$ & 2,050 & $13 / 39$ & 55,897 \\
\hline Utah & 38,022 & 7,658 & $91 / 554$ & 937 & $3 / 24$ & 303 & $2 / 14$ & 8,898 \\
\hline Out of Footprint & 610,284 & 122,424 & $1515 / 8119$ & 12,914 & $35 / 157$ & 5,658 & $40 / 166$ & 140,996 \\
\hline Total & 896,263 & 208,483 & $2316 / 24516$ & 23,046 & $63 / 341$ & 10,061 & $69 / 245$ & 241,590 \\
\hline
\end{tabular}


Table A- 18 Mega-Project Scenario, 30\% Wind In Footprint - Power Summary

\begin{tabular}{|c|c|c|c|c|c|c|c|c|c|c|c|c|c|c|}
\hline \multirow[b]{3}{*}{ Area } & \multicolumn{2}{|c|}{ Load } & \multicolumn{3}{|c|}{ Wind } & \multicolumn{3}{|c|}{ CSP with Storage } & \multicolumn{3}{|c|}{ PV } & \multicolumn{3}{|c|}{ Total Renewable } \\
\hline & \multirow{2}{*}{$\begin{array}{c}\text { Minimum } \\
\text { (MW) }\end{array}$} & \multirow{2}{*}{$\begin{array}{l}\text { Maximum } \\
(\mathrm{MW})\end{array}$} & \multirow{2}{*}{$\begin{array}{l}\text { Rating } \\
\text { (MW) }\end{array}$} & \multicolumn{2}{|c|}{ Penetration } & \multirow{2}{*}{$\begin{array}{c}\text { Rating } \\
\text { (MW) }\end{array}$} & \multicolumn{2}{|c|}{ Penetration } & \multirow{2}{*}{$\begin{array}{c}\text { Rating } \\
\text { (MW) }\end{array}$} & \multicolumn{2}{|c|}{ Penetration } & \multirow{2}{*}{$\begin{array}{c}\text { Rating } \\
\text { (MW) }\end{array}$} & \multicolumn{2}{|c|}{ Penetration } \\
\hline & & & & $\%$ Min & \%Max & & $\%$ Min & $\% \operatorname{Max}$ & & $\%$ Min & $\%$ Max & & $\%$ Min & $\% \operatorname{Max}$ \\
\hline Arizona & 6,995 & 23,051 & 1,890 & $27 \%$ & $8 \%$ & 1,500 & $21 \%$ & $7 \%$ & 1,100 & $16 \%$ & $5 \%$ & 4,490 & $64 \%$ & $19 \%$ \\
\hline Colorado East & 4,493 & 11,589 & 2,490 & $55 \%$ & $21 \%$ & 500 & $11 \%$ & $4 \%$ & 700 & $16 \%$ & $6 \%$ & 3,690 & $82 \%$ & $32 \%$ \\
\hline Colorado West & 712 & 1,526 & 90 & $13 \%$ & $6 \%$ & 0 & $0 \%$ & $0 \%$ & 200 & $28 \%$ & $13 \%$ & 290 & $41 \%$ & $19 \%$ \\
\hline New Mexico & 2,571 & 5,320 & 4,350 & $169 \%$ & $82 \%$ & 400 & $16 \%$ & $8 \%$ & 600 & $23 \%$ & $11 \%$ & 5,350 & $208 \%$ & $101 \%$ \\
\hline Nevada & 3,863 & 12,584 & 1,440 & $37 \%$ & $11 \%$ & 400 & $10 \%$ & $3 \%$ & 200 & $5 \%$ & $2 \%$ & 2,040 & $53 \%$ & $16 \%$ \\
\hline Wyoming & 2,369 & 4,016 & 13,770 & $581 \%$ & $343 \%$ & 0 & $0 \%$ & $0 \%$ & 100 & $4 \%$ & $2 \%$ & 13,870 & $586 \%$ & $345 \%$ \\
\hline In Footprint & 21,249 & 58,087 & 24,030 & $113 \%$ & $41 \%$ & 2,800 & $13 \%$ & $5 \%$ & 2,900 & $14 \%$ & $5 \%$ & 29,730 & $140 \%$ & $51 \%$ \\
\hline
\end{tabular}

\begin{tabular}{|c|c|c|c|c|c|c|c|c|c|c|c|c|c|c|}
\hline \multirow[b]{3}{*}{ Area } & \multicolumn{2}{|c|}{ Load } & \multicolumn{3}{|c|}{ Wind } & \multicolumn{3}{|c|}{ CSP with Storage } & \multicolumn{3}{|c|}{ PV } & \multicolumn{3}{|c|}{ Total Renewable } \\
\hline & \multirow{2}{*}{$\begin{array}{c}\text { Minimum } \\
\text { (MW) }\end{array}$} & \multirow{2}{*}{\begin{tabular}{|c} 
Maximum \\
(MW)
\end{tabular}} & \multirow{2}{*}{$\begin{array}{l}\text { Rating } \\
(\mathrm{MW})\end{array}$} & \multicolumn{2}{|c|}{ Penetration } & \multirow{2}{*}{$\begin{array}{l}\text { Rating } \\
\text { (MW) }\end{array}$} & \multicolumn{2}{|c|}{ Penetration } & \multirow{2}{*}{$\begin{array}{l}\text { Rating } \\
\text { (MW) }\end{array}$} & \multicolumn{2}{|c|}{ Penetration } & \multirow{2}{*}{$\begin{array}{l}\text { Rating } \\
\text { (MW) }\end{array}$} & \multicolumn{2}{|c|}{ Penetration } \\
\hline & & & & $\%$ Min & \%Max & & $\%$ Min & $\% \operatorname{Max}$ & & $\%$ Min & $\% \operatorname{Max}$ & & $\% \operatorname{Min}$ & $\% \operatorname{Max}$ \\
\hline $\mathrm{COB}$ & 138 & 294 & 180 & $131 \%$ & $61 \%$ & 100 & $73 \%$ & $34 \%$ & 100 & $73 \%$ & $34 \%$ & 380 & $276 \%$ & $129 \%$ \\
\hline Idaho East & 460 & 1,365 & 780 & $170 \%$ & $57 \%$ & 0 & $0 \%$ & $0 \%$ & 100 & $22 \%$ & $7 \%$ & 880 & $191 \%$ & $64 \%$ \\
\hline Idaho Southwest & 1,188 & 3,592 & 1,500 & $126 \%$ & $42 \%$ & 0 & $0 \%$ & $0 \%$ & 100 & $8 \%$ & $3 \%$ & 1,600 & $135 \%$ & $45 \%$ \\
\hline Montana & 1,149 & 2,337 & 1,050 & $91 \%$ & $45 \%$ & 0 & $0 \%$ & $0 \%$ & 100 & $9 \%$ & $4 \%$ & 1,150 & $100 \%$ & $49 \%$ \\
\hline Northern California & 10,297 & 28,319 & 11,790 & $114 \%$ & $42 \%$ & 800 & $8 \%$ & $3 \%$ & 800 & $8 \%$ & $3 \%$ & 13,390 & $130 \%$ & $47 \%$ \\
\hline Northwest & 14,278 & 30,953 & 12,930 & $91 \%$ & $42 \%$ & 0 & $0 \%$ & $0 \%$ & 1,300 & $9 \%$ & $4 \%$ & 14,230 & $100 \%$ & $46 \%$ \\
\hline Southern California & 9,557 & 26,864 & 14,490 & $152 \%$ & $54 \%$ & 2,300 & $24 \%$ & $9 \%$ & 1,300 & $14 \%$ & $5 \%$ & 18,090 & $189 \%$ & $67 \%$ \\
\hline Utah & 2,263 & 7,274 & 2,730 & $121 \%$ & $38 \%$ & 300 & $13 \%$ & $4 \%$ & 200 & $9 \%$ & $3 \%$ & 3,230 & $143 \%$ & $44 \%$ \\
\hline Out of Footprint & 46,328 & 119,696 & 45,450 & $98 \%$ & $38 \%$ & 3,500 & $8 \%$ & $3 \%$ & 4,000 & $9 \%$ & $3 \%$ & 52,950 & $114 \%$ & $44 \%$ \\
\hline
\end{tabular}

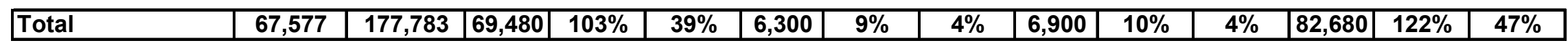


GE

Energy

Global Selection Algorithm (GSA) for Initial Siting of Wind and Solar Plants and Transmission for WWSIS

Version 10

(changes from $v 9$ highlighted)

September 29, 2008

GE Energy

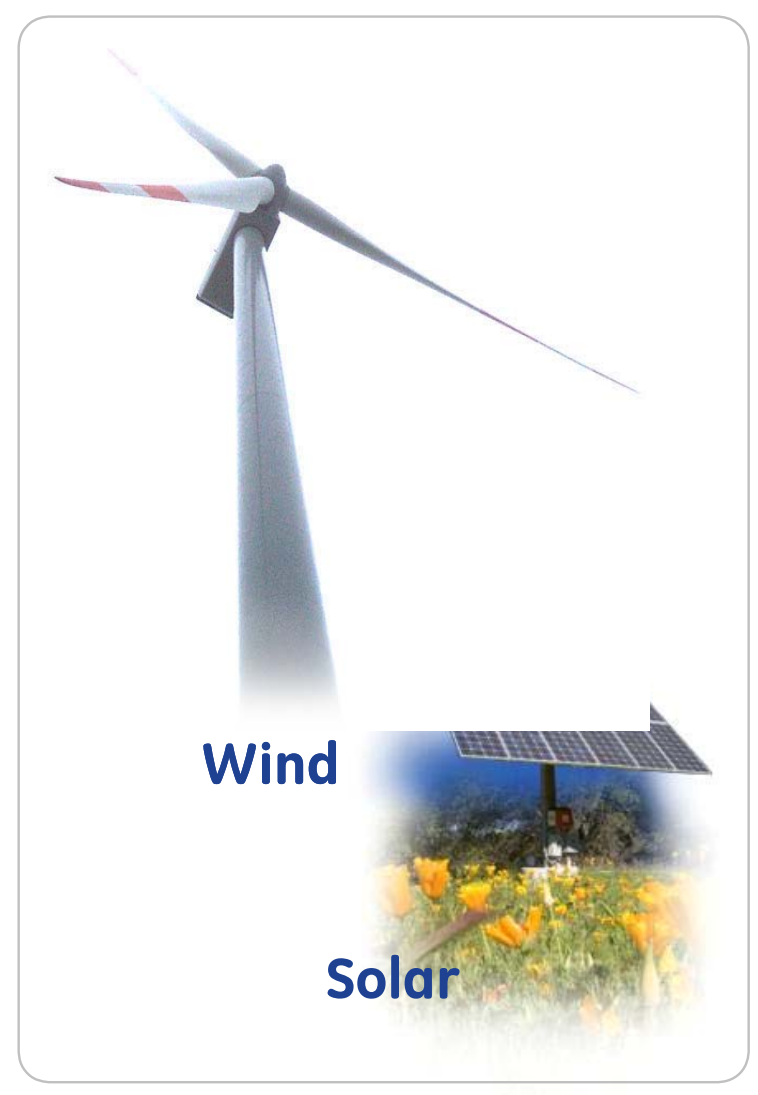




\section{Objectives and Constraints on Wind and Solar Plant Siting \\ Assumptions:}

1. Need to strike a balance between quality of wind and solar resources and distance from load centers

2. Geographic diversity within regions has value

3. Existing transmission limits apply to inter-area exchange of wind, but may be discounted due to other firm commitments

4. Cost of transmission can reasonably be approximated by $\$ / M W$-mile metrics

5. Exchange of wind (and solar) power between regions at less than available interarea transfer levels incurs no incremental transmission reinforcement cost

6. Hard limit/objectives

a. $30 \%$ wind energy inside footprint; $5 \%$ solar ( $70 \%$ of which is CSP, $30 \%$ PV)

b. $20 \%$ wind energy outside footprint; $3 \%$ solar (70\% of which is CSP, $30 \%$ PV)

c. Pre-selected wind plants are included (Baseline Scenario)

7. Given that Wind and Solar energy production and location of consumption are fixed, global optimization is primarily a cost minimization problem, i.e. balance capital costs of wind sites with transmission and losses

8. Given that Wind and the 2 types of Solar energy production are not interchangeable (due to the project hard limits), the optimization should be run independently for each of Wind, PV, and CSP.

9. Intra-area site selection for 8 areas inside footprint and 7 areas outside footprint will give In-Area Scenario

10. Inter-area site swapping for 8 areas inside footprint loutside footprint will not change) will give next study scenario 


\title{
Algorithm Overview
}

\author{
4 Stage process:
}

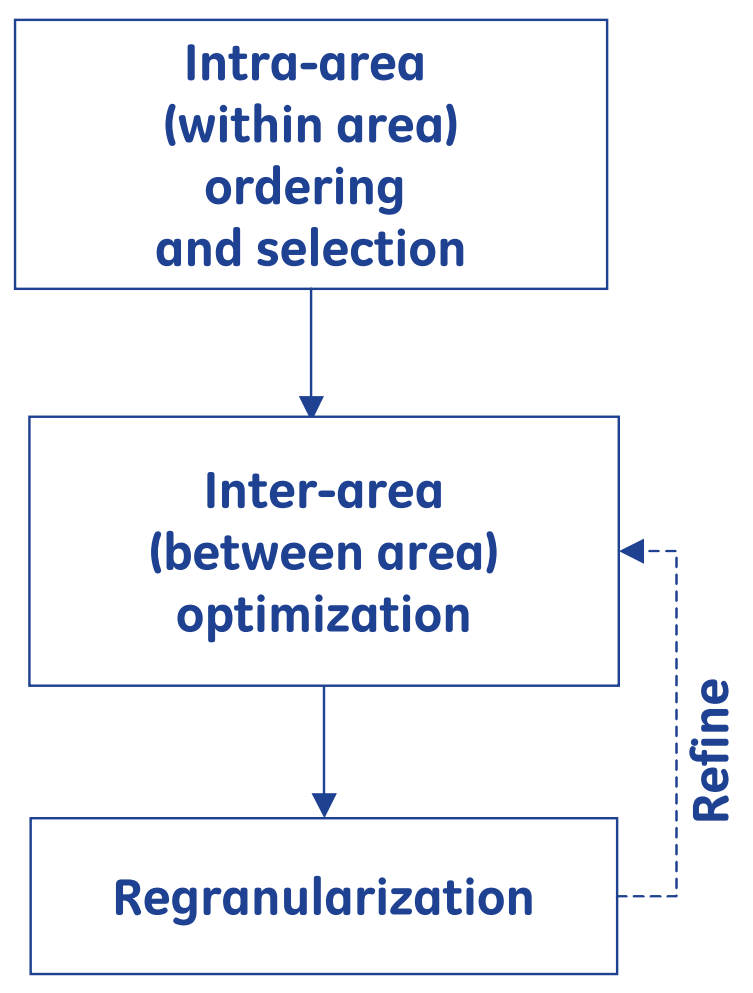

Order the available wind, PV and CSP sites within each area in descending order of preference, based on pre-selection of existing plants, and a mix of energy value, geographic diversity and capacity value. Select top sites necessary to meet energy target (e.g. 30\% for wind in-study footprint) with in-area resources. Intra-area selection is run once for each of wind, PV and CSP. Result is In Area Scenario.

Adjust selection of top sites, by replacing in-area site with remote sites, based on minimizing costs of wind, PV and CSP generation equipment, new transmission and losses. Interarea optimization is run once for each of wind, PV and CSP.

Heuristically adjust continuously optimized site selection and transmission reinforcements to reflect discrete nature of components, including whole wind and solar plants and realistically sized transmission reinforcements.

Regranularization is performed once, to combine results of all three optimization runs.

Refinement will run Inter-area optimization with rationalized transmission reinforcements, to better utilize the new transmission. Results are additional study scenarios. 


\section{Intra-area (within area) ordering}

Discussion of philosophy and notation.

The intent of the intra-area ordering is to create a list of candidate sites within each area that is sorted from best to worst, according to the criteria presented in the following pages.

For the study there are 8 areas within the study system and 7 outside. At any point in the intra-area ordering, we will be concentrating on the $j^{\text {th }}$ area.

Within that area, there will be a subset of sites selected. The count of sites in the $\mathrm{j}^{\text {th }}$ area at any point will be $\mathrm{N}_{\mathrm{j}}$. Any specific site within the stack is the $i^{\text {th }}$.

Thus, the value associated with any characteristic (say) $Z$ of the $i^{\text {th }}$ site of $j^{\text {th }}$ area will be $Z_{i j}$

And, the cumulative value associated the entire stack down to the $i^{\text {th }}$ site will be denoted with an underscore. For example, the sum of all values of $Z$ down to and including the $i^{\text {th }}$ site of $j^{\text {th }}$ area will be a $\underline{Z}_{\mathrm{ij}}$

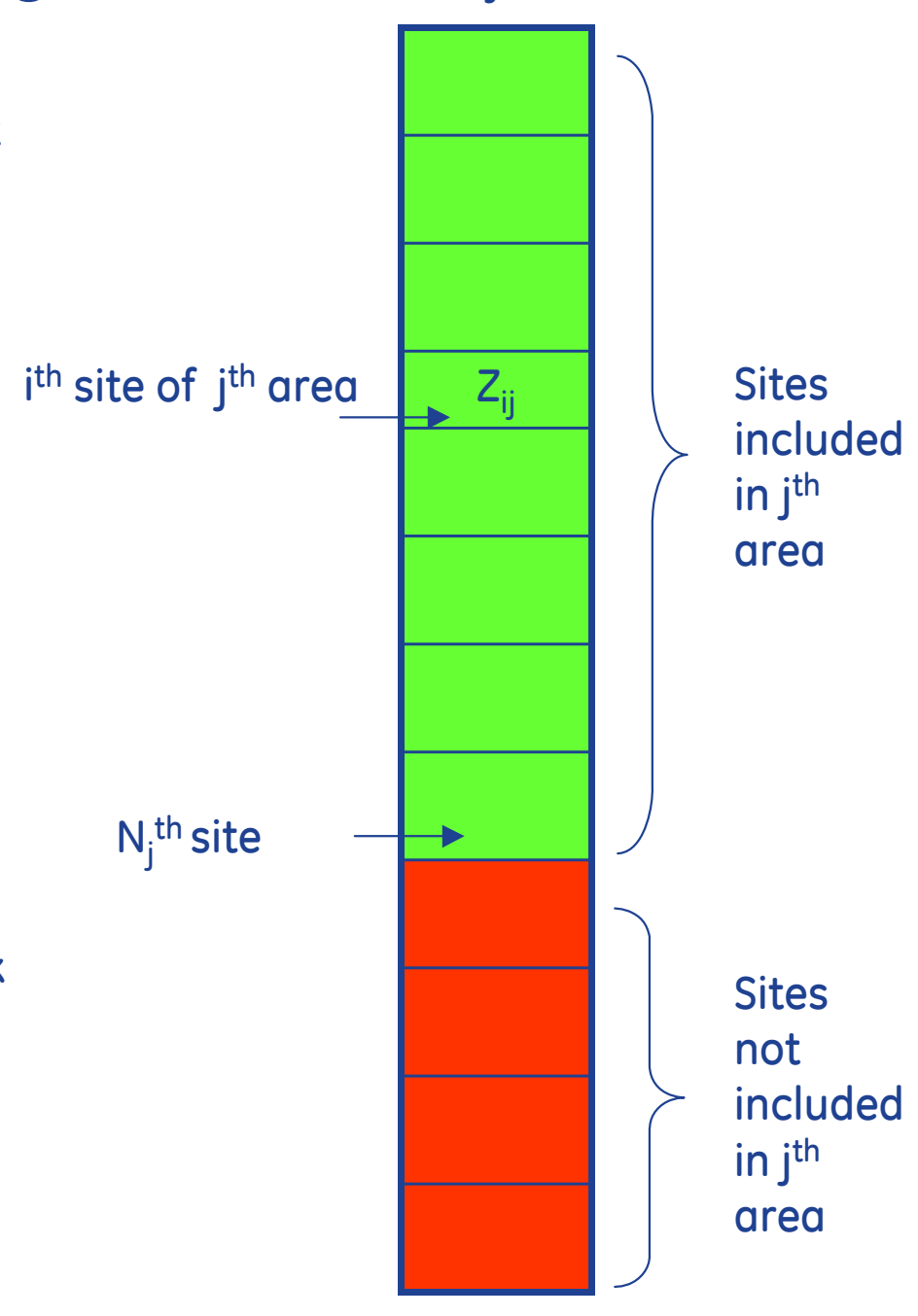




\section{Intra-area Geographic Diversity}

Postulate: Intra-area geographic diversity has value that is a proxy for wind and solar temporal diversity (e.g., lower ancillary services costs, but not market TOD benefits, which are covered by revenue calculation). Further speculate that direction is significant (i.e., East-West diversity is more valuable than North-South). (For Solar, only east-west diversity is of value)

A possible metric: "geographic diversity of $\mathrm{j}^{\text {th }}$ area" $=\underline{G D}_{\mathrm{j}}$

start with "center coordinates of $\mathrm{j}^{\text {th }}$ area" $=\underline{C}_{\mathrm{j}}=\underline{\mathrm{C}}_{\mathrm{j}}\left(\underline{\text { Lat }}_{\mathrm{j}}\right.$, $\left.\underline{\text { Long }}_{\mathrm{j}}\right)$

where the coordinates of the $\mathrm{i}^{\text {th }}$ wind plant of the $\mathrm{j}^{\mathrm{th}}$ area are given by $\mathrm{C}_{\mathrm{ij}}\left(\right.$ Lat $_{\mathrm{ij}}$, Long $\left._{\mathrm{ij}}\right)$

when there are $\mathrm{N}_{\mathrm{j}}$ wind plants in the $\mathrm{j}^{\text {th }}$ area,

$\underline{C}_{j}=\left(1 / N_{j}\right) * \sum\left(C_{i j}\left(\right.\right.$ Lat $_{i j}$, Long $\left._{i j}\right) \quad$ summed over $\left.N_{j}\right\}$

Then $\underline{G D}_{j}=\operatorname{sqrt}\left(\left(1 / N_{j}\right) * \sum\left(W_{d}(N S, E W) *(\right.\right.$ scale $\left.) *\left(C_{i j}\left(\text { Lat }_{i j}, \text { Long }_{i j}\right)-\underline{C}_{j}\left(\text { Lat }_{j}, \underline{L o n g}_{j}\right)\right)^{2}\right)$

where $\quad$ scale $=$ (miles per degree lat, miles per degree long)

$W_{d}(N S, E W)=$ relative east-west $v s$. north-south diversity benefit.

Initially $W_{d}$ is neutral $(1 ., 1$.$) .$

(for comparison a $W_{d}=(.707,1.22$ ) would give priority to $E-W$ over $N-S$ diversity), $\underline{G D}_{j}$ will have units of miles.

A statistical screening level evaluation of temporal diversity/coincidence was used to validate the geographic diversity proxy 


\section{Intra-area Capacity Value, ELCC}

Postulate: ELCC (Effective Load Carrying Capacity) of individual sites has value. The timing of energy production dominates the ELCC value (i.e., forced outage rates are not important). Generation during peak hours (i.e., the approach used to approximate ELCC in the NYS study) is a proxy for ELCC. Since the overall optimization question is focused on the exchange of energy between areas, it is reasonable to assign the same time window for assessment of ELCC to all candidate sites, regardless of area.

A possible metric: "capacity value of $\mathrm{j}^{\text {th }}$ area" $=\underline{\mathrm{CV}}_{\mathrm{j}}$

Since each individual site will have a single, independent, pre-calculated ELCC capacity value:

$\mathrm{CV}_{\mathrm{ij}}$ (with units of $\mathrm{MW}$ )

Then the total capacity value is:

$\underline{C V}_{j}=\sum \mathrm{CV}_{\mathrm{ij}} \quad$ ssummed over $\left.\mathrm{N}_{\mathrm{j}}\right\}$ 


\section{Intra-area Optimization}

Suggest an Energy Value for $\mathrm{j}^{\text {th }}$ area:

$\underline{E V}_{j}=\sum R_{i j} \quad$ summed over $\left.N_{j}\right\}$

where $R_{i j}$ is annual energy sale value of the $i$ th plant of the $j^{\text {th }}$ area (per Gary's screening, in units of $\$ / y r)$.

Also the cumulative energy:

$\underline{E}_{j}=\sum E_{i j} \quad$ ssummed over $\left.N_{j}\right\}$

where $E_{i j}$ is annual energy production of the $i^{i \text { th }}$ plant of the $j^{\text {th }}$ area (in units of $\left.M W h r / y r\right)$.

Then suggest a geographic diversity and capacity value weighted "adjusted energy value"

$\underline{E V A}_{j}=\underline{E V}_{j}\left(1+W_{G D}{ }^{*} \underline{G D}_{j}\right)+W_{c V}{ }^{*} \underline{C V}_{j}$

where $W_{G D}=$ benefit weight for geographic diversity, in units of pu/mile. For Wind, suggest an initial value of (say) 0.0002 , which would give a $2 \%$ bonus for 100 miles of diversity.

where $W_{c V}=$ benefit weight for capacity, in units of $\$ / M W$-year. Suggest an initial value of (say) $\$ 100,000$ (roughly $\$ 100 / \mathrm{kW}$ for new installed peaker capacity, $20 \%$ capital recovery factor). 


\section{Intra-area Optimization, sorting sites for each area into a area-optimum stack using gradient descent}

Using this adjusted energy value approach, a new "optimum" stack of plants for the $j^{\text {th }}$ area can be calculated, in which each successive plant adds the best marginal contribution to EVA, as follows:

Initially populate the stack with preselected sites*, then start with best site in area $\mathrm{j}$, i.e. the one with the (highest $R+W_{c v}{ }^{*} C V_{i j}$ ). That site becomes Site $(1, j)$, with coordinates $C_{i j}\left(\operatorname{Lat}_{\mathrm{ij}}\right.$, Long $\left._{\mathrm{ij}}\right)$.

Next will be the one for which $R_{i j}{ }^{*}\left(1+W_{G D}{ }^{*} \underline{G D}_{j}\right)+W_{c V}{ }^{*} C V_{i j}$, i.e. $\Delta E V A_{i j}$, is greatest.

That means, that $\underline{G D}_{j}$ will be evaluated for each next prospective site, and then saved when that selection is complete.

This stack will only need to be populated once (per choice of weights $W_{G D}$ and $W_{c v}$ ).

These, and several other measures and indices will be used below in the Inter-area search.

Once the stacks are populated, the initial pointer $N_{j}$ in each stack $j$ will correspond to the index $i$, to meet the target energy percentage of the total load energy for area $\mathrm{j}$. (e.g. such that $\underline{E}_{\mathrm{ij}}=30 \%$ for wind in-study areas; $\underline{E}_{i j}=2.1 \%=3 \% \times 0.70$ for CSP outside study area, etc. )

So for example, the index is N30j (i.e. the count of plants in area $\mathrm{j}$ that satisfies $30 \%$ energy of that area). The collection of areas all satisfying this is referred to as the In-Area Scenario.

* There may not be pre-selected solar sites 


\section{Intra-area Optimization,}

More on information saved during the sorting of sites for use in the inter-area gradient descent

Before proceeding to the Inter-area gradient descent, it is useful to record all of the total and incremental values determined in the course of the intra-area sorting.

Specifically, as each new member is added to the stack, and the value of $i$ is incremented, there will be a growing vector of

$\underline{E}_{j}, \underline{E V A} \underline{E V}_{j}, \underline{G D}_{j}, \underline{C V}, \underline{ }_{j}$, and $\underline{C}_{j}$

and incremental values:

$\Delta E_{i j}=\underline{E}_{i j}-\underline{E}_{i-1, j}=E_{i j}$

$\Delta \mathrm{EV}_{\mathrm{ij}}=\underline{\mathrm{EV}}_{\mathrm{ij}}-\underline{\mathrm{EV}}_{\mathrm{i}-1, \mathrm{j}}$

$\Delta \mathrm{EVA}_{\mathrm{ij}}=\underline{\mathrm{EVA}}_{\mathrm{ij}}-\underline{\mathrm{EVA}}_{\mathrm{i}-1, \mathrm{j}}$

$\Delta C V_{i j}=\underline{C V}_{i j}-\underline{C V}_{i-1, j}$

$\Delta \mathrm{GD}_{\mathrm{ij}}=\underline{G D}_{\mathrm{ij}}-\underline{G D}_{\mathrm{i}-1, \mathrm{j}}$ 


\section{Inter-area ordering}

The intent of the inter-area ordering is displace less valuable sites from one area, making it a net importer, while replacing the energy with more valuable sites in another area, making it a net exporter. The total renewable energy will be held constant. Thus, within the study, pairs of the 7 areas will be compared (repeatedly).

For notation purposes, throughout the following discussion, the area losing in-area renewables will always be the $j^{\text {th }}$ area, and the area adding renewables for export will always be the $k^{\text {th }}$ area

Thus, the value associate with any characteristic (say) $Z$ of the $i_{t h}$ site of $j$ th (importing) area will be $Z_{i j}$ or $Z_{i k}$ for the exporting area.

Parameters related to inter-area quantities will be denoted as $Z_{j k}$. For example, the power transfer to the $j^{\text {th }}$ area from the $k^{\text {th }}$ area, will be denoted as $\mathrm{PT}_{\mathrm{jk}}$.

Since energy production between sites is not constant, fractional sites will be added to the $k^{\text {th }}$ area when entire sites are deleted from the $\mathrm{j}^{\text {th }}$ area.

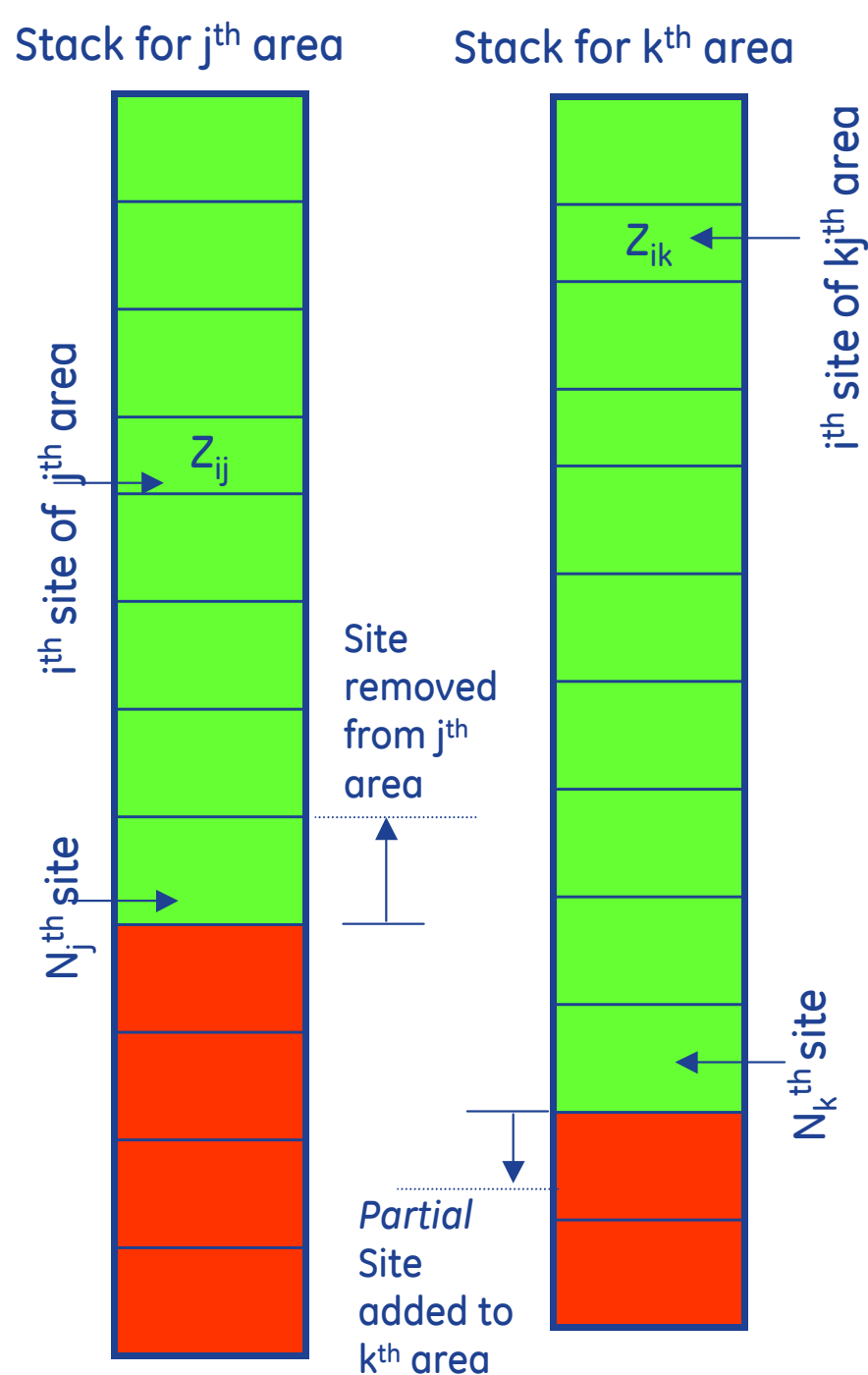




\section{Inter-area Incremental Transmission Costs}

Postulate the transmission reinforcement cost of excess power exchange between areas $j$ and $\mathrm{k}$.

Define inter-area exchange limit as:

$L_{j k}=T L_{j k}-C D_{j k}$

where $T L_{j k}$ is the path rating, and $C D_{j k}$ is any capability discounted le.g., reserved for other power plants). Set $C_{\mathrm{jk}}$ to zero for starters and plan to use all capability. For areas between which there are no existing connections, $\mathrm{TL}_{\mathrm{jk}}=$ zero.

Calculate the inter-area distance as $D_{j k}=$ scale * abs $\left(\underline{C}_{j}-\underline{C}_{k}\right)$.

Calculate a transmission cost for necessary reinforcements between areas $\mathrm{j}$ and $\mathrm{k}$ :

$C T_{j k}=\max \left(0,\left(P T_{j k}{ }^{*} T D F-L_{j k}\right)\right) * D_{j k} *\left(1+G D P * \underline{G D}_{k}\right) * T C F$

where

- $\mathrm{PT}_{\mathrm{jk}}$ is the net power exchange between areas (updated each algorithm pass)

- TCF is the annual carrying cost of transmission, units of \$/MW-mile-year (initially $\$ 160 / \mathrm{MW}$-mi-year, which reflects $\$ 800 / \mathrm{MW}$-mi with $20 \%$ capital recovery factor)

- TDF is the net discount on annual carrying cost of transmission reflecting a temporal diversity factor (a figure of $50-70 \%$ should be about right )

- GDP is transmission cost penalty associated with higher diversity in the exporting system (a figure of $.0005,5 \%$ per 100 miles should be about right ) 


\section{Inter-area Losses}

Strictly speaking, we should throw away energy for losses, and increase the $P$ rating of exporting systems. But, we really want to keep the energy generated fixed.

By designating losses as a transmission cost, we capture some aspect of the impact without the consequence of increasing the wind energy production. A loss term may be approximated as a penalty on net energy exchange between areas:

$C L_{j k}=E T_{j k} * D_{j k} * L C F * L D F$

where:

$\mathrm{ET}_{\mathrm{jk}}$ is the net inter-area energy transfer (as yet undefined)

$L C F$ is a loss cost factor in units of $\$ / M W h r / y r$. We may use $L C F_{j}=\Delta E V_{i j}$, the lost energy value to the receiving area. la simpler proxy would be the annual average value of wind energy, i.e., the average of all $\left.R_{i j}\right)$.

LDF is a loss distance factor, i.e., how much energy is lost per mile of transmission. A value of $1 \% / 100$ miles, or LDF $=0.0001$, is a reasonable place to start.

But, $\mathrm{ET}_{\mathrm{jk}}$ and therefore $\mathrm{CL}_{\mathrm{jk}}$ will only be apparent in a cumulative sense, and therefore must be tracked during optimization. For an exchange of power between the $j^{\text {th }}$ and $k^{\text {th }}$ areas, revalued at their respective pointer i for the stack, where $i=N_{i}$ or $N_{k}$. 


\section{Total Cost, considering Inter-area Power Exchange Limits}

Total value is fixed with energy calculation, so inter-area optimization is a question of minimizing costs only.

The cost of capital, represented as a fixed charge rate $\mathrm{FCR}_{\mathrm{j}}$ and total capital cost $T C C_{j}$, produce a per unit renewable plant annual carrying cost of $A C C_{j}=\mathrm{FCR}_{\mathrm{j}}{ }^{*} \mathrm{TCC}_{\mathrm{j}}$ in

$\$ / M W /$ year ${ }^{1}$.

Thus, the total capacity cost in area $j$ at pointer $i$ is:

$\operatorname{CCAP}_{j}=A C C_{j} * N_{j}$ * plant size 2

$A C C_{j}$ may reflect differences in total capital cost by area - including cost of land, labor, generation equipment, etc. However, initially ACC will be uniform across all areas.

Calculate a Total (annual) Cost:

$T C=\sum \mathrm{CCAP}_{\mathrm{j}}+\sum \mathrm{CT}_{\mathrm{jk}}+\sum \mathrm{CL}_{\mathrm{jk}}$

where CCAP is summed over $\mathrm{j}=1, \mathrm{~N}_{\text {areas }}$, and

$C T_{j k}$ and $C L_{j k}$ are summed over $j=1, N_{\text {areas }}$ and $k=1, N_{\text {areas }} ; j$ ? $k$

${ }^{1}$ total capital cost of solar in $\$ / M W$ is assumed to be uniform between plants.

${ }^{2}$ plant size is $30 \mathrm{MW}$ for all wind plant, $100 \mathrm{MW}$ for PV and CSP 


\section{Total Value, gradient descent}

For inter-area selection, start with In-Area Scenario, but set aside GD and CV stacks. Instead, re-sort stacks in two separate blocks: increasing (more sites) and decreasing (removing sites). Each of these blocks is sorted by CF (or EV) only. This allows a smooth gradient descent, without numerical discontinuities with the algorithm shown on the next page. I

Search for area pair, for which $\triangle T C$ is greatest, which should be for two top of the stack sites for the area pair, $j$ and $k$, such that the marginal cost of

$\sum \mathrm{CCAP}_{\mathrm{j}}+\sum \mathrm{CT}_{\mathrm{jk}}+\sum \mathrm{CL}_{\mathrm{jk}}$ is most negative

The best marginal capacity cost reduction will be for the pair for which $\left(\Delta E_{i j}-\Delta E_{i k}\right)$ is most negative. Specifically, for which $\left(\triangle E_{i j}{ }^{*} A C C_{j}-\Delta E_{i k}{ }^{*} A C C_{k}\right)$ is most negative when annual carrying cost is different between areas.

Since we must maintain constant energy, assume that an integer value of site count $\mathrm{N}_{\mathrm{j}}$ from area $j$ is decremented. Then, a non-integer value of site count $N_{k}$ from area k must be incremented, as given by:

$\Delta \mathrm{N}_{\mathrm{k}}=\Delta \mathrm{E}_{\mathrm{ij}} / \Delta \mathrm{E}_{\mathrm{ik}}$

Then $\triangle C C A P_{j k}=\left(\triangle N_{k} * A C C_{k}-A C C_{j}\right)^{*}$ plant size. \{which should be negative

This then allows calculation of marginal transmission cost as follows:

if $P T_{j k}{ }^{*} T D F \geq L_{j k}$, then $\Delta C T_{j k}=$ plant size ${ }^{*} \Delta N_{k}{ }^{*} D_{j k} *\left(1+G D P * \underline{G D}_{k}\right) * T C F * T D F$, else 0. \{which should be non-negative\}

(The asymmetry of the transfer limit equation means that we can assign different values to $L_{j k}$, and $L_{k j}$. Cleaner: no need to know a priori which areas will export or import) 


\section{Total Value, gradient descent, continued}

The incremental loss cost between areas (for an exchange of energy between the areas, at their respective pointer $\mathrm{i}$ for the $\mathrm{j}^{\text {th }}$ and $\mathrm{k}^{\text {th }}$ stack, will look like:

$\Delta C L_{j k}=\Delta E_{i j} * D_{j k} * L C F * L D F$

The next pair in the optimization is then the jk pair for which $\Delta C C A P_{j k}+\Delta C T_{j k}+\Delta C L_{j k}$ is most negative.

Search ends when no area pair has a negative $\triangle C C A P_{j k}+\Delta C T_{j k}+\Delta C L_{j k}$ (Optimization can't allow preselected sites to be dropped, even if descent shows they should be.)

At each search step, for the selected pair, site count $N_{j}$ drops by 1 , and site count $N_{k}$ increases by $\Delta \mathrm{N}_{\mathrm{k}}$. During the descent, all values of stack pointers (site counts) will become non-integer.

(Interpolation between the adjacent cells (indexed values) could be used. However, the granularity of the data is sufficiently fine so the nearest lower integer will be used. Minute errors in transmission costs might result.)

Once a new pair is selected in the descent, cumulative metrics need to be updated before the next iteration. Specifically:

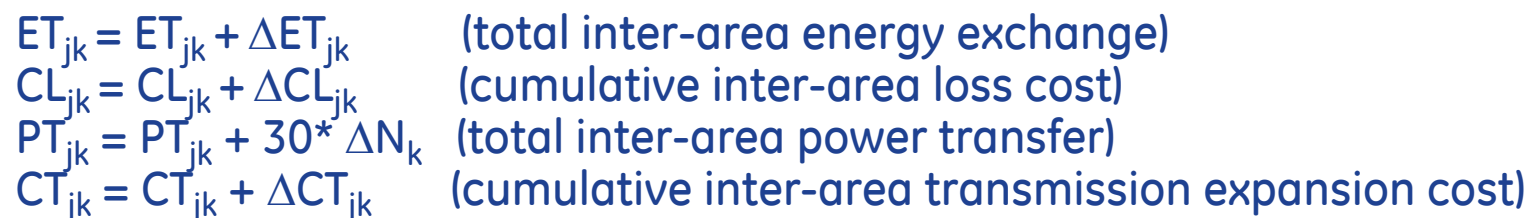




\section{Returning CV and GD to solved inter-area solution}

The results of the gradient decent with the CF-only stacks will not include benefits from CV and GD. The energy value of the redistributed system will be correct (I.e. total of $30 \%$ ).

Once the count and energy value of shifted sites is determined, return to the original area stacks. Select the count of plants from the original stacks that produces the same energy as gradient descent. For exporting areas, this will mean more new plants are needed; for importing systems, fewer plants will be removed.

The total capital cost savings calculated by the descent algorithm will need to be updated. However, the rationale for GD and (possibly) CV can be used to make the argument that loss and transmission cost terms need not be adjusted.

In future, it will be of significant interest to test the statistical variability of CF only distribution within an area (especially Wyoming), compared to the CV \& GD modified stack. The distribution of variability will help determine whether the modified distribution warrants the CF capital penalty. 


\section{Creating a physically sensible scenario}

At the conclusion of the descent, plant indices in each area and for each renewable technology must (obviously) be rounded off to integer values, but the more challenging need is for transmission to be rationalized:

In general, every area may be connected to every other area by new transmission, and rating (and possibly direction) of these connections will be different for each of the 3 renewable technologies.

For each pair, if $P T_{j k} \geq L_{j k}$, then $\left(P T_{j k}-L_{j k}\right)$ * TDF is the incremental amount of $M W$ rating needed between areas $j$ and $k$, with a length of $D_{j k}$.

The incremental ratings for PV and CSP will be added, then the largest incremental rating between the wind and the combined solar technologies will be considered. Alternative routings will be considered.

Lines of very low rating $\left(s m a l l \mathrm{PT}_{\mathrm{jk}}\right.$ ) can be discarded.

Lines of moderate rating and length, may be replaced with equivalent new capability representative of the type of transmission typical in the affected areas (e.g. $230 \mathrm{kV}$ or $345 \mathrm{kV}$ in much of the study areal.

Lines of substantial rating, may be replaced with new EHV or HVDC equivalent capability, suitably rated. This could be based on representative SIL, or perhaps multiples of $100 \mathrm{MW}$, in the case of HVDC. 


\section{Refinement}

After modification of the transmission plan, it may be desirable to return to the inter-area optimization for a refinement pass.

In this refinement stage, updated values of transmission limits, $L_{j k}$, can be input. These increased transmission limits would be treated at this step as sunk costs.

In future, the algorithm could then be run to refine the selection of sites. Under this refinement step, a high penalty on new transmission (i.e. a high value of TCF) is used. The net result will be a modified site selection that more effectively uses new transmission and avoids projects for which marginal (e.g. too small) transmission is necessary.

This refinement step may also prove to be valuable for populating other scenarios as well. This approach could be used for variations (e.g., different weights) from any case for which a fully populated vector of initial conditions (including $\mathrm{PT}_{\mathrm{jk}}$ and $\mathrm{ET}_{\mathrm{jk}}$ ) are available from earlier optimization runs. 


\section{Parameters}

Many parameters could be adjusted to examine sensitivity to various assumptions, and to help populate scenarios for later in the project.

Intra-area parameters :

$-W_{d}(E W, N S)$ initial value: (1., 1.) for Wind

- $W_{G D} \quad$ initial value: $0.0002 \mathrm{pu} / \mathrm{mile}$ (2\% bonus for 100 miles of diversity) for Wind

$-W_{d}$ (EW,NS) initial value: $(0.0,1.41)$ for Solar

- $W_{G D} \quad$ initial value: $0.0002 \mathrm{pu} / \mathrm{mile}$ (2\% bonus for 100 miles of diversity) - for Solar

- $W_{c v} \quad$ initial value: $\$ 100,000 \$ / M W$-year $(\$ 500 / \mathrm{kW}$ for new installed peaker capacity, $20 \%$ capital recovery factor)

Inter-area parameters:

- FCR $_{\mathrm{j}} \quad$ initial value: $0.20(20 \%)$ (by area)

- TCC $\quad$ initial value: $\$ 2 M / M W$ (by area) for Wind

- TCC $\quad$ initial value: $\$ 4 M / M W$ (by area) for CSP Solar

- TCC $\quad$ initial value: \$4M/MW (by area) for PV Solar

- TCF initial value: $\$ 160 / \mathrm{MW}$-mile-year (\$800/MW-mi, $20 \%$ capital recovery factor)

- TDF initial value: 0.7 (70\%, no dimensions)

- GDP initial value: 0.0005 ( $5 \%$ per 100 miles)

- LDF initial value: 0.0001 (1\% per 100 miles) 


\section{Appendix B Statistical Trend Analysis Plots}

\section{B.1 Monthly Energy}

\section{Footprint Monthly Energy from Wind and Solar by Year}
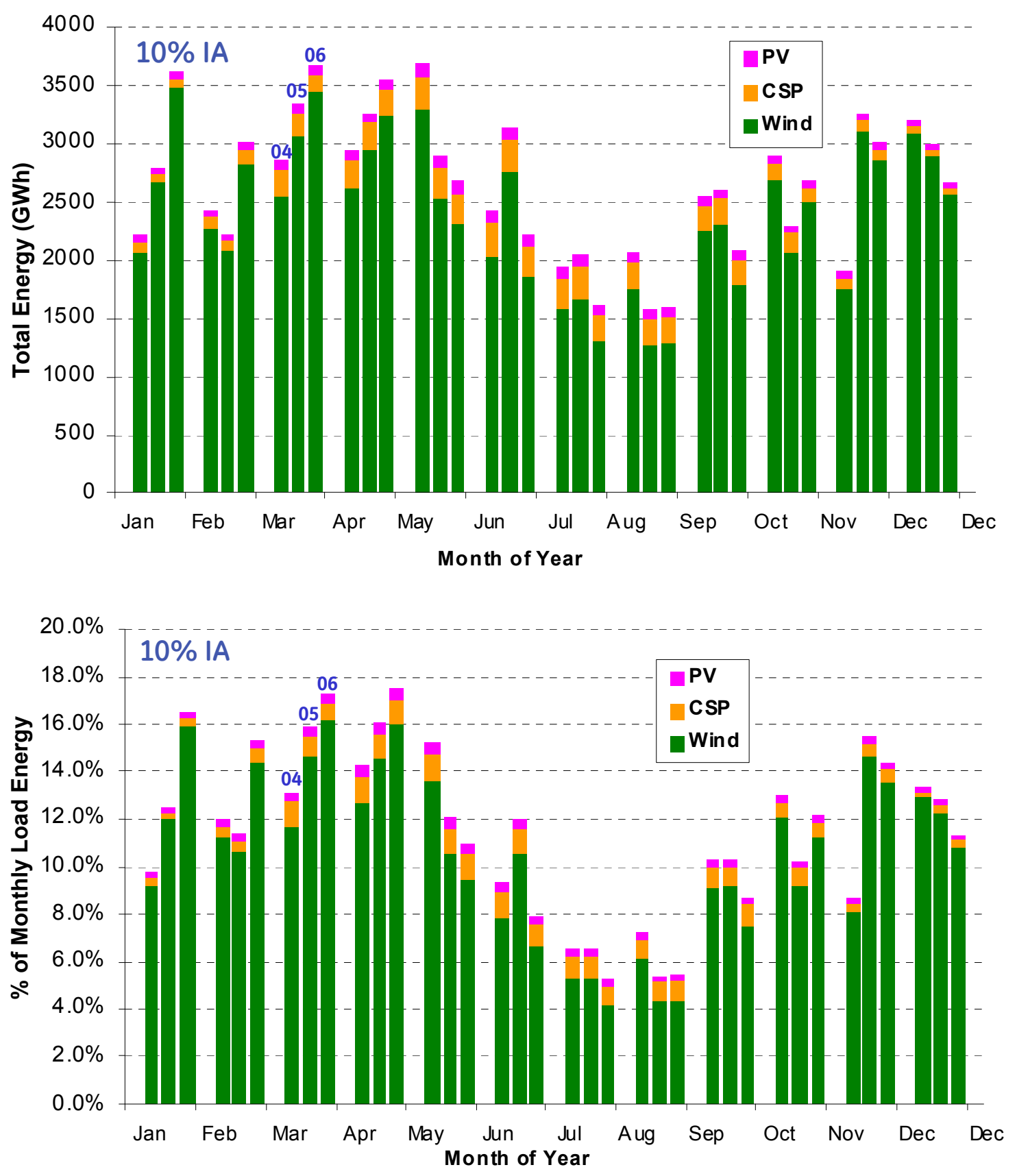

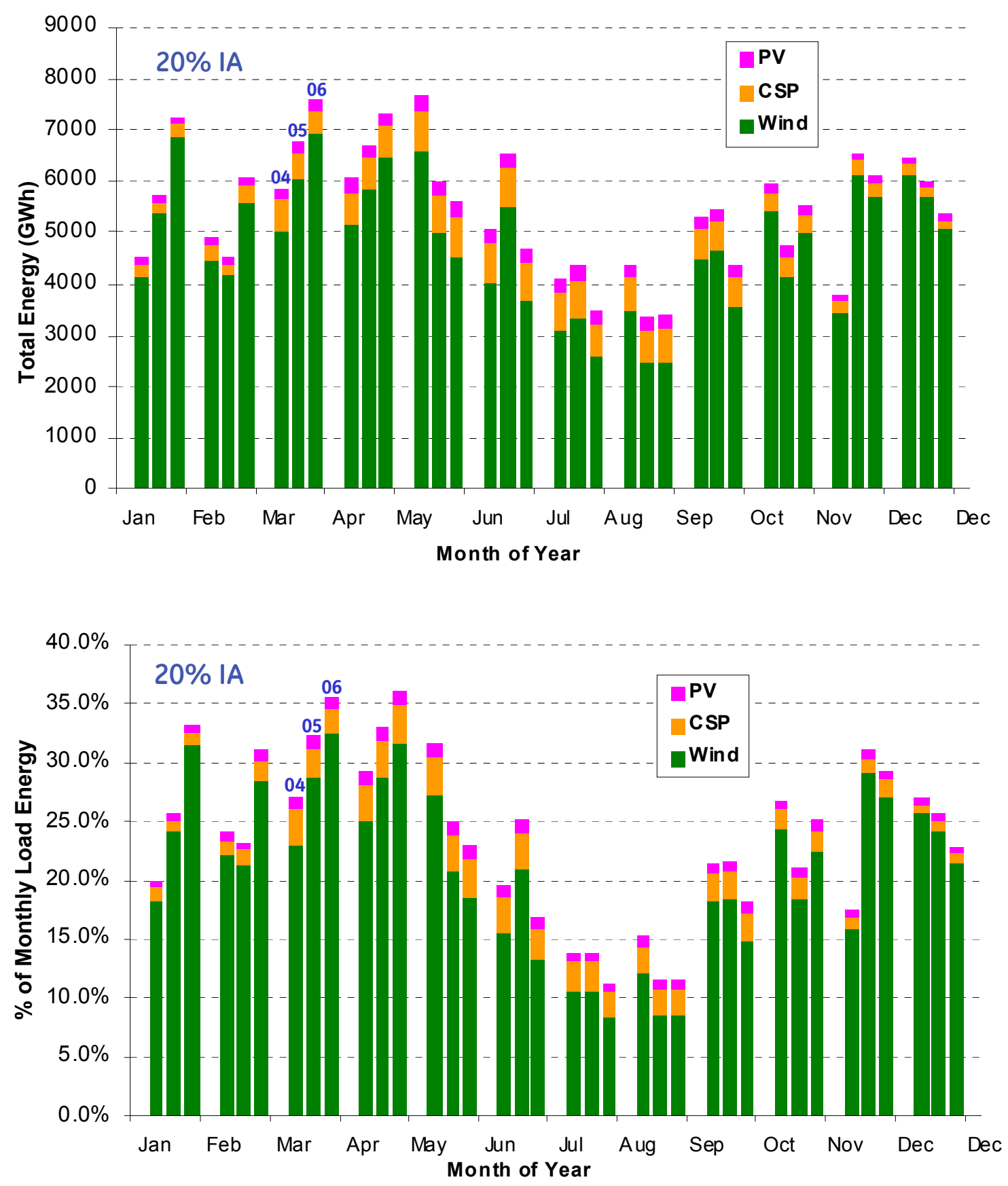
2006 Footprint Monthly Energy from Wind and Solar, 30\% Penetration

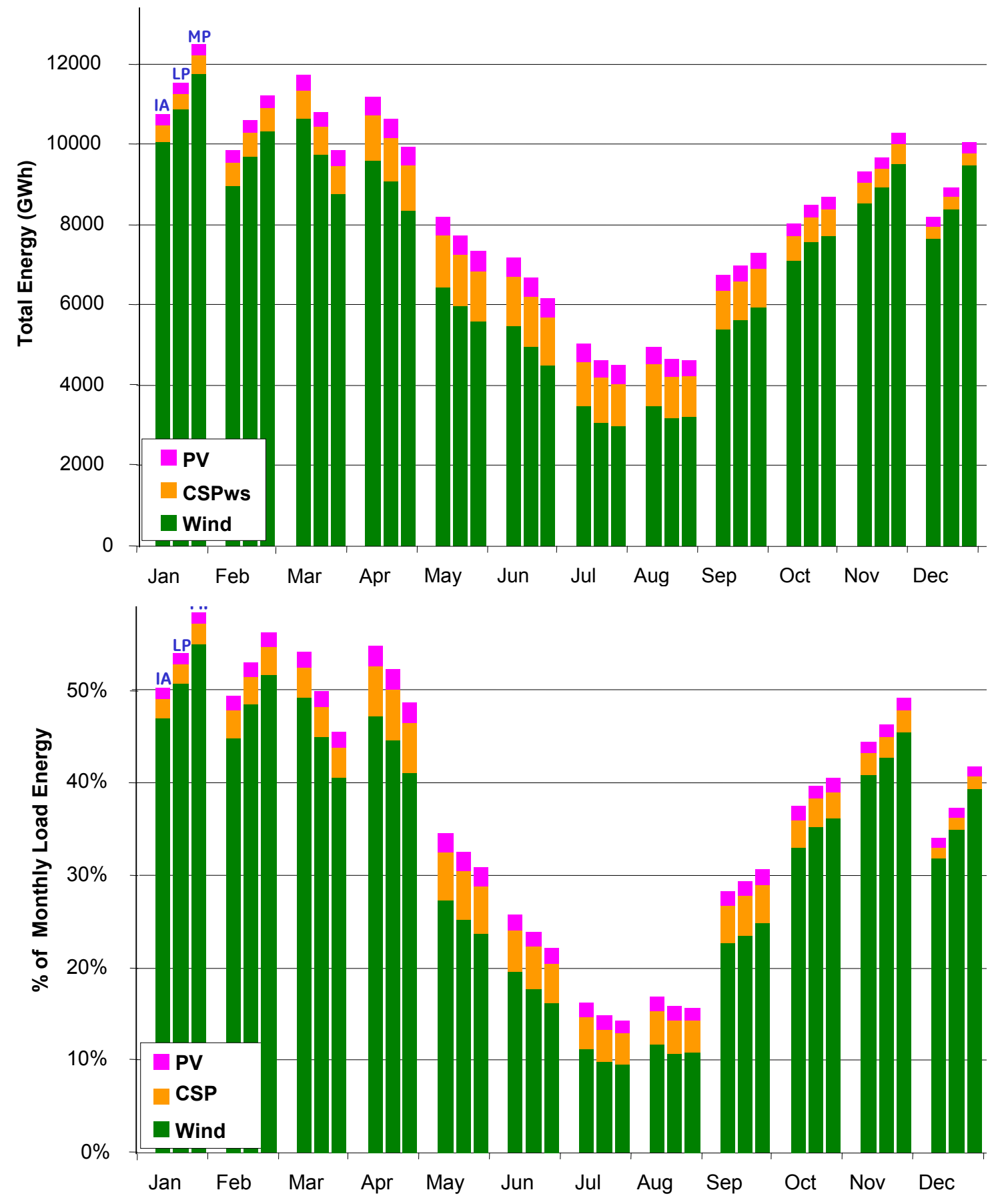


2006 Monthly Energy from Wind and Solar (30\% In-Area Scenario)
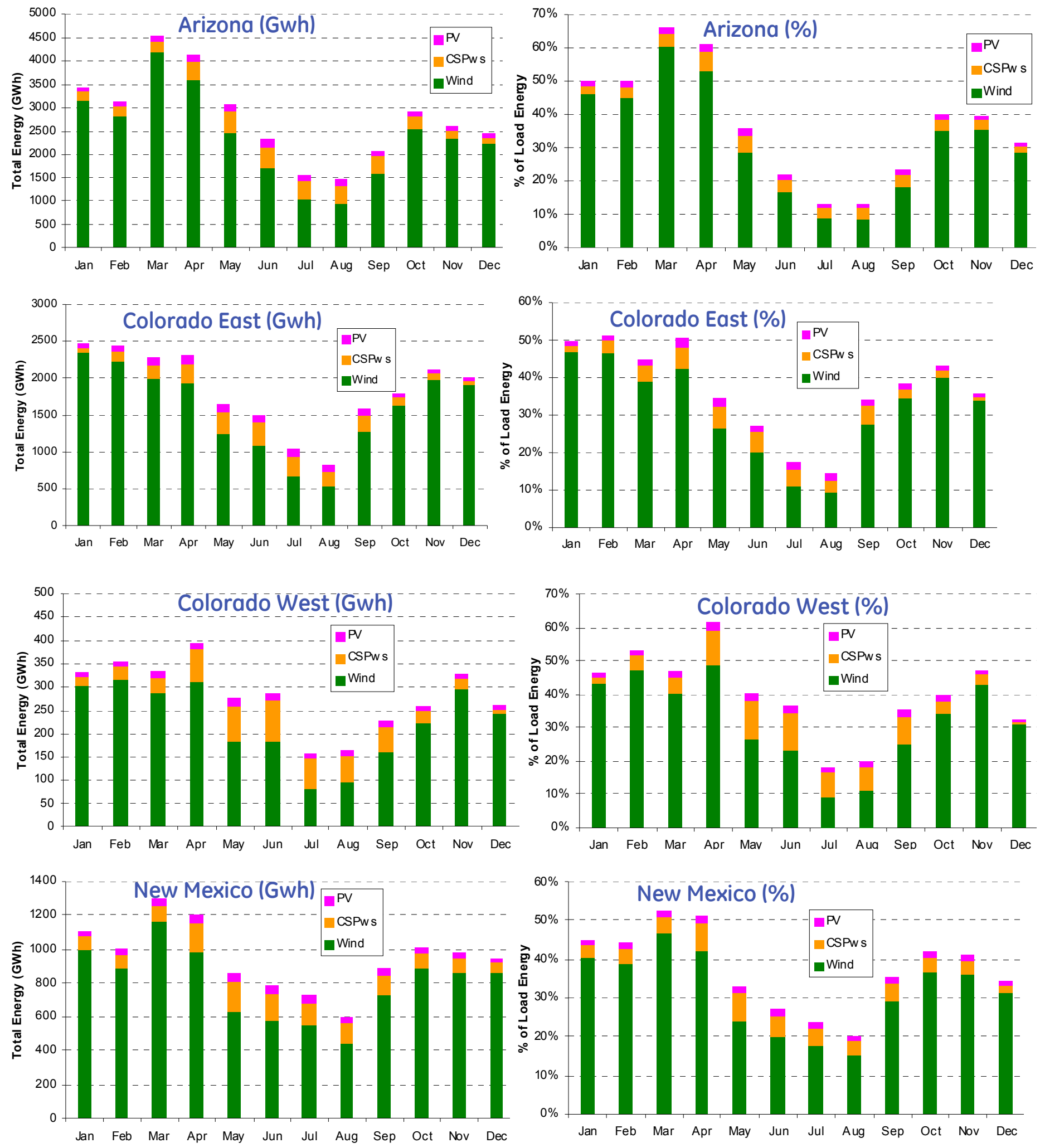

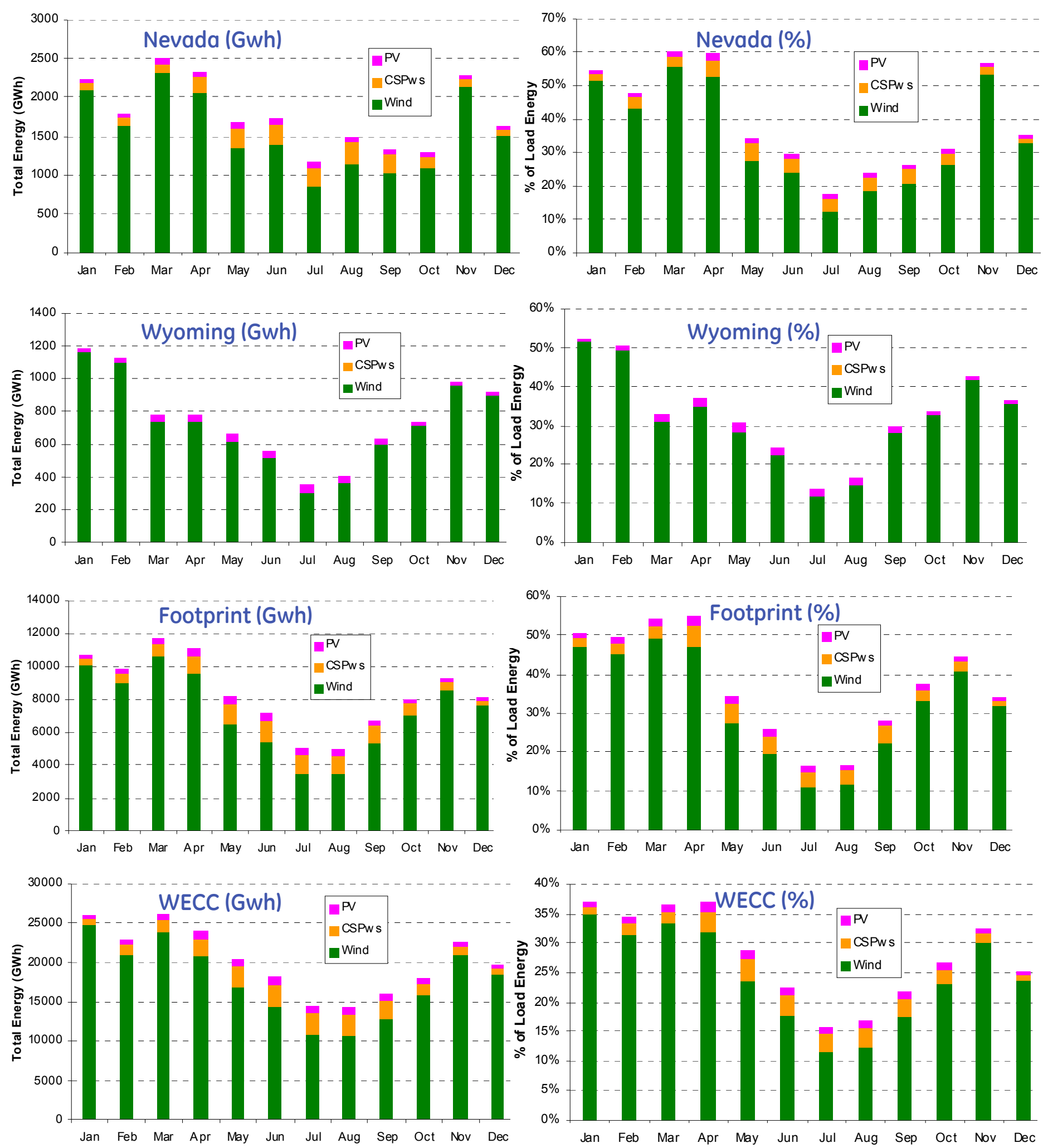


\section{Monthly Energy from Wind and Solar (30\% Local-Priority Scenario)}
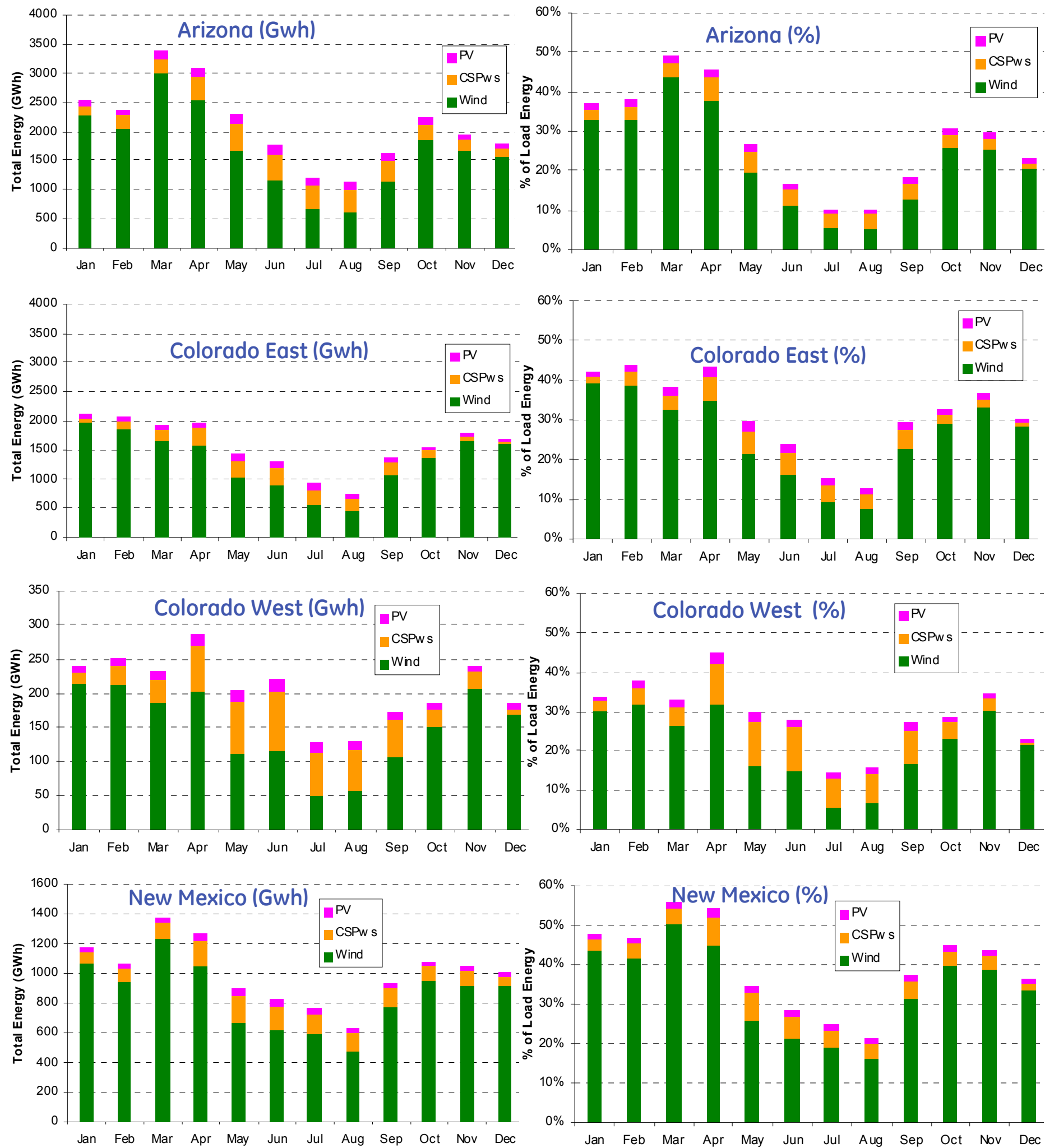

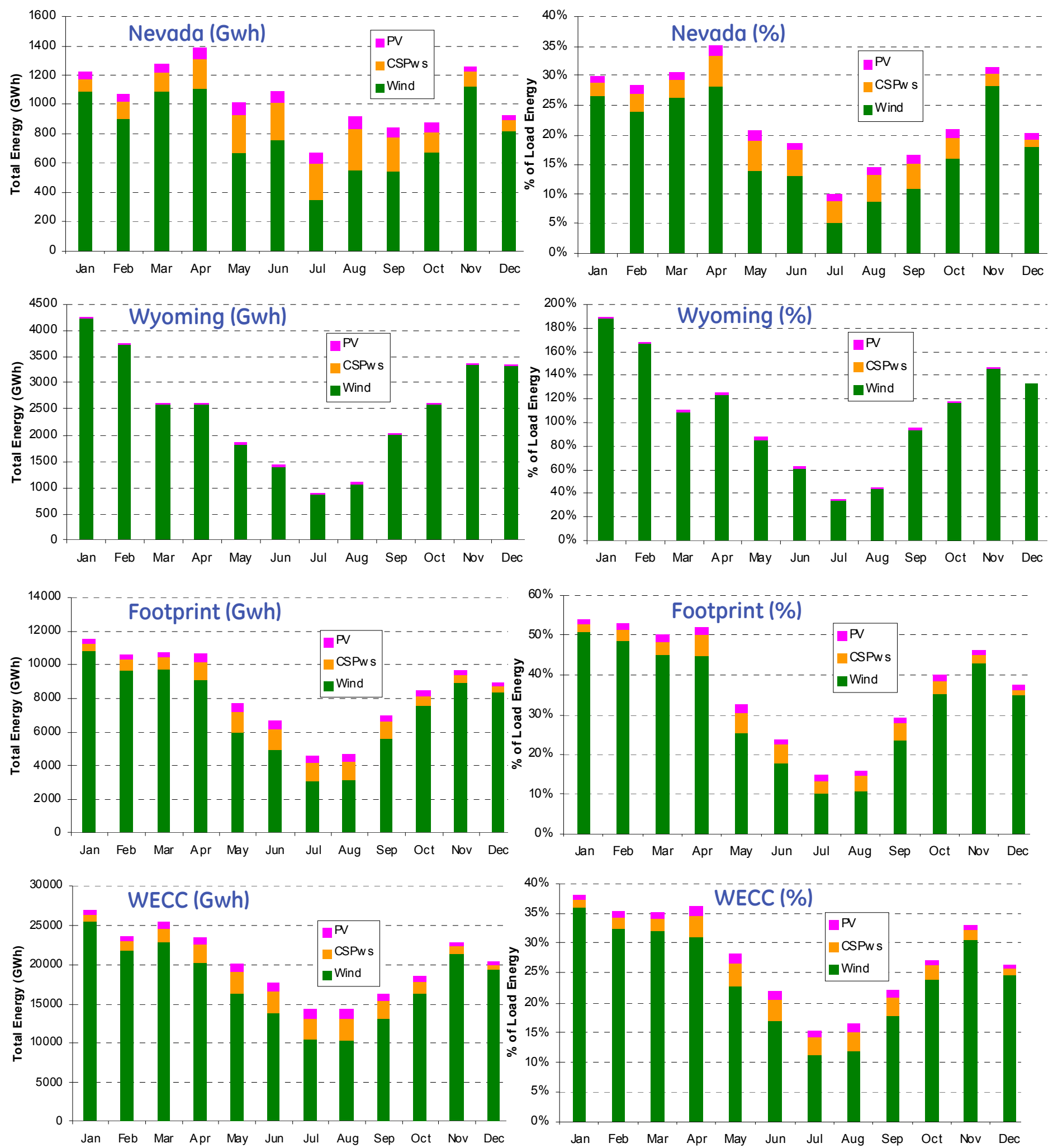
2006 Monthly Energy from Wind and Solar (30\% Mega-Project Scenario)
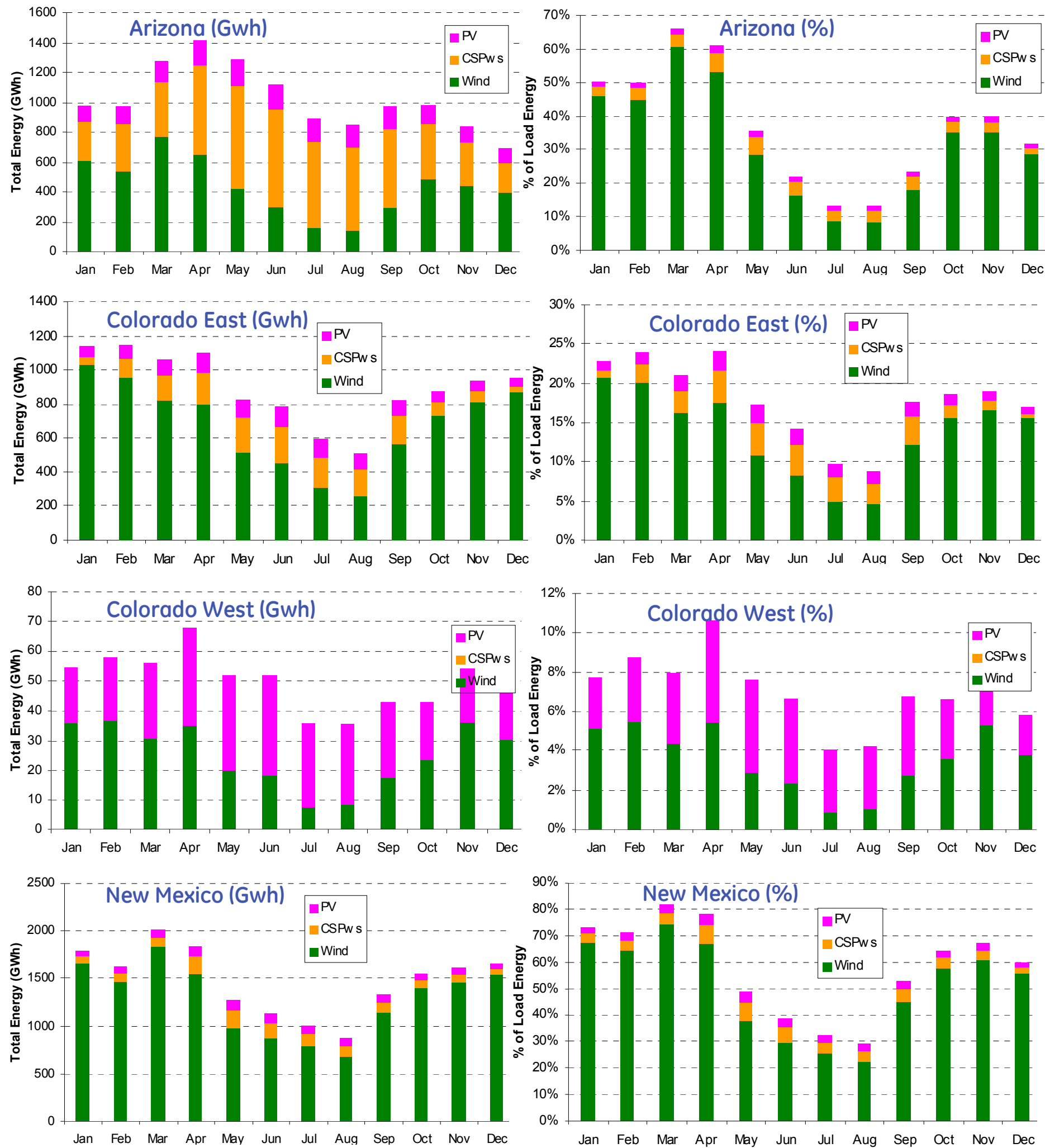

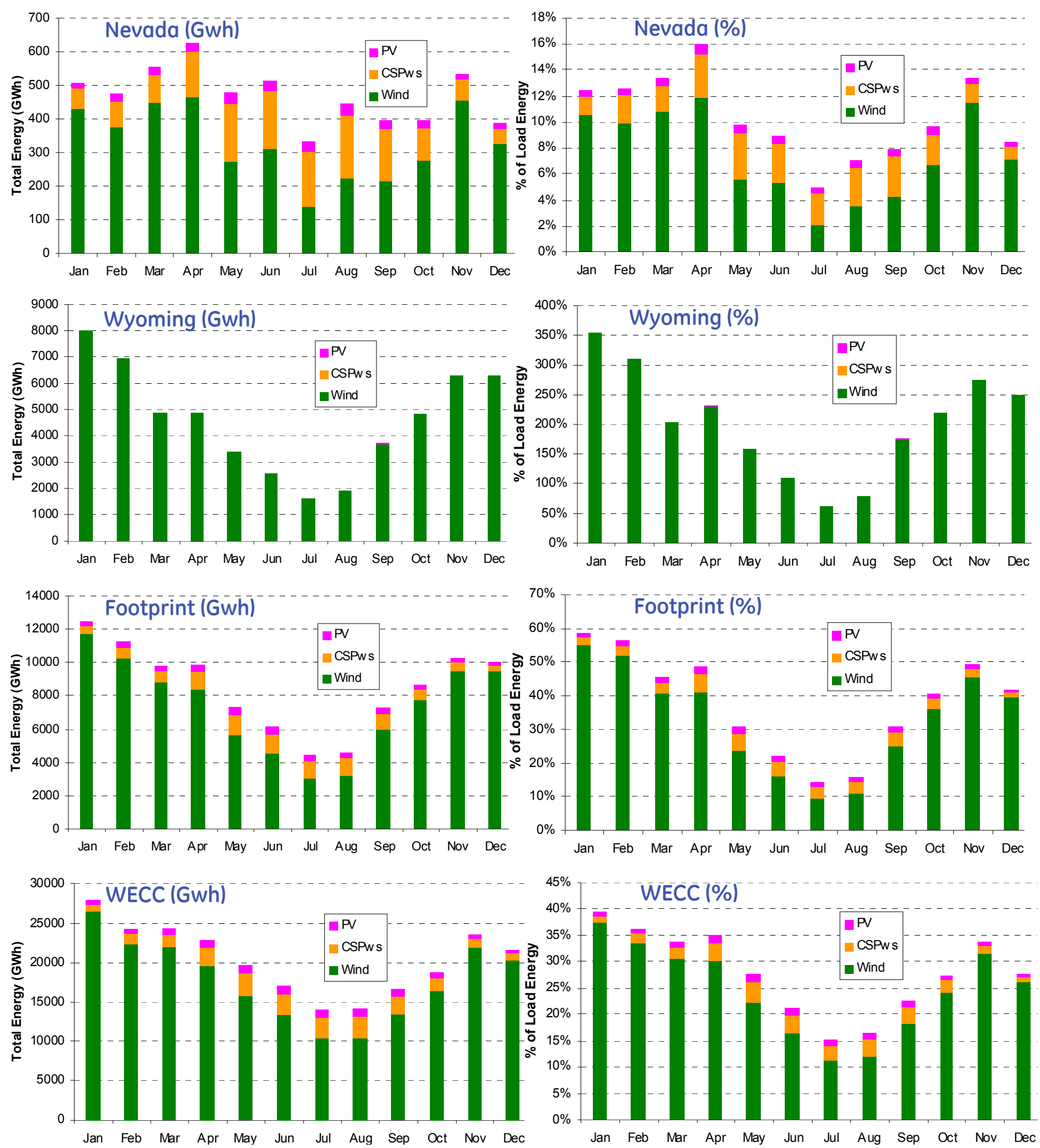


\section{B.2 Yearly Duration Plots}

Study Footprint 2006 Wind Duration Plots
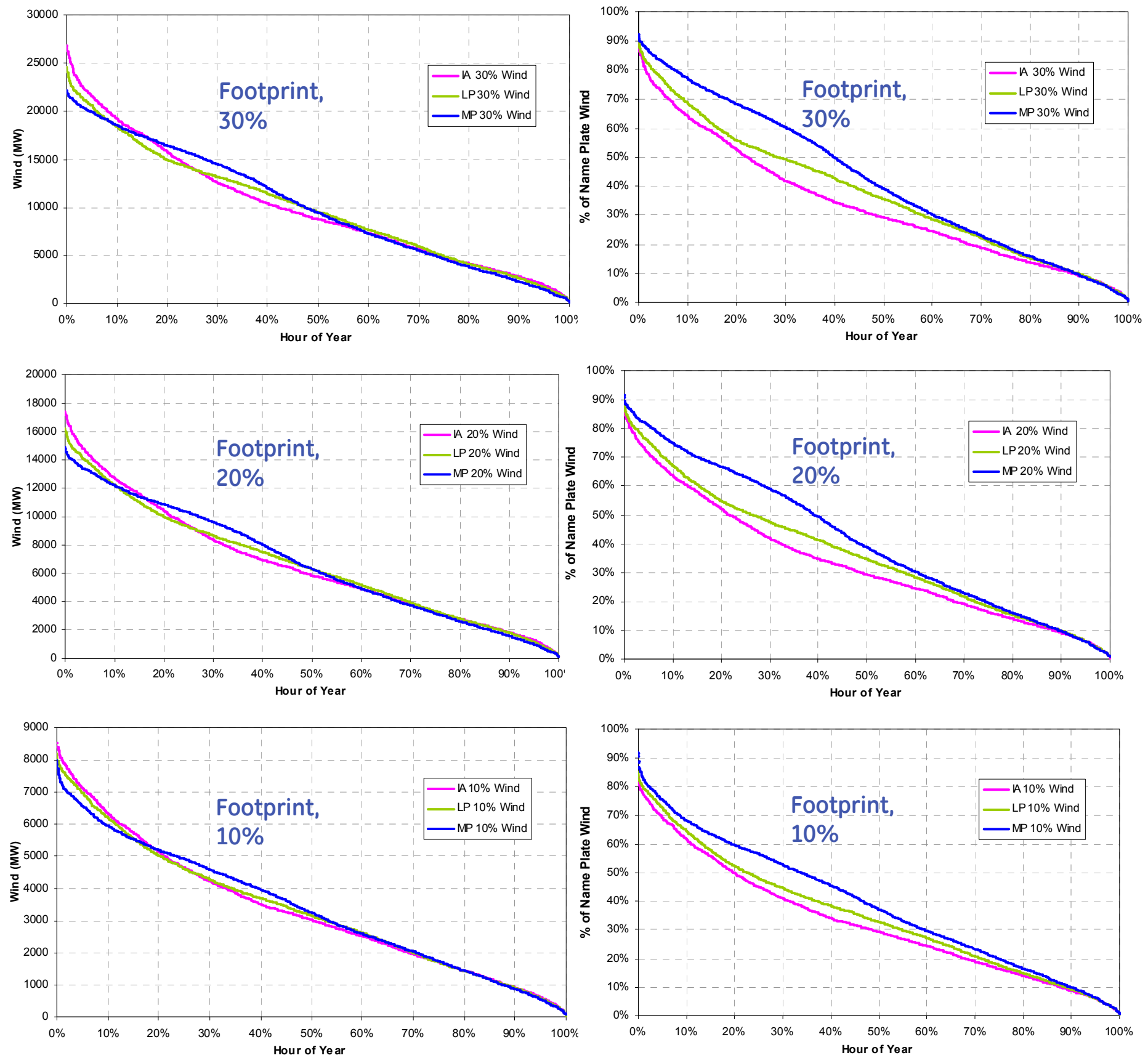

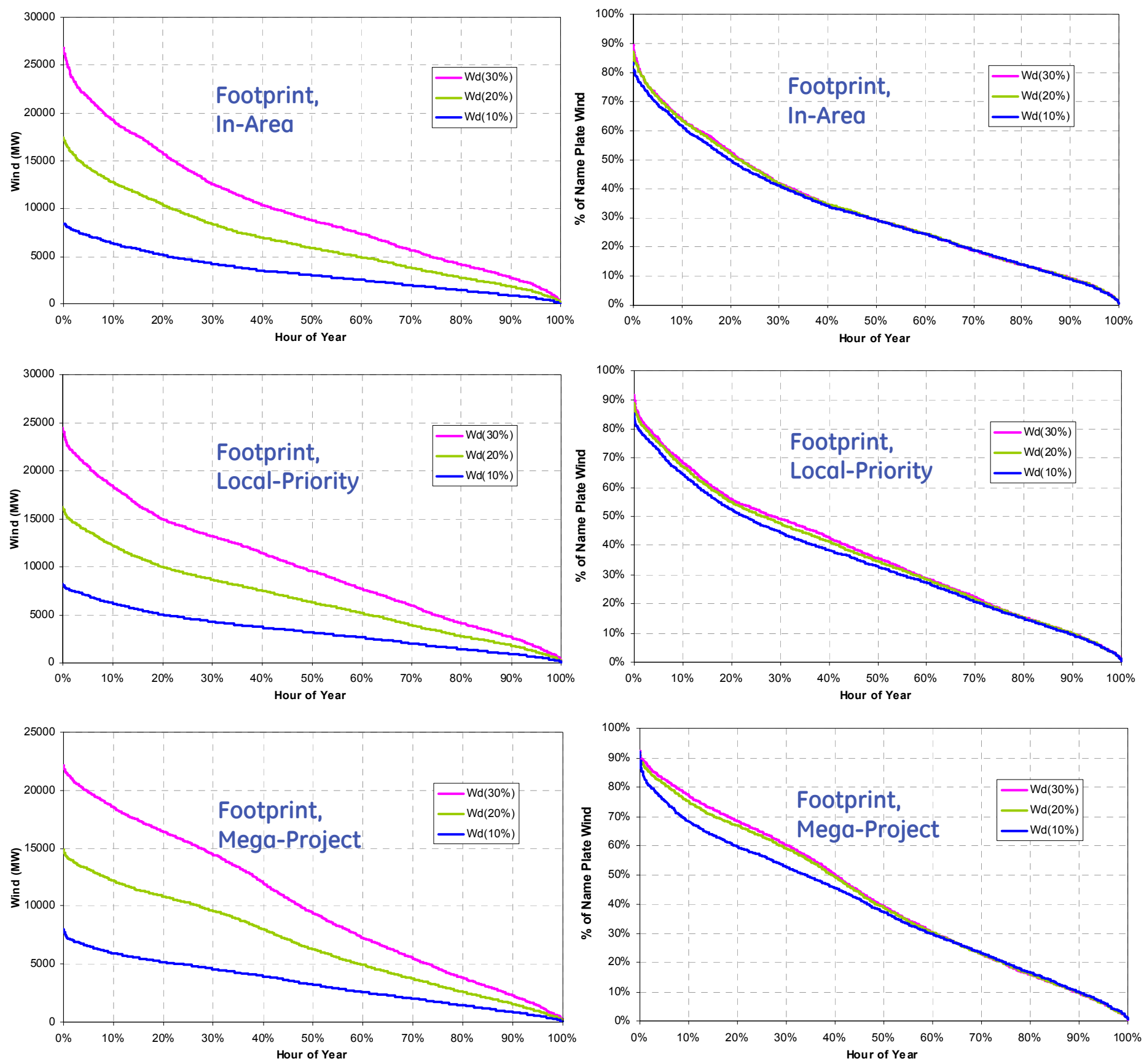


\section{In-Footprint State 2006 Wind Duration Plots}
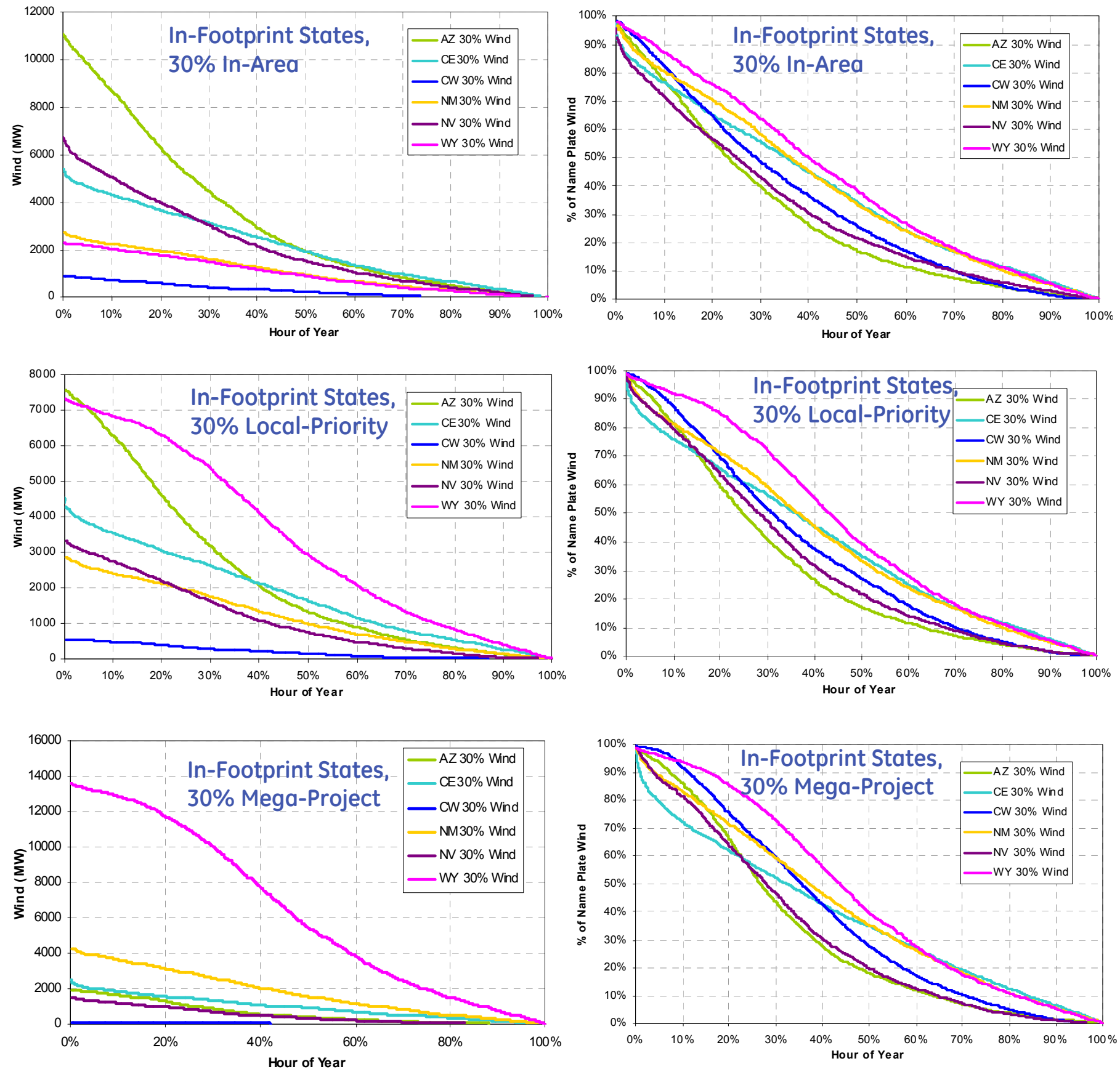


\section{Solar Duration and Penetration}
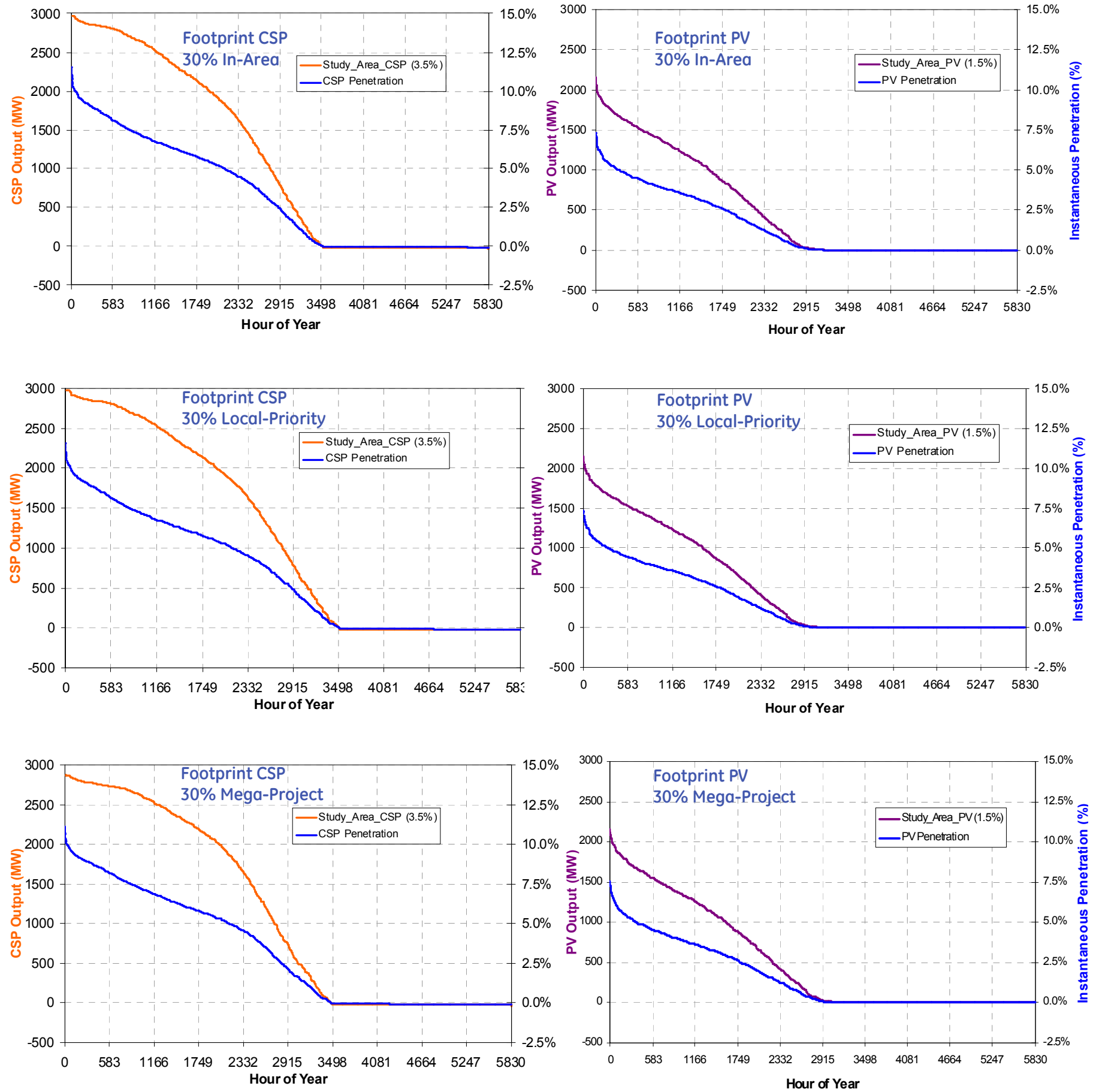


\section{Study Footprint 2006 Net Load Duration}
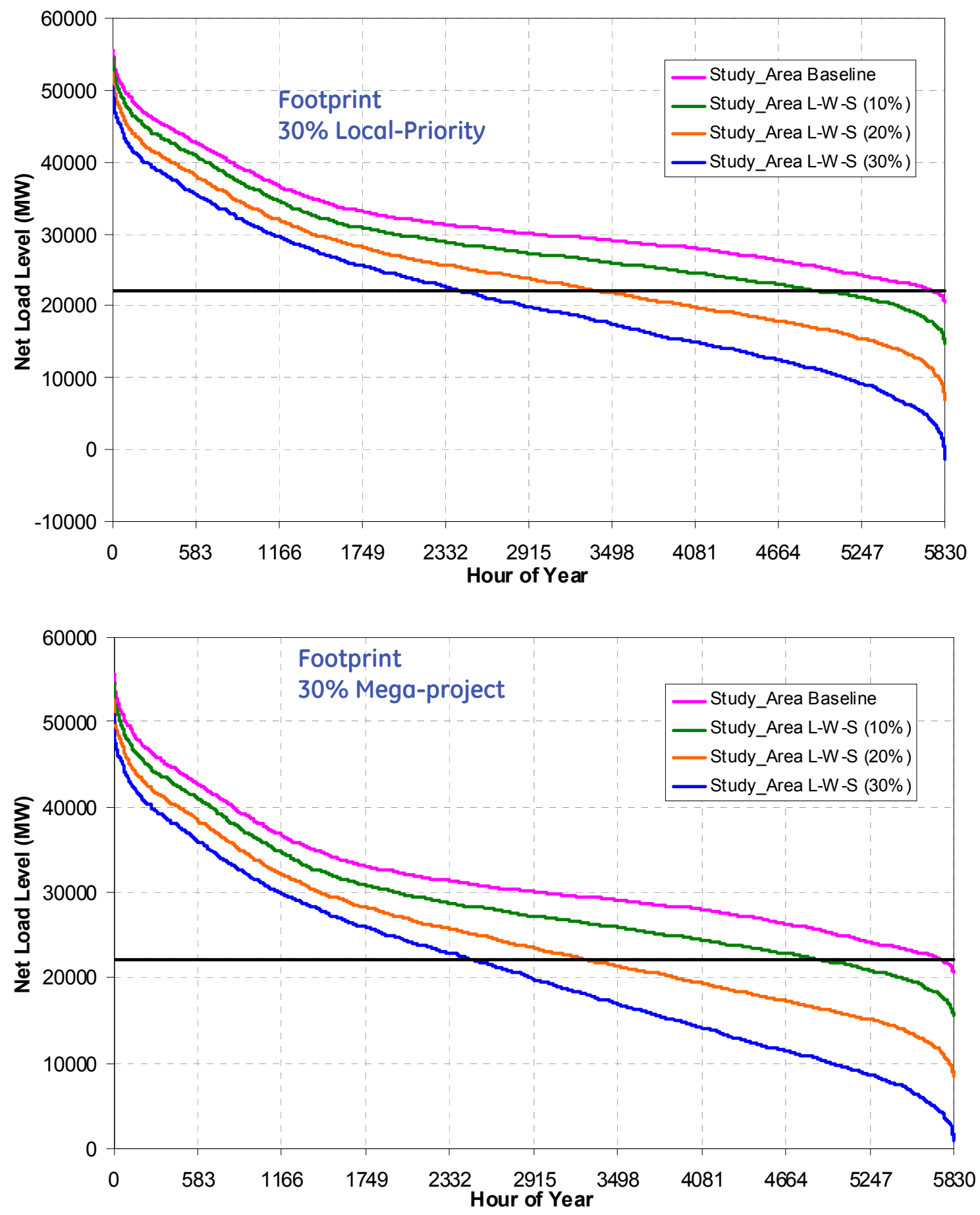
In-Footprint States 2006 Net Load Duration
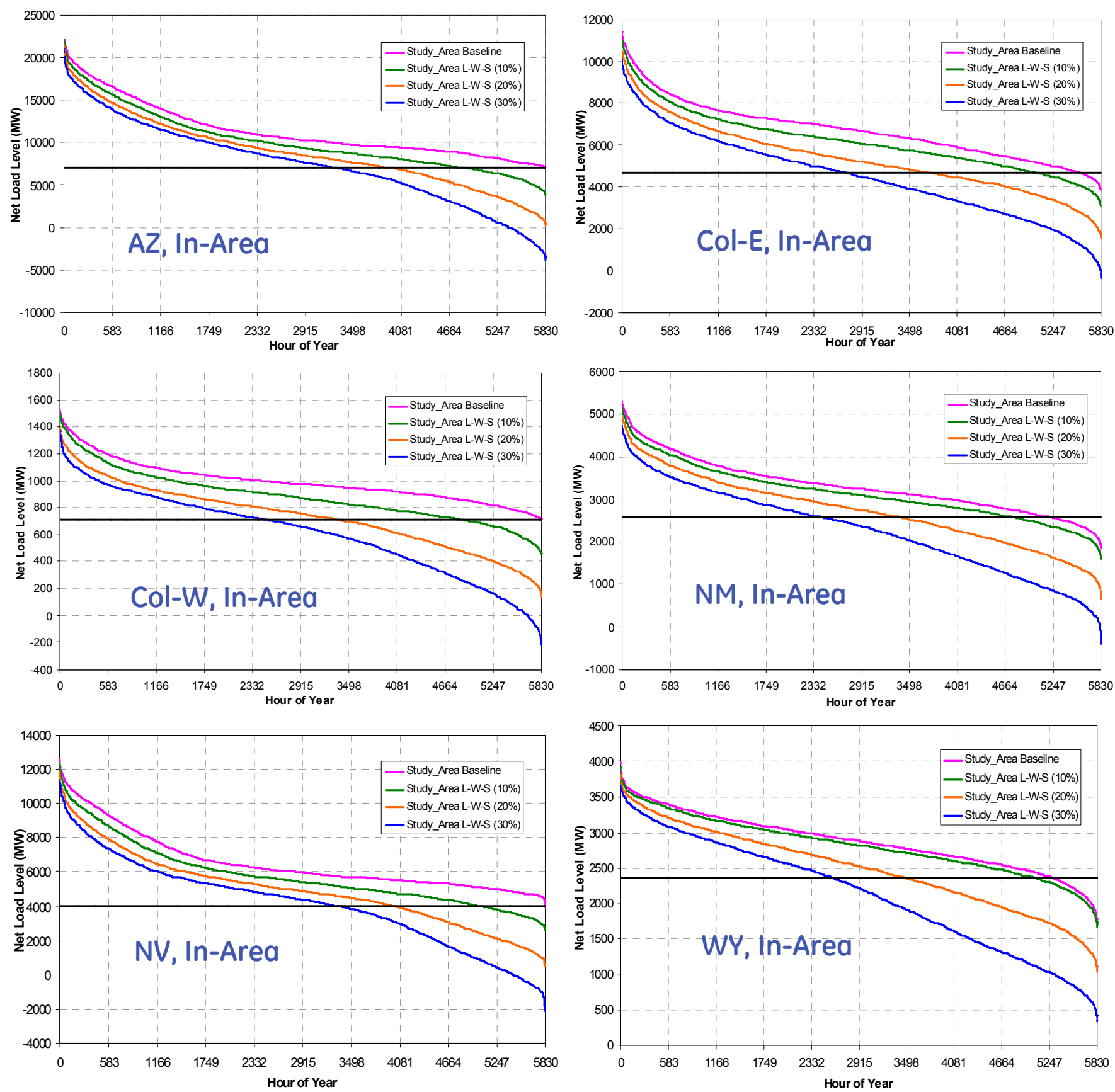

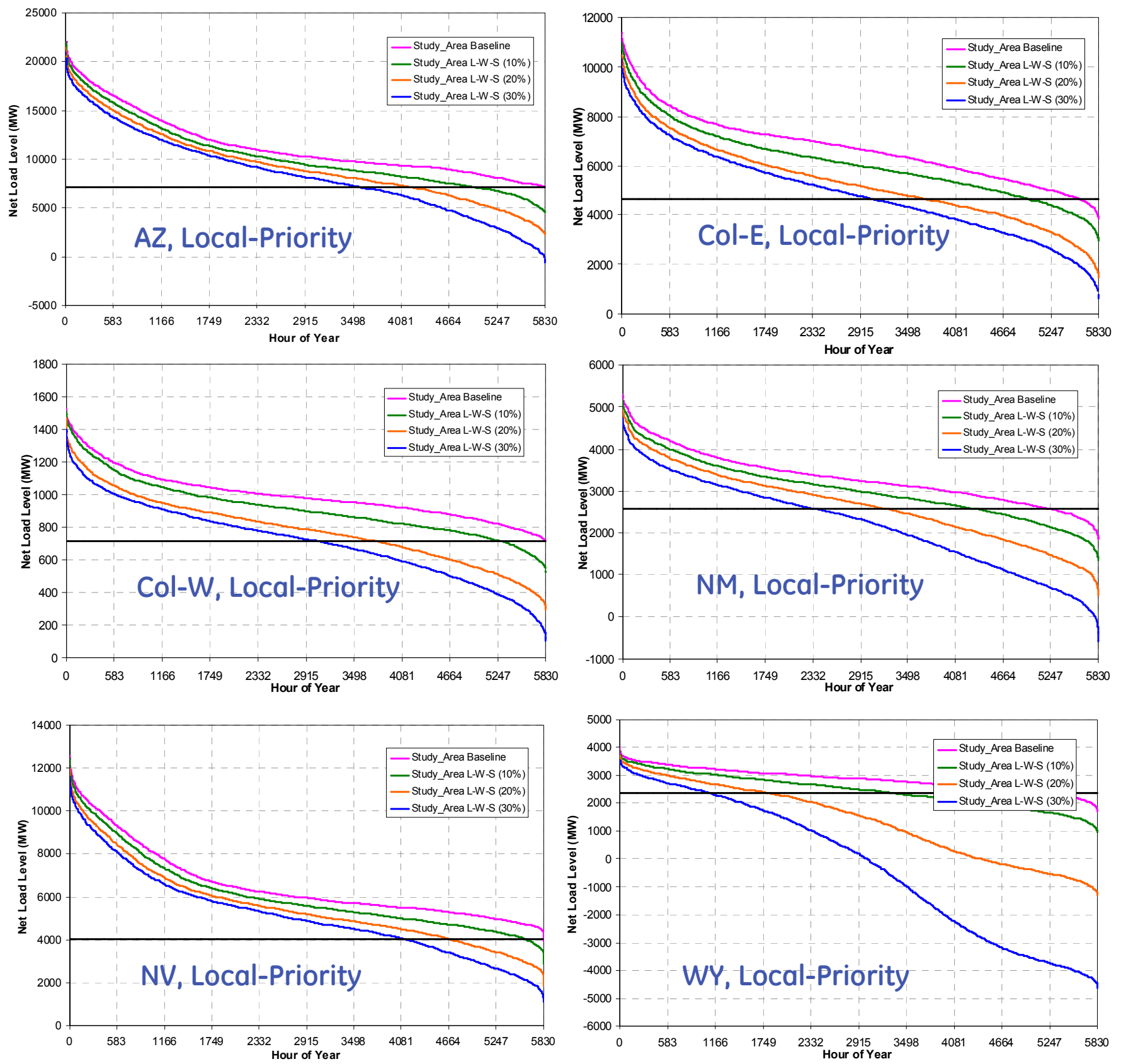

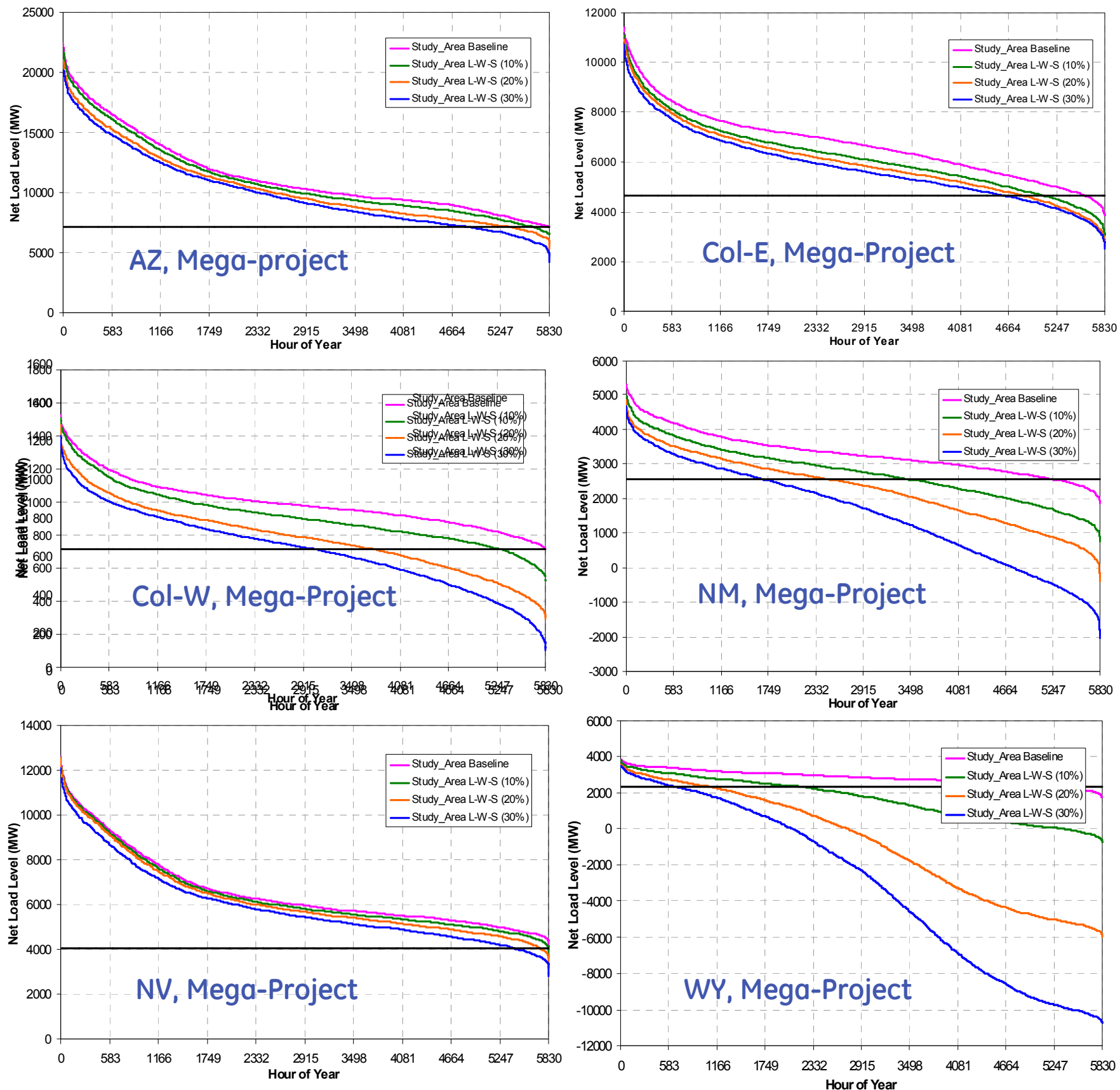
2006 Study Footprint Average Daily Profiles by Seasonal Month
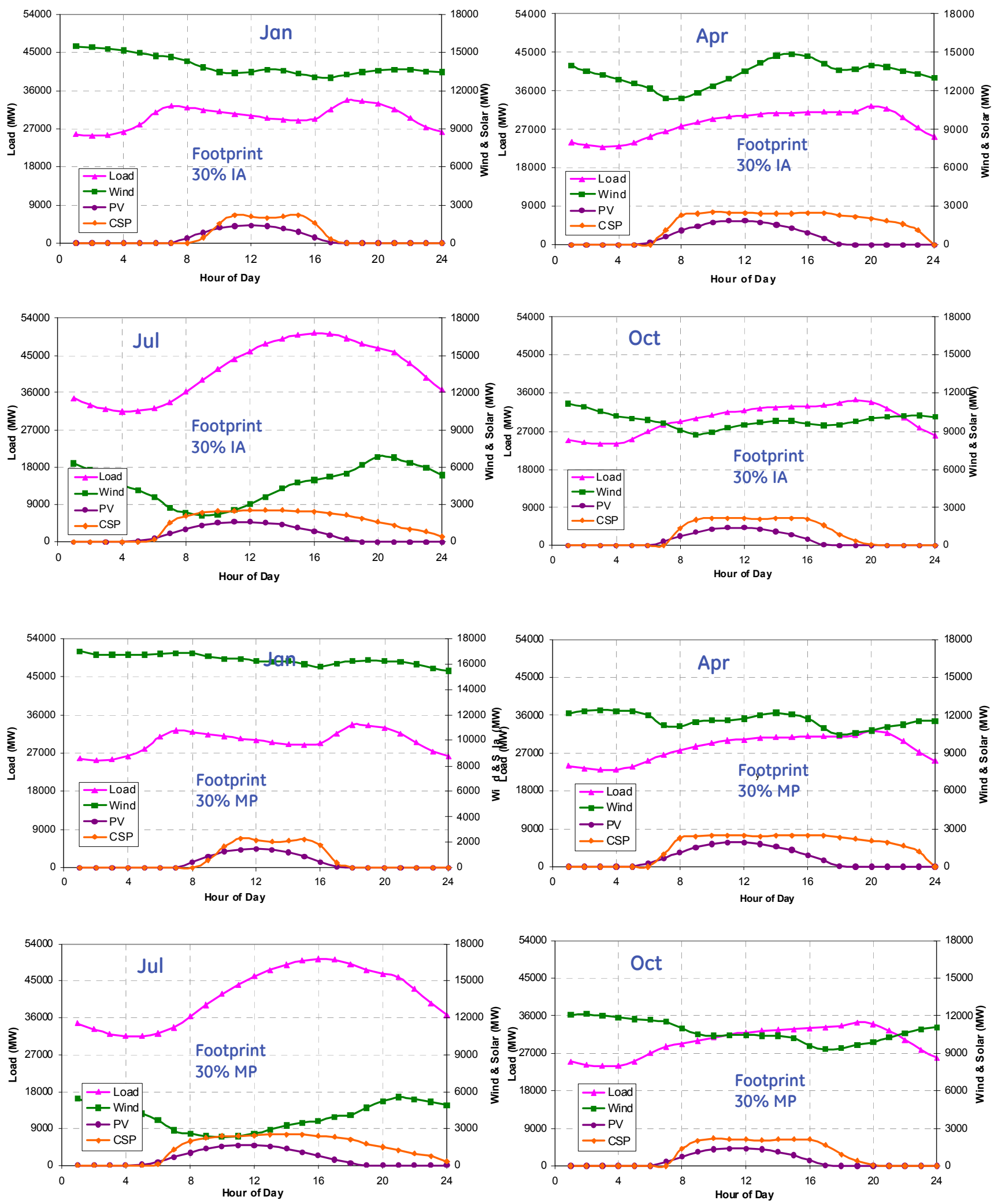


\section{B.3 Time Series Plots}

\section{Study Footprint July Week, In-Area Scenario}
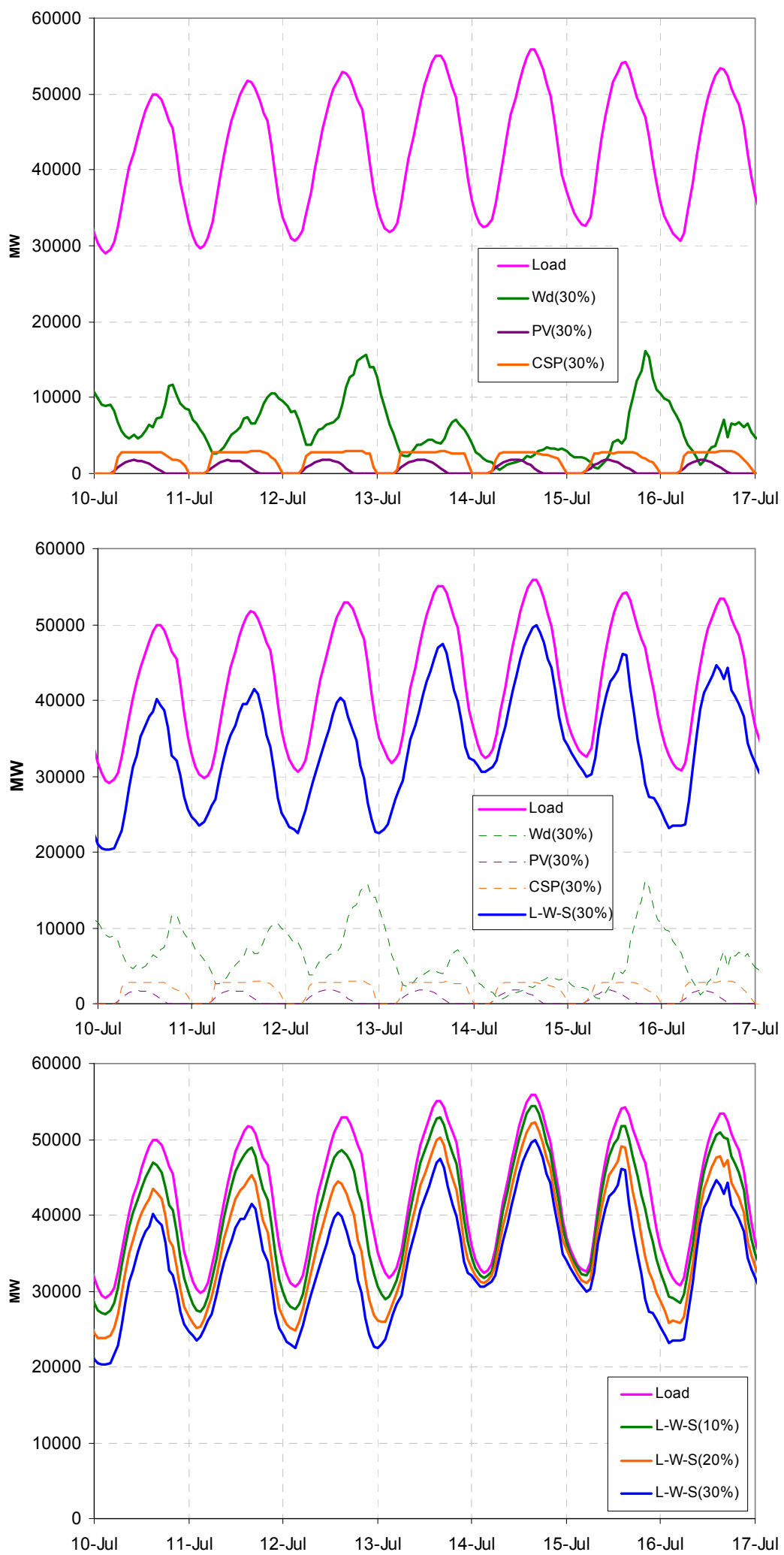

B-19 
Study Footprint April Week, In-Area Scenario
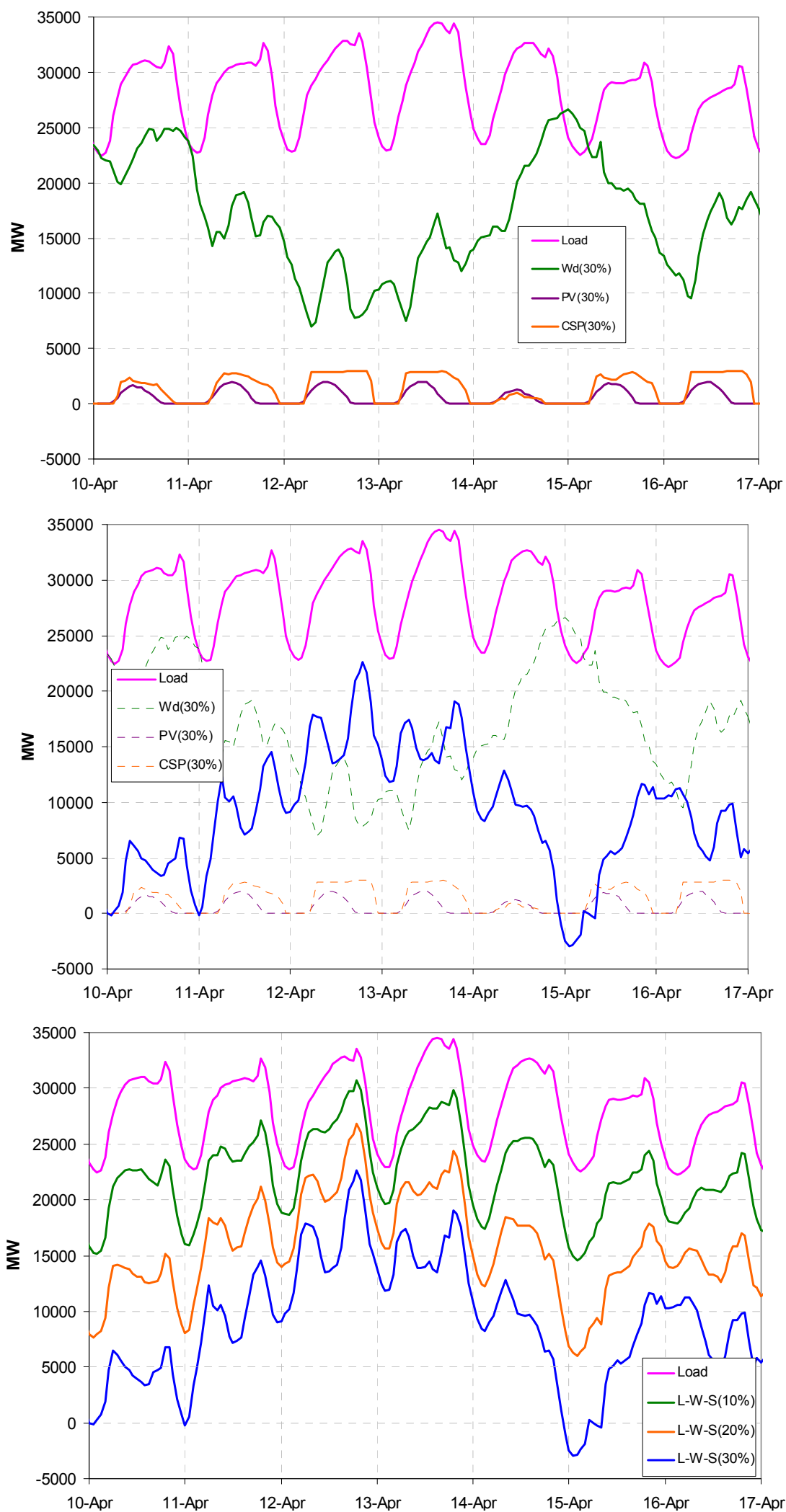
July and April Monthly Series, In-Area Scenario
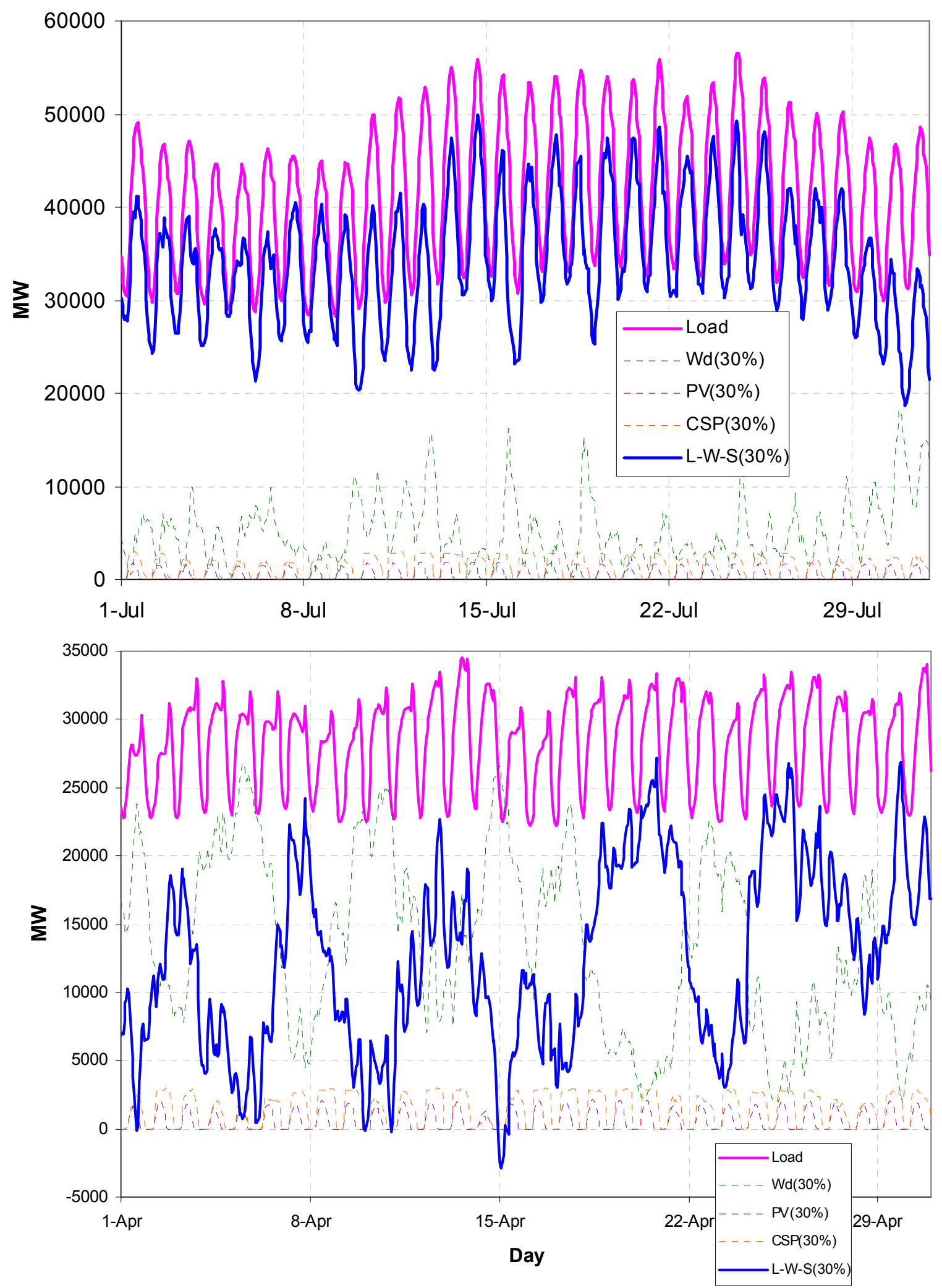



\section{Appendix C Statistical Delta Analysis Plots}

\section{C.1 Load-Wind Delta Scatter Plots}
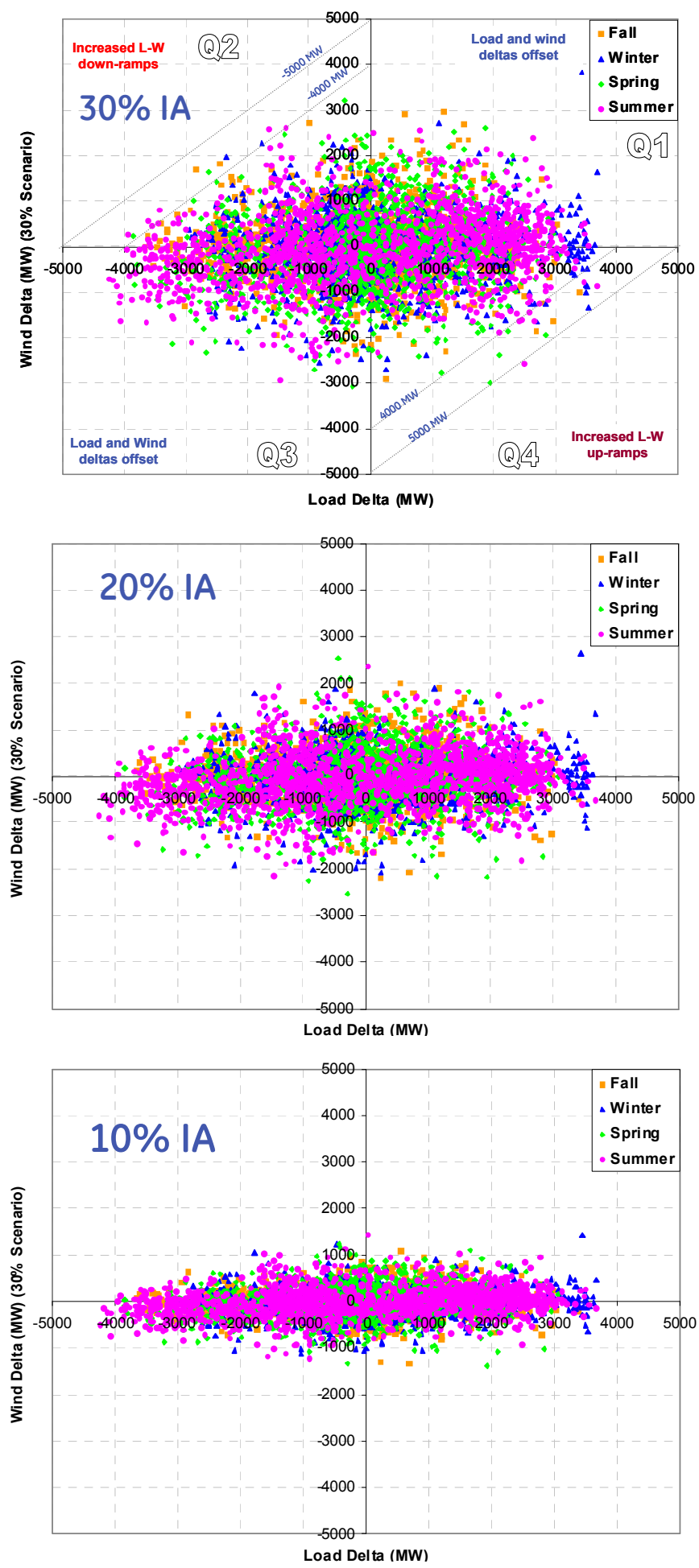

C-1 

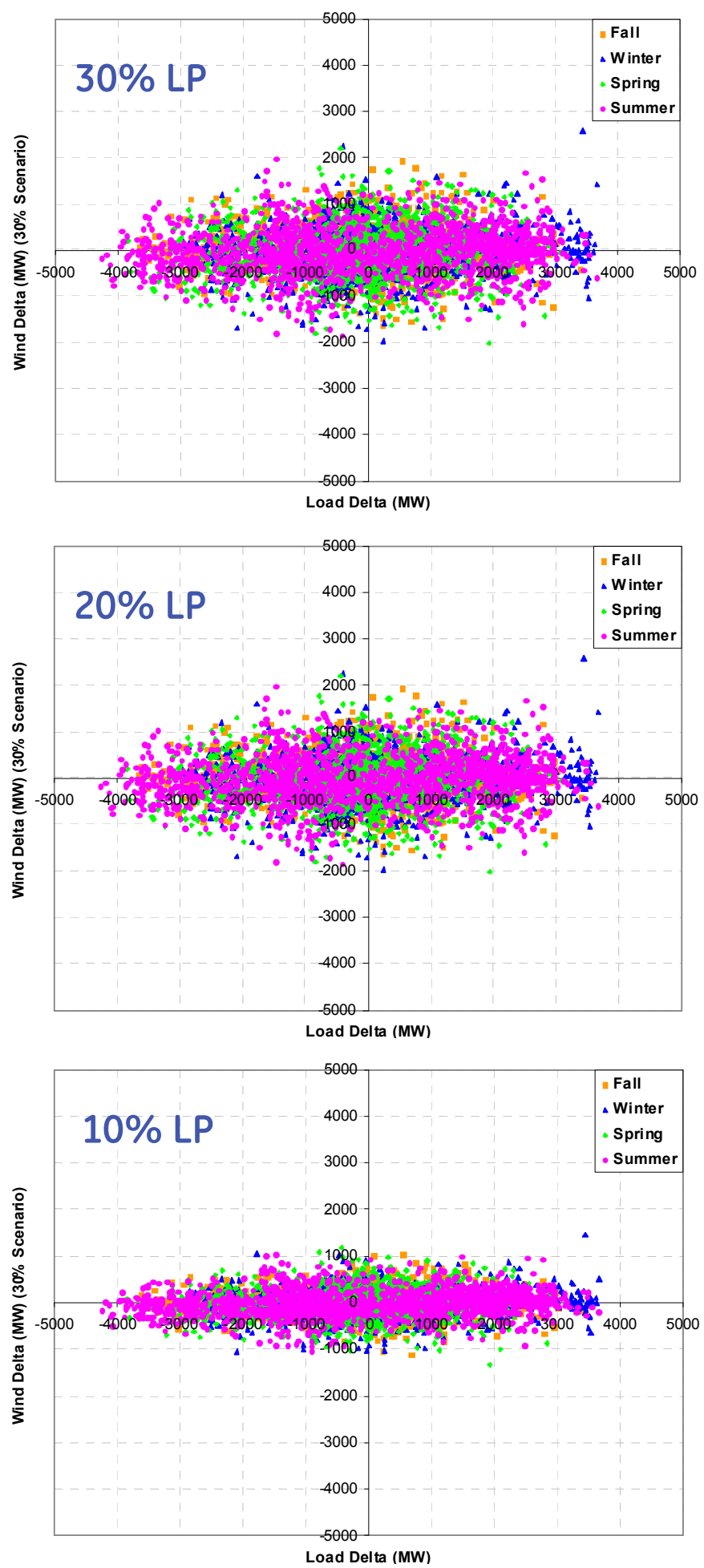

C-2 

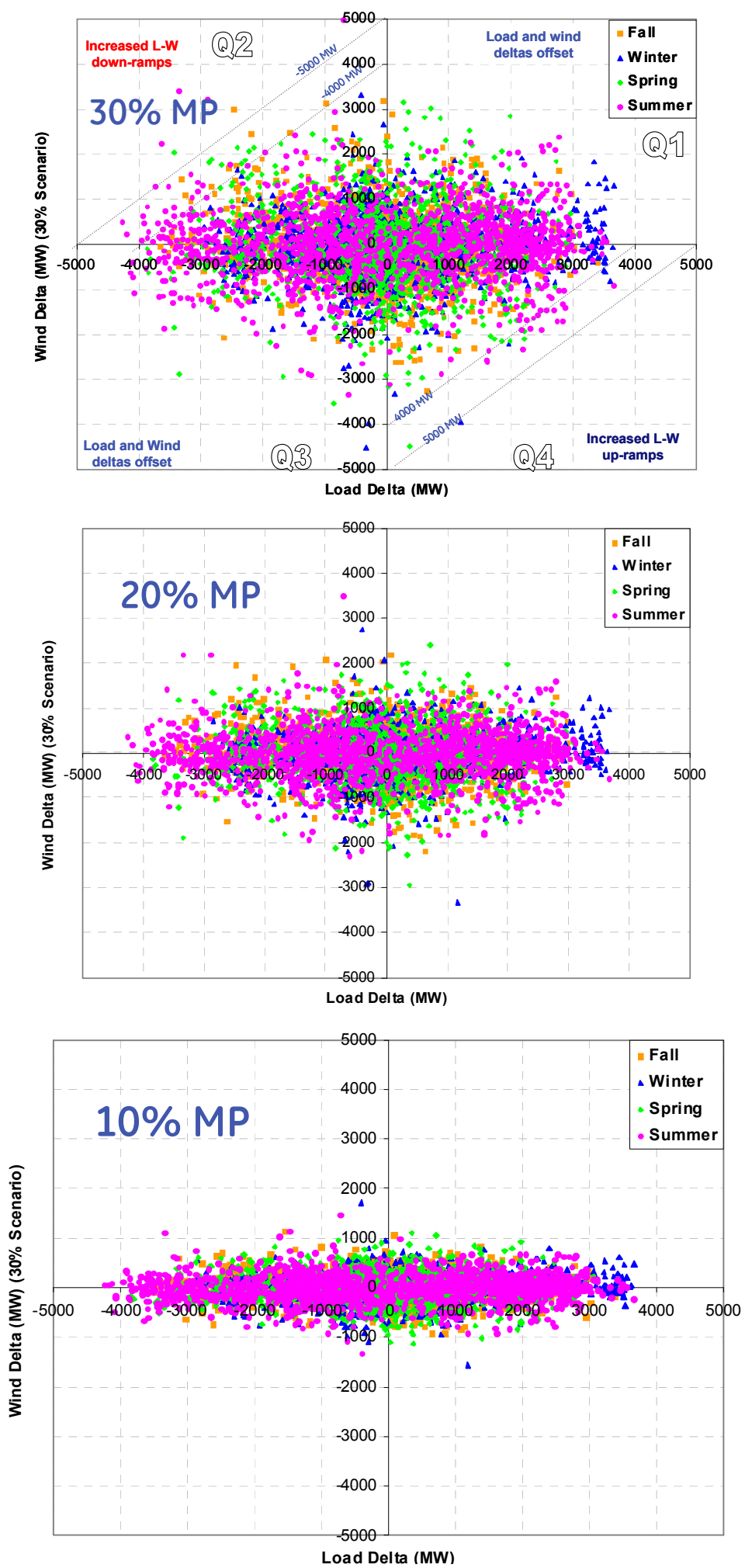

C-3 


\section{C.2 Net Load Variability by Hour-of-Day Plots}

\section{Study footprint Average Daily Profile of Deltas for Year 2006}
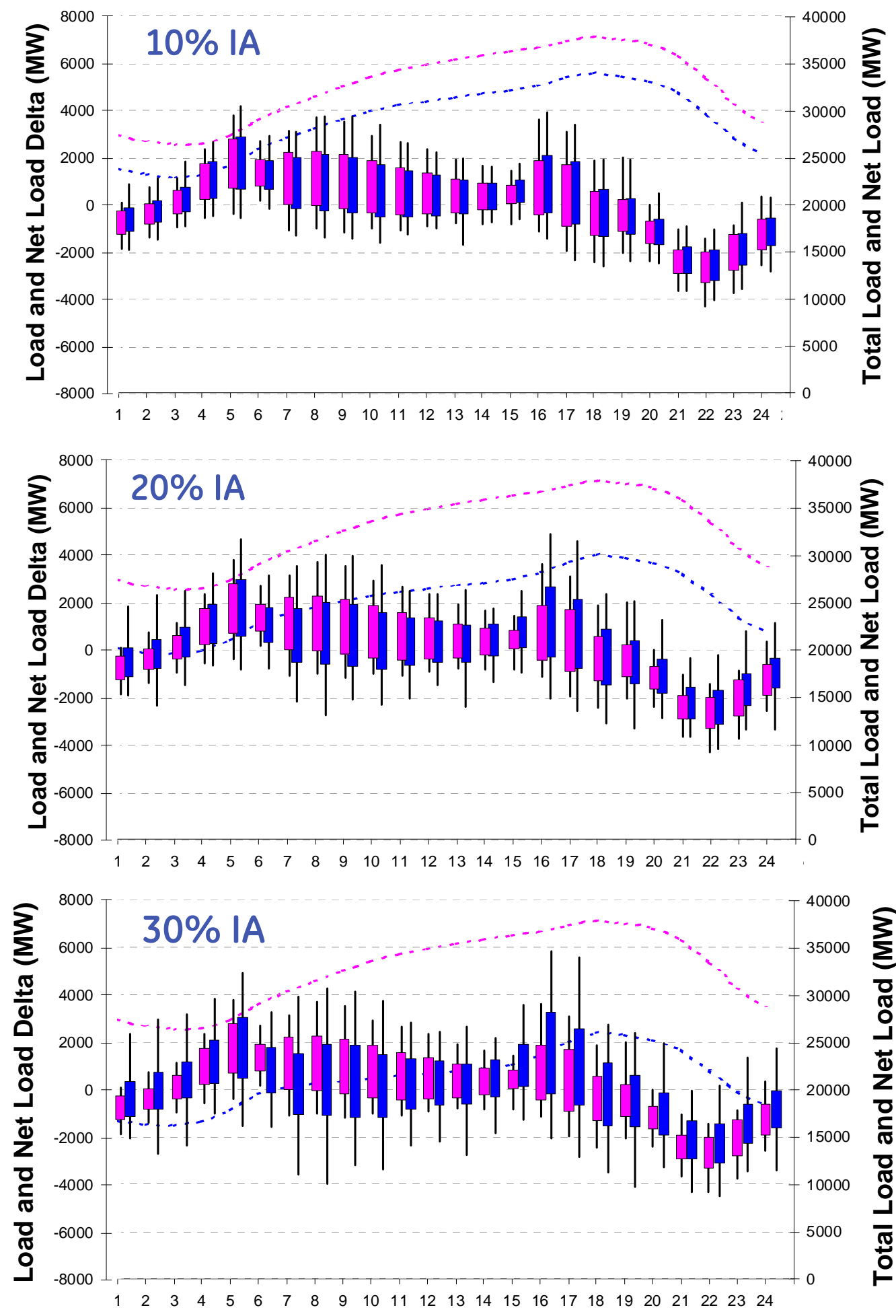

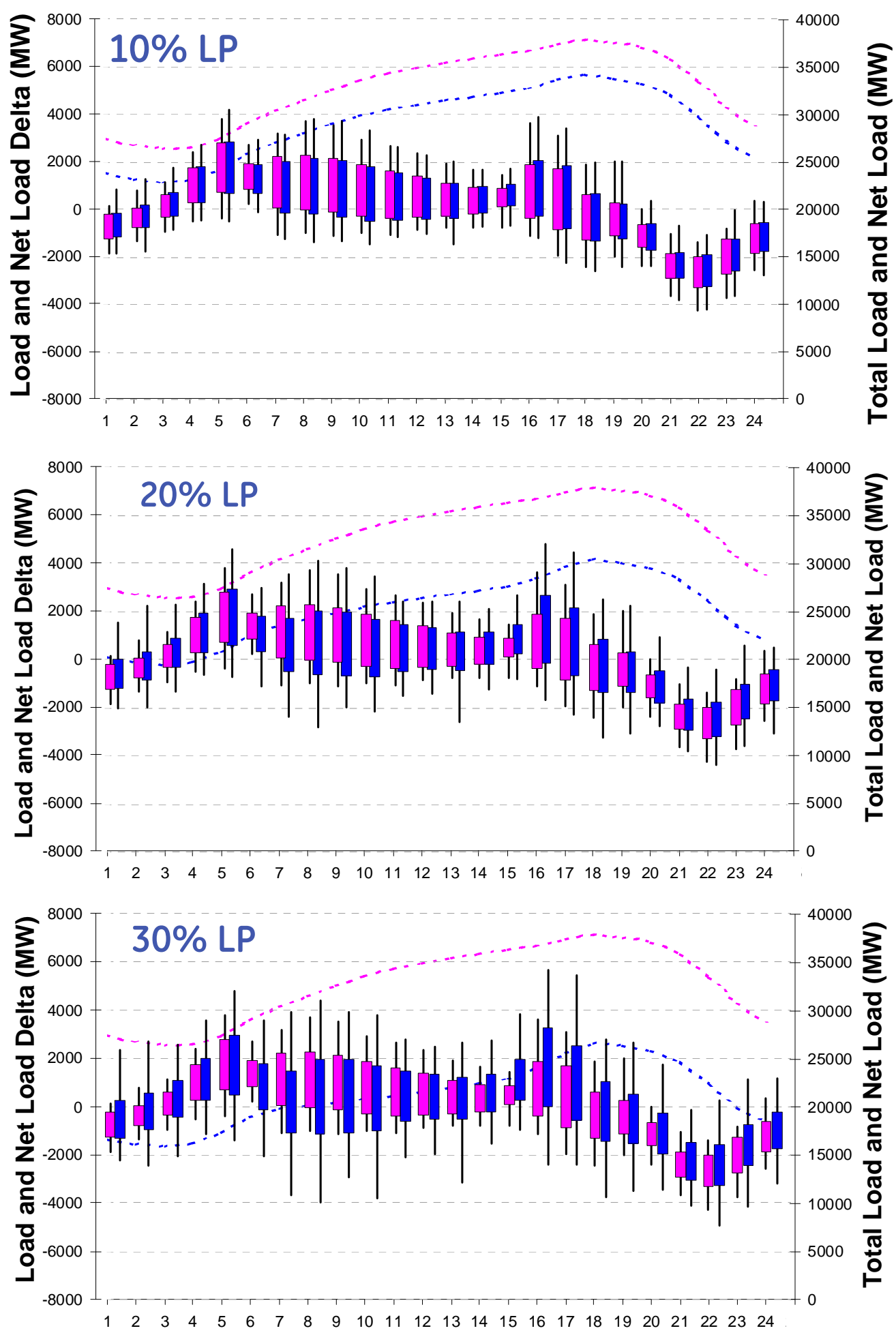

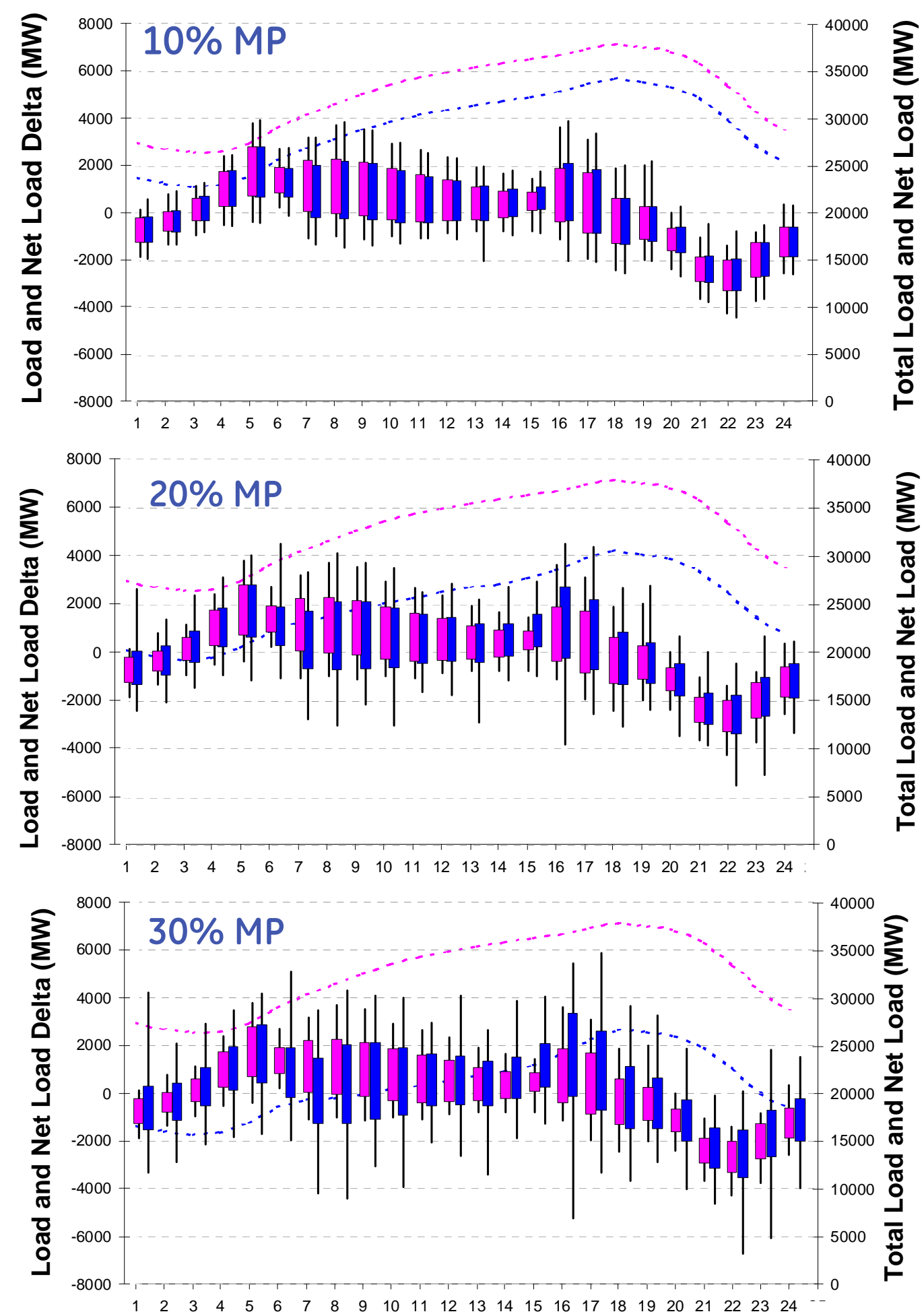
Study footprint Average Daily Profile of Deltas by 2006 Seasonal Month
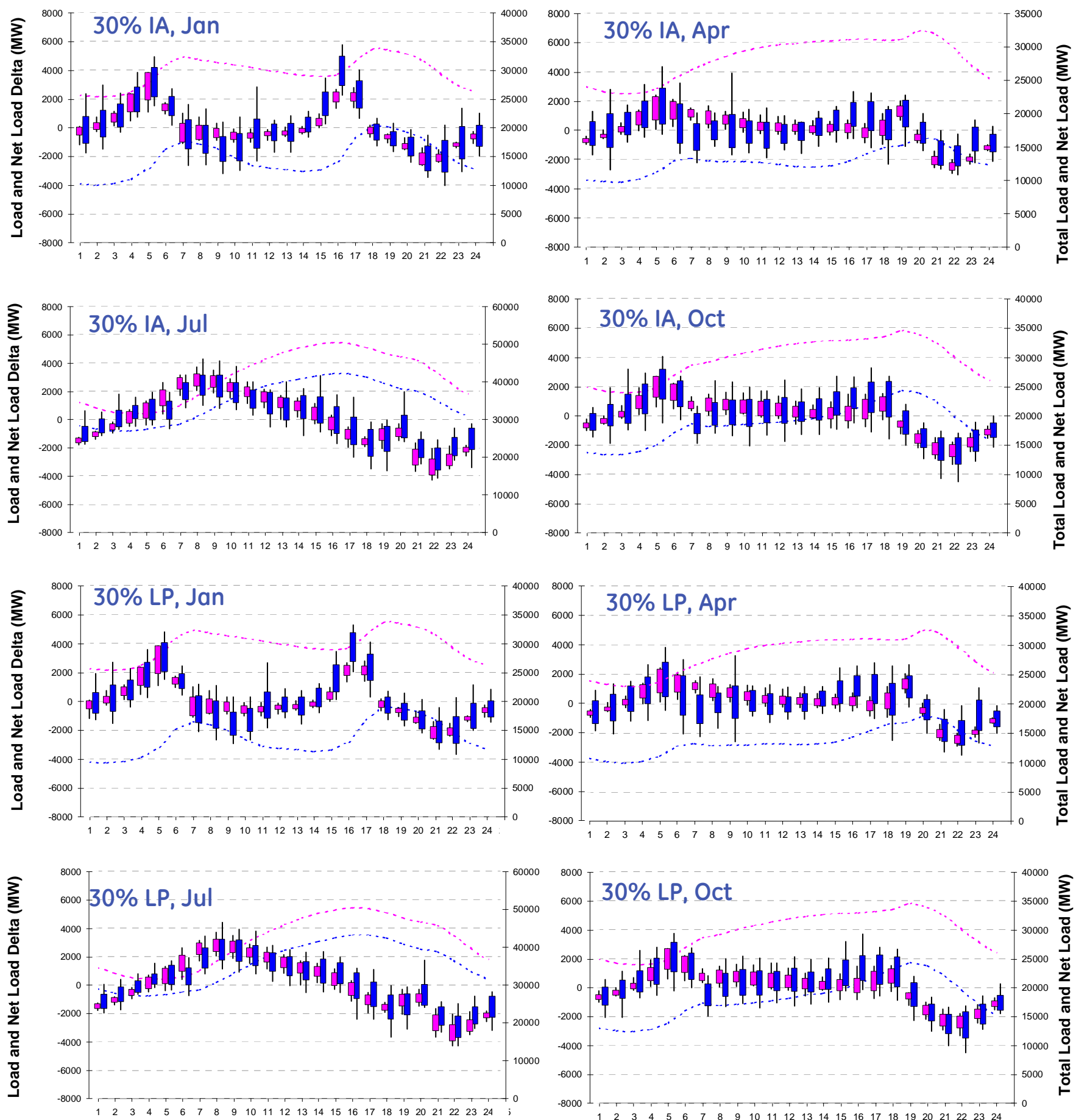

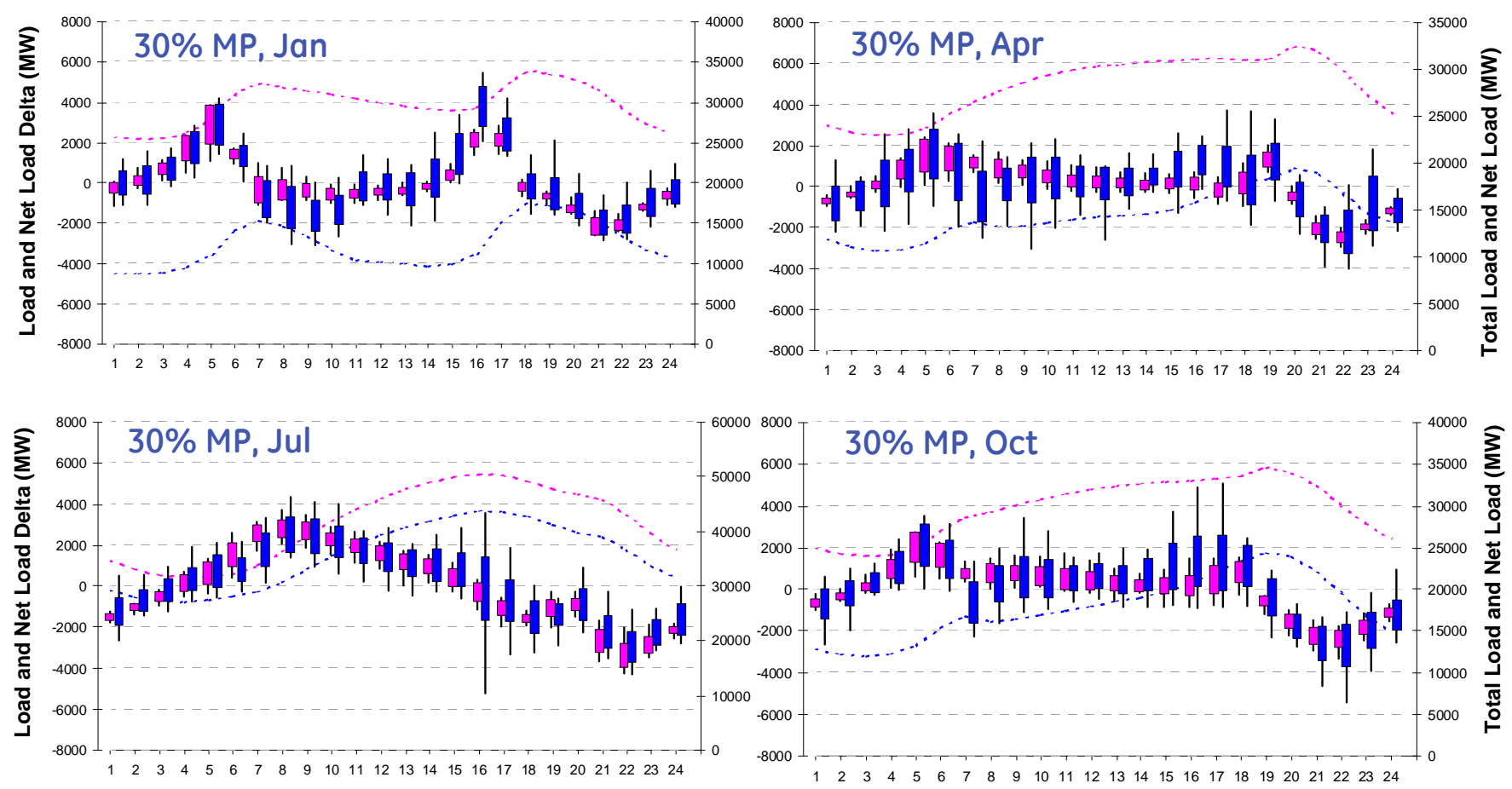
State/Area Average Daily Profile of Deltas for Year 2006
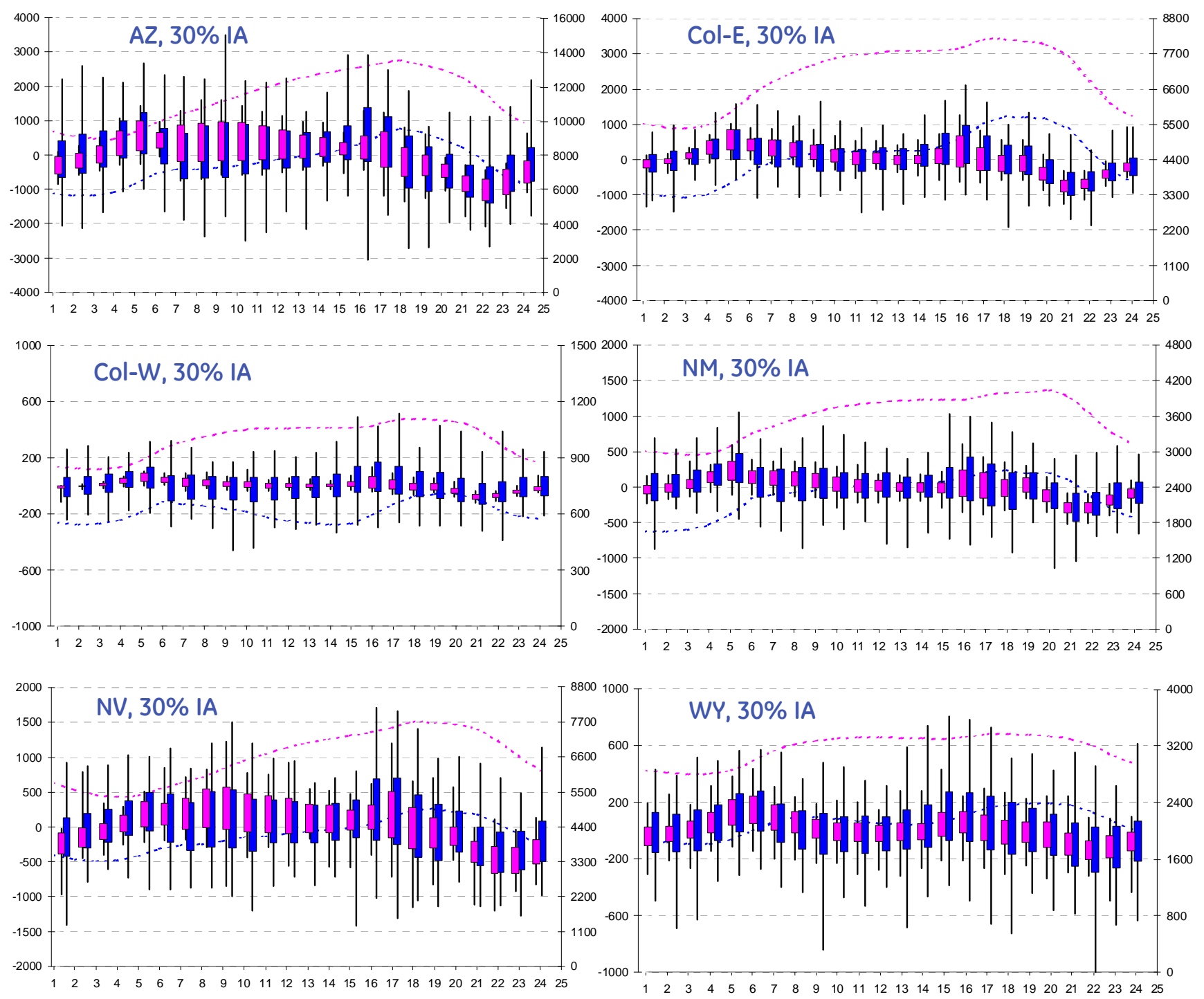

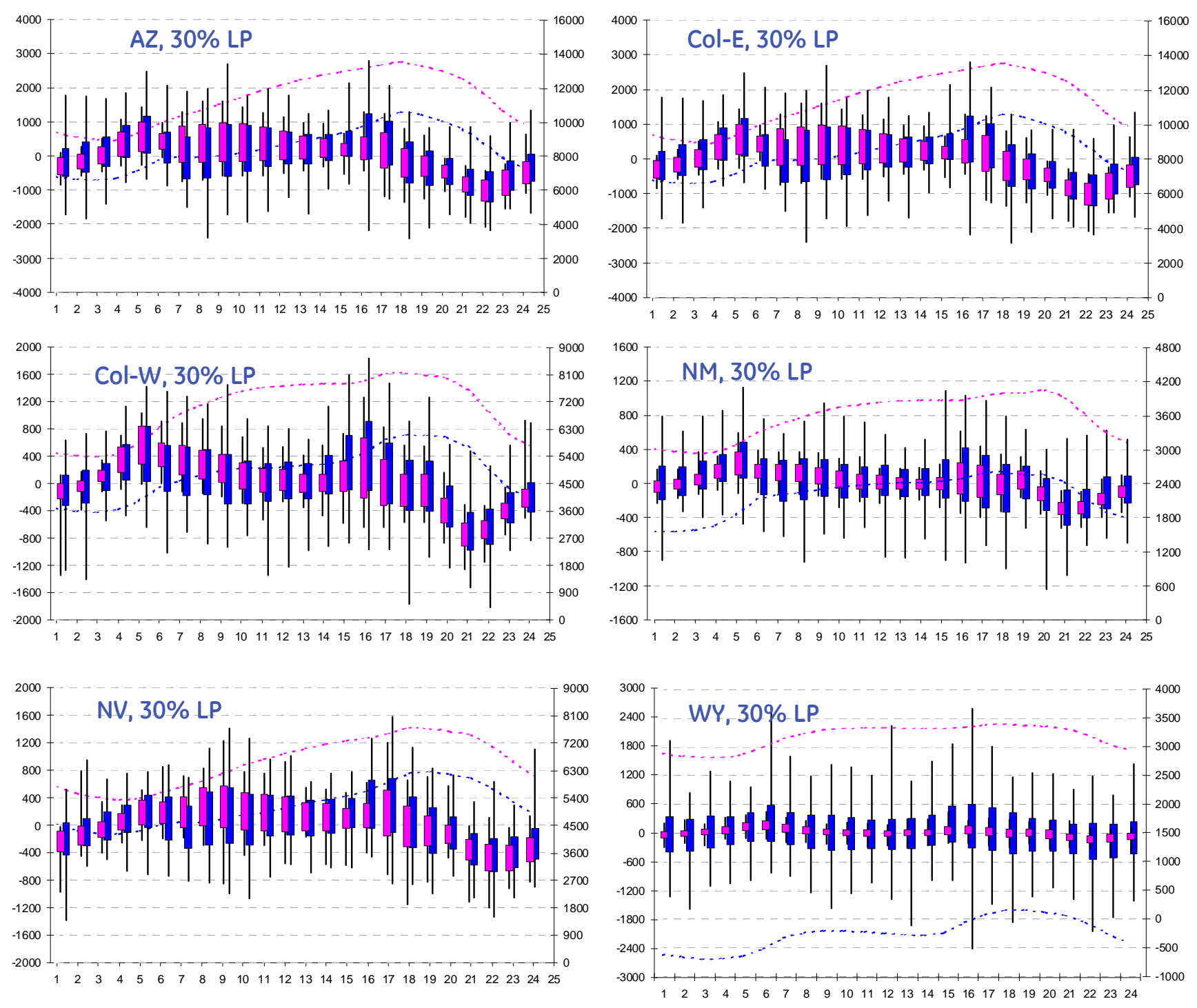

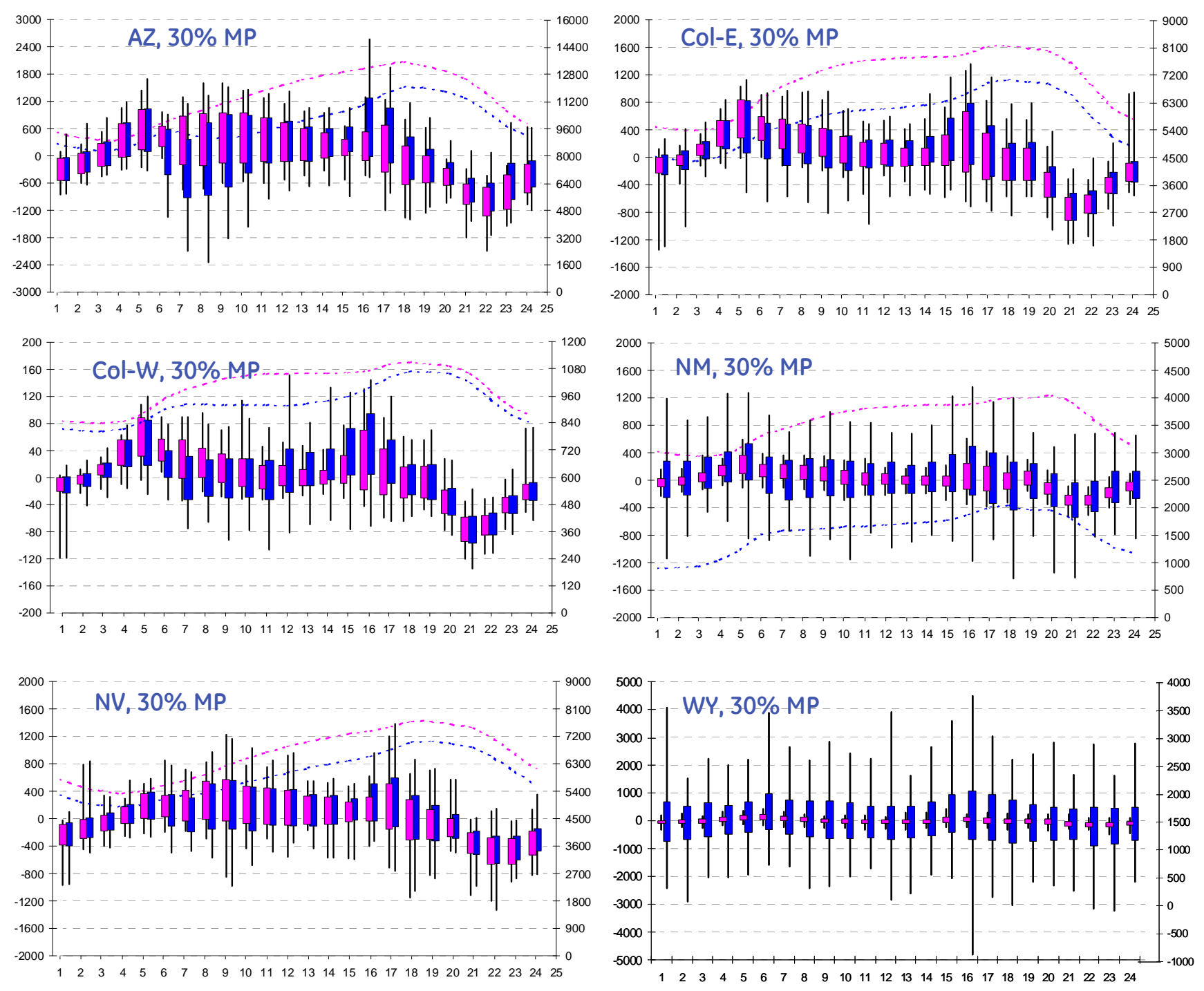


\section{C.3 Timing of 2006 Extreme Net Load Deltas}

\section{Footprint Positive Deltas}

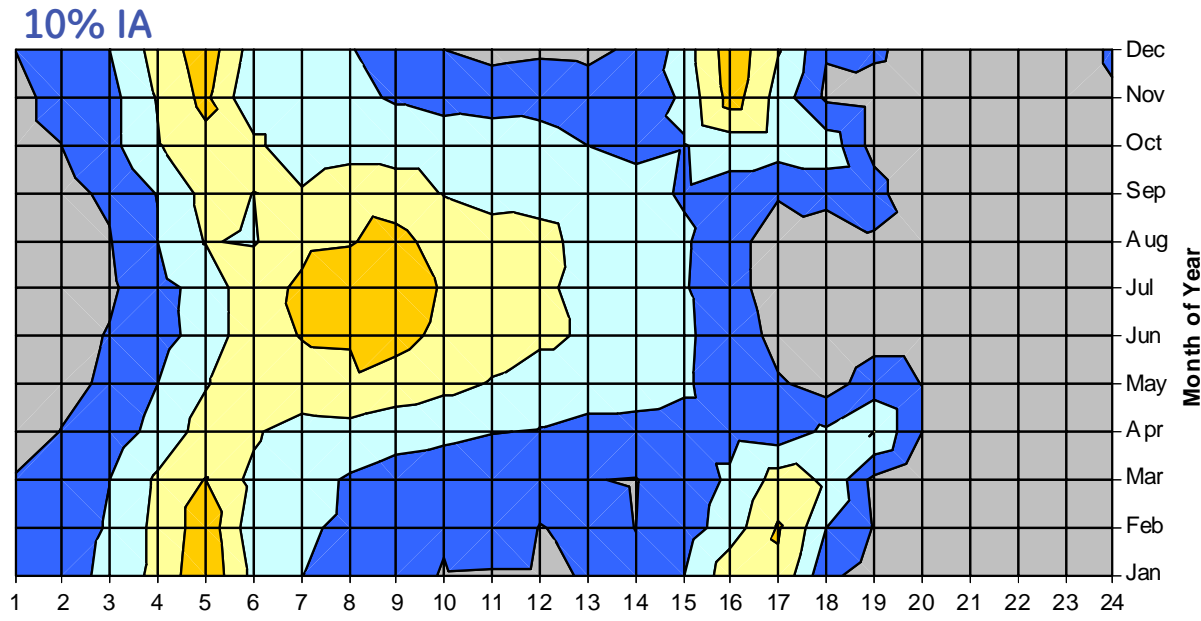

口5000-6000

4000-5000

3000-4000

प2000-3000

$\square$ 1000-2000

0-1000

$\square-1000-0$

$20 \%$ IA

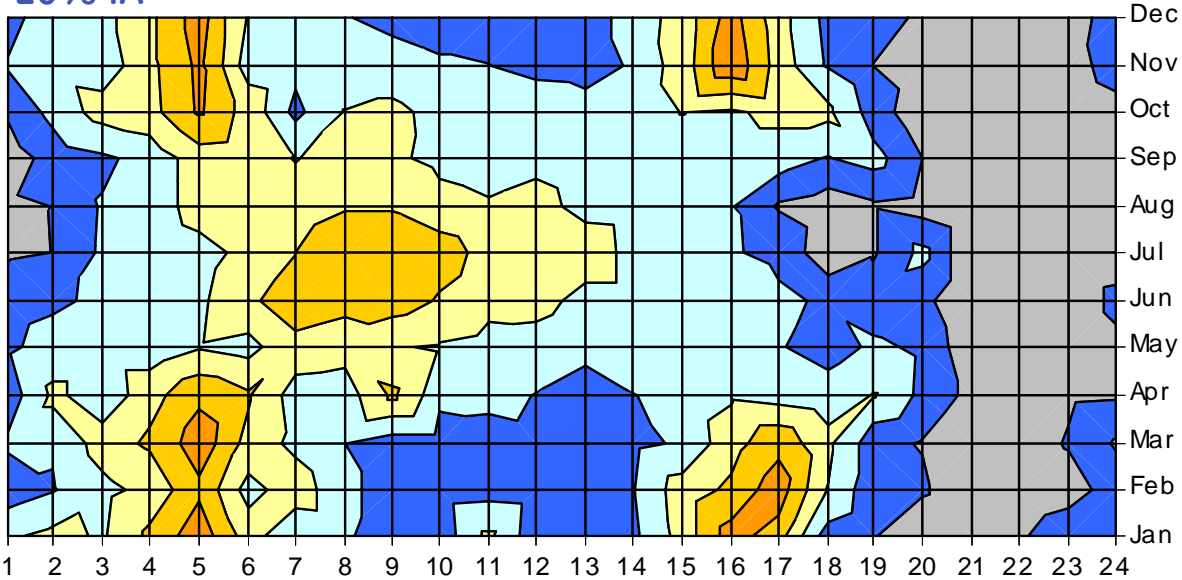

\begin{tabular}{|c|}
\hline$\square$ 5000-6000 \\
\hline$\square$ 4000-5000 \\
\hline$\square$ 3000-4000 \\
\hline$\square^{2000-3000}$ \\
\hline$\square$ 1000-2000 \\
\hline$\square^{0-1000}$ \\
\hline$\square-1000-0$ \\
\hline
\end{tabular}

$30 \%$ IA

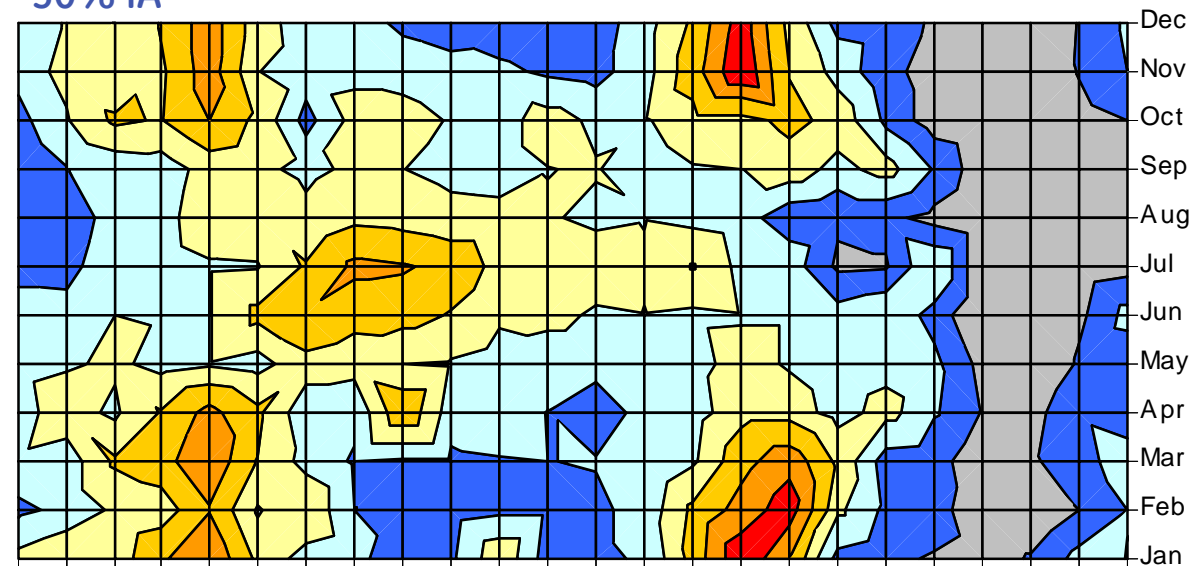

Dec

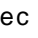

V

$\begin{array}{llllllllllllllllllllllll}1 & 2 & 3 & 4 & 5 & 6 & 7 & 8 & 9 & 10 & 11 & 12 & 13 & 14 & 15 & 16 & 17 & 18 & 19 & 20 & 21 & 22 & 23 & 24\end{array}$

Hour of Day 


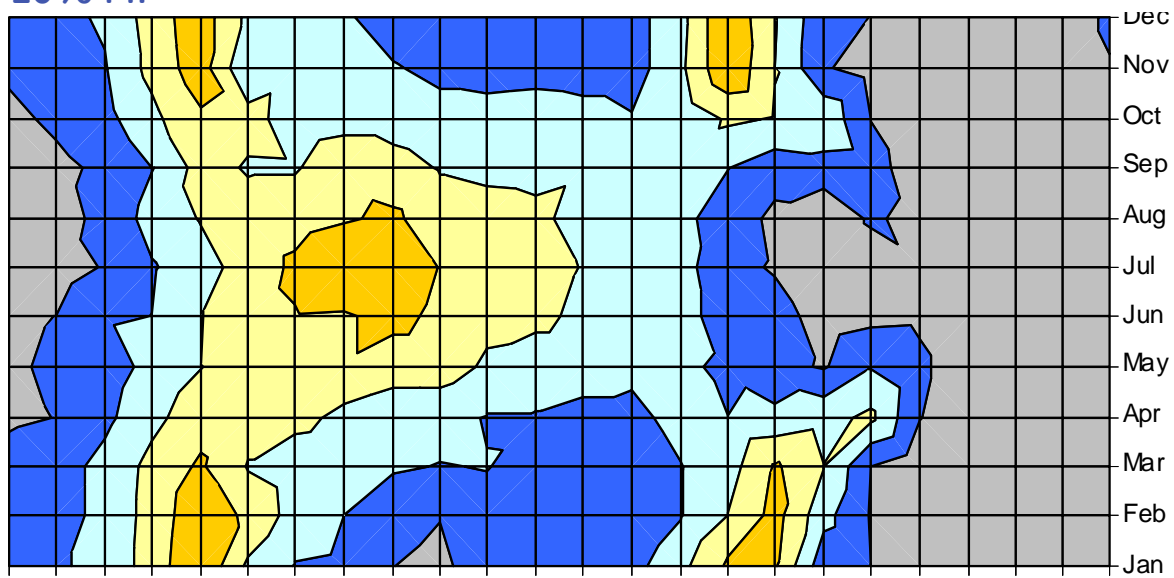

$\begin{array}{llllllllllllllllllllllll}1 & 2 & 3 & 4 & 5 & 6 & 7 & 8 & 9 & 10 & 11 & 12 & 13 & 14 & 15 & 16 & 17 & 18 & 19 & 20 & 21 & 22 & 23 & 24\end{array}$ 20\% MP

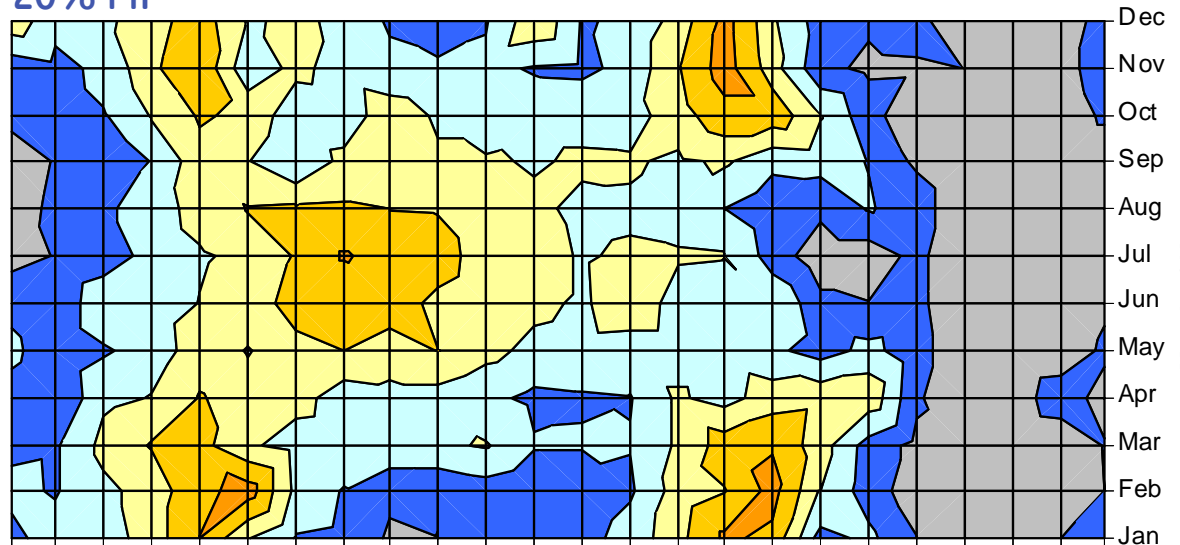

$\begin{array}{llllllllllllllllllllllll}1 & 2 & 3 & 4 & 5 & 6 & 7 & 8 & 9 & 10 & 11 & 12 & 13 & 14 & 15 & 16 & 17 & 18 & 19 & 20 & 21 & 22 & 23 & 24\end{array}$ $30 \% \mathrm{MP}$

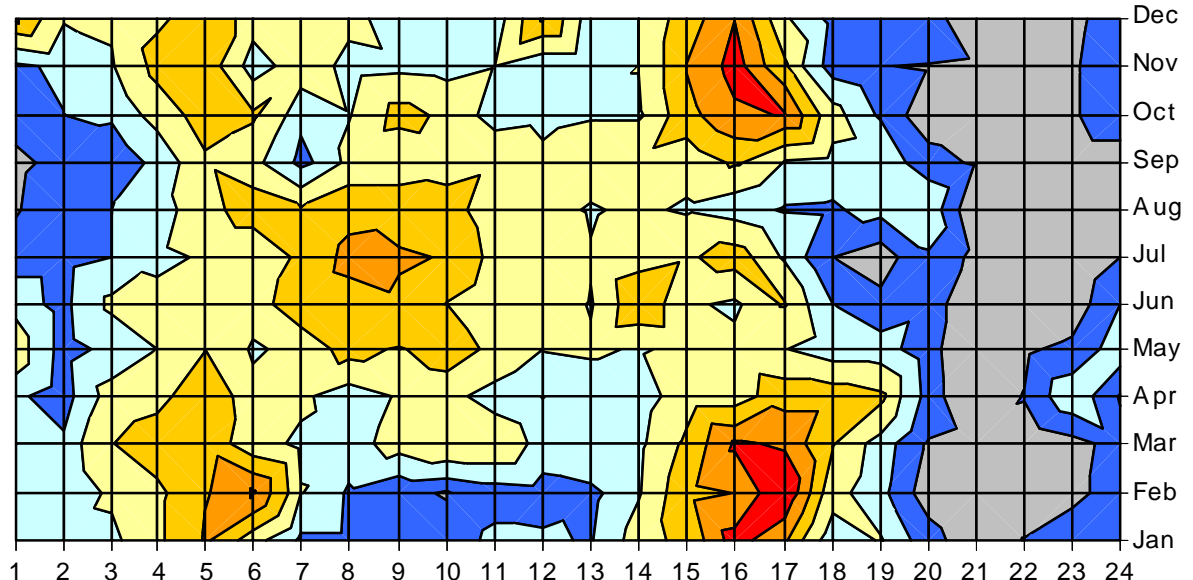

Hour of Day
口5000-6000 $\square$ 4000-5000 $\square 3000-4000$ $\square^{2000-3000}$ $\square^{1000-2000}$ 口 $0-1000$ -1000-0

$\square$ 5000-6000 $\square$ 4000-5000 $\square$ 3000-4000 $\square$ 2000-3000 $\square$ 1000-2000 $\square$ 0-1000 $\square-1000-0$ 
2006 Footprint Negative Deltas

$10 \% \mid A$

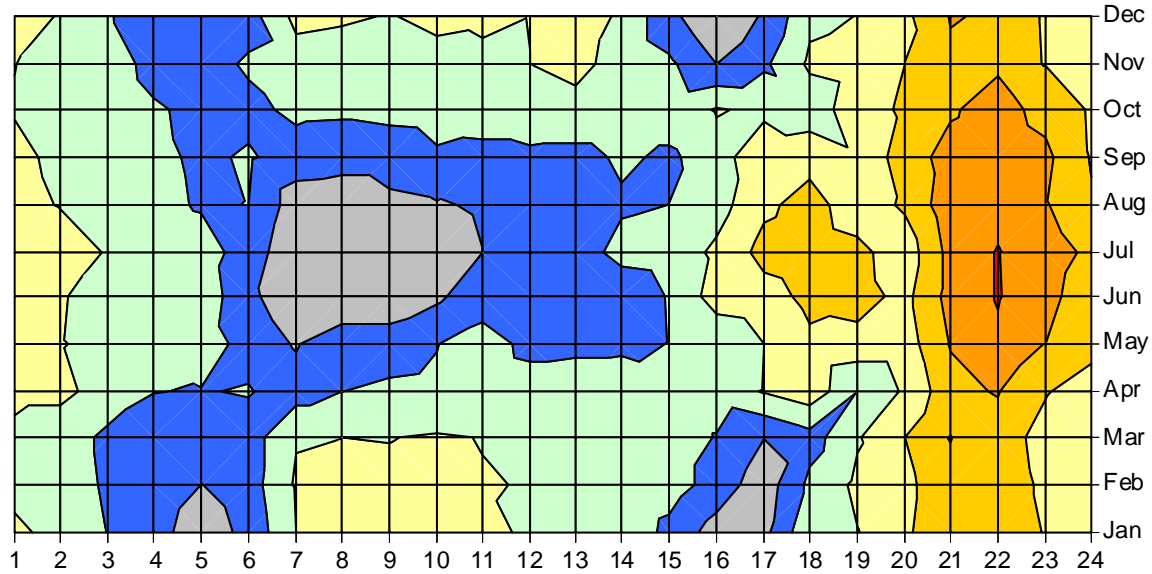

$\square$ 5000-6000

$\square$ 4000-5000

$\square 3000-4000$

$\square^{2000-3000}$

$\square$ 1000-2000

$\square$ 0-1000

$\square-1000-0$

$20 \%$ IA

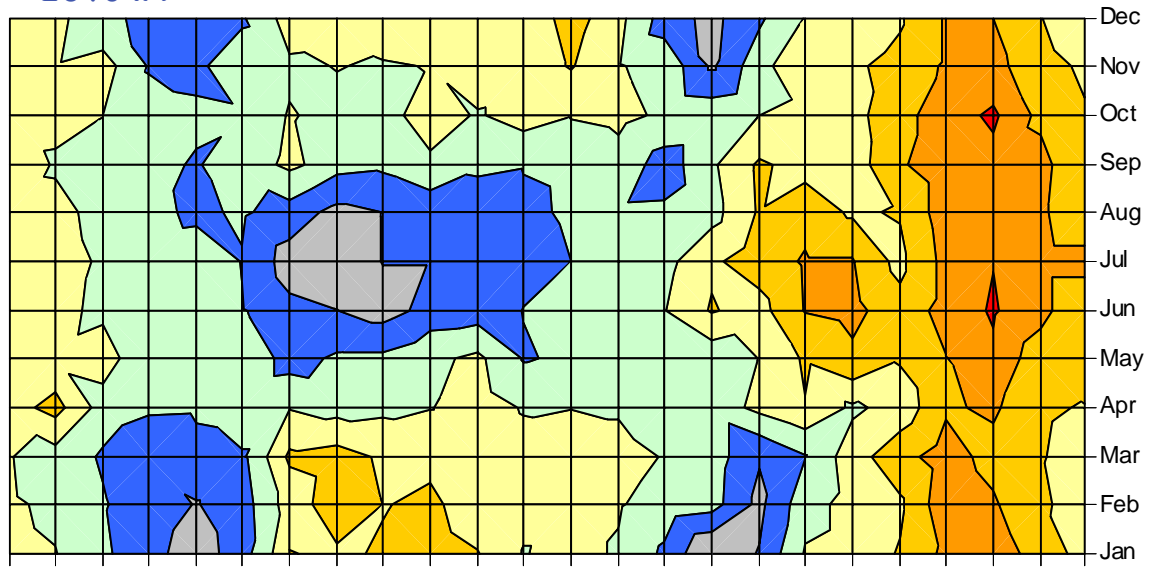

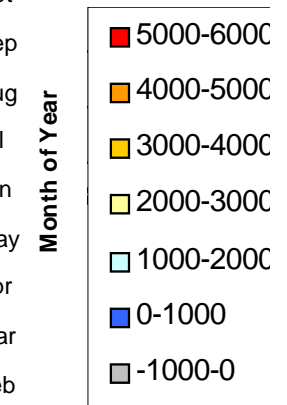

$30 \%$ IA

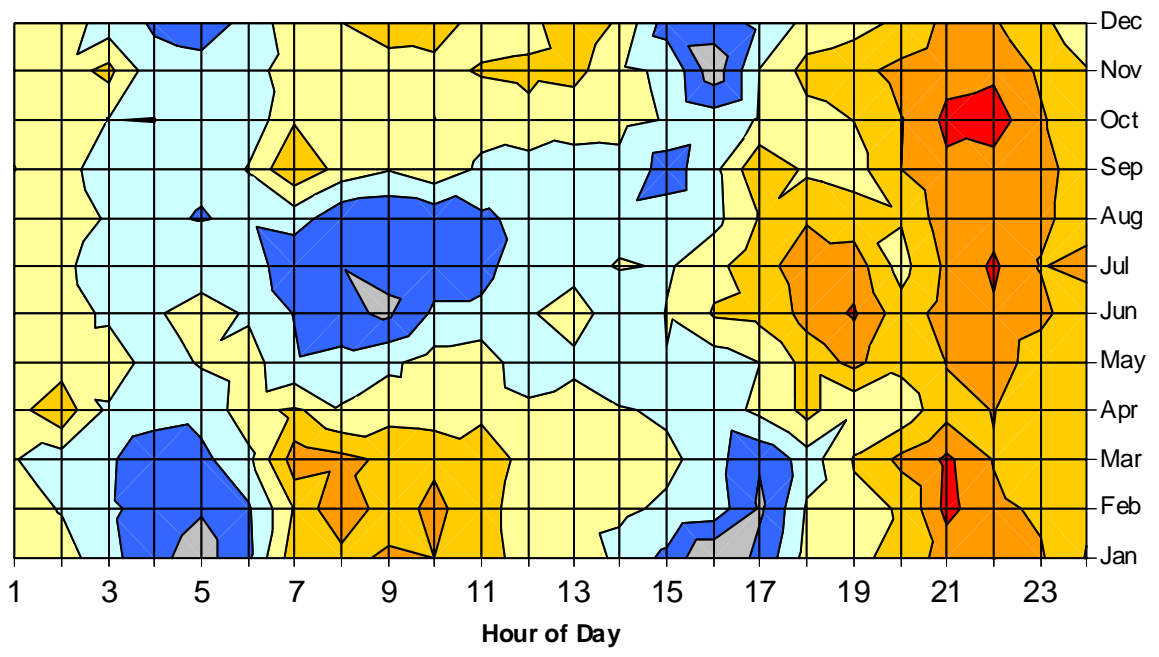

口5000-6000

$\square$ 4000-5000

ङ $\square 3000-4000$

$\square$ 2000-3000

$\square$ 1000-2000

口0-1000

$\square-1000-0$ 


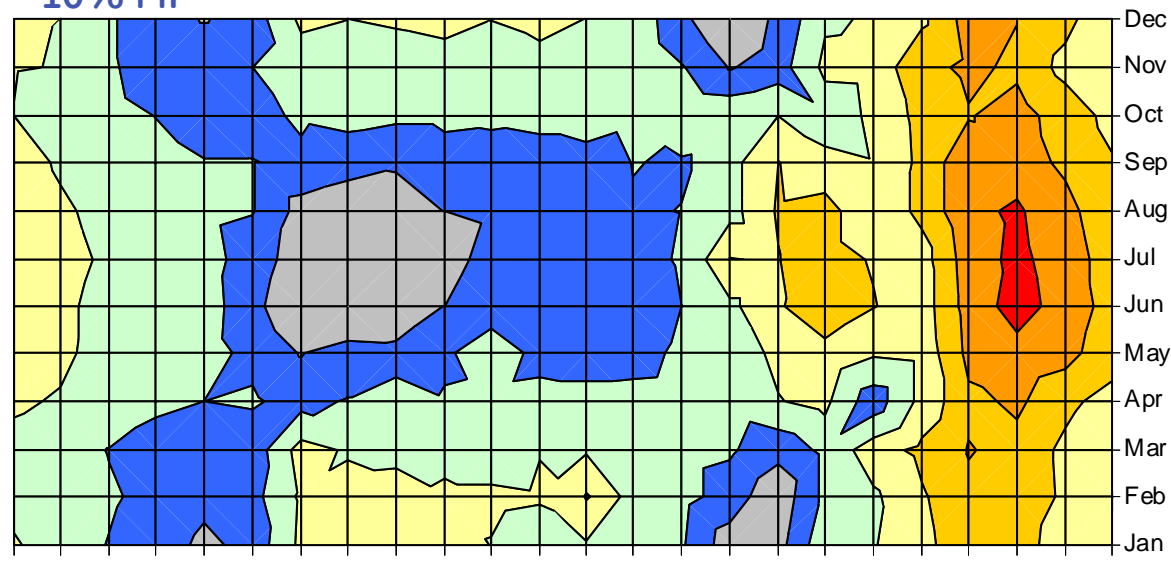

$$
\begin{aligned}
& \square 5000-6000 \\
& \square 4000-5000 \\
& \square 3000-4000 \\
& \square 2000-3000 \\
& \square 1000-2000 \\
& \square 0-1000 \\
& \square-1000-0
\end{aligned}
$$

$20 \%$ MP

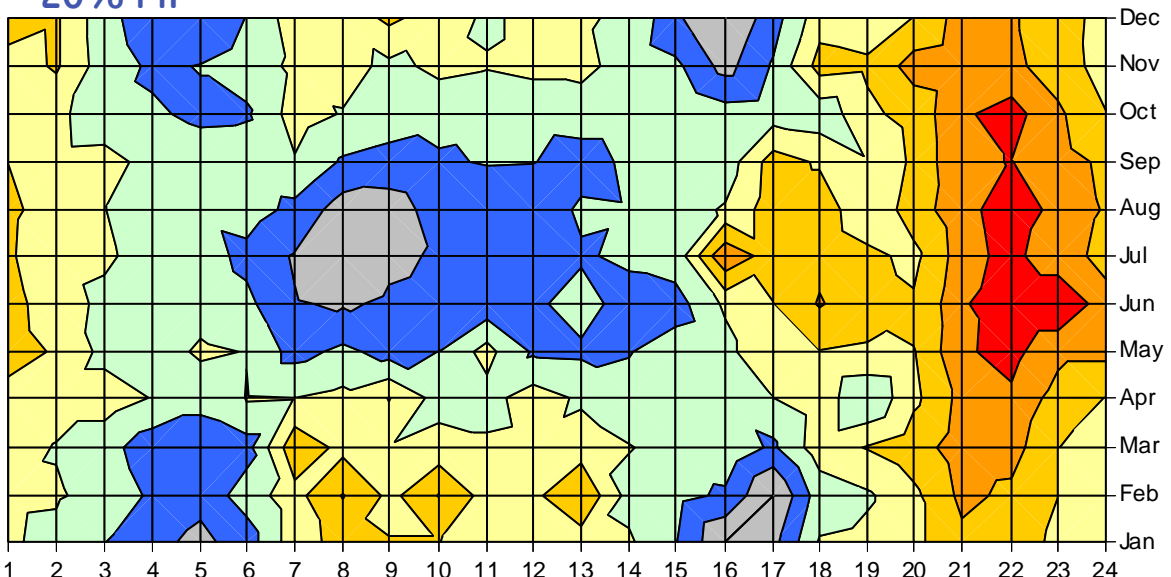

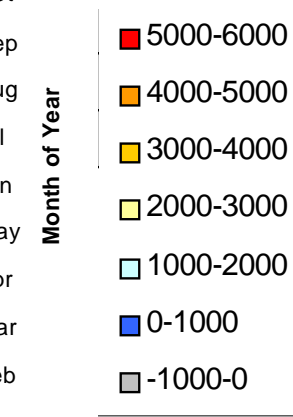

\section{$30 \% \mathrm{MP}$}

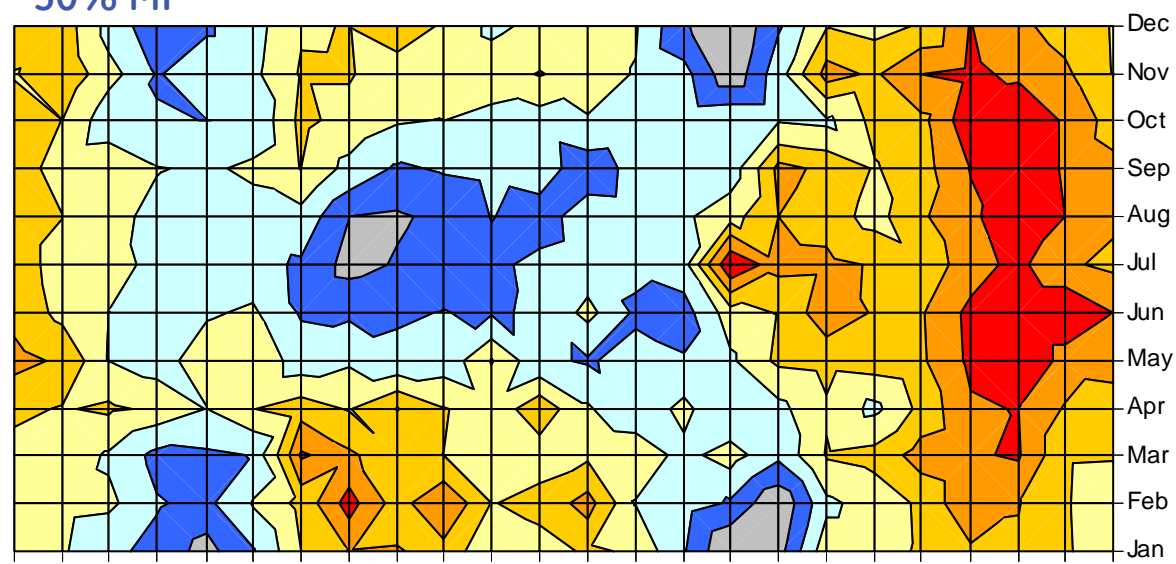

\footnotetext{
$\square 5000-6000$

$\square 4000-5000$

$\square 3000-4000$

$\square$ 2000-3000

$\square 1000-2000$

$\square 0-1000$

$\square-1000-0$
}

$\begin{array}{llllllllllllllllllllllll}1 & 2 & 3 & 4 & 5 & 6 & 7 & 8 & 9 & 10 & 11 & 12 & 13 & 14 & 15 & 16 & 17 & 18 & 19 & 20 & 21 & 22 & 23 & 24\end{array}$

Hour of Day 


\section{C.4 Distribution of Footprint Net Load Deltas}
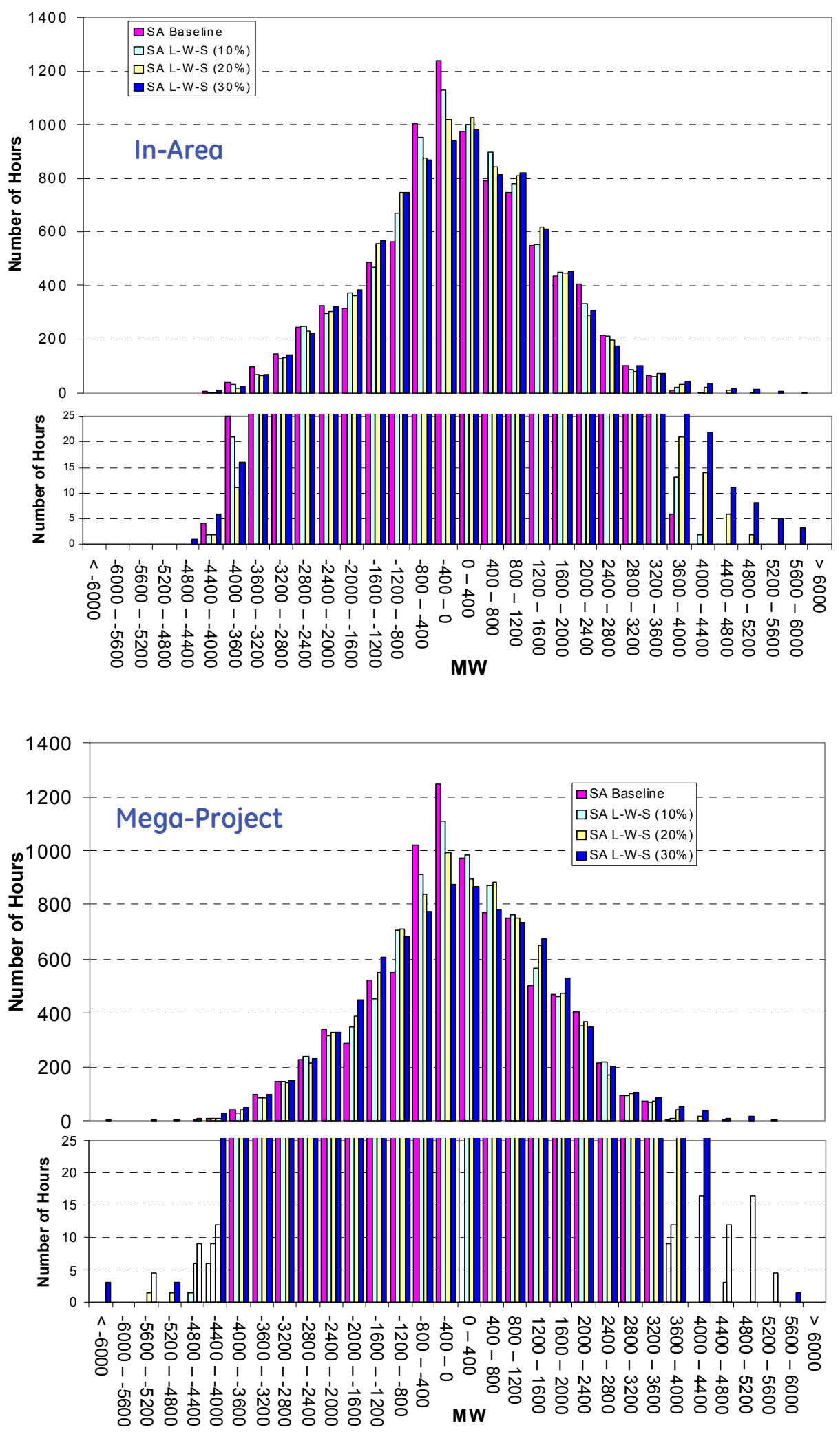


\section{C.5 Distribution of Extreme Footprint Net Load Deltas}
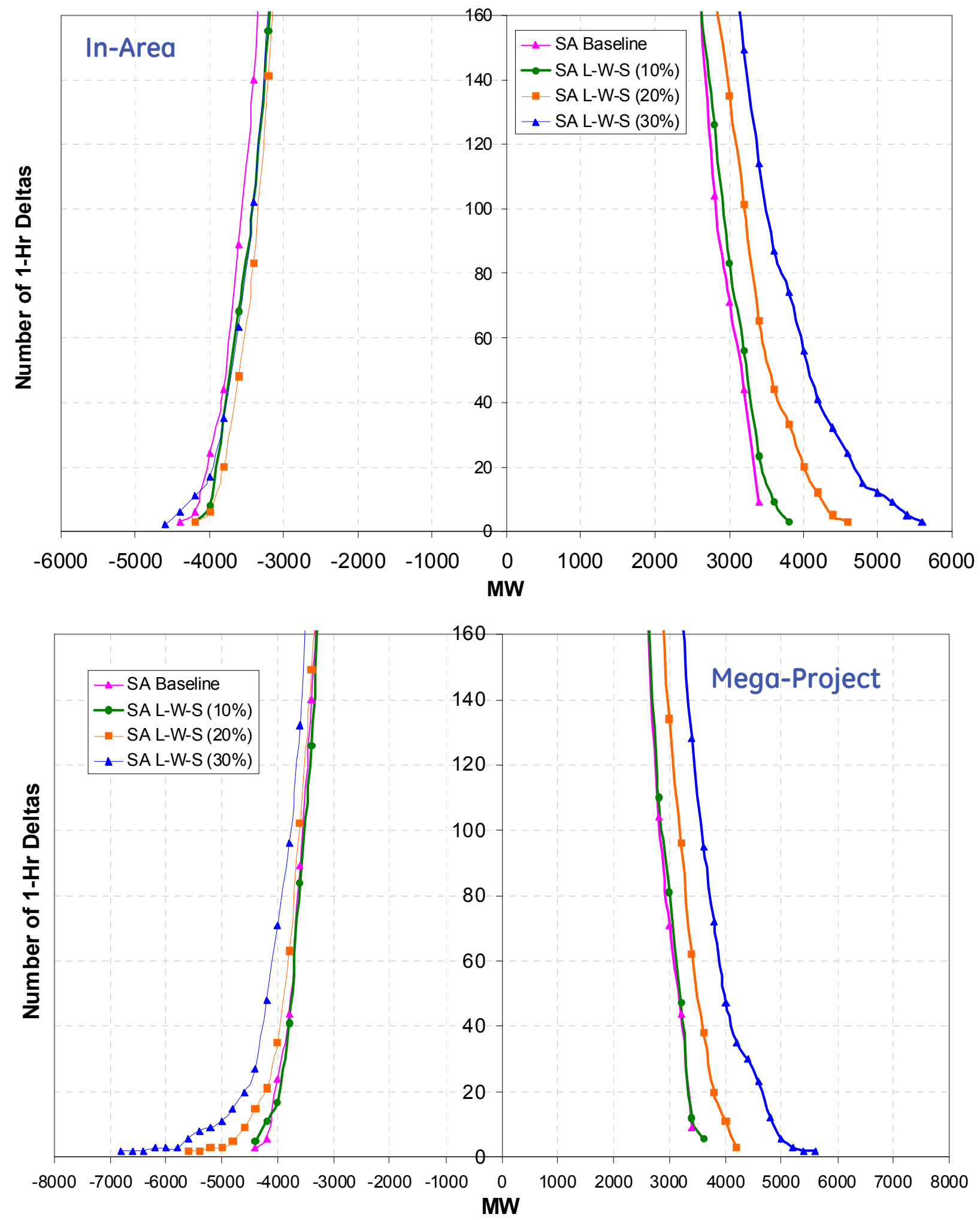



\section{Appendix D Transmission Path Flows}

The lines in Figure D.1 (reprinted from Chapter 3) show the base transfer capabilities assumed between the WECC transmission areas. Those labeled in red are within the study footprint and are adjusted for some of the scenarios. This Appendix shows the resulting flow distribution curves on these interfaces for many of the key scenarios.

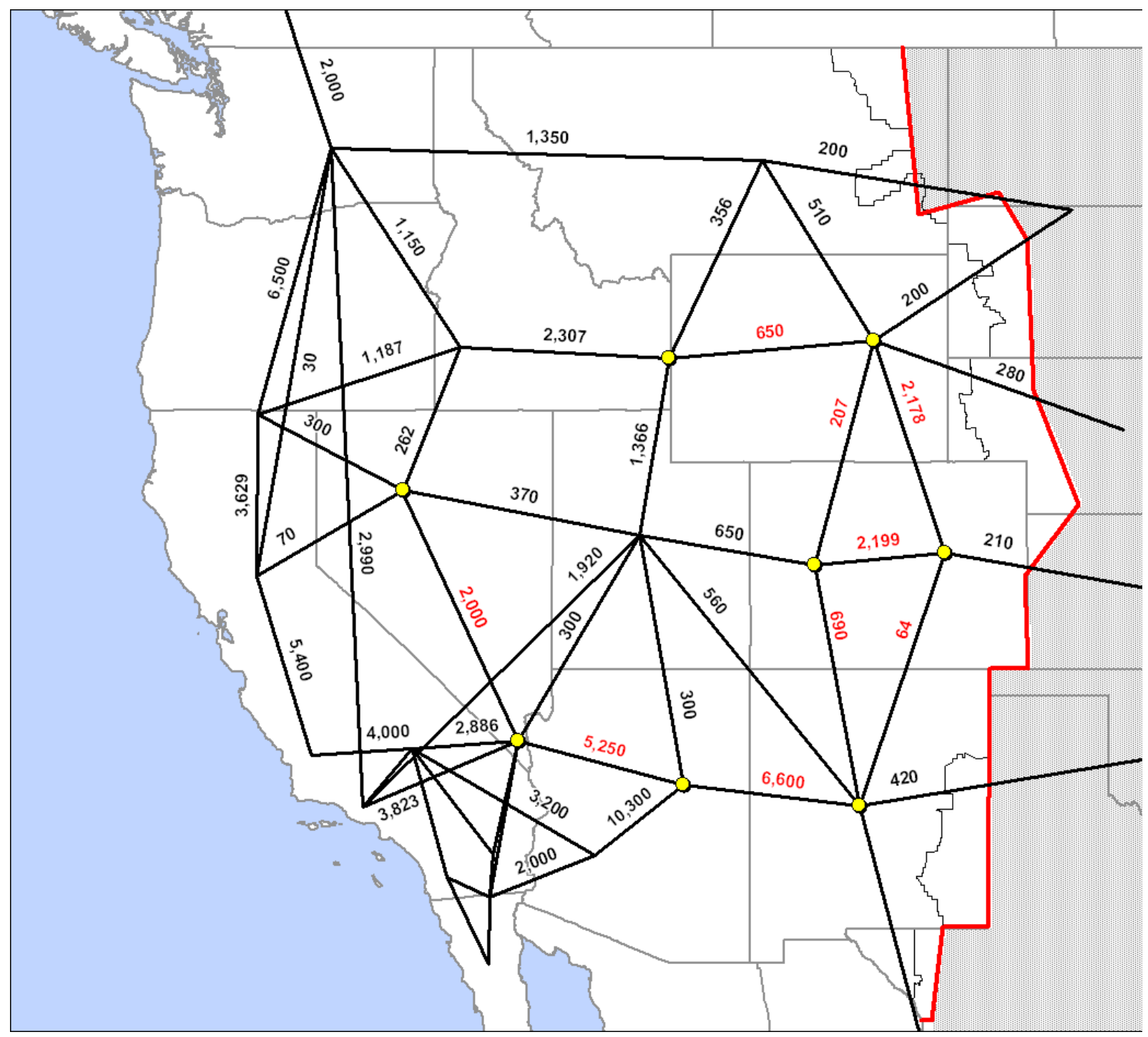

Figure D- 1 WECC base transfer limits 


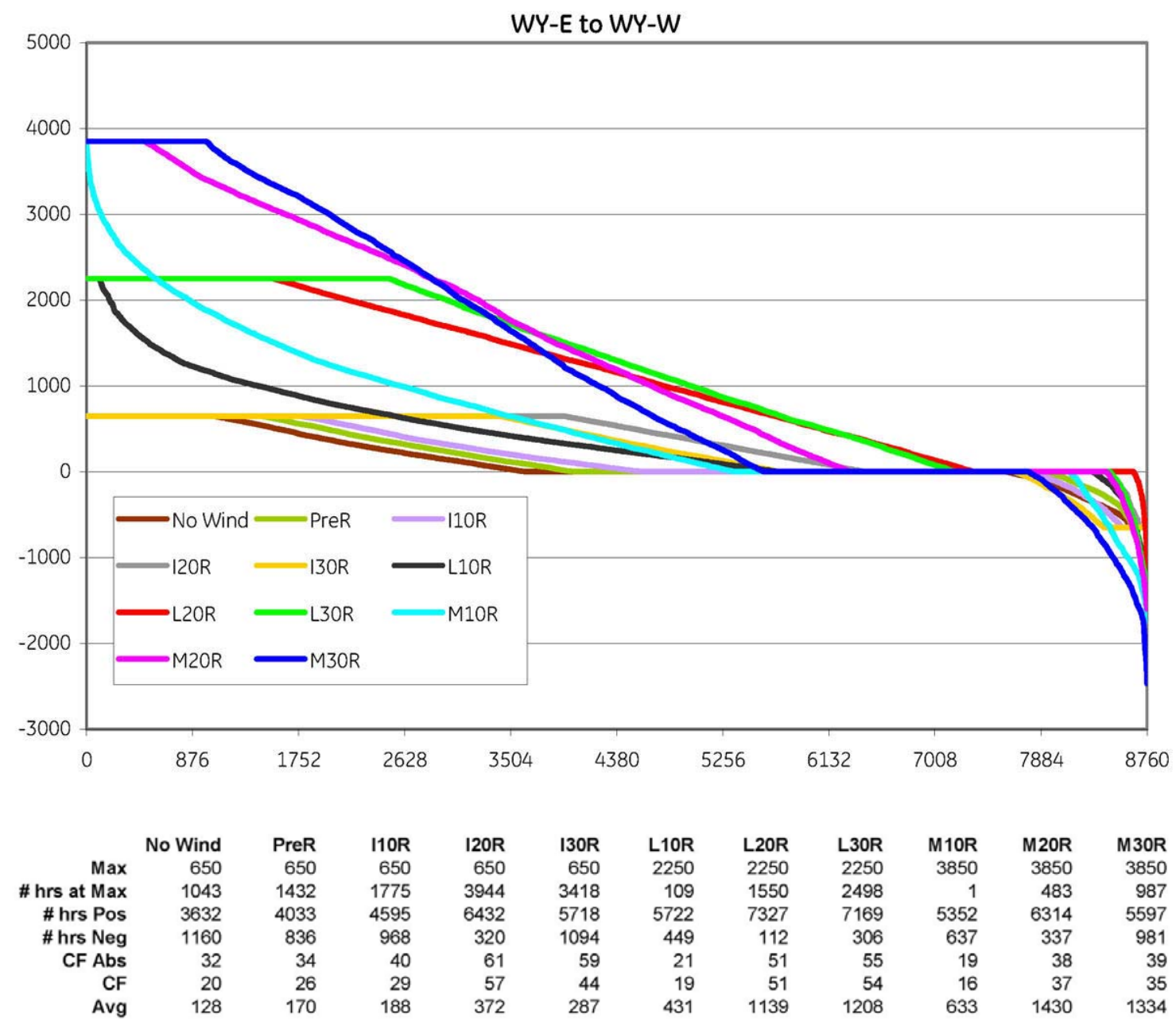

Figure D- 2 Wyoming East to Wyoming West flows 


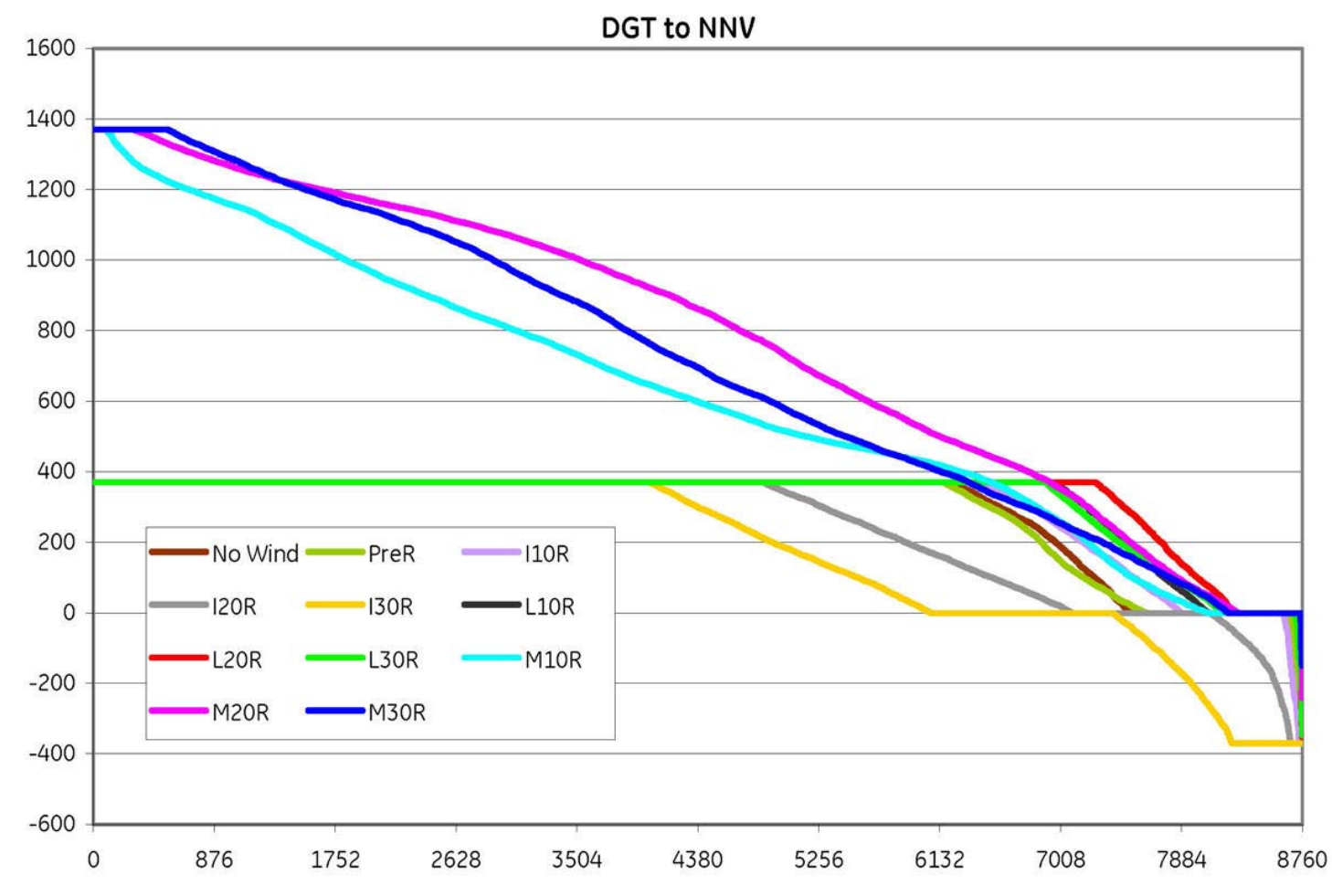

\begin{tabular}{|c|c|c|c|c|c|c|c|c|c|c|c|}
\hline & No Wind & PreR & I10R & I20R & I30R & L10R & L20R & L30R & M10R & M20R & M30R \\
\hline Max & 370 & 370 & 370 & 370 & 370 & 370 & 370 & 370 & 1370 & 1370 & 1370 \\
\hline \# hrs at Max & 6216 & 6162 & 6392 & 4858 & 4027 & 6958 & 7261 & 6901 & 96 & 292 & 539 \\
\hline \# hrs Pos & 7536 & 7631 & 7880 & 7093 & 6066 & 8085 & 8265 & 8181 & 8081 & 8295 & 8224 \\
\hline \# hrs Neg & 102 & 99 & 139 & 673 & 1371 & 13 & 16 & 54 & 7 & 5 & 15 \\
\hline CF Abs & 80 & 79 & 83 & 71 & 67 & 86 & 89 & 86 & 46 & 57 & 52 \\
\hline CF & 79 & 78 & 82 & 65 & 48 & 86 & 89 & 86 & 46 & 57 & 51 \\
\hline Avg & 293 & 290 & 302 & 241 & 176 & 318 & 328 & 317 & 624 & 775 & 706 \\
\hline
\end{tabular}

Figure D- 3 Deseret to Northern Nevada flows 


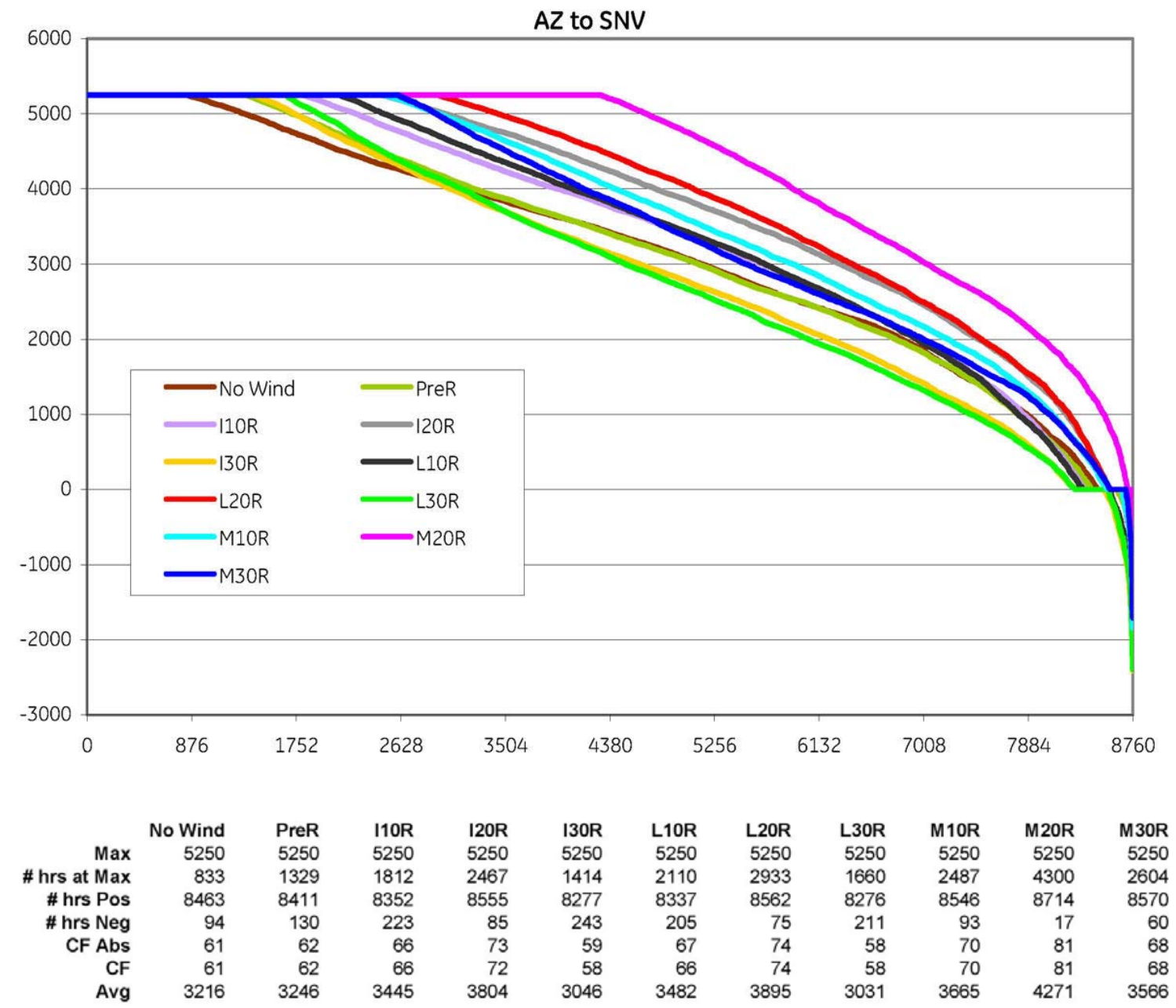

Figure D- 4 Arizona to Southern Nevada flows 


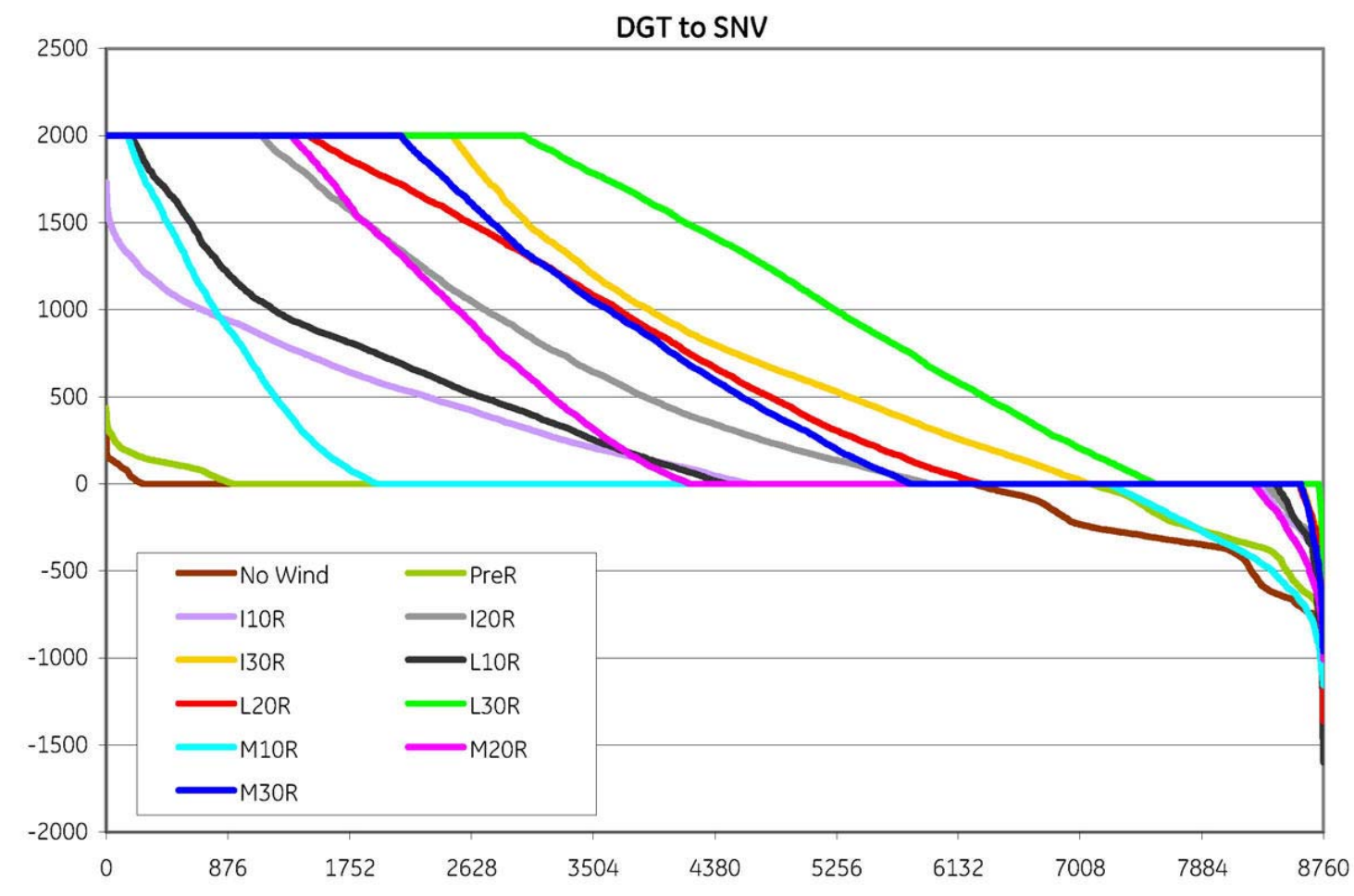

\begin{tabular}{|c|c|c|c|c|c|c|c|c|c|c|c|}
\hline & No Wind & PreR & I10R & I20R & I30R & L10R & L20R & L30R & M10R & M20R & M30R \\
\hline $\operatorname{Max}$ & 2000 & 2000 & 2000 & 2000 & 2000 & 2000 & 2000 & 2000 & 2000 & 2000 & 2000 \\
\hline \# hrs at Max & 0 & 0 & 0 & 1117 & 2495 & 175 & 1464 & 3003 & 158 & 1334 & 2124 \\
\hline \# hrs Pos & 266 & 912 & 4629 & 5917 & 7063 & 4463 & 6299 & 7543 & 1948 & 4198 & 5798 \\
\hline \# hrs Neg & 2518 & 1672 & 486 & 410 & 154 & 344 & 174 & 34 & 1541 & 503 & 163 \\
\hline CF Abs & 5 & 3 & 15 & 34 & 49 & 20 & 43 & 60 & 13 & 30 & 43 \\
\hline CF & -5 & -2 & 14 & 33 & 48 & 19 & 42 & 60 & 7 & 29 & 42 \\
\hline Avg & -90 & -42 & 279 & 665 & 963 & 371 & 843 & 1199 & 133 & 570 & 842 \\
\hline
\end{tabular}

Figure D- 5 Deseret to Southern Nevada flows 


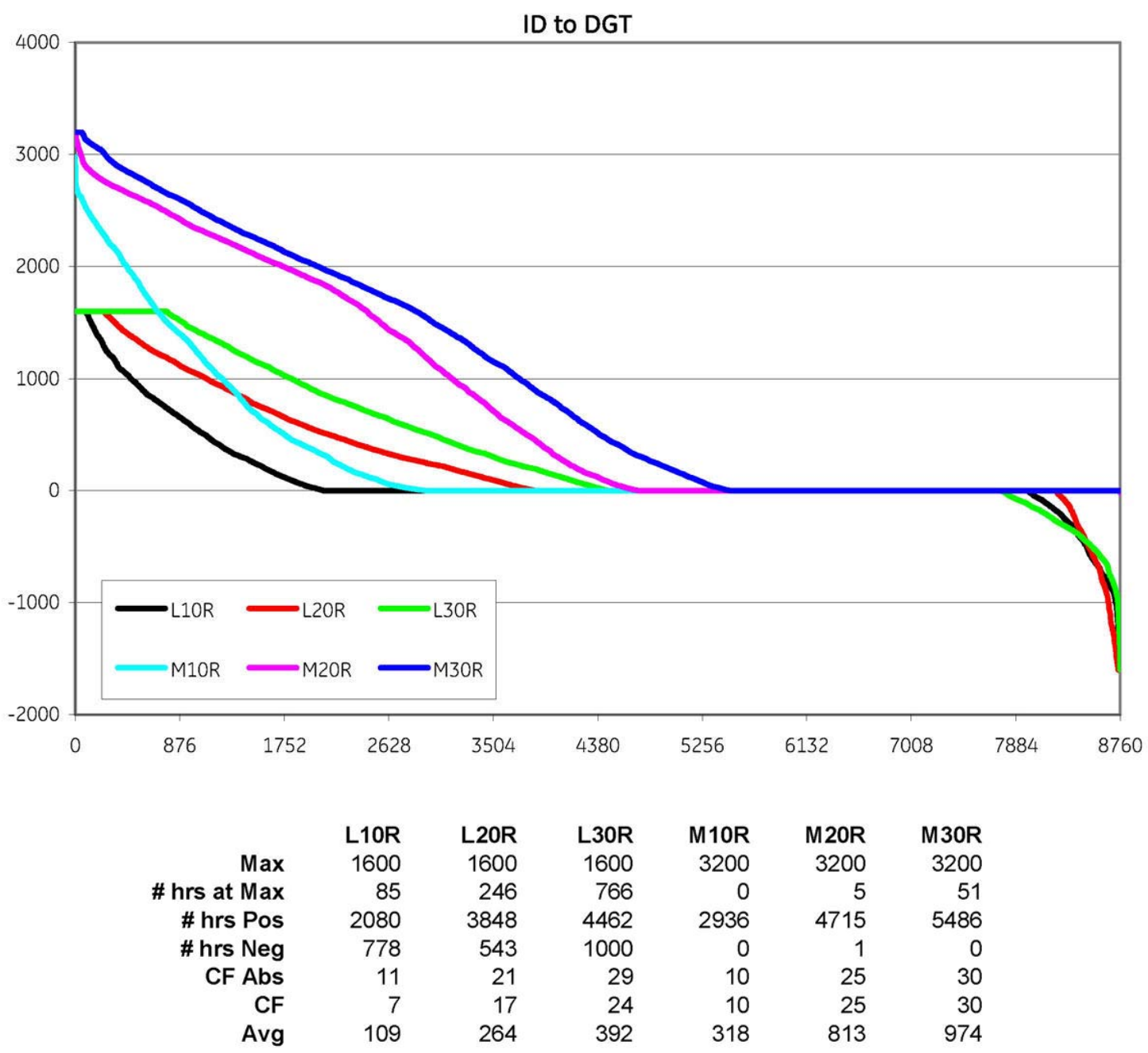

Figure D- 6 Idaho to Deseret flows 


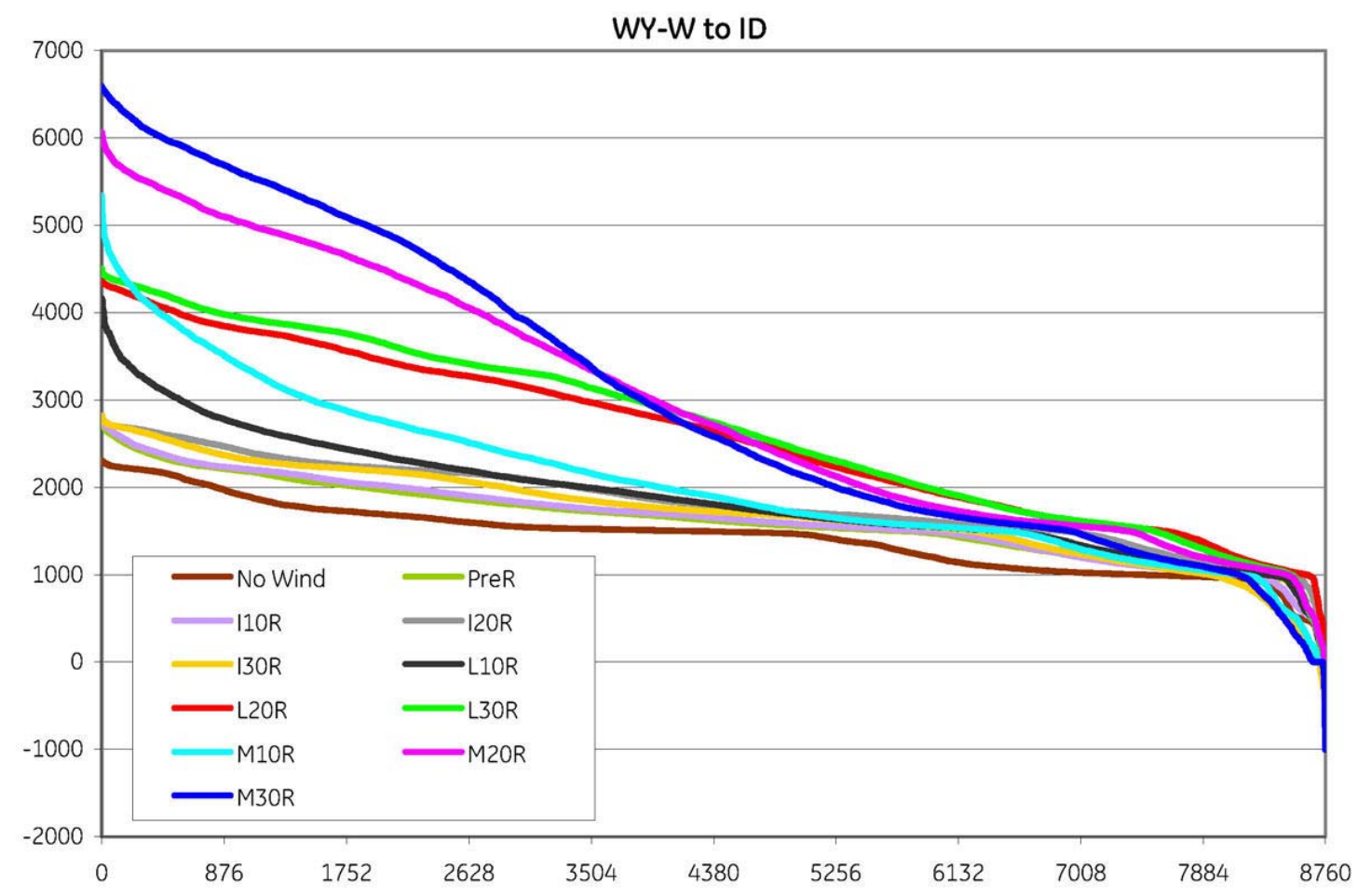

\begin{tabular}{|c|c|c|c|c|c|c|c|c|c|c|c|}
\hline & No Wind & PreR & I10R & I20R & I30R & L10R & L20R & L30R & M10R & M20R & M30R \\
\hline Max & 99999 & 99999 & 99999 & 99999 & 99999 & 99999 & 99999 & 99999 & 99999 & 99999 & 99999 \\
\hline \# hrs at Max & 0 & 0 & 0 & 0 & 0 & 0 & 0 & 0 & 0 & 0 & 0 \\
\hline \# hrs Pos & 8760 & 8760 & 8754 & 8759 & 8679 & 8746 & 8758 & 8733 & 8715 & 8753 & 8671 \\
\hline \# hrs Neg & 0 & 0 & 0 & 0 & 42 & 5 & 0 & 5 & 13 & 2 & 20 \\
\hline CF Abs & 1 & 2 & 2 & 2 & 2 & 2 & 3 & 3 & 2 & 3 & 3 \\
\hline $\mathrm{CF}$ & 1 & 2 & 2 & 2 & 2 & 2 & 3 & 3 & 2 & 3 & 3 \\
\hline Avg & 1428 & 1628 & 1646 & 1830 & 1708 & 1888 & 2596 & 2671 & 2090 & 2970 & 3056 \\
\hline
\end{tabular}

Figure D- 7 Western Wyoming to Idaho flows 


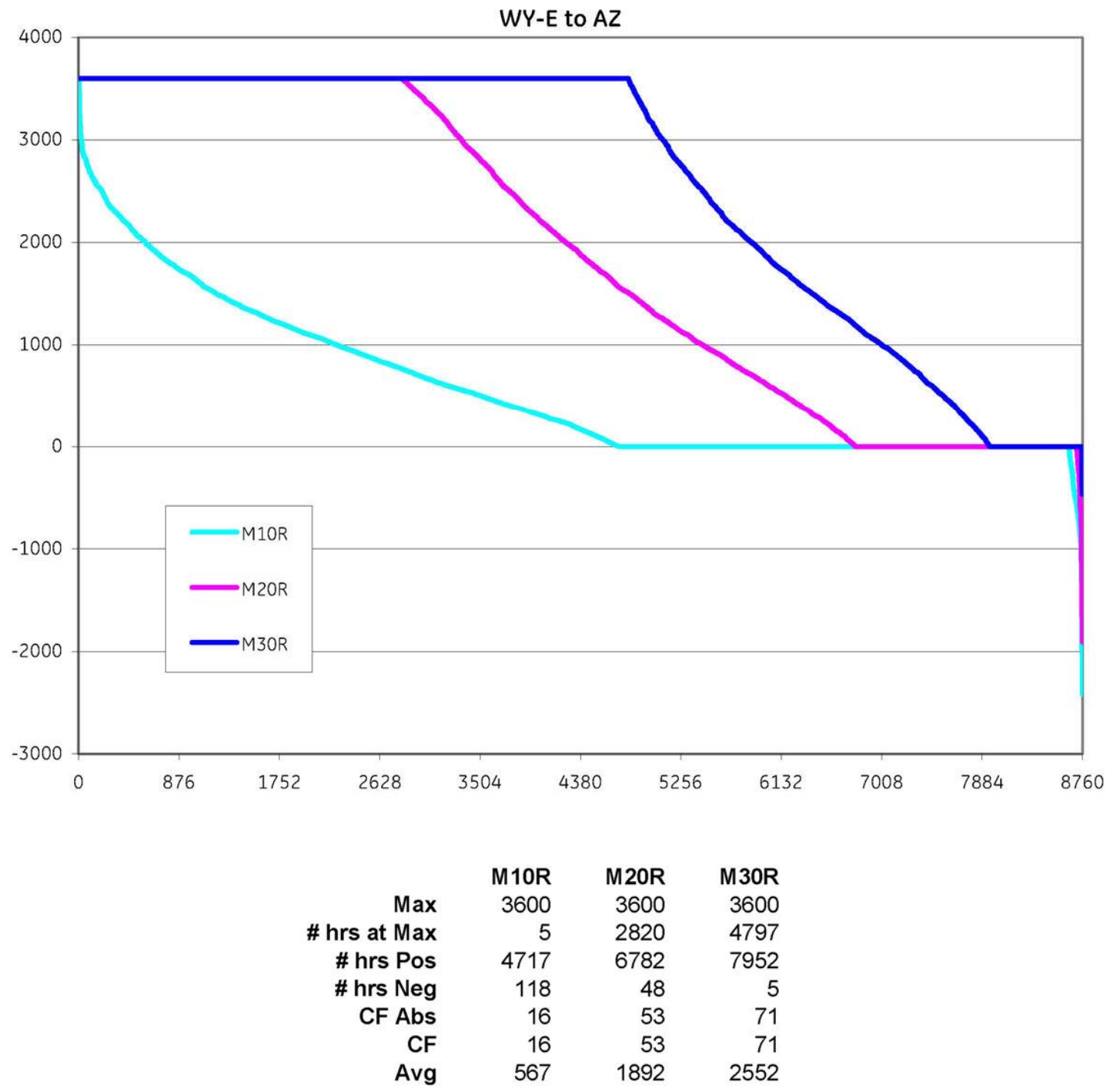

Figure D- 8 Eastern Wyoming to Arizona flows 


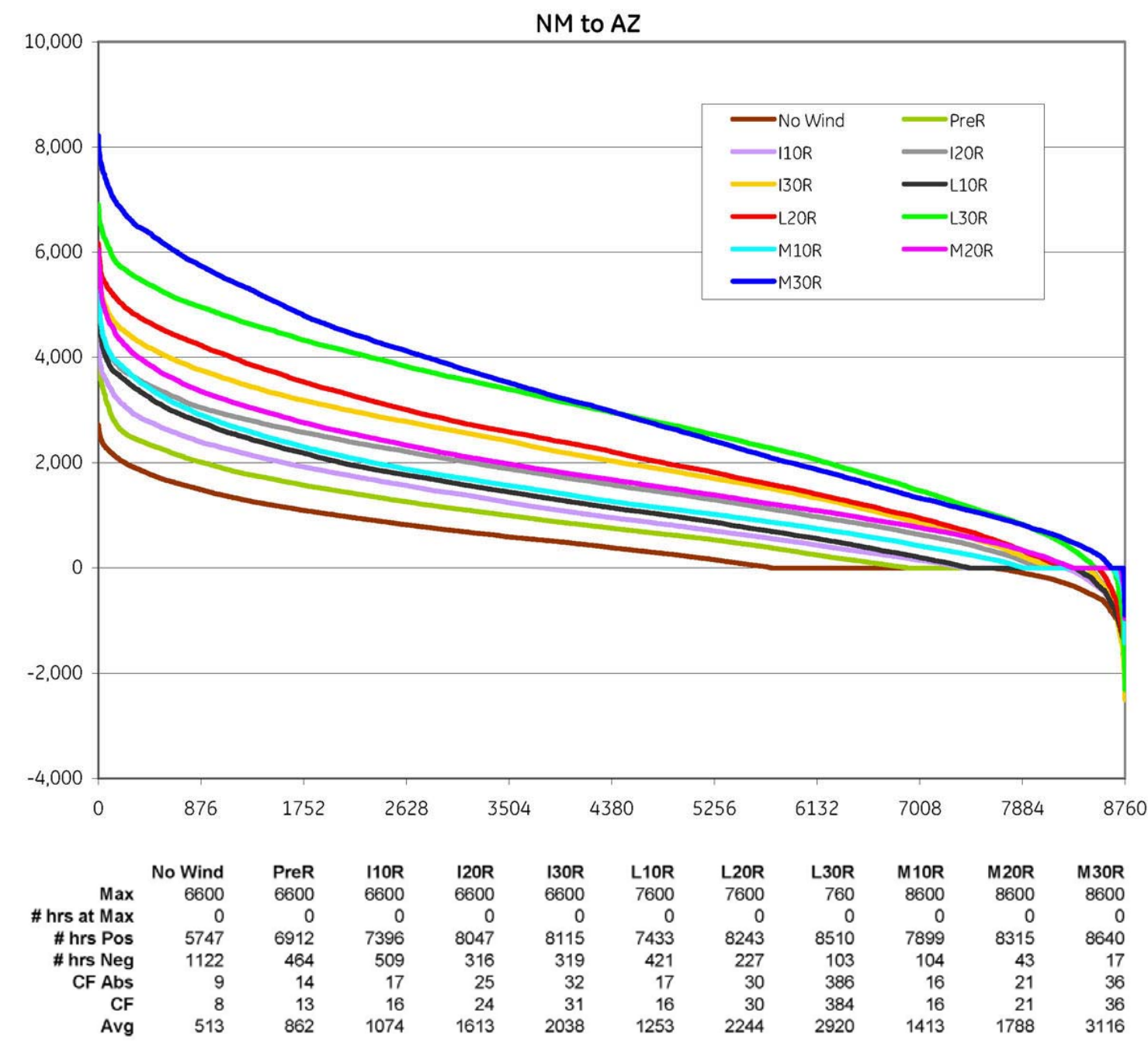

Figure D- 9 New Mexico to Arizona flows 


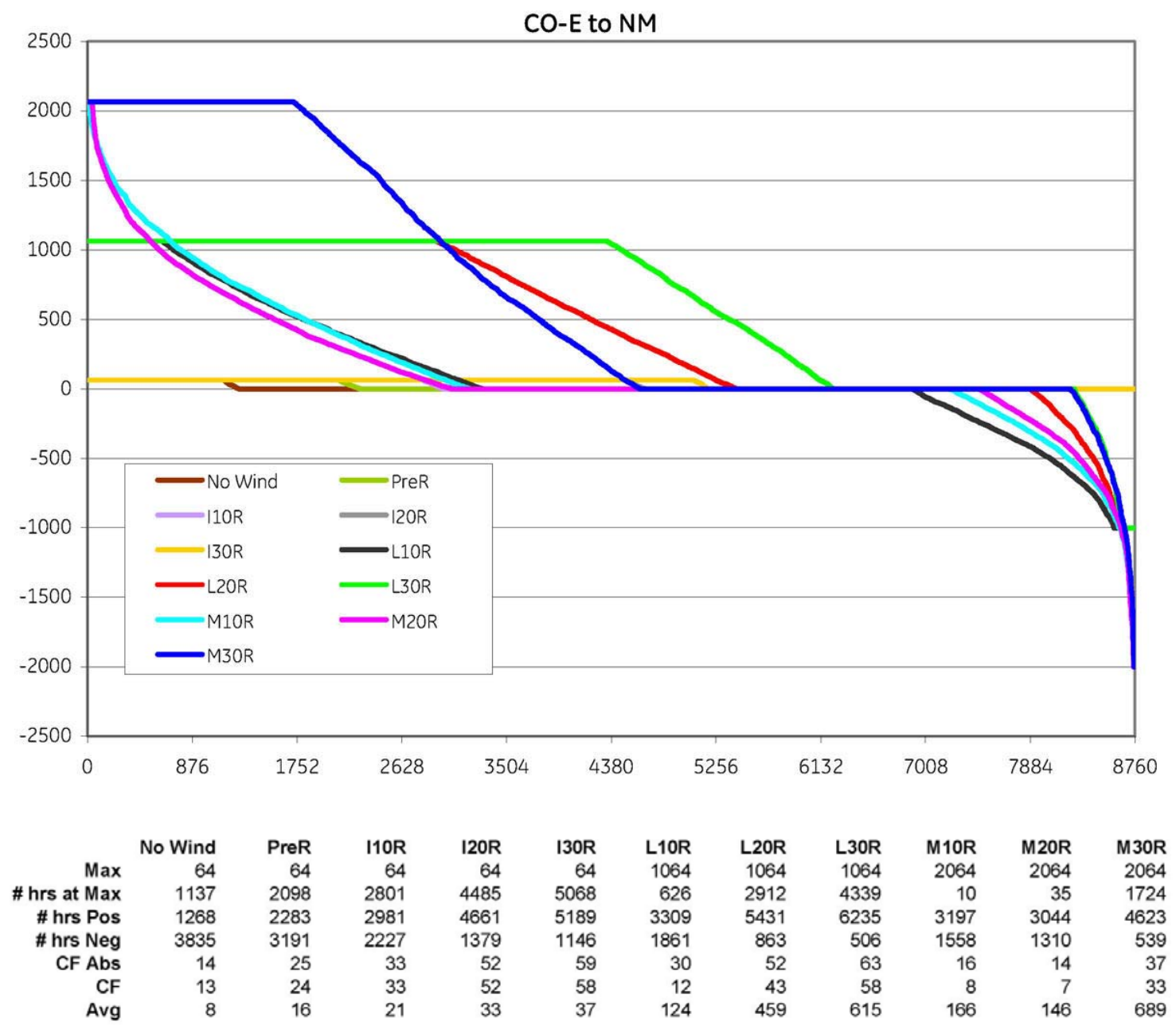

Figure D- 10 Eastern Colorado to New Mexico flows 


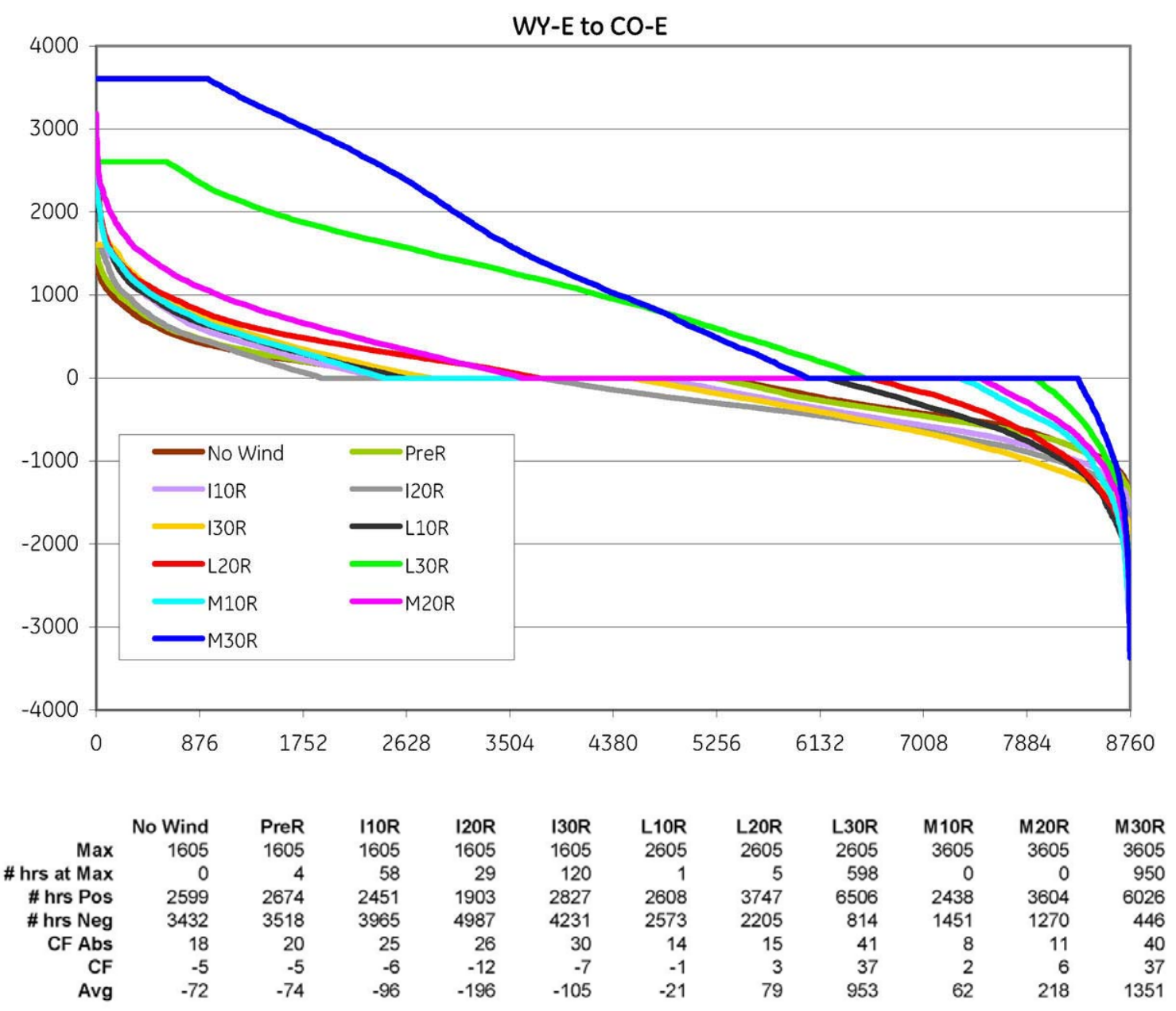

Figure D- 11 Eastern Wyoming to Eastern Colorado flows

The following group of figures present flow duration plots for all of the footprint interfaces for each of the scenarios. Where appropriate the interface limits have been increased. 


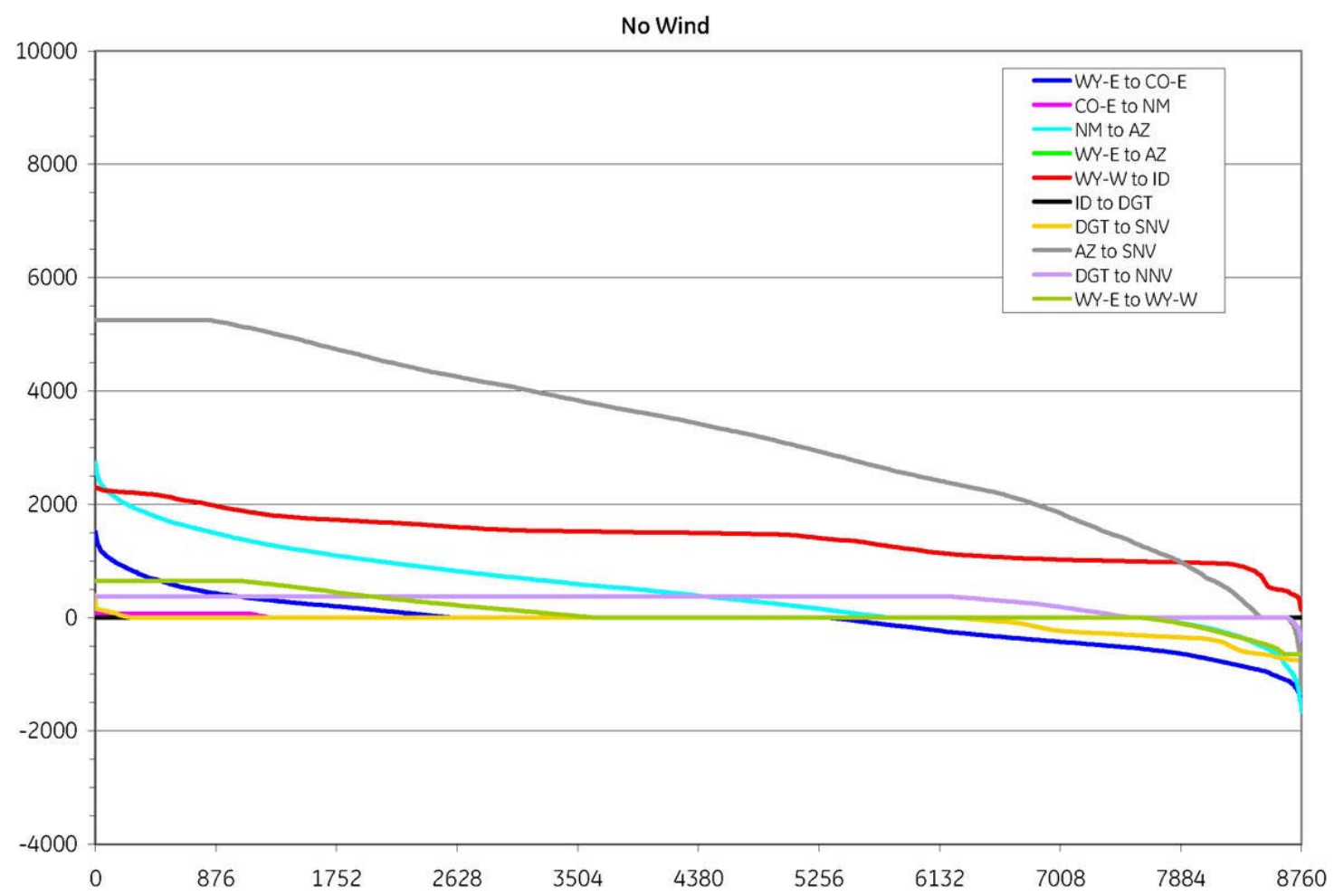

Figure D- 12 Interface flows - No wind scenario

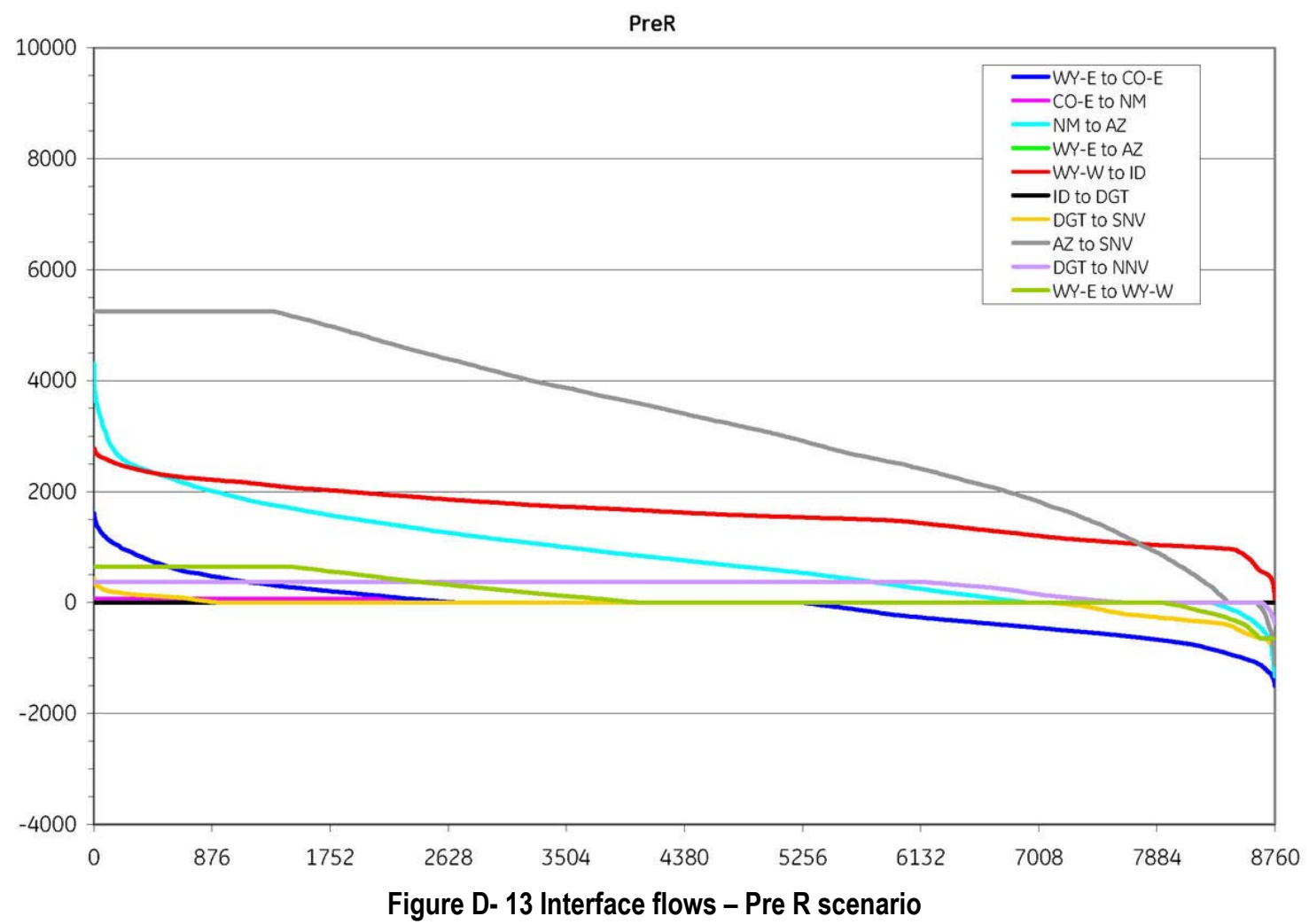



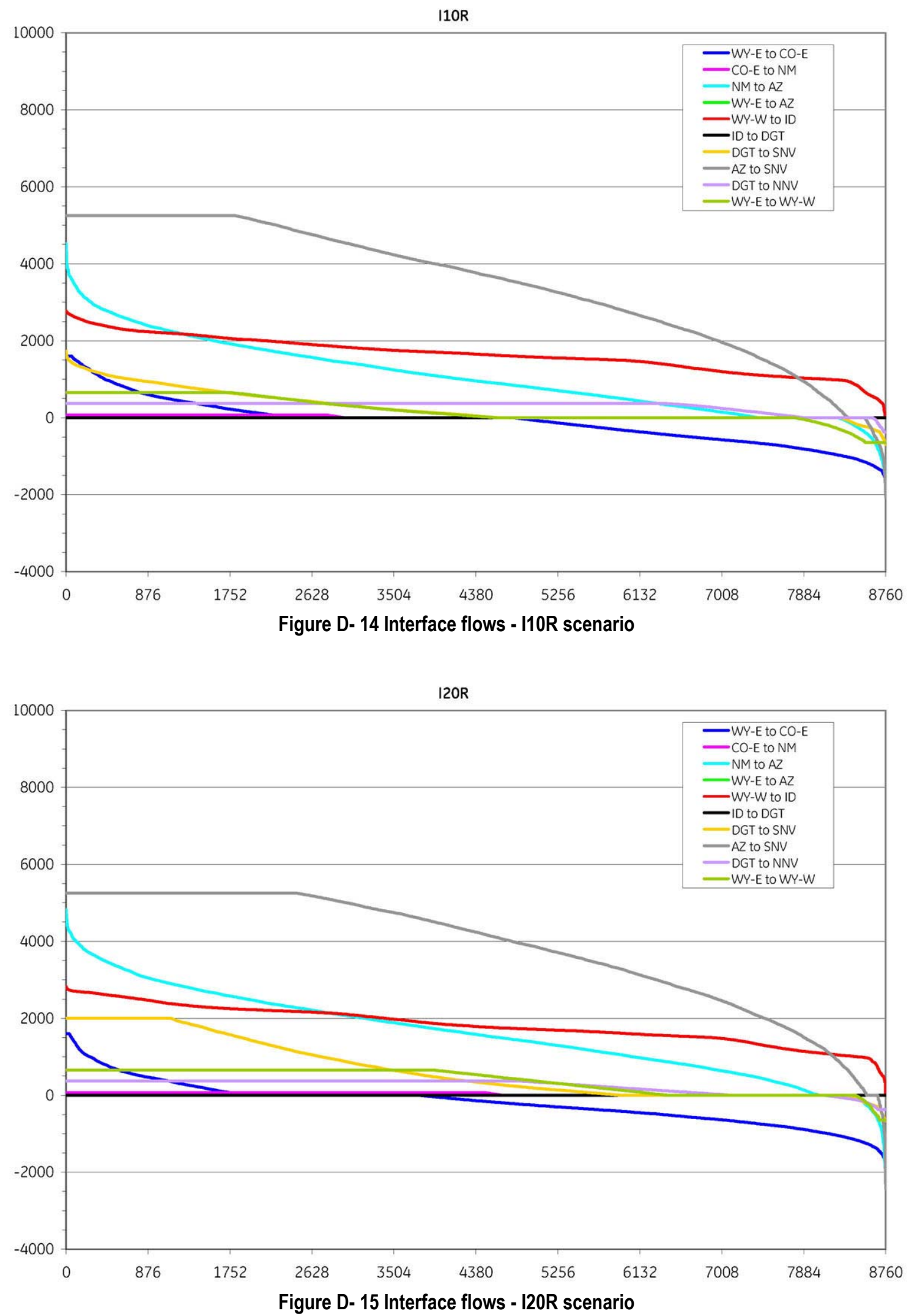

D-13 


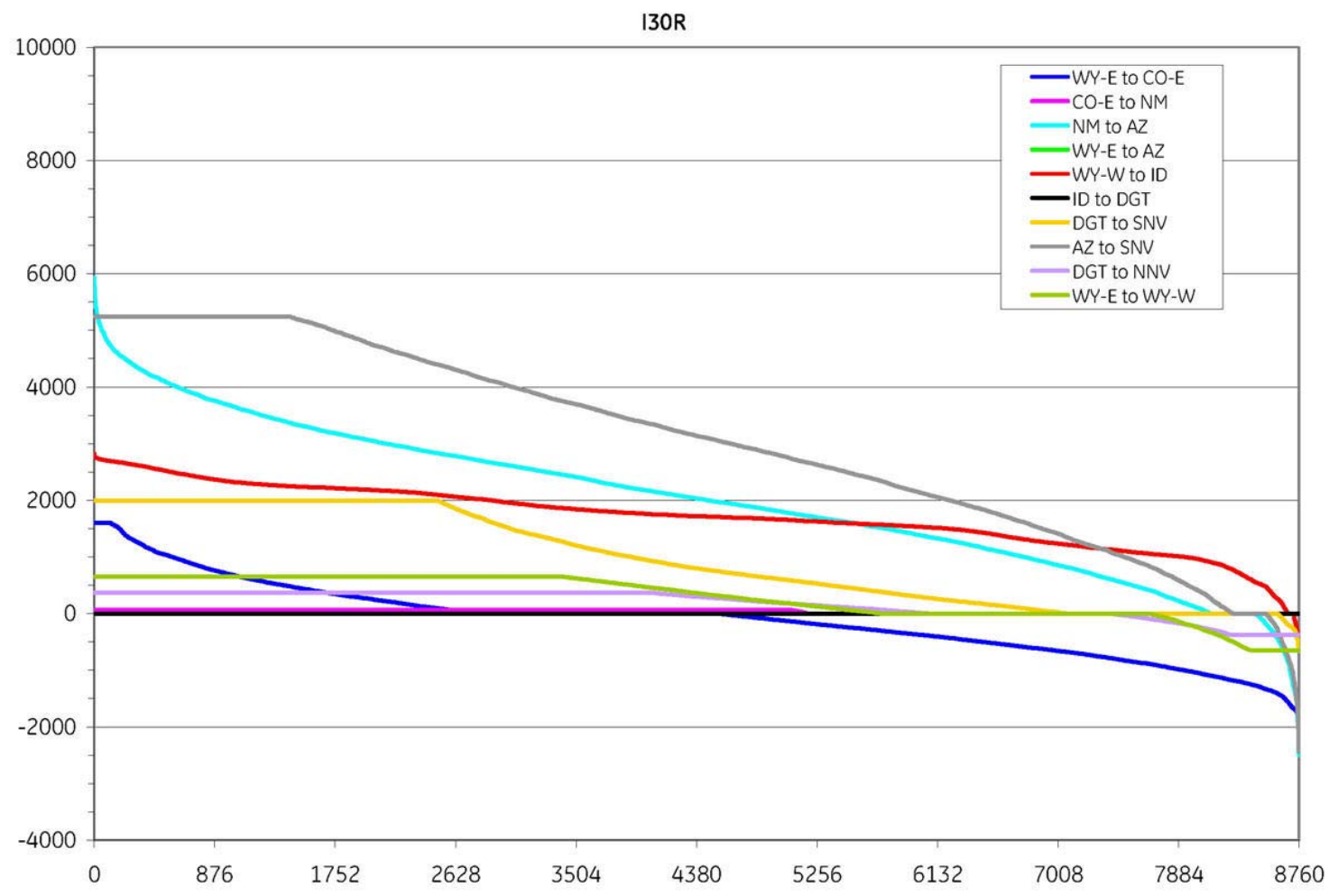

Figure D- 16 Interface flows - I30R scenario

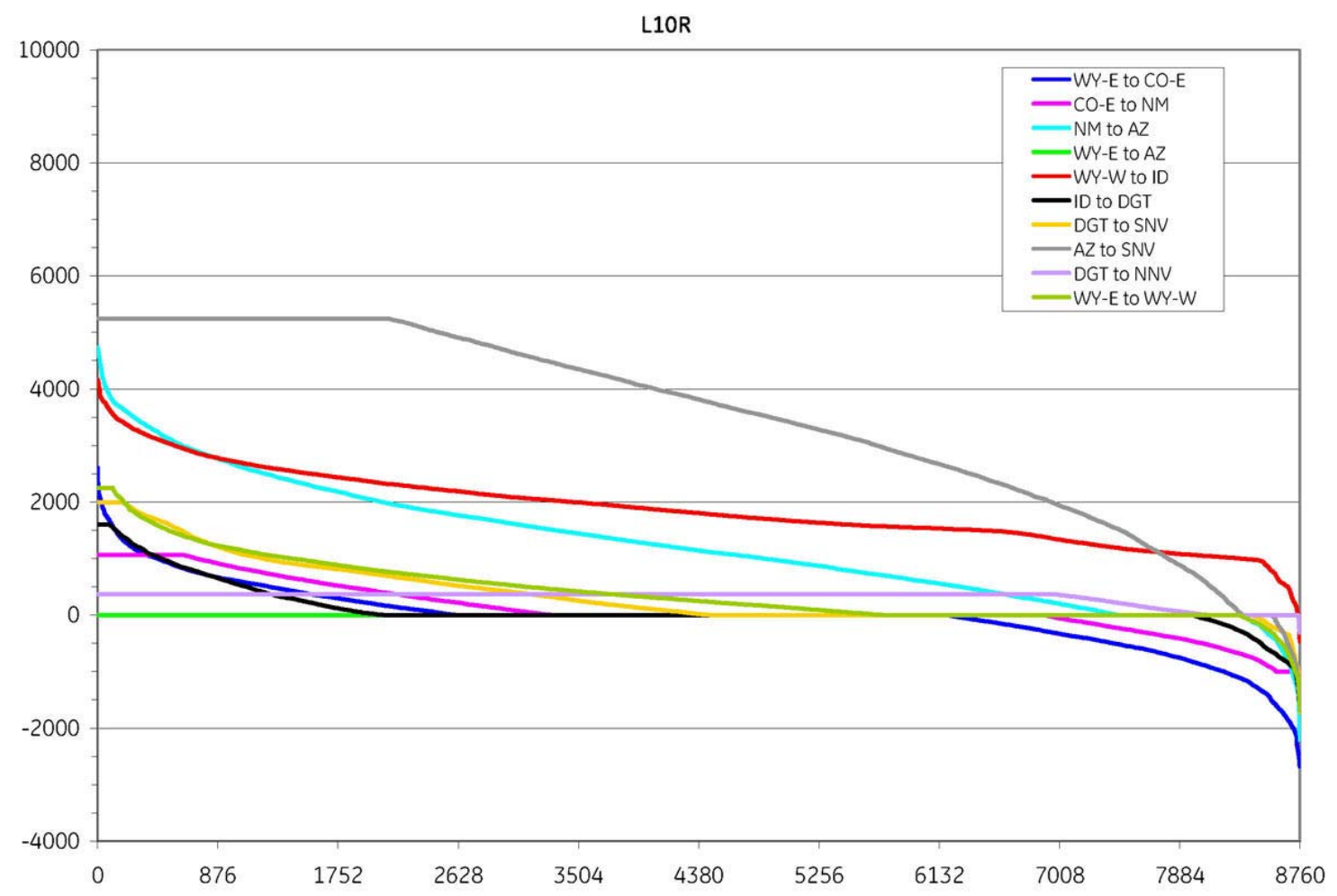

Figure D- 17 Interface flows - L10R scenario 

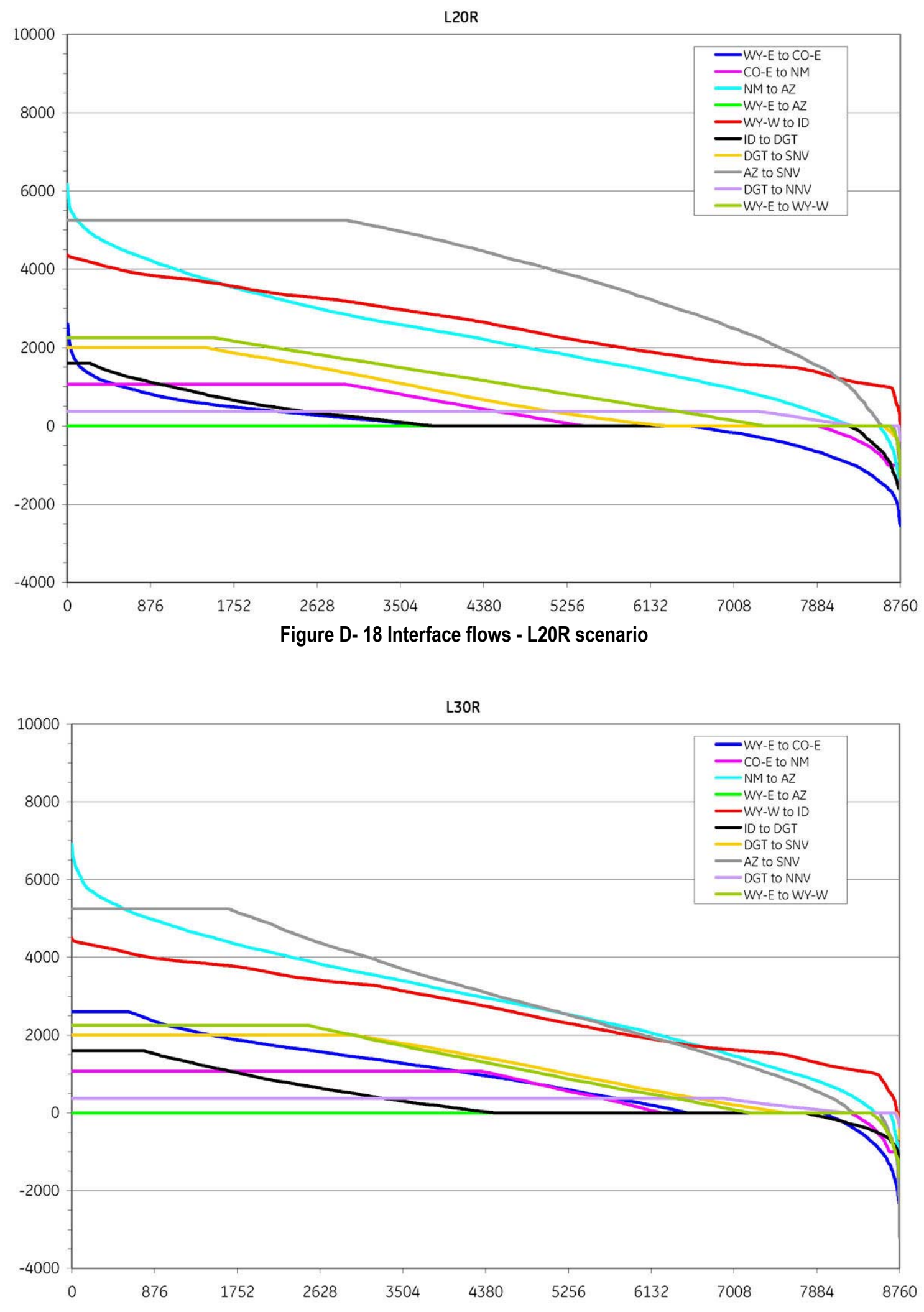

Figure D- 19 Interface flows - L30R scenario 


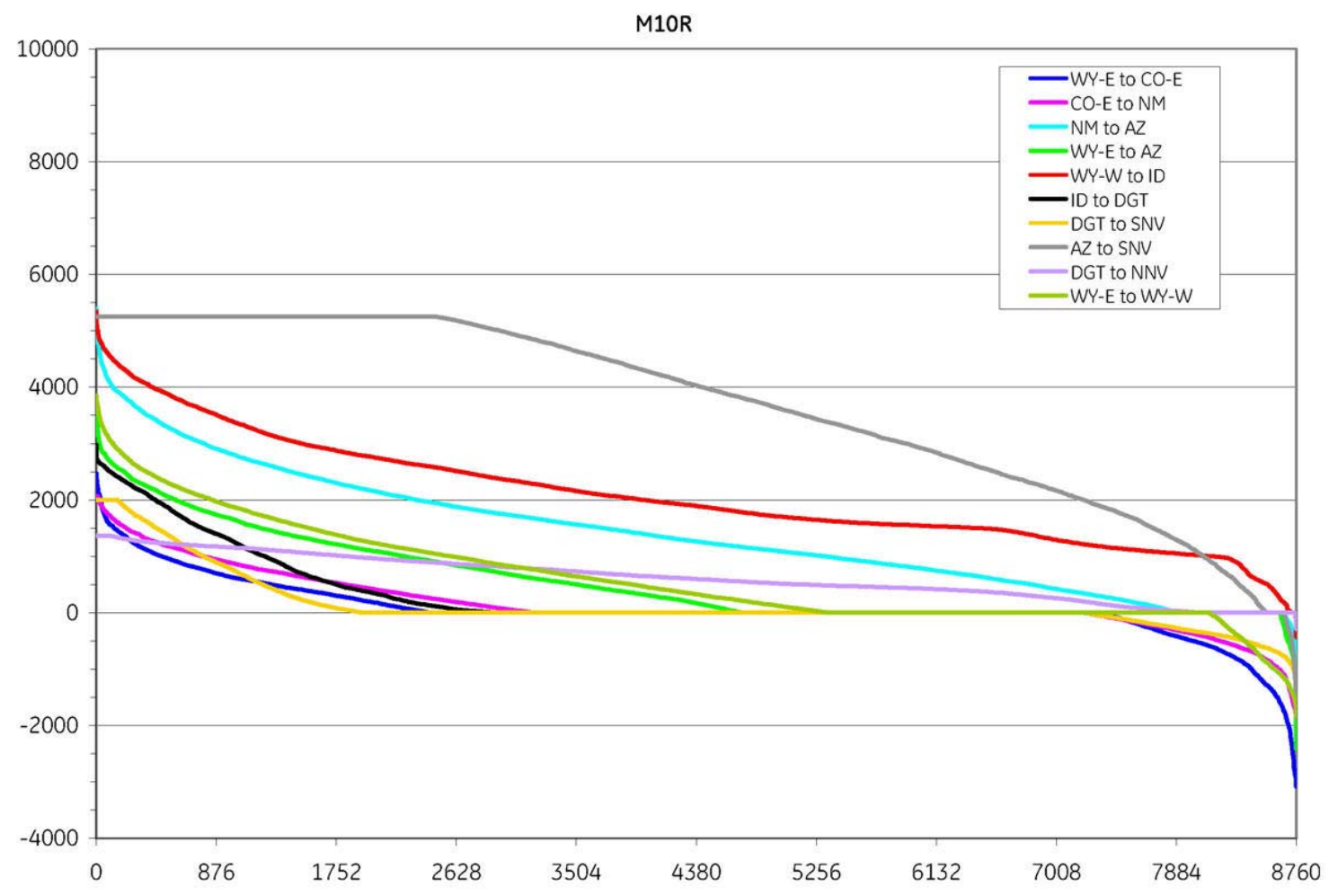

Figure D- 20 Interface flows - M10R scenario

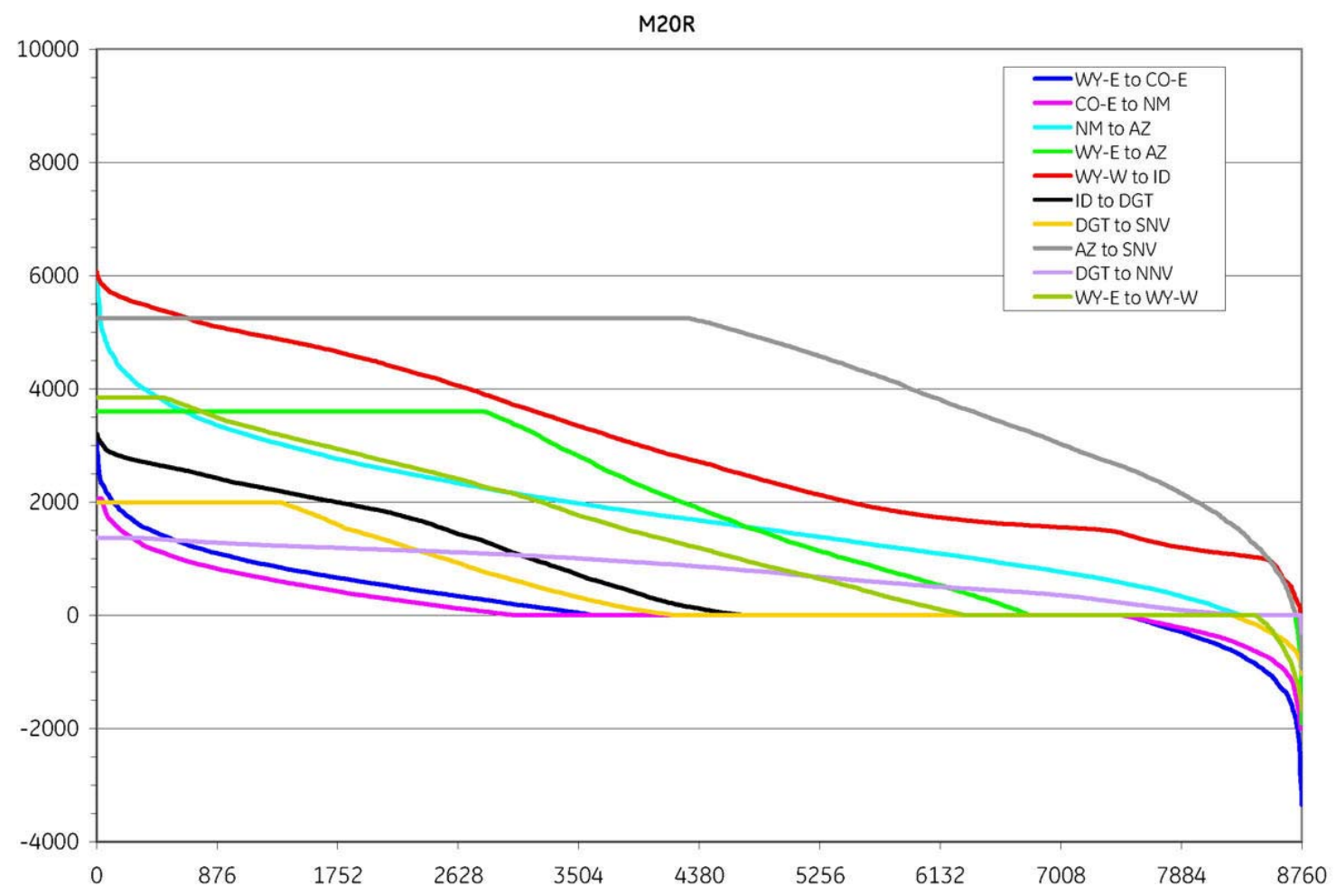

Figure D- 21 Interface flows - M20R scenario 


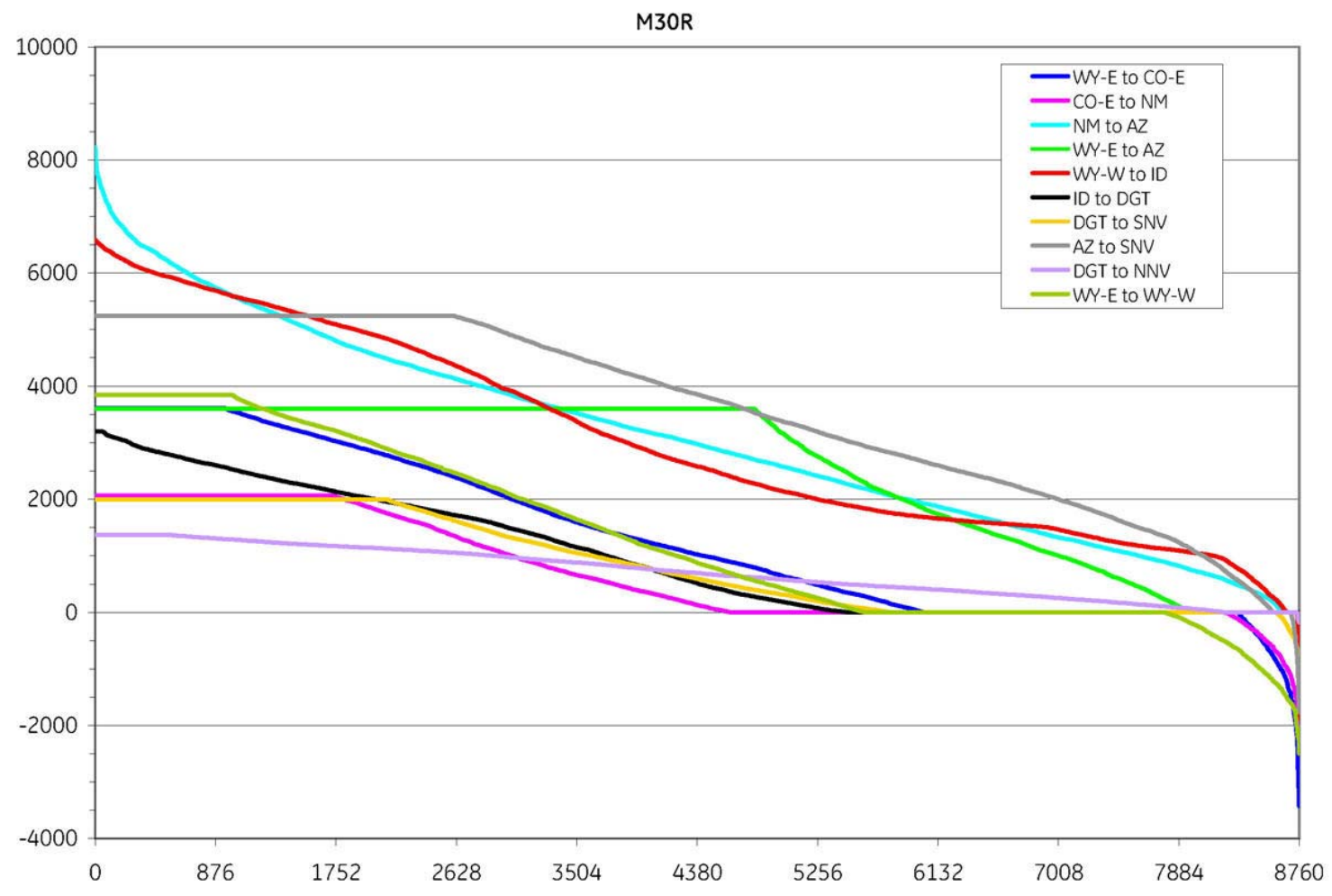

Figure D- 22 Interface flows - M30R scenario

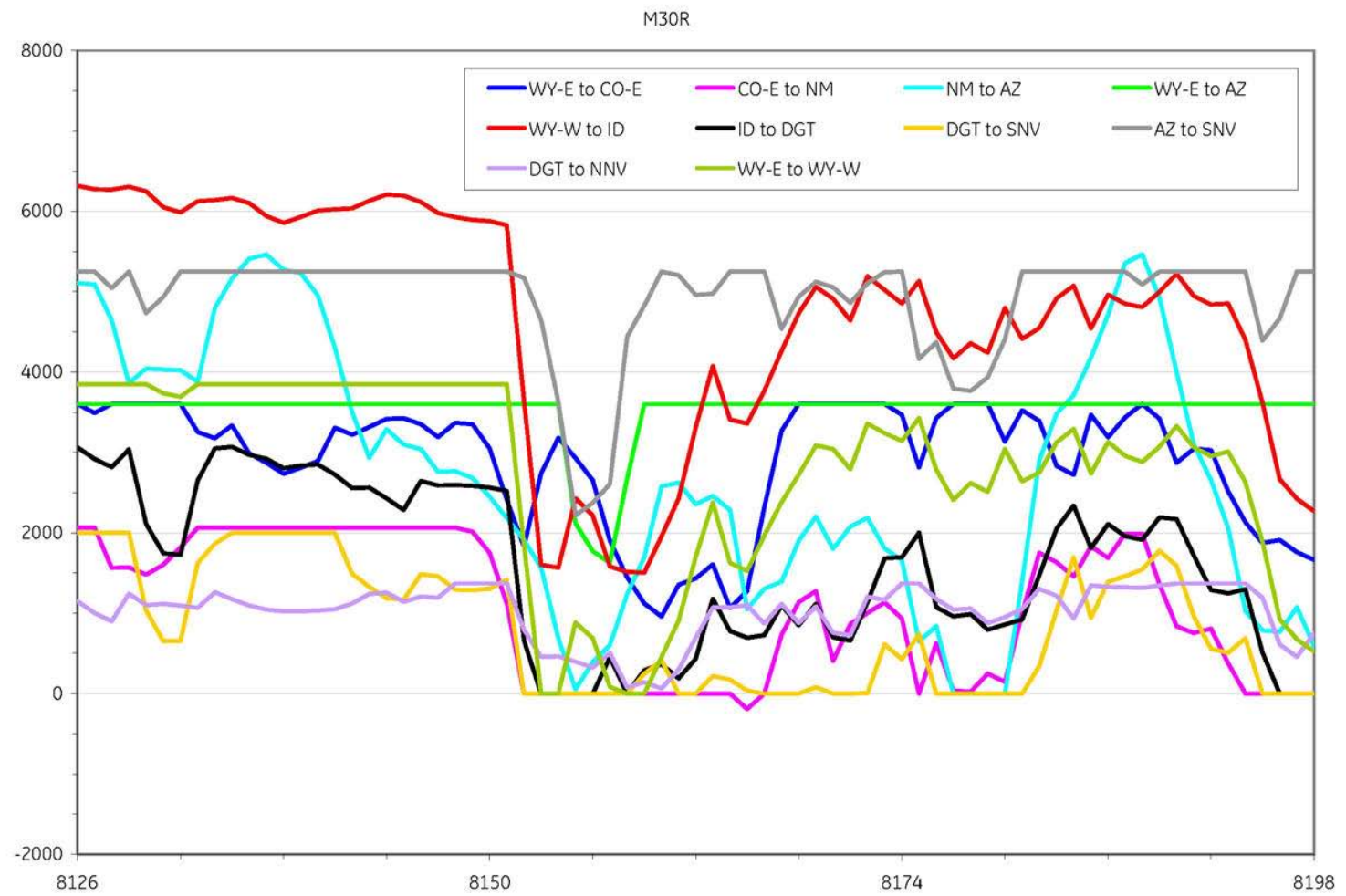

Figure D-23 Chronological interface flows - M30R scenario 


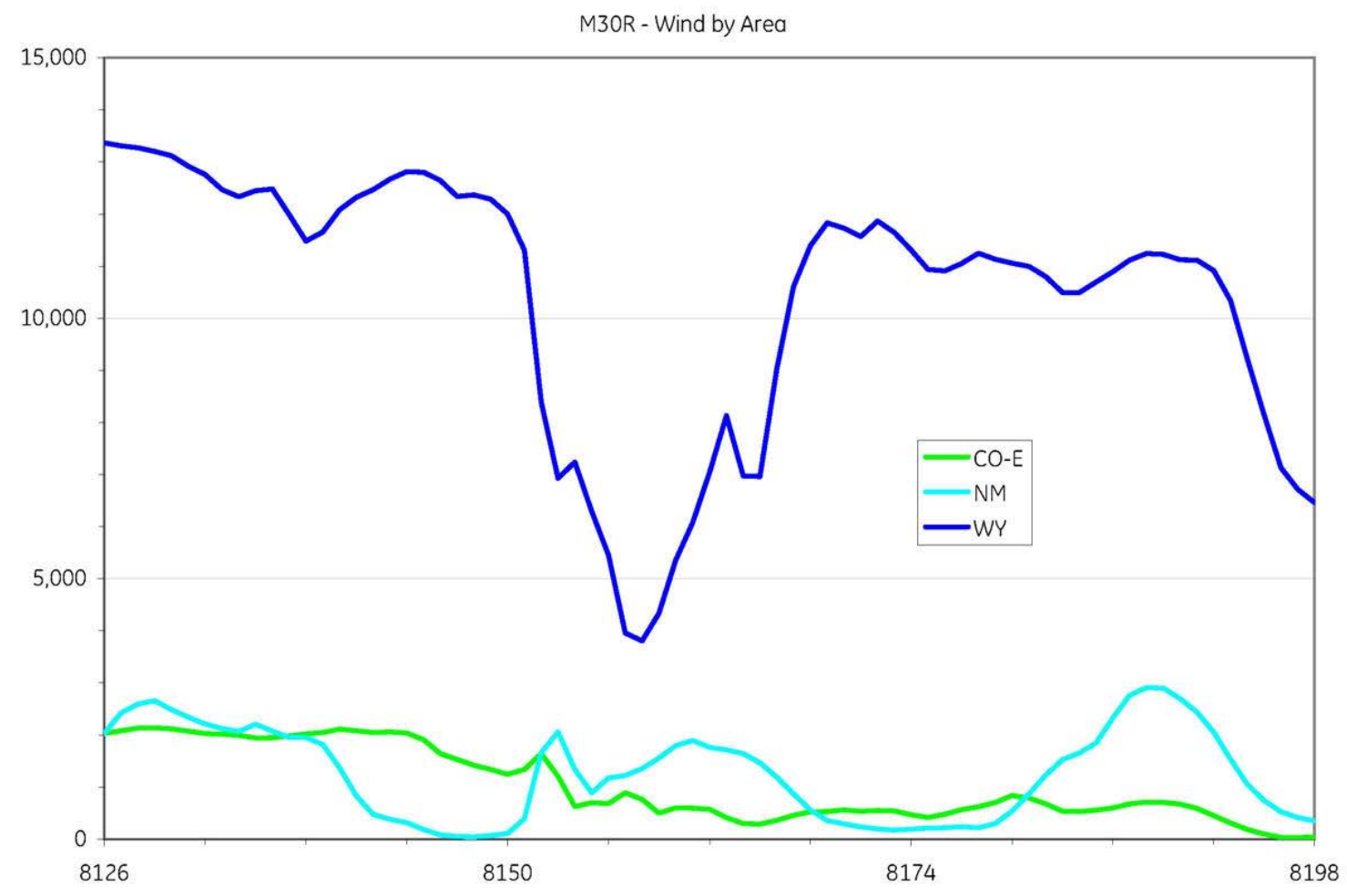

Figure D- 24 Chronological wind generation by area - M30R scenario

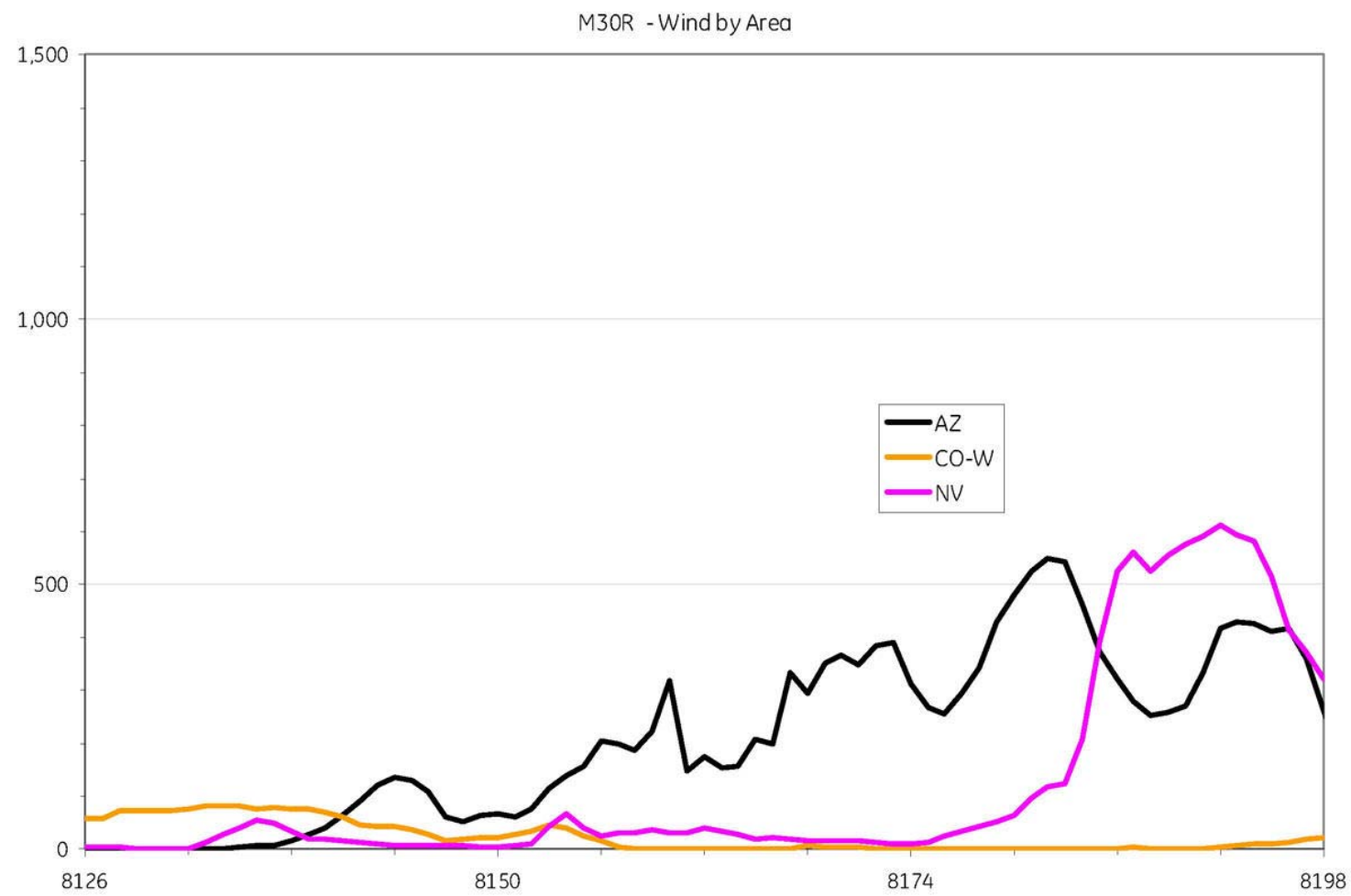

Figure D- 25 Chronological wind generation by area - M30R scenario 


\title{
Appendix E MAPS Flow-Duration
}

\author{
Summary Statistics for 10-Minute Data (2006, Day 1 Deleted)
}

(Scaled by $150 \%$ for "No. Drops" and "No. Rises")

\begin{tabular}{|c|c|c|c|c|c|c|c|c|c|c|c|c|c|c|c|c|c|c|c|c|c|c|c|c|}
\hline & \multicolumn{3}{|c|}{ Aniz } & \multicolumn{3}{|c|}{ COE.E } & \multicolumn{3}{|c|}{$\cos w$} & \multicolumn{3}{|c|}{$\operatorname{sim}$} & \multicolumn{3}{|c|}{ NV } & \multicolumn{3}{|c|}{$\mathrm{w} \gamma$} & \multicolumn{3}{|c|}{ In Foet Prist } & \multicolumn{3}{|c|}{ WECC } \\
\hline & IA & LP & Mp & IA & LP & MP & IA & LP & MP & IA & $L P$ & MP & IA & LP & MP & IA & LP & $M P$ & IA & LP & MP & IA & LP & MP \\
\hline \multicolumn{25}{|c|}{ Sigma (MW) } \\
\hline Load-alane & 97 & 97 & ज्ञा & 61 & 61 & 61 & 5 & $\overline{6}$ & 6 & 25 & 525 & z & 52 & 52 & ह2 & 15 & 15 & 15 & 239 & 259 & 239 & 731 & 731 & 731 \\
\hline Brreine (Existring) & 97 & 98 & 97 & 65 & ES & 와 & 6 & 6 & ef & 31 & it & 31 & 52 & 52 & 52 & 2 & 23 & 23 & 240 & 240 & 240 & 734 & 734 & 736 \\
\hline $10 \%$ in FP Scenaro & 107 & 105 & 97 & $\theta$ & 69 & 69 & 11 & 10 & 7 & 33 & 36 & 43 & 57 & 56 & 53) & 24 & 31 & of & 263 & 245 & 266 & 717 & 718 & 719 \\
\hline $20 \%$ in FP Scenaso & 128 & 116 & 100 & $\infty$ & of & 71 & 19 & 16 & 7 & 43 & 45 & 54 & 68 & 63 & 63 & 34 & 56 & 100 & 259 & 260 & 268 & 716 & 718 & 722 \\
\hline $30 \%$ in FP Scenato & 105 & 128 & 105 & 98 & 69 & 72 & 25 & 20 & 10 & 54 & 55 & ro & 79 & 69 & 5ा & 46 & 93 & $15 \pi$ & 286 & 206 & 301 & 728 & 728 & 734 \\
\hline \multicolumn{25}{|c|}{ Max Neg Detha (IMW) } \\
\hline Losistione & -363 & -348 & -365 & -223 & .223 & -223 & -20 & -20 & 20 & -6 & 58 & -80 & -200 & 200 & -200 & -53 & 53 & -53 & $\sqrt{08}$ & .708 & -700 & -2239 & 2239 & .2239 \\
\hline Dneline (Existrng) & $\$ 52$ & .952 & .52 & .412 & .412 & 412 & 20 & $=20$ & $x 0$ & 200 & .260 & 200 & .200 & 200 & .20 & .34 & .134 & .334 & 810 & ato & .010 & .2279 & 2279 & .22279 \\
\hline $10 \%$ in FP Scenato & -901 & $m 2$ & ext & -412 & .420 & $\infty$ & .134 & +109 & 41 & .252 & .240 & .34 & -367 & 300 & -250 & 300 & -340 & 900 & -843 & 967 & .991 & .3744 & $\$ 34$ & -306 \\
\hline $20 \%$ in FP Scentase & -1367 & .1062 & 30 & -ees & 829 & -20 & -234 & $4 \cdot 205$ & 60 & -394 & -108 & -532 & 568 & 565 & -305 & -401 & -962 & -1725 & -1360 & -1216 & -1821 & .3600 & $3 / 2$ & $-3 / 13$ \\
\hline $30 \%$ in FP Scenatio & .1616 & .1169 & 418 & -1062 & 956 & 580 & 200 & .259 & .91 & 516 & 5 coss & 700 & $\pi \theta$ & 633 & -305 & 501 & -1476 & .2274 & .1576 & -1641 & 2325 & .4265 & 4270 & .4300 \\
\hline \multicolumn{25}{|c|}{ Max Pes Della a (MM) } \\
\hline Cosd-alone & 257 & 267 & 267 & 196 & 196 & 196 & $\overline{22}$ & 22 & 22 & 106 & 5106 & 106 & 206 & 206 & 206 & 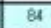 & 84 & 84 & 612 & 612 & 612 & 1706 & 1736 & 1786 \\
\hline Enselire (Existng) & 269 & 269 & 280 & 306 & 306 & 300 & 22 & 22 & 28 & 147 & 142 & 142 & 200 & 200 & 200 & 228 & 228 & 228 & 10 & 710 & 710 & 1865 & 1065 & 1008 \\
\hline 10\% in FP Scenaro & 711 & 500 & $m$ & 334 & $3 x$ & 564 & 100 & 93 & $x$ & tee & 20 & 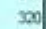 & 319 & $3 n$ & 200 & 203 & 280 & 56/ & 29 & aza & $m 0$ & $x \in 4$ & 2093 & 2118 \\
\hline $20 \%$ in FP Scenato & 917 & 784 & 897 & 500 & 555 & 360 & 211 & 197 & 51 & 323 & 358 & 460 & 455 & 49 & 233 & 459 & 630 & 105 & 1193 & 9075 & 1213 & 2211 & 2172 & 2003 \\
\hline $300 \%$ in FP Scenato & 1169 & 1041 & 1200 & 1010 & 804 & 428 & 296 & 221 & 9] & 252 & 456 & 54] & 562 & 519 & 293 & 849 & 994 & 1005 & 1430 & 1416 & 1542 & 2728 & 2001 & $26 \pi$ \\
\hline \multicolumn{25}{|c|}{ Ne, Dieps $>3^{*}$ Ld Sigma } \\
\hline Loud-viane & 18 & 18 & 18 & 36 & 5 & 36 & 90 & 59 & 90 & 45 & 54 & 45 & 54 & 54 & 54. & $\overline{45}$ & 45 & as & 0 & $\overline{0}$ & & 9 & 9 & \\
\hline Busebre (Exitting) & 18 & 18 & 18 & 155 & 155 & 155 & 90 & 50 & $x$ & 517 & 617 & 617 & 54 & 54 & 54 & 1458 & 1458 & 1458 & 12 & 12 & 12 & 9 & 9 & \\
\hline $105 \%$ in FP Scenato & 203 & 150 & 21 & 296 & 323 & $x_{2}$ & 2163 & 130 & 292 & 822 & 1121 & 1900 & 219 & 1as & 요 & 1649 & 2979 & 609 & 4 & 42 & $6=$ & 6 & E & 11 \\
\hline $20 \%$ in FP Scenato & 672 & 500 & 47 & 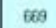 & 60 & 352 & 5428 & 54250 & 476 & 1977 & 2062 & $3+\infty$ & 600 & sit & 117 & 350 & 7836 & 12800 & 125 & 140 & 224 & 6 & 5 & 11 \\
\hline $30 \%$ in FP Scenatio & 1172 & 632 & 15: & 1300 & 995 & 344 & 7004 & 40074 & 415 & 3156 & 3652 & 5262 & 1317 & 684 & 150 & 6990 & 12185 & 15005 & 200 & 284 & 438 & 17 & 17 & 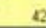 \\
\hline \multicolumn{25}{|c|}{ No. Rikes $>3^{\circ}$ La Sigma } \\
\hline Losd alane & 0 & 0 & 7 & 3 & 36 & 30 & 72 & 72 & 72 & 107 & 107 & 100 & 27 & 27 & 27 & 405 & 205 & 205 & 0 & $\bar{D}$ & & 0 & 0 & \\
\hline Bvetre (Existong) & 0 & 0 & d & $\omega$ & 66 & 60 & $n$ & 272 & 72 & 49 & 40 & 469 & 36 & 35 & 3) & 1563 & 1563 & 1563 & 0 & 0 & 0 & 0 & 0 & \\
\hline $10 \%$ in FP Scenaro & 165 & 123 & 1 & 194 & 256 & 204 & 1966 & 1194 & 235 & 656 & 95 & 1900 & 196 & 138 & as & 1779 & 3144 & क्रा1 & 15 & is & 12 & 0 & 0 & \\
\hline $20 \%$ in FP Scenato & 722 & 419 & 13 & $7 x$ & $\pi 4$ & 302 & 5603 & 2521 & $\mathbf{m}=$ & 2043 & 2357 & 311 & 714 & 259 & 74 & 3301 & 2039 & 13319 & 203 & 171 & 212 & 2 & D & 2 \\
\hline 30\% in FP Scenaro & 1204 & 762 & 357 & 1656 & 1209 & 235 & 7903 & 6146 & 1908 & 3497 & 304 & 5050 & 1350 & 824 & 191 & 6208 & 12513 & 16353 & 521 & 476 & 99 & 63 & 48 & 50 \\
\hline
\end{tabular}

\section{Summary Statistics for 10-Minute Delta Zone -> Arizona -> Footprint -> WECC (2006, Local Priority, Day 1 Deleted)}

\begin{tabular}{|c|c|c|c|c|c|c|c|c|c|c|c|c|}
\hline & \begin{tabular}{|l|l} 
AEPCA \\
\end{tabular} & APSCA & AZ.GCA & AZ.PhoA & SRPA & TEPA & AEPCA & AZEEA & AZ-NawA & $A Z$ & In FootPrint & WECC \\
\hline \multicolumn{13}{|l|}{ Sigma (MW) } \\
\hline Load-alone & 3 & 37 & 0 & 2 & 39 & 0 & 18 & 0 & 잉 & 97 & 239 & 731 \\
\hline Baseline (Existing) & 3 & 37] & 0 & 2 & 39 & 이 & 18 & 0 & o & 97 & 240 & 734 \\
\hline $10 \%$ In FP Scenario & 6 & 58 & 0 & 3 & 39 & 0 & 10 & 0 & o & 105 & 245 & 718 \\
\hline $20 \%$ In FP Scenario & 11 & 77 & 2 & 3 & 39 & 0 & 18 & 0 & of & 116 & 260 & 718 \\
\hline $30 \%$ In FP Scenario & 28 & 87 & 5 & 14 & 40 & 0 & 18 & 0 & o & 128 & 206 & 728 \\
\hline \multicolumn{13}{|l|}{ Max Neg Delta (MW) } \\
\hline Load-alone & 9 & -111 & -1 & -6 & .185 & 2 & -72 & 0 & 이 & 348 & 700 & .2239 \\
\hline Baseline (Existing) & 9 & -126 & -1 & -6 & -185 & -2 & -72 & 0 & of & -352 & -810 & .2279 \\
\hline $10 \%$ In FP Scenario & -911 & .705 & -1 & .60 & -185 & .2 & -72 & 0 & of & -772 & -867 & -3734 \\
\hline $20 \%$ In FP Scenario & -187 & -1000 & .30 & .60 & .261 & -2 & -72 & 0 & o & -1052 & -1216 & -3672 \\
\hline $30 \%$ In FP Scenario & .338 & .1137 & .52 & 80 & -400 & .2 & -72 & 0 & o & .1169 & -1641 & .4270 \\
\hline \multicolumn{13}{|l|}{ Max Pos Delta (MW) } \\
\hline Load-alone & 11 & 111 & 2 & 6 & 119 & 2 & 68 & 0 & 0 & 267 & 612 & 1786 \\
\hline Baseline (Existing) & 11 & 111 & 4 & 6 & 119 & 2 & 68 & 0 & 0 & 269 & 710 & 1865 \\
\hline $10 \%$ in FP Scenario & 77 & 505 & 2 & 55 & 119 & 2 & 68 & 0 & o & 590 & 873 & 2093 \\
\hline $20 \%$ in FP Scenario & 135 & 729 & 30 & 55 & 457 & 2 & 68 & 0 & 0 & 784 & 1075 & 2172 \\
\hline $30 \%$ in FP Scenario & 319 & 804 & 52 & 100 & 617 & 2 & 68 & 0 & 이 & 1041 & 1416 & 2681 \\
\hline
\end{tabular}


Summary Statistics for 10-Minute Delta Zone -> Wyoming -> Footprint -> WECC (2006, Local Priority, Day 1 Deleted)

\begin{tabular}{|c|c|c|c|c|c|c|c|c|c|c|}
\hline & BEPCWA & BHPLA & WYCENA & \begin{tabular}{|l} 
WYNWWA \\
\end{tabular} & WYSWA & TRSTWYOA & WACMA & $w r$ & In FootPrint & WECC \\
\hline \multicolumn{11}{|l|}{ Sigma (MW) } \\
\hline Load-alone & 2 & 2 & 2 & 3 & 3 & 1 & 2 & 15 & 239 & 731 \\
\hline Baseline (Existing) & 2 & 2 & 8 & 3 & 17 & 2 & 2 & 23 & 240 & 734 \\
\hline $10 \%$ in FP Scenano & 2 & 2 & 15 & 4 & 17 & 3 & 14 & 31 & 245 & 710 \\
\hline $20 \%$ in FP Scenario & 2 & 2 & 33 & 8 & 17 & 18 & 27] & 56 & 260 & 718 \\
\hline $30 \%$ in FP Scenario & 2 & 2 & 47. & 9] & 18 & 53 & 36] & 93) & 206 & 720 \\
\hline \multicolumn{11}{|l|}{ Max Neq Delta (MW) } \\
\hline Load-alone & 7 & 8 & .10 & -10 & -11 & 3 & 8 & -63 & .708 & .2239 \\
\hline Baseline (Existing) & -7) & 8 & 91 & -10 & 306 & .29 & $\theta$ & 334 & -810 & .2279 \\
\hline $10 \%$ in FP Scenario & -7 & 8) & -162 & 56 & 306 & 36 & 369 & 340 & -607 & .3734 \\
\hline $20 \%$ in FP Scenario & -7 & ?) & -409 & -170 & 305 & .439 & -660 & .962 & -1216 & -3672 \\
\hline $30 \%$ in FP Scenario & -7 & 2) & 590 & -170 & 300 & -809 & -1128 & .1476 & -1641 & -4270 \\
\hline \multicolumn{11}{|l|}{ Max Pos Delta (MW) } \\
\hline Load-alone & $\pi 1$ & 13 & 16 & 17 & 19 & E & 7 & 84 & 612 & 1786 \\
\hline Baseline (Existing) & 11 & 13 & 90 & 17] & 227 & 27 & 7 & 228 & 710 & 1865 \\
\hline $10 \%$ in FP Scenario & 11 & 13 & 168 & 50 & 227 & 31 & 264 & 280 & 873 & 2093 \\
\hline $20 \%$ in FP Scenario & 11 & 13 & 443 & 134 & 227 & 276 & 548 & 630 & 1075 & 2172 \\
\hline $30 \%$ in FP Scenano & 11] & 13 & 618 & 134 & 227 & 620 & 716 & 994 & 1416 & 2681 \\
\hline
\end{tabular}

Wind Deltas vs Load Deltas for Study Area (2006, 30\%, IA Scenario)

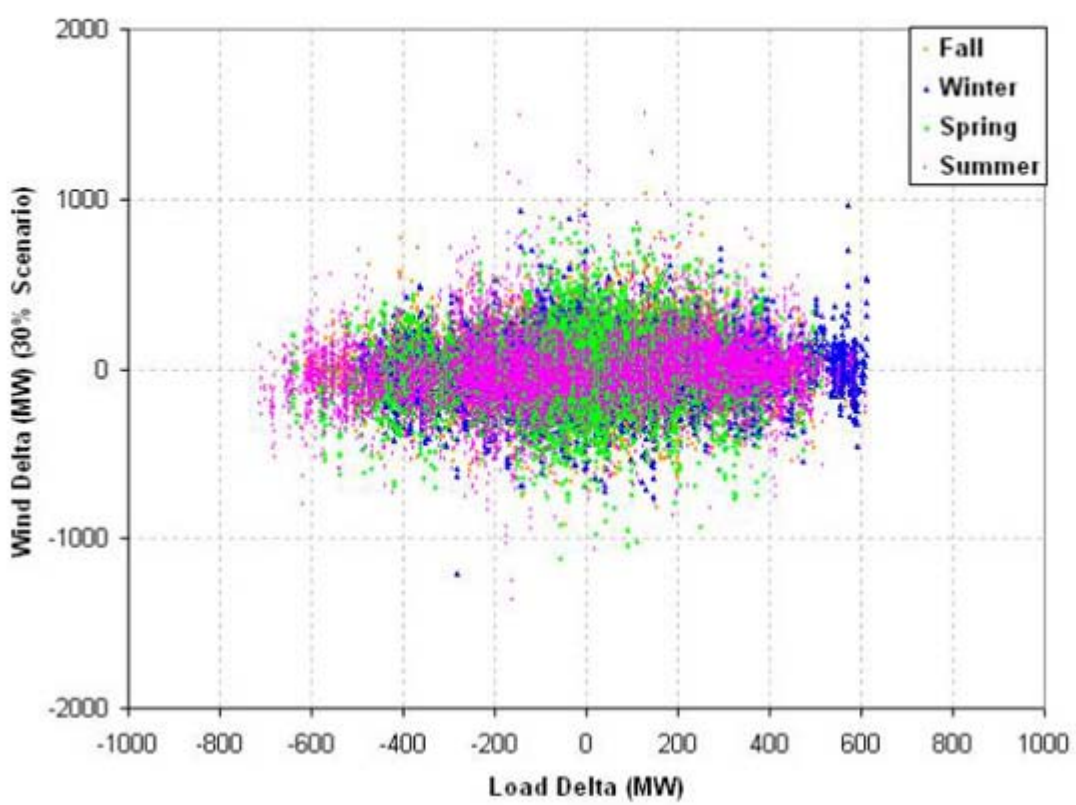


Wind Deltas vs Load Deltas by Season for Study Area (2006, 30\%, IA Scenario)
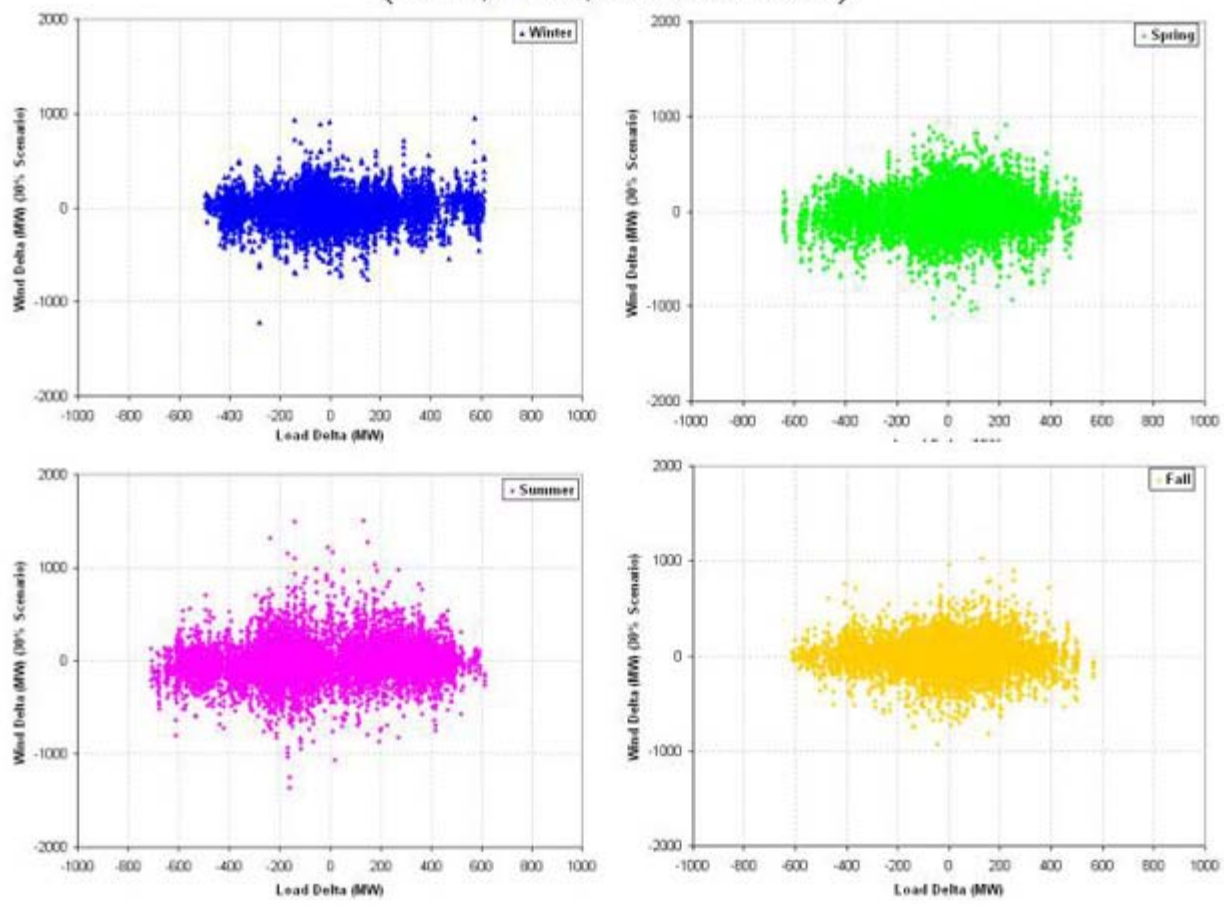

\section{Timing of Maximum Daily 10-Min Positive Deltas for Study Area (2006, IA Scenario)}

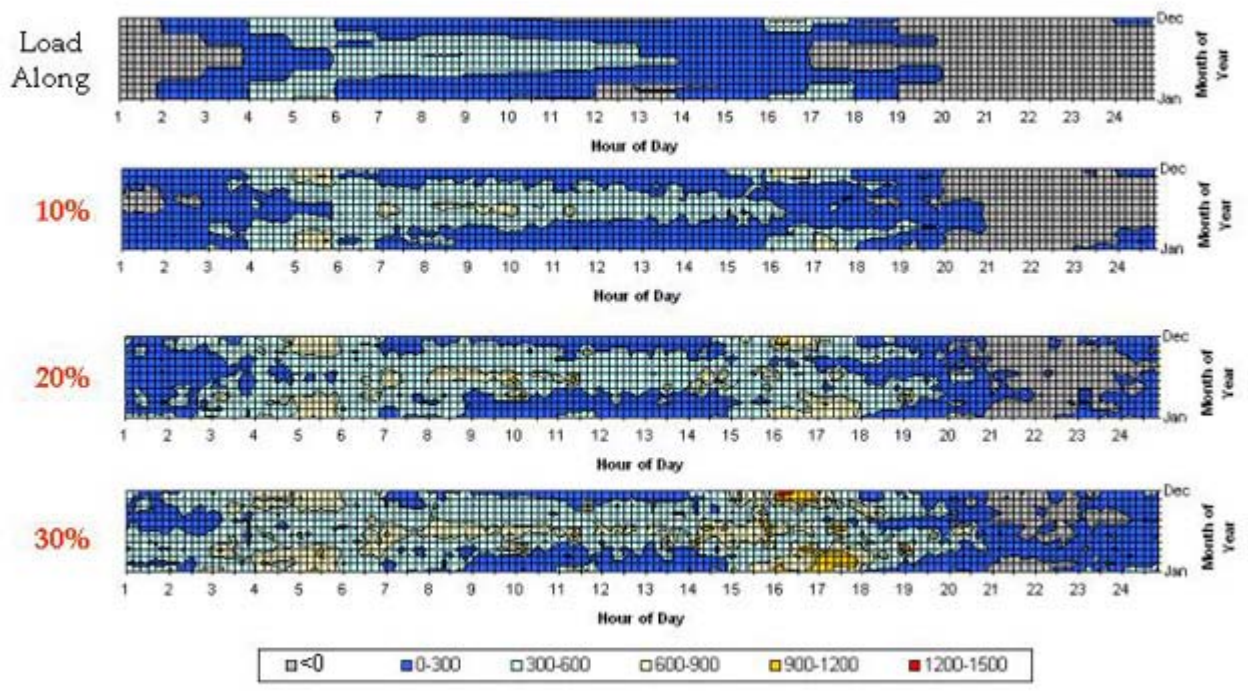


Timing of Maximum Daily 10-Min Negative Deltas for Study Area (2006, IA Scenario)

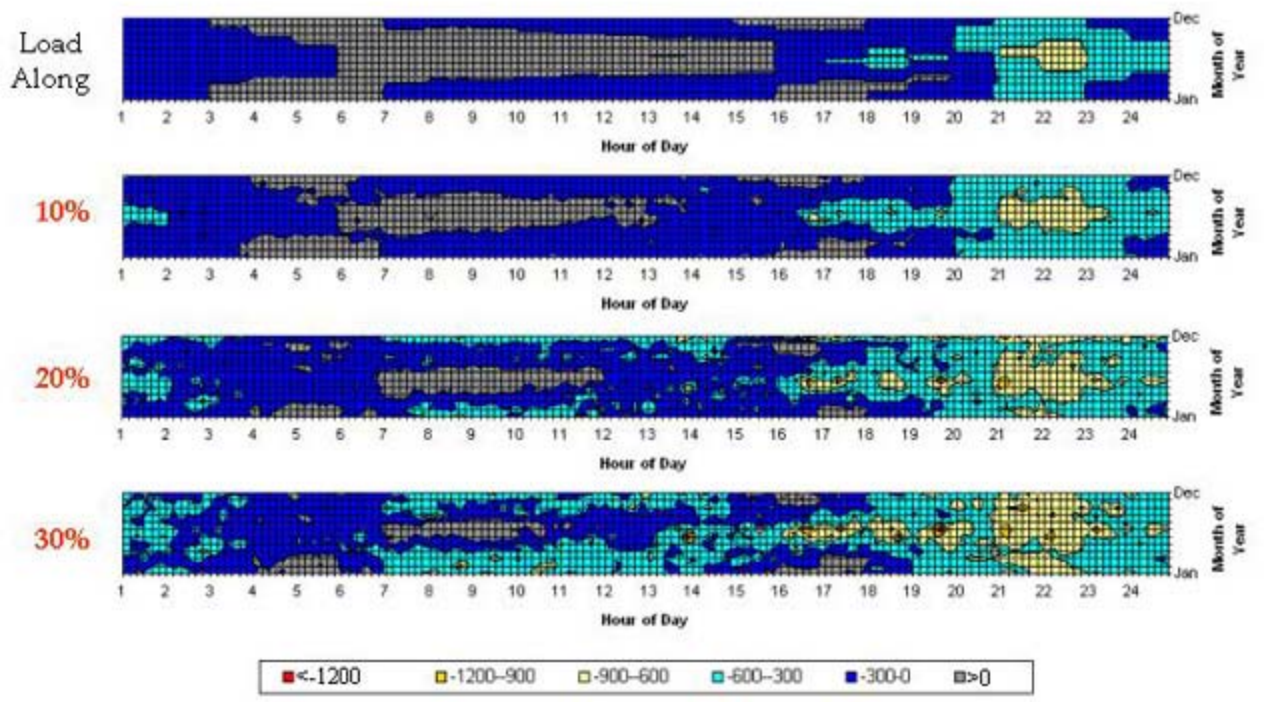

Effect of Wind Aggregation on Delta Distribution for Study Area (2006, IA Scenario, Scaled by $150 \%$ )

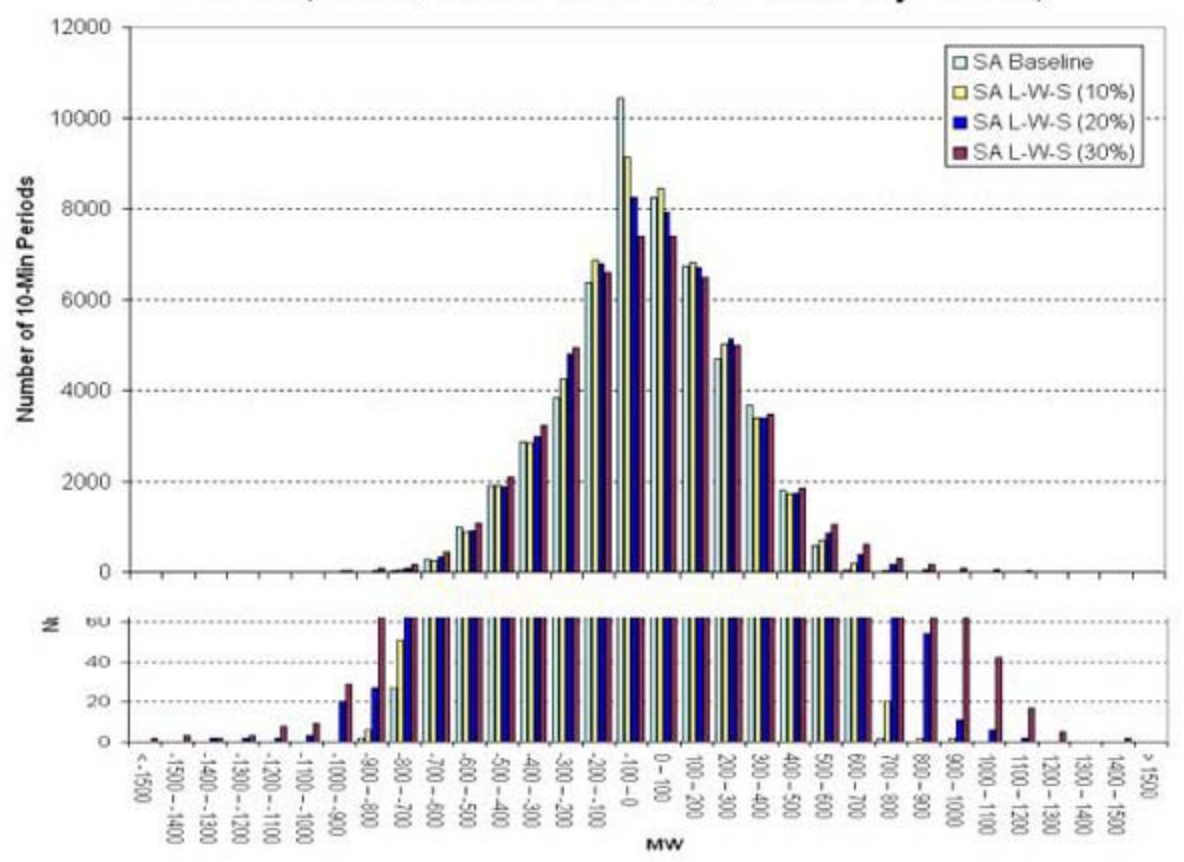




\section{Distribution of Extreme Hourly Wind Deltas for 2006 (2006, IA Scenario)}

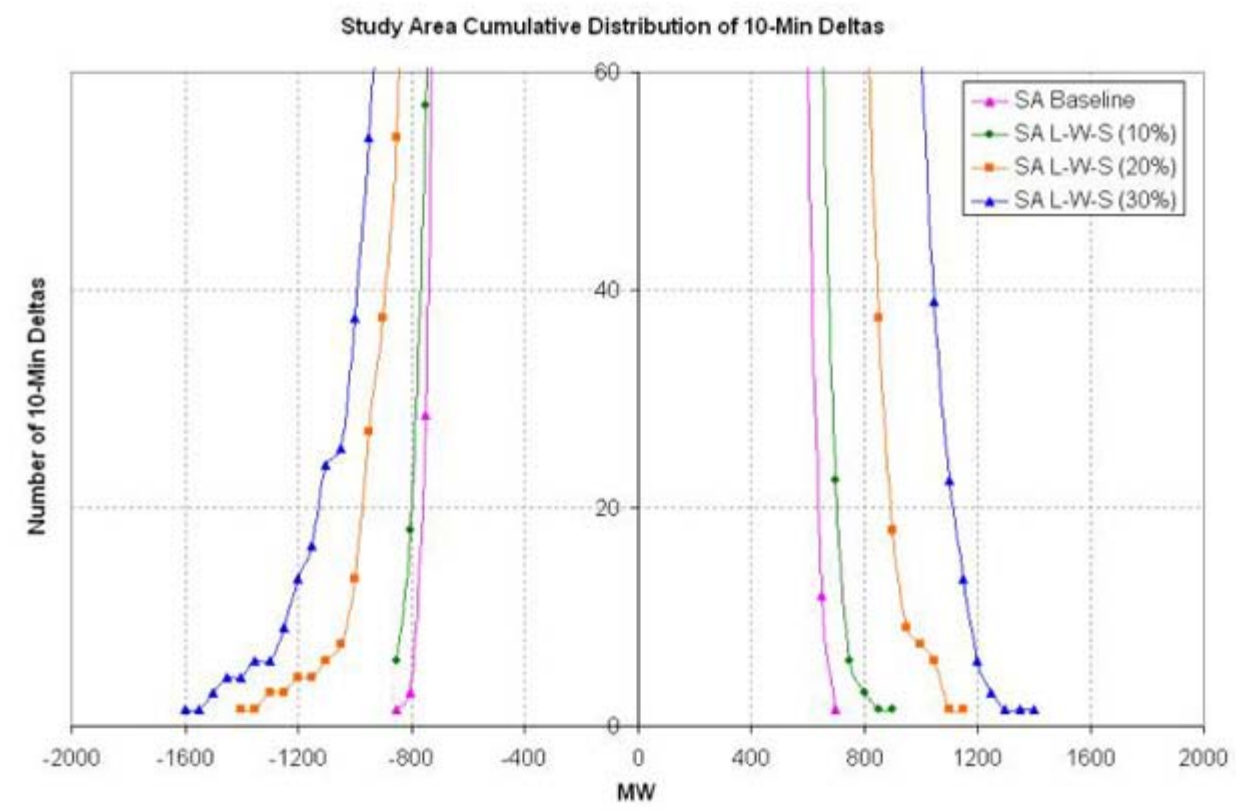

Wind Deltas vs Load Deltas for Study Area (2006, 30\%, LP Scenario)

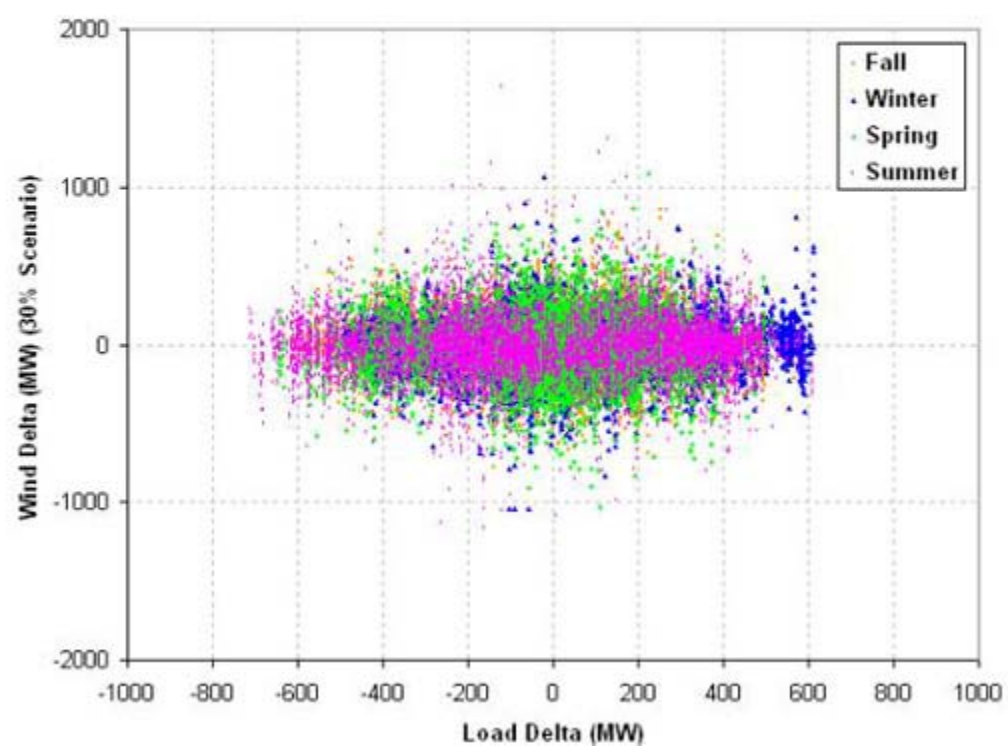


Wind Deltas vs Load Deltas by Season for Study Area (2006, 30\%, LP Scenario)
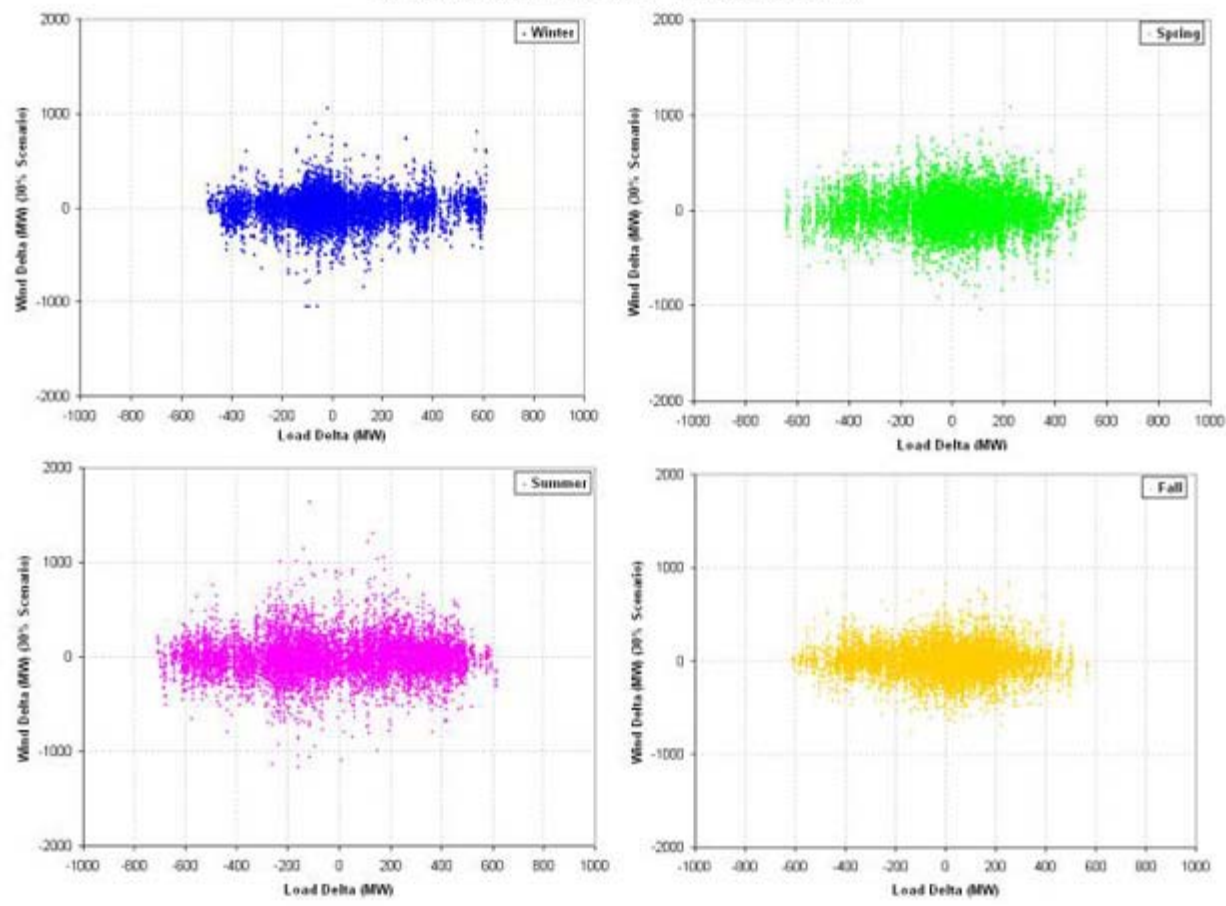

\section{Timing of Maximum Daily 10-Min Positive Deltas for Study Area (2006, LP Scenario)}

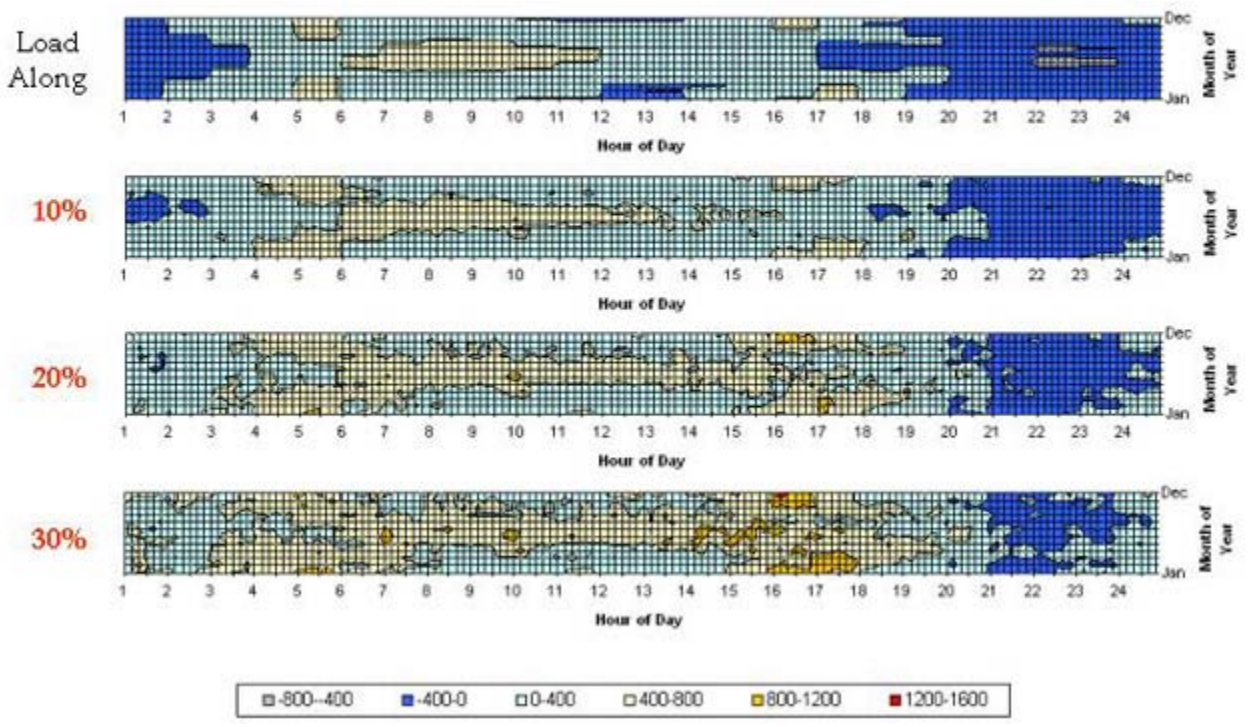


Timing of Maximum Daily 10-Min Negative Deltas for Study Area (2006, LP Scenario)

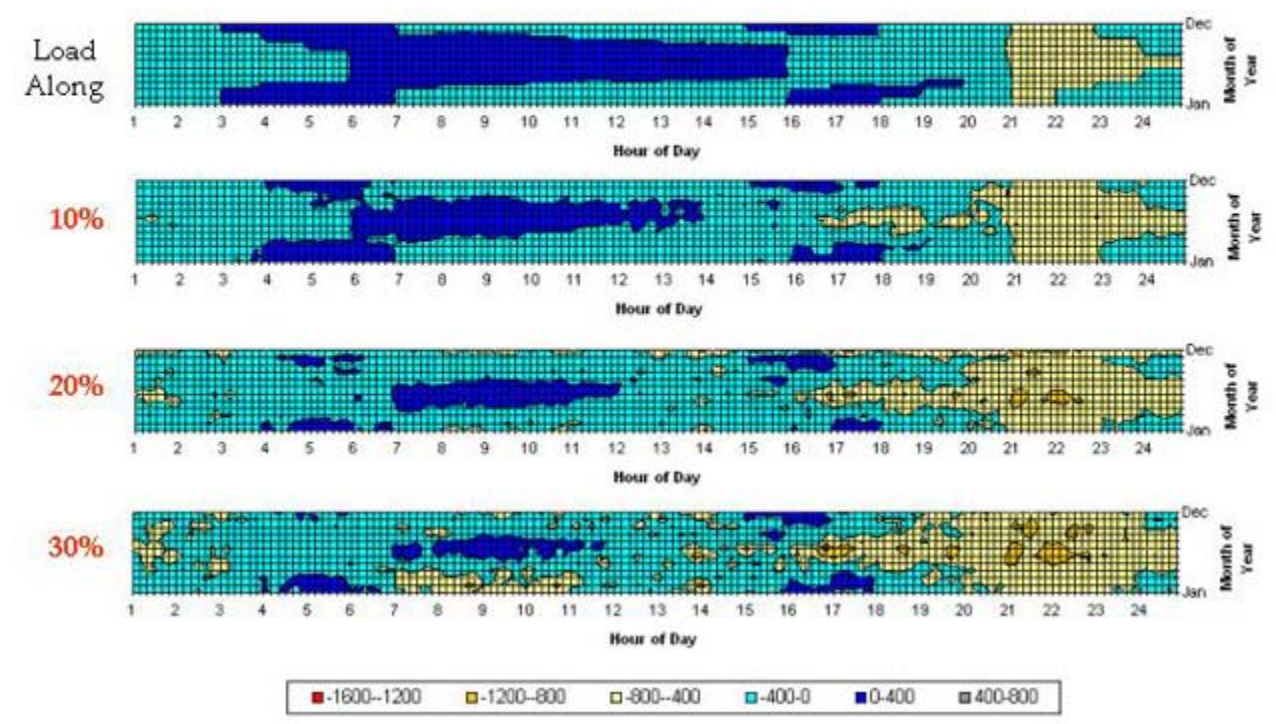

Wind Deltas vs Load Deltas by Season for Arizona (2006, 30\%, LP Scenario)
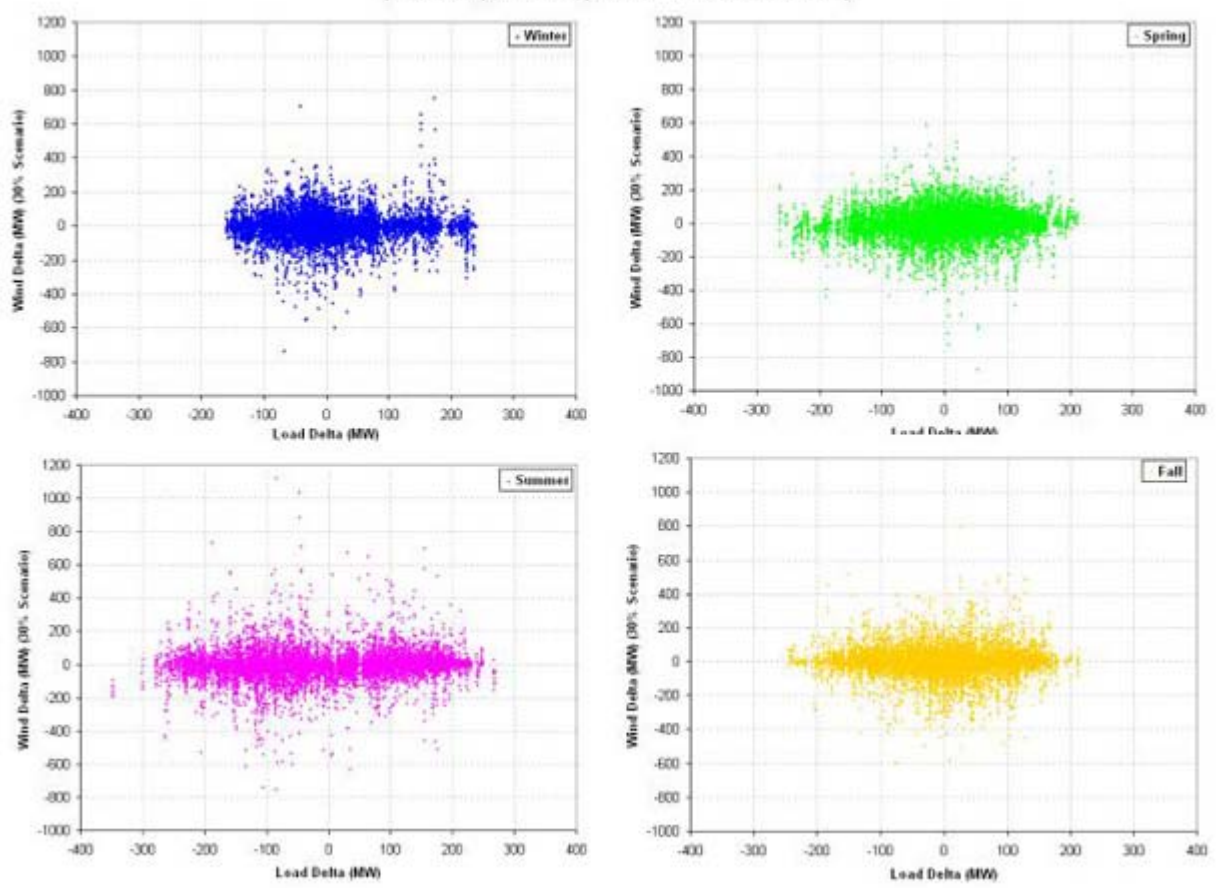
Timing of Maximum Daily 10-Min Positive Deltas for Arizona (2006, LP Scenario)

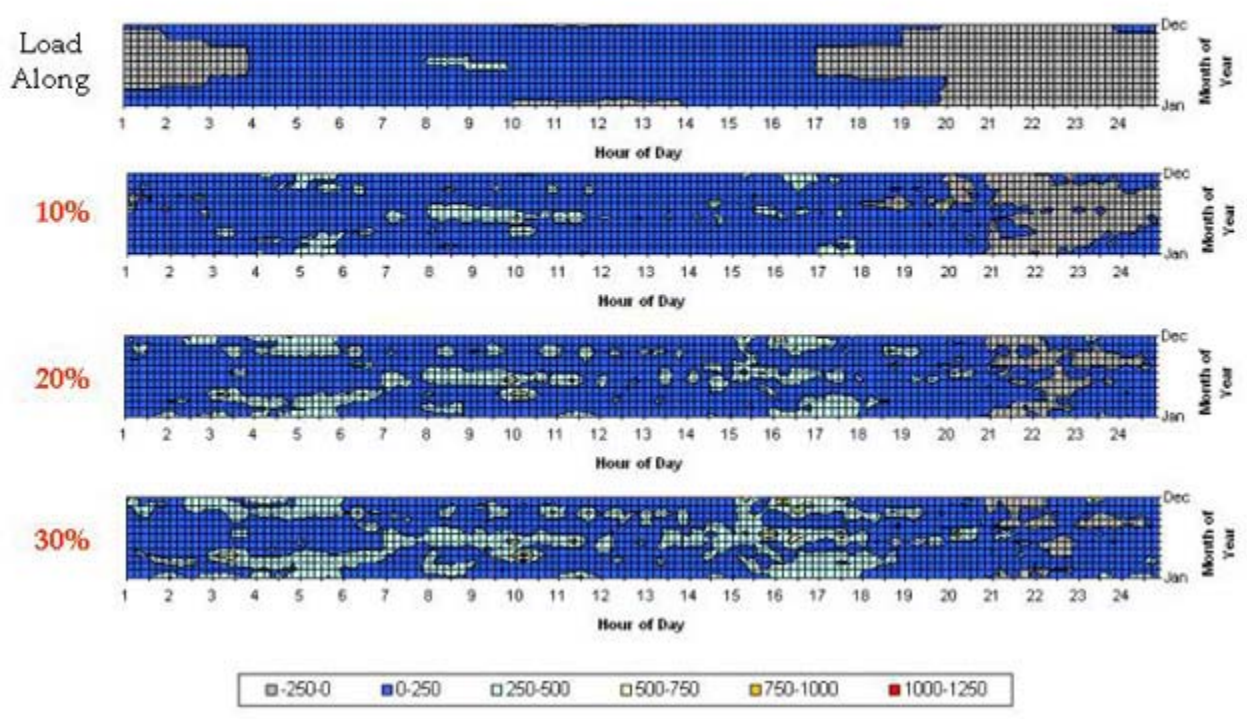

Timing of Maximum Daily 10-Min Negative Deltas for Arizona (2006, LP Scenario)

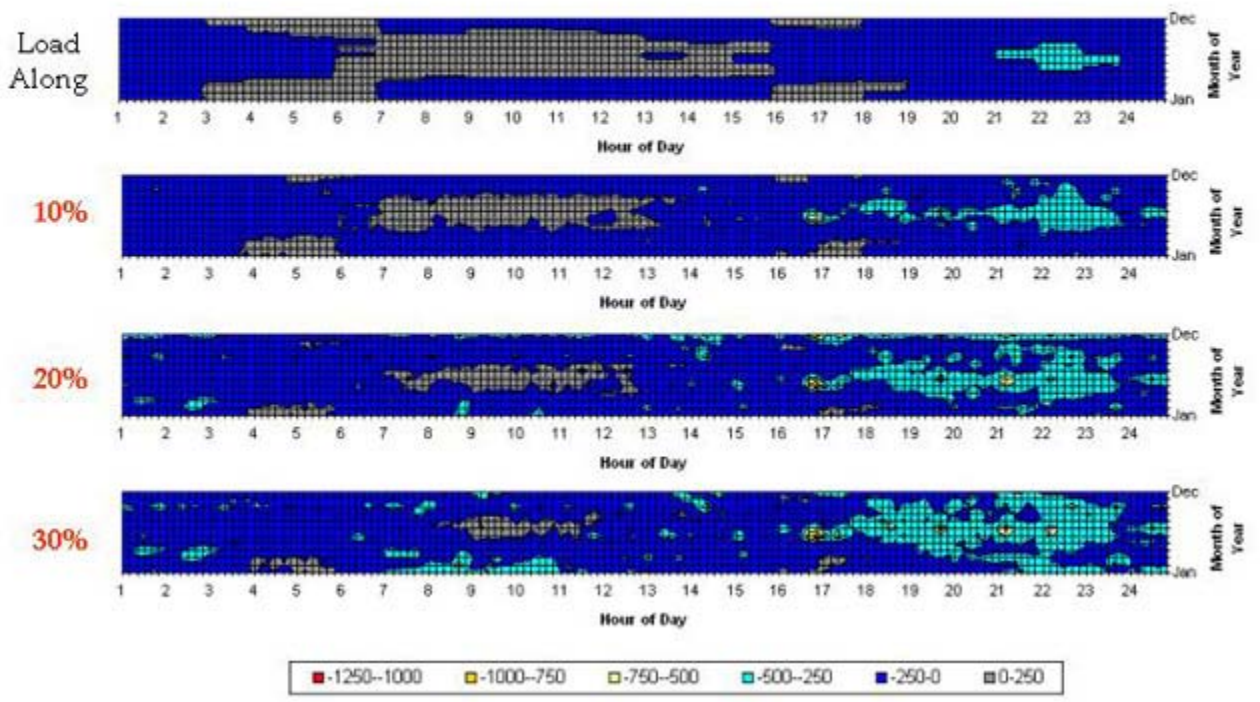


Effect of Wind Aggregation on Delta Distribution for Arizona(2006, LP Scenario, Scaled by $150 \%$ )

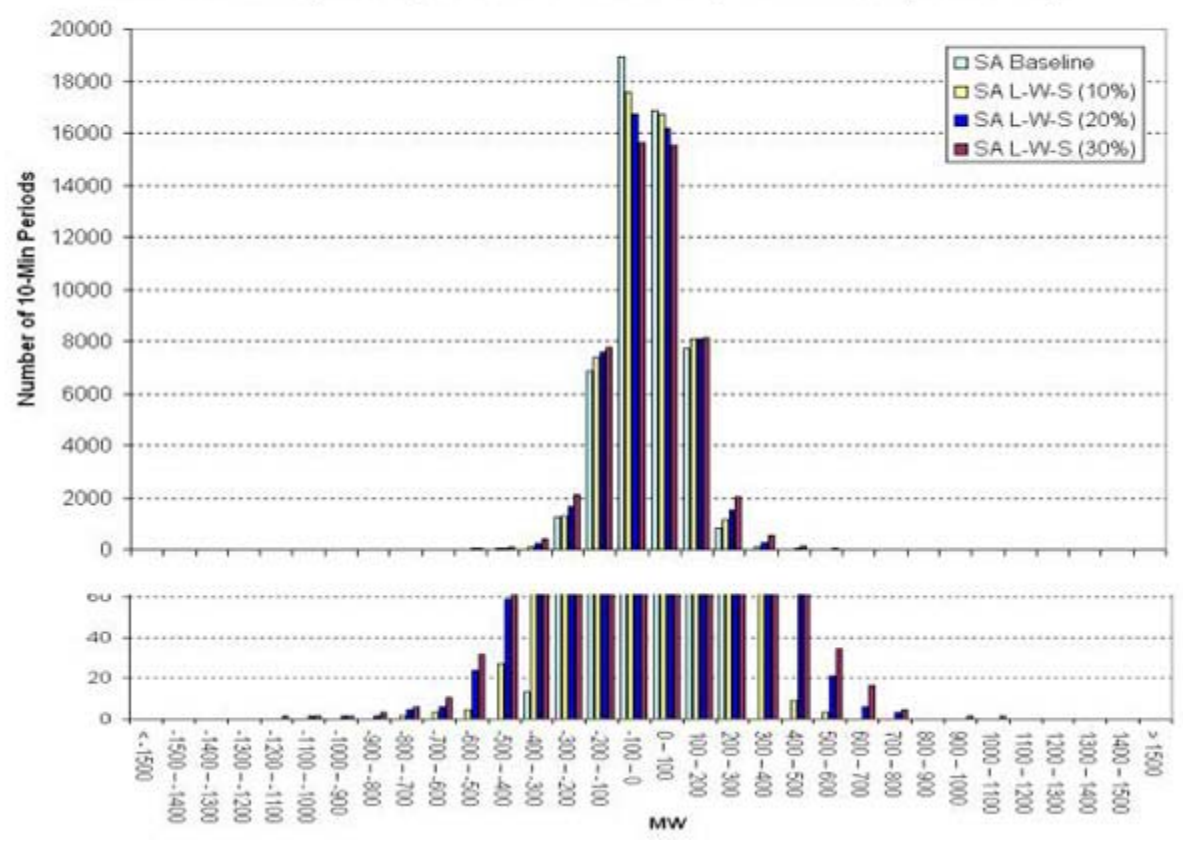

Distribution of Extreme Hourly Wind Deltas for Arizona (2006, LP Scenario)

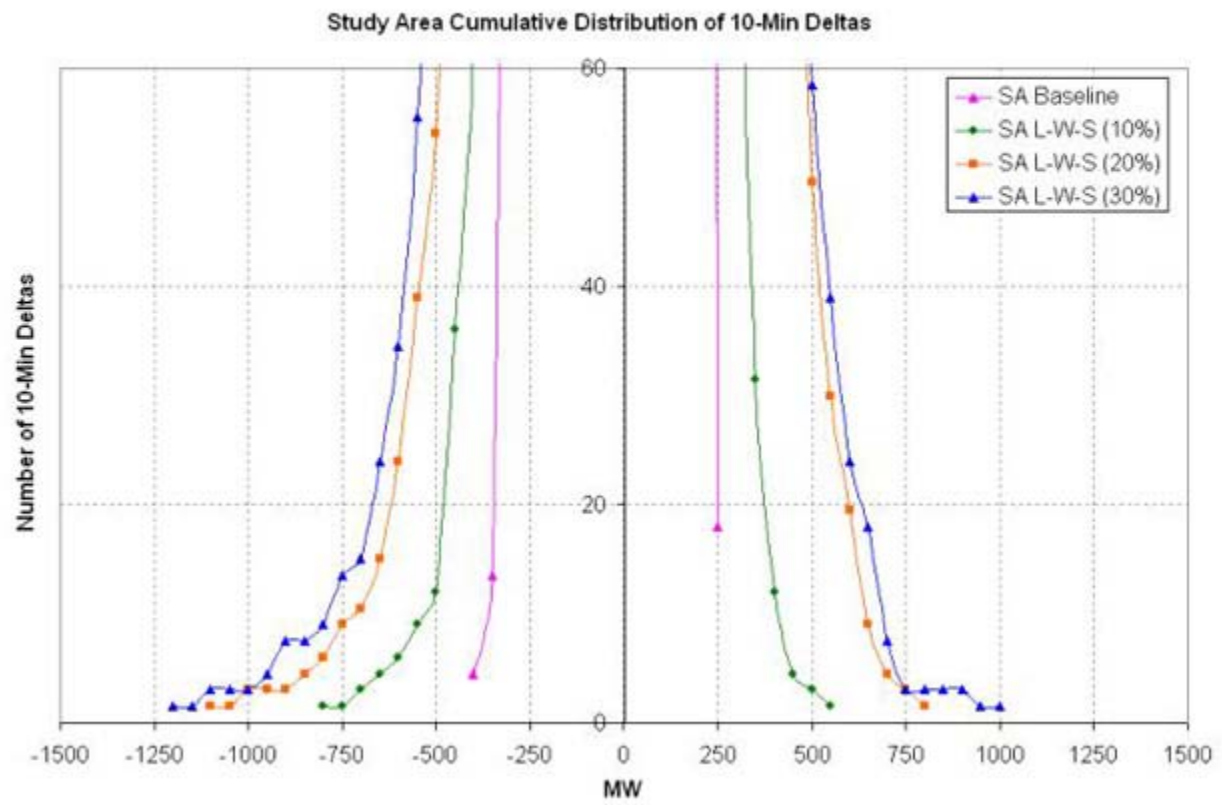




\section{Wind Deltas vs Load Deltas for Wyoming (2006, 30\%, LP Scenario)}

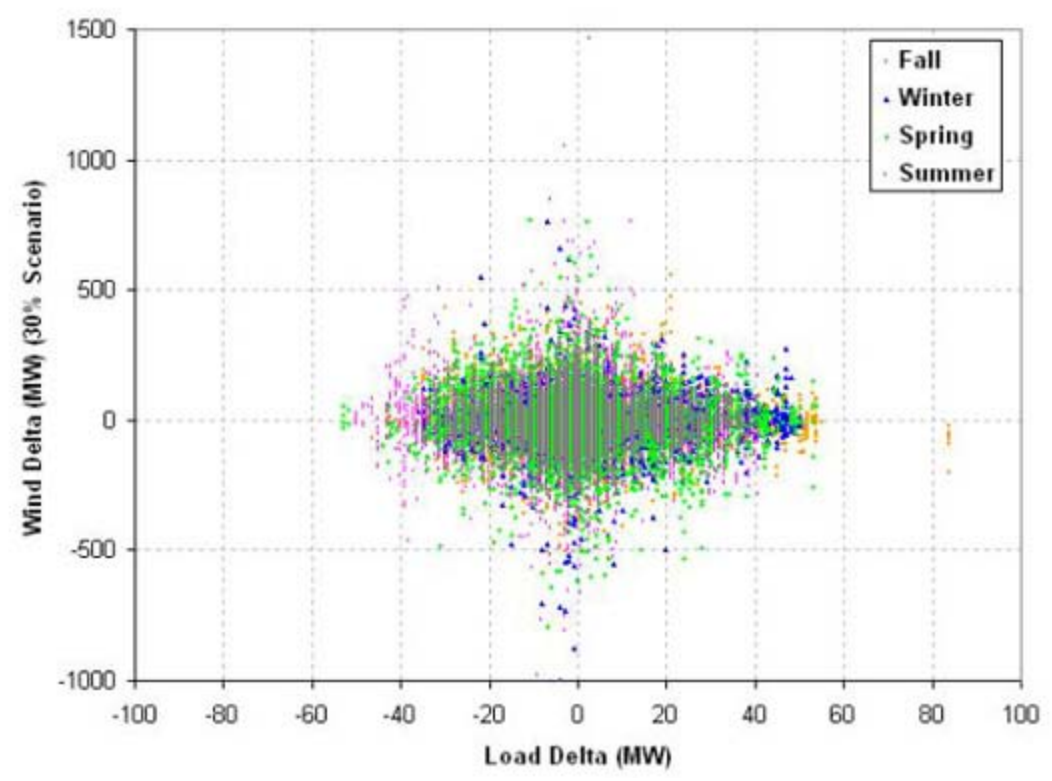

Wind Deltas vs Load Deltas by Season for Wyoming (2006, 30\%, LP Scenario)
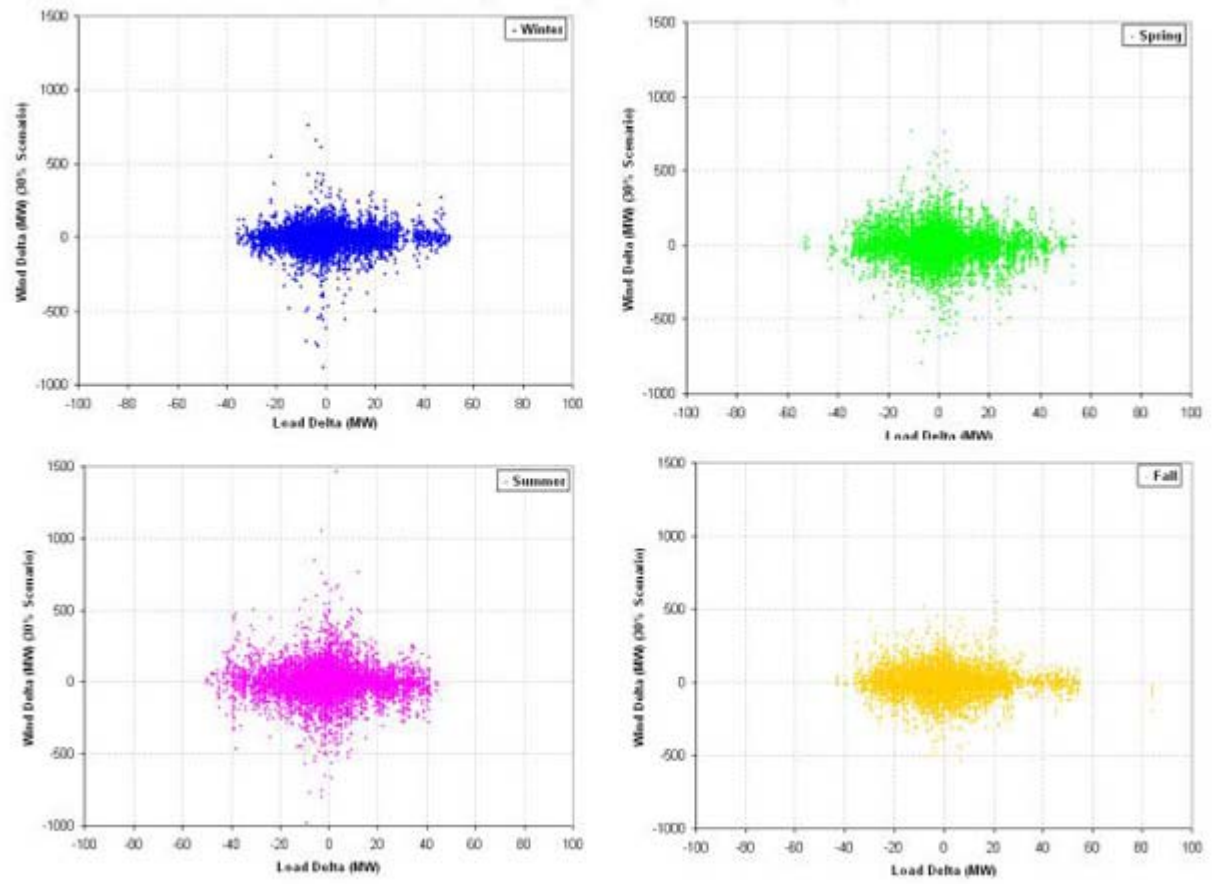
Timing of Maximum Daily 10-Min Positive Deltas for Wyoming (2006, LP Scenario)

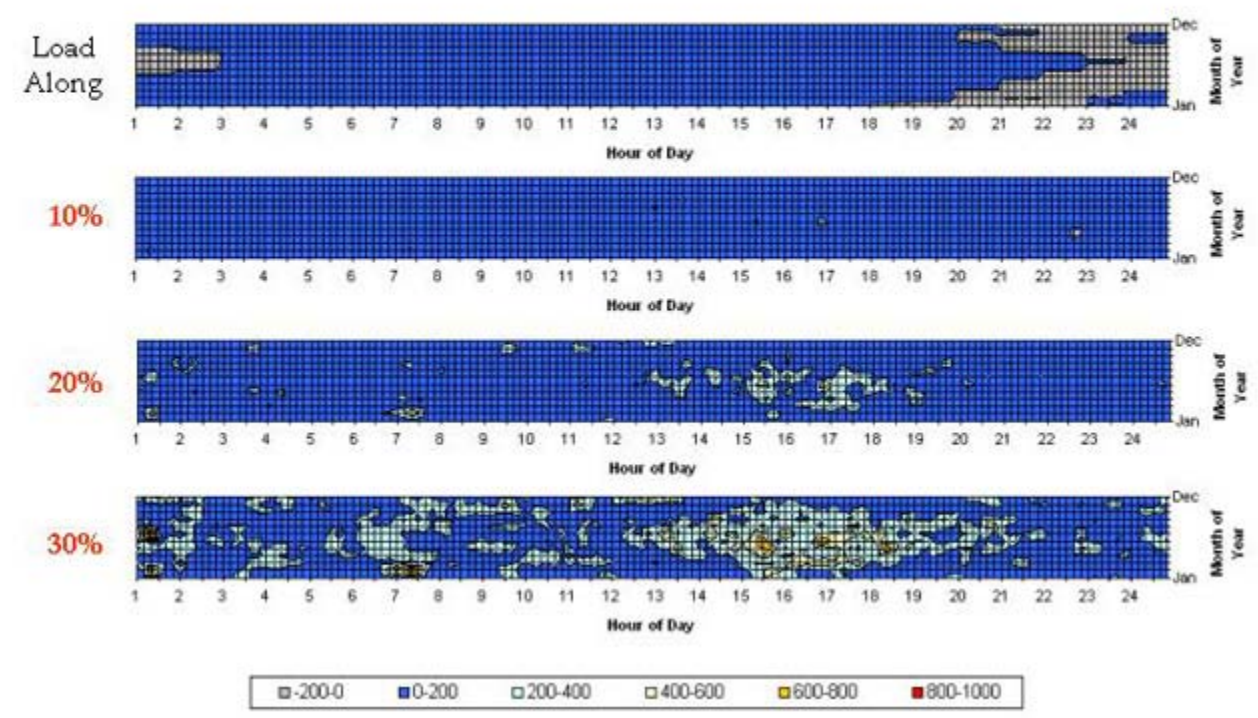

Timing of Maximum Daily 10-Min Negative Deltas for Wyoming (2006, LP Scenario)

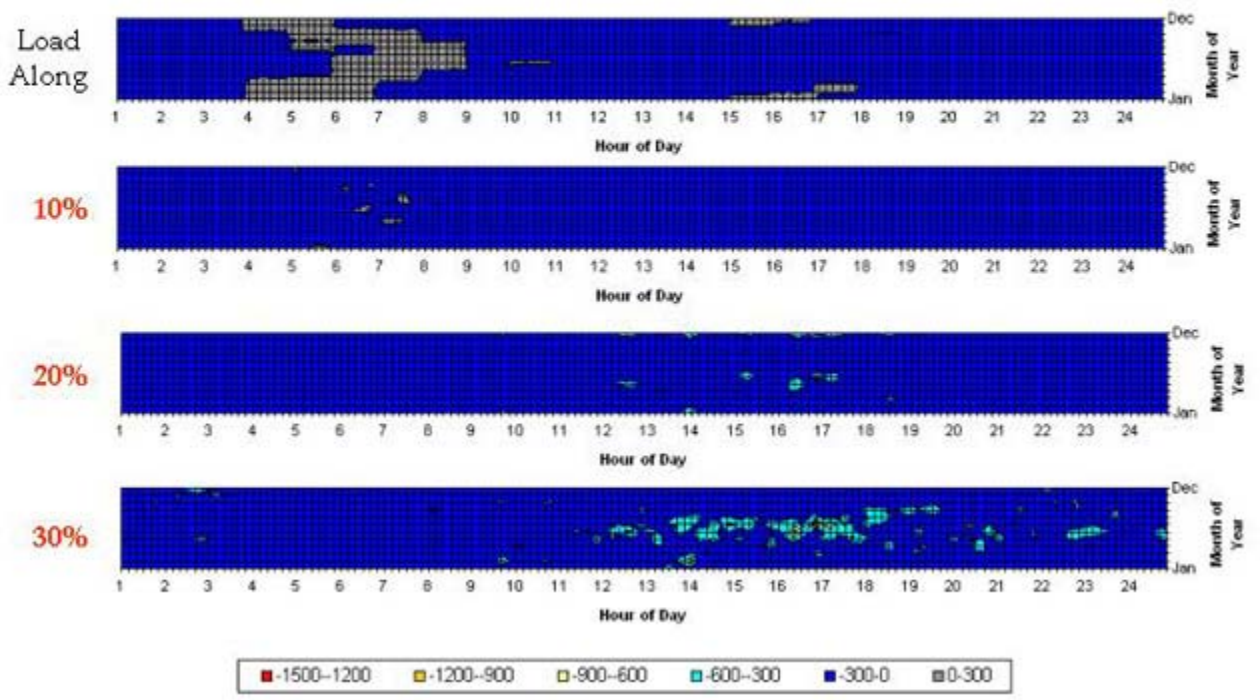


Effect of Wind Aggregation on Delta Distribution for

Wyoming (2006, LP Scenario, Scaled by 150\%)

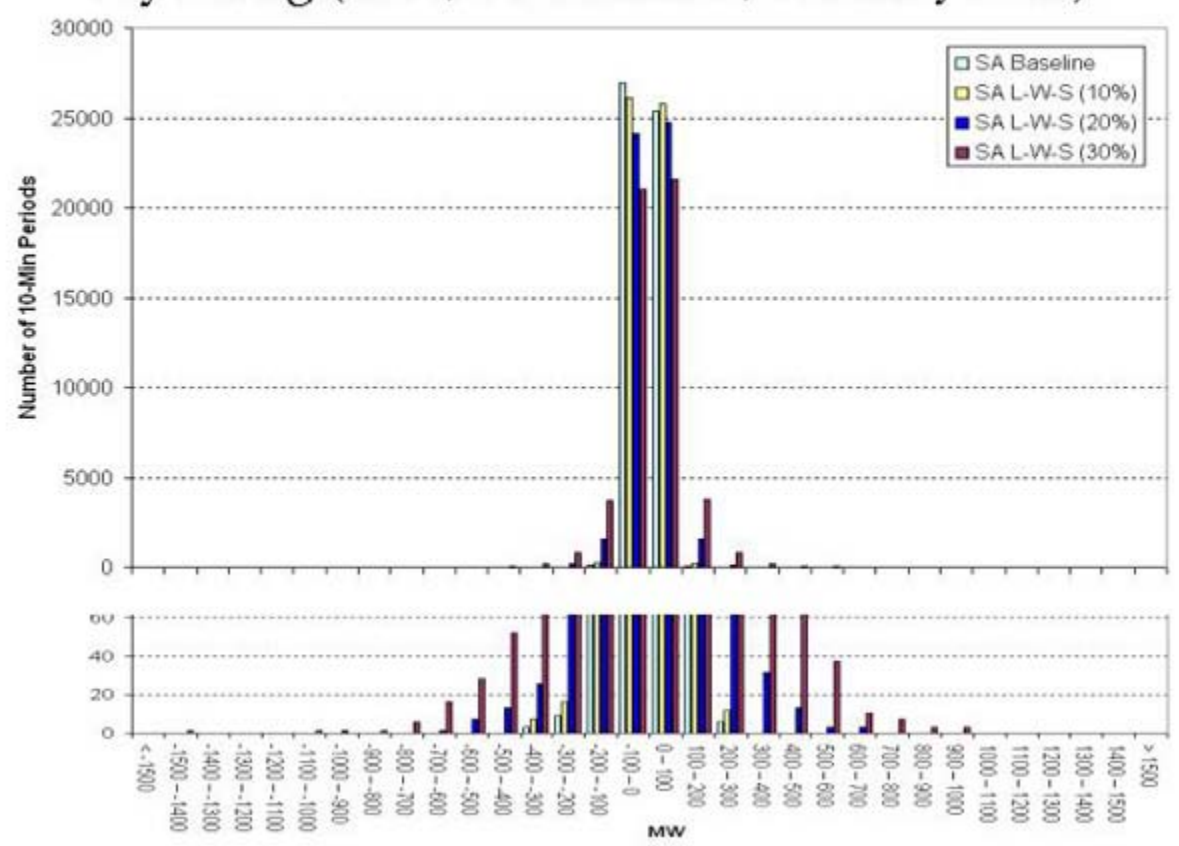

Distribution of Extreme Hourly Wind Deltas for Wyoming (2006, LP Scenario)

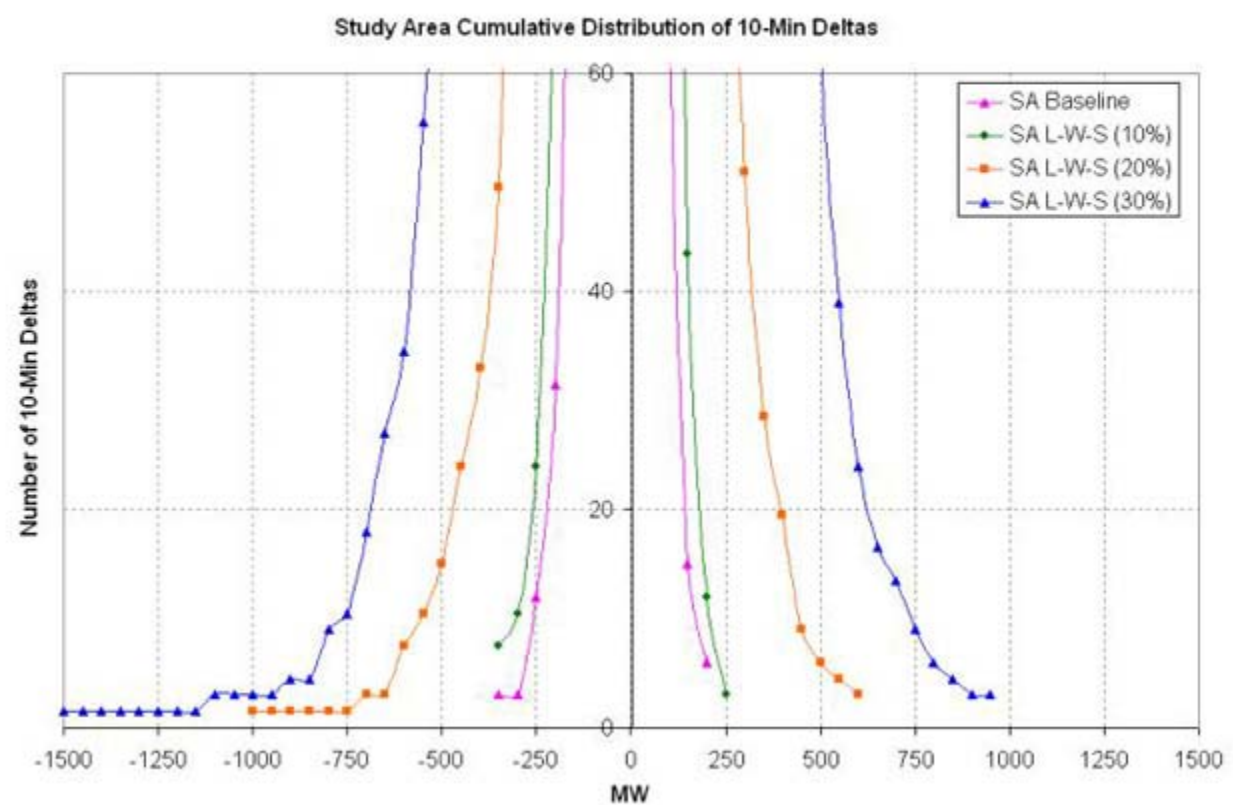


Effect of Wind Aggregation on Delta Distribution for Study Area (2006, LP Scenario, Scaled by $150 \%$ )

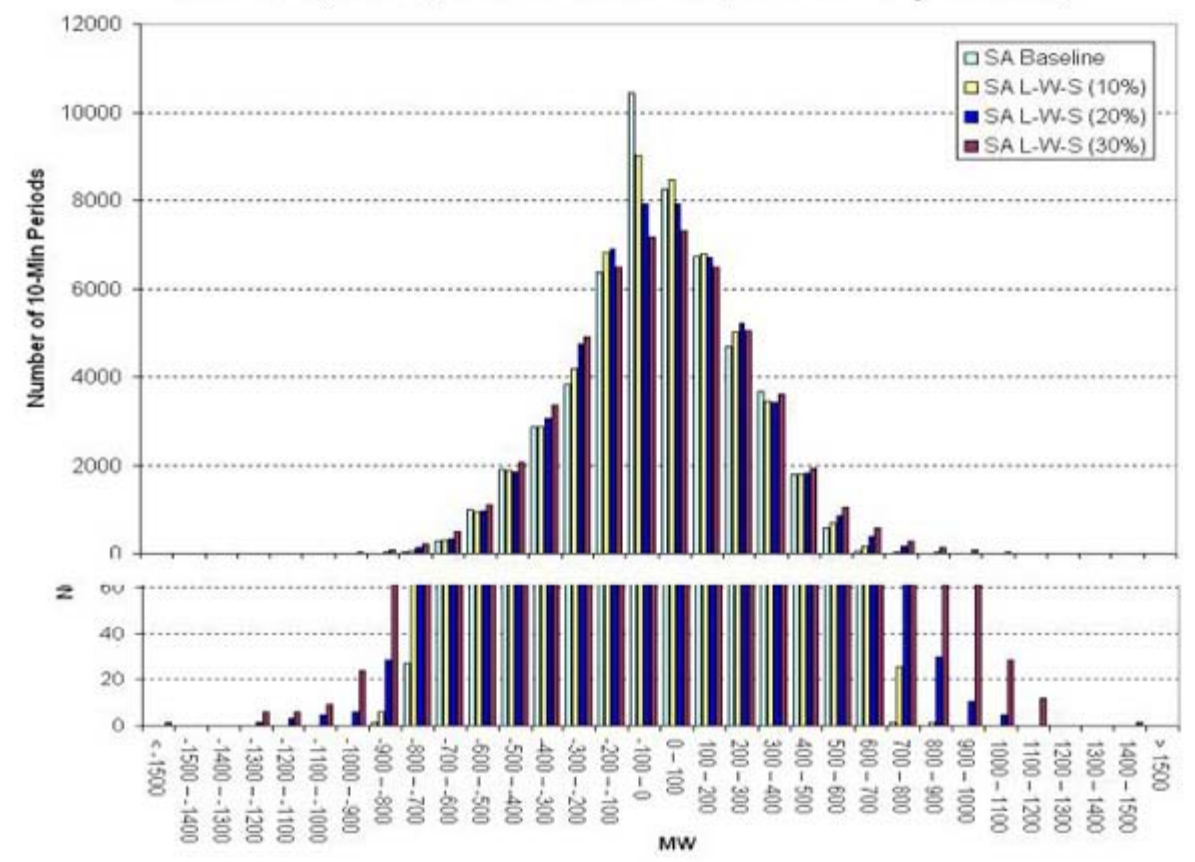

Distribution of Extreme Hourly Wind Deltas for 2006 (2006, LP Scenario)

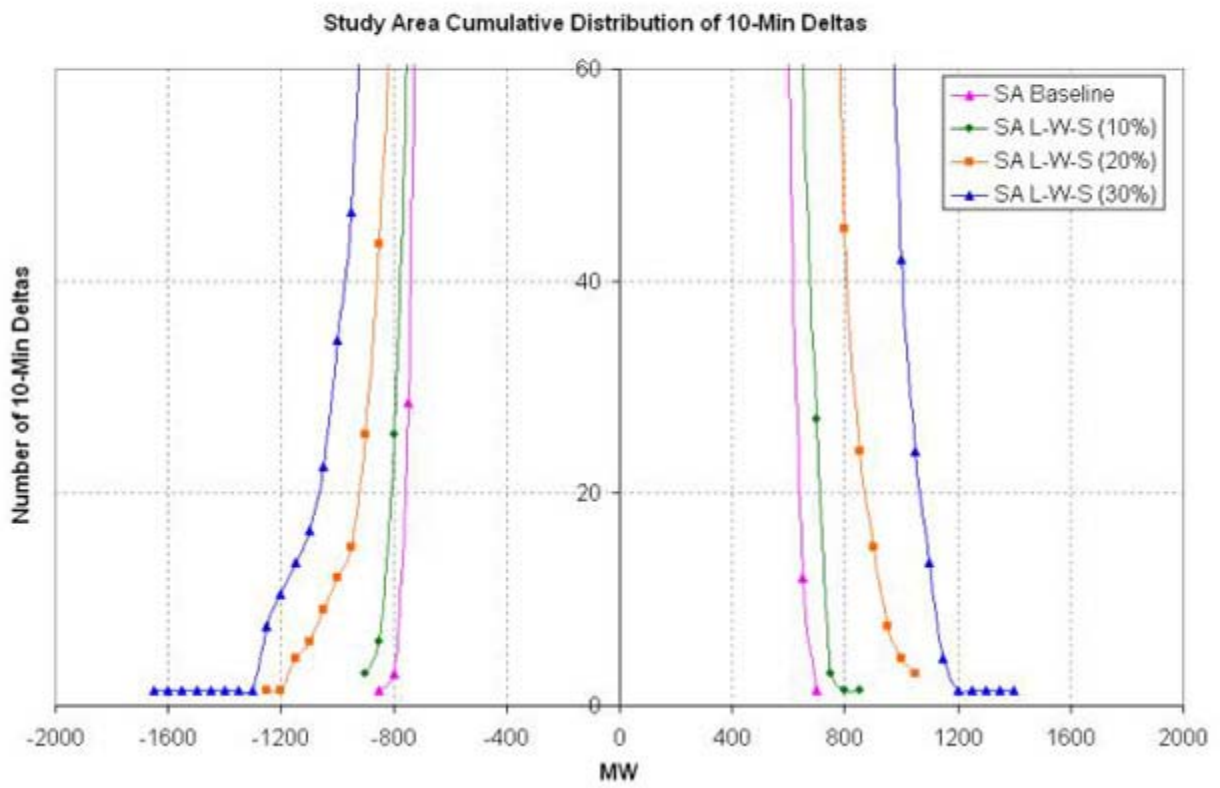




\section{Wind Deltas vs Load Deltas for Study Area (2006, 30\%, MP Scenario)}

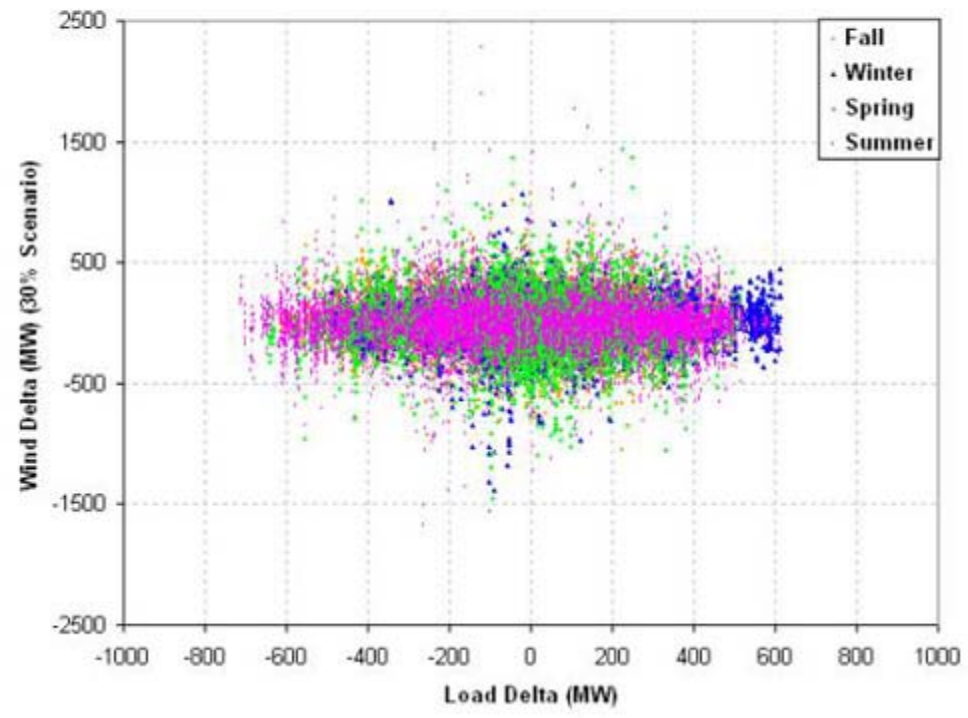

Wind Deltas vs Load Deltas by Season for Study Area (2006, 30\%, MP Scenario)
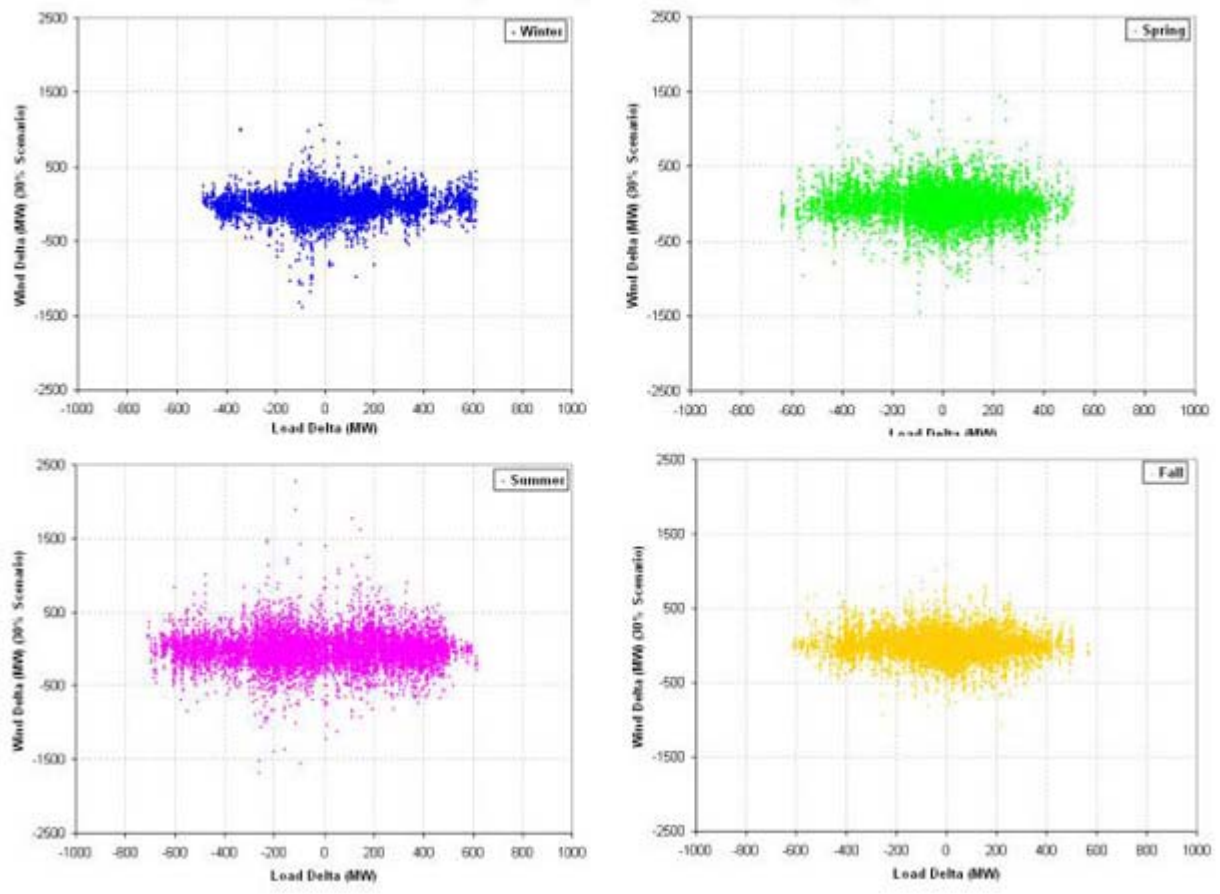
Timing of Maximum Daily 10-Min Positive Deltas for Study Area (2006, MP Scenario)

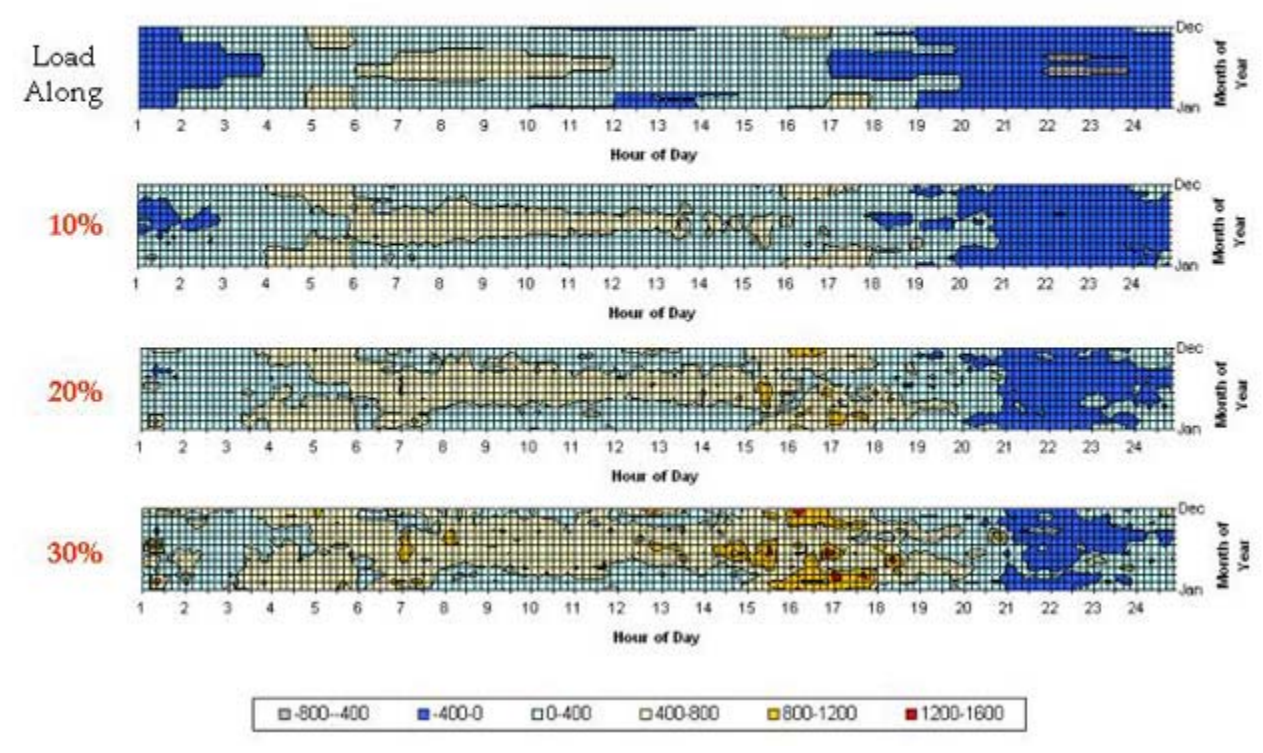

Timing of Maximum Daily 10-Min Negative Deltas for Study Area (2006, MP Scenario)

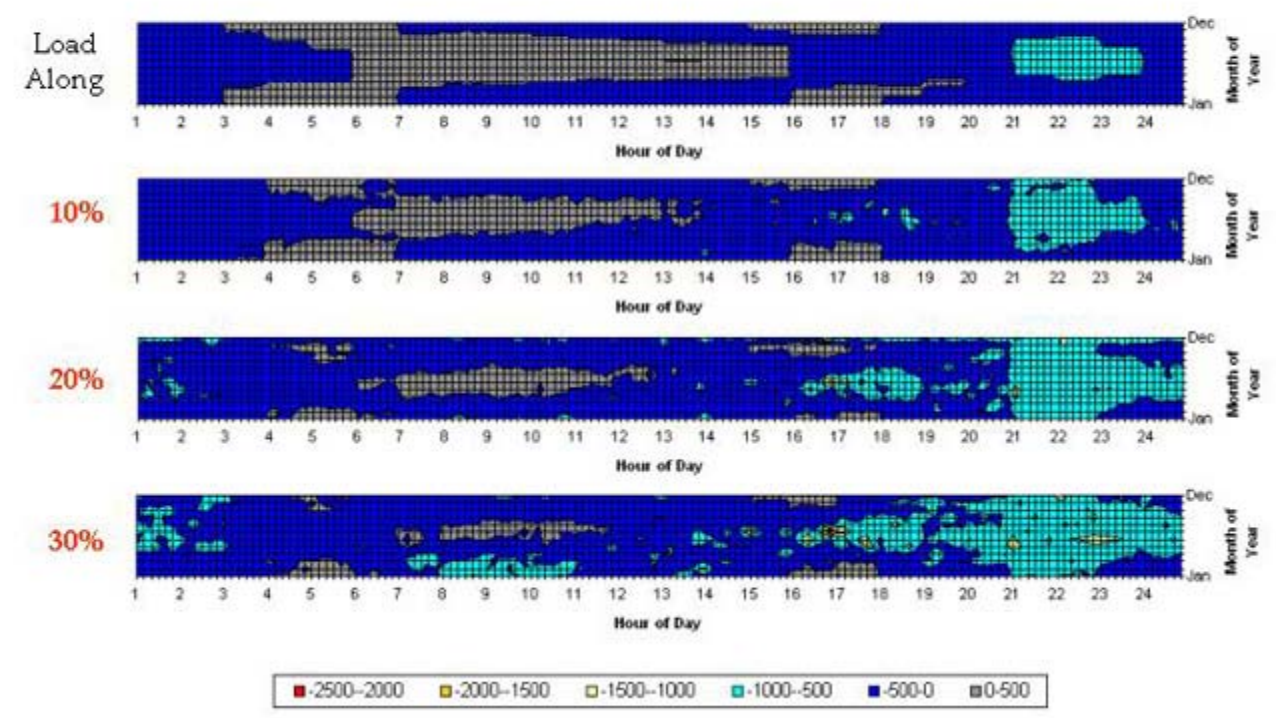


Effect of Wind Aggregation on Delta Distribution for Study Area (2006, MP Scenario, Scaled by $150 \%$ )

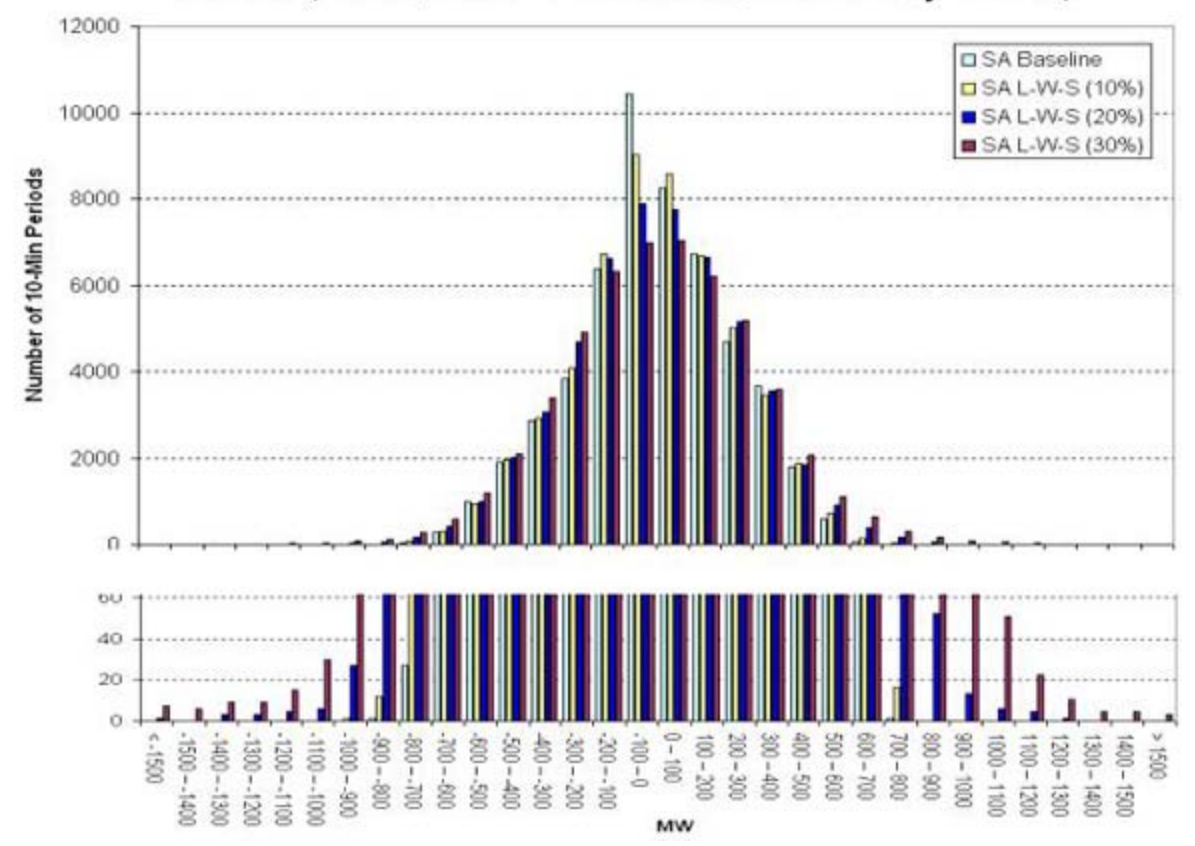

\section{Distribution of Extreme Hourly Wind Deltas for Study Area (2006, MP Scenario)}

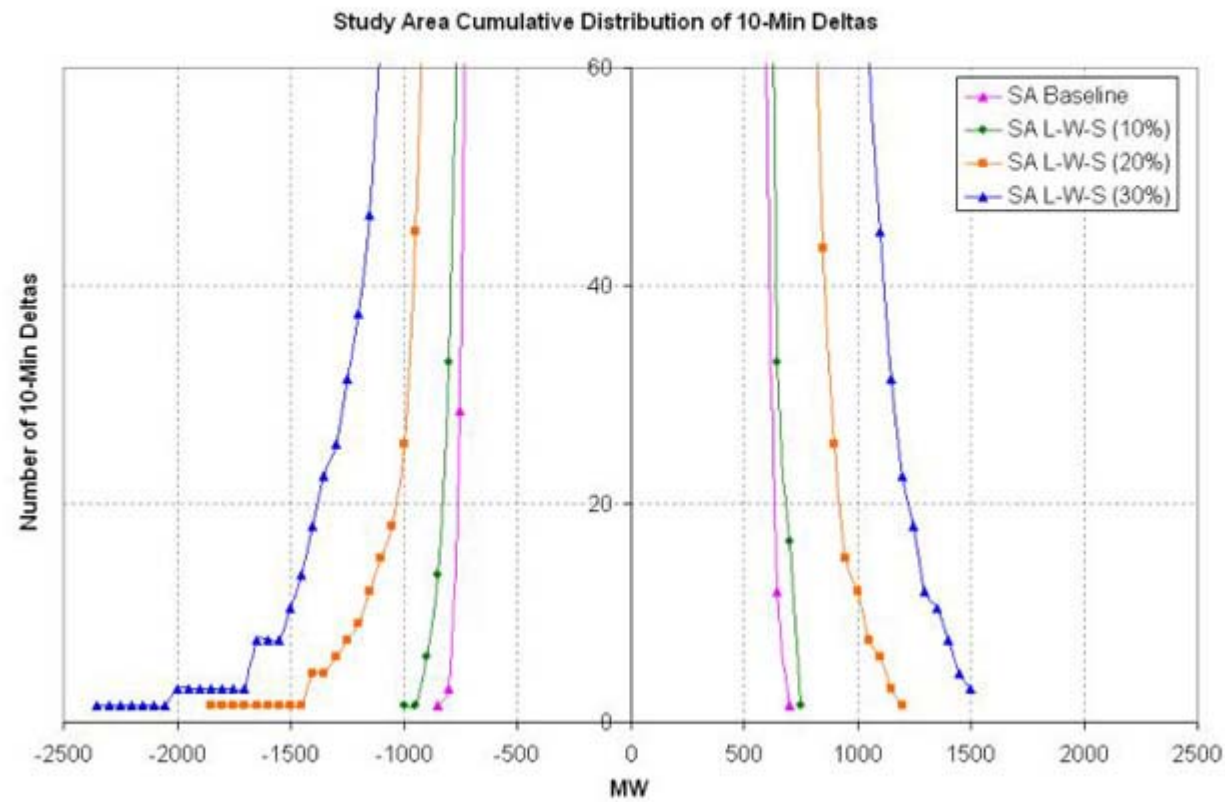




\section{Distribution of Extreme Hourly Wind Deltas for Study Area $(2006,30 \%)$}

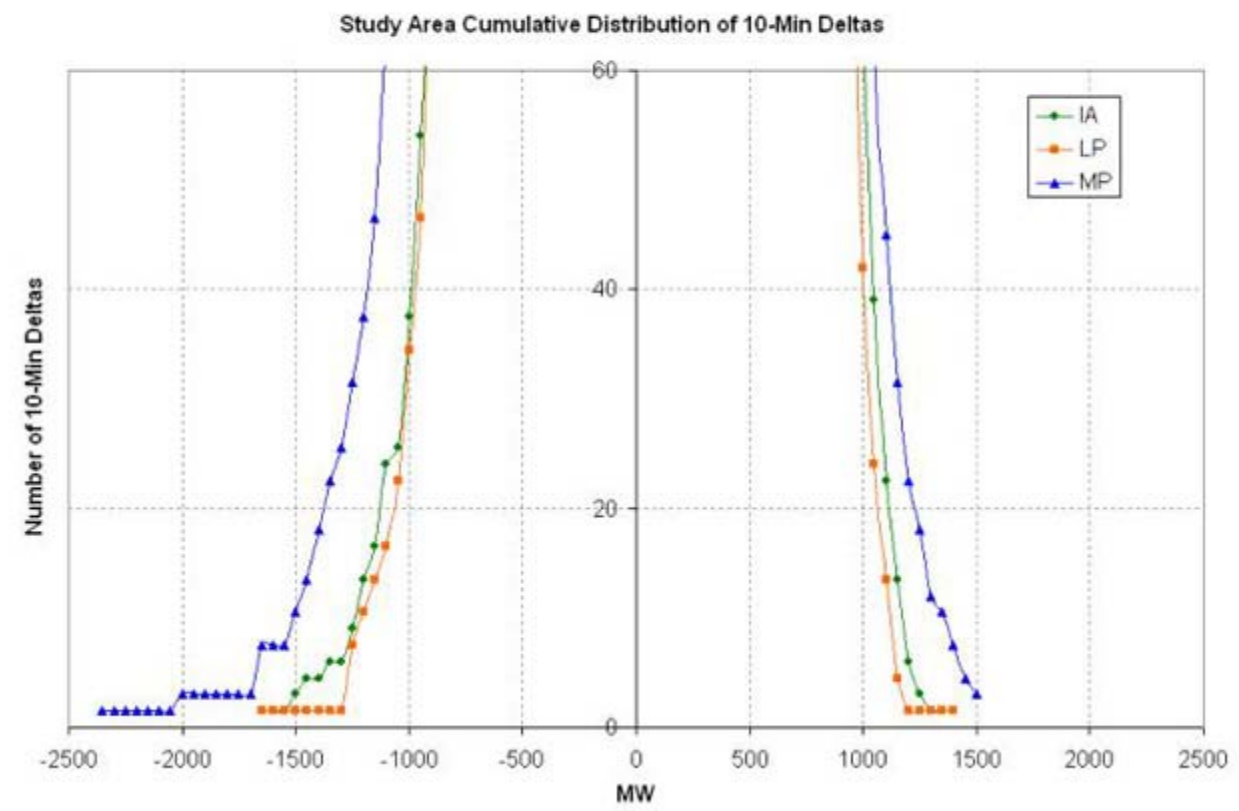

Wind Deltas vs Load Deltas for Arizona (2006, 30\%, LP Scenario)

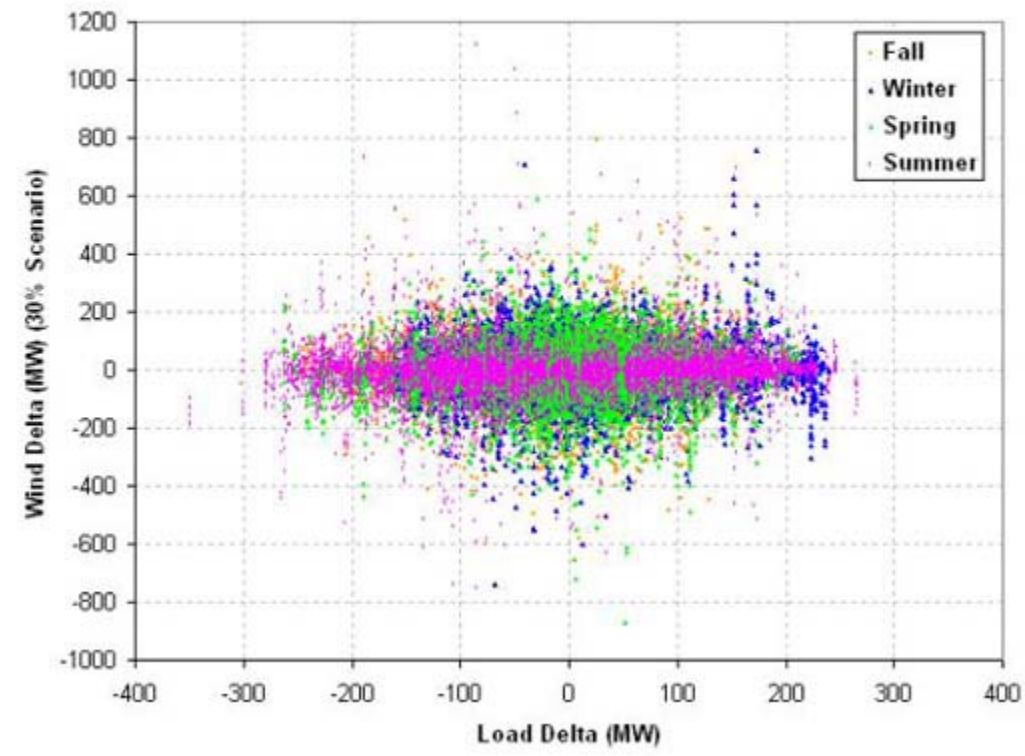





\section{Appendix F Additional QSS Simulation Results}

Appendix F presents additional QSS output from the 10\%, 20\%, 20/20\%, and $30 \%$ wind penetrations of various transmission areas on the April 15 and October 26 study periods. The QSS analysis in the main body of the report described in detail the results for Arizona and Colorado East. This appendix is an opportunity to share results of the QSS simulations for other transmission areas not previously described in the report. All of the results in this section are from the Local Priority scenario.

Each scenario is presented with plots that contain the unit dispatch, regulation and hourly schedules, and generation totals for each transmission area. Figures 1-9 detail the operation of Colorado East, Colorado West, and New Mexico for April 15 at 30\% penetration. Then plots of North and South Nevada are shown in Figures 10-15, again for April 15 at 30\% wind penetration. An additional plot shows the effect of balancing area consolidation for Nevada in Figure 16.

Output for Wyoming on April 15 for the 10\%, 20\%, 20/20\%, and 30\% penetrations are shown in Figures 17-28. A cross plot of the Wyoming ACE at each penetration level is given in Figure 29. Figures 30-32 display the effect of allocating 100MW of regulation to Wyoming.

Lastly, October 26 at $30 \%$ penetration is covered for Arizona, Colorado West, New Mexico, North and South Nevada, and Wyoming in Figures 33-50. 


\section{F.1 Colorado East April 15, 30\% Wind Penetration}

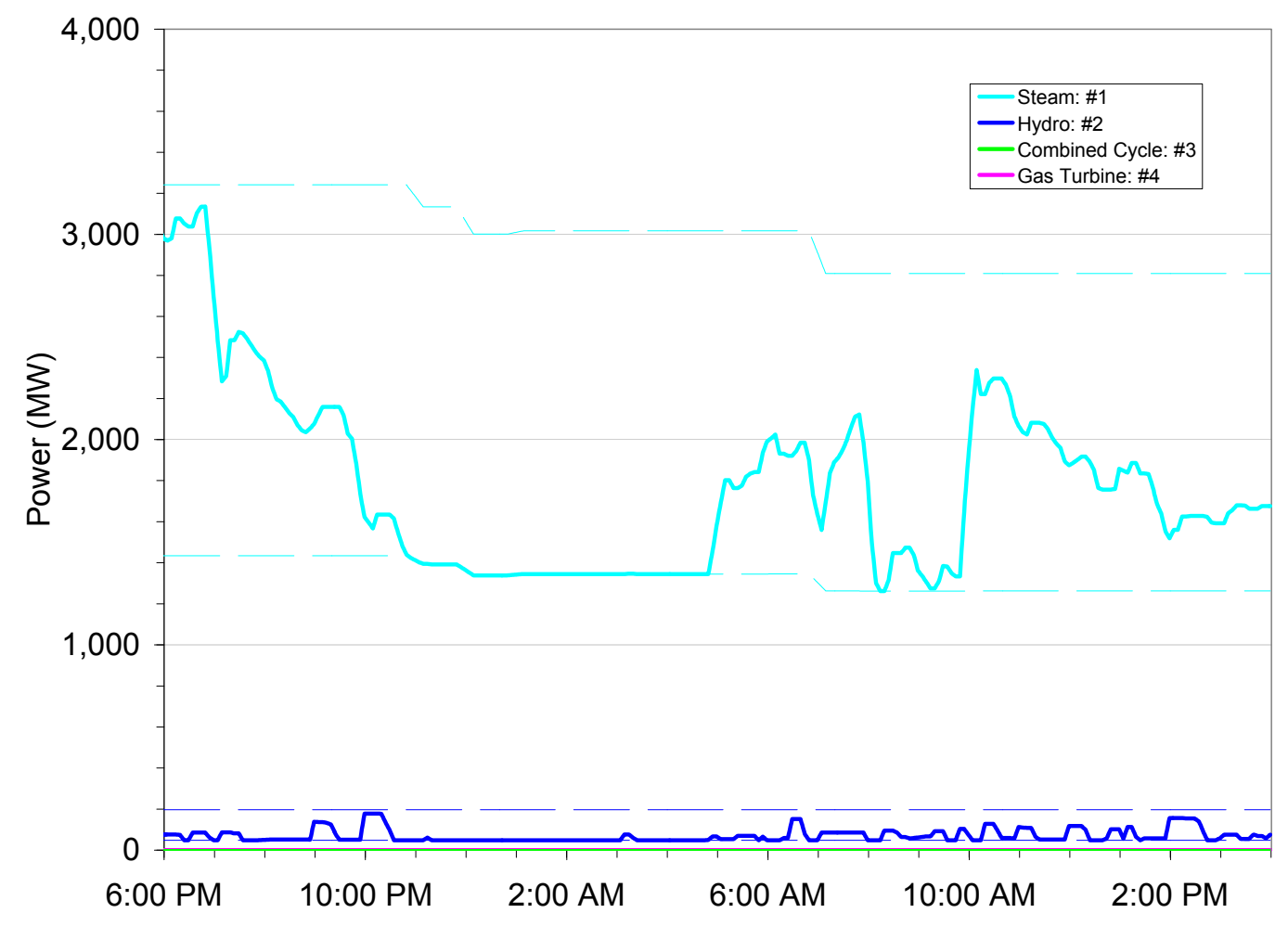

Figure F- 1 Colorado East Dispatch, 30\% Wind, Local Priority, April QSS Study Period 


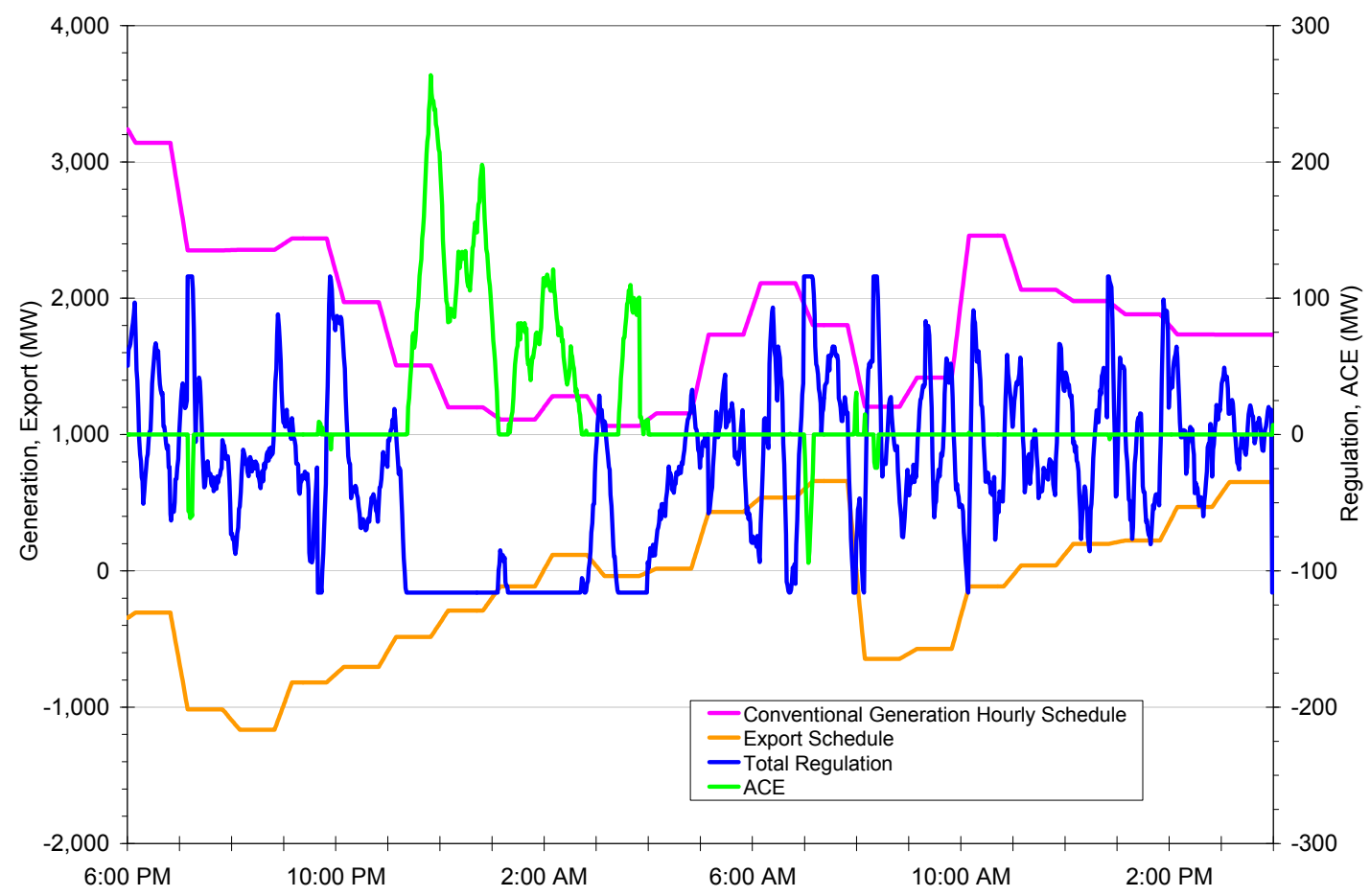

Figure F- 2 Colorado East Regulation, 30\% Wind, Local Priority, April QSS Study Period

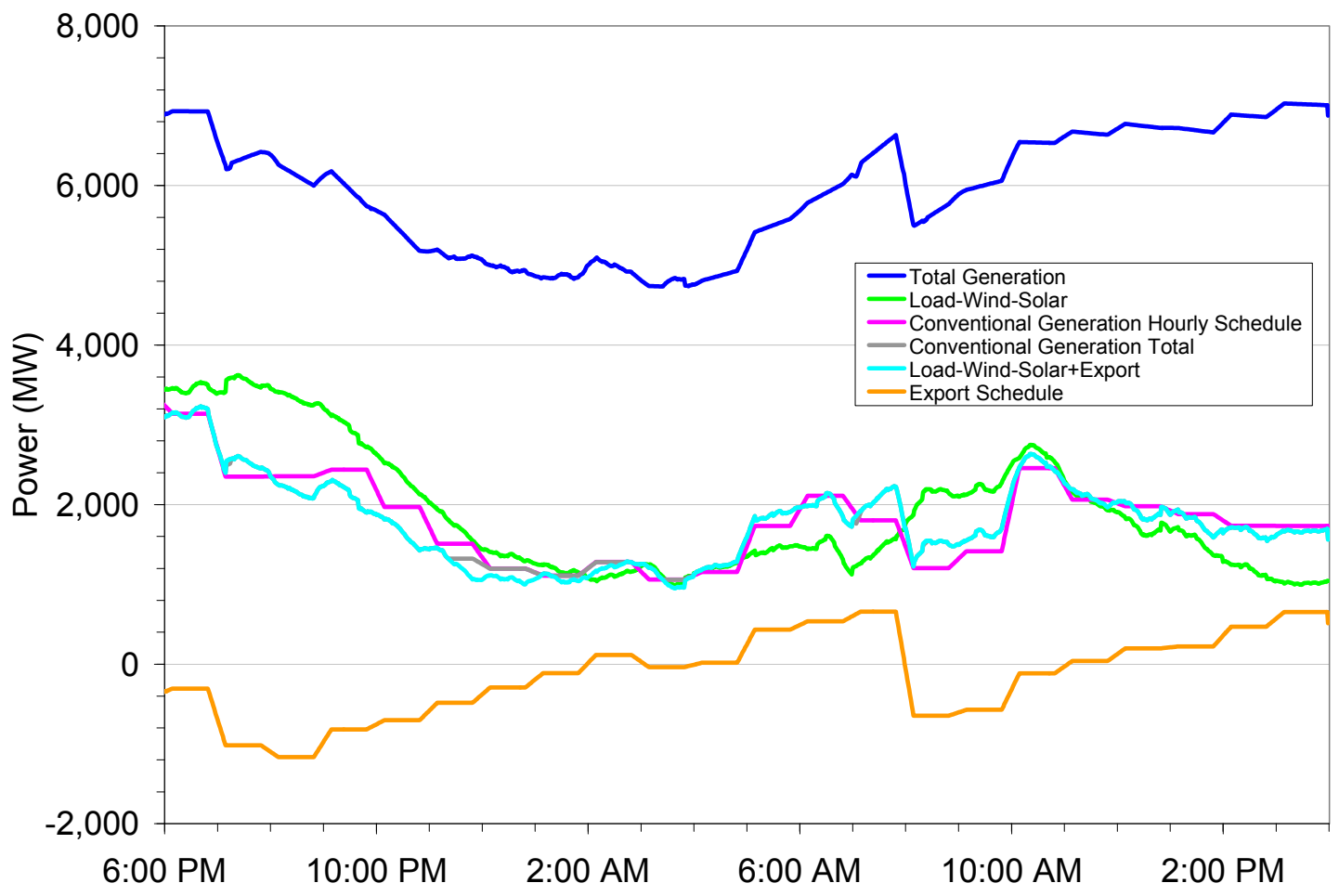

Figure F- 3 Colorado East Totals, 30\% Wind, Local Priority, April QSS Study Period 


\section{F.2 Colorado West April 15, 30\% Wind Penetration}

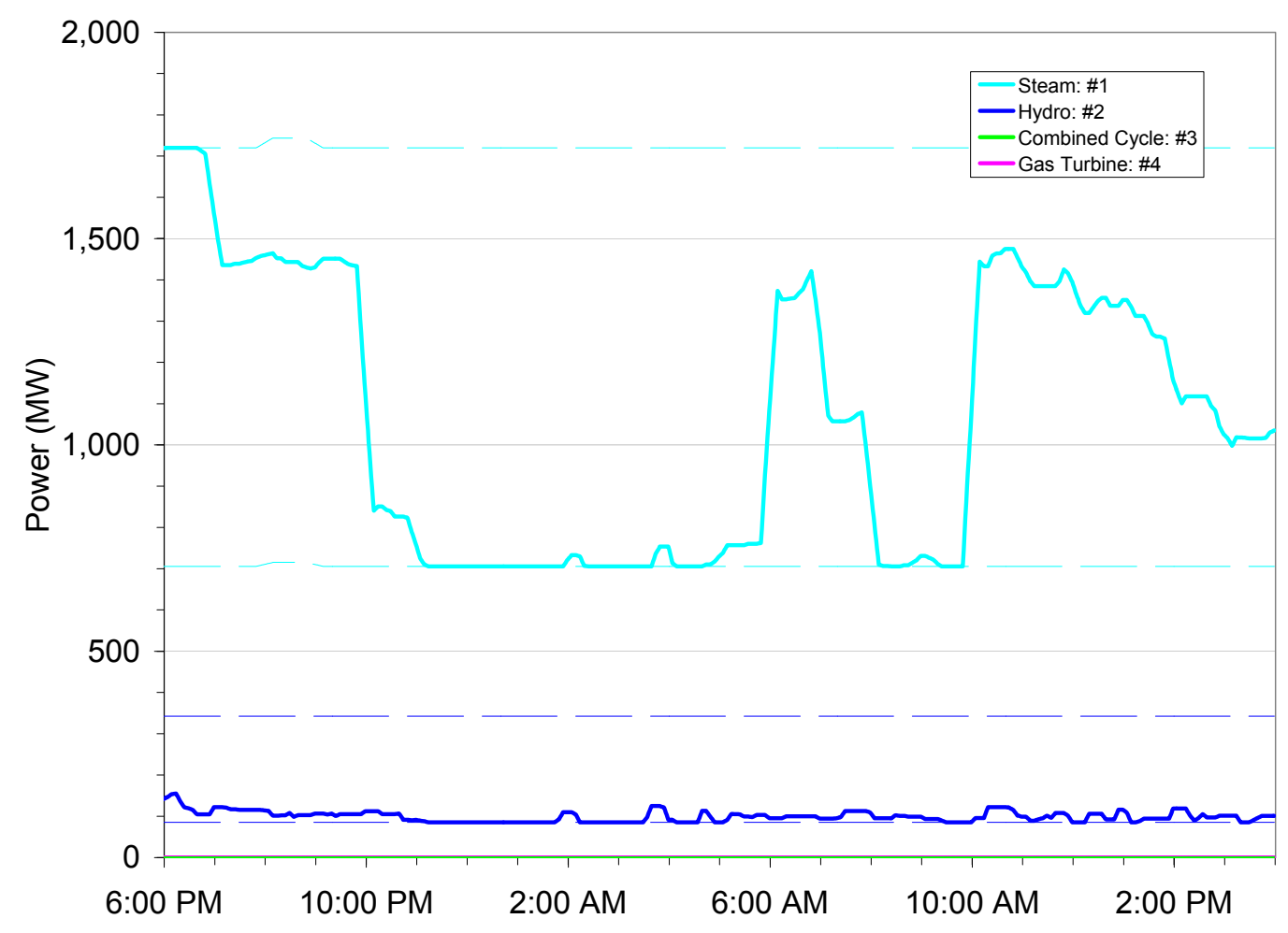

Figure F- 4 Colorado West Dispatch, 30\% Wind, Local Priority, April QSS Study Period

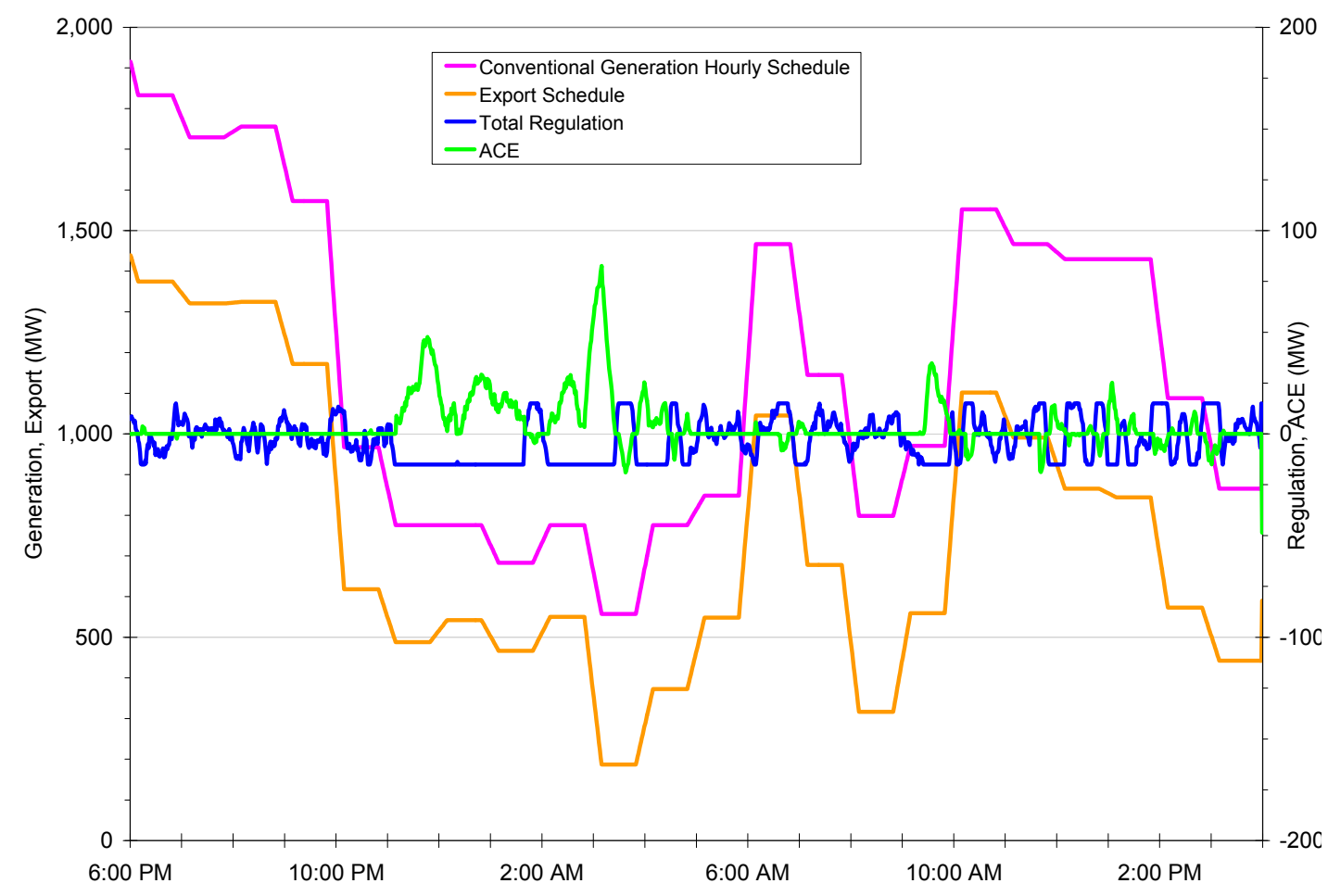

Figure F- 5 Colorado West Regulation, 30\% Wind, Local Priority, April QSS Study Period 


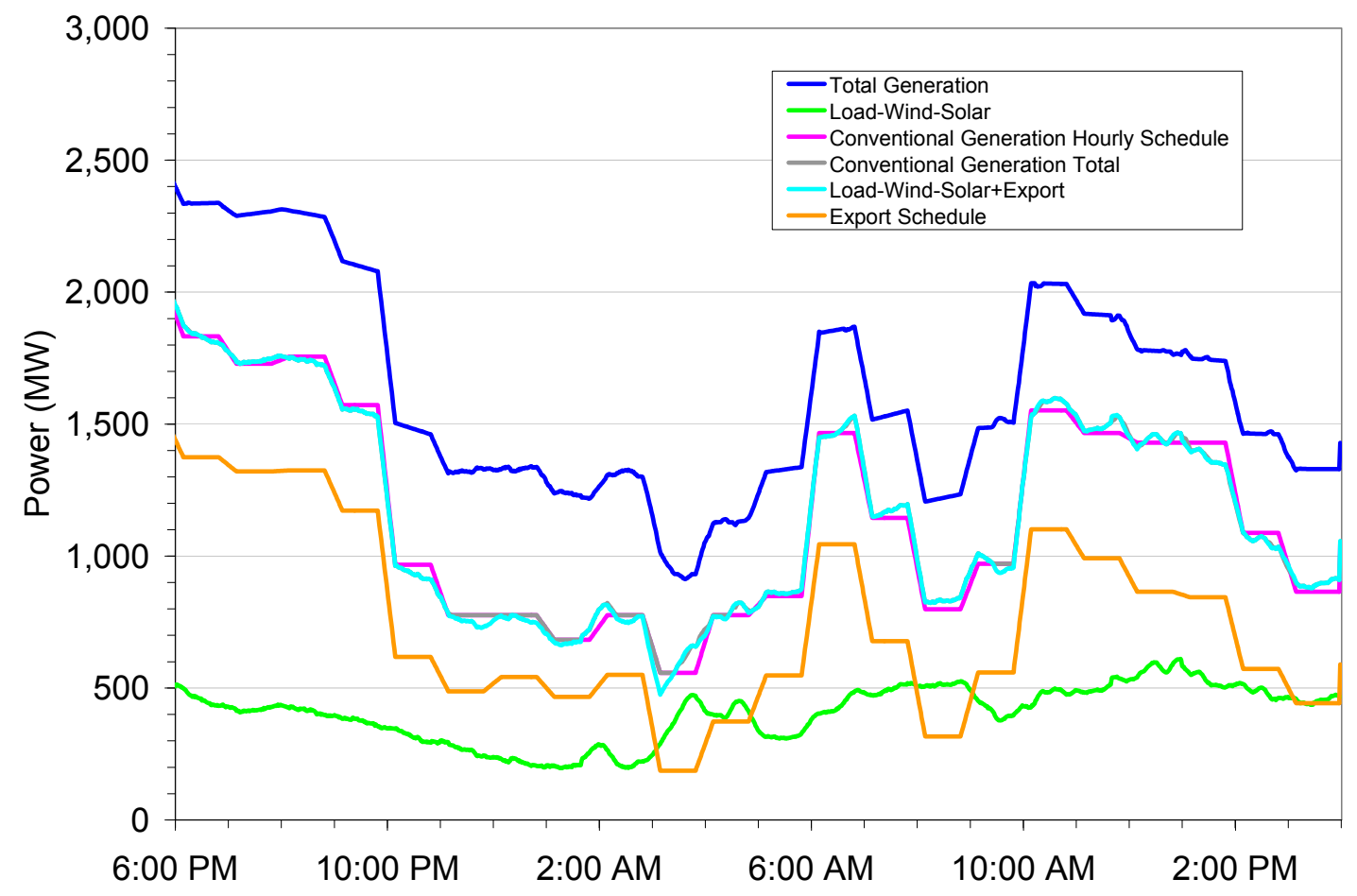

Figure F- 6 Colorado West Totals, 30\% Wind, Local Priority, April QSS Study Period

\section{F.3 New Mexico April 15, 30\% Wind Penetration}

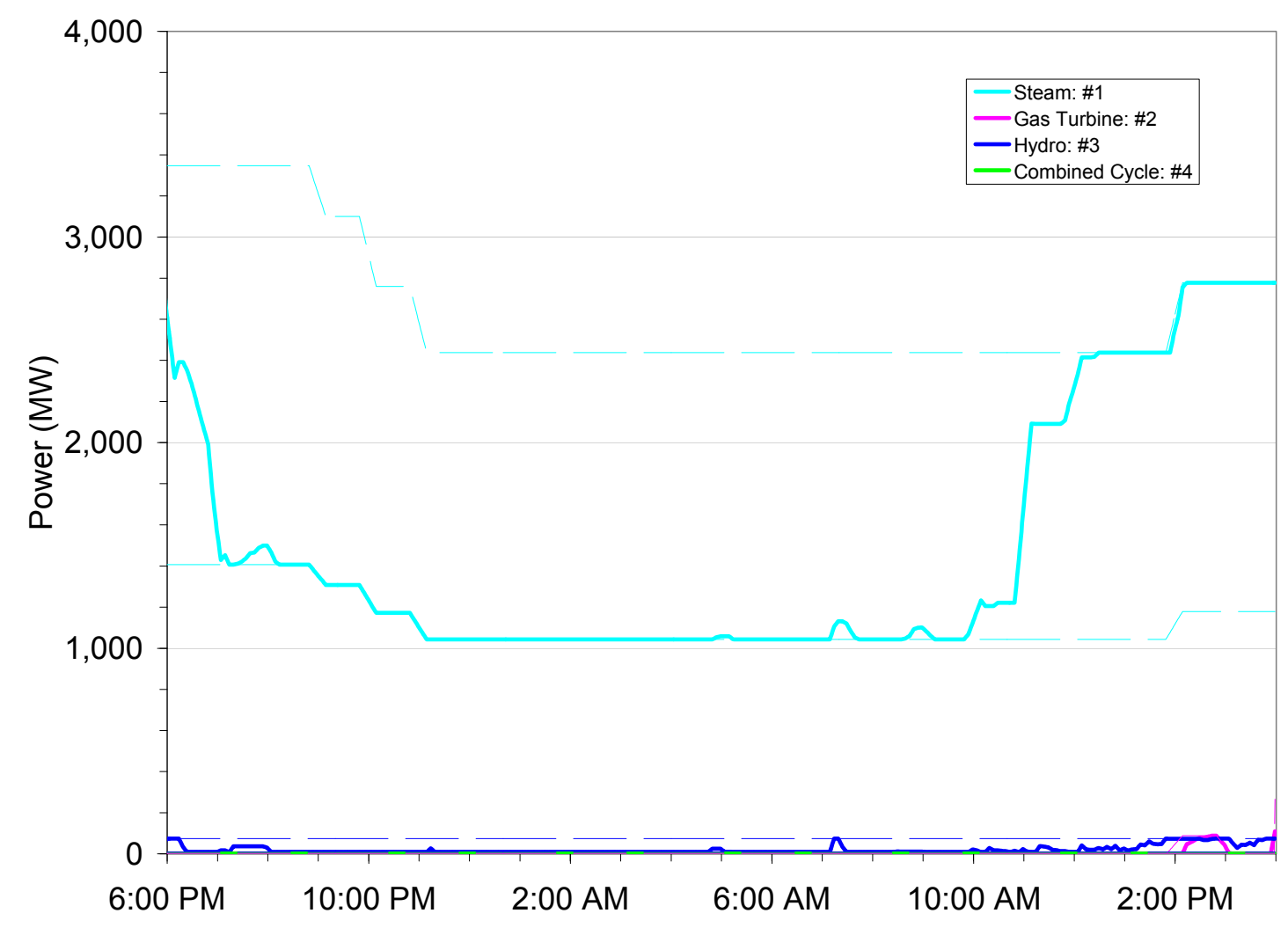

Figure F- 7 New Mexico Dispatch, 30\% Wind, Local Priority, April QSS Study Period 


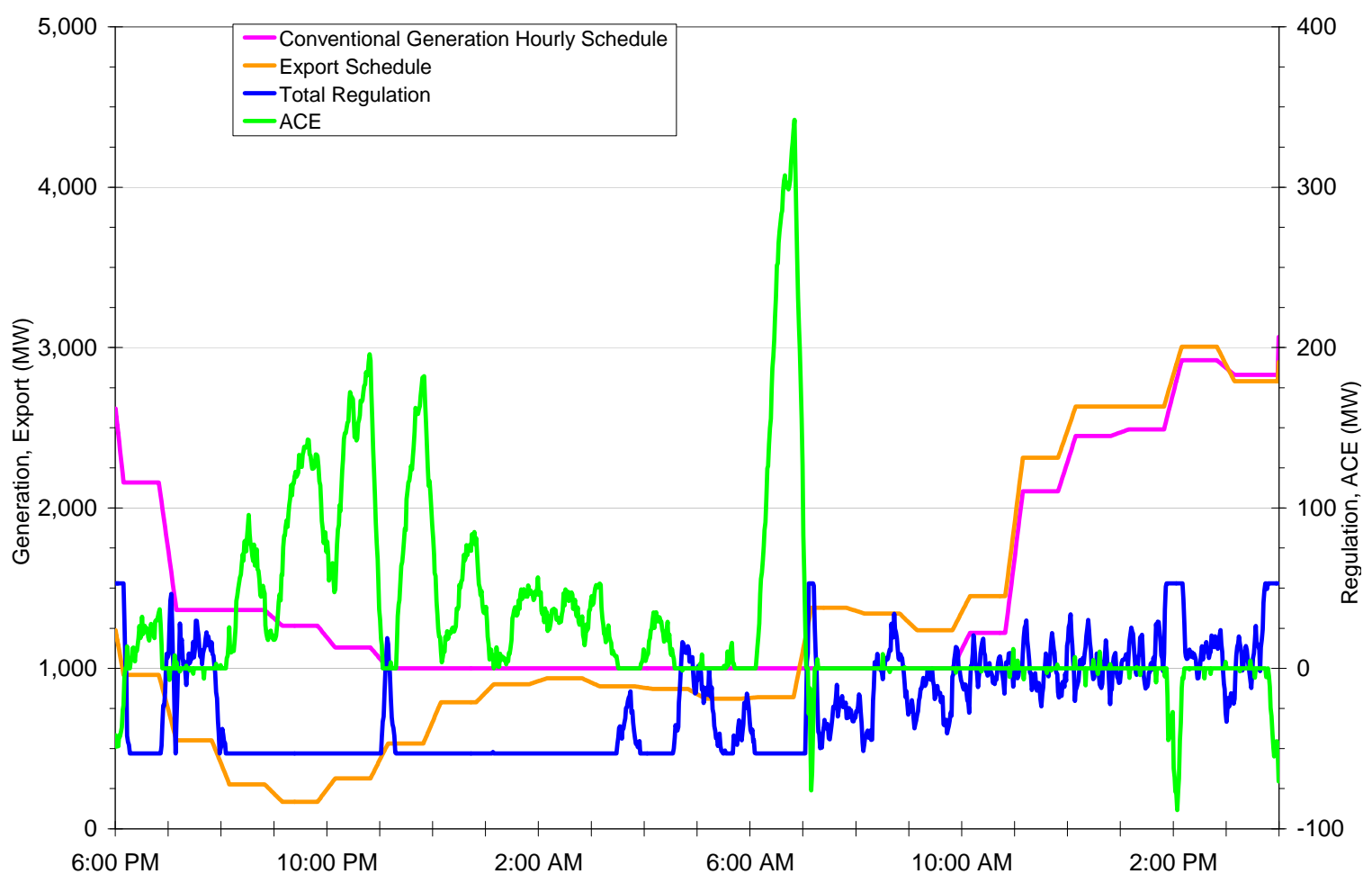

Figure F- 8 New Mexico Regulation, 30\% Wind, Local Priority, April QSS Study Period

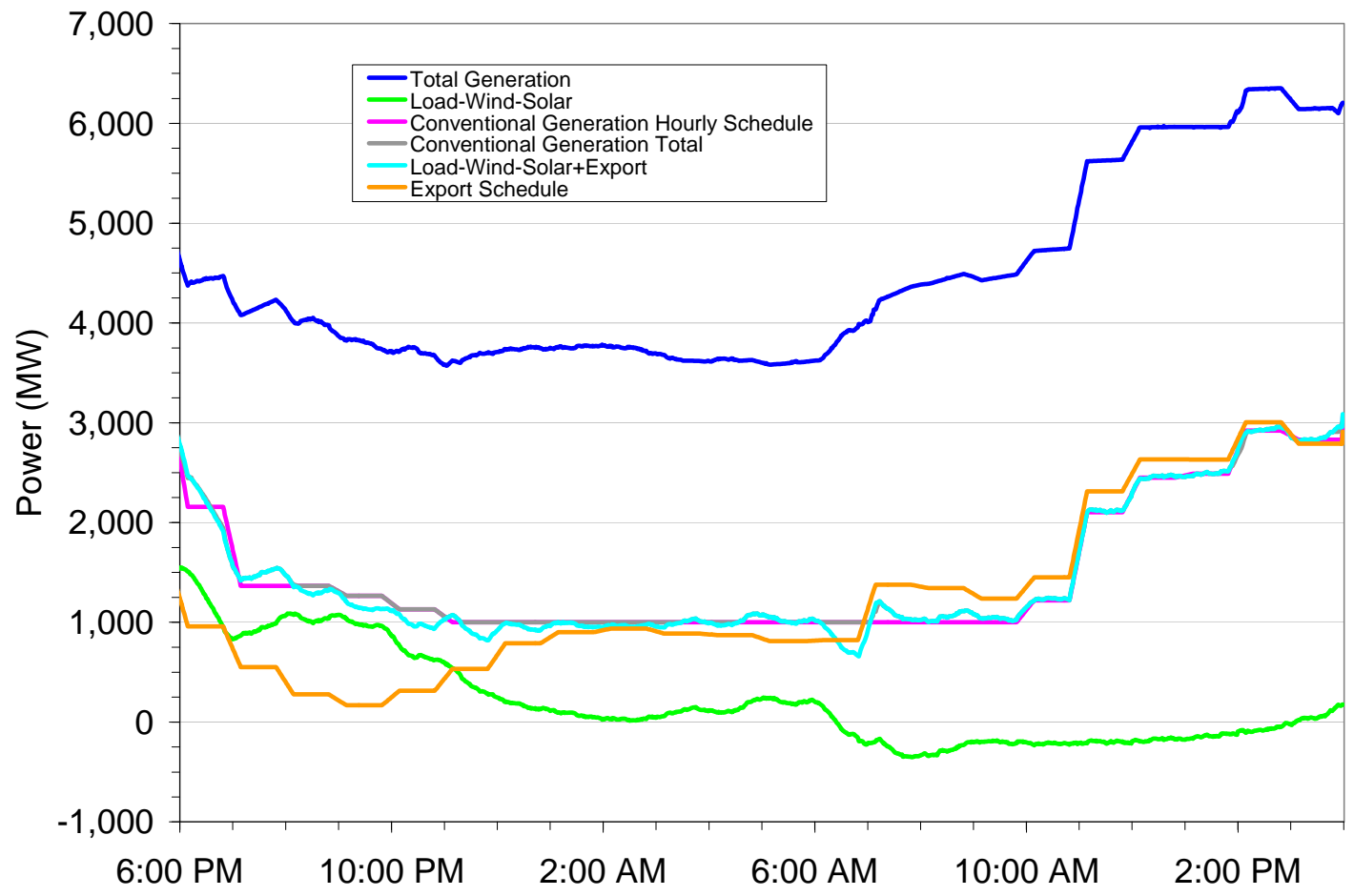

Figure F- 9 New Mexico Totals, 30\% Wind, Local Priority, April QSS Study Period 


\section{F.4 North Nevada April 15, 30\% Wind Penetration}

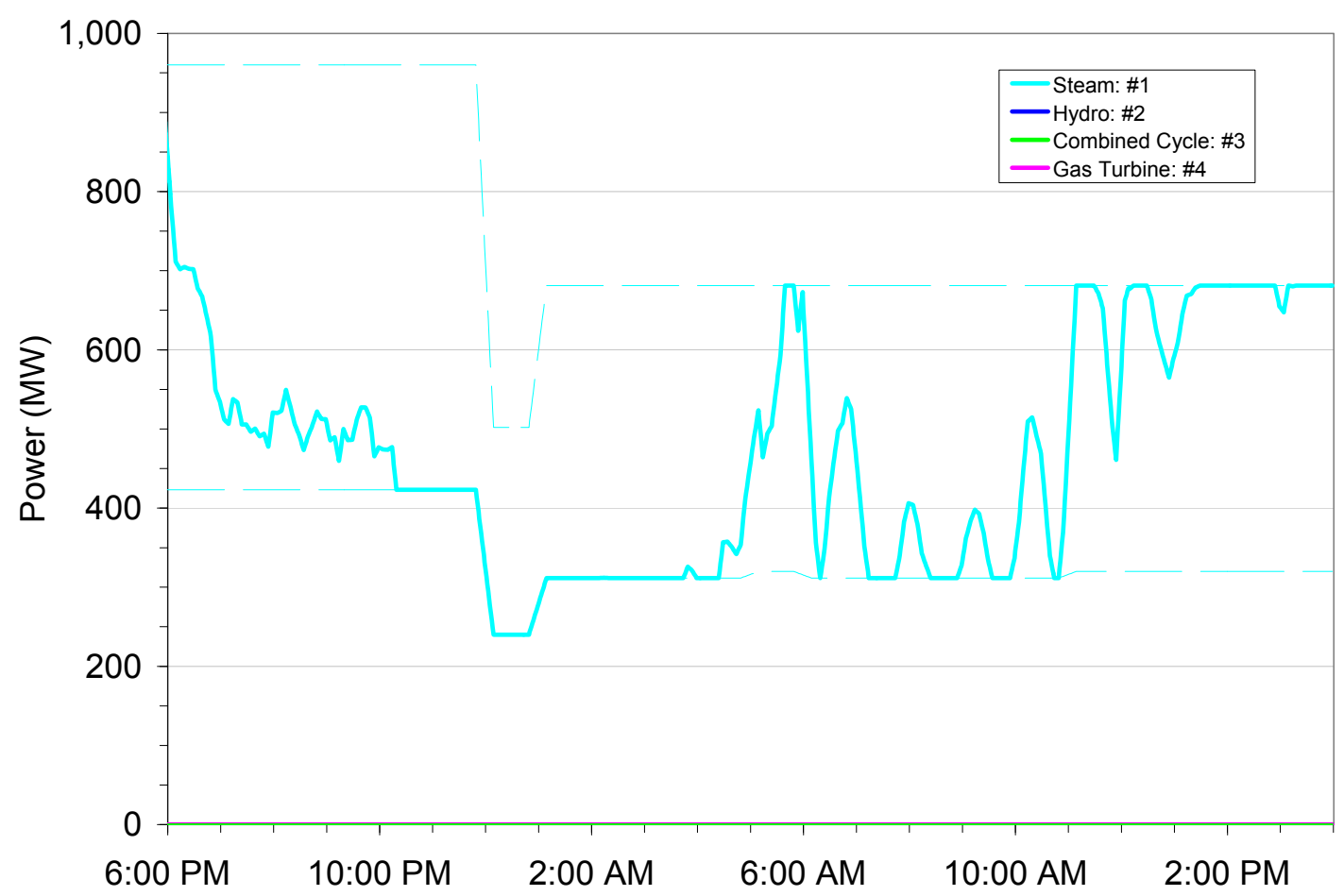

Figure F-10 North Nevada Dispatch, 30\% Wind, Local Priority, April QSS Study Period

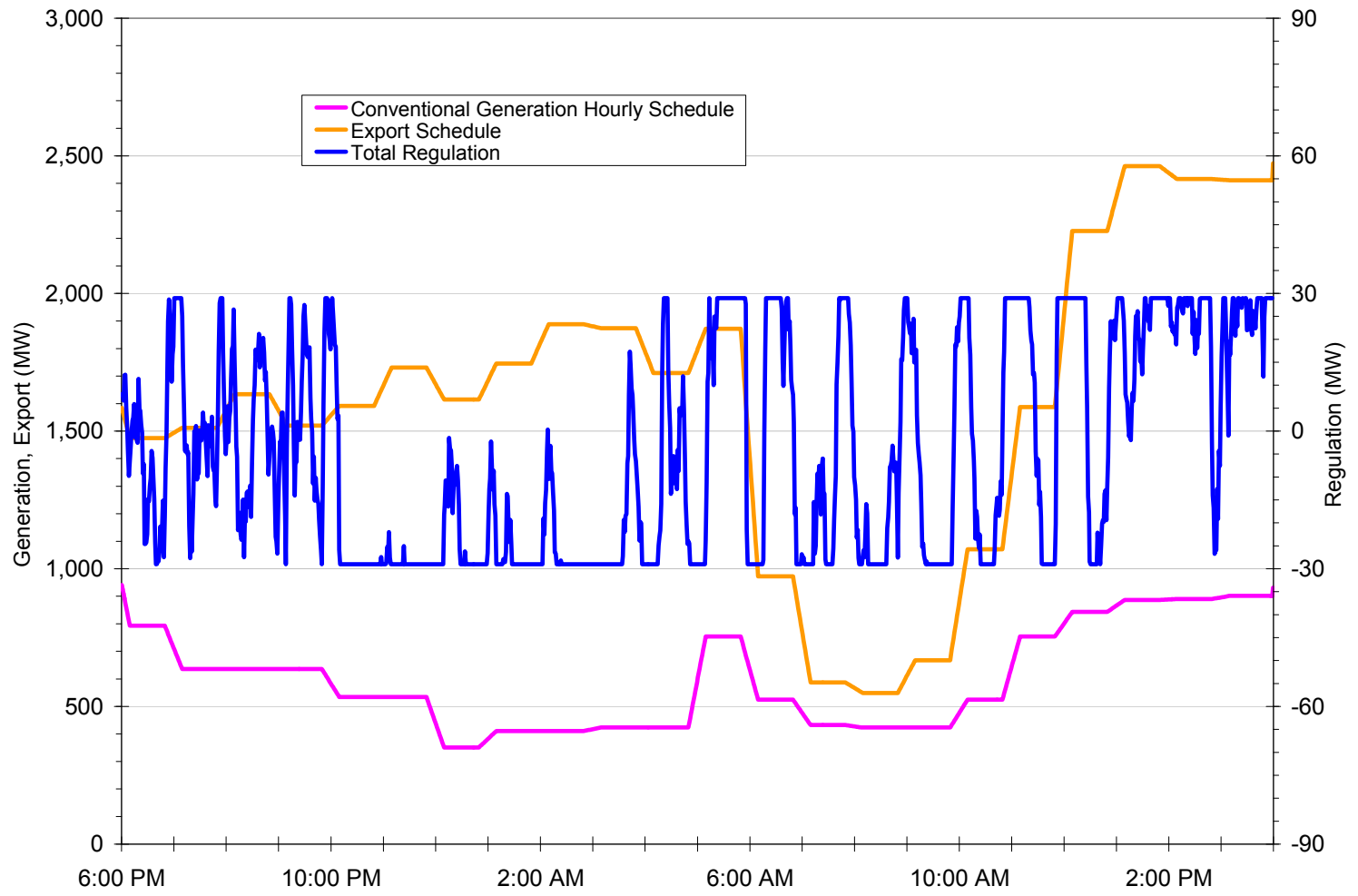

Figure F- 11 North Nevada Regulation, 30\% Wind, Local Priority, April QSS Study Period 


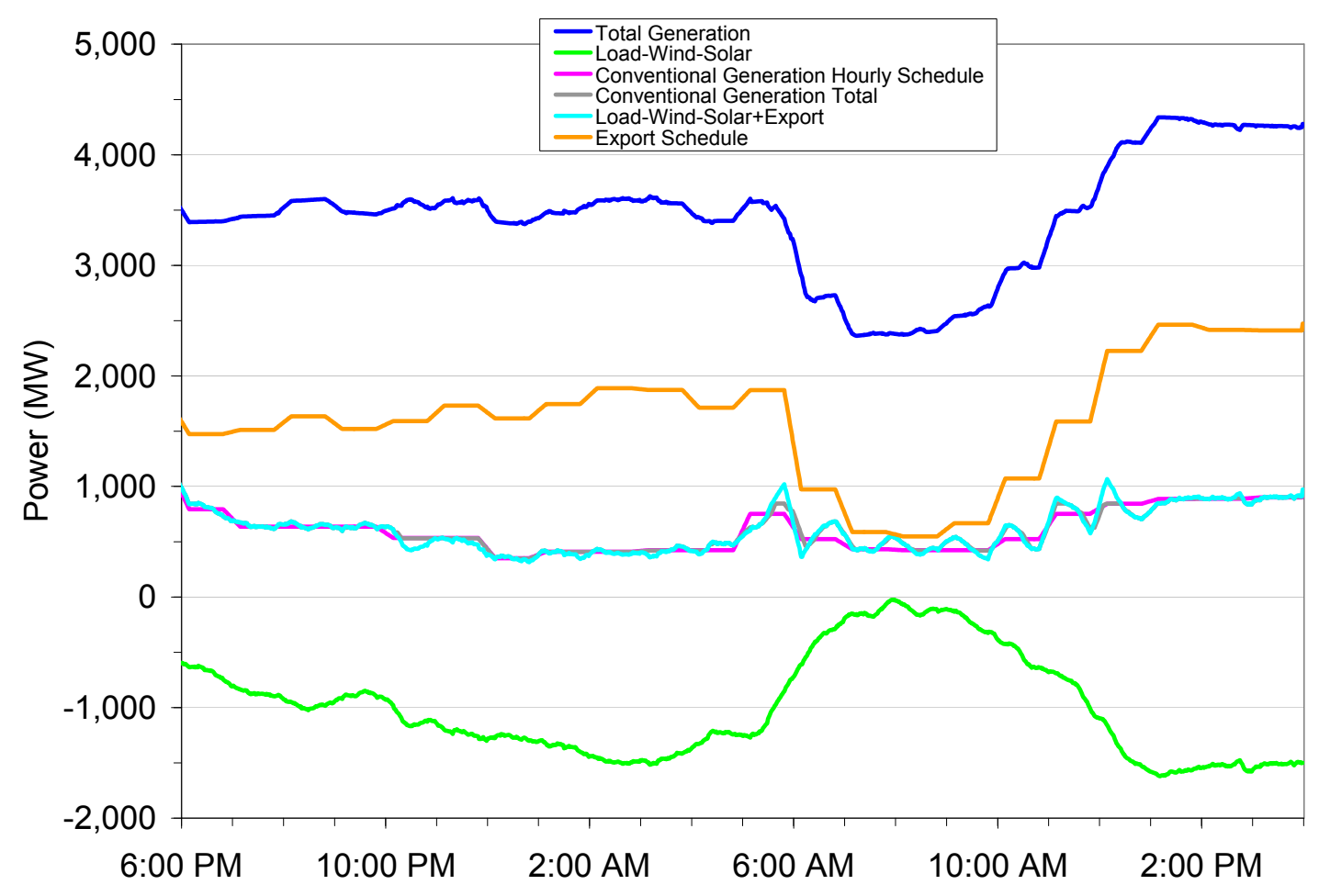

Figure F- 12 North Nevada Totals, 30\% Wind, Local Priority, April QSS Study Period

\section{F.5 South Nevada April 15, 30\% Wind Penetration}

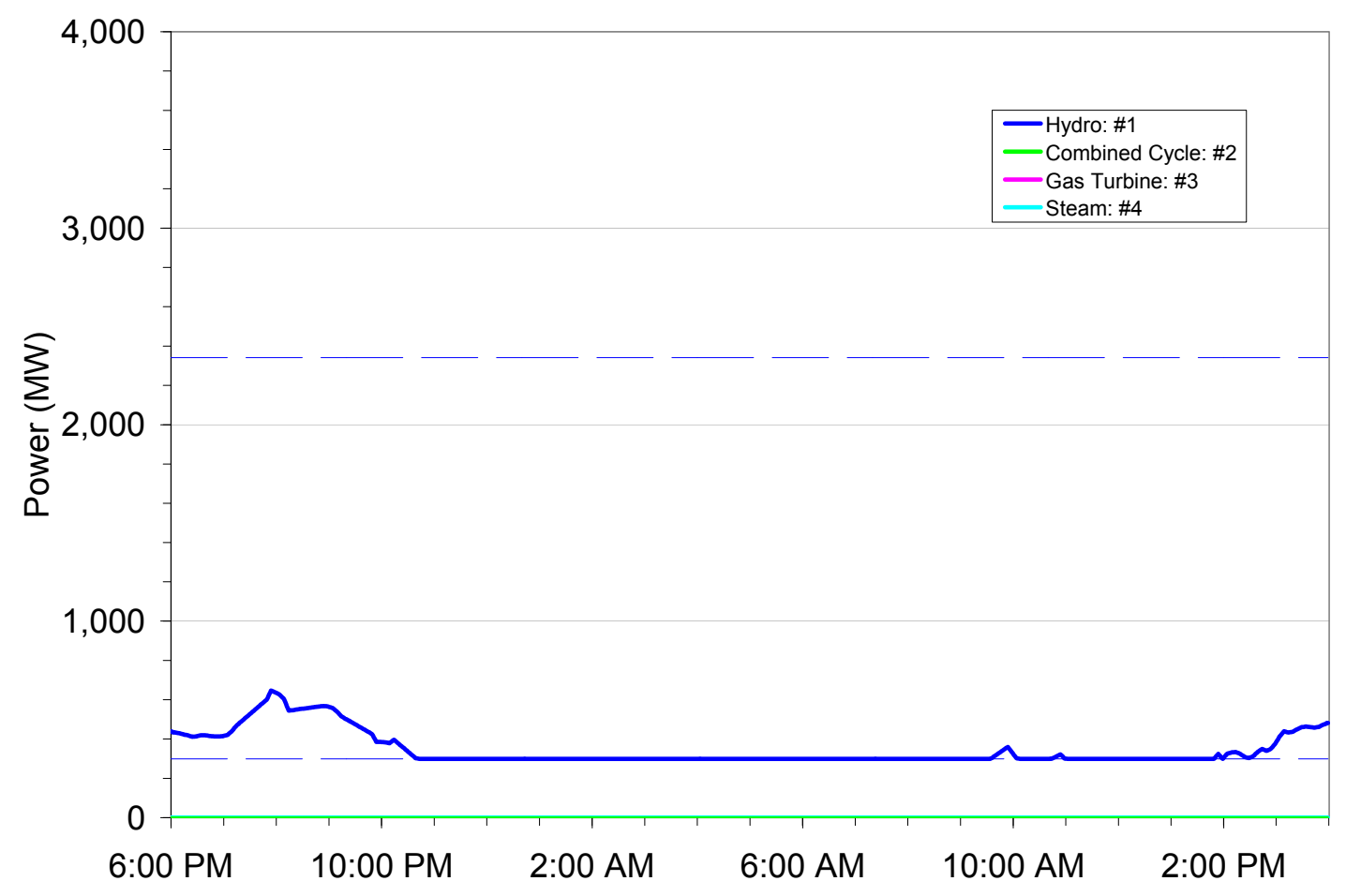

Figure F- 13 South Nevada Dispatch, 30\% Wind, Local Priority, April QSS Study Period 


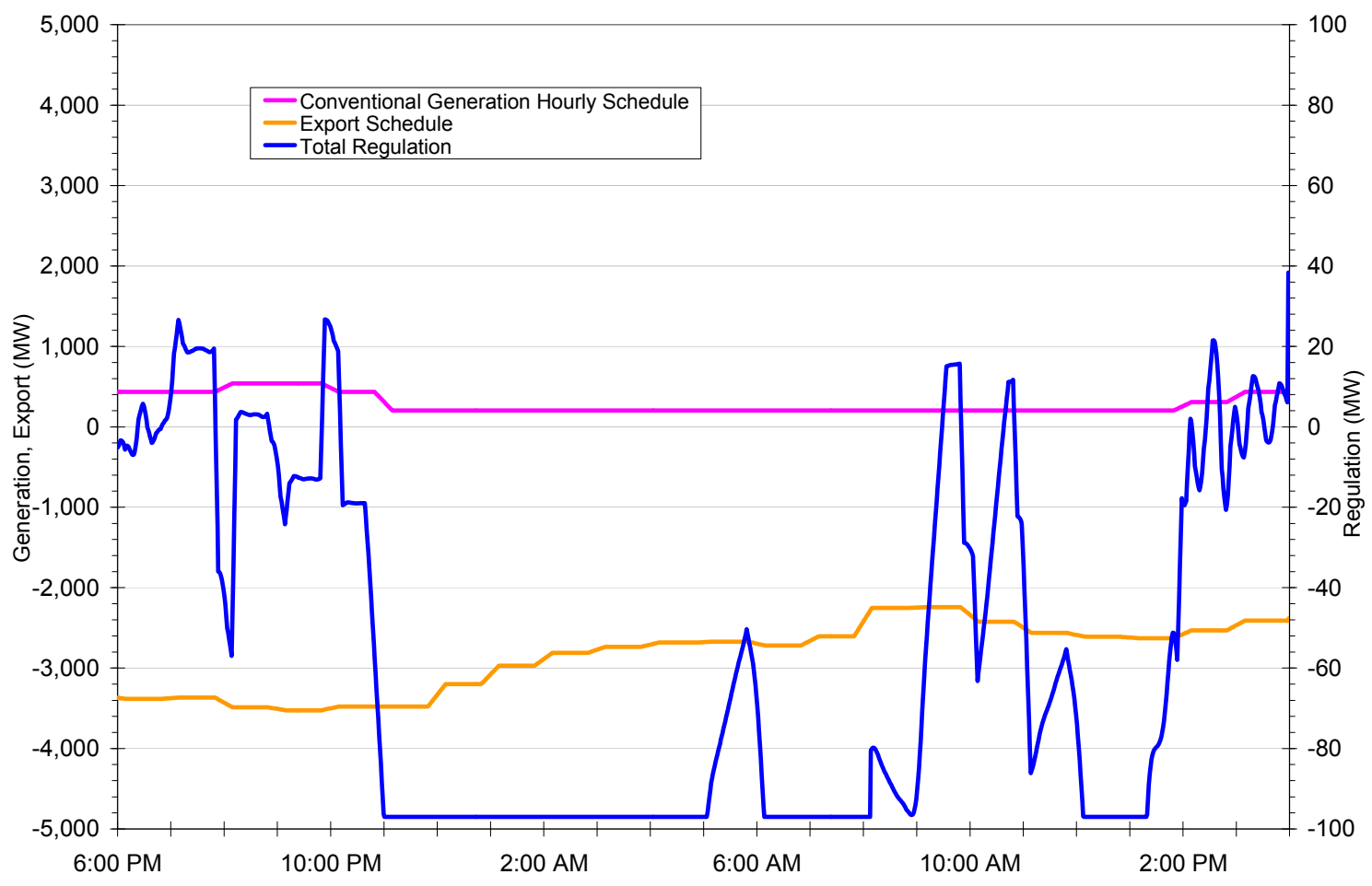

Figure F- 14 South Nevada Regulation, 30\% Wind, Local Priority, April QSS Study Period

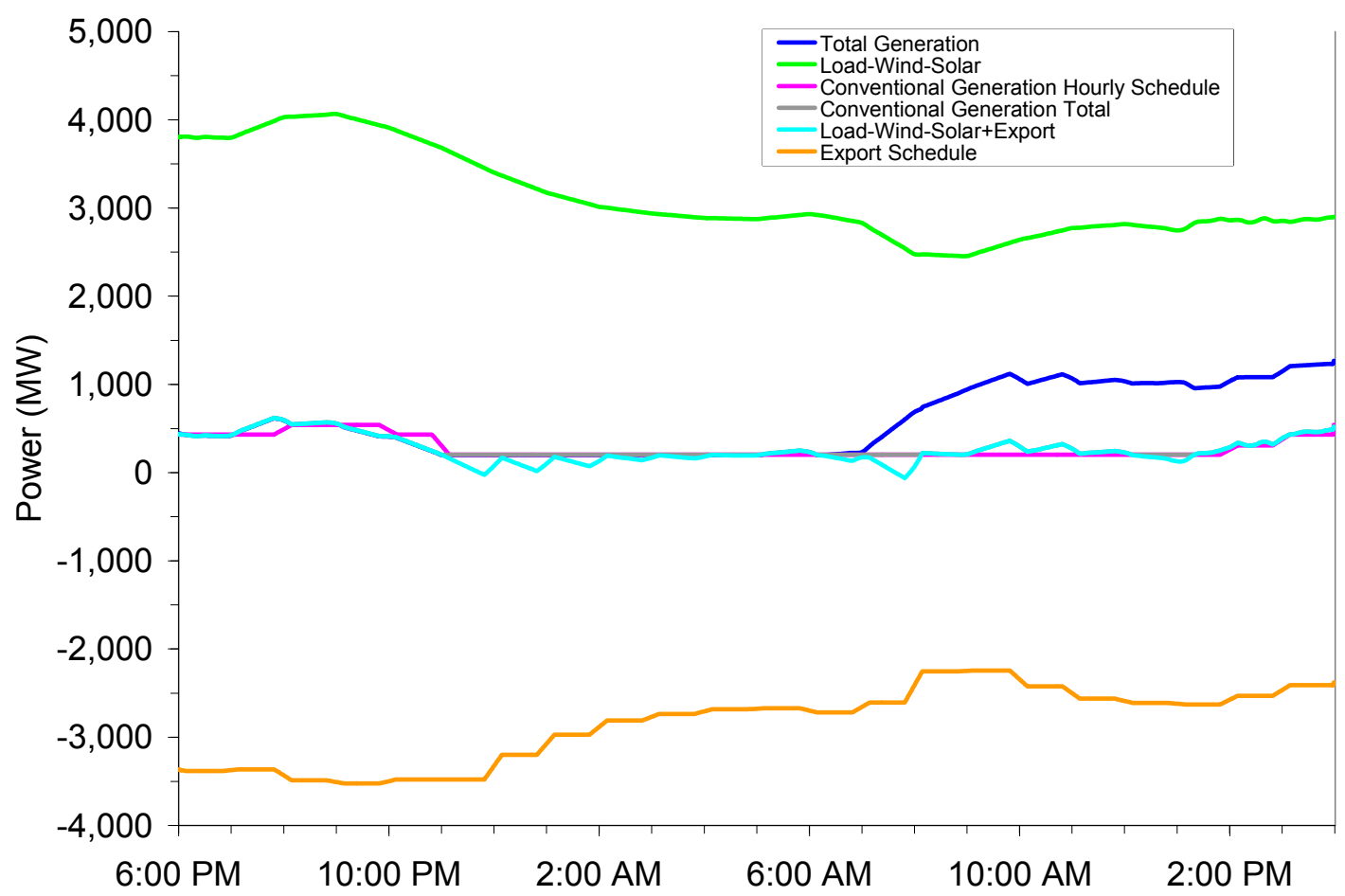

Figure F- 15 South Nevada Totals, 30\% Wind, Local Priority, April QSS Study Period 


\section{F.6 Consolidated North and South Nevada April 15, 30\% Wind Penetration}

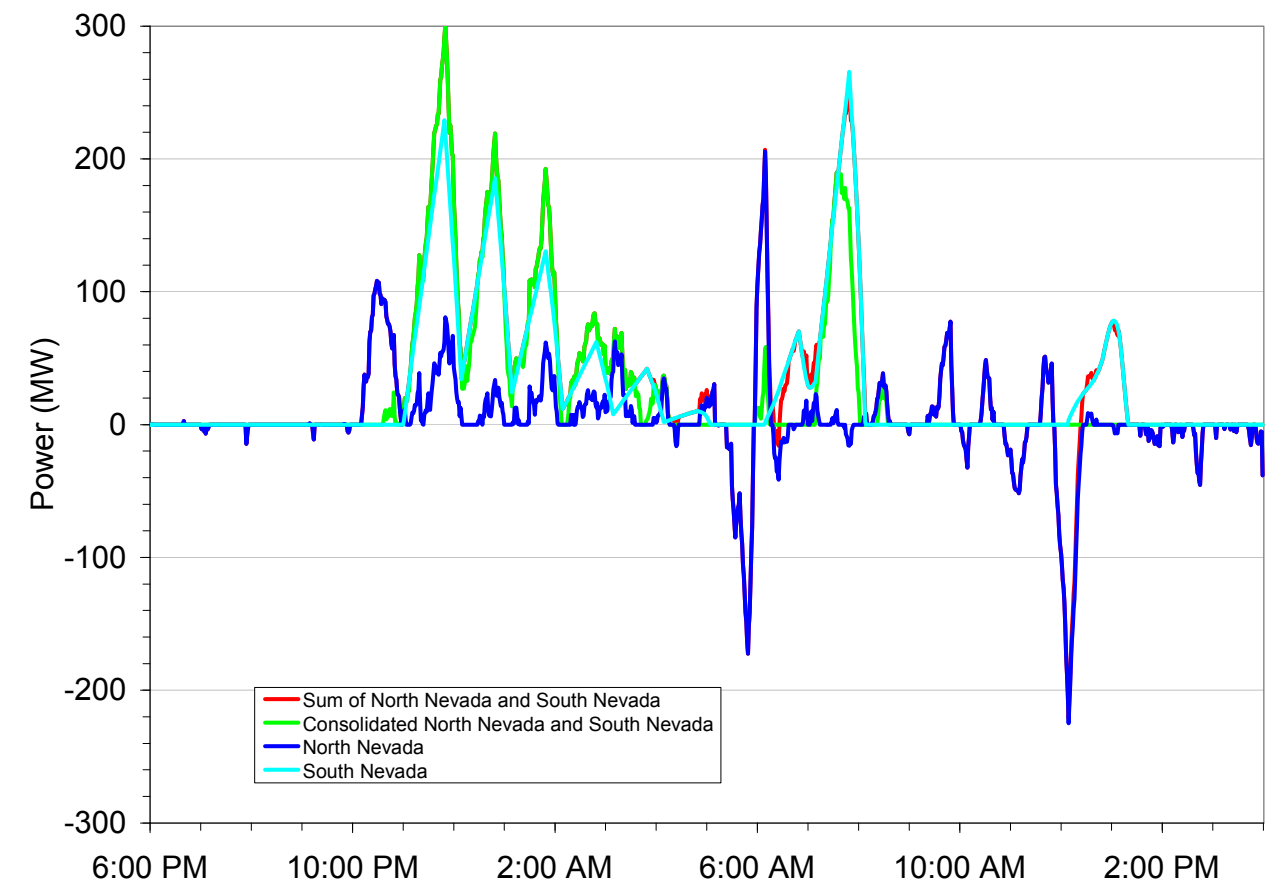

Figure F- 16 North and South Nevada ACEs, 30\% Wind, Local Priority, April QSS Study Period

\section{F.7 Wyoming April 15, 10\% Wind Penetration}

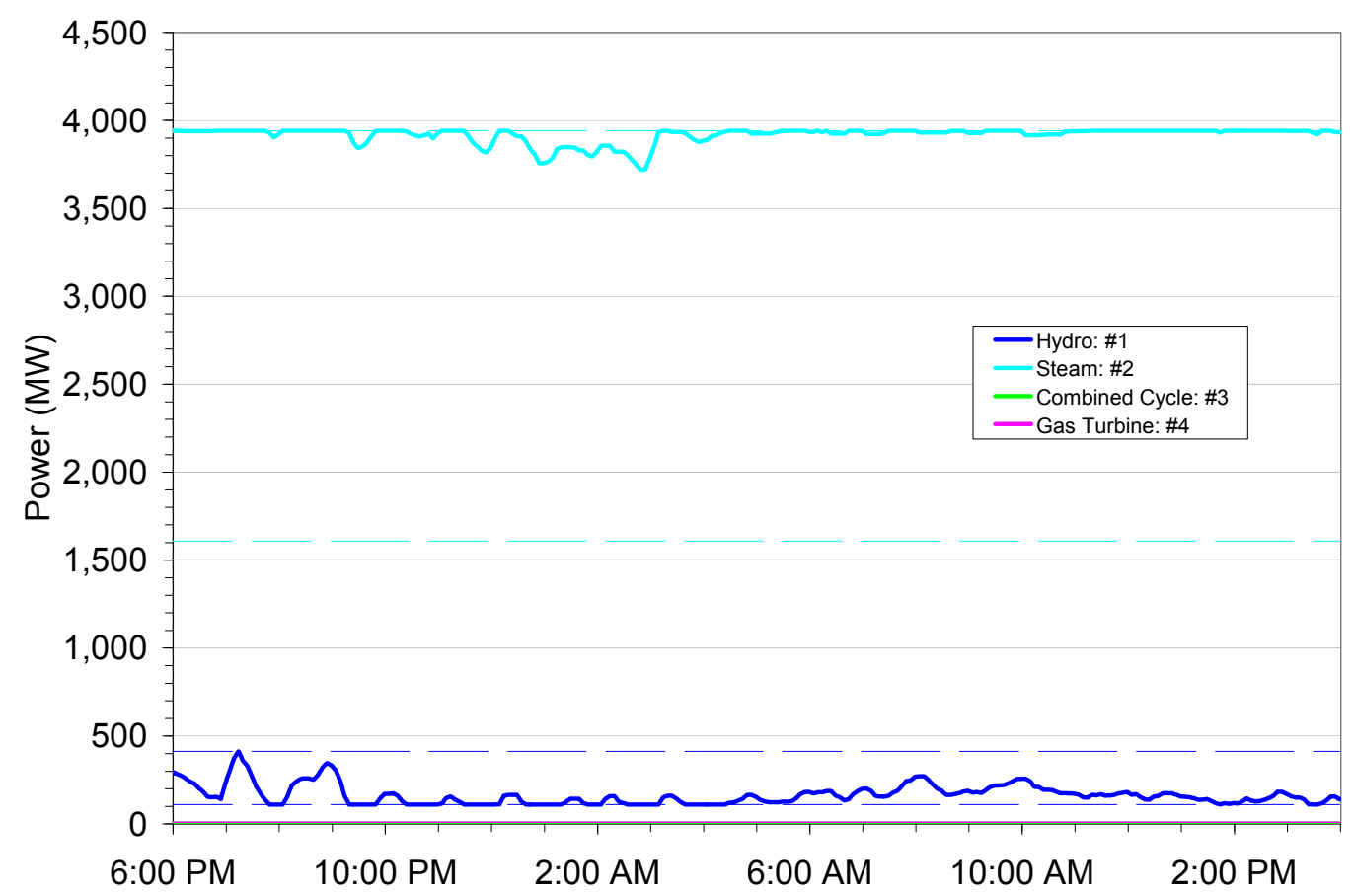

Figure F- 17 Wyoming Dispatch, 10\% Wind, Local Priority, April QSS Study Period 


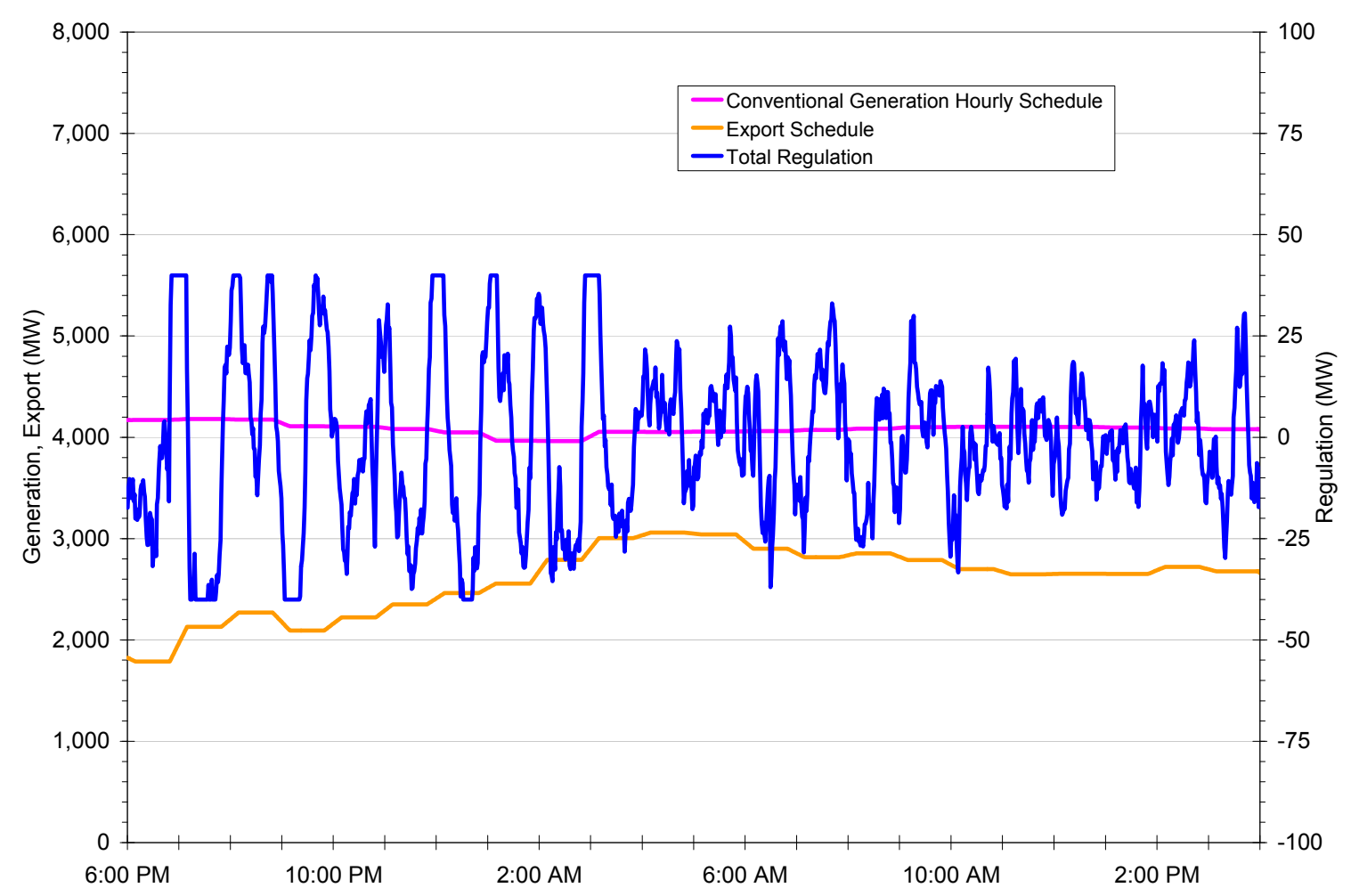

Figure F- 18 Wyoming Regulation, 10\% Wind, Local Priority, April QSS Study Period

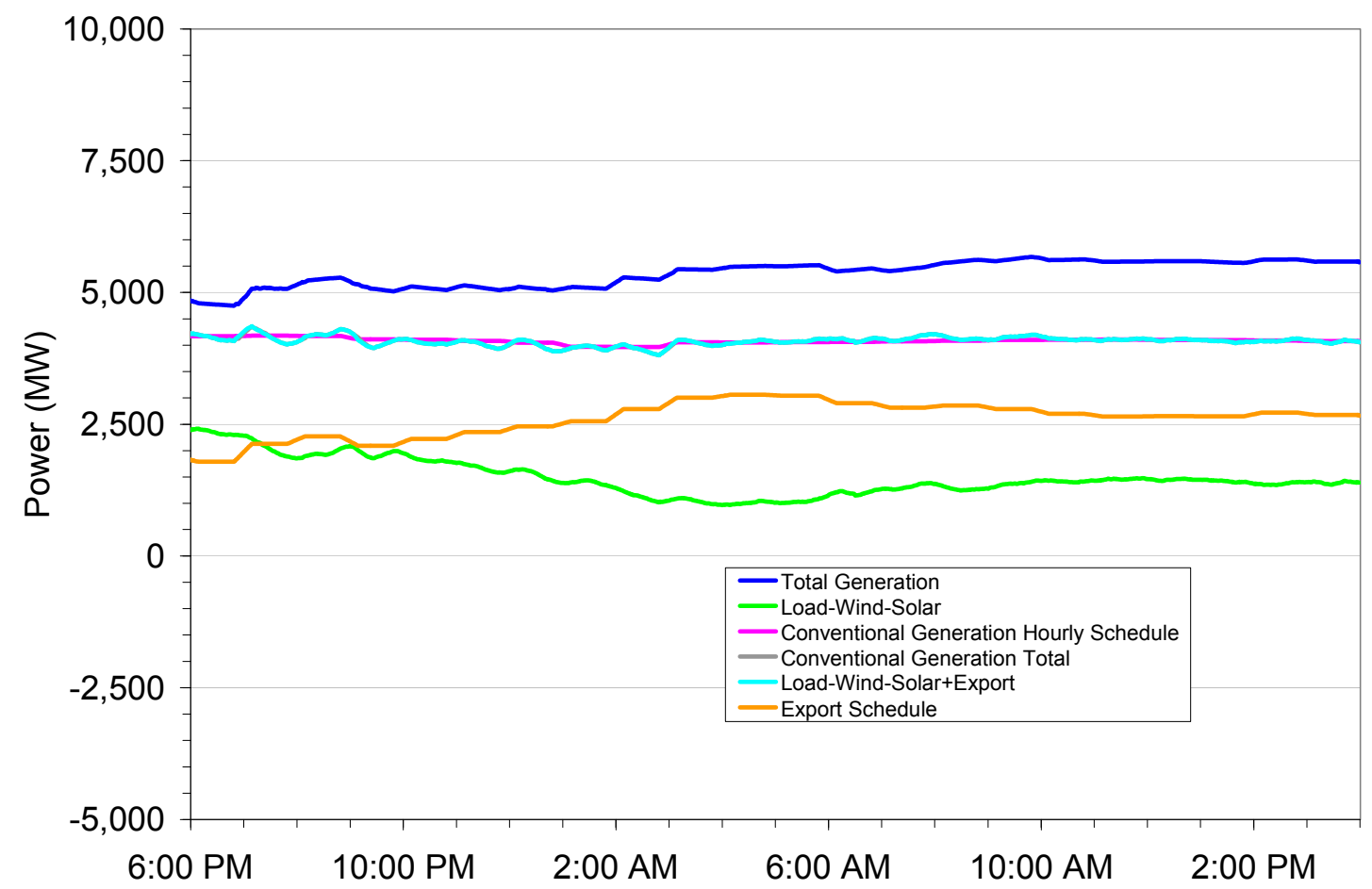

Figure F- 19 Wyoming Totals, 10\% Wind, Local Priority, April QSS Study Period 


\section{F.8 Wyoming April 15, 20\% Wind Penetration}

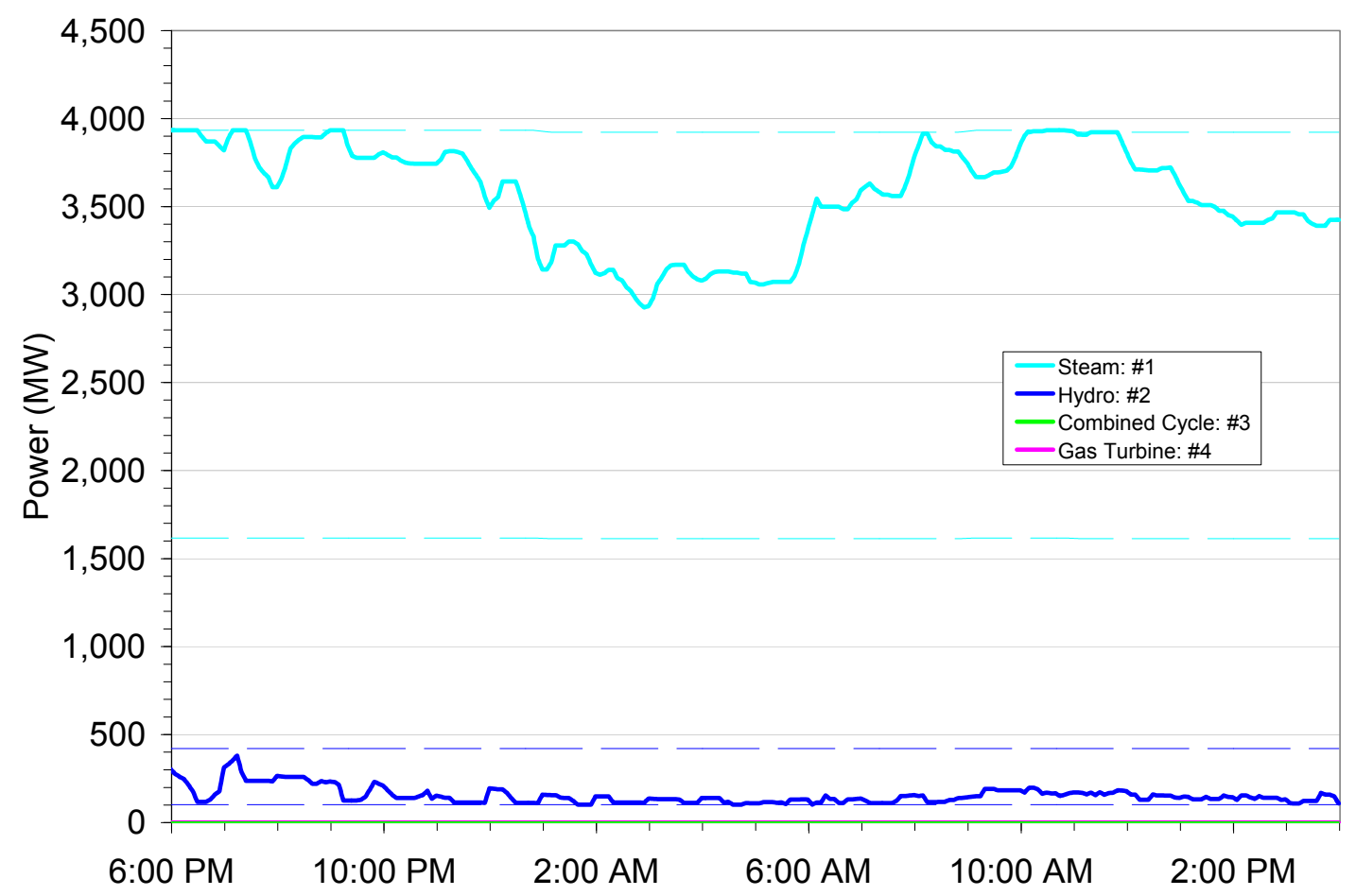

Figure F- 20 Wyoming Dispatch, 20\% Wind, Local Priority, April QSS Study Period

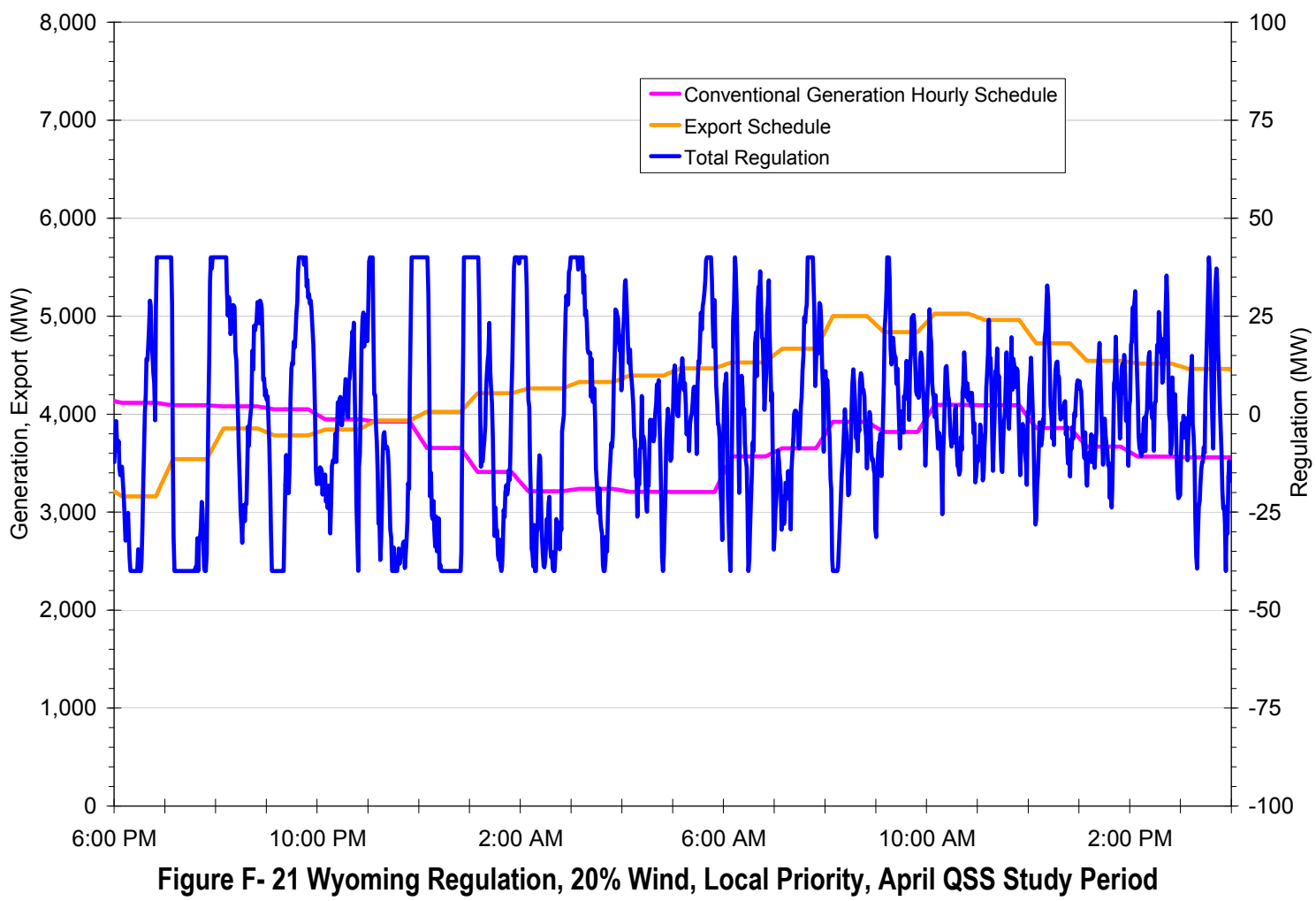




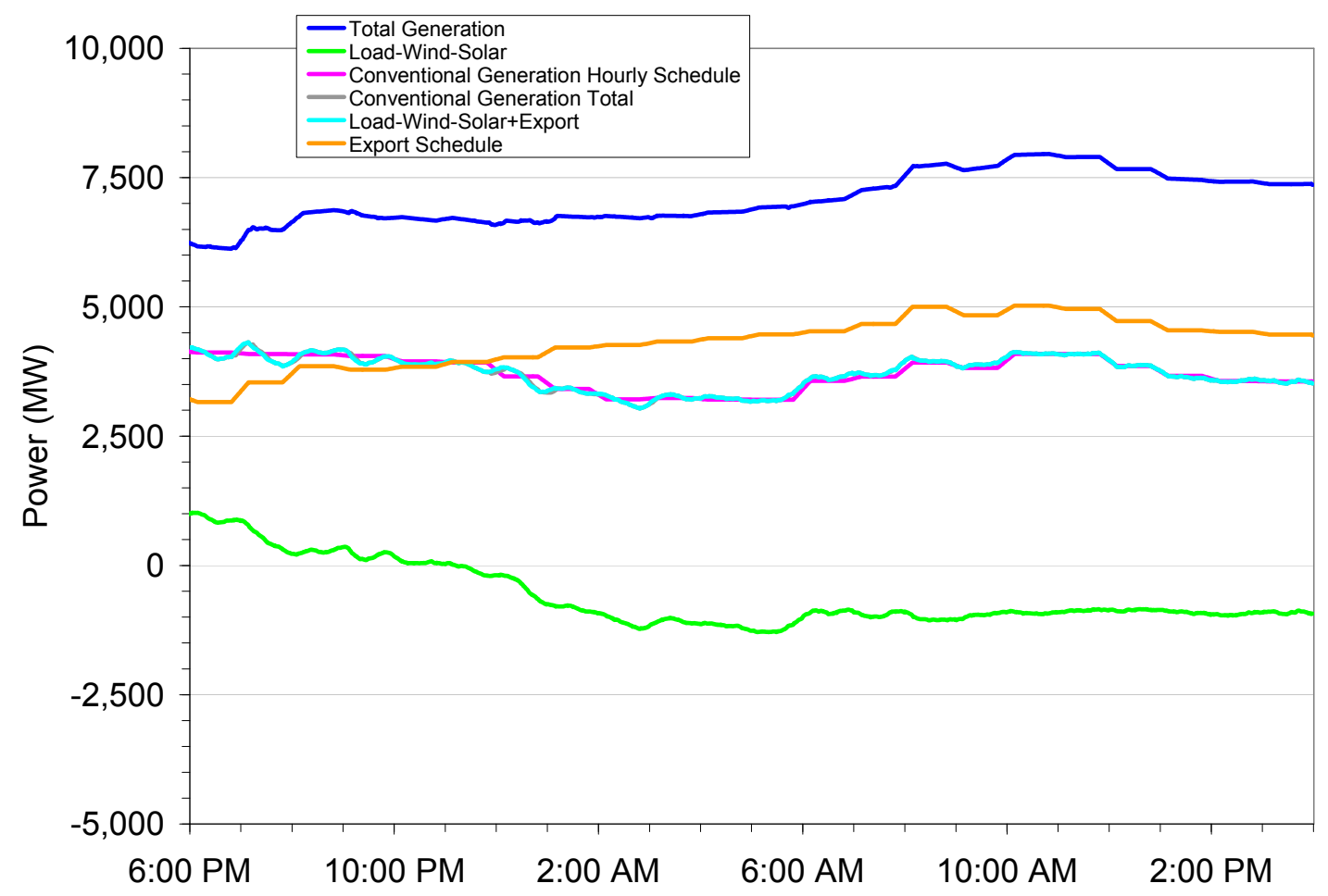

Figure F- 22 Wyoming Totals, 20\% Wind, Local Priority, April QSS Study Period

\section{F.9 Wyoming April 15, 20/20\% Wind Penetration}

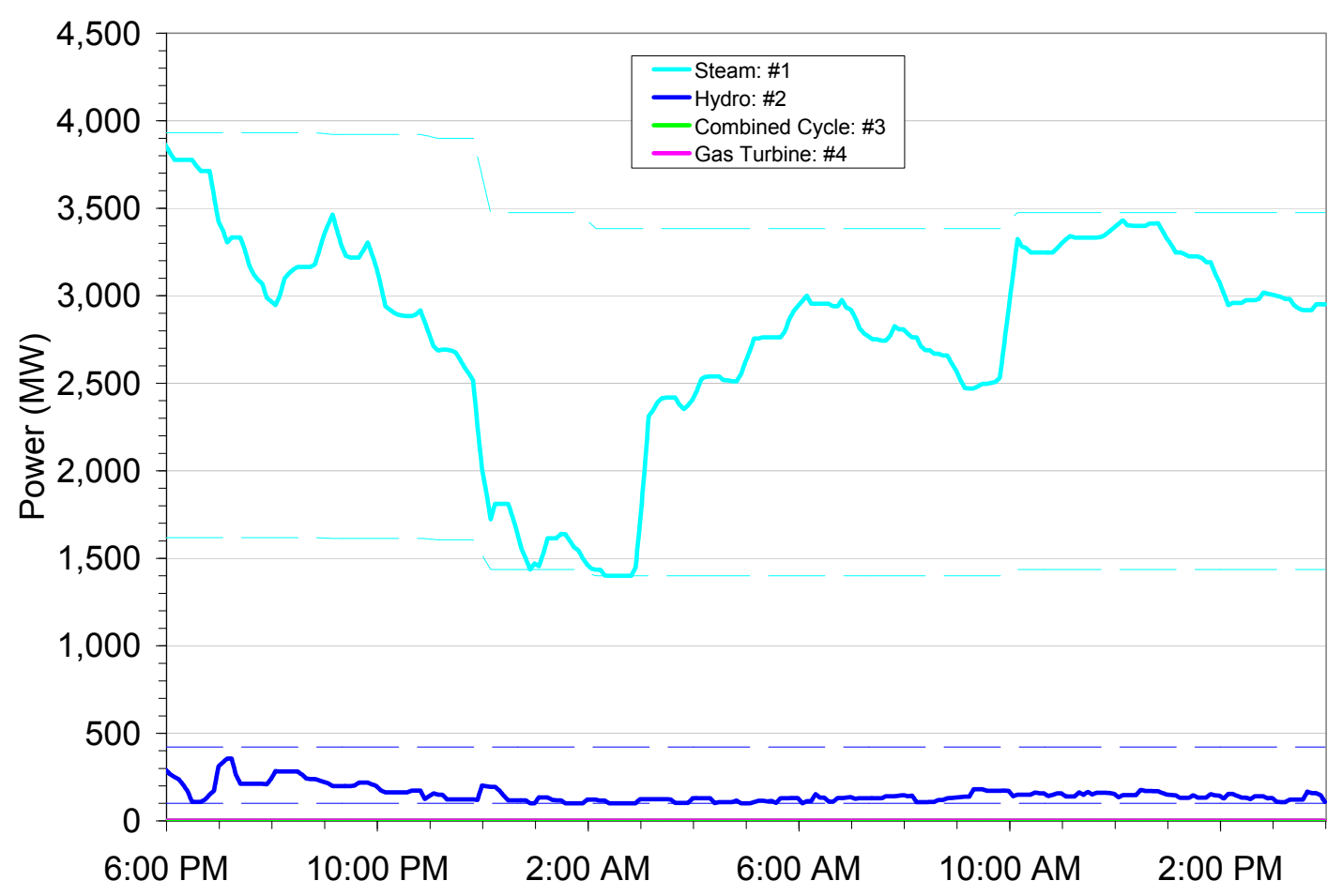

Figure F- 23 Wyoming Dispatch, 20/20\% Wind, Local Priority, April QSS Study Period 


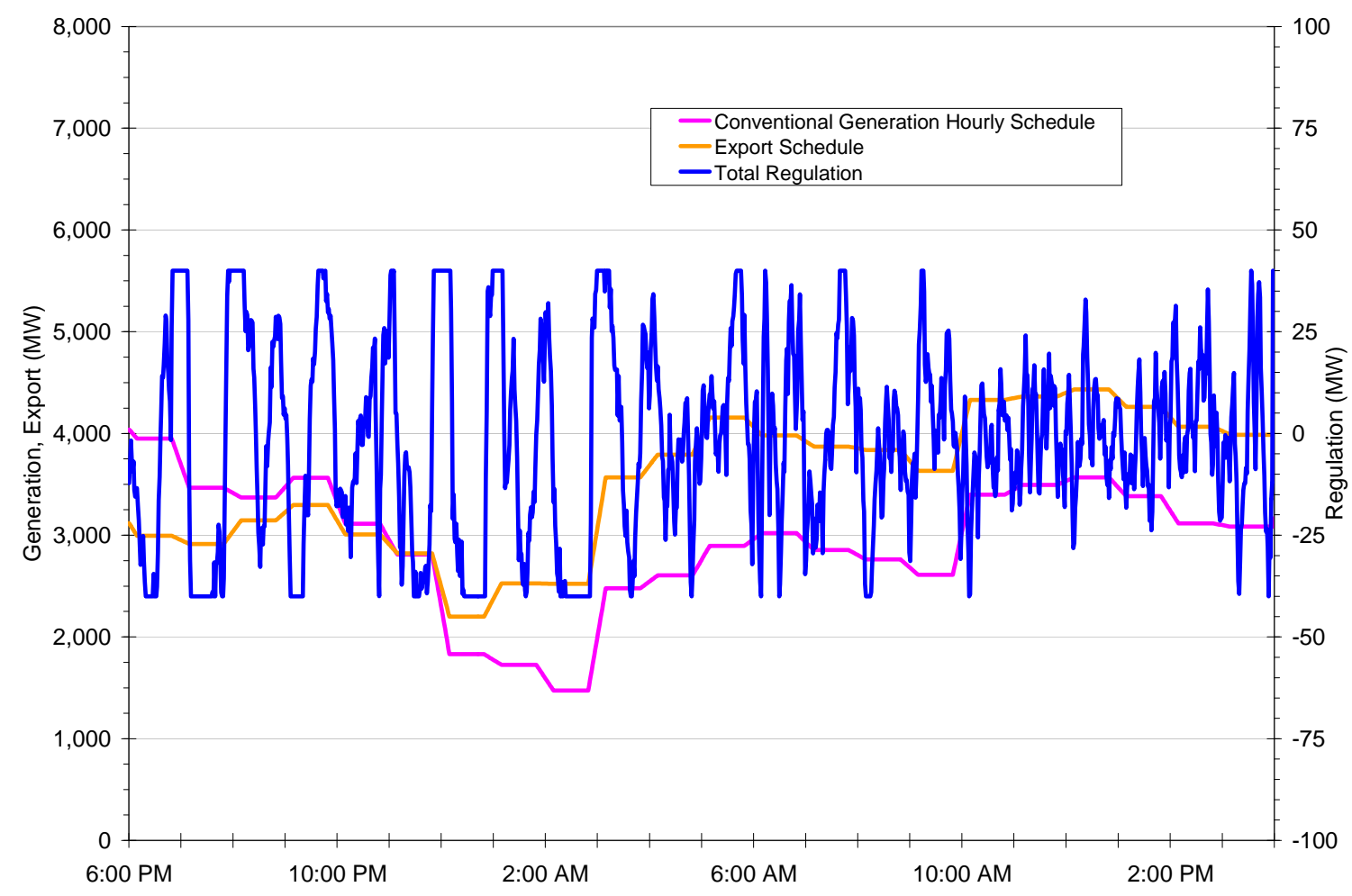

Figure F- 24 Wyoming Regulation, 20/20\% Wind, Local Priority, April QSS Study Period

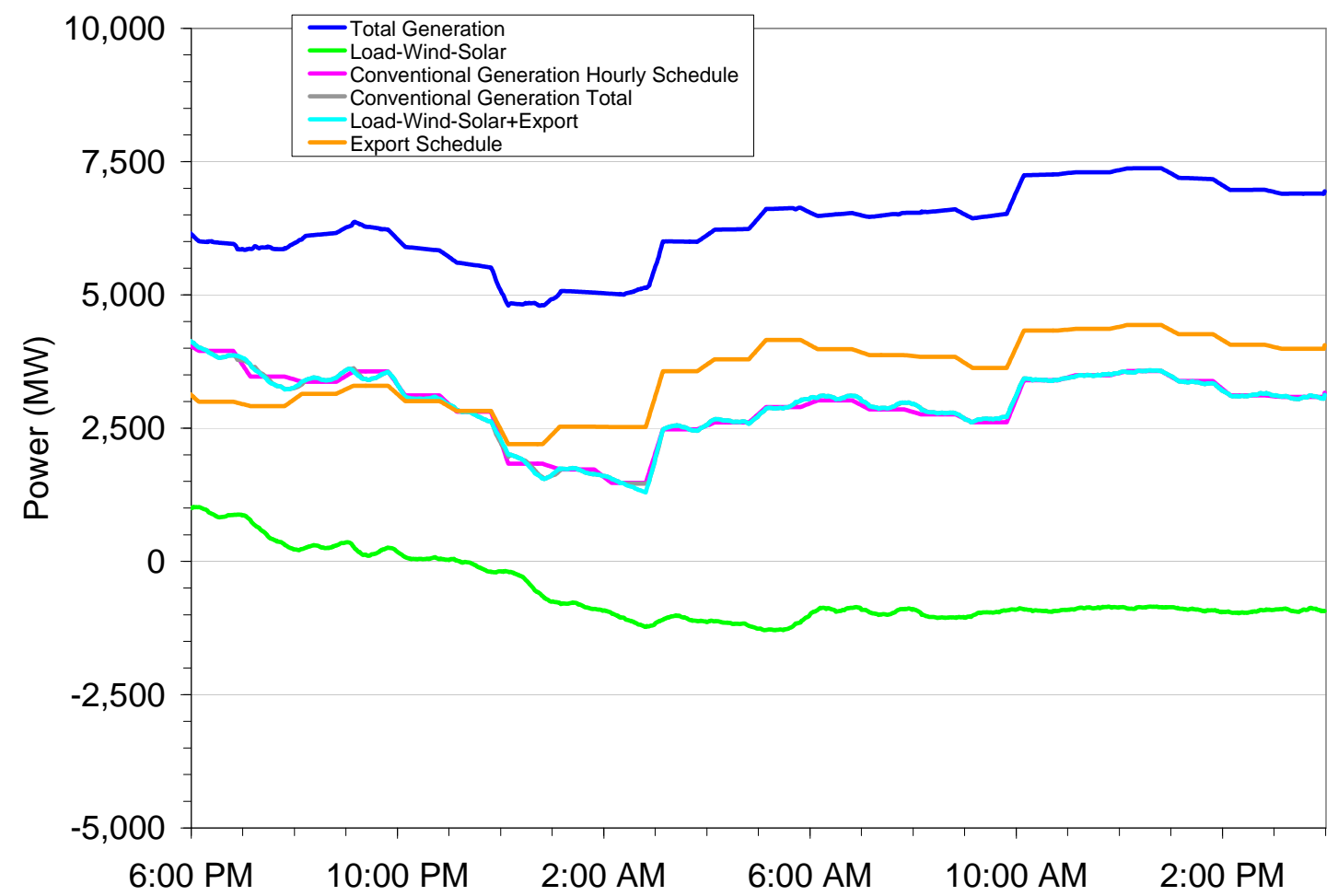

Figure F- 25 Wyoming Totals, 20/20\% Wind, Local Priority, April QSS Study Period 


\section{F.10 Wyoming April 15, 30\% Wind Penetration}

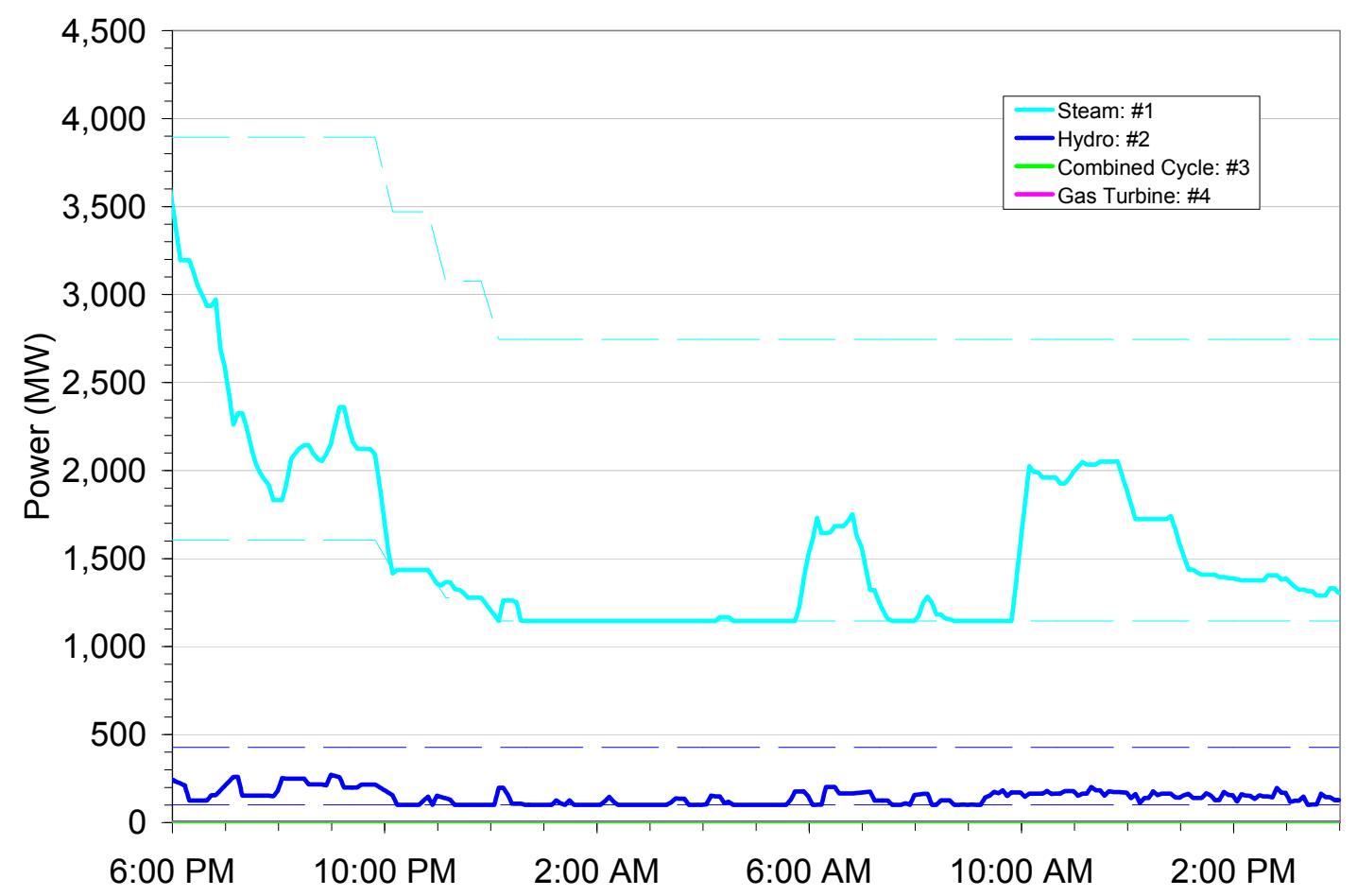

Figure F- 26 Wyoming Dispatch, 30\% Wind, Local Priority, April QSS Study Period

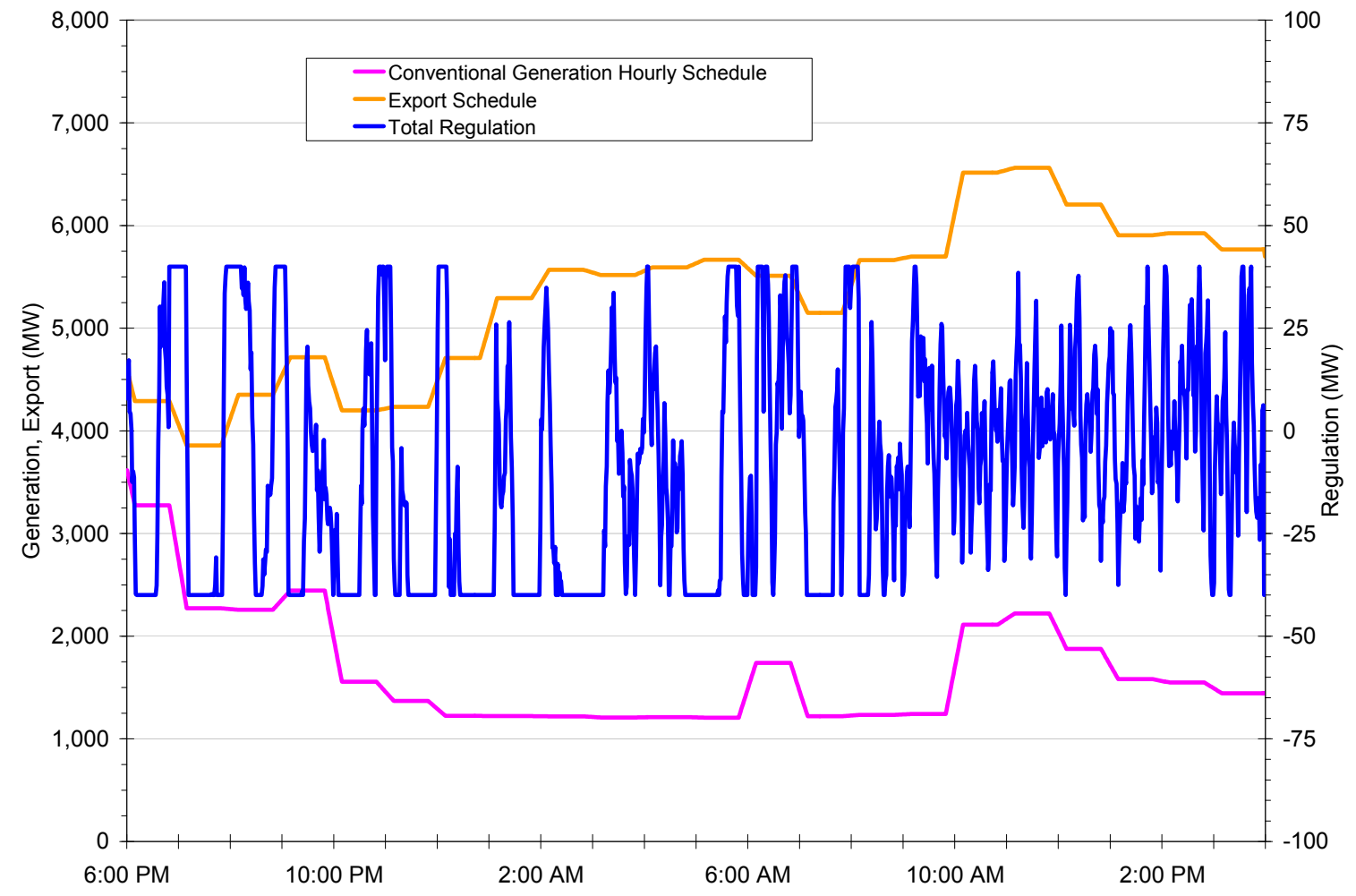

Figure F- 27 Wyoming Regulation, 30\% Wind, Local Priority, April QSS Study Period 


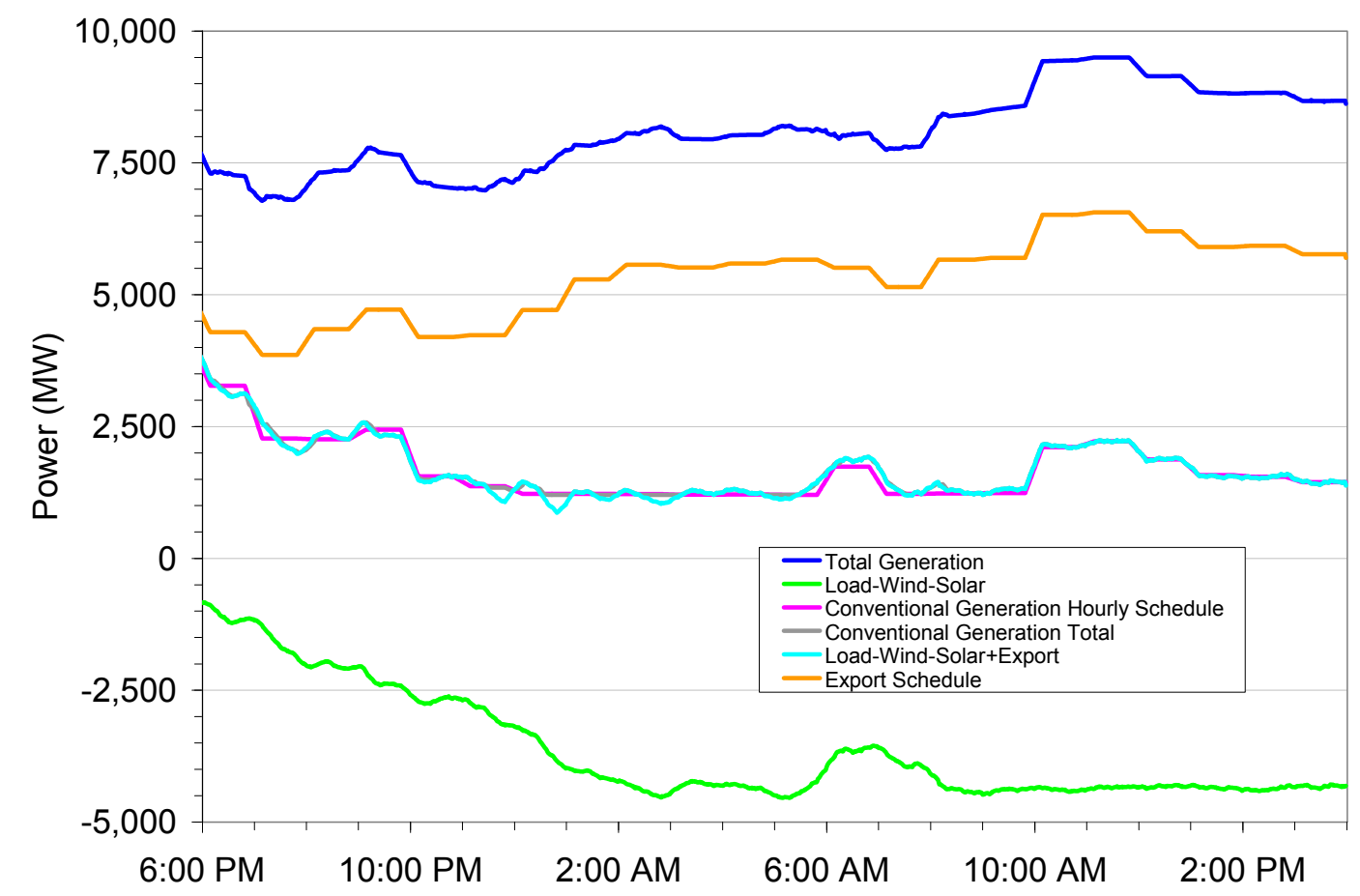

Figure F- 28 Wyoming Totals, 30\% Wind, Local Priority, April QSS Study Period

\section{F.11 Wyoming ACE April 15, 10\%, 20\%, 20/20\%, 30\% Wind Penetration}

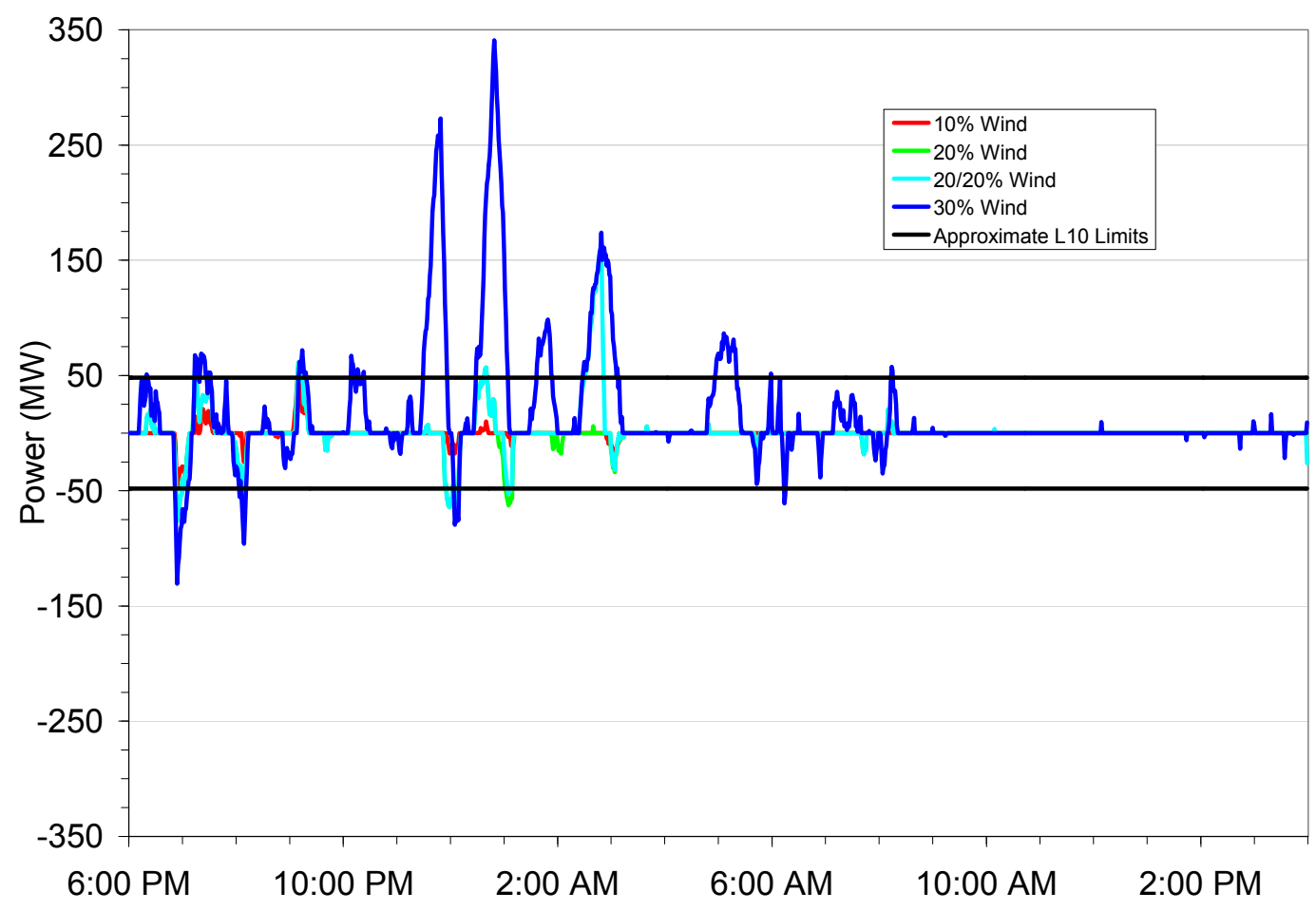

Figure F- 29 Wyoming ACE, All Penetration Levels, Local Priority, April QSS Study Period 


\section{F.12 Wyoming Extra Regulation (100 MW) April 15, 30\% Wind Penetration}

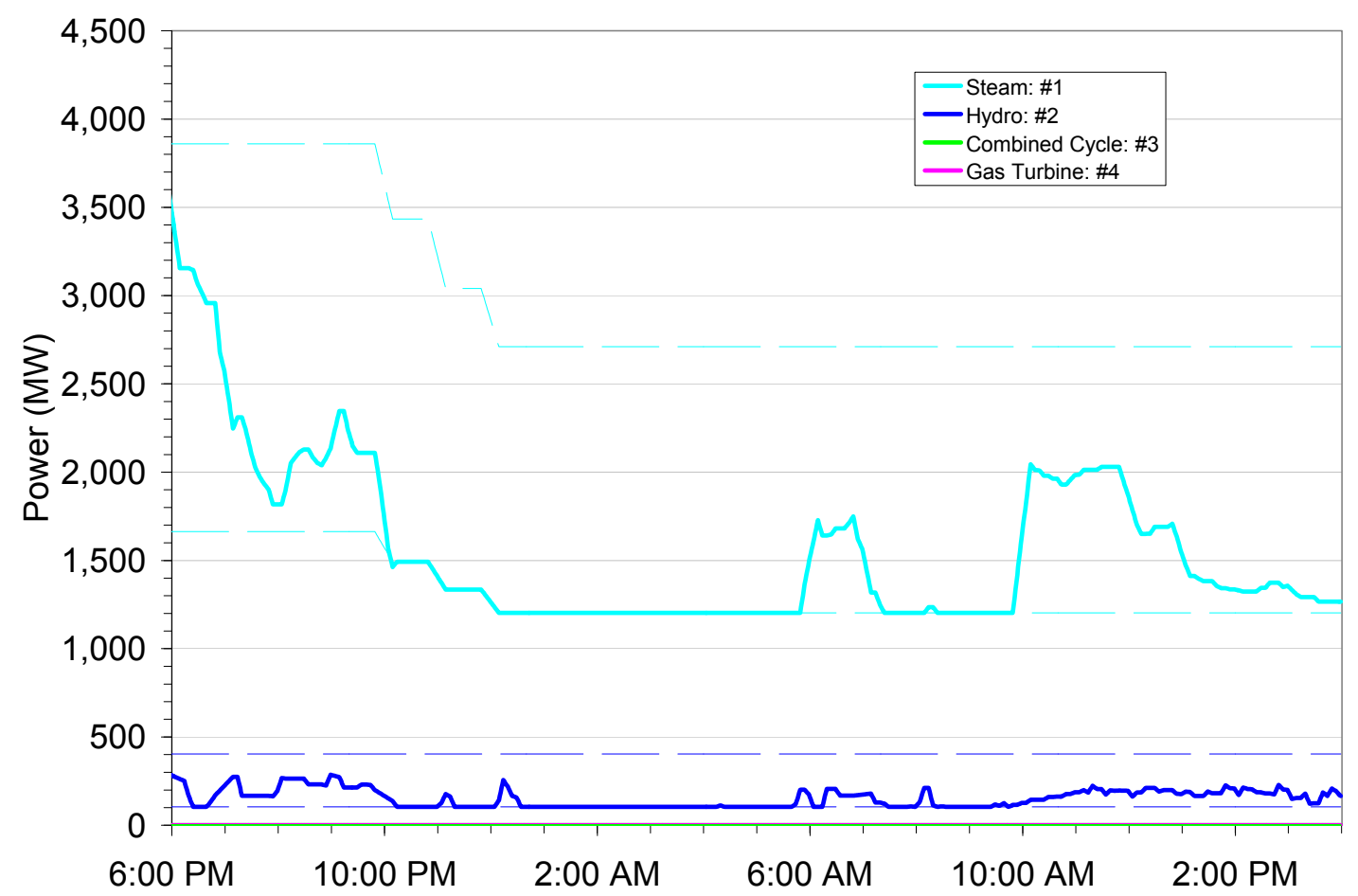

Figure F- 30 Wyoming Dispatch (100 MW), 30\% Wind, Local Priority, April QSS Study Period

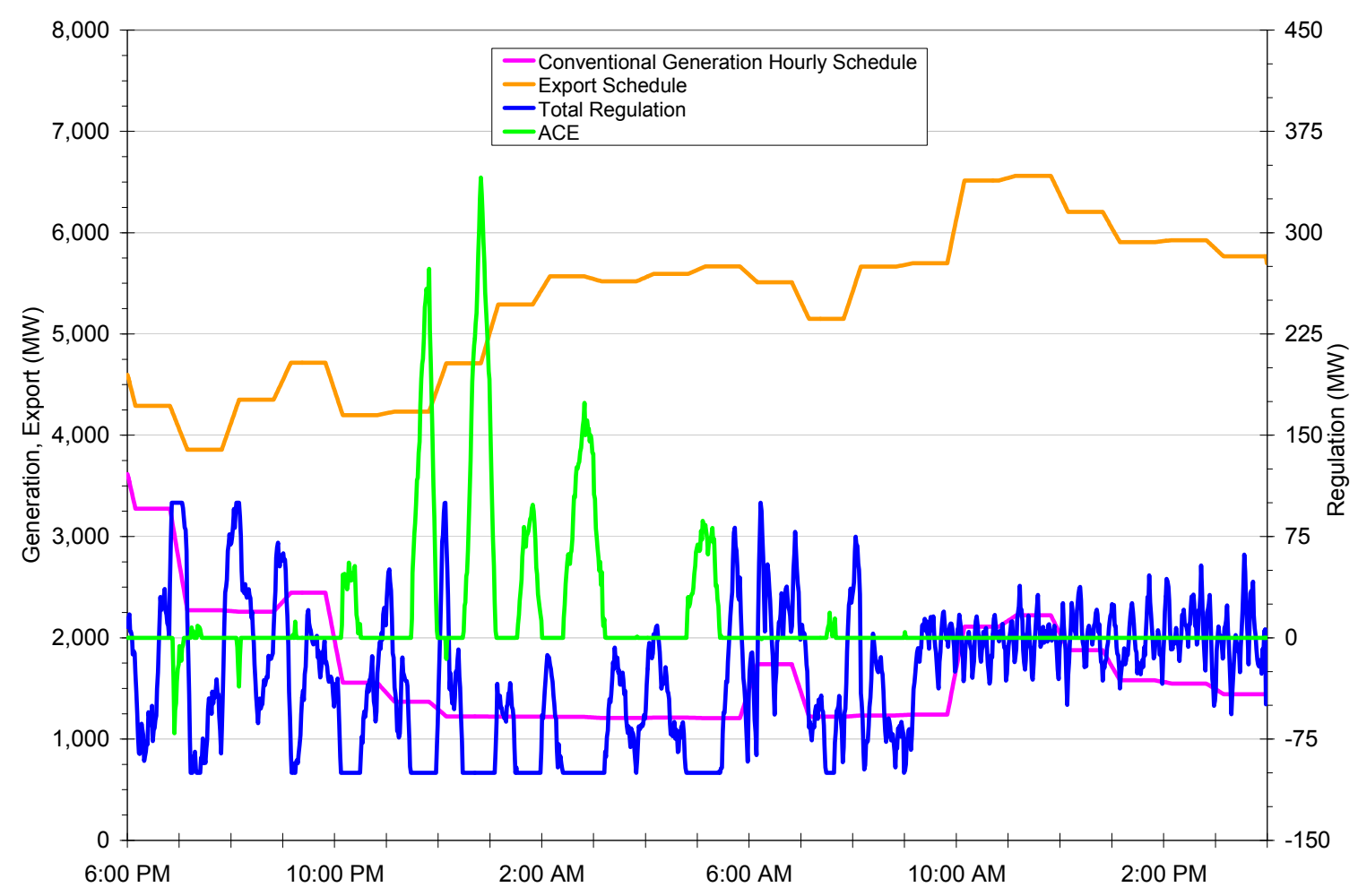

Figure F- 31 Wyoming Regulation (100 MW), 30\% Wind, Local Priority, April QSS Study Period 


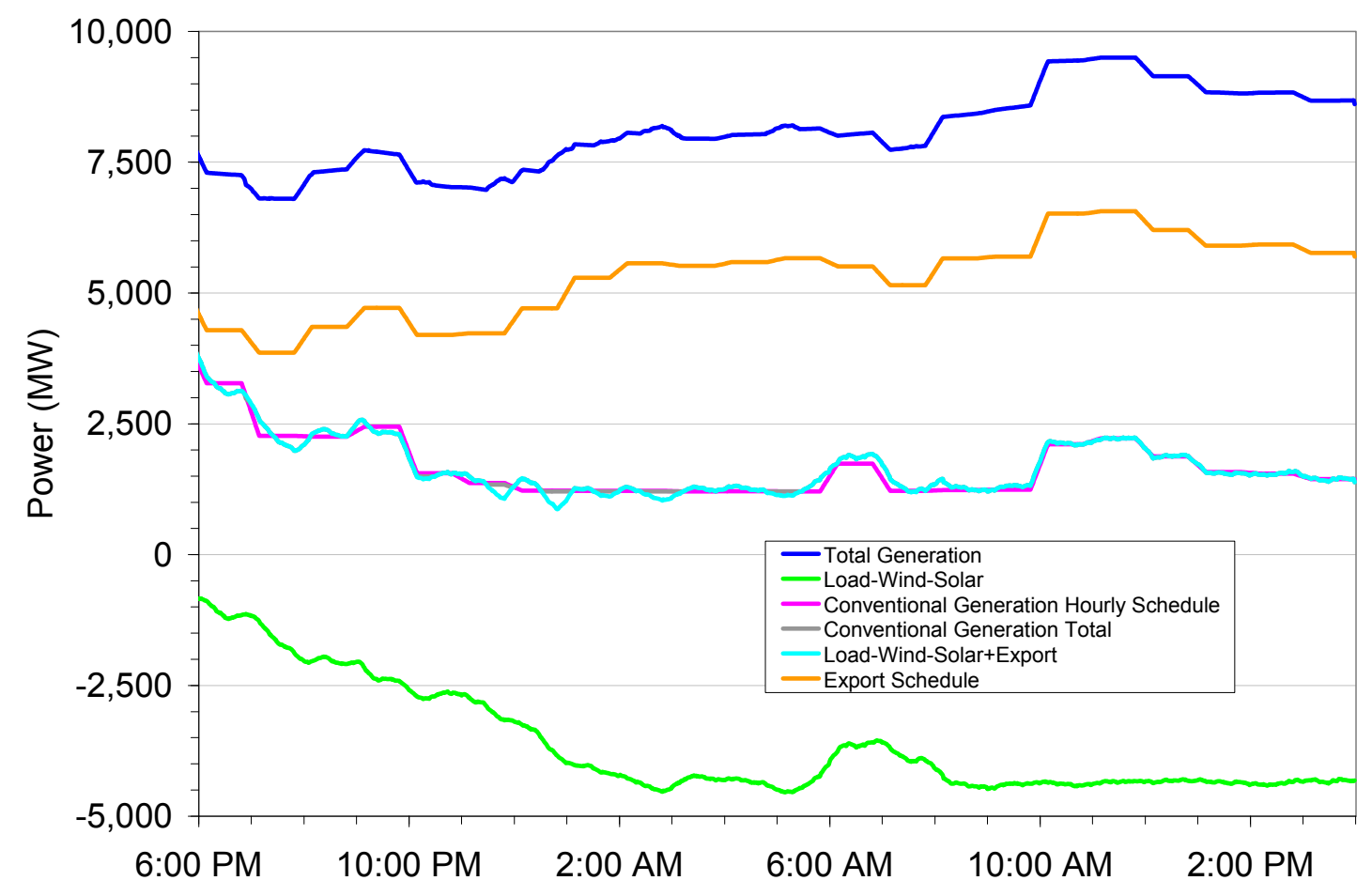

Figure F- 32 Wyoming Totals (100 MW), 30\% Wind, Local Priority, April QSS Study Period

\section{F.13 Arizona October 26, 30\% Wind Penetration}

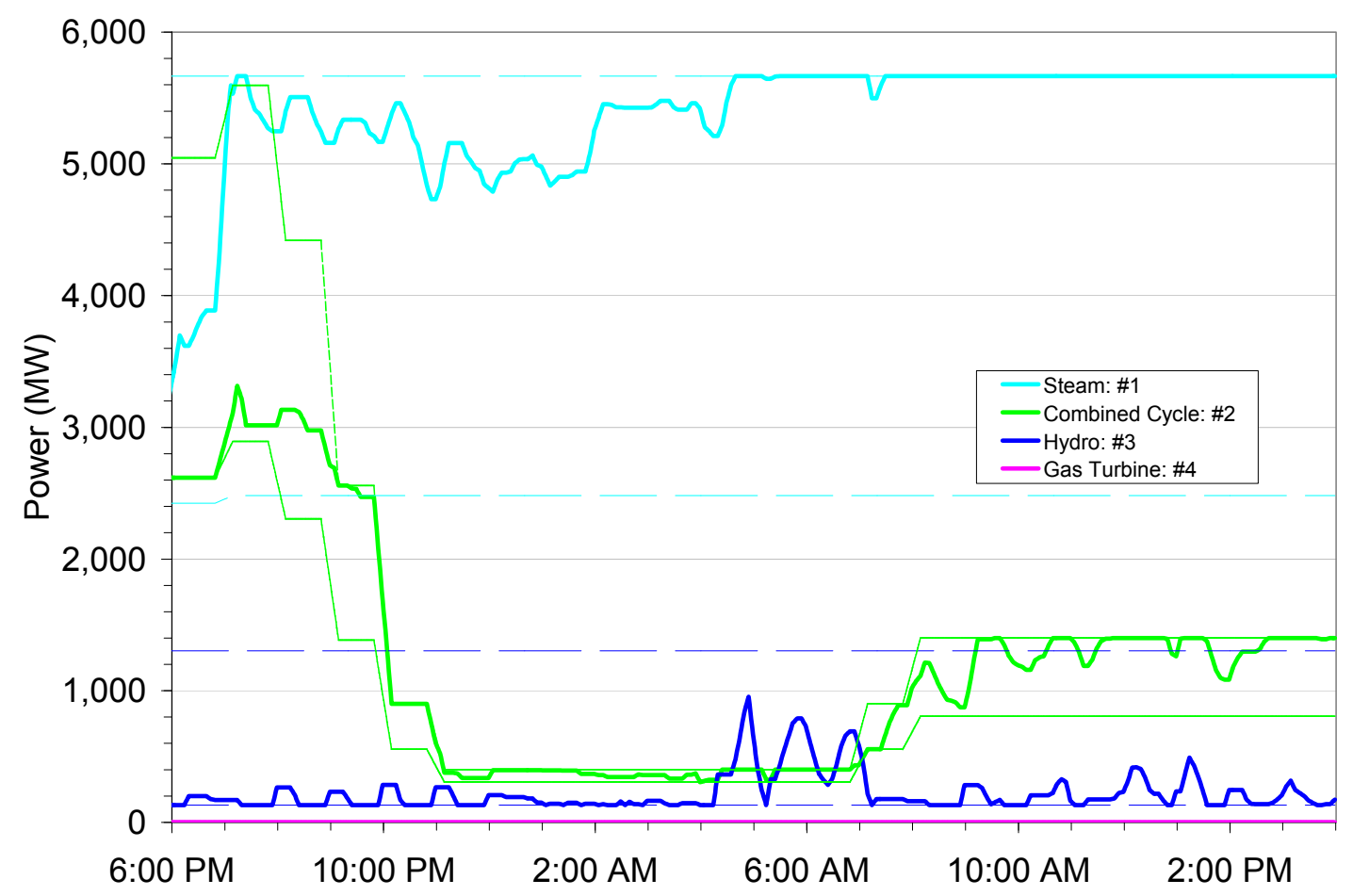

Figure F- 33 Arizona Dispatch, 30\% Wind, Local Priority, October QSS Study Period 


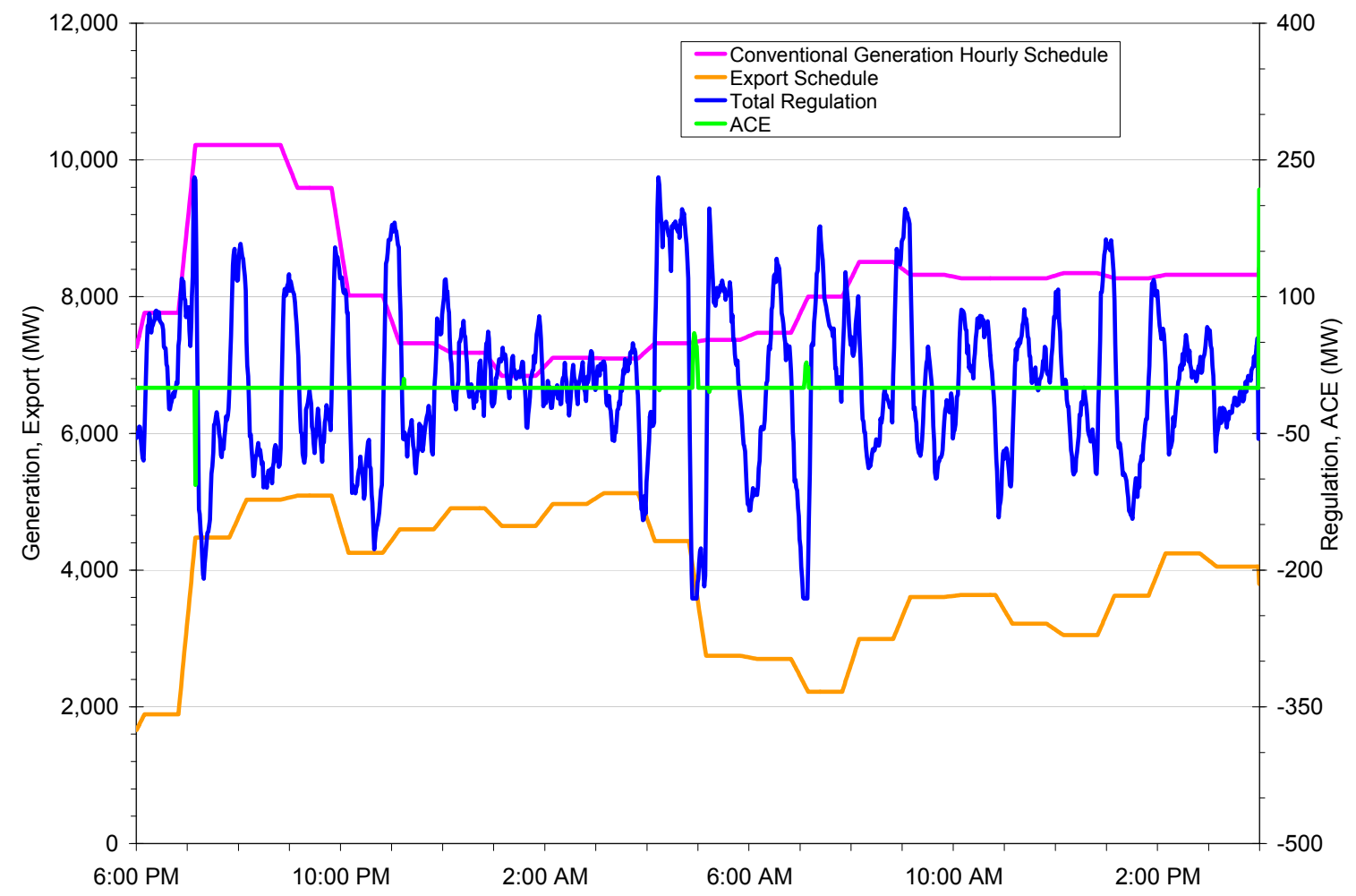

Figure F-34 Arizona Regulation, 30\% Wind, Local Priority, October QSS Study Period

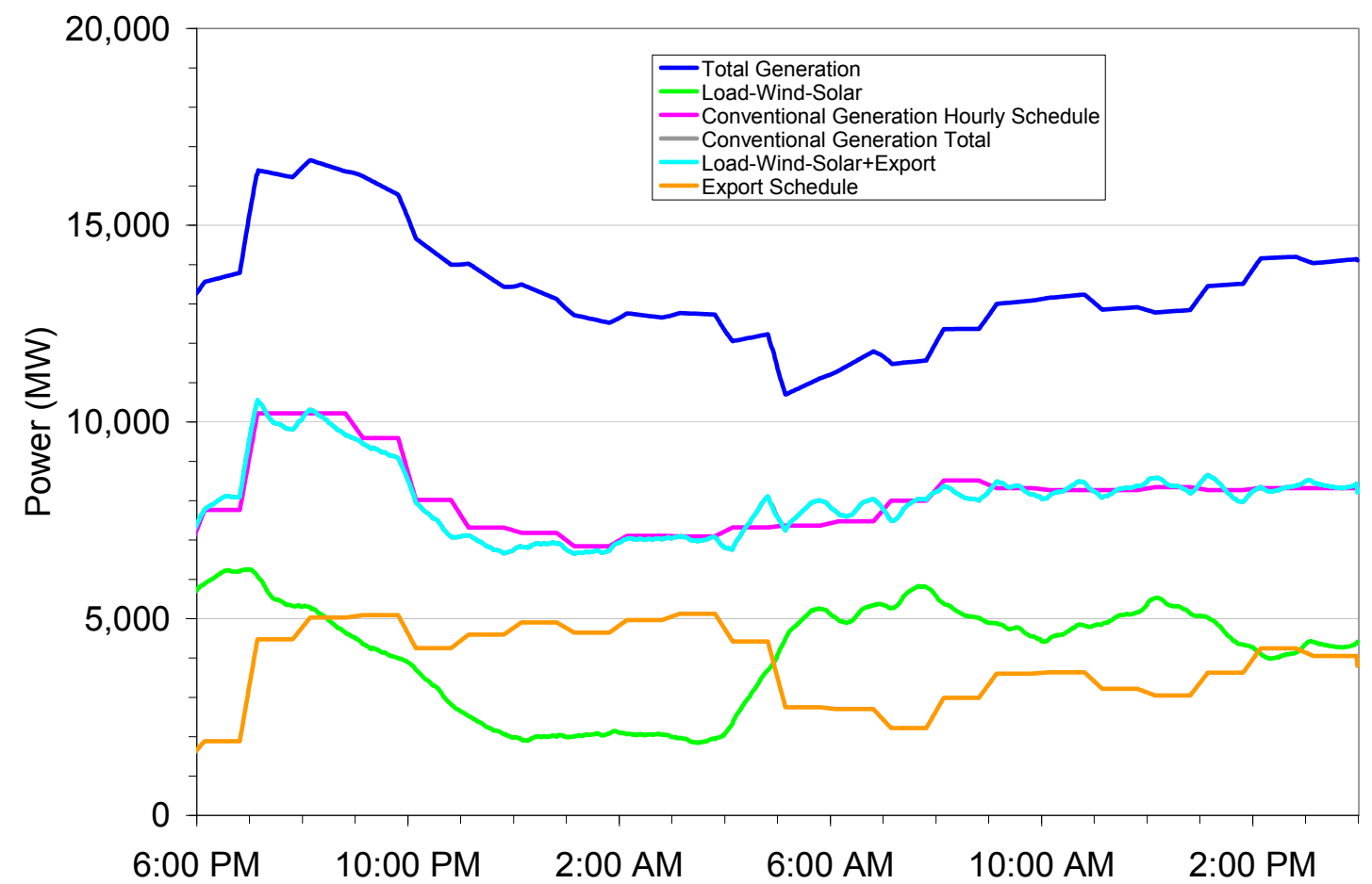

Figure F- 35 Arizona Totals, 30\% Wind, Local Priority, October QSS Study Period 


\section{F.14 Colorado West October 26, 30\% Wind Penetration}

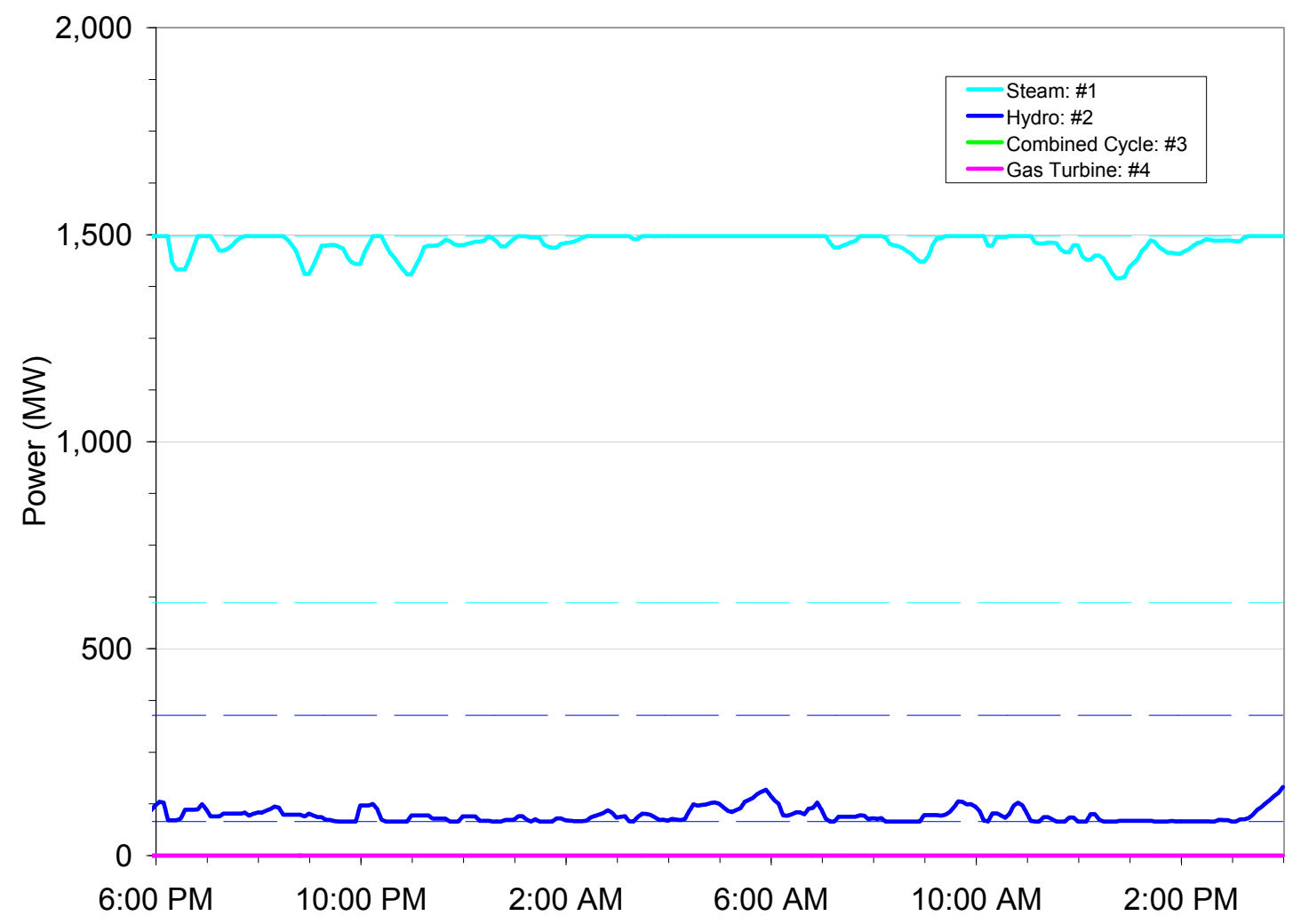

Figure F- 36 Colorado West Dispatch, 30\% Wind, Local Priority, October QSS Study Period

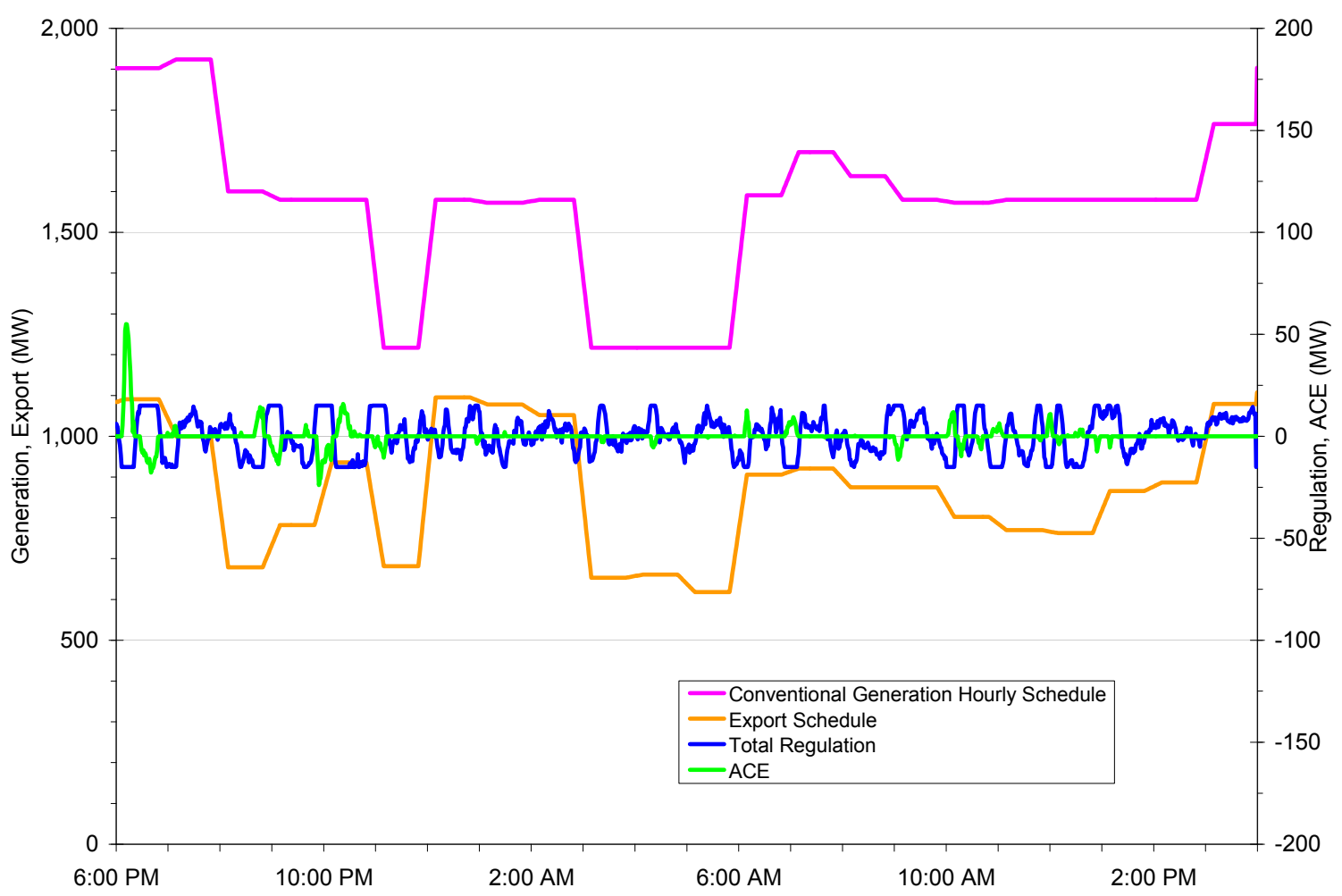

Figure F- 37 Colorado West Regulation, 30\% Wind, Local Priority, October QSS Study Period 


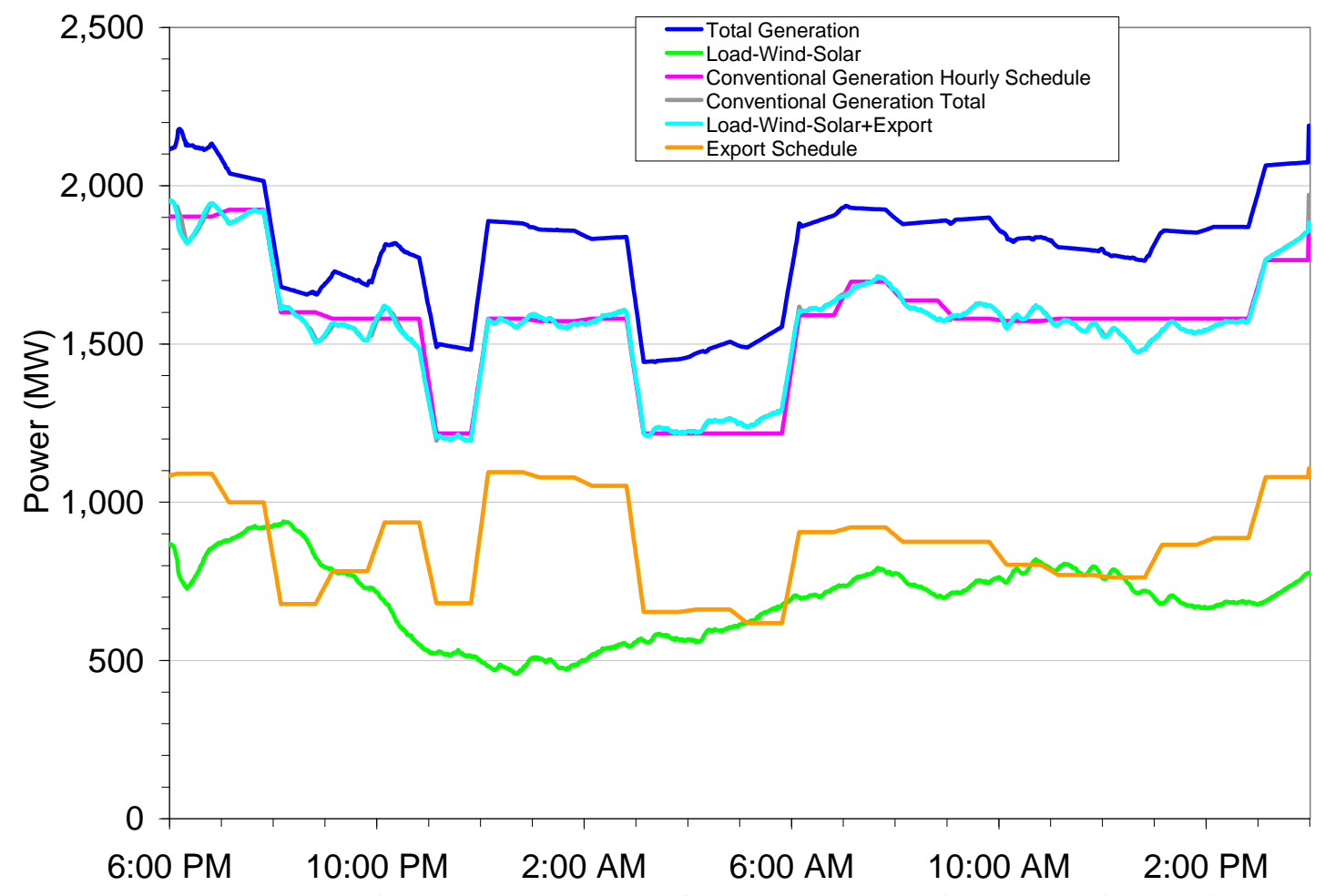

Figure F.38. Colorado West Totals, 30\% Wind, Local Priority, October QSS Study Period

\section{F.15 New Mexico October 26, 30\% Wind Penetration}

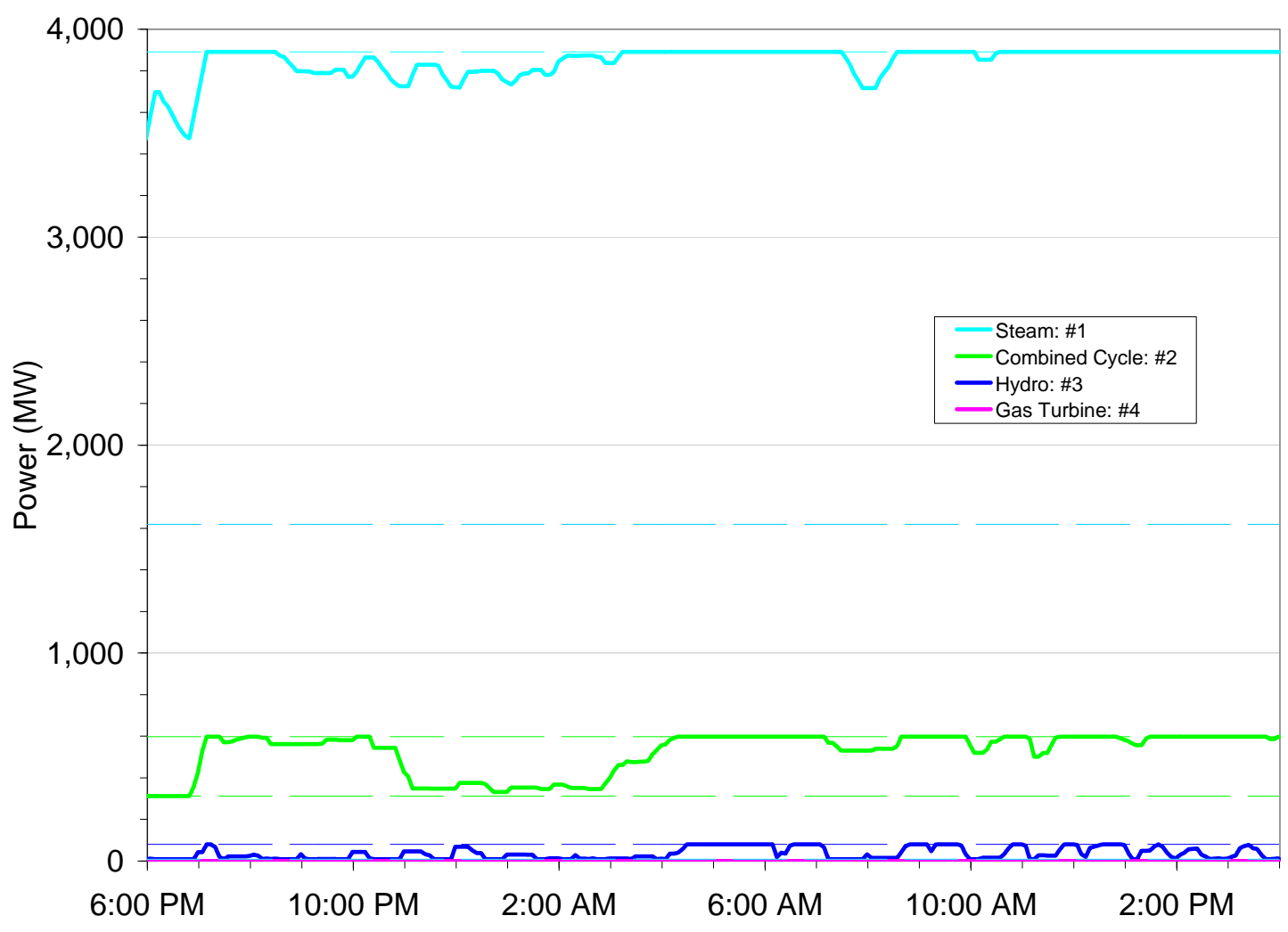

Figure F- 39 New Mexico Dispatch, 30\% Wind, Local Priority, October QSS Study Period 


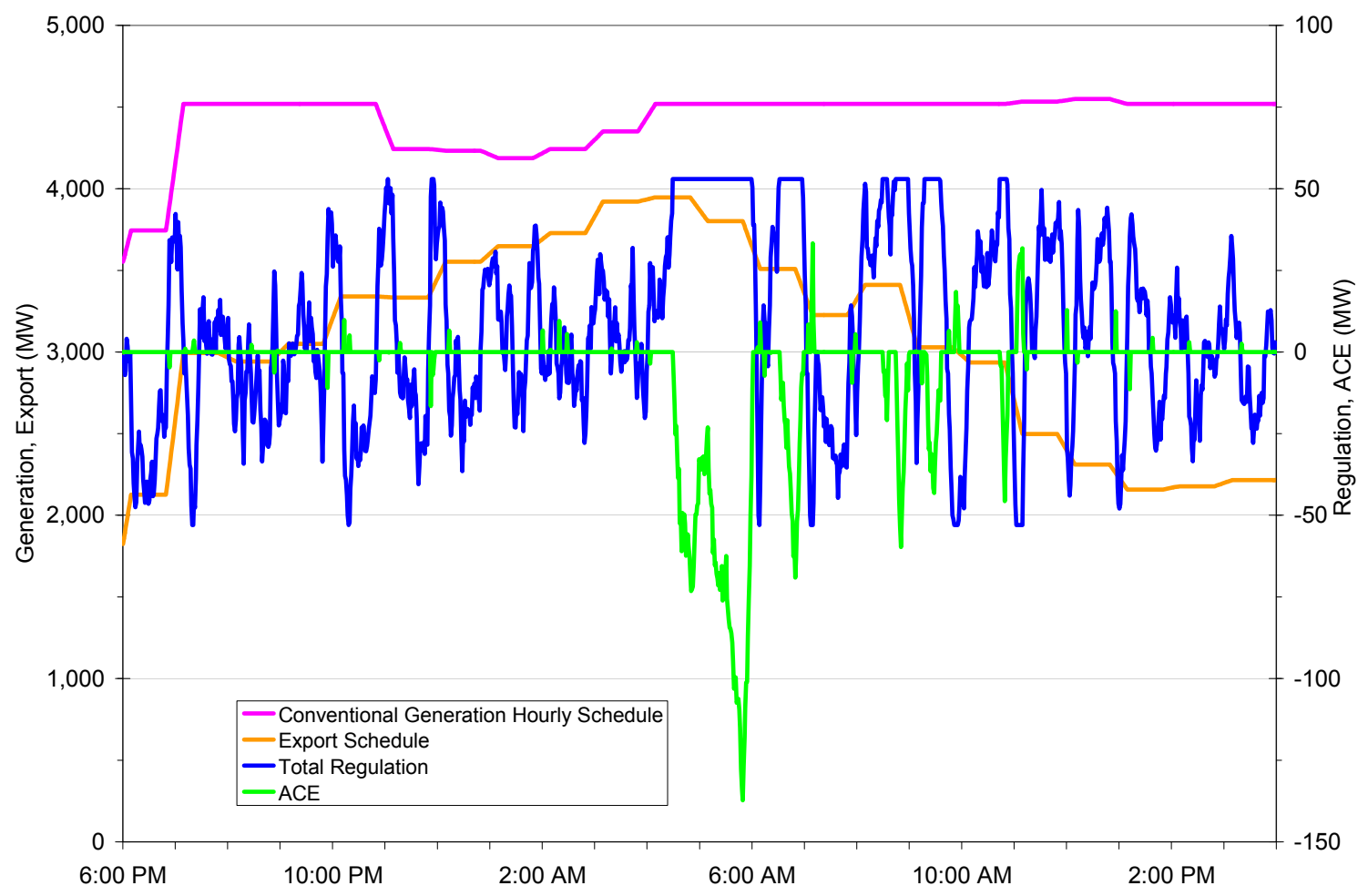

Figure F- 40 New Mexico Regulation, 30\% Wind, Local Priority, October QSS Study Period

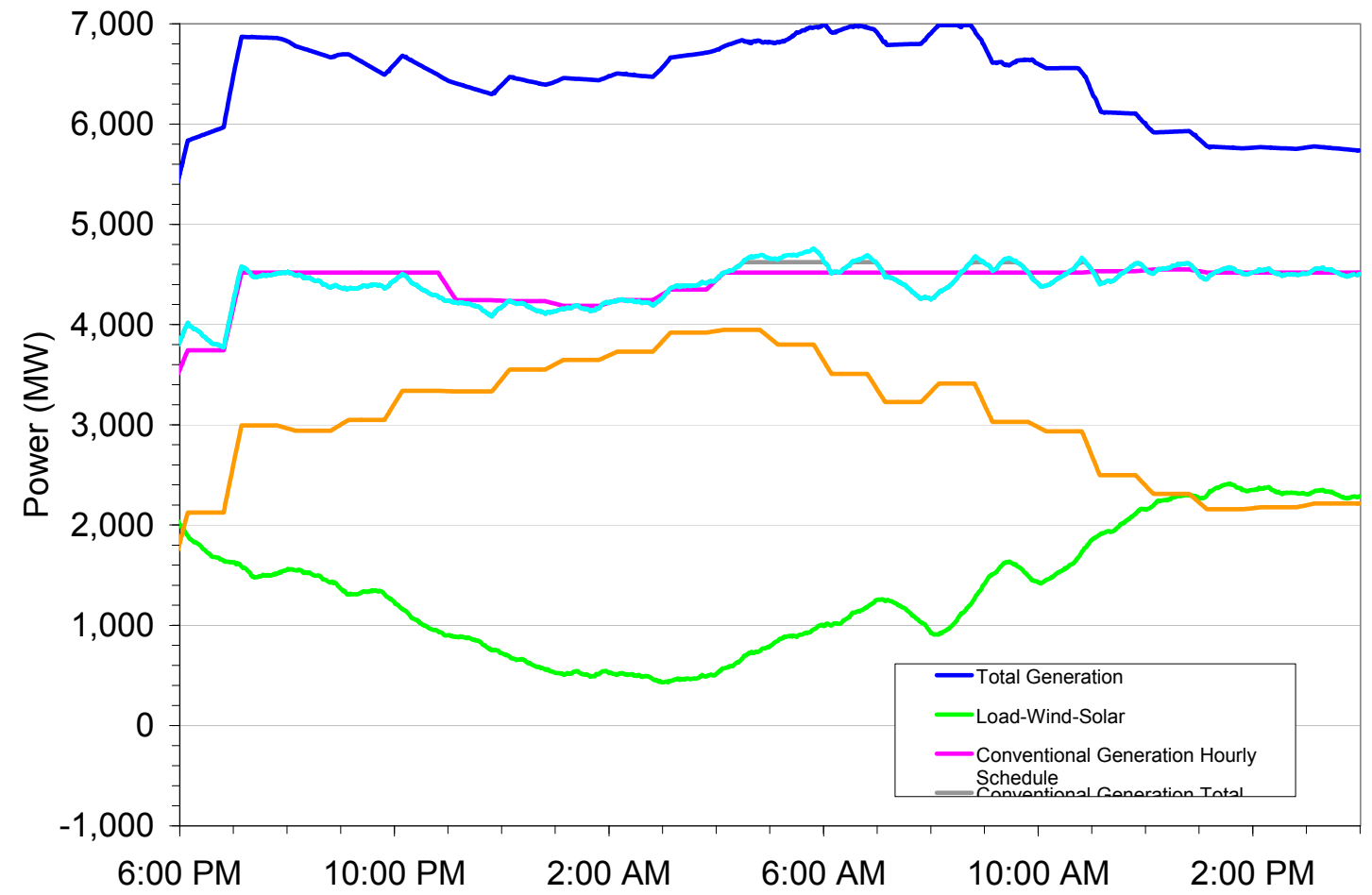

Figure F- 41 New Mexico Totals, 30\% Wind, Local Priority, October QSS Study Period 
F.16 North Nevada October 26, 30\% Wind Penetration

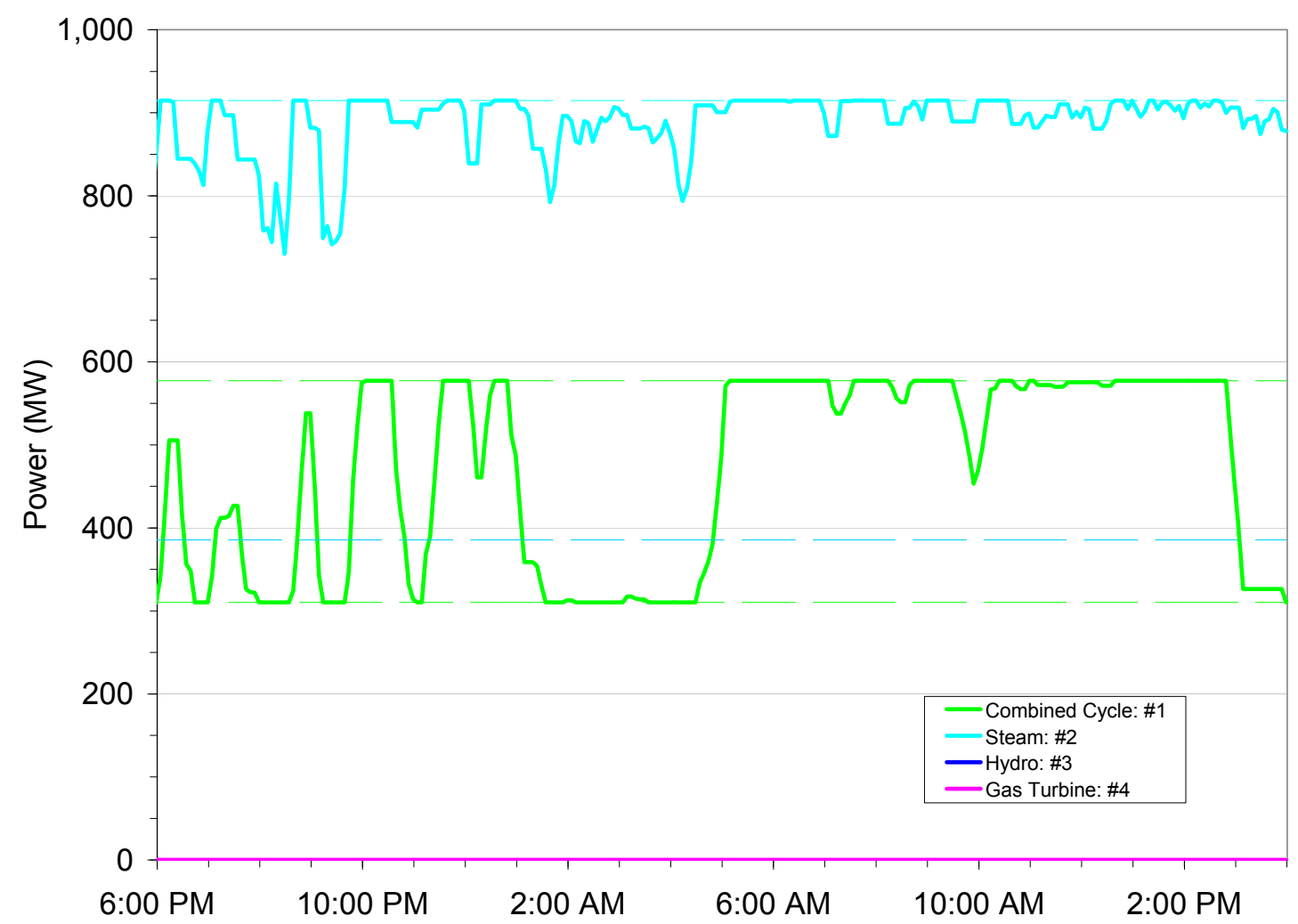

Figure F- 42 North Nevada Dispatch, 30\% Wind, Local Priority, October QSS Study Period

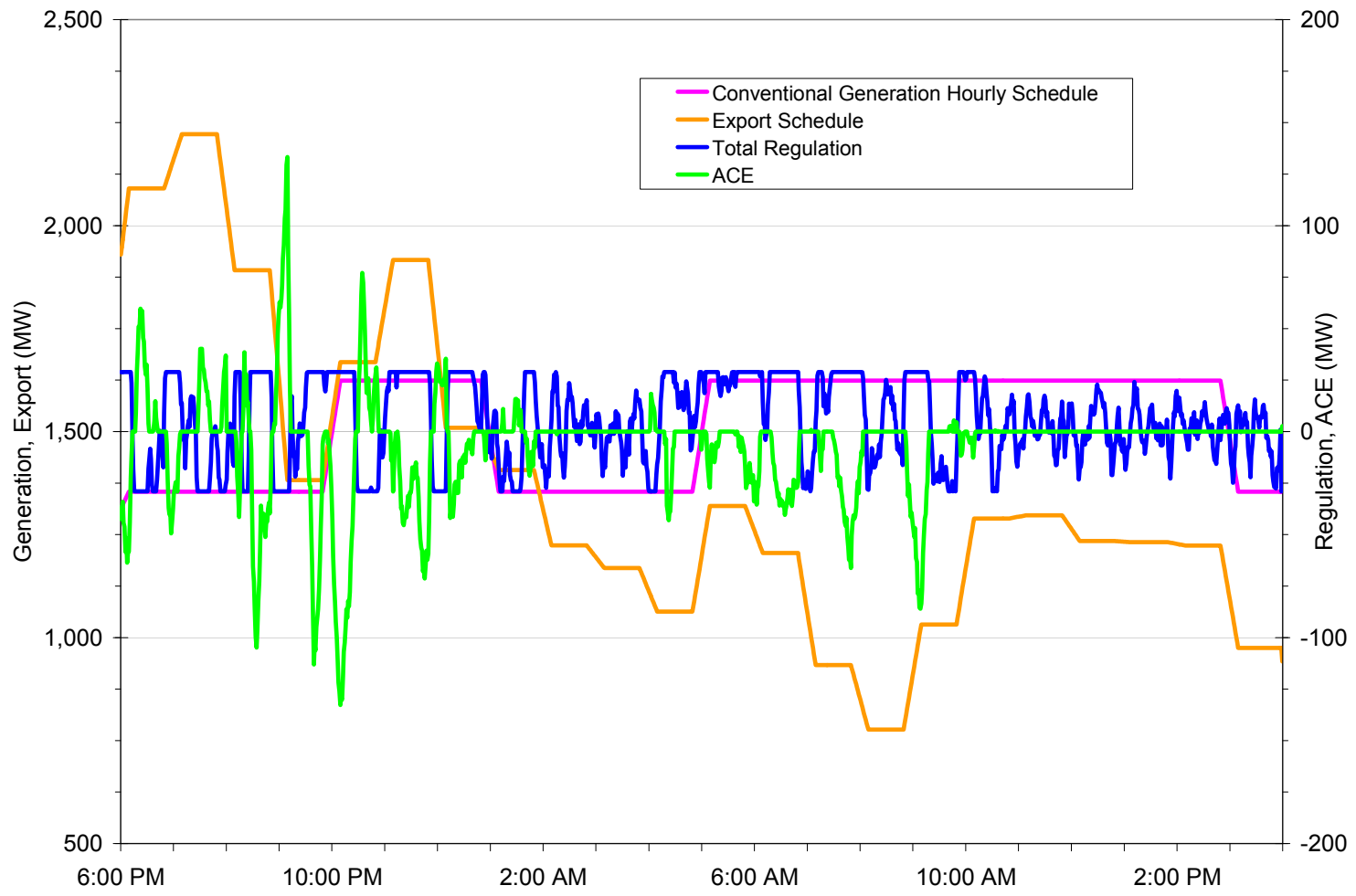

Figure F- 43 North Nevada Regulation, 30\% Wind, Local Priority, October QSS Study Period 


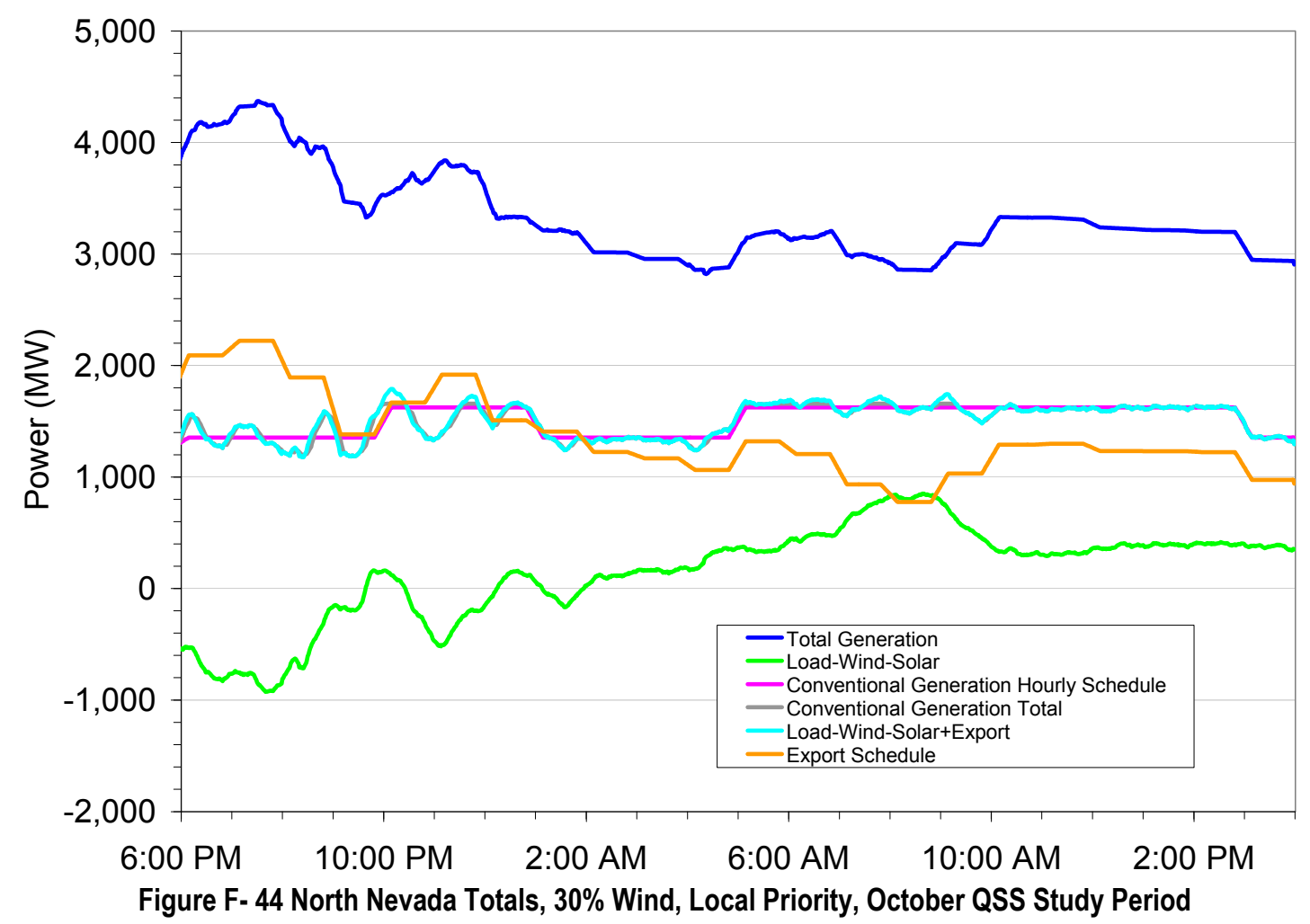

\section{F.17 South Nevada October 26, 30\% Wind Penetration}

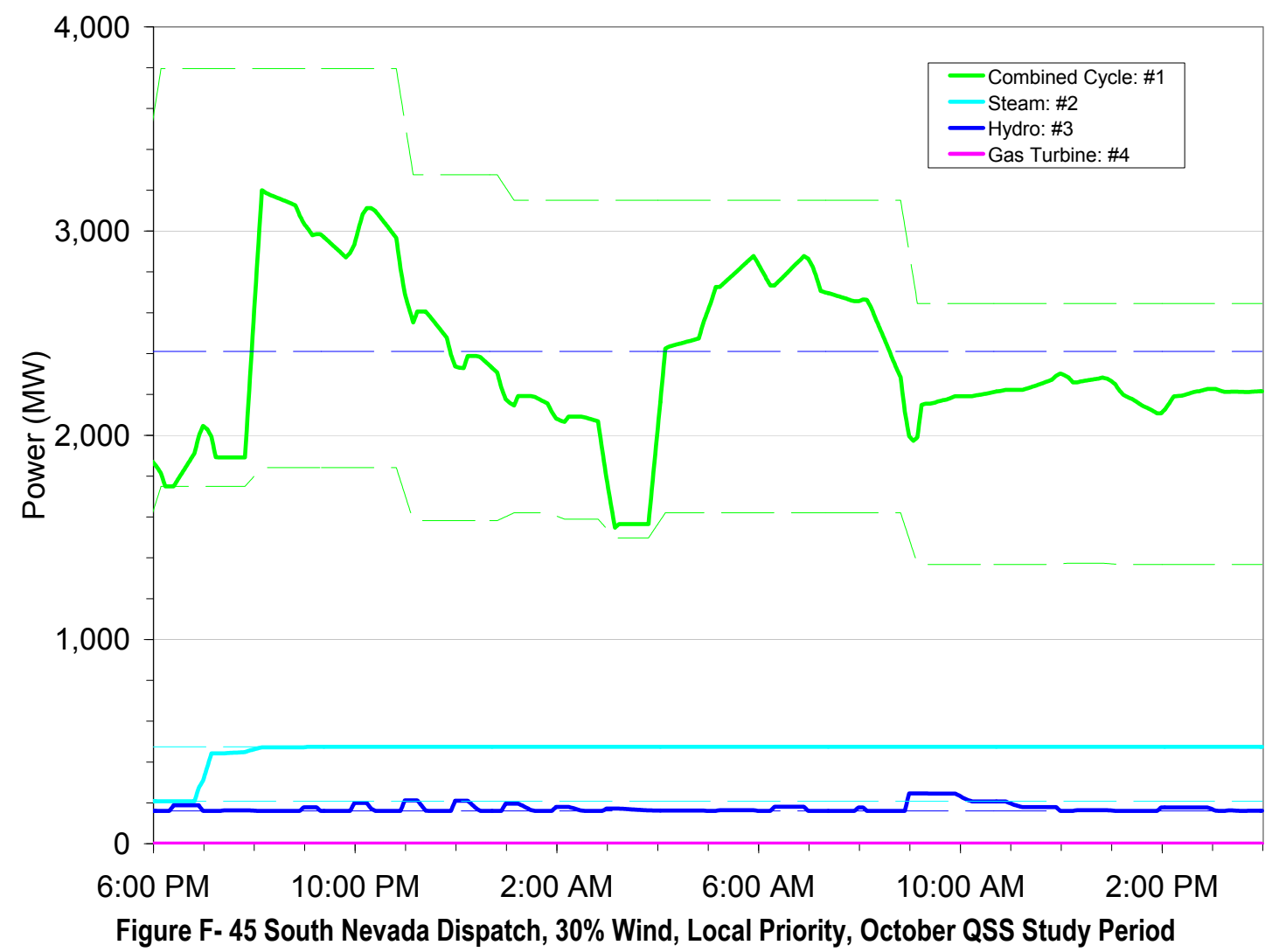




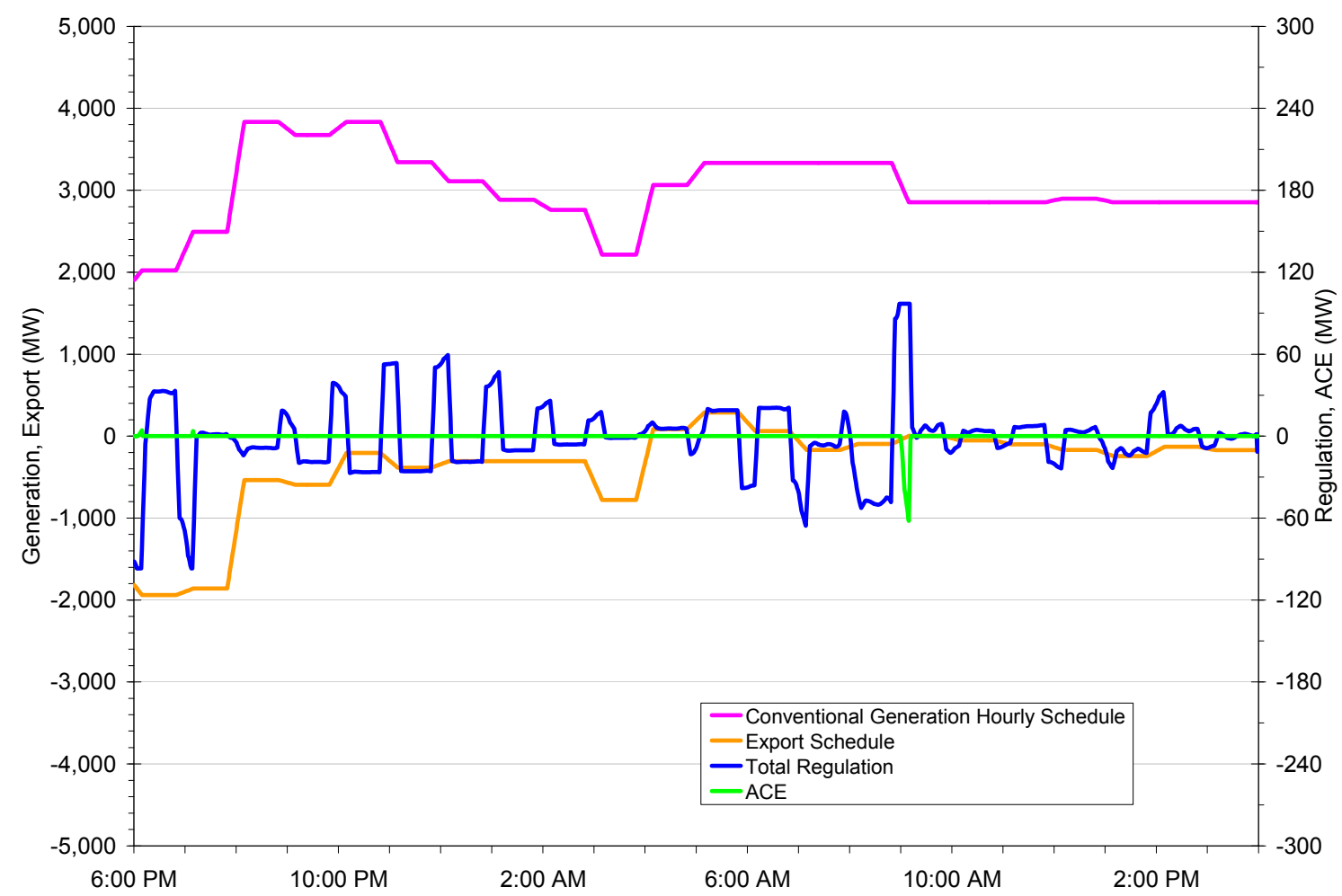

Figure F- 46 South Nevada Regulation, 30\% Wind, Local Priority, October QSS Study Period

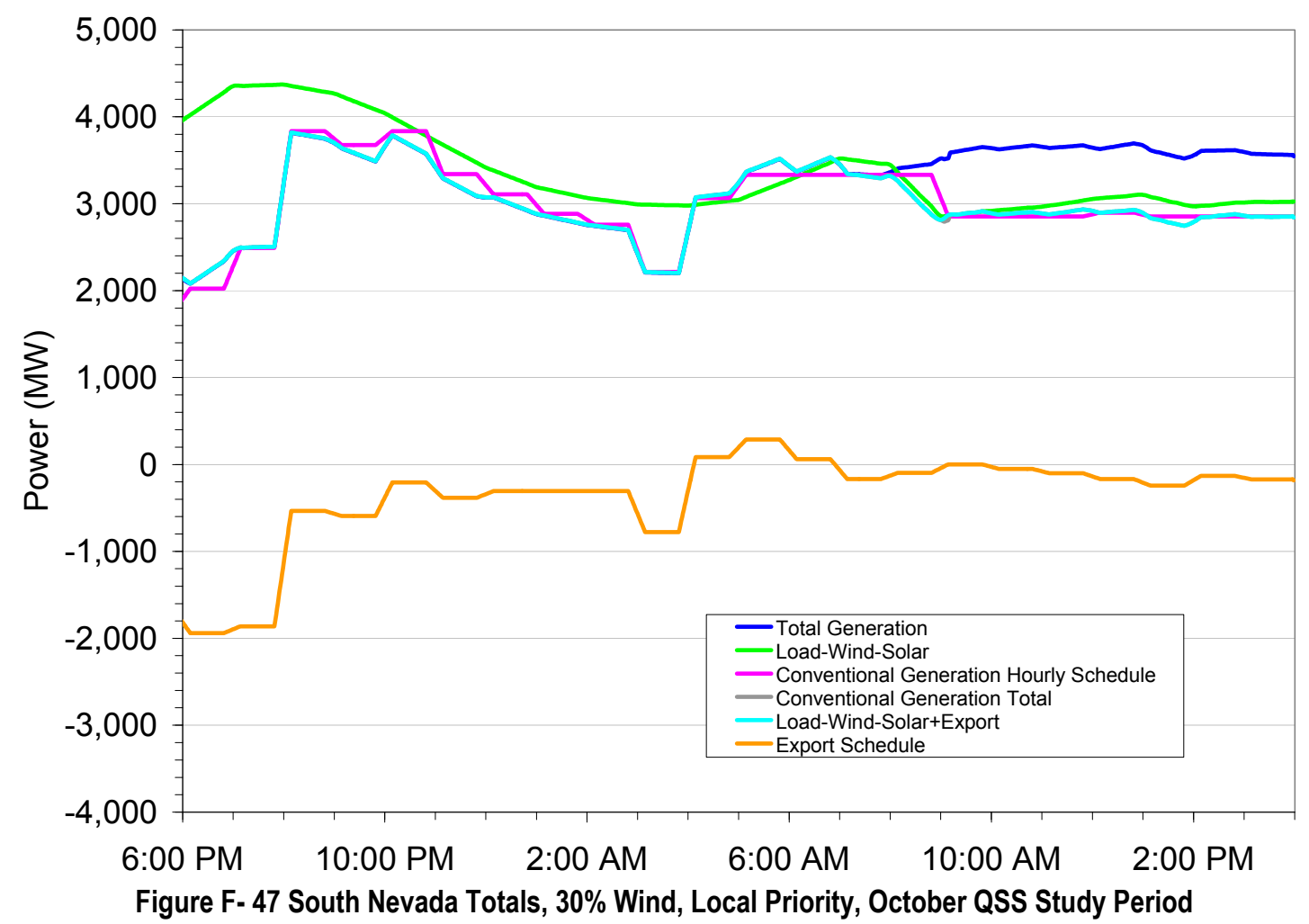


F.18 Wyoming October 26, 30\% Wind Penetration
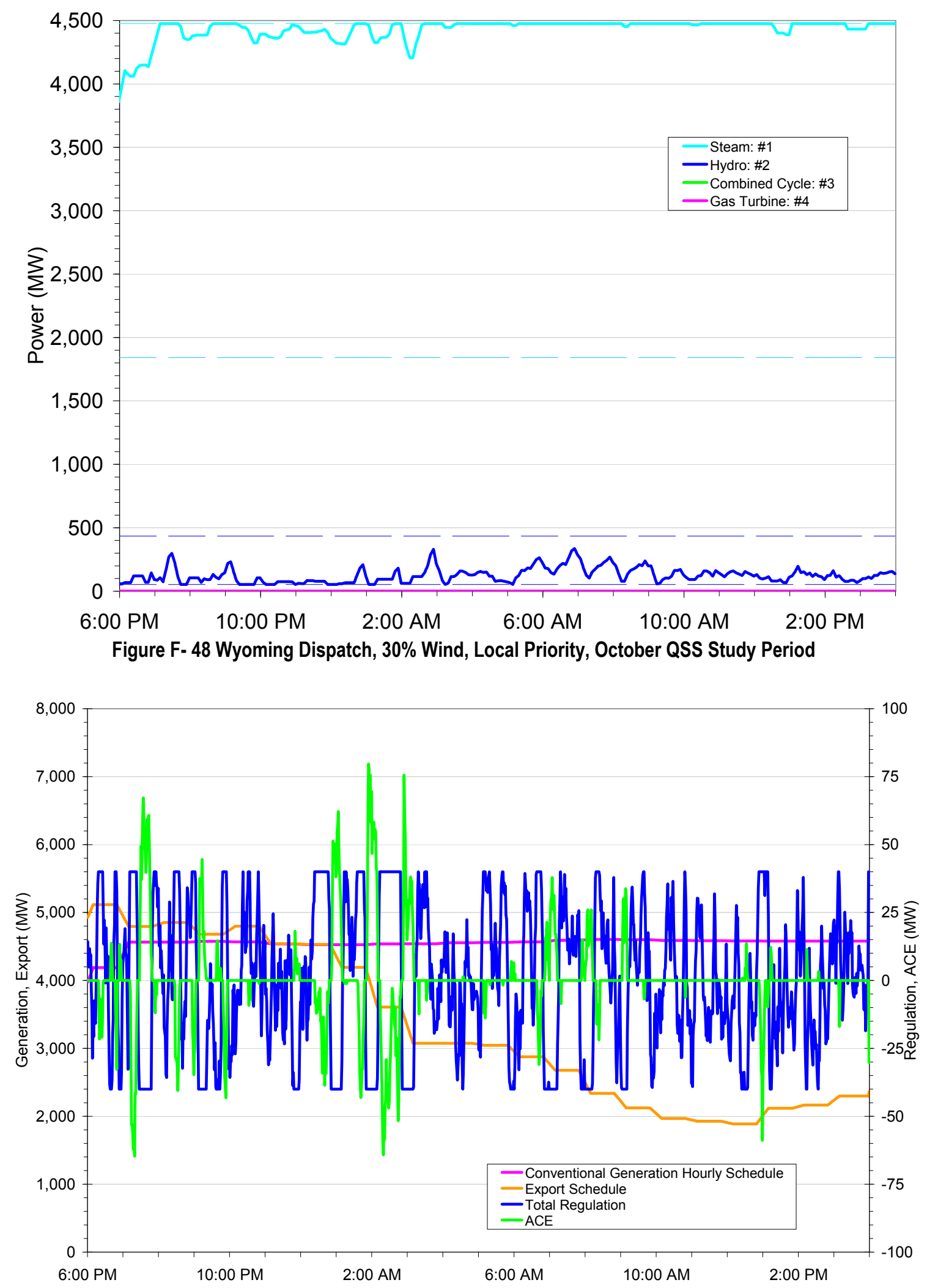

Figure F- 49 Wyoming Regulation, 30\% Wind, Local Priority, October QSS Study Period 


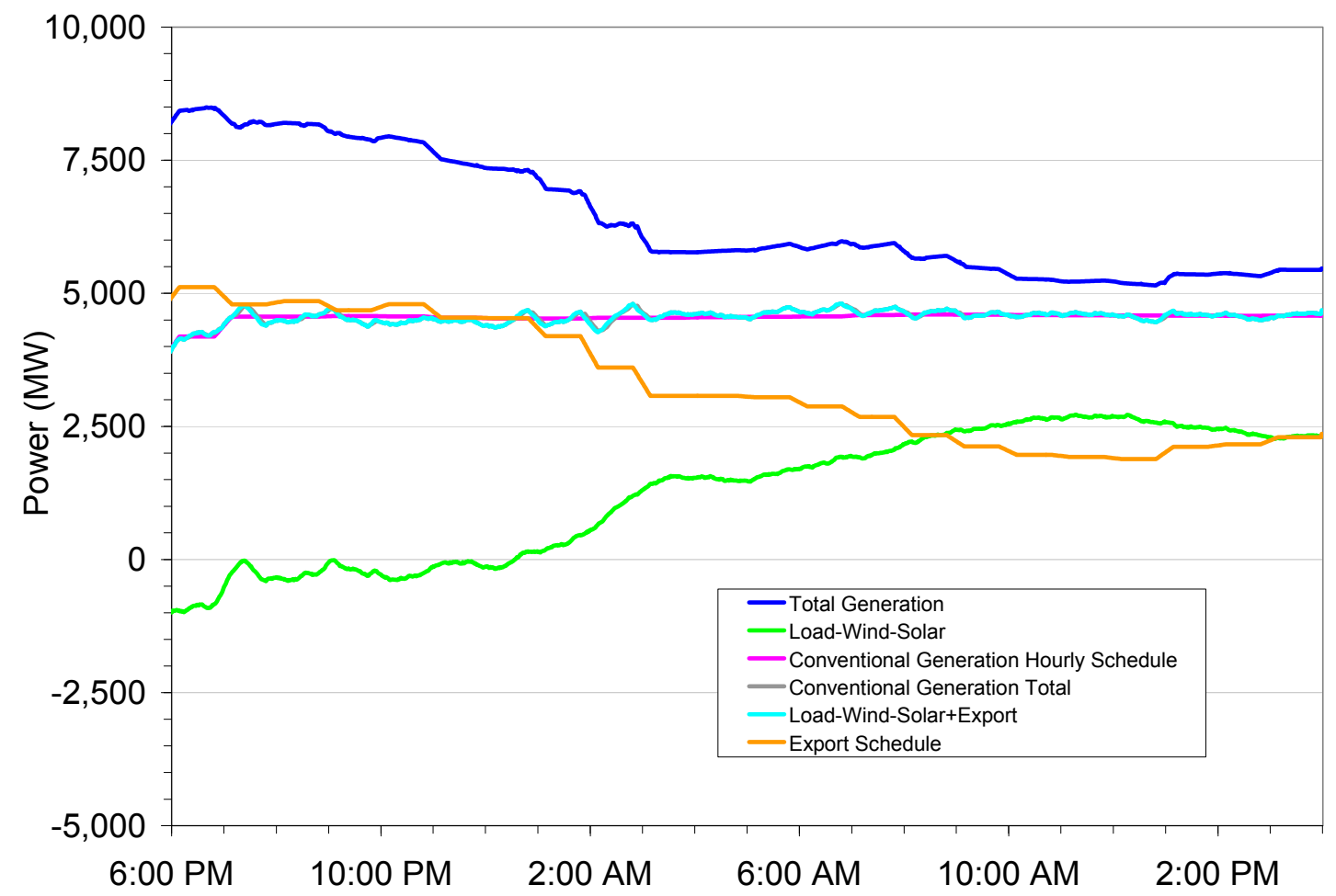

Figure F- 50 Wyoming Totals, 30\% Wind, Local Priority, October QSS Study Period 



\section{Appendix G Reserves}

This appendix provides additional results and observations from the investigation of possible simplified variability reserve rules presented in Section 7.4. For clarity and completeness, some figures from the body of section 7.4 are included again in the appropriate section.

\section{G.1 3+5 Rules}

This appendix provides additional supporting graphics and discussion for the material presented in Section 7.4.2. 


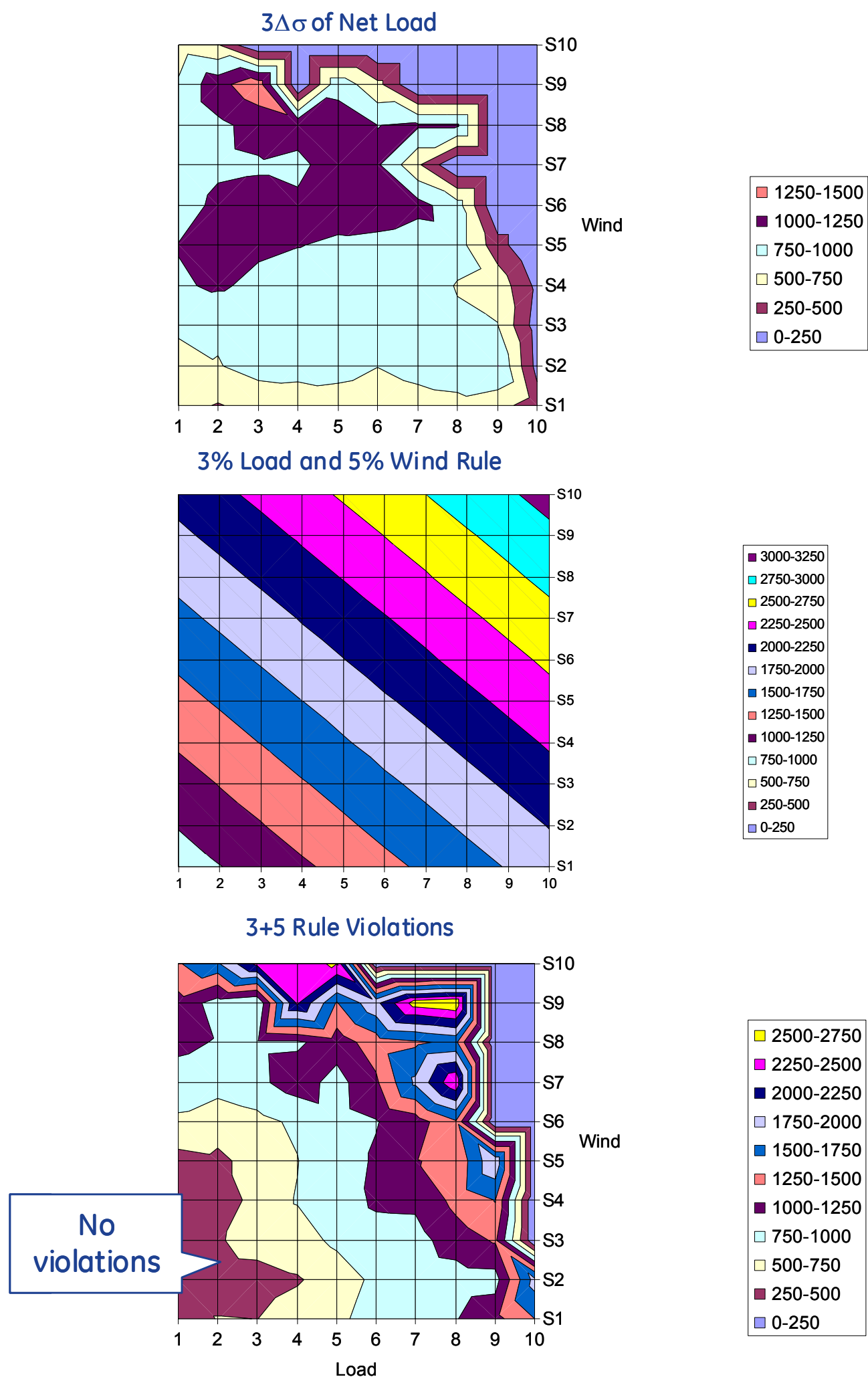

Figure G- 1 in Footprint L30R 3\% Load Plus 5\% Wind Rule 
Arizona - The 3+5 rule performance for Arizona is shown in Figure G- 1 . There are generally positive margins, although are some rare periods of high load and moderate wind (load 9 and wind 4) where there are pockets of violations.

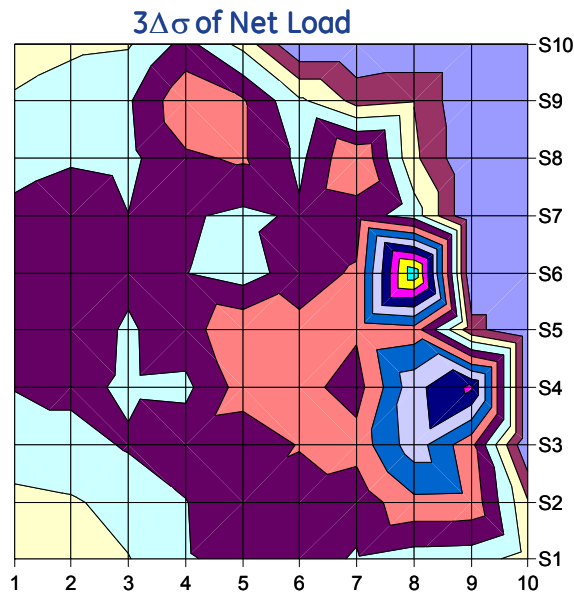

$$
\begin{array}{|l|}
\square \text { 1100.0-1200.0 } \\
\square \text { 1000.0-1100.0 } \\
\square 900.0-1000.0 \\
\square \text { 800.0-900.0 } \\
\square 700.0-800.0 \\
\square 600.0-700.0 \\
\square 500.0-600.0 \\
\square 400.0-500.0 \\
\square 300.0-400.0 \\
\square 200.0-300.0 \\
\square 100.0-200.0 \\
\square 0.0-100.0 \\
\hline
\end{array}
$$

$3 \%$ Load and $5 \%$ Wind Rule

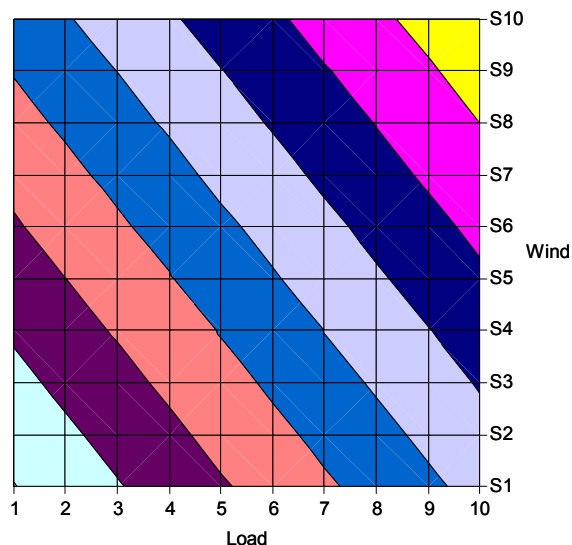

\begin{tabular}{|l|}
$\square$ \\
$\square 9000-1100$ \\
$\square 800-900$ \\
$\square 700-800$ \\
$\square 600-700$ \\
$\square 500-600$ \\
$\square 400-500$ \\
$\square 300-400$ \\
$\square 200-300$ \\
$\square 100-200$ \\
$\square 0-100$
\end{tabular}

$3+5$ Rule Violations

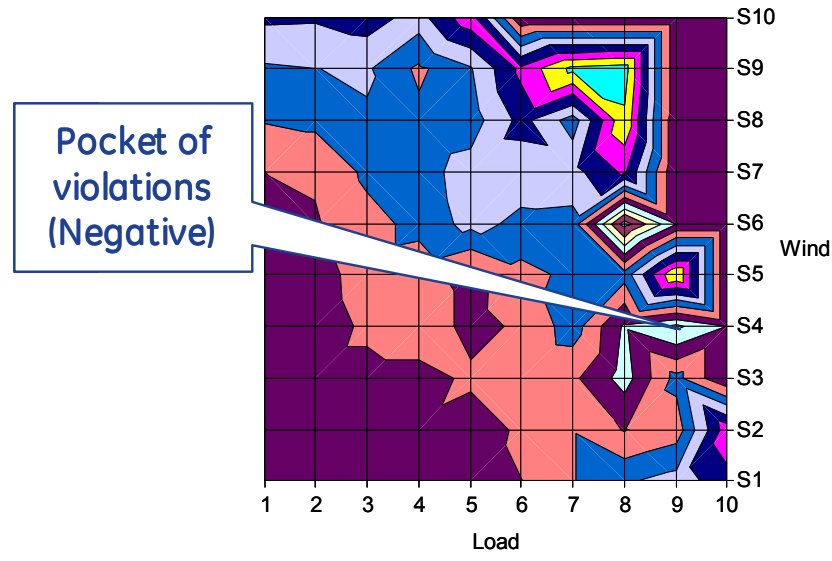

\begin{tabular}{|l|}
$\square 700-800$ \\
$\square 600-700$ \\
$\square 500-600$ \\
$\square 400-500$ \\
$\square 300-400$ \\
$\square 200-300$ \\
$\square 100-200$ \\
$\square 0-100$ \\
$\square-100-0$ \\
$\square-200--100$ \\
$\square-300--200$ \\
$\square-400--300$ \\
\hline
\end{tabular}

Figure G- 2 Arizona L30R 3\% Load Plus 5\% Wind Rule 
Wyoming - The 3+5 rule performance for Wyoming is shown in Figure G- 3. The reserves in this case are generally short of the requirements. They are severely negative in the area of very high load that was observed earlier to have limited statistical validity.

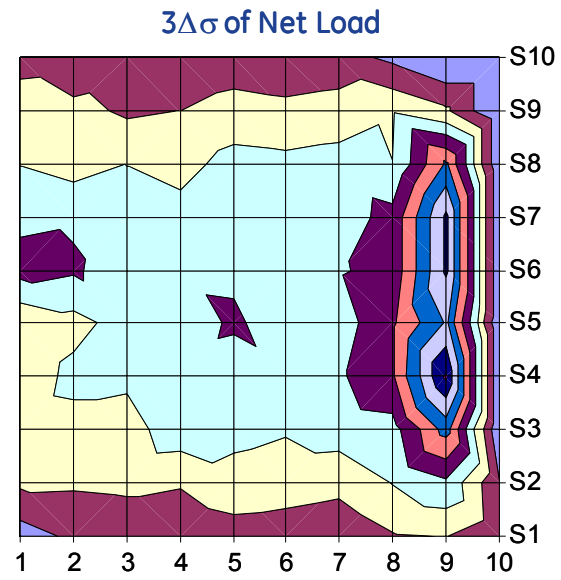

$$
\begin{aligned}
& \square 900-1000 \\
& \square 800-900 \\
& \square 700-800 \\
& \square 600-700 \\
& \square 500-600 \\
& \square 400-500 \\
& \square 300-400 \\
& \square 200-300 \\
& \square 100-200 \\
& \square 0-100
\end{aligned}
$$

$3 \%$ Load and $5 \%$ Wind Rule
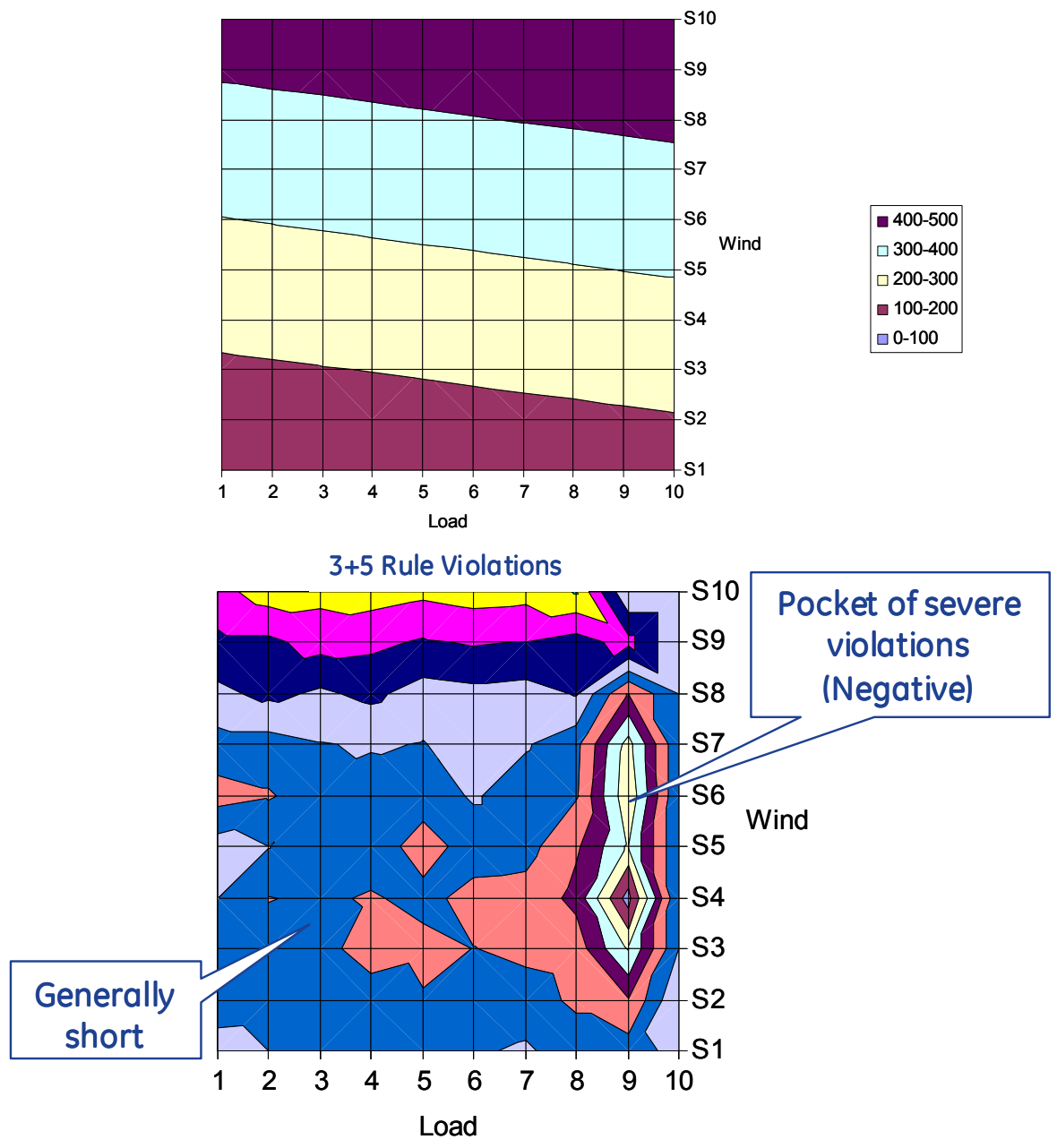

$$
\begin{array}{|l}
\square 400-500 \\
\square 300-400 \\
\square 200-300 \\
\square 100-200 \\
\square 0-100 \\
\square-100-0 \\
\square-200--100 \\
\square-300--200 \\
\square-400--300 \\
\square-500--400 \\
\square-600--500 \\
\square-700--600
\end{array}
$$

Figure G- 3 Wyoming L30R 3\% Load Plus 5\% Wind Rule 
Colorado East - The 3+5 rule performance for Colorado East is shown in Figure G- 4 . There are pockets of violation at high wind and moderately light load. Colorado East shows higher volatility at high wind than is observed in other areas. This reinforces the idea that reserve rules may benefit from customization by area.
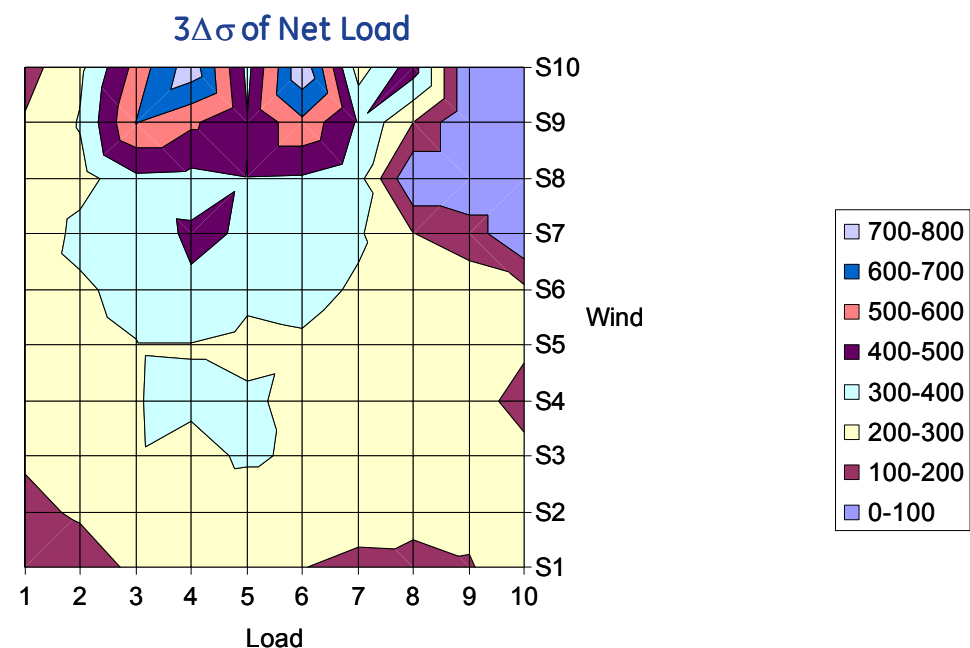

$3 \%$ Load and $5 \%$ Wind Rule

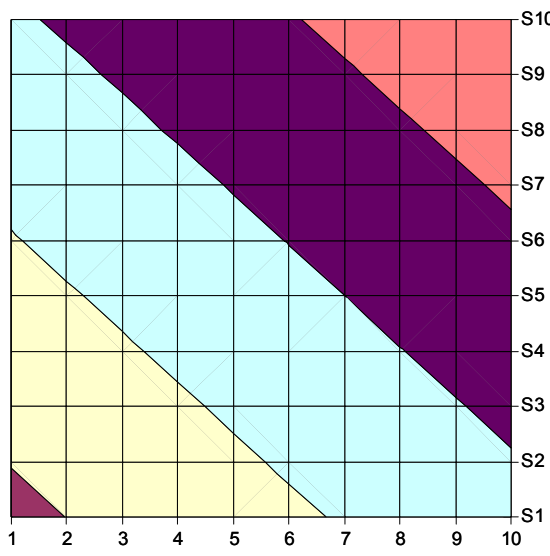

$3+5$ Rule Violations

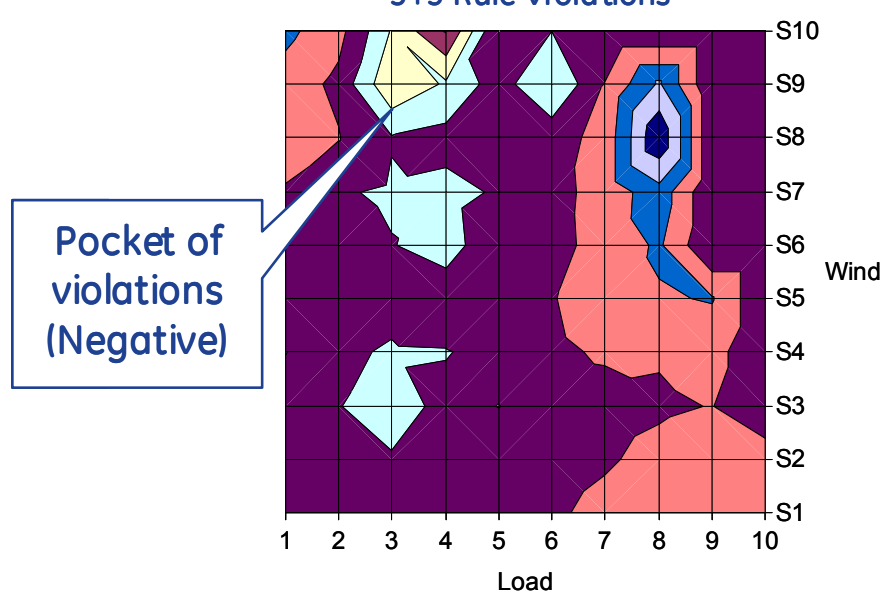

$\square 400-500$
$\square 300-400$
$\square 200-300$
$\square 100-200$
$\square 0-100$
$\square-100-0$
$\square-200--100$
$\square-300--200$
$\square-400--300$

Figure G- 4 Colorado East L30R 3\% Load Plus 5\% Wind Rule 
Nevada - The 3+5 rule performance for Nevada is shown in Figure G- 5. Some violations occur at moderate wind and light load.

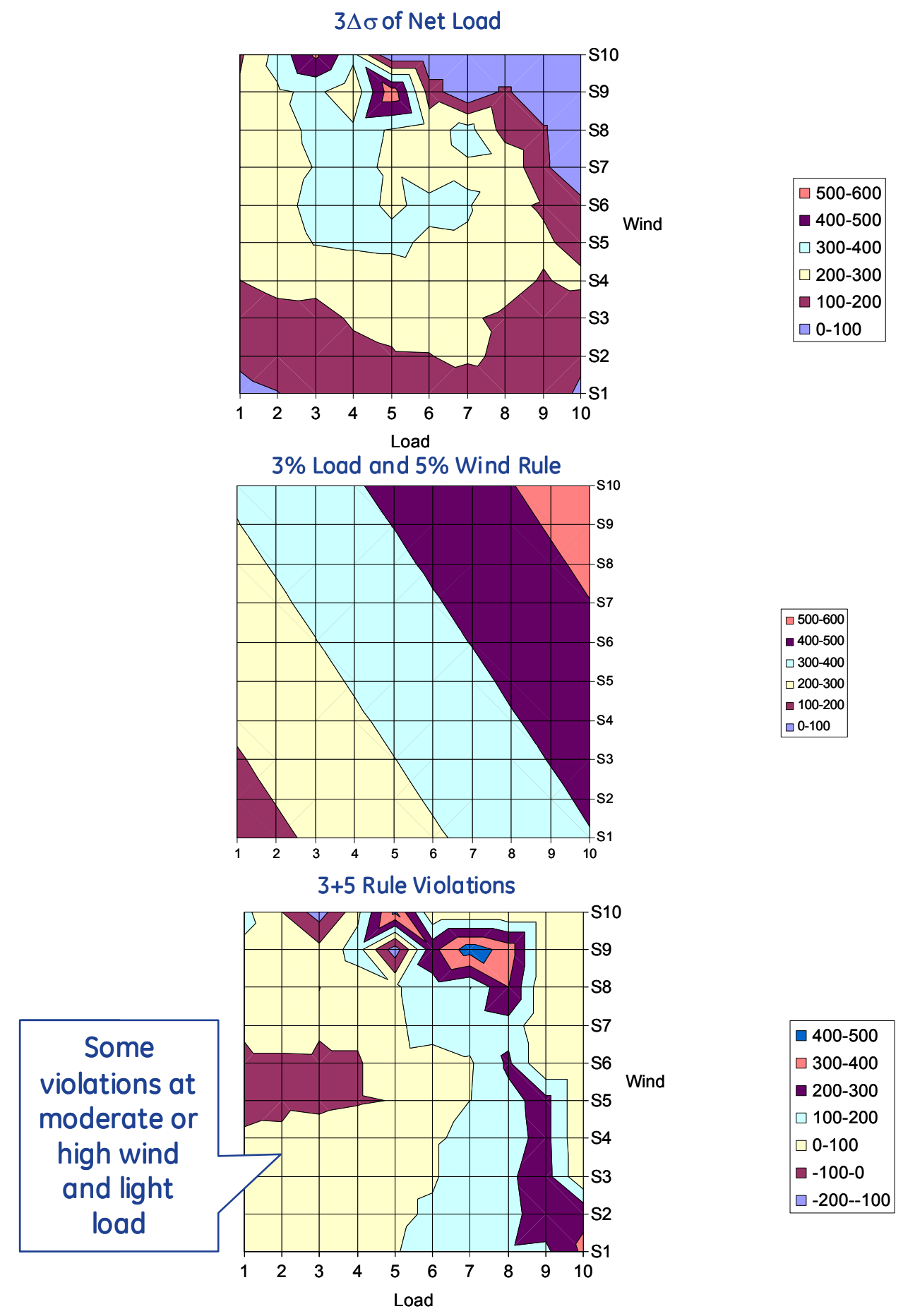

Figure G- 5 Nevada L30R 3\% Load Plus 5\% Wind Rule 
New Mexico - The 3+5 rule performance for New Mexico is shown in Figure G- 6. Small violations are shown at unusually high wind and light load conditions.
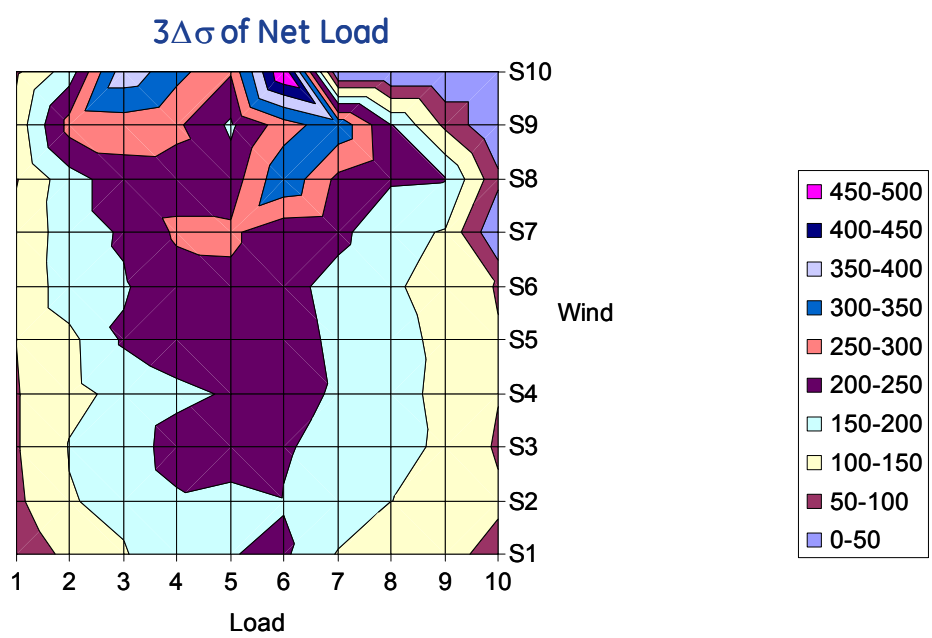

$3 \%$ Load and $5 \%$ Wind Rule

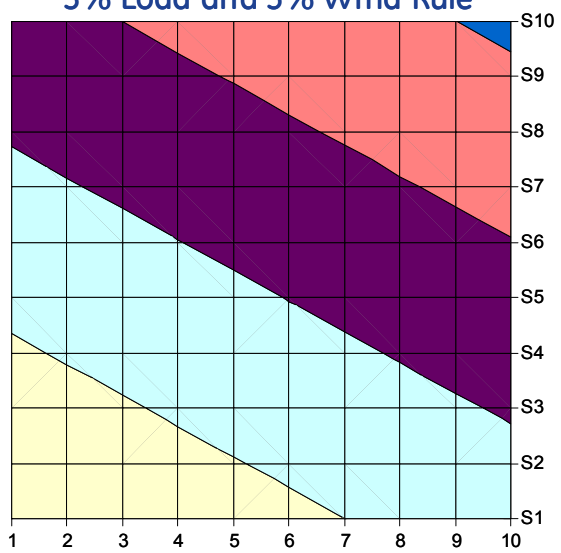

$3+5$ Rule Violations

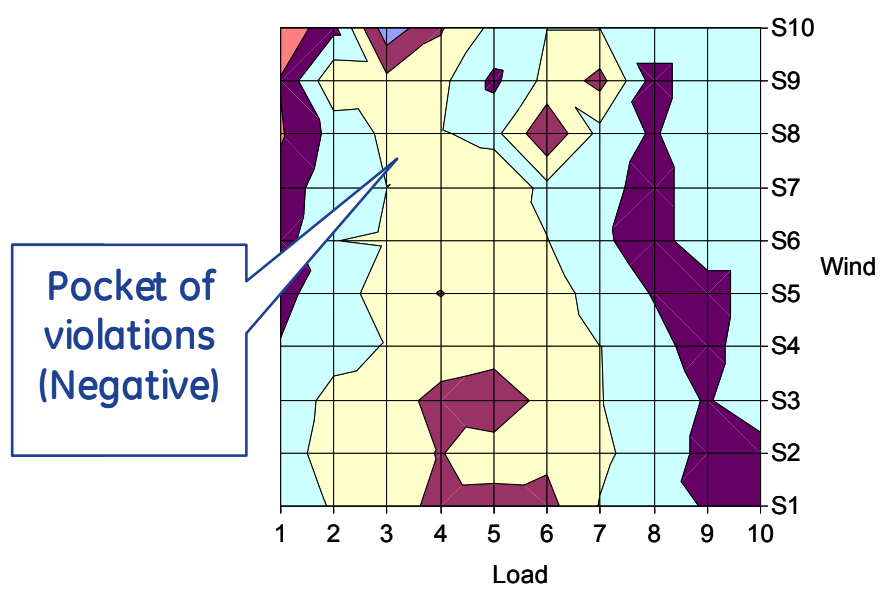

Figure G- 6 New Mexico L30R 3\% Load Plus 5\% Wind Rule 
Colorado West - The 3+5 rule performance for Colorado West is shown in Figure G- 7 . The reserves here are almost entirely inadequate across the entire load and wind space.

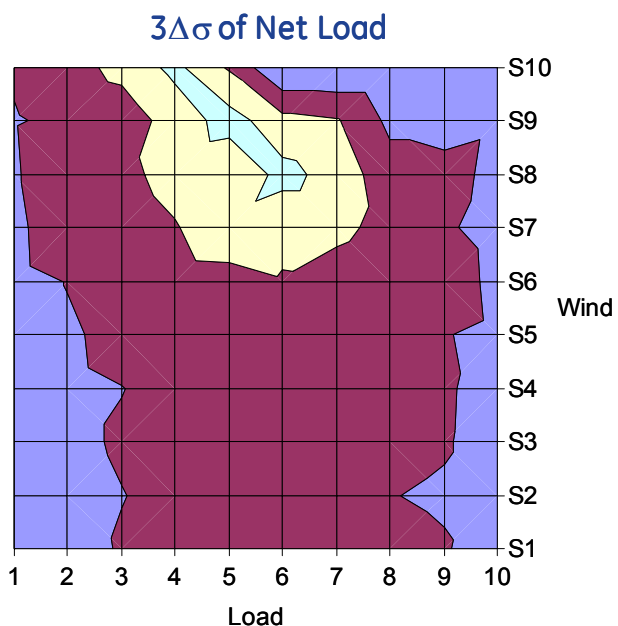

$3 \%$ Load and $5 \%$ Wind Rule

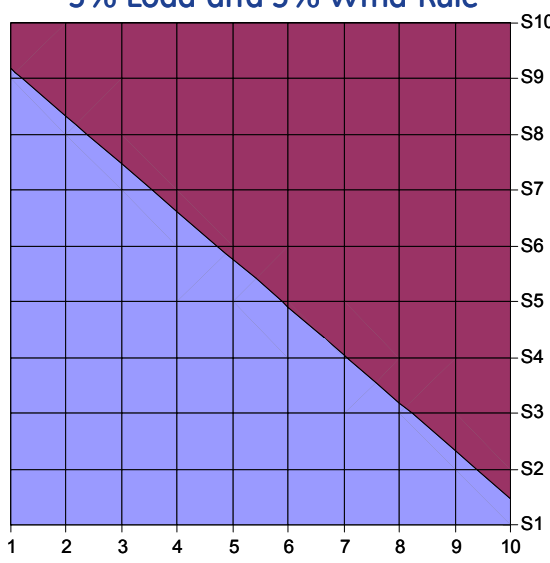

3+5 Rule Violations

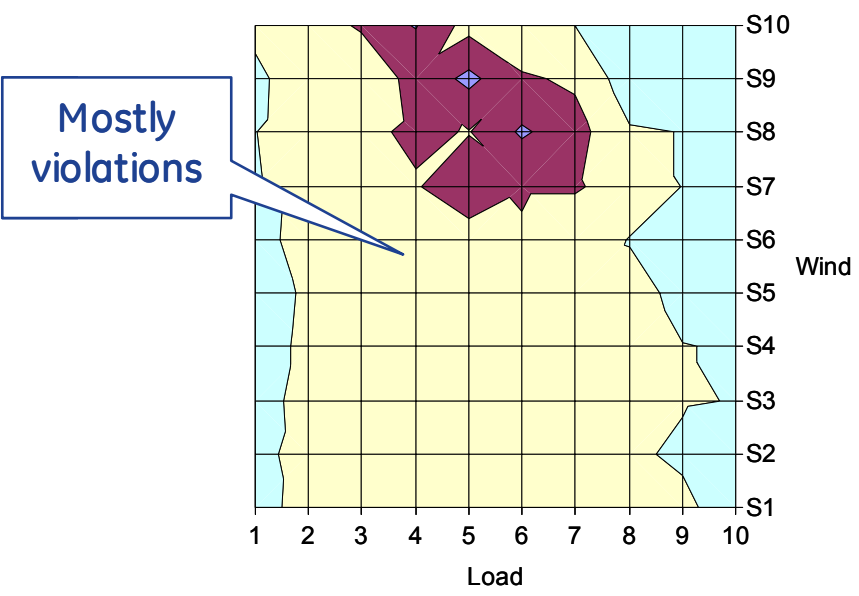

Figure G- 7 Colorado West L30R 3\% Load Plus 5\% Wind Rule 


\section{G.2 General Wind + Load Rules}

This appendix provides additional supporting graphics and discussion for the material presented in Section 7.4.3.

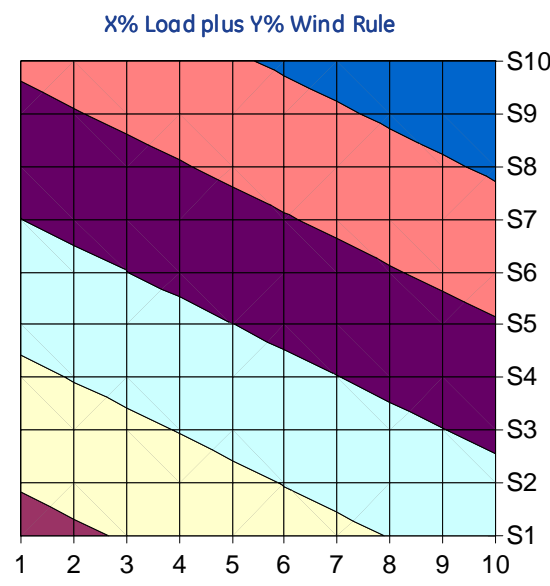

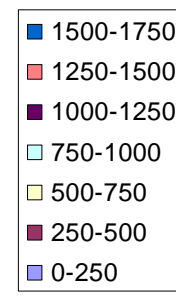

$X+Y$ rule RMS best fit: $1.3 \%$ Load $3.6 \%$ Wind

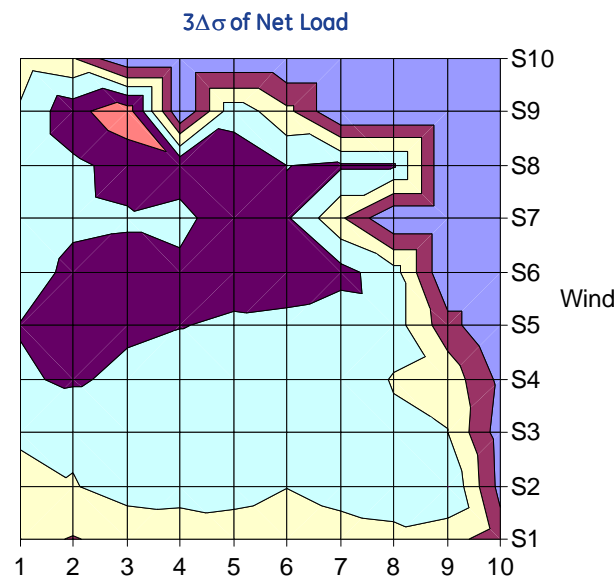

Figure G- 8 In Footprint L30R Best RMS Fit X\% Load Plus Y\% Wind Rule

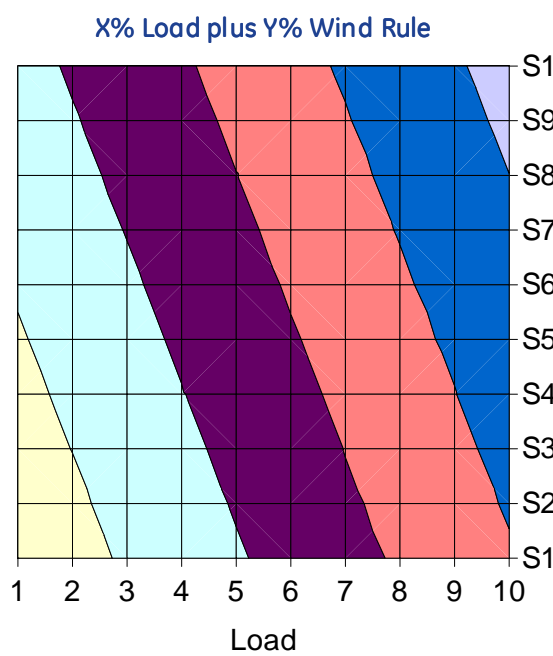

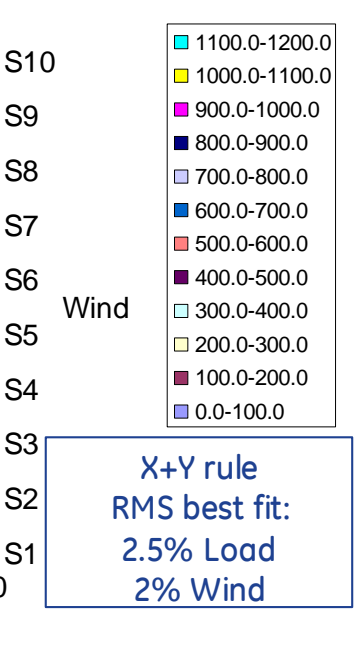

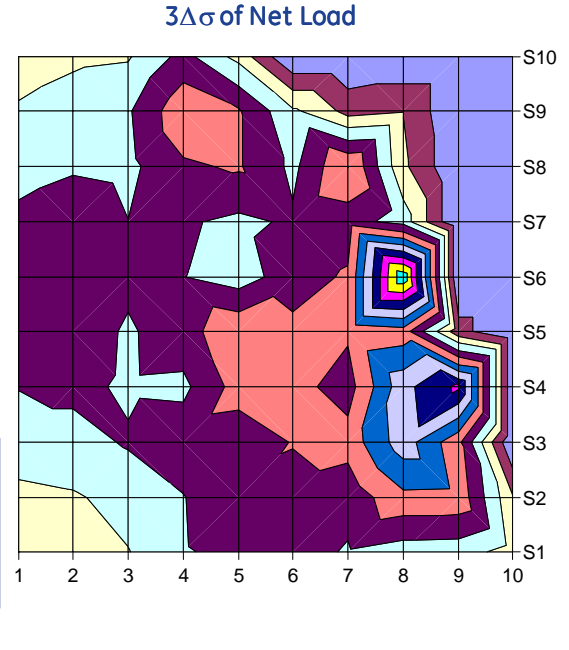

Figure G- 9 Arizona L30R Best RMS Fit X\% Load Plus Y\% Wind Rule 


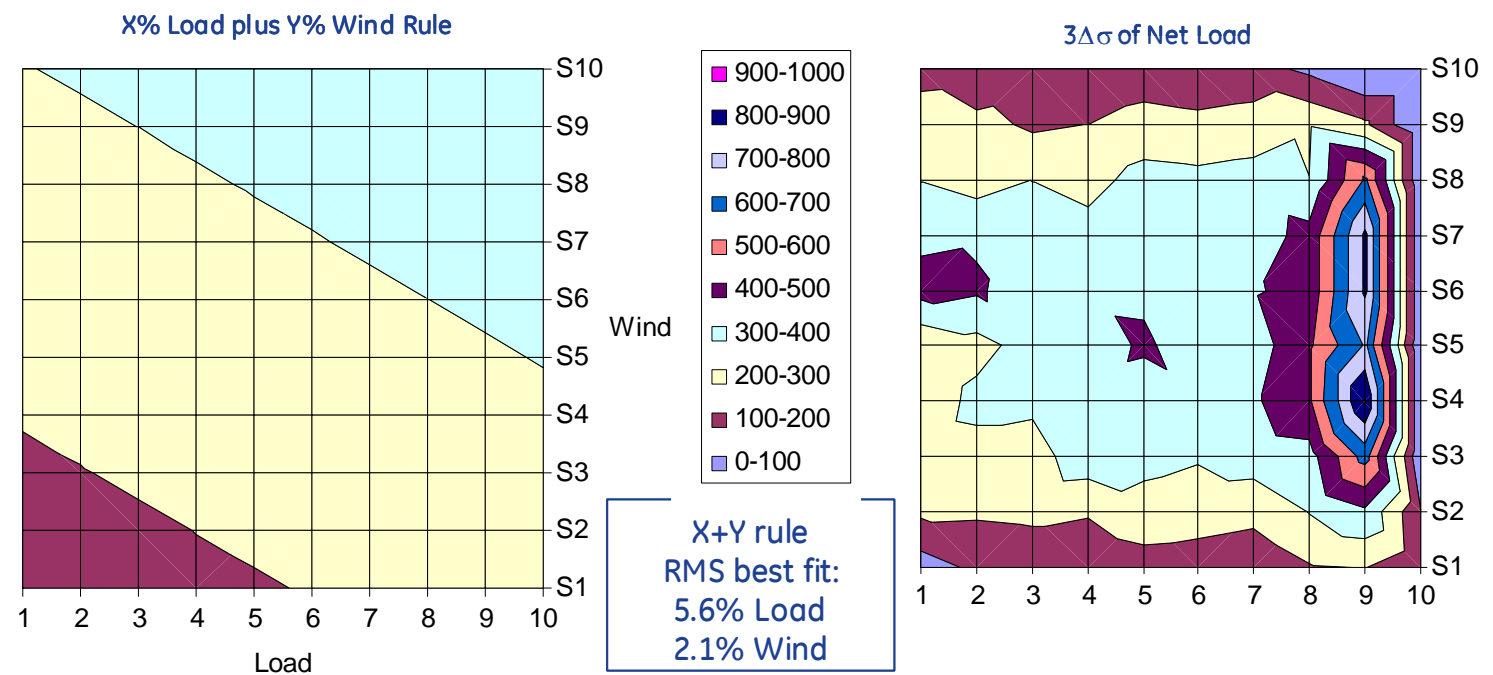

Figure G- 10 Wyoming L30R Best RMS Fit X\% Load Plus Y\% Wind Rule

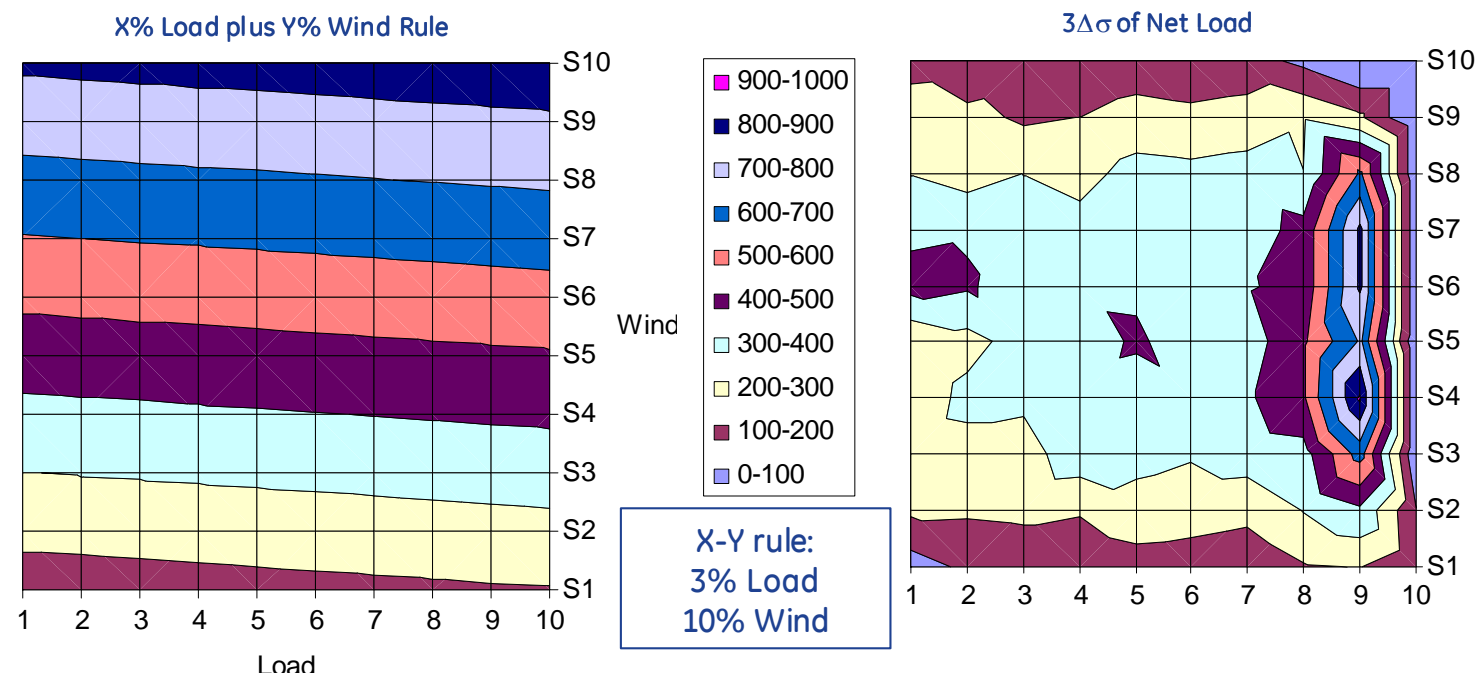

Figure G- 11 Wyoming L30R Heuristic 3\% Load Plus 10\% Wind Rule 

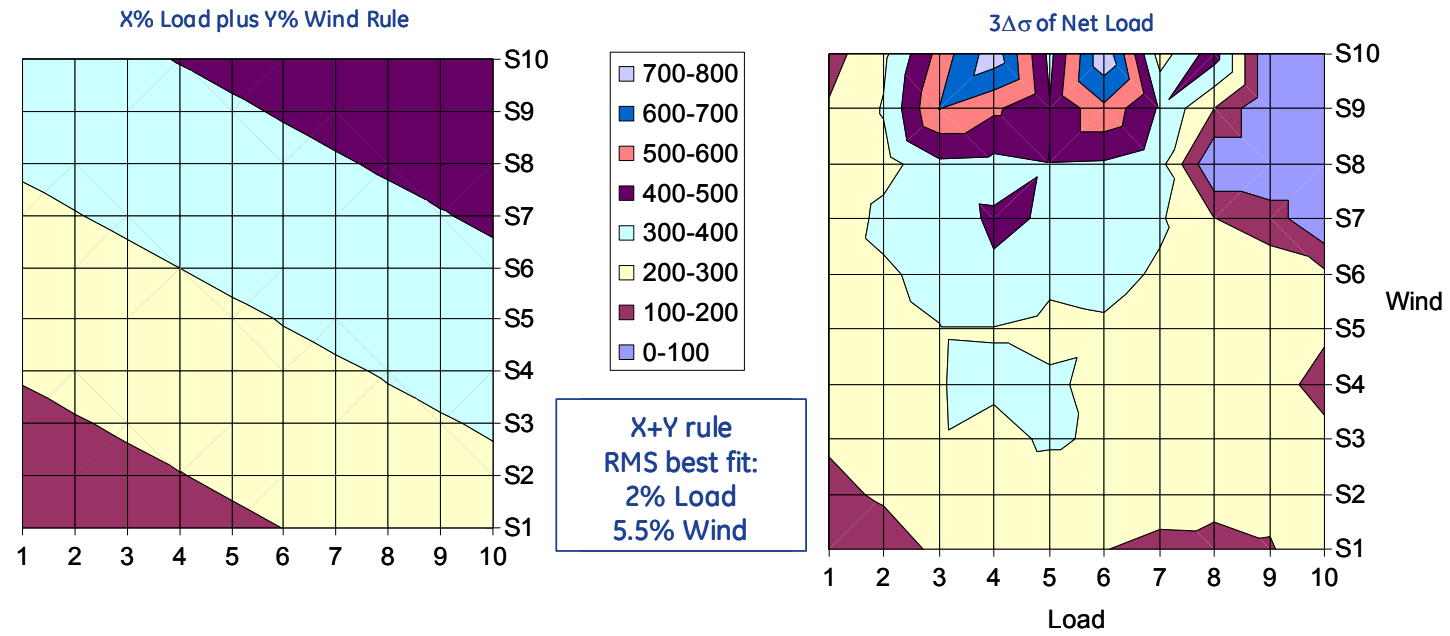

Figure G- 12 Colorado East L30R Best RMS Fit X\% Load Plus Y\% Wind Rule
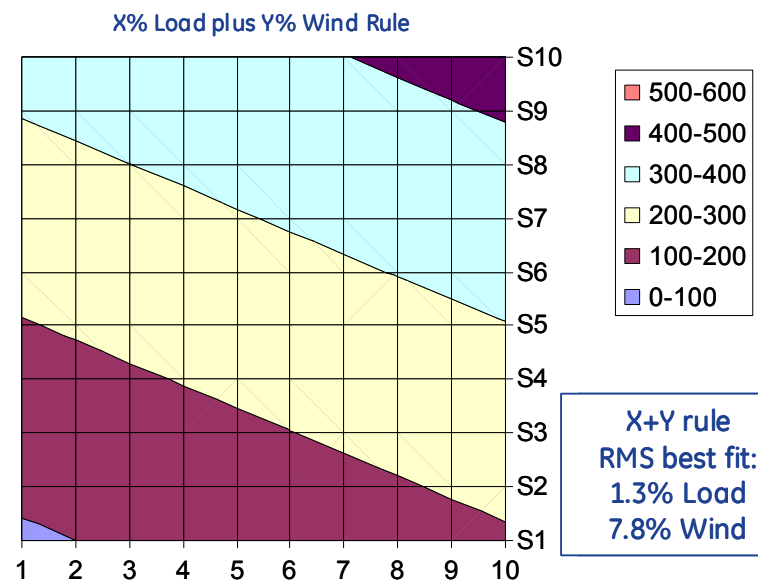

$X+Y$ rule RMS best fit: $1.3 \%$ Load $7.8 \%$ Wind

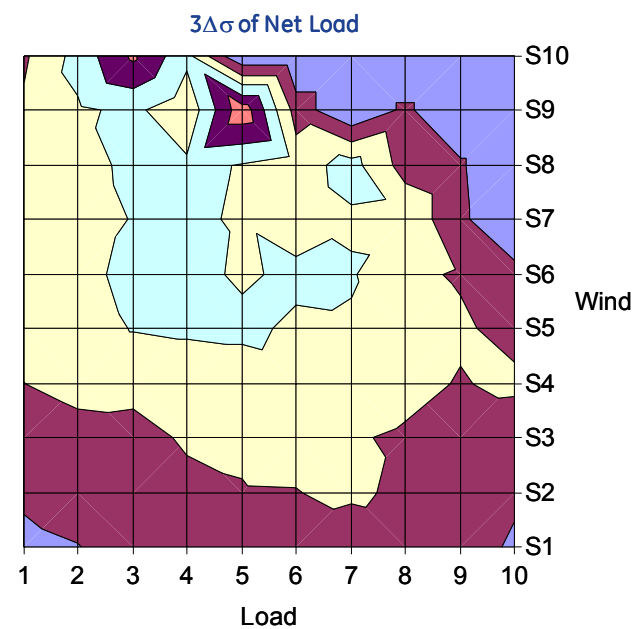

Figure G- 13 Nevada L30R Best RMS Fit X\% Load Plus Y\% Wind Rule
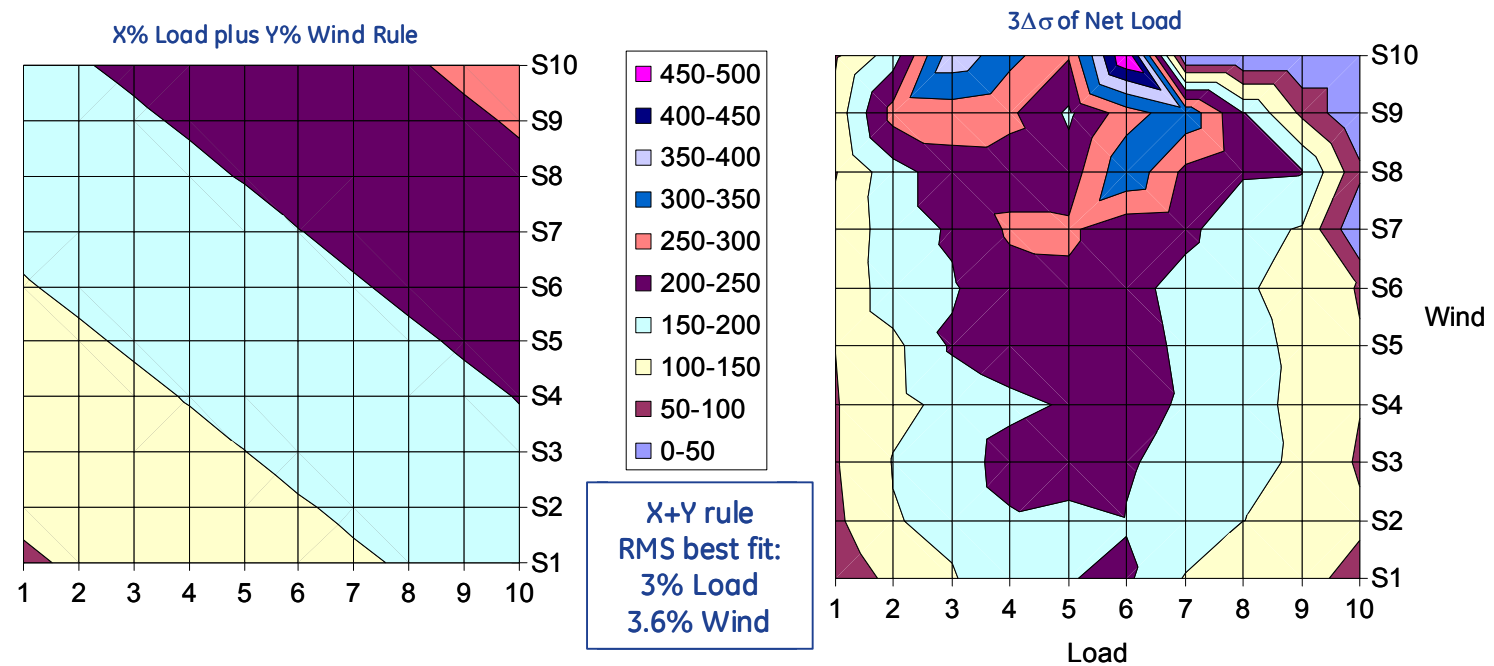

Figure G- 14 New Mexico L30R Best RMS Fit X\% Load Plus Y\% Wind Rule 

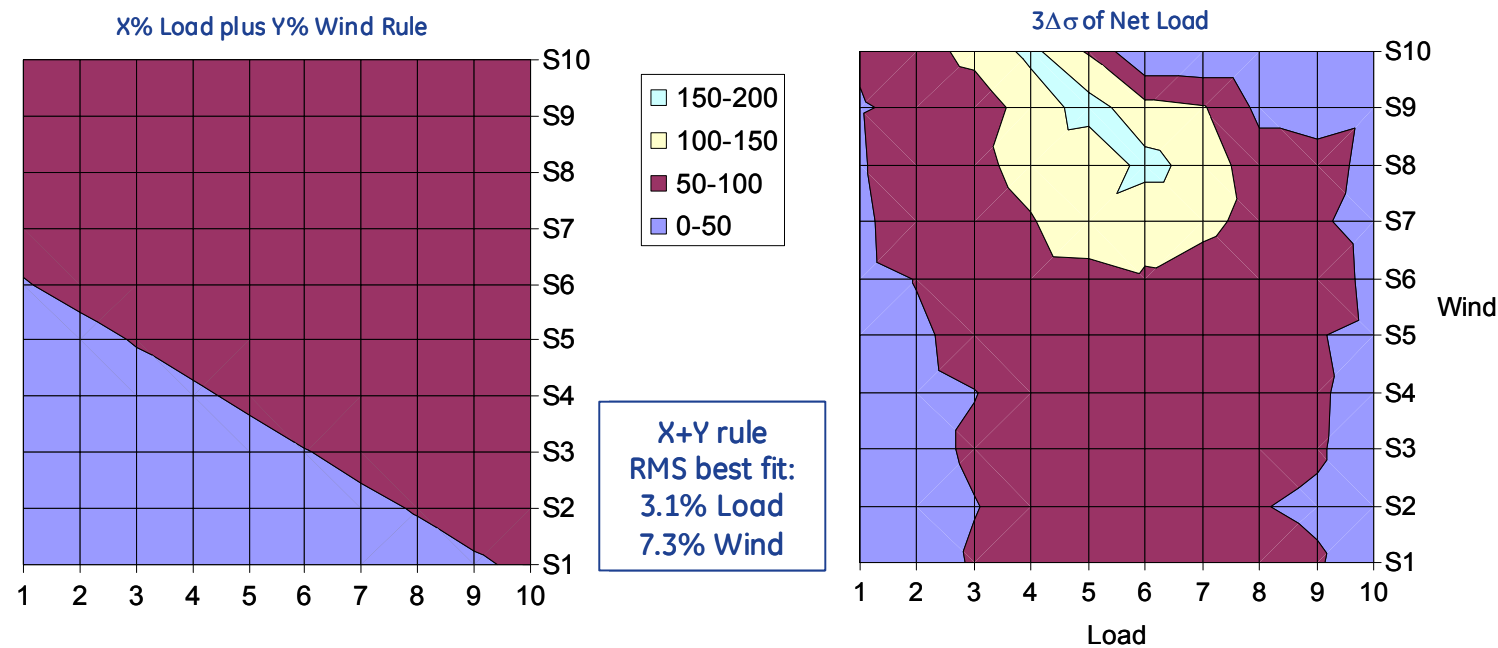

Figure G- 15 Colorado West L30R Best RMS Fit X\% Load Plus Y\% Wind Rule

\section{G.3 Conditional Wind + Load Rules}

This appendix provides additional supporting graphics and discussion for the material presented in Section 7.4.4.
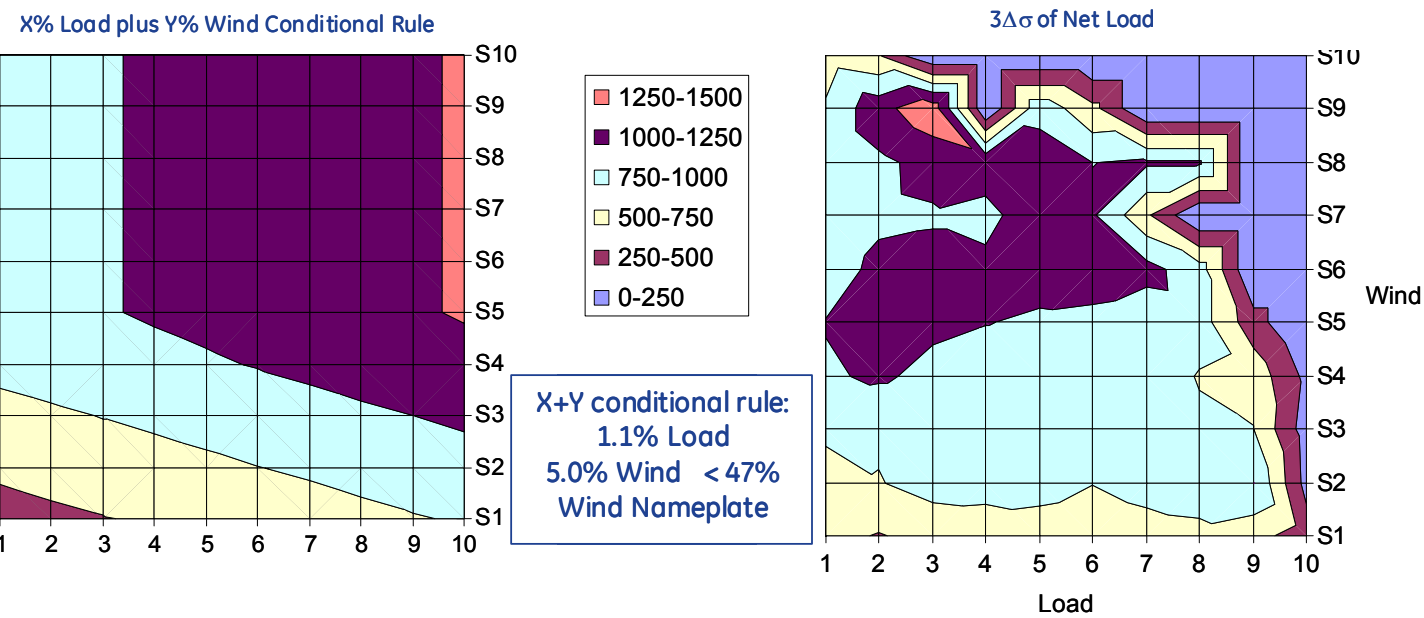

Figure G- 16 In Foot Print L30R X\% Load Plus Y\% Conditional Wind Rule 
X\% Load plus Y\% Wind Conditional Rule

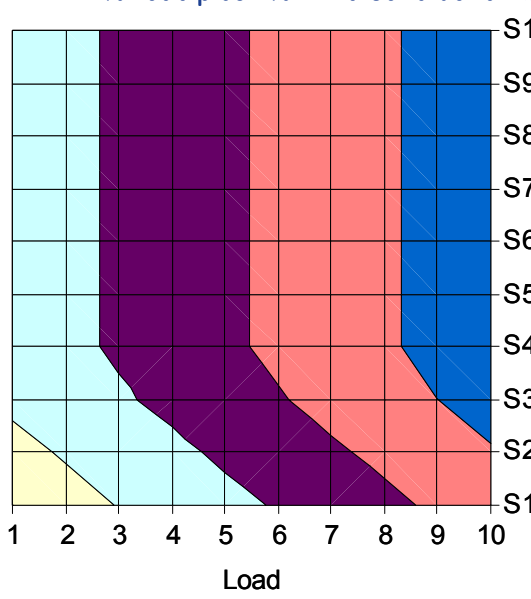

$\square$ 1100.0-1200.0

$\square$ 1000.0-1100.0

$\square$ 900.0-1000.0

- 800.0-900.0

$\square 700.0-800.0$

$\square 600.0-700.0$

$\square 500.0-600.0$

400.0-500.0

$\square 300.0-400.0$

$\square 200.0-300.0$

$\square$ 100.0-200.0

$\square 0.0-100.0$

$X-Y$ conditional rule:

$2.2 \%$ Load

$5.6 \%$ Wind $<36 \%$

Wind Nameplate

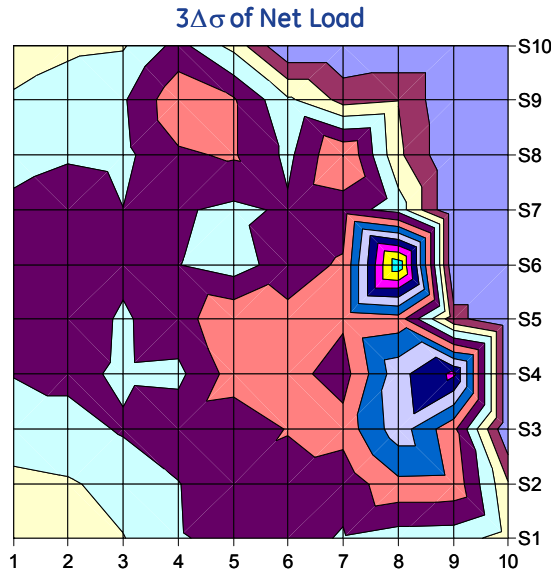

Figure G- 17 Arizona L30R X\% Load Plus Y\% Conditional Wind Rule

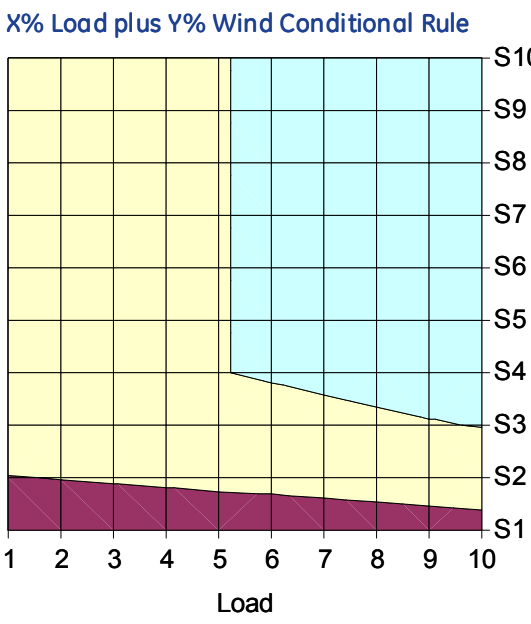

\begin{tabular}{|c|c|}
\hline & $\square 900-1000$ \\
\hline & - 800-900 \\
\hline & $\square 700-800$ \\
\hline & $\square 600-700$ \\
\hline & $\square 500-600$ \\
\hline & $\square 400-500$ \\
\hline Nin & $\square 300-400$ \\
\hline & $\square$ 200-300 \\
\hline & $\square$ 100-200 \\
\hline & $\square$ 0-100 \\
\hline & $\begin{array}{l}\text { conditionc } \\
2.2 \% \text { Loa }\end{array}$ \\
\hline & $\begin{array}{l}\% \text { Wind }< \\
\text { ind Namep }\end{array}$ \\
\hline
\end{tabular}

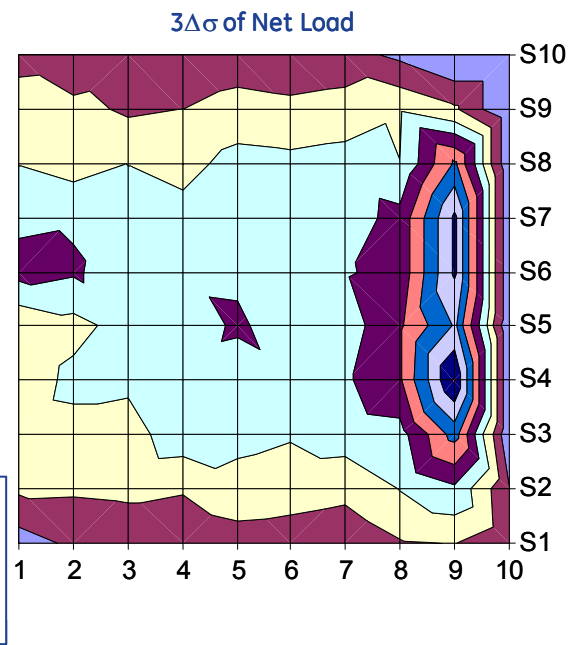

Figure G- 18 Wyoming L30R X\% Load Plus Y\% Conditional Wind Rule

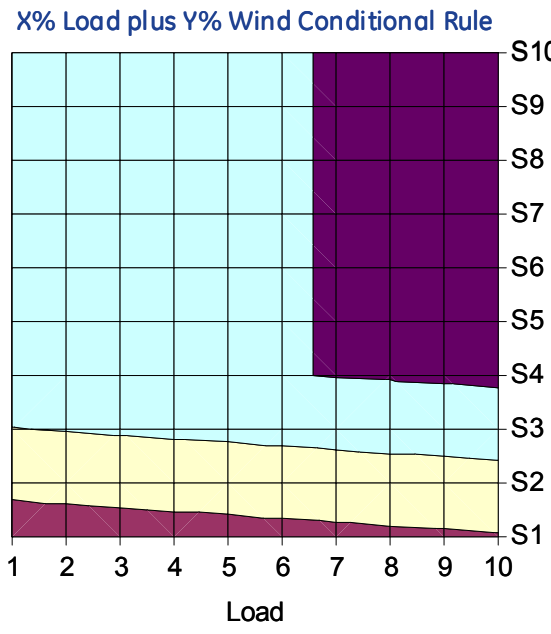

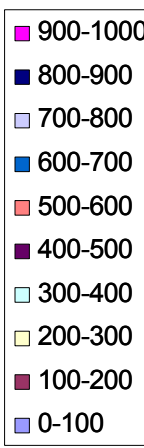

$X+Y$ conditional rule: $3 \%$ Load $10 \%$ Wind $<40 \%$ Wind Nameplate

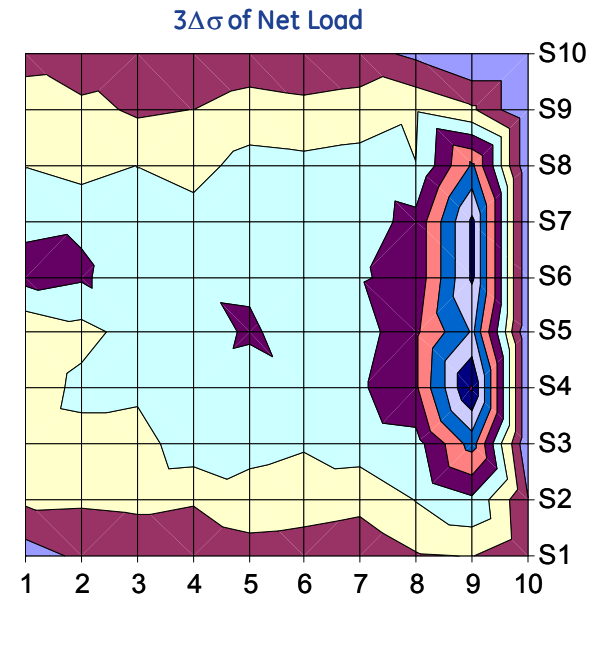

$3 \Delta \sigma$ of Net Load

Figure G- 19 Wyoming L30R X\% Load Plus Y\% Conditional Wind Rule, Low Power Priority 


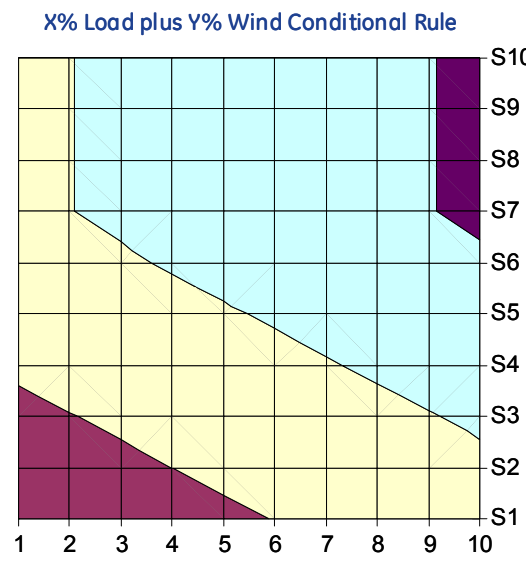

$X+Y$ conditional rule: $2.0 \%$ Load $5.7 \%$ Wind $<68 \%$ Wind Nameplate

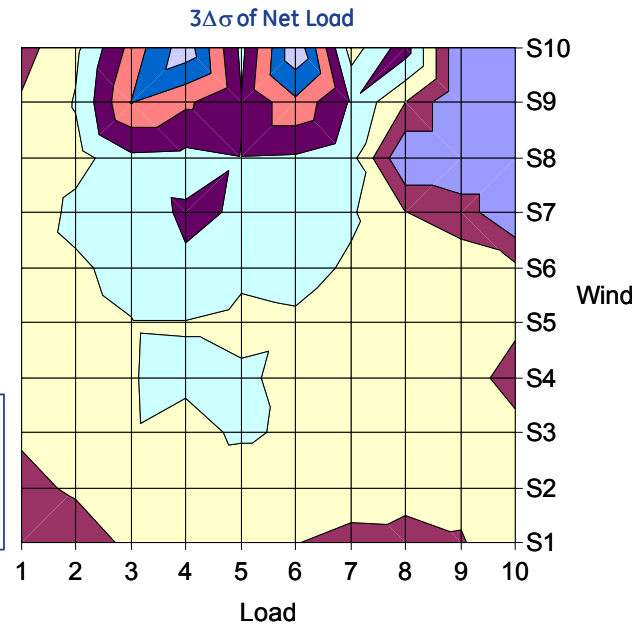

Figure G- 20 Colorado East L30R X\% Load Plus Y\% Conditional Wind Rule

X\% Load plus Y\% Wind Conditional Rule

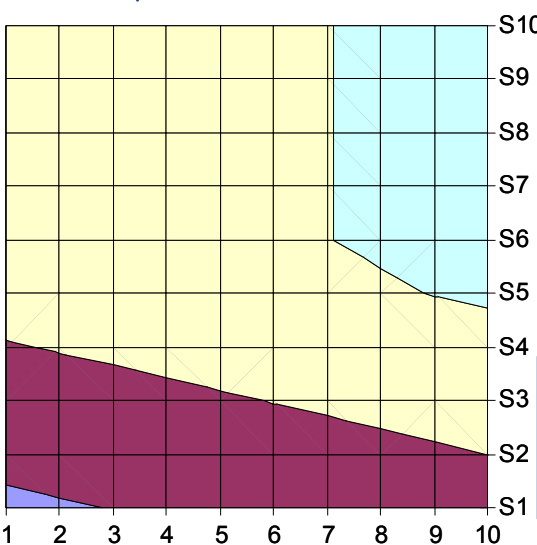

$\square 500-600$

- 400-500

$\square 300-400$

$\square 200-300$

$\square$ 100-200

$\square 0-100$

$X+Y$ conditional rule: $1.0 \%$ Load $10.7 \%$ Wind $<54 \%$ Wind Nameplate

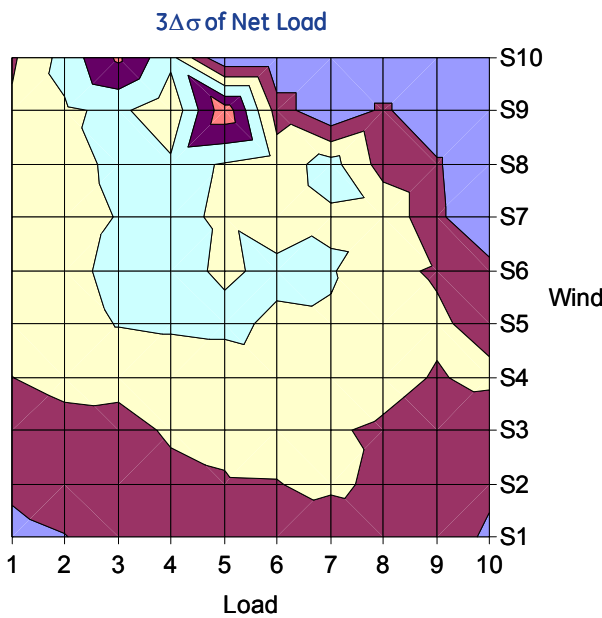

Figure G- 21 Nevada L30R X\% Load Plus Y\% Conditional Wind Rule

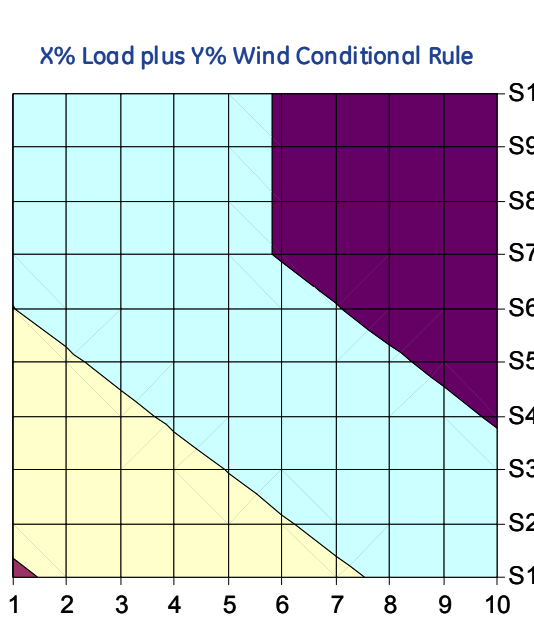

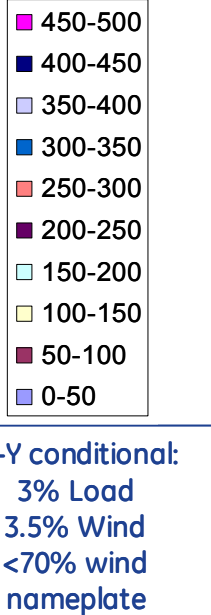

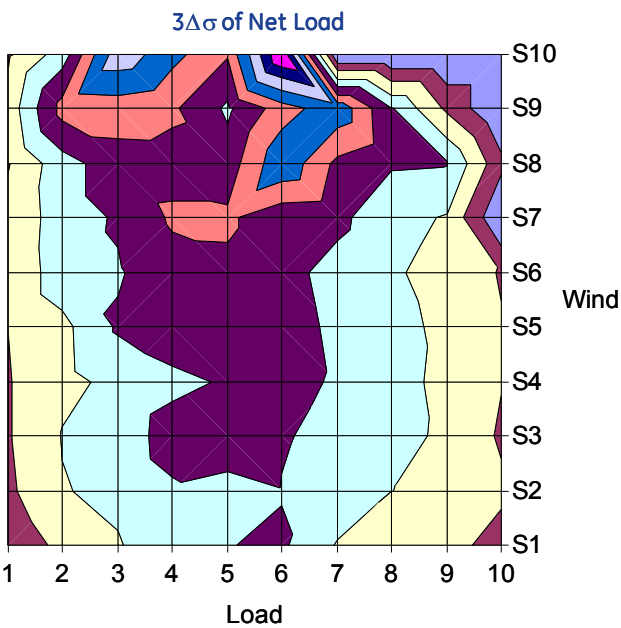

Figure G- 22 New Mexico L30R X\% Load Plus Y\% Conditional Wind Rule 
X\% Peak Load plus Y\% Wind Conditional Rule
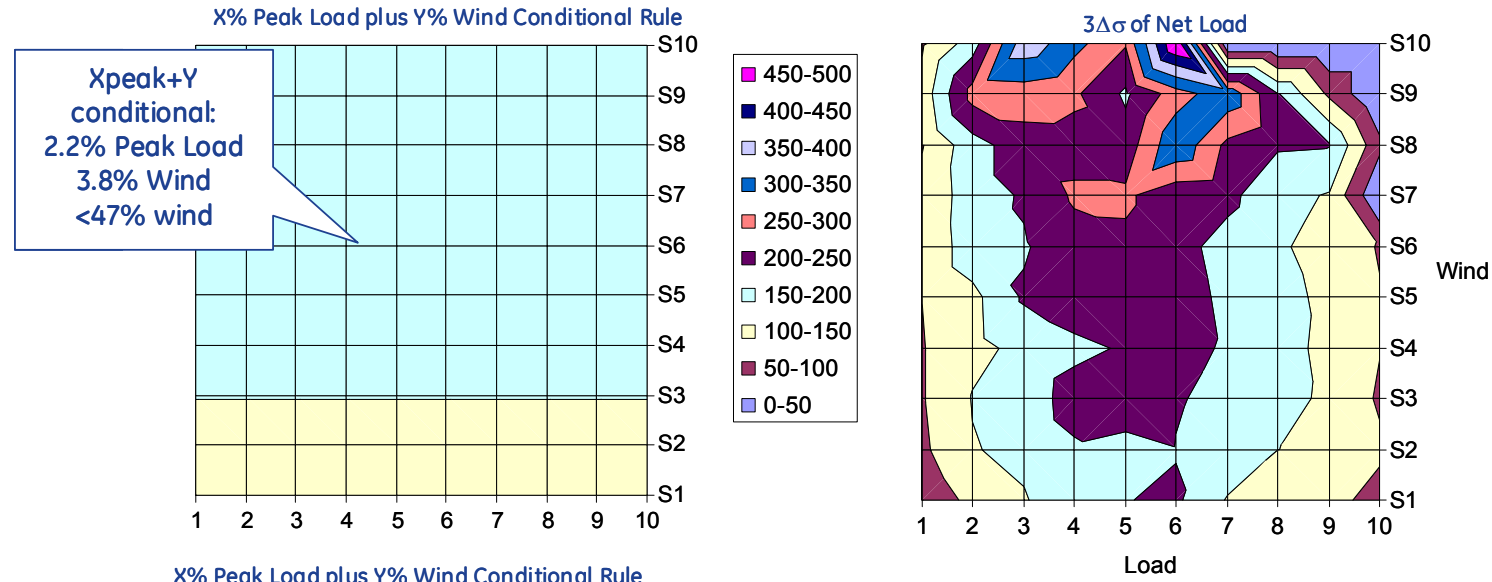

X\% Peak Load plus Y\% Wind Conditional Rule
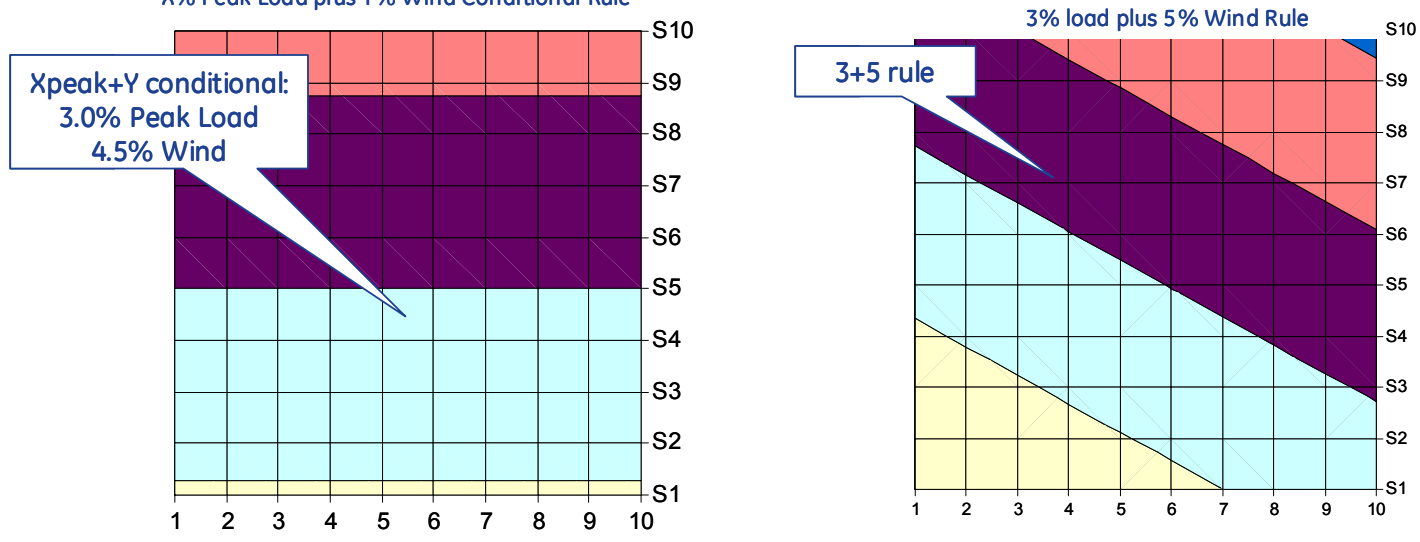

Figure G- 23 New Mexico L30R Other Heuristics on X+Y Conditional Rule

\section{G.4 Load Only Rules}

This appendix provides additional supporting graphics and discussion for the material presented in Section 7.4.5.
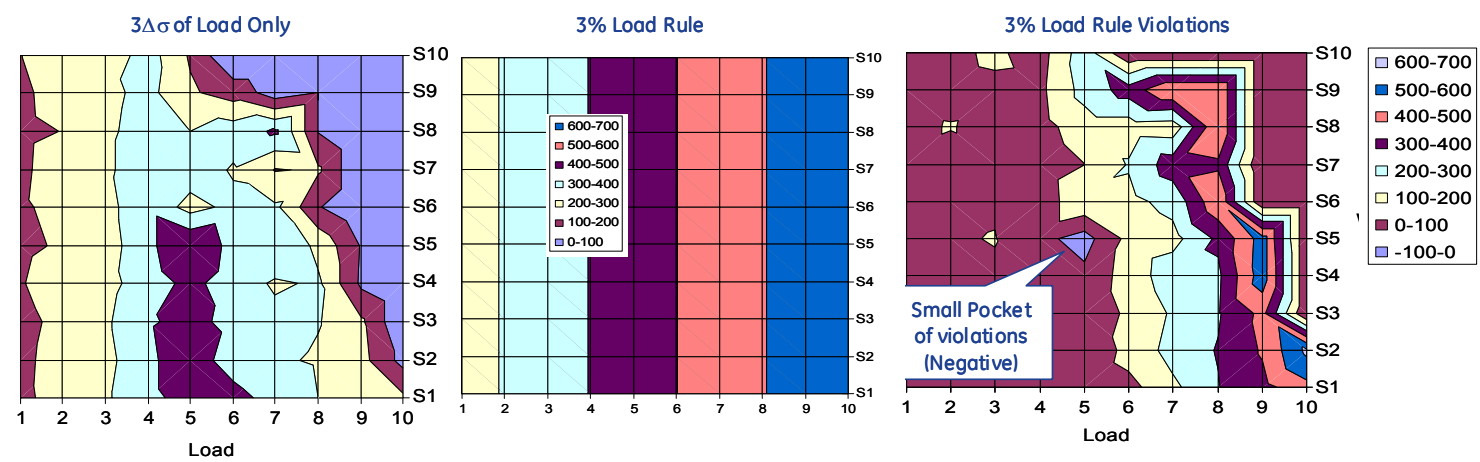

Figure G- 24 Arizona L30r Load-Only Variability 
$3 \Delta \sigma$ of Load Only

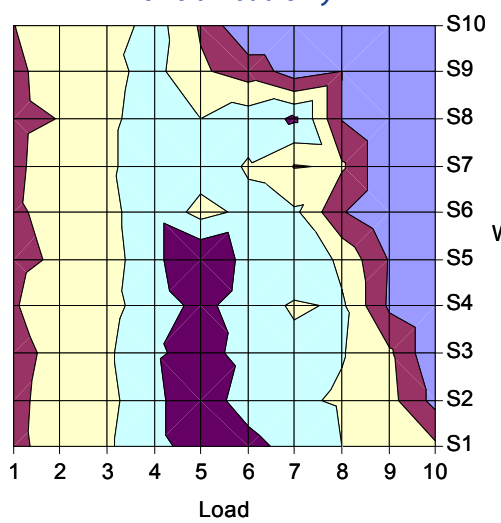

Load

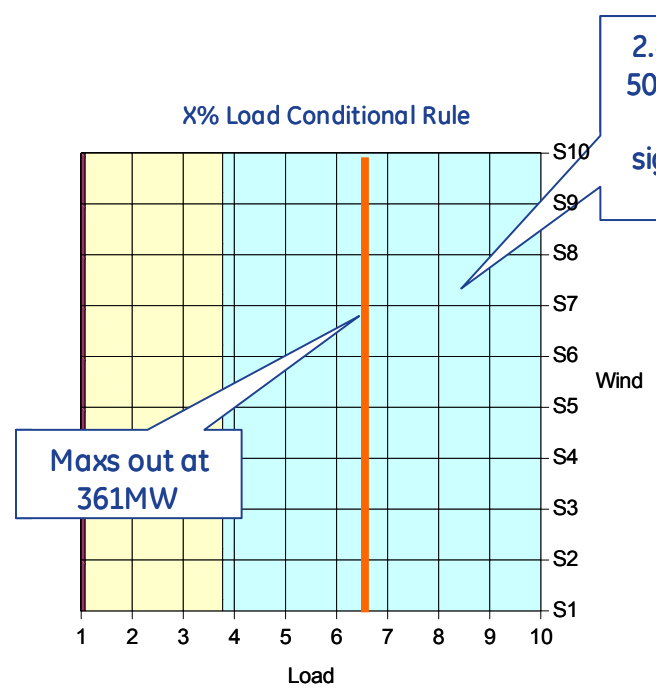

Figure G- 25 Arizona L30r Scenario Load-Only Variability ad Only Best Fit

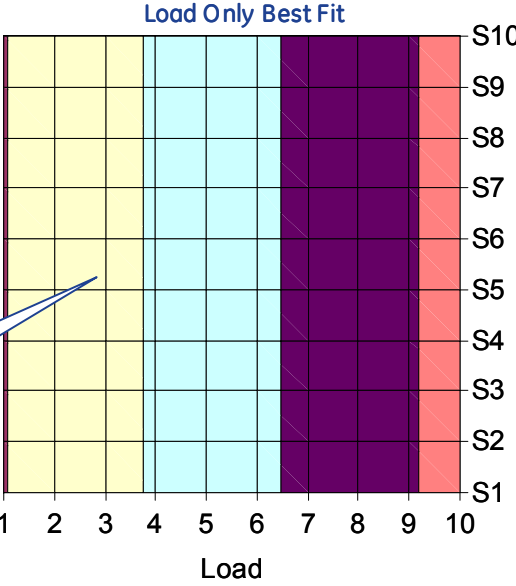

$.4 \%$ conditional to $0 \%$ of load range is best RMS fit to 3 sigma delta rule for load only

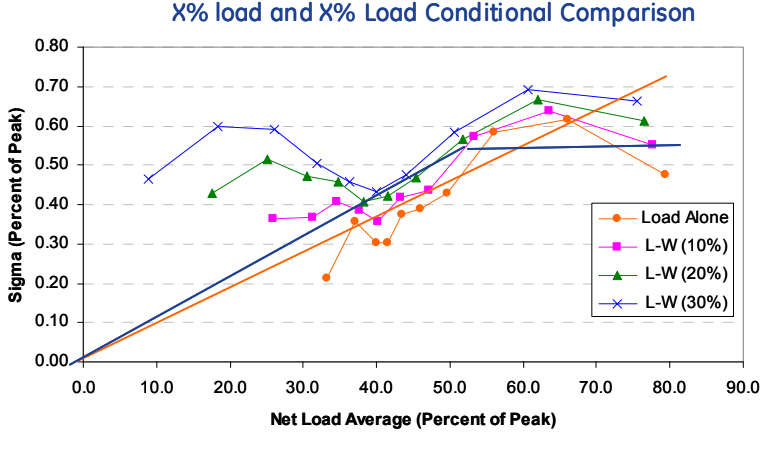



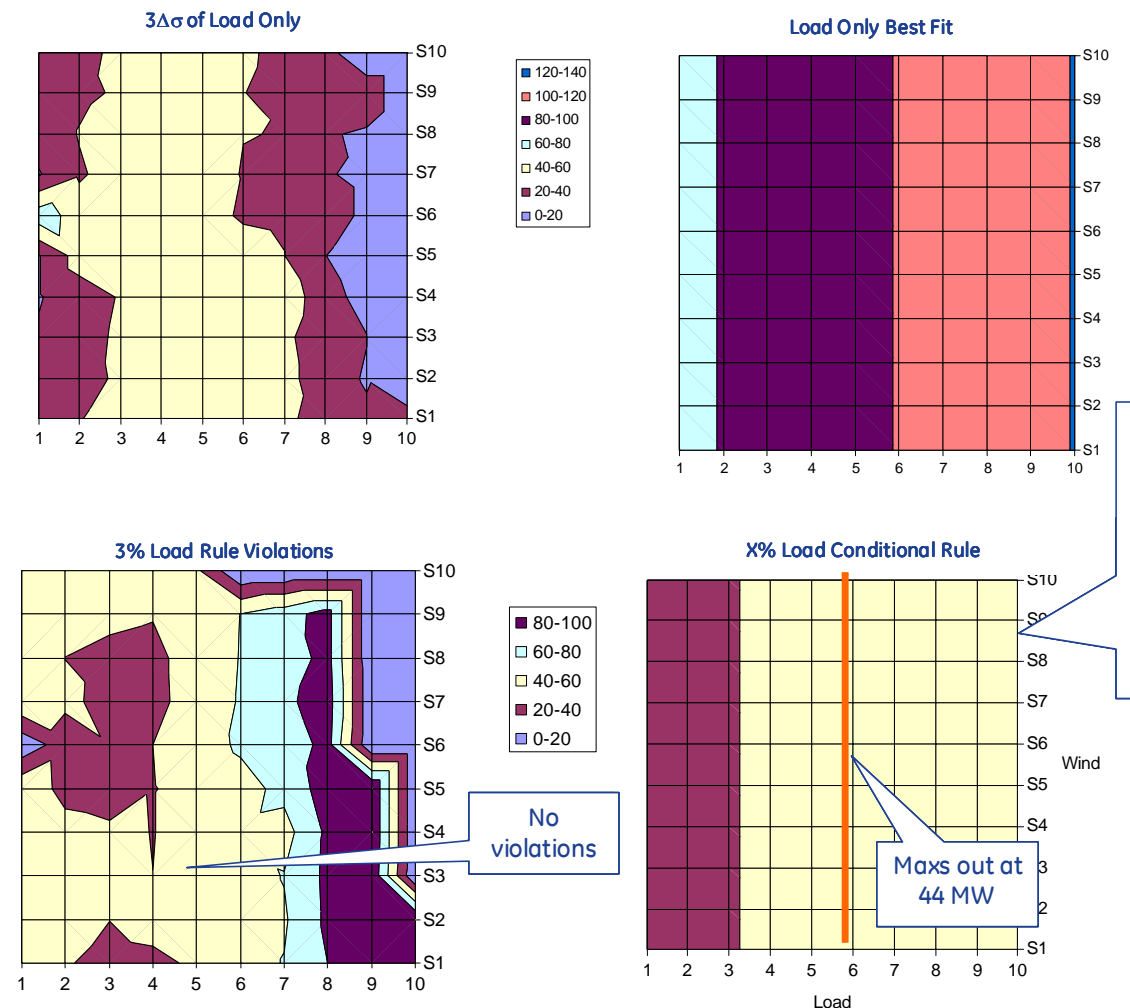

WY
$1.4 \%$
conditional to
$50 \%$ of load
range is best
RMS fit to 3
sigma delta
rule for load
only

Figure G- 26 Wyoming L30r Scenario Load-Only Variability
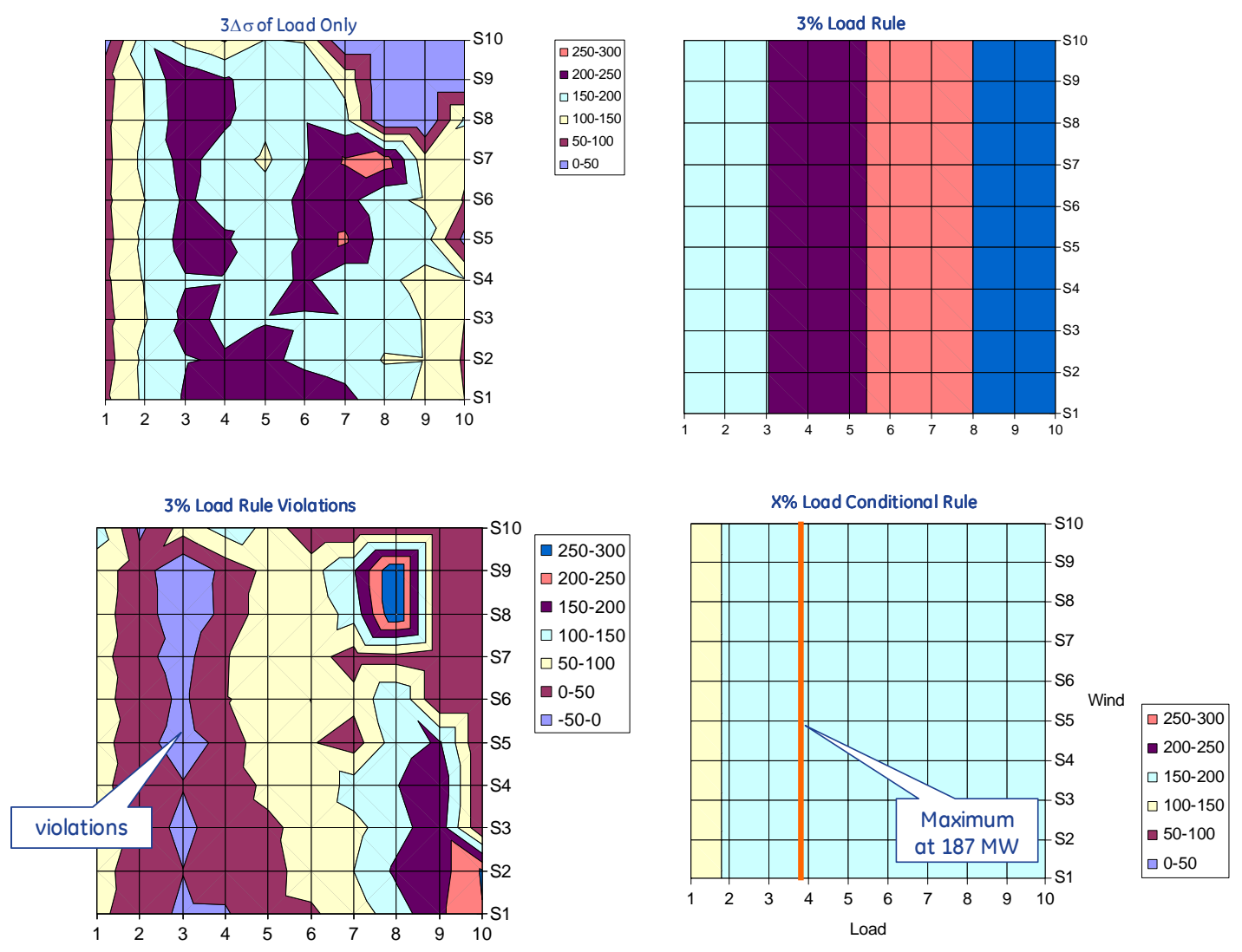

Figure G- 27 Colorado East L30r Scenario Load-Only Variability 
$3 \Delta \sigma$ of Load Only

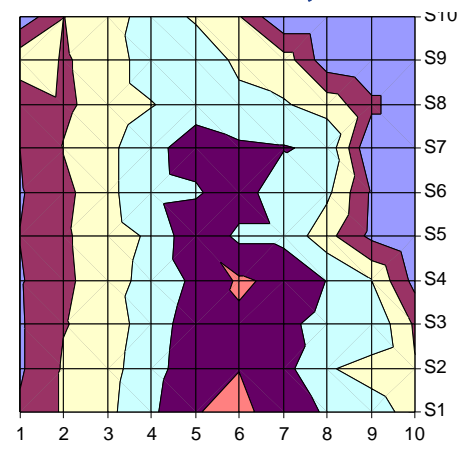

$3 \%$ Load Rule Violations

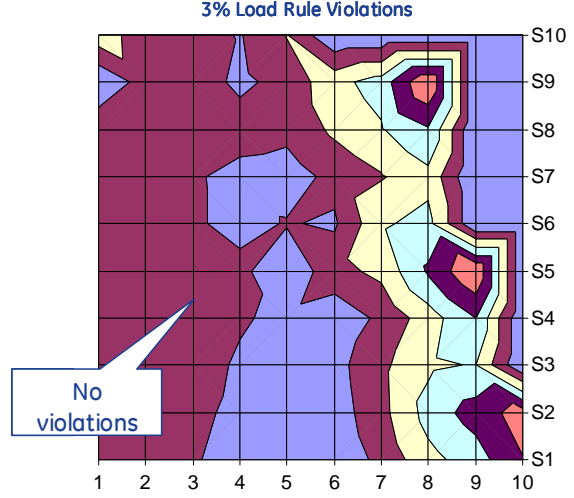

3\% Load Rule
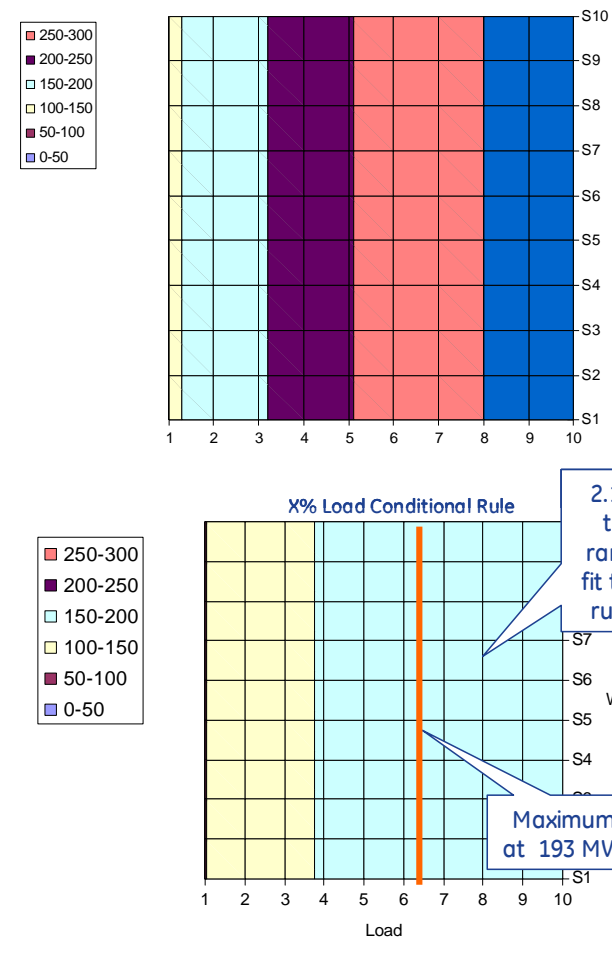

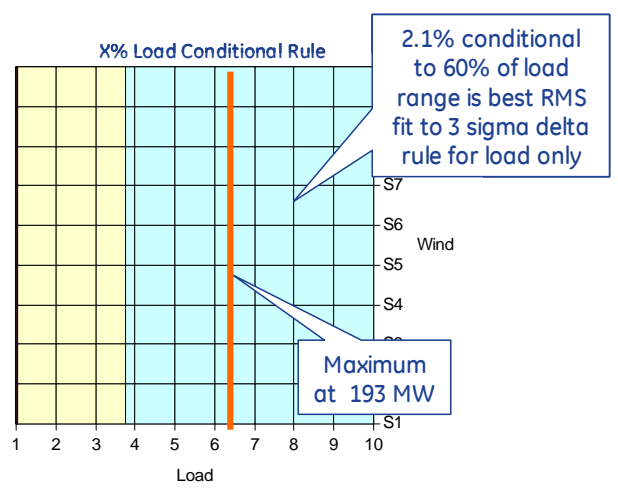

Figure G- 28 Nevada L30r Scenario Load-Only Variability

$3 \Delta \sigma$ of Load Only

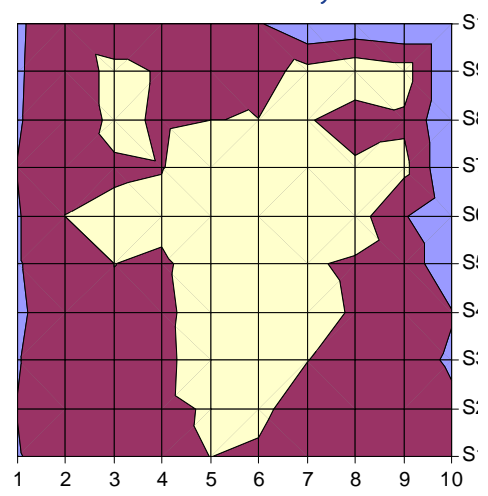

$3 \%$ Load Rule Violations

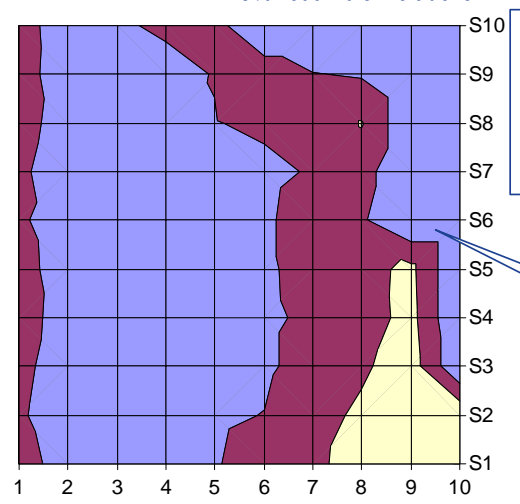

Figure G-29 New Mexico L30r Scenario Load-Only Variability

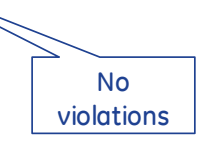

s\% Load kule
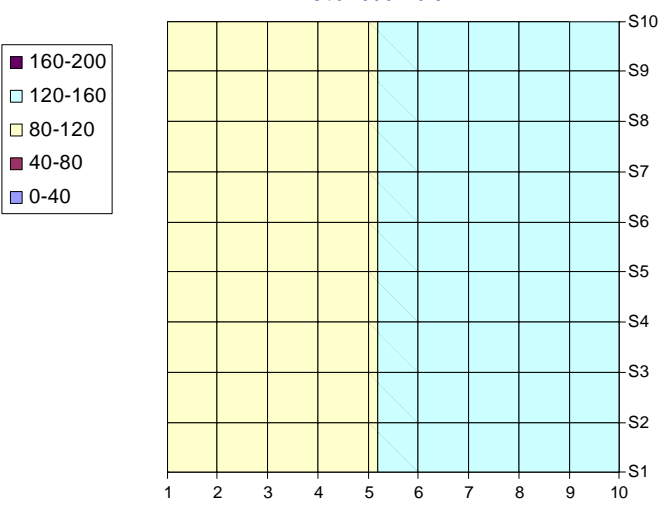

X\% Load Conditional Rule

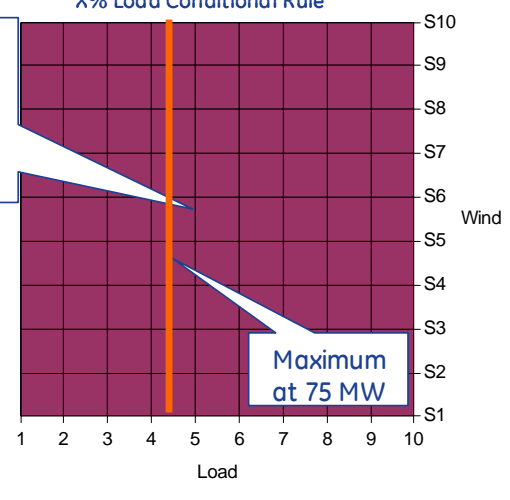

$2.1 \%$ conditiona to $43 \%$ of load range is best RMS fit to 3 sigma delta rule for load only

No
violations 

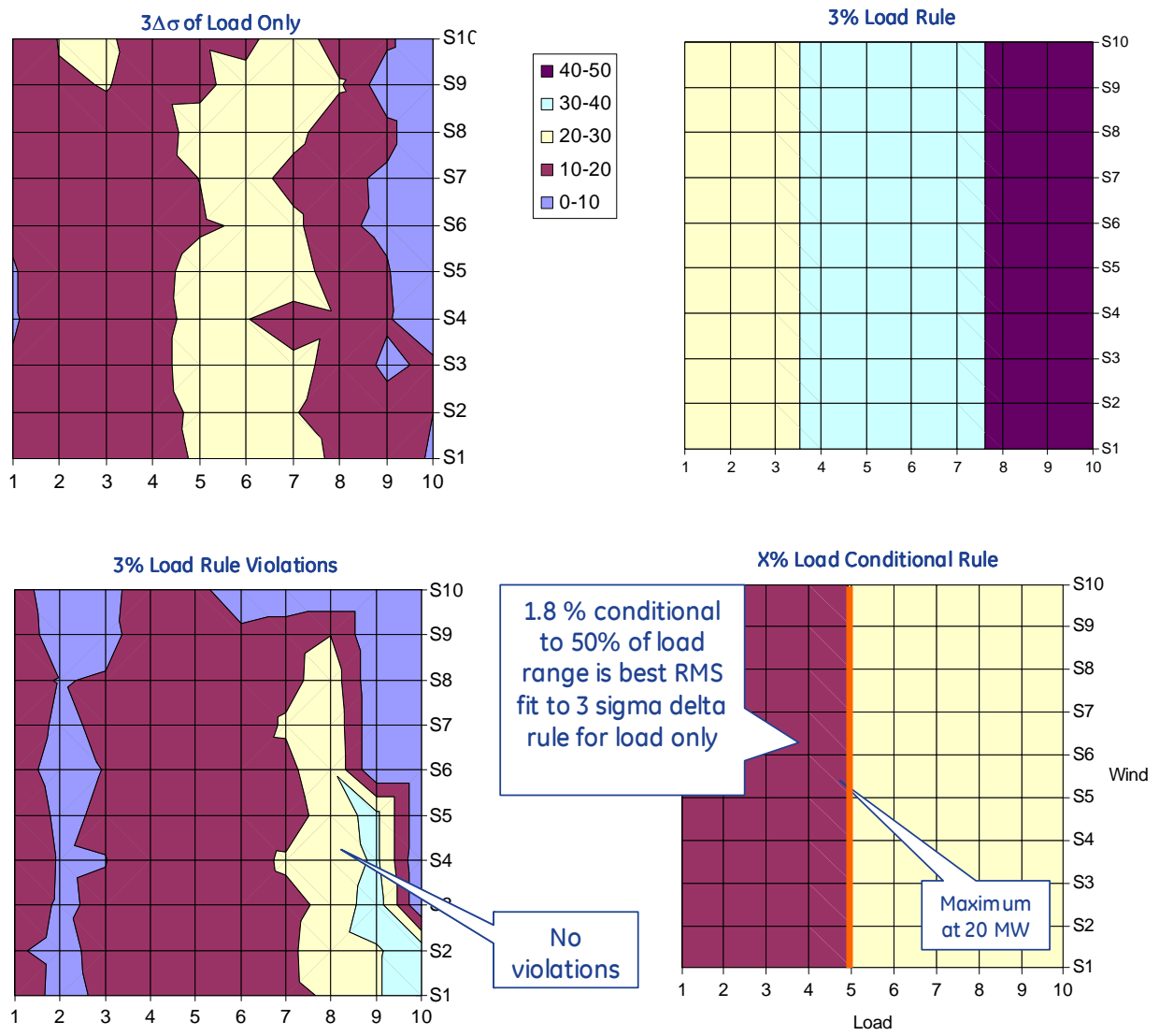

Figure G- 30 Colorado West L30r Scenario Load-Only Variability 


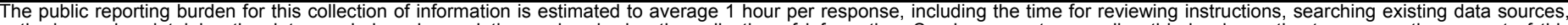

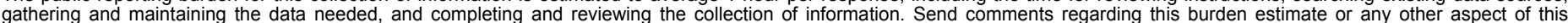

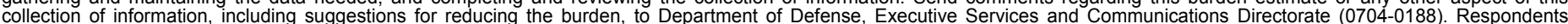

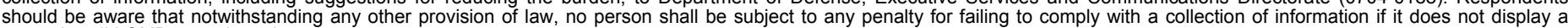

should be aware that notwithstanding

PLEASE DO NOT RETURN YOUR FORM TO THE ABOVE ORGANIZATION.

\begin{tabular}{l|l|l|l} 
1. REPORT DATE $(D D-M M-Y Y Y Y)$ & 2. & REPORT TYPE & 3. DATES COVERED (FrOm - TO)
\end{tabular}

May 2010

Subcontract Report

4. TITLE AND SUBTITLE

Western Wind and Solar Integration Study

5a. CONTRACT NUMBER

DE-AC36-08-GO28308

5b. GRANT NUMBER

5c. PROGRAM ELEMENT NUMBER

6. AUTHOR(S)

GE Energy

5d. PROJECT NUMBER

NREL/SR-550-47434

5e. TASK NUMBER

WW10.4311

5f. WORK UNIT NUMBER
7. PERFORMING ORGANIZATION NAME(S) AND ADDRESS(ES)

GE Energy

1 River Road

Schenectady, New York 12345

9. SPONSORING/MONITORING AGENCY NAME(S) AND ADDRESS(ES)

National Renewable Energy Laboratory

1617 Cole Blvd.

Golden, CO 80401-3393
8. PERFORMING ORGANIZATION REPORT NUMBER

AAM-8-77557-01

12. DISTRIBUTION AVAILABILITY STATEMENT

National Technical Information Service

U.S. Department of Commerce

5285 Port Royal Road

Springfield, VA 22161

13. SUPPLEMENTARY NOTES

NREL Technical Monitor: Debra Lew

14. ABSTRACT (Maximum 200 Words)

This report provides a full description of the Western Wind and Solar Integration Study (WWSIS) and its findings.

\section{SUBJECT TERMS}

WWSIS; GE Energy; WECC; grid integration; WestConnect; wind; solar; CSP; PV; photvoltaics; integration study; utilities; power industry; renewables.

\begin{tabular}{|c|c|c|}
\hline \multicolumn{3}{|c|}{ 16. SECURITY CLASSIFICATION OF: } \\
\hline $\begin{array}{l}\text { a. REPORT } \\
\text { Unclassified }\end{array}$ & $\begin{array}{l}\text { b. ABSTRACT } \\
\text { Unclassified }\end{array}$ & $\begin{array}{l}\text { c. THIS PAGE } \\
\text { Unclassified }\end{array}$ \\
\hline
\end{tabular}

\begin{tabular}{|c|c|}
\hline $\begin{array}{l}\text { 17. LIMITATION } \\
\text { OF ABSTRACT }\end{array}$ & $\begin{array}{ll}\text { 18. } & \text { NUMBER } \\
\text { OF PAGES }\end{array}$ \\
\hline UL & \\
\hline
\end{tabular}

19a. NAME OF RESPONSIBLE PERSON

19b. TELEPHONE NUMBER (Include area code) 